\title{
Data Recovery at Lake Alan Henry (Justiceburg Reservior), Garza and Kent Counties, Texas: Phase III, Season 3
}

Douglas K. Boyd

Prewitt and Associates, Inc.

Jay Peck

Prewitt and Associates, Inc.

Steve A. Tomka

Raba Kistner

Karl W. Kibler

Cross Timbers Geoarcheological Services

Martha Doty Freeman

Prewitt and Associates, Inc.

Follow this and additional works at: https://scholarworks.sfasu.edu/ita

Part of the American Material Culture Commons, Archaeological Anthropology Commons, Environmental Studies Commons, Other American Studies Commons, Other Arts and Humanities Commons, Other History of Art, Architecture, and Archaeology Commons, and the United States History Commons

Tell us how this article helped you.

This Article is brought to you for free and open access by the Center for Regional Heritage Research at SFA ScholarWorks. It has been accepted for inclusion in Index of Texas Archaeology: Open Access Gray Literature from the Lone Star State by an authorized editor of SFA ScholarWorks. For more information, please contact cdsscholarworks@sfasu.edu. 


\section{Data Recovery at Lake Alan Henry (Justiceburg Reservior), Garza and Kent}

Counties, Texas: Phase III, Season 3

\section{Creative Commons License}

\section{(c) (1) \&}

This work is licensed under a Creative Commons Attribution-NonCommercial 4.0 International License 


\section{DATA RECOVERY AT LAKE ALAN HENRY (JUSTICEBURG RESERVOIR), GARZA AND KENT COUNTIES, TEXAS: \\ PHASE III, SEASON 3}

by

Douglas K. Boyd

Jay Peck

Steve A. Tomka

Karl W. Kibler

and

Martha Doty Freeman

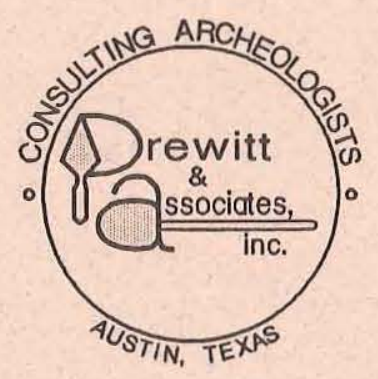

REPORTS OF INVESTIGATIONS, NUMBER 93

TEXAS ANTIQUITIES COMMITTEE PERMIT NUMBER 954 
DATA RECOVERY AT LAKE ALAN HENRY (JUSTICEBURG RESERVOIR), GARZA AND KENT COUNTIES, TEXAS: PHASE III, SEASON 3

by

\author{
Douglas K. Boyd \\ Jay Peck \\ Steve A. Tomka \\ Karl W. Kibler \\ and
}

- Martha Doty Freeman

with Contributions by
Barry W. Baker
J. Philip Dering
Karen M. Gardner
David G. Robinson
Brian S. Shaffer
and
D. Gentry Steele

Co-Principal Investigators: Elton R. Prewitt and Ross C. Fields

REPORTS OF INVESTIGATIONS, NUMBER 93

Prewitt and Associates, Inc.

Consulting Archeologists

Austin, Texas

July 1994

TEXAS ANTIQUITIES COMMITTEE PERMIT NO. 954 
Prewitt and Associates, Inc.

Consulting Archeologists

7701 N. Lamar, Suite 104

Austin, Texas 78752-1012

(512) 459-3349

Lake Allan Henry (Justiceburg Reservoir) Reports

Phase I Cultural Resources Investigations at Justiceburg Reservoir on the Double Mountain Fork of the Brazos River, Garza and Kent Counties, Texas, by Douglas K. Boyd, Martha Doty Freeman, Michael D. Blum, Elton R. Prewitt, and J. Michael Quigg. 2 vols. Reports of Investigations No. 66. 1989 (xiv + 643 pp., maps, illus., appendixes).

Phase II Investigations at Prehistoric and Rock Art Sites, Justiceburg Reservoir, Garza and Kent Counties, Texas, by Douglas K. Boyd, James T. Abbott, William A. Bryan, Colin M. Garvey, Steve A. Tomka, and Ross C. Fields. 2 vols. Reports of Investigations No. 71.1990 (xiv +571 pp., maps, illus., appendixes).

Phase II Historical Investigations at Justiceburg Reservoir, Garza and Kent Counties, Texas, by Martha Doty Freeman and Douglas K. Boyd. Reports of Investigations No. 72. 1990 (ix + 156 pp., maps, illus., appendix).

Archeological Survey of Wildlife Mitigation Lands, Justiceburg Reservoir, Garza County, Texas, by Douglas K. Boyd, C. Britt Bousman, and Martha Doty Freeman. Reports of Investigations No. 79. 1991 (vii + 75 pp., maps, illus., appendix).

Data Recovery at Justiceburg Reservoir (Lake Alan Henry), Garza and Kent Counties, Texas: Phase III, Season 1, by Douglas K. Boyd, Steve A. Tomka, C. Britt Bousman, Karen M. Gardner, and Martha Doty Freeman. Reports of Investigations No. 84. 1992 (xii + 256 pp., maps, illus., appendixes).

Data Recovery at Justiceburg Reservoir (Lake Alan Henry), Garza and Kent Counties, Texas: Phase III, Season 2, by Douglas K. Boyd, Jay Peck, Steve A. Tomka, and Karl W. Kibler. Reports of Investigations No. 88. 1993 (xxvi + 494 pp., maps, illus., appendixes).

Data Recovery at Lake Alan Henry (Justiceburg Reservoir), Garza and Kent Counties, Texas: Phase III, Season 3, by Douglas K. Boyd, Jack Peck, Steve A. Tomka, Karl W. Kibler, and Martha Doty Freeman. Reports of Investigations No. 93.1994 ( $\mathrm{xx}+395$ pp., maps, illus., appendixes).

Series Technical Editor: Linda Nance Foster 


\section{TABLE OF CONTENTS}

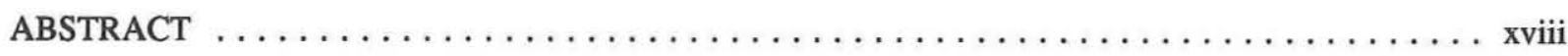

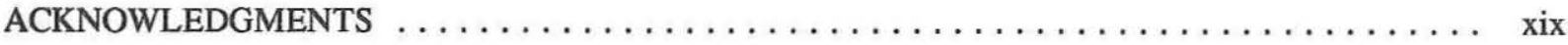

CHAPTER 1: INTRODUCTION AND ENVIRONMENTAL AND

ARCHEOLOGICAL BACKGROUND

Douglas K. Boyd $\ldots \ldots \ldots \ldots \ldots \ldots \ldots \ldots \ldots \ldots \ldots \ldots \ldots \ldots \ldots \ldots \ldots \ldots \ldots \ldots$

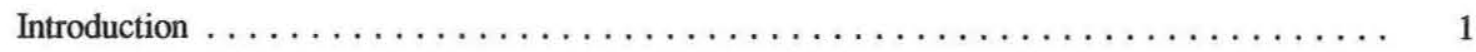

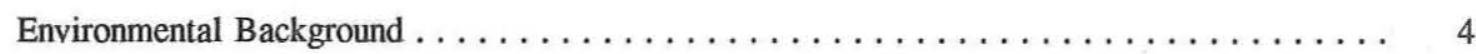

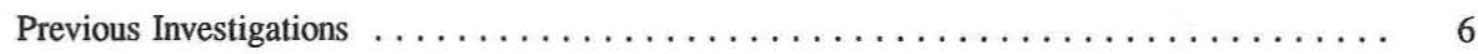

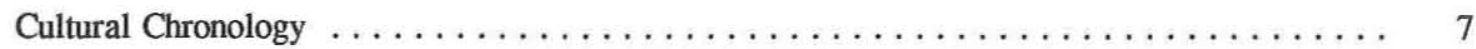

CHAPTER 2: RESEARCH ORIENTATION, OBJECTIVES, AND METHODS

Douglas K. Boyd, Steve A. Tomka, Martha Doty Freeman, and Jay Peck . . . . . . 9

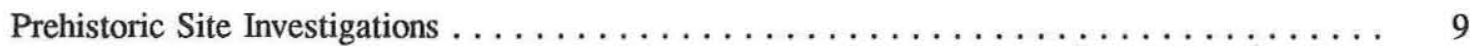

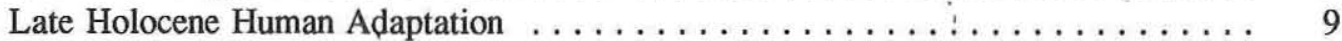

Settlement Patterns . . . . . . . . . 10

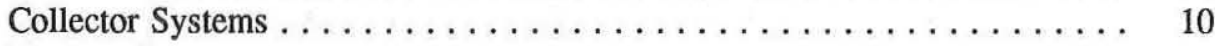

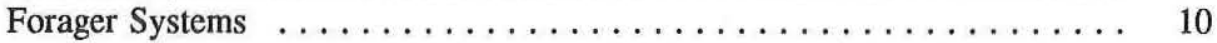

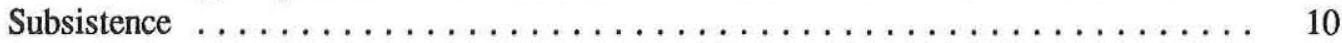

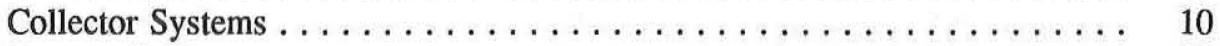

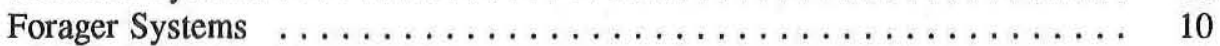

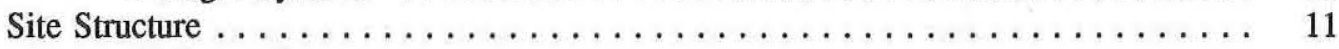

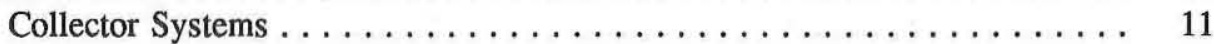

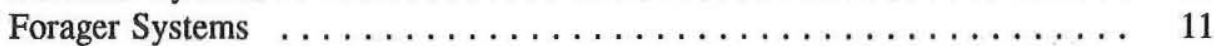

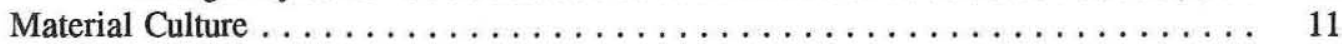

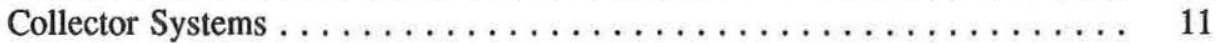

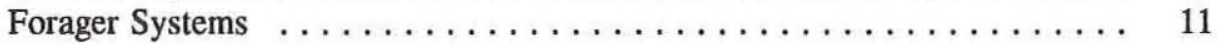

Excavations at Prehistoric Sites 41GR291, 41GR303B, 41GR546, and 41GR559 . . . 11

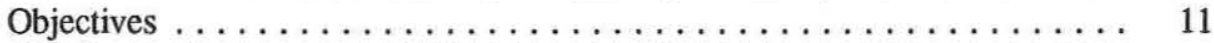

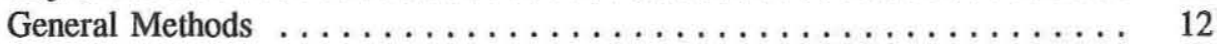

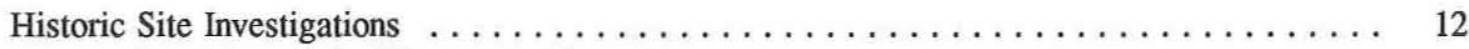

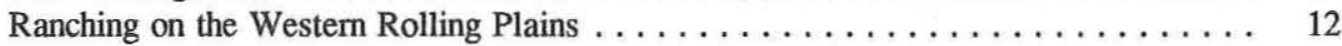

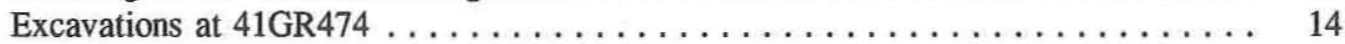

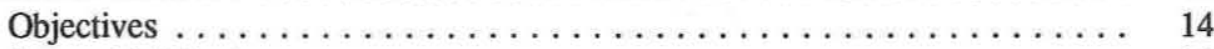

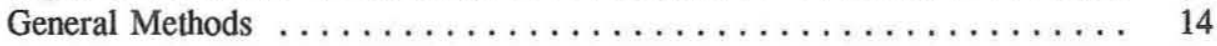

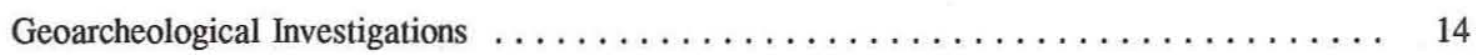

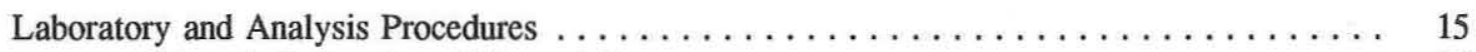

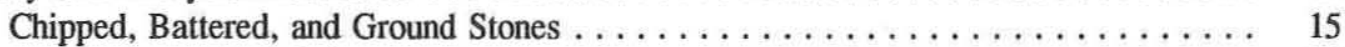

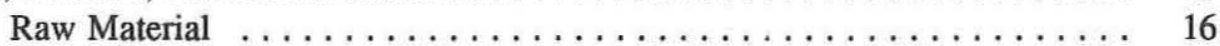

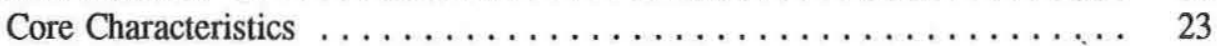

Nature of Tool Blank ...................... 23

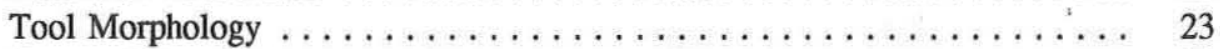

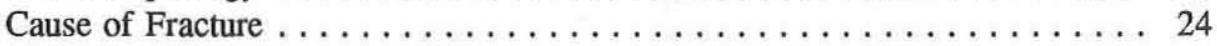

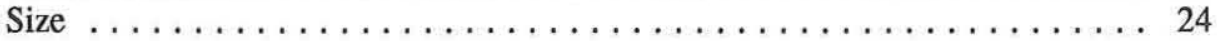

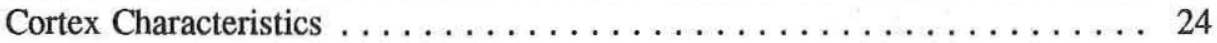

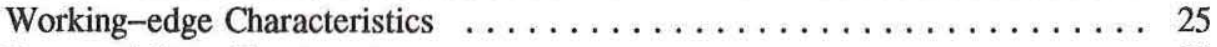

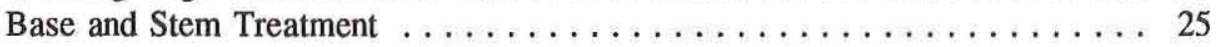




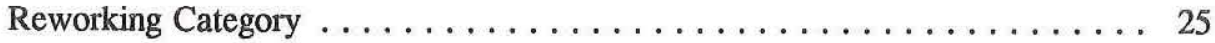

Platform Characteristics ..................... 25

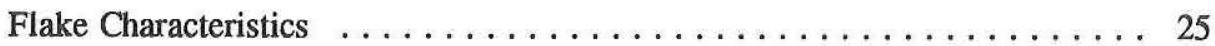

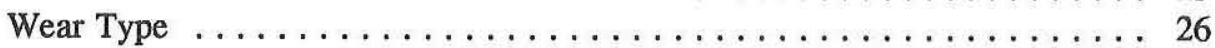

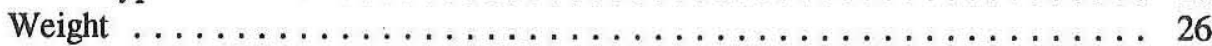

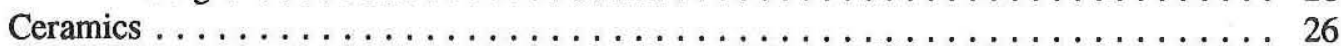

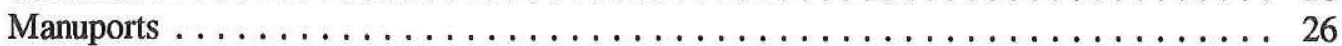

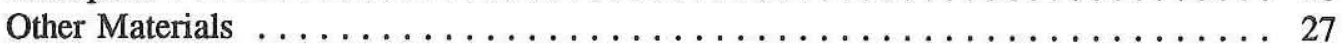

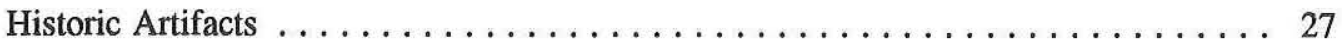

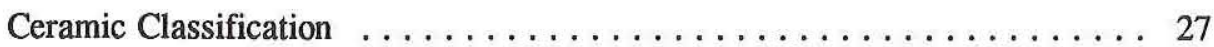

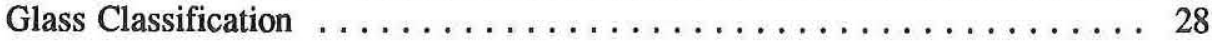

CHAPTER 3: THE SAM WAHL SITE, 41GR291

Douglas K. Boyd, Steve A. Tomka, and Karl W. Kibler . . . . . . . . . . . . . . 29

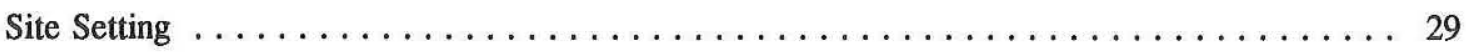

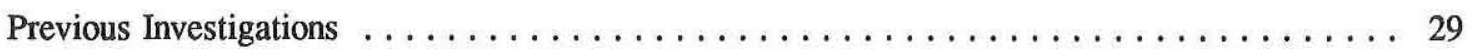

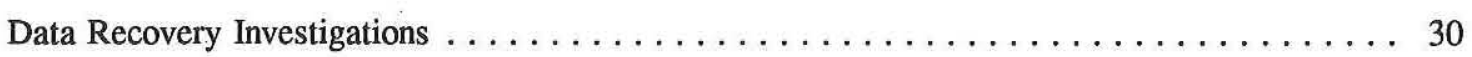

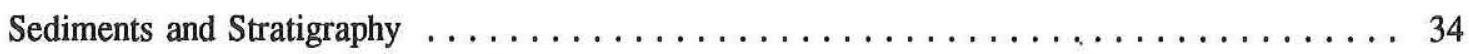

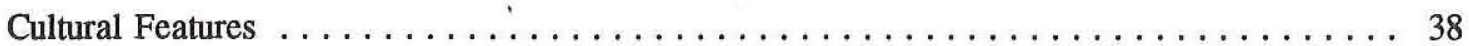

Bumed Rock and Fire-cracked Rock Clusters $\ldots \ldots \ldots \ldots \ldots \ldots \ldots \ldots$

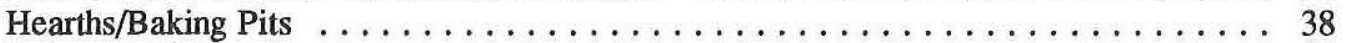

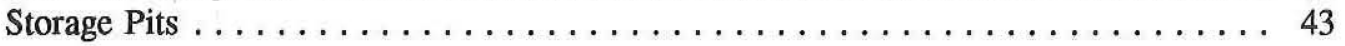

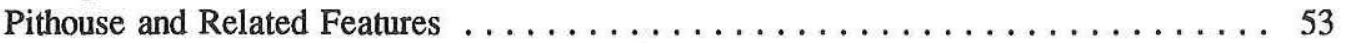

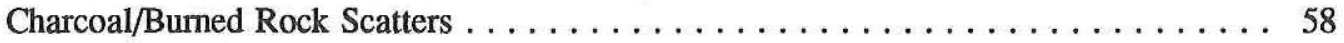

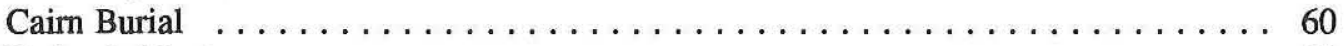

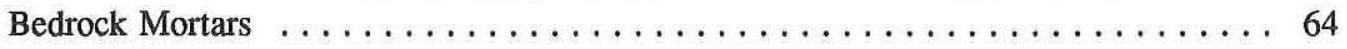

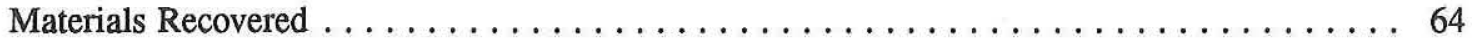

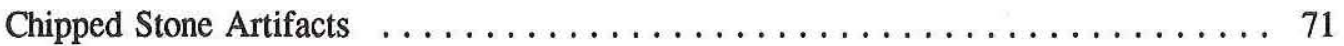

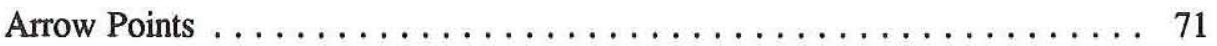

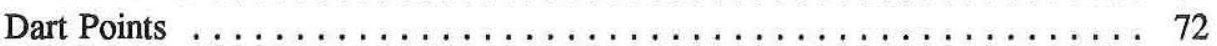

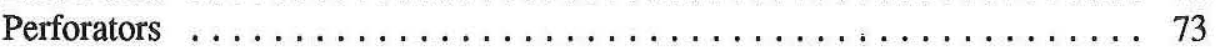

Gouges ............................ 73

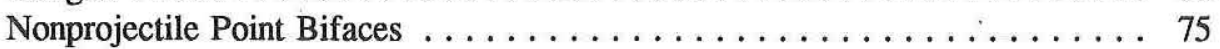

Cobble Tools . . . . . . . . . . . . . . . . . . . . . . . 76

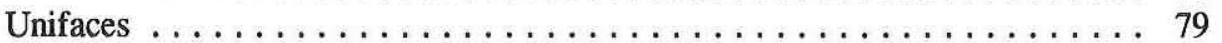

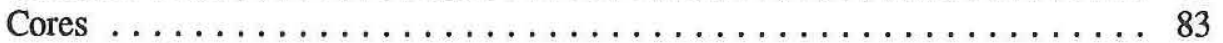

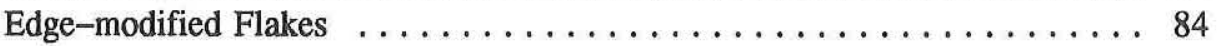

Unmodified Debitage $\ldots \ldots \ldots \ldots \ldots \ldots \ldots \ldots \ldots \ldots \ldots \ldots$

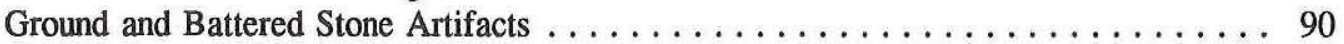

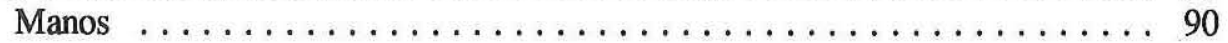

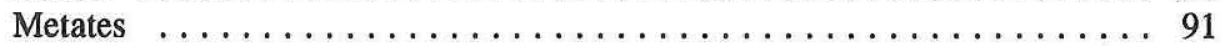

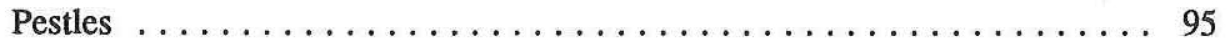

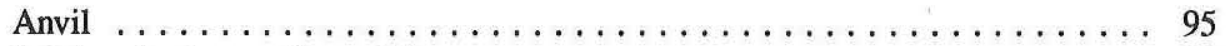

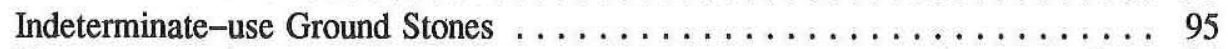

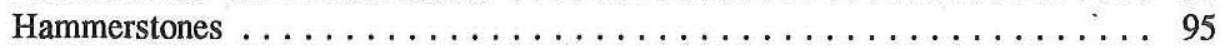

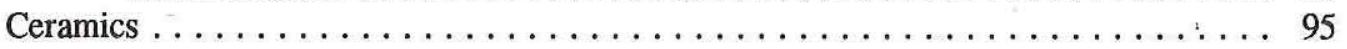

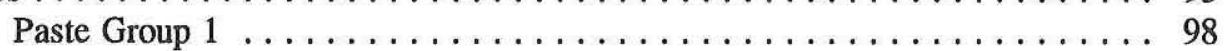

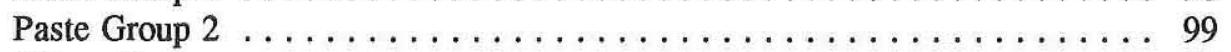

Modified Hematite . . . . . . . . . . . . . . . . . . . . . . 99 
Manuports . . . . . . . . . . . . . . . . . 100

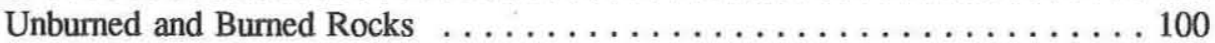

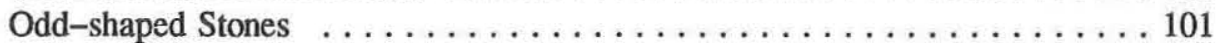

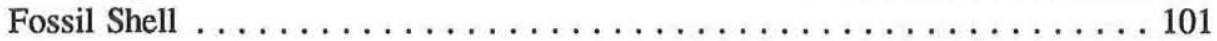

Vertebrate Faunal Remains . . . . . . . . . . . . . . . . . . . 103

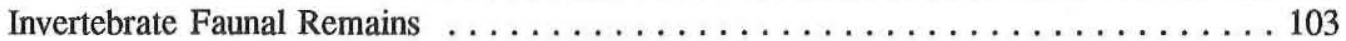

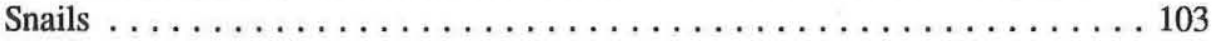

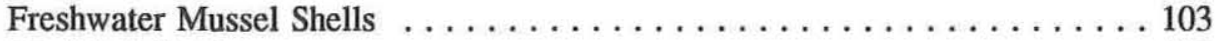

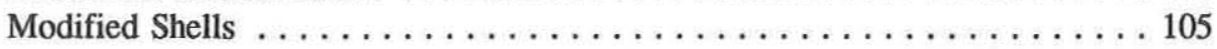

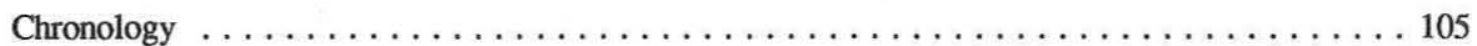

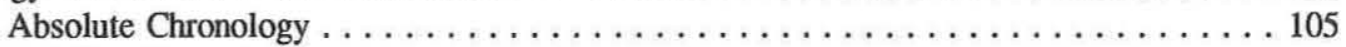

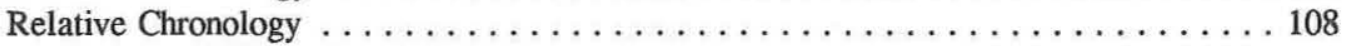

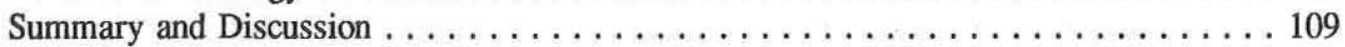

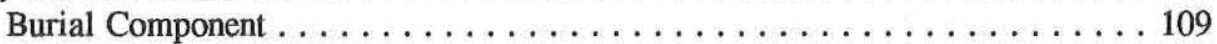

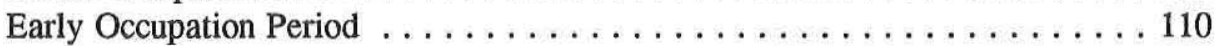

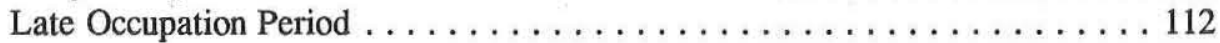

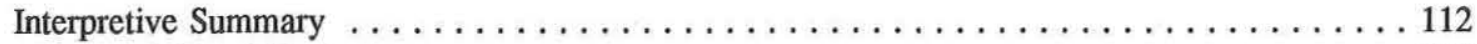

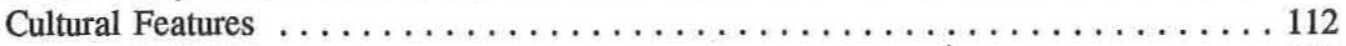

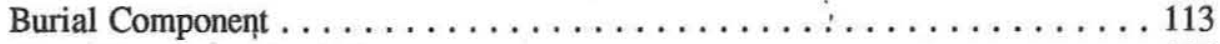

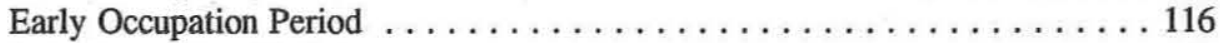

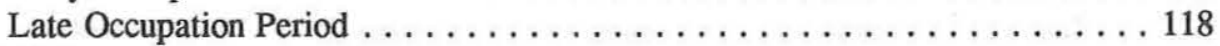

Material Culture . . . . . . . . . . . . . . . . . . . . . . . . 119

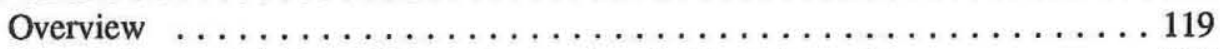

Comparisons of Early and Late Occupation Artifact Assemblages . . . . . . 122

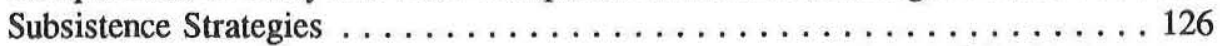

CHAPTER 4: THE CAT HOLLOW SITE, 41GR303B

Jay Peck, Steve A. Tomka, and Karl W. Kibler . . . . . . . . . . . . . . 129

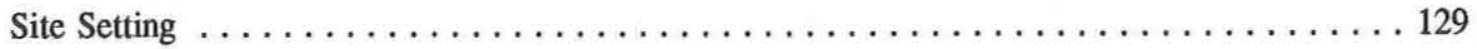

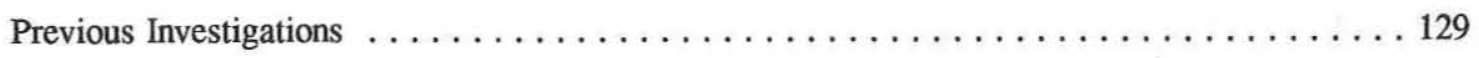

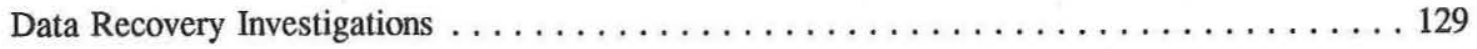

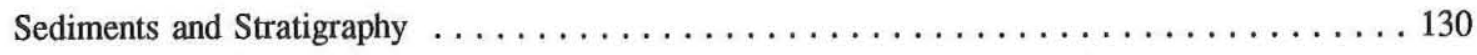

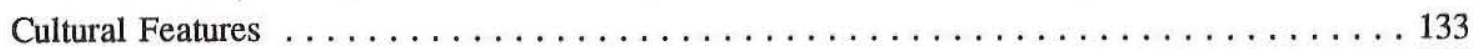

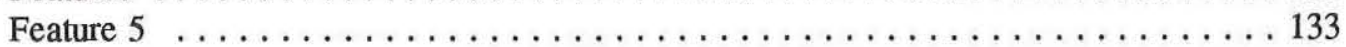

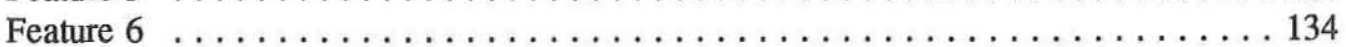

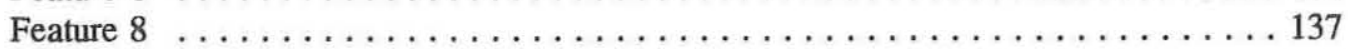

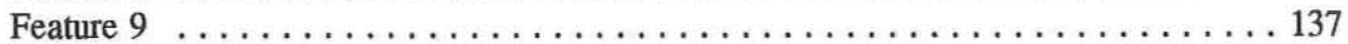

Discussion . . . . . . . . . . . . . . . 137

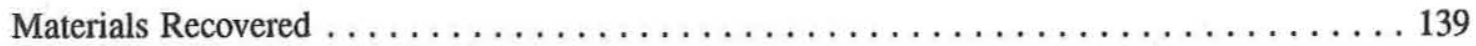

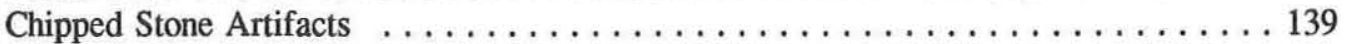

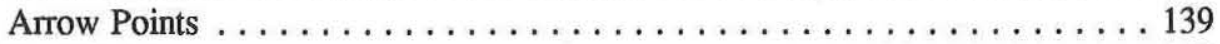

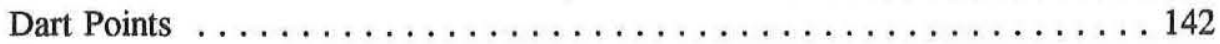

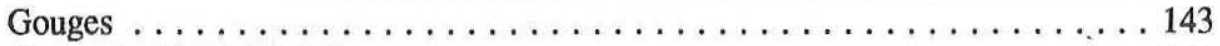

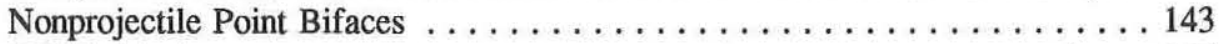

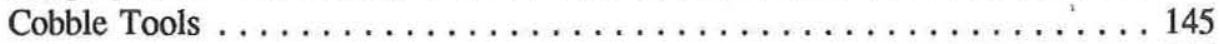

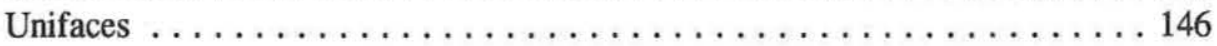

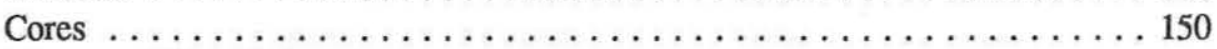

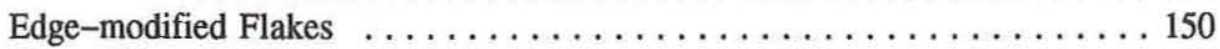

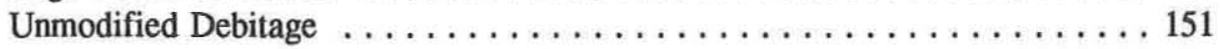


Ground and Battered Stone Artifacts . . . . . . . . . . . . . . . . . 154

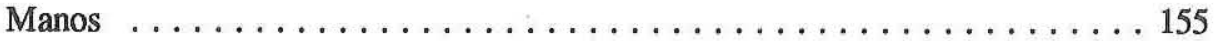

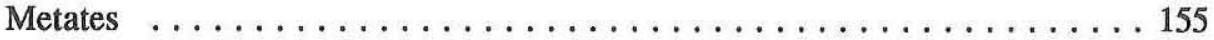

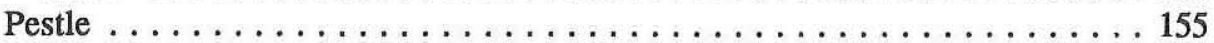

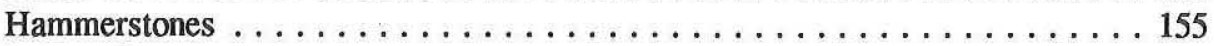

Modified Hematite . . . . . . . . . . . . . . . . . . . . . 155

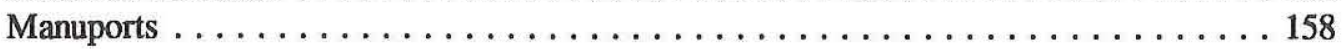

Burned and Fire-cracked Rocks $\ldots \ldots \ldots \ldots \ldots \ldots \ldots \ldots \ldots \ldots$

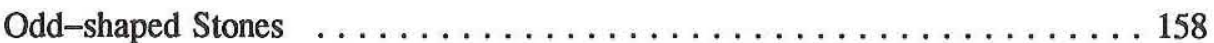

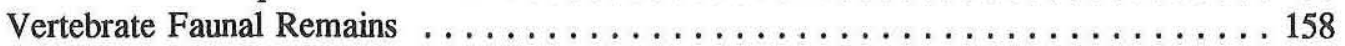

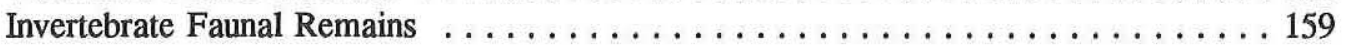

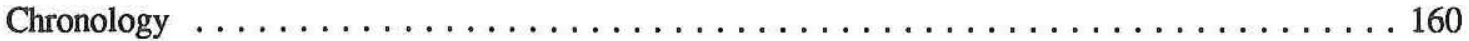

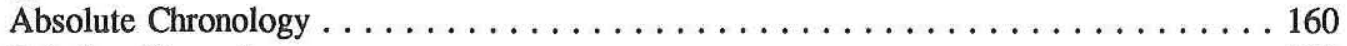

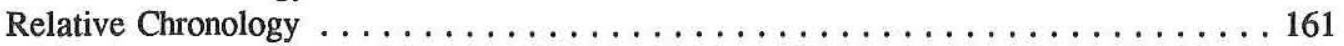

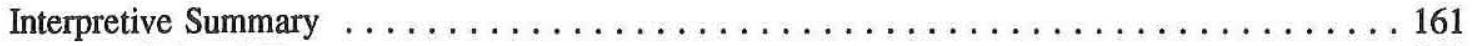

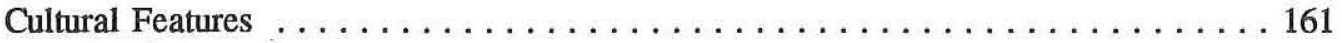

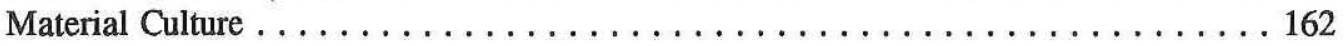

CHAPTER 5: THE JUSTICE DUGOUT, 41GR474

Douglas K. Boyd . . . . . . . . . . . . . . . . . . . 165

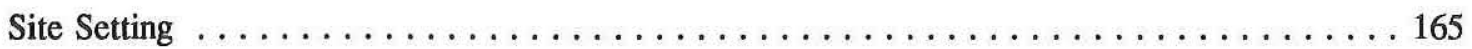

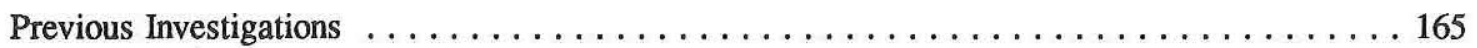

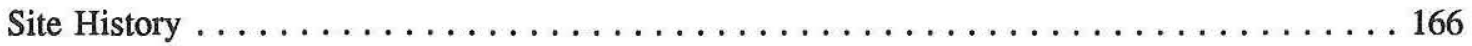

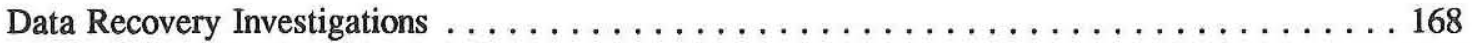

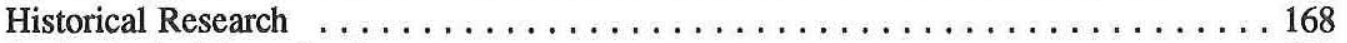

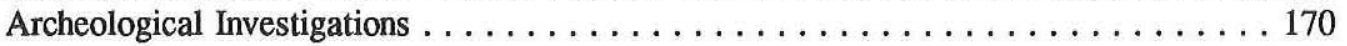

Stratigraphy and Definition of Components $\ldots \ldots \ldots \ldots \ldots \ldots \ldots \ldots \ldots \ldots \ldots \ldots \ldots \ldots \ldots \ldots \ldots$

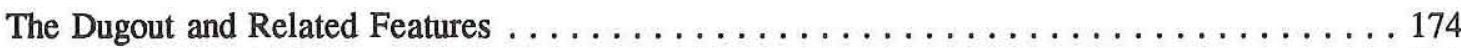

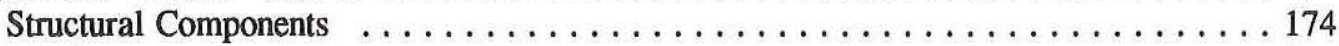

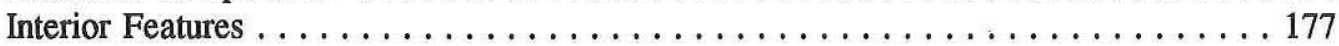

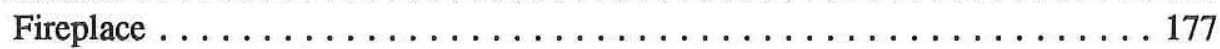

Ash Stains and Bone Concentrations $\ldots \ldots \ldots \ldots \ldots \ldots \ldots \ldots$

Materials Recovered . . . . . . . . . . . . . . . . . . . . . . . . . . . . . 178

Dugout Assemblage . . . . . . . . . . . . . . . . . . . . . . 179

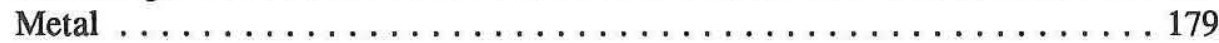

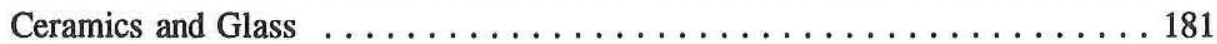

Bone . . . . . . . . . . . . . . . . . . . . . . . . . . . 182

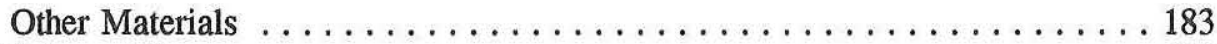

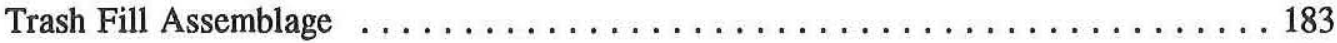

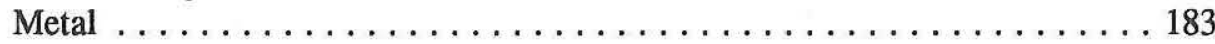

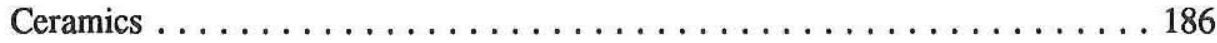

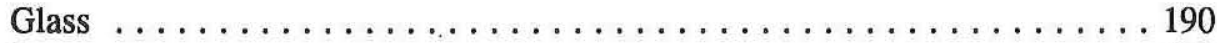

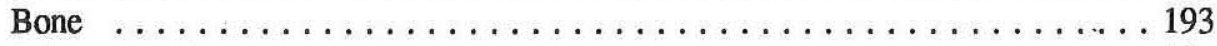

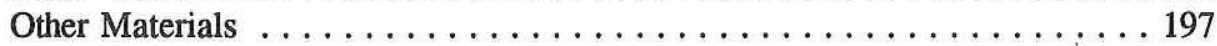

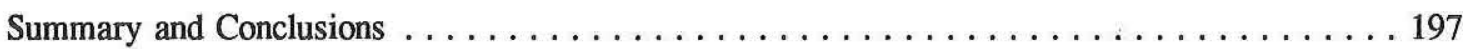

Site Chronology . . . . . . . . . . . . . . . . . . . . . . . . . . . . . . . . 197

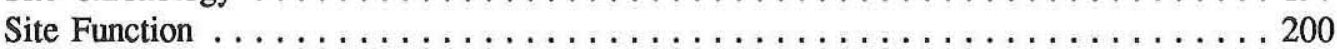

Subsistence and Technology ........................ 204

Socioeconomic Considerations . . . . . . . . . . . . . . . . . . . . . 205 
CHAPTER 6: BOREN SHELTER \#1, 41GR546

Jay Peck and Karl W. Kibler . . . . . . . . . . . . . . . . . . 207

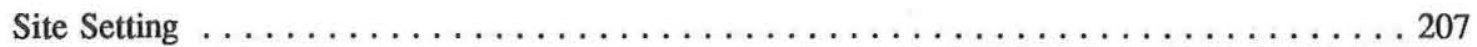

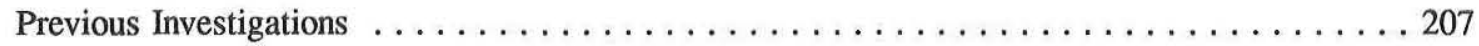

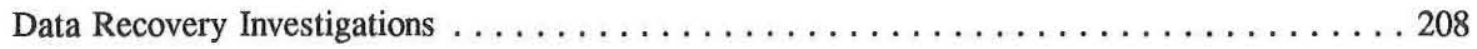

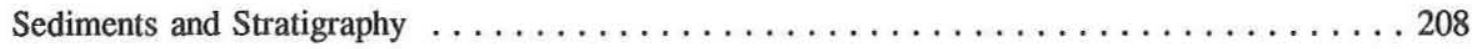

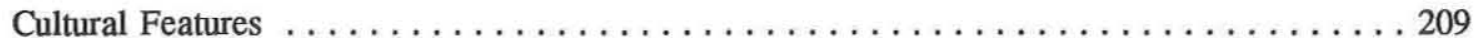

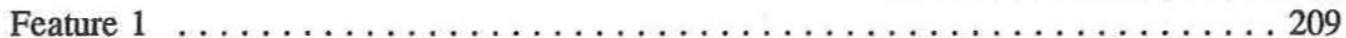

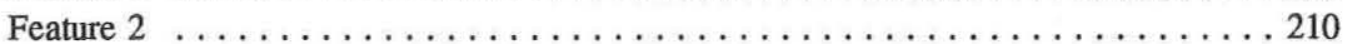

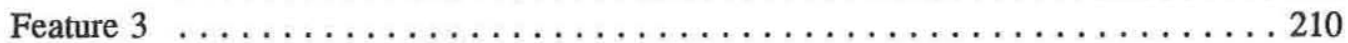

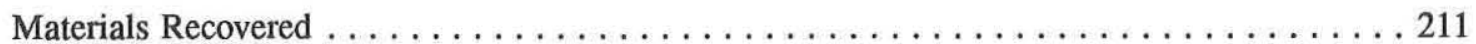

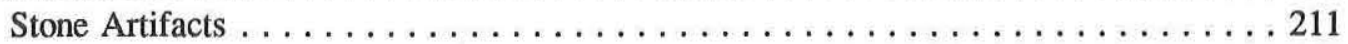

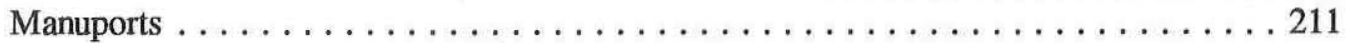

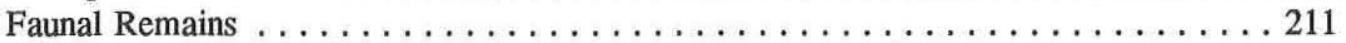

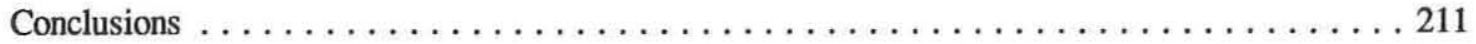

CHAPTER 7: BOREN SHELTER \#2, 41GR559

Jay Peck, Steve A. Tomka, and Karl W. Kibler . . . . . . . . . . . . 213

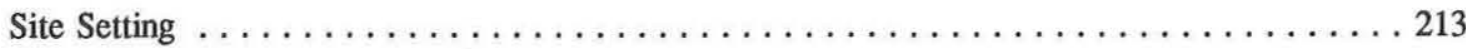

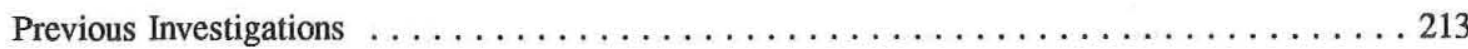

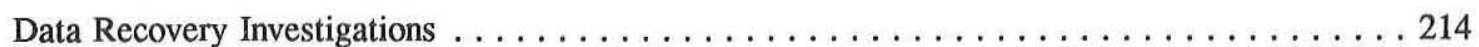

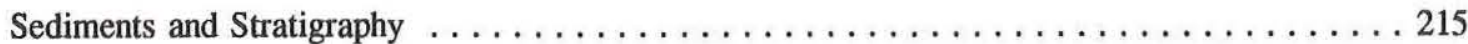

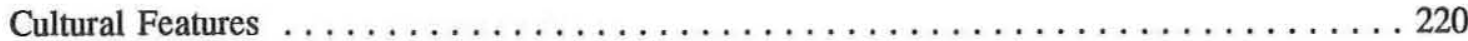

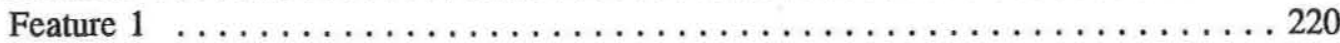

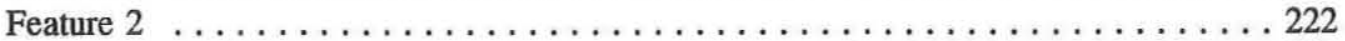

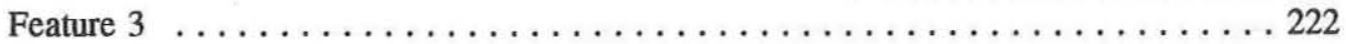

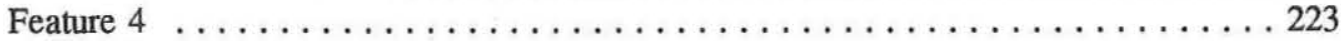

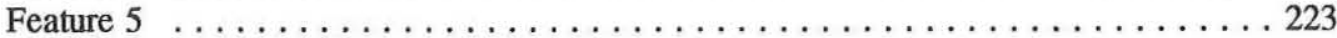

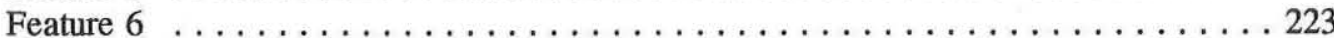

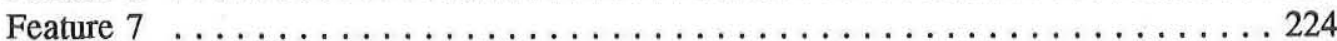

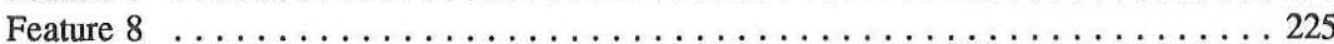

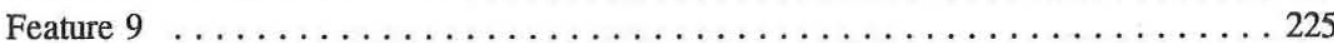

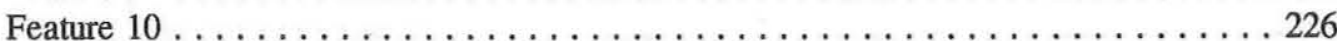

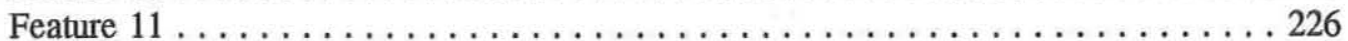

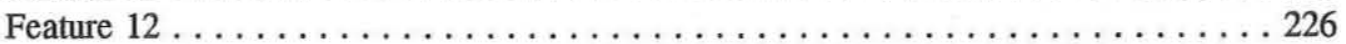

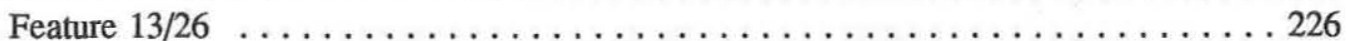

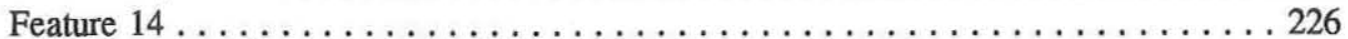

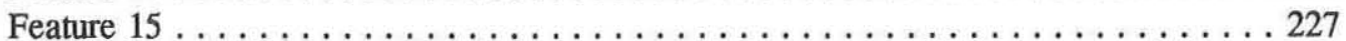

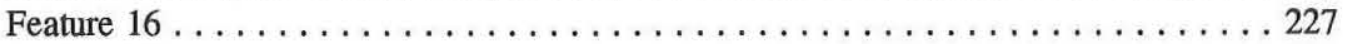

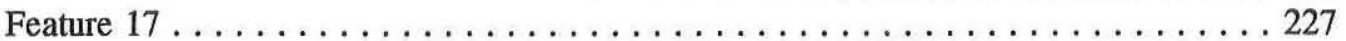

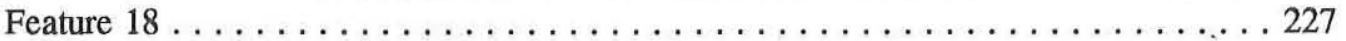

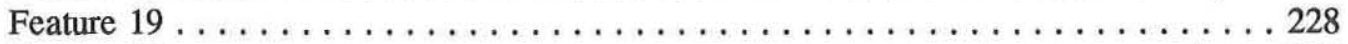

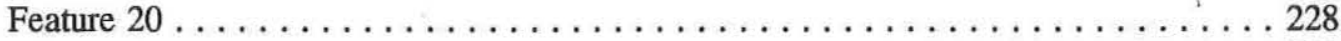

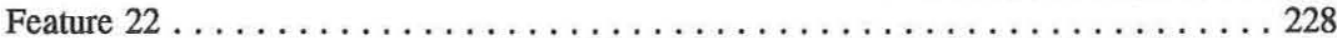

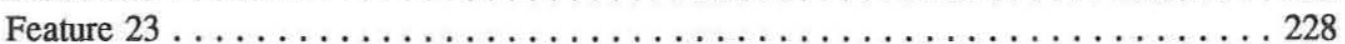

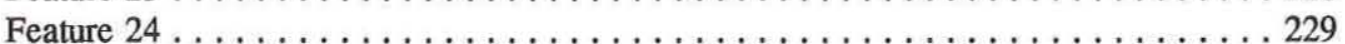

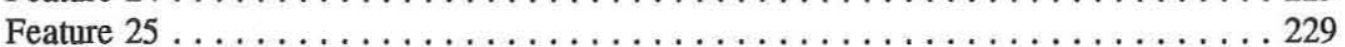

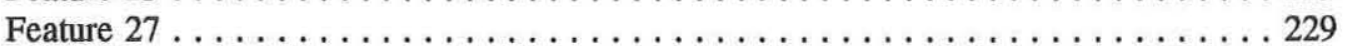


Discussion . . . . . . . . . . . . . . . . . . . . . . . . 229

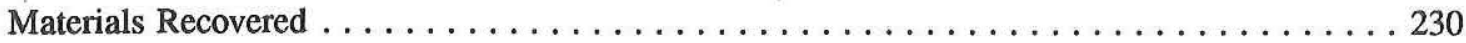

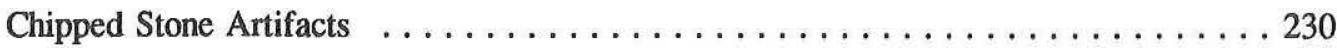

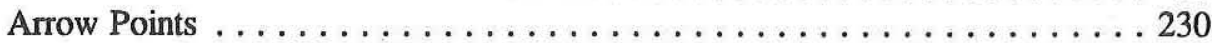

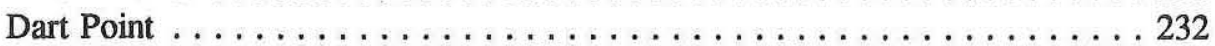

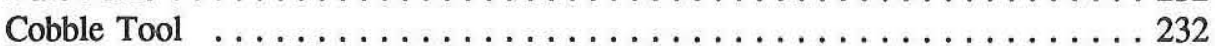

Unifaces ................................ 232

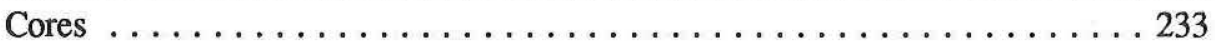

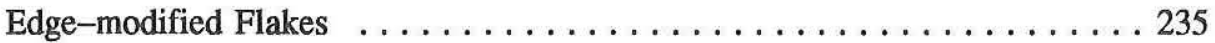

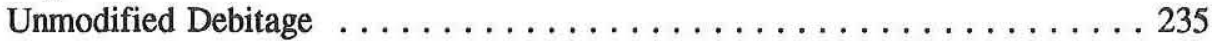

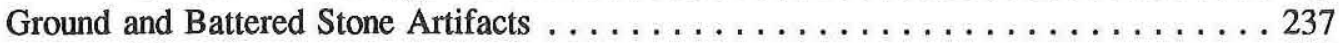

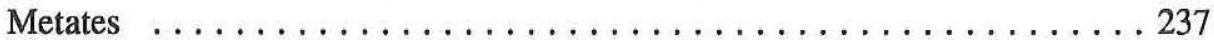

Hammerstones . . . . . . . . . . . . . . . . . . 237

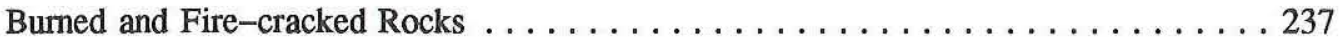

Vertebrate Faunal Remains . . . . . . . . . . . . . . . . . . . 237

Invertebrate Faunal Remains . . . . . . . . . . . . . . . . . . . . . . . 239

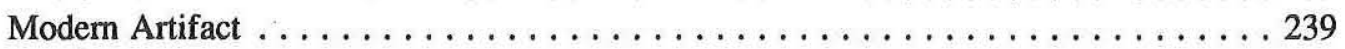

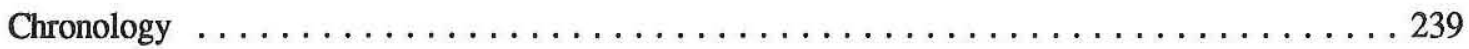

Absolute Chronology . . . . . . . . . . . . . . . . . . . . . . . . 239

Relative Chronology . . . . . . . . . . . . . . . . . . . . . . . 240

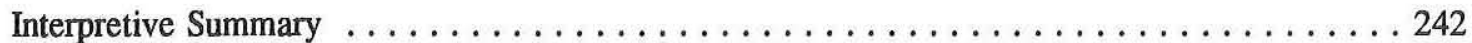

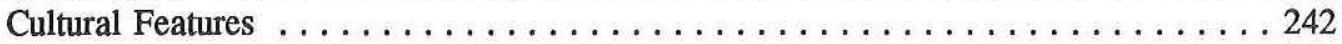

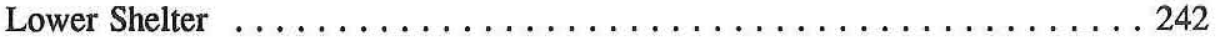

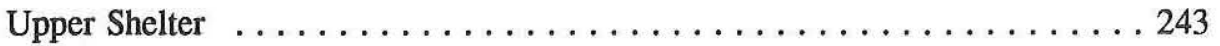

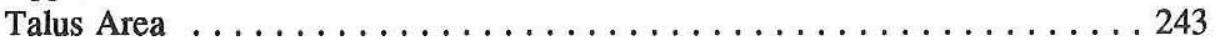

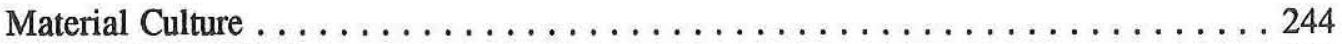

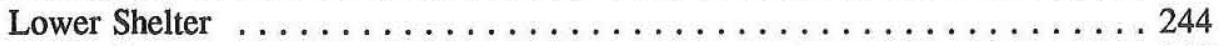

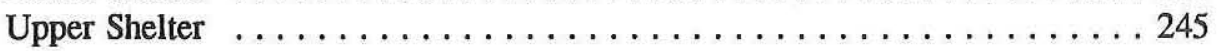

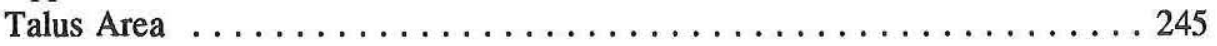

CHAPTER 8: SUMMARY AND CONCLUSIONS

Douglas K. Boyd, Steve A. Tomka, and Jay Peck . . . . . . . . . . . 247

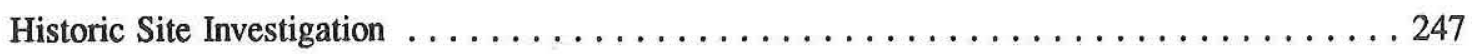

The Jeff Justice Homestead: Closed-range Ranching in the Western Rolling Plains . . . . . . 248

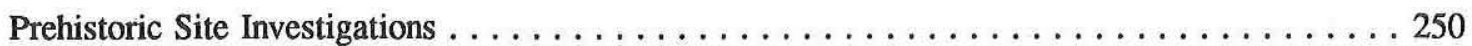

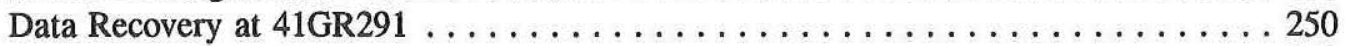

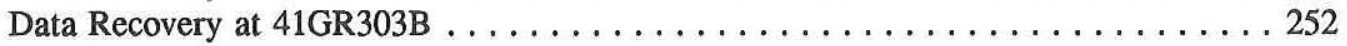

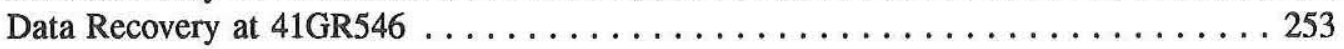

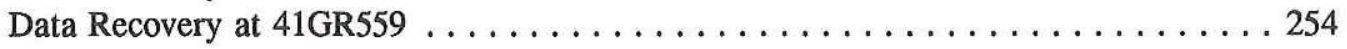

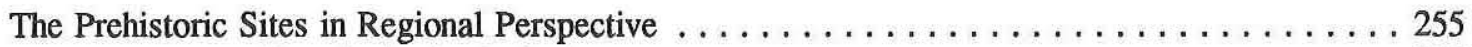

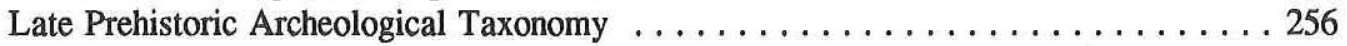

Late Prehistoric I Settlement Patterns and Subsistence . . . . . . . . . . . . . 260

Late Prehistoric II Settlement Patterns and Subsistence . . . . . . . . . . . . 268

Late Prehistoric Cultural Dynamics in the Lower Plains . . . . . . . . . . . . . . . . . 269

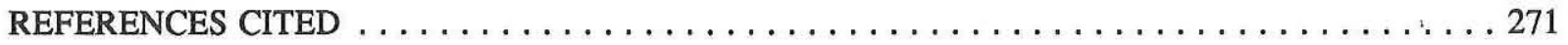


APPENDIX A: Geomorphic Profile Descriptions for 41GR291,

41GR303B, 41GR546, and 41GR559

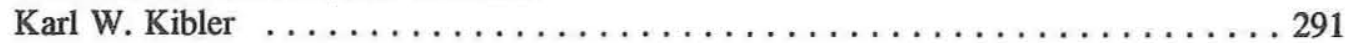

APPENDIX B: Textural and Chemical Analyses of Sediment Samples

from 41GR291, 41GR303B, and 41GR559

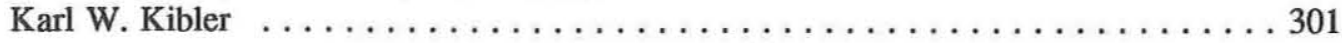

APPENDIX C: Analysis of the Vertebrate Remains from 41GR291, 41GR303B, 41GR474, and 41GR559

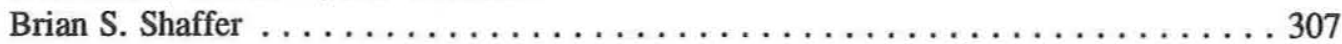

APPENDIX D: Analysis of Fish Otoliths and Invertebrate Fauna

from 41GR291, 41GR303B, and 41GR559

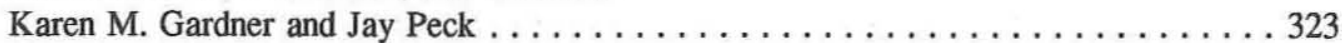

APPENDIX E: Analysis of Plant Remains from 41GR291 and 41GR559

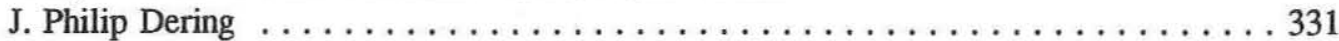

APPENDIX F: Analysis of Human Skeletal Remains from 41GR291

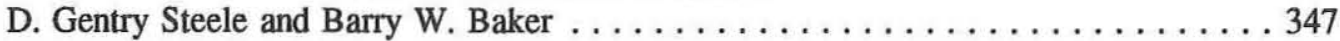

APPENDIX G: Petrographic Analysis of Plainwares from 41GR291

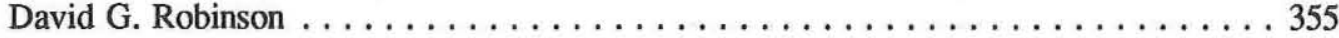

APPENDIX H: Provenience of Selected Artifact Classes from

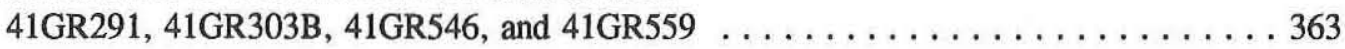

APPENDIX I: Discovery and Documentation of the Callahan Petroglyph Site, 41KT164

Douglas K. Boyd . . . . . . . . . . . . . . . . . . . . . . . . . . . . . . . . . . . . . 379 


\section{LIST OF FIGURES}

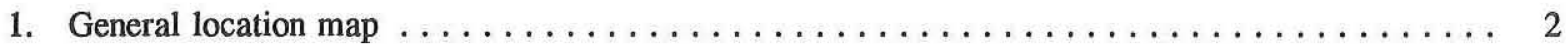

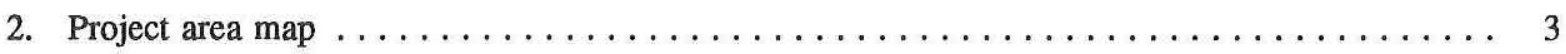

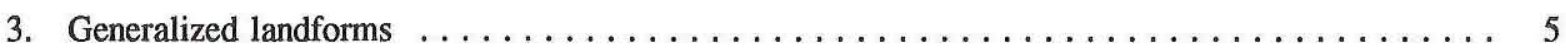

4. Map showing project area and locations of collection of regional lithic material comparative samples $\ldots \ldots \ldots \ldots \ldots \ldots \ldots \ldots \ldots \ldots \ldots \ldots$

5. Map of 41GR291 showing extent of site, 1988 test excavations and subsurface features, data recovery excavation blocks, and bedrock mortars $\ldots \ldots \ldots \ldots \ldots \ldots \ldots \ldots \ldots \ldots$

6. Map of north-central portion of 41GR291 showing locations of mechanical and hand excavations and data recovery cultural features $\ldots \ldots \ldots \ldots \ldots \ldots \ldots \ldots \ldots \ldots \ldots$

7. Map of 41 GR291 showing excavation block and unit numbers $\ldots \ldots \ldots \ldots \ldots \ldots \ldots \ldots$

8. Profiles of EUs 83, 88, and 95 and Backhoe Trenches 1992-1 and 1992-4, 41GR291 . . . . . 35

9. Plan, profile, and photograph of Feature 29,41 GR291 $\ldots \ldots \ldots \ldots \ldots \ldots \ldots \ldots \ldots \ldots$

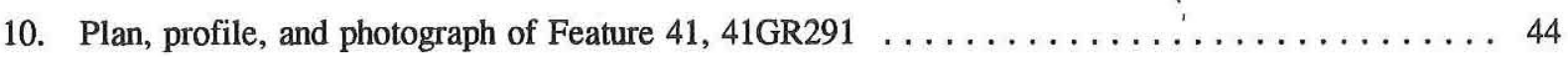

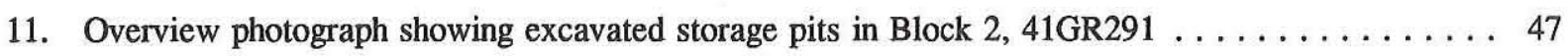

12. Storage pits at various stages of excavation, $41 \mathrm{GR} 291 \ldots \ldots \ldots \ldots \ldots \ldots \ldots \ldots \ldots$

13. Plan and profile drawings showing horizontal relationships and fill

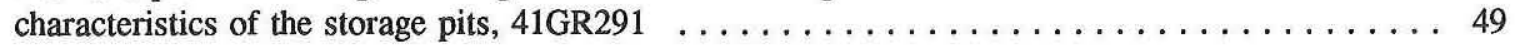

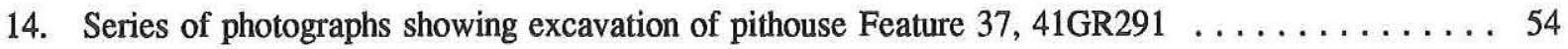

15. Plan and profile drawings of pithouse Feature 37,41 GR $291 \ldots \ldots \ldots \ldots \ldots \ldots \ldots \ldots$

16. Floor elevations and rocks in the fill of pithouse Feature $37,41 \mathrm{GR} 291 \ldots \ldots \ldots \ldots \ldots \ldots$

17. Plan and profile drawings of Feature 12,41 GR2 $291 \ldots \ldots \ldots \ldots \ldots \ldots \ldots \ldots \ldots \ldots$

18. Photograph of Feature 12 excavation in progress, $41 \mathrm{GR} 291 \ldots \ldots \ldots \ldots \ldots \ldots \ldots \ldots \ldots$

19. Plan and profile drawings of Feature $21,41 \mathrm{GR} 291 \ldots \ldots \ldots \ldots \ldots \ldots \ldots \ldots \ldots \ldots \ldots \ldots$

20. Photographs and plan and profile drawings of cairn burial, Feature $23,41 \mathrm{GR} 291 \ldots \ldots 2$

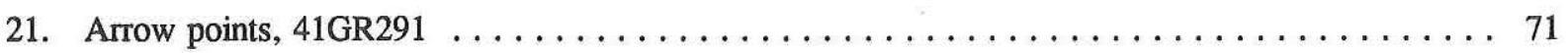

22. Untyped expanding-stem, convex-base dart points, $41 \mathrm{GR} 291 \ldots \ldots \ldots \ldots \ldots \ldots \ldots$

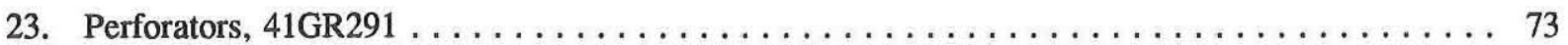

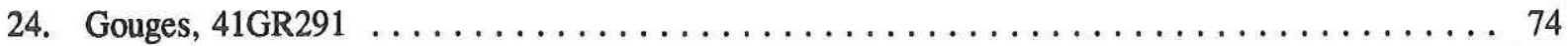

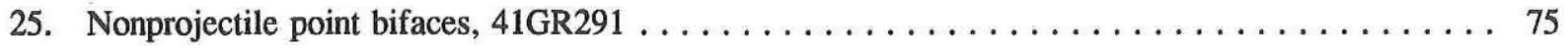

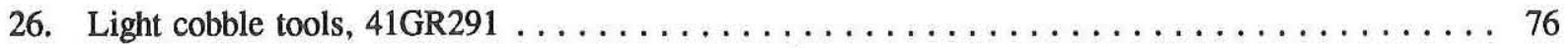

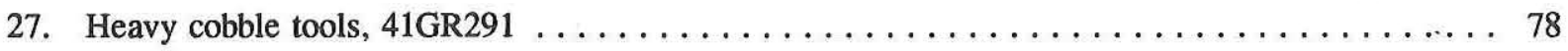

28. Heavy cobble tools with unusual use wear, $41 \mathrm{GR} 291 \ldots \ldots \ldots \ldots \ldots \ldots \ldots \ldots \ldots$

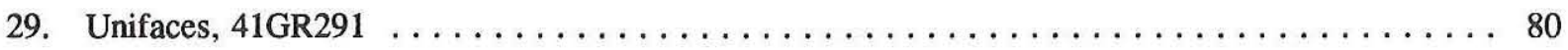

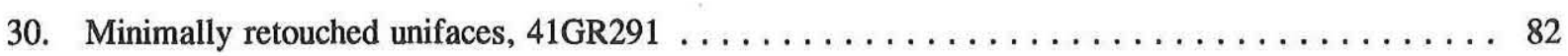

31. Indeterminate unifaces made on pebbles, $41 \mathrm{GR} 291 \ldots \ldots \ldots \ldots \ldots \ldots \ldots \ldots \ldots$ 


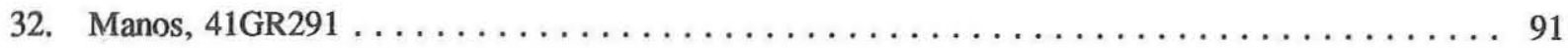

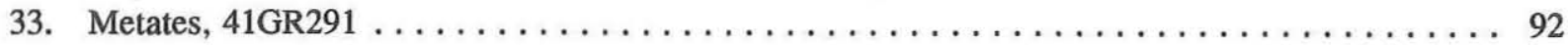

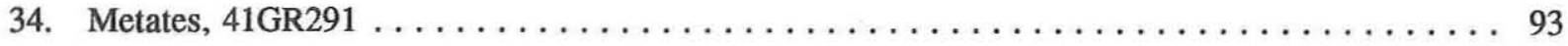

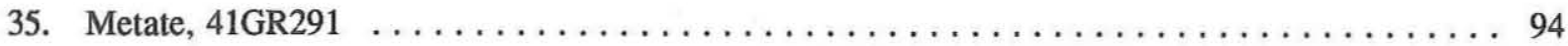

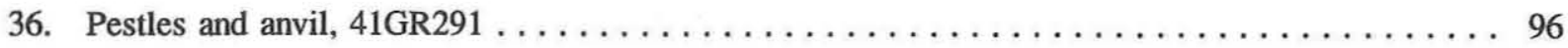

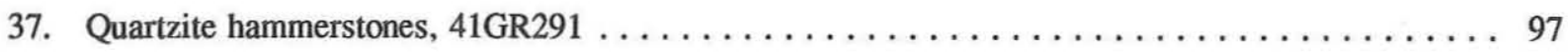

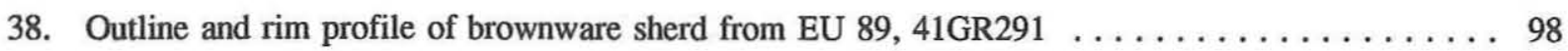

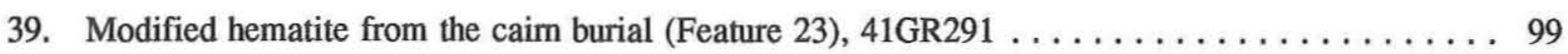

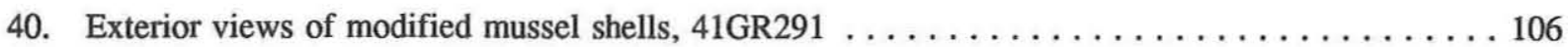

41. Graph of calibrated radiocarbon dates from features, 41GR291 $\ldots \ldots \ldots \ldots \ldots \ldots \ldots \ldots$

42. Map of 41GR303B showing topographic setting, 1988 test units, and 1992 excavation units $\ldots \ldots 131$

43. Plan of block excavation and nearby excavation units, 41GR303B $\ldots \ldots \ldots \ldots \ldots \ldots \ldots \ldots 132$

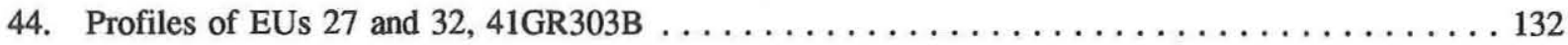

45. Three profiles showing the burned rock scatter in the block, 41GR303B $\ldots \ldots \ldots \ldots \ldots \ldots$

46. Photograph of EUs 32 and 33 showing the burned rock scatter in Level 2, 41GR303B . . . . . 135

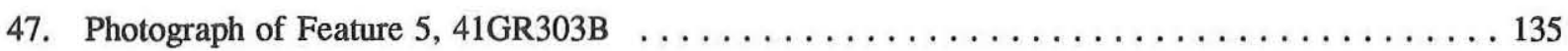

48. Cross section of EUs 13, 21, and 17 showing burned rock scatter and

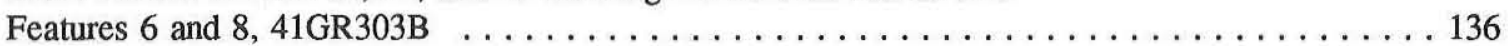

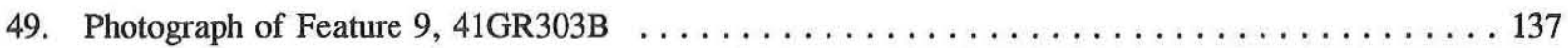

50. Profile of the north-south trench showing Features 6,8 , and 9 and the scattered burned rock zone, 41GR303B $\ldots \ldots \ldots \ldots \ldots \ldots \ldots \ldots \ldots \ldots \ldots \ldots \ldots \ldots \ldots \ldots \ldots$

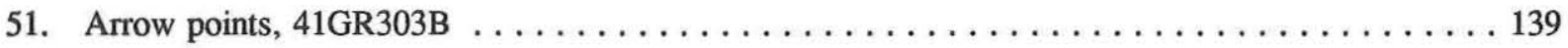

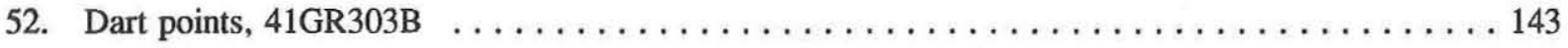

53. Unifacial gouges made on thick flake blanks, 41GR303B $\ldots \ldots \ldots \ldots \ldots \ldots \ldots \ldots \ldots \ldots$

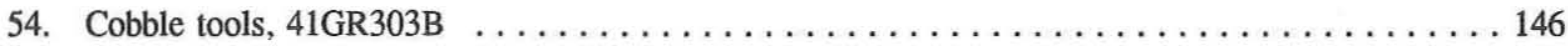

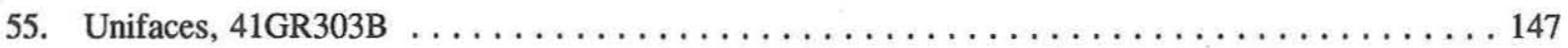

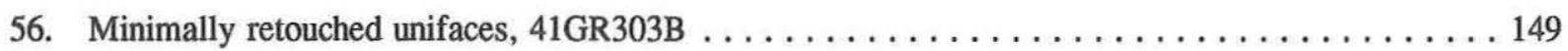

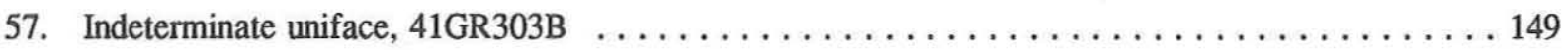

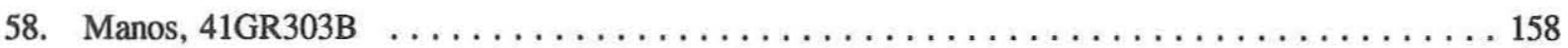

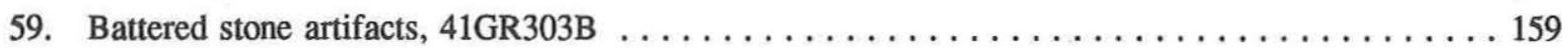

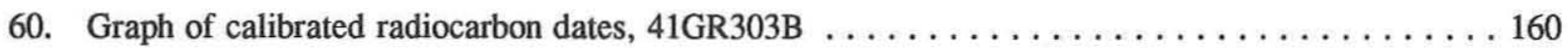

61. Densities of burned rocks, stone tools, and debitage by level in

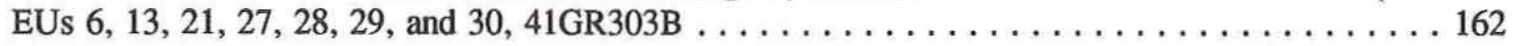

62. Photograph of dugout area at 41 GR474 as it appeared in $1987 \ldots \ldots \ldots \ldots \ldots \ldots \ldots \ldots \ldots$

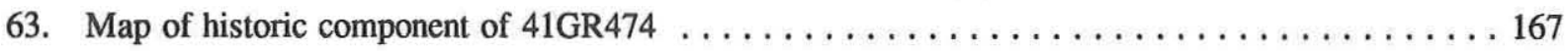

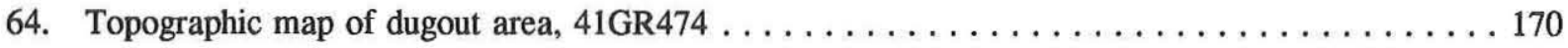

65. Photographs of excavation of the dugout at 41 GR474 in progress with rocks in situ $\ldots \ldots \ldots 172$ 
66. Photographs of the completed excavation of the dugout at $41 \mathrm{GR} 474 \ldots \ldots \ldots \ldots \ldots \ldots$

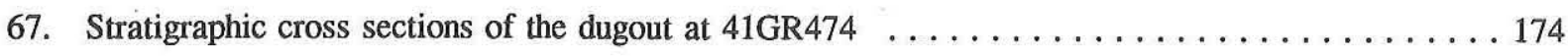

68. Plan views of the dugout at 41GR474 showing various structural elements $\ldots \ldots \ldots \ldots \ldots$

69. Photograph of firebox along the back wall of the dugout at 41 GR $474 \ldots \ldots \ldots \ldots \ldots \ldots$

70. Tobacco plug trademark in the dugout assemblage at $41 \mathrm{GR} 474 \ldots \ldots \ldots \ldots \ldots \ldots \ldots$

71. Ceramic vessel forms represented in the trash fill assemblage, 41 GR $474 \ldots \ldots \ldots \ldots \ldots 1$

72. Diagnostic ceramic marks in the trash fill assemblage, $41 \mathrm{GR} 474 \ldots \ldots \ldots \ldots \ldots \ldots \ldots \ldots \ldots$

73. Distribution of cultural features and artifacts on and

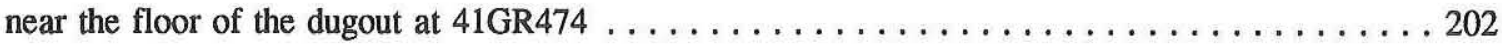

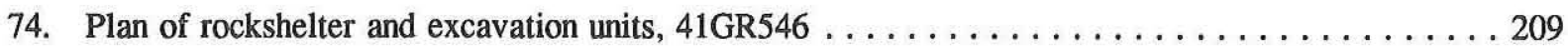

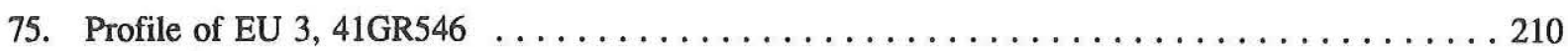

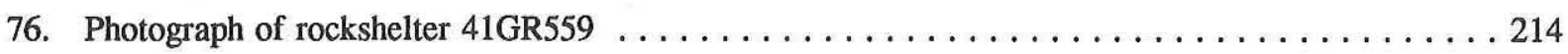

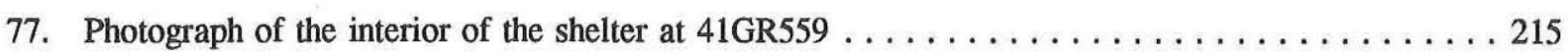

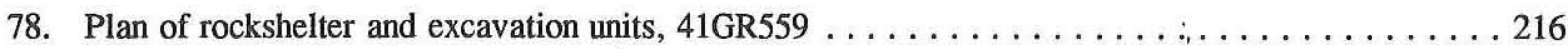

79. East-west profile of rockshelter fill depicting the two zones

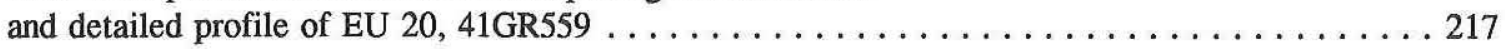

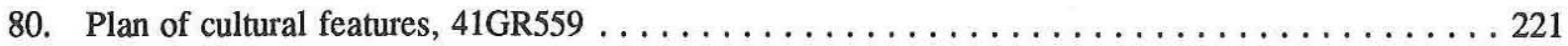

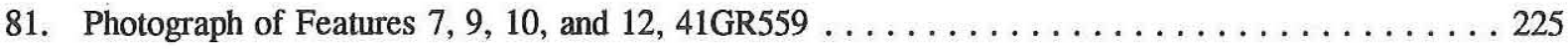

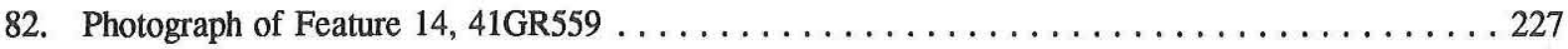

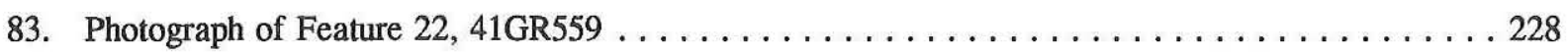

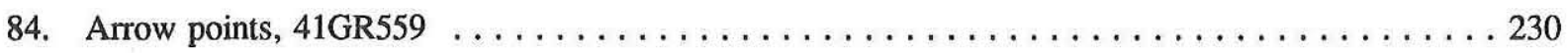

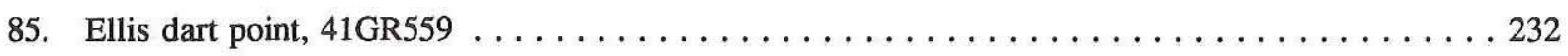

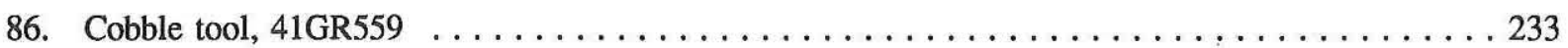

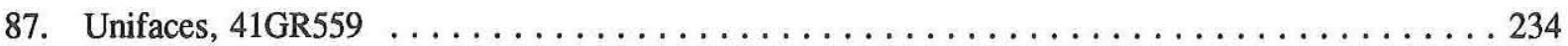

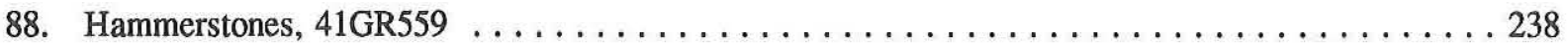

89. Graph of calibrated radiocarbon dates from features, 41 GR559 $\ldots \ldots \ldots \ldots \ldots \ldots \ldots \ldots 240$

90. Collapsed east-west profiles indicating the stratigraphic positions of

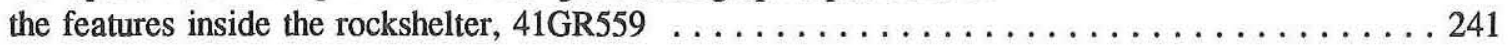

91. Collapsed north-south profile of bone midden area in front of the shelter, 41 GR559 $\ldots \ldots \ldots 241$

92. Comparison of calibrated radiocarbon dates from prehistoric sites

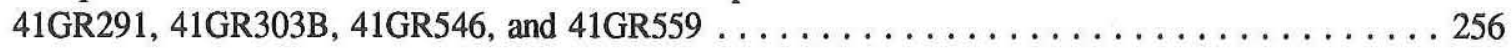

93. Plan and profile views and photograph of the overhang shelter, $41 \mathrm{KT} 164 \ldots \ldots \ldots \ldots 2$

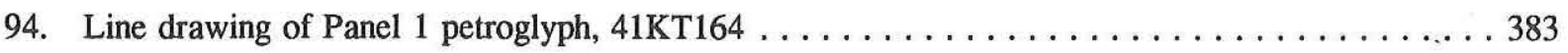

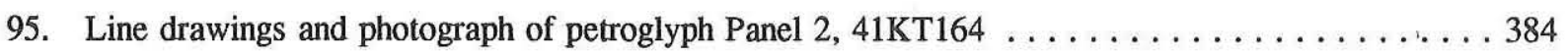

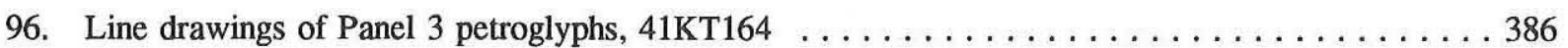

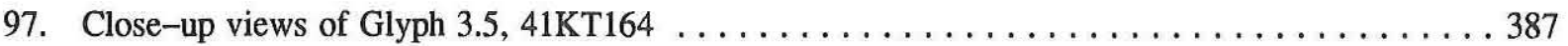

98. Line drawings of Panel 4 petroglyph, $41 \mathrm{KT} 164 \ldots \ldots \ldots \ldots \ldots \ldots \ldots \ldots \ldots \ldots \ldots$ 


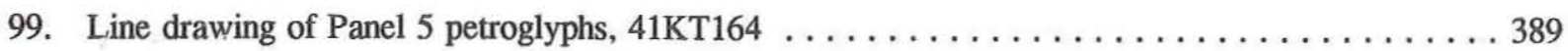

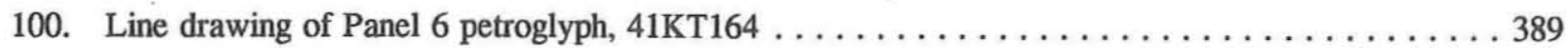




\section{LIST OF TABLES}

1. Data on lithic material comparative samples $\ldots \ldots \ldots \ldots \ldots \ldots \ldots \ldots \ldots \ldots \ldots \ldots$

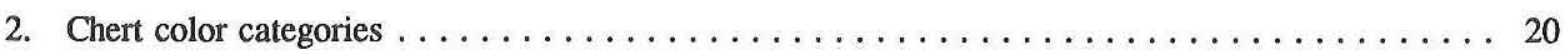

3. Decorative classification of historic ceramics $\ldots \ldots \ldots \ldots \ldots \ldots \ldots \ldots \ldots \ldots \ldots$

4. Artifacts recovered during survey and testing phases, 41GR291 $\ldots \ldots \ldots \ldots \ldots \ldots \ldots \ldots$

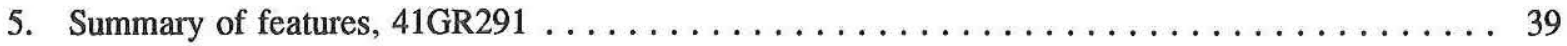

6. Results of radiocarbon dating of features, $41 \mathrm{GR} 291 \ldots \ldots \ldots \ldots \ldots \ldots \ldots \ldots \ldots \ldots$

7. Summary of feature flotation samples and macrobotanical recovery, $41 \mathrm{GR} 291 \ldots \ldots \ldots \ldots . .41$

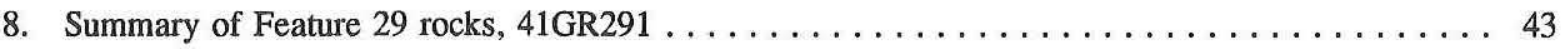

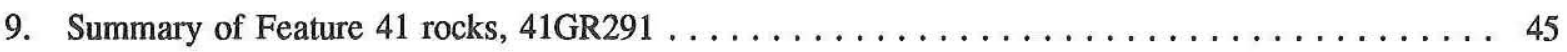

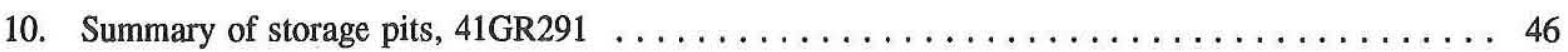

11. Summary of materials recovered from storage pit fill, $41 \mathrm{GR} 291 \ldots \ldots \ldots \ldots \ldots \ldots \ldots$

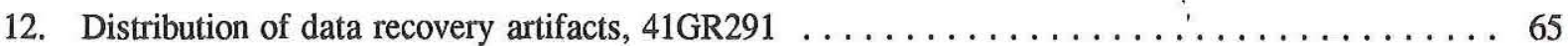

13. Breakdown of debitage attributes within the fine- and coarse-grained

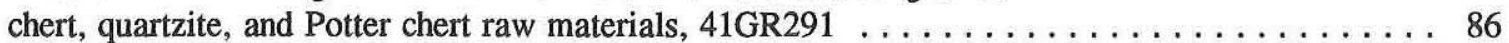

14. Breakdown of debitage attributes within the nonchert and

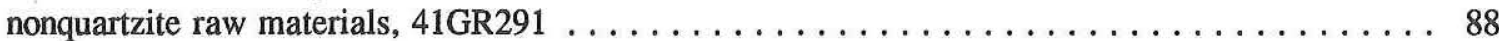

15. Summary of burned and unburned rocks by material type, $41 \mathrm{GR} 291 \ldots \ldots \ldots \ldots \ldots \ldots \ldots$

16. Summary of burned and unburned rocks by feature and material type, $41 \mathrm{GR} 291 \ldots \ldots \ldots \ldots$

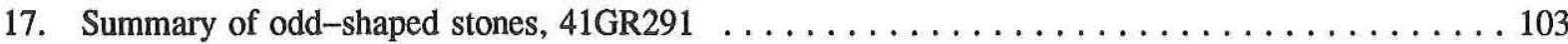

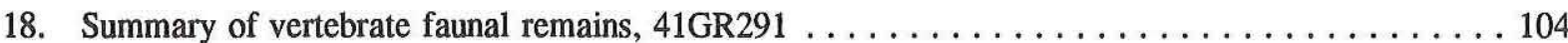

19. Summary of features by type and temporal affiliation, 41 GR2 $291 \ldots \ldots \ldots \ldots \ldots \ldots \ldots \ldots$

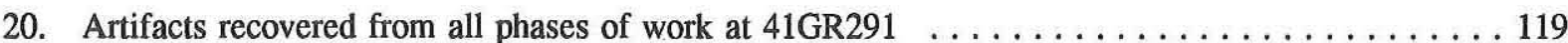

21. Summary of Phase III chipped stones by material type and origin, 41GR291 $\ldots \ldots \ldots \ldots \ldots 121$

22. Total chipped, ground, and battered stone tool samples for early and

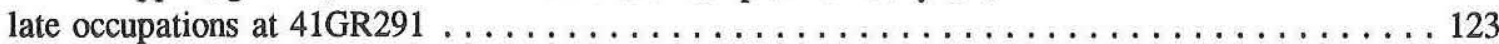

23. Average stone tool recovery per square meter for the early and late

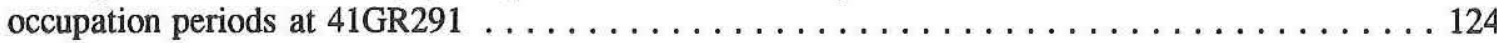

24. Comparisons of early and late occupation chipped stone collections by

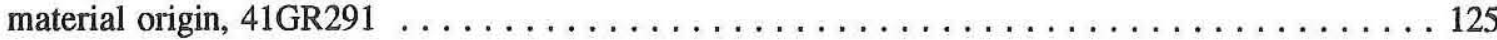

25. Comparison of stone artifact collections from the early and late

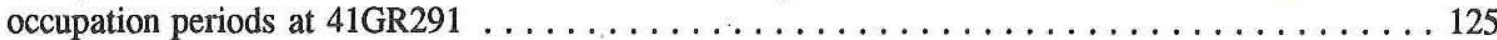

26. Comparison of other artifacts in the early and late occupation

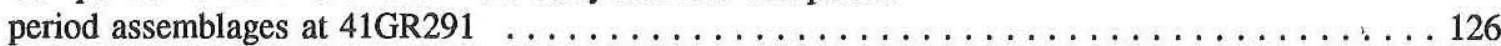

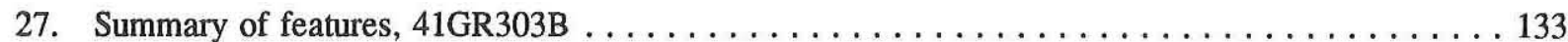

28. Results of radiocarbon dating of cultural contexts, $41 \mathrm{GR} 303 \mathrm{~B} \ldots \ldots \ldots \ldots \ldots \ldots \ldots \ldots$

29. Distribution of data recovery artifacts, 41GR303B $\ldots \ldots \ldots \ldots \ldots \ldots \ldots \ldots \ldots \ldots \ldots$ 
30. Breakdown of debitage attributes within the fine- and coarse-grained chert, quartzite, and Potter chert raw materials, 41GR303B

31. Breakdown of debitage attributes within the nonchert and

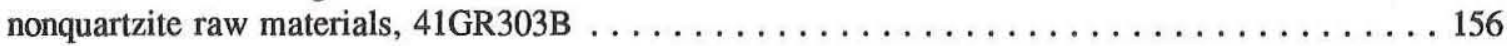

32. Comparison of stone artifact collections from the lower and upper zones at 41GR303B $\ldots \ldots 163$

33. Comparison of lower zone and upper zone chipped stone

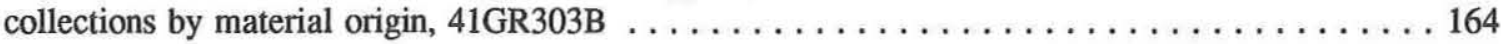

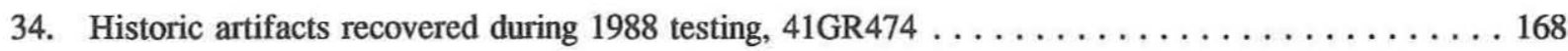

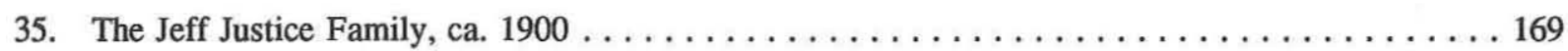

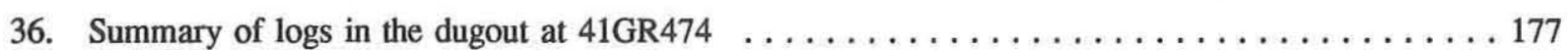

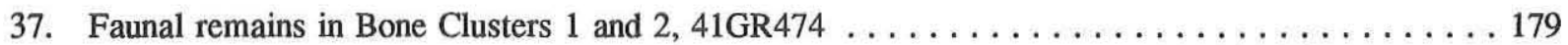

38. Historic artifacts recovered during data recovery, $41 \mathrm{GR} 474 \ldots \ldots \ldots \ldots \ldots \ldots \ldots \ldots$

39. Summary of the dugout artifact assemblage, $41 \mathrm{GR} 474 \ldots \ldots \ldots \ldots \ldots \ldots \ldots \ldots \ldots \ldots$

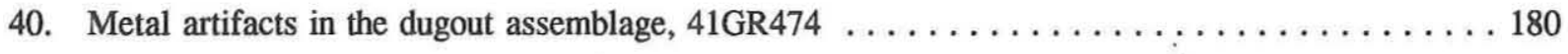

41. Ceramic, glass, bone, and miscellaneous material artifacts in the

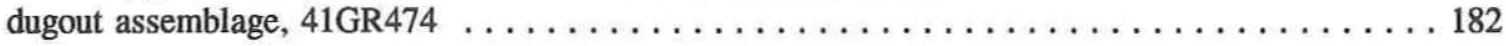

42. Summary of the trash fill artifact assemblage, 41 GR $474 \ldots \ldots \ldots \ldots \ldots \ldots \ldots \ldots \ldots$

43. Metal artifacts in the trash fill assemblage, $41 \mathrm{GR} 474 \ldots \ldots \ldots \ldots \ldots \ldots \ldots \ldots \ldots$

44. Ceramic artifacts in the trash fill assemblage, $41 \mathrm{GR} 474 \ldots \ldots \ldots \ldots \ldots \ldots \ldots \ldots \ldots$

45. Summary of ceramic vessels in the trash fill assemblage, 41 GR $474 \ldots \ldots \ldots \ldots \ldots \ldots \ldots 7$

46. Summary of ceramic vessel forms in the trash fill assemblage, $41 \mathrm{GR} 474 \ldots \ldots \ldots \ldots \ldots$

47. Glass artifacts in the trash fill assemblage, 41 GR $474 \ldots \ldots \ldots \ldots \ldots \ldots \ldots \ldots \ldots \ldots \ldots \ldots$

48. Summary of glass vessels in the trash fill assemblage, 41 GR474 $\ldots \ldots \ldots \ldots \ldots \ldots \ldots \ldots$

49. Diagnostic attributes of glass vessels in the trash fill assemblage, 41 GR474 . . . . . . . 197

50. Bone and other materials in the trash fill assemblage, $41 \mathrm{GR} 474 \ldots \ldots \ldots \ldots \ldots \ldots \ldots$

51. Functional comparison of historic artifacts in the dugout and

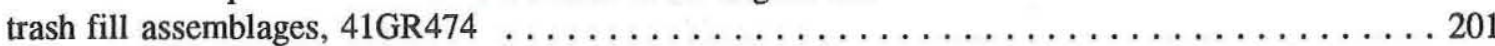

52. Functional comparison between $41 \mathrm{GR} 474$ artifacts and various artifact

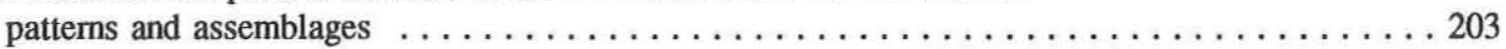

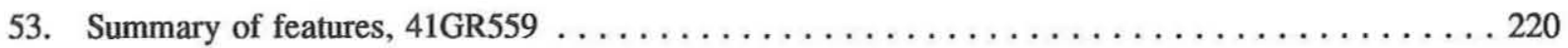

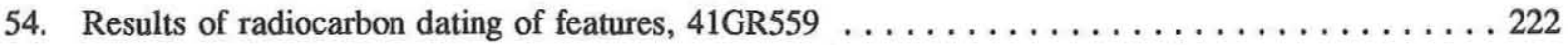

55. Summary of feature flotation samples and macrobotanical recovery, 41GR559 $\ldots \ldots \ldots \ldots 224$

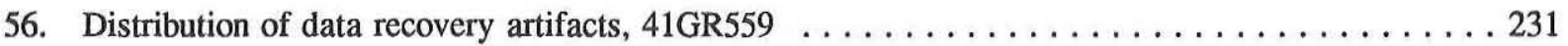

57. Breakdown of debitage attributes within the fine-grained chert and

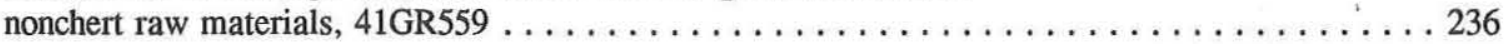

58. Summary of features by type and temporal affiliation, 41 GR559 $\ldots \ldots \ldots \ldots \ldots \ldots \ldots 242$

59. Artifacts recovered from all phases of work at $41 \mathrm{GR} 559 \ldots \ldots \ldots \ldots \ldots \ldots \ldots \ldots \ldots \ldots \ldots \ldots \ldots \ldots$

60. Vertebrate faunal remains from all phases of work at 41 GR559 $\ldots \ldots \ldots \ldots \ldots \ldots \ldots 245$ 


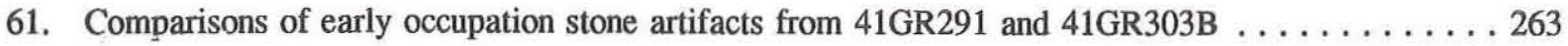

62. Comparisons of late occupation stone artifacts from 41GR291 and 41GR303B $\ldots \ldots \ldots \ldots 268$

63. Grain size, organic matter, and carbonate analysis of sediments from

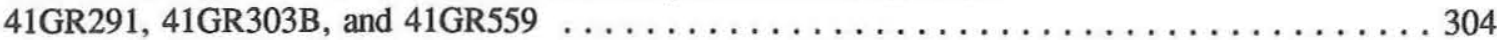

64. Dry sieve analysis of sediments from Boren Shelter \#2, 41GR559 $\ldots \ldots \ldots \ldots \ldots \ldots \ldots \ldots$

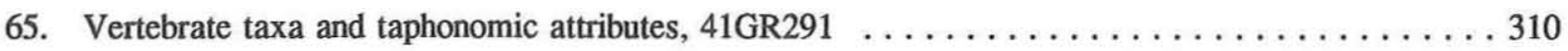

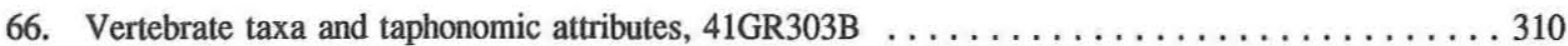

67. Vertebrate taxa and taphonomic attributes, 41GR474 $\ldots \ldots \ldots \ldots \ldots \ldots \ldots \ldots \ldots \ldots \ldots \ldots \ldots$

68. Number of individual specimens and minimum number of individuals in the

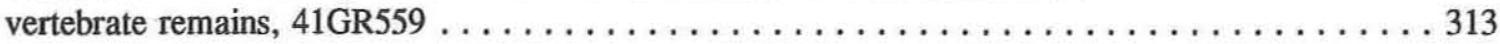

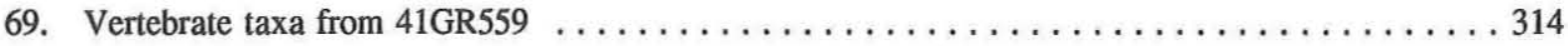

70. Canid elements recovered by area and feature, 41 GR559 $\ldots \ldots \ldots \ldots \ldots \ldots \ldots \ldots \ldots \ldots \ldots \ldots \ldots \ldots \ldots$

71. Vertebrate taxa by feature and nonfeature areas, 41GR559 $\ldots \ldots \ldots \ldots \ldots \ldots \ldots \ldots \ldots$

72. Vertebrate taxa with possible cultural modifications, 41 GR559 $\ldots \ldots \ldots \ldots \ldots \ldots \ldots \ldots 320$

73. Invertebrate faunal remains from 41GR291, 41GR303B, and 41GR559 $\ldots \ldots \ldots \ldots \ldots \ldots$

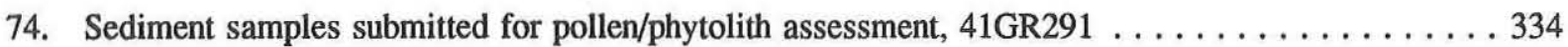

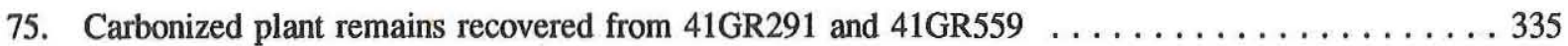

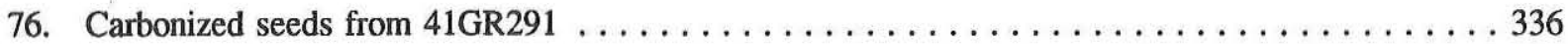

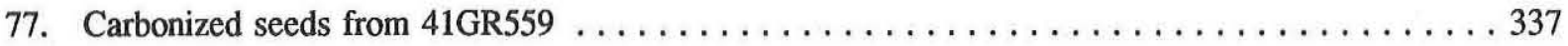

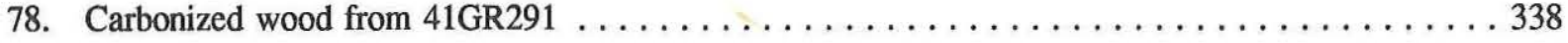

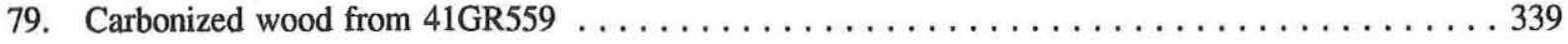

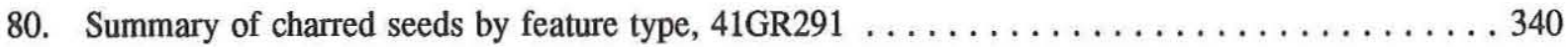

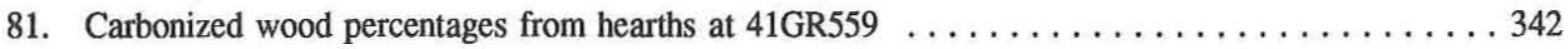

82. Summary of charred wood recovery by feature type at 41 GR $291 \ldots \ldots \ldots \ldots \ldots \ldots \ldots 34$

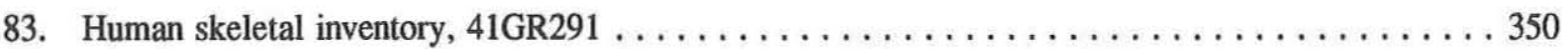

84. Tibia length and stature of prehistoric Texas populations $\ldots \ldots \ldots \ldots \ldots \ldots \ldots \ldots \ldots \ldots \ldots \ldots \ldots$

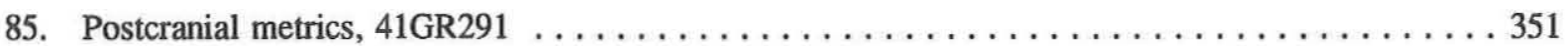

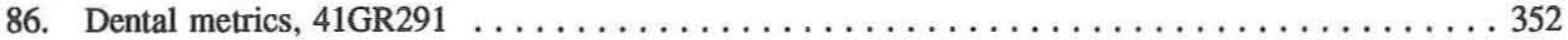

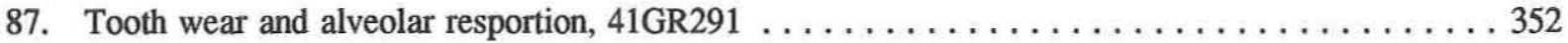

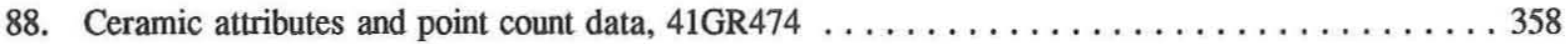

89. Provenience of projectile points from 41GR291, 41GR303B, and 41GR559 $\ldots \ldots \ldots \ldots \ldots 365$

90. Provenience of perforators and gouges from 41GR291 and 41GR303B $\ldots \ldots \ldots \ldots \ldots \ldots \ldots$

91. Provenience of nonprojectile point bifaces from 41GR291 and 41GR303B $\ldots \ldots \ldots \ldots \ldots \ldots 7$

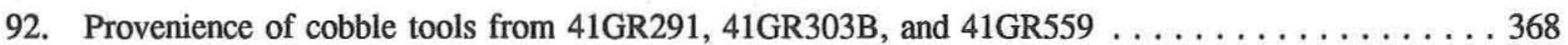

93. Provenience of unifaces from 41GR291, 41GR303B, 41GR546, and 41GR559 $\ldots \ldots \ldots \ldots 370$

94. Provenience of ground and battered stones from 41GR291,

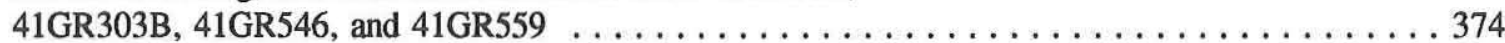


95. Provenience of ceramics, modified hematite, and modified shells from

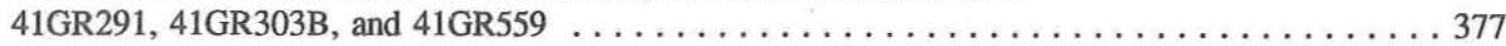

96. Historic and modern Euro-American inscriptions and vandalism at $41 \mathrm{KT} 164 \ldots \ldots \ldots \ldots 383$ 


\section{ABSTRACT}

The third and final season of Phase III data recovery at Lake Alan Henry (formerly Justiceburg Reservoir), located on the Double Mountain Fork of the Brazos River in Garza and Kent counties, Texas, was conducted during the fall of 1992. The work consisted of intensive archeological investigations at one historic site and four prehistoric sites. Subsequent to the Season 3 data recovery fieldwork, a newly discovered rock art site (41KT164) was documented.

The historic component at 41GR474 consists of a dugout depression and the ruins of a wood frame house and related complex of ranching structures. It was the homestead of Jeff Justice, Sr., who platted the community of Justiceburg in 1910. The Justice family lived in the dugout from 1900-1907. Excavations revealed a typical Plains-style half dugout with upper walls of native sandstone, a roof superstructure of juniper logs, and a sandstone fireplace. Artifacts and features on and near the floor include items from the dugout occupation period, but most represent a short episode (ca. 1907-1910) of reuse as a multifunctional workshop. Numerous artifacts in the upper fill are typical of early twentieth-century household debris and indicate use of the abandoned dugout for trash disposal.

The Sam Wahl site (41GR291) yielded an isolated secondary, cairn-covered interment of an adult male radiocarbon dated to A.D. $240-400$. The early occupation period, A.D. $600-1050$, is interpreted as a seasonal residential base related to the procurement, processing, and storage of plant foods; it may represent the Palo Duro complex. It is characterized by a pithouse, storage pits, hearths/baking pits, bedrock mortars, and an abundance of ground stones. The late occupation period, A.D. 1150-1400, lacks the distinctive features of the earlier period. A decrease in the number of ground stones and an increase in arrow point frequency may indicate a shift toward a hunting-oriented subsistence.

Two occupation periods recognized at the Cat Hollow site (41GR303B) approximate those at the Sam Wahl site. The Late Prehistoric I period is characterized by baking pits and an abundance of scattered burned rocks, apparently representing use of the site as a specialized plant processing area. The upper deposits, dating to the latter part of the Late Prehistoric I period or the early Late Prehistoric II period, lack evidence for intensive plant processing, and increases in some classes of chipped stone tools indicate an increased emphasis on hunting.

Two completely excavated rockshelters yielded evidence of Late Prehistoric and Protohistoric occupations. Boren Shelter \#1 (41GR546), only $12 \mathrm{~m}^{2}$, yielded few artifacts and three stone-lined hearths, one radiocarbon dated to ca. A.D. 1643. The site saw ephemeral use during the Protohistoric period. Boren Shelter \#2 (41GR559), with a sheltered area of $30 \mathrm{~m}^{2}$, contained sparse artifacts and numerous hearths; a midden area in front of the shelter contained a number of clusters of animal bones. The deposits inside the shelter can be separated into two periods of occupation, A.D. 0-1000 and A.D. 1000-1300, during which the site was used infrequently as a short-term campsite. The deposits in front of the shelter, with a single radiocarbon date of A.D. 1500-1660, reflect use of the site as a hunting camp/processing location during the Protohistoric period.

Collectively, these sites indicate that significant cultural changes took place around A.D. 1100, when people at the Sam Wahl and Cat Hollow sites changed their economic strategies. The Lake Alan Henry data correspond with other regional archeological data, and there is a growing body of evidence indicating that the Late Prehistoric I period (ca. A.D. 1-1100) was radically different from the Late Prehistoric II period (ca. A.D. 1100-1541). Intensive utilization of plant resources seems to characterize the occupations prior to A.D. 1100. Archeological evidence indicates that after A.D. 1100, subsistence strategies shifted away from intensive plant utilization toward more broad-based foraging with a greater emphasis on hunting. This change in subsistence strategies probably was related to regional climatic changes. 


\section{ACKNOWLEDGMENTS}

The final season of data recovery at Lake Alan Henry was a productive venture due to the efforts and contributions of many institutions and individuals. Foremost, the City of Lubbock provided the funding for these studies, and our work was coordinated by Dan A. Hawkins, Director of Water Utilities, and Mike Gilliland, Engineering Assistant. Barbara A. Nickerson of Freese and Nichols, Inc. served as Technical Representative for the City and acted as liaison, coordinating with the regulatory agencies represented by Robert F. Scott, IV of the Fort Worth District, U.S. Army Corps of Engineers, and James A. Bruseth and Nancy Kenmotsu of the Texas Historical Commission. Special thanks go to these people for sponsoring and promoting the Lake Alan Henry Archeology Open House on November 10, 1992. This event bolstered public awareness of Southern Plains archeology by allowing people to witness fieldwork in progress and through extensive media coverage.

The archeological investigations were a collaborative undertaking, and this report is the product of many contributors, from the field crew to the editors. Sincere thanks go to all of these people for their efforts. Field crewmembers for this season included Justiceburg veterans Wayne Chesser (who participated in the survey and in all three seasons of data recovery) and Rick Walter (for his second season of data recovery), and Rob Barros, David Cullom, Diane Kimbrell, Christina Leshley, Todd Reynolds, and Nick Valentine, who joined us for the first time. Two individuals, Ron Bornick, of Lake Ransom Canyon and long-time avocational archeologist Emmett Shedd, volunteered their labor on many days throughout the season, and their help is greatly appreciated. In addition, Dr. Grant Hall brought groups of his Texas Tech University anthropology students to volunteer, and some students came out again on other occasions. Students who volunteered on several days are Bonnie Alexander, Grace Brians, David Cross, Richard Forrester, Bob Hackeman, Stewart Hueston, Kent Richardson, Nancy Russell, Russ Shortes, and Chris Silman.

The Project Archeologists are especially indebted to two professional archeologists, retired Professor of Anthropology Dr. Jack T. Hughes (West Texas State University, Canyon) and Regge Wiseman (Office of Archeological Studies, Museum of New Mexico, Santa Fe), who visited the site and offered freely their extensive knowledge and advice.

Local landowners who contributed significantly to the project are Mr. and Mrs. Odie Hood, owners of the Hood Ranch at Justiceburg. Not only did they allow us access to the site on their ranch, but the Hoods provided valuable historical information during several site visits and in correspondence. Special thanks also go to John and Betty Boren, who gave us permission to excavate the two rockshelters located on their land within the reservoir's flood easement. Granite Construction Company employee Bryan Callahan is commended for reporting the discovery of a previously unknown rock art site in the project area.

During the analysis stage, Wiseman assisted in identifying the brownware ceramics, while Dr. David G. Robinson (Texas Archeological Research Laboratory, The University of Texas at Austin) conducted the petrographic analysis of selected sherds. Textural and chemical analyses of sediment samples were conducted by Michael Kolb (Soils and Physical Geography Laboratory, University of Wisconsin-Milwaukee), and radiocarbon assays were done by Beta Analytic, Inc. (Miami, Florida). Special studies conducted at the Department of Anthropology, Texas A\&M University were the macrobotanical analysis and assessment of pollen/phytolith preservation by Phil Dering, an analysis of vertebrate faunal remains by Brian S. Shaffer, and an analysis of human skeletal remains from 41GR291 by Dr. Gentry Steele and Barry Baker. Obsidian samples were submitted by Dr. Thomas R. Hester for the Texas Obsidian Project to the Lawrence Berkeley Laboratory of Berkeley, California, for nondestructive $\mathrm{x}$-ray fluorescence trace element analysis.

The staff at Prewitt and Associates, Inc. participated in all phases of this project and deserve special recognition for their professional assistance. Ross C. Fields and Linda Nance Foster did their usual superb job of editing and significantly improved the overall quality of this report. Fields also served as Co-Principal 
Investigator and had the daunting task of coordinating the work of the various authors and consultants and integrating their results into the report. His patience and perseverance are especially appreciated by the Project Archeologists. Adding significantly to the quality of the report, Sandra L. Hannum drafted the maps and figures, and E. Ellen Atha prepared the artifact illustrations. The laboratory processing and quantitative analysis of artifacts were efficiently directed by Jeanine McDonald and Karen M. Gardner. Jerrilyn B. McLerran catalogued the multitude of photographs taken during the field season. And finally, Co-Principal Investigator Elton R. Prewitt has overseen all phases of the cultural resource studies at Lake Alan Henry and has kept the project on track since 1987. 


\section{INTRODUCTION AND ENVIRONMENTAL AND ARCHEOLOGICAL BACKGROUND}

Douglas K. Boyd

\section{INTRODUCTION ,}

This report documents the third and final season of data recovery investigations at Lake Alan Henry (formerly Justiceburg Reservoir) ${ }^{1}$, located on the Double Mountain Fork of the Brazos River in Garza and Kent counties, Texas (Figures 1 and 2). The fieldwork conducted from September to December of 1992 consisted of intensive archeological investigations at prehistoric sites 41GR291, 41GR303B, 41GR546, and 41GR559 and historic site 41GR474. The historic site is located adjacent to the Double Mountain Fork in the upper portion of the reservoir and may be subject to periodic inundation, while all of the investigated prehistoric sites are located within the Sam Wahl Recreation Area and may be subject to a variety of impacts during future development of this public access area.

The archeological investigations entailed the

\footnotetext{
${ }^{1}$ The original name of Justiceburg Reservoir referred to the small community of Justiceburg located near the upstream end of the future lake. In 1987 the Lubbock City Council changed the name of the project to Lake Alan Henry in honor of the former mayor who led the efforts to fund construction of the city's future water-supply reservoir. Lake Alan Henry will be impounded behind the John Montford Dam, which is named for Lubbock's state senator who assisted in successfully promoting the project. Because of the change in the project name, some historical references to Justiceburg Reservoir are made in this report. However, for work done in 1987 and later, the label of Lake Alan Henry is used.
}

excavation of thirty-three $1-x-1-m$ units (ca. $24 \mathrm{~m}^{3}$ ) at rockshelters 41GR546 and 41GR559 and one hundred forty-one $1-\mathrm{x}-1-\mathrm{m}$ units (ca. $67 \mathrm{~m}^{3}$ ) at open sites 41GR291 and 41GR303B, and they resulted in the recovery of 6,043 prehistoric artifacts. In addition, ca. $25 \mathrm{~m}^{3}$ of fill was excavated to expose the dugout at 41GR474, and 2,321 historic artifacts were recovered.

This third season of data recovery at Lake Alan Henry, like all of the cultural resources investigations conducted since the inception of the project in 1987, was sponsored and financed by the City of Lubbock. This work has been performed under a Memorandum of Agreement among the U.S. Army Corps of Engineers, the State Historic Preservation Officer, and the Advisory Council on Historic Preservation, with the concurrence of the City of Lubbock, and since 1989 it has been guided by a comprehensive research design and data recovery plan developed by Prewitt and Associates, Inc. and reviewed and approved by cultural resources personnel of the City of Lubbock's Technical Representative (Freese and Nichols, Inc.) and the signatories to the Memorandum of Agreement. The investigations reported here are in accordance with the terms and stipulations of the Memorandum of Agreement and the Phase III data recovery plan.

The Phase III data recovery plan produced in 1989 identified the sites to be investigated during the mitigation phase and established the level of effort for each site. The plan proposed that the Phase III investigations be conducted in three 


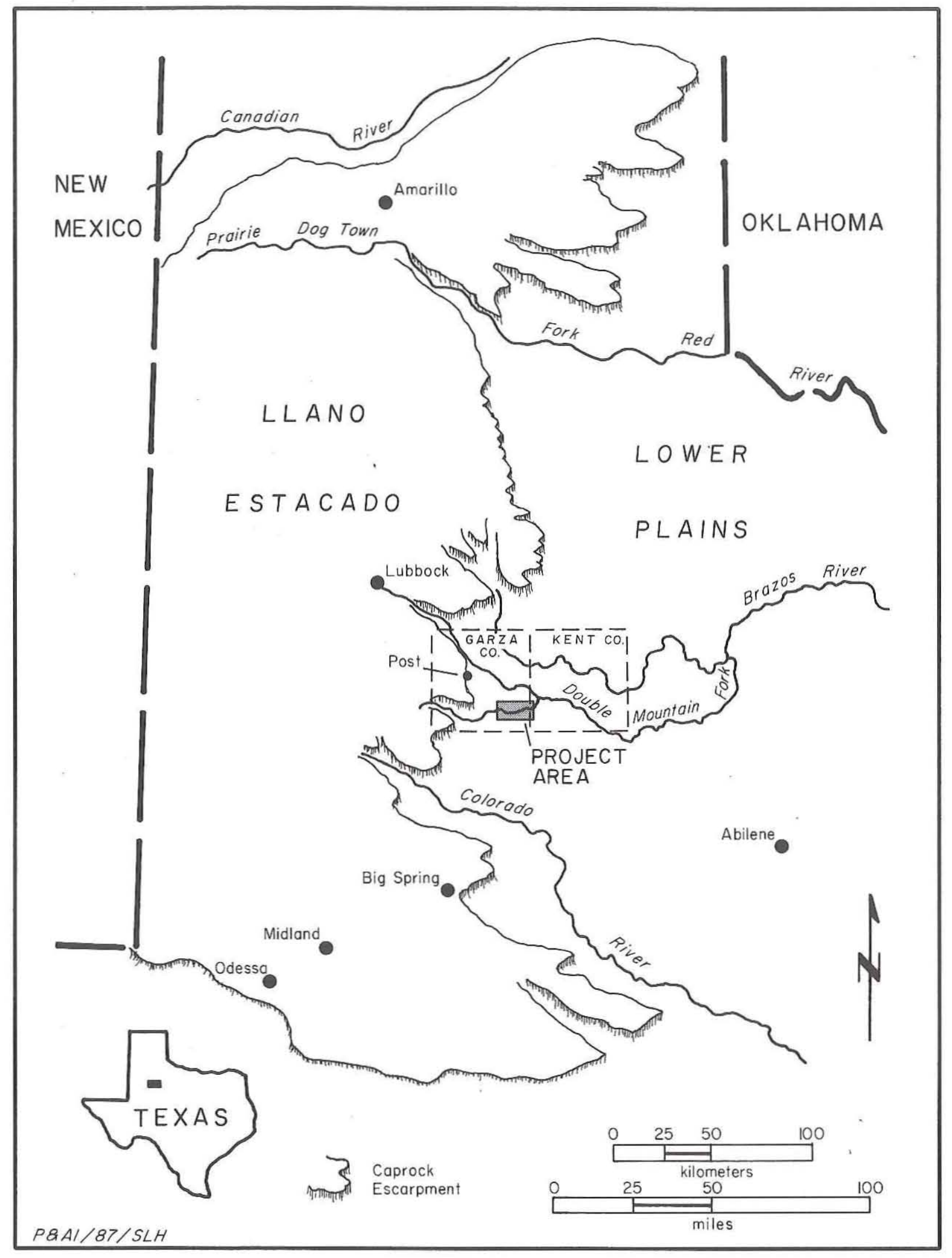

Figure 1. General location map. 


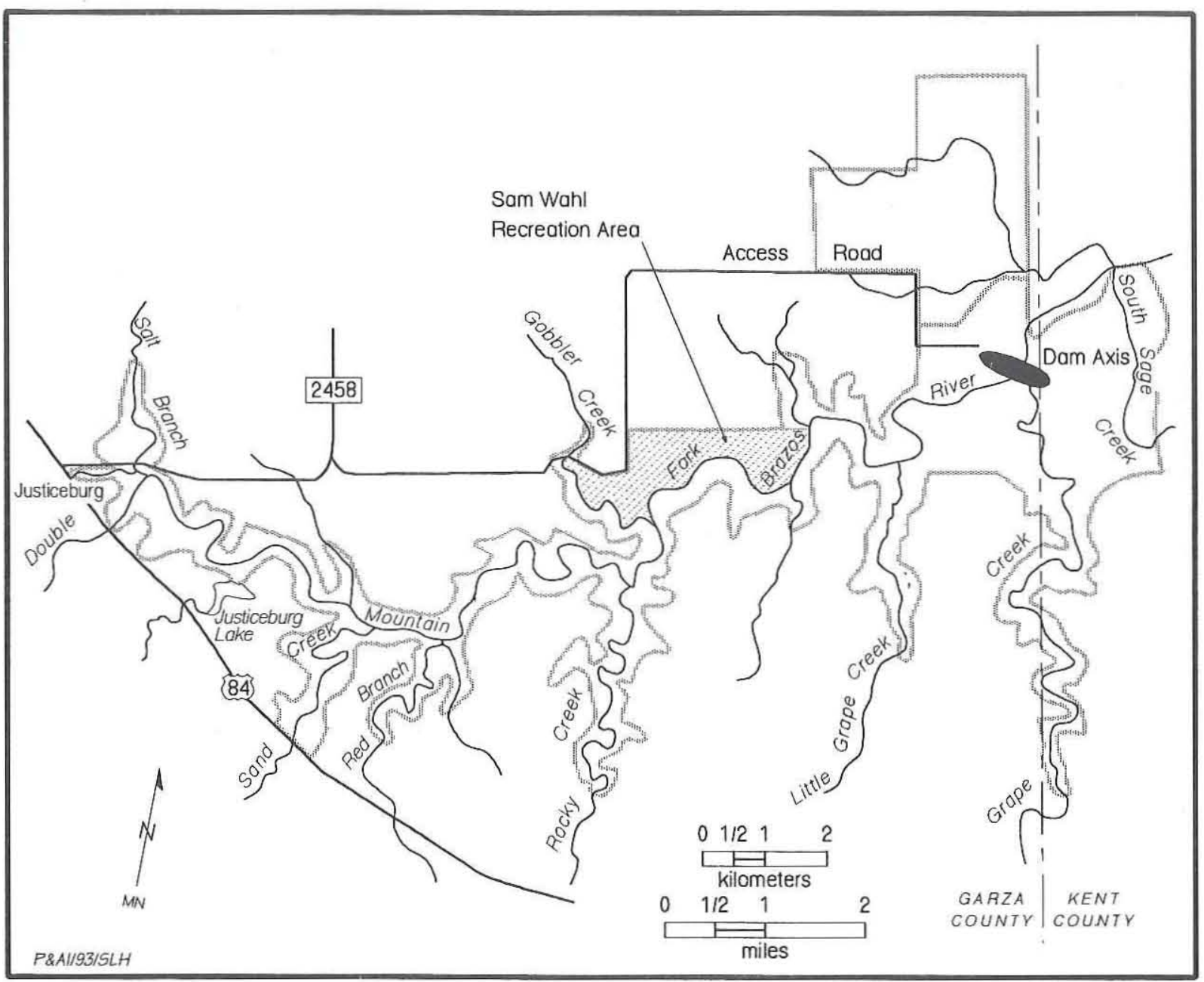

Figure 2. Project area map.

seasons of fieldwork, with work tasks prioritized according to the reservoir construction and inundation schedule. The plan also called for each field season to be followed by a period of laboratory processing and analysis, resulting in a descriptive report covering only the investigations undertaken during that season. The first and second field seasons of data recovery were conducted in 1990 and 1991, and descriptive reports have been completed (Boyd et al. 1992, 1993). This report deals only with the third season of work, and it will be followed by a period of comparative analytical research which will result in a final synthetic report. The final synthesis, scheduled for completion in 1995, will summarize all of the survey, testing, and data recovery investigations, place the work within a regional perspective, and address the specific and general research questions proposed in the research design.

This report consists of eight chapters and eight appendixes. The remainder of this chapter provides environmental and archeological background information for the project area. Chapter 2 summarizes pertinent portions of the research design, discusses the specific objectives that guided these investigations, and describes the methodologies used. Chapters 3 through 7 describe the data recovery investigations at the five sites. In each of these chapters, cultural features encountered and materials recovered are described and interpretations of the archeological evidence are offered. Chapters 3 and 4 describe the investigations at open prehistoric sites 41GR291 and 41GR303B, respectively; Chapter 5 presents the results of the investigation of a historic ranching dugout at 41GR474; and excavations at two prehistoric 
rockshelters, 41GR546 and 41GR559, are reported in Chapters 6 and 7, respectively. Chapter 8 summarizes the investigations and presents interpretations related to the research design.

The appendixes consist of reports on special studies and other supporting data. Appendixes A and B present profile descriptions and sediment data resulting from the on-site geomorphic investigations by Project Geomorphologist Karl W. Kibler. Analyses of the vertebrate and invertebrate fauna are described in Appendixes C and D, respectively, and an analysis of plant remains (macrobotanical and pollen/phytolith) is presented in Appendix E. Two appendixes relate specifically to 41GR291; human skeletal materials recovered from a burial are analyzed in Appendix F, and a petrographic analysis of nonlocal brownware ceramics is presented in Appendix G. Appendix H contains provenience data for selected artifact classes. Finally, Appendix I details the discovery and documentation of the Callahan petroglyph site (41KT164), a previously unknown rock art site discovered during the summer of 1993 after the Season 3 data recovery field season.

\section{ENVIRONMENTAL BACKGROUND}

Lake Alan Henry is located within the Lower Plains region of Texas (Brown et al. 1982), also referred to as the Rolling Plains (Lobeck 1948). The Lower Plains region is bordered on the north by the Canadian River in Oklahoma, on the east by the Western Cross Timbers region, on the south by the Edwards Plateau of Central Texas, and on the west by the Llano Estacado, the southernmost extension of the High Plains. The Llano Estacado, or High Plains, is the dominant physiographic feature, and the Caprock Escarpment clearly marks the boundary between the Lower Plains and the High Plains. The project area is several kilometers east of the Caprock Escarpment.

The flat, featureless plain of the Llano Estacado provides a stark contrast to the undulating, eroded badland topography of the Lower Plains. The rolling landscape is the result of the erosion and retreat of the High Plains. During the last several hundred thousand years, the Caprock Escarpment has been retreating westward, exposing to erosion the underlying Triassic and Permian beds. The region is further dissected by wellentrenched rivers and tributary drainages which head on the Llano Estacado or at the Caprock Escarpment. Three river systems - the Red, Brazos, and Colorado - flow generally eastward through the Lower Plains region. The surface waters originate mainly as rainfall or from spring seeps from groundwater aquifers.

The Lake Alan Henry project area lies mostly within Triassic exposures consisting of alternating layers of mudstones, conglomerates, and sandstones. Where the Double Mountain Fork has cut down through the erosion-resistant sandstone layers, the topography is quite rugged. This segment of the valley, in the middle portion of the reservoir from the mouth of Sand Creek to just below the mouth of Little Grape Creek, is a steepsided, narrow-valley canyon. The canyon rim and lower erosional remnants are capped by sandstone strata. The valley slope is nearly vertical in places, and the local relief varies from 18.3$30.5 \mathrm{~m}(60-100 \mathrm{ft})$. The canyon is less than $200 \mathrm{~m}$ wide at the bottom, and the river meanders back and forth within the valley, leaving only thin strips of alluvial floodplain between the canyon wall and stream.

The topography differs upstream and downstream from this canyon-confined segment of the river. The eastern end of the reservoir, near the mouth of Grape Creek, is also a steep-sided canyon, but the valley widens out considerably with an alluvial floodplain over $1 \mathrm{~km}$ wide. In the western upstream end of the reservoir near the community of Justiceburg, canyon cutting is not pronounced because the river has not cut down through the erosion-resistant sandstone strata. The river channel is within a wide shallow valley which slopes gradually into the eroded upland.

The rugged topography in the project area is characterized by a range of distinctive landforms (Figure 3). The upland and upland margin settings are found all along the canyon rim. In some areas, the flat upland ends abruptly at the canyon rim with little or no eroded upland margin. In other areas, the eroding upland margin extends hundreds of meters back from the canyon rim. Abrupt canyon rims are marked by erosion-resistant sandstone ledges which usually form a bluff several meters high. Erosion along the bluff has created numerous overhangs and shelters throughout the project area. The valley wall, the area between the upper bluff and the valley floor, consists of talus slopes, isolated mesas, erosional 


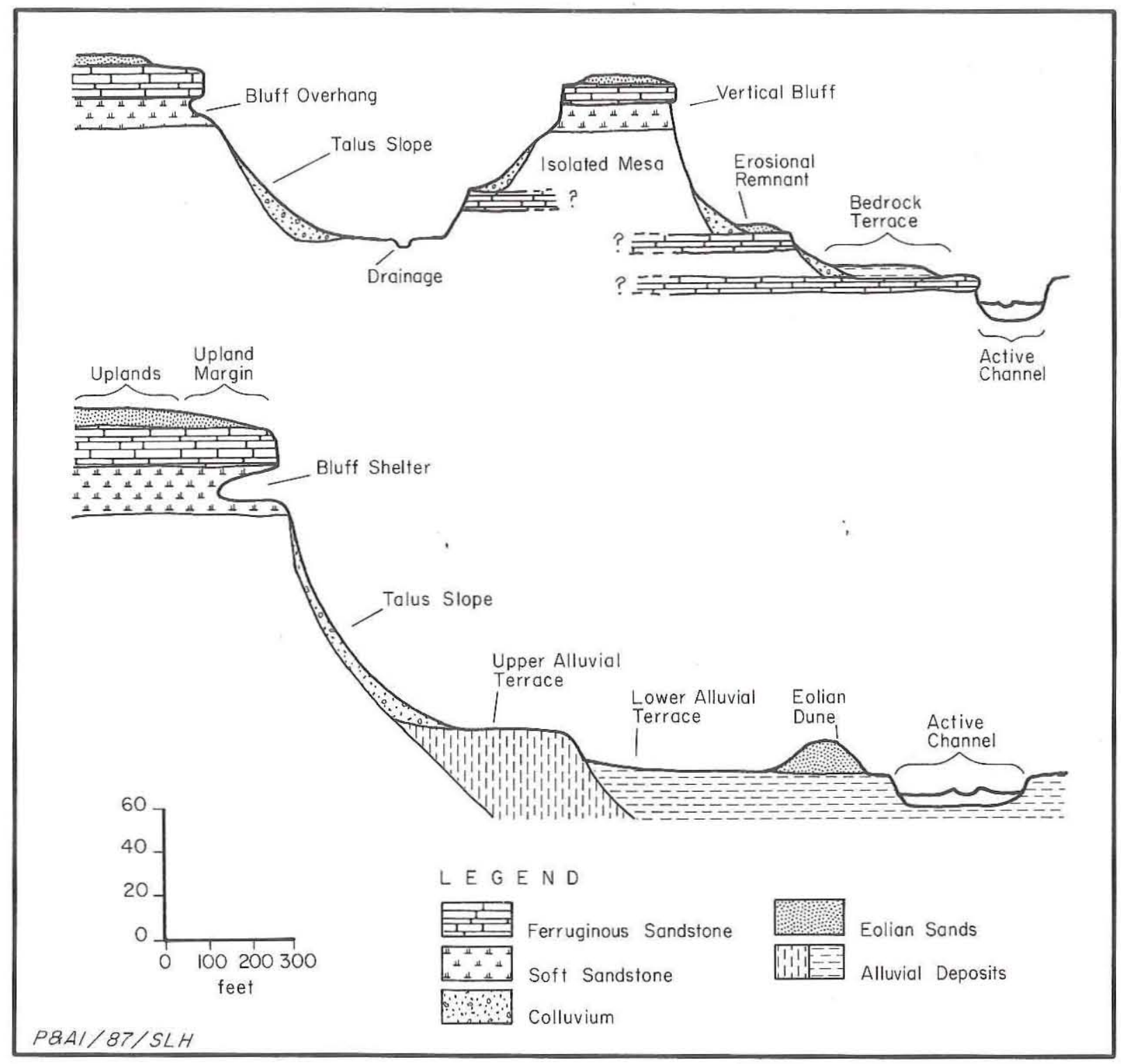

Figure 3. Generalized landforms.

remnants, bedrock benches, or any combination of these landforms. Talus slopes are composed of softer and more-easily eroded mudstones and siltstones and are characteristically very steep. Talus slopes may be $20-30 \mathrm{~m}$ high, extending from the canyon rim to the valley floor, or they may cover only a few meters between exposed ledges of sandstone. In heavily eroded areas with numerous layers of erosion-resistant sandstone, the valley wall stairsteps, forming projecting erosional remnants, bedrock terraces, and isolated mesas. The valley wall landforms constitute a considerable portion of the project area, and the surface distance between the canyon rim and the valley floor may be hundreds of meters. The valley floor consists of the meandering channel of the Double Mountain Fork or its tributaries and alluvial terraces. It varies from a few meters to over $1,000 \mathrm{~m}$ wide. In the lower portion of the reservoir, upper and lower alluvial terraces can be distinguished, but in the remainder usually only a single terrace is evident. A few eolian sand dunes have formed on the alluvial terraces at the upper end of the reservoir, and occasional levees are evident along the river 
and stream channel margins.

The modern climate in the project area (from Scurry County data in Pass 1981:122) is mild, with short periods of extreme heat or cold. The mean minimum temperature in January is $-2^{\circ} \mathrm{C}\left(28^{\circ} \mathrm{F}\right)$ and the mean maximum in July is $35.6^{\circ} \mathrm{C}\left(96^{\circ} \mathrm{F}\right)$, but extremes range from $-23.3^{\circ} \mathrm{C}\left(-10^{\circ} \mathrm{F}\right)$ to $46.1^{\circ} \mathrm{C}\left(115^{\circ} \mathrm{F}\right)$. The growing season averages 214 days. The average yearly rainfall is $49.07 \mathrm{~cm}$ (19.32 inches), with most occurring during the spring and summer months. Windspeed at Lubbock averages $20.6 \mathrm{kph}(12.8 \mathrm{mph})$, but a maximum windspeed of $143.3 \mathrm{kph}(89 \mathrm{mph})$ has been recorded (Pass 1981:111). The prevailing winds come from the south and southwest. Severe weather conditions, i.e., blizzards, droughts, and thunderstorms, are not uncommon.

The project area is within the Mesquite Plains district of the Kansan biotic province (Blair 1950). Vegetation in the region can be categorized generally into three floral assemblages occupying different topographic settings: (1) the juniper-mesquite/ upland slope assemblage; (2) the mesquiteoldfield/terrace-floodplain assemblage; and (3) the saltcedar/riparian floral assemblage (Kenmotsu 1981:343-346). Lists of flora found in the region are presented in Boyd et al. (1989:19-20), Wulfkuhle (1986:40-44), Kenmotsu (1981:343346), Etchieson et al. (1979:25-32), Hutchins (1968), and Rowell (1967). Recent modification to plant and animal communities is apparent throughout the region, but little effort has been made to identify native species, particularly those that are no longer extant. Portions of the project area have undergone severe alterations, but some locations appear to be relatively undisturbed.

\section{PREVIOUS INVESTIGATIONS}

The history of archeological investigations in the Lake Alan Henry area and the development of the region's cultural sequence are long and complex stories. A comprehensive archeological background is presented in Boyd et al. (1989:23-59), and only brief synopses are presented here and in the following section.

Archeological interest in the Justiceburg area began in the 1950s as members of the South Plains Archeological Society began documenting and investigating sites in Garza County. Professional archeological investigations, however, did not begin until Justiceburg Reservoir was in the early planning stages in the 1970s. The initial archeological work was a survey of the proposed reservoir area funded by the South Plains Association of Governments (SPAG). This work, conducted in 19741975 , produced an inventory of archeological sites and resulted in a series of reports (Campbell 1975, 1977; Campbell and Judd 1977a, 1977b; Judd 1977), but these reports made minimal recommendations for treatment of the resources. A second archeological investigation (Alexander 1982) documented selected sites in order to evaluate the results of the SPAG survey and their recommendations. Alexander (1982:3-4) concluded that the cultural resources were not adequately recorded and that their true significance had been underestimated. A complete resurvey of the proposed Justiceburg Reservoir area and a reevaluation of its cultural resources was recommended.

The proposed Lake Alan Henry area, including the construction zones, the flood pool, and a shoreline easement around the reservoir (ca. 8,600 acres), was intensively resurveyed by Prewitt and Associates, Inc. in 1987. This Phase I investigation included detailed regional prehistoric and historic overviews, subsurface geoarcheological testing, and an intensive pedestrian survey (Boyd et al. 1989). The survey resulted in the documentation of 330 sites (excluding 45 isolated finds), and site significance was reevaluated. Sites were assessed relative to specific prehistoric and historic research problems identified for the Lower Plains region of Texas. Most of the sites $(n=238)$ were considered to be potentially eligible for listing on the National Register of Historic Places because of their integrity and potential to provide data relevant to addressing regional research problems. It was recommended that a sample of the various site types be tested to determine their National Register significance.

Phase II testing of a sample of the cultural resources conducted by Prewitt and Associates, Inc. in 1988 included various levels of archeological testing and documentation at 67 sites (Boyd et al. 1990; Freeman and Boyd 1990). Intensive geoarcheological investigations were undertaken as well as the development of two regional historic contexts and a prehistoric research design to provide the framework for National Register assessments of the historic and prehistoric resources. Based on these contextual models, it 
was recommended that 17 prehistoric and rock art sites and 7 historic sites be considered eligible for listing on the National Register of Historic Places.

The next cultural resources investigation at Lake Alan Henry was an archeological survey of ca. 2,440 acres in Garza County immediately north of the dam site. The City of Lubbock was considering the purchase of this land to serve as wildlife habitat to mitigate the destruction of similar habitat in the reservoir. The wildlife mitigation lands survey (Boyd et al. 1991), conducted in August and September of 1990, documented 1 historic and 32 prehistoric archeological sites where none had been recorded previously. Nine of these sites were evaluated as being significant or potentially significant, and appropriate recommendations were made to protect the sites from impact during development of the wildlife area.

The final step in the Lake Alan Henry cultural resources program is the Phase III data recovery effort, which was initiated in the fall of 1990 . The Phase III effort encompassed three separate seasons of fieldwork and included archeological investigations of prehistoric, historic, and rock art sites as well as additional geomorphic and paleoenvironmental studies. The first and second seasons of Phase III work have been reported (Boyd et al. 1992, 1993), and this report documents the final season.

\section{CULTURAL CHRONOLOGY}

The Lower Plains of Texas have been inhabited by humans for at least the last ca. 12,000 years, but the archeological studies at Lake Alan Henry suggest that the region's archeological record is extremely biased. Severe erosion of the landscape over thousands of years has removed a considerable amount of the late Pleistocene and early/middle Holocene sediments and landforms. The result is an archeological record dominated by late Holocene (i.e., late Archaic, Late Prehistoric, and Historic) sites. This phenomenon has been noted in the past but only now is beginning to be understood. A growing body of paleoenvironmental, archeological, and geomorphological evidence for the Southern Plains strongly suggests that a long period of severe aridity during the mid Holocene, called the Altithermal period, was responsible for the accelerated erosion and removal of earlier sediments. While the evidence is far from conclusive, this phenomenon is observable in the geomorphic record at Lake Alan Henry.

The Paleoindian and early/middle Archaic periods (ca. 11,000-4500 B.P.) are not well represented in the Lower Plains. Diagnostic projectile points dating to these periods are sometimes found, but intact cultural deposits of this age are rare. In the Lake Alan Henry area, no intact components firmly dated to these time periods have been found, and only a few possible candidates exist in disturbed contexts. In the canyonland environment in and around the reservoir, it appears that erosion and retreat of the canyon rim and valley walls occur very rapidly, and it is doubtful if evidence of any early human occupations along the upland margin would be preserved. Likewise, the Double Mountain Fork of the Brazos River and its major tributaries are dynamic fluvial systems that apparently have stripped away all of the early sediments except for a few isolated remnants. Thus, it appears that the potential for Paleoindian and early/middle Archaic occupations now exists mainly in the uplands, and such occupations most likely would be found near large playa lakes or on high rises within a few hundred meters of the canyon rim.

The prehistoric archeological record at Lake Alan Henry dates almost entirely to the late Archaic (ca. 4500-2000 B.P.) and Late Prehistoric (ca. 2000 B.P. to European contact) periods, and sites from both periods are preserved along the eroding upland margin, on lower bedrock benches, and in the stream valley alluvial terraces. No late Archaic cultural phases or complexes have been adequately defined for the region, but late Archaic sites are very common. The Summers complex proposed for southwestern Oklahoma (Leonhardy 1966) may be a valid cultural unit in the Texas Lower Plains, but this remains to be demonstrated. Leonhardy (1966:32) noted similarities in the cultural materials between the Summers complex and late Archaic materials in Central Texas and in the Texas Panhandle.

The region's Late Prehistoric period is only somewhat better understood. Ceramics and arrow points appeared early in the first millenium A.D., and the Texas Panhandle-Plains sites show influence from two different areas. The Late Prehistoric I period (ca. 2000-1000 B.P.) in the northern Panhandle-Plains is characterized by 
occupations with demonstrated Woodland influence (e.g., cordmarked pottery), while contemporaneous occupations in the southern Panhandle-Plains show strong cultural ties with the Southwest. The Palo Duro complex defined for the southern Lower Plains (Willey and Hughes 1978) is characterized by Mogollon-style pithouse architecture and imported Jornada brownware pottery. Several sites in the Lake Alan Henry area have been recognized as belonging to the Palo Duro complex.

The Late Prehistoric II (ca. 1000 B.P. to European contact) and Protohistoric/Historic Native American (European contact to ca. A.D. 1875) periods include the Garza complex and historic Plains Indian occupations. The Garza complex, defined in the vicinity of Lake Alan Henry (Runkles 1964), dates to ca. 550-250 B.P. (A.D. 1400-1700) and is characterized as a bison- hunting nomadic lifestyle that may be associated with an influx of bison back into the Southern Plains after a period of relative scarcity. Only a few sites associated with the Garza complex have been investigated archeologically, however, and the complex remains poorly defined and understood. Many sites in the Lake Alan Henry area date to the Late Prehistoric II time period, and it is likely that at least some belong to the Garza complex, even though no Garza arrow points characteristic of the complex have been found. Protohistoric campsites have been identified in the Lake Alan Henry area, but they cannot yet be assigned to a cultural complex. The use of the area by protohistoric/historic Plains Indian groups also is evidenced in historic records and by the numerous rock art sites that contain historic Plains Biographic Style motifs. 


\section{RESEARCH ORIENTATION, OBJECTIVES, AND METHODS}

Douglas K. Boyd, Steve A. Tomka, Martha Doty Freeman, and Jay Peck

Archeological research in the southem Lower Plains has not been extensive, and the Lake Alan Henry studies constitute the largest single archeological data base in the region. In conjunction with the Phase II investigations, a detailed research design was developed to provide the necessary foundation for assessing the significance of late Archaic, Late Prehistoric, and Historic sites relative to regional research problems (Boyd et al. 1990). The research design was expanded following the Season 2 data recovery to account for the potential effects of the acquisition of horses and Euro-American goods during the Protohistoric period. The following section presents a synopsis of the prehistoric research design. Following this is a discussion of the research design, objectives, and methods employed in the investigations at historic site 41GR474 and descriptions of the objectives of the geoarcheological investigations and the laboratory and analysis procedures used.

\section{PREHISTORIC SITE INVESTIGATIONS}

\section{Late Holocene Human Adaptation}

The prehistoric site investigations were done according to the research goals set forth in "A Model of Late Holocene Human Adaptation" by Boyd et al. (1990:261-273). This model is based on work by Binford (1980), Kelly (1980, 1983), and Hayden $(1986,1987)$ and suggests that human subsistence strategies and settlement patterns are controlled in large part by resource distribution and that human social groups exploit resources using some combina- tion of two different resource acquisition patterns, i.e., as logistically oriented collectors or residentially mobile foragers. In a nutshell, collector strategies move food resources to residential camps through the employment of special task groups, while foragers move people to resources through frequent residential camp moves.

More specifically, the model proposes that a single resource, bison, was the main factor conditioning the selection of subsistence strategies and land-use patterns in the Lower Plains. The model suggests that climatic changes caused bison populations to fluctuate through time and that it was the abundance or scarcity of bison that controlled human subsistence strategy selection. Bison are viewed as the only sufficiently stable bulk resource in the Lower Plains that feasibly could have been exploited by humans under a logistically oriented collector strategy. During times of bison abundance, such as proposed for the late Archaic, Late Prehistoric II, and Protohistoric periods, human populations would have acted primarily as logistical collectors to exploit the bison, although shifts to forager-oriented strategies may have occurred in response to periodic bison scarcity as a result of the seasonal migration of herds within or out of the region. Thus, late Archaic, Late Prehistoric II, and Protohistoric period settlement systems may reflect a mix of collector and forager strategies. In prolonged times of bison scarcity, such as proposed for the Late Prehistoric I period, human populations would have operated primarily as foragers and exploited a wide range of less-abundant and less-dependable resources. Thus, the immediate 
goal of the Lake Alan Henry archeological research is to determine how individual sites fit within the collector-forager continuum, and the long-range goal is to identify, through intersite studies, the relationships between resource structure and land-use patterns through time and space.

Four major research topics pertinent to assessing this model at Lake Alan Henry have been defined: settlement patterns, subsistence, site structure, and material culture. Within each of these topics, two general hypotheses dealing with a regional level of interpretation were proposed in the research design. The first described archeological expectations under a collector-oriented resource procurement strategy, and the second discussed expectations under a forager-oriented strategy. These general hypotheses are reiterated in the following paragraphs. To a large extent, these discussions present idealized cases that are unlikely to mirror real archeological situations, as collector and forager strategies may have been employed in varying mixes. For this reason, elements of both sets of hypotheses may apply to a given time period.

\section{Settlement Patterns}

\section{Collector Systems}

Residential base camps within collector systems may be either single-season camps oriented toward procurement and processing of bison or multiseasonal base camps that were bison oriented on a seasonal basis but geared toward foraging for supplemental plant and animal resources during other parts of the year. In either case, lengthy site occupations and/or yearly reoccupations are likely, and low residential mobility and relatively high populations should be evident by site size, overall frequency of cultural materials and features, and intensity of site use. The presence of a relatively high percentage of nonlocal lithic materials and other exotic trade items may indicate large territory exploitation and participation in interregional exchange networks.

Logistically oriented collector systems may also include residential base camps that were season specific and oriented toward foraging for specific resources. Within the overall context of a collectordominated system, some residential base camps may have been solely foraging oriented and hence would appear no different archeologically than those described below.

\section{Forager Systems}

Residential base camps within an exclusively forager system should exhibit archeological evidence of highly mobile, relatively short term, seasonspecific occupations, although reoccupation on a seasonal basis is also likely. The sites served as base camps for daily foraging activities exploiting specific resource patches. It is possible that a single site may have been oriented toward a single resource, although it is more likely that multiple resources were exploited from a single base camp. Exclusive foragers exploited a relatively small overall territory that should be evident archeologically by low percentages of nonlocal lithic materials and few exotic trade items.

\section{Subsistence \\ Collector Systems}

Residential base camps that operated within a logistically oriented collector system will exhibit evidence of the bulk procurement, processing, and storage of bison, a high-yield, large-biomass resource. Exploitation of other plant and animal resources will also be evident in the flora and fauna recovered, especially at the residential sites occupied during seasons of relative bison scarcity. The overall faunal assemblages, however, will be dominated by bison and will be biased toward high-meat-yield body parts (e.g., ribs, scapulas, and femurs) that were selectively transported from kill sites back to the camps. Food storage of bulk resources is characteristic of collector systems but is not likely to include storage pits at bison-oriented sites. Meat drying and/or salting for storage probably were important activities at bison-oriented collector sites but may be difficult to recognize archeologically (e.g., postmolds indicating drying racks).

\section{Forager Systems}

Residential base camps generated by a forager system will exhibit evidence of exploitation of a diverse range of relatively low yield (small biomass) resources. Faunal assemblages will be diverse and may include deer, numerous small mammals such as rabbits and rodents, turtle, fish, etc. A low percentage of bison may be represented, but the overall faunal assemblage will not be dominated by any one 
resource. Macrobotanical and pollen remains will represent a diverse range of plant foods.

\section{Site Structure}

\section{Collector Systems}

Bison-oriented collector base camps will have well-defined site structure with spatially discrete activity areas and special-function features related to bison processing. Specific activity areas might include hide processing stations with evidence of unifacial scraping tool rejuvenation, tool manufacturing areas where the final stages of lithic reduction occurred, secondary butchering areas where selected cuts were further processed, bone grease manufacturing areas, and cooking areas where bison and/or other resources were prepared. Activity areas will be identifiable as high-frequency clusters of artifacts and/or faunal and floral remains. Horizontal patterning of activity areas may even provide evidence of community-level organization (i.e., habitation areas or structures). Due to the increased length of occupation, more-frequent site maintenance activities may have generated well-defined midden areas at these sites.

\section{Forager Systems}

Residential bases camps generated within an exclusively forager system will be characterized by multifunctional and overlapping features and activity areas representing exploitation of a single resource or contemporaneous exploitation of multiple resources. Site reoccupation for exploiting different resources at different times is also likely. Although individual base camps may exhibit various degrees of specialization, overall these sites will appear unspecialized due to the similarities in the procurement and processing strategies and material culture for exploiting various low-yield plant and animal resources.

\section{Material Culture \\ Collector Systems}

Material culture assemblages at bison-oriented collector residential base camps will include specialized tools reflecting the importance of bison hunting (projectile points), meat processing (cutting tools such as beveled knives), and hide processing (scraping tools such as end scrapers). Manufacture and rejuvenation of these tools will be important activities represented in the lithic debitage and in staged preforms. It is expected that hide processing may be particularly well represented in campsites, and resharpening of unifacial hide-scraping tools should be evident. Lithic debitage should reflect a strategy of nonlocal material procurement and transportation of early-stage tools to base camps for the final stages of tool manufacture. Specifically, the debitage should exhibit a high percentage of nonlocal materials and a disproportionately high frequency of decorticate flakes.

\section{Forager Systems}

Forager residential bașe camps will be characterized by a generalized stone tool assemblage and dominance of local material use. Chipped stone tools will be dominated by multifunctional and expedient tools, i.e., various types of bifacial and unifacial cutting and/or scraping tools exhibiting considerable morphological variability. Lithic debitage will be dominated by local materials, and all stages of lithic manufacture will be evident by the presence of cores and complementary percentages of corticate and decorticate flakes.

\section{Excavations at Prehistoric Sites 41GR291, 41GR303B, 41GR546, and 41GR559}

\section{Objectives}

The significance of prehistoric sites 41GR291, 41GR303B, 41GR546, and 41GR559 is derived from their potential to contribute data useful for testing the model of late Holocene human adaptation summarized above. The ultimate goal of the research is to reconstruct the broad patterns of interaction between human beings (i.e., cultures) and their environment and to understand the principles underlying the operation of cultural adaptive systems (e.g., Butzer 1982). To accomplish this, a wide range of site types (i.e., locationally and functionally), temporal periods, and cultural affiliations must be sampled, and all of the sites slated for data recovery during the Lake Alan Henry project fill a particular niche in the spatial/temporal/cultural continuum.

The data recovery investigations at these prehistoric sites were designed to recover the following: 
(1) adequate samples of artifacts and organic remains (faunal and macrobotanical) to support interpretations of site function, subsistence strategies, seasonality, cultural affiliations, and intercultural relationships; (2) cultural feature and artifact patterns to reveal site structure and function, occupation intensity, and local land-use patterns; (3) temporally diagnostic artifacts and datable samples to reveal site age and duration of occupation; and (4) paleoenvironmental data to contribute to reconstructions of past climates and relate human subsistence patterns to the changing resource base. Although ideally it is desirable to recover a variety of data on each of these research topics, the actual archeological remains recovered and their interpretive potential depend on the nature of the site occupations and the geomorphic context of the cultural deposits, as well as preservation and other factors.

Excavation strategies varied according to differences in the geomorphic context and the nature of the deposits containing the cultural materials at the various prehistoric sites. Minor variations in methodology are discussed in the respective chapters on the site investigations, but the overall methodology is described here.

\section{General Methods}

The archeological excavations at the prehistoric sites included both mechanical and hand excavations. Mechanical excavations using a maintainer and a backhoe were done only at 41GR291 and are discussed in more detail in Chapter 3. The standard methodology for hand excavations at the prehistoric sites is described below, and any deviations from these techniques are discussed within the individual site chapters. Horizontal and vertical controls were maintained using $1-\mathrm{x}-1-\mathrm{m}$ excavation units and $10-\mathrm{cm}$ levels. Site maps were prepared with the aid of a transit and stadia rod, and excavation units were laid out in a grid from one of the site datum points set in concrete during the 1988 testing. Each site had a primary datum point that was assigned an arbitrary elevation of $100.00 \mathrm{~m}$. Elevations of secondary datum points, all other grid points, and excavation levels were relative to the primary datum point. Arbitrary levels were set at even $10-\mathrm{cm}$ increments. For example, in an excavation unit starting at $97.58 \mathrm{~m}$ (the elevation of the surface at the highest corner of the unit), Level 1 was from 97.58 to $97.50 \mathrm{~m}$, Level 2 from 97.50 to $97.40 \mathrm{~m}$, Level 3 from 97.40 to $97.30 \mathrm{~m}$, and so on. Within a unit, excavation levels were measured using a transit and stadia rod, a level string line set at a known elevation, or a water level.

All excavated fill was screened through $1 / 4$-inchmesh hardware cloth, and all artifacts were bagged by unit and level provenience. Excavation Record Forms were completed for each level, and when a feature was encountered, it was assigned a unique number and recorded on a separate Feature Record Form. Additional feature documentation consisted of drawing plan and profile views, taking black-andwhite and color-slide photographs, and collecting special samples. In situ artifacts and features were mapped on grid paper, and elevations were shot by transit or taken from a string line or water level. For small artifacts, only one elevation was recorded, but for larger artifacts or features, top and bottom elevations were recorded. 'During excavation, field inventories of all collected artifacts were kept, and site maps showing units excavated and artifact densities were updated daily. These maps were reviewed periodically to select new areas for excavation.

Special samples include bulk sediment for flotation/macrobotanical analysis or radiocarbon dating, smaller sediment samples for pollen/phytolith analysis, and charcoal or other organic materials for radiocarbon dating. Special procedures, such as cleaning trowels with water and immediate sealing of samples in zip-lock plastic bags, were used for the pollen/phytolith samples to prevent contamination. In addition, all bones, shells, and charcoal fragments as well as fragile or unique artifacts were carefully collected in aluminum foil to prevent contamination or damage.

\section{HISTORIC SITE INVESTIGATIONS}

\section{Ranching on the Western Rolling Plains}

For historic resources in the Lake Alan Henry project area, two comprehensive regional historic contexts were developed as part of the Phase II investigations. The significance of the Historic period archeological remains at 41GR474 is related to one of these, the historic context "Ranching on the Western Rolling Plains, 1877-1945" by Freeman (1990), which falls within the general thematic context of "Agriculture in Texas" suggested by the Texas Historical Commission. 
Ranching in the western Rolling Plains area began in the late 1870s at a time when hostile Indians and large buffalo herds were disappearing. Utilization of the area accelerated during the early $1880 \mathrm{~s}$ as numerous ranchers from the Cross Timbers region moved their cattle herds westward to take advantage of the abundant grasses, springs, and shelter that the plains canyonlands provided. While cattle were the most frequently raised animals, a few ranchers brought sheep with them; however, the occurrence and distribution of sheep ranching in the region is poorly documented.

Favorable markets and weather resulted in a florescence of ranching on the western Rolling Plains between 1879 and 1885 . For the most part, ranching was carried on by a combination of individually and company-owned outfits that grazed animals on sections leased from railroad companies and the State of Texas. In a few cases, ranchers filed claims to sections along major water courses such as Grape Creek, but leases sufficed for the most part. Some ranchers brought their families with them; more commonly, headquarters were occupied by single males who hired other young men to work as hands on the range where they lived in shelters such as dugouts.

Goods and materials were acquired during the early years from Fort Griffin, Weatherford, Fort Worth, and the few small stores in the region that were a legacy of the 1870 s buffalo hunting trade. By 1881, however, most supplies came from Colorado City, a community that was established in anticipation of the construction of the Texas and Pacific Railroad from Fort Worth and eventually had the greatest impact on Rolling Plains ranching. Between 1881 when the first train arrived at the townsite and the mid 1880s when unfavorable weather and competition from other towns triggered an economic collapse, Colorado City was the shipping and supply headquarters for West Texas, serving ranches in a 27-county region. The nearest trade competitors were Dodge City, Kansas; Springer, New Mexico; and Trinidad, Colorado, which supplied the northern Texas Panhandle.

The connection between Colorado City and the western Rolling Plains was strong, with individuals such as "Uncle Pete" Snyder, who built a store on Deep Creek in Scurry County to supply buffalo hunters, later moving to Colorado City; and other Rolling Plains ranchers either making their permanent homes in the town or visiting there frequently. Boom turned to bust, however, and by the late 1880 s following a period of blizzard and drought, the economic situation worsened after the national panic of 1893 . Colorado City never regained its regional prominence.

Just as weather and economic issues affected the Rolling Plains, so also legal issues affected the ranching industry there. In 1895, the State Legislature passed the Four-Section Act. Designed to encourage permanent settlement and the acquisition of homestead tracts from the State, the Act forced openrange ranchers who had leased land for grazing to develop innovative methods of controlling sections that were sure to be purchased by new homesteaders from outside the immediate area. Many ranchers and ranching companies managed to retain their ranges for several years by encouraging employees to file on four sections. Assistance was provided to the employee when the ranch owner made the yearly payments required by the State and paid county taxes on the parcel as well. For their part, the employees agreed to allow the ranch owner to run his cattle on the sections. In addition, some hands agreed to allow the rancher or ranch company the privilege of first refusal should the hand decide to sell the sections. Hands who filed homesteads under such agreements usually remained employees of the larger ranch but were obligated to fulfill the terms of their agreements with the State by building improvements on the homestead section and occupying that section for three years.

A variety of property types was associated with ranching on the western Rolling Plains from 1877 to 1945. During the open-range period, ranch headquarters and line camps were the most prominent property types. During the closed-range period, the homestead/ranch headquarters was the common property type, and quite frequently a single property may have been occupied and in use during both periods.

Specific kinds of structures and features characterize the ranching period sites on the Rolling Plains. The dugout was by far the most common structure associated with the ranching period, followed by above-ground homes. During the openrange period, dugouts frequently functioned as line camps and often served as temporary ranch headquarters until a more permanent structure, usually a wood frame or rock house, could be constructed. The dugout also was a common structure associated with closed-range ranching since almost all new home- 
steaders constructed at least temporary dugouts before building permanent homes. Other features associated with ranching properties in the region included fences and corrals for horses and cattle; wells, windmills, water tanks, and stock ponds to provide water for home or livestock use; and outbuildings such as tack rooms for equipment storage and covered sheds for protecting livestock from severe weather.

The historic context summarized above is intended to place the ranching sites in the Justiceburg area within the context of the broader historic patterns that created them and to establish the identity and extent of other comparable cultural properties. The context is also essential for outlining the property types included within the region in general and the project area specifically.

\section{Excavations at 41GR474}

\section{Objectives}

Based on Freeman's (1990) historic context for ranching on the western Rolling Plains, it was recommended that the historic dugout at $41 \mathrm{GR} 474$ be considered eligible for listing on the National Register of Historic Places according to Criteria A, B, and D (Freeman and Boyd 1990:129-130). Due to its location and the potential for destructive impacts related to the construction of the reservoir and because of the integrity of its archeological deposits and architectural remains, the dugout was slated for data recovery during Season 3 . The general hypothesis proposed for the historic dugout is that it is a representative example of a homestead/ ranching headquarters which dates to the turn of the century, a transitional period from open- to closedrange ranching in West Texas. Archival research revealed that the dugout was constructed and used by the Jeff D. Justice family for a brief period, ca. 1899-1910. Jeff Justice was a prominent individual for whom the community of Justiceburg was named, and the short occupation at his dugout indicated that it is a time capsule of architectural and artifactual data for this critical period. Although dugouts are commonly associated with ranching and early settlement, no two were exactly alike and few archeological investigations of dugouts have been conducted in the region.

\section{General Methods}

The specific objective of the excavation of the
Justice dugout was to recover architectural details of its construction and the material culture associated with its occupation. Except for some deviations discussed below (and in more detail in Chapter 5), the excavation methods and field recording and collection procedures used at 41GR474 are generally the same as those employed for the prehistoric site investigations discussed above. One of the main deviations from the procedures outlined in the original research design is that a sample of cultural materials from the upper trash fill in the dugout was collected. Since the trash fill postdated the dugout occupation and appeared to represent a mix of materials dating from the early 1900 s to as late as the 1950 s, the original intent was to record and discard this material in the field. During the field investigations, however, it became clear that the trash fill dated mainly to the early 1900 s and would constitute a useful material culture assemblage for comparative purposes. A large sample of this material (including all diagnostics) was collected and processed for curation. The only other significant deviation from standard field techniques is that $10-\mathrm{cm}$ levels and $1-\mathrm{x}-1-\mathrm{m}$ units were not appropriate for the dugout, and thus excavation units and levels were altered (as discussed in Chapter 5).

\section{GEOARCHEOLOGICAL INVESTIGATIONS}

The Phase III, Season 3 geoarcheological investigations included on-site studies at all four prehistoric sites. These investigations had three primary objectives: (1) to assess the geological context and integrity of the archeological materials; (2) to establish the chronological sequence of deposition; and (3) to determine the nature of the depositional and postdepositional site formation processes and their influence on the archeological record. These investigations also contribute to the expanding late Pleistocene and Holocene geological record established for Lake Alan Henry by Blum (1989), Abbott (1990), Bousman (1991, 1992), Blum et al. (1992), and Kibler (Boyd and Kibler 1993), and they supplement the limited paleoenvironmental record for the Texas Southem Plains.

In order to accomplish these objectives, a number of methods and techniques were employed. Profiles were described according to the procedures and criteria presented by Buol et al. (1980:21-43), Bettis (1984), and Birkeland (1984), which provide field observations on color, texture, soil structure, consistence, boundary characteristics, and inclusions 
such as $\mathrm{CaCO}_{3}$ and manganese for each zone (see Appendix A). Selected sediments from backhoe trench and excavation unit profiles were subjected to textural and chemical analyses (see Appendix B). In addition, selected sediments were sampled for radiocarbon assay and microscopic analysis.

Microscopic sediment analysis involved examining the sand fractions (particles coarser than 4 phi) of selected samples from backhoe trench and excavation unit profiles, as well as pit feature fill. The purpose of this analysis was to identify depositional processes and intrusive objects that were not discernible at a macroscopic level. The samples were processed by placing 200-300 g of sediment in a small flask (300 ml) with tap water and approximately $20 \mathrm{~g}$ of sodium bicarbonate. The mixture was shaken vigorously for several minutes and let sit overnight. The flask was shaken again before being emptied into a U.S. Standard No. 230 mesh sieve (4 phi). The mixture was washed, after which the remaining sediment, the sand fraction, was collected and let dry over night at room temperature on paper. Once dry, a small portion of the same was microscopically (100x) examined on an opaque stage under a 100watt reflected light source.

The examination of each sample included preliminary documentation of sand grain characteristics (including angularity, rounding, and frosting) and identification and determination of the relative abundance of minerals and organics. While this analysis is not a quantitative study comparable to point counting in petrography, it is a simple qualitative technique that allows comparisons of the frequencies of certain minerals and their characteristics. Therefore, the results and interpretations of this analysis are not statistically valid and should be viewed with caution (see Appendix B).

\section{LABORATORY AND ANALYSIS PROCEDURES}

Laboratory procedures for prehistoric and historic cultural materials began with cleaning and cataloguing all items according to provenience. The only exception to the initial cleaning procedure is that some artifact classes, particularly historic artifacts such as metal or leather, could not be washed because they required special conservation measures to stabilize them for curation. Each specimen was labeled (or bagged with a label if it could not be labeled) with the site number, a lot number assigned to its particular provenience, and a unique specimen number within its lot. Lot numbers were assigned to every excavation level from which materials were recovered, to special proveniences associated with features, and to individually mapped specimens. A specimen inventory by lot number was maintained as the sequential lot numbers were assigned. All nonartifact samples (e.g., sediment and charcoal) were assigned a sequential sample number in the field and indexed by provenience.

After being catalogued, the cultural materials and special samples were sorted for analysis. Artifacts from flotation samples were sorted into those that would not pass through a $1 / 4$-inch screen and those that would; the former were added to the materials to be analyzed, while the microartifacts were counted but excluded from the analysis. Prehistoric artifacts were sorted according to material types: chipped, ground, battered, and other modified stones; unmodified manuports; ceramics; burned rocks; modified bones and shells; and unmodified faunal remains. Historic artifacts were sorted according to material type and further classified by function. The analysis of different artifact classes varied according to the nature of the materials and the kinds of data necessary for addressing different research problems. All historic artifacts (from 41GR474) were analyzed by Boyd; all chipped, battered, and ground stone tools from 41GR291, 41GR303B, and 41GR559 were analyzed by Tomka; and all other artifact classes (e.g., ceramics, modified shells, and burned rocks) were analyzed by Boyd (site 41GR291) or Peck (sites 41GR303B, 41GR546, and 41GR559). The chipped and battered stone tools from 41GR546 were analyzed by Peck. The objectives and methods of these various material culture analyses are described below, and the results are presented in Chapters 3 through 7. Various special studies were conducted on samples and artifacts, and some specialized technical analyses (e.g., macrobotanical and pollen analyses, petrographic analysis of ceramics, and radiocarbon assays) were performed by independent consultants. The methods and results of the special studies are reported in the appendixes. All radiocarbon assays are calibrated using the 20-year record of Stuiver and Pearson (1986) in the computer program by Stuiver and Reimer (1986).

\section{Chipped, Battered, and Ground Stones}

The objectives of the chipped, battered, and 
ground stone artifact analyses were to (1) characterize the collection in technological and morphological terms, (2) define site use and function if possible, and (3) relate patterns noted in the organization of technology to patterns defined in the research design. To achieve the first objective, a number of attributes were recorded for the various artifact categories. These attributes were chosen to describe the artifacts in morphological terms and help define the stage of reduction of the particular artifact.

The analysis of the lithic tools and debitage was intended, in part, to provide clues to aid in understanding the use of the sites and their functions within the regional settlement pattern. However, due to differential preservation of material remains and curation of tools, not all activities carried out at particular sites will be represented in the archeologically recovered assemblages. Site function, the role of the particular site in the overall land-use system, cannot be directly derived from artifacts recovered at a single site. Rather, a knowledge of the entire settlement/subsistence pattern is needed to provide information pertinent to the roles of specific sites within the overall system.

The chipped stone tools were classified into 10 categories: (1) arrow points; (2) dart points; (3) perforators; (4) gouges; (5) nonprojectile point bifaces; (6) cobble tools; (7) unifaces; (8) cores; (9) edge-modified flakes; and (10) unmodified debitage. The heat spalls encountered during the chipped stone tool analysis are included in the Manuports analytical category. The ground and battered stone artifacts were divided into six categories: (1) manos; (2) metates; (3) indeterminate-use ground stones; (4) anvils; (5) pestles; and (6) hammerstones.

The attributes recorded for the chipped, battered, and ground stone artifacts can be grouped under 14 headings: (1) raw material; (2) core characteristics; (3) nature of the tool blank; (4) tool morphology; (5) cause of fracture; (6) size; (7) cortex characteristics; (8) working-edge characteristics; (9) base and stem treatment; (10) reworking category; (11) platform characteristics; (12) flake characteristics; (13) wear type; and (14) weight. Each of these groups consists of individual attributes. Brief descriptions of attribute definitions, the states that each attribute can assume, and the class of artifacts on which the attribute was recorded are given below. To retain consistency, the majority of the attributes used in this analysis are the same as those employed during the Phase III, Season 1 and Season 2 analyses of lithic materials from Lake Alan Henry (Boyd et al. 1992, 1993).

\section{Raw Material}

Sixteen raw material types were defined to include the materials used for chipped, battered, and ground stone artifacts. The material types are (1) fine-grained chert, (2) coarse-grained chert, (3) finegrained Potter chert, (4) coarse-grained Potter chert, (5) fine-grained quartzite, (6) coarse-grained quartzite, (7) chalcedony, (8) silicified wood, (9) Tecovas jasper, (10) Alibates agate, (11) opalized caliche, (12) silicified caliche, (13) obsidian, (14) sandstone, (15) limestone, and (16) other.

\section{FINE-GRAINED LITHIC MATERIALS}

To characterize the fine-grained cherts from the project area proper and contrast them with finegrained cherts found in the immediate region as well as those from more-distant areas, a systematic collection of materials at natural occurrences was undertaken. Fine-grained cherts were collected from the project area proper, from the upstream drainage of the Double Mountain Fork of the Brazos River, and from the Cretaceous limestones of the Edwards Plateau and the Callahan Divide, a northern outlier of the Edwards Plateau located between 97 to $161 \mathrm{~km}$ (60 to 100 miles) south-southeast of the project area in a long linear outcrop (Figure 4). Consistent differences were noted in colors and textures among the specimens from the project area and the Double Mountain Fork drainage versus the cherts from the Edwards Plateau and Callahan Divide. These color and texture differences can aid in the definition of lithic raw material procurement practices, especially when contrasting the procurement of local versus distant nonlocal raw materials (Tomka and Fields 1990).

Twenty-five chert samples were collected from primary and secondary geologic contexts (Table 1). Eight of these are from Lingos Formation gravels (as defined by Caran and Baumgardner [1988, 1990]) within the Lake Alan Henry project area proper, seven are from upstream of the project area in the Double Mountain Fork drainage, and two are from southwest of the project area in the Colorado River drainage. Eight samples of Cretaceous cherts were collected from the Callahan Divide and adjacent portions of the Edwards Plateau. Two of these are 


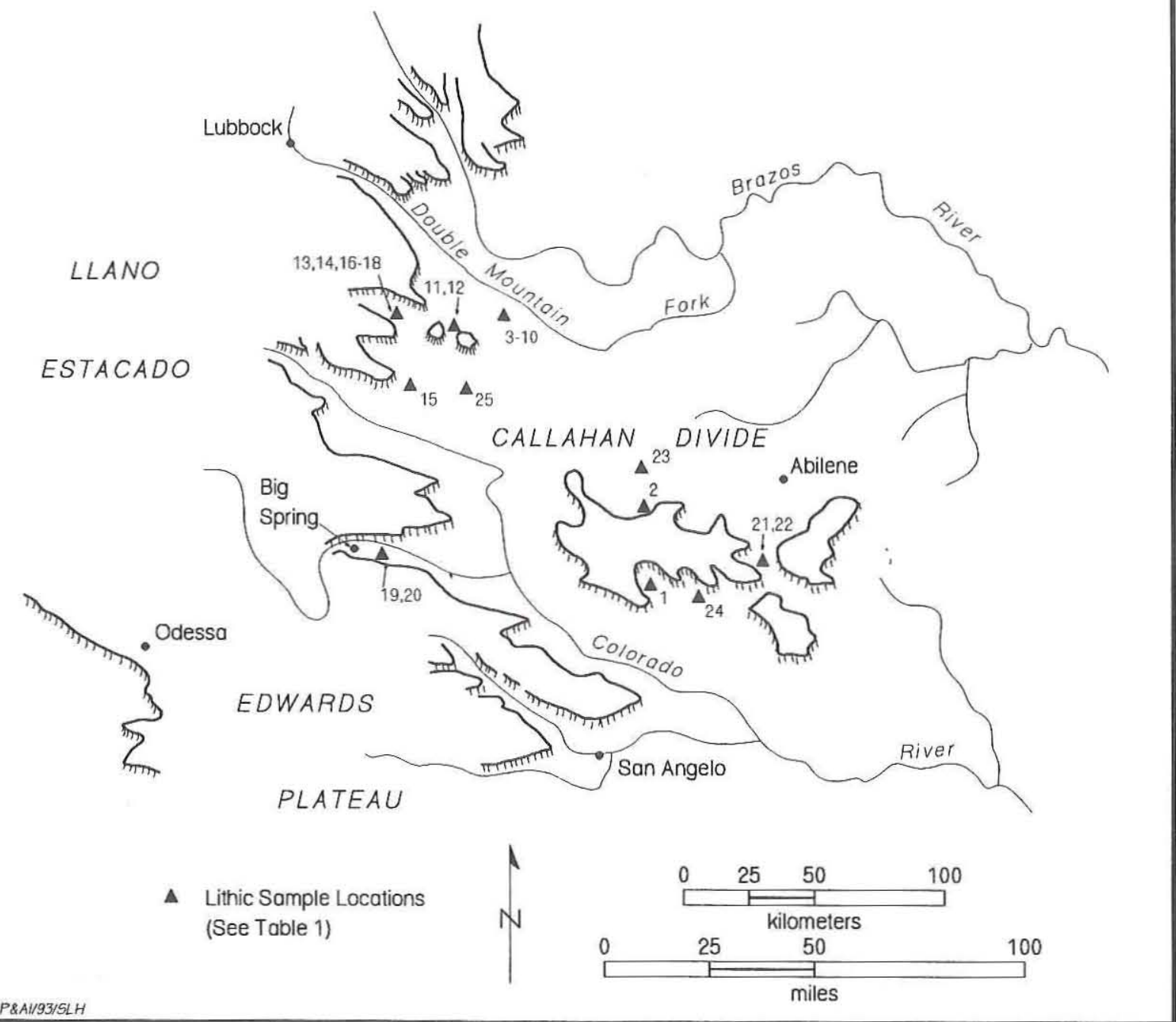

Figure 4. Map showing project area and locations of collection of regional lithic material comparative samples (adapted from Raisz 1957).

from the northwestern corner of the Edwards Plateau at Big Spring in Howard County, three are from the western portion of the Callahan Divide just south of Sweetwater in Nolan County, two are from the eastern edge of the divide near Buffalo Gap in Taylor County, and one is from north of Sweetwater in Fisher County.

To characterize the comparative raw material collection, flakes were removed from each chert nodule, labeled by locality and nodule, and then sorted into distinct color categories (Table 2). Fiftyfour color categories were defined; Colors 1-37 represent local chert specimens, and Colors 38-54 represent nonlocal sources. Three additional color categories were employed: local indeterminate
(Color 55), nonlocal indeterminate (Color 56), and indeterminate (Color 57). The local color categories are characteristic of Sample Localities 3-18, which are all within $40 \mathrm{~km}$ of the project area. Nonlocal colors are characteristic of Sample Localities 1, 2, and 19-25. All of these, except Sample Locality 25, are over $75-125 \mathrm{~km}$ from the project area. Sample Locality 25 , however, is only $30 \mathrm{~km}$ from Lake Alan Henry and is unique in that its color variations clearly match those of the Callahan Divide. The implication is that some of the distinctive chert variations characteristic of the Callahan Divide actually occur in close proximity to the project area. In spite of this, none of the nonlocal chert colors have been found to occur naturally in the immediate vicinity of the 


\begin{tabular}{|c|c|c|c|}
\hline \multicolumn{4}{|c|}{ TABLE 1} \\
\hline $\begin{array}{l}\text { Sample } \\
\text { Locality } \\
\text { No. }\end{array}$ & Sample Description & Color(s) (see Table 2) & Sample Location (see Figure 4) \\
\hline 1 & Primary(?) Cretaceous & $40,41,42,47,50$ & $\begin{array}{l}\text { Nolan County; north of Oak Creek } \\
\text { Reservoir }\end{array}$ \\
\hline 2 & $\begin{array}{l}\text { Secondary Cretaceous; } \\
\text { Quaternary alluvium }\end{array}$ & 45,51 & Nolan County; south of Sweetwater \\
\hline 3 & Secondary Lingos gravels & $16,18,19,25,30,34$ & $\begin{array}{l}\text { Garza County; Justiceburg } \\
\text { project area }\end{array}$ \\
\hline 4 & Secondary Lingos gravels & 12,22 & $\begin{array}{l}\text { Garza County; Justiceburg } \\
\text { project area }\end{array}$ \\
\hline 5 & $\begin{array}{l}\text { Primary Lingos gravels; } \\
\text { gravel pit }\end{array}$ & $\begin{array}{l}5,7,12,14,15,21,28,31,36 \\
37\end{array}$ & $\begin{array}{l}\text { Garza County; Justiceburg } \\
\text { project, area }\end{array}$ \\
\hline 6 & Secondary Lingos gravels & $2,9,28,31,37$ & $\begin{array}{l}\text { Garza County; Justiceburg } \\
\text { project area }\end{array}$ \\
\hline 7 & Secondary Lingos gravels & $13,17,25,27,29$ & $\begin{array}{l}\text { Garza County; Justiceburg } \\
\text { project area }\end{array}$ \\
\hline 8 & Secondary Lingos gravels & $2,3,4,6,7,26,28,35,37$ & $\begin{array}{l}\text { Garza County; Justiceburg } \\
\text { project area }\end{array}$ \\
\hline 9 & Secondary Lingos gravels & $4,6,11,20,27,28,30,35,37$ & $\begin{array}{l}\text { Kent County; Justiceburg } \\
\text { project area }\end{array}$ \\
\hline 10 & Secondary Lingos gravels & $5,6,7,12,26,33,35$ & $\begin{array}{l}\text { Kent County; Justiceburg } \\
\text { project area }\end{array}$ \\
\hline 11 & Stream gravels; mixed & $\begin{array}{l}\text { no fine-grained cherts in } \\
\text { sample }\end{array}$ & $\begin{array}{l}\text { Garza County; Double Mountain } \\
\text { Fork at Highway } 84\end{array}$ \\
\hline 12 & Stream gravels; mixed & $1,3,18$ & $\begin{array}{l}\text { Garza County; Sand Creek at } \\
\text { Highway } 84\end{array}$ \\
\hline 13 & Stream gravels; mixed & $3,10,11,24,27,29,32$ & $\begin{array}{l}\text { Garza County; Double Mountain } \\
\text { Fork at FM } 669\end{array}$ \\
\hline 14 & Stream gravels; mixed & $4,5,6,8,10,12,22,29,31,36$ & Garza County; creek at FM 669 \\
\hline 15 & Stream gravels; Cretaceous & $6,14,27,28$ & Borden County; creek north of Gail \\
\hline 16 & Stream gravels; mixed & $5,8,9,10,12,15,24,29$ & $\begin{array}{l}\text { Garza County; creek on Macy } \\
\text { Ranch }\end{array}$ \\
\hline 17 & Stream gravels; mixed & $6,12,22,23,30,31$ & $\begin{array}{l}\text { Garza County; Double Mountain } \\
\text { Fork on Macy Ranch }\end{array}$ \\
\hline 18 & Stream gravels; mixed & $4,8,11,24,28,35,36$ & $\begin{array}{l}\text { Garza County; Double } \\
\text { Mountain Fork on Macy Ranch }\end{array}$ \\
\hline 19 & $\begin{array}{l}\text { Secondary Cretaceous; } \\
\text { colluvial slope }\end{array}$ & 43,44 & $\begin{array}{l}\text { Howard County; south of Big } \\
\text { Spring }\end{array}$ \\
\hline
\end{tabular}




\begin{tabular}{|c|c|c|c|}
\hline \multicolumn{4}{|c|}{ Table 1, continued } \\
\hline $\begin{array}{l}\text { Sample } \\
\text { Locality } \\
\text { No. }\end{array}$ & Sample Description & Color(s) (see Table 2) & Sample Location (see Figure 4) \\
\hline 20 & $\begin{array}{l}\text { Primary Cretaceous gravels; } \\
\text { gravel pit }\end{array}$ & 43,44 & $\begin{array}{l}\text { Howard County; south of Big } \\
\text { Spring }\end{array}$ \\
\hline 21 & Stream gravels; Cretaceous & $40,41,42,46,48,49,52$ & $\begin{array}{l}\text { Taylor County; Buffalo Gap/Lake } \\
\text { Abilene }\end{array}$ \\
\hline 22 & $\begin{array}{l}\text { Primary Cretaceous gravels; } \\
\text { gravel pit }\end{array}$ & $40,42,46,49,52$ & $\begin{array}{l}\text { Taylor County; Buffalo Gap/Lake } \\
\text { Abilene }\end{array}$ \\
\hline 23 & $\begin{array}{l}\text { Secondary Cretaceous; } \\
\text { colluvial slope }\end{array}$ & $38,39,42,50,53$ & $\begin{array}{l}\text { Fisher County; adjacent to FM } \\
\text { 2744/U.S. Highway } 70 \text { intersection }\end{array}$ \\
\hline 24 & $\begin{array}{l}\text { Secondary Cretaceous; } \\
\text { stream gravels }\end{array}$ & $38,39,40,47,49,52,54$ & $\begin{array}{l}\text { Nolan County; } 2.1 \text { miles north of } \\
\text { FM 153/U.S. Highway } 277 \\
\text { intersection }\end{array}$ \\
\hline 25 & $\begin{array}{l}\text { Secondary Cretaceous; } \\
\text { colluvial slope }\end{array}$ & $40,43,49,51$ & $\begin{array}{l}\text { Borden County; between } 1-3 \text { miles } \\
\text { north of U.S. Highway } 80 \text { and } 14 \\
\text { miles east of Gail }\end{array}$ \\
\hline
\end{tabular}

project area.

To investigate lithic procurement patterns, all archeological fine-grained chert specimens were compared to these samples. Archeological specimens that visually appeared to be exact matches to the modern collected specimens were assumed to have originated from the documented local or nonlocal source; however, the main purpose of this exercise was to distinguish between local and nonlocal finegrained cherts rather than to identify specific sources. Those artifacts with colors that are similar in range and character to the local samples but do not have an exact match in the raw materials collected from the project area were classified as local indeterminate. Those archeological cherts that are similar to nonlocal specimens in terms of color and texture but have no exact match in the comparative color chart were classified as nonlocal indeterminate.

Finally, specimens that are so small or so heavily burned that the true color could not be discerned with certainty were classified as indeterminate. In classifying individual chert flakes into the available color categories, it was assumed that heat treatment (not burning) would not have changed the original color of the raw material from one color category to another and that the before-heat-treatment color can be identified with certainty. In support of this assertion, limited heat treatment experiments conducted on some chert specimens showed no substantial color changes in the cherts.

Although none of the comparative specimens of chert collected from the project area developed patina from exposure to air, a number of the nonlocal cherts collected from the Callahan Divide began patinating almost immediately after removal from the core. This process, which most likely is dehydration, resulted in a light blue to bluish gray tint acquired by at least two of the nonlocal color categories (Colors 43 and 44) that were originally dark to light brown. The rapid rate of alteration suggests that most archeological specimens from these cherts would be light blue to bluish gray. Because none of the local specimens are of this color or patinated to this color, it is assumed that most archeological light blue to bluish gray flakes are of a nonlocal origin matching Color 43 in character.

The local cherts range in color from light gray to yellowish brown and darker gray, with all specimens containing a variety of bands of lighter and darker colors. These fine-grained specimens occur in the form of angular fragments and small to mediumsized rounded or lenticular pebbles. One specimen collected from the project area in Garza County is $14 \mathrm{~cm}$ long, although most specimens tend not to exceed $6 \mathrm{~cm}$ in maximum size. The large amount of swirling and banding, together with the smaller size 


\begin{tabular}{|c|c|c|}
\hline & & $\begin{array}{c}\text { TABLE } 2 \\
\text { CHERT COLOR CATEGORIES }\end{array}$ \\
\hline Color No. & Sample Locality Nos. & Description \\
\hline 1 & 12 & flat white with gray brown translucent mottles \\
\hline 2 & 6,8 & light yellowish gray with gray specks \\
\hline 3 & $8,12,13$ & light gray with brown mottles, fossiliferous \\
\hline 4 & $8,9,14,18$ & light yellow brown with light gray mottles, fossiliferous \\
\hline 5 & $5,10,14,16,23$ & yellow brown with white and brown mottles \\
\hline 6 & $8,9,10,14,15,17$ & light brownish gray with white mottles or specks \\
\hline 7 & $5,8,10$ & light gray to white translucent \\
\hline 8 & $14,16,18$ & $\begin{array}{l}\text { blue gray with black, brown, blue specks, mottles and veins, fossiliferous, } \\
\text { translucent }\end{array}$ \\
\hline 9 & 6,16 & blue gray with gray and white mottling \\
\hline 10 & $13,14,16$ & gray with white mottling or banding \\
\hline 11 & $9,13,18$ & light gray \\
\hline 12 & $4,5,10,14,16,17$ & gray \\
\hline 13 & 7 & brownish gray, coarse \\
\hline 14 & 5,15 & brownish gray with brown or white specks \\
\hline 15 & 5,16 & brownish gray with large light gray mottles \\
\hline 16 & 3 & light yellow red \\
\hline 17 & 7 & light to dark greenish brown with dark blue specks \\
\hline 18 & 3,12 & black with milky quartz veins \\
\hline 19 & 3 & dark gray, coarse \\
\hline 20 & 9 & black \\
\hline 21 & 5 & light olive brown with dark blue and red specks \\
\hline 22 & $4,14,17$ & $\begin{array}{l}\text { dark gray mottled with light gray and black; central core is reddish yellow to } \\
\text { light red }\end{array}$ \\
\hline 23 & 17 & dark gray mottled with red, yellowish red, light gray, fossiliferous \\
\hline 24 & $13,16,18$ & light to dark olive brown mottled with red \\
\hline 25 & 3,7 & red, can have black, yellow, light gray mottles, coarse \\
\hline 26 & 8,10 & milky white with reddish yellow tint, translucent \\
\hline 27 & $7,9,13,15$ & brown gray with red specks \\
\hline 28 & $5,6,8,9,15,18$ & brownish yellow with light gray mottles, can be translucent \\
\hline
\end{tabular}




\begin{tabular}{|c|c|c|}
\hline Color No. & Sample Locality Nos. & Description \\
\hline 29 & $7,13,14,16$ & yellow brown with dark brown or white mottling \\
\hline 30 & $3,9,17$ & mottled yellow brown, dark brown, light brown \\
\hline 31 & $5,6,14,17$ & yellow brown mottled with white and red, fossiliferous \\
\hline 32 & 13 & light brownish yellow with light red mottles and white specks \\
\hline 33 & 10 & light yellowish brown with dark reddish brown mottles \\
\hline 34 & 3 & light reddish gray mottled with dark red, translucent \\
\hline 35 & $8,9,10,18$ & milky white with brown specks, translucent \\
\hline 36 & $5,14,18$ & light gray with red or white mottles, translucent \\
\hline 37 & $5,6,8,9$ & light gray with dark gray mottles \\
\hline 38 & 23,24 & light gray to glossy chert with small to medium white to lighter gray specks \\
\hline 39 & 23,24 & $\begin{array}{l}\text { dark gray with light blue tint, irregular light blue to white specks, dull to } \\
\text { glossy finish }\end{array}$ \\
\hline 40 & $1,21,22,24,25$ & blue gray with small red, light gray, and light blue mottles \\
\hline 41 & 1,21 & blue gray with brown mottles and white specks \\
\hline 42 & $1,21,22,23$ & dark blue gray with small red and white specks \\
\hline 43 & $19,20,25$ & brown, translucent with few red specks, patinates to pinkish gray \\
\hline 44 & 19,20 & light brown, coarse, translucent, patinates to light gray \\
\hline 45 & 2 & light gray with many small brown specks \\
\hline 46 & 21,22 & grayish brown with yellowish brown mottling \\
\hline 47 & 1,24 & blue gray with bands of small brown and dark gray mottles \\
\hline 48 & 21 & mottled light gray, brown, to dark brown with white and dark gray specks \\
\hline 49 & $21,22,24,25$ & gray to light gray with brown or white mottles, translucent \\
\hline 50 & 1,23 & dark brownish gray with large blue gray mottles, translucent \\
\hline 51 & 2,25 & $\begin{array}{l}\text { very pale brown to light gray with large mottles of gray and gray or brown } \\
\text { specks }\end{array}$ \\
\hline 52 & $21,22,24$ & dark brownish gray, translucent \\
\hline 53 & 23 & brownish gray chert with white specks \\
\hline 54 & 24 & dark blue chert with heavy concentration of light blue specks and mottling \\
\hline 55 & & local indeterminate \\
\hline 56 & & nonlocal indeterminate \\
\hline 57 & & indeterminate \\
\hline
\end{tabular}


and the lack of extremely fine grained, highly silicified, and translucent materials, sets these specimens apart from the nonlocal Cretaceous cherts collected from the Edwards Plateau and Callahan Divide. The latter are more highly silicified, are generally larger, and lack the swirling and narrow banding noted in the specimens collected from the project area. Also, the nonlocal Cretaceous cherts contain dark brown, dark gray, and bluish gray colors that are not represented in specimens collected from within the project area.

Other nonlocal fine-grained materials are documented archeologically, and these include Alibates agate and Tecovas jasper. Alibates agate is mottled, multicolored, silicified dolomite originating in the Alibates Dolomite Lentil of the Quartermaster Formation of Permian age. It occurs northeast of Amarillo and has a relatively limited, localized distribution. Tecovas jasper is somewhat similar to Alibates agate in broad visual characteristics. It originates from the Tecovas Formation of Triassic age and is encountered in outcrops along the eastern escarpment from Quitaque to Palo Duro Canyon and northwest of Amarillo (Holliday and Welty 1981:207). A single outcrop of moderate-quality Tecovas jasper may be present near the project area (Emmett Shedd, personal communication 1991), but its existence has not been confirmed. Small tabular chunks of Tecovas jasper also were recovered from the main channel of the Devil's Mountain Fork of the Brazos River as well as a number of its tributaries; however, the majority of these specimens as well as those from the primary outcrop do not appear to be of workable quality.

\section{OTHER LITHIC MATERIALS}

A number of other lithic resources of varying quality are abundant in Quaternary Lingos gravel deposits found on the uplands, canyon rims, and erosional remnants of the project area (Boyd et al. 1989:Figure 23). The most abundant include quartzites and Potter chert. Many of these gravels are composed of pink, purple, and red quartzites derived from the Ogallala Formation. These materials range from small spherical pebbles to large elongated, and even flat biconvex, nodules. The small to medium-sized pebbles are ideal for hammerstones and occur in the immediate vicinity of most sites. The larger specimens which could serve as blanks for ground stone manos are not common, however, and their procurement would involve greater search times.

Also contained in the Quaternary gravels are Potter chert nodules varying in diameter from ca. 8 to $20 \mathrm{~cm}$ or more. This material ranges in quality from extremely coarse nodules that can be split only with extreme force to very fine grained pieces which fracture relatively easily and predictably. Heat treatment improves the fracture quality of both types, but more improvement is seen in the fine-grained Potter chert.

Less-frequent lithic raw materials in the Quaternary gravels in the project area are silicified wood, opalized caliche, silicified caliche, and coarse-grained chert. The silicified wood specimens are relatively coarse to fine grained, small $(3-5 \mathrm{~cm})$ to medium-sized $(8-12 \mathrm{~cm})$ tabular chunks. Chalcedony specimens varying in size from 2 to $20 \mathrm{~cm}$ have been recovered from gravel bars of the Double Mountain Fork of the Brazos River and its tributary creeks. The chalcedony specimens have a barklike corticate surface that is reminiscent of silicified wood. They range in quality from very fine grained and translucent specimens to coarse-grained and internally fractured chunks. Opalized and silicified caliche occur frequently in the project area and its vicinity. While the finer-quality opalized variety is less frequent, some single chunks can contain portions that grade from silicified to opalized in quality.

These raw materials range in size from small angular nodules to specimens greater than $20 \mathrm{~cm}$ in maximum dimension. The coarse-grained cherts occur in the form of small $(5-7 \mathrm{~cm})$ lenticular nodules and range in color from dark gray to white or red. Their small size and internal fracture planes and lines make them an inferior raw material compared to the locally occurring finegrained cherts.

Sandstone, a widely utilized raw material in the project area, occurs in abundance and forms the bluff edges in most of the reservoir area (Boyd et al. 1989:126). Triassic-age sandstones extend through a large portion of the region, including Palo Duro Canyon (Matthews 1969:23) and Mackenzie Reservoir (Hughes and Willey 1978: 21). Tabular pieces of sandstone, together with lenticular and oval nodules formed as resistant concretions in softer limestone, occur often on solid sandstone bedrock. Although their distribution is 
patchy, they occur throughout the project area. Limestone occurs in the project area as streamworn cobbles, ranging in size from a few centimeters to well over $10 \mathrm{~cm}$, in the Lingos gravels. They originated in Cretaceous formations which are no longer present in the immediate vicinity, and the closest primary source of bedrock limestone is many kilometers south of the project area.

\section{LOCAL AND NONLOCAL LITHIC MATERIALS}

Based on the geomorphic history and the comparative collections of lithic raw materials from the project area, the following raw material types are considered to be local: (1) fine-grained Potter chert; (2) coarse-grained Potter chert; (3) fine-grained quartzite; (4) coarse-grained quartzite; (5) a variety of fine-grained cherts (i.e., Colors 1-37 and 55); (6) coarse-grained chert; (7) silicified wood; (8) opalized caliche; (9) silicified caliche; (10) chalcedony; and (11) sandstone. The origins of the miscellaneous materials are uncertain but are probably local. Referring to the regional collection of raw materials and other raw material studies, the following presently are presumed to be nonlocal: (1) Alibates agate; (2) Tecovas jasper; (3) obsidian; and (4) a variety of fine-grained cherts (i.e., Colors 38-54 and 56). Indeterminate-color fine-grained cherts (Color 57) cannot be classified in terms of origin.

\section{Core Characteristics}

Three attributes are included in this group: (1) core type; (2) removal directionality; and (3) number of flake removals. These attributes were recorded only on cores. Core type refers to the nature of the raw material from which flakes have been removed (flake, nodule/pebble, or indeterminate). Flake removal directionality (unidirectional, bidirectional, opposed bidirectional, multidirectional, or indeterminate) was recorded to gauge the degree of systematic core reduction practiced by the prehistoric knapppers. The number of flake removals consists of the count of flake scars not resulting from platform crushing. Platform preparation scars 1-10 $\mathrm{mm}$ in length were excluded.

\section{Nature of Tool Blank}

This attribute refers to the initial nature or condition of the raw material used in the manufacture of tools such as projectile points, perforators, gouges, bifaces, and unifaces. Three possible blank types were defined: (1) cobble/pebble; (2) flake; and (3) indeterminate. The presence of cortex on both faces of a biface or on unreduced corticate margins was considered indicative of cobble/pebble blanks. Smaller bifaces with unreduced platform remnants and/or unreduced flake ventral surfaces as remnants were assumed to have been made from flake blanks. Numerous bifacial artifacts that were substantially reduced and show none of the characteristics described were classified as indeterminate blank types.

\section{Tool Morphology}

Four attributes are used to characterize tool morphology: (1) completeness; (2) outline shape; (3) transverse cross section; and (4) morphofunctional category. Completeness is used to differentiate fragments from complete artifacts. Incomplete projectile points, perforators, gouges, bifaces, unifaces, and cobble tools were classified into the following fragment categories: (1) proximal; (2) medial; (3) distal; (4) longitudinal; (5) triangular wedges; (6) edges; and (7) barbs. Completeness for cores, edge-modified flakes, hammerstones, and ground stone artifacts was recorded as complete or fragmentary. Outline shape was recorded for ground stone artifacts. It assumes the following categories: (1) flat with rounded edges; (2) oval; (3) angular; (4) spherical; (5) rectangular; and (6) indeterminate. Outline shape could be determined with certainty for complete specimens or when only a small portion of the artifact was missing, and thus most fragments were classified as having indeterminate shapes. Transverse cross section categories include the following: (1) lenticular; (2) biconvex; (3) biconcave; (4) planoconvex; (5) planoconcave; (6) beveled; (7) tabular; (8) biconvex or planoconvex with medial ridge; (9) cylindrical; and (10) indeterminate. Transverse cross sections were recorded only on gouges and ground stone artifacts.

Morphofunctional categories were employed in the classification of perforators, bifaces, gouges, 
unifaces, and ground stones. The categories designate specific functional categories established primarily on the basis of morphological characteristics; they are subgroupings within specific tool types. Two categories of perforators were distinguished: drills and reamers. Drills are assumed to have been utilized to perforate materials, while reamers were used to enlarge already-existing holes. Bifaces are grouped into three morphological categories: (1) unutilized bifaces; (2) bifacial knives; and (3) indeterminate fragments. Unifaces are classified into the following morphofunctional categories: (1) end scrapers; (2) side scrapers; (3) combination end/side scrapers; (4) minimally retouched end scrapers; (5) minimally retouched side scrapers; (6) minimally retouched combination end and side scrapers; and (7) indeterminate unifaces and indeterminate-function edge fragments. Marginal retouch evident on scrapers is extensive and usually results in changes in the outline shape of the blank on which the tool was manufactured. Minimally retouched unifaces contain retouch on only small (maximum of $3 \mathrm{~cm}$ ) portions of their working edges. In addition, this retouch never results in substantial changes in blank outline shape. Gouges are grouped into the following morphofunctional categories: (1) unifacial triangular (Clear Fork); (2) bifacial triangular (Clear Fork); (3) unifacial ovate; (4) bifacial ovate; (5) unifacial rectangular; (6) bifacial rectangular; and (7) indeterminate. It is recognized that some unifacial gouges probably were utilized in scraping as opposed to planing tasks (see Hester et al. 1973; Shiner 1975). Although these specimens could be incorporated with the end scrapers, the lack of microwear analysis restricts their classification strictly on morphological characteristics.

Ground stone artifacts were subdivided into the following morphofunctional analytical categories: (1) mano; (2) metate; (3) anvil; (4) pestle; and (5) unknown/indeterminate. The presence/absence of grinding wear was established based on examination with a binocular microscope under 40x magnification. Specimens exhibiting macroscopic or microscopic striations; polish; flat surfaces; beveled, planoconcave, or ridged transverse cross sections; or evidence of working-surface rejuvenation (i.e., pecking) were classified as ground. These characteristics were readily observable on quartzite specimens. On sandstone specimens, striations and polish could not be observed at $40 \mathrm{x}$ magnification. Rather, specimens with characteristic flat surfaces and either diagnostic cross-sectional configurations or surface rejuvenation were classified as ground stones. Many of the ground stone tools are extensively weathered, and this results in a conservative classification of this class of tools.

\section{Cause of Fracture}

This attribute identifies the cause of fracture of incomplete projectile points, perforators, bifaces, gouges, and unifaces. Four types were distinguished: (1) use; (2) manufacture; (3) postdepositional; and (4) indeterminate. Some use-generated breaks and a large variety of manufacturing breaks have been reproduced experimentally (Tomka 1986). Others are taken from studies of manufacture and use-generated tool failures (e.g., Callahan 1979; Crabtree 1972; Johnson 1979, 1981; Muto 1971; Odell and Cowan 1986). The diagnostic morphologies defined from these replications are the comparative bases used in identifying fracture causes on the archeological specimens. Break morphologies that share characteristics of more than one cause of fracture or that were not replicated experimentally were assigned to an indeterminate category.

\section{Size}

Nine size measurements are included under this attribute: (1) maximum length; (2) maximum width; (3) maximum thickness; (4) maximum dimension; (5) blade length; (6) blade width; (7) base width; (8) neck width; and (9) stem/haft length. Maximum length, maximum width, and maximum thickness were measured on complete specimens and fragments with appropriate intact portions. Blade length, blade width, base width, neck width, and haft length were measured only when the appropriate portion of the artifact was complete. Maximum dimension categories consist of 10-mm-increment size classes ranging from 1-10 $\mathrm{mm}$ to $61 \mathrm{~mm}$ and greater. Edgemodified flakes and unmodified debitage are grouped into dimension categories. In all of the artifact categories, only measurements taken on complete specimens or complete portions of fragmentary artifacts are used in the statistical manipulation of the data.

\section{Cortex Characteristics}

Three attributes were used to characterize the 
nature of cortex on lithic artifacts: (1) cortex amount; (2) cortex presence/absence; and (3) cortex backing. Cortex amount was recorded in four increments: $0 \%$, $1-50 \%, 51-99 \%$, and $100 \%$. It was recorded on unmodified debitage (flakes, proximal fragments, and chips) and edge-modified flakes. Cortex presence/ absence is self-explanatory. It was recorded on unmodified debitage chunks or angular debris. Cortex was recorded as present on gouge, biface, uniface, core, cobble tool, and perforator fragments with cortex. Cortex presence/absence was recorded as indeterminate on fragments without cortex. The cortex backing attribute is an indication of the character of the surface of the tool opposite the working edge. It was recorded on cobble tools.

\section{Working-edge Characteristics}

This group consists of three attributes: (1) number of modified edges; (2) edge shape; and (3) inferred manner of use. The first attribute is selfexplanatory; it was recorded on edge-modified flakes and cobble tools. The edge shape attribute refers to the outline shape of the working edge. It consists of the following values: (1) pointed; (2) linear; and (3) combination pointed/linear. The inferred manner of use, on the majority of the specimens, was determined by comparison with replicated use wear on experimental tools in conjunction with diagnostic wear patterns described in the literature (Keeley 1980; Tringham et al. 1974; Yerkes 1987). The following use categories were identified: (1) cut/saw; (2) scrape; (3) engrave; (4) bore; (5) saw/scrape; (6) scrape/engrave; (7) saw/engrave; (8) scrape/saw/ engrave; and (9) other combination.

\section{Base and Stem Treatment}

This attribute was recorded on projectile points. It has two states: (1) stem smoothing; and (2) base smoothing. These attributes were recorded as present or absent.

\section{Reworking Category}

This analytical category consists of two attributes: (1) resharpening; and (2) previous use. Resharpening, recorded on projectile points and bifaces as present/absent, gauges the occurrence of artifact maintenance. Two criteria were used to establish the presence of resharpening on a specimen:
(1) morphological asymmetry along the longitudinal or transverse cross section; and (2) changes in flake removal patterns along the length of the projectile point or biface. The analytical category of previous use refers to the penultimate use of an artifact- that is, the functional or morphological category preceding the one into which it is classified. It consists of the following states: (1) core; (2) mano; (3) cobble tool; (4) hammerstone; (5) none; and (6) indeterminate. Previous use was recorded for cores and cobble tools. The attribute was not recorded for artifacts that had a single use throughout their use life.

\section{Platform Characteristics}

Three attributes were recorded to characterize striking platforms on unmodified lithic debitage: (1) cortex; (2) grinding; and (3) faceting. Platform cortex and grinding were recorded as either present or absent, while faceting refers to the number of platform facets. Platform faceting was recorded in three groups: (1) single; (2) two; and (3) three or more.

\section{Flake Characteristics}

Two attributes are included in this analytical category: (1) completeness; and (2) type. Four flake completeness categories were distinguished: (1) complete; (2) proximal; (3) chip; and (4) chunk. Complete flakes retain both a striking platform and a feathered and/or hinged termination. Proximal fragments have only a platform. All medial and distal fragments were classified as chips. Angular debris lacking clear flake features was placed in the chunk category. Flake completeness was identified for edge-modified flakes and unmodified debitage. Five flake type categories were distinguished: (1) biface; (2) uniface; (3) core preparation; (4) other; and (4) indeterminate. The flake type attribute was recorded on unmodified debitage and was used to categorize the reduction or manufacture strategy represented by a specific flake and, when possible, flake fragment. Complete flakes and proximal flake fragments with multifaceted striking platforms, multidirectional dorsal flake scars, parallel to slightly expanding flake margins, and slight to moderate longitudinal curvatures were classified as biface manufacture flakes. Complete flakes and proximal fragments with single-faceted striking platforms, parallel unidirectional flake scars on their dorsal 
surfaces, step fracturing immediately dorsal to the striking platform, and a longitudinal curvature that is most pronounced near the distal end of the flake were considered uniface manufacturing and/or resharpening flakes. Bladelike flakes and blades with corticate or decorticate single-faceted striking platforms, parallel unidirectional or opposed bidirectional dorsal flake scarring, dorsally trimmed platform preparation (Lintz 1978:Figure 4), and straight rather than curving longitudinal cross sections were considered core preparation flakes. These flakes are most indicative of bladelike flake or blade core rather than multidirectional or bifacial core preparation. Complete flakes and proximal flake fragments with rounded and heavily step-fractured striking platforms and specimens with ground or polished dorsal surfaces were considered cobble tool rejuvenation and ground stone tool manufacture flakes. They were categorized into a combined other flake type category. Complete flakes that did not fit into these four types and flake fragments with insufficient diagnostic traits for a confident determination of flake type were classified as indeterminate. Flake fragments with sufficient diagnostic elements to allow a secure determination were classified to the appropriate flake type category.

\section{Wear Type}

The attributes in this group identify the presence/absence of wear on selected bifacial, unifacial, and ground stone artifacts and the type and degree of wear on ground stones, hammerstones, and cobble tools. The attributes are as follows: (1) use wear; (2) haft wear; (3) degree of battering; (4) pecking; (5) step fracturing; and (6) edge rounding. The first attribute identifies the presence/absence of use wear on ground stone artifacts. It is recorded as either unifacial, bifacial, or indeterminate. The presence of haft wear was recorded on perforators, gouges, nonprojectile point bifaces, and unifaces. Haft wear was observed under $10 \mathrm{x}$ to $40 \mathrm{x}$ magnification. It consisted of ventral and/or dorsal face polish and flake ridge rounding and/or lateral edge rounding. The degree of battering was recorded on hammerstones and manos. It consists of the following values: (1) slight; (2) moderate; (3) extensive; and (4) absent. The first category consists of battering covering an area of only $1 \mathrm{~cm}^{2}$. Battering covering between $1-3 \mathrm{~cm}^{2}$ was included in the second category, while battering over more than $3 \mathrm{~cm}^{2}$ was considered extensive. Pecking had the following values: (1) unifacial; (2) bifacial; (3) absent; and (4) indeterminate. It was recorded on ground stone artifacts. Step fracturing was recorded on cobble tools and is indicative of the degree of working-edge use in conjunction with the hardness of the worked material. It was recorded as a presence/absence attribute. Finally, edge rounding, also recorded only on cobble tools, is indicative of the degree of working-edge wear. While some edge rounding similar to use wear may result from platform preparation (i.e., of a core), the wear noted on these tool edges extends onto the two faces of the working edge. This distribution should not occur in the case of core platform preparation.

\section{Weight}

Weight was recorded to the nearest gram with an O'Hause Triple Beam Bálance scale. Weight was measured for complete cobble tools, hammerstones, and manos.

\section{Ceramics}

Two independent types of analyses, typological and petrographic, were employed on ceramics. A descriptive ceramic analysis was done in-house using published data and in consultation with regional ceramic experts, and the petrographic analysis was conducted by Dr. David G. Robinson of the Texas Archeological Research Laboratory (see Appendix G). Since ceramics were recovered only at the Sam Wahl site (41GR291), the specific details of the ceramic analyses are presented in Chapter 3 and in Appendix G.

\section{Manuports}

The manuport category contains all objects that were brought onto the sites by humans but that cannot be assigned to any specific functional classes. All specimens in this category are unmodified (i.e., they lack intentional modification) with the exception of evidence of heat alteration, which is generally considered to be an unintentional form of modification. The manuport category varies somewhat from site to site depending upon differences in the depositional environments and various cultural factors, but it generally includes burned and unburned rocks and various classes of unusual objects (i.e., odd-shaped stones and fossils). The manuport 
categories for specific sites are discussed in more detail within the respective site chapters, but the following definitions are applicable for all of the prehistoric sites.

Unburned rocks are considered to be manuports if they are obviously a material that does not occur in the vicinity of the site or are too large to occur naturally in a specific depositional context. The distinction of what rocks are considered to be manuports was made on a site-by-site basis. The size criterion also generally applies to burned rocks, particularly sandstones, but other types are burned materials are treated differently. The burned rock category also includes (1) fire-cracked siliceous materials (quartzites and Potter chert) and limestone that have angular fractures, (2) fire-cracked cherts often called heat shatter, and (3) heat-altered cherts that are not fire cracked (i.e., heat spalls or heated specimens that are probably flake fragments but lack diagnostic attributes and cannot be classified as flakes). In some contexts, all of these materials are considered to be manuports regardless of size.

The manuport category also includes classes of unusual objects that are considered to be novelty items. Specimens are classified as odd-shaped stones or fossils and are unique because of their physical characteristics (i.e., size, shape, and/or material type).

\section{Other Materials}

Other materials that are considered separately are modified hematite specimens and the floral and faunal remains. Modified hematite is a distinct class of artifacts which includes all hematite specimens that exhibit evidence of having been ground (i.e., faceted surfaces with striations). This class is not included within the ground stone category since the modified hematite specimens are thought to represent a different functional group (i.e., rocks ground to obtain red pigment as opposed to rocks used as grinding tools).

Vertebrate faunal remains from standard collections (i.e., 1/4-inch-screen recovery) and flotation samples were analyzed by Brian Shaffer of Texas A\&M University (see Appendix C), while invertebrate remains were analyzed in-house and identified by comparison with reference collections or published data (see Appendix D). Floral remains consist of macrobotanical materials recovered as standard collections or as flotation samples and special samples (e.g., pollen/phytolith) from feature contexts. Selected samples were processed and analyzed by J. Philip Dering of Texas A\&M University (see Appendix E).

\section{Historic Artifacts}

After they were washed and/or stabilized and catalogued, the historic artifacts were classified into material and functional categories. All materials were initially sorted into five material groups - metal, ceramics, glass, bone, and other materials - and into nine functional categories - kitchen, bone, architecture, furniture, arms, clothing, personal, tobacco, and activities (includes ranching- and transportationrelated items) — following South (1977:Table 4). In addition to South's functional categories, this analysis employed an unknown functional group for all unidentified specimens. Within each material/ functional category, artifacts were further classified by specific identifications. For example, metal artifacts in the arms category were classified as cartridges, primers, and conical bullets or round balls. For the most part, these identifications and functional assignments are self-explanatory and are discussed in Chapter 5, but the classification of ceramics and glass is more detailed and is described below.

\section{Ceramic Classification}

Except for a few ceramic buttons assigned to the clothing category, all other ceramics represent vessels related to the preparation, storage, and/or serving of food and were assigned to the kitchen functional category. Kitchen ceramics were further classified into ware types and then into decorative categories. Classifications based on ware types are much debated, and definitions are often disputed because ware types represent arbitrary divisions along a continuum of technological developments (Majewski and O'Brien 1987:104-106). A simple ware type classification, however, is useful and is generally employed as an initial level of classification for descriptive purposes. The ceramic ware types that were used in the historic artifact analysis are stoneware, whiteware, and semiporcelain.

Stonewares are refined earthenwares with nonwhite paste, generally vitrified to various degrees, but as used here the stoneware category also includes some low-fired wares that grade into coarse (lowfired, nonvitreous) earthenware. Greer (1981:14-16) 
notes the continuum between earthenwares and stonewares and differentiates the two only by firing temperature (above or below $1100^{\circ} \mathrm{C}$ ) and relative vitrification. Greer (1981:263) also notes, however, that "American stoneware is theoretically vitrified" but that many pieces are in fact only partially vitrified. Hence, as the stoneware category is used here, it does include some poorly fired specimens that are actually earthenwares but whose forms are generally considered to be stonewares (e.g., crock/ jars).

Whitewares are refined earthenwares with white paste and generally include nonvitreous, semivitreous, and vitreous wares (Majewski and O'Brien 1987:116124). All of the whitewares in this analysis are semivitreous. This category includes wares that are often called "ironstones" (Majewski and O'Brien 1987:120).

Semiporcelains (or soft-paste porcelain) are an imitation of true porcelain. They have a highly vitrified, glasslike paste that is fused with the glaze and is translucent (Majewski and O'Brien 1987:124125).

Decorative classifications are somewhat more useful in that they are less arbitrary and more closely approximate the ceramic "types" recognized by merchants and consumers. Decorative types also reflect some degree of economic scaling (i.e., simple undecorated wares being less expensive than elaborately decorated wares) as demonstrated by Miller's (1980) cost-based decorative classification. Miller's classification, however, is not used since it is generally applicable only for the first half of the nineteenth century (Majewski and O'Brien 1987:132133). The decorative classification employed follows Majewski and O'Brien (1987:131-138), as shown in Table 3.

\section{Glass Classification}

Historic glass was classified into broad categories following Jones and Sullivan (1985:9-10). The categories used are (1) container (a general term for

\begin{tabular}{|l|l|}
\hline \multicolumn{2}{|c|}{ TABLE 3} \\
DECORATIVE CLASSIFICATION OF \\
HISTORIC CERAMICS
\end{tabular}

commercial bottles and jars), (2) tableware (a general term for vessels used to serve food and drink or as decorative items), (3) flat glass (i.e., window glass), and (4) unidentified. Closures (i.e., devises for sealing containers or tablewares) were not classified separately but were included within the container or tableware categories. These glass categories include specimens that were used for many differentpurposes, and they are assigned to functional classes accordingly.

Glass specimens in the container category were assigned to a functional class only if their specific identifications allowed it. For example, fruit jars and food containers were assigned to the kitchen functional class, while liquor and medicine bottles were assigned to the personal class. Container glass (including closures) was assigned to the unidentified functional class if no specific identifications were possible. Tablewares were assigned to the several different functional classes depending upon their identifications. Glass tablewares that are food related (e.g., tumblers, bowls, and pitchers) were assigned to the kitchen class, those that are decorative (e.g., vases) were assigned to the furniture class, and those that could not be specifically identified were assigned to the unidentified functional class. The final glass category, that of flat or window glass, was assigned to the architecture functional class. 


\section{THE SAM WAHL SITE, 41GR291}

Douglas K. Boyd, Steve A. Tomka, and Karl W. Kibler

\section{SITE SETTING}

Located within the Sam Wahl Recreation Area at Lake Alan Henry, the Sam Wahl site, 41GR291, is on the north side of the Double Mountain Fork of the Brazos River, approximately $4 \mathrm{~km}$ (2.5 miles) west of the dam. The site occupies the upland flat and eroding upland margin, ca. $150-300 \mathrm{~m}$ from the river (Figure 5). The northem portion is shallowly buried in the upland except for artifacts and features exposed in an east-west ranch road that cuts down into the cultural zone. The site extends onto two southward-projecting ridges that are separated by lower bedrock benches and a small drainage and flanked by larger drainages. Between the two ridges but south of the site, a small pool of water is found at a freshwater spring where the smaller drainage is cut down deeply into the bedrock. In the southern part of the site, cultural materials are shallowly buried in some places but are exposed by eolian deflation and sheet erosion along the edges and lower ends of the ridges, and some artifacts have been washed down into the drainages.

Elevations range from $2290 \mathrm{ft} \mathrm{msl}$ in the upland flat to $2250 \mathrm{ft} \mathrm{msl}$ in the lower eroded portions of the site, while the riverbed is ca. $27 \mathrm{~m} \mathrm{(90} \mathrm{ft)} \mathrm{lower,} \mathrm{at}$ $2160 \mathrm{ft}$ msl. Vegetation cover is moderate to heavy in the upland and sparse in the lower areas, consisting mainly of grasses, junipers, and mesquites, with lesser amounts of cholla, tasajillo, prickly pear, yucca, Mormon tea, and catclaw acacia.

\section{PREVIOUS INViESTIGATIONS}

Site 41GR291 was discovered in 1987 (Boyd et al. 1989) and tested in 1988 (Boyd et al. 1990). When it was initially recorded, artifacts and burned rock features were noted in the eroded lower areas and were exposed in the ranch road. No diagnostics were recovered during the survey, but shovel testing confirmed that the site had a high probability of containing intact buried cultural remains. It was assessed as being eligible for listing on the National Register of Historic Places, and further work (i.e., controlled surface collection, mapping, and testing) was recommended.

Archeological investigations in 1988 consisted of detailed site mapping, surface collection of selected diagnostic artifacts and all materials from a $3-x-15-m$ segment of the ranch road, and mechanical and hand excavations. Mechanical excavations consisted of 1 backhoe trench and 24 auger tests, while hand excavations were limited to six $1-x-1-m$ test units. Three hundred eighty-seven artifacts were recovered during the testing phase, bringing the total number of specimens to 392 (Table 4), with most of these $(66 \%)$ being surface collected.

The testing confirmed that a shallowly buried cultural zone with intact features was present in the upland portion of the site. Untyped brownware sherds, along with a Scallorn and a Deadman's arrow point, indicated an affiliation with the Palo Duro complex and a Late Prehistoric I temporal designa- 


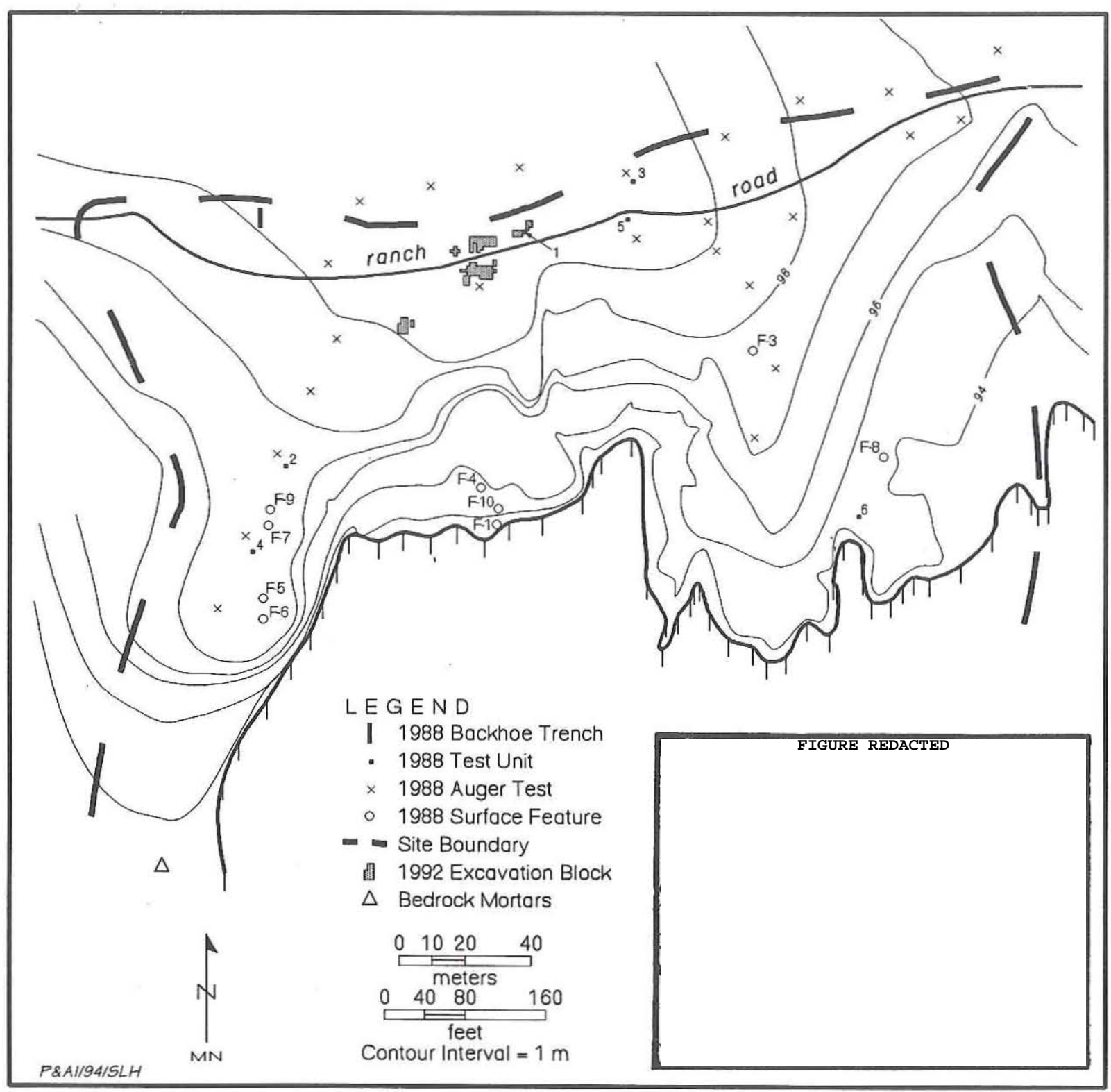

Figure 5. Map of 41GR291 showing extent of site, 1988 test excavations and surface features (Features 1 and 3-10 are shown; Feature 2 is in Test Unit 5), data recovery excavation blocks, and bedrock mortars (Features 26 and 27).

tion. A kaolinite pendant fragment provided evidence of extraregional trade or group mobility. Although the possibility of structures at 41GR291 was acknowledged, no evidence of their existence was found during the testing. It was recommended that the site be considered eligible for listing on the National Register of Historic Places and data recovery was recommended. The upland portion in the vicinity of Test Units 1 and 5 was considered to have the best potential for contributing important information, but mechanical testing was suggested as an appropriate means of further defining the upland cultural deposits.

\section{DATA RECOVERY INVESTIGATIONS}

The Phase III investigations consisted of mechanical testing with a maintainer (road grader) and a backhoe and hand excavation of 1-x-1-m units (Figures 6 and 7). The maintainer was used first to reblade the ranch road to provide a fresh exposure, and two additional cuts - one east-west parallel to the 
TABLE 4

ARTIFACTS RECOVERED DURING SURVEY AND TESTING PHASES, 41GR291

\begin{tabular}{|c|c|c|c|c|c|c|c|c|c|c|c|c|c|}
\hline Provenience & $\begin{array}{l}\text { Arrow } \\
\text { Points }\end{array}$ & $\begin{array}{l}\text { Dart } \\
\text { Point }\end{array}$ & Bifaces & $\begin{array}{l}\text { Cobble } \\
\text { Tools }\end{array}$ & Unifaces & Cores & $\begin{array}{l}\text { Edge-modified } \\
\text { Debitage }\end{array}$ & $\begin{array}{l}\text { Unmodified } \\
\text { Debitage }\end{array}$ & Hammerstones & $\begin{array}{l}\text { Ground } \\
\text { Stones }\end{array}$ & Ceramics & $\begin{array}{l}\text { Kaolinite } \\
\text { Pendant }\end{array}$ & Totals \\
\hline $\begin{array}{l}1987 \text { Survey } \\
\text { Surface } \\
\text { Shovel Test } 1\end{array}$ & $\begin{array}{l}- \\
-\end{array}$ & $\begin{array}{l}- \\
-\end{array}$ & $\begin{array}{l}- \\
-\end{array}$ & $\begin{array}{l}1 \\
-\end{array}$ & $\begin{array}{l}- \\
-\end{array}$ & $\begin{array}{l}- \\
-\end{array}$ & - & $\overline{3}$ & $\begin{array}{l}- \\
-\end{array}$ & 1 & $\begin{array}{l}- \\
-\end{array}$ & $\begin{array}{l}- \\
-\end{array}$ & $\begin{array}{l}2 \\
3\end{array}$ \\
\hline $\begin{array}{l}1988 \text { Testing } \\
\text { Surface } \\
\text { Surface Collection Unit } \\
\text { Auger Test } 1 \\
\text { Auger Test } 2 \\
\text { Auger Test } 4 \\
\text { Auger Test } 10 \\
\text { Auger Test } 18 \\
\text { Auger Test } 19 \\
\text { Auger Test } 22 \\
\text { Auger Test } 24 \\
\text { Test Unit } 1 \\
\text { Test Unit } 2 \\
\text { Test Unit } 3 \\
\text { Test Unit } 4 \\
\text { Test Unit } 5 \\
\text { Test Unit } 6\end{array}$ & $\begin{array}{l}2 \\
1 \\
- \\
- \\
- \\
- \\
- \\
- \\
- \\
- \\
- \\
- \\
- \\
- \\
- \\
1\end{array}$ & $\begin{array}{l}1 \\
- \\
- \\
- \\
- \\
- \\
- \\
- \\
- \\
- \\
- \\
- \\
- \\
- \\
- \\
-\end{array}$ & $\begin{array}{l}6 \\
2 \\
- \\
- \\
- \\
- \\
- \\
- \\
- \\
- \\
1 \\
- \\
- \\
- \\
- \\
-\end{array}$ & $\begin{array}{l}1 \\
5 \\
- \\
- \\
- \\
- \\
- \\
- \\
- \\
- \\
- \\
- \\
- \\
- \\
1 \\
-\end{array}$ & $\begin{array}{l}9 \\
8 \\
- \\
- \\
- \\
- \\
- \\
- \\
- \\
- \\
2 \\
- \\
- \\
- \\
- \\
-\end{array}$ & $\begin{array}{l}2 \\
5 \\
- \\
- \\
- \\
- \\
- \\
- \\
1 \\
- \\
- \\
- \\
- \\
- \\
- \\
-\end{array}$ & $\begin{array}{l}5 \\
7 \\
1 \\
- \\
- \\
- \\
- \\
- \\
- \\
- \\
- \\
2 \\
- \\
1 \\
1 \\
-\end{array}$ & $\begin{array}{r}4 \\
186 \\
1 \\
2 \\
1 \\
3 \\
3 \\
1 \\
1 \\
2 \\
36 \\
7 \\
6 \\
7 \\
16 \\
28\end{array}$ & $\begin{array}{l}1 \\
- \\
- \\
- \\
- \\
- \\
- \\
- \\
- \\
- \\
- \\
- \\
- \\
- \\
1 \\
-\end{array}$ & $\begin{array}{l}8 \\
1 \\
- \\
- \\
- \\
- \\
- \\
- \\
- \\
- \\
- \\
- \\
- \\
1 \\
2 \\
1\end{array}$ & $\begin{array}{l}- \\
1 \\
- \\
- \\
- \\
- \\
- \\
- \\
- \\
- \\
1 \\
- \\
- \\
- \\
- \\
-\end{array}$ & $\begin{array}{l}1 \\
- \\
- \\
- \\
- \\
- \\
- \\
- \\
- \\
- \\
- \\
- \\
- \\
- \\
- \\
-\end{array}$ & $\begin{array}{r}40 \\
216 \\
2 \\
2 \\
1 \\
3 \\
3 \\
1 \\
2 \\
2 \\
40 \\
9 \\
6 \\
9 \\
21 \\
30\end{array}$ \\
\hline Totals: & 4 & 1 & 9 & 8 & 19 & 8 & 17 & 307 & 2 & 14 & 2 & 1 & 392 \\
\hline
\end{tabular}




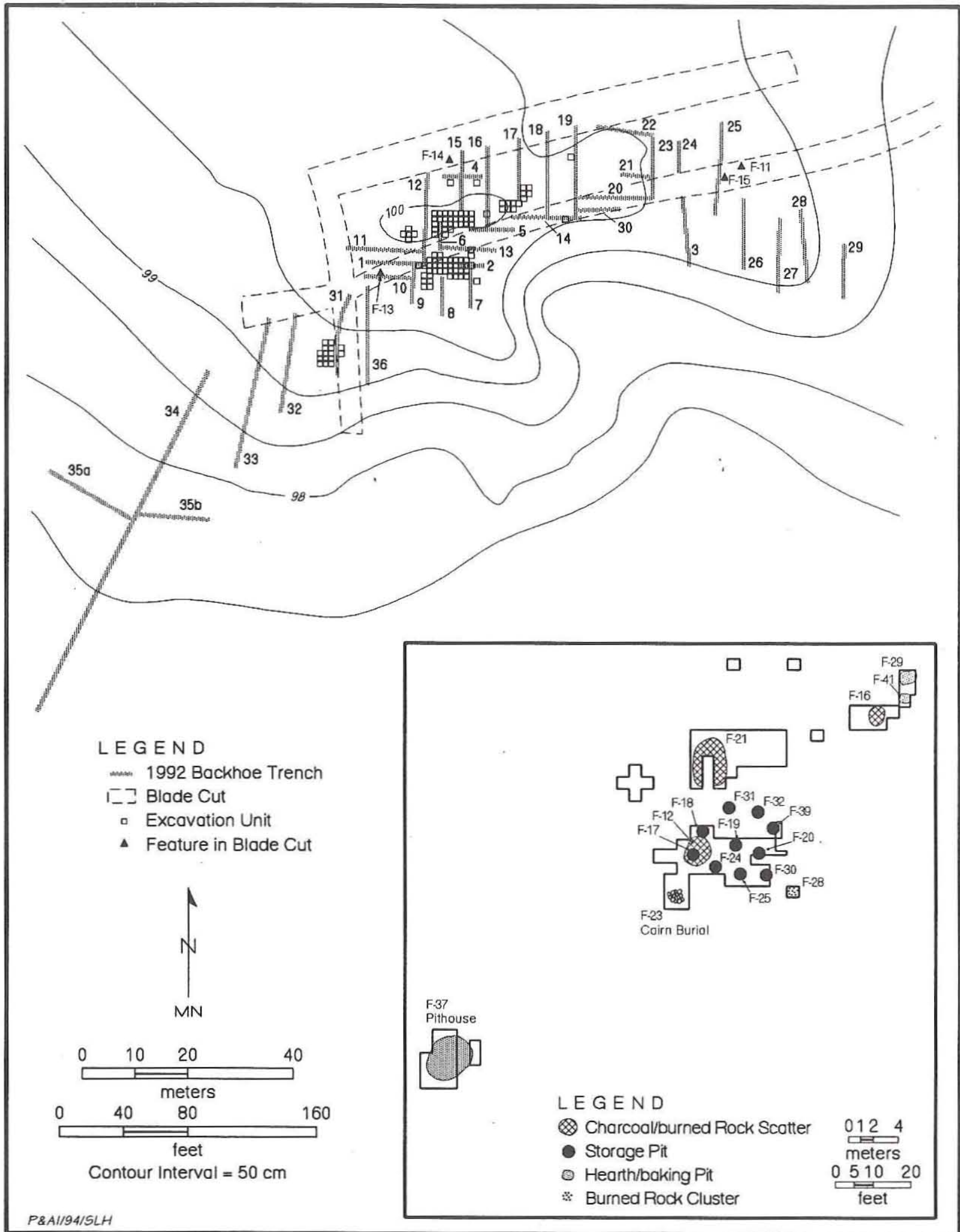

Figure 6. Map of north-central portion of 41GR291 showing locations of mechanical and hand excavations and data recovery cultural features. Note that bedrock mortars (Features 26 and 27) and isolated EUs 108-112 (renumbered 1988 Test Units 2-6) are not shown (see Figure 5). 


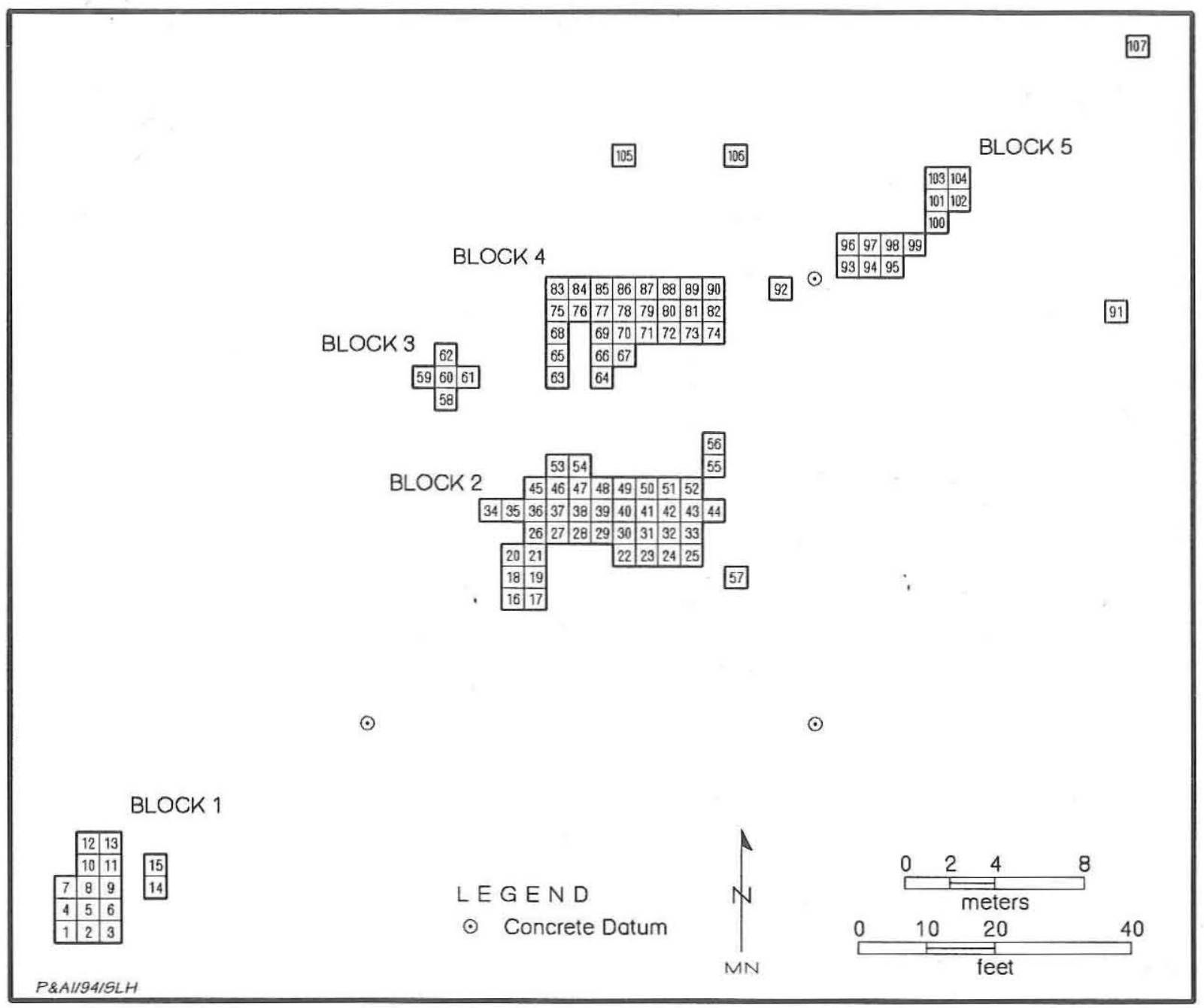

Figure 7. Map of 41GR291 showing excavation block and unit numbers; isolated EUs 108-112 (renumbered 1988 Test Units 2-6) are not shown.

road and one north-south - were made. The blading was monitored closely and was stopped when in situ features requiring mapping and description were encountered. After completing the blading, hand excavations were begun in several locations in the vicinity of 1988 Test Unit 1 . Hand excavations also were begun adjacent to the blade cut in the road, where the maintainer exposed a large sandstone rock that was found to be a metate associated with a pit later designated as Feature 18 and a dark stained area designated as Feature 12.

Several times during the field season, a backhoe was used to trench different parts of the site. The initial use of the backhoe was to obtain deep profiles to provide geomorphic data, but when the hand excavations revealed pit features, the backhoe also was used to search for additional buried features. The backhoe successfully located several more pits (Features 20, 30, 31, 32, and 39) and a large charcoal/burned rock scatter (Feature 21). When the nature of the pits became apparent, the presence of structures was considered likely, and extensive backhoe trenching was conducted specifically to look for structural remains. The trenching was effective, as a pithouse (Feature 37) was discovered in Backhoe Trench 1992-31.

Three blade cuts, 36 backhoe trenches, and 106 excavation units were dug at 41GR291 during 1992. The excavations were conducted over a 12-week period from September through December and required ca. 282 person-days of effort. A total of $45 \mathrm{~m}^{3}$ of sediment was hand excavated and screened 
through 1/4-inch-mesh hardware cloth. All data recovery work was tied into the site grid and datum points used during the 1988 testing, and five permanent datum markers (aluminum caps set in concrete at N980/E980, N980/E1000, N1000/E1000, N1000/E1020, and N1000/E1050) were established. Units were designated by their southwest grid coordinates in the field but were redesignated by sequential excavation unit (EU) numbers for analysis purposes. The 1988 test units also were redesignated with sequential numbers (TUs 1-6 were redesignated as EUs 99 and 108-112, respectively). Of the 112 excavation units (includes the 6 redesignated test units), 94 are complete $1-x-1-m$ units, 9 are $1-x-1-m$ units that had some portion of the upper levels removed by blading (EUs 14, 15, 34, 35, 4547, 53, and 54), and 9 are partial units smaller than $1 \times 1 \mathrm{~m}$ (EUs 25, 33, 42-44, 55, 56, 102, and 104). Eleven of the excavation units are isolated, while the others are clustered into five excavation blocks of five or more units that are contiguous, or nearly so (Block 1, $\mathrm{n}=15$; Block 2, $\mathrm{n}=41$; Block $3, \mathrm{n}=5$; Block 4, $\mathrm{n}=28$; and Block 5, $\mathrm{n}=12$ ).

\section{SEDIMENTS AND STRATIGRAPHY}

The sediments and stratigraphy at 41GR291 were observed and analyzed in the profiles of three backhoe trenches and three archeological excavation units. Detailed descriptions of these profiles are provided in Appendix A. Forty-three sediment samples from the profiles $(n=40)$ and one feature $(n$ $=3$ ) were subjected to analyses of sediment grain size, organic matter content, and carbonate content (see Appendix B), and one sediment sample was submitted for radiocarbon dating. These observations and analyses reveal a late Holocene soil that has developed on in situ weathered bedrock and a minor amount of eolian sediments overlying Triassic Dockum Group sandstones and mudstones.

Artifacts and cultural features were recovered from within a 50-60-cm-thick, strongly developed, cumulic sandy loam to sandy clay loam soil with an argillic horizon. Richardson et al. (1965:78) describe the soil as an Inceptisol and classify it as a Typic Ustocrept. However, by definition Inceptisols do not have argillic horizons, especially those formed in resistant quartzose parent materials (Buol et al. 1980:241-242). Argillic horizons are usually limited to Ultisols or Alfisols, whose definitions clearly do not fit the soil at 41GR291. Such inconsistencies are common in geomorphological studies and should be disregarded when soils are studied for purposes other than agronomy (Birkeland 1984:43). This inconsistency might also be explained by a unique set of circumstances at play in the region and on the site (discussed below).

The profiles of EUs 83, 88, and 95 and Backhoe Trenches 1992-1 and 1992-4 are typical of the stratigraphy across the site (Figure 8). The five profiles provide a view of the sediments and stratigraphy across east-west and north-south axes of the site.

Excavation Units 83, 88, and 95 are along an east-west axis. The following profile record from EU 83 is from the south wall of the unit and extends to $36 \mathrm{~cm}$ below the surface. Three zones were identified. Zone $1(0-20 \mathrm{~cm})$ is a brown to dark brown (10YR 4/3) fine sandy loam with moderate medium subangular blocky structure. Zone 1 is an A horizon. Zone 2 is an 11-cm-thick brown to dark brown (10YR 4/3) fine sandy loam with moderate medium subangular blocky structure. Zone 2 is a transitional $\mathrm{AB}$ horizon containing cultural materials and features. Zone $3(31-36+\mathrm{cm})$ is a dark yellowish brown (10YR 4/4) sandy clay loam with moderate to strong medium subangular blocky structure. Zone 3 is an argillic horizon and is termed a $\mathrm{Bt}$ horizon.

To the east is EU 88. The south wall of EU 88 was recorded, extending to $60 \mathrm{~cm}$ below the surface. Four zones were identified. Zone $1(0-19 \mathrm{~cm})$ is a brown to dark brown (10YR 4/3) fine sandy loam with moderate medium subangular blocky structure. It is an A horizon with a clear to gradual smooth lower boundary. Zone $2(19-33 \mathrm{~cm})$ consists of a brown to dark brown (10YR 4/3) fine sandy loam with moderate medium subangular blocky structure. Zone 2 is a transitional AB horizon. Charcoal flecks, sandstone gravels and pebbles, and cultural debris are dispersed commonly throughout the zone. Zone 3 $(33-53 \mathrm{~cm})$ is a yellowish brown (10YR 5/4) sandy clay loam with moderate to strong medium subangular blocky structure. Many voids and vertical cracks are filled with illuvial clays, representive of an argillic or Bt horizon. Zone $4(53-60+\mathrm{cm})$ is a massive light yellowish brown (10YR 6/4) loam. Many sandstone gravels, which are disintegrating and broken up pieces of bedrock, are scattered throughout the zone. Zone 4 is a $\mathrm{Cr}$ horizon.

Farther to the east is EU 95. The south wall of EU 95 was recorded, extending to $33 \mathrm{~cm}$ below the 


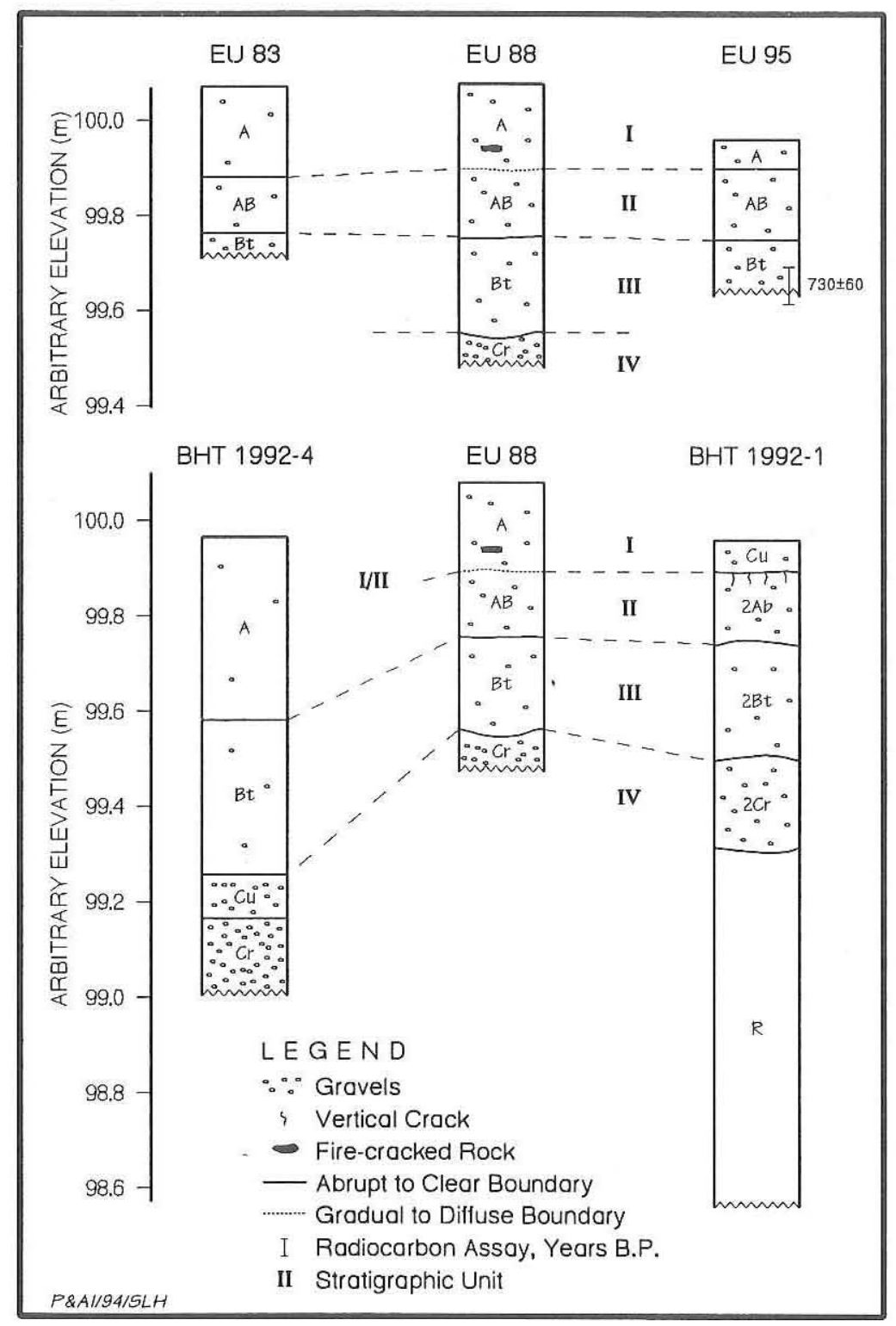

Figure 8. Profiles of EUs 83, 88, and 95 and Backhoe Trenches 1992-1 and 1992-4, 41GR291.

surface. Three zones were identified. Zone 1 $(0-7 \mathrm{~cm})$ is a structureless brown to dark brown (10YR 4/3) fine sandy loam. Zone 1 is an A horizon. Zone $2(7-22 \mathrm{~cm})$ is a brown to dark brown (10YR 4/3) fine sandy loam with moderate medium subangular blocky structure. Zone 2 is a transitional $\mathrm{AB}$ horizon. Zone $3(22-33+\mathrm{cm})$ is a yellowish brown (10YR 5/4) to dark yellowish brown (10YR 4/4) sandy clay loam with moderate medium subangular blocky structure. Illuvial clays were observed filling voids and vertical cracks. Zone 3 is an argillic or Bt horizon. A sediment sample from the top of this horizon was dated by radiocarbon assay to $730 \pm 60$ B.P. (Beta-59824; uncorrected age $=$ $630 \pm 60$ B.P.; $\delta^{13} \mathrm{C}=-18.8 \%$ o). Its one-sigma calibrated range based on the 20-year calibration curve of Stuiver and Pearson (1986) is 698-665 B.P. (A.D. 1252-1285).

A similar set of profiles was observed along the north-south axis of the site and includes the previously described profile of EU 88, Backhoe Trench 1992-4, and the east end of Backhoe Trench 19921. Backhoe Trench 1992-4 is north of EU 88. The north wall was recorded, extending to $97 \mathrm{~cm}$ below the surface. Four zones were identified. Zone $1(0-40 \mathrm{~cm})$ is a dark yellowish brown (10YR 4/4) fine sandy loam with moderate medium to coarse subangular blocky structure. Zone 1 is an A horizon. It overlies Zone 2 (40$72 \mathrm{~cm}$ ), a brown to dark brown (7.5YR 4/4) sandy clay loam with moderate medium subangular blocky structure. Zone 2 is an argillic or Bt horizon. Zone 3 (72$81 \mathrm{~cm})$ is a massive yellowish brown (10YR 5/4) loam with many sandstone gravels. It represents a $\mathrm{Cu}$ horizon. Zone $4(81-97+\mathrm{cm})$ is a massive very pale brown (10YR 7/4) gravelly sandy clay loam. It is a $\mathrm{Cr}$ horizon.

Backhoe Trench 1992-1 is southeast of EU 88. The south wall of the east end of the trench (located along the south wall of EU 35) was recorded, extending to $140 \mathrm{~cm}$ below the surface. Five zones were identified. Zone $1(0-7 \mathrm{~cm})$ is a weakly planar-laminated yellowish brown (10YR 5/4) fine sandy loam. Zone 1 is a Cu horizon. An abrupt and smooth boundary separates this recent deposit from the underlying buried soil (Zone 2). Zone $2(7-22 \mathrm{~cm})$ is a yellowish brown (10YR 5/4) fine sandy loam with moderate medium to coarse subangular blocky structure. A few small vertical cracks extend down from the surface of this buried soil. A rodent burrow at the bottom of the zone is 
filled with many sandstone gravels. Zone 2 is a $2 \mathrm{Ab}$ horizon. Zone $3(22-47 \mathrm{~cm})$ is a strong brown (7.5YR 4/6) sandy clay loam. It has strong coarse subangular blocky structure and is an argillic or $2 \mathrm{Bt}$ horizon. Zone $4(47-65 \mathrm{~cm})$ is a massive light yellowish brown (10YR 6/4) sandy clay loam to fine sandy loam. Many sandstone gravels and pebbles, representing disintegrating and broken pieces of bedrock, are dispersed throughout the zone. Zone 4 is a $2 \mathrm{Cr}$ horizon. Zone $5(65-140+\mathrm{cm})$ is an $\mathrm{R}$ horizon, representing Triassic Dockum Group friable sandstones and mudstones.

Two inconsistencies or contradictions arise from the sedimentological and chronometric data from 41GR291 when compared to other soils data and data from other upland localities of previous studies at Lake Alan Henry (cf. Abbott 1990; Blum 1989; Blum et al. 1992): (1) the radiocarbon assay from the top of the Bt horizon at 41GR291 is extremely recent for an argillic horizon and is also recent when compared to two other assays from upland B soil horizons; and (2) the microscopic examination of sediments from the locality of 41GR291 questions the predominance of an eolian and colluvial origin for upland soils in the Lake Alan Henry area.

The development of argillic soil horizons is generally believed to take thousands, if not tens of thousands, of years (Birkeland 1984:208; Waters 1992:54). However, a bulk sediment sample from the Bt horizon at $41 \mathrm{GR} 291$ produced a $\delta^{13} \mathrm{C}$-corrected radiocarbon age of $730 \pm 60$ B.P., and Holliday (1983:57) reports that argillic horizons have developed at the Lubbock Lake Site within as little as 350 years. Such rapid development is considered to be the result of mechanical infiltration of aerosolic dust or clays in sandy sediments (Holliday 1983:57). A considerable amount of aerosolic clay is available on the Llano Estacado or Caprock to the west and northwest of the project area, derived through the abrasion of clay films or argillans on sand grains during eolian transport (Gillette 1981; Gillette and Walker 1977). Dust storms on the Llano Estacado are common, especially during the spring months. Once airborne or suspensed in the atmosphere, the dust could be carried the short distance to the project area and flushed out of the atmosphere by eastwardmoving frontal thunderstorms. The shallow bedrock and sandy sediments at 41GR291 would be conducive to the rapid percolation of meteoric water, illuviation of associated aerosolic clays, and hence the quick development of an argillic horizon. Were it not for this regional phenomenon, the soil at 41GR291 could be truly classified as an Inceptisol.

The rapid development of the argillic horizon provides a possible explanation for the relatively young radiocarbon ages obtained from the $\mathrm{Bt}$ horizon at 41GR291 and other upland B soil horizons (4730 \pm 70 B.P. [Blum 1989:101] and $2770 \pm 80$ B.P. [Abbott 1990:53]) within the project area. The differences between the most recent assay (730 \pm 60 B.P.) and the two previous assays may simply be a matter of provenience. The sample from Zone 3 of EU 95 was taken from the top of the $\mathrm{Bt}$ horizon $(27-35 \mathrm{~cm}$ below the surface), whereas the sediment samples of Blum and Abbott were taken from deep within the soil profiles $(90$ and $80 \mathrm{~cm}$ below the surface, respectively).

One other possible explanation for the great differences between the radiocarbon ages may be contamination of the bulk' sediment sample from EU 95. Microscopic examination (100x) of the sand fraction of four sediment samples from EU 95 found that charcoal flecks are prevalent throughout the cultural zone and the underlying Bt horizon. If clays are being rapidly translocated throughout the soil profile, small flecks of wood charcoal probably also are being illuviated, resulting in contamination of the bulk sediment sample with more-recent carbon. Comparing the sample's $\delta^{13} \mathrm{C}$ value of $-18.8 \%$ to that of $-15.4 \%$ oo for the $2770 \pm 80$ B.P. radiocarbon age also suggests that wood charcoal possibly is responsible for contamination of the sample. Since it is generally believed that the uplands were predominantly grasslands (e.g., Fredlund 1993; Hatch et al. 1990; Kenmotsu 1981) prior to historical disturbances, soil organic inputs from $\mathrm{C}_{3}$ plants (which would result in lower $\delta^{13} \mathrm{C}$ values) are less likely to be responsible for the lower $\delta^{13} \mathrm{C}$ value of the bulk sediment sample from 41GR291. It is possible that wood charcoal from Feature 16, which is just north of the area where the sediment sample was collected and at a similar elevation, contaminated the sample. Although the date is coincidentally similar to other dated features at the site (see Cultural Features), it is felt that the potential mixing of wood charcoal and soil organics may not accurately reflect the age of Feature 16 or provide an accurate age of the $\mathrm{Bt}$ horizon.

The second contradiction or inconsistency that was revealed during the examination of the sediments and stratigraphy at 41GR291 is the proposed eolian and/or colluvial origin for upland soils in the project 
area (cf. Abbott 1990:51-53; Blum 1989:99-101; Blum et al. 1992:359-360). While eolian and colluvial processes are responsible for the deposition of sediment upon which soils have formed in many upland areas within the project area, substantial eolian or colluvial deposits do not appear to be present at 41GR291. Microscopic examination $(100 x)$ of the sand fractions of four sediment samples from the three zones of EU 95 revealed increasingly sorted sand populations with depth, associated with a relative decrease in medium sand grains downprofile. The medium sand grains tend to be more rounded and frosted, while the finer quartzose sand grains are more angular. Coarse sand-sized aggregrates of very fine sand cemented with silica are relatively more common with depth. Flecks of mica, which are fragile and tend to be winnowed out of eolian deposits, also increase in frequency with depth. It is believed that these characteristics do not represent eolian- or colluvial-deposited sediments but rather in situ weathering of the underlying sandstone regolith, although the cultural stratigraphy makes it clear that Zone 2 of EU 95 contains eolian sediments that resulted in slow aggradation during the time the site was occupied and Zone 1 may be entirely eolian in origin. The large increase in sandstone gravels and pebbles with depth also supports the hypothesis that the sediments at 41GR291 partly represent in situ weathering of the regolith. The relatively few gravels scattered throughout the upper zones can be attributed to rodent burrowing, which is prevalent throughout the site, moving smaller stones to old surfaces (e.g., Johnson 1989).

The in situ weathering of the bedrock and associated soil formation imply that the surface at 41GR291 was nearly stable for a considerable period of time. Whether the current soil is one of a series of soils that has formed on the upland exposures of Triassic bedrock and then deflated to start the cycle all over again is not known. It is most likely that the modern soil has been forming since the end of the middle Holocene, given the late Holocene radiocarbon assays (minimum dates) on two other upland soils in the project area. This time also marks the cessation of the severe deflation of upland surfaces and xeric conditions of the middle Holocene (Holliday 1989; Meltzer 1991).

The profiles from across the site can be organized into four stratigraphic units (see Figure 8) and can be correlated to the occupational and culturally sterile zones of 41GR291. The deepest unit, Stratigraphic Unit IV, is represented by Zone 4 of Backhoe Trench 1992-1, Backhoe Trench 1992-4, and EU 88, as well as Zone 3 of Backhoe Trench 1992-4. It consists of sands and weathered and disintegrating bedrock and is pedogenically unaltered, except for the presence of a small amount of illuvial clays. Stratigraphic Unit III is represented by Zone 3 of Backhoe Trench 1992-1 and EUs 83, 88, and 95, as well as Zone 2 of Backhoe Trench 1992-4. This unit is the $\mathrm{Bt}$ or argillic horizon of a soil that has formed on the weathered bedrock sediments. Stratigraphic Unit II is represented by Zone 2 of Backhoe Trench 1992-1 and EUs 83, 88, and 95 and contains the cultural components of the Sam Wahl site. Prior to its burial by Stratigraphic Unit I, it was the eluvial horizon of the soil formed on the weathered bedrock sediments. The strongly developed soil (Stratigraphic Units II and III) depicts a long period of surface stability. Stratigraphic Unit II varies in thickness and contains a minor eolian component. Although the addition of these eolian sediments resulted in slow aggradation of what was essentially a stable surface, the cultural components of the site are not clearly separable stratigraphically.

Postoccupational deposition is represented by Stratigraphic Unit I (Zone 1 of Backhoe Trench 1992-1 and EUs 83, 88, and 95), which tends to become progressively thicker in the northern portion of the site. Pedogenic alteration of this unit varies across the site. Zone 1 of Backhoe Trench 1992-1, a loosely consolidated fine sandy loam, is virtually unweathered and has buried the old surface or soil. In other profiles, the sediments of Stratigraphic Unit I have welded to the underlying soil forming a cumulic soil or thickened A horizon (Zone 1 of Backhoe Trench 1992-4), or they have developed into a discrete A horizon, transforming the old underlying A horizons of Stratigraphic Unit II into AB horizons (Zone 2 in EUs 83, 88, and 95). Eolian processes are credited with this depositional episode, as suggested by the relative increase in rounded and frosted medium sand grains in the surficial zones. Eolian deposition probably commenced during or shortly after the latest occupation of the site (ca. A.D. 1300; see Chronology). Eolian processes became a dominant sedimentary process in the region during this time period (Abbott 1990:57) as a possible response to increasingly xeric conditions (e.g., Ferring 1986; Hall 1982, 1988; Holliday 1985). Sedimentation has continued and probably has 
accelerated in historic times due to ranching and agricultural activities. At present, a large agricultural field lies just north of the site and undoubtedly has supplied sediment that was transported to the site. This field appears on aerial photographs from 1941 and was probably in existence even earlier.

\section{CULTURAL FEATURES}

Seven natural and 37 cultural features have been recorded at the Sam Wahl site (Table 5). ${ }^{2}$ The seven natural features were considered to be of possible cultural origin when they were discovered, but subsequent testing proved them to be of noncultural origin. One of these (Feature 22) is a rodent burrow, while the other six (Features 33-36, 38, and 40) are natural undulations in the bedrock substrate (e.g., old gullies). No cultural materials are associated with the noncultural features, and since they have little relevance to the site, no further description or consideration is warranted.

The 37 cultural features include a variety of feature types representing a wide range of cultural activities and are scattered across the site (see Figures 5 and 6). Burned rock features $(n=17)$ are the most frequent, but most of these were exposed on the surface or by mechanical testing and were not fully investigated. Other feature types include storage pits $(n=10)$, a pithouse with three related interior features, charcoal/burned rock scatters $(n=$ 3 ), a cairn burial, and bedrock mortars $(n=2)$. Each class of feature is described below, followed by a summary discussion. Ten radiocarbon dates (nine on charcoal and one on bone) were obtained from these features (Table 6), and 29 flotation samples were processed for macrobotanical remains (Table 7).

\section{Burned Rock and Fire-cracked Rock Clusters}

Fourteen burned rock features are classified as either burned rock clusters or fire-cracked rock clusters. These include 13 features that were not investigated and for which incomplete data exist: Features 1 and 3-10 are surface features that were mapped during the 1988 testing (Boyd et al. 1990: 66); and Features 11, 13, 14, and 15 were exposed in

\footnotetext{
${ }^{2}$ Excludes 14 possible postholes, rodent disturbances, or root disturbances found in or near Feature 37.
}

the data recovery blade cuts. Of these 13 features, the majority $(n=9)$ are burned rock clusters composed of sandstone. These features are ca. 50$100 \mathrm{~cm}$ in maximum dimension and may represent various kinds of activities (e.g., hearths, baking pits, secondary dumps). Three (Features 1,6 , and 10) are small burned or fire-cracked rock clusters, less than $50 \mathrm{~cm}$ in diameter, composed of angular Potter chert fragments that may represent boiling stone dumps; and one burned rock cluster (Feature 4) is a ca. 1-mdiameter concentration of burned sandstone and firecracked quartzite and Potter chert of unknown function.

One burned rock cluster was excavated, but the investigation failed to adequately define its form and/or function. Feature 28 is a $75-x-100-\mathrm{cm}$ surficial cluster of sandstone rocks that was exposed from $0-12 \mathrm{~cm}$ below the surface in EU 57 . It is composed of ca. 73 loose fragments ranging in size from 3-24 cm, of which only $23(32 \%)$ appear to be burned. The fragments were found lying directly on an outcrop of fractured sandstone, and all of the loose fragments are of the same type of sandstone as the bedrock. This feature cannot be classified with any certainty but could represent a small hearth constructed on the edge of an exposed bedrock bench; the lack of any definable patterning suggests that the feature is disturbed.

\section{Hearths/Baking Pits}

Three burned rock features that were partially or completely excavated are classified as hearths/baking pits. While it is suspected that these features may have been baking pits (rather than hearths), the ambiguous classification of hearth/baking pit is used because this interpretation cannot be fully supported. Feature 2 was partially excavated in 1988 (Boyd et al. 1990:66) in an isolated unit (Test Unit 5, now designated as EU 111). The investigated portion is approximately one-third of a circular, shallow basin of burned sandstone from $40-55 \mathrm{~cm}$ below the surface and may have been intrusive from the bottom of the cultural zone (Stratigraphic Unit II/III contact) into the weathered bedrock (Stratigraphic Unit IV). Although the feature originally was interpreted as a basin hearth, it is quite similar to Feature 41 (described below) and may represent the lower portion of a baking pit. As with Feature 41, numerous burned sandstone fragments scattered above the basin (from $30-40 \mathrm{~cm}$ below the surface) 


\begin{tabular}{|c|c|c|c|c|}
\hline \multicolumn{5}{|c|}{ SUMMARY OF FEATURES, 41GR291* } \\
\hline $\begin{array}{l}\text { Feature } \\
\text { No. }\end{array}$ & Type & Provenience & $\begin{array}{l}\text { Top } \\
\text { Elevation (m) }\end{array}$ & $\begin{array}{l}\text { Bottom } \\
\text { Elevation (m) }\end{array}$ \\
\hline 1 & fire-cracked rock cluster & surface, mapped in 1988 & 93.98 & - \\
\hline 2 & hearth/baking pit & EU 111 (1988 TU 5) & 99.16 & 99.01 \\
\hline 3 & burned rock cluster & surface, mapped in 1988 & 97.83 & - \\
\hline 4 & burned rock cluster & surface, mapped in 1988 & 94.66 & - \\
\hline 5 & burned rock cluster & surface, mapped in 1988 & 97.45 & - \\
\hline 6 & burned rock cluster & surface, mapped in 1988 & 97.58 & - \\
\hline 7 & burned rock cluster & surface, mapped in 1988 & 97.35 & - \\
\hline 8 & burned rock cluster & surface, mapped in 1988 & 93.52 & - \\
\hline 9 & burned rock cluster & surface, mapped in 1988 & 97.28 & - \\
\hline 10 & fire-cracked rock cluster & surface, mapped in 1988 & 94.50 & - \\
\hline 11 & burned rock cluster & exposed in blade cut & 99.12 & - \\
\hline 12 & charcoal/burned rock scatter & EUs $26,27,36-38,45-47,53$, and 54 & 100.00 & 99.60 \\
\hline 13 & burned rock cluster & exposed in blade cut & 99.61 & - \\
\hline 14 & bumed rock cluster & exposed in blade cut & 99.52 & - \\
\hline 15 & bumed rock cluster & exposed in blade cut & 99.22 & - \\
\hline 16 & charcoal/burned rock scatter & EUs $94,95,97$, and 98 & 99.76 & 99.62 \\
\hline 17 & storage pit & EUs 36 and 37 & 99.75 & 98.95 \\
\hline 18 & storage pit & EUs 53 and 54 & 99.75 & 98.97 \\
\hline 19 & storage pit & EUs 40,49 , and 50 & 99.80 & 99.15 \\
\hline 20 & storage pit & EUs $42,43,51$, and 52 and BHT 1992-2 & 99.83 & 99.17 \\
\hline 21 & charcoal/burned rock scatter & EUs $63-66,68,69,75-78$, and $84-86$ & 99.98 & 99.82 \\
\hline $22 * *$ & rodent burrow & EU 47 & 99.70 & - \\
\hline 23 & caim burial & EUs $16-19$ & 99.90 & 99.25 \\
\hline 24 & storage pit & EUs 28 and 29 & 99.75 & 99.13 \\
\hline 25 & storage pit & EUs 22 and 23 & 99.75 & 99.04 \\
\hline 26 & bedrock mortar & surface & 92.74 & 99.47 \\
\hline 27 & bedrock mortar & surface & 92.74 & 92.66 \\
\hline 28 & burned rock cluster & EU 57 & 99.87 & 99.75 \\
\hline 29 & hearth/baking pit & EUs 103 and 104 & 99.71 & 99.51 \\
\hline 30 & storage pit & EUs 25 and 33 & 99.80 & 99.25 \\
\hline 31 & storage pit & BHT 1992-13 & 99.80 & 99.50 \\
\hline 32 & storage pit & BHT 1992-13 & 99.75 & 99.25 \\
\hline $33 * *$ & natural gully or depression & EU 91, BHT 1992-14 & 99.40 & - \\
\hline $34 * *$ & natural gully or depression & BHT 1992-16 & 99.45 & - \\
\hline $35^{* *}$ & natural gully or depression & BHT 1992-15 & 99.40 & - \\
\hline $36 * *$ & natural gully or depression & EU 107, BHT 1992-17 & 99.80 & - \\
\hline 37 & pithouse structure & EUs $1-15$ & $99.40-20$ & 98.82 \\
\hline $38 * *$ & natural gully or depression & BHT 1992-14 & 99.60 & 99.35 \\
\hline 39 & storage pit & exposed by backhoe & 99.82 & 99.10 \\
\hline $40 * *$ & natural gully or depression & BHTs $1992-20$ and $1992-30$ & 99.95 & - \\
\hline 41 & hearth/baking pit & EU 100 & 99.68 & 99.42 \\
\hline 42 & structure floor (Feature 37) & EU 8 & 98.92 & 98.89 \\
\hline 43 & structure floor (Feature 37) & EUs 5 and 6 & 98.91 & 98.89 \\
\hline 44 & structure floor (Feature 37) & EUs $5,6,8$, and 9 & 98.90 & 98.88 \\
\hline
\end{tabular}




\begin{tabular}{|l|l|l|l|l|}
\hline \multicolumn{5}{|c|}{ RESULTS OF RADIOCARBON DATING OF FEATURES, 41GR291 } \\
\hline \multirow{2}{*}{ Lab No. } & Provenience & $\begin{array}{l}\text { Uncorrected } \\
\text { Age B.P. }\end{array}$ & $\begin{array}{l}\text { Corrected Age } \\
\text { B.P.* }\end{array}$ & $\begin{array}{l}\text { Calibrated Date A.D. } \\
\text { (Intercepts and } \\
\text { 1-Sigma Range** }\end{array}$ \\
\hline Beta-61497 & Feature 12 & $720 \pm 110$ & $730 \pm 110(-24.7)$ & $1216(1272) 1379$ \\
\hline Beta-59821 & Feature 12 & $810 \pm 60$ & $820 \pm 60(-24.4)$ & $1166(1225) 1265$ \\
\hline Beta-61498 & Feature 17 & $1210 \pm 80$ & $1250 \pm 80(-22.6)$ & $669(772) 883$ \\
\hline Beta-61499 (CAMS-5824) & Feature 19 & $* * * *$ & $1390 \pm 60$ & $609(651) 669$ \\
\hline Beta-59820 & Feature 21 & $690 \pm 60$ & $710 \pm 60(-23.8)$ & $1262(1277) 1290$ \\
\hline Beta-61496 & Feature 23 & $1530 \pm 60$ & $1720 \pm 60(-13.6)$ & $238(265,281,333) 399$ \\
\hline Beta-59822 & Feature 29 & $1370 \pm 90$ & $1380 \pm 90(-24.7)$ & $600(654) 685$ \\
\hline Beta-61501 & Feature 37 & $980 \pm 80$ & $970 \pm 80(-25.1)$ & $990(1027) 1163$ \\
\hline Beta-59823 & Feature 37 & $1020 \pm 90$ & $1030 \pm 90(-24.5)$ & $897(1007) 1039$ \\
\hline Beta-61500 & Feature 41 & $1180 \pm 80$ & $1200 \pm 80(-23.5)$ & $689(812,847,852) 897$ \\
\hline $\begin{array}{l}* \text { Ages uncalibrated; } \delta^{13} \text { C values in parentheses. } \\
* * \text { Calibrations use 20-year record of Stuiver and Reimer (1986). }\end{array}$ \\
$\begin{array}{c}* * * \text { Date done by Lawrence Livermore National Laboratory in California using AMS technique; uncorrected age and } \\
\delta^{13} \text { C value not given. } \\
\text { NOTE: Beta-61496 (Feature 23) is on bone; all other dates are on charcoal. }\end{array}$ \\
\hline
\end{tabular}

probably are associated and may represent the upper part of Feature 2. The 33 sandstone rocks that comprise this feature (i.e., all rocks from $30-60 \mathrm{~cm}$ below the surface) are tabular fragments, ranging in size from small pieces less than $4 \mathrm{~cm}$ in diameter to large slabs up to $25 \mathrm{~cm}$. These rocks (total weight $=$ $18 \mathrm{~kg}$ ) all exhibit evidence of heat discoloration and include one mano fragment. In addition, a quartzite mano/hammerstone and a Potter chert cobble tool were found among the scattered upper rocks and may be associated with the feature.

Feature 29 is a large, oval burned rock feature measuring $85 \times 140 \mathrm{~cm}$ and composed almost entirely of various types of sandstone $(n=79)$, with a total rock weight of $98 \mathrm{~kg}$ (Figure 9 and Table 8). It was discovered in Backhoe Trench 1992-17, which disturbed an estimated $16-27 \%$ of the feature (by weight and number of rocks, respectively). Thus, the actual size may have been as large as $85 \times 175 \mathrm{~cm}$. The feature rocks extend from $20-41 \mathrm{~cm}$ below the surface and are found in two distinct layers. In plan view, the top of the feature appears as a single layer of rocks from $20-30 \mathrm{~cm}$. No charcoal staining was observed at or above the upper rocks, but staining is intensive below the first layer of rocks. A second layer of rocks from $31-41 \mathrm{~cm}$ is somewhat smaller and confined to the central or perhaps western half of the feature. The most intensive charcoal staining was noted among and below the second-layer rocks, and some staining extends down as much as $70 \mathrm{~cm}$ below the surface, although this lowest portion of the feature (i.e., 45-70 cm) appears to be rodent disturbance.

Of all the rocks in the feature, $89 \%(n=73)$ exhibit evidence of heating, and some apparently were subjected to intensive heat. It should be noted, however, that several types of sandstone are represented, and, based on heating experiments on the local materials, it is clear that some sandstones exhibit thermal discoloration and/or fracturing more readily than others. Thus, it is likely that all of the rocks in the feature have been heated, but it is not possible to determine the relative intensities. 


\begin{tabular}{|c|c|c|c|c|c|}
\hline \multicolumn{6}{|c|}{$\begin{array}{l}\text { SUMMARY OF FEATURE FLOTATION SAMPLES AND } \\
\text { MACROBOTANICAL RECOVERY, 41GR291 }\end{array}$} \\
\hline $\begin{array}{l}\text { Feature } \\
\text { No. }\end{array}$ & $\begin{array}{l}\text { Flotation } \\
\text { Sample } \\
\text { No. }\end{array}$ & Description of Archeological Context & $\begin{array}{l}\text { Sediment } \\
\text { Volume } \\
\text { Processed } \\
\text { (liters) }\end{array}$ & $\begin{array}{l}\text { No. of } \\
\text { Charred } \\
\text { Seeds } \\
\text { Recovered }\end{array}$ & $\begin{array}{l}\text { Carbonized } \\
\text { Wood } \\
\text { Identified }\end{array}$ \\
\hline 12 & $\begin{array}{r}4 \\
5 \\
14 \\
15 \\
36 \\
37 \\
38 \\
43\end{array}$ & $\begin{array}{l}\text { EU 46, 99.85-99.80 m } \\
\text { EU 46, 99.80-99.75 m } \\
\text { EU 37, } 99.86-99.82 \mathrm{~m} \\
\text { EU 37, } 99.80-99.77 \mathrm{~m} \\
\text { EU 27, } 99.80-99.70 \mathrm{~m} \text {, lower portion of feature } \\
\text { EU 27, } 99.90-99.80 \mathrm{~m} \text {, lower portion of feature } \\
\text { EU 27, } 99.80-99.70 \mathrm{~m} \text {, lower portion of feature } \\
\text { EU 27, } 99.80-99.70 \mathrm{~m} \text {, lower portion of feature }\end{array}$ & $\begin{array}{l}5.0 \\
5.5 \\
5.0 \\
5.0 \\
6.0 \\
5.0 \\
4.5 \\
8.0\end{array}$ & $\begin{array}{l}19 \\
- \\
4 \\
3 \\
- \\
5 \\
6 \\
7\end{array}$ & $\begin{array}{l}\text { multiple taxa } \\
\text { single taxa } \\
\text { multiple taxa } \\
\text { multiple taxa } \\
\text { no } \\
\text { single taxa } \\
\text { single taxa } \\
\text { multiple taxa }\end{array}$ \\
\hline 17 & 52 & Upper pit fill, $99.60-99.50 \mathrm{~m}$ & 4.0 & 8 & no \\
\hline 18 & $\begin{array}{l}44 \\
45\end{array}$ & $\begin{array}{l}\text { Bottom pit fill, } 99.03-98.97 \mathrm{~m} \\
\text { Lower pit fill, } 99.10-98.97 \mathrm{~m}\end{array}$ & $\begin{array}{l}4.0 \\
4.0\end{array}$ & $\begin{array}{r}6 \\
12\end{array}$ & $\begin{array}{l}\text { multiple taxa } \\
\text { multiple taxa }\end{array}$ \\
\hline 19 & 48 & Upper pit fill, $99.60-99.50 \mathrm{~m}$ & 4.0 & 180 & multiple taxa \\
\hline 20 & $\begin{array}{l}18 \\
31\end{array}$ & $\begin{array}{l}\text { Upper pit fill, } 99.70-99.60 \mathrm{~m} \\
\text { Lower pit fill, } 99.30-99.21 \mathrm{~m}\end{array}$ & $\begin{array}{l}5.0 \\
5.0\end{array}$ & $\begin{array}{r}29 \\
-\end{array}$ & $\begin{array}{l}\text { no } \\
\text { no }\end{array}$ \\
\hline 21 & $\begin{array}{l}32 \\
39\end{array}$ & $\begin{array}{l}\text { EU } 68,99.86-99.82 \mathrm{~m} \\
\text { EU } 66,100.00-99.95 \mathrm{~m}\end{array}$ & $\begin{array}{l}5.5 \\
6.0\end{array}$ & $\begin{array}{l}38 \\
36\end{array}$ & $\begin{array}{l}\text { no } \\
\text { single taxa }\end{array}$ \\
\hline 23 & 34 & Fill above bone cluster, $99.43-99.40 \mathrm{~m}$ & 3.5 & - & single taxa \\
\hline 24 & 47 & Bottom pit fill, $99.17-99.13 \mathrm{~m}$ & 5.0 & 2 & multiple taxa \\
\hline 29 & $\begin{array}{l}56 \\
69\end{array}$ & $\begin{array}{l}\text { Fill among first layer of rocks, } 99.70-99.60 \mathrm{~m} \\
\text { Fill below first layer of rocks, } 99.63-99.59 \mathrm{~m}\end{array}$ & $\begin{array}{l}21.0 \\
30.0\end{array}$ & $\begin{array}{l}14 \\
15\end{array}$ & $\begin{array}{l}\text { multiple taxa } \\
\text { multiple taxa }\end{array}$ \\
\hline 30 & 54 & Lower pit fill, $99.20-99.00 \mathrm{~m}$ & 4.0 & - & no \\
\hline 31 & 55 & Lower pit fill, $99.66-99.56 \mathrm{~m}$, under large rock & 4.0 & - & no \\
\hline 32 & 57 & Lower pit fill, $99.38-99.25 \mathrm{~m}$ & 10.0 & 13 & multiple taxa \\
\hline 37 & $\begin{array}{l}72 \\
74 \\
76 \\
\\
80\end{array}$ & $\begin{array}{l}\text { Flotation column in EU } 8,99.30-98.88 \mathrm{~m} \text { (floor) } \\
\text { EU } 8 \text { floor, } 98.95-98.90 \mathrm{~m} \\
\text { EUs } 5 \text { and } 8 \text {, lower fill below large metate, } \\
\quad 99.05-98.87 \mathrm{~m} \\
\text { EU } 9 \text { floor in possible hearth (Feature } 44 \text { ), } \\
\quad 99.90-98.88 \mathrm{~m}\end{array}$ & $\begin{array}{r}20.0 \\
5.5 \\
17.0 \\
\\
6.5\end{array}$ & $\begin{array}{r}5 \\
7 \\
19 \\
\\
12\end{array}$ & $\begin{array}{l}\text { no } \\
\text { single taxa } \\
\text { no } \\
\text { single taxa }\end{array}$ \\
\hline 39 & 65 & Lower pit fill, $99.30-99.10 \mathrm{~m}$ & 5.0 & - & multiple taxa \\
\hline 41 & 70 & Lower fill among bottom rocks, $99.50-99.45 \mathrm{~m}$ & 9.0 & 15 & single taxa \\
\hline
\end{tabular}




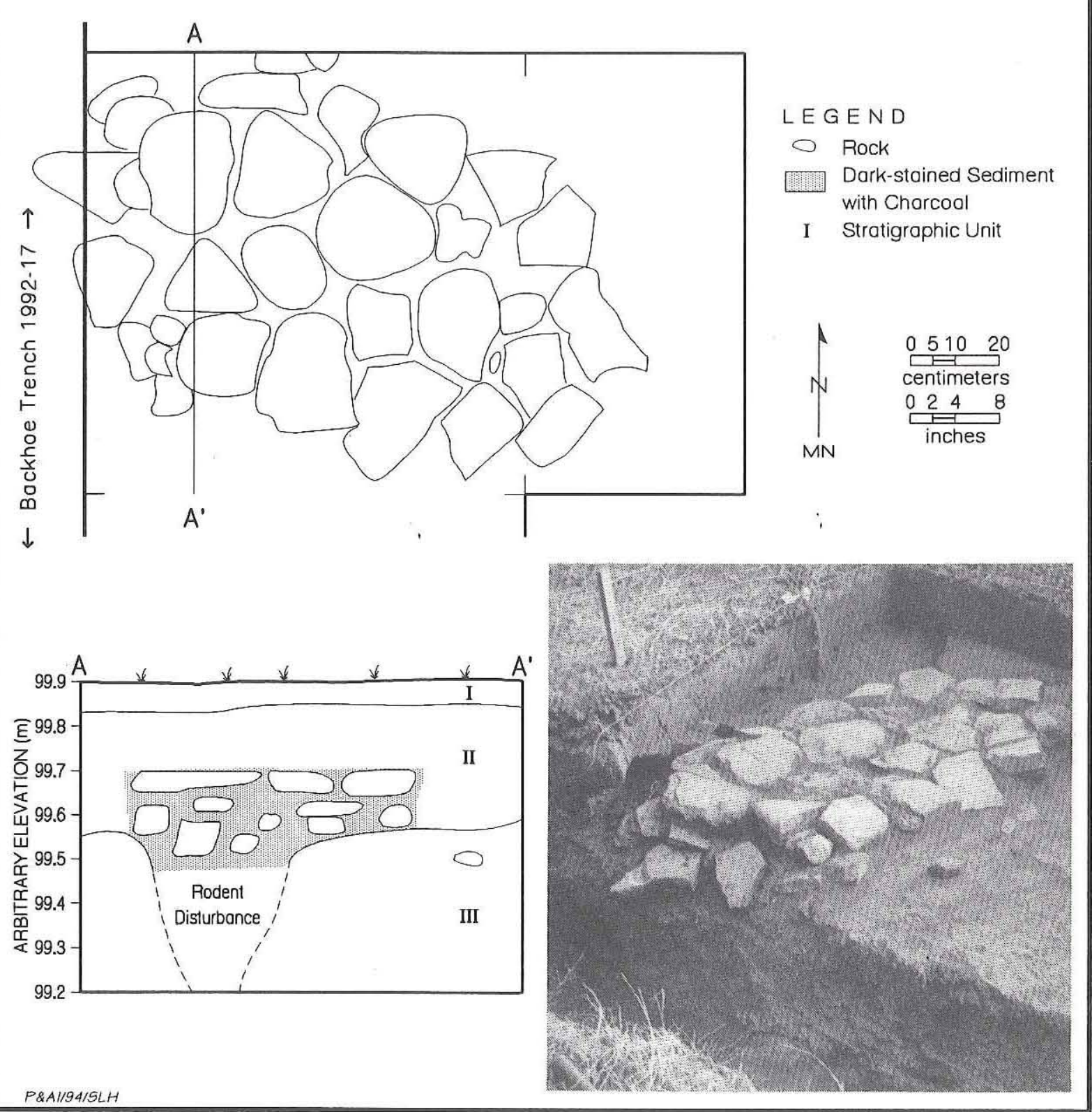

Figure 9. Plan, profile, and photograph (view northeast) of Feature 29, 41GR291.

Interestingly, $31 \%(\mathrm{n}=25)$ of the sandstone rocks are fragments of ground stones ( 2 manos, 21 metates, and 2 indeterminate) that were reused in the feature. One notable specimen is a fragment of a large basin metate that exhibits extensive bifacial wear.

Several lines of evidence suggest that Feature 29 represents a baking pit rather than a hearth. The upper layer of rocks is flat with no hint of a basin shape, and its elongated oval shape is unusual. While the near absence of charcoal staining above or in the upper rocks could be due to exposure, the extensive staining confined to the central lower portion of the feature may indicate that the burning was at and below the lower rock layer. In addition, the upper rocks are considerably larger than the lower rocks, as evidenced by their average weights (average weight of the 29 upper rocks is $2.2 \mathrm{~kg}$ compared to $0.6 \mathrm{~kg}$ average weight of the 31 lower rocks). This size difference may be due to more-intensive heating and fracturing of the lower rocks. Thus, it is likely that 


\begin{tabular}{|l|c|c|c|c|c|c|}
\hline \multicolumn{7}{|c|}{ TABLE 8 } \\
\hline & $\begin{array}{l}\text { No. of } \\
\text { Bumed } \\
\text { Sandstone* }\end{array}$ & $\begin{array}{l}\text { No. of } \\
\text { Unburned } \\
\text { Sandstone }\end{array}$ & $\begin{array}{l}\text { No. of } \\
\text { Fire-cracked } \\
\text { Potter Chert }\end{array}$ & $\begin{array}{l}\text { Size } \\
\text { Range (cm) }\end{array}$ & $\begin{array}{l}\text { Total No. } \\
\text { of Rocks }\end{array}$ & $\begin{array}{l}\text { Total } \\
\text { Weight of } \\
\text { Rocks (kg) }\end{array}$ \\
\hline $\begin{array}{l}\text { BHT 1992-17 } \\
\text { (undifferentiated } \\
\text { upper and lower) }\end{array}$ & $22(10)$ & - & - & $8-31$ & 22 & 16.1 \\
\hline $\begin{array}{l}\text { Upper layer } \\
\text { 20-30 cm }\end{array}$ & $26(13)$ & 3 & - & $12-29$ & 29 & 63.7 \\
\hline $\begin{array}{l}\text { Lower layer } \\
31-41 \mathrm{~cm}\end{array}$ & $24(2)$ & 6 & 1 & $6-23$ & 31 & 18.4 \\
\hline Totals: & 72 (25) & 9 & 1 & $6-31$ & 82 & 98.2 \\
\hline *Number of ground specimens is indicated in parentheses. & \\
\hline
\end{tabular}

the upper rocks are a capping layer over a baking pit.

Besides the recycled ground stones, 1 uniface, 1 cobble tool, 1 gouge, and 32 flakes were found in and immediately around the feature, but their occurrence may be incidental. A radiocarbon assay (see Table 6) on charcoal from below the first layer of rocks (from $29-36 \mathrm{~cm}$ in EU 103) yielded a calibrated date of A.D. 600 (654) 685.

Feature 41 is an irregular, $85-\mathrm{x}-90-\mathrm{cm}$ burned rock feature found from ca. $24-53 \mathrm{~cm}$ below the surface in EU 100 (Figure 10 and Table 9). Most of the rocks appear to be burned, and sandstone is the dominant material, comprising $95 \%$ of all the rocks. There are roughly three layers of rocks, but the upper and middle layers appear to have no formal arrangement and may represent only scattered feature rocks. The lower rocks form a single layer tightly clustered within a circular charcoal-stained area $60 \mathrm{~cm}$ in diameter. As for Feature 29, a large number of ground stone fragments $(\mathrm{n}=11,27.5 \%)$ were reused in Feature 41. A Potter chert cobble tool and a complete quartzite mano also were found among the upper-level rocks.

Diffuse charcoal staining was observed in the upper and middle layers, and intensive staining was noted among and immediately below the lowest rock layer. The lower half of the feature appears to be intrusive into sterile sediments. Although it could be a hearth, Feature 41 is more appropriately interpreted as a baking pit, with the lower portion being intact and the upper and middle rocks being scattered.
Numerous chipped stone artifacts (i.e., 32 flakes, a uniface, and a gouge) were recovered in and around the feature, but these cultural materials may not be functionally associated with the feature, and their occurrence may be incidental. A radiocarbon assay (see Table 6) on charcoal from the bottom of Feature 41 (from $42-47 \mathrm{~cm}$ in EU 100) yielded a calibrated date of A.D. $689(812,847,852) 897$.

\section{Storage Pits}

Ten features are pits that were dug down from the cultural zone into the bedrock (Table 10). All of these pits are clustered in a small area in and to the north of Block 2 (Figure 11). Four (Features 17, 18, 19, and 24) were completely excavated. Three pits were partially excavated (Features 20, 25, and 39), and only minimal excavations to define dimensions and obtain samples were done on three pits (Features 30,31 , and 32) discovered in backhoe trenches. Flotation and sediment samples were obtained from the bottoms of all pits, and selected pits were extensively sampled. For a variety of reasons discussed below, these features are interpreted as storage pits.

These pits are consistent in size, morphology, and fill characteristics (Figures 12 and 13). All are circular in plan view, ranging from at least $74 \mathrm{~cm}$ to $120 \mathrm{~cm}$ in diameter. When first encountered, the pits appeared as circular stains of brownish sandy loam (generally observed as 10YR $5 / 4$ to $5 / 6$ ), which 


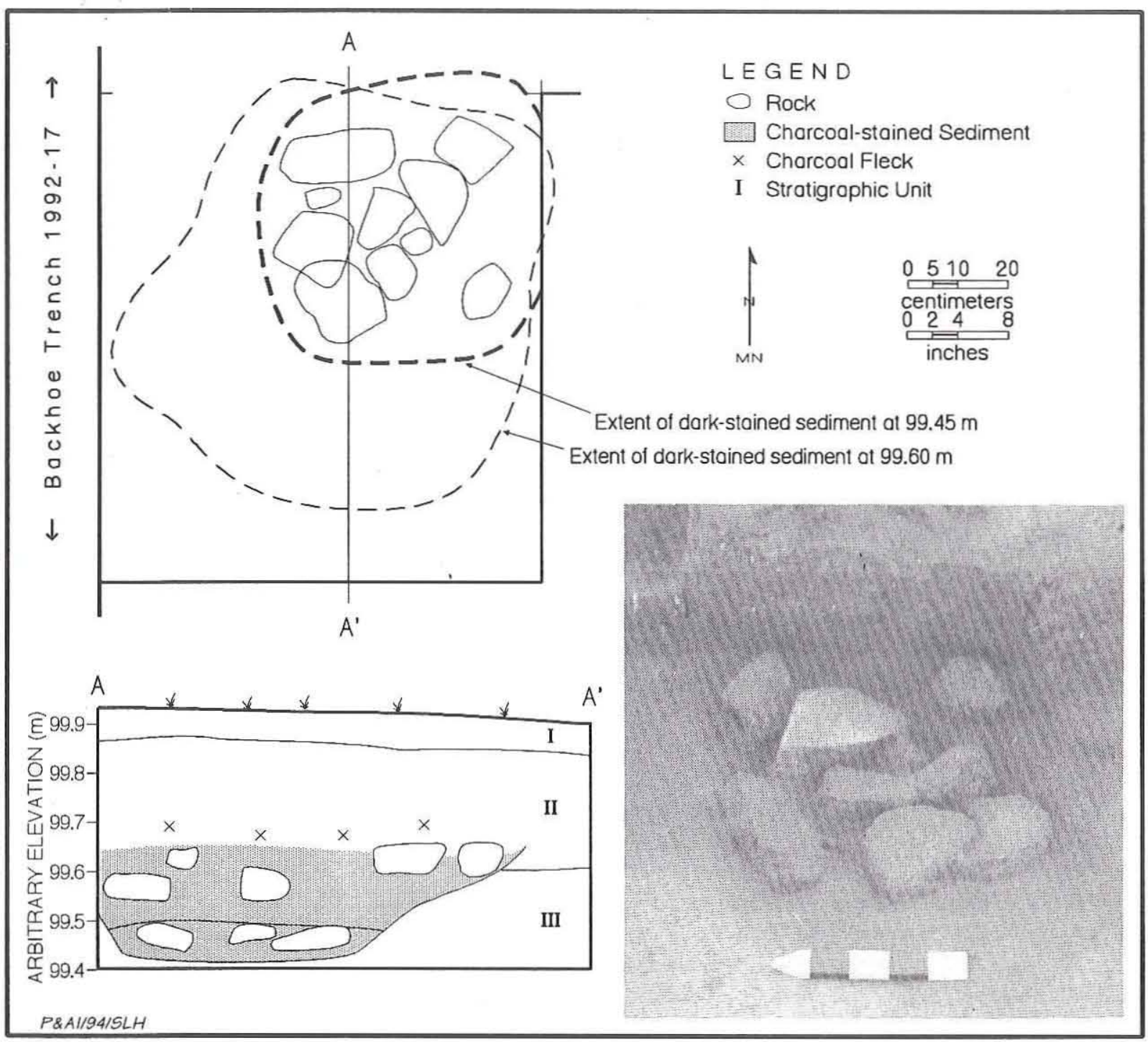

Figure 10. Plan, profile, and photograph of Feature 41, 41GR291. Photo shows lower rocks with north arrow at $99.45 \mathrm{~m}$ (view east).

contrasted with the uppermost weathered bedrock (Stratigraphic Unit IV) characterized by grayish white (10YR $7 / 2$ to $8 / 1$ ) sand and white (5Y 8/1) calcareous fragments. The pit openings could have been slightly higher and larger than indicated on Table 10, since the absolute tops of the pits seemed to disappear into the cultural zone. Thus, the pit measurements are conservative and were taken at the highest elevations where full pit outlines were visible, although in some cases indications of pit edges were noted at higher elevations. The overall morphology of the pits conforms to the general cylindrical shape described for subterranean storage features. DeBoer (1988:5) notes that storage pits are "likely to assume shapes that maximize volume while minimizing surface and access area."

Upon excavation, the pits were found to be more or less cylindrical with side walls that taper slightly to moderately inward and with rounded to flattened bottoms. The nine larger pits range from $50-80 \mathrm{~cm}$ in depth, while one pit was quite shallow, being only ca. $30 \mathrm{~cm}$ deep. Although some cultural materials were recovered or noted in the fill of all but one of the pits (Table 11), their fill is generally clean with only minor amounts of charcoal flecking observed. Overall, the fill appeared to have very little cultural staining compared to the overlying cultural zone (i.e., Stratigraphic Unit II), but Features 17 and 31 were 


\begin{tabular}{|l|c|c|c|c|c|c|}
\hline \multicolumn{7}{|c|}{ TABLE 9 } \\
\hline & $\begin{array}{l}\text { No. of } \\
\text { Burned } \\
\text { Sandstone* }\end{array}$ & $\begin{array}{l}\text { No. of } \\
\text { Unbumed } \\
\text { Sandstone* }\end{array}$ & $\begin{array}{l}\text { No. of } \\
\text { Fire-cracked } \\
\text { Potter Chert** }\end{array}$ & $\begin{array}{l}\text { Size } \\
\text { Range (cm) }\end{array}$ & $\begin{array}{l}\text { Total No. } \\
\text { of Rocks }\end{array}$ & $\begin{array}{l}\text { Total } \\
\text { Weight of } \\
\text { Rocks (kg) }\end{array}$ \\
\hline Upper, 22-32 cm & $8(4)$ & $1\left(1^{* * *}\right)$ & $1(1)$ & $8-29$ & 10 & 18.7 \\
\hline Middle, 32-42 cm & $19(5)$ & - & 1 & $7-19$ & 20 & 6.4 \\
\hline Lower, 42-52 cm & $10(2)$ & - & - & $7-25$ & 10 & 9.7 \\
\hline Totals: & $37(11)$ & 1 (1) & $2(1)$ & $7-29$ & 40 & 34.8 \\
\hline
\end{tabular}

exceptions to this.

The upper $15 \mathrm{~cm}$ of the fill in Feature 17 was considerably darker than the lower portion of the fill. This charcoal staining appears to be associated with Feature 12, a dark sediment stain which stratigraphically overlies and blends in with the top of Feature 17 (see Charcoal/Burned Rock Scatters described below). In addition, the Feature 17 fill contained numerous rocks, including eight large unburned sandstone rocks found at various depths and angles. These rocks originally may have been part of the pit's cover and are discussed in more detail below.

Feature 31 is the most aberrant of the pits. Although its dimensions are somewhat questionable, it is certainly the smallest pit and was not dug as deep as the others, apparently because the bedrock was significantly harder and more difficult to dig. Feature 31 is also different in that its fill contains areas of darker organic-rich sediment, charcoal staining, and/or ashy sediment. Although the fill was not excavated, burned rocks also were observed. Unlike any of the other pits, it appears that Feature 31 was backfilled with cultural trash. Its small size probably made Feature 31 less useful for storage than the other pits, perhaps explaining why it was abandoned and filled with trash while the others were not. It should be noted that the apparent absence of charred macrobotanical remains from Feature 31 (see Table 7 and Appendix E) is misleading, since the flotation sample was taken from an area at the bottom of the pit where very little charcoal staining was observed.
Two of the pits, Features 18 and 19, are unique in that both had very large complete oval basin metates lying upside down at the tops of the pits (see Figure 12). The Feature 18 metate measures $59 \times 37 \times 9.5 \mathrm{~cm}$ and weighs $32.6 \mathrm{~kg}$; the Feature 19 metate is $57 \times 36 \times 10 \mathrm{~cm}$ and weights $27.2 \mathrm{~kg}$. Feature 18 also had four large sandstone rocks in its fill. Three were lying immediately under the metate near the top of the pit, and a fourth rock was found near the bottom of the pit. Two large rocks also were found on top of Feature 19 at approximately the same level as the metate.

One significant characteristic of these features is that all were excavated into bedrock. The bedrock underlying the cultural zone consists of $5-10 \mathrm{~cm}$ of calcareous weathered fragments grading into hard sandstone and mudstone. A test excavation into the underlying strata confirmed that the bedrock is very hard and difficult to dig. Clearly, the excavation of these large pits into hard bedrock represents a significant labor investment, thus raising the question of motive. A plausible explanation is that the bedrock walls and floors of the pits provided a solution to a number of serious problems in subsurface pit storage. Wedel (1986:122-123) states that the two most important concerns with pit storage are controlling moisture (to prevent rotting of foods due to bacteria or fungi growth) and preventing contamination or consumption of foods by burrowing rodents. A bedrock storage pit would not allow moisture or rodents to get in from the bottom or sides, and if the pits were properly capped, they would have been 


\begin{tabular}{|c|c|c|c|c|c|}
\hline \multicolumn{6}{|c|}{ TABLE 10} \\
\hline $\begin{array}{l}\text { Feature } \\
\text { No. }\end{array}$ & $\begin{array}{l}\text { Diameter } \\
(\mathrm{cm})\end{array}$ & $\begin{array}{l}\text { Depth } \\
(\mathrm{cm})\end{array}$ & Elevation $(\mathrm{m})$ & $\begin{array}{l}\text { Volume } \\
\left(\mathrm{m}^{3}\right)^{*}\end{array}$ & Description of Investigation ${ }^{* *}$ \\
\hline 17 & 110 & 80 & 99.75-98.95 & $0.53-0.68$ & Entire pit excavated; stratified samples taken \\
\hline 18 & 104 & 78 & $99.75-98.97$ & $0.46-0.59$ & Entire pit excavated; stratified samples taken \\
\hline 19 & 98 & 65 & $99.80-99.15$ & $0.34-0.44$ & Entire pit excavated; stratified samples taken \\
\hline 20 & 110 & 66 & $99.83-99.17$ & $0.44-0.57$ & $\begin{array}{l}\text { Found in BHT 1992-2, ca. one-half of the pit } \\
\text { was destroyed; the other half was excavated, and } \\
\text { stratified samples were taken }\end{array}$ \\
\hline 24 & 85 & 62 & $99.75-99.13$ & $0.24-0.31$ & Entire pit excavated; stratified samples taken \\
\hline 25 & 110 & 71 & $99.75-99.04$ & $0.47-0.60$ & $\begin{array}{l}\text { One-half of pit excavated and sampled; the other } \\
\text { half of the fill was left intact }\end{array}$ \\
\hline 30 & 120 & 55 & $99.80-99.25$ & $0.48-0.61$ & $\begin{array}{l}\text { Found in BHT 1992-7, ca. one-third of the pit } \\
\text { was destroyed; remainder was sampled but } \\
\text { otherwise left intact }\end{array}$ \\
\hline 31 & $74(?)$ & $30(?)$ & $99.80-99.50$ & $0.09-0.12$ & $\begin{array}{l}\text { Found in BHT 1992-13, ca. one-half of the pit } \\
\text { was destroyed; remainder was sampled but } \\
\text { otherwise left intact }\end{array}$ \\
\hline 32 & 100 & 50 & $99.75-99.25$ & $0.27-0.35$ & $\begin{array}{l}\text { Found in BHT 1992-13, ca. two-thirds of the pit } \\
\text { was destroyed; remainder was sampled but } \\
\text { otherwise left intact }\end{array}$ \\
\hline 39 & 92 & 72 & $99.82-99.10$ & $0.33-0.42$ & $\begin{array}{l}\text { One-half of the pit was excavated and sampled, } \\
\text { but the fill was not screened; the other half of the } \\
\text { fill was left intact }\end{array}$ \\
\hline Average & 100.3 & 62.9 & - & $0.37-0.47$ & \\
\hline \multicolumn{6}{|c|}{$\begin{array}{l}\text { **All excavated fill was screened through } 1 / 1 \text {-inch mesh except where otherwise noted. "Sampled" refers to flotation } \\
\text { and sediment samples taken from the bottoms of pits, while "stratified samples" refers to flotation and sediment } \\
\text { samples taken at different levels in the pit fill. }\end{array}$} \\
\hline
\end{tabular}

quite secure.

Also, the pits appear to be clustered intentionally, and their location probably is related to the need for waterproof and rodent-proof storage. The pits are located on a very slight rise on the upland edge, and water drains away in all directions. This rise is also the only area investigated where consolidated weathered bedrock is shallowly buried. At the time the pits originally were dug, only a thin layer of sandy sediment $(\mathrm{ca} .10-20 \mathrm{~cm}$ ) would have capped the bedrock, while the sediment cover increases in thickness in all directions from the pit cluster. Thus, only minimal effort was needed to remove the sandy overburden to dig the pits, whereas in any other part of the site significant amounts of sediment $(50 \mathrm{~cm}$ or more) would have to have been removed.

Further, the exposed bedrock ledges around the site (in the eroded areas all along the edge of the upland flat) are not suitable for pits for several reasons. The exposed bedrock is either badly 


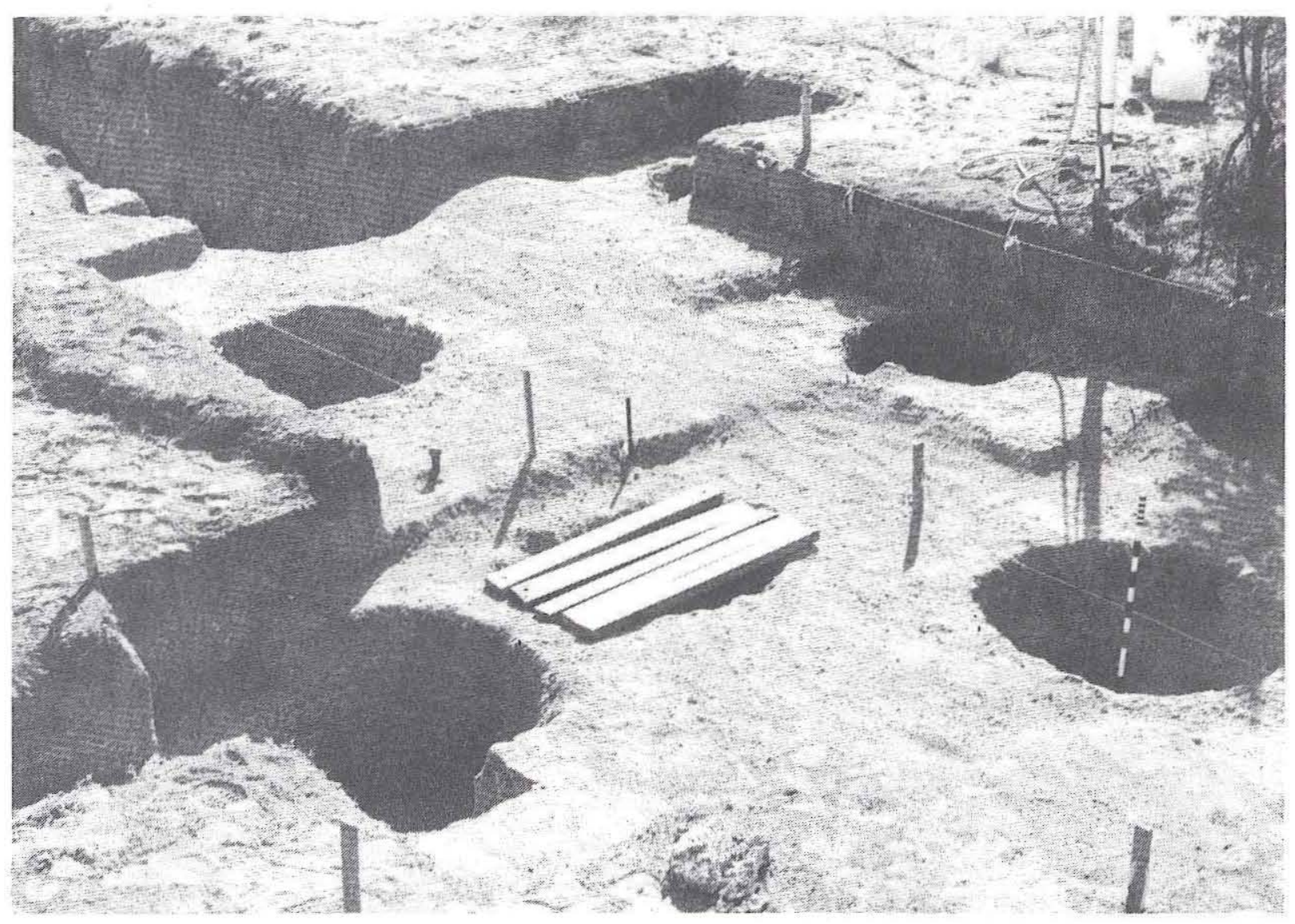

Figure 11. Overview looking southeast showing excavated storage pits in Block 2,41GR291. Feature 17 is in right foreground (with scale), Feature 18 is in left foreground, Feature 24 is center right, Feature 19 is center left, and Feature 20 is in backhoe trench at upper left. Features 25 and 30 were not excavated at the time.

fractured and friable, or it is intact and hard as concrete. This is in sharp contrast to the bedrock layers that have been shallowly buried and weathering in situ for a long time. These strata are still solid enough to be extremely firm and stable yet friable enough to be dug.

Taking into account the effects of a wide range of postdepositional alterations, the nature of the fill inside pits should reflect different abandonment or postabandonment processes such as those described by Wilson (1985). There are four potential explanations to account for the condition of the pits at the time of abandonment: (1) they were intentionally backfilled with trash; (2) they were full of stored products; (3) they were left empty (or only partially full) and filled by natural processes; and (4) they were intentionally backfilled with clean fill. Interpretations of abandonment conditions for the pit features at the Sam Wahl site are confused by postdepositional factors such as recent bioturbation and hundreds of years of pedogenic alteration, but differences in the fill attributes suggest that more than one explanation is applicable. While the abandonment and postabandonment processes are probably more complex than these simplified explanations suggest, they are useful as working models that relate the archeological attributes (i.e., characteristics of pit fill) to the cultural and natural processes which produced them. DeBoer (1988:4) notes that defining the nature and "associational status" of pit fill is critical to interpreting their use and/or abandonment histories. Each explanation is presented below with a discussion of its relative merits for explaining the pit features at the Sam Wahl site.

The first explanation is that the pits were intentionally backfilled with trash at the time they were abandoned. As mentioned above, Feature 31 appears to be the only one that was reused as a trash pit, while all the other pits had relatively clean fill. Although small quantities of lithic debris, charred 


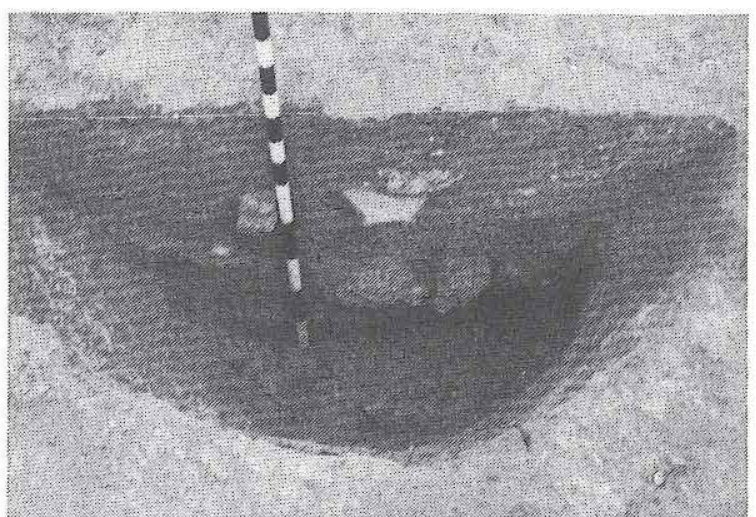

a

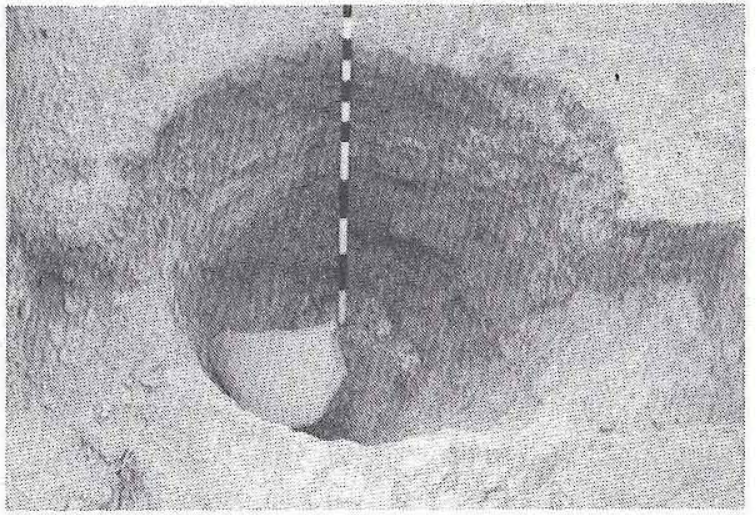

C

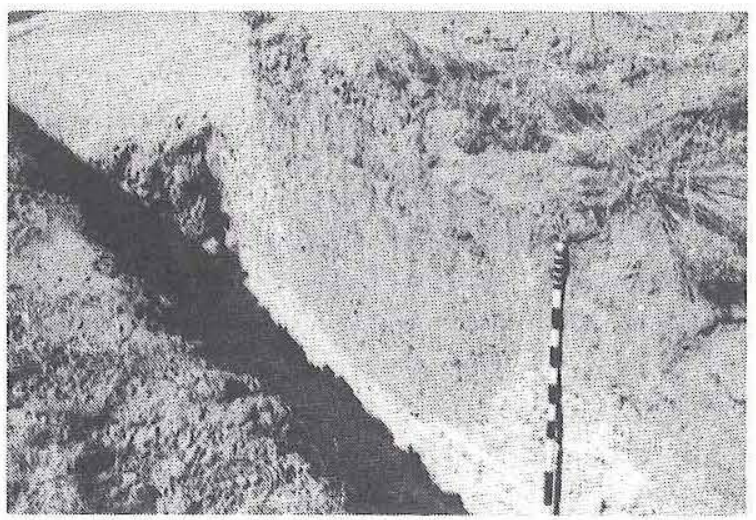

e

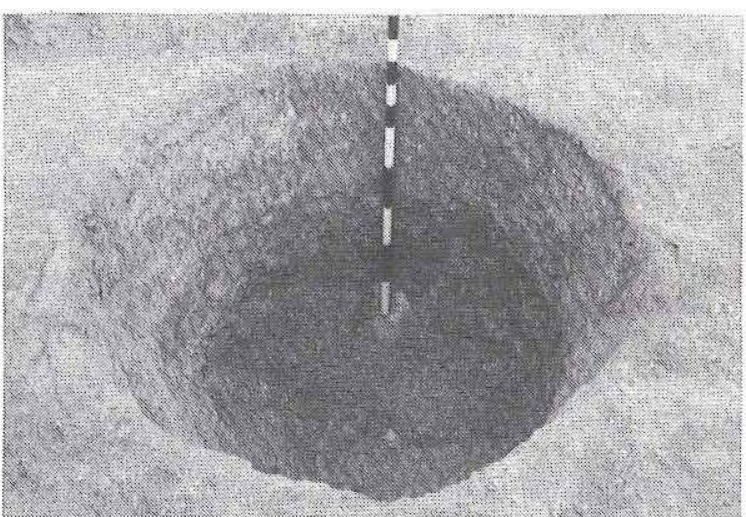

b

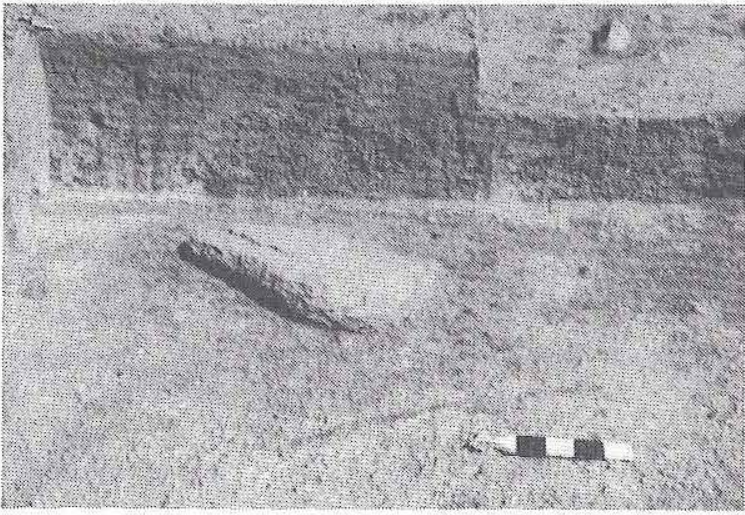

d

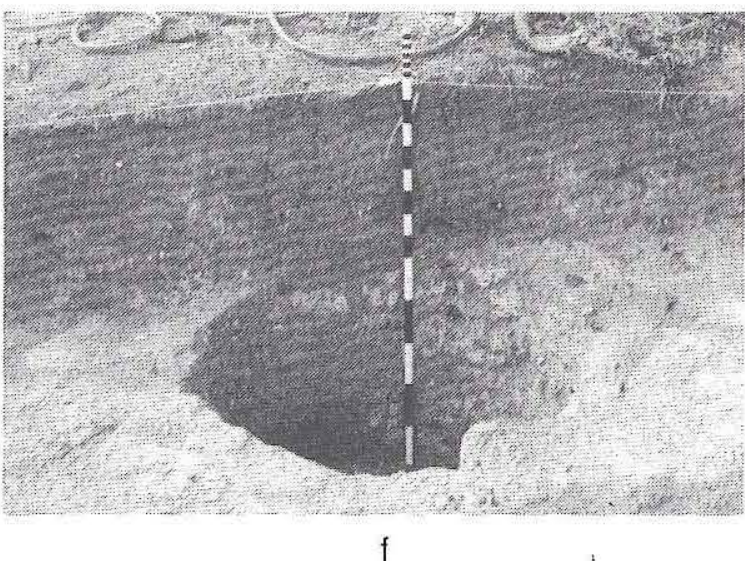

f

Figure 12. Storage pits at various stages of excavation, 41GR291. (a) Feature 17, south half excavated showing rocks in fill; (b) Feature 17, completely excavated; (c) Feature 18, completely excavated with rock in bottom; (d) Feature 19, unexcavated with pit outline defined and metate exposed; (e) Feature 20, exposed in Backhoe Trench 1992-2; $f$ ) Feature 24, completely excavated showing relationship of pit and overlying sediment. 

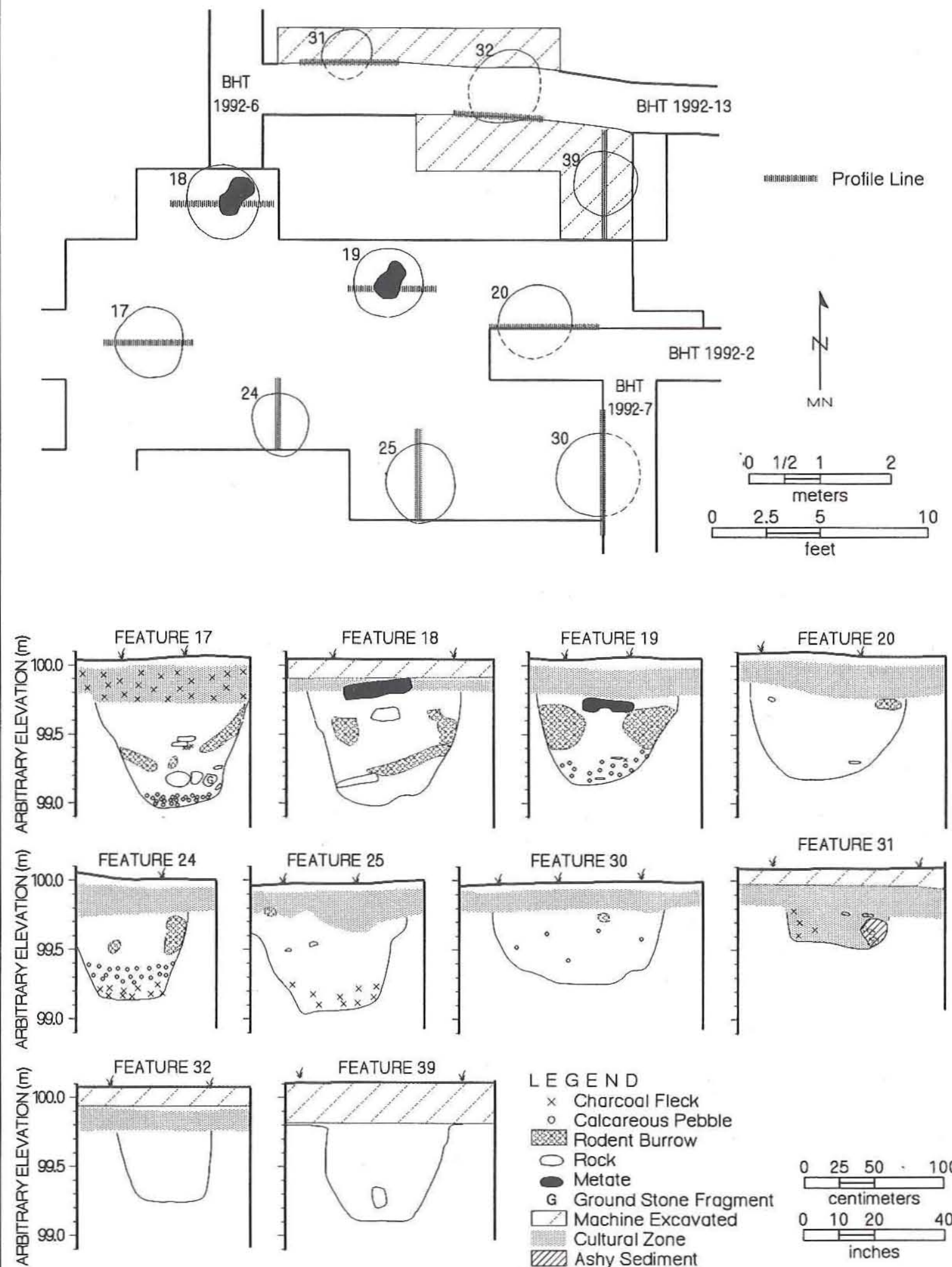

LEGEN D

$\times$ Charcoal Fleck

- Calcareous Pebble

rax Rodent Burrow

$\checkmark$ Rock

- Metate

G Ground Stone Fragment

Machine Excavated

Cultural Zone

VIIA Ashy Sediment

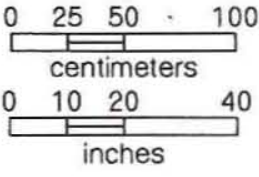

P\&AI/94/SLH

Figure 13. Plan and profile drawings showing horizontal relationships and fill characteristics of the storage pits, 41 GR291. 


\begin{tabular}{|c|c|c|c|c|c|}
\hline \multicolumn{6}{|c|}{ TABLE 11} \\
\hline \multicolumn{6}{|c|}{ SUMMARY OF MATERIALS RECOVERED FROM STORAGE PIT FILL, $41 G R 291$} \\
\hline $\begin{array}{l}\text { Feature } \\
\text { No. }\end{array}$ & $\begin{array}{l}\% \text { of Fill } \\
\text { Represented* }\end{array}$ & Lithic Artifacts & $\begin{array}{l}\text { Unmodified } \\
\text { Rocks }\end{array}$ & $\begin{array}{l}\text { Burned } \\
\text { Rocks }\end{array}$ & $\begin{array}{l}\text { Total Rock } \\
\text { Weight }(\mathrm{kg})\end{array}$ \\
\hline 17 & 100 & 1 uniface, 2 ground stone fragments, 17 flakes & 18 & 24 & 15 \\
\hline 18 & 100 & complete metate, 1 core, 28 flakes & 18 & 8 & 16 \\
\hline 19 & 100 & complete metate, 2 cores, 18 flakes & 10 & 15 & 11 \\
\hline 20 & 50 & 11 flakes, 1 flake from flotation & 6 & 8 & 2 \\
\hline 24 & 100 & 1 cobble tool, 2 ground stone fragments, 5 flakes & 3 & 8 & 1 \\
\hline 25 & 50 & 9 flakes & 11 & 9 & 2 \\
\hline 30 & 0 & none & - & - & - \\
\hline $31^{* *}$ & 0 & 1 flake from flotation & - & - & - \\
\hline 32 & 0 & 2 flakes from flotation & 1 & - & 10 \\
\hline 39 & $* * *$ & 1 flake from flotation & & - & - \\
\hline \multicolumn{6}{|c|}{$\begin{aligned} * 100 \% & =\text { Entire feature excavated and screened. } \\
50 \% & =\text { Half of feature was excavated and screened. } \\
0 \% & =\text { Feature was found in backhoe trench and fill was sampled but not excavated. }\end{aligned}$} \\
\hline \multicolumn{6}{|c|}{ **Intensive charcoal staining was observed in the Feature 31 fill. } \\
\hline
\end{tabular}

seeds, and charred wood were recovered from most of the pits, intensive charcoal staining, which generally characterizes trash-filled pits (Dickens 1985:37), was observed only in Feature 31. Trashfilled pits are common in many archeological sites. But Dickens (1985) points out that it is unlikely that such pits were dug specifically to serve as trash repositories and that the original function of trashfilled pits was probably as storage facilities. Dickens (1985:42-43) also suggests that such pits were filled with trash soon after they were permanently abandoned.

The second explanation is that the pits may have been filled with something that was being stored, such as dried plant foods, which subsequently deteriorated without leaving any physical evidence. Archeological evidence of pits abandoned in this state might be limited. Perhaps pollen or phytolith remains would be preserved in the fill, but charred macrobotanical remains would not be expected unless cooked or roasted foods were being stored. Pits that were full at the time of abandonment would have been capped, but Wedel (1986:122) suggests that it is unlikely that any evidence of storage pit coverings, such as alternating layers of wood, grass, hide, and sediment, would survive. For example, layered pit coverings, described for Hidatsa storage pits by Wilson (1987:87-95) and for Pawnee storage pits by Weltfish (1965:69), included only organic materials and sediment and thus would leave little or no archeological evidence. If rocks, perhaps used as weights or markers, were incorporated into the pit covering, however, some contextual evidence would survive in the form of rocks that collapsed into the pit as the organic materials deteriorated. As previously noted, this is a plausible interpretation for Feature 17 at the Sam Wahl site, and Dickens (1985:38) also interprets rocks found in the fill of some storage pits as covering slabs. The abandonment of producefilled pits would suggest that the inhabitants intended to return and use the contents but, for whatever reason, never did.

The third explanation is that the pits may have been left empty (with or without having been capped) the last time they were used and then subsequently filled with sediment through natural processes. If the pits were empty and not capped at abandonment, they would have filled rapidly with wind-blown sands, 
and some cultural materials (e.g., small flakes and charcoal) from the surrounding area easily could have been introduced. Likewise, a pit that was capped when empty (or partially filled with produce) eventually could have deteriorated to the point that its covering collapsed, with the remainder of the pit subsequently filling with eolian materials. In some cases, differential layering of pit fill should be expected to indicate the sequence of events (as noted by Wilson 1985), but this requires certain soil conditions and limited postdepositional disturbance. Unfortunately, the sandy loam sediments at the Sam Wahl site are not conducive to such preservation.

It is unlikely that the pits would have been left open during periods of site abandonment if the occupants intended to return and reuse them for storage. Ethnographic evidence suggests that concealment was an important aspect of pit storage (Raymer 1988; Ward 1985:99), and leaving pits open would have revealed their locations. Covering and concealing of partially filled storage pits is documented for the Pawnee by Weltfish (1965:238-239), whose informants noted that upon returning to the village for the fall harvest, ladders were put down into a pit so that it could be entered to retrieve stored items and inspected. Thus, it is possible that empty or partially filled pits were capped during periods of abandonment. Perhaps this is the case for Feature 17, which had a large number of rocks scattered throughout its fill. Although the rocks could have been thrown in if the pit was intentionally backfilled with clean fill or trash, their placement suggests that they represent a capping rock layer that collapsed into an empty or nearly empty pit.

The fourth explanation is that the pits may have been intentionally backfilled with sediment when they were abandoned. As mentioned above, archeological data from other parts of North America indicate that pits that were intentionally backfilled without the intent of being used again generally were filled in with trash. Conversely, one would expect backfilling with clean sediment if pits were intended to be used again. There are several aspects of intentional backfilling that make it an appealing interpretation. During periods of temporary village abandonment, backfilling might have served to hide pit locations, prevent water from collecting and deteriorating pit floors and walls, and possibly even rejuvenate pits that had been soured by spoiled produce. In addition, backfilled pits would not be in danger of being discovered because their coverings collapsed.
Although sparse cultural materials (e.g., flakes and charcoal flecks) probably would be present even in relatively clean fill, this type of backfilling is quite different from reusing the pit as a trash repository. Backfilling with clean fill suggests that the inhabitants intended to use the pit for storage again.

Two of the Sam Wahl site storage pits are good candidates for having been intentionally backfilled. Features 18 and 19 had metates, which apparently were cached upside-down, on top of the pits. If the pits were empty or filled with produce, one would expect the metates to have fallen into the pits at angles when the coverings collapsed. If, however, the pits were intentionally backfilled with sediment (which may have been compacted as the pits were filled), the metates cached near the tops would not have moved to any significant degree. This scenario is also appealing because the cached metates, which probably were covered over by sediment so the pit locations were concealed, also could have served as markers for re-locating the pits upon returning to the site. The caching of metates on storage pits suggests that the inhabitants expected to return and reuse the metates and the pits. Collins (1968:73-79) notes five cases where metates and/or manos were cached at the Salt Cedar site in Andrews County; in two of the cases, the items were cached inside small claycapped pits.

One possible alternative explanation for Features 18 and 19 is that they were empty, partially full, or full of produce but were capped in such a way that the pits filled with sediment without the metates collapsing. While this scenario may seem implausible at first, it is easily explained if the pits were capped with wooden covers consisting of branches or split logs laid across the mouths of the pits. The Hidatsa covered storage pits with a thick hide and a layer of grass at the top of the pit and a wooden "puncheon cover" over the mouth; on top of that, another layer of grass was "trampled down hard, well into the mouth of the pit," followed by a second hide which was covered over with "earth until the pit was filled level with the ground" (Wilson 1987:93-94). Weltfish (1965:69) describes a similar technique used by a Pawnee woman for covering a storage pit: "First she covered everything with a skin cover, then a layer of thatch grass, then she wedged some sticks across the opening into the side walls, then another layer of grass and then earth that she trampled down hard to keep out the rain and to conceal the place of the opening." These types of layered coverings 
apparently supported a considerable amount of weight (at least the weight of a person, and Wilson [1987: 93] indicates that Hidatsa pits could support the weight of "any horse"). After abandonment, the wooden portion of such coverings probably would outlast the rest of the layers, and sediment could wash and/or blow into the pit between the logs and effectively fill up the pit while an item such as a cached metate was held in place at its mouth. It is also possible that the metates were not merely cached but functioned as lids laid on top of the pit covering so that they could be removed to provide access to the pits. Amsden (1949:Figure 3) illustrates this type of covering, found intact on top of slab-lined storage cists in a dry cave in Utah. He notes that the cists "usually were roofed with a crisscross of poles and brush, overlaid with bark, grass, or leaves, and sometimes capped with a layer of adobe mud. A little square hatchway in the center, covered with a single slab, was the entrance" (Amsden 1949:49).

Aside from the pits specifically mentioned in the discussions above (i.e., Features 17, 18, 19, and 31), the remainder of the pits have relatively clean fill and no associated metates or significant concentrations of rocks. At the time of their abandonment, these pits could have been empty (or even full of produce) and subsequently filled in when their coverings collapsed, or they could have been intentionally filled with clean sediment. The preceding discussions suggest that there are several possible origins for the fill in the pits at the Sam Wahl site.

The construction of two of the pits apparently occurred prior to A.D. $600-670$ and $670-880$. This assessment is supported by roughly contemporaneous radiocarbon dates on charcoal from the fill of two of the pits (see Table 6). A calibrated AMS date of A.D. 609 (651) 669 was obtained from the bottom of Feature 19 at $99.27-99.14 \mathrm{~m}$, and a calibrated date of A.D. 669 (772) 883 was obtained from Feature 17 on charcoal from below a large rock in the middle of the pit at $99.38 \mathrm{~m}$. These dates are interpreted as representing the approximate time of pit abandonment, but this interpretation makes two critical assumptions.

First, it assumes that the pit fill represents rapid accumulation, and second, it assumes that the charcoal samples represent wood that is roughly contemporaneous with the abandonment/filling episode(s). The first assumption is well founded because pits that were intentionally backfilled with clean sediment (e.g., Feature 19?) certainly represent rapid accumulation; pit filling through natural processes (i.e., wind- and water-lain sediments) also would have occurred rapidly in the windy Southern Plains, and even pits that were capped (whether empty or full of produce) probably would not have survived for many years before their coverings collapsed and they filled in with sediment (e.g., Feature 17?).

The second assumption, however, is more problematic. The possibility that older charcoal (i.e., from earlier occupations) or charcoal from old wood (i.e., burning of wood that is considerably older than the burning event) were introduced cannot be ruled out. The potential for this type of bias is difficult to evaluate, and one can only suggest that it is more likely that wood charcoal introduced into pits (by cultural or natural processes) is roughly contemporaneous with the filling process. Quite simply, the likelihood of such biases decreases as the significance of the error factor increases (i.e., the older the sample, the less likely it is to survive and be introduced into later contexts). Consequently, the dates for the two pits at the Sam Wahl site are considered to represent approximate abandonment dates.

The chronology of the construction and use of the pits, however, is a different matter. The two dated pits may have been in use for a long time before they were abandoned, and as Wilson (1987:95) notes, "[a] cache pit lasted for a long time, used year after year." Unfortunately, it is impossible to determine when the Sam Wahl site pits were constructed, and it cannot be assumed that all of them were constructed at the same time. However, three lines of evidence suggest that all of the pits may have been constructed by the same group of people and may have been in use at approximately the same time. First, all of the pits appear to have been excavated from the same level, suggesting approximate contemporaneity. Although the precise top of each pit was sometimes questionable, they all appear to originate consistently from the lower $15 \mathrm{~cm}$ of the cultural zone, at ca. $18-30 \mathrm{~cm}$ below the surface. It is likely that this level represents the prehistoric surface at the time the pits were excavated. Second, the uniformity of the pit morphology suggests that the people who constructed these pits were following a well-defined mental template (i.e., a traditional style for their culture). And finally, the pits are clustered within a small (ca. $8 \times 8 \mathrm{~m}$ ) area and are neatly arranged in rows. This spatial patterning probably reflects 
intentional planning. While it does not necessarily mean that they all were constructed at the same time, it could indicate that each time a new pit was constructed, the spatial patterning was followed.

Since there is no particular reason why any of the storage pits (except for aberrant trash-filled Feature 31) could not have been reused indefinitely, it is possible that all of the pits may have been in use at one time. Perhaps their precise spatial arrangement was necessary because they were all used contemporaneously. The edge-to-edge distance between adjacent pits (excluding diagonal measurements) is between 0.9 and $2.0 \mathrm{~m}$ (average distance is $1.37 \mathrm{~m}$ ). This $1-2-\mathrm{m}$ distance between pits is a reasonable spacing to insure adequate access to all of the pits if they were in use at the same time. If this is the case, then the entire storage pit complex would have been constructed some time prior to A.D. 600-670, the date when Feature 19 was abandoned.

Alternatively, it is possible that some of the storage pits were abandoned before others were constructed, in which case not all of the pits had to be constructed prior to A.D. 600-670. The logical problem with this latter scenario is that, if some pits were abandoned, there would have been no need to maintain the spatial arrangement as new pits were constructed.

Using the same line of reasoning as above, it cannot be assumed that all of the storage pits were abandoned at the same time. In fact, the opposite is probably true, and the abandonment dates for the pits could vary considerably. Interestingly, the calibrated dates for Features 17 and 19 suggest that they could have been abandoned ca. 120 years apart, and it is possible that some of the other pits were still in use long after these were abandoned. It is impossible to know when the last storage pit was abandoned, but most, if not all, probably were abandoned before A.D. 1200. This assessment is based on the two radiocarbon dates from Feature 12, which yielded calibrated dates ranging from A.D. 1166-1379. Stratigraphically, Feature 12 is above and clearly postdates Features 17 and 18, and it appears to postdate the entire storage pit complex.

A substantial volume of storage capacity is represented by these pit features (see Table 10). The nine larger pits (excluding Feature 31) range in individual volume from a minimum of $0.24 \mathrm{~m}^{3}$ to a maximum of $0.68 \mathrm{~m}^{3}$, with an average capacity of $0.40-0.51 \mathrm{~m}^{3}$. All 10 of the pits would have provided a total storage capacity of $3.65-4.69 \mathrm{~m}^{3}$.
Although Schroedl (1980:33, cited in DeBoer 1988:4) suggests that pits used for bulk food storage are characteristically large $\left(>1.12 \mathrm{~m}^{3}\right)$, it may have been easier and more efficient to make larger numbers of smaller pits in many circumstances. A great deal of variability has been noted for storage pits in the American Southwest (Woodbury and Zubrow 1979: 52 ), and there are many occurrences of storage pits with capacities less than $1 \mathrm{~m}^{3}$ (e.g., DeBoer 1988:67).

Although the question of what was stored in these pits is addressed later in this chapter, Binford (1990:141-144) suggests that plant foods are much more likely to be stored in moderate climates than is meat, which tends to be stored in regions with colder climates. This suggests that subsurface pits in the Lake Alan Henry area, with an effective temperature of $14.85^{\circ} \mathrm{C}$, probably would have been used to store plant foods. The direct association of the metates cached on top of storage pits provides further circumstantial evidence that they were used for storage of plant foods. While there is no definitive macrobotanical evidence for what was stored in these pits, the large number of charred Chenopodium seeds recovered from Feature 19 (see Appendix E) is particularly intriguing. Although these seeds do not necessarily mean that Chenopodium was stored in the pit, the fact that they are charred suggests that they may represent a food resource that was utilized at approximately the same time as the storage pits.

\section{Pithouse and Related Features}

Feature 37 is a pithouse first encountered in a maintainer blade cut which exposed darkly stained sediments and artifacts. Backhoe Trench 1992-31 was then excavated along the west edge of the blade cut to reveal the nature of the stained area. The west profile of the trench clearly revealed the shallow pithouse depression, and hand clearing of the eolian sand to the west exposed the dark oval stain in plan view at ca. 8-10 cm below the surface (ca. $99.20 \mathrm{~m}$ elevation). Although ca. one-quarter of the structure was disturbed by the backhoe, the rest of the feature remained intact and was completely excavated by hand in 1-x-1-m units (Block 1, EUs 1-15). Figure 14 reveals the excavation sequence of Feature 37.

Feature 37 is an ovate, $3.5-x-3.0-\mathrm{m}$, basinshaped depression filled with dark charcoal-stained sediment and cultural debris (Figure 15). The pithouse floor is at $58 \mathrm{~cm}$ below the surface (ca. 


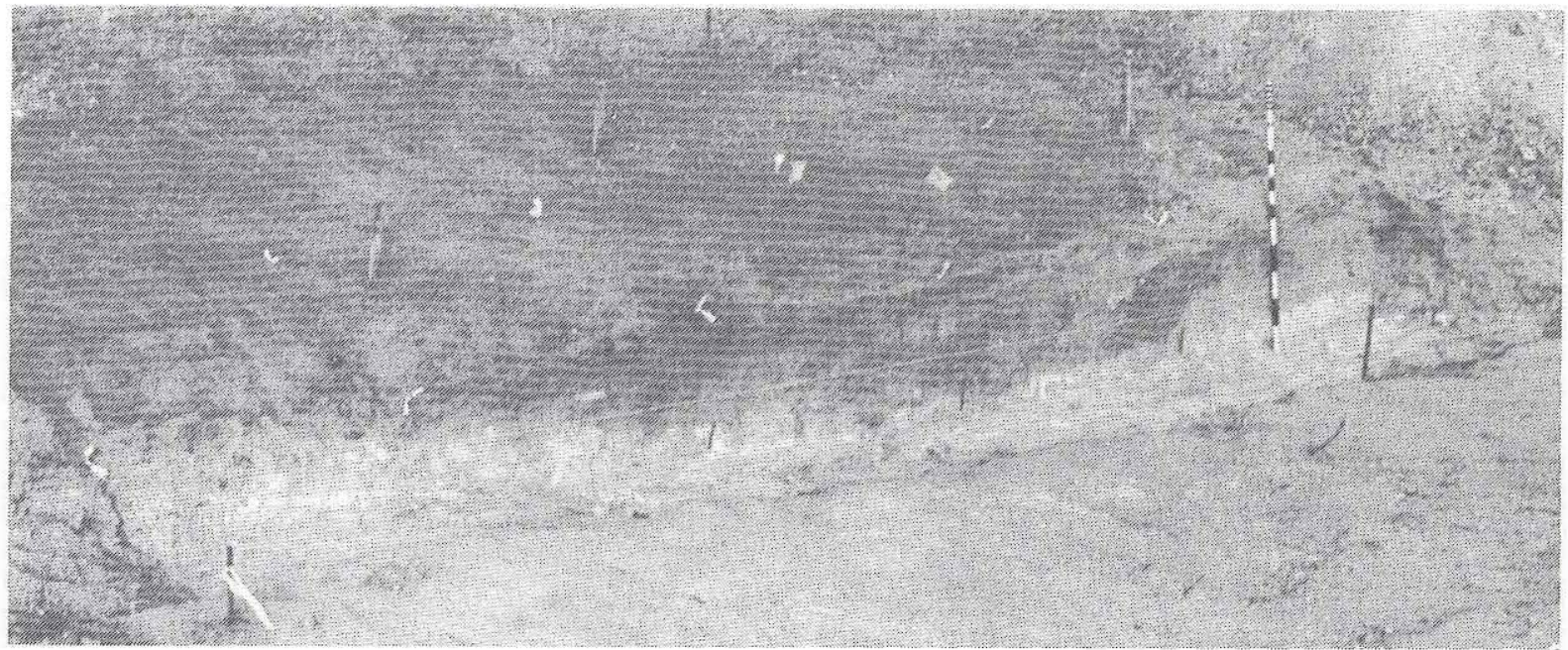

a

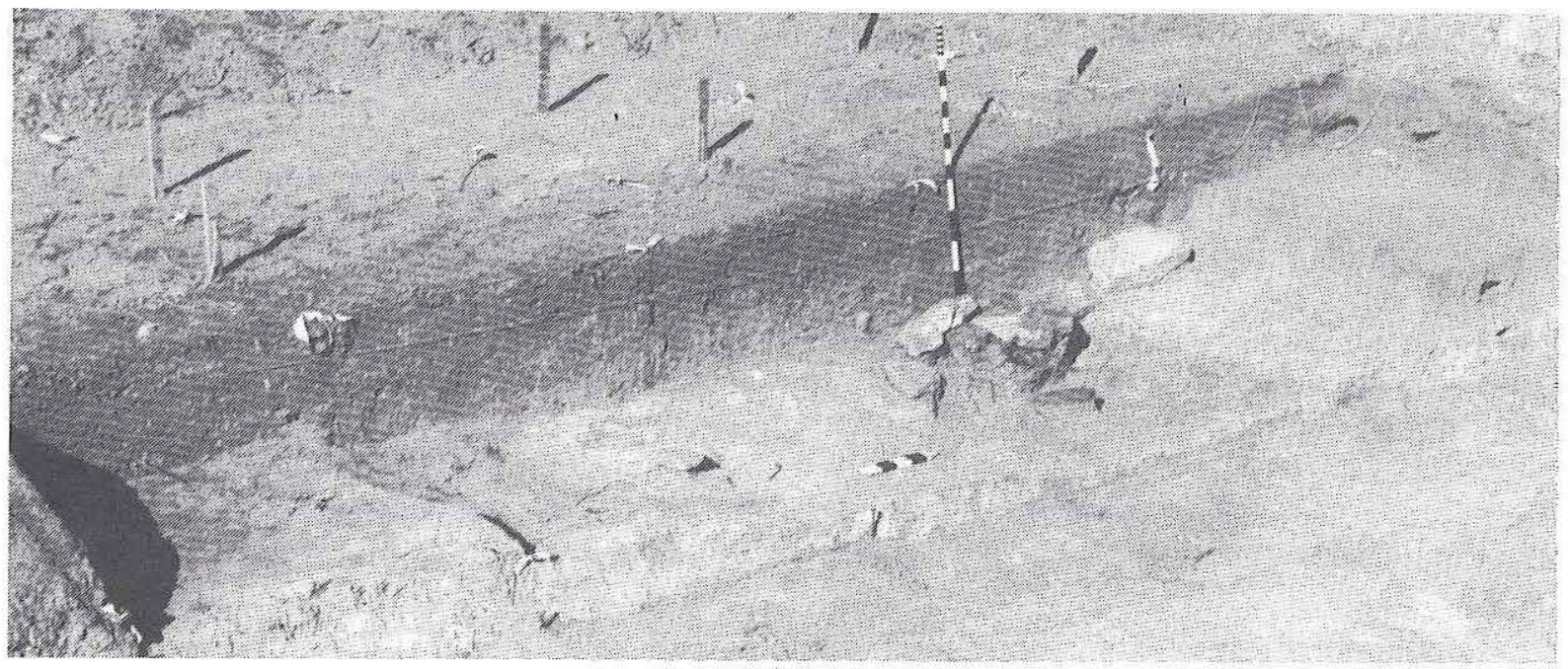

b

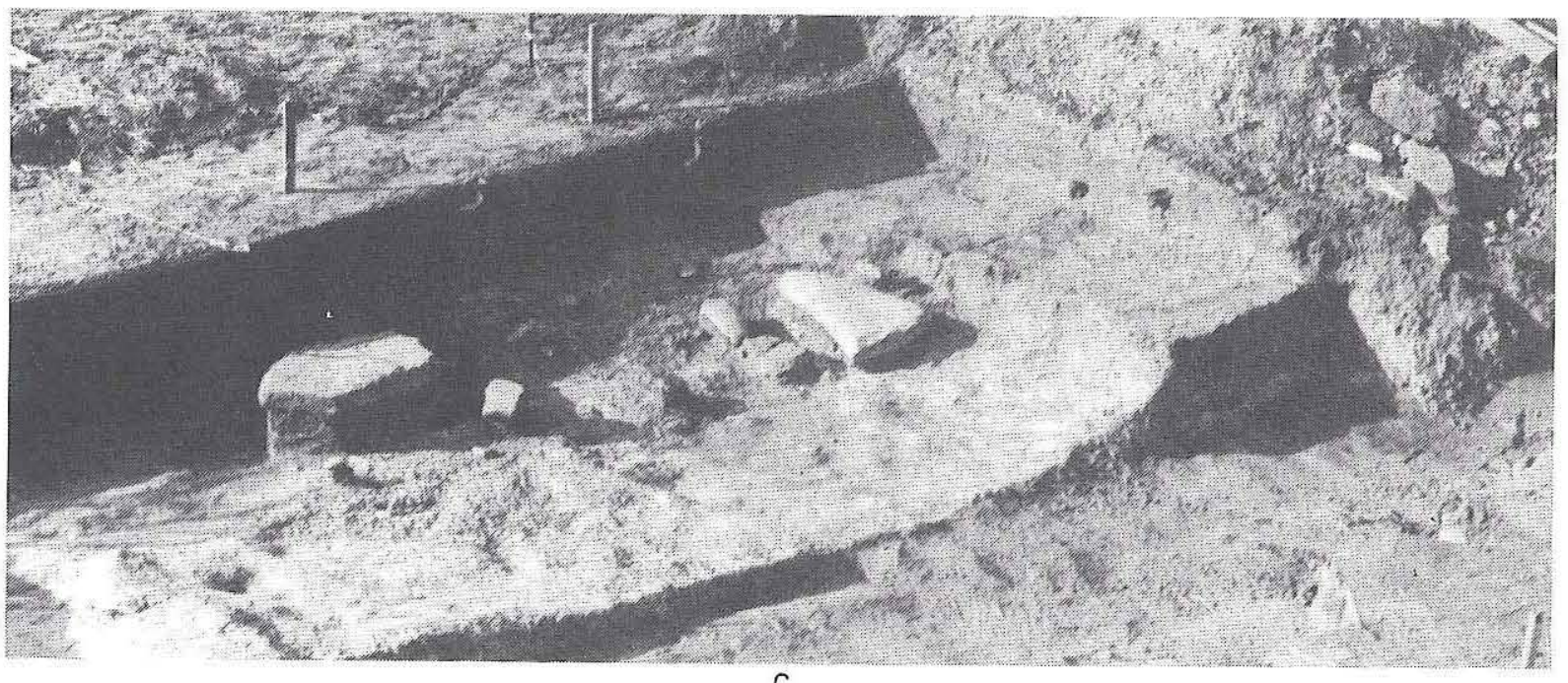

Figure 14. Series of photographs showing excavation of pithouse Feature 37,41GR291. (a) Profile of pithouse as first seen after cleaning the west wall of Backhoe Trench 1992-31; $(b)$ pithouse after first row of $1-\mathrm{x}-1-\mathrm{m}$ units was excavated; $(c)$ pithouse after second row of $1-x-1-m$ units was excavated. The two largest rocks are upside-down basin metates. 


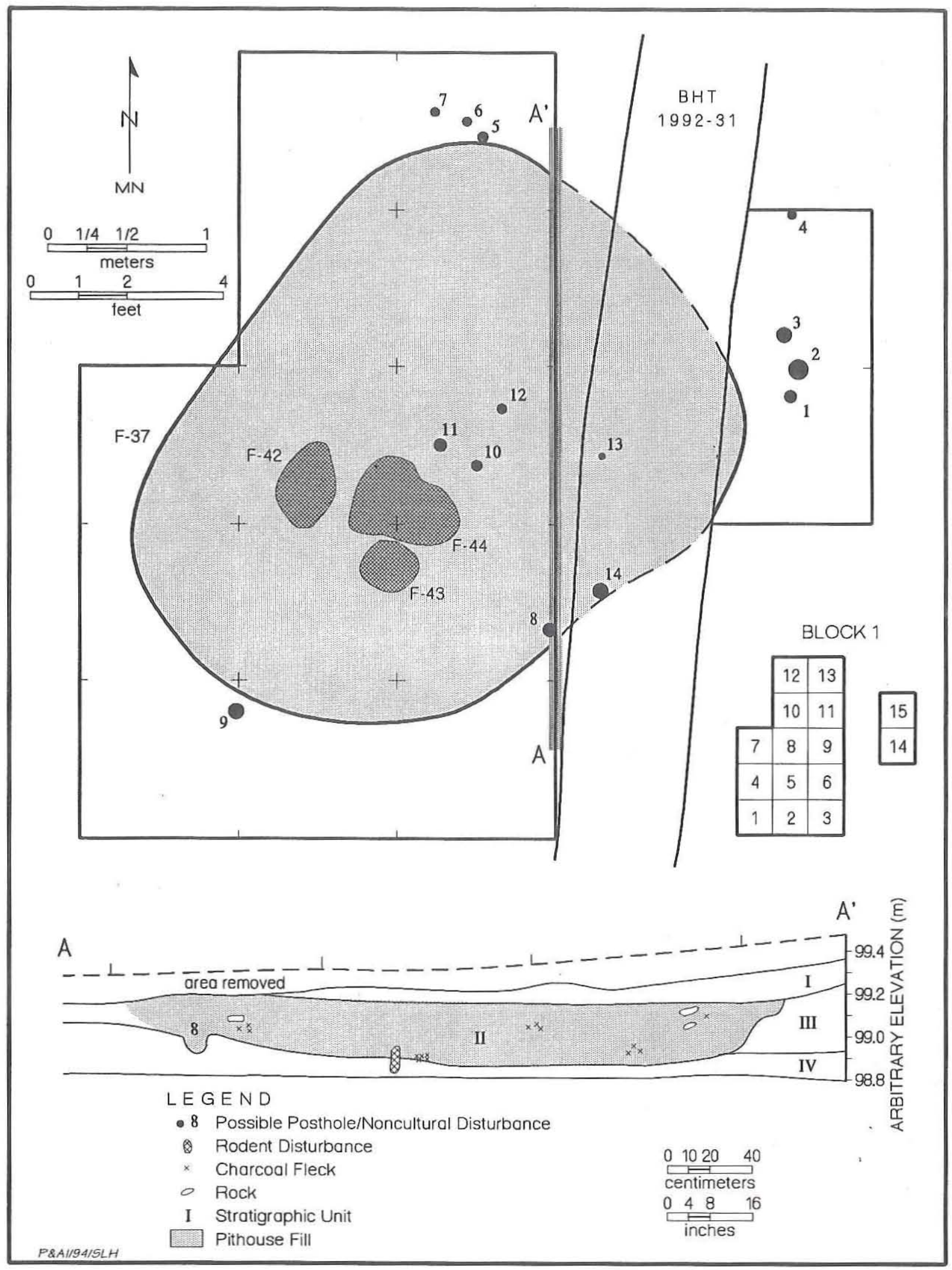

Figure 15. Plan and profile drawings of pithouse Feature 37, 41GR291. 
$98.82 \mathrm{~m})$ at it lowest point, located in the northcentral part of the structure (in EU 9). At this point, the dark cultural fill is well defined at $10-13 \mathrm{~cm}$ (99.30-99.27 m). Thus, Feature 37 extends 45 and $48 \mathrm{~cm}$ below the original ground surface (which was at ca. $99.40 \mathrm{~m}$ ).

Figure 16 illustrates the topography of the structure floor and the horizontal and vertical distributions of the large rocks in the fill (elevations are relative to the arbitrary $100.00 \mathrm{~m}$ elevation of the site datum). Except for some rodent-disturbed areas in the southwest end of the pithouse, the floor surface was easily defined as the contact between the charcoal-stained fill and the friable weathered bedrock. The floor zone was more distinct in the north half of the structure than in the southern half, where large areas of rodent disturbance were noted. Generally, however, the floor zone is rather uniform, with slight undulations observed in some areas.

The north wall of the structure is quite distinct because the floor turns abruptly to form a vertical edge which is clearly defined by the contact between the dark cultural fill and the sterile yellowish red (7.5YR 4/6) loam. The south and west edges of the structure are less well defined because the floor slopes gradually upward in these directions and no absolute wall contact was apparent. The approximate south and west edges of the structure, however, were defined as the point were the bedrock substrate levels off and the dark-stained sediment (i.e., the fill) becomes less apparent.

A significant number of large rocks were present in the pithouse fill, and their elevations correspond to the contours of the floor, with the highest rocks found along the edges of the structure and the lowest ones in the central area. The locational relationship between the rocks and the structure is apparent, but their contextual relationship is less obvious. Many of the rocks may be unrelated to the pithouse occupation and could represent materials discarded into the pithouse depression after it was abandoned. Conversely, most or all of the rocks could be directly related to the structure. Some could have been used as weights, braces, or shims along the base of the walls or on the superstructure, while others could have been used inside the structure for various purposes. The large cluster of rocks in the northcentral part of the structure (in EUs 9 and 11) is particularly intriguing since they are in the lowest part of the structure (see Figures 14 and 16). These rocks could represent an interior feature or perhaps were on top of the superstructure. While the origins and possible functions of most of these rocks remain a mystery, at least three specimens are functional tools that probably were stored (i.e., cached) inside, or on top of, the structure.

Two of these are oval basin metates (see Figures 14 and 16) that were found face down on or near the floor, and the third is a slab metate that was brought up by the backhoe from the eastern half of the structure. The smaller of the two basin metates $(56 \times 35 \times 8.9 \mathrm{~cm})$ weighs $21.5 \mathrm{~kg}$ and was found lying directly on the sloping floor/north wall of the pithouse at $99.13-98.94 \mathrm{~m}$. The larger basin metate (75 x $47.5 \times 9.1 \mathrm{~cm}$ ) weighs $38.8 \mathrm{~kg}$ and was found lying on some smaller rocks at ca. $8-11 \mathrm{~cm}$ above the floor at $99.12-98.99 \mathrm{~m}$. The slab metate $(44 \times 34 \times 8.2 \mathrm{~cm}$ ) weighs $17.2 \mathrm{~kg}$ and was found by the backhoe inside the structure fill, between EUs 9 and 14 at approximately $99.10-99.00 \mathrm{~m}$. The slab metate also appears to have been lying on or near the floor along the east wall and probably was cached there.

In addition to the metates, a complete sandstone mano found lying on the floor at $98.92-98.90 \mathrm{~m}$ in EU 8 was probably a functional tool also, but it is too small ( $75 \times 54 \times 29 \mathrm{~mm} ; 0.15 \mathrm{~kg}$ ) to have been used on the large basin metates. None of the other artifacts found in the fill (11 ground stone fragments, 4 cobble tools, 10 unifaces, 3 edge-modified flakes, 8 cores, 204 flakes, and 4 potsherds) can be associated confidently with the use of the structure, and they may have been dumped there after abandonment. The artifacts recovered from the pithouse fill are discussed in more detail later in this chapter.

Fourteen possible postholes and three interior features were recorded in or near the pithouse (see Figure 15). A considerable amount of modern rodent disturbance was apparent in the fill, and in some cases obvious rodent burrows went through the floor zone. Less obvious were faint traces of older rodent burrows and probable root disturbances. Except in the most obvious cases, however, all small circular stains were treated as possible cultural features until they could be profiled. Upon profiling, it was determined that most of these probably represent rodent burrows or root disturbances (only Nos. 1, 8, and 9 did not appear to be rodent or root disturbed), although the possibility that they are disturbed postholes cannot be ruled out. While none of these can be identified securely as postholes, this does not mean that no posts were associated with the pithouse. 


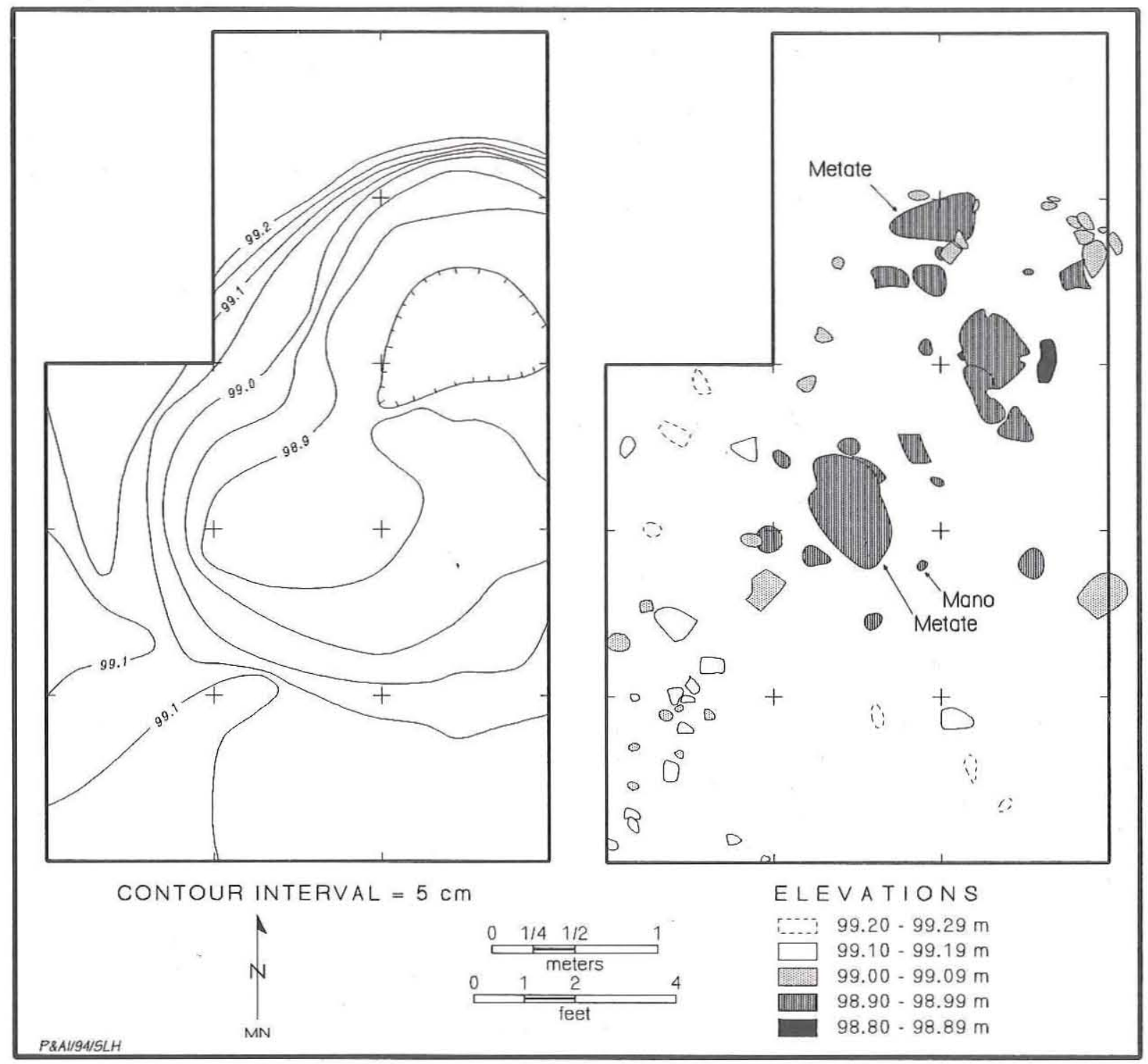

Figure 16. Floor elevations and rocks in the fill of pithouse Feature 37, 41GR291.

It is unlikely that any interior postholes extending into bedrock were missed, but it may be reasonable to assume that 1,000-year-old exterior postholes in the loose sandy loam sediments would be archeologically unrecognizable.

The three interior features (Features 42, 43, and 44) are located in the south-central part of the pithouse at the floor level (see Figure 15). These features are 2-cm-thick layers of hard sediment mottled with tiny flecks of charcoal and calcareous pebbles immediately overlying the bedrock substrate. They were significantly more compact than the loose fill above. Feature 42 covers an ovate $42-x-35-\mathrm{cm}$ area at $98.92-98.89 \mathrm{~m}$, Feature 43 covers a roughly circular $35-\mathrm{x}-31-\mathrm{cm}$ area at $98.91-98.89 \mathrm{~m}$, and Feature 44 covers an amorphous $70-x-50-\mathrm{cm}$ area at 98.90-98.88 m (see Figure 16 for floor elevations). Feature 43 is somewhat different from the other two in that it is almost circular, it is thinner and contained less charcoal, and the bedrock below appears to be discolored (i.e., slightly darker than the surrounding bedrock).

These hard-packed patches of cultural sediment are interpreted as remnants of the floor surface. Why such remnants were observed only in these locations is not known. Their preservation may be due, in part, to the large metate which covers Feature 42 and part of Feature 44. This explanation is not completely 
satisfactory, however, since Feature 43 and much of Feature 44 were not protected by overlying rocks. Another possible interpretation is that these features represent burned areas that were preserved due to partial oxidation of the sediments. If this is correct, then Features 42, 43, and 44 could represent the general location of a central fireplace inside the pithouse. While there certainly was no formal basinshaped hearth in the structure, small fires directly on the floor could have resulted in burning of the floor, and this may explain the slight discoloration of the bedrock below Feature 43 .

Two radiocarbon dates obtained for the fill of Feature 37 are roughly contemporaneous (see Table 6). An assay on scattered charcoal from the lower fill $(0-15 \mathrm{~cm}$ above the floor) beneath the large metate (99.05-98.87 $\mathrm{m}$ in EUs 5 and 8) yielded a calibrated date of A.D. 897 (1007) 1039. A second assay, on charcoal from within $2-3 \mathrm{~cm}$ of the floor (98.95-98.90 $\mathrm{m}$ in EU 9), yielded a calibrated date of A.D. 990 (1027) 1163. These dates (with a maximum range of A.D. 897-1163) represent the age of the floor during the latest occupations and/or the fill that accumulated shortly after the structure was abandonded. The considerable overlap between these dates (i.e., A.D. 990-1039) provides an approximate age of the final occupation/abandonment of the pithouse, but it represents a minimum age for its construction.

\section{Charcoal/Burned Rock Scatters}

Three features are tentatively classified as charcoal/burned rock scatters and consist of confined areas of dark charcoal-stained sediments with scattered burned rocks. They are variable in size and in the amount of burned rocks associated. Each feature is described separately below.

Feature 12 consists of a dark organic stain covering an area $3 \mathrm{~m}$ northeast-southwest by $2 \mathrm{~m}$ northwest-southeast in EUs 26, 27, 36-38, 45-47, 53, and 54 (Figure 17). Charcoal flecks and artifacts (e.g., chipped stone tools and debitage) are common throughout the stained area, but no significant quantity of burned rocks is associated. The stain generally appears at ca. $10 \mathrm{~cm}$ below the surface (between 99.90 and $100.00 \mathrm{~m}$, being highest on the north edge) and is generally $15-20 \mathrm{~cm}$ thick. The outer edges of the stain end rather abruptly, forming an irregular long oval. In the southern portion, the staining becomes more intensive with depth and extends down into a circular depression or basin-shaped pit. The stain is ca. $35 \mathrm{~cm}$ thick at this point and extends from 5-40 cm below the surface $(99.95-99.60 \mathrm{~m})$. At ca. $20 \mathrm{~cm}(99.80 \mathrm{~m})$, the stain was confined to a roughly ovate $100-\mathrm{x}-125-\mathrm{cm}$ area centered over storage pit Feature 17. At ca. $35-40 \mathrm{~cm}(99.65-99.60 \mathrm{~m})$, the dark stain was confined to a small $15-\mathrm{cm}$-diameter basin-shaped area just south of Feature 17 (Figure 18). While the pit edge was first defined at ca. $25 \mathrm{~cm}(99.75 \mathrm{~m})$, it appeared that some dark staining from Feature 12 extended down into the pit fill to ca. $39 \mathrm{~cm}(99.61 \mathrm{~m})$. Clearly, Feature 12 postdates Feature 17, and it seems likely that the slight organic staining observed in the upper part of Feature 17 is due to the overlying stain being compressed and compacted into the upper part of pit.

In the lowest part of Feature 12 in the northeast corner of EU 27, the sediments became increasingly darker with depth, and charcoal staining was most intensive inside the small basin-shaped depression (see Figures 17 and 18). Although no oxidized sediments were observed, the intensity of staining in this area suggests in situ burning. The lack of definitive evidence of in situ burning probably is due to the sandy nature of the sediments, which, unlike clay sediments, would not be expected to discolor due to oxidation. Notably, no oxidized sediments were observed in association with any of the burned rock or hearth/baking pit features.

Feature 12 is interpreted as an unlined basinshaped hearth and its associated charcoal staining. There are, however, two different interpretations for the archeological context of the hearth. It may represent an outdoor activity area such as a small hearth or baking pit with a scatter of charcoal staining downwind from it. Alternatively, it may represent an ephemeral surface structure with an interior hearth and charcoal staining on the floor surface. The latter explanation is preferred for two reasons. First, the general shape and discreteness of the edges of the stained area suggest that it was confined, such as inside a structure or windbreak, rather than open and exposed. And second, exterior hearths and baking pits usually are lined with rocks, while small unlined hearths are more characteristic of interior features.

Two radiocarbon assays from Feature 12 yielded overlapping dates suggesting approximate contemporaneity (see Table 6). A calibrated date of A.D. 1166 (1225) 1265 was obtained on charcoal from the southern portion of the feature $(9-19 \mathrm{~cm}$ below the surface in EU 27), while a calibrated date 


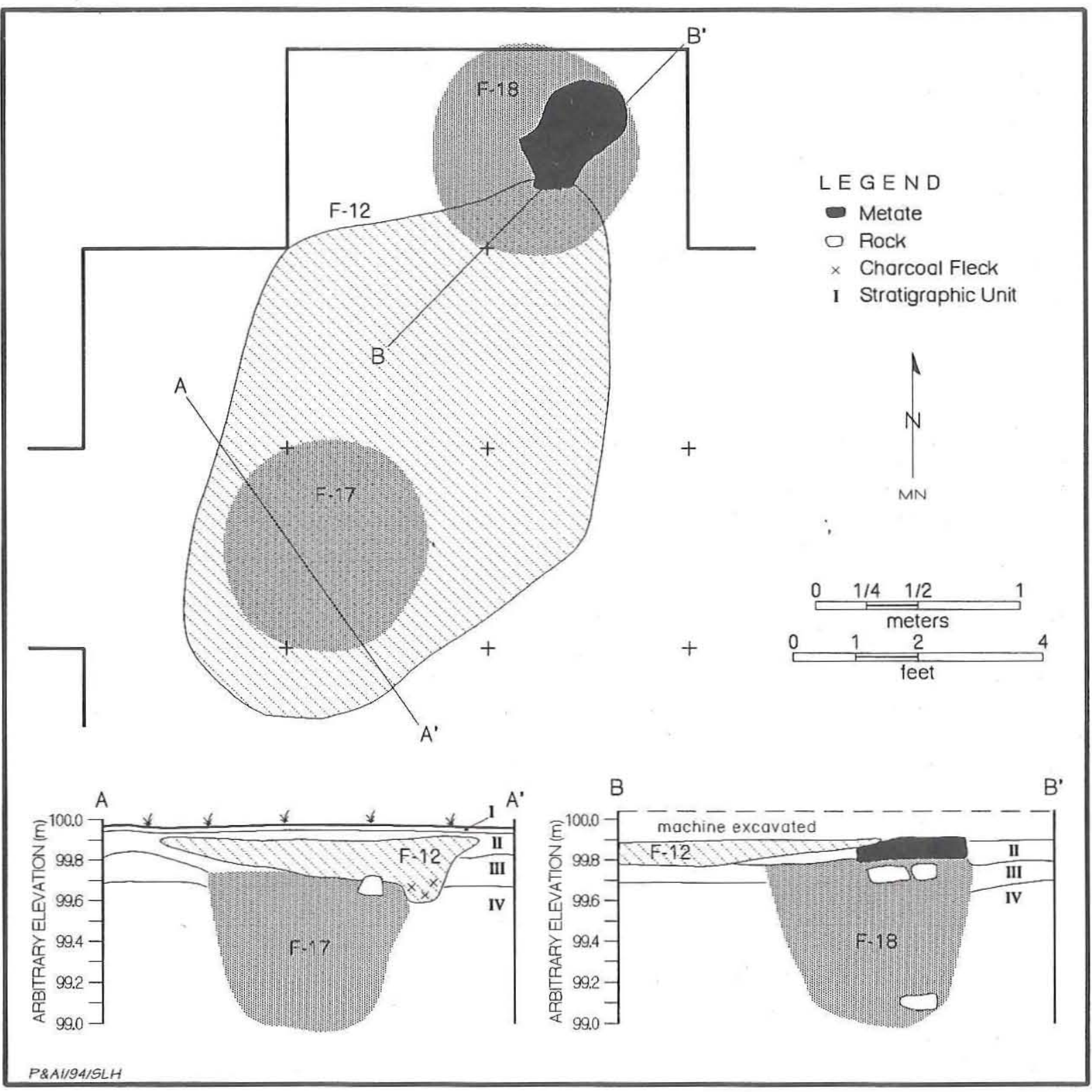

Figure 17. Plan and profile drawings of Feature 12, 41GR291. Note relationship of Feature 12 to storage pit Features 17 and 18.

of A.D. 1216 (1272) 1379 was obtained on scattered charcoal from the northern end of the feature (19-29 cm below the surface in EU 47). An approximate date of A.D. 1200 appears reasonable given the ca. A.D. $669-883$ date for the fill inside storage pit Feature 17, which is stratigraphically below the southern half of Feature 12 .

Feature 16 is a relatively small oval charcoal stain, $2 \mathrm{~m}$ north-south by $1.5 \mathrm{~m}$ east-west, with a considerable amount of scattered burned rocks associated. Slight charcoal staining extends from 18-
$30 \mathrm{~cm}$ below the surface over much of EUs 94,95 , 97 , and 98 , but the exact edges of the stain are difficult to determine since it grades into the surrounding sediments. The burned rocks, mostly sandstone but also including some fire-cracked quartzite, are found from $12-34 \mathrm{~cm}$ below the surface and appear to be randomly scattered. Numerous artifacts (e.g., flakes, potsherds, and mussel shells) were collected from in and around the feature area, but they are not necessarily associated.

The nature and function of Feature 16 are 


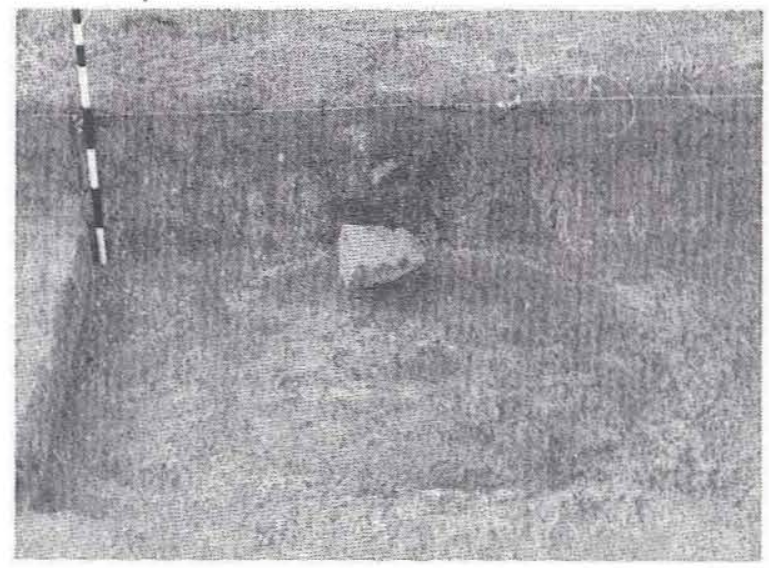

Figure 18. Photograph of Feature 12 excavation in progress, 41GR291. View is to the south (scale is in the southeast comer of EU 37). Feature 17 is in the forground with the large rock along its south edge, and the lower portion of Feature 12 is the dark stained area in the profile immediately behind the rocks.

unclear, but it most likely represents a disturbed hearth area or secondary dump of hearth debris. Within the oval stain, there appears to be a $60-\mathrm{x}-50-\mathrm{cm}$ central area of darker sediment with more charcoal flecking. Perhaps this represents an area of in situ burning, i.e., the former location of a burned rock feature, from which the rocks and charcoal were scattered.

A radiocarbon date on sediment adjacent to Feature 16 may approximate the age of this feature, but it is somewhat problematic. A calibrated date of A.D. 1252 (1272) 1285 was obtained for a sediment sample from $99.68-99.60 \mathrm{~m}$ (ca. $27-35 \mathrm{~cm}$ below the surface) along the south wall of EU 95 (see assay for Beta-59824 in Sediments and Stratigraphy). Although this sample was from $25 \mathrm{~cm}$ south of Feature 16 and no charcoal staining was visually obvious, microscopic examination revealed a significant quantity of wood charcoal throughout the sediment. The charcoal in the sample presumably originated from Feature 16 and apparently skews the date toward the age of the feature. Unfortunately, it is impossible to determine whether the resulting date was derived mainly from organics associated with the feature or whether a significant amount of older soil organics might have been present.

Feature 21 is a large, amorphous, dark charcoal stain with abundant scattered burned rocks (Figure 19). It was first encountered in Backhoe Trench 1992-6, and subsequent excavation units revealed its extent. It is present in parts of 13 units (EUs 63-66,
$68,69,75-78$, and 84-86). This large stained area extends approximately $3.5 \mathrm{~m}$ north-south and $3 \mathrm{~m}$ east-west at $10-28 \mathrm{~cm}$ below the surface. Darkstained sediment, numerous burned rocks, ground stone fragments, miscellaneous chipped stone tools and debris, and mussel shell fragments are scattered throughout this area. There are a few concentrated areas of intensive charcoal staining or clusters of burned rocks within the feature area, but none of these appear to represent in situ hearths.

Feature 21 cannot be classified with any degree of confidence. It may represent a series of disturbed hearth features within an activity area or living surface. Conversely, it could represent a refuse disposal area (i.e., a midden) adjacent to an activity area. Or perhaps most likely, Feature 21 could represent a combination of both-that is, an intensively used (and reused) activity/refuse area for which no interpretable patterning would be expected. A radiocarbon assay on scattered charcoal from Feature 21 (23-25 cm below the surface in EU 66) yielded a calibrated date of A.D. 1262 (1277) 1290 (see Table 6). This date should approximate the age of the activities represented by this feature, but it cannot be taken to mean that all of Feature 21 necessarily dates to this time period.

\section{Cairn Burial}

Feature 23 is a cairn-covered human burial inside a $98-\mathrm{x}-75-\mathrm{cm}$ ovate pit (Figure 20). Some of the rocks were exposed on the surface, and it originally was thought to be a hearth. Excavation revealed many vertical rocks and scattered human bone fragments and teeth in the upper part of the cairn, obviously brought up by rodents. The caim rocks extended from $9 \mathrm{~cm}$ above the surface $(99.98 \mathrm{~m})$ down to $43 \mathrm{~cm}$ below the surface $(99.46 \mathrm{~m})$ inside the pit. The top of the pit is estimated to have been at $15-20 \mathrm{~cm}(99.74-$ $99.69 \mathrm{~m})$, and it was dug down to $64 \mathrm{~cm}(99.25 \mathrm{~m})$. Thus, the pit intruded ca. $44-49 \mathrm{~cm}$ below the original surface, and it was dug at least $30 \mathrm{~cm}$ into the weathered bedrock. The oval pit is quite different from the round storage pit features described above, however, and it apparently was dug specifically for the burial rather than being a reused storage pit. Unlike the storage pits, the burial pit walls and floor are irregular and undulating, and the bedrock is significantly softer (i.e., more weathered).

The rock cairn consisted of 79 rocks with a total 


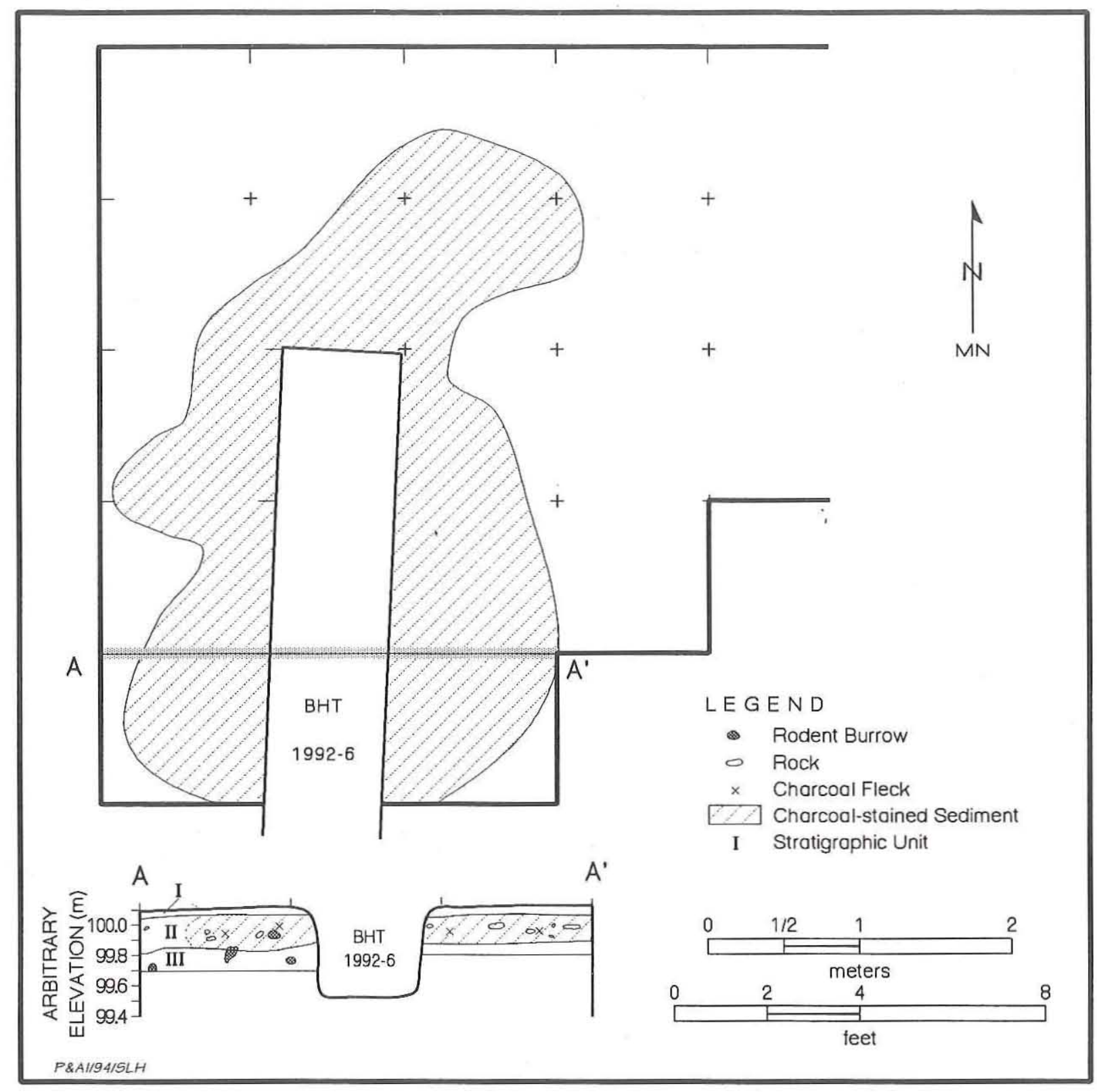

Figure 19. Plan and profile drawings of Feature 21, 41GR291.

weight of $166.5 \mathrm{~kg}$. They range in size from $6-53 \mathrm{~cm}$ (maximum dimension) and in weight from less than a kilogram to $22.6 \mathrm{~kg}$. Seventy-six of the rocks $(96 \%)$ are sandstone, and all are unburned except for three of the smaller fragments. The other rocks consist of an unmodified chert nodule, a firecracked Potter chert fragment, and a burned Potter chert cobble tool. The latter is complete and was found among the lower cairn rocks at $35-44 \mathrm{~cm}$ below the surface (99.54-99.45 m); its occurrence in the fill may be incidental, or it could have been used during the digging of the burial pit.

It is clear that many of the cairn rocks were intentionally selected because of their size, and 10 of them are large sandstone slabs weighing over $5 \mathrm{~kg}$. Some of the slabs were oriented horizontally, while several were oriented vertically - most notably, a large slab found at the northeast end of the oval pit which extended from $2 \mathrm{~cm}$ above to $22 \mathrm{~cm}$ below the surface (from 99.91-99.67 m, see Figure 20b).

Below the caim rocks, a mixed fill consisted of yellowish brown (10YR 5/4 to 6/5) sandy loam 


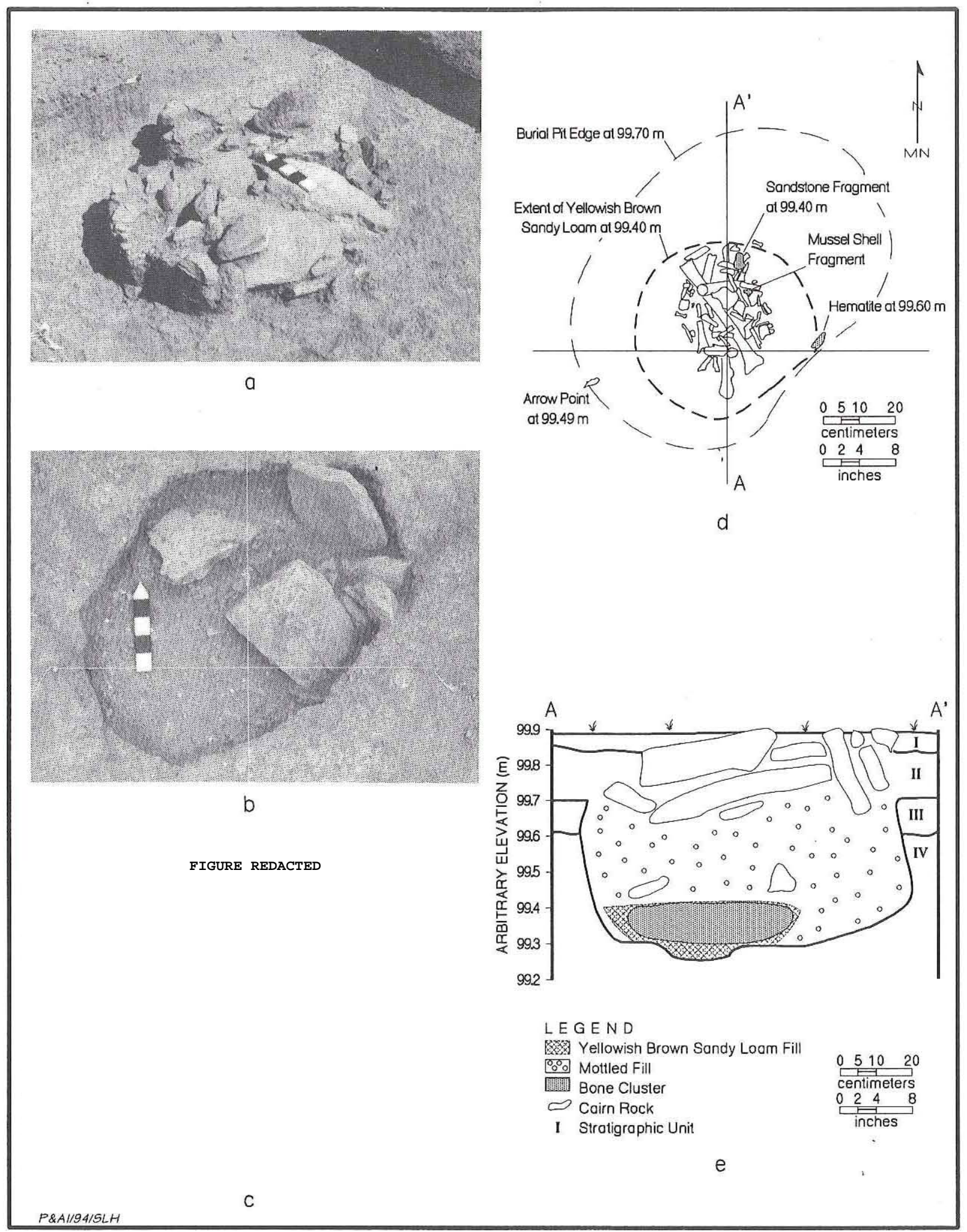

Figure 20. Cairn burial, Feature 23, 41GR291. (a) Rock caim as first exposed; (b) lower cairn rocks inside ovate pit; (c) bone cluster at bottom of pit; $(d)$ plan view of burial pit; $(e)$ profile view of burial pit. 
mottled with fragments of weathered sandstone and mudstone of various colors ranging from pale brown (10YR 7/3) to light gray (5Y 7/2) or white (5Y 8/1). Some rodent disturbance was noted in the fill, and a few bone fragments were found in obvious burrow contexts. Other materials noted in the fill were a few small siliceous pebbles and burned rock fragments, an occasional charcoal fleck, 2 small cores, and 21 flakes.

The fill apparently represents mixing of bedrock and cultural sediments, but only two artifacts - a hematite paint stone and an unusually large comernotched arrow point-are identifiable as grave inclusions. The hematite artifact (see Figure 39) was found along one edge of the pit (see Figure 20d) at $29 \mathrm{~cm}$ below the surface $(99.60 \mathrm{~m})$, but it was in close proximity to a rodent burrow and could have been moved a short distance. The arrow point, typed as a Scallorn, was found along the southwest edge at $40 \mathrm{~cm}$ below the surface $(99.49 \mathrm{~m})$ with its tip pointing outward as if an arrow had been laid in along the long axis of the pit (see Figure 20d).

In the south-central part of the pit, a pile of human bones (hereafter referred to as the bone cluster) was encountered at $49-56 \mathrm{~cm}$ below the surface (see Figure $20 c$ and $d$ ). The bone cluster was concentrated within a larger circular stain of yellowish brown (10YR 5/4 to 6/4) sandy loam. The sediment within this ca. 48-cm-diameter area (and throughout the bone cluster) is relatively clean and free of mottling compared to the mixed fill around it. The bone cluster consists of ca. 500 bones and bone fragments in a 10-cm-thick layer within a $45-x-30-\mathrm{cm}$ area. The bone cluster was lying near the bottom of the pit at ca. $46-56 \mathrm{~cm}$ below the surface (99.43-99.33 m). These remains, described in detail in Appendix F, represent a nearly complete skeleton of a middle-aged male. They are badly fragmented, and except for a few hand and foot elements, none of the bones are complete. Modern rodent disturbance was confined to the very top of the bone cluster, and the cluster was largely intact.

Feature 23 represents a secondary interment, although the condition of the bones does not necessarily mean that they were heavily weathered or in a fragmented state prior to burial, and no gnawing was evident. Aside from the north-south orientation of the more complete long bones, the remainder of the bones appeared to be randomly distributed. One unmodified flake, a small $8-x-6-\mathrm{cm}$ unmodified sandstone fragment, and a freshwater mussel umbo fragment also were found in the bone cluster, but their occurrence probably is incidental.

Based on the evidence described above, the following sequence of events is proposed. The ovate burial pit was dug into the soft bedrock substrate, and the fill removed became mixed in the process (i.e., sandy loam mottled with weathered bedrock and some cultural debris). Apparently a layer of loose mixed fill was left in the bottom of the pit, perhaps indicating that the pit was left open for a short time preceding the interment. Prior to the actual burial, a small circular area was scooped out in the loose pit fill. The disarticulated human bones, possibly enclosed in a container (such as a hide bag or a basket that later decayed, leaving an area of clean fill around the bones), were then placed inside the circular depression and perhaps were covered over with loose fill. At this time, the grave inclusions may have been placed in the pit. The archeological evidence is limited to an arrow point and a hematite paint stone, but other perishable items may have been included as well. The projectile point - an unusually large and well-made specimen that may have had special significance in its mortuary context-suggests that an arrow was laid in the grave.

The rest of the pit was subsequently covered over with more mixed fill. A few smaller rocks, including a Potter chert cobble tool, were either accidentally or intentionally thrown in with the fill. The pit was backfilled with sediment to within $15-20 \mathrm{~cm}$ of the ground surface, and then sandstone rocks and large slabs obtained from nearby outcrops were placed inside the pit. Some large slabs were placed horizontally, while others were placed vertically, held upright by the pit edges and other rocks. Rocks continued to be piled on until a large circular caim completely capped the burial.

A radiocarbon assay on collagen from the right tibia yielded a calibrated date of A.D. 238 (265, 281, 333) 399 (see Table 6). This date is considered to be the approximate age of the burial and suggests that the interment predates the earliest storage pit by ca. 350 years and the pithouse by ca. 700 years.

The $\delta{ }^{13} \mathrm{C}$ value obtained on the collagen fraction of the bone is $-13.6 \%$, and this value can be used to estimate the contribution of $\mathrm{C}_{4}$ plants or animals that ate $\mathrm{C}_{4}$ plants to the diet of this individual using the following formula:

$$
\text { Estimated \% of } C_{4} \text { Plants in Diet }=\frac{\left[\left(\delta^{13} C-5\right)+27\right]}{(0.14)} \text {. }
$$

This formula assumes that the mean $\delta{ }^{13} \mathrm{C}$ value for $\mathrm{C}_{3}$ plants is $-27 \%$ (Cerling and Hay 1986; Cerling 
$\mathrm{C}_{3}$ plants is $-27 \%$ (Cerling and Hay 1986; Cerling et al. 1989) and that carbon isotopes in human collagen are fractioned $+5.0 \%$ above either the plant foods directly ingested by humans or the plant foods ingested by herbivore prey animals eaten by humans (Krueger and Sullivan 1984). It is then estimated that $60 \%$ of the diet of the individual from the Sam Wahl site consisted of $\mathrm{C}_{4}$ plants or animals that ate $\mathrm{C}_{4}$ plants.

The dominant presence of $\mathrm{C}_{4}$ plants in prehistoric diets has been used as an indicator of subsistence practices (maize horticulture) and social complexity (e.g., Bender et al. 1981; Katzenberg and Kelley 1991; Matson and Chisholm 1991; Schurr 1992; Schwarcz et al. 1985). However, these studies have been carried out on large burial populations or samples, and with only one burial from the Sam Wahl site, any interpretation of diet and subsistence practices is tenuous. Further, the ethnographically documented dietary patterns of the Southern Plains and Southwest, unlike the Eastern Woodlands, consisted of many wild $\mathrm{C}_{4}$ plants, including Amaranthus graecizans, A. hybridus, and Atriplex canescens (Basehart 1960; Castetter and Opler 1936:40, 46; Wallace and Hoebel 1986:73). Also documented was use of Opuntia spp. and Yucca spp., two Crassulacean Acid Metabolism (CAM) plants that tend to operate as $\mathrm{C}_{4}$ plants in semiarid to arid environments. Bison, which feed predominantly on $\mathrm{C}_{4}$ grasses, also could have been a dietary source resulting in relatively higher $\delta^{13} \mathrm{C}$ values in human collagen. Human coprolites from Archaic-age rockshelter deposits in southeastern Utah reveal a predominance of Opuntia and wild $\mathrm{C}_{4}$ plant remains, which coincide with a $\delta{ }^{13} \mathrm{C}$ value of $-13.9 \%$ from an Archaic-age burial in the same area (Matson and Chisholm 1991).

The absence of conclusive evidence of maize at 41GR291, such as pollen, charred kemels, and cobs, suggests that the $\delta^{13} \mathrm{C}$ value from Feature 23 does not reflect any significant consumption of maize. This is supported by a comparison with the $\delta^{13} \mathrm{C}$ values of individuals associated with intensive maize horticultural sites in the Sierra Blanca region of New Mexico, which reveals that the $\delta^{13} \mathrm{C}$ value of $-13.6 \%$ is relatively low (cf. mean values of -7.8 , $-7.3,-7.4,-6.7,-10.5,-8.4$, and $-7.1 \%$ in Katzenberg and Kelley 1991:Table 1). While large differences in $\delta^{13} \mathrm{C}$ values (i.e., dietary patterns) among maize horticulturalists have been documented and attributed to differences in social status in
Mississippian populations (e.g., Schurr 1992), social complexity resulting in differing diets for prehistoric groups on the Southern Plains is highly unlikely.

\section{Bedrock Mortars}

Two bedrock mortars were discovered by local avocational archeologist Emmett Shedd many years prior to the data recovery investigations. Shedd relocated the mortar holes, which were filled with sediment and vegetation, and brought them to our attention. An extensive search in the vicinity failed to locate additional mortars, but several ground stone tools, including a pestle, were found within $30 \mathrm{~m}$ of the mortars.

Both are pointed oval mortars on an exposed sandstone ledge at the southernmost edge of the site, approximately $170 \mathrm{~m}$ south-southwest of the pithouse at an elevation of ca. $92.70 \mathrm{~m}$. Feature 26 is the larger of the two, being $40 \times 19 \mathrm{~cm}$ and at least $27 \mathrm{~cm}$ deep (it was not cleaned out, however), while Feature 27 is considerably smaller at $25 \times 16 \mathrm{~cm}$ and $8 \mathrm{~cm}$ deep. Although they cannot be dated, the presence of pestle fragments (several of which are large enough to have been used in bedrock mortars) in the excavations suggests that mortars were used by the site inhabitants. One specimen in particular-a distal fragment recovered in the pithouse fill dated to approximately A.D. 1000-provides a tentative chronological link between the use of the pithouse and bedrock mortars, although not necessarily the two mortars located by Shedd.

\section{MATERIALS RECOVERED}

This section discusses all of the materials recovered from the Sam Wahl site during the Season 3 data recovery investigations. Where appropriate, materials recovered from previous phases of work are mentioned (see Table 4), but they are not described again nor are they included in the artifact counts presented here. Some of these materials, however, are added later for analysis purposes in the summary discussions. The data recovery work produced 3,578 artifacts, of which the majority ( $n=3,356,93.8 \%$ ) are from the five excavation blocks. The remainder are from nonblock excavation units $(n=134,3.8 \%)$, backhoe trenches $(n=66,1.8 \%)$, blade cuts $(n=16$, $0.5 \%$ ), and general surface proveniences $(n=4$, $0.1 \%$ ). Table 12 shows the distribution of all the data recovery artifacts by excavation unit, and more- 
TABLE 12

\begin{tabular}{|c|c|c|c|c|c|c|c|c|c|c|c|c|}
\hline \multicolumn{13}{|c|}{$\begin{array}{c}\text { TABLE } 12 \\
\text { DISTRIBUTION OF DATA RECOVERY ARTIFACTS, 41GR291 }\end{array}$} \\
\hline $\begin{array}{l}\text { Unit/Maximum } \\
\text { Depth Below } \\
\text { Surface }\end{array}$ & $\begin{array}{l}\text { Projectile } \\
\text { Points }\end{array}$ & Bifaces & \begin{tabular}{|l|} 
Cobble \\
Tools
\end{tabular} & Unifaces & Cores & \begin{tabular}{|l|} 
Edge- \\
modified \\
Flakes
\end{tabular} & $\begin{array}{l}\text { Unmodified } \\
\text { Debitage }\end{array}$ & $\begin{array}{l}\text { Ground } \\
\text { Stone } \\
\text { Tools }\end{array}$ & Other Lithics & Ceramics & Other Materials & Totals \\
\hline \multicolumn{13}{|l|}{ Block 1* } \\
\hline EU $1,30 \mathrm{~cm}$ & _- & - & - & - & 1 & - & 32 & 1 & - & 1 & - & 35 \\
\hline EU $2,45 \mathrm{~cm}$ & - & - & - & 2 & 1 & - & 18 & 1 & - & - & - & 22 \\
\hline $\mathrm{EU} 3,32 \mathrm{~cm}$ & - & - & 1 & 1 & 1 & - & 12 & - & - & - & |- & 15 \\
\hline EU $4,46 \mathrm{~cm}$ & - & - & - & 1 & - & - & 14 & 1 & - & 1 & - & 17 \\
\hline EU $5,54 \mathrm{~cm}$ & - & - & - & 2 & - & 1 & 24 & 3 & - & - & - & 30 \\
\hline EU $6,43 \mathrm{~cm}$ & - & - & - & 1 & - & 1 & 17 & - & - & - & - & 19 \\
\hline EU $7,49 \mathrm{~cm}$ & - & - & - & 2 & 1 & - & 10 & - & $=$ & - & 1 modified shell & 14 \\
\hline EU $8,60 \mathrm{~cm}$ & - & - & - & - & 2 & - & 16 & 1 & - & 1 & - & 20 \\
\hline EU $9,52 \mathrm{~cm}$ & - & - & - & - & - & - & 2 & - & - & - & |- & 2 \\
\hline EU $10,60 \mathrm{~cm}$ & - & - & 1 & - & 1 & - & 16 & 2 & - & - & - & 20 \\
\hline EU $11,63 \mathrm{~cm}$ & - & - & - & - & - & - & 16 & 2 & - & - & - & 18 \\
\hline $\mathrm{EU} 12,41 \mathrm{~cm}$ & - & - & - & 1 & - & 1 & 15 & - & - & 1 & - & 18 \\
\hline EU $13,46 \mathrm{~cm}$ & - & - & 2 & - & 1 & - & 12 & - & - & - & - & 15 \\
\hline EU $14,6 \mathrm{~cm}^{* *}$ & - & - & - & - & - & - & - & - & - & - & - & 0 \\
\hline EU $15,11 \mathrm{~cm}^{* *}$ & - & - & - & - & - & - & 1 & - & - & - & - & 1 \\
\hline BHT 1992-31 & - & - & - & - & - & - & - & 2 & - & - & - & 2 \\
\hline Block 1 Totals: & 0 & 0 & 4 & 10 & 8 & 3 & 205 & 13 & 0 & 4 & 1 modified shell & 248 \\
\hline \multicolumn{13}{|l|}{ Block 2} \\
\hline EU $16,19 \mathrm{~cm}$ & - & - & 1 & - & - & - & 7 & - & - & - & - & 8 \\
\hline EU $17,19 \mathrm{~cm}$ & - & - & - & - & - & - & 9 & - & $-\cdots$ & - & 1 modified hematite & 10 \\
\hline EU $18,23 \mathrm{~cm}$ & - & - & - & 2 & - & - & 10 & - & - & - & - & 12 \\
\hline EU $19,24 \mathrm{~cm}$ & - & - & - & 1 & 3 & - & 9 & 1 & - & - & - & 14 \\
\hline EU $20,35 \mathrm{~cm}$ & 1 & - & - & - & - & - & 13 & - & - & - & - & 34 \\
\hline
\end{tabular}

*All materials recovered from Block 1 and two specimens from BHT 1992-31 are associated with Feature 37.

**Upper levels removed by blading; depth refers to thickness of excavated levels.

****Partial unit smaller than $1 \times 1 \mathrm{~m}$. 


\begin{tabular}{|c|c|c|c|c|c|c|c|c|c|c|c|c|}
\hline \multicolumn{13}{|l|}{ Table 12 , continued } \\
\hline $\begin{array}{l}\text { Unit/Maximum } \\
\text { Depth Below } \\
\text { Surface }\end{array}$ & $\begin{array}{l}\text { Projectile } \\
\text { Points }\end{array}$ & Bifaces & $\begin{array}{l}\text { Cobble } \\
\text { Tools } \\
\end{array}$ & Unifaces & Cores & \begin{tabular}{|l} 
Edge- \\
modified \\
Flakes \\
\end{tabular} & $\begin{array}{l}\text { Unmodified } \\
\text { Debitage }\end{array}$ & $\begin{array}{l}\text { Ground } \\
\text { Stone } \\
\text { Tools } \\
\end{array}$ & Other Lithics & Ceramics & Other Materials & Totals \\
\hline EU 21, $27 \mathrm{~cm}$ & - & - & - & 1 & - & 1 & 31 & - & - & - & 1 modified hematite & 34 \\
\hline EU 22, $34 \mathrm{~cm}$ & 1 & - & - & - & 1 & - & 22 & - & - & - & - & 24 \\
\hline EU $23,34 \mathrm{~cm}$ & 1 & - & - & 2 & 2 & 1 & 36 & - & - & - & - & 42 \\
\hline EU $24,28 \mathrm{~cm}$ & 1 & - & 1 & 4 & 1 & - & 32 & 1 & - & - & - & 40 \\
\hline EU $25,30 \mathrm{~cm}^{* * * *}$ & - & - & - & 2 & - & - & 33 & - & $\begin{array}{l}1 \text { hammerstone; } \\
1 \text { gouge }\end{array}$ & - & - & 37 \\
\hline EU $26,32 \mathrm{~cm}$ & - & - & 1 & 1 & 1 & 1 & 19 & - & - & - & - & 23 \\
\hline EU 27, $39 \mathrm{~cm}$ & - & 1 & - & 1 & 1 & - & 38 & 1 & - & 3 & - & 45 \\
\hline EU $28,37 \mathrm{~cm}$ & - & - & - & 1 & 2 & 1 & 14 & - & - & - & - & 18 \\
\hline EU $29,47 \mathrm{~cm}$ & - & - & - & - & - & 1 & 28 & - & - & - & - & 29 \\
\hline EU $30,37 \mathrm{~cm}$ & - & - & - & 1 & - & - & 16 & 1 & - & - & - & 18 \\
\hline EU $31,34 \mathrm{~cm}$ & - & - & - & 1 & - & - & 44 & 1 & - & - & - & 46 \\
\hline EU $32,32 \mathrm{~cm}$ & - & - & - & - & 2 & 1 & 43 & 2 & - & - & 1 modified shell & 49 \\
\hline EU $33,30 \mathrm{~cm}^{* * * *}$ & - & - & - & 1 & 1 & - & 21 & - & - & - & - & 23 \\
\hline EU $34,36 \mathrm{~cm}^{* * *}$ & - & - & 1 & 1 & 2 & - & 23 & - & - & - & - & 27 \\
\hline EU $35,40 \mathrm{~cm}^{* *}$ & - & - & - & - & - & - & 35 & - & - & 1 & - & 36 \\
\hline EU $36,45 \mathrm{~cm}$ & - & - & - & - & 1 & 1 & 31 & - & - & - & 1 modified hematite & 34 \\
\hline EU $37,48 \mathrm{~cm}$ & - & - & - & 1 & 3 & - & 30 & - & - & 1 & 1 modified shell & 36 \\
\hline EU $38,39 \mathrm{~cm}$ & 1 & - & 1 & - & - & - & 35 & 1 & 1 hammerstone & - & - & 39 \\
\hline EU $39,36 \mathrm{~cm}$ & - & - & - & 1 & - & - & 24 & - & - & - & - & 25 \\
\hline $\mathrm{EU} 40,37 \mathrm{~cm}$ & - & - & - & - & 1 & 1 & 20 & 1 & 1 gouge & - & - & 24 \\
\hline EU $41,36 \mathrm{~cm}$ & - & - & - & - & - & - & 28 & 1 & - & - & 1 modified shell & 30 \\
\hline EU $42,47 \mathrm{~cm}^{* * * *}$ & - & - & - & - & - & - & 11 & - & - & - & - & 11 \\
\hline EU $43,47 \mathrm{~cm}^{* * *}$ & - & - & - & - & 1 & - & 16 & - & - & - & - & 17 \\
\hline EU $44,38 \mathrm{~cm}^{* * * *}$ & - & - & 1 & 1 & - & - & 9 & - & - & - & - & 11 \\
\hline EU 45, $35 \mathrm{~cm}^{* *}$ & - & - & - & - & - & - & 6 & - & - & - & - & 6 \\
\hline EU $46,38 \mathrm{~cm}^{* *}$ & - & 1 & - & 1 & 1 & - & 19 & - & - & - & - & 22 \\
\hline EU $47,39 \mathrm{~cm}$ & 2 & 2 & - & 2 & - & - & 38 & 3 & - & - & - & 47 \\
\hline $\mathrm{EU} 48,39 \mathrm{~cm}$ & 1 & 1 & - & 1 & - & - & 61 & - & - & - & - & 64 \\
\hline EU $49,39 \mathrm{~cm}$ & - & - & - & - & - & - & 34 & - & - & - & - & 34 \\
\hline EU $50,41 \mathrm{~cm}$ & - & - & - & - & - & 1 & 32 & - & - & - & - & 33 \\
\hline EU $51,31 \mathrm{~cm}$ & - & 1 & - & 2 & - & - & 31 & - & 1 perforator & - & - & 35 \\
\hline $\mathrm{EU} 52,31 \mathrm{~cm}$ & 1 & - & - & - & 1 & 2 & 37 & - & - & - & - & 41 \\
\hline EU 53, $20 \mathrm{~cm}^{* * *}$ & - & - & - & - & 1 & 1 & 23 & - & - & - & - & 25 \\
\hline EU $54,26 \mathrm{~cm}^{* * *}$ & - & - & - & 1 & - & - & 7 & - & - & - & - & 8 \\
\hline
\end{tabular}




\begin{tabular}{|c|c|c|c|c|c|c|c|c|c|c|c|c|}
\hline \multicolumn{13}{|l|}{ Table 12 , continued } \\
\hline $\begin{array}{l}\text { Unit/Maximum } \\
\text { Depth Below } \\
\text { Surface }\end{array}$ & \begin{tabular}{|l} 
Projectile \\
Points
\end{tabular} & Bifaces & $\begin{array}{l}\text { Cobble } \\
\text { Tools }\end{array}$ & Unifaces & Cores & \begin{tabular}{|l|}
$\begin{array}{l}\text { Edge- } \\
\text { modified } \\
\text { Flakes }\end{array}$ \\
\end{tabular} & $\begin{array}{l}\text { Unmodified } \\
\text { Debitage } \\
\end{array}$ & \begin{tabular}{|l} 
Ground \\
Stone \\
Tools \\
\end{tabular} & Other Lithics & Ceramics & Other Materials & Totals \\
\hline $\begin{array}{l}\text { EU } 55,44 \mathrm{~cm}^{* * *} \\
\text { EU } 56,44 \mathrm{~cm} \\
\text { Feature } 17 \\
\text { Feature } 18 \\
\text { Feature } 19 \\
\text { Feature } 20 \\
\text { Feature } 23 \\
\text { Feature } 24 \\
\text { Feature } 25 \\
\text { Feature } 39 \\
\end{array}$ & $\begin{array}{l}- \\
- \\
- \\
- \\
- \\
- \\
1 \\
- \\
- \\
-\end{array}$ & $\begin{array}{l}- \\
- \\
- \\
- \\
- \\
- \\
- \\
- \\
- \\
-\end{array}$ & $\begin{array}{l}- \\
- \\
- \\
- \\
- \\
- \\
1 \\
1 \\
- \\
-\end{array}$ & $\begin{array}{l}- \\
\overline{1} \\
- \\
- \\
- \\
- \\
- \\
- \\
-\end{array}$ & $\begin{array}{l}- \\
- \\
\overline{1} \\
2 \\
- \\
2 \\
- \\
- \\
-\end{array}$ & $\begin{array}{l}1 \\
- \\
- \\
- \\
- \\
- \\
- \\
- \\
- \\
-\end{array}$ & $\begin{array}{r}15 \\
17 \\
17 \\
28 \\
18 \\
12 \\
22 \\
5 \\
9 \\
1 \\
\end{array}$ & $\begin{array}{l}\overline{1} \\
2 \\
1 \\
1 \\
- \\
\overline{2} \\
- \\
-\end{array}$ & $\begin{array}{l}- \\
- \\
- \\
- \\
- \\
- \\
- \\
- \\
- \\
-\end{array}$ & $\begin{array}{l}- \\
- \\
- \\
- \\
- \\
- \\
- \\
- \\
- \\
-\end{array}$ & $\begin{array}{l}- \\
- \\
- \\
- \\
- \\
- \\
1 \text { modified hematite } \\
- \\
- \\
-\end{array}$ & $\begin{array}{r}16 \\
18 \\
20 \\
30 \\
21 \\
12 \\
27 \\
8 \\
9 \\
1\end{array}$ \\
\hline Block 2 Totals: & 10 & 6 & 8 & 30 & 30 & 13 & 1119 & 20 & \begin{tabular}{|l}
2 hammerstones \\
2 gouges \\
1 perforator \\
\end{tabular} & 5 & $\begin{array}{l}4 \text { modified hematite } \\
3 \text { modified shells }\end{array}$ & 1253 \\
\hline \multicolumn{13}{|l|}{ Block 3} \\
\hline $\begin{array}{l}\text { EU } 58,45 \mathrm{~cm} \\
\text { EU } 59,46 \mathrm{~cm}\end{array}$ & - & - & 1 & 1 & 2 & 1 & $\begin{array}{l}54 \\
67\end{array}$ & 2 & - & - & - & 61 \\
\hline EU $60,46 \mathrm{~cm}$ & - & - & 1 & 1 & 1 & - & 95 & - & $\begin{array}{l}1 \text { perforator; } \\
1 \text { gouge }\end{array}$ & - & - & 100 \\
\hline $\begin{array}{l}\text { EU } 61,43 \mathrm{~cm} \\
\text { EU } 62,46 \mathrm{~cm}\end{array}$ & $\begin{array}{l}1 \\
1\end{array}$ & - & $\overline{3}$ & $\begin{array}{l}2 \\
2\end{array}$ & $\overline{2}$ & $\overline{-}$ & $\begin{array}{l}21 \\
31\end{array}$ & $\overline{1}$ & $\begin{array}{l}1 \text { gouge } \\
-\end{array}$ & - & - & $\begin{array}{l}25 \\
40\end{array}$ \\
\hline Block 3 Totals: & 2 & 0 & 5 & 6 & 5 & 1 & 268 & 4 & $\begin{array}{l}1 \text { perforator } \\
2 \text { gouges }\end{array}$ & 0 & 1 modified hematite & 295 \\
\hline \multicolumn{13}{|l|}{ Block 4} \\
\hline EU $63,42 \mathrm{~cm}$ & - & - & - & 1 & 1 & - & 42 & - & 1 gouge & - & - & 45 \\
\hline EU $64,32 \mathrm{~cm}$ & 1 & - & - & 1 & - & - & 23 & - & - & - & - & 25 \\
\hline EU $65,22 \mathrm{~cm}$ & - & - & - & 1 & 1 & 1 & 10 & - & - & - & - & 13 \\
\hline $\mathrm{EU} 66,37 \mathrm{~cm}$ & - & - & - & - & - & - & 41 & - & - & - & - & 41 \\
\hline EU $67,35 \mathrm{~cm}$ & - & - & - & 2 & - & - & 48 & - & - & - & - & 50 \\
\hline EU $68,35 \mathrm{~cm}$ & - & - & - & - & - & - & 18 & 1 & - & - & - & 19 \\
\hline EU $69,41 \mathrm{~cm}$ & 1 & - & - & 1 & 3 & - & 59 & 1 & - & - & - & 65 \\
\hline
\end{tabular}




\begin{tabular}{|c|c|c|c|c|c|c|c|c|c|c|c|c|}
\hline \multicolumn{13}{|c|}{ Table 12 , continued } \\
\hline $\begin{array}{l}\text { Unit/Maximum } \\
\text { Depth Below } \\
\text { Surface }\end{array}$ & \begin{tabular}{|l} 
Projectile \\
Points
\end{tabular} & Bifaces & $\begin{array}{l}\text { Cobble } \\
\text { Tools }\end{array}$ & Unifaces & Cores & $\begin{array}{l}\text { Edge- } \\
\text { modified } \\
\text { Flakes }\end{array}$ & $\begin{array}{l}\text { Unmodified } \\
\text { Debitage }\end{array}$ & $\begin{array}{l}\text { Ground } \\
\text { Stone } \\
\text { Tools }\end{array}$ & Other Lithics & Ceramics & Other Materials & Totals \\
\hline EU $70,41 \mathrm{~cm}$ & - & - & - & 2 & - & 1 & 72 & 2 & - & - & - & 77 \\
\hline EU $71,49 \mathrm{~cm}$ & 1 & - & - & 1 & 1 & 1 & 44 & 2 & 1 gouge & - & - & 51 \\
\hline EU $72,39 \mathrm{~cm}$ & - & 1 & - & - & - & 1 & 36 & - & 2 gouges & 1 & - & 41 \\
\hline EU $73,38 \mathrm{~cm}$ & - & 1 & 1 & - & - & - & 38 & 1 & - & - & - & 41 \\
\hline EU $74,38 \mathrm{~cm}$ & - & 2 & - & 1 & - & 1 & 43 & - & 1 hammerstone & - & - & 48 \\
\hline EU $75,38 \mathrm{~cm}$ & - & - & - & - & 1 & - & 47 & 3 & - & - & - & 51 \\
\hline EU $76,38 \mathrm{~cm}$ & - & - & 1 & 3 & 2 & 1 & 56 & - & - & - & - & 63 \\
\hline EU $77,37 \mathrm{~cm}$ & 2 & - & - & 1 & 1 & - & 34 & - & - & - & - & 38 \\
\hline EU $78,49 \mathrm{~cm}$ & - & 1 & - & 3 & 1 & - & 42 & - & - & - & - & 47 \\
\hline EU $79,49 \mathrm{~cm}$ & 1 & - & 1 & - & 1 & - & 36 & - & - & - & - & 39 \\
\hline EU $80,39 \mathrm{~cm}$ & - & - & - & - & - & - & 31 & 1 & - & - & - & 32 \\
\hline EU $81,51 \mathrm{~cm}$ & 1 & - & - & 1 & 1 & - & 30 & - & - & - & - & 33 \\
\hline EU $82,38 \mathrm{~cm}$ & - & - & - & 1 & - & 1 & 30 & 2 & - & 2 & 1 modified hematite & 37 \\
\hline EU $83,35 \mathrm{~cm}$ & 1 & - & - & 1 & - & - & 28 & - & - & - & - & 30 \\
\hline EU $84,36 \mathrm{~cm}$ & - & - & - & - & 1 & - & 32 & - & - & - & - & 33 \\
\hline EU $85,37 \mathrm{~cm}$ & - & - & 2 & - & - & - & 26 & - & - & - & - & 28 \\
\hline EU $86,42 \mathrm{~cm}$ & - & 1 & 2 & - & - & 1 & 23 & 1 & - & - & - & 28 \\
\hline EU $87,40 \mathrm{~cm}$ & 1 & - & - & 1 & 1 & 2 & 40 & - & 1 gouge & - & - & 46 \\
\hline $\mathrm{EU} 88,59 \mathrm{~cm}$ & 3 & 1 & - & 1 & - & 1 & 52 & - & 1 perforator & - & - & 59 \\
\hline EU $89,34 \mathrm{~cm}$ & - & - & - & - & 1 & 2 & 40 & - & - & 2 & - & 45 \\
\hline EU $90,38 \mathrm{~cm}$ & - & - & - & 1 & - & - & 50 & - & - & 1 & - & 52 \\
\hline Block 4 Totals: & 12 & 7 & 7 & 23 & 16 & 13 & 1071 & 14 & $\begin{array}{l}5 \text { gouges } \\
1 \text { hammerstone } \\
1 \text { perforator }\end{array}$ & 6 & 1 modified hematite & 1177 \\
\hline \multicolumn{13}{|l|}{ Block 5} \\
\hline EU 93, $37 \mathrm{~cm}$ & - & - & 1 & 2 & 1 & 1 & 30 & - & 1 gouge & - & - & 36 \\
\hline EU $94,37 \mathrm{~cm}$ & - & - & - & - & 1 & - & 20 & 1 & - & - & - & 22 \\
\hline EU $95,36 \mathrm{~cm}$ & - & - & 1 & 2 & 1 & 1 & 36 & 4 & - & 1 & - & 46 \\
\hline EU $96,36 \mathrm{~cm}$ & - & - & - & 2 & - & - & 28 & 1 & - & 1 & - & 32 \\
\hline EU 97, $36 \mathrm{~cm}$ & - & 1 & 2 & - & 1 & - & 39 & - & - & 1 & - & 44 \\
\hline EU $98,39 \mathrm{~cm}$ & - & 1 & 1 & - & 1 & - & 18 & 1 & - & - & - & 22 \\
\hline EU $100,53 \mathrm{~cm}$ & - & - & 1 & - & - & 2 & 24 & 12 & - & - & - & 39 \\
\hline
\end{tabular}




\begin{tabular}{|c|c|c|c|c|c|c|c|c|c|c|c|c|}
\hline \multicolumn{13}{|l|}{ Table 12, continued } \\
\hline $\begin{array}{l}\text { Unit/Maximum } \\
\text { Depth Below } \\
\text { Surface }\end{array}$ & $\begin{array}{l}\text { Projectile } \\
\text { Points }\end{array}$ & Bifaces & $\begin{array}{l}\text { Cobble } \\
\text { Tools }\end{array}$ & Unifaces & Cores & $\begin{array}{l}\text { Edge- } \\
\text { modified } \\
\text { Flakes }\end{array}$ & $\begin{array}{l}\text { Unmodified } \\
\text { Debitage }\end{array}$ & $\begin{array}{l}\text { Ground } \\
\text { Stone } \\
\text { Tools }\end{array}$ & Other Lithics & Ceramics & Other Materials & Totals \\
\hline $\begin{array}{l}\text { EU 101, } 48 \mathrm{~cm} \\
\text { EU 102, } 47 \mathrm{~cm}^{* * *} \\
\text { EU 103, } 72 \mathrm{~cm} \\
\text { EU 104, } 49 \mathrm{~cm}^{* * * *} \\
\text { Feature } 29\end{array}$ & $\begin{array}{l}- \\
- \\
- \\
- \\
-\end{array}$ & $\begin{array}{l}- \\
- \\
- \\
- \\
-\end{array}$ & $\begin{array}{l}1 \\
1 \\
1 \\
- \\
-\end{array}$ & $\begin{array}{l}1 \\
- \\
- \\
- \\
1\end{array}$ & $\begin{array}{l}2 \\
- \\
- \\
- \\
-\end{array}$ & $\begin{array}{l}1 \\
- \\
- \\
- \\
-\end{array}$ & $\begin{array}{l}37 \\
14 \\
30 \\
15 \\
10\end{array}$ & $\begin{array}{c}- \\
\overline{1} \\
\overline{2} \\
25\end{array}$ & $\begin{array}{l}1 \text { gouge } \\
- \\
- \\
1 \text { gouge } \\
-\end{array}$ & $\begin{array}{l}- \\
- \\
- \\
- \\
-\end{array}$ & $\begin{array}{l}- \\
- \\
- \\
- \\
-\end{array}$ & $\begin{array}{l}43 \\
15 \\
32 \\
16 \\
36\end{array}$ \\
\hline Block 5 Totals: & 0 & 2 & 9 & 8 & 7 & 5 & 301 & 45 & 3 gouges & 3 & 0 & 383 \\
\hline \multicolumn{13}{|l|}{ Nonblock } \\
\hline $\begin{array}{l}\text { EU } 57,33 \mathrm{~cm} \\
\text { EU } 91,52 \mathrm{~cm} \\
\text { EU } 92,44 \mathrm{~cm} \\
\text { EU } 105,58 \mathrm{~cm} \\
\text { EU } 106,56 \mathrm{~cm} \\
\text { EU } 107,60 \mathrm{~cm} \\
\text { Feature } 31 \\
\text { Feature } 32\end{array}$ & $\begin{array}{l}- \\
- \\
- \\
- \\
- \\
- \\
- \\
-\end{array}$ & $\begin{array}{l}- \\
- \\
- \\
- \\
- \\
- \\
-\end{array}$ & $\begin{array}{l}- \\
- \\
1 \\
- \\
1 \\
- \\
- \\
-\end{array}$ & $\begin{array}{l}- \\
- \\
- \\
1 \\
- \\
- \\
- \\
-\end{array}$ & $\begin{array}{l}2 \\
- \\
- \\
2 \\
- \\
- \\
- \\
-\end{array}$ & $\begin{array}{l}2 \\
- \\
- \\
- \\
- \\
- \\
- \\
-\end{array}$ & $\begin{array}{r}41 \\
6 \\
28 \\
20 \\
19 \\
3 \\
1 \\
2\end{array}$ & $\begin{array}{l}- \\
- \\
- \\
2 \\
2 \\
- \\
- \\
-\end{array}$ & $\begin{array}{l}- \\
- \\
1 \text { gouge } \\
- \\
- \\
- \\
- \\
-\end{array}$ & $\begin{array}{l}- \\
- \\
- \\
- \\
- \\
- \\
-\end{array}$ & $\begin{array}{l}- \\
- \\
- \\
- \\
- \\
- \\
-\end{array}$ & $\begin{array}{r}45 \\
6 \\
30 \\
25 \\
22 \\
3 \\
1 \\
2\end{array}$ \\
\hline $\begin{array}{l}\text { Nonblock } \\
\text { Totals: }\end{array}$ & 0 & 0 & 2 & 1 & 4 & 2 & 120 & 4 & 1 gouge & 0 & 0 & 134 \\
\hline \multicolumn{13}{|l|}{$\begin{array}{l}\text { Backhoe } \\
\text { Trenches }\end{array}$} \\
\hline $1992-1$ & - & - & - & - & - & - & - & - & 1 gouge & - & - & 1 \\
\hline $1992-2$ & - & - & - & - & - & - & 1 & - & 1 gouge & - & - & 2 \\
\hline $1992-5$ & - & - & 1 & - & - & - & - & - & - & - & - & 1 \\
\hline $1992-20$ & - & - & - & - & 2 & - & 17 & 1 & - & - & - & 20 \\
\hline $1992-24$ & - & 1 & - & - & - & - & 13 & 3 & - & - & - & 17 \\
\hline $1992-25$ & - & - & 1 & 1 & 1 & - & 12 & - & - & - & 1 modified hematite & 16 \\
\hline $1992-28$ & - & - & - & - & - & - & 7 & 1 & 1 hammerstone & - & - & 9 \\
\hline $1992-31$ & - & - & - & - & - & - & - & 2 & - & - & - & 2 \\
\hline
\end{tabular}




\begin{tabular}{|c|c|c|c|c|c|c|c|c|c|c|c|c|}
\hline \multicolumn{13}{|c|}{ Table 12 , continued } \\
\hline $\begin{array}{l}\text { Unit/Maximum } \\
\text { Depth Below } \\
\text { Surface }\end{array}$ & \begin{tabular}{|l} 
Projectile \\
Points
\end{tabular} & Bifaces & \begin{tabular}{|l} 
Cobble \\
Tools
\end{tabular} & Unifaces & Cores & \begin{tabular}{|l} 
Edge- \\
modified \\
Flakes
\end{tabular} & $\begin{array}{l}\text { Unmodified } \\
\text { Debitage }\end{array}$ & \begin{tabular}{|l} 
Ground \\
Stone \\
Tools
\end{tabular} & Other Lithics & Ceramics & Other Materials & Totals \\
\hline Blade Cuts & 4 & - & 1 & 1 & - & - & - & 8 & 2 gouges & - & - & 16 \\
\hline $\begin{array}{l}\text { General } \\
\text { Surface }\end{array}$ & - & - & - & - & - & - & - & 3 & 1 gouge & - & - & 4 \\
\hline Grand Totals: & 28 & 16 & 38 & 80 & 73 & 37 & 3134 & 118 & $\begin{array}{l}18 \text { gouges } \\
4 \text { hammerstones } \\
3 \text { perforators }\end{array}$ & 18 & $\begin{array}{l}4 \text { modified shells } \\
7 \text { modified hematite }\end{array}$ & 3578 \\
\hline
\end{tabular}


detailed provenience data for selected classes of materials are provided in Appendix $\mathrm{H}$.

\section{Chipped Stone Artifacts}

A total of 26 arrow points and fragments, 2 dart points, 3 perforators and fragments, 18 gouges, 16 nonprojectile point bifaces, 80 unifaces and fragments, 38 cobble tools, 73 cores, 37 edge-modified flakes, and 3,134 pieces of unmodified lithic debitage was recovered. Of these, 3 arrow points, 1 dart point, 6 gouges, 1 nonprojectile point biface, 3 unifaces, 2 edge-modified flakes, 5 cobble tools, 7 cores, and 170 pieces of unmodified debitage are from nonblock units, backhoe trenches, blade cuts, or general surface proveniences. The remaining chipped stone artifacts are from the five excavation blocks.

\section{Arrow Points}

Of the 26 arrow points, 5 are complete, 3 are proximal fragments, 8 are medial fragments, 5 are distal fragments, 2 are longitudinally broken, and 3 are wedge fragments. All but one are of fine-grained chert. The single nonchert specimen is of finegrained Potter chert. Five of the 26 specimens are typed, 2 are untyped, 13 are untypeable fragments, and 6 are arrow point preforms. Of the 5 typed arrow points and fragments, 1 is a Bonham and 4 are Scallorn.

\section{BONHAM}

A single complete specimen, made of nonlocal fine-grained chert (Color 39), is typed as Bonham (Suhm et al. 1954:496). It has straight blade edges, moderate barbs, a narrow parallel-sided stem, and a straight base (Figure 21a). Although it is bifacially shaped, a large portion of the ventral surface of the parent flake still remains on one of its faces. It is made on a flake or blade blank that has substantial longitudinal curvature and also might have had a medial ridge along its ventral surface. The point is $24 \mathrm{~mm}$ long, the blade measures $19 \mathrm{~mm}$, and the haft is $5 \mathrm{~mm}$ long. The blade is $13 \mathrm{~mm}$ wide, while its neck and base are only $4 \mathrm{~mm}$ wide. The point is $4 \mathrm{~mm}$ thick.

\section{SCALLORN}

Three complete points and a proximal fragment

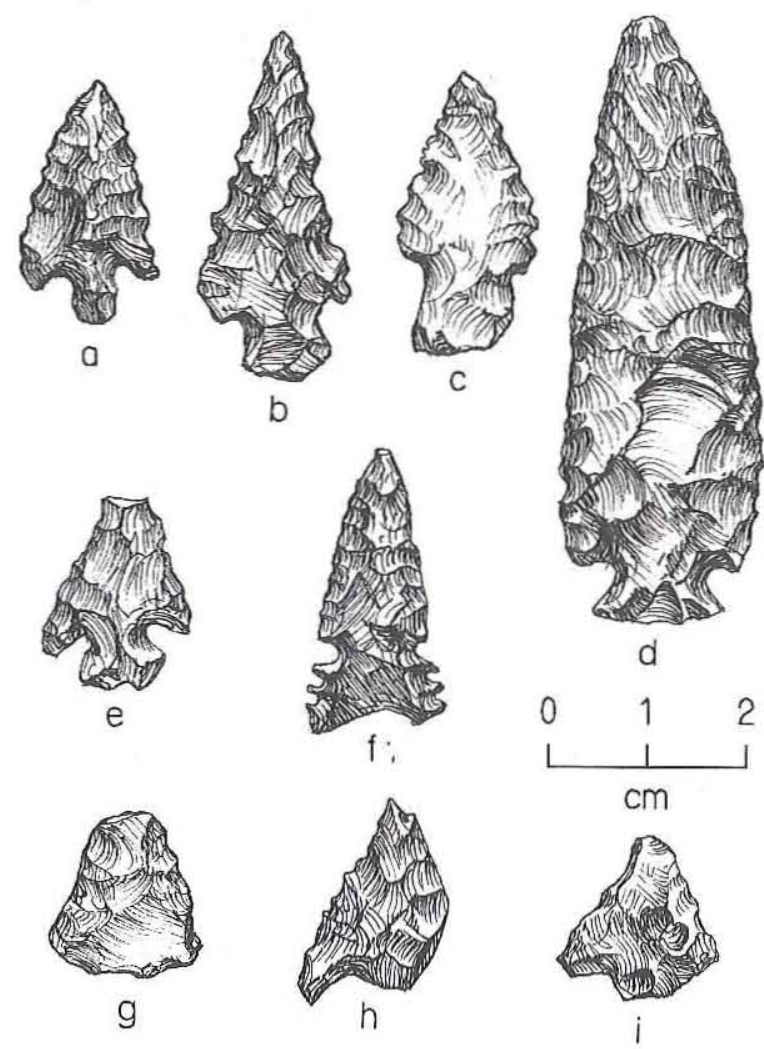

Figure 21. Arrow points, 41GR291. (a) Bonham; $(b-e)$ Scallom; $(f)$ untyped triangular, side notched; $(g)$ untyped triangular; $(h-i)$ untyped medial fragments.

are included in this type (Jelks 1962). Two of the four are relatively similar. They have straight to slightly concave serrated blade edges and slightly expanding corner-notched stems (Figure 21b,c). They are similar to another Scallorn arrow point recovered from the site during the Phase II investigations (Boyd et al. 1990:Figure 66a). Although they are considered complete, both are missing barbs and one also has a broken ear. These specimens are similar to the Scallorn eddy variety identified by Jelks (1962:27-29) at the Kyle site. The third complete point has long convex blade edges and a moderately expanding corner-notched stem (Figure 21d). It is missing one barb, and its distal blade has been reworked to rejuvenate a probable use-break. The fourth Scallorn point, a proximal fragment, has short recurved blade edges, moderate barbs, and a moderately expanding corner-notched stem (Figure $21 e$ ). Its blade edges and one of the barbs have been reworked. The specimen was discarded before the rejuvenation was completed. The third and fourth Scallorn points (see Figure 21d,e) are similar to the 
coryell variety defined by Jelks (1962:27-29). The specimen with slightly concave blade edges (see Figure $21 b$ ) was made on a flake blank and appears to have been reworked. Although the nature of the core used in the manufacture of the other three points could not be determined, it is likely that they also were made on flake blanks. All four are of local fine-grained chert (Colors 6, 15, and $32[\mathrm{n}=2]$ ). The two complete specimens with serrated blades (Scallorn eddy) have a mean length of $31.0 \mathrm{~mm}(\sigma=$ 4.2 ), a mean blade length of $22.5 \mathrm{~mm}(\sigma=6.4)$, a mean haft length of $8.5 \mathrm{~mm}(\sigma=2.1)$, and a mean neck width of $6.5 \mathrm{~mm}(\sigma=2.1)$. The mean neck width of the two Scallom coryell specimens is $8.0 \mathrm{~mm}(\sigma=1.4)$, and their mean thickness is $4.5 \mathrm{~mm}(\sigma=0.7)$. The third complete arrow point, found in association with the cairn burial (Feature 23), is $60 \mathrm{~mm}$ long, its blade measures $54 \mathrm{~mm}$, and it has a 6-mm-long haft, an 8-mm-wide neck, and a 12-mm-wide base. It is made on a flake that is $5 \mathrm{~mm}$ thick. The proximal fragment has a reworked blade width of $15 \mathrm{~mm}$, a haft length of $6 \mathrm{~mm}$, and a neck width of $5 \mathrm{~mm}$. It also is $5 \mathrm{~mm}$ thick.

\section{UNTYPED TRIANGULAR}

One complete arrow point and a proximal fragment are included in this category. The complete specimen (Figure 21f), of local fine-grained chert (Color 15), is a triangular side-notched arrow point with a concave base and two additional notches on each side of the stem. With the exception of the multiple notches, the specimen resembles Washita arrow points. The point is similar to other untyped multinotched specimens recovered in Crosby and Garza counties (Watts 1972:28). It also resembles Huffaker points found in Washita River focus and Custer focus sites throughout Oklahoma (Bell 1960: 58-59). Huffaker points usually have one or, more rarely, two pairs of shallow notches below the deeper pair of notches that define the stem. The point has a haft length of $9 \mathrm{~mm}$, and its blade is $19 \mathrm{~mm}$ long. Its base is missing a small portion of one ear. Its blade is $11 \mathrm{~mm}$ wide, while its neck is only $8 \mathrm{~mm}$ wide. The maximum thickness of the specimen is $4 \mathrm{~mm}$.

The proximal fragment (Figure 21g), of local fine-grained chert (Color 32), is an unnotched triangular specimen with a slightly convex base and reworked blade edges. Although the point retains a portion of the ventral surface of the parent flake, the use-broken distal end suggests that it was a function- ing arrow point rather than a preform. The base is $15 \mathrm{~mm}$ wide, and the specimen is made on a 3- $\mathrm{mm}-$ thick flake blank.

\section{UNTYPEABLE FRAGMENTS}

A total of 13 arrow point fragments, consisting of 8 medial fragments, 3 distal fragments, and 2 wedge fragments, are included in this category. Two of the medial fragments (Figure $21 h, i$ ), broken at the neck and extreme distal end of the blade, are characterized by relatively long barbs and, in one case, by finely serrated blade edges. These are similar to the shorter of the two Scallom coryell specimens (see Figure 21e) recovered from the site.

Seven medial and one distal fragment were broken in use. One of the distal and one of the medial fragments appear to have been broken in manufacture. The break cause could not be identified on the third distal fragment and the two wedge fragments. Ten of the 13 fragments are of local finegrained chert (Colors $2[n=2], 22[n=2], 32[n=$ 4], 36, and 55). Two medial fragments are of nonlocal fine-grained chert (Colors 47 and 52). One specimen is of fine-grained Potter chert.

\section{PREFORMS}

Six bifacially and/or unifacially shaped flake fragments are considered to be arrow point preforms, given the extent and nature of the retouch and shaping. Three are distal fragments, and one each is a proximal, a longitudinal, and a wedge fragment. All of the preforms are shaped primarily unifacially with only minor marginal flaking present on the ventral surfaces of the flake blanks. Three are of local fine-grained chert (Colors 32 and $55[n=2]$ ). Two specimens are of nonlocal fine-grained chert (Colors 38 and 52), while the sixth fragment is of indeterminate-origin fine-grained chert (Color 57).

\section{Dart Points}

Two proximal dart point fragments were recovered from 41GR291. Both are untyped expanding-stem, convex-base points of local finegrained chert (Colors 6 and 32). The larger point (Figure 22a) has a relatively broad blade and usebroken but partially reworked barbs. The distal blade appears to have been broken during blade rejuvenation. The haft is $16 \mathrm{~mm}$ long; the neck is $17 \mathrm{~mm}$ 

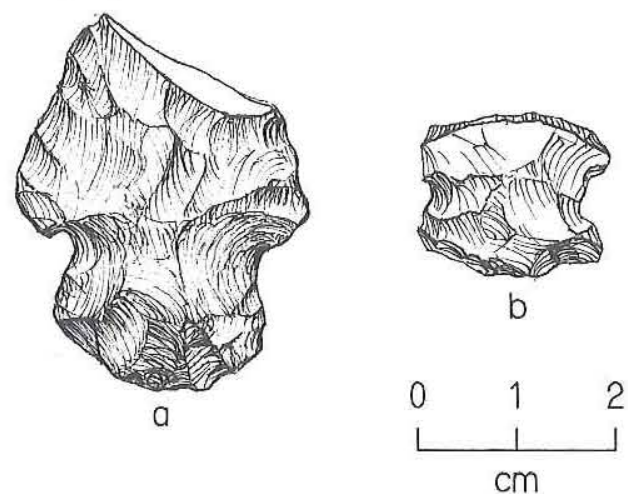

Figure 22. Untyped expanding-stem, convex-base dart points, 41 GR291.

wide, expanding to $20 \mathrm{~mm}$ at the base. The maximum thickness of the specimen is $8 \mathrm{~mm}$.

The smaller dart point is broken immediately above the neck (Figure 22b). Both ears have been sheared off by use-related impact, although subsequently the break was partially reworked. The blade also was broken in use. With the exception of the 15-mm-wide neck, no other metric attributes could be obtained from this specimen.

\section{Perforators}

One complete perforator and two perforator fragments were recovered. All three are made on secondary flakes of local fine-grained chert (Colors 5, 6, and 55). The complete specimen has a relatively short $(7 \mathrm{~mm})$ and broad $(3 \mathrm{~mm})$ pointed working end (Figure 23a). Use-related microflake scarring is distributed on alternate faces and margins of the tip. Its proximal end is $18 \mathrm{~mm}$ wide, $8 \mathrm{~mm}$ thick, and $19 \mathrm{~mm}$ long. Based on its morphology and use-wear distribution, this specimen can be classified

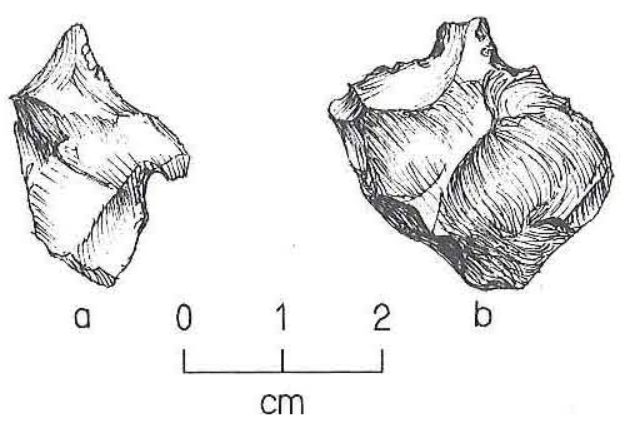

Figure 23. Perforators, 41GR291. (a) Complete reamer made on a secondary flake; $(b)$ proximal perforator fragment made on a secondary flake. as a reamer. The two perforator fragments consist of a proximal and a medial specimen. The proximal fragment is made on a broad $(29 \mathrm{~mm}), 8$ - $\mathrm{mm}$-thick secondary flake (Figure $23 b$ ). Its working end, which at its neck is $6 \mathrm{~mm}$ wide, appears to have been unifacially shaped. It was broken in use. The medial fragment consists of a small portion of the neck and proximal end of the specimen. The breaks appear to have been caused by postdiscard or postdepositional factors. The retouch on the small blade remnant suggests that the working end was unifacially shaped. The blade is $7 \mathrm{~mm}$ wide at its neck.

\section{Gouges}

Of the 18 gouges, 10 are primarily unifacially shaped, while the other 8 , are bifacial specimens. Some of the unifacial specimens are similar in morphology to Clear Fork tools recovered from sites throughout Texas (Turner and Hester 1985). Of the 10 unifacial specimens, 4 are complete, 4 are proximal, and 2 are distal fragments. With the exception of two distal fragments for which shape could not be determined, the remaining unifacial specimens are roughly triangular. Three complete unifacial gouges (Figure $24 a-c$ ) and two of the proximal fragments are made on large thick secondary flakes. The other complete specimen (Figure $24 d$ ) and a third proximal fragment are made on small chert pebbles. The two distal fragments and the fourth proximal fragment are made on thinner flakes that have very little or no cortex. The working edges of all four of the proximal fragments appear to have been intentionally removed in the process of edge rejuvenation. It is possible that these rejuvenation attempts removed too much material, and as a result the gouges were discarded. The four complete unifacial gouges have a mean length of $69.3 \mathrm{~mm}$ $(\sigma=18.1)$, a mean width of $43.0 \mathrm{~mm}(\sigma=9.4)$, and a mean thickness of $23.5 \mathrm{~mm}$ ( $\sigma=6.1)$. Haft wear was noted on two complete specimens and two proximal fragments. The ratios of complete length to haft length are 1.3:1 and 1.4:1 on the two complete specimens with haft wear. The total hafted portion of the larger proximal fragment measures $48 \mathrm{~mm}$; the break occurs before the termination of haft wear on the smaller proximal fragment. The mean maximum width and thickness of the four complete and four proximal fragments together is $40.3 \mathrm{~mm}(\sigma=7.7)$ and $21.4 \mathrm{~mm}(\sigma=6.3)$, respectively. 

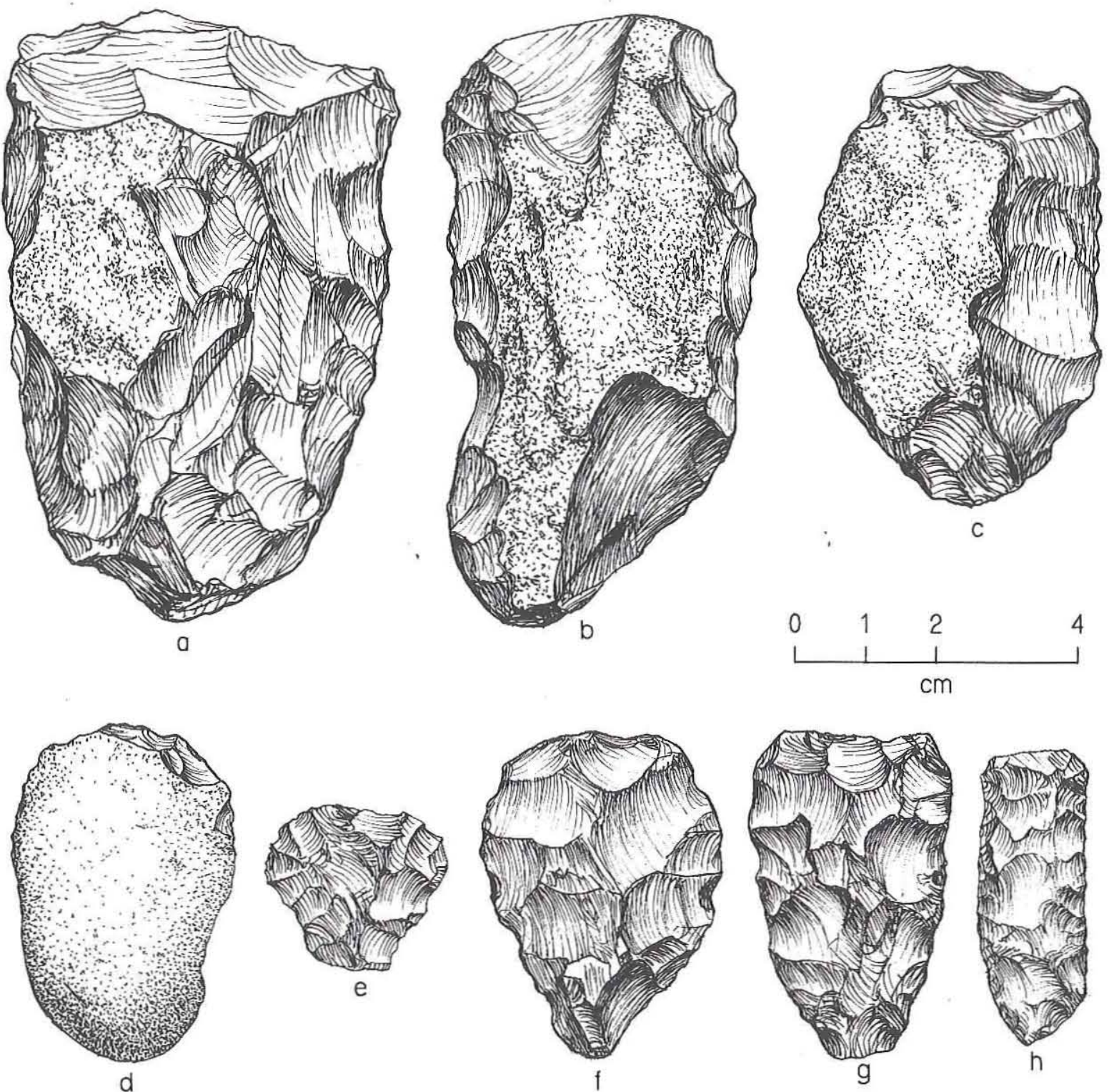

Figure 24. Gouges, 41GR291. (a-c) Unifacial gouges made on thick secondary flakes; $(d)$ unifacial gouge made on chert pebble; $(e-h)$ bifacial gouges.

The eight bifacially shaped gouges normally would have been classified as nonprojectile point bifaces. However, an initial examination of their broad beveled distal ends revealed the presence of use wear in the form of use polish, micro-stepfractured working edges, and minimal to moderate rounding. A further examination of flake scars on the faces of these gouges indicated that these bifacial artifacts were hafted. An additional characteristic of at least three of the bifacial specimens is the rather abrupt narrowing of the lateral edges behind the broad beveled distal end (Figure 24e). This feature is similar to bifacial gouges from sites at Cooper Lake (Fields et al. 1993:Figure 17c). Based on the combination of use- and haft-wear location and morphology, it is clear that these artifacts were used as gouges rather than bifacial knives.

The eight bifacial gouges consist of five distal fragments and three complete or nearly complete specimens. The breaks on all five of the distal fragments are use related; two are broken immediately behind the working edges, and the other three are broken adjacent to the narrow neck of the tool. Two of the complete specimens have roughly 
triangular shapes and planoconvex transverse cross sections (Figure 24f, g). Their working edges are slightly convex and slightly concave, respectively. While the third complete specimen has a similar transverse cross section and roughly triangular shape, it is much narrower than the other gouges (Figure 24h). The steep bevel along one lateral edge indicates substantial reworking, which might have been necessary to rejuvenate a longitudinally broken gouge. The nature of the blanks used in their manufacture could be determined on only two specimens - one is on a secondary flake and the other is on a tertiary flake. The three complete specimens have a mean length of $43.7 \mathrm{~mm}(\sigma=2.3)$. Haft wear was noted on five of the bifacial gouges, including the three complete specimens on which the ratios of complete length to haft length range from 1.3:1 (on two specimens) to 1.2:1. The mean maximum width of the three complete specimens and three of the larger distal fragments is $27.2 \mathrm{~mm}(\sigma=8.2)$; their mean thickness is $6.5 \mathrm{~mm}(\sigma=1.8)$.

With the exception of the two unifacial gouges made on small local fine-grained chert pebbles (Colors 17 and 55), the more bulky unifacial gouges are made of coarse raw materials (i.e., coarsegrained Potter chert [ $=4]$, coarse-grained quartzite $[\mathrm{n}=3$ ], and silicified caliche). The bifacial gouges are made of relatively finer grained materials such as locally available fine-grained cherts (Colors 3, 23, 28 , and $32[\mathrm{n}=2]$, and 55), indeterminate-origin fine-grained chert (Color 57), and fine-grained Potter chert. The morphological and raw material differences between these two groups suggest that the bulky gouges made of durable raw materials were used in different tasks or at different stages of the same task compared to the slender gouges made of materials that have sharper but less-durable edges.

\section{Nonprojectile Point Bifaces}

Sixteen bifaces consisting of 1 complete specimen and 15 fragments were recovered. Six of the 15 fragments are wedges, 5 are proximal fragments, 3 are medial specimens, and 1 is a distal fragment.

The complete biface is an early-reduction-stage specimen made on a secondary flake blank (Figure $25 a$ ). Cortex is present on the platform of the parent flake, and the remainder of its edges are only minimally flaked. Differential patina suggests that it was reworked sometime after initial discard. At

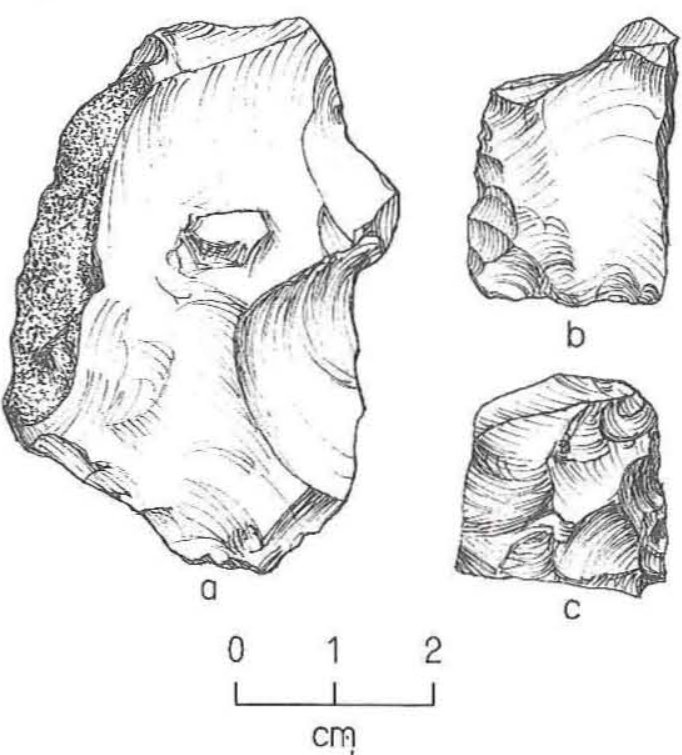

Figure 25. Nonprojectile point bifaces, 41GR291. (a) Complete early-reduction-stage specimen; (b) longitudinally burinated proximal fragment; $(c)$ longitudinally burinated medial fragment.

least five flakes were removed during the reworking episode. The biface is $55 \mathrm{~mm}$ long, $37 \mathrm{~mm}$ wide, and $19 \mathrm{~mm}$ thick.

None of the five proximal fragments retain cortex, and all exhibit heavy reworking. Given the differential degree of patina present on two specimens, it appears that some time elapsed between the initial manufacture and subsequent reworking of these specimens. In addition to the transverse blade breaks on the five proximal fragments, one is also longitudinally burinated (Figure $25 b$ ). This specimen retains some polish and light rounding on flake scar ridges on both faces. These wear traces are characteristics of haft wear. The other four proximal fragments do not exhibit haft wear. Two of the fragments were broken in manufacture; the break cause could not be determined with certainty on the other proximal fragments. However, the presence of reworking on all of the fragments suggests that they were functional specimens that were broken during reworking or under postdepositional circumstances. Metric attributes could be measured on only a single proximal fragment that is $32 \mathrm{~mm}$ wide and $8 \mathrm{~mm}$ thick.

Two of the three medial fragments appear to represent use-broken bifacial knives. Following use breakage, one of these specimens was longitudinally split by a blow directed to its proximal end (Figure $25 c$ ). This longitudinal removal is similar to burin 
resharpening flakes removed from broken bifacial artifacts used as gravers. However, based on the differential patina developed on the specimen, the burinlike resharpening flake was removed sometime after the artifact was initially manufactured and discarded. The third specimen is an early-reductionstage manufacture-broken flake blank. The three medial fragments have a mean thickness of $7.3 \mathrm{~mm}$ $(\sigma=0.6)$. No other metric measurements could be obtained.

The single distal fragment is an early-reductionstage manufacture-broken specimen. A small portion of cortex is present on this relatively thick $(26 \mathrm{~mm})$ fragment.

Of the six wedge fragments, two retain cortex and appear to represent early-reduction-stage bifaces; one of these was broken in manufacture. Break cause could not be established on the remaining five wedges. However, all four of the decorticate specimens have small resharpened bifacial edge segments indicative of a finished manufacture stage and tool use prior to breakage and discard.

Fifteen $(94 \%)$ of the bifaces and fragments are of fine-grained chert; the only specimen not made of this raw material is of fine-grained Potter chert. The majority of the fine-grained chert specimens are of locally available materials $(\mathrm{n}=9,60 \%$; Colors 2,5 $[\mathrm{n}=2], 11,31[\mathrm{n}=2], 32[\mathrm{n}=2]$, and 55). Four (27\%) specimens are made of nonlocal fine-grained cherts (Colors 47, $49[n=2]$, and 54), and two (13\%) are of indeterminate-origin fine-grained cherts.

\section{Cobble Tools}

Of the 38 cobble tools, $23(61 \%)$ are complete and $15(39 \%)$ are fragmentary. Most $(n=24,63 \%)$ are made of coarse-grained Potter chert: Nearly one-fourth are made of coarse-grained quartzite $(n=$ $10,26 \%$ ), while specimens made of fine-grained chert $(\mathrm{n}=3,8 \%)$ and an unidentified basaltic material $(n=1,3 \%)$ are infrequent. The three finegrained chert specimens are of local origin (Colors 9, 22 , and 32 ).

Although it is probable that the 38 specimens represent a number of different uses, it is difficult to separate them into distinct functional categories based on the types of use wear that they exhibit. Previous classifications of cobble tools from East Texas (e.g., Fields et al. 1991:72-73) into wedges and choppers relied on size differences between the two and the presence of rounded edges on choppers and their absence on wedges. Both tool types exhibited step fracturing.

The 23 complete cobble tools can be separated into two broad size categories: (1) less than $100 \mathrm{~g}$ (n $=4)$, and (2) greater than $100 \mathrm{~g}(\mathrm{n}=19)$. Normally, specimens in the lighter tool group would be considered wedges, while those in the heavier group would be chopping tools. However, two of the specimens in the lighter group exhibit heavy edge rounding derived from crushing, and all four have step-fractured working edges (Figure 26a,b). The two specimens with heavy edge rounding are made on coarse-grained quartzite flake blanks and have two working edges each. The remaining two specimens in the light tool group are made on local fine-grained chert pebbles (Colors 9 and 22). Both have single working edges that are not rounded but exhibit step fracturing (Figure 26c, d). Three of the four light tools have cortex backing opposite at least one working edge.

Based on the nature of the use wear (i.e., edge rounding vs. step fracturing), it is possible that the light tool group contains two functionally distinct subgroups: (1) specimens with edge rounding; and (2) specimens with step-fractured working edges but no edge rounding. It is also possible that these two groups represent specimens in different stages of their use lives and that eventually, with increased use, all of the light tools also would have developed edge rounding. In either case, it is not known how these specimens were used to develop the degree and type of wear described above. It is doubtful that the use of the specimens as wedges would have generated the degree of edge rounding noted on at least two of the four light tool specimens. It also is unlikely that the cobble tools that weigh a mean of $54.0 \mathrm{~g}$ (minimum $=44$, maximum $=77, \sigma=15.4$ ) could have been used effectively as chopping tools. These specimens are also rather small as evidenced by their mean length $(\overline{\mathrm{x}}=53.8 \mathrm{~mm}, \sigma=5.6)$, mean width $(\overline{\mathrm{x}}=40.3 \mathrm{~mm}$, $\sigma=2.5)$, and mean thickness $(\overline{\mathrm{x}}=24.0 \mathrm{~mm}, \sigma=4.5)$.

The 19 complete cobble tools that are heavier than $100 \mathrm{~g}$ are characterized by a mean weight of $454.6 \mathrm{~g}$ (minimum $=122$, maximum $=1,425, \sigma=$ 334.4). These specimens are also considerably larger than their counterparts in the lighter group as evidenced by their mean length $(\overline{\mathrm{x}}=99.3 \mathrm{~mm}, \sigma=$ $30.2)$, mean width $(\bar{x}=76.3 \mathrm{~mm}, \sigma=19.8)$, and mean thickness $(\bar{x}=48.0 \mathrm{~mm}, \sigma=14.3)$. Nine (47\%) have single working edges (Figure $27 a$ ), four (21\%) have two working edges (Figure $27 b$ ), three 


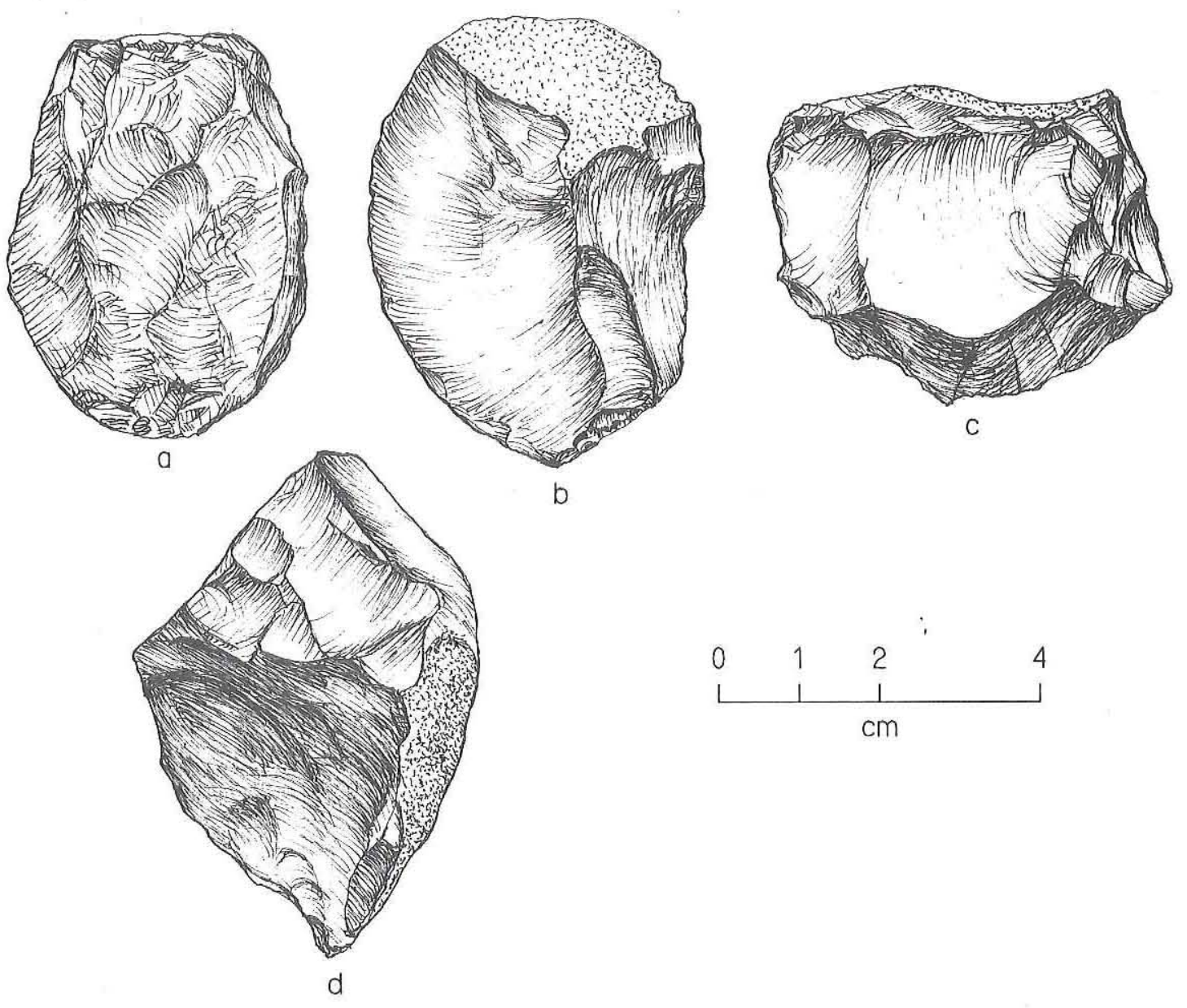

Figure 26. Light cobble tools, 41GR291. ( $a-b)$ Rounded/crushed working edges; $(c-d)$ step-fractured working edges.

(16\%) have three working edges (Figure 27c), and three $(16 \%)$ have four working edges (Figure $27 d$ ). Of the 19 cobble tools in this weight group, $5(26 \%)$ have pointed working edges, $6(32 \%)$ have straight to convex working edge shapes, and $8(42 \%)$ have multiple working edges of which some are pointed and others are straight to slightly convex. Cortex backing of all working edges is found on 12 tools, while some working edges are cortex backed and others are not on 6 tools with multiple working edges. A single tool with multiple working edges does not have cortex backing opposite any of the working edges.

Eleven of the 19 heavy complete cobble tools are made of coarse-grained Potter chert. Seven others are of coarse-grained quartzite, and the remaining specimen is of an unidentified basaltic material.

Although this group of cobble tools is distinguishable from the lighter group on the basis of weight, the differences between the two groups are not consistent in terms of use-wear characteristics. For instance, although 14 of the heavy cobble tools exhibit edge rounding on at least one of their working edges, edge rounding is entirely absent on 5 specimens. One of these five heavy cobble tools also lacks step fracturing on its working edge (Figure $28 a$ ). In addition, of the 14 tools with rounded working edges, 1 specimen entirely lacks step fracturing (Figure 28b). This variability in use-wear characteristics among the heavy cobble tools may indicate that these specimens represent tools along the entire continuum of use lives, including tools that have not seen a great deal of use, tools that have been heavily worn but not rejuvenated, and tools that have been worn and subsequently rejuvenated prior to discard and/or abandonment.

In addition to this variability in use lives, it is probable that the specimens included in the heavy 

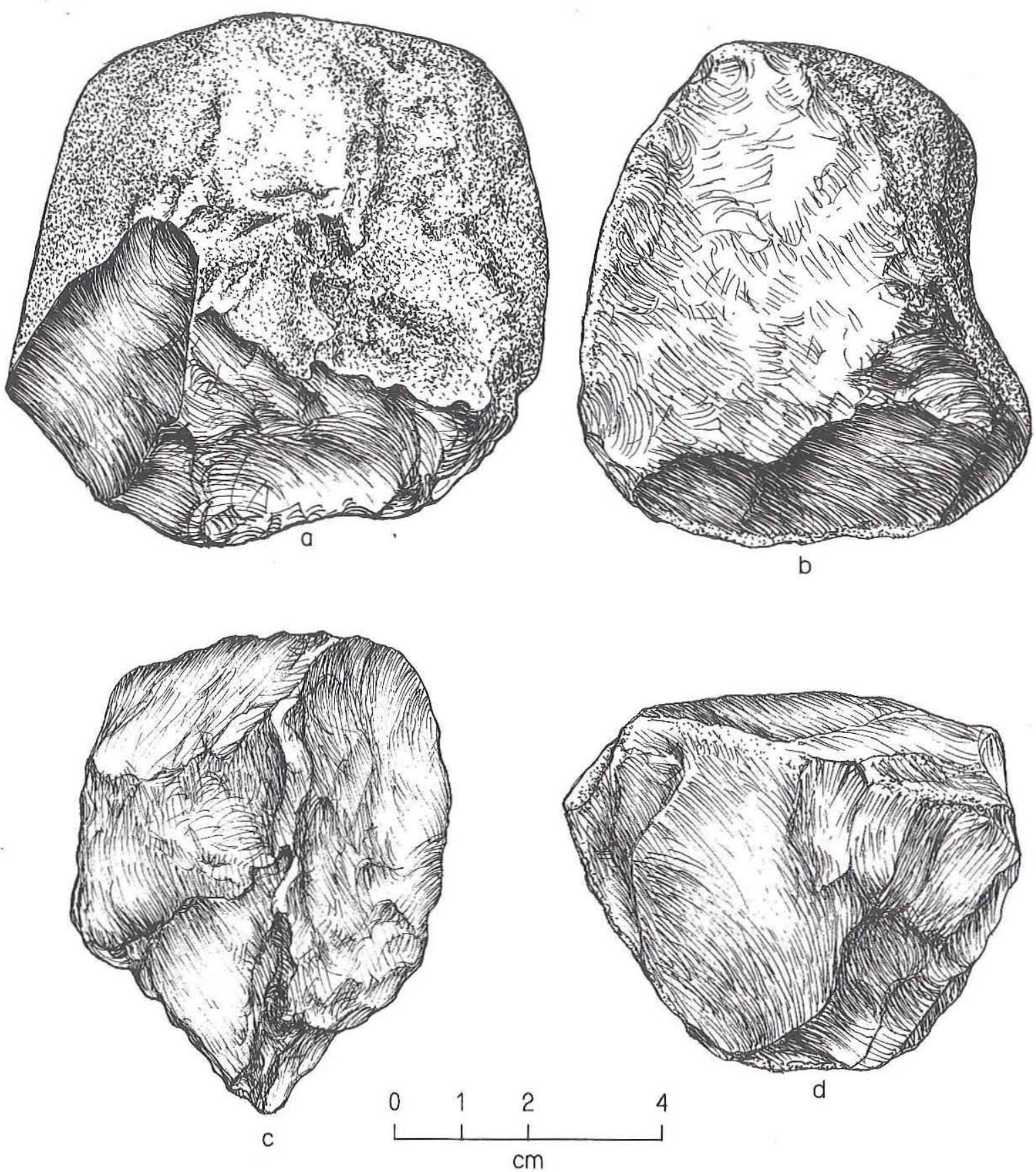

Figure 27. Heavy cobble tools, 41GR291. (a) Single working edge; (b) two working edges; $(c)$ three working edges; $(d)$ four working edges.

cobble tool category may represent a number of distinct uses. Given the coarseness of most of the raw materials from which these cobble tools are made, microscopic use-wear analysis could not be used to aid in the identification of tool use. However, limited experimental replication of ground stone tool refurbishing (i.e., pecking) with a large bifacially flaked Potter chert cobble generated usewear characteristics that were identical to those noted on most of the heavy cobble tool working edges. The early stages of pecking produced numerous step fractures along the rather thin and weak working edge. As the weaker portions of the working edge stabilized, the edge began to abrade and became 


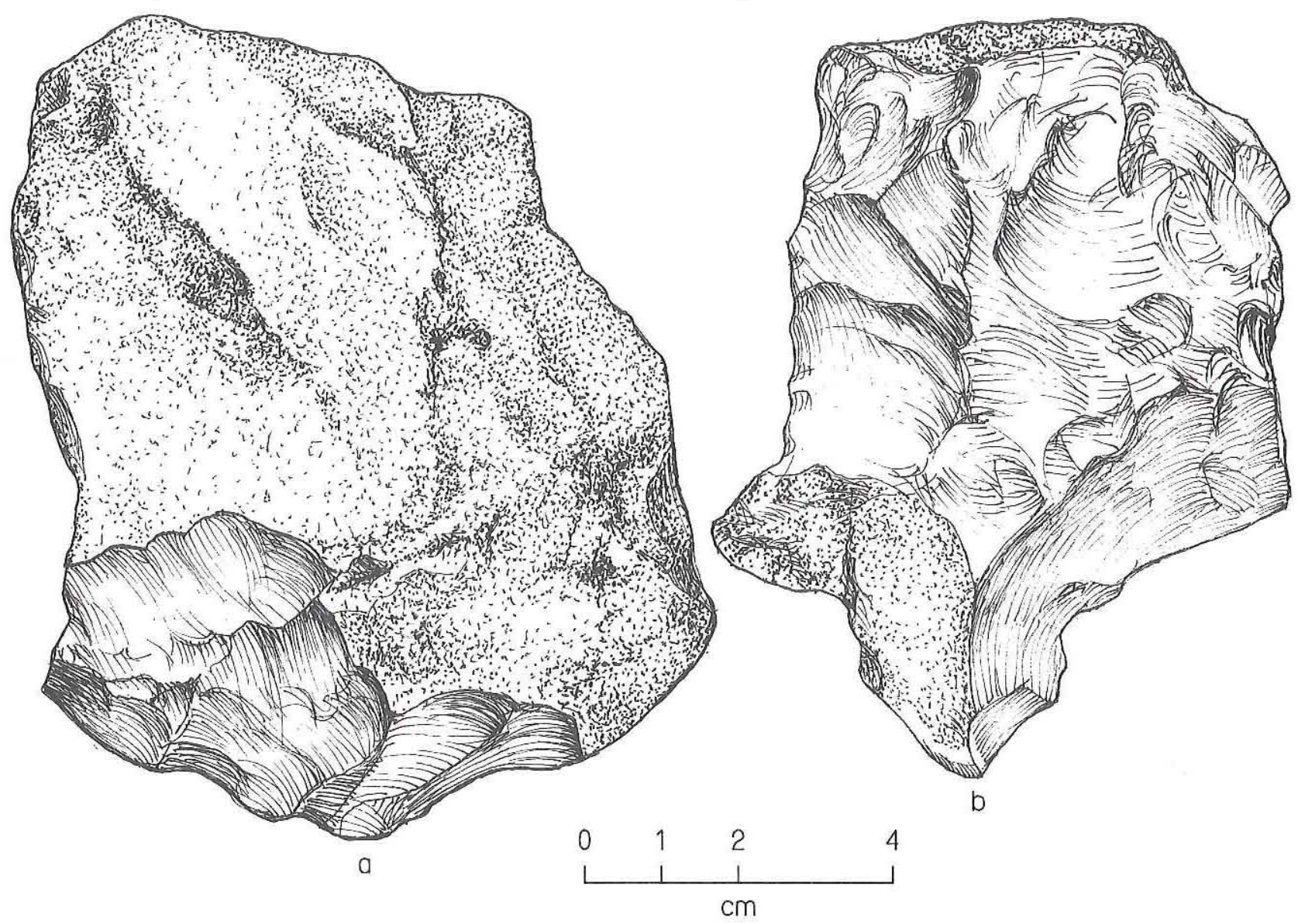

Figure 28. Heavy cobble tools with unusual use wear, 41GR291. (a) No rounding or step fracturing on working edge; $(b)$ rounded but not step fractured working edge.

rounded in a fashion similar to the rounding noted on many of the heavy cobble tools. The similarities in use wear between the archeological specimens and the experimental piece suggest that at least some of the heavy cobble tools from 41GR291 were used as ground stone refurbishing tools.

\section{Unifaces}

The 80 unifaces and fragments recovered during the Phase III data recovery consist of 8 end scrapers, 6 side scrapers, 3 combination end and side scrapers, 2 gravers, 11 minimally retouched end scrapers, 10 minimally retouched side scrapers, and 40 indeterminate unifaces and uniface edges.

\section{END SCRAPERS}

The eight end scrapers have heavily retouched working edges, but their lateral margins exhibit little retouch. Although no attempts were made to standardize the shape of the blanks used in their manufacture, the end scrapers are rather short and trapezoidal (Figure $29 a, b$ ). Five (63\%) of the blanks retain cortex; the other three $(37 \%)$ are decorticate flakes. Four specimens are complete or nearly complete, three were broken in use, and the break cause could not be established on the remaining specimen. Haft wear is present on a single complete specimen and absent on three others; its presence/ absence could not be established on three distal fragments and a nearly complete proximal fragment made of coarse-grained quartzite. Five $(63 \%)$ of the end scrapers are made of fine-grained chert, while the remaining three are made of fine-grained Potter chert, coarse-grained quartzite, and Tecovas jasper. Four of the five fine-grained chert pieces are of local origin (Colors 5, 6, 31, and 35); the remaining 


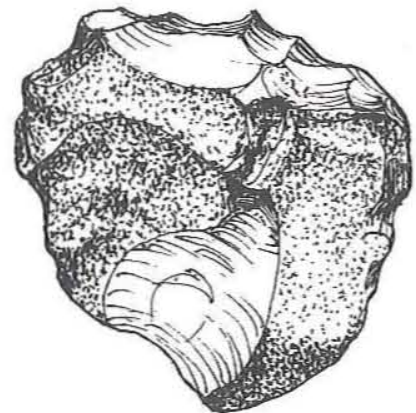

a
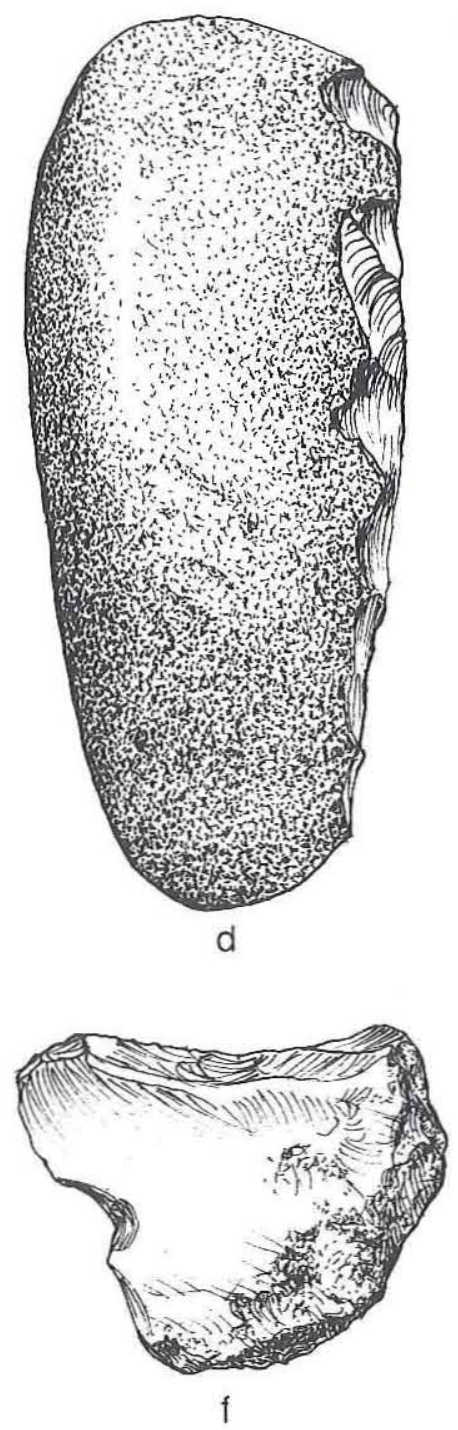

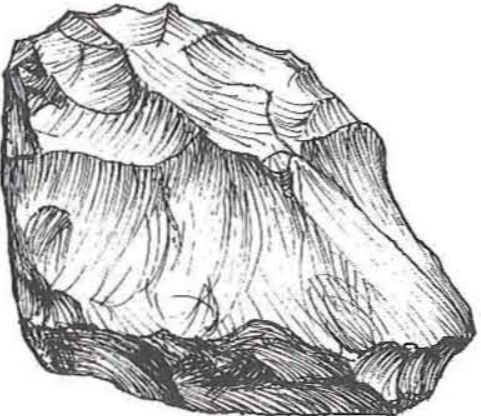

b

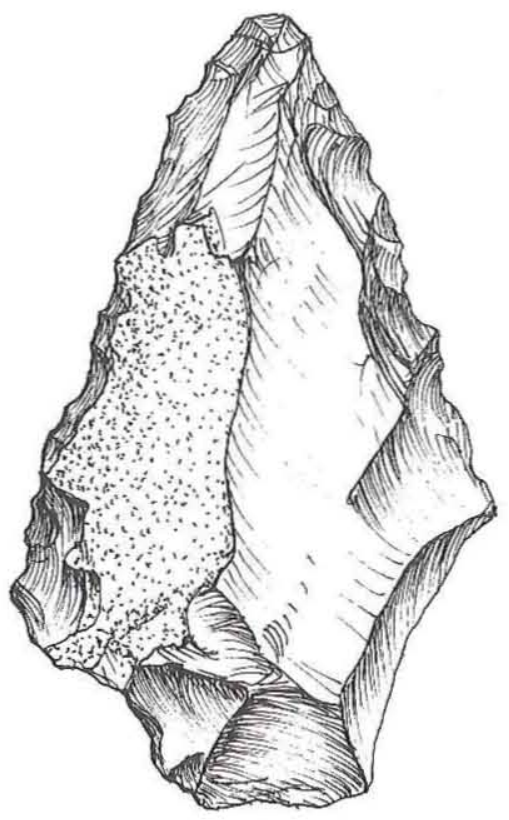

C

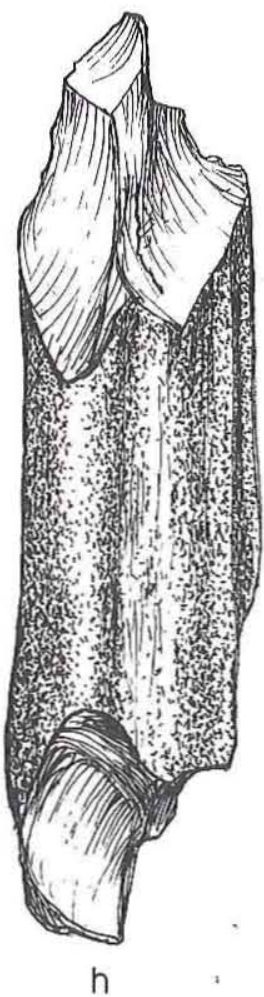

Figure 29. Unifaces, 41GR291. ( $a-b)$ End scrapers made on short trapezoidal secondary flakes; $(c-d)$ side scrapers made on large secondary flakes; $(e-g)$ combination end and side scrapers with concave lateral edges; $(h)$ graver with two working edges. 
specimen is of nonlocal origin (Color 42). The four complete specimens have a mean length of $33.0 \mathrm{~mm}$ ( $\sigma=5.8)$, a mean width of $39.5 \mathrm{~mm}(\sigma=10.3)$, and a mean thickness of $11.5 \mathrm{~mm}(\sigma=5.3)$.

\section{SIDE SCRAPERS}

The six side scrapers consist of four complete specimens and two distal fragments. Three of the six are made on large corticate flakes (Figure 29c, $d$ ), two are made on short thick corticate flakes, and one is made on a thin corticate blank. The range of flake blank shapes and sizes again shows little standardization, suggesting that these blanks perhaps were selected from bulk debitage rather than having been made purposefully. The two incomplete specimens were broken in use or in the process of rejuvenation. Haft wear is absent on four of the six end scrapers and could not be determined on two complete specimens of coarse-grained Potter chert and coarsegrained quartzite. The latter side scraper has a strong longitudinal curvature and appears to represent one end of a lenticular quartzite nodule (see Figure 29d). One of its lateral edges is unifacially retouched, and the narrower end is rounded and smooth. In addition, the apex of the dorsal surface exhibits considerable polish similar to that noted on quartzite manos from the site. However, this type of wear on manos is found on their flat grinding surfaces, while the margins are either unused or are lightly battered rather than exhibiting use polish. These differences suggest that the dorsal polish on this side scraper might have derived from use or, more unlikely, hafting. Two (33\%) specimens are made on finegrained materials - one is a nonlocal chert (Color 52), and the other is a silicified wood piece. The other four $(67 \%)$ are made of coarse-grained materials, including two of coarse-grained quartzite, one of fine-grained quartzite, and one of coarse-grained Potter chert. The two side scrapers made on short blanks measure $29 \times 36$ x $16 \mathrm{~mm}$ and $36 \times 28 \times 17 \mathrm{~mm}$. The two complete side scrapers made on large thick flake blanks measure $80 \times 46 \times 20 \mathrm{~mm}$ and $88 \times 38 \times 28 \mathrm{~mm}$.

\section{COMBINATION END AND SIDE SCRAPERS}

Three combination end and side scrapers were recovered. All are made on short corticate flake blanks. In addition to two scraping edges, one specimen has a small pointed graver beak protruding from the intersection of its lateral margin and distal end (Figure 29e). A second specimen, made of Tecovas jasper, has a slightly convex distal working edge and a notched lateral working margin (Figure 29f). The third combination end and side scraper has a heavily reworked straight distal end and a slightly concave lateral working edge (Figure $29 \mathrm{~g}$ ). The two nonjasper specimens are made of local fine-grained chert (Colors 15 and 28). The three specimens have a mean length of $32.7 \mathrm{~mm}(\sigma=7.5)$, a mean width of $37.7 \mathrm{~mm}(\sigma=2.3)$, and a mean thickness of $16.0 \mathrm{~mm}$ $(\sigma=5.6)$.

\section{GRAVERS}

A complete graver and a distal graver fragment were recovered. The complete specimen has two graver bits on opposite ends of an elongated, angular silicified wood pebble (Figure 29h). The two working bits were manufactured by removals from all four of the pebble facets. The center of the pebble remains entirely corticate. The tool is $90 \mathrm{~mm}$ long, $23 \mathrm{~mm}$ wide and $23 \mathrm{~mm}$ thick.

The distal graver fragment is made of local finegrained chert (Color 6). It appears to have been made on a corticate blade or bladelike flake blank. The specimen was broken by a blow directed onto its dorsal surface while it was seated on an anvil. This method suggests a purposeful break, perhaps in an attempt to rejuvenate the worn edge.

\section{MINIMALLY RETOUCHED END SCRAPERS}

Minimally retouched end scrapers constitute the second largest $(n=11)$ analytical category. All of the specimens are complete tools. Nearly all $(n=9$, $82 \%$ ) are made on secondary flake blanks; only two $(18 \%)$ are made on tertiary flakes. The flake blanks are irregular in shape and size (Figure $30 a-c$ ), suggesting that they are not the products of standardized uniface blank manufacture. Their mean length is $35.0 \mathrm{~mm}(\sigma=21.5)$; they have a mean width of $40.7 \mathrm{~mm}(\sigma=15.1)$ and a mean thickness of $13.2 \mathrm{~mm}(\sigma=6.6)$. The majority $(\mathrm{n}=6,55 \%)$ are made of fine-grained chert, with coarse-grained quartzite $(n=3,27 \%)$ and coarse- and fine-grained Potter chert ( $\mathrm{n}=1$ each) occurring in lower frequencies. Among the fine-grained cherts, local-origin specimens are the most common $(n=5$; Colors 6 


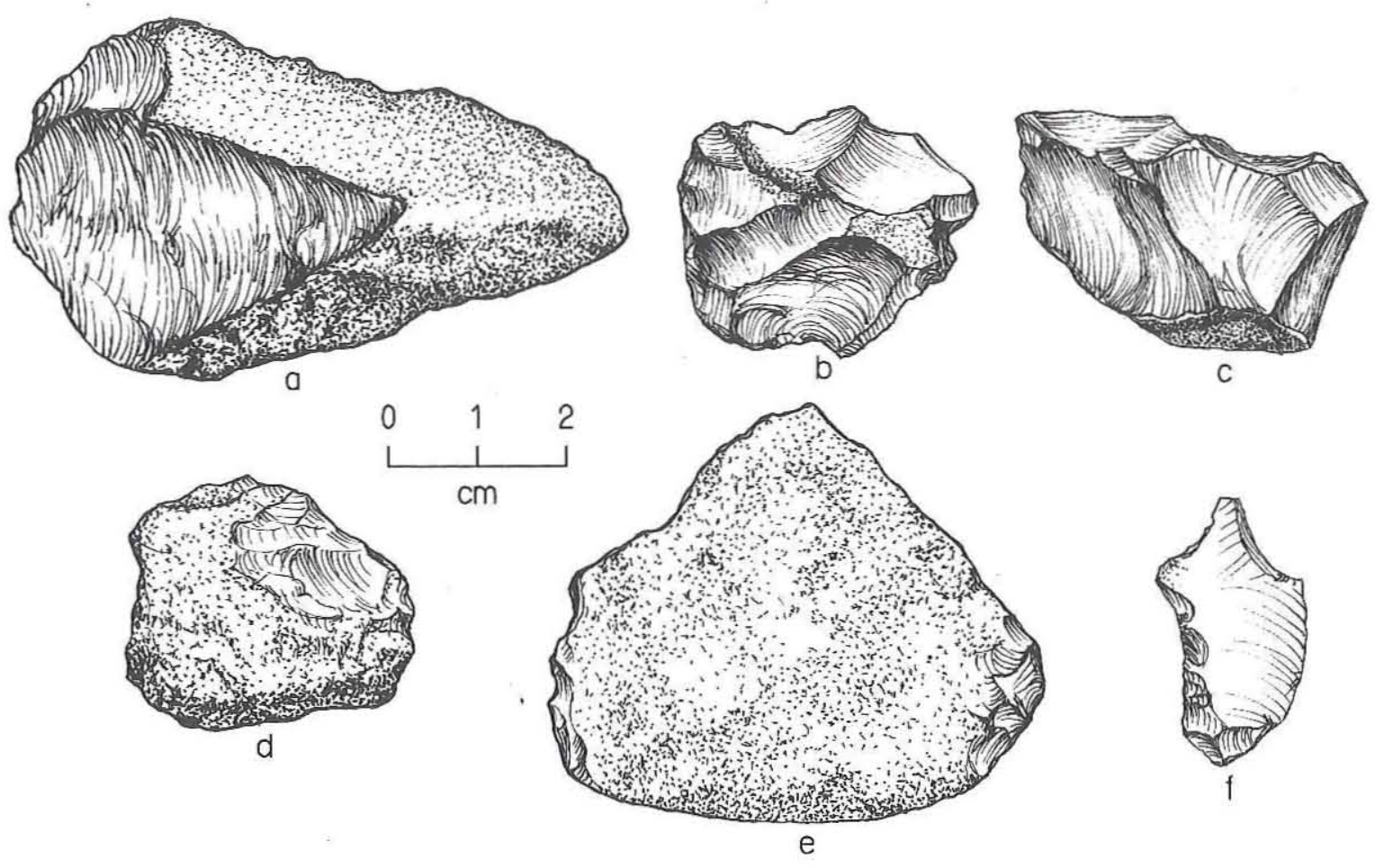

Figure 30. Minimally retouched unifaces, 41GR291. $(a-c)$ End scrapers made on secondary flakes; $(d-e)$ side scrapers made on split pebble and natural flake, respectively; $(f)$ side scraper made on flake.

$[\mathrm{n}=2], 31,36$, and 55). The remaining specimen is of nonlocal chert (Color 52).

\section{MINIMALLY RETOUCHED SIDE SCRAPERS}

Minimally retouched side scrapers are nearly as numerous $(n=10)$ as the previous analytical category. One is made on a naturally split, locally available, fine-grained chert (Color 26) pebble (Figure 30d). A second specimen has two short retouched segments and is made on a natural opalized caliche flake (Figure 30e). The remaining eight specimens are made on flake blanks (Figure 30f). All of the blanks retain some cortex, and as in the previous category, the blanks are highly irregular in shape and size. They have a mean length of $33.9 \mathrm{~mm}$ $(\sigma=10.2)$, a mean width of $31.0 \mathrm{~mm}(\sigma=15.0)$, and a mean thickness of $11.3 \mathrm{~mm}(\sigma=5.0)$. With the exception of one fine-grained Potter chert and one coarse-grained quartzite (10\% each) specimen, the remainder $(n=8,80 \%)$ are made of fine-grained chert. All are of local origin (Colors 3, 6, 8, $26[\mathrm{n}=$ $2], 28$, and $55[n=2])$.

\section{INDETERMINATE UNIFACES}

These 40 specimens consist of $4(10 \%)$ complete tools, $16(40 \%)$ distal fragments, $6(15 \%)$ proximal fragments, $3(7 \%)$ medial specimens, and $11(28 \%)$ indeterminate uniface edges. The four complete specimens form a homogeneous group of small locally available chert $(n=3)$ and quartzite $(n=1)$ pebbles unifacially flaked along one $(n=2)$ or two ( $n$ $=2$ ) lateral margins (Figure 31). Given that their surfaces lack haft wear, it appears that they were hand-held tools. However, since they differ from previously identified tool types, it is not possible to determine whether they were used as scraping or cutting tools or for other functions. The four specimens have a mean length of $42.3 \mathrm{~mm}(\sigma=5.1)$, a mean width of $32.5 \mathrm{~mm}(\sigma=6.2)$, and a mean thickness of $12.5 \mathrm{~mm}(\sigma=2.4)$.

The remainder of the indeterminate unifaces consist of proximal, medial, distal, and lateral edge fragments that could not be assigned with certainty to other unifacial tool categories given the absence of key portions that might have allowed correct classification. Some of these fragments might 

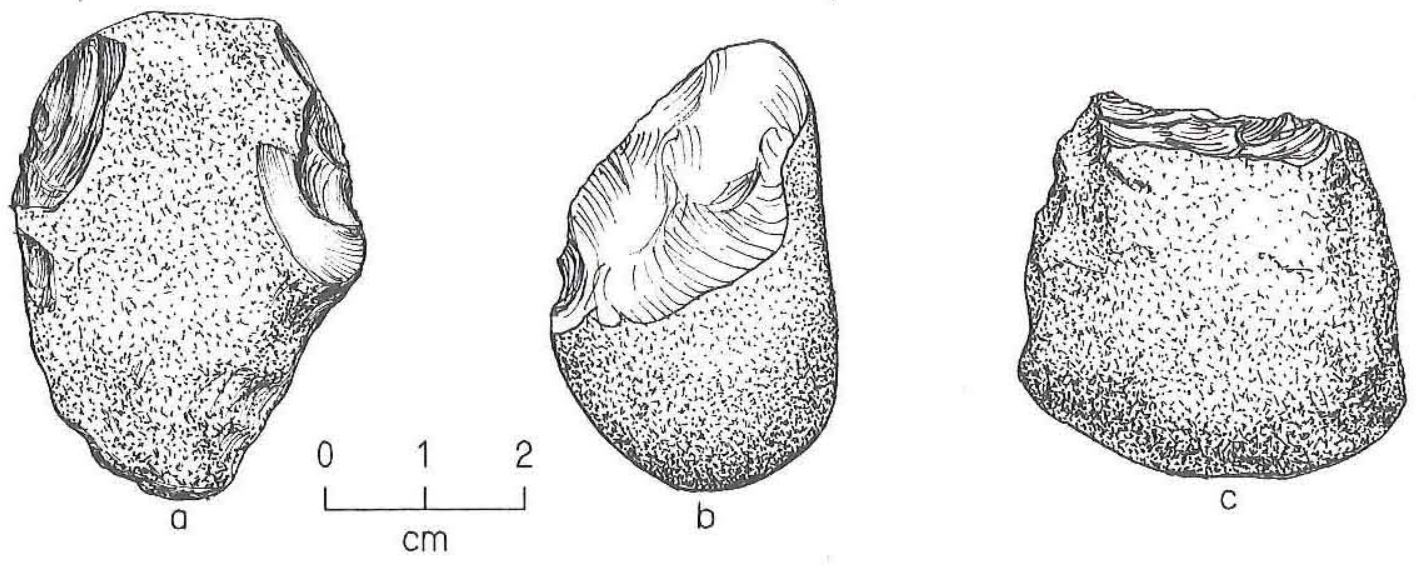

Figure 31. Indeterminate unifaces made on pebbles, 41GR291.

represent minimally retouched unifaces, while others are portions of more extensively shaped end, side, and/or combination end and side scrapers. The majority of the indeterminate unifaces and edge fragments $(n=27,68 \%)$ are made of fine-grained chert. Specimens made of fine-grained Potter chert $(\mathrm{n}=5,13 \%)$ and coarse-grained quartzite $(\mathrm{n}=4$, $10 \%$ ) occur in low frequencies, while other raw materials are infrequent. Local-origin specimens represent the majority of the fine-grained cherts $(\mathrm{n}=$ 19, 70\%; Colors 2, 4, $5[\mathrm{n}=3$ ], $6[\mathrm{n}=5], 8[\mathrm{n}=2]$, $16[n=2], 31,37[n=2]$, and $55[n=2])$. Slightly less than one-third of the fine-grained cherts are of nonlocal origin $(n=8,30 \%$; Colors $41[n=2], 42$, 43 [n $=2], 49,51$, and 54).

\section{Cores}

Seventy-three cores and core fragments were recovered. Forty $(55 \%)$ of these are of fine-grained chert, and $12(16 \%)$ each are of fine-grained and coarse-grained quartzite. Silicified wood, finegrained Potter chert, and silicified caliche each occur in low frequencies $(\mathrm{n}=2,3 \%$, each), while coarsegrained chert, Tecovas jasper, and opalized caliche are infrequent ( $\mathrm{n}=1,1 \%$, each). Local-origin specimens represent $88 \%$ of the fine-grained chert cores (Colors $3[n=4], 4[n=2], 5[n=2], 6[n=$ 4], 7 [n=4], 8 [n=2], 9, 10, 13, 15, 19, 24, 26, 27 $[\mathrm{n}=3], 32,37$, and $55[\mathrm{n}=5])$. Only one $(2 \%)$ is of nonlocal origin (Color 39), while four other finegrained chert specimens $(10 \%)$ are of indeterminate origin (Color 57).

Of the 40 fine-grained chert specimens, 34 (85\%) are complete and $6(15 \%)$ are fragmentary.
Seventeen (43\%) are pebble cores, $10(25 \%)$ are flake cores, and the nature of the core could not be established on $13(33 \%)$ specimens either due to their incompleteness or degree of reduction. The majority $(\mathrm{n}=23,58 \%)$ are multidirectional, about a third $(\mathrm{n}=$ $12,30 \%)$ are unidirectional, and a small number $(\mathrm{n}=$ $5,12 \%$ ) are bidirectional. Cortex is present on 35 $(88 \%)$ of the 40 cores; the remaining $5(12 \%)$ are decorticate. The number of flake removals ranges from 1 to 16 , with the mean being 6.7 ( $\sigma=3.8$ ). None of the cores appear to have been recycled into this functional category from other tool or artifact categories. The fine-grained chert cores are relatively small, having a mean length of $37.3 \mathrm{~mm}$ $(\sigma=10.8)$, a mean width of $26.6 \mathrm{~mm}(\sigma=8.1)$, and a mean thickness of $17.1 \mathrm{~mm}(\sigma=3.8)$.

A preliminary analysis of the cores made of coarse-grained materials (i.e., coarse-grained chert, fine- and coarse-grained quartzite, fine-grained Potter chert, silicified caliche, and silicified wood) indicated that, with the exception of size differences between fine- and coarse-grained quartzites, a great degree of similarity existed in core attributes among these materials. In general, the fine-grained quartzite cores are larger than the coarse-grained quartzite cores. The fine-grained quartzite cores have a mean length of $49.2 \mathrm{~mm}(\sigma=14.2)$, a mean width of $31.5 \mathrm{~mm}(\sigma=13.8)$, and a mean thickness of $20.0 \mathrm{~mm}(\sigma=6.0)$. On the other hand, the coarsegrained quartzite cores have a mean length of $32.0 \mathrm{~mm}(\sigma=9.2 \mathrm{~mm})$, a mean width of $24.7 \mathrm{~mm}$ $(\sigma=7.7)$, and a mean thickness of $17.1 \mathrm{~mm}(\sigma=$ 6.3). The similarities make it possible to combine the cores made of these raw materials into a single group for the purposes of discussion. 
Of the 31 coarse-grained cores, $21(68 \%)$ are complete, and $10(32 \%)$ are fragmentary. The percentage of fragmentary cores is more than double that noted in the fine-grained chert cores. Pebble cores constitute a much larger proportion of the coarse-grained cores $(n=22,71 \%)$, while flake cores represent a relatively small percentage $(n=7$, $23 \%$ ). In striking contrast to the fine-grained chert cores, the coarse-grained collection consists primarily of unidirectional specimens $(n=24,77 \%)$. Multi- and bidirectional cores are found in small numbers. Cortex is present on all but a single coarse-grained core $(n=30,97 \%)$. Corticate specimens represent a higher proportion of the core collection than among the fine-grained chert cores. Finally, in terms of functional categories, four of the coarse-grained specimens represent manos recycled as cores, and another piece is a hammerstone recycled as a core.

The single Tecovas jasper specimen is a small corticate flake core $(38 \times 23 \times 15 \mathrm{~mm})$. Its dorsal surface has eight flake scars removed from multiple directions.

The opalized caliche specimen is a small $(16 \times 15 \times 14 \mathrm{~mm})$ corticate pebble core fragment. Its surface exhibits four bidirectionally removed flake scars.

If it is assumed that cores with a single flake removal represent tested specimens discarded prior to further reduction, it is interesting to note that $10 \%$ $(n=4)$ of the fine-grained chert cores fall into the tested core category. On the other hand, $45 \%$ ( $n=$ 14) of the coarse-grained raw material cores have not progressed beyond the tested core stage. In terms of the other end of the reduction continuum, while $43 \%$ $(n=17)$ of the fine-grained chert cores have eight or more flake removals, only one (3\%) coarse-grained raw material core has eight or more removals. These differences indicate that fine-grained chert cores tended to be used relatively intensively.

\section{Edge-modified Flakes}

Slightly more than half of the 37 edge-modified flakes are complete flakes $(n=19,51 \%)$, followed by chips $(n=16,43 \%)$ and proximal flake fragments $(n=2,5 \%)$. A large majority $(n=26,70 \%)$ are complete tools. Of these, $6(23 \%)$ are chips, $1(4 \%)$ is a proximal flake fragment, and the rest $(n=19$, $73 \%)$ are complete flakes. Nearly half $(n=18,49 \%)$ of the edge-modified flakes are tertiary specimens,
$11(30 \%)$ have between $1-50 \%$ cortex, 7 (19\%) have between $51-99 \%$ cortex, and $1(3 \%)$ specimen is entirely corticate. Seven (27\%) are between $21-30 \mathrm{~mm}$, and only one (4\%) specimen is less than $20 \mathrm{~mm}$.

Forty-three working edges were noted on the 37 edge-modified flakes. The majority $(n=31,84 \%)$ of the specimens have single working edges. Only six $(16 \%)$ have two working edges. Working edges used for scraping tasks alone occur on 35 (95\%) flakes. A single tool has a working edge used in scraping tasks and a small pointed protrusion used as a graver beak. A second edge-modified flake has a single edge used in both sawing and scraping tasks.

The great majority $(n=30,81 \%)$ are of finegrained chert. Fine-grained Potter chert constitutes the next most frequent group $(n=3,8 \%)$, although it occurs in low frequencies. Edge-modified flakes of coarse-grained quartzite, silicified wood, Tecovas jasper, and opalized caliche are each represented by a single specimen (3\% each). Twenty-six $(87 \%)$ of the fine-grained chert specimens are of locally available cherts (Colors 3 [n $=2$ ], $5[n=3], 6[n=$ $3], 16,22,23,24$ [n = 2], 26, 28, 29, 31 [ $n=2], 32$ $[\mathrm{n}=2]$, and $55[\mathrm{n}=6])$. The remaining four $(13 \%)$ are of nonlocal fine-grained chert (Colors $43[n=2]$ and $52[n=2])$.

\section{Unmodified Debitage}

A total of 3,134 pieces of unmodified lithic debitage was recovered. The majority $(n=1,916$, $61 \%)$ are of fine-grained chert. Coarse-grained Potter chert $(n=333,11 \%)$, fine-grained Potter chert $(\mathrm{n}=320,10 \%)$, and coarse-grained quartzite $(\mathrm{n}=$ $319,10 \%$ ) occur in smaller but nearly equal quantities. Similarly, unidentified material types $(\mathrm{n}=$ $45)$, fine-grained quartzite $(n=37)$, Tecovas jasper $(\mathrm{n}=36)$, silicified caliche $(\mathrm{n}=34)$, sandstone $(\mathrm{n}=$ 33 ), and opalized caliche $(n=30)$ occur in even lower but equal percentages (1\% each). Materials such as coarse-grained chert $(n=5)$, obsidian $(n=2)$, and Alibates agate $(n=1)$ are infrequent and together comprise less than $0.1 \%$ of the collection. The two obsidian specimens have been sourced to the Malad, Idaho, area (Thomas R. Hester, personal communication 1993). The majority $(n=1,729,90 \%)$ of the fine-grained chert debitage is of local origin. Less than $10 \%(n=161,8 \%)$ consists of nonlocal-origin specimens, and a small fraction $(n=26,1 \%)$ of the collection could not be identified in terms of origin. 
Table 13 shows the breakdown of debitage attributes within the fine- and coarse-grained cherts, quartzites, and Potter cherts.

Chips represent the largest percentage among the fine- and coarse-grained cherts and quartzites and the fine-grained Potter chert. Complete flakes are slightly more common than chips in the coarsegrained Potter chert. With the exception of the coarse-grained quartzite and the coarse-grained Potter chert, complete flakes are the second most common category. Compared to the other flake completeness categories, chunks are infrequent in all raw material types. They are most common among the fine- and coarse-grained quartzites.

Entirely decorticate complete flakes and flake fragments are the most common category in all of the raw materials. The highest percentages of entirely decorticate debitage occur in the coarse-grained quartzite, fine-grained Potter chert, and fine-grained chert. Somewhat surprisingly, the coarse-grained Potter chert and fine-grained chert debitage contain nearly identical percentages of entirely decorticate specimens. These similarities suggest that, as a group, artifacts made of fine-grained chert were not reduced more substantially than artifacts made of coarse-grained Potter chert. Entirely corticate debitage is relatively infrequent in all raw materials, with the highest percentages occurring in the coarsegrained quartzite specimens. Among the fine-grained chert and the coarse-grained quartzite, the materials with the two largest numbers of chunks, the majority of the specimens are corticate.

In general, entirely decorticate striking platforms are more common than corticate platforms in the platform-bearing flakes (i.e., complete flakes) and flake fragments (i.e., proximal fragments). The highest percentages $(81 \%)$ of decorticate platforms occur in the fine-grained chert platform-bearing debitage and the lowest in the coarse-grained quartzite.

Platform grinding is found on slightly more than one-third of the platform-bearing debitage. The highest percentages occur in the fine-grained chert and quartzite (17\%), followed by the fine- and coarse grained Potter cherts $(14 \%)$. The lowest percentage of ground platforms occurs in the coarse-grained quartzite debitage.

Among all of the raw material types, the majority of the platform-bearing complete flakes and flake fragments are single faceted. The highest percentages of single-faceted specimens occur in the fine- and coarse-grained quartzites. The two grades of Potter chert contain the two lowest percentages of single-faceted platform-bearing specimens. Correspondingly, the fine- and coarse-grained Potter cherts and the fine-grained chert and quartzite contain the highest percentages of platform-bearing debitage with more than two platform facets.

In each raw material type, the largest percentage of debitage falls in the 11-20-mm size category. Debitage greater than $40 \mathrm{~mm}$ in maximum dimension is most frequent in the coarse-grained Potter chert and quartzite and the fine-grained Potter chert.

Unfortunately, the majority of the complete flakes and flake fragments could not be categorized into flake type (reduction technique) category; some interesting patterns are evident nonetheless. For instance, the largest percentages of flakes derived from bifacial and unifacial reduction strategies occur in the fine-grained chert debitage. On the other hand, debitage derived from bifacial reduction is infrequent in the fine- and coarse-grained Potter cherts, and specimens derived from unifacial reduction represent insignificant portions of the collections of these materials. Among both grades of Potter chert and the coarse-grained quartzite, specimens grouped into the other flake type category represent the largest percentages. The other flake type category includes debitage derived from cobble tool rejuvenation and ground stone tool shaping.

The breakdowns of lithic debitage attributes for raw materials other than chert, quartzite, and Potter chert are shown in Table 14. As in the case of the previously discussed raw material groups, chips also tend to form the largest category among the sandstone, opalized caliche, silicified caliche, silicified wood, and unidentified materials debitage. Complete flakes constitute equal or slightly higher percentages than chips among the combined Tecovas jasper/ Alibates agate and silicified caliche debitage, respectively. The highest percentages of chunks occur in the silicified wood and combined obsidian/ unidentified raw material debitage. These percentages are similar to those noted for the fine-grained quartzite specimens.

High percentages of entirely decorticate debitage are found in the combined obsidian/unidentified materials, the silicified caliche, and the combined Tecovas jasper/Alibates agate. The percentages of entirely decorticate debitage are higher in these raw material categories than in any of the other raw material categories mentioned. The lowest percent- 
TABLE 13

BREAKDOWN OF DEBITAGE ATTRIBUTES WITHIN THE FINE- AND COARSE-GRAINED CHERT, QUARTZITE, AND POTTER CHERT RAW MATERIALS, 41GR291

\begin{tabular}{|c|c|c|c|c|c|c|c|c|c|c|c|c|c|}
\hline \multirow[b]{3}{*}{ Attribute } & \multicolumn{12}{|c|}{$\begin{array}{l}\text { BREAKDOWN OF DEBITAGE ATTRIBUTES WITHIN THE FINE- AND COARSE-GRAINED } \\
\text { CHERT, QUARTZITE, AND POTTER CHERT RAW MATERIALS, } 41 \text { GR2 } 21\end{array}$} & \multirow[b]{3}{*}{ Totals } \\
\hline & \multicolumn{2}{|c|}{$\begin{array}{l}\text { Fine-grained } \\
\text { Chert }\end{array}$} & \multicolumn{2}{|c|}{$\begin{array}{l}\text { Coarse-grained } \\
\text { Chert }\end{array}$} & \multicolumn{2}{|c|}{$\begin{array}{l}\text { Fine-grained } \\
\text { Quartzite }\end{array}$} & \multicolumn{2}{|c|}{$\begin{array}{l}\text { Coarse-grained } \\
\text { Quartzite }\end{array}$} & \multicolumn{2}{|c|}{$\begin{array}{l}\text { Fine-grained } \\
\text { Potter Chert }\end{array}$} & \multicolumn{2}{|c|}{$\begin{array}{l}\text { Coarse-grained } \\
\text { Potter Chert }\end{array}$} & \\
\hline & $\#$ & $\%$ & $\#$ & $\%$ & $\#$ & $\%$ & $\#$ & $\%$ & $\#$ & $\%$ & $\#$ & $\%$ & \\
\hline \begin{tabular}{l} 
Flake Completeness \\
Complete \\
Proximal \\
Chips \\
Chunks \\
\multicolumn{1}{c}{ Totals: }
\end{tabular} & $\begin{array}{r}717 \\
308 \\
814 \\
\frac{77}{1916}\end{array}$ & $\begin{array}{r}37 \\
16 \\
42 \\
4 \\
99\end{array}$ & $\begin{array}{r}1 \\
1 \\
3 \\
\overline{-}\end{array}$ & $\begin{array}{r}20 \\
20 \\
60 \\
\overline{-} \\
100\end{array}$ & $\begin{array}{r}12 \\
6 \\
15 \\
\frac{4}{37} \\
\end{array}$ & $\begin{array}{r}32 \\
16 \\
41 \\
11 \\
100\end{array}$ & $\begin{array}{r}61 \\
70 \\
167 \\
21 \\
319\end{array}$ & $\begin{array}{r}19 \\
22 \\
52 \\
7 \\
100\end{array}$ & $\begin{array}{r}86 \\
74 \\
154 \\
\quad 6 \\
320\end{array}$ & $\begin{array}{r}27 \\
23 \\
48 \\
2 \\
100\end{array}$ & $\begin{array}{r}132 \\
67 \\
129 \\
\frac{5}{333}\end{array}$ & $\begin{array}{r}40 \\
20 \\
39 \\
2 \\
101\end{array}$ & $\begin{array}{r}1009 \\
526 \\
1282 \\
1113 \\
2930\end{array}$ \\
\hline $\begin{array}{c}\text { Cortex Percent } \\
0 \\
1-50 \\
51-99 \\
100 \\
\text { Totals: } \\
\end{array}$ & $\begin{array}{r}1009 \\
447 \\
253 \\
130 \\
1839 \\
\end{array}$ & $\begin{array}{r}55 \\
24 \\
14 \\
7 \\
100 \\
\end{array}$ & $\begin{array}{l}3 \\
- \\
- \\
\frac{2}{5}\end{array}$ & $\begin{array}{r}60 \\
- \\
- \\
40 \\
100\end{array}$ & $\begin{array}{r}20 \\
4 \\
8 \\
1 \\
33 \\
\end{array}$ & $\begin{array}{r}61 \\
12 \\
24 \\
3 \\
100 \\
\end{array}$ & $\begin{array}{r}191 \\
49 \\
28 \\
30 \\
298 \\
\end{array}$ & $\begin{array}{r}64 \\
16 \\
9 \\
10 \\
99 \\
\end{array}$ & $\begin{array}{r}195 \\
54 \\
46 \\
19 \\
314 \\
\end{array}$ & $\begin{array}{r}62 \\
17 \\
15 \\
6 \\
100 \\
\end{array}$ & $\begin{array}{r}176 \\
72 \\
55 \\
25 \\
328\end{array}$ & $\begin{array}{r}54 \\
22 \\
17 \\
8 \\
101\end{array}$ & $\begin{array}{r}1594 \\
626 \\
390 \\
207 \\
2817\end{array}$ \\
\hline $\begin{array}{l}\text { Chunk Cortex } \\
\text { Absent } \\
\text { Present } \\
\text { Totals: } \\
\end{array}$ & $\begin{array}{r}26 \\
51 \\
77 \\
\end{array}$ & $\begin{array}{r}34 \\
66 \\
100 \\
\end{array}$ & $\begin{array}{l}- \\
\overline{0}\end{array}$ & $\begin{array}{l}- \\
\overline{0}\end{array}$ & $\begin{array}{r}2 \\
2 \\
4 \\
\end{array}$ & $\begin{array}{r}50 \\
50 \\
100 \\
\end{array}$ & $\begin{array}{r}9 \\
12 \\
21 \\
\end{array}$ & $\begin{array}{r}43 \\
57 \\
100 \\
\end{array}$ & $\begin{array}{r}2 \\
\frac{4}{6} \\
\end{array}$ & $\begin{array}{r}33 \\
67 \\
100 \\
\end{array}$ & $\begin{array}{r}3 \\
2 \\
5 \\
\end{array}$ & $\begin{array}{r}60 \\
40 \\
100 \\
\end{array}$ & $\begin{array}{r}42 \\
71 \\
113 \\
\end{array}$ \\
\hline $\begin{array}{l}\text { Platform Cortex } \\
\text { Absent } \\
\text { Present } \\
\text { Totals: } \\
\end{array}$ & $\begin{array}{r}830 \\
195 \\
1025 \\
\end{array}$ & $\begin{array}{r}81 \\
19 \\
100 \\
\end{array}$ & $\begin{array}{l}1 \\
\frac{1}{2} \\
\end{array}$ & $\begin{array}{r}50 \\
\frac{50}{100} \\
\end{array}$ & $\begin{array}{r}12 \\
\frac{6}{18} \\
\end{array}$ & $\begin{array}{r}67 \\
33 \\
100 \\
\end{array}$ & $\begin{array}{r}79 \\
52 \\
131 \\
\end{array}$ & $\begin{array}{r}60 \\
40 \\
100 \\
\end{array}$ & $\begin{array}{r}121 \\
39 \\
160 \\
\end{array}$ & $\begin{array}{r}76 \\
24 \\
100 \\
\end{array}$ & $\begin{array}{r}134 \\
65 \\
199\end{array}$ & $\begin{array}{r}67 \\
33 \\
100\end{array}$ & $\begin{array}{r}1177 \\
\quad 358 \\
1535\end{array}$ \\
\hline $\begin{array}{l}\text { Platform Grinding } \\
\text { Absent } \\
\text { Present } \\
\text { Totals: }\end{array}$ & $\begin{array}{r}855 \\
170 \\
1025\end{array}$ & $\begin{array}{r}83 \\
17 \\
100\end{array}$ & $\begin{array}{l}1 \\
\frac{1}{2}\end{array}$ & $\begin{array}{r}50 \\
50 \\
100\end{array}$ & $\begin{array}{r}15 \\
\frac{3}{18}\end{array}$ & $\begin{array}{r}83 \\
17 \\
100\end{array}$ & $\begin{array}{r}125 \\
\frac{6}{131}\end{array}$ & $\begin{array}{r}95 \\
\frac{5}{100}\end{array}$ & $\begin{array}{r}138 \\
22 \\
160\end{array}$ & $\begin{array}{r}86 \\
14 \\
100\end{array}$ & $\begin{array}{r}171 \\
\underline{28} \\
199\end{array}$ & $\begin{array}{r}86 \\
14 \\
100\end{array}$ & $\begin{array}{r}1305 \\
230 \\
1535\end{array}$ \\
\hline
\end{tabular}




\begin{tabular}{|c|c|c|c|c|c|c|c|c|c|c|c|c|c|}
\hline Table 13, continued & & & & & & & & & & & & & \\
\hline \multirow[b]{2}{*}{ Attribute } & \multicolumn{2}{|c|}{$\begin{array}{l}\text { Fine-grained } \\
\text { Chert }\end{array}$} & \multicolumn{2}{|c|}{$\begin{array}{l}\text { Coarse-grained } \\
\text { Chert }\end{array}$} & \multicolumn{2}{|c|}{$\begin{array}{l}\text { Fine-grained } \\
\text { Quartzite }\end{array}$} & \multicolumn{2}{|c|}{$\begin{array}{l}\text { Coarse-grained } \\
\text { Quartzite }\end{array}$} & \multicolumn{2}{|c|}{$\begin{array}{l}\text { Fine-grained } \\
\text { Potter Chert }\end{array}$} & \multicolumn{2}{|c|}{$\begin{array}{l}\text { Coarse-grained } \\
\text { Potter Chert }\end{array}$} & \multirow[b]{2}{*}{ Total } \\
\hline & $\#$ & $\%$ & $\#$ & $\%$ & $\#$ & $\%$ & $\#$ & $\%$ & $\#$ & $\%$ & $\#$ & $\%$ & \\
\hline $\begin{array}{l}\text { Platform Facet Count } \\
1 \\
2 \\
>2 \\
\text { Totals: }\end{array}$ & $\begin{array}{r}728 \\
124 \\
173 \\
1025\end{array}$ & $\begin{array}{r}71 \\
12 \\
17 \\
100 \\
\end{array}$ & $\begin{array}{l}1 \\
1 \\
\overline{2}\end{array}$ & $\begin{array}{r}50 \\
50 \\
- \\
100\end{array}$ & $\begin{array}{r}14 \\
1 \\
3 \\
18 \\
\end{array}$ & $\begin{array}{r}78 \\
6 \\
17 \\
101\end{array}$ & $\begin{array}{r}108 \\
12 \\
11 \\
131 \\
\end{array}$ & $\begin{array}{r}82 \\
9 \\
8 \\
100 \\
\end{array}$ & $\begin{array}{r}106 \\
26 \\
28 \\
160\end{array}$ & $\begin{array}{r}66 \\
16 \\
18 \\
100\end{array}$ & $\begin{array}{r}129 \\
33 \\
37 \\
199\end{array}$ & $\begin{array}{r}65 \\
17 \\
19 \\
101\end{array}$ & $\begin{array}{r}1086 \\
197 \\
252 \\
1535 \\
\end{array}$ \\
\hline $\begin{array}{l}\text { Maximum Dimensions } \\
1-10 \mathrm{~mm} \\
11-20 \mathrm{~mm} \\
21-30 \mathrm{~mm} \\
31-40 \mathrm{~mm} \\
>40 \mathrm{~mm} \\
\text { Totals: }\end{array}$ & $\begin{array}{r}63 \\
1406 \\
362 \\
73 \\
12 \\
1916 \\
\end{array}$ & $\begin{array}{r}3 \\
73 \\
19 \\
4 \\
1 \\
100 \\
\end{array}$ & $\begin{array}{l}- \\
3 \\
1 \\
1 \\
\overline{5}\end{array}$ & $\begin{array}{r}- \\
60 \\
20 \\
20 \\
-\overline{100} \\
\end{array}$ & $\begin{array}{r}1 \\
20 \\
11 \\
3 \\
2 \\
37 \\
\end{array}$ & $\begin{array}{r}3 \\
54 \\
30 \\
8 \\
5 \\
100 \\
\end{array}$ & $\begin{array}{r}8 \\
181 \\
70 \\
32 \\
28 \\
319 \\
\end{array}$ & $\begin{array}{r}3 \\
57 \\
22 \\
-10 \\
\frac{9}{101} \\
\end{array}$ & $\begin{array}{r}8 \\
190 \\
64 \\
37 \\
21 \\
320 \\
\end{array}$ & $\begin{array}{r}3 \\
59 \\
20 \\
12 \\
7 \\
101 \\
\end{array}$ & $\begin{array}{r}3 \\
149 \\
75 \\
50 \\
56 \\
333 \\
\end{array}$ & $\begin{array}{r}1 \\
45 \\
23 \\
15 \\
17 \\
101\end{array}$ & $\begin{array}{r}83 \\
1949 \\
583 \\
196 \\
119 \\
2930 \\
\end{array}$ \\
\hline $\begin{array}{l}\text { Flake Type } \\
\text { Biface } \\
\text { Uniface } \\
\text { Core Preparation } \\
\text { Other } \\
\text { Indeterminate } \\
\quad \text { Totals: }\end{array}$ & $\begin{array}{r}325 \\
231 \\
3 \\
6 \\
1274 \\
1839\end{array}$ & $\begin{array}{l}18 \\
13 \\
<1 \\
<1 \\
\frac{69}{100}\end{array}$ & $\begin{array}{l}- \\
- \\
- \\
- \\
\frac{5}{5}\end{array}$ & $\begin{array}{r}- \\
- \\
- \\
- \\
\frac{100}{100}\end{array}$ & $\begin{array}{r}2 \\
- \\
\overline{1} \\
\frac{30}{33}\end{array}$ & $\begin{array}{r}6 \\
- \\
- \\
3 \\
91 \\
100\end{array}$ & $\begin{array}{r}6 \\
3 \\
- \\
18 \\
271 \\
298\end{array}$ & $\begin{array}{r}2 \\
1 \\
- \\
6 \\
91 \\
100\end{array}$ & $\begin{array}{r}23 \\
6 \\
- \\
24 \\
\underline{261} \\
314\end{array}$ & $\begin{array}{r}7 \\
2 \\
\overline{8} \\
83 \\
100\end{array}$ & $\begin{array}{r}21 \\
1 \\
1 \\
43 \\
\underline{262} \\
328\end{array}$ & $\begin{array}{r}6 \\
<1 \\
<1 \\
13 \\
80 \\
100\end{array}$ & $\begin{array}{r}377 \\
241 \\
4 \\
92 \\
2103 \\
2817\end{array}$ \\
\hline
\end{tabular}




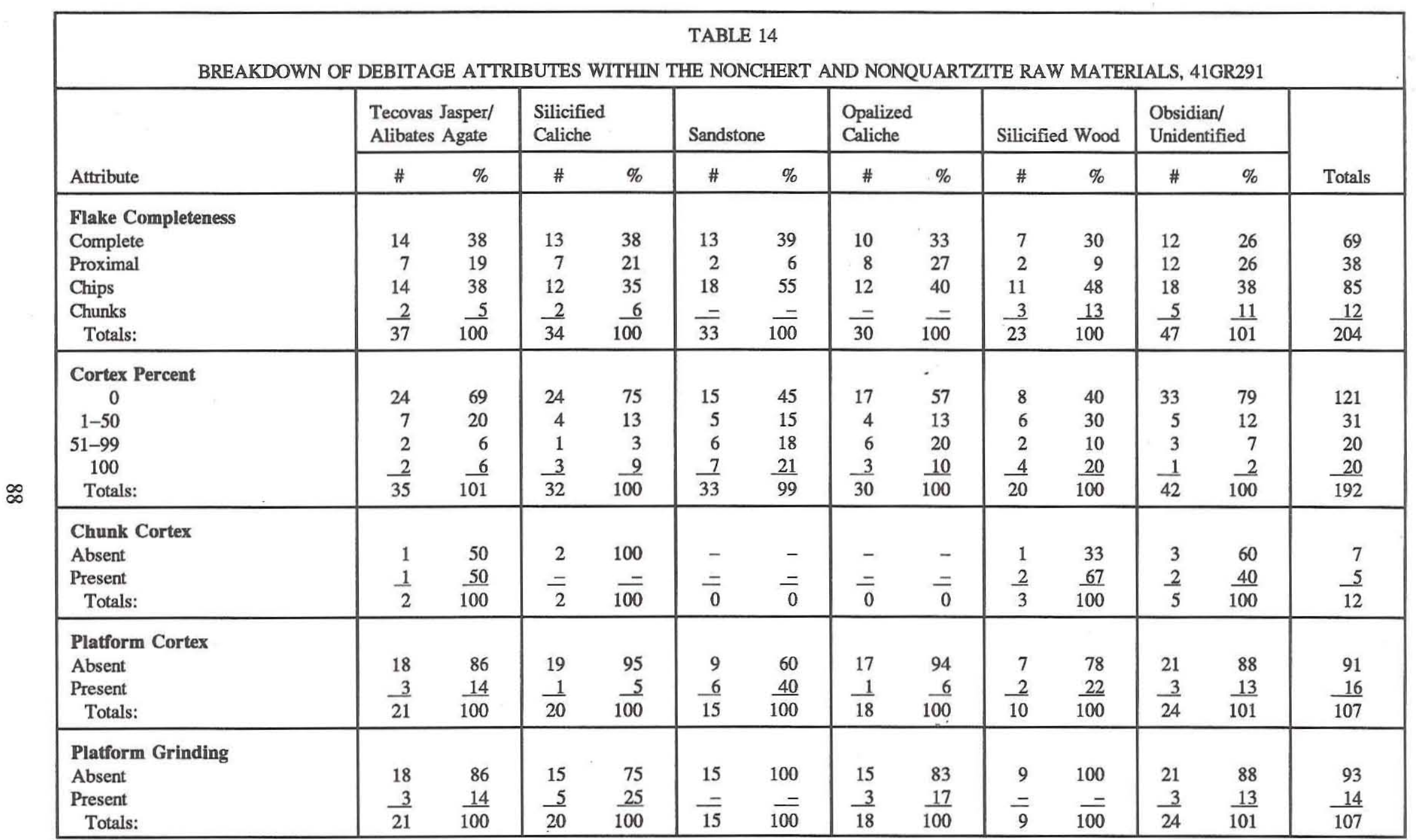




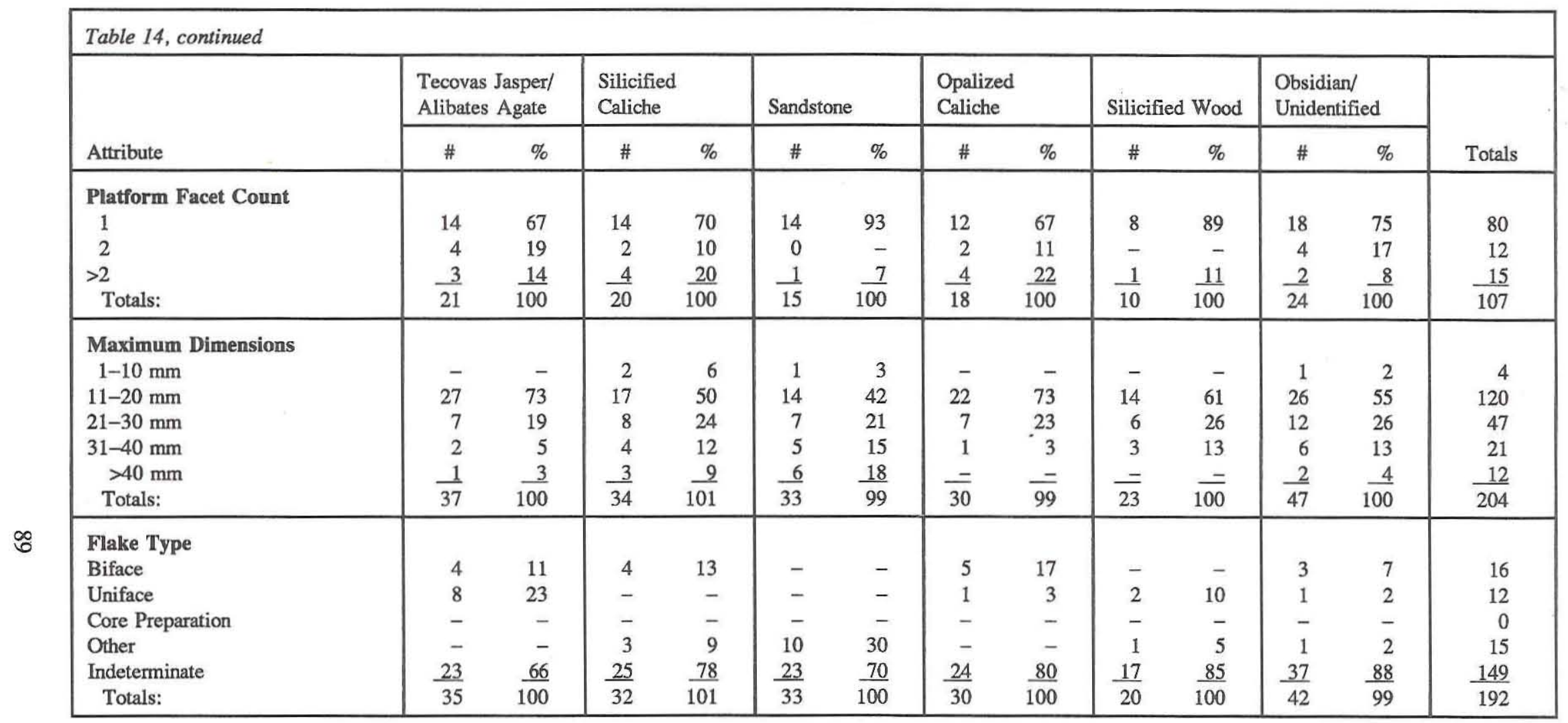


age of decorticate debitage occurs among the silicified wood specimens. The small numbers of chunks found in these less common materials range from entirely decorticate (i.e., silicified caliche) to mainly corticate (i.e., silicified wood) specimens.

The large majority of the platform-bearing debitage have decorticate striking platforms. The highest percentages of decorticate striking platforms occur in the silicified caliche and opalized caliche debitage. All but two (i.e., sandstone and silicified wood) of these less common raw materials have higher percentages of complete flakes and proximal fragments with decorticate striking platforms than the fine-grained chert, one of the more common raw materials with the highest percentage of decorticate striking platforms. The lowest percentage of decorticate flakes occurs in the sandstone debitage.

Among these raw material types, the highest percentage of ground striking platforms occurs in the silicified caliche debitage. This percentage is higher than in any of the raw materials from the previously discussed group. None of the sandstone and silicified wood platform-bearing debitage have ground striking platforms.

Debitage with single-faceted striking platforms is relatively common among the sandstone and silicified wood specimens. Both percentages are higher than those for the cherts, quartzites, and Potter cherts. The largest percentages of striking platforms with three or more platform facets occur in the opalized caliche and silicified caliche debitage. These percentages are also higher than those for the previously discussed group of raw materials.

The $11-20-\mathrm{mm}$ size category is the largest among all raw material types. However, while nearly three-fourths of the combined Tecovas jasper/Alibates agate and opalized caliche debitage fall in the 11-20-mm size category, less than half of the sandstone debitage is this small. Rather, the debitage of sandstone, combined obsidian/unidentified materials, silicified caliche, and silicified wood contain moderate percentages of debitage measuring $30 \mathrm{~mm}$ or larger in maximum dimension. These latter percentages are similar to those observed for the fine- and coarse-grained Potter chert and coarsegrained quartzite.

The majority of the debitage could not be classified in terms of reduction strategy, but of those specimens that could be identified, the highest percentages produced through bifacial reduction are in the opalized caliche, silicified caliche, and the combined Tecovas jasper/Alibates agate. Moderate percentages of debitage derived from unifacial reduction are found in the Tecovas jasper/Alibates agate debitage, and smaller percentages also occur among the silicified wood specimens. The largest percentage of debitage in the other flake type category occurs in the sandstone debitage. Since all of the metates and most of the manos recovered from the site are of sandstone, this inflated percentage most likely represents ground stone tool manufacture (i.e., shaping).

\section{Ground and Battered Stone Artifacts}

A total of 122 ground and battered stone artifacts was recovered. They are divided into six categories: manos, metates, pestles, anvils, indeterminate-use ground stones, and hammerstones. Of these, 22 ground stones and 1 hammerstone are from isolated units; the remaining ground and battered stones are from the five excavation blocks. Manos $(n=35)$, metates $(n=65)$, and indeterminate-use ground stone specimens $(n=11)$ constitute the largest categories. The remaining specimens consist of six pestles and an anvil. The only battered stone artifacts are one fragmentary and three complete hammerstones.

\section{Manos}

Fifteen $(43 \%)$ of the 35 manos are complete (Figure 32). Twenty (57\%) specimens exhibit bifacial use wear; the others $(n=15,43 \%)$ have unifacial wear patterns. Pecking is unifacial on 6 (17\%) specimens, bifacial on $12(34 \%)$, absent on 8 $(23 \%)$, and indeterminate on $9(26 \%)$. Twelve $(60 \%)$ of the 20 manos with bifacial use wear also exhibit bifacial pecking. Two (10\%) of the 20 have unifacial pecking, pecking is entirely absent on $3(15 \%)$, and its presence could not be determined with certainty on $3(15 \%)$ others. Only $4(27 \%)$ of the 15 manos with unifacial wear have pecking. Pecking is absent on five $(33 \%)$ others, and its presence could not be determined with certainty on six $(40 \%)$ specimens. Battering is absent on $14(41 \%)$ manos and indeterminate on $9(26.5 \%)$ specimens. Of the remaining 12 manos, 8 (21\%) exhibit slight battering, $3(9 \%)$ have moderate battering, and $1(3 \%)$ has extensive battering. Seventeen (49\%) of the manos are biconvex in transverse cross section, while 8 (23\%) are lenticular. Of the remaining $10,3(9 \%)$ are planoconvex, $1(3 \%)$ has two beveled surfaces that 

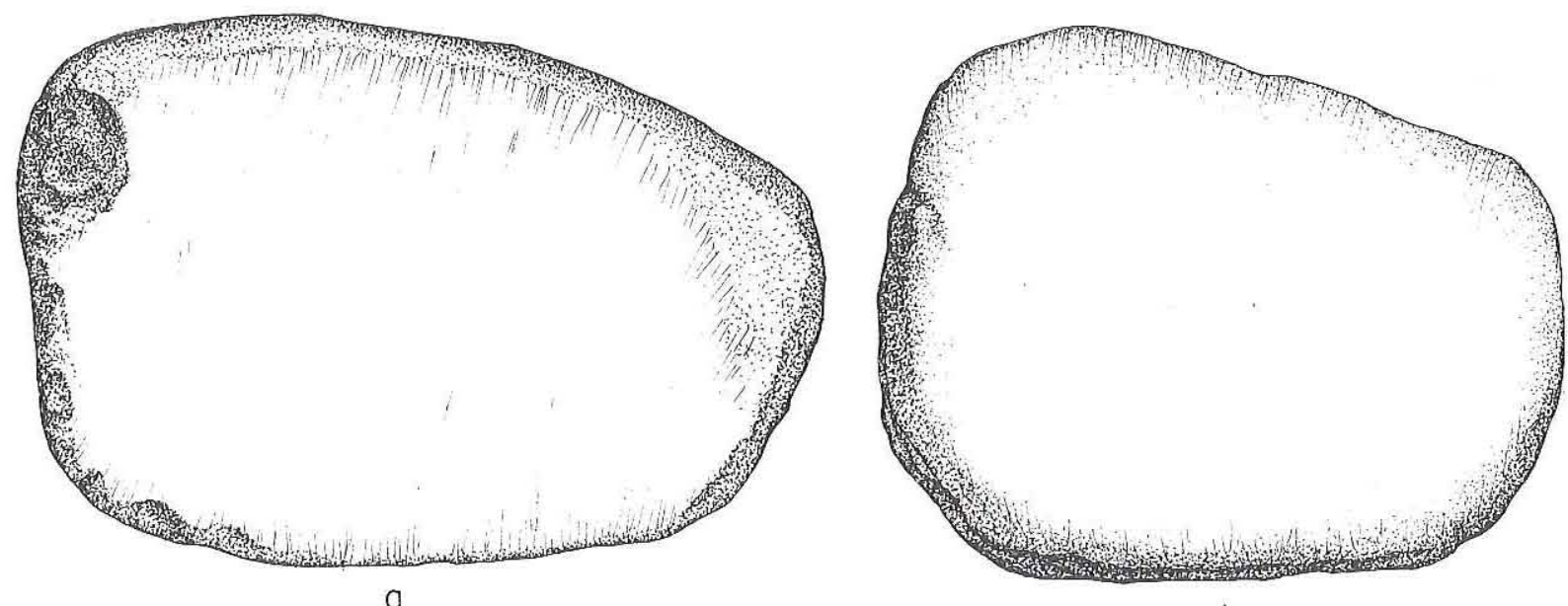

a

b

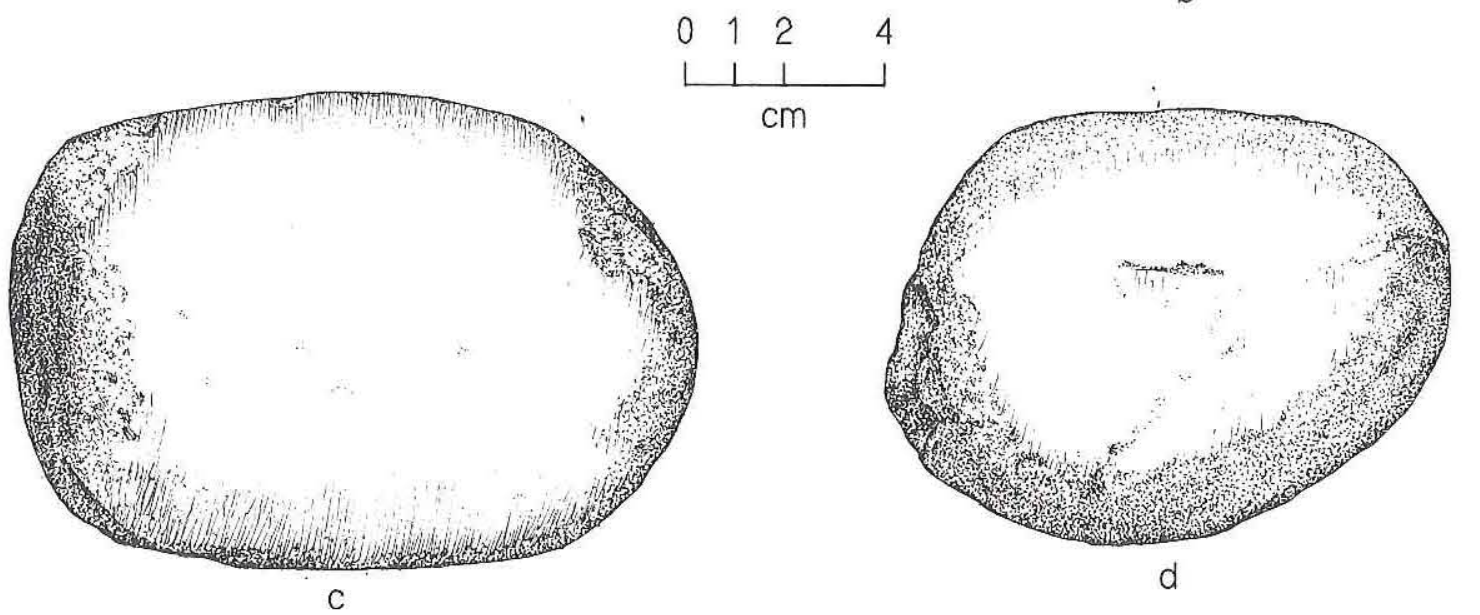

Figure 32. Manos, 41GR291. ( $a-b)$ Sandstone manos; $(c-d)$ quartzite manos.

form a medial ridge on its working face, and cross section shape could not be determined on $6(17 \%)$ small fragments. The shape of $24(69 \%)$ manos could be determined, including 9 relatively large fragments. The majority $(\mathrm{n}=22,63 \%)$ appear to have been oval in shape, while $2(6 \%)$ are rounded and flat.

The 15 complete manos have a mean length of $119.8 \mathrm{~mm}(\sigma=33.6)$, a mean width of $95.7 \mathrm{~mm} \mathrm{(} \sigma=$ $26.8)$, and a mean thickness of $47.1 \mathrm{~mm}(\sigma=11.7)$. They have a mean weight of $844.3 \mathrm{~g}(\sigma=489.5)$, ranging from a minimum of $150 \mathrm{~g}$ to a maximum of $2,096 \mathrm{~g}$. Three $(20 \%)$ of the complete manos weigh less than $650 \mathrm{~g}, 10(67 \%)$ weigh between 650 $1,150 \mathrm{~g}$, and $2(13 \%)$ are heavier than $1,150 \mathrm{~g}$. The mean working surface area of the 15 complete manos is $7,894.4 \mathrm{~mm}^{2}(\sigma=3,772.1)$, ranging from a minimum of $3,102 \mathrm{~mm}^{2}$ to a maximum of
$16,740 \mathrm{~mm}^{2}$. Four specimens have working surfaces larger than $11,000 \mathrm{~mm}^{2}$, three have working surfaces smaller than $5,000 \mathrm{~mm}^{2}$, and the remaining eight have grinding surfaces measuring between 5,000 and $10,000 \mathrm{~mm}^{2}$.

\section{Metates}

Only $5(8 \%)$ of the 65 metates are complete (Figures 33, 34, and 35). The majority ( $n=41,63 \%)$ exhibit unifacial use wear; the remainder $(n=24$, $37 \%$ ) have use wear on two faces. Pecking is absent on two (3\%) specimens, and its presence could not be determined with certainty on an additional fragment. Of the 24 metates with bifacial use wear, 19 (79\%) also exhibit bifacial pecking, and the remaining 5 (21\%) are unifacially pecked. Of the 41 metates with unifacial use wear, $38(93 \%)$ are also unifacially 

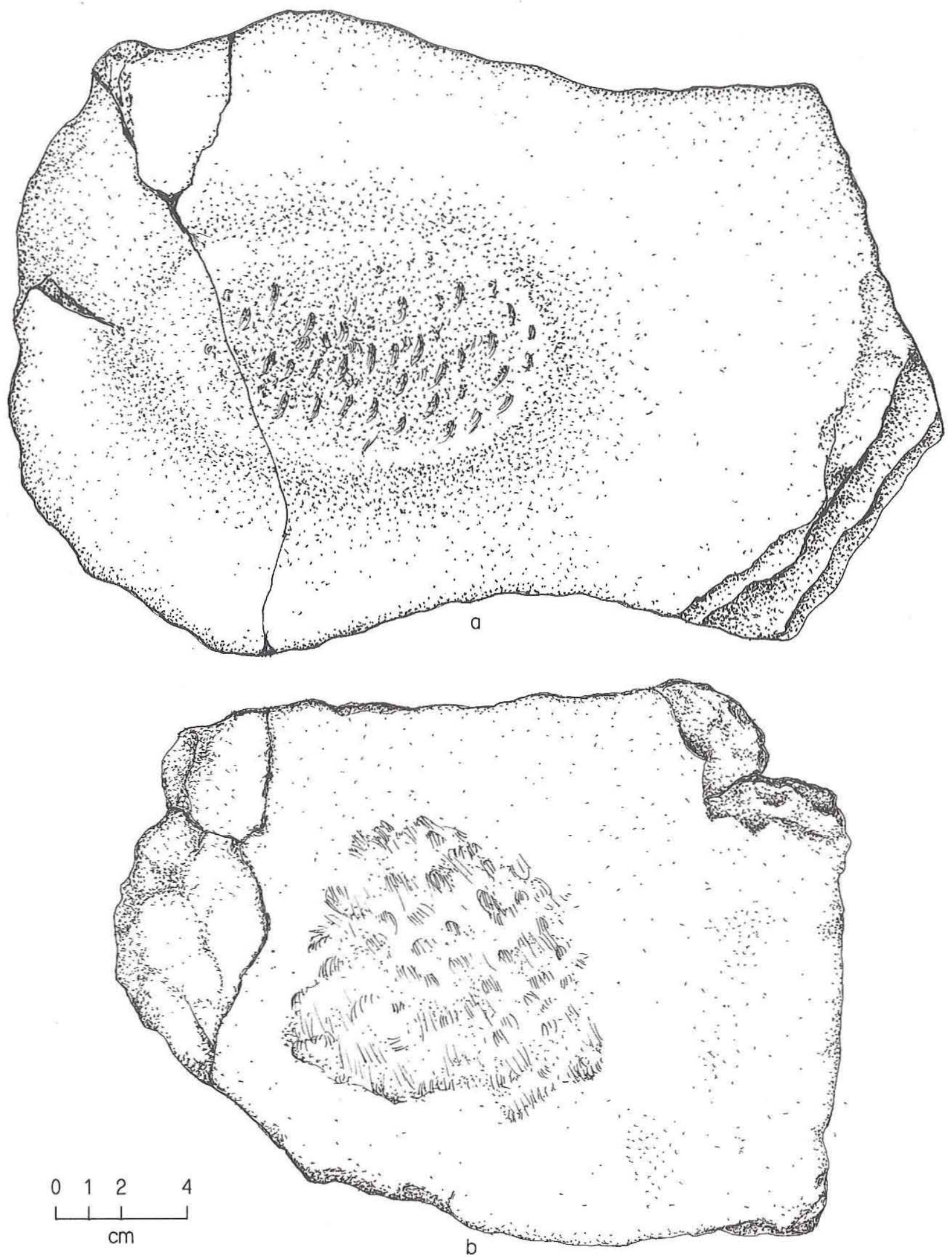

Figure 33. Metates, 41 GR291. 


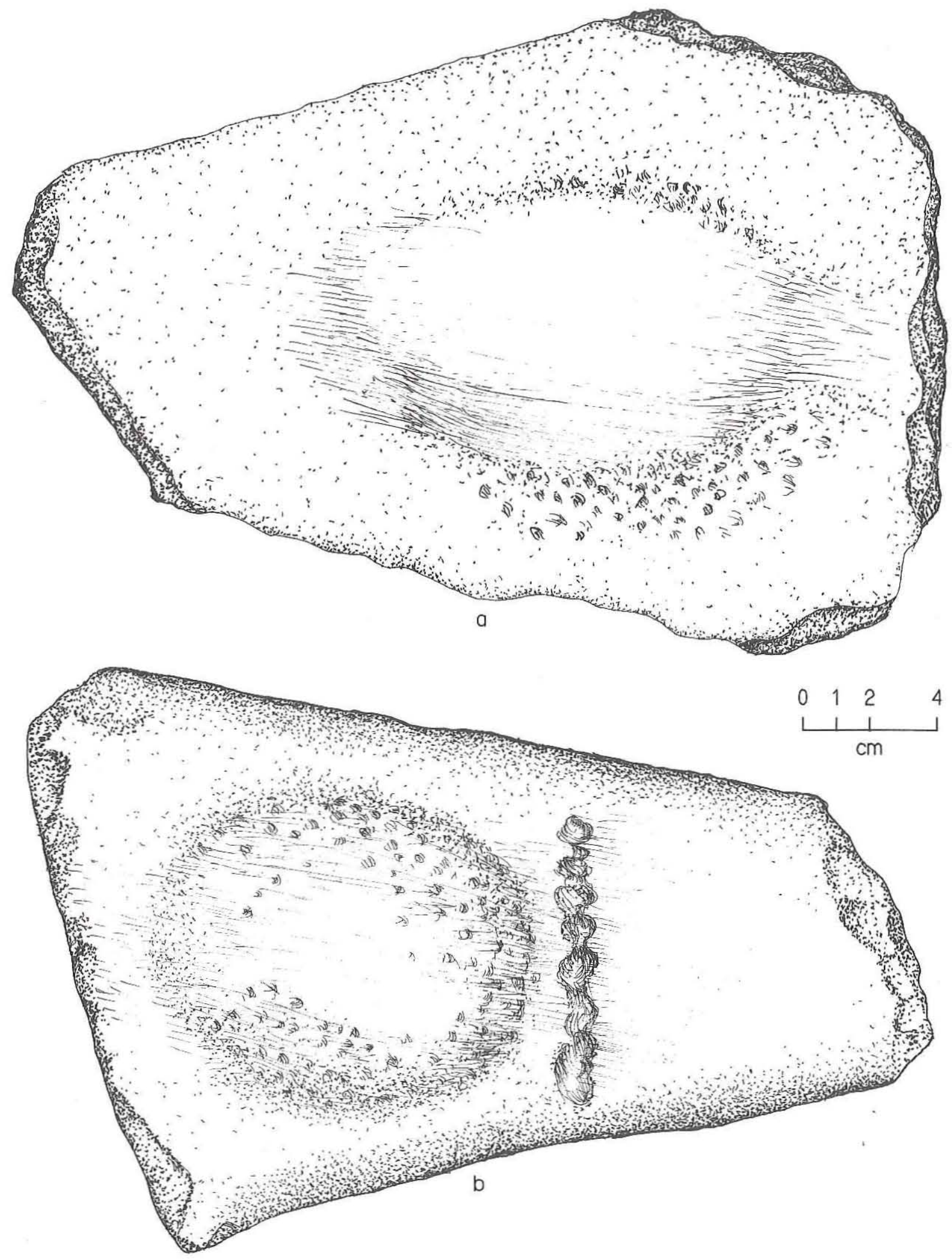

Figure 34. Metates, 41GR291. 
Phase III Data Recovery, Season 3, Lake Alan Henry

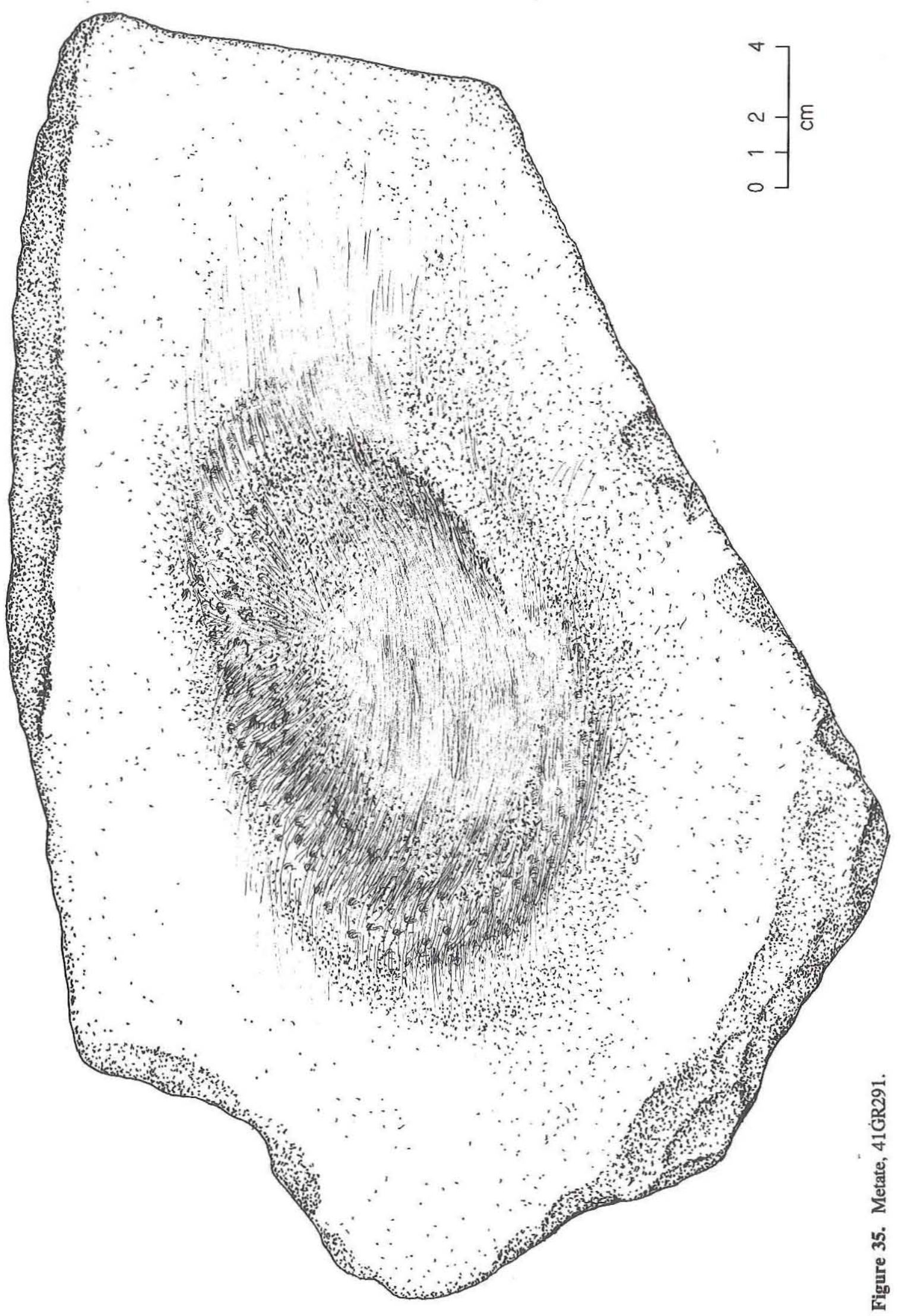


pecked; pecking is absent on $2(5 \%)$ and indeterminate on $1(2 \%)$ fragment. Battering is not present on the faces of any of the metates. The five complete metates are rectangular in shape. Two rather large fragments appear to have an oval shape. The original shape of the other fragmentary specimens cannot be determined. The majority $(\mathrm{n}=30,46 \%)$ have planoconvex transverse cross sections, although specimens with tabular $(\mathrm{n}=18,28 \%)$ and biconcave $(\mathrm{n}=16,25 \%)$ cross sections are also common.

The five complete metates have a mean length of $581.0 \mathrm{~mm}(\sigma=112.4)$ and a mean maximum width of $378.0 \mathrm{~mm}(\sigma=55.7)$. Maximum thickness was measured on 62 metates; their mean thickness is $51.2 \mathrm{~mm}(\sigma=21.9)$. The mean working surface area for the five complete metates is $138,820.0 \mathrm{~mm}^{2}$ ( $\sigma=$ $39,765.2$ ), ranging from a minimum of $90,300 \mathrm{~mm}^{2}$ to a maximum of $185,600 \mathrm{~mm}^{2}$. Notably, all five of the complete metates were recovered from archeological contexts that suggest that they were stored or cached tools. Two are oval basin metates found upside down on top of storage pit Features 18 (see Figure $33 a$ ) and 19 (see Figure 34a), while a slab metate (see Figure $33 b$ ) and two other oval basin metates (see Figures $34 b$ and 35) were recovered from on or near the floor of pithouse Feature 37.

\section{Pestles}

Six fragmentary specimens made of sandstone are included in this functional category (Figure 36a, b). They have elongated oval shapes with blunt points and lenticular transverse cross sections. Their working edges exhibit light $(n=4)$ to moderate $(n=$ 2) battering on their extreme distal portions and some light smoothing of both faces immediately proximate to the working edge proper. Pecking is not evident on any of the specimens. Maximum width $(\overline{\mathrm{x}}=$ $129.5 \mathrm{~mm}, \sigma=2.1$ ) was measured on two specimens. The mean maximum thickness of the six fragments is $34.7 \mathrm{~mm}(\sigma=5.3)$.

\section{Anvil}

A single fragmentary lenticular sandstone specimen is identified as an anvil (Figure 36c). Use wear in the form of light pitting and striations is distributed on one face of the artifact. Pecking is not evident on either face of the specimen. Although it is broken longitudinally, it appears to have had a roughly rectangular shape. The specimen is $410 \mathrm{~mm}$ long and $37 \mathrm{~mm}$ thick.

\section{Indeterminate-use Ground Stones}

Eleven sandstone fragments could not be classified into any of the preceding categories, mainly due to their small size or inconclusive use-wear characteristics. Seven (64\%) were used unifacially; four have bifacial use wear. Pecking is present on single faces of four fragments, it occurs on both faces of three specimens, and it is absent on the remaining four fragments. Three of the seven specimens with unifacial use wear also have unifacial pecking. The other four fragments lack pecking. Three of the four fragments with bifacial wear also exhibit bifacial pecking; the remaining specimen has pecking on a single face. None of the fragments have battering. Seven fragments appear to have been oval. The original shape of the other four incomplete specimens could not be determined. The majority $(\mathrm{n}=7,64 \%)$ of the fragments are lenticular in transverse cross section. Two additional specimens are planoconvex, and one each is biconvex and tabular. The mean maximum thickness of the 11 specimens is $29.5 \mathrm{~mm}$ $(\sigma=10.1)$.

\section{Hammerstones}

Three complete and one fragmentary hammerstone were recovered. All four are of coarse-grained quartzite. Two of the complete specimens are medium-sized ( $56 \times 49 \times 46 \mathrm{~mm}$ and $70 \times 63 \times 37 \mathrm{~mm}$ ) rounded to ovate nodules, weighing 173 and $236 \mathrm{~g}$, respectively. The heavier of the two has moderate battering on both faces of the ends (Figure 37a); the other hammerstone is only lightly battered on one end (Figure 37b). The largest $(89 \times 74 \times 49 \mathrm{~mm})$ and heaviest of the complete hammerstones weighs $437 \mathrm{~g}$. It has moderate battering on one end and is lightly battered on the other (Figure 37c). The incomplete specimen is a longitudinally split ovate pebble. It has moderate battering on its two ends and the remaining lateral margin. The pebble is $68 \mathrm{~mm}$ long.

\section{Ceramics}

Eighteen plainware ceramic sherds were recovered during data recovery. With the 2 sherds recovered during the 1988 testing, the total number of sherds from 41GR291 is 20, and three to five vessels 


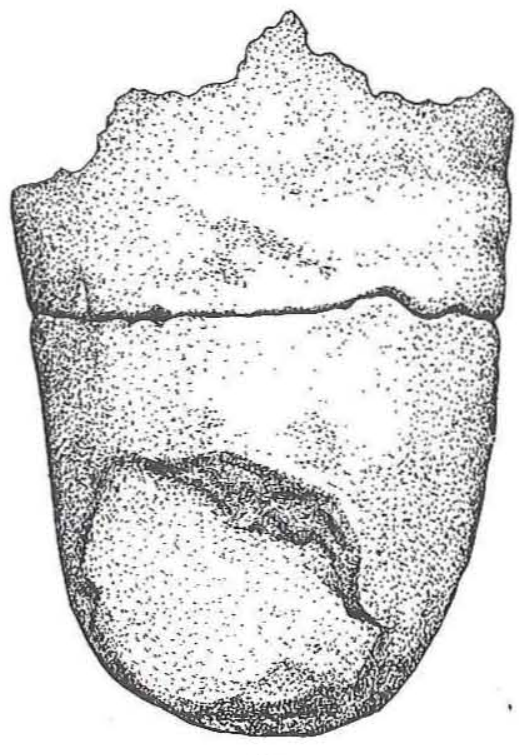

a
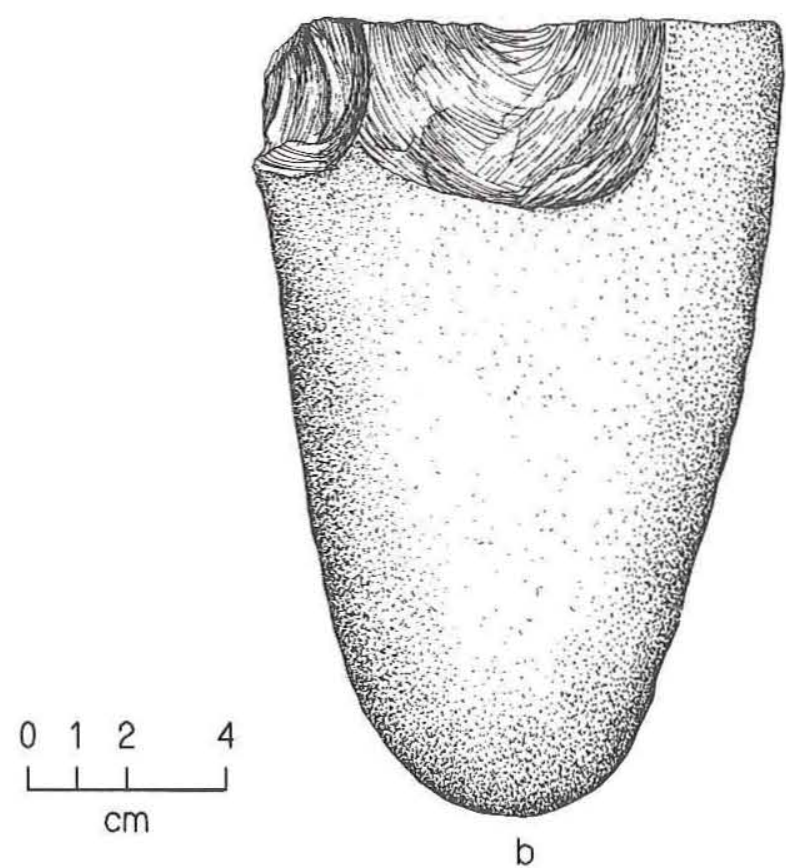

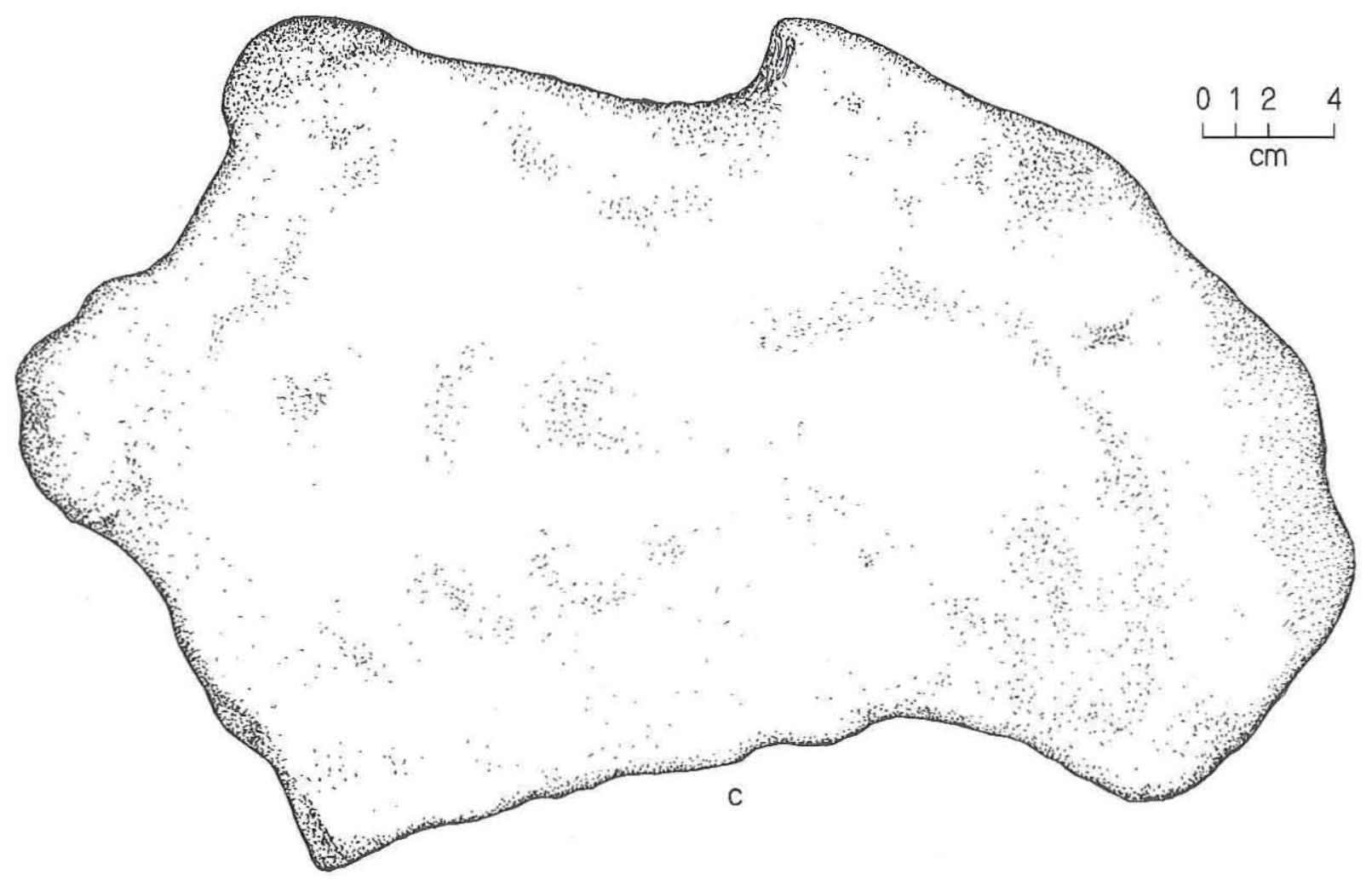

Figure 36. Pestles and anvil, 41GR291. (a-b) Pestle fragments; $(c)$ anvil. 

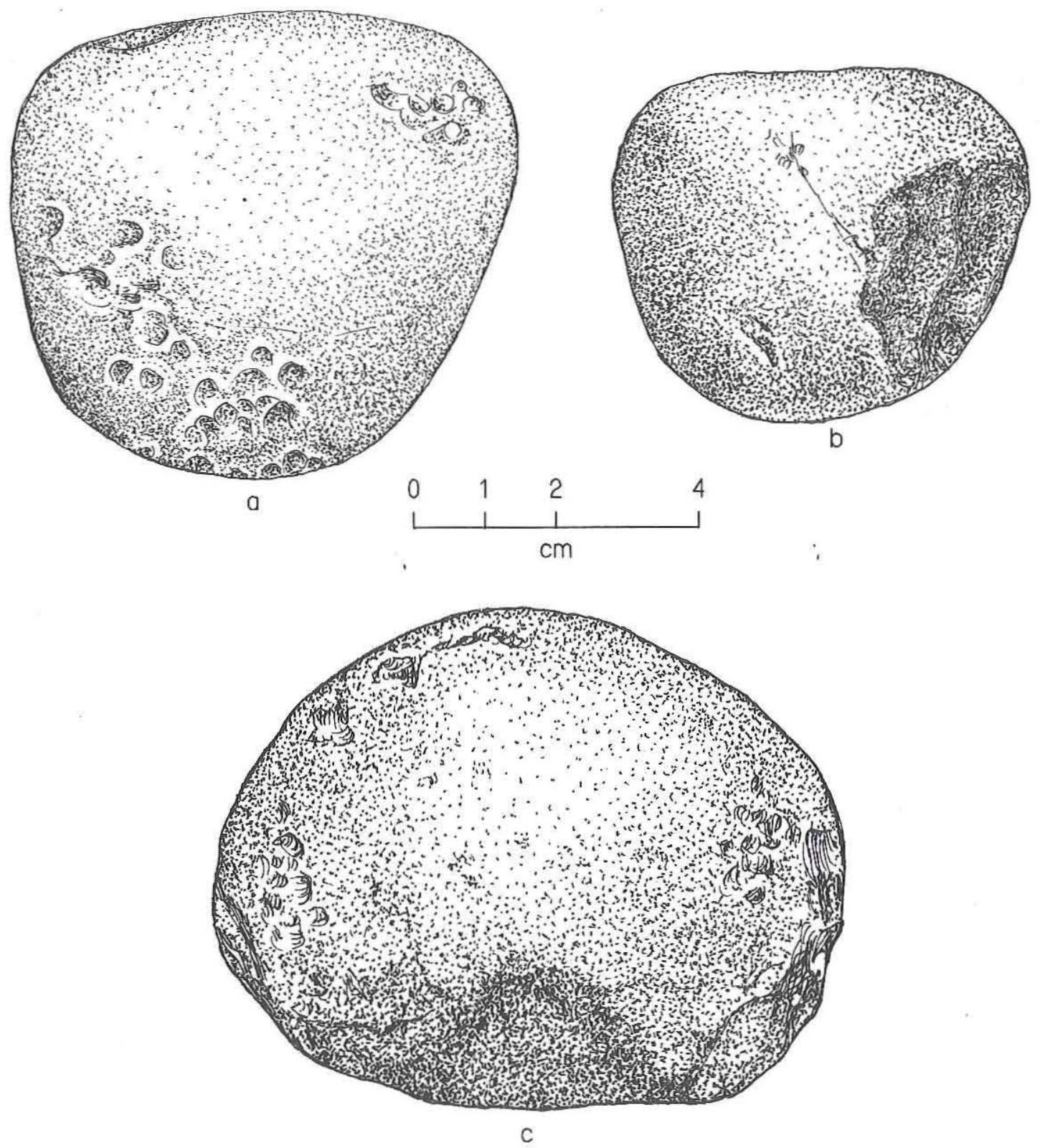

Figure 37. Quartzite hammerstones, 41GR291.

appear to be represented. The sherds are all small, ranging in size from $8 \times 8 \mathrm{~mm}$ to $45 \times 36 \mathrm{~mm}$. All are classified generally as brownwares and were fired under oxidizing conditions, although a few specimens exhibit dark gray (10YR 4/1), very dark gray (7.5YR $3 / 0)$, or black (7.5YR $2 / 0$ ) surfaces and were fired in a reducing atmosphere. It is possible that the latter represent intentional smudging during manufacture, but the darker colors also could be the result of userelated firings. Scratches and cracking on the interior surfaces of these sherds are probably use related and support this interpretation. Surface colors for most of the specimens, however, range from pale brown (10YR $6 / 3$ to $7 / 3$ ) to reddish brown (5YR $5 / 4$ or 10YR 4/4) and include brown (10YR 5/3), dark yellowish brown to yellowish brown (10YR $4 / 4$ to $5 / 4$ ), gray (10YR $4 / 1$ ), and grayish brown (10YR $5 / 2$ ).

No decorations are present on any of the specimens, but many of the sherds appear to have been slipped and polished. This glossy appearance is misleading, however, and is due to postdepositional 
build-up of a thin, white, calcareous deposit. It is difficult to determine the extent of surface smoothing or polishing on most specimens, but it appears that the surface treatments are limited to smoothing or burnishing. The largest sherd is a notable exception and has intact surfaces (lacking the glossy carbonate coating) that are smoothed but not polished. Paste colors are generally darker than are the surface colors and include dark gray to very dark gray (10YR $4 / 1$ to $3 / 1$ or $3 / 0,7.5 \mathrm{YR} 4 / 0$, and $5 \mathrm{YR} 3 / 1$ ), dark grayish brown to very dark grayish brown (10YR $4 / 2$ to $3 / 2$ ), dark brown (7.5YR $4 / 2$ to $3 / 2$ ), and reddish gray (5YR 4/2). Most of the sherds have a consistent paste color, but a few have a central core that is much darker.

All of the specimens are body sherds except for a single specimen identified as a bowl rim (Wiseman 1993). It is a straight rim that tapers from 5 to $3 \mathrm{~mm}$, has a rounded to slightly flattened lip, and has an estimated rim diameter of $20-25 \mathrm{~cm}$. Its rim profile (Figure 38) closely matches the "rounded direct" profile for middle Pecos brownwares illustrated by Jelinek (1967:Figure 10C) and the "pinched-flattened" form described for late brownwares in western Texas by Whalen (1981:217-219). The body sherds range in thickness from $3.5-7.0 \mathrm{~mm}$ but average $5.0 \mathrm{~mm}$. The curvature of the largest body sherd indicates a diameter of at least $25 \mathrm{~cm}$ (which corresponds with the estimated rim diameter above).
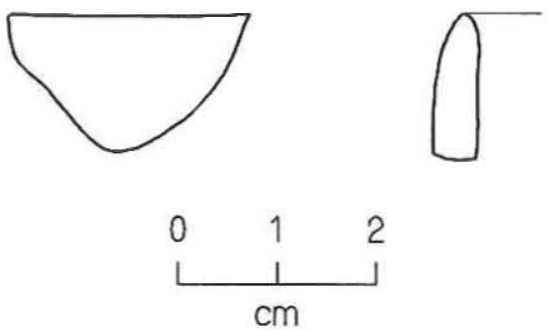

Figure 38. Outline and rim profile of brownware sherd from EU 89, 41 GR291.

Two distinctive paste groups are represented in this ceramic assemblage. These groups are broadly similar in that they are characterized as having angular to subangular grains, ranging in size from medium to coarse sand, that appear to be crushed rock fragments. The larger and more angular inclusions are interpreted as tempering additives and are dominated by granular white or gray feldspar fragments and platy colorless or clear quartz fragments and/or crystals. The paste groups, described in more detail below, originally were defined based on compositional differences observed under low-power magnification but were refined further based on observations made by Wiseman (1993). The paste group definitions are also supported by point count data derived during an independent petrographic study (see Appendix G).

One notable point with regard to the distribution of ceramics is that eight of the Paste Group 1 sherds can be dated tentatively by their feature associations. Four were found in the fill of pithouse Feature 37, dated to ca. A.D. 900-1160, and four were found in and around charcoal/burned rock scatter Feature 12, dated to ca. A.D. 1160-1380. Paste Group 2 sherds were not found in dated feature contexts but were recovered from Levels 2, 3, and 4. Thus, none of the sherds definitely predate A.D. 900 , but some of the Paste Group 2 specimens could be earlier.

\section{Paste Group 1}

A very sandy, poorly sorted paste characterizes the nine body sherds in Paste Group 1. Most of the smaller nonplastics (in the very fine sand to fine sand sizes) are rounded to subrounded; they appear to represent natural inclusions and include a variety of different materials. Colorless and white grains (presumably quartz and feldspars) are most common, but black, brown, and reddish brown grains also are present. The larger inclusions (ranging from medium to coarse sand) are generally subangular or angular, and the dominant materials are white feldspars and clear quartz. Paste Group 1 is interpreted as a sandy clay with crushed rock temper.

The average thickness of these nine sherds is $5.7 \mathrm{~mm}$ (range is $5.0-6.5 \mathrm{~mm}$ ), and the largest sherd suggests a maximum body diameter of at least $25 \mathrm{~cm}$, but only two or possibly three vessels are represented. Whalen (1981:217-219) suggests that early brownwares in western Texas tend to be smaller vessels (average rim diameter $13.2 \mathrm{~cm}$ ) with thicker walls (average $5.6 \mathrm{~mm}$ ). The average thickness would indicate that the Paste Group 1 sherds represent early brownwares, but vessel rim size cannot be evaluated.

Wiseman (1993) identified the Paste Group 1 sherds as Jornada Brown, originally described as "unnamed brownware" or "plain brown ware" by Jennings (1940:5-6), named and defined by Mera (1943:12), and further defined by Jelinek (1967:47- 
49). While Jomada Brown generally is well polished, smoothed or slightly polished examples do occur, and the apparent absence of polish observed on the Group 1 specimens may be due to postdepositional alteration (e.g., weathering). Wiseman (1993) notes that the Group 1 sherds have white feldspar inclusions and are generally redder in surface and paste color than the Group 2 specimens.

\section{Paste Group 2}

This paste group includes one rim and eight body sherds ${ }^{3}$ and is characterized as well sorted with a bimodal grain size distribution. The clay component is very fine with very little fine or very fine sand. The larger (medium and coarse sand sizes) nonplastic inclusions are subangular and angular and are dominantly white feldspars and colorless quartz grains. The paste is laminated parallel to the sherd surfaces (giving them a platy appearance) and appears to be fairly porous. A few fine mica flecks were observed on the surface of one specimen. Paste Group 2 is interpreted as a fine sandy clay with crushed rock temper.

These sherds average $4.3 \mathrm{~mm}$ in thickness (range is $3.5-5.0 \mathrm{~mm}$ ) and represent one or perhaps two vessels. The bowl rim is from a vessel with a maximum diameter of $20-25 \mathrm{~cm}$. These dimensions generally conform to those described by Whalen (1981:217-219) for late brownwares (after ca. A.D. 800), which tend to be larger vessels (average rim diameter of $17 \mathrm{~cm}$ ) with thinner walls (average thickness of $5 \mathrm{~mm}$ ) than are the earlier brownwares. As a cautionary note, the attributes described by Whalen (1981) were derived from El Paso Brown, and although the same characteristics may be generally applicable for other brownwares in the Jomada region, this remains to be demonstrated.

Wiseman (1993) identified the Paste Group 2 sherds as South Pecos Brown as defined by Jelinek (1967:53-54). Relative to Jornada Brown, South Pecos Brown tends to be thinner, and the temper inclusions in South Pecos Brown are larger and more angular but are more sparsely distributed throughout the paste. In addition, two specimens exhibit minute

\footnotetext{
${ }^{3}$ The two sherds from the 1988 testing also belong in this group.
}

radiating cracks (due to shrinkage of the clay) around temper fragments which protrude through the surfaces of the sherds. Wiseman (1993) noted that the Pecos Brown specimens generally have a grayish brown surface and paste color (as opposed to the reddish color of the Jornada Brown sherds) and that the feldspars are gray rather than white. The platy appearance of the paste, radiating surface cracks, and presence of grayish feldspars seem to be characteristic attributes of South Pecos Brown (Regge Wiseman, personal communication 1993).

\section{Modified Hematite}

Seven specimens of modified hematite were recovered. All are of the specular variety of hematite often called "paint ore" (Pough 1960:124) and exhibit some striations from grinding. The earthy luster and relative softness (hardness 1-4) make this a preferred material for producing bright red pigment, and it is likely that all of these specimens were used in this way. Six are small fragments (ranging in size from $16-40 \mathrm{~mm}$ in maximum dimension) from various parts of the site (i.e., EUs 17, 21, 36, 59, and 82; Backhoe Trench 1992-25), but one complete specimen (Figure 39) was found in association with the caim burial (Feature 23). It is an elongated, wedge-shaped piece $(58 \times 22 \times 12 \mathrm{~mm})$ with extensive wear resulting in numerous facets with parallel striations. Its three main faces are relatively flat, but there are at least six smaller facets along its lateral edges and ends.

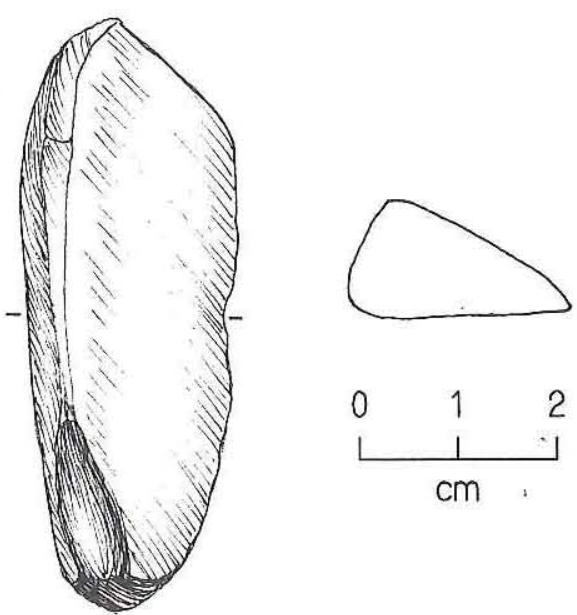

Figure 39. Modified hematite from the caim burial (Feature 23), 41GR291. 


\section{Manuports}

\section{Unburned and Burned Rocks}

Large numbers of unburned and burned rocks (including fire-cracked rocks, heat shatter, and heat spalls) found throughout the site are considered to be manuports and were analyzed prior to being discarded. Given the depostional context of the upland sediments at the Sam Wahl site (i.e., relatively well sorted fine sandy loam with no gravel inclusions), objects of any size were almost certainly introduced by human activities. Hence, all burned and unburned rocks were collected if they were over $25 \mathrm{~mm}$ in maximum dimension. For analysis purposes, however, the sample was conservatively limited to only those specimens over $38 \mathrm{~mm}$ in maximum dimension (equivalent to Wentworth's large pebble class). Observations on these materials consisted of identification of material type, presence or absence of evidence of burning, and count and weight by material/buming group (weights were rounded to the nearest tenth of a kilogram, with the minimum recorded being $0.1 \mathrm{~kg}$ ).

The exception to the above rule is that all siliceous materials (quartzites, Potter chert, and cherts) and limestone that exhibited evidence of burning were collected regardless of size. These materials, which include fire-cracked rocks, heat shatter, and heat spalls, are much less frequent than burned sandstone but are still considered to be manuports. Hence, the unburned and burned rock category includes all materials above a minimum size and all fire-cracked siliceous materials regardless of size.

It should be mentioned that occasional unmodified cobbles (mostly quartzites) were found in the lower levels of some units in Block 4, apparently below the cultural zone and usually within the upper weathered bedrock. In these cases, it was unclear whether they were natural (i.e., in situ or redeposited gravels of the Lingos Formation) or cultural (i.e., manuports). These materials were collected and are included in the manuport analysis. In other cases where it was not so obvious (i.e., the gravels found within the sandy loam), the specimens were collected and analyzed. Rocks of questionable origin, however, are limited to a few dozen specimens, and even if they are of natural origin, they would not alter the interpretations significantly.

A total of 3,618 rocks (weighing $807 \mathrm{~kg}$ ) is considered to be manuports, and a large percentage of these ( $56 \%$ by count, $44 \%$ by weight) are burned (Table 15). Sandstone is the dominant material (78\% by count, $89 \%$ by weight), and it is almost exclusively tabular fragments from outcrops of various materials around the site. Sandstone is followed in order of decreasing frequency by quartzite $(12 \%$ by count, $6 \%$ by weight), Potter chert ( $6 \%$ by count, $4 \%$ by weight), chert ( $3 \%$ by count, $1 \%$ by weight), and limestone ( $1 \%$ by count, $1 \%$ by weight). All of these materials are available locally in gravel outcrops. Of the burned and unburned rocks collected, those of sandstone tend to be much larger than the other materials. The average weight of the sandstone specimens is ca. $0.25 \mathrm{~kg}$, while the average weights of each of the other materials are less than $0.1 \mathrm{~kg}$. The weights of individual specimens are much higher for sandstone (ca. $10 \mathrm{~kg}$ maximum, with many specimens in the range of $2-5 \mathrm{~kg}$ ) than for any of the other material types (maximum weights of individual specimens: quartzite, $0.6 \mathrm{~kg}$; Potter chert, $3.0 \mathrm{~kg}$; chert, $0.4 \mathrm{~kg}$; and limestone, $0.6 \mathrm{~kg}$ ).

To accurately identify the presence or absence of burning on the sandstone specimens, samples of the various types of sandstone cropping out around the site were subjected to heating experiments. Minimal heating often did not result in any visible alteration of the sandstone, and it is likely that the frequency of burned sandstone is underestimated. Various degrees of thermal discoloration (i.e., different shades of reddening, graying, or blackening) were noted on different sandstones that were intensively heated, but they tended not to fracture. Unlike the sandstone, thermal fracturing is the dominant attribute noted for the limestone and siliceous materials (i.e., quartzite, Potter chert, and chert). Thermal discoloration was observed on the Potter chert and cherts, but no discoloration was noted on most of the quartzites, which generally are naturally dark (dark red, maroon, or purple are most common). While the coarser grained materials generally are angular fragments (i.e., heat-shattered chunks), the chert specimens include both angular fragments and smooth fragments with internal fractures or potlidding (i.e., burned flake fragments).

Presumably, these rocks were brought onto the site to serve a variety of functions, but specific functions can be inferred only for those found in feature contexts (Table 16). Those rocks found in association with Feature 23 are obviously part of the rock cairn covering the burial. Most of these rocks 


\begin{tabular}{|l|c|c|c|c|c|c|}
\hline \multicolumn{7}{|c|}{ TABLE 15 } \\
\multicolumn{7}{|c|}{ SUMMARY OF BURNED AND UNBURNED ROCKS BY MATERIAL TYPE, 41GR291 } \\
\hline & Sandstone & Quartzite & Potter Chert & Chert & Limestone & Totals \\
\hline Burned Rocks & & & & & & \\
Count & 1,635 & 63 & 209 & 93 & 9 & 2,009 \\
Weight (kg) & 321.3 & 5.3 & 21.4 & 6.3 & 0.8 & 355.1 \\
\hline Unburned Rocks & & & & & & \\
Count & 1,189 & 365 & 20 & 8 & 27 & 1,609 \\
Weight (kg) & 396.4 & 42.3 & 8.7 & 1.3 & 3.8 & 452.5 \\
\hline Total Rocks & & & & & & \\
Count & 2,824 & 428 & 229 & 101 & 36 & 3,618 \\
Weight (kg) & 717.7 & 47.6 & 30.1 & 7.6 & 4.6 & 807.6 \\
\hline
\end{tabular}

are unburned and appear to have been selected for that purpose, particularly the larger sandstone slabs. Some of the rocks associated with the storage pits may have served as weights on top of pit covers, and the large metates on top of Features 18 and 19 may have functioned in this manner also. In particular, large rocks found in Feature 17 may represent a collapsed pit cover.

Burned rocks were most frequently associated with two types of features - hearths/baking pits and charcoal/burned rock scatters. In the case of the hearths/baking pits, the rocks probably were used to line the features to retain heat, and it is likely that the burned rocks scattered in the charcoal/burned rock scatters and throughout much of the site were originally associated with these types of features. It should be noted that the counts and weights of burned rocks in these features (see Table 16) are considerably higher when the burned fragments of ground stones recycled as hearthstones are taken into account (see Cultural Features).

The large quantity of burned and unburned rocks inside pithouse Feature 37 is intriguing, but the functions of these rocks cannot be definitely determined. The presence of these rocks may be fortuitous (e.g., they were discarded into the pithouse depression after abandonment), or they may have been associated with the structure (e.g., used as shims or weights along the edges of the structure or parts of interior features), or they may have come from both of these sources.

\section{Odd-shaped Stones}

Within the manuport category, 10 specimens are classified as odd-shaped stones and are considered to be novelty items (Table 17); All appear to be natural concretions of micaceous sandstone, found locally throughout the Triassic Trujillo Formation. Similar concretions are found downslope from the site, but they are manuports when found in the upland sandy sediments (i.e., they do not occur naturally in that depositional context). Notably, 3 of the 10 specimens were recovered on or near the floor of pithouse Feature 37.

None of the specimens are definitely modified, but five specimens have either facets or scars along their ends or edges which are suggestive of modification. It is possible that some of these functioned as tools (e.g., those with faceted edges may have been small grinding tools, while specimens with end scars may have been hammerstones), but the coarse texture of these sandstones is not amenable to recognizing minimal use modification. Lacking any definitive modification, these specimens cannot be assigned to any functional class, and any of the specimens could have been picked up simply as oddities (e.g., because of their unusual shapes). Unmodified odd-shaped stones (i.e., "novelty items") have been recovered in cultural contexts at other Lake Alan Henry sites (41KT51 and 41KT53 in Boyd et al. 1993:73-74, 190-191).

\section{Fossil Shell}

One complete fossil shell $(71 \times 46 \times 18 \mathrm{~mm})$ was recovered in EU 6 from the fill of pithouse Feature 37 , and its occurrence there is certainly due to cultural processes. It is identified as Exogyra texana, a common Cretaceous pelecypod found in 
TABLE 16

SUMMARY OF BURNED AND UNBURNED ROCKS BY FEATURE AND MATERIAL TYPE, 41GR291*

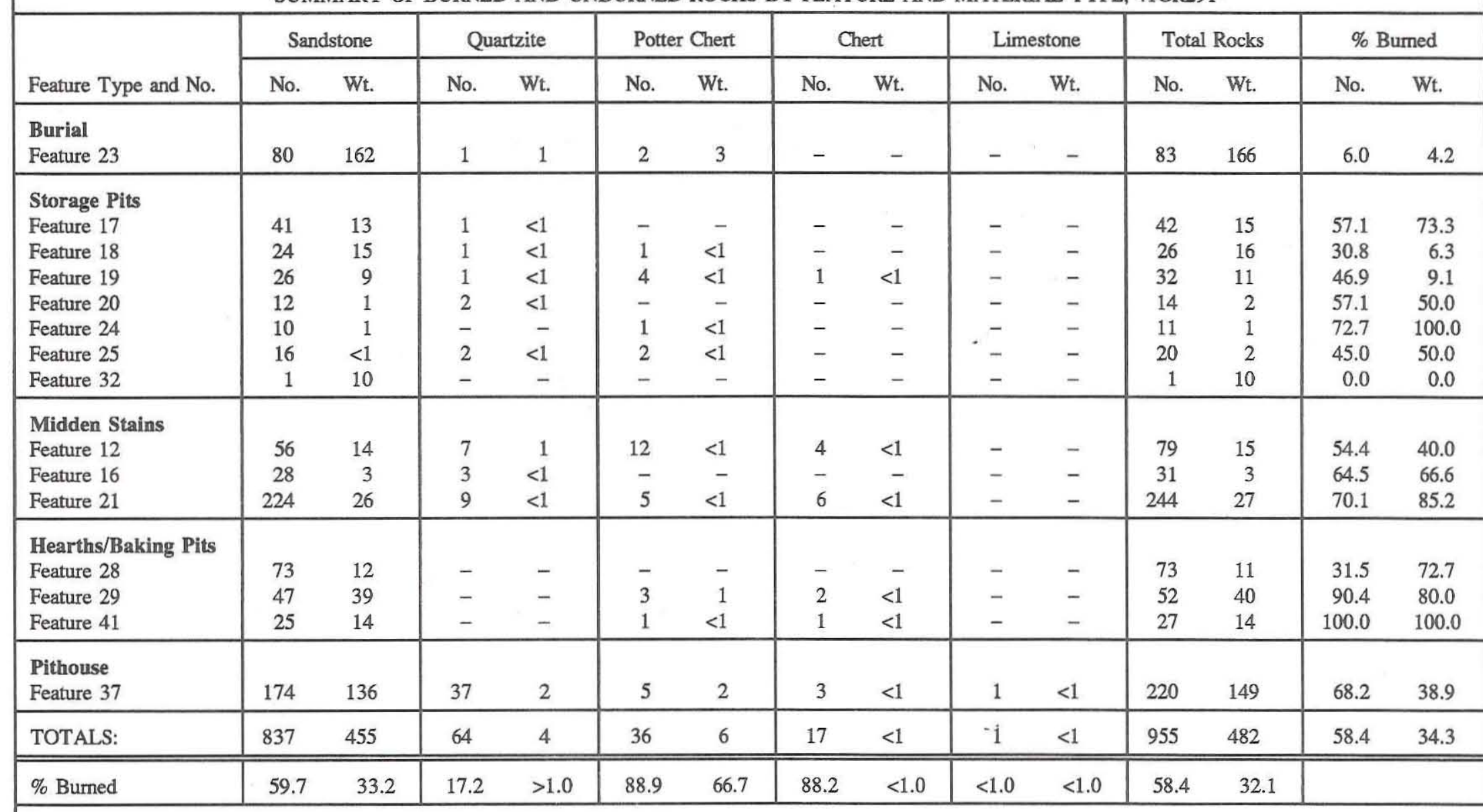

*Weight is rounded to the nearest kilogram. 


\begin{tabular}{|l|l|r|l|}
\hline \multicolumn{2}{|c|}{ SUMMARY OF ODD-SHAPED STONES, 41GR291 } \\
\hline Provenience & Shape & Dimensions $\left(\mathrm{cm}^{2}\right)^{*}$ & Comments \\
\hline EU 6, Feature 37 & Elongated/ovate & $48 \times 25 \times 15$ & \\
EU 8, Feature 37 & Elongated/irregular & $(90) \times 43 \times 18$ & possible ground facet on end \\
EU 11, Feature 37 & Discoidal & $35 \times 32 \times 16$ & possible ground facets on edges \\
EU 16 & Ovate & $48 \times 39 \times 28$ & possible flake scars on end \\
EU 42 & Ovate & $46 \times 33 \times 15$ & possible ground facet on one edge \\
EU 60 & Elongated/ovate & $67 \times 26 \times 13$ & \\
EU 65 & Elongated/pointed & $64 \times 14 \times 10$ & \\
EU 75 & Elongated/ovate & $113 \times 40 \times 22$ & \\
EU 79 & Discoidal & $(80 \times 80 \times 42$ & \\
BHT 1992-25 fill & Elongated/ovate & p 20 & possible flake scar on end \\
\hline *Dimensions in parentheses are partial measurements and indicate that the specimen is incomplete.
\end{tabular}

nearby limestone outcrops and locally in the Lingos Formation gravels. Although no modifications are apparent, limited use wear would not be identifiable on this type of fossil shell, which has naturally stepfractured and rounded edges. Thus, this specimen might have functioned as a tool (cf., a fossil Exogyra scraper found in the previous season's work at 41KT53; Boyd et al. 1993:148, 191-192), or it may have been picked up simply as a curiosity.

\section{Vertebrate Faunal Remains}

The Sam Wahl site yielded a meager vertebrate faunal assemblage of only 183 specimens (see Appendix C). Of these, 114 unidentifiable and unburned fragments are from in and around Feature 23 (EUs 16, 17, and 18), and most, if not all, are probably human bone fragments brought up by rodents (Table 18). If these are excluded, then the faunal assemblage is limited to 69 specimens, of which 27 $39 \%)$ exhibit evidence of burning and none show evidence of butchering. It is likely that the majority of these specimens are associated with the prehistoric occupations, particularly since most $(n=38,55 \%)$ were recovered through flotation of feature fill, but only two unburned specimens are identifiable. There is no reason to doubt that the fragmentary bones of a medium artiodactyl (probably a deer or longhorn) and a Lepus sp. (rabbit) recovered from feature contexts are cultural in origin.

A serious bias is evident in this sample, and it appears that most of the bones that did survive are either burned or were preserved in unusual feature contexts (e.g., in the fill of features intrusive into bedrock). Because of this preservation bias, the interpretability of the collection is limited. Not only are the bones badly fragmented and largely unidentifiable, they surely represent only a fraction of the bones that were associated with the prehistoric occupations. Thus, the faunal collection cannot be considered an accurate reflection of the importance of game animals in the diet of the site inhabitants.

In addition to the terrestrial faunal remains, two fish otoliths were recovered from EUs 58 and 62 . These are identified as Aplodinotus grunniens Rafinesque, a common freshwater drum (see Appendix D). Although these otoliths are not from feature contexts, their presence in the cultural zone probably indicates that freshwater drum was utilized as a food resource by the prehistoric inhabitants.

\section{Invertebrate Faunal Remains}

\section{Snails}

Five terrestrial snail shells were recovered, but they are considered to be noncultural. All are identified as being in the family Succinidae or as Catinella sp. (see Appendix D). These snails are not particularly sensitive environmental indicators and are common in archeological sites of various ages throughout the Lake Alan Henry project area (Boyd et al. 1990:566).

\section{Freshwater Mussel Shells}

Freshwater mussel shells are well represented (see Appendix D). Some fragments were recovered 


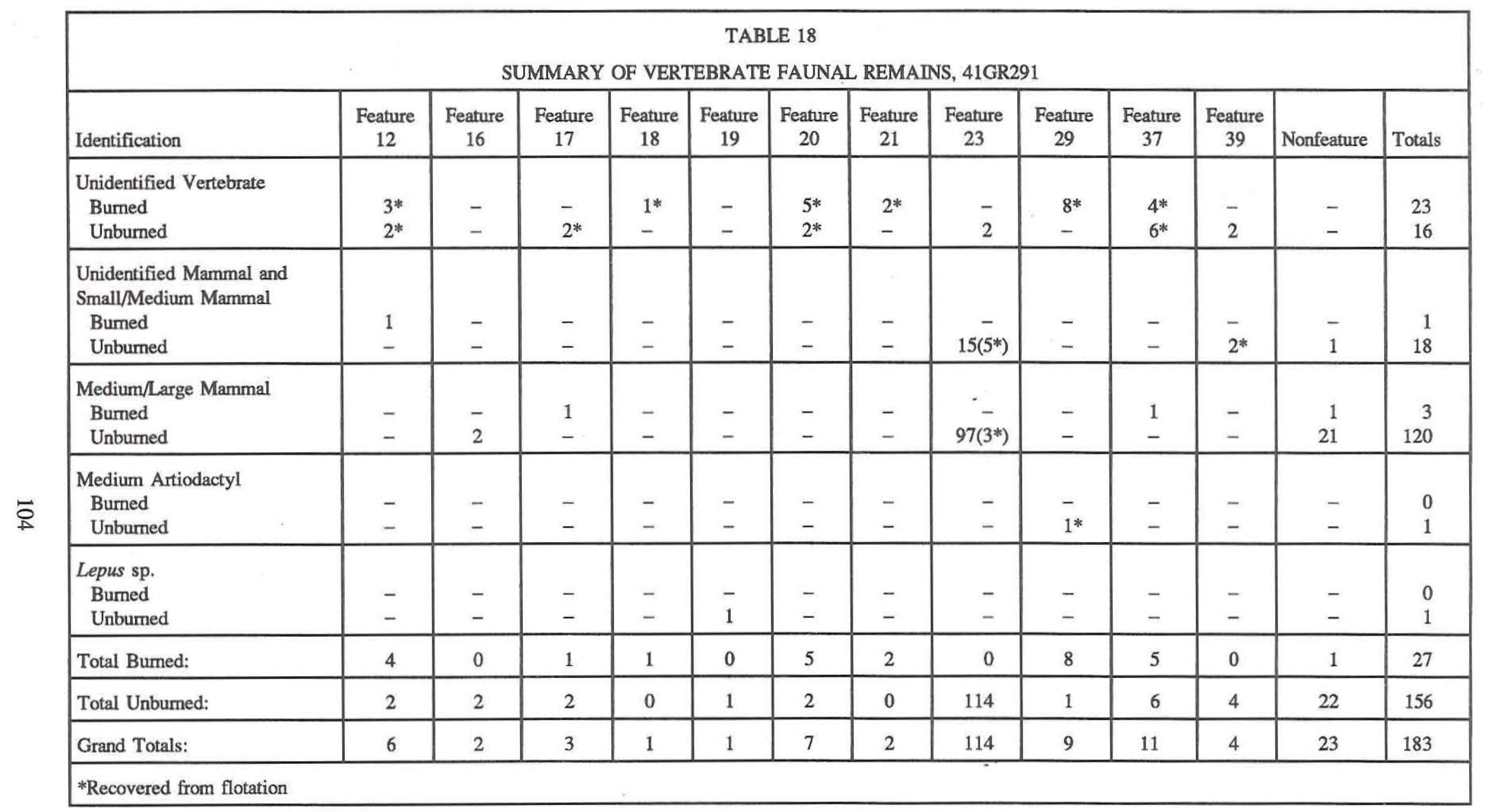


from almost every excavation unit, but only six units (EUs 4, 10, 63, 64, 67, and 86) yielded $5 \mathrm{~g}$ or more. Only two specimens are complete or nearly complete valves (a whole valve from EU 63 and a 75\% complete valve from EU 4); both are Uniomerus declivus. In addition, at least 18 umbo fragments are identifiable as $U$. declivus, which is the only species of freshwater mussel that has been identified in the Lake Alan Henry project area.

Two shell fragments from EUs 43 and 101 exhibit evidence of burning (i.e., thermal discoloration). These specimens may indicate that freshwater mussels were used as a food resource. In particular, one is a burned umbo fragment (from EU 43), which is more diagnostic of food use since live mussels are easily opened by placing their dorsal surface (hinge line) on a hot rock or coals. Heated umbo fragments also were recovered from the South Sage Creek site (41KT33) during the first season of data recovery (Boyd et al. 1992:127, 206).

While it is likely that some freshwater mussels were brought to the site to be eaten, the overall paucity of shell remains suggests that mussels were not a significant food resource. The presence of modified pieces (see Modified Shells) indicates that the mussel shells may have been used as tools and manufactured into ornaments. It is also possible that the shells themselves were of more importance than were the mussels they contained.

\section{Modified Shells}

Four shell specimens are intentionally modified and indicate that the shells of freshwater mussels were used for decorative purposes (i.e., jewelry) and possibly for utilitarian purposes as well (i.e., tools). Three specimens have cut and/or cut and smoothed or ground edges, while one specimen exhibits possible use wear along natural edges (Figure 40). Although none of these can be positively identified, they are probably Uniomerus declivus since this is the only species of freshwater mussel represented at the site (see Appendix D). Modified specimens of this species also were found at the South Sage Creek site during the first season of data recovery (Boyd et al. 1992:126-127).

Specimen 1 (from EU 7) is an ovate, $24-\mathrm{x}-21-\mathrm{mm}$ piece with cut and smoothed edges. Approximately $70 \%$ of its edges are ground smooth, while the remaining edges are jagged. It appears to be a pendant blank that may have been discarded because of its broken distal edge. Specimen 2 (from EU 37) is a complete, $22-x-21-m m$ trapezoidal shell piece with three cut edges and smoothing and rounding along one edge (the ventral margin). The differential edge treatment suggests that this piece was cut from a valve that may have been used previously as a scraping tool. Due to its small size and irregular shape, this specimen may have been discarded as a by-product of the manufacture of shell pendant blanks. Specimen 3 (from EU 32) is an irregular-shaped, $49-x-33-m m$ piece with four cut edges opposite its unmodified dorsal margin. It is relatively thick (2-4 $\mathrm{mm}$ ) and curved and may represent a valve section that was discarded after removal of the flatter portions that were more suitable for manufacturing decorative items such as beads or pendants.

Unlike the other modified shells, Specimen 4 (from EU 41) is a $48-\mathrm{x}-35-\mathrm{mm}$ valve fragment that has no cut edges but exhibits smooth, rounded edges along its posterior margin. This valve fragment most likely represents the distal end of a scraping tool.

\section{CHRONOLOGY}

Radiocarbon dating of organic remains associated with cultural features and relative dating through artifact typology are employed to define the chronology of the Sam Wahl site. A third technique, thermoluminescence dating of burned rocks, was attempted during the Phase II investigations, but the result of that attempt (a date of $5170 \pm 1040$ B.P. on a burned sandstone rock from Feature 2) is questionable (Boyd et al. 1990:368-373) and is not considered here.

\section{Absolute Chronology}

Ten radiocarbon dates associated with cultural features constitute the evidence for defining the absolute chronology. As seen in Figure 41, the dates span a long period of time from A.D. 238-1379. All of these dates were obtained on materials from good archeological contexts and have relatively small 1sigma ranges. They are discussed below in chronological order from oldest to youngest.

The cairn burial (Feature 23) is the oldest cultural event at the site, and it is roughly $200-350$ years earlier than the next closest dates (from Features 19 and 29). The Feature 23 date is on bone and should reflect the time of the individual's death 

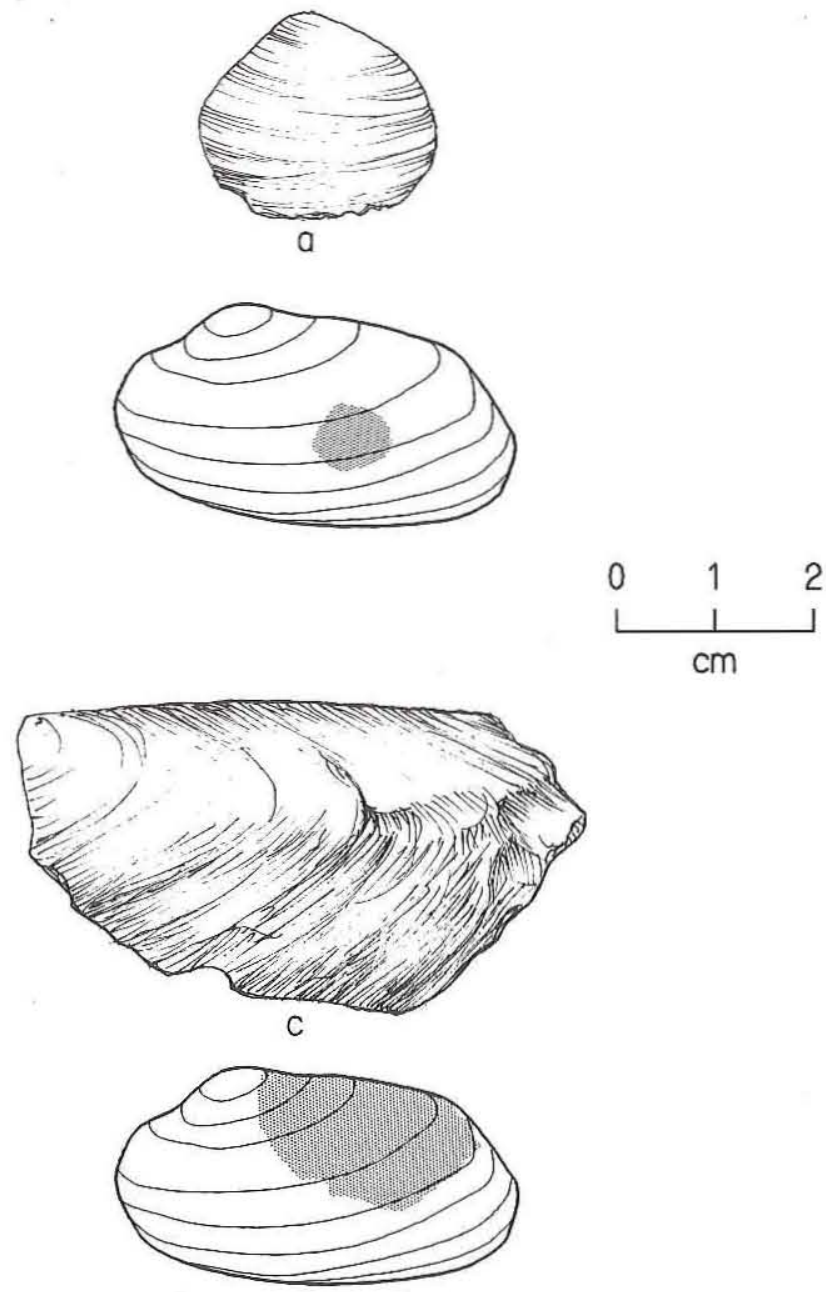

Figure 40. Exterior views of modified mussel shells, 41GR291. Specimen 4. Shaded areas indicate location of specimen on valve.

and presumably his interment, although Feature 23 is a secondary burial and an unknown period of time elapsed between death and burial. The burial appears to represent a separate and discrete component that may be unrelated to the other activities at the site.

The next four dates fall within the A.D. $600-900$ time span and are clustered into two pairs. The assays constituting the first pair have single intercepts at ca. A.D. 650 and ranges that overlap between A.D. $600-669$. These dates are from a hearth/baking pit (Feature 29) and a storage pit (Feature 19) and strongly suggest the contemporaneity of these features. The second pair of dates is also from a storage pit (Feature 17) and a hearth/baking pit (Feature 41) and suggest contemporaneity. These two dates have ranges that overlap between A.D. $689-883$, and all of the intercepts fall between

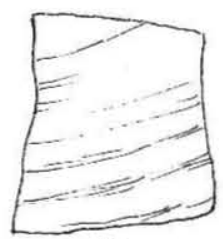

b
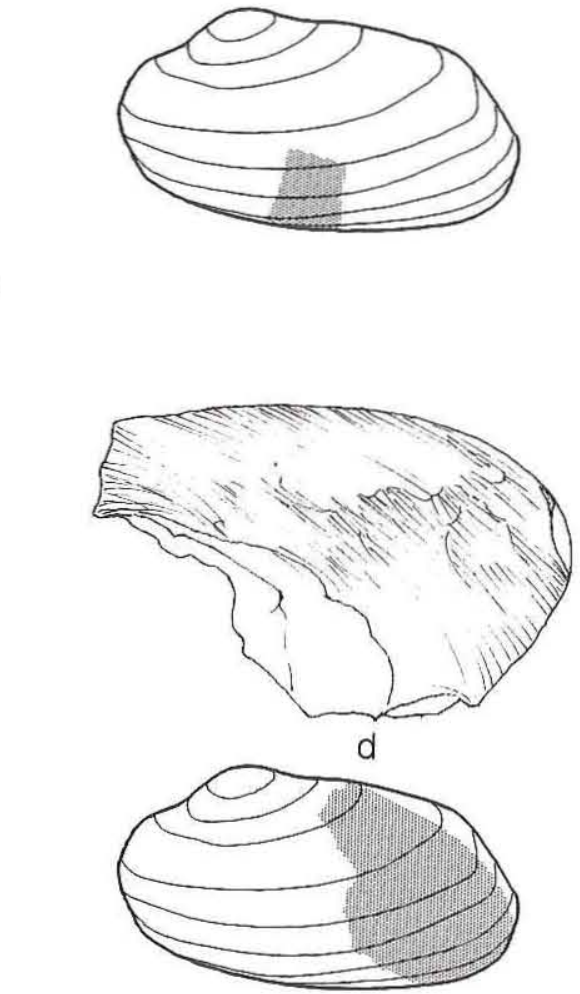

(a) Specimen 1; (b) Specimen 2; (c) Specimen 3; (d)

A.D. $772-852$. The 1-sigma ranges of the two pairs of dates do not overlap appreciably, and they suggest that the same kinds of activities were performed at 41GR291 over an extended period of time, perhaps as much as 300 years.

Several points about the storage pit and hearth/ baking pit dates merit mention. If two assumptions are made, then the dated charcoal associated with the hearths/baking pits can be seen as accurately reflecting the actual age of the construction and use of the features. First, it can be assumed that the wood burned in these features was not of great antiquity (i.e., burning of extremely old wood), and second, even though it is acknowledged that these hearths/ baking pits could have been reused many times, it can be assumed that their total life spans were relatively short (i.e., it is not likely that such features would 


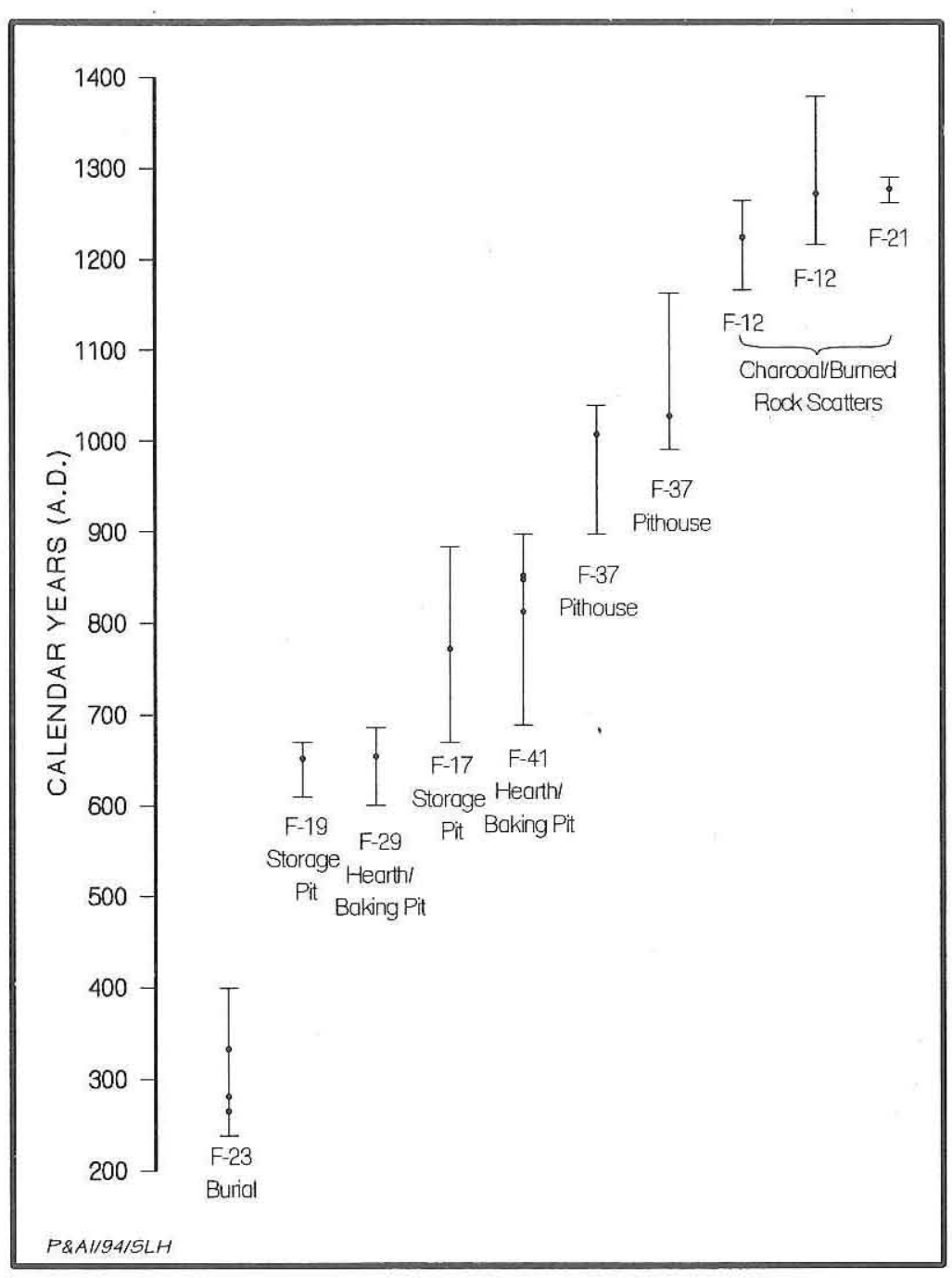

Figure 41. Graph of calibrated radiocarbon dates (intercepts and 1-sigma ranges) from features, 41GR291.

have been used repeatedly over many years). Conversely, the storage pits could have been reused over a long period of time, and the associations between the dates and construction and use of the pits is less obvious. Both dates are on charcoal from pit fill (the Feature 17 date is from middle fill, and the Feature 19 date is from bottom fill), and they do not necessarily date the construction of the features. As discussed previously (see Storage Pits in Cultural Features), it can be assumed that the pit fill accumulated at or soon after the time of final abandonment and that the dates represent minimum ages for the use of the storage pits. If this is the case, then Feature 19 may have been abandoned long before Feature 17.

Alternatively, it is possible that the dated charcoal from the bottom of Feature 19 was introduced sometime during use of the pit and remained there during subsequent use episodes. Another possibility is that the pits filled naturally (i.e., by eolian processes) soon after final abandonment but that some of the charcoal in the fill originated from much older occupations. If either of these two scenarios is the case, then the fill dates would not accurately reflect the time of final use and abandonment, and the pits could have been used much longer than the dates would indicate. These two scenarios seem unlikely since the ;irst assumes that the pits were not cleaned out very well between uses and the second assumes that old charcoal survived exposure to the elements and was blown into the features.

Still another possibility is that the site's inhabitants intentionally backfilled the pits, perhaps to conceal their locations, at the time they were last used. If this is the case, then it is possible that the fill is a mix of cultural sediments and could contain some older charcoal, and thus the dates might not reflect minimum ages for the pits. Even this scenario, however, seems unlikely since it assumes the survival of older as well as contemporaneous charcoal. While the exact relationships of the charcoal dates to the storage pits cannot be fully resolved, it seems most likely that the radiocarbon dates for the pit features approximate the times of final abandonment. These dates do not, however, approximate the time of their construction, and it is possible that the pits were used intermittently for many decades or even hundreds of years.

The next two dates are on charcoal from the pithouse fill (Feature 37). The earlier date is on scattered charcoal within $15 \mathrm{~cm}$ of the floor and underneath the large metate that was stored upside down in the structure, while the later date is on concentrated charcoal fragments lying directly on the floor. While the ranges of these two dates do not completely correspond, they do overlap between 
A.D. 990-1039 and both have single intercepts that are very close at A.D. 1007 and 1027. The contemporaneity of these two dates suggests that the fill probably accumulated quickly after the last occupation episode, and they provide a minimum age for the final use/abandonment of the pithouse at ca. A.D. 1000-1050. These dates, however, may not approximate the construction of the pithouse, and it is possible that the structure was used for a long time prior to A.D. 1000.

The latest set of dates consists of three assays from two charcoal/burned rock scatters. The two dates from Feature 12 range from A.D. 1166-1397 and overlap at A.D. 1216-1265, and the date from Feature 21 is approximately contemporaneous at A.D. 1262-1290. These appear to represent the latest occupation(s) at the site, but the activities represented by these features are not well understood (see Cultural Features).

\section{Relative Chronology}

The material culture provides limited temporal information about the occupations of the Sam Wahl site. Projectile points and ceramics are the only two artifact classes that have any temporal sensitivity, and they clearly indicate that the majority of the materials date to the Late Prehistoric period. Three fragmentary dart points (one from testing and two from data recovery) suggest that the site may have had an ephemeral late Archaic occupation. It is also possible that these points were picked up and brought to the site by the Late Prehistoric occupants or that dart points were used contemporaneously with arrow points for a time, but this issue has yet to be resolved for the Southern Plains (e.g., Hughes [1991:20] suggests that dart and arrow points may co-occur from ca. A.D. 0-500, and Taylor [1987] reports that a corner-notched arrow point is associated with radiocarbon dates of $940-740$ B.C. and 600-480 B.C. in Canadian County, Oklahoma) or the Southwest (e.g., Newton and Upham [1988] suggest that some "archaic" point styles were actually used as late as ca. A.D. 1400).

The majority $(91 \%)$ of the Sam Wahl site projectile points, however, are arrow points $(n=30)$, and the more diagnostic specimens include both early and late styles. A barb fragment recovered during the testing phase was classified as a Deadman's arrow point, a type that is diagnostic of the Palo Duro complex. Deadman's points have been found in well- dated contexts at only two sites, but they apparently date to the first millineum A.D. (Cruse 1992:125; Willey and Hughes 1978:185-190). Interestingly, an almost identical form of arrow point is found, also in association with Scallorn points, in Late 18 Mile phase sites (ca. A.D. 900-1000) in the middle Pecos region of New Mexico (cf. style C6 in Jelinek 1967: 96, 104).

Five Scallorn specimens from 41GR291 (one from testing and four from data recovery) are considered to be early-style arrow points and include both the classic coryell variety (comer notched and barbed with expanding stem) and the less common eddy variety (corner notched with short or no barbs and slightly expanding to bulbar stem) described by Jelks (1962:27-31). The burial specimen in particular appears to be older than the others, an assessment that is substantiated by the associated radiocarbon date. In the Southern Plains, Scallorn and Scallom-like arrow points are extremely common and are associated with the Plains Woodland (A.D. 200-800) and early Plains Village (A.D. 8001200) periods (Brooks 1989; Hofman 1978, 1984a; Hofman and Brooks 1989; Vehik 1984) and locally in the Texas Panhandle-Plains with the Palo Duro complex at ca. A.D. 200-1000 (Cruse 1992; Etchieson 1979; Wedel 1975; Willey and Hughes 1978). Although they are not typed as such, Scallorn-like arrow points also are common in the eastern extension of the Jornada Mogollon region (ca. A.D. 900-1250) in southeastern New Mexico (cf. Type 3 in Leslie 1978:106-114, 116), and they are found in Jelinek's (1967:103-104, 144-148) Early and Late 18 Mile phases (ca. A.D. 700 or $800-1000$ ) in the middle Pecos River valley in eastern New Mexico.

Notably, the Scallorn point from the 41GR291 burial is quite large (see Figure 21d) and may be a transitional Archaic style. Its overall morphology is similar to some of the corner-notched dart points associated with late Archaic bison kills in the Upper Red River Drainage of the Texas Panhandle-Plains (Hughes 1977, 1989; Lintz et al. 1991). These sites have yielded a variety of points (cf. Castroville, Marcos, Williams, Ellis, Ensor, Trinity, Palmillas), but the burial point is most similar to Variety III points at the Twilla site in Hall County (Hughes 1989:Figure 3, L and M).

A stemmed Bonham point from the Sam Wahl site is similar to arrow points that characterize the newly defined Blow Out Mountain phase (ca. 
A.D. 800 or 900-1300) of west-central Texas (cf. Group E and F points; Creel 1990:15-18, 95-96). Bonham points are also characteristic of Plains Woodland occupations (prior to A.D. 450 to ca. 800) in south-central Oklahoma (Vehik 1984:194). Also from the Sam Wahl site are several small triangular points (cf. Fresno) and one multiple-side-notched specimen (cf. Washita and Huffaker) that are considered to be late arrow point styles. According to Hughes (1991:28-29), the transition from cornernotched to side-notched arrow points is one of the diagnostic hallmarks differentiating the early and late parts of the Neo-Indian or Late Prehistoric periods in the Texas Panhandle-Plains at ca. A.D. 1100. This replacement of projectile point styles apparently occurred over most of the Southern Plains at about the same time - at ca. A.D. 1250 in the middle Pecos region of New Mexico (Jelinek 1967:154), in the latter half of the Custer phase (ca. A.D. 1100-1300) in western Oklahoma (Brooks 1989:76-77), and in the eastern extension of the Jornada Mogollon at ca. A.D. 1200 (Leslie 1978:91). While this stylistic transition probably took place over a period of time and may have occurred at different times in different areas, the presence of a side-notched arrow point at the Sam Wahl site suggests that the occupations there did extend until at least ca. A.D. 1100-1200. Such an assessment is substantiated by the latest radiocarbon dates from the site.

The 20 ceramic sherds (including 2 from the testing phase) are all plain brownware sherds representing wares imported from the Jornada Mogollon region of southern New Mexico or western Texas (see Appendix G). Such wares are generally classified as Jornada Brown (or some related variant such as South Pecos Brown), but it is clear that the frequently cited dates of A.D. 900-1350 (Runyan and Hedrick 1973:23, 1987:29) for their occurrence in the Texas Panhandle-Plains should not be applied to the Sam Wahl site ceramics. While the ending date for Jornada Brown probably is applicable and generally is not contested, the beginning date of A.D. 900 cannot be substantiated. Although there is a paucity of absolute chronological data for the Jornada Mogollon region(Whalen 1985:142), most researchers have departed from Lehmer's (1948:89) and Jelinek's (1967:144-145) original estimates of A.D. 800-900 for the earliest brownwares in the Jornada Mogollon and Middle Pecos regions. There is now good evidence indicating that brownwares were manufactured before A.D. 500 and perhaps as early as
A.D. 200 (e.g., Fields and Girard 1983:233; Lintz et al. 1988:37; Miller and Stuart 1991:12-13, 117; O'Laughlin 1980:25; Whalen 1978:7). Consequently, it is not surprising that recent evidence indicates that Jornada Mogollon brownwares were exported to the Southern Plains much earlier than A.D. 900. Of particular interest, Jornada brownwares have been found in dated contexts in the Texas Southern Plains at A.D. $60-250$ and 395-775 at Deadman's Shelter (based on the association of ceramics with four radiocarbon dates from Strata D and B, respectively; Willey and Hughes 1978:185, 189), at A.D. 590-830 at the Kent Creek site (based on the association of a radiocarbon date from the floor of a pithouse and ceramics in the fill; Cruse 1992:57, 103), and at A.D. $400-850$ at Chalk Hollow (based on the association of brownware ceramics with six radiocarbon dates from the upper midden; Wedel 1975: 272-273).

\section{Summary and Discussion}

The absolute and relative chronological data indicate that the Sam Wahl site was utilized for a long period of time, perhaps as early as the terminal Archaic and definitely spanning much of the Late Prehistoric period. The radiocarbon dates are considered much more reliable than the material culture for defining the ages of the site components. Clearly, the full time span of human activities at the Sam Wahl site is extensive (ca. 1,200-1,400 years), but the absolute dates indicate that the activities can be separated into three periods of use: (1) an early and unrelated component consisting of the burial feature; (2) an early occupation period that includes the pithouse, storage pits, and hearths/baking pits; and (3) a late occupation period that includes two charcoal/burned rock scatters.

\section{Burial Component}

Burial Feature 23 stands alone as the earliest event at the site, with a radiocarbon assay dating it to A.D. 238-399. This date appears reasonable since the burial was accompanied by an unusual Scallorn arrow point. Although some of the other arrow points also are classified as Scallorn, they are of a different variety and are distinct from the large burial specimen. No definite connection can be made between the burial and the early occupation period, which appears to have begun ca. A.D. 600 . 
This burial is perplexing for many reasons, and it is unclear where it fits into the regional picture. The radiocarbon assay indicates that it falls within the time frame suggested for the late Archaic bisonhunting peoples in the Texas Panhandle-Plains (Hughes 1977, 1989; Lintz et al. 1991), and the large Scallorn point is morphologically similar to some of the corner-notched dart points associated with these sites. It appears to be a transitional style between dart and arrow points that may represent continuity in projectile point technology between the late Archaic and Late Prehistoric periods. The Sam Wahl site burial does not necessarily represent the same people as the late Archaic bison hunters in the Texas Panhandle-Plains, but its age and the projectile point similarities suggest a possible connection.

\section{Early Occupation Period}

Although the relationships between the pithouse, the storage pits, and the hearths/baking pits are not clear, the intercepts for six radiocarbon dates for these feature types fall within the A.D. $650-1050$ range. The radiocarbon dates indicate that storage pits and hearths/baking pits may have been in use by ca. A.D. 600 (Features 29 and 19) and continued to be used perhaps at late as ca. A.D. 900 (Features 17 and 41). The date ranges for the latest storage pit (Feature 17) and hearth/baking pit (Feature 41) do not overlap with the ranges for the dates from pithouse Feature 37, and the intercepts suggest that Features 17 and 41 were abandoned ca. 200 years before the final occupation of pithouse Feature 37 (ca. A.D. 1000-1050). For the pithouse to have been occupied contemporaneously with the use of Features 17 and 41 , it must be assumed that the structure was occupied repeatedly for a substantial period of time. Even though there is no evidence for multiple use episodes (e.g., multiple floors) of the pithouse, this does not mean that it was not rebuilt and reused many times, although the time mentioned above makes it seem unlikely that Feature 37 was contemporaneous with the dated storage pits or hearths/ baking pits. Perhaps the more important issue is what classes of features, rather than what specific features, were contemporaneous, since the excavated features are only a sample of what may have been present at the site, and for most feature classes, only a sample of the excavated features has been dated.

In terms of the population of cultural features at 41GR291 as a whole, the 10 identified storage pits probably represent a good sample of this class of feature, while the 3 hearths/baking pits probably represent a very poor sample. The storage pits seem to be spatially confined to one area, and the excavations may have uncovered all, or at least the majority, of them. Although isolated storage pits could have been missed, it is unlikely that the closely spaced backhoe trenches would have missed a similar cluster of storage pits in the main part of the site. Only 2 of the 10 storage pits were dated, however, and as previously concluded (see Cultural Features), these dates are not necessarily representative of the abandonment ages for all of the pits. Conversely, it is likely that undiscovered hearths/baking pits may yet exist at the site, and many of the uninvestigated burned rock clusters found on the surface and in mechanical excavations could be similar features. As was suggested for the storage pits, the two radiocarbon dates on hearths/baking pits probably are not representative of the full age range for this feature type.

It is possible that Feature 37 is not the only pithouse at the Sam Wahl site. While the closely spaced backhoe trenching in the main portion of the site was done specifically to look for structures, it is possible that some were missed or that some were present but were not recognizable archeologically. Similar ephemeral pithouse structures at Keystone Dam near El Paso were extremely difficult to recognize archeologically. Carmichael (1985:146) reported that these structures were difficult to recognize because (1) they were "ephemeral and lack many of the formal attributes characterizing longterm residential structures," (2) the feature boundaries were very subtle in the "sandy soils," (3) the fill sediments were locally derived and were very similar to the surrounding matrix, and (4) the floors and walls were "obscured by root and rodent disturbances and water percolation through the soils." These characteristics also are applicable to the Sam Wahl site, and every amorphous stain encountered in the backhoe trenches could not be tested. One of these, however, a large charcoal-stained area encountered in Backhoe Trench 1992-4, was thought to be a candidate for a pithouse. Unfortunately, testing of this area (EU 105) was inconclusive because this amorphous stain was severely disturbed by root and rodent activity, and its diffuse boundaries could not be adequately defined. It is possible that Feature 37 was recognizable archeologically because it was dug into bedrock but that pithouses that were entirely 
within the sandy loam strata would have been disturbed beyond recognition.

In addition to the possibility of unrecognizable structures, pithouses could be present away from the main site area that was intensively trenched. Bearing in mind that the entire site is considerably larger than the mechanically tested area, pithouses located downslope could have eroded away or, if they are preserved, would not have been discovered. In conclusion, given the limited nature of the archeological sampling, it is suggested that other pithouses and hearths/baking pits are likely. Further, it is suggested that the six radiocarbon dates for the pithouse, storage pits, and hearths/baking pits are not necessarily representative of the full age range for these feature classes. Hence, if the problems of archeological sampling are considered in relation to the contemporaneity of pithouses, storage pits, and hearths/baking pits, two opposing scenarios 'emerge.

The first scenario is that the storage pits and hearths/baking pits actually predate the use of pithouses at this site. This would mean that storage pits and hearths/baking pits were used contemporaneously, perhaps from ca. A.D. 600 to 800 or 900 , but by the time the pithouse(s) was constructed at the site at ca. A.D. 1000 , these feature types were no longer in use. This scenario suggests that some important cultural change led to the transition between feature types. This could represent an influx of different peoples, or it could represent cultural change within a group, perhaps in the form of subsistence strategy shifts in response to climatic changes or population pressures. For example, the storage pits and hearths/ baking pits could represent a mobile hunter-gatherer society that gradually intensified its subsistence activities (possibly by incorporating horticulture) to the point where they became semisedentary. A shift to a more permanent type of dwelling (i.e., pithouses) might have been accompanied by a corresponding shift from subsurface cooking and pit storage to above-ground cooking and storage in structures.

The second scenario is that the storage pits, hearths/baking pits, and pithouse are contemporaneous and represent a complex of related activities. This view suggests that the activities represented by these features are complementary and occurred simultaneously at the Sam Wahl site for a long period of time, perhaps from ca. A.D. 600 to ca. 1000-1100. For a variety of reasons discussed below, the second scenario is favored while the first is less appealing. The most perplexing problem related to pit storage and pithouses is that one is hard to explain without the other. The presence of numerous storage pits at the Sam Wahl site indicates a certain degree of sedentism and suggests that the people who used them probably lived at the site long enough to gather or harvest, process, and store a surplus of food. Such activities would have required at least a brief residential occupation on-site, in which case some type of structure should be expected. It is possible that structures other than pithouses were utilized in conjunction with the storage pits, but there is no archeological evidence of this. Thus, within the framework of the first scenario, one must explain the absence of structures associated with the storage pits.

Using ethnographic data, Gilman (1987:547) suggests four important archeological implications for the use of pithouses: (1) pit structures indicate a biseasonal settlement system; (2) pit structures will be occupied at least during cold months; (3) stored foods will be the dietary mainstay during coldweather occupation of pit structures; and (4) pit structures should be accompanied by storage facilities. Other researchers have suggested that residentially mobile groups need to conceal their stored foods and that subsurface pits are the preferred storage facility (DeBoer 1988; Raymer 1988). If these assumptions are true, then one would expect pithouses and storage pits commonly to occur together, which is indeed the case in the American Southwest. Hence, within the framework of the first scenario, one must also explain the absence of storage pits associated with the pithouse.

Also arguing for contemporaneity of the storage pits and pithouses at 41GR291 is the fact that pithouse architecture was widespread during the time that the two dated storage pits at the Sam Wahl site were in use. A variety of subterranean structures is well documented in the surrounding areas during the first millenium A.D. - in southwestern Oklahoma by Plains Woodland/Plains Village cultures (Brooks 1989; Hofman and Brooks 1989) and in southeastern New Mexico and western Texas by Jornada Mogollon peoples (LeBlanc and Whalen 1980). It is likely that pithouse architecture was not a foreign concept to the Sam Wahl site inhabitants prior to A.D. 1000 , a conclusion that is supported by the presence of at least one pithouse dating to ca. A.D. 700 at the Kent Creek site in Hall County, less than $160 \mathrm{~km}$ to the north of Lake Alan Henry (Cruse 1992:124). This conclusion is further supported by the fact that the Sam Wahl site ceramics were 
imported from the Jornada Mogollon and/or Middle Pecos regions and that similar brownwares at the Chalk Hollow, Deadman's Shelter, and Kent Creek sites are dated between A.D. 60-850 (Cruse 1992:57; Wedel 1975:272-273; Willey and Hughes 1978:185, 189). The relative abundance of early Jornada Mogollon brownwares in the Texas Panhandle-Plains indicates the intensity of the interaction between the two regions during the first millennium A.D., thus increasing the likelihood that the Plains groups were well aware of pithouse architecture during that time.

The second scenario is more convincing because a number of researchers have noted the interrelationship of pithouses and pit storage and their relationship to residential mobility (e.g., Gilman 1987:547548; O'Laughlin 1993; Raymer 1988:3). It has been suggested that residentially mobile people with a low dependence upon agriculture lived in pithọuses and used storage pits to conceal surplus foods during periods of absence. The relevance of this to the early occupation period at 41GR291, which may have involved the use of pithouses, storage pits, and hearths/baking pits, is discussed in more detail later in this chapter.

\section{Late Occupation Period}

The late occupation period is defined by two charcoal/burned rock scatters that are dated by three radiocarbon assays to A.D. 1166-1379. This component is less definable than the early component in terms of the activities represented because the function of these two features cannot be determined with confidence (see Cultural Features). Feature 21 could represent a secondary refuse area (i.e., a midden), a highly disturbed primary activity area (i.e., a series of disturbed burned rock features), or a combination of these. Feature 12 is interpreted as a small unlined basin hearth within a confined charcoal-stained surface. It could represent an outdoor activity area or a structural floor. No other features can be associated confidently with the late occupation period.

The apparent gap between the early and late occupation periods is roughly $100-150$ years from ca. A.D. 1050 to 1150 or 1200 . This gap may reflect a lack of occupation, or it may be a product of archeological sampling, with the site having been used more or less continuously from the early to the late occupation periods. If Feature 12 does represent a surface structure, then its date (ca. A.D. 1270) corresponds with the shift from pithouses to surface structures noted throughout the American Southwest (Gilman 1983, 1987) and in the Jornada Mogollon region at ca. A.D. 1100-1200 (Whalen 1980a, 1980b). If this same architectural transition occurred among the pithouse dwellers in the Texas Southern Plains, then it may have many implications for corresponding shifts in subsistence strategies, settlement patterns, and social structure that should be reflected in the archeological record (Gilman 1987). Consequently, much of the discussion in the remainder of this chapter is concerned with whether the early and late occupation periods at the Sam Wahl site reflect cultural continuity or change.

\section{INTERPRETIVE SUMMARY}

The chronological evidence indicates that human activities at the Sam Wahl site spanned over a millennium, from the second century A.D. until the thirteenth or possibly fourteenth century. The site consists of a discrete early component, represented by an isolated burial, followed by two sequential occupation periods that may or may not be related to each other. While many of the features can be assigned confidently to temporal/analytical units, it is unfortunate that the geomorphic and stratigraphic context of the cultural deposits is not conducive to sorting the artifacts into discrete assemblages. With the exception of the burial component, which is characterized by a definable but meager artifact assemblage, the material culture from most of the site can be separated only into gross assemblages that approximate the occupation periods defined by the radiocarbon-dated features. Hence, the occupation periods can be distinguished only in vague terms, and their overall interpretability is somewhat limited. Because of this ambiguity, the use of the term "occupation period" is preferred over component since the latter has explicit connotations. As LeRoy Johnson (1987:3) suggests, a component designation requires a "close contextual relationship among artifacts" that cannot be convincingly demonstrated for the two occupation periods at the Sam Wahl site.

\section{Cultural Features}

Most $(n=16)$ of the cultural features can be separated, either directly through radiocarbon dating or indirectly through stratigraphic associations, into components or periods of occupation (Table 19), and 


\begin{tabular}{|c|c|c|c|c|c|}
\hline \multirow[b]{3}{*}{ Feature Type } & \multicolumn{4}{|c|}{$\begin{aligned} \text { TABLE } 19 \\
\end{aligned}$} & \multirow[b]{3}{*}{$\begin{array}{l}\text { Total No. of } \\
\text { Features }\end{array}$} \\
\hline & \multicolumn{4}{|c|}{ Feature Nos.* } & \\
\hline & $\begin{array}{l}\text { Burial } \\
\text { Component }\end{array}$ & $\begin{array}{l}\text { Early } \\
\text { Occupation } \\
\text { Period }\end{array}$ & $\begin{array}{l}\text { Late } \\
\text { Occupation } \\
\text { Period }\end{array}$ & $\begin{array}{l}\text { Temporally } \\
\text { Unidentified }\end{array}$ & \\
\hline Burial & 23 & & & & 1 \\
\hline Pithouse** & & 37 & & & 1 \\
\hline Storage pit & 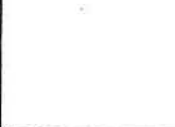 & $\begin{array}{c}17,18,19,20 \\
24,25,30 \\
31,32,39\end{array}$ & & & 10 \\
\hline Hearth/baking pit & & 29,41 & & 2 & 3 \\
\hline Charcoal/burned rock scatter & & & 12,21 & 16 & 3 \\
\hline Fire-cracked rock cluster & & ' & & 1,10 & 2 \\
\hline Burned rock cluster & & & & $\begin{array}{c}3,4,5,6,7 \\
8,9,11,13 \\
14,15,28 \\
\end{array}$ & 12 \\
\hline Bedrock mortar & & $26(?), 27(?)$ & & & 2 \\
\hline Total No. of Features & 1 & 15 & 2 & 16 & 34 \\
\hline
\end{tabular}

two others (bedrock mortar Features 26 and 27) are assigned to the early occupation period based on the recovery of functionally related artifacts from the fill of the Feature 37 pithouse. Fourteen features cannot be assigned to a temporal/analytical group because they are undated fire-cracked and burned rock clusters for which specific functions are unknown. The two remaining features cannot be assigned confidently to any temporal group. Feature 2 is a hearth/baking pit that is very similar to Features 29 and 41 , which are radiocarbon dated to the early occupation period. It is tempting to assume that Feature 2 is also from this time period, but its stratigraphic position cannot be related to the main part of the site since it was in a distant isolated unit. Likewise, it is tempting to associate Feature 16, a charcoal/burned rock scatter, with the late occupation period because of its similarities to Features 12 and 21. This interpretation is tentatively supported by a radiocarbon assay on soil humates from $25 \mathrm{~cm}$ south of the Feature 16 stain. This assay yielded a calibrated date of A.D. 1252 (1272) 1285 and is heavily influenced by charcoal from Feature 16. Unfortunately, it is problematic because of the potential for mixing of younger soil organics (see Sediments and Stratigraphy).

\section{Burial Component}

The burial component consists of a single feature, a cairn-covered secondary burial (Feature 23). A partial skeleton of an adult male was found in an oval pit, covered with a substantial pile of unmodified sandstone rocks. The disarticulated and fragmented remains, represented by 521 elements and fragments (see Appendix F), were found in a small cluster representing a bundle burial, which originally may have been inside a container or wrapped. The remains are too incomplete for most osteological purposes, but no unusual pathological conditions were noted. The only identifiable grave inclusions are a large corner-notched Scallorn arrow point, the 
orientation of which suggests that an arrow was laid across the bone bundle, and a modified hematite fragment (i.e., a paint stone). It dates to ca. A.D. $240-400$ and may be transitional between the late Archaic and Late Prehistoric periods.

This burial appears to be unrelated to the early occupation period and apparently predates it by at least 200 years. Two burials that are contemporaneous with and culturally similar to the early occupation period at the Sam Wahl site suggest that secondary burials should not be expected during this time. A subfloor burial in a pithouse at the Kent Creek site (Cruse 1992:54-55) and a burial in Deadman's Shelter (Willey and Hughes 1978:154) are both primary flexed or semiflexed burials with similar grave inclusions (i.e., mussel shells and modified deer bones were found in both). The Kent Creek burial, in Hall County, probably occurred at approximately the same time that the structure was abandoned, ca. A.D. 590-830 (Cruse 1992:124). The tip of an arrow point found beneath the left shoulder could have been the cause of death (Cruse 1992:55), which is interesting given the evidence of violence in other Late Prehistoric burials in the region (discussed below). The Deadman's Shelter burial, in Swisher County, could date to the earlier occupation of the shelter, ca. A.D. $60-250$, but it more likely is intrusive from later occupations, ca. A.D. 395-775 (Willey and Hughes 1978:154, 187). The secondary burial at 41GR291 is quite different from these and may reflect the mortuary practices of an earlier unrelated group.

The Feature 23 burial is somewhat unusual for the Southern Plains because, although isolated cairncovered burials are relatively common, secondary bundle burials are rather rare. Owsley (1989:128) lists only 10 sites with secondary bundle burials in his bioarcheological data base, which includes all of the Texas Panhandle-Plains, western Oklahoma, and southwestern Kansas. Although an exhaustive literature search was not conducted, a number of burials in the Southern Plains are similar to the 41GR291 burial and warrant mention. Of particular interest are two bundle burials found in the upper part of "Roberts Covered Mound \#3" near Abilene, Texas. Ten burials were excavated and reported by Cyrus Ray (1933) and apparently represent separate rockcovered interments (eight primary and two secondary) within a small area that appears to be a mound. One of the bundle burials had no grave inclusions, but an Alba-like arrow point was found among the bones of the second bundle burial. This arrow point (Ray 1933:Plate 8) is apparently as large (ca. $60 \mathrm{~mm}$ in length) as the specimen found with the Feature 23 burial. Ray (1933:19) notes that the skull from this bundle burial (described in Hooten [1933] as RM-3S10) was "of a type never before found in a stone slab grave" in the Abilene region. Another interesting point is that although no diagnostic artifacts were associated with any of the primary burials in Roberts Covered Mound \#3, they are likely of Late Prehistoric age, and five of the individuals had missing mandibles. Ray (1933:19-20) notes this unusual trait and suggests that the jaws were removed as war trophies.

Three burials, including one bundle burial, in Shackelford County (Forrester 1951) have large (ca. 60-75 cm long), stemmed, Alba-like and Sabinallike arrow points associated (Forrester [1987] now suggests that all of these stemmed points should be typed as Moran arrow points and notes that they generally occur in burials as the cause of death). Burial Plot II was a rock cairn containing two individuals who apparently met violent deaths and were buried together. Skeleton 1 was a 20 -year-old male, semiflexed, with an arrow point found near the right hip. The charred mandible of a child was found in the chest area of Skeleton 1. Its context and the absence of any other juvenile bones suggest that it could have been worn as a pendant (a war trophy?). Skeleton 2, an incomplete bundle burial of an 18year-old male, was found between the knees and shoulders of flexed Skeleton 1. A stemmed arrow point was found embedded in the distal end of its right humerus (i.e., the elbow). Forrester (1951:136) notes that the distal end of the right ulna had cut marks and speculates that Skeleton 2 may have had a hand removed as a war trophy. A third burial nearby, Skeleton 4 in Burial Plot III, had yet another stemmed arrow point that was not found with the other grave goods (i.e., a cluster of modified deer bone tools and blanks) and may have been the cause of death.

Several caim burials have been found in and near O. H. Ivie Reservoir in Concho, Coleman, and Runnels counties (Lintz et al. 1994:Chapter 4), and two are probably contemporaneous with the 41GR291 burial. One primary burial (41CN94), excavated by the Concho Valley Archeological Society, is similar to the Shackelford County burials and yielded a Sabinal arrow point (J. A. Jaquier, personal communication 1993). At 41CC237, a child cremation and 
a probable secondary burial of an aduit male probably were interred at the same time in a single cairn. Although the artifacts found in the burial pit (i.e., thin bifacial preforms, unifacial tools, debitage, modified limonite fragments, and 19 Olivella shell beads) are not temporally sensitive, a radiocarbon date of A.D. $280-570$ on bone collagen from the adult skeleton indicates that this caim is probably contemporaneous with the 41 GR291 cairn burial. A possible cut mark observed on one rib may be evidence of dismemberment (Lintz et al. 1994:665675). Notably, the mandible was among the elements missing from this adult skeleton.

Several other primary (flexed or semiflexed) burials have large early-style arrow points found in contexts that indicate they may have been the cause of death. Seven arrow points found with two individuals in a cairn-covered "double burial" in Donley County (Witte 1955) are large Scallorn-like specimens with expanding stems and serrated blades. Collins $(1968: 68-73,167)$ reports that Burial 3 at the Salt Cedar site in Andrews County was an adult male with 187 Olivella shell beads, a Canis claw, several pigment stones including hematite, and 9 arrow points. The arrow points, one of which was embedded in a vertebra, include several fragments of barbed points, a stemmed specimen similar to Alba or Bonham, a stemmed specimen similar to the eddy variety of Scallorn, and a Harrell point (Collins 1968:Figure 18).

A few other primary and secondary burials in the region are of interest. Two primary burials have yielded early-style arrow points but lack any evidence of violence and could be temporally and/or culturally similar to the Sam Wahl site burial. An infant burial in Potter County yielded a "Scallornlike" arrow point, an obsidian "pendant," and numerous bone, shell, and hackberry seed beads (Harrison and Griffin 1973). Stickney and Stickney (1984:53-55) report an isolated burial in Crane County (41CR4) that contained three corner-notched points which might be small dart points or large arrow points. Bundle burials reported by Hughes (1942:39-41) at the Harrell site in Young County are probably of Late Prehistoric age, but no associated diagnostic artifacts were recovered.

One multiple burial in western Oklahoma is contemporaneous with the 41GR291 burial. Gettys (1991) reports that Burial 2 at 34RM668 in Roger Mills County consisted of three flexed skeletons in a single interment. One of the individuals was beheaded prior to burial, and corner- and sidenotched dart points associated with two individuals may have been the cause of death. Two radiocarbon dates on bone collagen indicate that these individuals were killed and buried between A.D. 210-440 (Oklahoma Archeological Society 1993).

At Lake Altus in Greer County, Oklahoma, numerous burials from two sites (34GR5 and 34GR6) represent a Late Prehistoric cemetery with evidence of violent deaths (Agogino and Button 1985; Boyd 1982; Button and Agogino 1987; LeVick and LeVick 1966). According to Button and Agogino (1987), 26 individuals recovered from these sites are thought to be from the early Plains Village period, and some or all of them may be associated with the Custer phase. A single burial is dated to A.D. 470-650, and a multiple interment of five individuals is dated to A.D. 590-770 (Button and Agogino 1987:19, 31), but the precise age of the others is not known. At least one of the interments is a bundle burial (and perhaps as many as five are secondary), and it had a sherd of Stafford Cordmarked pottery in its fill (Boyd 1982: 10-16; Button and Agogino 1987:31). Two cases of violent deaths are represented in the Greer County burials. One individual, possibly a bundle burial, had an untyped fragmentary arrow point embedded in its ulna (Boyd 1982:15-16; LeVick and LeVick 1966), and one of the five individuals in the multiple burial had received a fatal blow to the head. The composition of this group - an elderly male with the head injury, two juvenile males, a female child, and a middle-aged female-led Button and Agogino (1987:30-31) to speculate that the entire group had been killed.

All of the burials mentioned above are similar to the Sam Wahl site burial either because they are bundle burials or because they could be roughly contemporaneous. Beyond these broad similarites, however, there do not appear to be any strong parallels in mortuary characteristics or material culture (e.g., no Scallorn points exactly matching the 41GR291 specimen have been reported). The Feature 23 burial appears to be unique in many respects and may not be related culturally to any of the reported burials in the region. The regional mortuary data do suggest that violence was prominent during the Late Prehistoric period. Given the relative scarcity of Late Prehistoric bundle burials (bundle burials account for only ca. 5\% of Owsley's [1989:128] osteological sample), perhaps this was not a culturally distinctive mortuary practice but was a 
necessary procedure in situations where people met violent or accidental deaths and their remains could not be interred immediately. It may not be purely coincidental that evidence of violence is present at several sites where bundle burials occur in the Southern Plains (e.g., Forrester 1951; Hughes 1942; Ray 1933). The possibility that human mandibles were taken as war trophies during Late Prehistoric times seems likely, and Hughes $(1969: 39,53)$ notes that an isolated child's mandible recovered in Level 4 of the Canyon City Club Cave dates to ca. A.D. $300-680$.

\section{Early Occupation Period}

The early occupation period is characterized by a complex of pithouse, storage pit, and hearth/baking pit features. Archeological evidence from the nearby Kent Creek site indicates that at least two (and possibly three) pithouses are approximately contemporaneous with a storage pit (Feature 8) and a large hearth/baking pit (Feature 5). Cruse (1992:125) suggests that these features date to ca. A.D. 6901010. Thus, the pithouse/storage pit/baking pit complex at the Kent Creek site is contemporaneous with the early occupation period at the Sam Wahl site. In addition, a fourth feature type-bedrock mortars - also may be associated with this feature complex at the Sam Wahl site.

The pithouse (Feature 37$)$ is a small $(3 \times 3.5 \mathrm{~m})$, shallow, oval basin filled with cultural debris and charcoal-stained sediment and is radiocarbon dated to ca. A.D. 1000-1050. Definite postholes and an entryway were not identified, but the floor was easily defined since it was dug down into sandstone bedrock. Three large sandstone metates appear to have been cached in the structure. This pithouse is interpreted as an ephemeral structure which may have been little more than a brush- or hide-covered hut (i.e., something akin to a wickiup). Macrobotanical remains recovered from flotation of sediments from on and near the floor provide evidence that mesquite beans were utilized as a food resource at the time the pithouse was last occupied or abandoned (see Appendix E). In addition, a pestle fragment from the floor suggests that bedrock mortars may have been used contemporaneously with the pithouse.

While circular or ovate pithouses are not necessarily distinctive of a particular culture or region, they appear to be quite rare in Plains Woodland or Plains Village sites, which are generally characterized by square or rectangular structures. In the Texas Panhandle, no Woodland period structures have been identified for the Lake Creek complex (Hughes 1962, 1991:25), and Plains Village structures of unknown affiliation at the Greenbelt site in Donley County are rectangular (Campbell 1983:39-41). Although circular and oval structures are found in the Antelope Creek phase of the northern Panhandle (Brooks 1989:80-81; Lintz 1986:85-148), they are always slab lined and are quite different from the 41GR291 pithouse. Other than slab-lined structures, circular or ovate pit structures are described for only two Plains Woodland or Plains Village complexes in the Southern Plains (Brooks 1989; Hofman and Brooks 1989). The Keith phase (or focus) of northcentral Kansas is characterized by "basin-like pit houses" 4.0 to $5.5 \mathrm{~m}$ in diameter (Hofman and Brooks 1989:65). Wedel (1986:85) suggests that Keith phase houses were "lightly built shelters of poles, presumably covered with rush mats, thatch, hides, or other perishables." A possible Plains Village pit structure - "an oval floor area measuring 6.6 by $4.8 \mathrm{~m}$ within a saucer-like depression" - at the Glass site may be associated with the Henrietta complex of north-central Texas, but it more likely was constructed by "later occupants of the site" (Brooks 1989:85; also see Lorrain 1967). The problem with these two comparisons is that the Keith focus is located over $650 \mathrm{~km}$ to the north of Lake Alan Henry, and pithouse architecture is not defined for the Henrietta complex (Brooks 1989:85). Hofman and Brooks (1989:67) note that no definite structural remains are associated with Plains Woodland sites in central or western Oklahoma, and Brooks (1989:76, 78) notes only square or rectangular structures for the Plains Village period in that region.

The closest architectural parallels to the Sam Wahl site pithouse are in the Jornada Mogollon region, less than $350 \mathrm{~km}$ to the west and southwest. Features of similar size and morphology discovered at Keystone Dam in the El Paso area (Carmichael 1985:142-18; O'Laughlin 1980:135-149) are interpreted as ephemeral pithouses and are dated to ca. 550 B.C. to A.D. 150 (O'Laughlin 1980:149) and ca. A.D. 1000-1400 (Carmichael 1985:149-150).

Miller and Stuart (1991:25-36, 52) report two ovate Mesilla phase pithouses (i.e., Structures 1 and 2 at BK4) in Dona Ana County, New Mexico, that are slightly larger than the Sam Wahl site pithouse but are similar in configuration and are radiocarbon dated to between A.D. 540-660. Closer to the South 
Plains, Wiseman (1981:174-175) describes a similar circular pithouse at the King Ranch site on the Pecos River near Roswell. This structure is slightly smaller, with a floor area of $5.1 \mathrm{~m}^{2}$, than the 7.0 $7.5-\mathrm{m}^{2}$ floor area of the Feature 37 pithouse. Like Feature 37, the King Ranch structure lacks evidence of an entryway or superstructure (i.e., postholes), and it has a slightly off-center hearth defined only by a 2-cm-thick charcoal stain. Wiseman (1981:192-193) suggests that it dates to A.D. $1150-1250$ or 1300 but notes that circular pithouses at several sites in the Jornada Mogollon region (e.g., Green 1955; Hammack 1962; Honea 1965; Lehmer 1948:26-27; O'Laughlin 1980:135-149; Whalen 1977:164-168, 1978:170-174) generally date much earlier than the King Ranch structure.

Circular pithouses also have been reported as far east as south-central Lea County, ca. $240 \mathrm{~km}$ west of the Lake Alan Henry project area. Leslie (1965) reports a $6.5-\mathrm{x}-6.2-\mathrm{m}$ pithouse excavated at the Merchant site. Hughes (1991:27-29) notes that the cultural affiliation of village sites in southeastern New Mexico (i.e., the eastern extension of the Jornada Mogollon proposed by Corley [1965a, 1965b]) is uncertain but suggests that pithouses are characteristic of the Maljamar phase, which dates to ca. A.D. 1100-1300. This temporal assessment corresponds with some of the Keystone Dam pithouses (Carmichael 1985:149-150) and with Wiseman's (1981) suggested age of the King Ranch pithouse. While it has been proposed that circular pithouses are generally earlier than rectangular ones in the Mogollon region (Anyon 1980:166; Martin 1979:66; Whalen 1980a:370), there is clear evidence that they were used until quite late in southeastern New Mexico and western Texas.

The 10 storage pits (Features 17, 18, 19, 20, 24, $25,30,31,32$, and 39) exhibit a great deal of uniformity. All were located within a small area $(8 \times 8 \mathrm{~m})$, and they appear to have been systematically spaced in rows. This patterned cluster of pits is interpreted as a specialized activity area since it is likely that some (or perhaps all) of the pits were in use at the same time. Although only two of these pits are radiocarbon dated, all are considered to be associated with the early occupation period because of their consistent morphology, patterned arrangement, and stratigraphic positions below late occupation period Feature 12.

The pits are generally cylindical and are ca. $1 \mathrm{~m}$ wide at the mouth and $50-80 \mathrm{~cm}$ deep. These well- constructed pits were dug down into the friable sandstone bedrock, and as such they may have provided solutions to some of the serious problems associated with pit storage, such as controlling moisture, insects, and rodents (Wedel 1986:122-123). Of particular interest is the apparent caching of large basin metates on top of two of the pits, supporting an interpretation that they were used for storage of plant foods. Macrobotanical remains recovered from the storage pits include charred Chenopodium and grass seeds (see Appendix E), but it is unclear whether these remains represent plants used to line the pits, stored plant foods, or materials introduced at the time of or after abandonment.

Storage pit size and morphology are highly variable across North America, and cylindrical pits, such as those at the Sam Wahl site, are a common form and cannot be considered specific to a particular culture or region, as the following examples illustrate. Storage pits at a site in North Carolina are described as being "circular in outline" with sides that are "usually straight" and average "3 feet in diameter and 3 feet deep" (Ward 1985:86). In the Central Plains, Wedel (1986:122) states that some storage pits are "vertical flat-floored cylindrical holes that seldom exceed one meter in depth and floor diameter." Cylindrical storage pits are common in Plains Village period sites in western Oklahoma, the Texas Panhandle, and southern Kansas (Brooks 1989:76, 78, $81,88)$. Cylindrical to very slightly bell shaped storage pits are common in Henrietta complex sites in north-central Texas (Brooks 1989:85), and those at the Coyote (Woodall 1967:18-20) and Glass (Lorrain 1967:28) sites are good examples. Storage pits associated with the Custer phase are "cylindrical in form with a flat bottom, averaging about $1 \mathrm{~m}$ in diameter and ranging from .5 to $1 \mathrm{~m}$ in depth," and those of the Washita River phase are similarly described (Brooks 1989:76-78). Storage pits also are common in the Mogollon area of the Southwest (Martin 1979:67; Wiseman 1991:Table 8). Excavations at the Bent site in Otero County, New Mexico, revealed slightly bell shaped to straight-sided pits with diameters ranging from 50-165 cm and depths of $90-140 \mathrm{~cm}$ (Wiseman 1991:11, 17, Table 2, Figures 6-8).

Clearly, cylindrical and slightly bell shaped storage pits are common in many areas, and minor variations in size and morphology do not appear to be particularly diagnostic characteristics. Given the wide archeological distribution of subterranean 
storage pits, along with the extensive ethnographic evidence for their use (e.g., Raymer 1988), attempts to define culture-specific attributes should be made cautiously.

In many areas, storage pits are found both inside and outside structures (e.g., Brooks 1989:78, 81; Martin 1979:67), but clusters of three or more extramural storage pits do not appear to be common in the Southern Plains. Although no extensive literature search was undertaken and there may be more cases, a cluster of storage pits similar to those at the Sam Wahl site was found ca. $340 \mathrm{~km}$ northeast of Lake Alan Henry. Woodall (1967:17, Figure 18) reports a "cache pit cluster" of seven pits in a linear arrangement within an 11-x-4-m area at the Coyote site on the Red River in Montague County, Texas. No structures were located (a few isolated storage pits and hearths were the only other features reported), but the pits are quite similar to those at 41GR291. The Coyote site pits are circular in plan view, ranging from 118 to $200 \mathrm{~cm}$ in diameter, and are straight walled, ranging in depth from 45 to $80 \mathrm{~cm}$ (Woodall 1967:17-20, Figures 18 and 19). Unlike the pits reported here, most of the Coyote site pits were filled with trash (Woodall 1967:17-20). The site is dominantly Late Prehistoric in age as indicted by abundant ceramics and Scallorn, Alba, Fresno, and Harrell arrow points, with the latter being most frequent. No dates are available for the Coyote site, but projectile points and pottery found in the fill of the pits (Woodall 1967:Table 2) link them with the Late Prehistoric occupation(s). Projectile points recovered from the pits include an Alba (in Feature 6), a Harrell (in Feature 4), and seven Fresnos (three in Feature 1, two in Feature 4, and two in Feature 11). Sherds of Nocona Plain $(n=107)$ were recovered in six of the seven pits. Most of these materials suggest that the Coyote site pits are approximately contemporaneous with the late occupation period at the Sam Wahl site, since the Harrell and Fresno points and Nocona Plain pottery apparently date no earlier than ca. A.D. 1200 (Prikryl 1990:60-62, 80). The Alba style, however, appears to date to ca. A.D. 700-1200 in north-central Texas (Prikryl 1990:58, 62) and could indicate that some of the Coyote site pits are contemporaneous with the early occupation period at 41GR291.

Two burned rock concentrations (Features 29 and 41) are classified as hearths/baking pits and are associated with the early occupation period by radiocarbon dates. While the exact function of these features is uncertain, they appear to be intrusive pits with charcoal staining and multiple layers of burned sandstone rocks. One (Feature 41) was partially disturbed, while the other (Feature 29) was nearly intact with a layer of rocks capping a layer of charcoal-stained sediment and a lower cluster of burned rocks. These features are interpreted as baking pits and probably were used for cooking plant foods (see Chapter 4 for a more detailed discussion of baking pits). Macrobotanical remains recovered from flotation do not provide evidence of what may have been cooked in the features, but charred fragments indicate that juniper was the dominant wood fuel (see Appendix E).

Bedrock mortars are a fourth feature type that probably is associated with the early occupation period. While the two pointed-oval mortars found at the Sam Wahl site are not dated and cannot be linked positively to the early occupation period, a general association is made based on the presence of pestles found in excavations, particularly a pestle fragment found in the pithouse fill. This suggests that mortars were approximately contemporaneous with the occupation of the pithouse.

Collectively, the early occupation period features appear to represent a set of activities that occurred from perhaps ca. A.D. 600 to 1050 . They are interpreted generally as representing a residential site occupied intermittently and perhaps seasonally where the inhabitants were involved in a variety of activities related to the procurement, cooking, consumption, and storage of plant foods. A more detailed discussion of the specific plants that may have been utilized is presented later in this chapter.

\section{Late Occupation Period}

Only two features can be associated confidently with the late occupation period. Charcoal/burned rock scatter Features 12 and 21 have been radiocarbon dated to ca. A.D. 1160-1380, and they occur stratigraphically higher than all of the early occupation features. While the function of Feature 21 is unclear, it probably represents a series of disturbed burned rock features and/or refuse dumped on a living surface. It has been suggested that Feature 12 could represent a structural floor, but if so it would have been a very different type of structure (i.e., a surface house) than the early occupation pithouse. The stratigraphic relationship between Feature 12 and the underlying pits (Features 17 and 18) clearly indicates 
that some of the storage pits were abandoned prior to ca. A.D. 1150. A third charcoal/ burned rock scatter, Feature 16, also may be associated with the late occupation period. As mentioned above, however, this association is problematic given the nature of the radiocarbon date on soil organics. Macrobotanical remains associated with Features 12 and 21 include a wide variety of charred seeds of unknown origin (i.e., they could reflect plants utilized as food resources, bumed as fuel, or accidentally burned), but juniper was the dominant wood fuel (see Appen$\operatorname{dix} E)$.

While the nature of the feature-related activities during the late occupation period is unclear, there appear to be some substantial differences from the earlier pithouse, storage pit, and hearth/baking pit complex. Consequently, the late occupation period, ca. A.D. 1150-1400, may reflect different subsistence and land-use practices than those of the earlier occupations. The material culture, described below, appears to support the interpretation of two distinct occupation periods and exhibits significant differences that appear to reflect changes in site function.

\section{Material Culture}

The 3,578 artifacts described in this report constitute $90 \%$ of all of the materials recovered from the Sam Wahl site (Table 20). All of the artifact classes and types represented by the materials recovered during the survey and testing phases are represented by materials recovered during the Phase III investigations, with the exception of two specimens that expand the data recovery classification/ typology. One is a Deadman's arrow point fragment that was recovered during the 1988 testing phase, but since it was found in an isolated unit (Test Unit 6, now EU 112), its association with the main part of
TABLE 20

ARTIFACTS RECOVERED FROM ALL PHASES OF WORK AT 41GR291

\begin{tabular}{|c|c|c|c|c|}
\hline Artifact Class & $\begin{array}{l}\text { Phase I } \\
\text { Survey }\end{array}$ & $\begin{array}{l}\text { Phase II } \\
\text { Testing }\end{array}$ & $\begin{array}{l}\text { Phase III } \\
\text { Data Recovery }\end{array}$ & Totals \\
\hline Chipped Stones & & & & \\
\hline Arrow points & - & $4^{*}$ & 26 & 30 \\
\hline Dart points & - & $1 * *$ & 2 & 3 \\
\hline Perforators & - & - & 3 & 3 \\
\hline Gouges & - & 2 & 18 & 20 \\
\hline Nonprojectile point bifaces & 1 & 9 & 16 & 26 \\
\hline Cobble tools & - & 7 & 38 & 45 \\
\hline Unifaces & - & 17 & 80 & 97 \\
\hline Cores & - & 8 & 73 & 81 \\
\hline Edge-modified flakes & - & 17 & 37 & 54 \\
\hline Unmodified debitage & 3 & 304 & 3,134 & 3,441 \\
\hline Ground and Battered Stones & & $\therefore$ & & \\
\hline Manos & 1 & 11 & 35 & 47 \\
\hline Metates & - & - & 65 & 65 \\
\hline Indeterminate-use ground stones & - & 2 & 11 & 13 \\
\hline Anvils & - & - & 1 & 1 \\
\hline Pestles & - & - & 6 & 6 \\
\hline Hammerstones & - & 2 & 4 & 6 \\
\hline Other Materials & & & & \\
\hline Ceramics & - & 2 & 18 & 20 \\
\hline Modified hematite & - & - & 7 & 7 \\
\hline Modified shells & - & - & 4 & 4 \\
\hline Kaolinite pendant & - & 1 & - & 1 \\
\hline Totals: & 5 & 387 & 3,578 & 3,970 \\
\hline
\end{tabular}

the site is unclear. The other specimen is a kaolinite pendant fragment surface collected $50 \mathrm{~m}$ due south (downslope) of the pithouse (Boyd et al. 1990:392, 458-459, Figure 74). The pendant is unusual in that the high-quality kaolinite is visually identical to materials from the Burro Mesa outcrop in Big Bend National Park, which is currently the only known source for this material and was exploited prehistorically (Alex 1990). Although the pendant fragment probably is associated with one of the occupation periods at the Sam Wahl site, its age and relationship to the site are uncertain.

\section{Overview}

In functional terms, the material assemblage is 
varied and includes a wide range of bifacial and unifacial chipped stone tools, several different types of ground stone tools, and ceramics. Although the quantities of these artifacts are not large, particularly in view of the long temporal span of the occupations, they probably are indicative of a multifunctional residential base camp. There is considerable evidence that repeated and/or lengthy occupations occurred at the site. The stone artifacts, ranging from hammerstones and debitage to finished bifaces and arrow points, represent all stages of manufacture and indicate that bifacial and unifacial reduction techniques were employed. Many tool types, such as arrow points, nonprojectile point bifaces, and gouges, exhibit evidence of various stages of reworking, indicating that extensive tool refurbishing and reuse occurred on-site. Differential patination on some fine-grained chert tools indicates some time lapse between initial discard and reworking, which probably relates to repeated site use, although it could represent procurement of lithic resources from other (i.e., older) sites. Of particular interest, the ground stone assemblage is quite variable, including manos, metates, pestles, anvils, and indeterminate-use specimens, and is indicative of an intensive grinding technology. Five large complete metates and 15 complete manos exhibit extensive wear and evidence of refurbishing (i.e., pecking), indicating that they were in use for a long time. The caching of complete metates, the high frequency of ground stone fragments, and the recycling of useless fragments in feature contexts are further evidence of repeated or lengthy occupations. Related to the grinding technology is the suggestion, based on experimental replication of use wear, that many of the coarsegrained cobble tools were used to refurbish ground stone tools.

As might be expected at a residential site, some of the artifacts are related to personal adornment and/or jewelry manufacture and represent activities beyond mere subsistence. The kaolinite pendant is a unique decorative item that probably was obtained in trade, and the modified mussel shell fragments suggest that some jewelry was manufactured on-site. The modified hematite specimens, probably representing paint stones, also hint at artistic endeavors.

Although only 20 ceramic sherds have been recovered, three to five vessels are represented, and two distinctive paste groups are described. Paste Groups 1 and 2 are defined on the basis of compositional differences, and their validity was confirmed by Wiseman (1993), who provided typological identifications for the groups. A petrographic analysis of five of these sherds (see Appendix G), conducted independent of the descriptive/typological analyses, supports the definition of these paste groups and provides possible evidence of different manufacturing sources.

Petrographic Group 1 includes three sherds (Specimens 367-3, 472-1, and 521-1) from Paste Group 1 that were visually identified as Jomada Brown. The petrographic data for this group indicate that $36-40 \%$ of the paste is composed of nonplastic inclusions that are dominated by granitic rock comprising $14-23 \%$ of the nonplastics and its major constituent minerals-orthoclase and quartzcomprising $71-74 \%$ of the nonplastics. Petrographic Group 2 includes two sherds (Specimens 306-1 and 8-0) from Paste Group 2 that were visually identified as South Pecos Brown. Petrographic data for this group indicate that $41 \%$ of the paste is composed of nonplastics that also include orthoclase and/or quartz (comprising $27-60 \%$ of the nonplastics). The most important variation in the Petrographic Group 2 specimens, however, is the presence of basaltic rocks comprising $4-7 \%$ of the nonplastics. One of the samples also includes a large amount $(38 \%$ of the nonplastics) of celadonite, a mineral commonly occurring in basaltic rocks.

The petrographic analysis indicates a significant difference between the Group 1 (i.e., Jornada Brown) and Group 2 (i.e., South Pecos Brown) ceramic types in that the former is characterized by the exclusive use of crushed granitic rock temper while the latter also includes crushed basaltic rock temper. Granitic rocks are of intrusive igneous origin, basaltic rocks are of extrusive igneous origin, and the two generally are not found together (Prinz et al. 1978:416-417), although they can occur in close proximity. There are three important implications of the petrographic data.

First, the presence of igneous rock fragments confirms the nonlocal origin of these samples and indicates that the most likely general source area for these wares was the Jornada Mogollon area of southcentral and southeastern New Mexico and western Texas. Second, the petrographic data support the division of the 41GR291 ceramics into two types based on variability in composition. Although the petrographic evidence cannot confirm the type identifications, it does support the recognition of Jornada Brown and South Pecos Brown as distinctive ceramic types. 
The third implication is that the Group 2 specimens, which are defined by the presence of basaltic rocks and related mineral inclusions that may reflect a distinctive tempering, potentially can be linked geologically to a source area or manufacturing locality. No conclusive interpretations are offered, but it is possible that the Group 2 sherds originated from an area where extrusive basaltic rocks are abundant in either primary or secondary exposures. The closest potential source area is just north of the Tularosa Basin along the western edge of the Sierra Blanca Mountains (i.e., west of the town of Carrizozo), which is characterized by extensive Quaternary basalt flows (New Mexico Geological Society 1982). While there are other potential source areas where basalt flows are common, such as northeastern New Mexico and the Trans-Pecos region of Texas (New Mexico Geological Society 1982; Bureau of Economic Geology 1992), the basaltic area north of the Tularosa Basin is relatively close to the area where South Pecos Brown commonly is found, i.e., in the Pecos River valley south of Roswell (Jelinek 1967:53).

The presence of basaltic rocks in two sherds tentatively identified as South Pecos Brown does not indicate that this is a diagnostic trait for this ceramic type, nor does it positively identify a source area for the sherds. While the results of this limited study are not conclusive, they do provide data that suggest that some variants of the Jornada brownwares are distinctive enough to be recognizable petrographically and potentially can be linked to manufacturing source areas. As Kelley (1984:122) notes, "one of many problems facing future archeologists is the adequate typological and geographical differentiation of the various brown pottery types." While some researchers have lamented the many problems of visually classifying brownware ceramics into ill-defined types, others have noted that petrographic analysis has "a tremendous potential" for unraveling some of the complexities (Dulaney and Pigott 1977:274-275).

In terms of lithic raw materials, the chipped stones at the Sam Wahl site are dominantly of local origin (i.e., materials originating in the Quaternary Lingos gravels that are ubiquitous in the project area; see Chapter 2). Using only the data recovery artifacts, 92\% ( $\mathrm{n}=3,164)$ are of local materials, while only $7 \%(\mathrm{n}=230)$ are of nonlocal origin, and ca. $1 \%$ are of indeterminate origin (Table 21). Although the overall percentage of nonlocal materials is low, there was a preference for the use of selected materials for certain types of artifacts. Gouges and cobble tools, for example, are made almost exclusively of coarser-grained local materials, in contrast

TABLE 21

SUMMARY OF PHASE III CHIPPED STONES BY MATERIAL TYPE AND ORIGIN, 41GR291

\begin{tabular}{|c|c|c|c|c|c|c|c|c|c|}
\hline \multirow[b]{2}{*}{ Artifact Type } & \multicolumn{2}{|c|}{ Local } & \multicolumn{4}{|c|}{ Nonlocal } & \multirow{2}{*}{$\begin{array}{l}\text { Indeterminate } \\
\text { Fine- } \\
\text { grained } \\
\text { Chert }\end{array}$} & \multirow[b]{2}{*}{ Totals } & \multirow[b]{2}{*}{$\begin{array}{l}\% \\
\text { Nonlocal }\end{array}$} \\
\hline & $\begin{array}{l}\text { Fine- } \\
\text { grained } \\
\text { Chert }\end{array}$ & Other* & $\begin{array}{l}\text { Fine- } \\
\text { grained } \\
\text { Chert }\end{array}$ & $\begin{array}{l}\text { Alibates } \\
\text { Agate }\end{array}$ & $\begin{array}{l}\text { Tecovas } \\
\text { Jasper }\end{array}$ & Obsidian & & & \\
\hline Arrow points & 19 & 1 & 5 & - & - & - & 1 & 26 & 19.2 \\
\hline Dart points & 2 & - & - & - & - & - & - & 2 & 0.0 \\
\hline Perforators & 3 & - & - & - & - & - & - & 3 & 0.0 \\
\hline Gouges & 8 & 9 & - & - & - & - & 1 & 18 & 0.0 \\
\hline Bifaces & 9 & 1 & 4 & - & - & - & 2 & 16 & 25.0 \\
\hline Cobble tools & 3 & 35 & - & - & - & - & - & 38 & 0.3 \\
\hline Unifaces & 38 & 28 & 11 & - & 3 & - & - & 80 & 17.5 \\
\hline Cores & 35 & 32 & 1 & - & 1 & - & 4 & 73 & 2.7 \\
\hline Edge-modified flakes & 26 & 6 & 4 & - & 1 & - & - & 37 & 10.8 \\
\hline Unmodified debitage & 1,729 & 1,179 & 161 & 1 & 36 & 2 & 26 & 3,134 & 6.4 \\
\hline Totals: & 1,872 & 1,291 & 186 & 1 & 41 & 2 & 34 & 3,427 & 6.7 \\
\hline
\end{tabular}


to projectile points, perforators, and bifaces, which are made almost exclusively of fine-grained cherts. The arrow point, biface, uniface, and edge-modified flake categories have the highest percentages of nonlocal materials (i.e., greater than $10 \%$ each).

Nonlocal materials in the Phase III assemblage include $5 \%$ fine-grained chert and 1\% Tecovas jasper. The chert specimens exhibit colors that are representative of nonlocal Cretaceous origin, and most of them apparently came from primary or secondary outcrops along the Callahan Divide, ca. $75-100 \mathrm{~km}$ south of the project area. The Tecovas jasper probably came from primary outcrops located $75 \mathrm{~km}$ or less to the north of the project area. Since the sources of these materials are relatively close, it is likely that the nonlocal cherts and Tecovas jasper were obtained directly by the Sam Wahl site inhabitants. The other two nonlocal materials, Alibates agate and obsidian, are represented by less than $0.1 \%(\mathrm{n}=3)$ and were brought in from greater distances. The primary source for Alibates agate is $290 \mathrm{~km}$ north of the project area, and the obsidian was identified as being from Malad, Idaho. These materials may not have been obtained directly by the Sam Wahl site inhabitants but probably were procured indirectly through trade.

The overall variability in the material culture assemblage indicates a variety of activities and supports the interpretation that, except for the isolated burial, the Sam Wahl site served as a multifunctional residential site. Feature analysis and radiocarbon dating demonstrate that a variety of activities occurred over an extended period of time, perhaps from ca. A.D. 600 to as late as A.D. 1300, but within this time frame the picture gets more complex. It has been suggested that two discrete periods of occupation are represented and that significant changes in site function may have occurred at ca. A.D. 10501150. The recognition of substantial differences between the early and late occupation periods has many implications for interpreting subsistence strategies and settlement patterns. The separation of the artifacts into corresponding assemblages, then, becomes a crucial issue for interpreting 41GR291.

\section{Comparisons of Early and Late Occupation Artifact Assemblages}

Unlike the features, the cultural materials are much more difficult to sort into interpretable temporal/analytical units. Except for a Scallorn arrow point and a modified hematite stone directly associated with the burial and a few artifacts mixed in the burial fill, the remainder of the cultural materials cannot be easily separated into assemblages based on archeological contexts. There is essentially a single cultural zone throughout the site, and no natural stratigraphic units could be defined. Thus, the only hope for separating the cultural materials into meaningful assemblages is to split the cultural zone by arbitrary excavation levels that roughly correspond to the time periods indicated. Since the cultural zone was generally $25-35 \mathrm{~cm}$ thick and appears to represent pedogenically altered sediments on a slowly aggrading surface, the potential for separating cultural materials into gross assemblages roughly corresponding to the early and late occupation periods was good. The method of defining these assemblages is described below.

The first step was to define the analysis universe. Initially it was decided to limit the assemblage analysis to the materials recovered in the excavation blocks, thereby excluding all surface collections and materials recovered in mechanical excavations or isolated excavation units. The materials recovered from 1988 Test Unit 1, now designated as EU 99 in Block 5, also were added to the analysis. By adding and excluding artifacts from these proveniences, the analysis data set included only those artifacts that could be associated with excavation levels that are consistent from block to block and eliminated those that could not be correlated. Within the excavation blocks, except for unusual cases (e.g., intrusive features such as the pithouse and storage pits), all cultural materials were contained in the upper four (and occasionally five) 10-cm excavation levels. Criteria for separating the artifact assemblages were developed on a block-by-block basis.

In Block 1, all of the cultural materials are considered to be related to the pithouse occupation(s) or postoccupational filling of the structural depression. Since the pithouse fill probably represents a rapid accumulation that occurred soon after abandonment, all of the materials from Block 1, regardless of level, are considered to be roughly contemporaneous. There is no evidence of later occupations in the vicinity of the pithouse. Thus, all Block 1 materials are assigned to the early occupation assemblage.

For all of the other blocks, the cultural materials are separated by arbitrary excavation levels. The late occupation features consistently appear in Levels 1 and 2 and are confined mainly to the upper 
20-25 cm. Conversely, the early occupation features consistently appear somewhere in Level 3 or the top of Level 4 and are generally below $20-25 \mathrm{~cm}$. Although the excavation levels follow absolute elevations rather than the slope of the modern ground surface, it appears that the contact between Levels 2 and 3 closely approximates the transition between the early and late occupation periods. By separating the artifacts at the Level $2 / 3$ contact, which varied from $15-24 \mathrm{~cm}$ below the surface because of variations in surface elevations, the materials were grouped into assemblages that grossly correspond with the early and late occupation features. It is acknowledged that this break is an arbitrary one and that some artifacts from Levels 1 and 2 could go with the early occupation and that some late occupation materials could be found below Level 2. Lacking any natural stratigraphic breaks, however, dividing the cultural materials in this manner is an appropriate means for defining gross assemblages that, allowing for some degree of error, generally correspond to the different time periods. Thus, all materials from Levels 1 and 2 in Blocks $2-5$ are assigned to the late occupation assemblage, and all materials from Levels 3, 4, and 5 in these blocks are assigned to the early occupation assemblage.

Only two exceptions to the above rule were made. First, the artifacts recovered from the fill of all of the storage pits were included in the early occupation assemblage for Block 2. The materials from Features 31 and 32 were included in Block 2 although they are not actually in the block. This exception was made because all of the storage pits are considered to date to the early occupation period. The second exception is that one artifact from Block 4 , Level 3 was excluded from the data base. It is an untypeable, reworked dart point stem fragment (see Figure $22 b$ ), but its occurrence in the early occupation strata is problematic. There are at least three plausible explanations for its apparent association with the early occupation period. Dart points could have been in use during this time, or it may have been picked up from a nearby Archaic site and subsequently reused by the early occupation inhabitants. In either of these cases, the dart point actually belongs in the early occupation period. The third alternative is that the dart point indicates that there was an ephemeral Archaic occupation whose cultural materials cannot be separated from those of the early occupation period. Because of this uncertainty, this specimen was excluded from analysis. Notably, however, evidence that a previous Archaic component might exist at 41GR291 is limited to three untypeable dart point fragments (see Table 20), two of which were surface collected, and two of the three are reworked.

When the early and late occupation assemblages are defined, the analysis data set consists of 3,333 stone tools, constituting $85 \%$ of the total stone tools $(n=3,938)$ recovered from all phases of work (Table 22). The remainder of this section compares and contrasts the early and late occupation assemblages.

\begin{tabular}{|c|c|c|c|c|c|c|}
\hline \multicolumn{7}{|c|}{$\begin{array}{c}\text { TABLE } 22 \\
\text { TOTAL CHIPPED, GROUND, AND BATTERED } \\
\text { STONE TOOL SAMPLES FOR EARLY AND } \\
\text { LATE OCCUPATIONS AT 41GR291 }\end{array}$} \\
\hline \multirow[b]{2}{*}{ Block No. } & \multicolumn{2}{|c|}{$\begin{array}{c}\text { Early } \\
\text { Occupation }\end{array}$} & \multicolumn{2}{|c|}{$\begin{array}{l}\text { : Late } \\
\text { Occupation }\end{array}$} & \multicolumn{2}{|c|}{ Total } \\
\hline & No. & $\%$ & No. & $\%$ & No. & $\%$ \\
\hline 1 & 243 & 17.0 & - & - & 243 & 7.3 \\
\hline 2 & 275 & 19.2 & 944 & 49.7 & 1,219 & 36.6 \\
\hline 3 & 164 & 11.5 & 130 & 6.8 & 294 & 8.8 \\
\hline 4 & 462 & 32.3 & 704 & 37.0 & 1,167 & 35.0 \\
\hline 5 & 287 & 20.0 & 123 & 6.5 & 410 & 12.3 \\
\hline Totals: & 1,431 & 100.0 & 1,901 & 100.0 & 3,333 & 100.0 \\
\hline
\end{tabular}

Of the 3,333 stone tools in the analysis data set, 1,431 specimens (43\%) are assigned to the early occupation assemblage, and 1,901 specimens (57\%) are assigned to the late occupation assemblage. When the interblock distributions of the early and late occupation assemblages are compared, an interesting pattern emerges. While the average artifact frequencies remain fairly consistent from the early to late occupation periods in Block 3, there is an increase through time in the amount of materials in Blocks 2 and 4 and a decrease in Block 5 (Table 23).

The twofold decrease in artifact density in Block 5 suggests that this portion of the site saw limited refuse-generating activities or refuse disposal during the late occupation period. The features in Block 5 may date to both occupation periods; two are hearths/ baking pits (Features 29 and 41) that date to the early occupation period, and the third is a charcoal/ burned rock scatter (Feature 16) that could date to the late occupation period, although the latter association is not conclusive. Thus, there is evidence that cooking activities occurred in Block 5 during the 


\begin{tabular}{|c|c|c|c|c|}
\hline \multicolumn{5}{|c|}{$\begin{array}{l}\text { AVERAGE STONE TOOL RECOVERY PER SQUARE } \\
\text { METER FOR THE EARLY AND LATE } \\
\text { OCCUPATION PERIODS AT 41GR291 }\end{array}$} \\
\hline & \multicolumn{2}{|c|}{ Early Occupation Period } & \multicolumn{2}{|c|}{ Late Occupation Period } \\
\hline Block No. & $\begin{array}{c}\text { Area } \\
\text { Excavated }\left(\mathrm{m}^{2}\right)\end{array}$ & Artifacts $/ \mathrm{m}^{2}$ & $\begin{array}{c}\text { Area } \\
\text { Excavated }\left(\mathrm{m}^{2}\right)\end{array}$ & Artifacts $/ \mathrm{m}^{2}$ \\
\hline $\begin{array}{l}1^{*} \\
2^{* * *} \\
3 \\
4 \\
5^{* * * *}\end{array}$ & $\begin{array}{r}13 \\
37 \\
5 \\
28 \\
11\end{array}$ & $\begin{array}{r}18.7 \\
7.4 \\
32.8 \\
16.5 \\
26.1\end{array}$ & $\begin{array}{r}13 \\
30 \\
5 \\
28 \\
11\end{array}$ & $\begin{array}{c}- \\
31.5 \\
26.0 \\
25.1 \\
11.2\end{array}$ \\
\hline All Blocks & 94 & 15.2 & 87 & 21.8 \\
\hline \multicolumn{5}{|c|}{$\begin{array}{l}\text { *Excludes Excavation Units } 14 \text { and } 15 \text { which were almost totally } \\
\text { removed by the maintainer. } \\
\text { **Number of units is adjusted to account for maintainer blade removal } \\
\text { of some upper levels and counts seven partial units as three full } \\
\text { units. } \\
\text { *** Counts two half units as one full unit. }\end{array}$} \\
\hline
\end{tabular}

may have been kept clean or maintained during the early occupation period, although not to the extent of Block 2. Refuse-generating activities or disposal increased in Block 4 during the late occupation period, presumably after the abandonment of the storage area.

There do not appear to be any significant differences between the early and late occupation periods in terms of the lithic sources used (Table 24). Nearly equal frequencies of nonlocal materials are represented in both assemblages (i.e., $7.3 \%$ in the early occupation period vs. $5.7 \%$ in the late occupation period). In contrast, the various' artifact classes are not represented equally in the two assemblages, and this difference may be functionally related.

When the ground stones are included and the early and late occuearly occupation period, and these tasks may have been accompanied by refuse-generating activities such as stone tool manufacture and maintenance or refuse disposal. In contrast, the Block 5 area may have been utilized only sparsely during the late occupation period.

The strongest and perhaps most interpretable pattern is the fourfold increase in artifact density in Block 2, which indicates more refuse-generating activities or refuse dumping in the late occupation period. The late occupation period data show that Block 2 has only a slightly higher artifact frequency than do Blocks 3 and 4, while the early occupation data show that Block 2 has the lowest artifact frequency of all the blocks. One interpretation of this difference is that Block 2 served as a specialized storage area during the early occupation period and that this area was intentionally kept clean. This interpretation is logical in that one would not expect any refuse-generating activities or refuse disposal to have occurred in a storage area until it had been largely abandoned.

The $52 \%$ increase in artifact density in Block 4 also may be related to the early occupation storage pits, although this relationship is less clear. Relative to Blocks 1,3 , and 5, the early occupation artifact density in Block 4 is low, perhaps due to its close proximity to the storage area in Block 2. Block 4 pation assemblages are compared in greater detail, there are statistically significant differences in some classes of artifacts (Table 25). Most notably, the early occupation assemblage is characterized by a relative paucity of arrow points and debitage and a high frequency of ground stones compared to the late occupation assemblage. These differences have important implications for interpreting site function and suggest that subsistence activities may have changed between the early and late occupation periods. In general, the early occupation period might be characterized by a greater dependence upon plant processing (i.e., more ground stones), and the late occupation period may be characterized by an increase in the manufacture of chipped stone tools (i.e., more unmodified debitage) and a greater reliance upon hunting (i.e., more arrow points). These interpretations are supported by several other lines of evidence and are discussed in more detail in the following section.

In addition to the stone tools, artifacts of other material classes were sorted by occupation period (Table 26). Modified shells and modified hematite artifacts are associated with both occupation periods, and sherds of ceramic Paste Group 1 (i.e., Jornada Brown) and Paste Group 2 (i.e., South Pecos Brown) are found in approximately equal numbers in both assemblages. As previously mentioned, Paste Group 


\begin{tabular}{|c|c|c|c|c|c|c|c|c|c|}
\hline \multirow[b]{4}{*}{ Artifact Class } & \multicolumn{8}{|c|}{$\begin{array}{l}\text { TABLE } 24 \\
\text { AND LATE OCCUPATION CHIPPED STONE } \\
\text { BY MATERIAL ORIGIN, } 41 \text { GR } 291\end{array}$} & \multirow[b]{4}{*}{ Totals } \\
\hline & \multicolumn{4}{|c|}{ Early Occupation Period* } & \multicolumn{4}{|c|}{ Late Occupation Period** } & \\
\hline & \multicolumn{2}{|c|}{ Local } & \multicolumn{2}{|c|}{ Nonlocal } & \multicolumn{2}{|c|}{ Local } & \multicolumn{2}{|c|}{ Nonlocal } & \\
\hline & No. & $\%$ & No. & $\%$ & No. & $\%$ & No. & $\%$ & \\
\hline Arrow points & 1 & 0.1 & 3 & 3.1 & 17 & 1.0 & 1 & 1.0 & 22 \\
\hline Perforators & 1 & 0.1 & - & - & 1 & 0.1 & - & - & 3 \\
\hline Gouges & 3 & 0.2 & - & - & 8 & 0.5 & - & - & 11 \\
\hline Bifaces & 4 & 0.3 & 1 & 1.0 & 6 & 0.3 & 3 & 2.8 & 14 \\
\hline Cobble tools & 17 & 1.4 & - & - & 15 & 0.9 & - & - & 32 \\
\hline Unifaces & 23 & 1.9 & 5 & 5.1 & 40 & 2.3 & 8 & 7.5 & 76 \\
\hline Cores & 33 & 2.7 & - & - & 29 & 1.6 & 1 & 1.0 & 63 \\
\hline Edge-modified flakes & 14 & 1.1 & 2 & 2.0 & 16 & 0.9 & 3 & 2.8 & 35 \\
\hline Unmodified debitage & 1,140 & 92.2 & 87 & 88.8 & 1,626 & 92.4 & 90 & 84.9 & 2,943 \\
\hline Totals: & 1,236 & 100.0 & . 98 & 100.0 & 1,759 & 100.0 & 106 & 100.0 & 3,199 \\
\hline
\end{tabular}

\begin{tabular}{|c|c|c|c|c|}
\hline \multicolumn{5}{|c|}{$\begin{array}{l}\text { TABLE } 25 \\
\text { ARTIFACT COLLECTIONS FROM THE } \\
\text { CCUPATION PERIODS AT 41GR } 291\end{array}$} \\
\hline Artifact Class & Early Occupation Period & $\begin{array}{l}\text { Adjusted Standardized } \\
\text { Residuals for Early } \\
\text { Occupation Assemblage* }\end{array}$ & Late Occupation Period & Totals \\
\hline $\begin{array}{l}\text { Arrow points** } \\
\text { Bifaces } \\
\text { Perforators } \\
\text { Gouges } \\
\text { Cobble tools } \\
\text { Unifaces } \\
\text { Cores } \\
\text { Edge-modified flakes } \\
\text { Unmodified debitage } \\
\text { Ground stone tools } \\
\text { Hammerstones }\end{array}$ & $\begin{array}{r}4 \\
5 \\
1 \\
3 \\
17 \\
31 \\
33 \\
16 \\
1,242 \\
78 \\
1\end{array}$ & $\begin{array}{l}-2.35 \\
-0.95 \\
-0.34 \\
-1.26 \\
+1.17 \\
-0.67 \\
+1.41 \\
+0.33 \\
-3.77 \\
+7.69 \\
-0.34\end{array}$ & $\begin{array}{r}18 \\
11 \\
2 \\
9 \\
15 \\
48 \\
31 \\
19 \\
1,728 \\
18 \\
2\end{array}$ & $\begin{array}{r}22 \\
16 \\
3 \\
12 \\
32 \\
79 \\
64 \\
35 \\
2,970 \\
96 \\
3 \\
\end{array}$ \\
\hline Totals: & 1,431 & & 1,901 & 3,332 \\
\hline
\end{tabular}




\begin{tabular}{|l|l|l|}
\hline \multicolumn{3}{|c|}{ TABLE 26 } \\
COMPARISON OF OTHER ARTIFACTS IN THE \\
EARLY AND LATE OCCUPATION PERIOD \\
ASSEMBLAGES AT 41GR291 \\
\hline \multicolumn{2}{|c|}{ Early } & Late \\
& Occupation & Occupation \\
Artifact Class & Period & Period \\
\hline Paste Group 1 ceramics & 4 & 5 \\
Paste Group 2 ceramics & 4 & 6 \\
Modified hematite & 3 & 2 \\
Modified shells & 2 & 2 \\
\hline Totals: & 13 & 15 \\
\hline
\end{tabular}

1 sherds include specimens from feature contexts dated to both the early and late occupation periods. Four sherds are associated with pithouse Feature 37 and radiocarbon dates ranging from A.D. 900-1160, and four sherds are associated with charcoal/burned rock scatter Feature 12 and radiocarbon dates ranging from A.D. 1160-1380. None of the sherds definitely predate A.D. 900, although it is possible that some of the Paste Group 2 sherds are earlier.

While some stone tool classes appear to indicate that subsistence activities changed at A.D. 10501150 , the ceramic, shell, and hematite artifacts may indicate a degree of continuity between the early and late occupation periods. In particular, the presence of imported Jornada Mogollon ceramics during both occupation periods may be evidence that interregional mobility and/or exchange systems were maintained during this time.

\section{Subsistence Strategies}

One of the main research goals for understanding Southem Plains prehistory is to define changing subsistence strategies and their relationships to extemal environmental factors (i.e., changing climates). The data recovery excavations at the Sam Wahl Site provide some intriguing evidence for changing subsistence practices after the first millennium A.D.

\section{BURIAL COMPONENT (A.D. 240-400)}

The burial at 41GR291 (Feature 23) is perplexing for many reasons. It appears to predate the early occupation period, but it is unclear where it fits into the regional picture. A radiocarbon assay on bone
(A.D. 238-399) indicates that the burial dates within the time frame suggested for the late Archaic bisonhunting peoples in the Texas Panhandle-Plains (Hughes 1977, 1989; Lintz et al. 1991), and its associated Scallorn point shows some morphological characteristics similar to some of the comer-notched dart points associated with the late Archaic bison kills. The burial also dates to Dillehay's (1974:182) proposed bison Presence Period II (2500 B.C. to A.D. 500). The $\delta^{13} \mathrm{C}$ value of $-13.6 \%$ obtained on the collagen fraction of a bone from the 41GR291 burial may indicate that ca. $60 \%$ of the individual's diet was composed of $\mathrm{C}_{4}$ plants or animals that ate $\mathrm{C}_{4}$ plants. This interpretation is not conclusive, however, since this moderately high $\delta^{13} \mathrm{C}$ value probably resulted from a mixed diet that included wild $\mathrm{C}_{4}$ and CAM plants (e.g., Amaranthus spp. and Opuntia spp.) and may or may not have included $\mathrm{C}_{4}$ cultigens (e.g., maize) and/or bison.

\section{EARLY OCCUPATION PERIOD (A.D. 600 to 1050 )}

A considerable amount of evidence, much of it indirect or circumstantial, suggests that the early occupation period inhabitants were heavily involved with the procurement, processing, storage, and consumption of plant foods while they were at 41GR291. While it is unlikely that they excluded animal resources from their diet (e.g., a freshwater drum otolith may indicate the use of fish as a food resource), there is little evidence indicating that they were of much importance. Although the faunal remains certainly are biased by poor preservation, the relative paucity of arrow points and formal cutting and scraping tools suggests that hunting, butchering, and hide processing were not important activities prior to A.D. 1150. This does not imply that the early occupation inhabitants did not conduct such activities elsewhere, simply that they are not represented to any significant degree at this site.

All of the hearths/baking pits and storage pits, which are suggestive of plant food processing and utilization, are associated with the early occupation. This period also is characterized by abundant ground stone tools, including all five of the complete metates. The complete metates are directly associated with the pithouse and storage pits, and the nature and distribution of the early occupation ground stone assemblage has implications concerning site function and subsistence. 
Based on analyses of ground stones from archeological contexts in southwestem Colorado, Schlanger $(1990,1991)$ reaches several relevant conclusions: (1) complete tools are often found in use contexts (i.e., cached or left in primary activity areas), and structure floors generally have more complete specimens than other contexts; (2) as occupation length increases, the frequency of ground stones in reuse and discard contexts increases; and (3) the frequency of ground stone fragments is related to the overall intensity of plant food grinding since most breakage occurred as a result of maintenance and refurbishing rather than use. At 41GR291, the frequency of ground stones is higher in the early occupation assemblage $(6 \%$, or 78 of 1,431 stone tools) than in the late occupation assemblage $(1 \%$, or 18 of 1,901 stone tools). It also is high compared to assemblages from other contemporaneous sites ( $2 \%$, or 50 of 2,158 stone tools at $41 \mathrm{GR} 383 ; 2 \%$, or 30 of 2,104 stone tools at $41 \mathrm{KT} 33$ ) in the project area (Boyd et al. 1992:Tables 16 and 27). The percentage of complete ground stone specimens also is relatively high in the early occupation assemblage $(14 \%$, or 11 of 78 ground stones) compared to the late occupation assemblage at $41 \mathrm{GR} 291(11 \%$, or 2 of 18 ground stones) and assemblages from other contemporaneous sites $(10 \%$, or 5 of 50 ground stones at 41GR383; $13 \%$, or 5 of 38 ground stones at $41 \mathrm{KT} 33$ ) in the project area (Boyd et al. 1992:72-73, 120-123). Further, many more ground stone fragments in the 41GR291 early occupation assemblage were recovered from reuse contexts (i.e., from hearths/ baking pits). These differences can be interpreted as indicating greater intensity or duration of occupation during the early occupation period, or perhaps that early occupation period subsistence strategies placed a greater emphasis upon plant food processing. Certainly, the presence of numerous recycled ground stone fragments $(n=37)$ in hearth/baking pit Features 29 and 41 indicates that intensive grinding activities were performed on the site at or before A.D. 600685 and 689-897, respectively.

The size of the complete metates at 41GR291 also suggests more-intensive plant processing than has been documented at other sites in the Texas Panhandle-Plains, with the exception of the horticultural Plains Village occupations of the Antelope Creek phase in the northern Panhandle (Lintz 1982:43, 1986:33-35). Many researchers have noted the relationship between the size and style of ground stones, their grinding efficiency, and intensity of plant use (e.g., Adams 1993; Calamia 1991; Hard 1990; Mauldin 1991, 1993; Mauldin and Tomka 1988). In particular, it has been suggested that differences in the size and style of ground stone tools (e.g., onehand vs. two-hand manos and basin vs. trough metates) are related to differences in the intensity of processing rather than what was being processed. Hence, intensive processing does not necessarily indicate agriculture (Morris 1990:186-187, 191).

While the evidence indicates intensive plant processing at 41GR291 during the early occupation period, the basin and slab metates from the site, although quite large, denote a less intensive grinding technology than do the trough metates used by sedentary agriculturalists in the Southwest. Except for mesquite beans, the specific plants utilized as food resources at 41GR291 are not well documented (see Appendix E). Charred seeds of a few other plants (e.g., Chenopodium' and Cucurbitaceae) may represent food resources, but their archeological contexts do not necessarily confirm this interpretation. While their charring probably resulted from cultural activities, some may represent plants used as fuel in fires or plants used for nonfood purposes (e.g., gourds used as containers). In any case, the feature evidence suggests that some plant resource(s) was present in sufficient quantities to yield a surplus for storage.

The few charred cucurbit seeds (see Appendix E) constitute the only evidence that horticulture might have been practiced at the Sam Wahl site. These remains are inconclusive, however, since they cannot be identified to species and could have come from wild rather than cultivated varieties. Given the presence of storage pits and a pithouse, however, it is tempting to think that the absence of cultigens may be due to factors such as differential preservation or plant processing techniques that did not result in charred remains.

For the Southwest United States as a whole, the initial introduction of domesticated plants has been characterized as a "monumental nonevent" that had "little immediate impact on native human populations" (Minnis 1985:310). While maize appears to have been established throughout the Southwest by A.D. 1, significant cultural changes did not take place until much later when populations increased their reliance upon agriculture (Minnis 1985:310, 338). Thus, it was not the introduction of agriculture but instead the intensification of agricultural production that had widespread effects upon prehistoric economies. Following Bronson's (1977) terminology 
(cited in Minnis 1985:338), it is suggested that the early occupants at the Sam Wahl site were not agriculturalists ("those dependent upon cultivated plants"), but that they could have been cultivators ("those for whom crops are not necessarily major economic items"). Lacking definitive evidence of cultigens at 41GR291, it can only be suggested that one possible scenario for the early occupation period is that limited plant cultivation was incorporated into a seasonal pattern of resource collection (Minnis 1985:331). Certainly, it is likely that the early occupation inhabitants were at least aware of farming since agricultural systems were well established in the Southwest by A.D. 500-700 (Minnis 1985:310; Woodbury and Zubrow 1979:50-51), in the Central Plains by A.D. 900 (Adair 1988:114), and in southcentral Oklahoma (Vehik 1984:196) and northeast Texas (Pertulla 1992:13; Perttula and, Bruseth 1983:17) by A.D. 800 . Many of the important domesticated plants appear originally to have diffused northward out of Mexico, and Ford (1985: 352, 362-364) suggests that the Southern Plains may have been a corridor for the continued eastward spread of cultigens (specifically maize and beans), and presumably farming technologies, from the American Southwest.

\section{LATE OCCUPATION PERIOD}

(A.D. 1150 to 1400 )

Evidence for subsistence practices during the late occupation period at the Sam Wahl site is limited. Only two features can be confidently associated with this period, and their functions are not clear (see charcoal/burned rock scatter Features 12 and 21). The faunal remains are meager because of poor preservation (see Appendix C), but a freshwater drum otolith may indicate that fish were eaten. The floral remains are also meager, and interpretations of the food resources used are questionable. Charred seeds that may represent food resources include Chenopodium, Cucurbitaceae, and perhaps mesquite (see Appendix E).

Indirect evidence for late occupation period subsistence strategies is found in the material culture. As previously discussed (see Table 23), a high frequency of arrow points and debitage and a low frequency of ground stones suggest that the late occupation inhabitants may have hunted more and processed plant foods less intensively than their predecessors. The apparent absence of two important feature types - hearths/baking pits and storage pits - in the late occupation period supports an interpretation of the decreased importance of plant resources.

Although the evidence discussed so far is limited to 41GR291, it is interesting that the dramatic change in site function that may have occurred at ca. A.D. 1150 seems to correspond with other data from the Lake Alan Henry project area and the surrounding region. While a shift in site function at a single site could be fortuitous or could indicate an influx of new people into a region, much of the evidence suggests that a regional climatic change at ca. A.D. 10001250 may have been the impetus for widespread cultural changes seen in the archeological record for the Southern Plains. 


\section{THE CAT HOLLOW SITE, 41GR303B}

Jay Peck, Steve A. Tomka, and Karl W. Kibler

\section{SITE SETTING}

Site 41GR303 is situated in the upland margin west of Cat Hollow and north of the Double Mountain Fork of the Brazos River, ca. $0.5 \mathrm{~km}$ east of Cedar Hill. The site occupies an isolated, eastward-projecting upland ridge and the surrounding lower benches ca. $300 \mathrm{~m}$ north of the Double Mountain Fork. The edges of the ridge and the lower benches are severely eroded and contain abundant sandstone bedrock exposures. The lower benches north and east of the ridge are exposed bedrock with isolated remnants of sandy sediments, while the lower benches to the west and south contain larger remnants of sediments but are also dissected by numerous small drainages. A dirt road runs east from Cedar Hill onto the main ridge, but no other land alteration is apparent. Vegetation consisting of grasses, junipers, prickly pears, agaritas, and small shrubs is moderate to heavy on the rise and on lower bench sediment remnants but sparse to absent in the eroded areas. Site elevations range from 2240 to $2310 \mathrm{ft}$ msl. Site 41GR303A occupies the flat portion of the upper ridge to the south of 41GR303B. Site 41GR303B is situated on the lower bench north of 41GR303A. All work in 1992 took place at 41GR303B.

\section{PREVIOUS INVESTIGATIONS}

Area $B$ is an isolated $25-x-75-m$ remnant of sediments on the bench to the north of the ridge. Considerable amounts of burned rocks and lithic artifacts are exposed on the surface and eroding from the edges of the bench. In the 1988 testing, diagnostic artifacts were individually plotted and collected, and a 10-x-10-m surface collection unit was situated in an area where cultural materials were exposed on bedrock. Cull piles from artifact collecting were noted, but the amount of disturbance by relic hunters is unknown (Boyd et al. 1990).

Two test units were excavated in Area B. Test Unit 4 on the westem portion of the bench yielded 2 bifaces, 1 drill, 3 unifaces, 1 core, 5 modified flakes, and 160 pieces of unmodified debitage. Test Unit 5 on the eastern, more-deflated portion of the bench yielded 2 cobble tools, 2 unifaces, 1 core, 7 modified flakes, 1 ground stone, and 112 pieces of unmodified debitage. From the controlled $10-x-10-m$ surface collection unit, 1 biface, 8 cobble tools, 18 unifaces, 12 cores, 12 modified flakes, 3 hammerstones, 2 ground stones, and 84 pieces of unmodified debitage were recovered. Other surface-collected artifacts consist of 4 bifaces, 1 cobble tool, 5 unifaces, 5 cores, 7 modified flakes, 4 ground stones, and 16 pieces of unmodified debitage.

\section{DATA RECOVERY INVESTIGATIONS}

Excavation of 41GR303B was undertaken over a period of 3.8 work-weeks and required ca. 94.5 person-days of effort. A total of $22.14 \mathrm{~m}^{3}$ of sediment was excavated and screened through 1/4-inch-mesh hardware cloth. Initially, an arbitrary grid was reestablished based on a grid point designated N100/E100 set during the 1988 testing. 
This grid point was marked with a piece of rebar driven into the ground and assigned an arbitrary elevation of $100 \mathrm{~m}$ above an imaginary datum plane. All subsequent three-dimensional measurements were taken relative to this point. The grid increases north and east from a zero point $100 \mathrm{~m}$ south and $100 \mathrm{~m}$ west of the rebar. The grid was oriented to magnetic north and projected across the entire site, and this orientation was maintained during excavation. A permanent horizontal and vertical marker consists of an aluminum cap set in concrete at N100/E114.

Thirty-five units were excavated at 41GR303B in 1992 (Figure 42). Initially, a two-pronged strategy was implemented involving the excavation of nine scattered units to sample the site, define its extent, and locate concentrated cultural deposits and the excavation of eight contiguous units to expose a deep north-south profile across the dome-shaped bench containing the site. Of the nine dispersed units, EUs 1-3 and 32-36 were concentrated in the western portion which appeared least disturbed by erosion and deflation, and EU 31 was excavated in the eastcentral portion of the site to determine the degree of disturbance in this deflated area. Excavation Units 4-6, 13, and 27-30 were excavated to create a trench extending $4 \mathrm{~m}$ north and $4 \mathrm{~m}$ south of Test Unit 4 excavated in 1988 (renumbered as EU 21).

As a result of these initial excavations, it was determined that the most productive area for placement of a block excavation was west of the central portion of the trench. Eighteen additional units were placed west from EUs 13, 21, and 27, the central units of the north-south trench. Test Unit 4 (EU 21) was not reexcavated. The resulting block consists of 27 contiguous units averaging $61 \mathrm{~cm}$ in depth (Figure 43).

Generally, all units were excavated in 10-cmthick arbitrary levels, although levels less than or greater than $10 \mathrm{~cm}$ in thickness sometimes were used for the upper proveniences to level off the excavations at even increments relative to the site datum. Units were excavated to depths ranging from 28 to $143 \mathrm{~cm}$, with the mean depth of all block and nonblock units being $63 \mathrm{~cm}$. When the 1988 test units are added (renumbered as EUs 21 and 37), a total of 37 units was excavated at 41GR303B (see Figure 42).

\section{SEDIMENTS AND STRATIGRAPHY}

The sediments and stratigraphy of 41GR303B were examined in the walls of the excavation units. In all, five profiles were recorded. Ten sediment samples were collected and submitted for analyses of sediment grain size, organic matter content, and carbonate content (see Appendix B). Two other sediment samples were submitted for radiocarbon dating.

These observations and analyses reveal that the site occupies an erosional remnant or knoll of colluvial sediments that have accumulated on a lower erosional bedrock bench or strath terrace of Triassic Dockum Group sandstone (Barnes 1967). The underlying friable sandstone bedrock dips sharply to the east and north and is the source of the colluvial sediments that contain the cultural remains. The overlying colluvial deposits are extremely thin $(<20 \mathrm{~cm})$ along the western margins of the site, and beyond the western edge the bedrock is commonly exposed. Along the eastern margin of the site, the bedrock lies well over a meter beneath the surface. A weakly developed cumulic soil has formed on the sediments. It is an Inceptisol classified as a Typic Ustochrept. Such soils are characterized by thin mollic epipedons and cambic horizons and belong to a ustic soil moisture regime (Buol et al. 1980:243245).

General descriptions and geomorphic interpretations of two of the five profiles are presented here (Figure 44). More-detailed descriptions of the sediments and stratigraphy of all five excavation unit profiles can be found in Appendix A.

Excavation Unit 27 was placed on the east end of the site and excavated to bedrock: The following profile record is from the west wall of the unit and extends to $125 \mathrm{~cm}$ below the surface. Three zones were identified. Zone $1(0-62 \mathrm{~cm})$ is a structureless brown to dark brown (10YR 4/3) fine sandy loam. Many sandstone gravels and pebbles are dispersed throughout the zone. Zone 1 is an A horizon. Soil humates from $25-35 \mathrm{~cm}$ below the surface produced a $\delta^{13} \mathrm{C}$-corrected radiocarbon assay of $730 \pm 70$ B.P. (Beta-59825; uncorrected age $=670 \pm 70$ B.P.; ${ }^{13} \mathrm{C}$ $=-21.5 \%$ o). Its one-sigma calibrated range based on the 20-year calibration curve of Stuiver and Pearson (1986) is 706-662 B.P. (A.D. 1244-1288). Zone 1 grades to a moderate medium blocky brown to dark brown (7.5YR 4/4) fine sandy loam between $62-125 \mathrm{~cm}$ (Zone 2). Many sandstone gravels and pebbles are scattered throughout the zone. Filaments of pedogenic carbonates are common. Zone 2 is a Bwk horizon. A sediment sample from $95-105 \mathrm{~cm}$ 


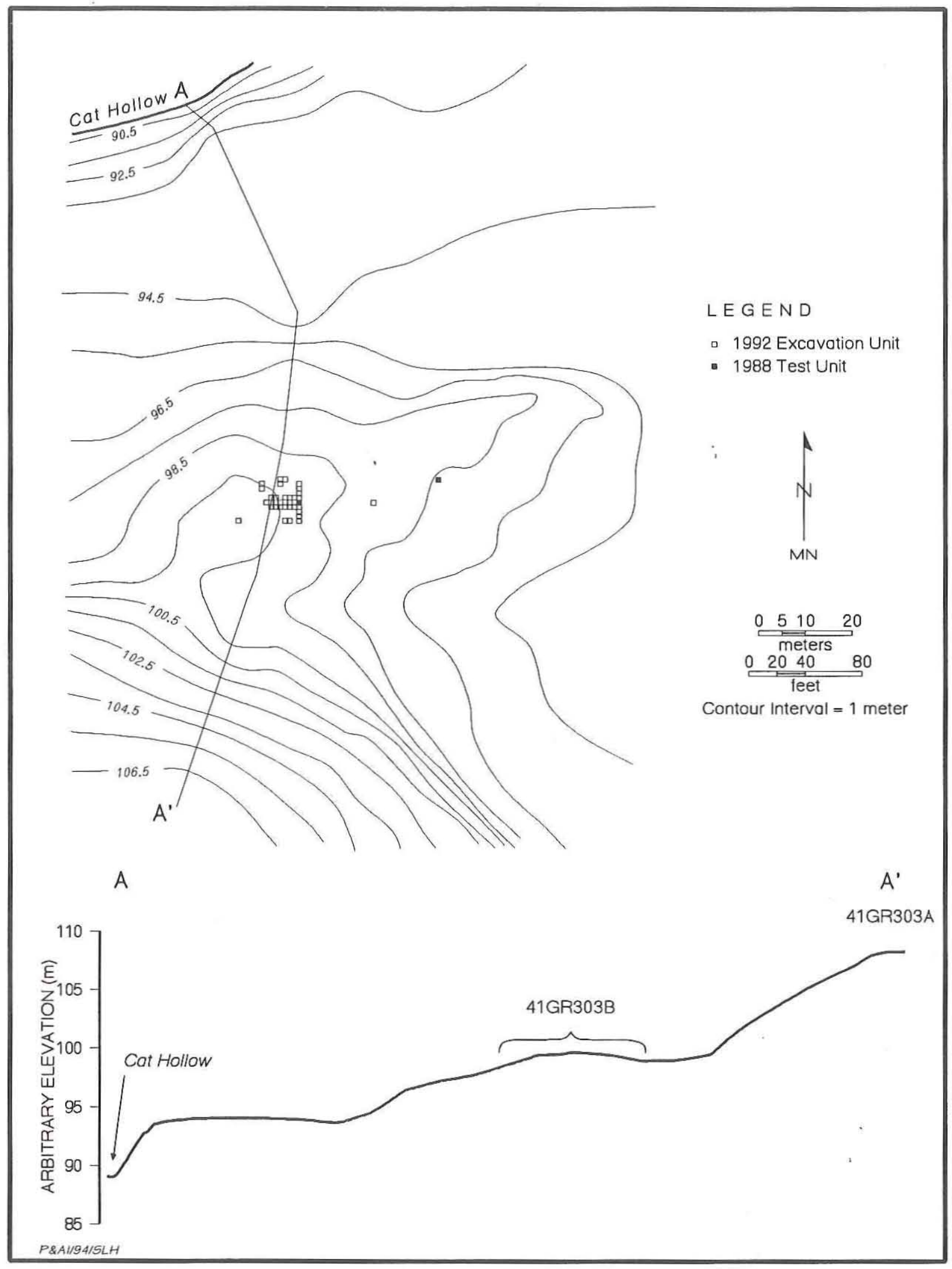

Figure 42. Map of 41GR303B showing topographic setting, 1988 test units, and 1992 excavation units. 


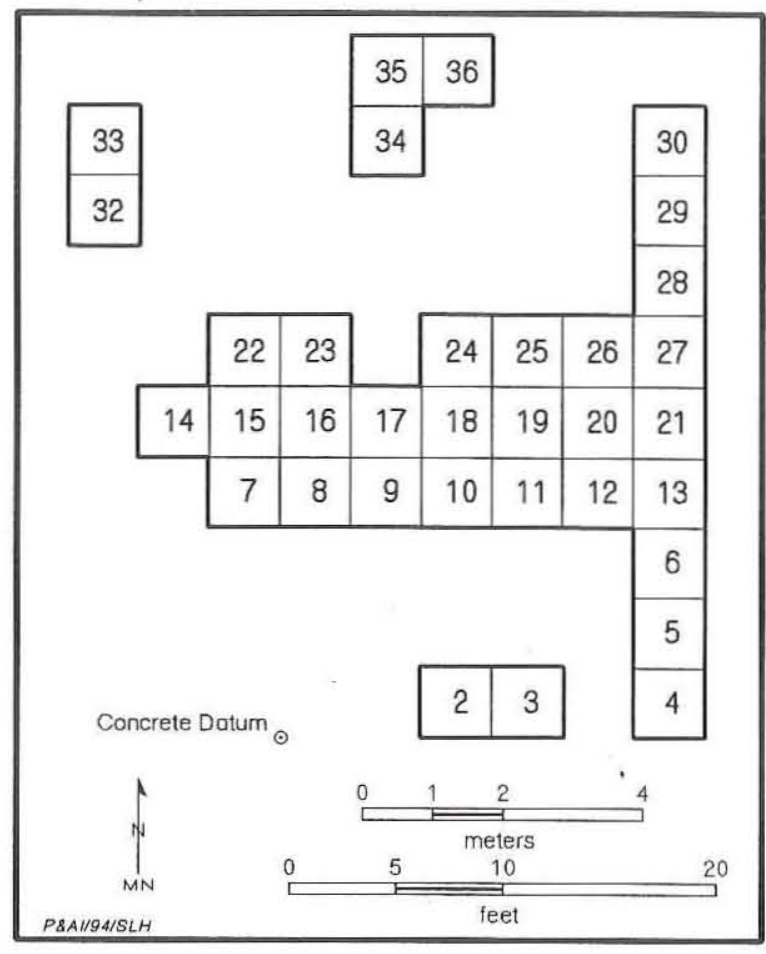

Figure 43. Plan of block excavation and nearby excavation units, 41GR303B.

did not yield enough carbon for analysis; however, charcoal from a similar depth in the unit produced a $\delta{ }^{13} \mathrm{C}$-corrected assay of $1880 \pm 50$ B.P. by AMS. If the charcoal is not intrusive, it suggests that the sediments began accumulating in the late Holocene. Zone 2 rests unconformably on Triassic-age sandstone (Zone 3).

Excavation Unit 32 is located west and upslope of EU 27 and was excavated to bedrock. The following profile record is from the west wall of the unit and extends to $81 \mathrm{~cm}$ below the surface. Zone 1 $(0-48 \mathrm{~cm})$ is a weak fine blocky brown to dark brown (10YR 4/3) fine sandy loam. Zone 1 is an A horizon. A cultural zone is evident from commonly occurring flecks of charcoal and a pavement of sandstone cobbles at $25 \mathrm{~cm}$ below the surface. Fine planar laminations occur above and below the rock pavement. This zone grades into a strong brown (7.5YR 4/6) fine sandy loam between $48-81 \mathrm{~cm}$ (Zone 2). Zone 2 is a Bwk horizon with moderate medium blocky structure and common filaments of $\mathrm{CaCO}_{3}$. Many sandstone gravels and pebbles are dispersed throughout the zone. It rests unconformably on Triassic-age sandstone (Zone 3).

The age of the formation of the strath terrace is

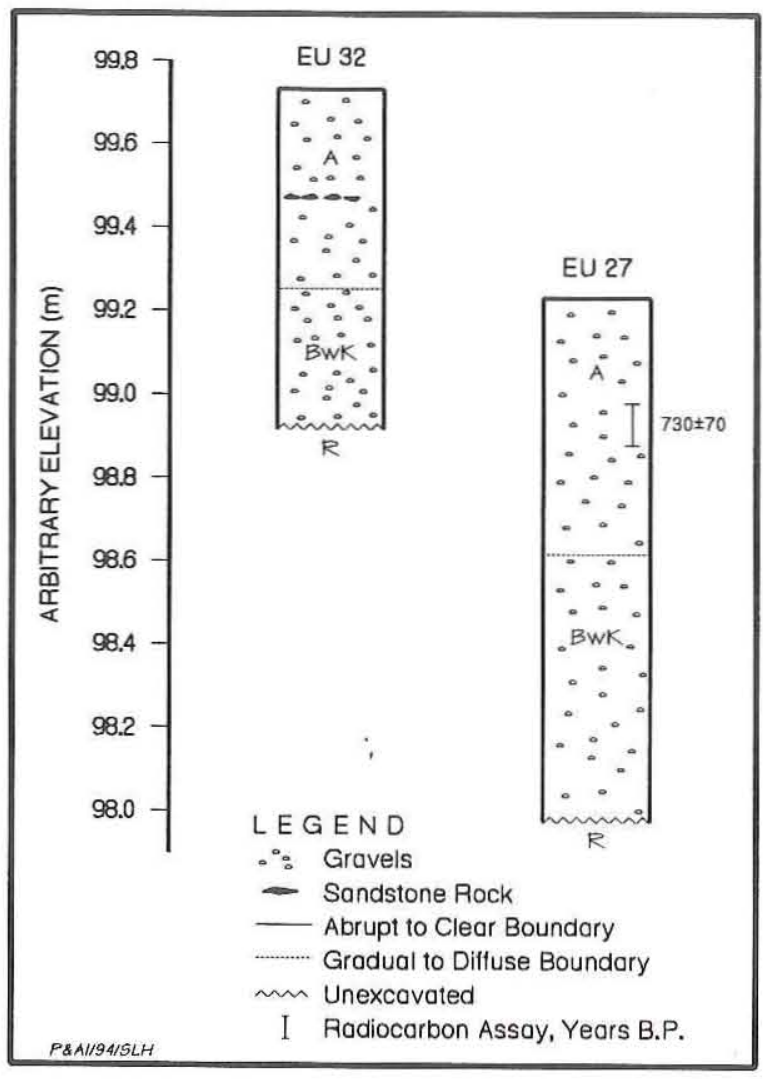

Figure 44. Profiles of EUs 27 and 32, 41GR303B.

not known. Bousman (1992:138) estimates that the previously overlying Mio-Pliocene Ogallala Formation was removed from the project area ca. 225,000-735,000 years ago, based on the Caprock Escarpment retreat rates of Gustavson and Simpkins (1989:42). From this, he estimates that the initial incision of the Triassic-age deposits may have occurred ca. 100,000-150,000 years ago (Bousman 1992:138). Given the hard resistant nature of the Triassic conglomeratic sandstone that overlies the more friable micaceous sandstone in the site area and the fact that the strath terrace is approximately 10 $11 \mathrm{~m}$ above the streambed of Cat Hollow, a considerable period of time for down-cutting is inferred.

The deposition of the knoll at 41GR303B occurred between ca. 2000-1000 B.P., based on radiocarbon assays on soil humates and charcoal. Based on the radiocarbon ages and the position of the knoll or erosional remnant $10-11 \mathrm{~m}$ above the streambed, the initial assessment of the deposit as an alluvial terrace remnant of Cat Hollow by Boyd et al. (1990:173) is highly improbable. At site 41GR383, 
which is approximately $2 \mathrm{~km}$ west of site 41GR303B, colluvial processes are credited with the formation of culturally relevant deposits by $2000-1000$ B.P. on a similar bedrock terrace above Gobbler Creek (Boyd et al. 1992:155). Similar sedimentary processes are indicated at 41GR303B by the vast number of matrix-supported gravels and pebbles throughout the deposits. Microscopic examination (100x) of the sand fractions of three sediment samples from various elevations and localities within the site revealed many sharp angular quartzose sand grains throughout the samples. Numerous flecks of mica also are present and are derived from the highly micaceous, friable Triassic sandstones. The dominant presence of mica tends to rule out an eolian origin for the knoll; however, rounded and subrounded quartzose sand grains are present in very small numbers in the upper deposits, suggesting that there is a minor eolian component to the later deposits.

It is likely that the knoll is an erosional remnant of a much more extensive colluvial deposit derived from upper slopes of the Triassic sandstones that has since undergone erosion. Lag deposits of lithic artifacts are scattered extensively across exposed areas of bedrock. A weakly developed soil and radiocarbon assays suggest that the surface of the deposit aggraded rapidly for at least a millenium (ca. 2000-1000 B.P.), providing a degree of stratigraphic separation in the cultural remains. A minimum radiocarbon age of $730 \pm 70$ B.P. on soil humates suggests that aggradation may have ceased around 1000 B.P. Erosional processes, channel incision, and increased alluvial/colluvial fan activity due to the onset of xeric conditions are well documented at this time throughout the project area (e.g., Abbott 1990; Blum et al. 1992; Boyd et al. 1993). The susceptibility of the thin, permeable, colluvial deposit to erosion is only accelerated by the underlying impermeable bedrock.

\section{CULTURAL FEATURES}

Four anomalies at 41GR303B were recorded as features. Three were discovered during the 1988 testing (Features 5, 6, and 8; Boyd et al. 1990:173) and were designated as burned rock concentrations. Feature 9 was identified during the Phase III excavations (Table 27). Features 1-4 and 7 were recorded at 41GR303A during the 1988 testing and hence are not discussed further here. A dominant feature at 41GR303B is a scatter of burned and

\begin{tabular}{|c|c|c|c|c|}
\hline \multicolumn{5}{|c|}{ TABLE 27 } \\
\hline \multicolumn{5}{|c|}{ SUMMARY OF FEATURES, 41GR303B } \\
\hline $\begin{array}{c}\text { Feature } \\
\text { No. }\end{array}$ & Feature Type & $\begin{array}{l}\text { Excavation } \\
\text { Unit }\end{array}$ & $\begin{array}{l}\text { Top } \\
\text { Elevation } \\
(\mathrm{m})\end{array}$ & $\begin{array}{l}\text { Bottom } \\
\text { Elevation } \\
(\mathrm{m})\end{array}$ \\
\hline 5 & $\begin{array}{l}\text { Burned rock } \\
\text { concentration }\end{array}$ & 37 & 97.43 & 97.25 \\
6 & $\begin{array}{l}\text { Burned rock } \\
\text { concentration }\end{array}$ & 21 & 98.61 & 98.37 \\
8 & $\begin{array}{l}\text { Baking pit } \\
\text { Baking pit }\end{array}$ & 21 & 98.37 & 98.22 \\
9
\end{tabular}

unburned sandstone that covers most of the site at varying levels and densities. This burned rock scatter is more deeply buried in the eastern portion of the block than elsewhere. Figure 45 illustrates the burned rock scatter in a south-facing profile running eastwest $\left(A-A^{\prime}\right)$, an east-facing profile running northsouth (B-B'), and a west-facing profile running south-north $\left(\mathrm{C}-\mathrm{C}^{\prime}\right)$. To the west, the burned rocks appear in the first or second level of excavation. To the east, they occur in deeper levels, probably due to the sloping nature of the old ground surface upon which the rocks were laid down. This overall scatter is not considered a discrete cultural feature because it is ubiquitous across the excavated area. The depths at which the burned rocks occur in the block and nearby units vary systematically. The highest point on the site is near EU 32, and the block and all of the 1-x-1-m units are east of this point, with the exception of EU 1. The depths at which burned rocks were encountered increase from west to east. Figure 46 illustrates the top of the burned rock layer in Level 2 of EUs 32 and 33.

\section{Feature 5}

Feature 5 was discovered in the deflated eastern portion of the site and consists of a burned rock concentration that occurs as two clusters $5-23 \mathrm{~cm}$ below the modem ground surface in Test Unit 5 (EU 37) (Figure 47). The weight of the burned rocks is estimated to be $13 \mathrm{~kg}$. The measured dimensions are $100 \times 100 \mathrm{~cm}$. Flotation of sediments from Feature 5 recovered burned and unburned juniper wood, burned oak wood, Chenopodium fruit, and snail shells. A radiocarbon assay on charcoal from this concentration yielded a calibrated date of A.D. 1020 (1230) 1382 (Table 28). These clusters may be part 


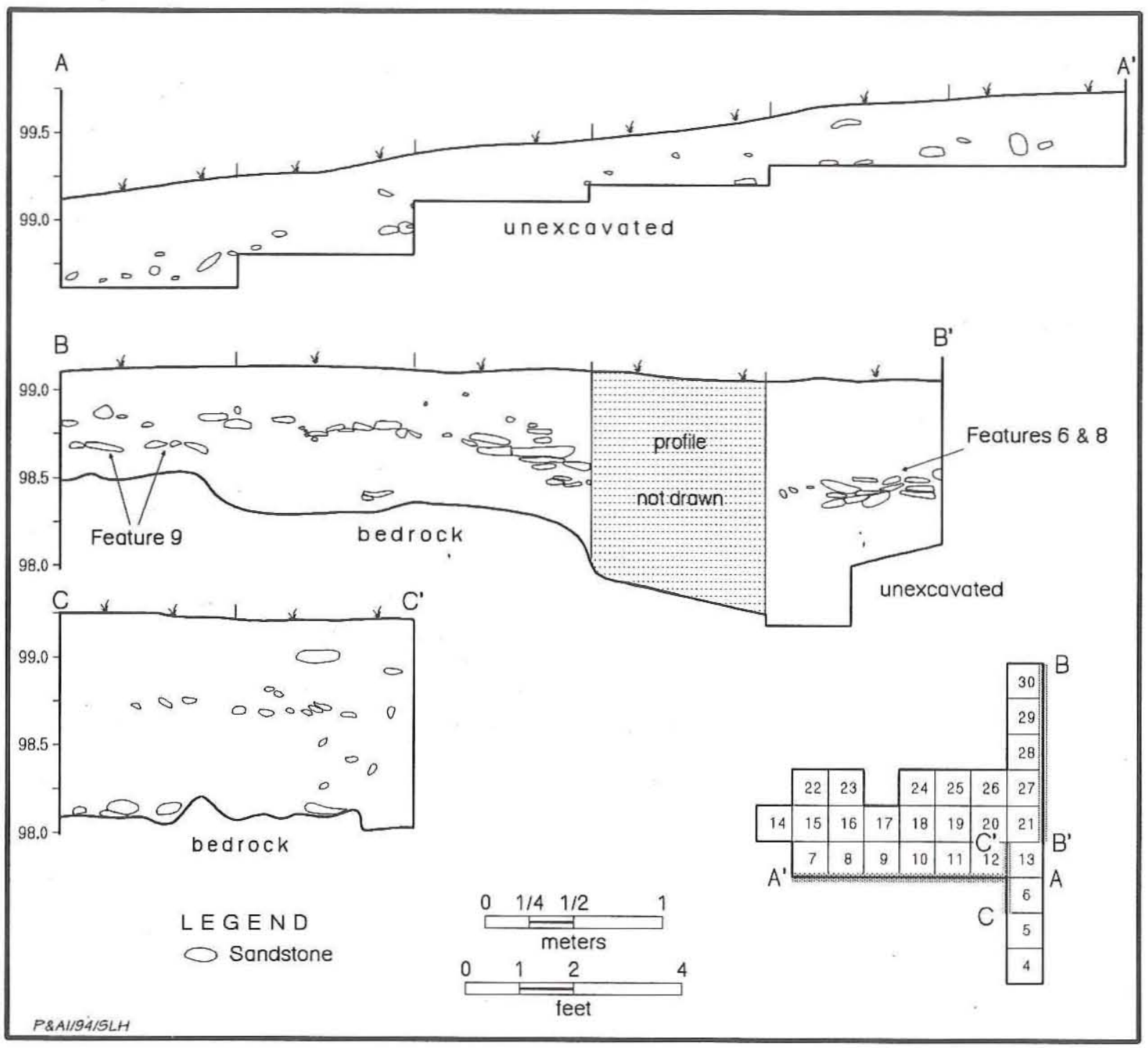

Figure 45. Three profiles showing the burned rock scatter in the block, 41GR303B.

of a disaggregated hearth, or they may be part of the sitewide burned sandstone scatter. Without further excavation around the periphery of the feature, it is not possible to be more explicit. This portion of the site was avoided during the Phase III excavations due to extreme deflation of the area and the strong possibility of mixing of cultural materials.

\section{Feature 6}

Feature 6, discovered in Test Unit 4 (EU 21) in the western portion of the site, consists of a burned rock concentration at $56-80 \mathrm{~cm}$. The measured dimensions are $90 \mathrm{~cm}$ east-west and $100 \mathrm{~cm}$ north- south. The elevations given for this feature are somewhat deceptive because burned rocks occur higher in the west part of the unit than in the east, and the feature begins sparsely with the tops of a few rocks at $56 \mathrm{~cm}$ and the main body of the feature at $70-80 \mathrm{~cm}$. This concentration covers the entire level, generally to a thickness of one rock, and slopes down slightly to the east. The weight of the burned rocks is estimated to be $10 \mathrm{~kg}$.

Although Feature 6 could be part of the sitewide scatter of burned rocks, another interpretation is favored here. Specifically, Feature 6 appears to be a discrete layer of rocks covering a stone-lined baking pit, the bottom of which is represented by 


\begin{tabular}{|l|l|l|l|l|}
\hline \multicolumn{5}{|c|}{ RESULTS OF RADIOCARBON DATING OF CULTURAL CONTEXTS, 41GR303B } \\
\hline Lab No. & Provenience & $\begin{array}{l}\text { Uncorrected } \\
\text { Age B.P. }\end{array}$ & $\begin{array}{l}\text { Corrected Age } \\
\text { B.P.* }\end{array}$ & $\begin{array}{l}\text { Calibrated Date A.D. (Intercepts } \\
\text { and 1-Sigma Range)** }\end{array}$ \\
\hline GX-14597 & Feature 5, 5-23 cm & $775 \pm 195$ & $810 \pm 195(-22.9)$ & $1020(1230) 1382$ \\
\hline Tx-6295*** & Feature 8, 80-95 cm & $1150 \pm 50$ & $1250 \pm 50(-18.6)$ & $681(772) 852$ \\
\hline $\begin{array}{l}\text { Beta-59827 } \\
\text { CAMS-5168 }\end{array}$ & EU 27, 93-103 cm & $* * * *$ & $1880 \pm 50$ & $71(118) 197$ \\
\hline $\begin{array}{l}* \text { Ages uncalibrated; } \delta^{13} \mathrm{C} \text { values in parentheses. } \\
* * \text { Calibrations use 20-year record of Stuiver and Reimer (1986). }\end{array}$ \\
***Radiocarbon date on soil humates; remainder are on charcoal. \\
****Date done by Lawrence Livermore National Laboratory in California using AMS technique; uncorrected age and \\
$\delta^{13}$ C value not given.
\end{tabular}

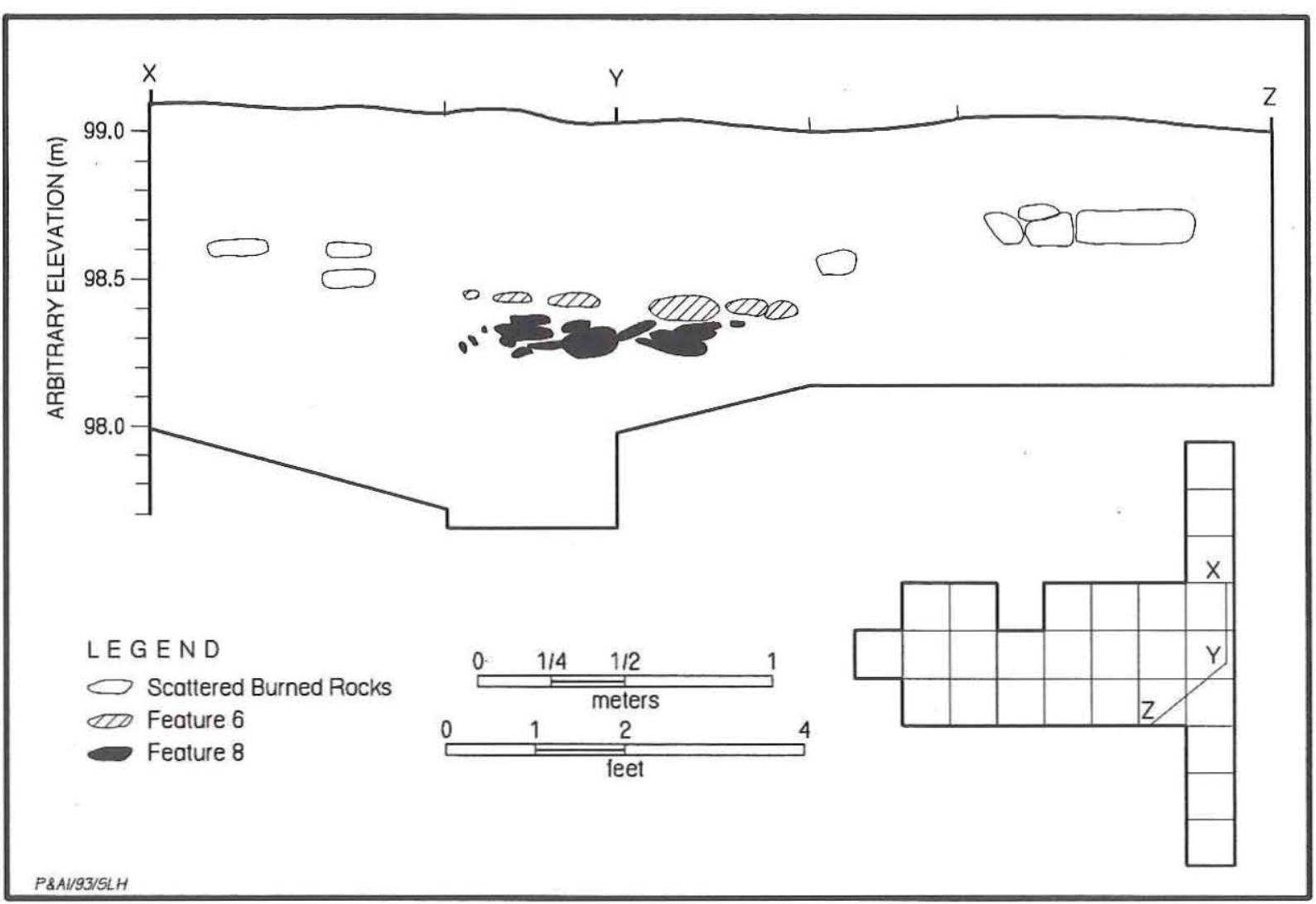

Figure 48. Cross section of EUs 13, 21, and 17 showing burned rock scatter and Features 6 and 8, 41GR303B.

were placed on a layer of grass, and the pit was subsequently capped with grass and dirt (Basehart 1960:31-32, 41; Castetter and Opler 1936:35-38). Thus, Feature 6 could represent an undisturbed middle layer of heated rocks in a rock-lined baking pit which had its cooked contents removed. Perhaps no capping layer of rocks was used or a capping layer of rocks may have been removed. Other ethnographic accounts describe similar methods of pit baking that used rock layers above stone-lined fire 
pits to cook agave or sotol (Pennington 1963:130, 1969:110, 141; Sonnichsen 1958:16-20; Tunnell and Madrid 1990:145-162), yucca (Basehart 1960:40; Castetter and Opler 1936:39), pricky pear (Basehart 1960:38-39), and com (Castetter and Opler 1936:29). Unfortunately, there is no direct evidence of what plants might have been cooked in the Feature $6 / 8$ pit.

\section{Feature 8}

Feature 8 consists of a concentration of ca. 27 pieces of burned sandstone in EU 21 (Test Unit 4). The total weight of the observable rocks is $11.8 \mathrm{~kg}$. The central feature rocks are generally horizontal, while those on the perimeter slant slightly inward. The rocks occur in two to four overlapping layers that form a tight half-circle cluster extending into the east and south walls of the unit. The feature occurs at a depth of 80-95 cm. The measured dimensions are $73 \mathrm{~cm}$ north-south and $35 \mathrm{~cm}$ east-west, and the estimated total diameter is ca. $100 \mathrm{~cm}$.

No soil staining or charcoal was observed, and a radiocarbon assay on humates extracted from sediments directly associated with the feature yielded a calibrated date range of A.D. 681 (772) 852 (see Table 28). Humate dates are generally considered to be younger than the artifacts with which they are associated as they date the formation of the soil matrix, which may contain organic matter leached from higher in the profile (Stein 1992:204). An AMS radiocarbon assay on charcoal below, and not directly associated with, Feature 8 indicates that it is no older than A.D. 71-97 (see Table 28). Hence, the true age of Feature 8 probably is between A.D. 79-852. A thermoluminescence assay obtained in 1989 on burned sandstone yielded an age of $4150 \pm 850$ B.P., and although the humate date represents a minimum age, the discrepancy of 2,200-3,000 years suggests that the thermoluminescence date is considerably too old. Phytolith analysis of sediment samples from Feature 8 confirmed the presence of Opuntia calcium oxylate crystals, suggesting the processing of prickly pear as a function of the feature (Cummings 1990:541).

Excavation Units 13 and 27, adjacent to EU 21, were monitored for possible extensions of burned rocks at the levels of Feature 8, but none were found. The arrangement of the rocks (i.e., the central ones lying horizontal and the peripheral ones slanting inward) and their relationship to overlying Feature 6 (see Figure 48) suggest that Feature 8 is the bottom of a stone-lined baking pit (see Feature 6).

\section{Feature 9}

Feature 9 consists of a tight cluster of ca. 11 pieces of burned sandstone at a depth of $49-61 \mathrm{~cm}$ in EU 30 (Figure 49). The total weight of the observed rocks is $11.5 \mathrm{~kg}$. The feature contains a single horizontal layer of rocks, with those on the outer margin slanted inward toward the center of the feature. This arrangement forms a tight half-circle cluster extending into the east wall of the unit. The observed portion measures $75 \mathrm{~cm}$ north-south and $45 \mathrm{~cm}$ east-west, and the estimated total diameter is $75-90 \mathrm{~cm}$. No soil staining or charcoal was observed in or around the feature, and there is no discernible extension of the feature into EU 29, the excavated unit south of EU 30. Feature 9 lies $10-22 \mathrm{~cm}$ below the bottom of the burned rock scatter in EU 30 and EU 29 just to the south, and thus it may be the bottom of a stone-lined baking pit comparable to Feature 8.

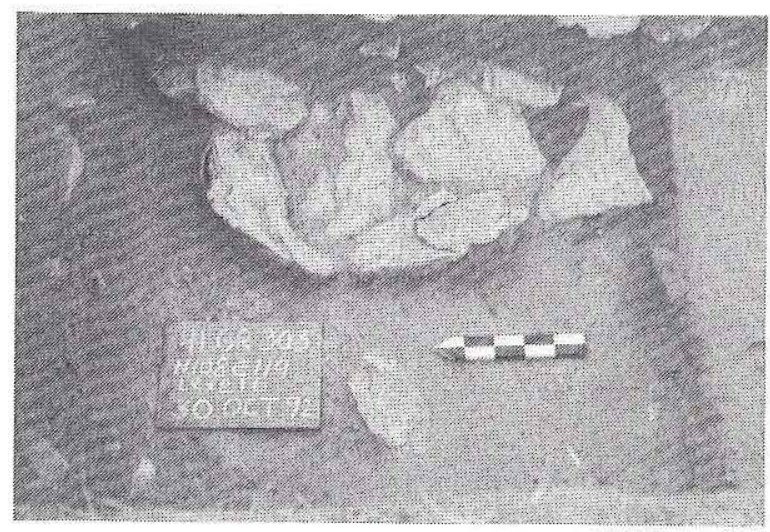

Figure 49. Feature 9, 41GR303B.

\section{Discussion}

The identified features at 41GR303B consist of a burned rock concentration (Feature 5) discovered in the deflated area east of the block, two baking pit bottoms (Features 8 and 9) discovered in EUs 21 and 30 in the block, and one concentration of burned rocks above Feature 8 in EU 21 that is an upper layer of rocks covering the Feature 8 baking pit (Feature 6).

A scatter of burned and unburned sandstone rocks occurs at varying depths across the entire excavated area. While the burned rock scatter occurs in all units and appears to be sitewide, there are some areal differences in its appearance. In some areas, for example in EUs 32-36, the scatter is thicker than in other areas, as if the rocks were piled 
there, while some small areas contain no rocks. Unburned rocks are mixed with burned rocks in every unit. It appears that the piles represent stockpiling of sandstone slabs or discarded hearth or baking pit rocks, while the clear areas could be walkways between features and between stockpiles of fresh rocks and scatters of exhausted burned rocks.

The relationship of the sitewide scatter to the cultural features can be seen in a cross section through the block (Figure 50). Feature 8 is the bottom, or firebox, of a roasting pit at a depth of 80 $95 \mathrm{~cm}$. Feature 6 is a roasting rack of sandstone just above the firebox at $70-80 \mathrm{~cm}$. Both Features 6 and 8 lie just below the burned rock scatter in the surrounding units, and the baking pit appears to have been dug from a surface marked by the burned rock zone. Feature 9 exhibits the same relationship with the burned rock scatter, but it lacks an upper rock layer comparable to Feature 6 . Features 6 and 8 are radiocarbon dated to A.D. 71-852 (see Feature 8), and this date range is probably representative of the age of the sitewide scatter of burned rocks.

While the features and burned rock scatter at 41GR303B are not comparable to the classic ring or crescent middens described for western Texas by Greer (1968), they may have been used in similar ways to process plant foods. Carmichael (1985:130-
131) discusses similar features in the El Paso area and relates them to the processing of desert succulent plants, noting the necessity of locating such stations near water. Cat Hollow Spring, just north of 41GR303B, is considered to be perennial (Emmett Shedd, personal communication 1992) and was flowing well during the period of excavation in 1992. O'Laughlin (1979:18-33), also working in the El Paso area, investigated many burned rock features with associated pits and indicates that some were lined with flat stone slabs. In northwest El Paso, O'Laughlin (1980:105-107) observed burned rock features that included a rock layer above the firebox to support the baked product, and he indicates both the long-term use of such features and their use to process desert succulent plants.

Wulfkuhle (1986:36) documents the present of sotol (Dasylirion sp.), yucca (Yucca sp.), and Texas prickly pear (Opuntia lindheimeri) in the region in recent times. Her source, McMahan et al. (1984:10), verifies their presence among the mesquite-juniper brush association in the highland areas of Garza County, but their presence in the area in the past is not documented. The portions of these plants most commonly associated with baking are the bulb (or heart) of sotol, the peeled stalk of yucca, and the pads of prickly pear, although other portions of these

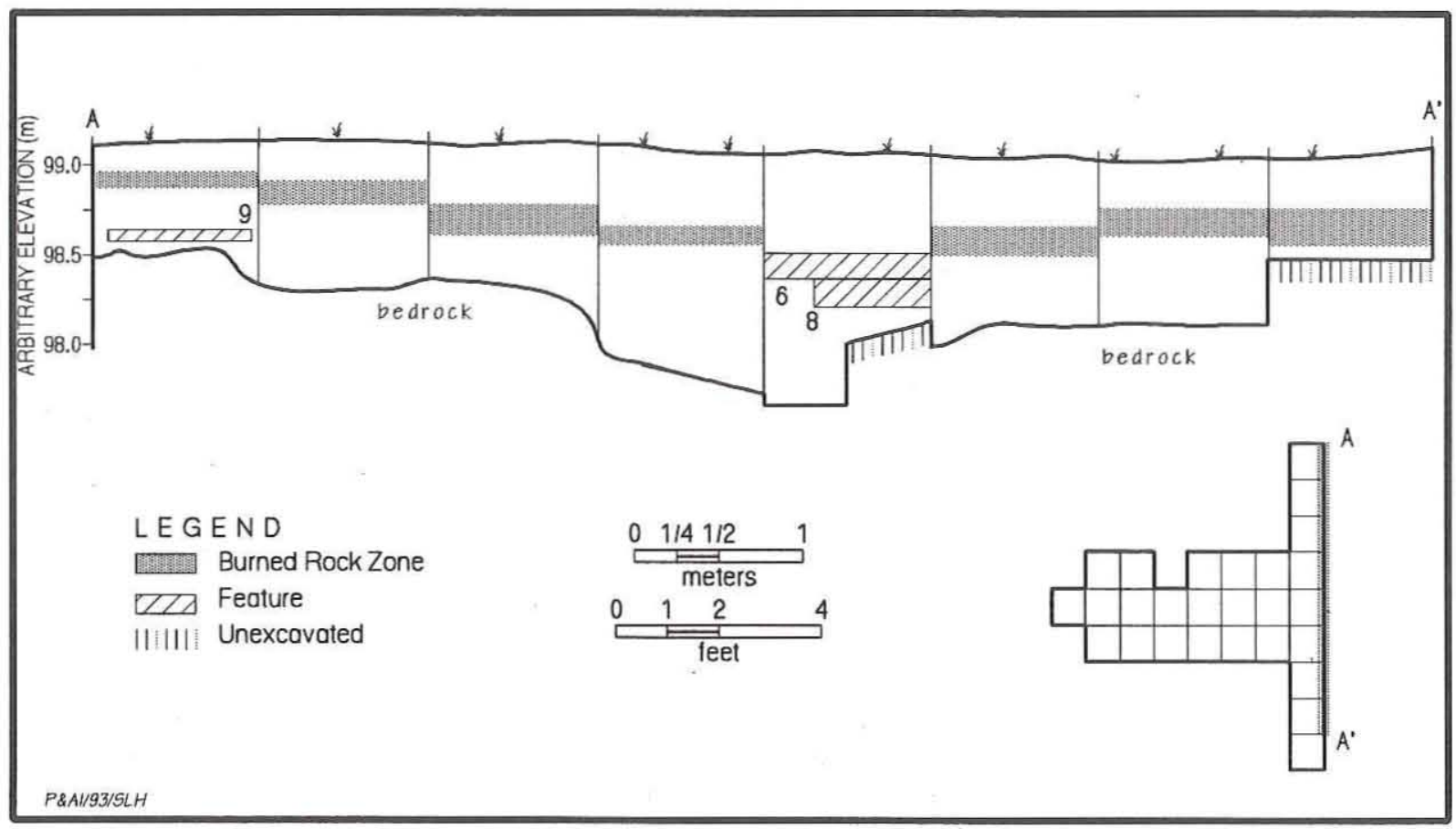

Figure 50. Profile of the north-south trench showing Features 6, 8, and 9 and the scattered burned rock zone, 41GR303B. 
plants are edible. The fruit of the datil (Yucca baccata) is also storable in its dried and ground form. While there are no data on the presence or absence of this species of yucca in the area, Kirkpatrick (1992:149-152) notes the abundance of another species (Yucca angustifolia).

\section{MATERIALS RECOVERED}

This section discusses the materials recovered from 41GR303B during 1992. Of the total 2,317 specimens, the majority $(\mathrm{n}=1,711,74 \%)$ are from the excavation block. Others $(n=564,24 \%)$ are from isolated excavation units located away from the block. The remaining $42(2 \%)$ specimens are from the general surface collection. Table 29 shows the provenience of these artifacts.

\section{Chipped Stone Artifacts}

Six arrow points and fragments, 2 dart point fragments, 8 nonprojectile point bifaces, 27 cobble tools, 68 unifaces and fragments, 17 gouges, 59 cores, 37 edge-modified flakes, and 2,064 pieces of unmodified lithic debitage were recovered.

\section{Arrow Points}

Of the six arrow points, one is complete, three are proximal fragments, one is a distal fragment, and one is a longitudinally broken specimen. All but one are of fine-grained chert. The single nonchert specimen is of Tecovas jasper. Two of the six specimens are typed as Scallorn, one is untyped, one is an untypeable fragment, and two are arrow point preforms.

\section{SCALLORN}

A nearly complete point and a proximal fragment are included in this type (Jelks 1962). Although both are corner-notched expanding-stem points, their blade morphologies differ significantly. The nearly complete specimen (Figure $51 a$ ) has a broad blade that has nearly parallel sides for about two-thirds of its length. Near the distal end, the blade edges bend rapidly to form a rather rounded distal end. The barbs are moderate in length and downward pointing. Both stem ears are broken, and the base is slightly convex. The specimen is well made and appears to have been lost or discarded

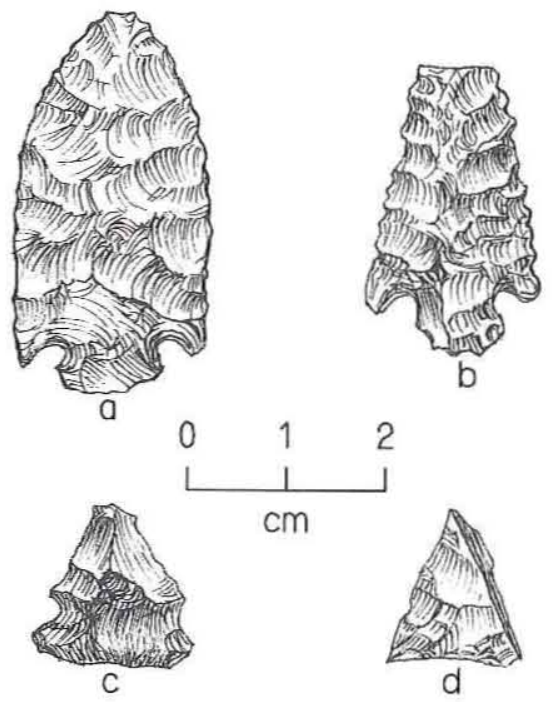

Figure 51. Arrow points, 41GR303B. (a-b) Scallorn; (c) untyped expanding stem, straight base; (d) untypeable longitudinally broken proximal fragment.

without having been resharpened or reworked in any manner after its initial manufacture. The nature of the blank used in making the point cannot be determined; it is of nonlocal fine-grained chert (Color 56). The point has a $32-\mathrm{mm}$-long blade and a 6-mm-long stem. Its maximum blade width is $19 \mathrm{~mm}$, while its neck is $10 \mathrm{~mm}$ wide. Because of the missing stem ears, the original maximum stem width could not be measured. The point is $4 \mathrm{~mm}$ thick.

The proximal fragment (Figure 51b) has straight blade edges and moderate downward-pointing barbs. Based on flake-scar patterning, the point appears to have undergone some resharpening, although this did not appreciably alter the blade edge outline. One ear is missing, and both the distal blade break and the ear break are use related. Although no blank diagnostics are visible, it most likely was made on a flake blank. The point is made of Tecovas jasper. The specimen has a 6-mm-long stem, a 17-mm-wide blade, and a neck that is $7 \mathrm{~mm}$ wide. Its maximum thickness is $4 \mathrm{~mm}$.

These specimens are similar to the Scallorn coryell variety identified by Jelks (1962:27-29) at the Kyle site. Both are also similar to the two coryell variety Scallorn points recovered from 41GR291. In particular, the larger of the two Scallorn points from each site are quite similar morphologically (see Figures $21 d$ and 51a). The two smaller Scallorns from each site are somewhat different from each 


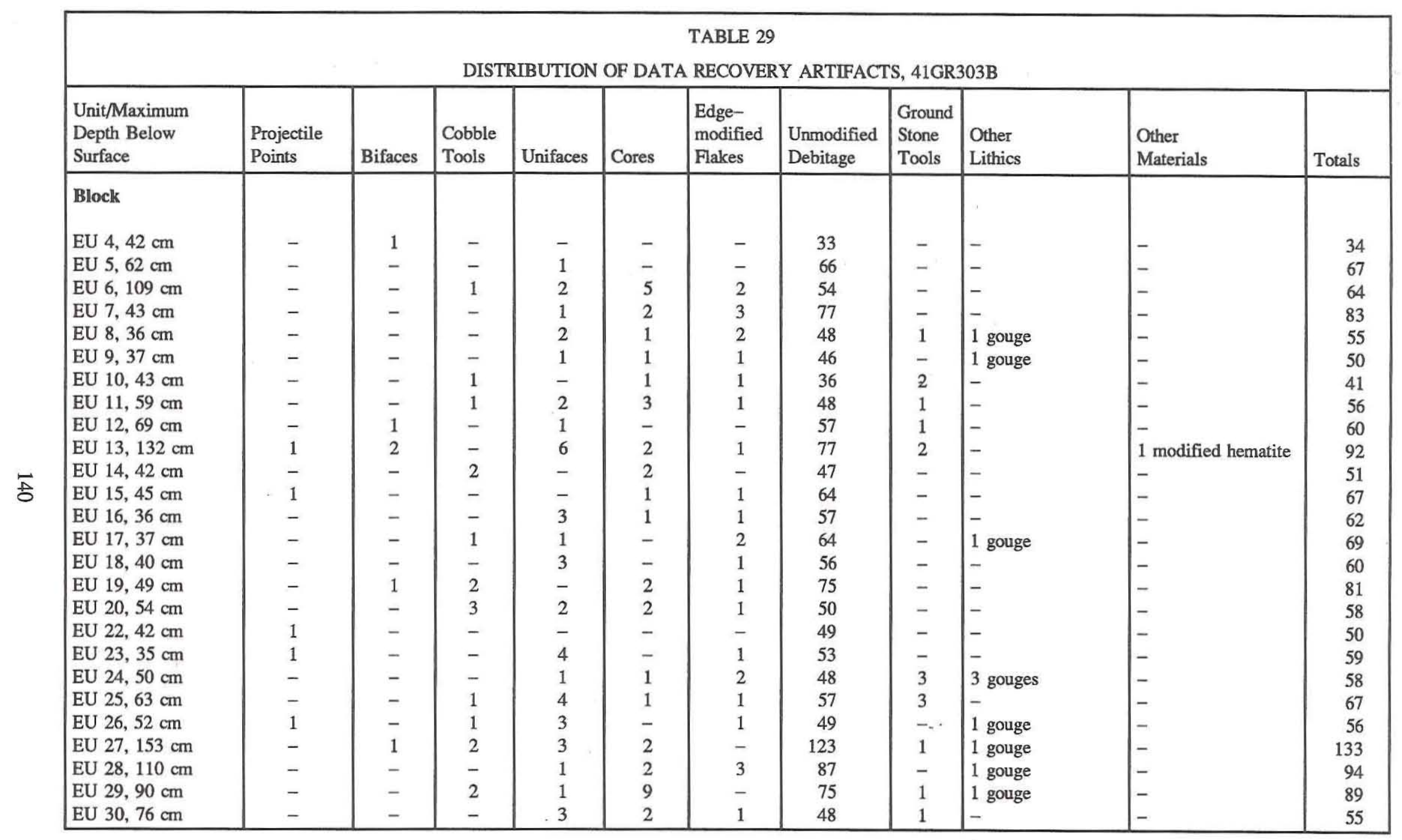




\begin{tabular}{|c|c|c|c|c|c|c|c|c|c|c|c|}
\hline \multicolumn{12}{|c|}{ Table 29 , continued } \\
\hline $\begin{array}{l}\text { Unit/Maximum } \\
\text { Depth Below } \\
\text { Surface }\end{array}$ & \begin{tabular}{|l} 
Projectile \\
Points
\end{tabular} & Bifaces & \begin{tabular}{|l} 
Cobble \\
Tools \\
\end{tabular} & Unifaces & Cores & \begin{tabular}{|l} 
Edge- \\
modified \\
Flakes \\
\end{tabular} & $\begin{array}{l}\text { Unmodified } \\
\text { Debitage }\end{array}$ & $\begin{array}{l}\text { Ground } \\
\text { Stone } \\
\text { Tools }\end{array}$ & $\begin{array}{l}\text { Other } \\
\text { Lithics }\end{array}$ & $\begin{array}{l}\text { Other } \\
\text { Materials }\end{array}$ & Totals \\
\hline \multicolumn{12}{|l|}{ Nonblock Units } \\
\hline EU $1,28 \mathrm{~cm}$ & - & - & - & 1 & - & - & 15 & - & - & - & 16 \\
\hline EU $2,48 \mathrm{~cm}$ & - & - & 1 & 1 & - & - & 53 & - & 1 hammerstone & 1 modified hematite & 57 \\
\hline EU $3,57 \mathrm{~cm}$ & - & - & 3 & - & 2 & 2 & 59 & - & 1 gouge & - & 67 \\
\hline EU $31,67 \mathrm{~cm}$ & - & 1 & - & - & 1 & 1 & 60 & 2 & - & - & 65 \\
\hline EU $32,73 \mathrm{~cm}$ & - & - & - & 2 & - & - & 39 & - & $\begin{array}{l}1 \text { gouge; } \\
1 \text { hammerstone }\end{array}$ & - & 43 \\
\hline EU $33,92 \mathrm{~cm}$ & - & - & 1 & 5 & 1 & - & 39 & 2 & 1 gouge & - & 49 \\
\hline EU $34,74 \mathrm{~cm}$ & - & - & - & 3 & 3 & 3 & 88 & 1 & 1 gouge & - & 99 \\
\hline EU $35,80 \mathrm{~cm}$ & - & - & 2 & - & 2 & 2 & 66 & 1 & 1 gouge & - & 74 \\
\hline EU $36,105 \mathrm{~cm}$ & - & 1 & - & 5 & 6 & 1 & 81 & - & - & - & 94 \\
\hline General Surface & 3 & - & 3 & 6 & 4 & 1 & 20 & 3 & 2 gouges & - & 42 \\
\hline Totals: & 8 & 8 & 27 & 68 & 59 & 37 & 2,064 & 25 & $\begin{array}{l}17 \text { gouges } \\
2 \text { hammerstones }\end{array}$ & 2 modified hematite & 2,317 \\
\hline
\end{tabular}


other, although this difference may be a function of the degree of resharpening of the 41GR291 specimen (see Figure 21e) compared to the specimen from 41GR303B (see Figure 51b).

\section{UNTYPED EXPANDING STEM, STRAIGHT BASE}

A proximal arrow point fragment constitutes this category (Figure $51 \mathrm{c}$ ). It is missing a small portion of its distal blade. Even in its original complete form, the blade appears to have been relatively short. The diminutive size is in part due to heavy resharpening which also removed the barbs. In its present form, even with one ear missing, the stem is wider than the blade.

This corner-notched point is made of local finegrained chert (Color 20). Its haft is $6 \mathrm{~mm}$ long, and the neck is $11 \mathrm{~mm}$ wide. With the exception of maximum thickness $(5 \mathrm{~mm})$, no other measurements could be obtained. Based on morphological criteria (i.e., stem as broad as the blade and strongly expanding), the point is similar to the sattler variety of Scallorn arrow points found at the Kyle site (Jelks 1962:29-30). However, the heavy resharpening of the blade, to the degree that even the barbs have been removed, prevents its identification as a sattler variety Scallorn with certainty.

\section{UNTYPEABLE FRAGMENTS}

A single proximal fragment makes up this category (Figure 51d). Although it also is broken longitudinally, enough remains to determine that the fragment represents a triangular arrow point. The base is slightly concave, and the blade edges appear to have been straight. The break face traverses a coarse inclusion in the fine-grained chert material. It is not possible to establish whether the break occurred as a result of use or manufacture or to establish with certainty whether the point represents a finished specimen or a triangular preform, although the lightly smoothed base suggests that it was finished. Because of the uncertainty regarding the manufacture stage of the specimen, it is not typed as a Fresno arrow point. It is possible that, if the specimen represents a triangular preform, it might have been side and/or base notched in its finished form. In that case, the point would be classified as either a Washita or Harrell. The point is made on a locally available fine-grained chert (Color 6) flake blank.

\section{PREFORMS}

One of the two arrow point preforms is a distal fragment, and the other is a longitudinally split specimen. The distal fragment, made on a nonlocal fine-grained chert (Color 52) flake blank, exhibits primarily unifacial retouch. The longitudinal fragment was broken while the point was being notched. It is made on a locally available finegrained chert (Color 32) flake blank. No metric measurements could be recorded on these fragments.

\section{Dart Points}

A complete dart point and a proximal dart point fragment were recovered. The complete specimen is typed as Darl (Miller and Jelks 1952; Suhm et al. 1954); the proximal fragment is an untyped expanding-stem specimen.

\section{DARL}

The point has a parallel to slightly expanding stem and a concave base (Figure 52a). The stem edges are moderately ground, while the base has not been smoothed. The blade edges have been resharpened numerous times, resulting in only a small shoulder at the juncture of the blade and stem. At some stage of its use life, one ear and a portion of the distal blade were removed by an impact break that left large impact scars on both faces. The distal break subsequently was reworked into a functional point. Given the lack of differential patina development, it appears that the reworking occurred immediately or soon after the break. As a result, the blade rejuvenation is not an indicator of reuse of the dart point by the Late Prehistoric occupants of the site. The point is of nonlocal fine-grained chert (Color 43). The blade is $20 \mathrm{~mm}$ long, and the stem is $10 \mathrm{~mm}$ long. The blade is $17 \mathrm{~mm}$ wide, the neck and stem are $14 \mathrm{~mm}$ wide, and the maximum thickness is $4 \mathrm{~mm}$.

\section{UNTYPED EXPANDING STEM}

This small proximal fragment (Figure $52 b$ ) has three breaks - one at the junction of the blade and stem, another along the stem edge, and a third on the base adjacent to the ear. Although the point has a moderately expanding stem, it cannot be established whether it had a straight or a convex base. Although 


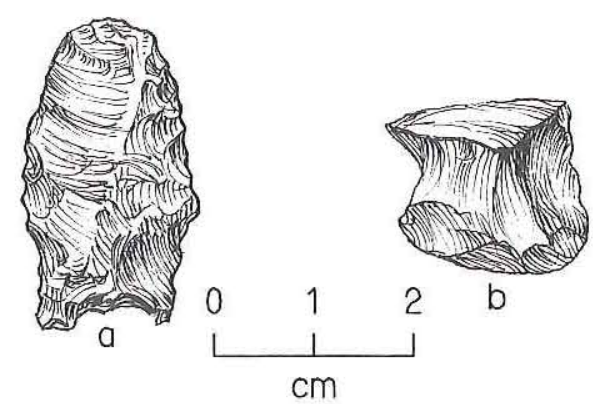

Figure 52. Dart points, 41GR303B. (a) Darl; (b) untyped expanding-stem proximal fragment.

it is roughly similar to the larger of the untyped expanding-stem, convex-base dart points recovered from 41GR291 (see Figure 22a) and it is reminiscent of the Marcos and Williams types, neither of these connections can be established with certainty. The dart point is of nonlocal fine-grained chert (Color 42). No measurements were obtained.

\section{Gouges}

Seventeen gouges were recovered. Of these, 10 are primarily unifacial and 7 are bifacial. Of the 10 unifacial specimens, 7 are complete and 3 are distal fragments. Four of the complete unifacial specimens are roughly triangular (Figure $53 a, b$ ) and three (Figure $53 c, d$ ) are roughly rectangular, while shape could not be determined for the distal fragments. With the exception of one specimen made on a small secondary flake, the unifacial gouges (see Figure $53 a-c)$ are made on large, thick, secondary $(n=6)$ or tertiary $(n=3)$ flakes. One of the three distal specimens appears to be an intentionally removed working-edge fragment. This method of workingedge rejuvenation also was employed on some of the gouges recovered from 41GR291. Break cause could not be determined on the other two distal fragments.

The seven complete unifacial gouges have a mean length of $54.1 \mathrm{~mm}(\sigma=8.9)$, a mean width of $43.9 \mathrm{~mm}(\sigma=7.2)$, and a mean thickness of $16.9 \mathrm{~mm}$ $(\sigma=4.3)$. Haft wear could be identified on two complete tools made of fine-grained chert; the ratios of complete length to haft length are 1.2:1 and 1.1:1 on these specimens. The mean maximum width of the seven complete and one distal fragment together is $44.1 \mathrm{~mm}(\sigma=6.7)$, and the mean maximum thickness of the seven complete specimens and two distal fragments is $17.1 \mathrm{~mm}(\sigma=4.1)$.

None of the seven bifacially shaped gouges are complete. Two are distal fragments, three are medial specimens, one is a proximal fragment, and the remaining specimen is a longitudinally broken distal fragment. They are identified as bifacial gouges based on their planoconvex transverse cross sections, beveled working edges (on the distal fragments), the presence of haft wear (on two medial and one proximal fragment), and their general morphological similarity to bifacial gouges from 41GR291 and other sites in Texas (e.g., Fields et al. 1993:Figure 17c).

The breaks on all seven are use related. Two of the three medial fragments and the longitudinally broken distal fragment were broken in use and subsequently broken again in the process of reworking. Given the bifacial manufacture technique and the degree of reduction, the nature of the blanks used in their manufacture could not be determined.

The full complement, of metric attributes could not be obtained on any of the fragments. Maximum width could be measured on a single fragment $(28 \mathrm{~mm})$, while the mean maximum thickness of the five measurable specimens is $7.6 \mathrm{~mm}(\sigma=1.8)$.

With the exception of two primarily unifacial gouges of local fine-grained chert (Colors 7 and 55), the bulkier unifacial gouges are of coarse raw materials (i.e., fine-grained $[n=5]$ and coarsegrained $[n=2]$ Potter chert and silicified caliche [ $n=$ 1]). Three of the bifacial gouges are of fine-grained chert; two of these are of local origin (Color 5), and the other is of nonlocal origin (Color 48). One specimen is of Tecovas jasper, two are of silicified caliche, and one is of very fine grained Potter chert.

\section{Nonprojectile Point Bifaces}

Eight biface fragments, consisting of five proximal, one medial, one distal, and one wedge fragment, were recovered. Two of the five proximal fragments retain cortex; one of these is an earlyreduction-stage specimen, and the other has been reworked and appears to be a finished specimen. The three decorticate proximal fragments also exhibit substantial reworking, suggesting that they too are finished artifacts. In addition to the transverse blade breaks on the five proximal fragments, one is also longitudinally broken, and one decorticate specimen exhibits haft wear. The corticate early-reductionstage fragment was broken in manufacture; the second corticate fragment exhibits a use break. The break cause could not be determined with certainty on the three decorticate proximal fragments; however, the 

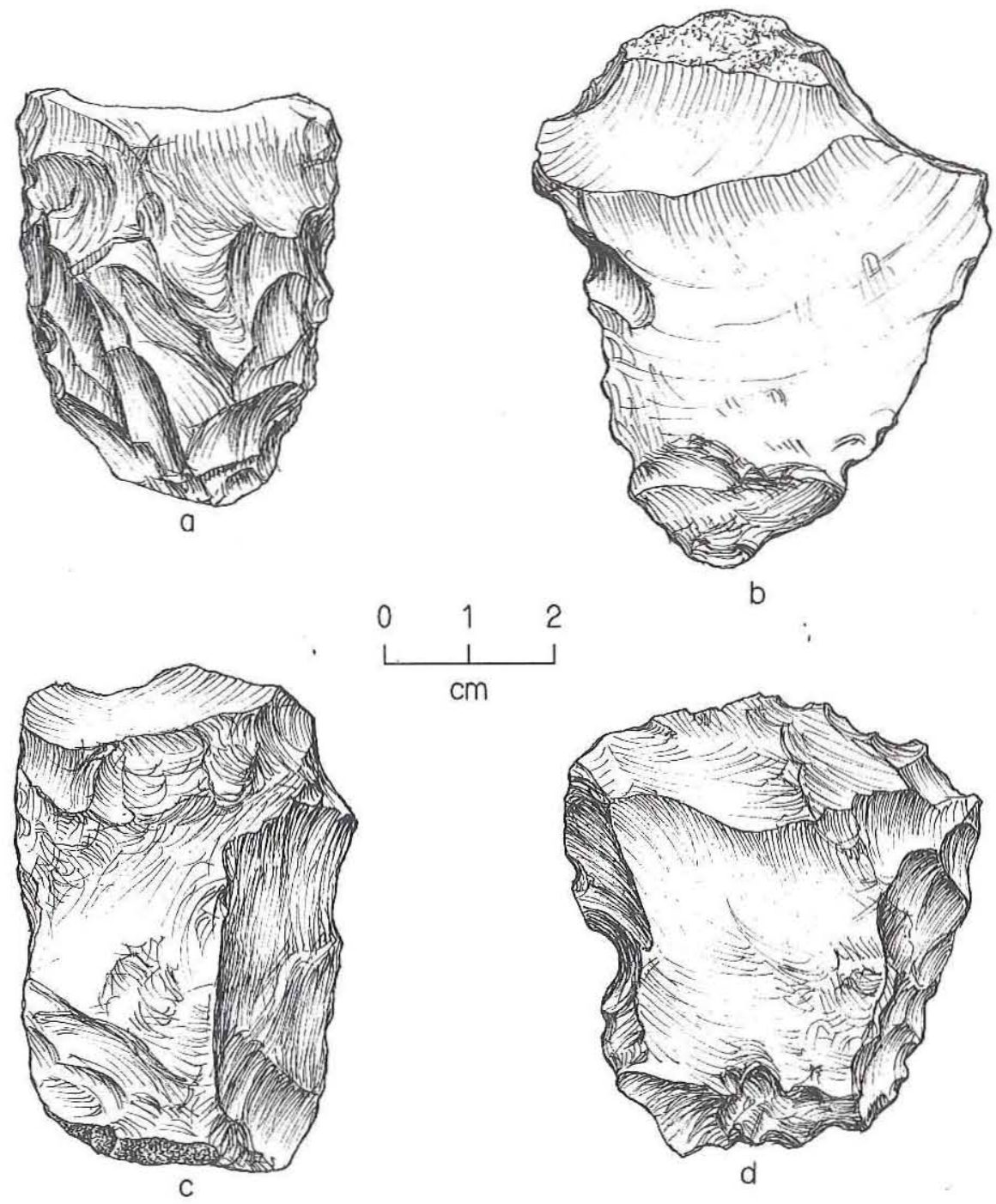

Figure 53. Unifacial gouges made on thick flake blanks, 41GR303B.

presence of reworking on these fragments suggests that they were functional specimens that were broken during reworking or under postdepositional circumstances. Maximum thickness could be measured only on two fragments $(\bar{x}=12.5 \mathrm{~mm}, \sigma=2.1)$. No other measures could be obtained on the remaining proximal fragments. All five fragments are of finegrained chert; three are of local origin (Colors 3, 5, and 6), one of the corticate fragments is nonlocal (Color 40), and the remaining decorticate specimen is of indeterminate origin (Color 57).

The single medial fragment is broken longitudinally. Based on the large amount of cortex and the sinuous bifacial edge, the fragment appears to represent an early-reduction-stage biface. Due to postdiscard heating, the break face has been altered so that the original break cause cannot be established with certainty. The medial fragment is of local finegrained chert (Color 10).

The single distal fragment is of silicified caliche. The presence of reworking on the blade edges, coupled with the use break, indicates that the fragment represents a finished biface. No cortex is present on the small fragment, and the nature of the blank used in its manufacture cannot be determined.

The wedge fragment appears to have been substantially reworked prior to discard. Although no measurements could be obtained, this fragment appears to represent a broad biface unlike the others in the small collection. Its morphology suggests that 
it was manufactured as a bifacial knife and that even after resharpening it continued to be used as a bifacial knife. Although the break morphology indicates a transverse break generated by a blow delivered to the lateral edge of the specimen, the resharpened blade edge suggests that this break may have occurred while the biface was being rejuvenated. It is of indeterminate-origin fine-grained chert (Color 57), although its original size appears to have exceeded that of most of the locally available cherts.

\section{Cobble Tools}

Twenty-seven cobble tools were recovered. Of these, $23(85 \%)$ are complete and $4(15 \%)$ are fragmentary. Although it is probable that these represent a number of different uses, it is difficult to separate them into distinct functional categories based on the types of use wear.

Based on their weight, the 23 complete tools can be separated into two broad size categories: (1) less than $100 \mathrm{~g}(\mathrm{n}=8$; Figure $54 a, b)$; and (2) greater than $100 \mathrm{~g}(\mathrm{n}=15$; Figure $54 c, d)$. While the lighter cobble tool group from 41GR291 contains a mix of functional categories, the eight light cobble tools from 41GR303B appear to form a more homogeneous group. All of the tools exhibit step-fractured working edges, and while some have heavily step fractured edges, none exhibit the edge rounding noted on some of the light cobble tools from 41GR291. Each tool has a single working edge, which is cortex backed on six of the eight specimens. Most $(n=$ $5,63 \%$ ) of the light cobble tools have straight to convex working edges; pointed working edges are present on three (37\%). Seven of the eight specimens in the light tool group are made on local fine-grained chert pebbles (Colors 5, 11, $27[n=3]$, and $55[n=$ 2]). The remaining specimen is made on a secondary fine-grained Potter chert flake.

As a group, the eight light cobble tools are relatively small. They have a mean length of $43.9 \mathrm{~mm}(\sigma=9.8)$, a mean width of $45.4 \mathrm{~mm}(\sigma=$ $6.2)$, and a mean thickness of $24.4 \mathrm{~mm}(\sigma=5.7)$. They are also relatively light, having a mean weight of $55.5 \mathrm{~g}(\sigma=24.8)$, ranging from a minimum of $21 \mathrm{~g}$ to a maximum of $87 \mathrm{~g}$.

The 15 complete cobble tools that are heavier than $100 \mathrm{~g}$ are characterized by a mean weight of $433.8 \mathrm{~g}$ (minimum $=151$, maximum $=1,186, \sigma=$ 296.1). These specimens are also considerably larger than their counterparts in the lighter group, as evidenced by their mean length $(\overline{\mathrm{x}}=87.0 \mathrm{~mm}, \sigma=$ 21.6), mean width $(\bar{x}=74.7 \mathrm{~mm}, \sigma=15.4)$, and mean thickness $(\overline{\mathrm{x}}=51.4 \mathrm{~mm}, \sigma=10.6)$.

Only four $(27 \%)$ specimens have single working edges; the majority ( $n=6,40 \%)$ have two working edges, three (20\%) have three working edges, and one each (7\%) has four and six working edges. Of the 15 tools in this weight group, $4(27 \%)$ have pointed working edges, 6 (40\%) have straight to convex working edges, and 5 (33\%) have multiple working edges of which some are pointed and others are straight to slightly convex. Cortex backing of all working edges is found on seven tools, while some working edges are cortex backed and others are not on six tools with multiple working edges. Three tools with multiple working edges do not have cortex backing opposite any of the working edges.

In addition to being distinguishable on the basis of weight, and unlike the cobble tools from 41GR291, the two groups of tools are distinct in terms of usewear characteristics. For instance, all 15 of the heavy cobble tools exhibit step fracturing on at least one of their working edges. In addition, all but three of these heavy cobble tools have edge rounding on all or at least some of their multiple working edges. This is less variability in use-wear characteristics among the heavy cobble tools than at 41GR291. This suggests that the 41GR303B cobble tool collection contains a large proportion of tools that are heavily worn but not rejuvenated, while tools that were worn and subsequently rejuvenated prior to discard and/or abandonment are fewer in number.

None of the heavy cobble tools are of finegrained chert. Specimens of coarse-grained Potter chert $(\mathrm{n}=7,47 \%)$ are most common, followed by those of coarse-grained quartzite $(n=4,27 \%)$. Two (13\%) heavy cobble tools are of fine-grained quartzite, and one $(7 \%)$ each is of coarse-grained chert and fine-grained Potter chert.

As mentioned earlier, four cobble tool fragments also were recovered. Three of these appear to represent the distal ends of heavy cobble tools. They are of fine-grained Potter chert. The fourth fragment is of locally available fine-grained chert (Color 31) and appears to represent the distal end of a light cobble tool or wedge.

Similarities between the experimentally produced use wear and the 41GR291 and 41GR303B specimens with rounded edges suggest that at least some of the heavy cobble tools from 41GR303B also were used as ground stone refurbishing tools. Given 

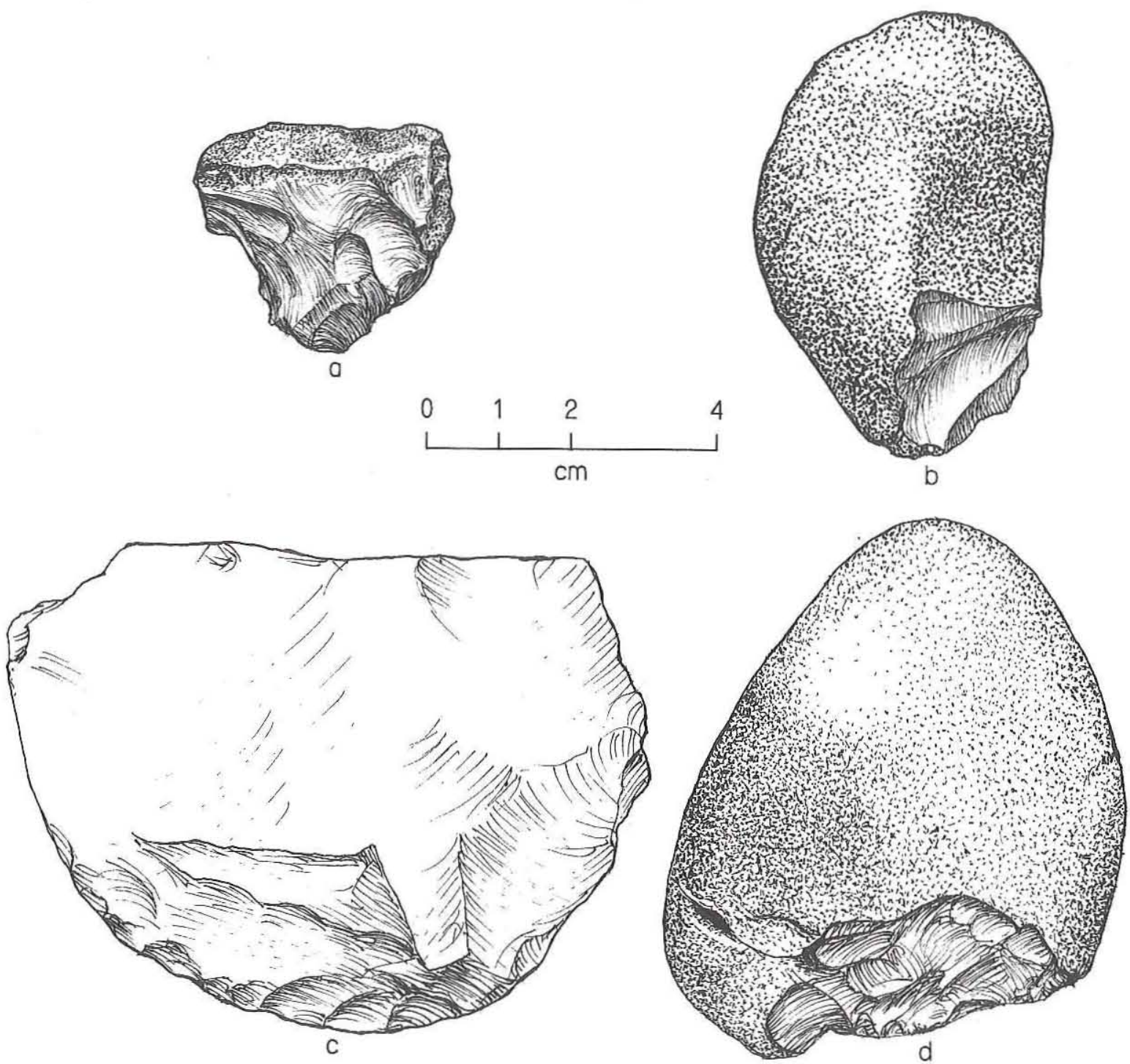

Figure 54. Cobble tools, 41GR303B. $(a-b)$ Light cobble tools with step-fractured working edges; $(c-d)$ heavy cobble tools with rounded working edges.

the lack of edge rounding on the lighter cobble tool working edges, it is possible that these tools were used as wedges.

\section{Unifaces}

Sixty-eight unifaces and fragments were recovered. They consists of 6 end scrapers, 1 side scraper, 2 combination end and side scrapers, 1 graver, 11 minimally retouched end scrapers, 5 minimally retouched side scrapers, 5 minimally retouched combination end and side scrapers, and 37 indeterminate unifaces and uniface edges.

\section{END SCRAPERS}

The six end scrapers have heavily retouched working edges, but their lateral margins exhibit little retouch. Two complete (Figure 55a,b) end scrapers and one nearly complete distal fragment are rather short and tear-drop shaped; the others are made on short and broad flake blanks. Five $(83 \%)$ of the blanks retain cortex; the remaining specimen is made on a tertiary fine-grained Potter chert flake blank. This specimen and two others are unusual in that they were made by using the dorsal face of the flake blank as the ventral face of the tool; as a result, two have 

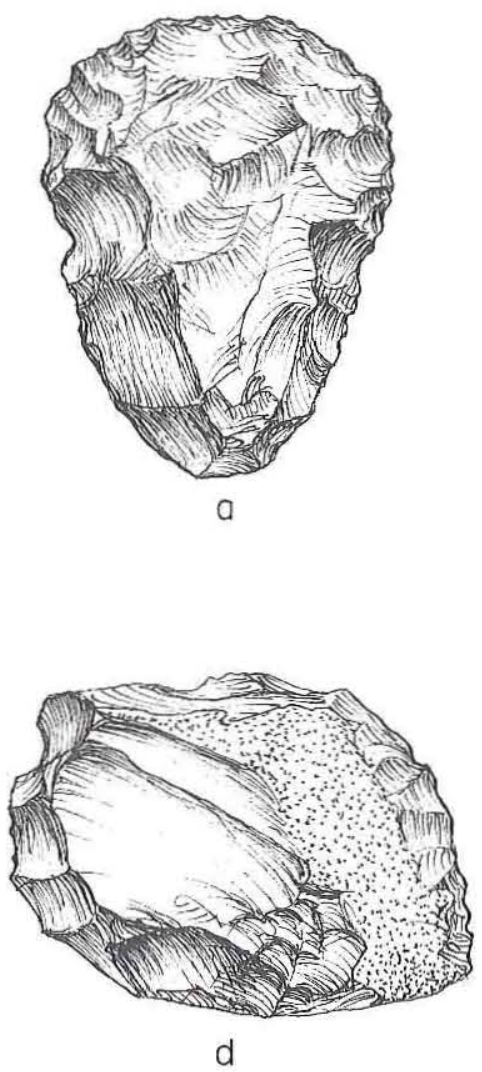
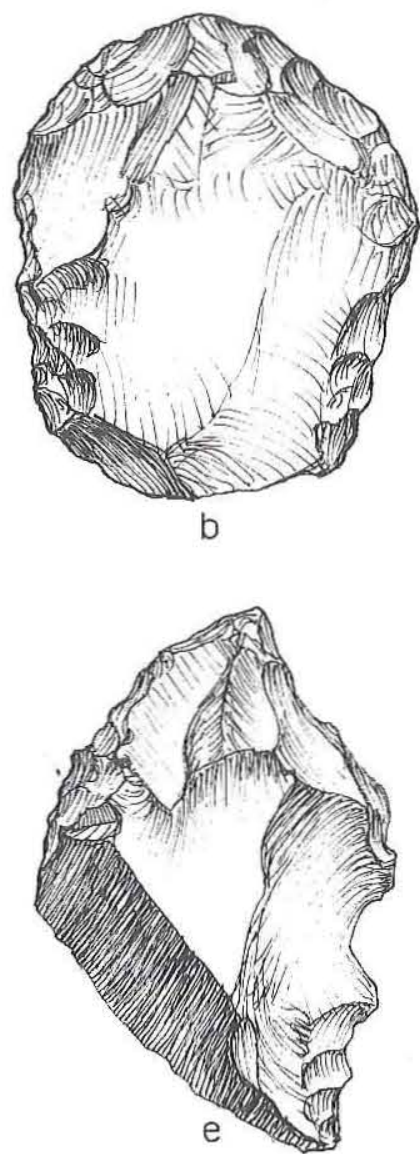
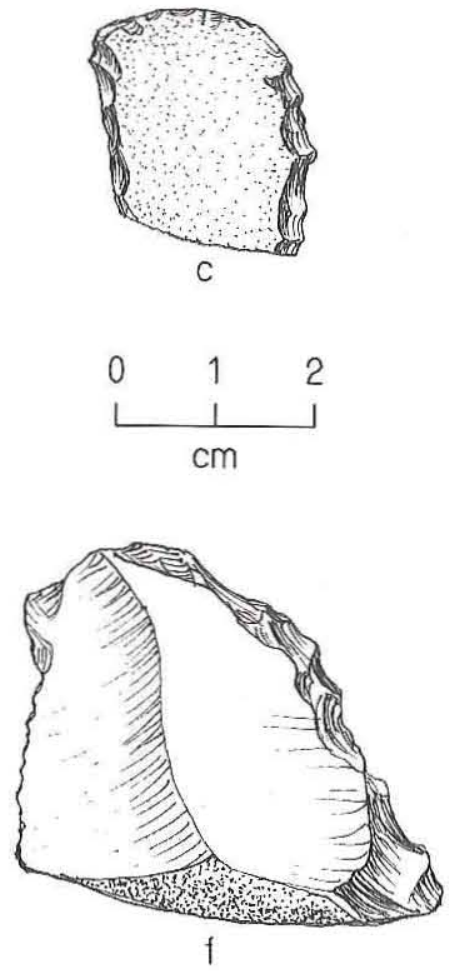

Figure 55. Unifaces, 41GR303B. ( $a-b)$ End scrapers; (c) side scraper with two working edges; $(d-e)$ combination end and side scrapers; $(f)$ graver.

corticate ventral surfaces, and the third has flake scars and intersecting flake scar ridges on its ventral face. Four specimens are complete, and two are distal fragments. One of the fragments was a functional specimen that appears to have been broken in the process of rehafting or reshaping of the haft portion. Due to substantial postdiscard heat shattering, the break cause could not be determined with certainty on the second fragment. Haft wear is present on one of the complete specimens and the nearly complete distal fragment. It is absent on two other complete specimens, and its presence/absence could not be established on the smaller distal fragment and a complete specimen of fine-grained Potter chert. Four $(67 \%)$ of the end scrapers are of local fine-grained chert (Colors 5, 27, 32, and 33). The remaining two are of fine-grained Potter chert. The four complete specimens have a mean length of $40.0 \mathrm{~mm}(\sigma=5.9)$, a mean width of $35.3 \mathrm{~mm} \mathrm{(} \sigma=$ 2.1 ), and a mean thickness of $16.0 \mathrm{~mm}(6.2)$.

\section{SIDE SCRAPERS}

The single side scraper is a distal fragment with two retouched lateral margins (Figure $55 c$ ). It is made on a secondary, local, fine-grained chert flake (Color 5). It appears to have been broken in use, and no haft wear is evident. The side scraper is $20 \mathrm{~mm}$ wide; no other measurements could be obtained.

\section{COMBINATION END AND SIDE SCRAPERS}

Both combination end and side scrapers are made on short, corticate, fine-grained chert flake blanks. One is of local chert (Color 29). and is complete; the other, of indeterminate-origin chert (Color 57), is a nearly complete proximal fragment. The complete specimen was made on a short $(47 \mathrm{~mm})$ and rather broad $(33 \mathrm{~mm})$ flake (Figure $55 d)$. It has substantial retouch on both lateral margins, and its distal end has been heavily 
reworked. The nearly complete specimen, made on a triangular flake blank (Figure 55e), is missing a small portion of its distal end. One lateral edge is heavily retouched, with the flaking extending onto the distal end. The opposite lateral margin is lightly retouched on the alternate face. The two specimens have a mean length of $41.5 \mathrm{~mm}(\sigma=7.8)$, a mean width of $38.0 \mathrm{~mm}(\sigma=7.1)$, and a mean thickness of $15.0 \mathrm{~mm}(\sigma=1.4)$.

\section{GRAVER}

A single complete graver (Figure 55f) was recovered. It has a single graver bit manufactured on the distal end of a thick, decorticate, local finegrained chert (Color 6) flake blank. The working bit was manufactured through minor modifications to the distal end of the parent flake. The tool is $53 \mathrm{~mm}$ long, $35 \mathrm{~mm}$ wide, and $18 \mathrm{~mm}$ thick.

\section{MINIMALLY RETOUCHED END SCRAPERS}

With the exception of the indeterminate unifaces, the minimally retouched end scrapers constitute the largest $(n=11)$ category. All but one specimen, a proximal fragment, are complete tools. Nine $(82 \%)$ are made on secondary flake blanks; the remaining two $(18 \%)$ are made on tertiary flakes. The flake blanks are irregular in shape and size (Figure 56a-d), ranging from small and mediumsized narrow flakes to small and medium-sized broad flakes. In general, however, the amount of retouch observed on their edges is significantly less in both amount and regularity than that observed on specimens categorized as end scrapers, side scrapers, and combination end and side scrapers. Like the 41GR291 uniface collection, the range of shapes and sizes suggests a lack of standardization of the flake blanks employed in uniface manufacture. The mean length of the 10 complete specimens is $33.4 \mathrm{~mm}$ ( $\sigma=$ 11.2). Maximum width $(\overline{\mathrm{x}}=33.6 \mathrm{~mm}, \sigma=12.1)$ and thickness $(\overline{\mathrm{x}}=11.8 \mathrm{~mm}, \sigma=3.8)$ were recorded on all 11 specimens. Seven (64\%) specimens are of fine-grained chert of local origin (Colors $3[n=2]$, 12,14 , and 55). Two of the remaining four are of fine-grained Potter chert, and one each is of coarsegrained quartzite and silicified caliche.

\section{MINIMALLY RETOUCHED SIDE SCRAPERS}

Of the five minimally retouched side scrapers, one is a large, longitudinally split, fine-grained quartzite heat spall with marginal retouch. The remaining specimens are made on complete secondary flake blanks (Figure 56e,f). The flake blanks appear to represent debitage derived from the early stages of biface manufacture rather than the purposeful removal of standardized blanks used for uniface manufacture. Only one specimen is of local fine-grained chert (Color 5); two are of coarse-grained quartzite, and one each is of fine-grained quartzite and fine-grained Potter chert. They have a mean length of $53.4 \mathrm{~mm}$ $(\sigma=19.9)$, a mean width of $33.0 \mathrm{~mm}(\sigma=12.0)$, and a mean thickness of $16.4 \mathrm{~mm}(\sigma=2.7)$.

\section{MINIMALLY RETOUCHED COMBINATION END AND SIDE SCRAPERS}

Five minimally retouched combination end and side scrapers were recovered. All are complete and are made on secondary flake blanks. One specimen is made on a short fine-grained Potter chert flake (Figure $56 \mathrm{~g}$ ). It has lightly retouched lateral and distal working edges. A second tool, of nonlocal fine-grained chert, is similar in morphology (Figure $56 h)$. It has alternately retouched lateral working edges and a lightly retouched distal working edge that retains use wear on its unresharpened portion. The three remaining specimens are made on irregularly shaped flake blanks. Two of these have one notched working edge in combination with a second straight to slightly convex working edge.

The two morphologically similar combination end and side scrapers suggest that minimally retouched unifaces may represent edge-modified flakes that have undergone subsequent resharpening rather than a distinctive class of expediently manufactured unifaces. That is, the retouch evident on minimally retouched unifaces results from the resharpening of worn unmodified working edges rather than shaping of the margins of the parent flake or generating a particular working-edge morphology prior to use of the tool.

Three of the five specimens are of fine-grained chert. As previously mentioned, one of these is of nonlocal origin (Color 51); the other two are of local origin (Color 6). One of the remaining two tools is of Tecovas jasper; the other is of fine-grained Potter chert. As with the other minimally retouched uniface categories, these unifaces are relatively small. They have a mean length of $40.2 \mathrm{~mm}(\sigma=15.7)$, a mean width of $30.4 \mathrm{~mm}(\sigma=8.0)$, and a mean thickness of 

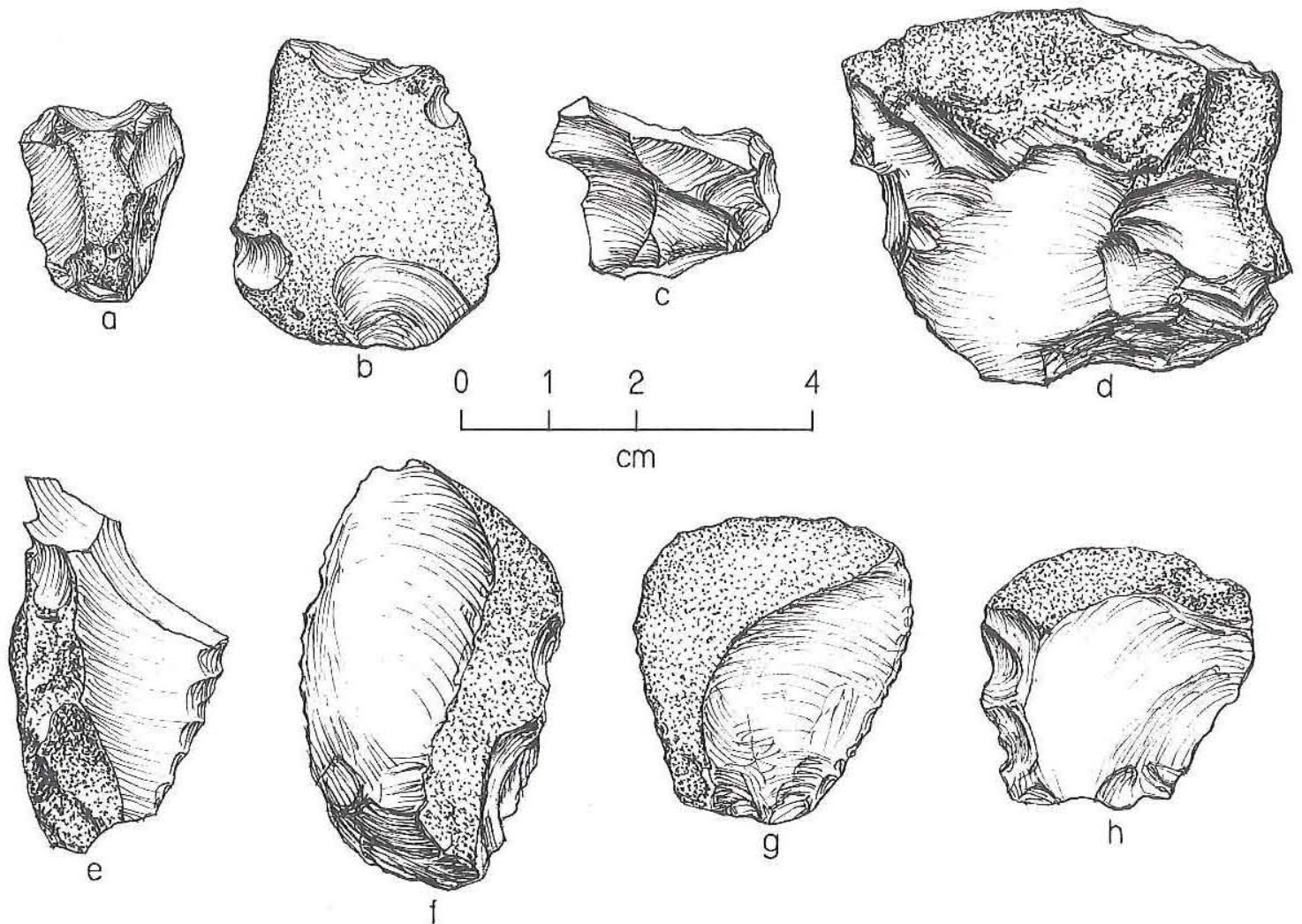

$\mathrm{cm}$
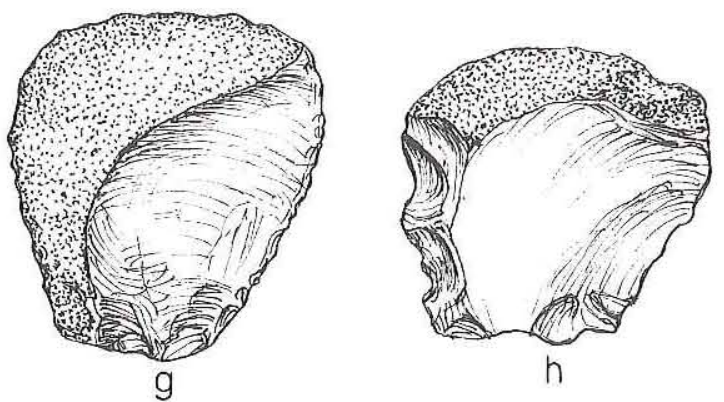

Figure 56. Minimally retouched unifaces, 41GR303B. (a-d) Small and medium-sized end scrapers made on narrow and broad flake blanks; $(e-f)$ side scrapers made on secondary flake blanks; $(g-h)$ combination end and side scrapers made on secondary flake blanks.

$10.6 \mathrm{~mm}(\sigma=3.3)$.

\section{INDETERMINATE UNIFACES}

The 37 indeterminate unifaces consist of $1(3 \%)$ complete tool, $12(32 \%)$ proximal fragments, 10 (27\%) distal fragments, $4(11 \%)$ medial specimens, 3 (8\%) longitudinally broken fragments, and 7 (19\%) indeterminate edges. The complete specimen is made on a short $(31 \mathrm{~mm})$ and rather broad $(57 \mathrm{~mm})$, local, fine-grained chert flake blank. It is heavily retouched on its distal end and one of its lateral margins. Because much of the dorsal surface is corticate and the remainder is highly patinated, the presence of haft and use wear is difficult to determine. Although it is illustrated with its distal end oriented toward the top of the page (Figure 57), as if it were an end scraper, the cortex and patina make it difficult to establish whether it is an end scraper, a combination end and side scraper, or a unifacial gouge with the working edge located along the shorter

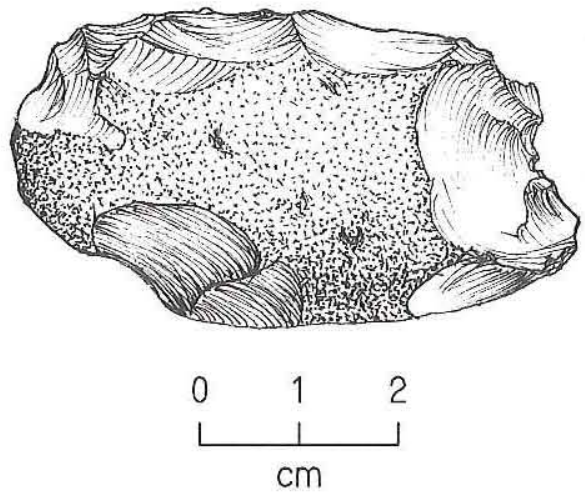

Figure 57. Indeterminate uniface, 41GR303B.

retouched edges.

The remainder of the indeterminate unifaces consist of proximal, medial, distal, and lateral edge fragments that could not be assigned with certainty to any other unifacial tool category. These fragments might represent minimally retouched unifaces, while others are portions of more extensively shaped end, 
side, and/or combination end and side scrapers.

The majority of the indeterminate unifaces and edge fragments are of fine-grained chert $(n=27$, $73 \%$ ). Specimens of locally available fine-grained chert are most common among these specimens $(\mathrm{n}=$ 21, 78\%; Colors 3, 5, $6[\mathrm{n}=4], 11,17,26[\mathrm{n}=2]$, $28,31[n=2], 32,33$, and $55[n=6])$. Only four (15\%) of the fine-grained chert specimens are of nonlocal cherts (Colors $43[n=2], 49$, and 56). The remaining two fine-grained chert fragments are of indeterminate origin (Color 57). Specimens of coarse-grained Potter chert $(\mathrm{n}=3,8 \%)$ and silicified caliche $(n=2,5 \%)$ occur in low frequencies. The remaining indeterminate uniface fragments consist of single specimens of coarse-grained chert, coarsegrained quartzite, Tecovas jasper, fine-grained Potter chert, and opalized caliche.

\section{Cores}

Fifty-nine cores and core fragments were recovered. Nearly half $(n=28,48 \%)$ of these are of fine-grained chert. Fine-grained Potter chert $(n=16$, $27 \%)$ and coarse-grained quartzite $(\mathrm{n}=10,17 \%)$ cores are the next most abundant categories. Finegrained quartzite, chalcedony, coarse-grained Potter chert, opalized caliche, and the indeterminate raw material category are each represented by a single specimen. With the exception of a single indeterminate-origin core, the remaining fine-grained chert cores are of local materials (Colors 2, 3 [ $n=4], 5,6$, $9,14[n=2], 16,22,26[n=2], 27[n=5], 31,32$, and $55[n=6])$.

Half $(n=14)$ of the 28 fine-grained chert specimens are complete; the others are fragmentary. Eleven (39\%) are pebble cores, 9 (32\%) are flake cores, and the nature of the core could not be established on 8 (29\%) specimens due to their incompleteness or degree of reduction. Multidirectional cores are most common $(n=13,46 \%)$, although unidirectional $(n=9,32 \%)$ and bidirectional specimens $(n=6,21 \%)$ are also frequent. Cortex is present on all but one (4\%) of the fine-grained chert cores.

The number of flake removals ranges from 1 to 13 , with the mean being $4.9(\sigma=3.4)$. None of the cores appear to have been recycled into this functional category from other tool or artifact categories. The fine-grained chert cores are relatively small; they have a mean length of $33.5 \mathrm{~mm}(\sigma=7.6)$, a mean width of $24.6 \mathrm{~mm}(\sigma=4.7)$, and a mean thickness of
$16.8 \mathrm{~mm}(\sigma=4.6)$.

Because a preliminary analysis of cores made of nonchert raw materials indicated substantial similarities between raw material types and because a number of the other raw materials are represented by single specimens, the discussion of the remaining cores combines the nonchert specimens into a single group. Of the 31 nonchert cores, 11 (35\%) are complete and $20(65 \%)$ are fragmentary. The percentage of fragmentary cores is somewhat higher than in the fine-grained chert cores. Pebble cores constitute a higher percentage of the nonchert cores $(\mathrm{n}=16,52 \%)$ than among the fine-grained chert specimens. On the other hand, flake cores represent a smaller percentage of the nonchert cores $(n=6$, $19 \%)$. Nearly equal percentages of the nonchert cores are multidirectional $(\mathrm{n}=13,42 \%)$ and bidirectional $(\mathrm{n}=12,39 \%)$. Unidirectional cores represent a smaller percentage of the nonchert cores $(\mathrm{n}=6,19 \%)$ than among the fine-grained chert specimens. Most $(n=28,90 \%)$ of the cores are corticate; only three $(10 \%)$ are entirely decorticate. Three $(10 \%)$ of the nonchert cores are manos that were broken and subsequently recycled into cores.

Assuming that cores with single flake removals represent tested specimens discarded prior to further reduction, six $(21 \%)$ of the fine-grained chert specimens fall into the tested core category. Somewhat surprisingly, only three $(10 \%)$ of the nonchert cores have not progressed beyond the tested core stage. On the other hand, while five $(16 \%)$ nonchert cores have eight or more flake removals, only three $(11 \%)$ fine-grained chert cores have eight or more flake removals. Unlike the collection of cores from 41GR291, these differences in the 41GR303B collection indicate that nonchert raw materials tended to be reduced relatively more intensively than fine-grained chert cores.

\section{Edge-modified Flakes}

Thirty-seven edge-modified flakes were recovered. Nearly three-quarters $(n=27,73 \%)$ are complete tools; the remaining $10(27 \%)$, are tool fragments. Most $(n=23,85 \%)$ of the complete tools represent complete flakes. Only four (15\%) are flake fragments; of these, three $(75 \%)$ are proximal fragments and one $(25 \%)$ is a chip. Slightly more than half $(n=19,51 \%)$ of the edge-modified flakes are tertiary specimens, nine $(24 \%)$ have between $1-50 \%$ cortex, seven (19\%) have between $51-99 \%$ 
cortex, and two (5\%) specimens are entirely corticate. Nearly equal numbers of specimens are in the 21-30-mm $(\mathrm{n}=13,35 \%)$ and $31-40-\mathrm{mm}(\mathrm{n}=12$, $32 \%)$ size categories. Seven (19\%) are greater than or equal to $41 \mathrm{~mm}$, and five (14\%) are less than $20 \mathrm{~mm}$.

Forty-six working edges were noted on the 37 edge-modified flakes. The majority $(n=29,78 \%)$ of the specimens have single working edges. Seven (19\%) edge-modified flakes have two working edges, and a single (3\%) tool has three utilized edges. Working edges used for scraping tasks alone occur on 32 (86\%) edge-modified flakes. Four $(11 \%)$ have working edges that were used in sawing/cutting tasks, and a single tool has working edges used in both sawing/cutting and scraping tasks.

The majority ( $\mathrm{n}=29,78 \%)$ of the edgemodified flakes are of fine-grained chert. Two specimens each $(3 \%)$ are of fine-grained Potter chert and silicified wood. The remaining four edgemodified flakes are of coarse-grained quartzite, Tecovas jasper, coarse-grained Potter chert, and silicified caliche. Twenty-three $(79 \%)$ of the finegrained chert specimens are of locally available cherts (Colors $5[n=4], 6[n=3], 7,16[n=3], 26$, $30[n=2], 32[n=3]$, and $55[n=6])$. Five $(17 \%)$ are of nonlocal fine-grained chert (Colors 39, 40, 42, and $51[n=2])$, and one $(3 \%)$ specimen is of indeterminate origin.

\section{Unmodified Debitage}

A total of 2,064 pieces of unmodified lithic debitage was recovered. Slightly more than half $(n=$ $1,094,53 \%)$ are of fine-grained chert. Fine-grained Potter chert $(n=415,20 \%)$, coarse-grained Potter chert $(n=229,11 \%)$, and coarse-grained quartzite $(n=150,7 \%)$ are the next most frequent raw materials. Specimens of fine-grained quartzite $(\mathrm{n}=$ $46,2 \%)$, silicified caliche $(n=27,1 \%)$, unidentified material types $(n=24,1 \%)$, opalized caliche $(n=22$, $1 \%)$, silicified wood $(\mathrm{n}=17,1 \%)$, Tecovas jasper $(\mathrm{n}=17,1 \%)$, sandstone $(\mathrm{n}=12,1 \%)$, coarse-grained chert $(\mathrm{n}=8,<1 \%)$, and chalcedony $(\mathrm{n}=3,<1 \%)$ occur in significantly lower quantities. Table 30 shows the breakdown of debitage attributes within the fine- and coarse-grained chert, quartzite, and Potter chert.

Chips are relatively common among the fineand coarse-grained chert and quartzite and the finegrained Potter chert. Complete flakes are slightly more common than chips in the coarse-grained Potter chert. This pattern is identical to that noted in the 41GR291 debitage collection, suggesting that raw material coarseness rather than behavioral factors conditioned the breakage of debitage during removal, assuming that most of the chips were broken during removal rather than postdepositionally. Except in the coarse-grained Potter chert, complete flakes are the second most common category. Chunks are infrequent or absent in all raw material types.

Entirely decorticate complete flakes and flake fragments are most common among all the raw materials. This pattern is also identical to that noted in the 41GR291 collection. It suggests not only a general similarity in the degree of reduction or manufacture of artifacts between the sites but also similarities in the degree of reduction of different grades of raw materials. The highest percentages of entirely decorticate debitage occur in the fine-grained quartzite, fine-grained Potter chert, and fine-grained chert. The lowest percentage of entirely decorticate debitage occurs in the coarse-grained Potter chert collection. Entirely corticate debitage is relatively infrequent in all raw materials, with the highest percentages occurring in the coarse-grained quartzite and coarse-grained Potter chert collections. The lowest percentages of entirely corticate debitage are found in the fine-grained chert, fine-grained Potter chert, and fine-grained quartzite; in these categories, the majority of the chunks are decorticate.

Among the flakes and flake fragments with platforms, entirely decorticate striking platforms are more common than corticate platforms in all of the raw materials. The highest percentages of decorticate platforms occur among the fine-grained chert (78\%) and fine-grained Potter chert (71\%) specimens. The lowest percentages of decorticate platforms are found among the coarse-grained quartzite and coarsegrained Potter chert.

Overall, platform grinding is not common in any of the raw material types. The highest percentages occur in the fine-grained chert and fine-grained Potter chert (9\% each). Somewhat surprisingly, the coarse-grained Potter chert debitage has only a slightly lower percentage (8\%) of ground platforms. The lowest percentage of ground platforms occurs in the coarse- and fine-grained quartzite debitage.

The majority of the platform-bearing flakes are single faceted in all of the raw material types. The highest percentages of single-faceted specimens occur in the coarse-grained quartzite $(82 \%)$, fine-grained 


\begin{tabular}{|c|c|c|c|c|c|c|c|c|c|c|c|c|c|}
\hline \multirow[b]{2}{*}{ Attribute } & \multicolumn{12}{|c|}{$\begin{array}{l}\text { TABLE } 30 \\
\text { UTES WITHIN THE FINE- AND COARSE-GRAINED } \\
\text { TTER CHERT RAW MATERIALS, 41GR303B }\end{array}$} & \multirow[b]{2}{*}{ Totals } \\
\hline & $\#$ & $\%$ & $\#$ & $\%$ & $\#$ & $\%$ & $\#$ & $\%$ & $\#$ & $\%$ & $\#$ & $\%$ & \\
\hline $\begin{array}{l}\text { Flake Completeness } \\
\text { Complete } \\
\text { Proximal } \\
\text { Chips } \\
\text { Chunks } \\
\text { Totals: }\end{array}$ & $\begin{array}{r}443 \\
167 \\
467 \\
17 \\
1094\end{array}$ & $\begin{array}{r}40 \\
15 \\
43 \\
2 \\
100\end{array}$ & $\begin{array}{l}- \\
3 \\
5 \\
\overline{8}\end{array}$ & $\begin{array}{r}\overline{38} \\
63 \\
\overline{-} \\
101\end{array}$ & $\begin{array}{r}16 \\
9 \\
20 \\
\frac{1}{46}\end{array}$ & $\begin{array}{r}35 \\
20 \\
43 \\
\frac{2}{100}\end{array}$ & $\begin{array}{r}41 \\
37 \\
68 \\
4 \\
150\end{array}$ & $\begin{array}{r}27 \\
25 \\
45 \\
\frac{3}{100}\end{array}$ & $\begin{array}{r}127 \\
107 \\
173 \\
-8 \\
415\end{array}$ & $\begin{array}{r}31 \\
26 \\
42 \\
\frac{2}{101}\end{array}$ & $\begin{array}{r}105 \\
42 \\
82 \\
\overline{-}\end{array}$ & $\begin{array}{r}46 \\
18 \\
36 \\
\overline{-}\end{array}$ & $\begin{array}{r}732 \\
365 \\
815 \\
\quad 30 \\
1942\end{array}$ \\
\hline $\begin{array}{c}\text { Cortex Percent } \\
0 \\
1-50 \\
51-99 \\
100 \\
\text { Totals: }\end{array}$ & $\begin{array}{r}670 \\
259 \\
105 \\
43 \\
1077\end{array}$ & $\begin{array}{r}62 \\
24 \\
10 \\
4 \\
100\end{array}$ & $\begin{array}{l}5 \\
\overline{1} \\
\frac{2}{8}\end{array}$ & $\begin{array}{r}63 \\
- \\
13 \\
25 \\
101 \\
\end{array}$ & $\begin{array}{r}31 \\
7 \\
4 \\
3 \\
45\end{array}$ & $\begin{array}{r}69 \\
16 \\
9 \\
7 \\
101 \\
\end{array}$ & $\begin{array}{r}85 \\
30 \\
17 \\
14 \\
146 \\
\end{array}$ & $\begin{array}{r}58 \\
21 \\
12 \\
10 \\
101 \\
\end{array}$ & $\begin{array}{r}264 \\
82 \\
40 \\
21 \\
407\end{array}$ & $\begin{array}{r}65 \\
20 \\
10 \\
5 \\
100\end{array}$ & $\begin{array}{r}108 \\
61 \\
39 \\
21 \\
229\end{array}$ & $\begin{array}{r}47 \\
27 \\
17 \\
9 \\
100\end{array}$ & $\begin{array}{r}1163 \\
439 \\
206 \\
104 \\
1912\end{array}$ \\
\hline $\begin{array}{l}\text { Platform Cortex } \\
\text { Absent } \\
\text { Present } \\
\text { Totals: }\end{array}$ & $\begin{array}{l}478 \\
132 \\
610\end{array}$ & $\begin{array}{r}78 \\
22 \\
100\end{array}$ & $\begin{array}{l}2 \\
\frac{1}{3}\end{array}$ & $\begin{array}{r}67 \\
33 \\
100\end{array}$ & $\begin{array}{r}15 \\
10 \\
25\end{array}$ & $\begin{array}{r}60 \\
40 \\
100\end{array}$ & $\begin{array}{r}43 \\
35 \\
78\end{array}$ & $\begin{array}{r}55 \\
45 \\
100\end{array}$ & $\begin{array}{r}165 \\
\frac{69}{234}\end{array}$ & $\begin{array}{r}71 \\
29 \\
100\end{array}$ & $\begin{array}{r}81 \\
66 \\
147\end{array}$ & $\begin{array}{r}55 \\
45 \\
100\end{array}$ & $\begin{array}{r}784 \\
313 \\
1097\end{array}$ \\
\hline $\begin{array}{l}\text { Platform Grinding } \\
\text { Absent } \\
\text { Present } \\
\text { Totals: }\end{array}$ & $\begin{array}{r}556 \\
54 \\
610\end{array}$ & $\begin{array}{r}91 \\
\frac{9}{9} \\
100\end{array}$ & $\begin{array}{l}\frac{3}{3} \\
\frac{-}{3}\end{array}$ & $\begin{array}{l}\frac{100}{100} \\
\frac{\sigma}{10}\end{array}$ & $\begin{array}{r}24 \\
\frac{1}{25}\end{array}$ & $\begin{array}{r}96 \\
\quad 4 \\
100\end{array}$ & $\begin{array}{r}76 \\
\frac{2}{78}\end{array}$ & $\begin{array}{r}97 \\
\frac{3}{100}\end{array}$ & $\begin{array}{r}214 \\
20 \\
234\end{array}$ & $\begin{array}{r}91 \\
\frac{9}{100}\end{array}$ & $\begin{array}{r}135 \\
12 \\
147\end{array}$ & $\begin{array}{r}92 \\
\frac{8}{100}\end{array}$ & $\begin{array}{r}1008 \\
\frac{89}{1097}\end{array}$ \\
\hline
\end{tabular}




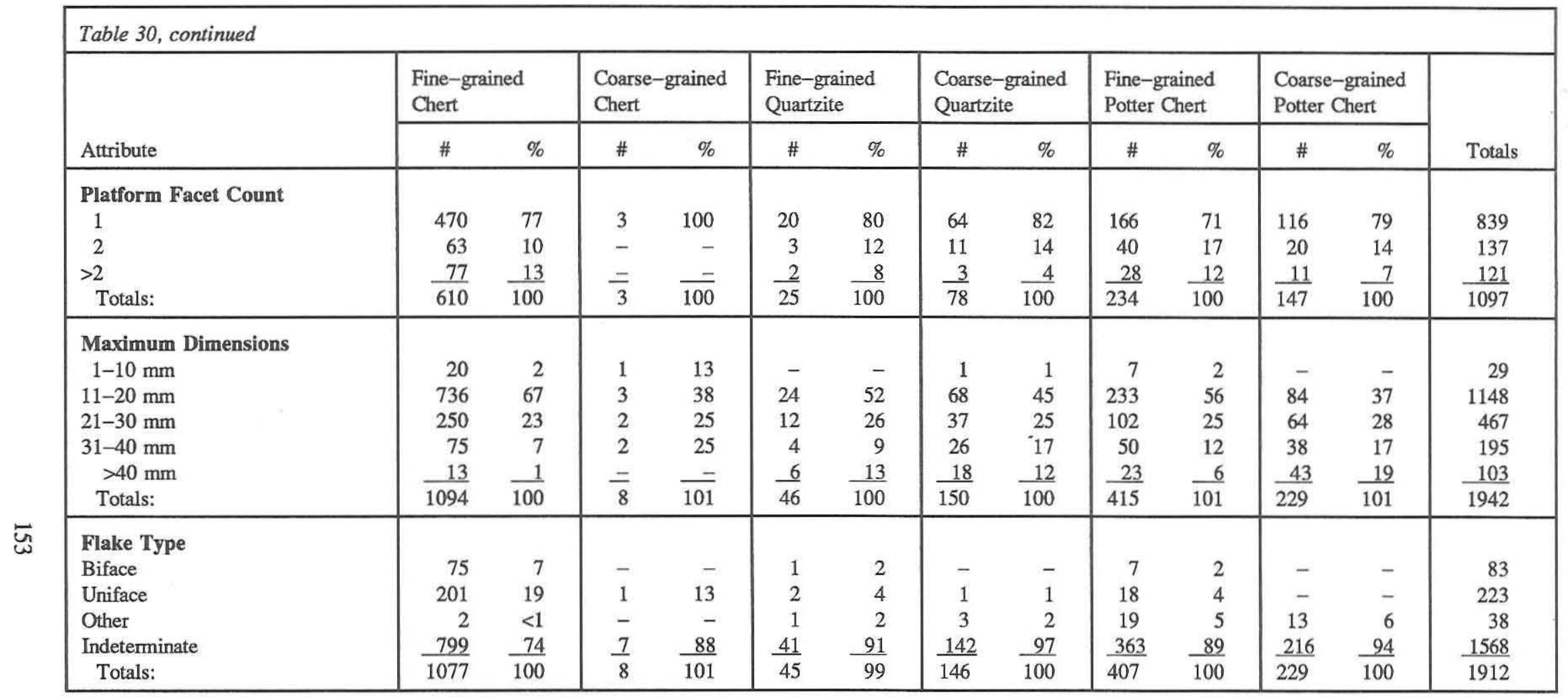


quartzite $(80 \%)$, and coarse-grained Potter chert (79\%). Fine-grained chert $(13 \%)$ and fine-grained Potter chert $(12 \%)$ contain the highest percentages of platform-bearing debitage with more than two platform facets.

In each raw material type, the largest percentage of debitage falls in the 11-20-mm size category. This pattern is also similar to that noted at 41GR291. It appears to confirm the initial conclusion regarding similarities in stages of reduction, as well as implying that the raw materials used at the two sites were of similar size ranges. Debitage greater than $40 \mathrm{~mm}$ in maximum dimension is most frequent in the coarse-grained Potter chert and the fine- and coarsegrained quartzite.

As at 41GR291, the majority of the complete flakes and flake fragments recovered from 41GR303B could not be grouped into any flake type (reduction technique) category. However, some interesting patterns are evident within the small number of specimens that were assigned. For instance, the largest percentages of flakes derived from bifacial and unifacial reduction strategies are in the fine-grained chert debitage. Debitage derived from bifacial reduction is much less frequent in the fine-grained quartzite and the fine-grained Potter chert, and none of the specimens of coarse-grained chert, coarse-grained quartzite, and coarse-grained Potter chert are in this category. Similarly, specimens derived from uniface reduction occur in low percentages or are absent in the fine- and coarsegrained quartzite and the fine- and coarse-grained Potter chert collections. Specimens grouped into the other flake type category are relatively frequent in the fine- and coarse-grained Potter cherts. These flakes most probably are from cobble tool rejuvenation.

The majority $(n=939,86 \%)$ of the fine-grained chert debitage is of local origin. This percentage is slightly lower than that noted at 41GR291. Finegrained chert specimens of nonlocal origin comprise $12 \%$ of the 41 GR $303 B$ collection, while only $2 \%(n=$ 28) are of indeterminate origin.

The breakdown of lithic debitage attributes for raw materials other than chert, quartzite, and Potter chert is shown in Table 31. Because only three chalcedony specimens were recovered, they are combined with the unidentified materials. As with the previously discussed raw material groups, chips tend to form the largest category among the silicified caliche, sandstone, opalized caliche, and unidentified materials debitage. Complete flakes constitute slightly higher percentages than chips among the Tecovas jasper and silicified wood debitage. Chunks are infrequent or absent in the various raw materials.

High percentages of entirely decorticate debitage are found in the Tecovas jasper (76\%) and combined chalcedony/unidentified $(70 \%)$ materials. These percentages are higher than those for any of the other raw material categories recovered from the site. The lowest percentage of decorticate debitage occurs in the sandstone debitage ( $25 \%$ ), followed by silicified wood $(50 \%)$. The small number of chunks in these less-common raw materials range from entirely decorticate (i.e., silicified caliche) to entirely corticate (i.e., opalized caliche and silicified wood).

Most of the platform-bearing flakes have decorticate striking platforms. The highest percentages of decorticate striking platforms occur in the Tecovas jasper (90\%), opalized caliche $(90 \%)$, and combined chalcedony/unidentified materials (86\%). These percentages are higher than the percentages of decorticate striking platforms among the chert, quartzite, and Potter chert debitage. None of the platform-bearing sandstone debitage have decorticate striking platforms. Platform grinding is an insignificant preparation technique in these raw material types. None of the Tecovas jasper, silicified caliche, and sandstone flakes have ground striking platforms. Flakes with single-faceted striking platforms are most common among the sandstone $(100 \%)$, silicified caliche $(87 \%)$, and Tecovas jasper (80\%) specimens. Flakes with three or more striking platform facets are few to absent in all of these raw materials.

In general, the highest percentages of debitage are in the 11-20-mm size category (i.e., sandstone and combined chalcedony/unidentified materials) or the 21-30-mm size category (i.e., opalized caliche and silicified wood). Within the Tecovas jasper and silicified caliche debitage, equal percentages of specimens are found in these two size categories. The highest percentages of debitage larger than $40 \mathrm{~mm}$ are found in the sandstone and combined chalcedony/unidentified materials.

Very few of the specimens of these raw material types could be identified in terms of manufacturing technique. Most are included in the indeterminate flake type category.

\section{Ground and Battered Stone Artifacts}

Twenty-five ground and battered stone artifacts were recovered. They are divided into four catego- 
ries: mano, metate, pestle, and hammerstone. Manos $(n=16)$ and metates $(n=8)$ constitute the largest categories. The remaining ground stone specimen is a pestle. The only battered stone artifacts are two complete hammerstones.

\section{Manos}

The majority ( $n=13,81 \%)$ of the manos are of coarse-grained quartzite; the remaining three (19\%) are of sandstone. Only four (25\%) are complete (Figure 58). Seven (44\%) specimens exhibit bifacial use wear, two (13\%) have unifacial use wear, and seven others have unifacial wear patterns but are too small to establish with certainty whether use wear is present on both faces of the artifact. Pecking is present on both faces of four (25\%) manos; it is unifacial on three others, and absent on a large fragment. The presence and/or extent of pecking could not be established with certainty on eight $(50 \%)$ specimens. Battering is absent on three (19\%) and indeterminate on nine $(56 \%)$. Of the remaining four, one $(6 \%)$ has slight battering and three $(19 \%)$ have moderate battering. Seven (44\%) manos have biconvex transverse cross sections, and two (23\%) are lenticular. Transverse cross sections could be determined on only two of the remaining seven specimens; one has a beveled cross section and the other is tabular. The shape of five (31\%) small fragments could not be determined. Nine $(56 \%)$ of the remaining specimens are oval in shape, while two $(13 \%)$ are rounded and flat. The four complete manos have a mean length of $105.0 \mathrm{~mm}(\sigma=23.6)$ and a mean weight of $473.3 \mathrm{~g}$, ranging from 185 to $534 \mathrm{~g}$. Maximum width was measured on five specimens; their mean is $76.8 \mathrm{~mm}(\sigma=14.7)$. The mean maximum thickness of 10 manos is $38.3 \mathrm{~mm}$ $(\sigma=9.1)$. The mean working surface area of the four complete manos is $4,835.0 \mathrm{~mm}^{2}(\sigma=1,477.7)$, ranging from a minimum of $2,900 \mathrm{~mm}^{2}$ to a maximum of $6,232 \mathrm{~mm}^{2}$.

\section{Metates}

The eight metates recovered are of sandstone. Only two (25\%) are complete. Four (50\%) exhibit unifacial use wear, and three $(38 \%)$ others have use wear on two faces. The extent of use wear could not be determined with certainty on the remaining fragment. Pecking is present on the single use-worn face of the four specimens with unifacial use wear.
It is present on both faces of the three metates with bifacial use wear. The presence of use wear could not be determined with certainty on one small fragment. Battering is absent on the two complete metates, and it cannot be determined on the fragments, although it is probable that none of these were battered. The two complete metates are rectangular in shape. Five larger fragments appear to have been oval, and the original shape of the remaining fragmentary specimen cannot be determined. Three of the metates have biconcave transverse cross sections, and three others are planoconvex in cross section. A single specimen has a lenticular cross section, while the cross section could not be determined with certainty on the small metate fragment. The two complete metates have a mean length of $405.5 \mathrm{~mm}(\sigma=46.0)$. Maximum width ( $\overline{\mathrm{x}}=210.7 \mathrm{~mm}, \sigma=11.2)$ could be measured on three specimens. Maximum thickness $(\overline{\mathrm{x}}=43.1 \mathrm{~mm}, \sigma=$ 8.8) was measured on seven metates. The working surfaces of the two complete metates measure $42,900 \mathrm{~mm}^{2}$ and $61,200 \mathrm{~mm}^{2}$.

\section{Pestle}

A single complete sandstone specimen is included in this category (Figure 59a). It has an elongated hourglass shape with one broad and one narrow end and a lenticular transverse cross section. Only the broad end appears to have been used. It exhibits light battering on its extreme distal portion, although the two faces are not smoothed as on the pestle fragments from 41GR291. Pecking is not evident. The specimen is $207 \mathrm{~mm}$ long; it has a maximum width of $83 \mathrm{~mm}$ and a maximum thickness of $30 \mathrm{~mm}$. It weighs $648 \mathrm{~g}$.

\section{Hammerstones}

Two complete hammerstones were recovered; both are of coarse-grained quartzite. One is small $(60 \times 60 \times 20 \mathrm{~mm})$ and light $(110 \mathrm{~g})$, and the other is a medium-sized $(76 \times 74 \times 73 \mathrm{~mm}$ ) rounded nodule weighing $616 \mathrm{~g}$. The smaller hammerstone exhibits light battering around its periphery (Figure $59 b$ ); the heavier specimen has moderate battering concentrated primarily on one end (Figure 59c).

\section{Modified Hematite}

Two pieces of modified hematite were recov- 


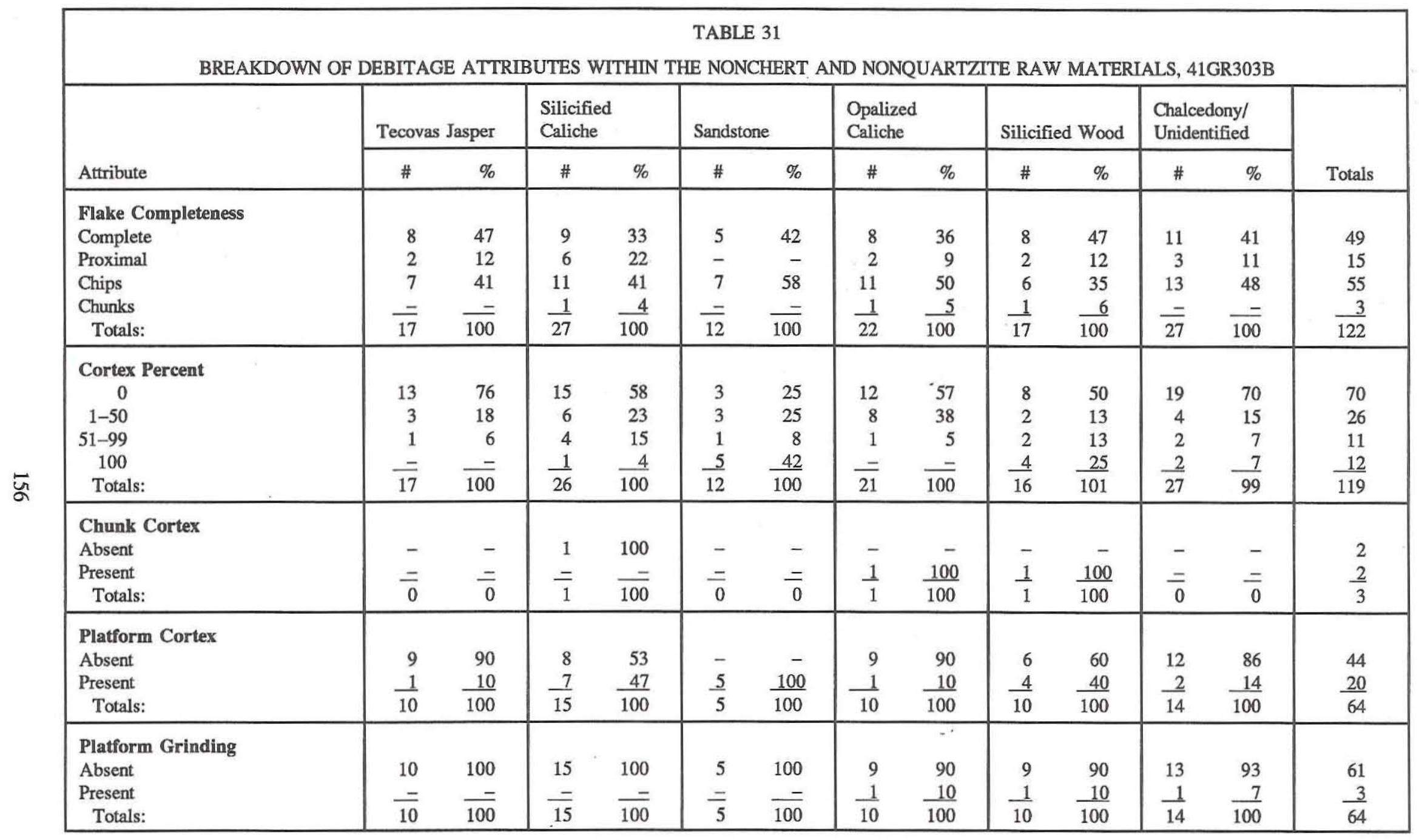




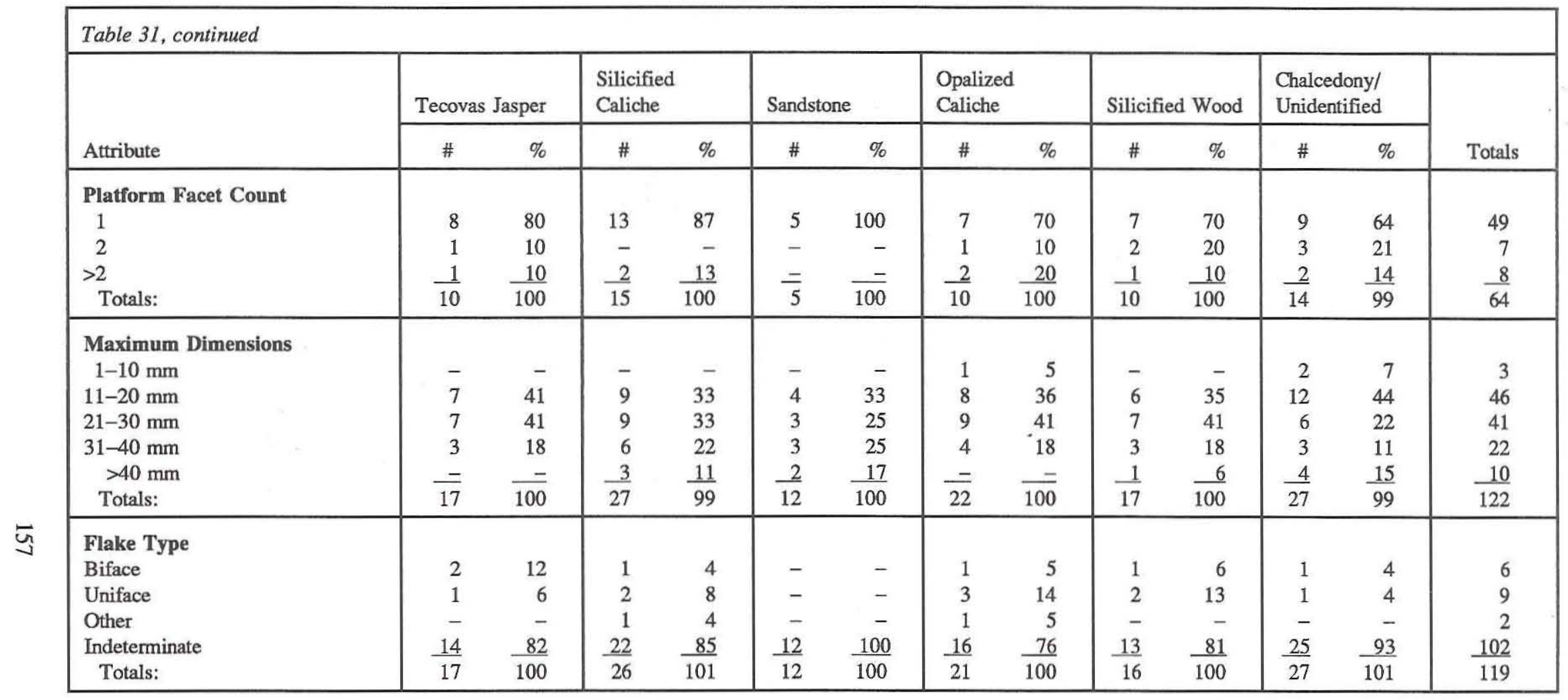




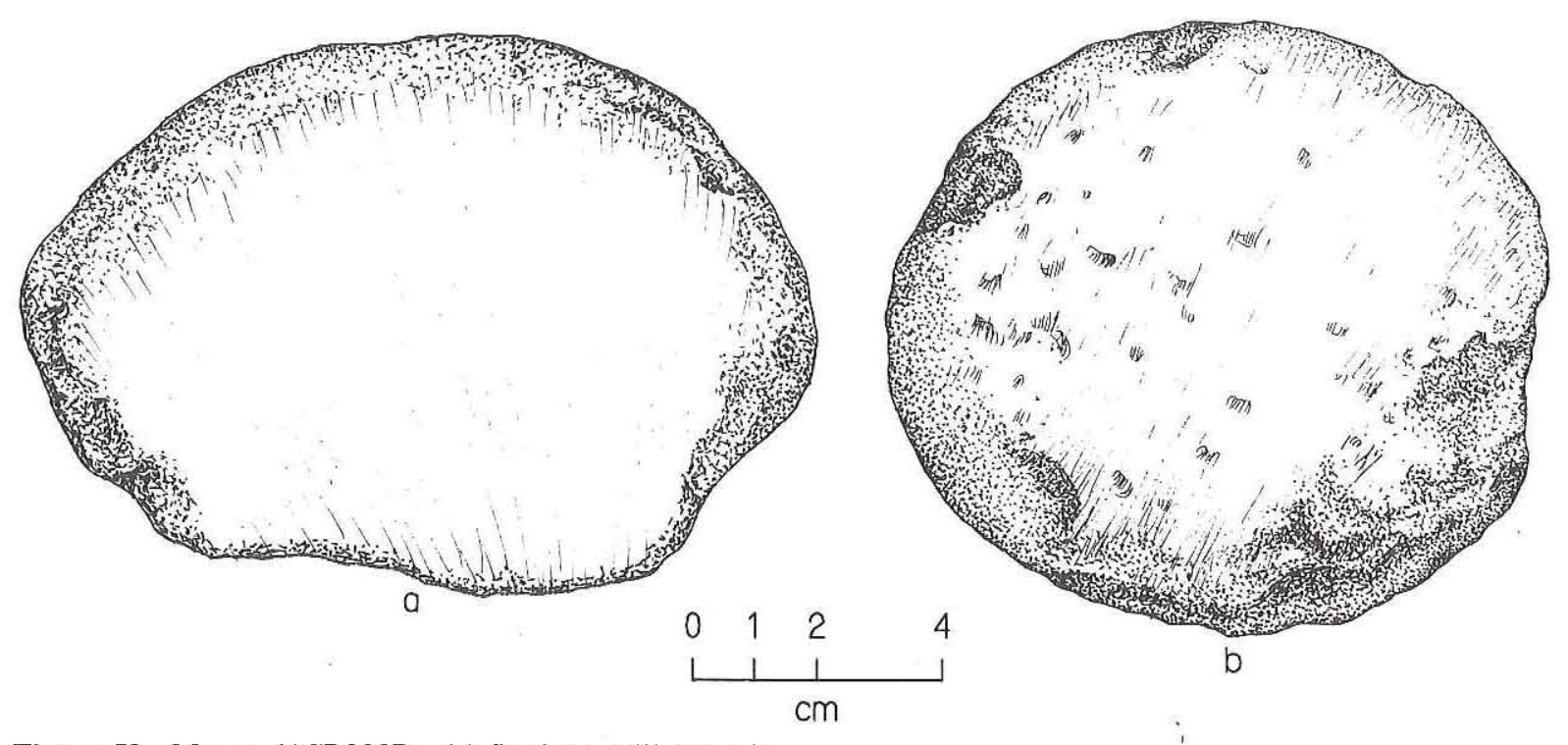

Figure 58. Manos, 41GR303B. (a) Sandstone; (b) quartzite.

ered. Specular hematite is considered to be a paint stone because of its relative softness, and when ground it yields a bright red powder (Pough 1960: 124). Two specimens exhibit indications of such grinding on their flat surfaces. One specimen, from Level 7 of EU 13, has striations on its broadest surface and measures $33.4 \mathrm{~mm}$ in length, $15.2 \mathrm{~mm}$ in width, and $7.5 \mathrm{~mm}$ in thickness; it weighs $4.9 \mathrm{~g}$. A second specimen, from Level 2 of EU 2, has high polish on its flattest side and measures $22.2 \mathrm{~mm}$ in length, $15.3 \mathrm{~mm}$ in width, and $11.1 \mathrm{~mm}$ in thickness; it weighs $4.4 \mathrm{~g}$. This material is quite common in the project area, and these specimens probably were obtained locally.

\section{Manuports}

These specimens do not fit into any artifactual categories and do not occur naturally within the site limits. The category consists of burned sandstone and fire-cracked chert and quartzite and small pieces of interestingly shaped unburned sandstone.

\section{Burned and Fire-cracked Rocks}

The category of burned and fire-cracked rocks at 41GR303B consists of burned sandstone and firecracked chert, Potter chert, and quartzite. Burned sandstone is ubiquitous, occurring in every excavation unit. A total of $654 \mathrm{~kg}$ of burned sandstone was documented during the 1992 season.
A total of $2.5 \mathrm{~kg}$ of fire-cracked rocks was recovered from the entire site. This category consists of heavily burned chert chunks, Potter chert, and quartzite. All of these materials are of local origin; they were scattered over the entire site and were not particularly concentrated in any specific areas.

\section{Odd-shaped Stones}

This category consists of 15 pieces of unburned sandstone with maximum dimensions of less than $15 \mathrm{~cm}$, minimum dimensions of more than $5 \mathrm{~cm}$, and shapes described as round balls, flat ovals, flat rectangles, and elongated bipoints. These specimens exhibit no wear patterns and have no obvious use, and thus they are classified as curiosities.

\section{Vertebrate Faunal Remains}

Sixteen vertebrate faunal specimens were recovered from 41GR303B during the 1992 excavations (see Appendix C). Possible human alteration of the bones consists of spiral fracturing and burning. Spiral fracturing is present on one specimen, a large artiodactyl proximal radius fragment recovered from EU 29 at $52 \mathrm{~cm}$. Five specimens exhibit burning: two charred medium/large mammal fragments recovered from EU 31 at $7-17 \mathrm{~cm}$; a charred element only identifiable to the level of subphylum Vertebrata recovered from EU 34 at $34-44 \mathrm{~cm}$; a calcined fragment of a medium artiodactyl tooth recovered 

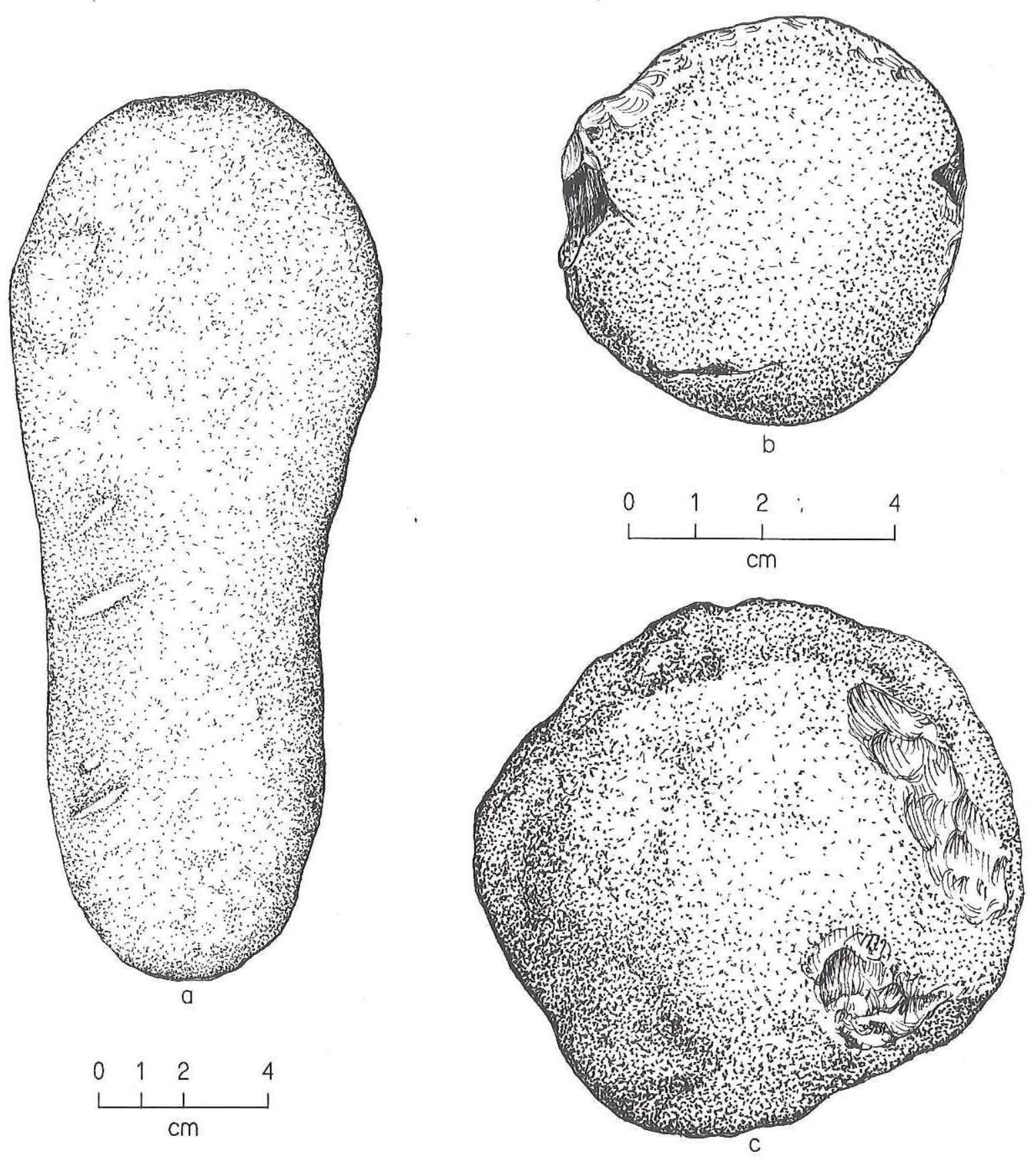

Figure 59. Battered stone artifacts, 41GR303B. (a) Pestle; $(b-c)$ coarse-grained quartzite hammerstones.

from EU 35 at $11-21 \mathrm{~cm}$; and a calcined mammal element recovered from EU 17 at $6-16 \mathrm{~cm}$. Unmodified bones consist of the following: three fragments of medium/large mammal from EU 4 (Level 4), EU 6 (Level 4), and EU 7 (Level 1); a bison astragalus from EU 6 (Level 11); a pelvis fragment of a rabbit from EU 27 (Level 11); and a Geomys sp. mandible with four articulated teeth from
EU 5 (Level 6) that is thought to be intrusive.

\section{Invertebrate Faunal Remains}

The invertebrate faunal assemblage consists of 51 fragments of freshwater mussel shells and 4 gastropod shells. The gastropods are identified to the family Succineidae (see Appendix D). These snails 
are native to the area, and their presence is not considered to be cultural. The freshwater mussel shell fragments are similar to those previously identified in this locality as Uniomerus declivus by Raymond Neck (Boyd et al. 1990:566). None are modified, and it appears that they were utilized as a food source.

\section{CHRONOLOGY}

The primary technique employed for defining the chronology of 41GR303B is radiocarbon dating of organic remains associated with feature and nonfeature proveniences. Of secondary importance are the temporally sensitive artifacts. Thermoluminescence dating of burned rocks was used in a previous phase of the project, but the results were inconsistent with the radiocarbon dates, and it was concluded that this technique is not a reliable chronological tool for the Lake Alan Henry project area.

\section{Absolute Chronology}

Site $41 G R 303 B$ is dated by three radiocarbon assays from cultural contexts, two of which are associated with features (see Table 28; Figure 60). Two dates are from assays on charcoal, and one is from humates extracted from sediments associated with a cultural feature.

Feature 5 in EU 37 (Test Unit 5) at $5-23 \mathrm{~cm}$ yielded charcoal that produced a calibrated 1-sigma date range of A.D. 1020-1382 with a single intercept at A.D. 1230. This represents the most recent dated cultural manifestation at the site. But this date and its context are difficult to interpret because EU 37 is located 29 m east of the 1992 excavation block in a severely deflated area. As discussed below, this assay may pertain to a late period of site use, but this cannot be demonstrated stratigraphically.

Feature 8 in EU 21 (Test Unit 4) at $80-95 \mathrm{~cm}$ yielded no charcoal, but a radiocarbon assay on humates from sediments associated with the feature produced a calibrated 1-sigma date range of A.D. $681-852$ with a single intercept at A.D. 772 . Because this date is on humates, it probably represents a minimum age for the cultural feature with which it is associated. Since Feature 8 appears to be the bottom of a baking pit extending down from the burned rock zone, this date may also provide a minimum age for the sitewide scatter of burned rocks.

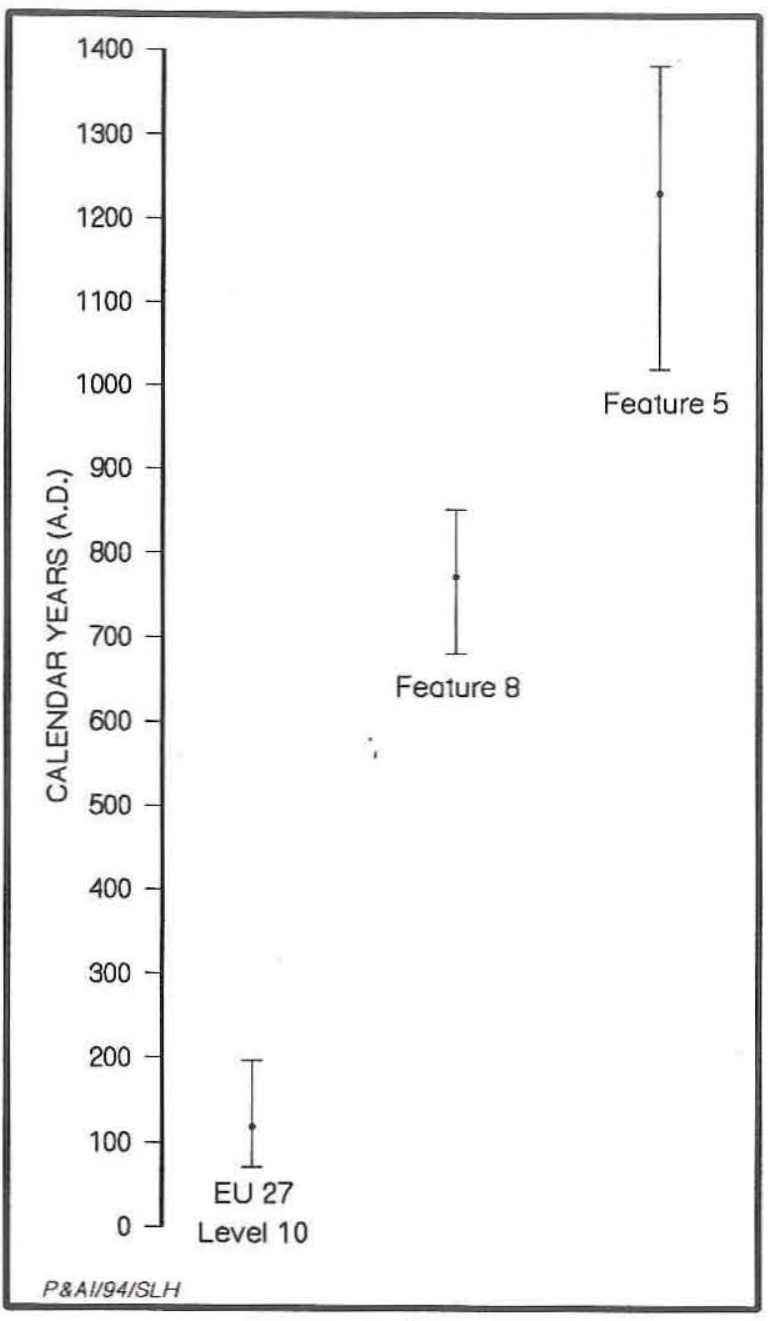

Figure 60. Graph of calibrated radiocarbon dates (intercepts and 1-sigma ranges), 41GR303B.

Excavation Unit 27 yielded one of the few charcoal samples recovered from 41GR303B. This unit is immediately north of EU 21 (Test Unit 4), and the dated sample was recovered from Level 10 at 93-103 cm, slightly lower in the profile than Feature $8(80-95 \mathrm{~cm})$. Because the sample was small $(1.3 \mathrm{~g})$, accelerator dating was required, producing a calibrated 1-sigma date range of A.D. 71-197 with a single intercept at A.D. 118. The stratigraphic position of this sample, i.e., below Feature 8 and the burned rock zone (and below the zones containing most of the artifacts), indicates that this assay predates most of the cultural deposits at 41GR303B, and it probably relates to the earliest cultural activities at the site.

In sum, the limited radiocarbon data suggest that the burned rock features, the scatter of burned rocks 
found across the block, and the artifacts from the levels associated with these features and below date to sometime between A.D. 71 and 852, or the Late Prehistoric I period. As discussed below, artifacts were found in high densities above the burned rock zone as well, however, and dating these remains is more problematical. They may postdate the burned rock scatter only slightly and thus be assignable to the Late Prehistoric I period, or they may date to the early part of the Late Prehistoric II period, as suggested by the assay from Feature 5 .

\section{Relative Chronology}

The small collection of potentially diagnostic artifacts consists of two Scallorn arrow points, one untyped expanding-stem arrow point, one fragment of a triangular arrow point or preform, a heavily reworked Darl dart point, and a fragment of an untyped expanding-stem dart point. As a group, they are consistent with occupations primarily during the Late Prehistoric I period, especially if the dart points are viewed as being intrusive (i.e., recycled) and the triangular arrow point represents a preform rather than a Fresno point.

Scallom arrow points are dated by Suhm and Jelks (1962:285) to approximately A.D. 500-1200. On the Southern Plains they occur somewhat earlier at A.D. 200-1200 (Hofman 1978). In the Jornada Mogollon region, Leslie $(1978: 106-114,116)$ dates Scallorn-like arrow points to A.D. 900-1250.

The triangular arrow point fragment may be a preform, or it may be part of a completed specimen similar to the Fresno type. Fresno points are defined in southeastern New Mexico by Leslie (1978:91-92) as dating to A.D. 950-1500. If the specimen is a preform, it is most likely the basis for a Washita or a Harrell, which Leslie (1978:98-102) dates between A.D. 1200-1500. These types are dated somewhat later in Oklahoma (Hofman 1984b:357).

The dart point base fragment is untypeable and undatable. The reworked Darl dart point is most likely either intrusive or an heirloom. Darl dart points are dated by Suhm and Jelks (1962:179-180) to A.D. 1-1000, which fits part of the time range at this site; however, this dating is not necessarily applicable to the Southern Plains. In any case, the original manufacturing scars and the reworking scars are equally patinated, and it is doubtful that the reworking was done by the occupants of 41GR303B.

Unfortunately, the distributions of these specimens do little to clarify the chronological picture. One of the Scallorns and the expanding-stem arrow point were found in the upper levels of the block above the burned rock scatter, while the other Scallorn and the triangular fragment were surface finds in the eastern portion of the site. The reworked Darl dart point was recovered from below the burned rock zone, and the untypeable dart point base was recovered from the upper levels of the block.

\section{INTERPRETIVE SUMMARY}

After analysis of the data recovered in the 1988 testing, it appeared that at least two separable components were represented in Test Unit 4 (EU 21), with Feature 8 representing an early occupation and Feature 6 representing a later occupation. Feature 6 also appeared to be directly associated with the sitewide scatter of burned and unburned sandstone. After the 1992 excavations, the relationships between these two burned rock anomalies were reanalyzed. Feature 6 appears to be the upper part of a baking pit, the bottom of which is represented by Feature 8 . Feature 9 also appears to be the bottom of a baking pit. The following sections address the question of multicomponency at 41GR303B through analyses of the cultural features and material culture.

\section{Cultural Features}

Four cultural features were investigated at 41GR303B. All are burned rock features. A burned and unburned rock scatter appears in every excavation unit across the site. Feature 5, found in a test unit not associated with the excavation block, consists of scattered burned rocks that may be part of a disaggregated hearth; it was radiocarbon dated to A.D. 1230. This date apparently associates the feature with an occupation of the site postdating the deposition of the sitewide burned rock layer. Three more cultural features bear direct relationships to this deposition. Features 8 and 9 are fireboxes which comprise the bottoms of baking pits that were dug down from the level of the burned rock scatter. Feature 6 , a layer of rocks just above Feature 8 , probably held products being baked above the coals. Feature 9 was not dated, but radiocarbon assays on soil humates from the context of Feature 8 and charcoal recovered from just below and north of Feature 8 date the Feature $6 / 8$ complex between A.D. 71-852. 
The relationships between Features 6, 8, and 9 and the burned rock scatter indicate that they are all related functionally and probably temporally. The burned rock scatter represents rocks that were removed from pits after baking of vegetal products. The unburned rocks scattered amongst it represent stockpiled rocks for replacing exhausted components of baking pits. The volume of this scatter indicates that there are probably more baking pits below portions of the burned rock layer that were not excavated.

No cultural features were discovered above the burned rock layer. However, the majority of the artifacts were recovered from above the burned rock layer. Thus, it appears that the site was utilized for different purposes by groups occupying the site during more recent times.

\section{Material Culture}

Figure 61 shows the vertical distributions of three classes of cultural materials (burned rocks, stone tools, and debitage) in seven of the deeper excavation units in the block. The highest densities of tools and debitage are in Levels 2 or 3, with a secondary peak in Level 5 and decreasing densities below that. In contrast, the burned rocks are nearly restricted to the middle levels with a single peak in Level 6, and this marks the burned rock scatter that was found across the entire block. These distributions suggest that the deposits represent at least two periods of use.

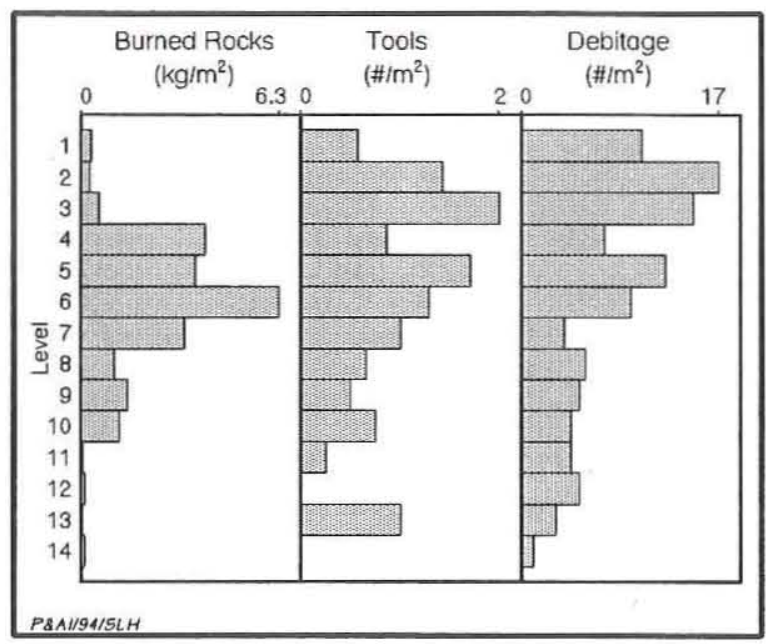

Figure 61. Densities of burned rocks, stone tools, and debitage by level in EUs $6,13,21,27,28,29$, and 30, 41GR303B.
Only small quantities of burned rocks were found in the levels above the burned rock scatter, but there are enough artifacts in these upper levels to indicate that the site was occupied. Such occupations did not include the utilization of burned rock features, however, which indicates a difference in the manner in which the site was used later in time. This change in site use dictates dividing the block into two analytic units: (1) the upper zone, i.e., all levels above the burned rock layer; and (2) the lower zone, i.e., the burned rock layer and all levels below this. While the distributions hint that a third unit may be isolable in the lowermost levels (i.e., small numbers of lithic artifacts but very few burned rocks), the sample from these deepest deposits is too small to allow confident interpretations. Hence, these materials are lumped with those from the burned rock scatter. As discussed above, the lower zone appears to date to the Late Prehistoric I period. The chronology of the upper zone deposits is less clear, but they probably date to the latter part of the Late Prehistoric I period and/or the early part of the Late Prehistoric II period. Only the artifacts from the contiguous excavation units of Block 1 are utilized for this analysis $(n=1,827)$. One broken dart point base from the upper zone and one heavily reworked Darl dart point from the lower zone were removed from the sample because they may be intrusive or heirlooms.

Table 32 compares the numbers of artifacts of each type (excluding two dart points) in the upper and lower zones of the block. The total of 1,827 artifacts includes Test Unit 4 (EU 21) from the 1988 testing. ${ }^{4}$

The adjusted standardized residuals account for variations in sample size, and show statistically significant differences between the upper and lower zones in the numbers of cores, cobble tools, ground stones, and unmodified debitage. Although not significant statistically, arrow points are present only in the upper zone. Among the cobble tools in the lower zone, more small wedgelike tools are represented ( 7 of $13,54 \%$, as opposed to 1 of $4,25 \%$, in the upper zone). Indications are that the early

\footnotetext{
${ }^{4}$ Boyd et al. (1990:Table 45) report that 172 specimens were recovered from Test Unit 4 . For this reanalysis, only 119 specimens ( 2 bifaces, 2 unifaces, 1 core, 5 edgemodified flakes, and 109 pieces of unmodified debitage) were added. Fifty-three specimens (1 uniface, 1 drill, and 51 pieces of unmodified debitage) could not be re-located.
} 


\begin{tabular}{|c|c|c|c|c|}
\hline \multicolumn{5}{|c|}{$\begin{array}{c}\text { COMPARISON OF STONE ARTIFACT COLLECTIONS FROM } \\
\text { THE LOWER AND UPPER ZONES AT 41GR303B }\end{array}$} \\
\hline Artifact Type* & $\begin{array}{l}\text { Lower } \\
\text { Zone }\end{array}$ & $\begin{array}{l}\text { Adjusted Standardized } \\
\text { Residual for the Lower } \\
\text { Zone** }\end{array}$ & $\begin{array}{l}\text { Upper } \\
\text { Zone }\end{array}$ & Totals \\
\hline Arrow point & - & -1.24 & 3 & 3 \\
\hline Biface & 4 & +0.96 & 4 & 8 \\
\hline Uniface & 18 & +0.63 & 29 & 47 \\
\hline Core & 22 & +2.69 & 19 & 41 \\
\hline Edge-modified flake & 10 & -0.33 & 22 & 32 \\
\hline Cobble tool & 13 & +3.72 & 4 & 17 \\
\hline Ground stone & 11 & +2.95 & 5 & 16 \\
\hline Gouge & 4 & +0.40 & 6 & 10 \\
\hline Unmodified debitage & 539 & -3.85 & 1,114 & 1,653 \\
\hline Totals: & 621 & & 1,206 & 1,827 \\
\hline $\begin{array}{l}\text { *Excludes } 1 \text { dart poi } \\
\text { **Everitt (1977:46-48 } \\
\text { significant at } .05 \text { le }\end{array}$ & Numb & $\begin{array}{l}\text { zone. } \\
\text { above }+1.95 \text { or below }- \\
\text { nce. }\end{array}$ & are $\mathrm{s}$ & tically \\
\hline
\end{tabular}

processing by the later occupants.

There do not appear to be any significant differences in the origins of the raw materials in the upper zone and lower zone chipped stone assemblages (Table 33). Both are dominated by local materials, which constitute $94 \%$ of the lower zone and $92 \%$ of the upper zone, and all of the cores in both assemblages are local materials. Nonlocal materials in the lower zone consist of 2 Tecovas jasper specimens (both unmodified flakes) and 31 fine-grained chert specimens of nonlocal colors ( 1 uniface, 2 edge-modified flakes, and 28 unmodified flakes). Nonlocal materials in

occupants were more dedicated to plant processing and perhaps woodworking and that the later occupants were more inclined toward hunting.

There are five metates and metate fragments in the lower zone and one in the upper zone. The one metate fragment in the upper zone could have originated from one level below, which is in the lower zone. This indicates that plant processing is better represented in the lower zone, and it suggests that products of the baking pits were processed further on the site by grinding. Some of the large cobble tools in the lower zone may be related to refurbishing metates.

Along with more lithic debitage in the upper zone, there is evidence of more late-reduction-stage debitage in the presence of more small flakes (64\% in the upper zone, $52 \%$ in the lower zone), more tertiary flakes $(63 \%$ in the upper zone, $57 \%$ in the lower zone), and more multifaceted platforms (26\% in the upper zone, $18 \%$ in the lower zone). There are also more uniface resharpening flakes $(13 \%$ in the upper zone, $10 \%$ in the lower zone) and bifacial flakes (5\% in the upper zone, $3 \%$ in the lower zone). In total, these conditions indicate that the occupants of the upper zone carried reduction further and produced more completely finished products. The earlier tool kit may have been more expedient. The unifacial resharpening flakes may indicate more hide the upper zone consist of 15 Tecovas jasper specimens (1 arrow point, 1 uniface, 1 gouge, 1 edgemodified flake, and 11 unmodified flakes) and 78 fine-grained chert specimens of nonlocal colors (1 uniface, 1 gouge, 4 edge-modified flakes, and 72 unmodified flakes). The Tecovas jasper probably originated somewhere north of the project area, while the nonlocal fine-grained cherts probably came from the south or southwest, most likely from sources along or near the Callahan Divide (see Chapter 2). Notably, no obsidian or Alibates agate are represented in either assemblage.

The absence of projectile points and other hunting-oriented artifacts and the abundance of burned rocks indicate a subsistence strategy focused on gathering and plant processing during the period of occupation represented by the lower zone. Keeping in mind the possibility of on-site habitation areas outside of the portion of the site that was excavated, the evidence from the block indicates that 41GR303B was a special-purpose location dedicated to plant processing. Feature morphology, the limited macrobotanical remains, and ethnographic' comparisons suggest that the plants processed may have included prickly pear, sotol, and yucca. Direct evidence for the possible prehistoric use of these plants, however, is limited to prickly pear calcium oxylate crystals recovered from Feature 8. 


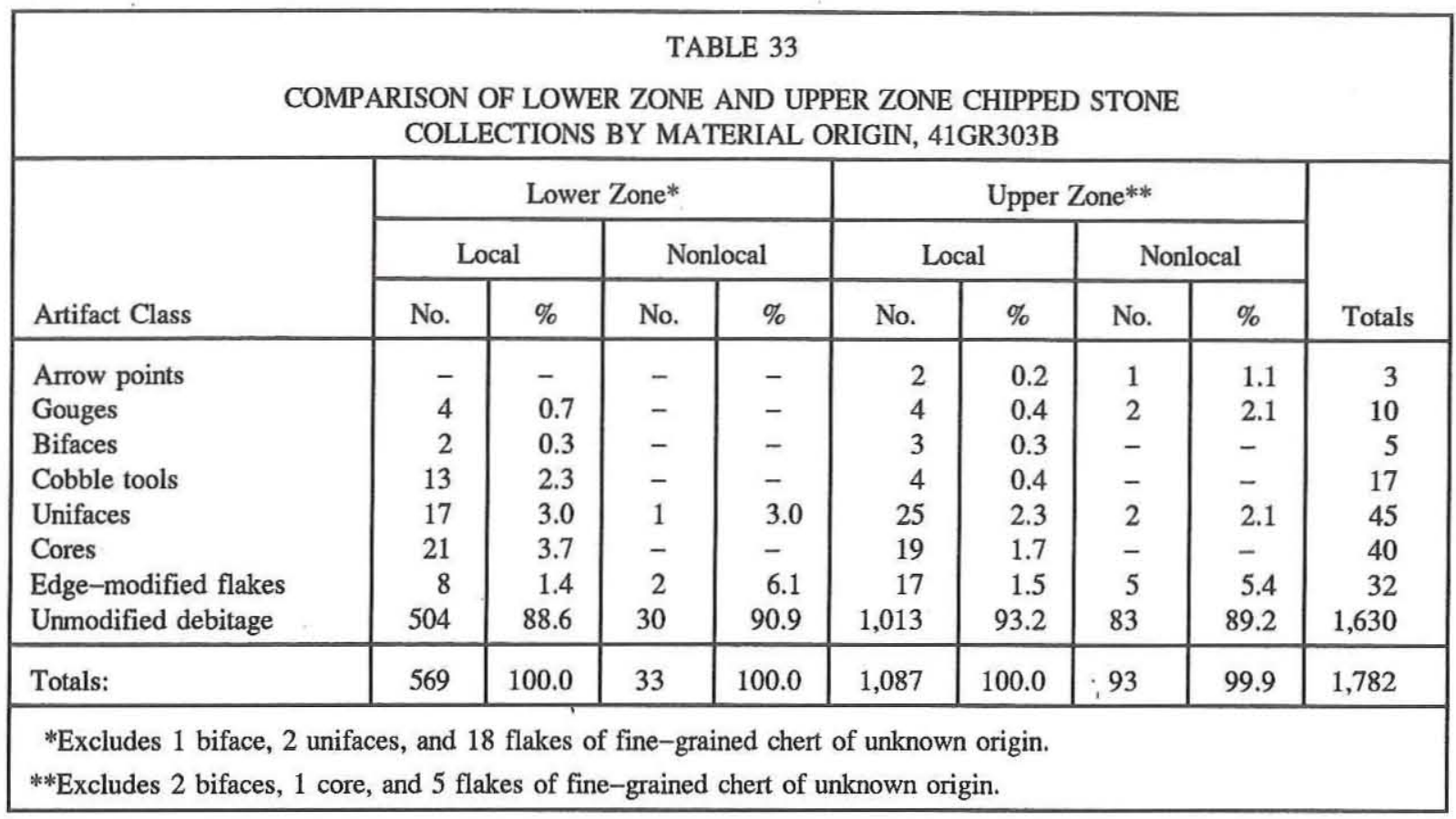

The activities represented by the materials from the upper zone are somewhat more difficult to identify. Temporally sensitive artifacts are sparse, being represented by Scallorn and possibly triangular arrow points. While these two types may not be out of place together, neither do they necessarily belong together. Scallorn is a ubiquitous Late Prehistoric I type, and triangular arrow points are generally thought of as Plains bison-hunting points placed somewhat later in time. There are very few ground stones and plentiful late-reduction-stage debitage and uniface resharpening flakes in the upper zone. This suggests that the latest occupations at the site were a series of short-term layovers that included the manufacture and maintenance of stone tools but not intensive processing of plant foods. The permanent springs in the area were probably one of the attractions. 


\section{THE JUSTICE DUGOUT, 41 GR474}

Douglas K. Boyd

\section{SITE SETTING}

Site 41GR474 is situated on an erosional remnant bedrock terrace in the upper portion of the reservoir, $2 \mathrm{~km}$ east-southeast of the post office in the community of Justiceburg. The erosional remnant is a northeast-projecting ridge which forms a prominent meander bend in the Double Mountain Fork of the Brazos River. The surface of the ridge varies from a few meters to $15 \mathrm{~m}$ above the riverbed, and Triassic sandstone crops out along the margins of the landform and sporadically within its interior. Alluvial and/or eolian sediments cover most of the ridge, and substantial dunes occur on the northeastern end of the landform. Site elevation is $2220-2260 \mathrm{ft}$ msl.

The historic component is confined to the north-central portion of the ridge. The housesite and related features are located along the crest of the ridge, while the dugout is located approximately $100 \mathrm{~m}$ to the north on the sloping edge of the ridge overlooking the river.

\section{PREVIOUS INVESTIGATIONS}

The site was recorded in 1987 as a Late Prehistoric open campsite with a nineteenth-century historic component consisting of a dugout depression and a nearby house foundation and associated complex of features (Boyd et al. 1989). Archival and informant research documented two separate historic occupations, the earliest being at the dugout (Figure 62 ) and the later and more extensive occupation represented by the house complex. Historic documents indicated that the dugout probably was constructed in 1899 and subsequently occupied by Jeff D. Justice and his family for only a short time. The house, a much larger and more elaborate structure, was built by the Justice family while they lived in the dugout. In the early 1900s, the house was completed and the dugout was abandoned. Archeologically, features relating to both historic occupations were considered to be relatively intact and to warrant further work.

Investigation of the historic component in 1988 included mapping of all structures and features within a ca. 150-x-250-m area (Figure 63), surface collection of selected diagnostic artifacts from the house complex and the dugout, and hand excavation of two $1-\mathrm{x}-1-\mathrm{m}$ test units and a $0.5-\mathrm{x}-3.0-\mathrm{m}$ test trench (Freeman and Boyd 1990). In addition to the archeological work, archival and informant research was continued with two main goals. One was to define a detailed, site-specific history for 41GR474 (see discussion below, derived from Boyd et al. [1989] and Freeman and Boyd [1990]). A second goal was to develop a historic context to be used for evaluating sites for National Register eligibility, relating to ranching in the western Rolling Plains during the period from 1877 to 1945 . The archival research confirmed that the earlier dugout occupation was brief, ca. 1899-1900, while the later house was occupied from the early 1900 s on, possibly until the structure was razed in the 1960s.

Archeological excavations cross-sectioned the dugout and reached the floor in one small area and 


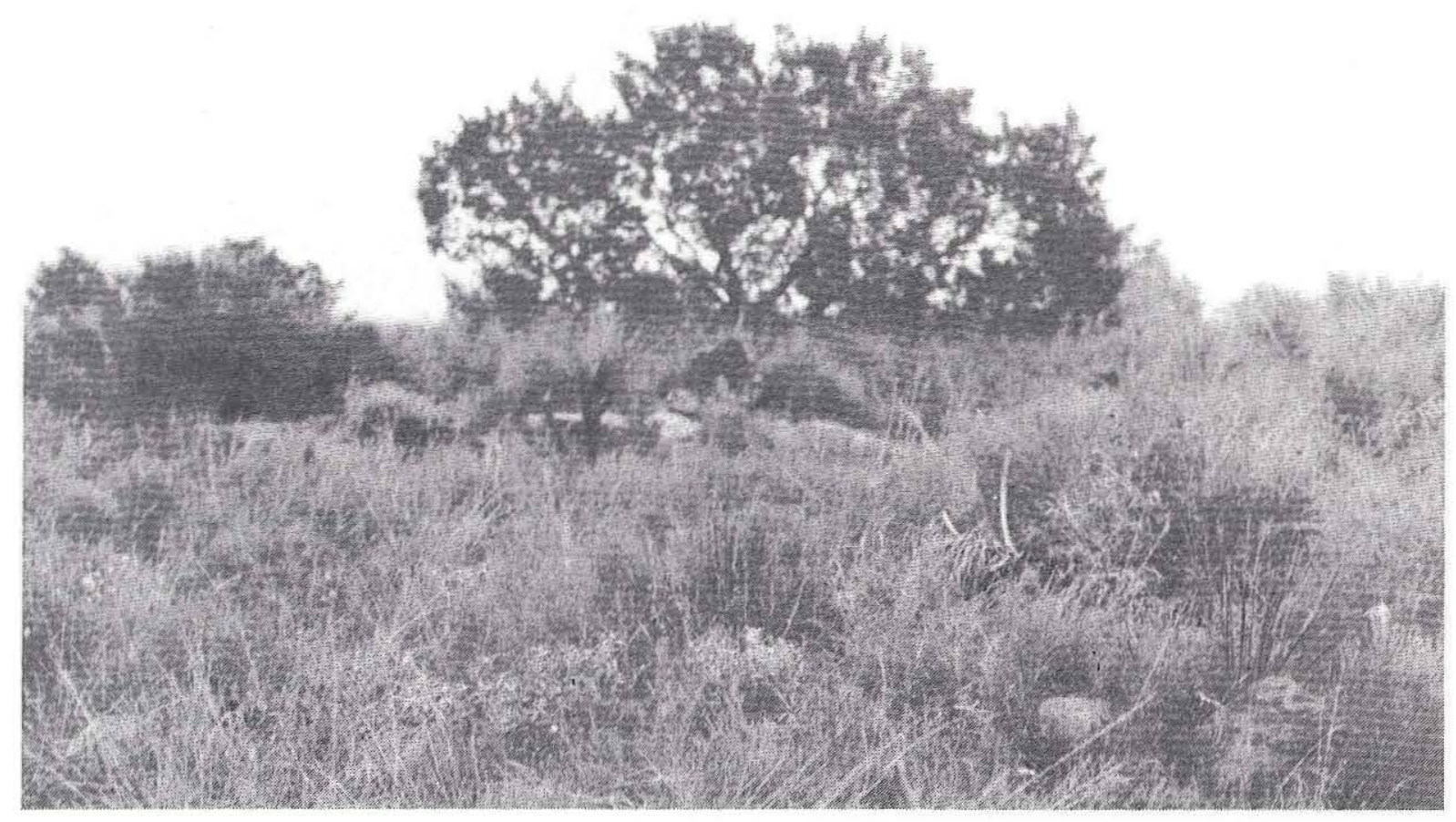

Figure 62. Dugout area at 41GR474 as it appeared in 1987. View is to the east toward the rocks along the back wall of the structure.

recovered cultural materials associated with the dugout and later housesite occupations (Table 34). It was clear that the structure was a relatively intact half dugout, its upper rock walls having collapsed inward. A wooden beam, presumably from the roof, was found near the floor level. Shortly after its abandonment, the roof and walls of the dugout apparently collapsed, thus sealing in the cultural materials associated with its occupation. Subsequently, the dugout depression was backfilled with wind-blown sand and trash from the later occupation. The dugout was determined to be the only physical evidence of the initial site occupation, and no other surface features or artifacts could be attributed with confidence to the pre-1900 time period.

The 1988 work also documented the housesite and its associated features (see Figure 63). The house area consists of a foundation and a chimney brick scatter where a large multiroom wooden structure once stood, a cistern, and a surrounding yard partially outlined by rocks. Other features include a concrete platform and a metal windmill tower, barbed wire fences, a corral, and a scatter of wooden boards denoting a former barn. The house complex was limited to surficial archeological evidence except for the cistern.
Following the testing phase, the historic component at 41GR474 was again evaluated as being eligible for listing on the National Register of Historic Places. Using the historic context "Agriculture in Texas: Ranching on the Western Rolling Plains, 1877-1945" (Freeman 1990), site 41GR474 derives its significance from three criteria: (1) it represents a pivotal period in the agricultural development of the region (i.e., the shift from openrange to closed-range ranching); (2) it is associated with Jeff $\mathrm{D}$. Justice, an individual who was prominent in the initial settlement of the region; and (3) the integrity of the site's archeological remains, particularly the dugout, allowed for additional research questions to be addressed through continued investigations (Freeman and Boyd 1990:129-130).

\section{SITE HISTORY}

The history of the property containing the Justice homestead is well documented, but the precise timing and details of the construction and abandonment of the structures are rather vague. The following site history is summarized from information provided in Boyd et al. (1989:61-67, 133-156, 618619) and Freeman and Boyd (1990:104-106). Site 


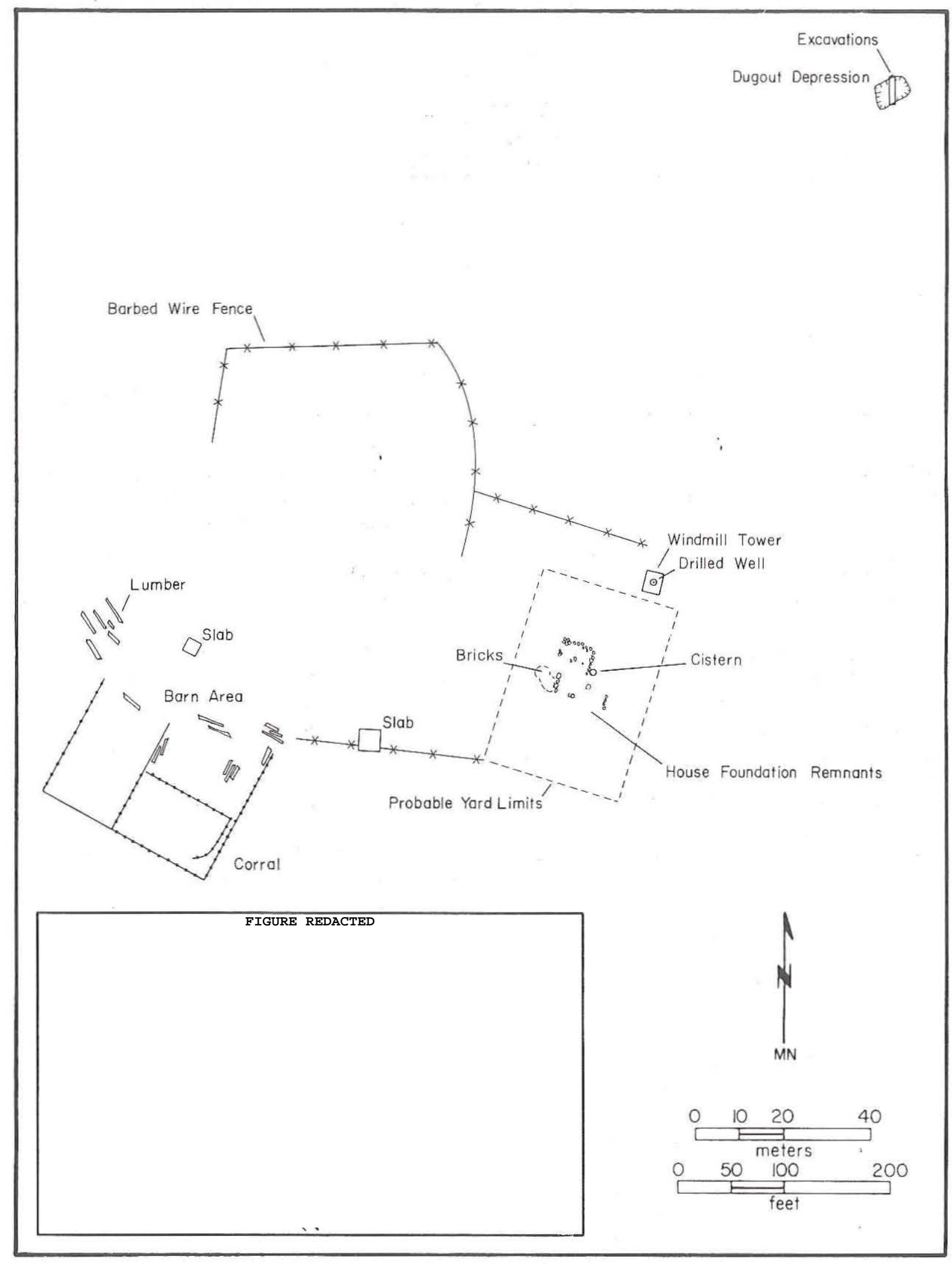

Figure 63. Map of historic component of 41GR474. 


\begin{tabular}{|c|c|c|c|c|c|c|}
\hline \multicolumn{7}{|c|}{$\begin{array}{l}\text { TABLE } 34 \\
\text { HISTORIC ARTIFACTS RECOVERED DURING } 1988 \text { TESTING, 41GR474 }\end{array}$} \\
\hline \multirow[b]{2}{*}{$\begin{array}{l}\text { Material } \\
\text { Classification }\end{array}$} & \multirow{2}{*}{$\begin{array}{c}\text { Early Occupation } \\
\text { Dugout Floor } \\
\end{array}$} & \multirow{2}{*}{$\begin{array}{c}\text { Mixed Occupation } \\
\begin{array}{c}\text { Surface near } \\
\text { Dugout }\end{array}\end{array}$} & \multicolumn{3}{|c|}{ Late Occupation } & \multirow[b]{2}{*}{ Totals } \\
\hline & & & BHT 9 & $\begin{array}{c}\text { Surface } \\
\text { near House }\end{array}$ & $\begin{array}{l}\text { Trash Fill } \\
\text { in Dugout }\end{array}$ & \\
\hline Metal & 1 & - & - & - & 94 & 95 \\
\hline Ceramic & - & 3 & - & 2 & 58 & 63 \\
\hline Glass & - & 2 & 1 & 9 & 148 & 160 \\
\hline Rubber & - & - & - & - & 1 & 1 \\
\hline Leather & - & - & - & - & 54 & 54 \\
\hline Bone & 11 & - & - & - & 70 & 81 \\
\hline Shell & - & - & - & - & 1 & 1 \\
\hline Other & - & - & - & - & 2 & 2 \\
\hline Totals & 12 & 5 & 1 & 11 & 428 & 457 \\
\hline
\end{tabular}

41GR474 is located in the south half of Section 20, Block 6. Prior to any permanent settlement in the vicinity, this 320-acre tract was leased to the Lexington Ranch Company of Nolan County by the General Land Office from 1888 to ca. 1896 (Garza County Deed Record 4:83-85, 280-282, 302-304, 5:159-161). In response to the Four-Section Act of 1895 , many individuals began filing applications for land with the General Land Office and settling in the Justiceburg area. In order to obtain a homestead section and three additional sections for grazing or farming, the act required applicants to live on and improve the land and, after 3 years, to file proof of occupancy. On February 17, 1900, Jeff D. Justice made an application and filed an affidavit stating that he was living on the land and desired to purchase the homestead from the State (Texas. General Land Office 1939). Justice apparently had moved onto the land the previous year because an affidavit made by T. J. Payne on nearby Section 26 stated that he, T. E. Payne, Jeff Justice, and W. B. Justice had made camps in the area for the purpose of establishing homestead claims by December of 1899 (Texas. General Land Office 1936).

Occupation of the dugout appears to have been very brief, and a Garza County history states that Jeff Justice moved into a new ranch house in 1900 (Didway 1973:24). Interestingly, none of the informants who had lived at the site, even those whose knowledge of the area dated back to World War I, knew anything about the dugout. The dugout apparently was abandoned soon after the turn of the century and probably collapsed quickly after its abandonment, and as the depression began to fill in with blown sand, it was used temporarily as a dump for household refuse. Within a few years of its abandonment, even the depression was no longer obvious, and the dugout was soon forgotten.

The new ranch house, built with materials hauled from Colorado City, was an elaborate affair, and the site was eventually expanded into an extensive ranch headquarters. Freeman and Boyd (1990:104-106) describe this complex in greater detail, but it consisted of the house, a cistern, a drilled water well, an outhouse, a smokehouse, stock pens, and a barn with attached tack rooms. In later years, a concrete-slabbed garage was added to house automobiles.

Jeff D. Justice lived at 41GR474 when the town of Justiceburg was platted on August 9, 1910 (Garza County Map and Plat Record No. 1:18) and during the time that the Santa Fe Railroad constructed a line through the area from 1909-1911. Jeff Justice helped promote the community of Justiceburg during its early days and apparently occupied site 41GR474 until about 1920, when he moved to Fort Worth and Mrs. Justice moved to Post. Jeff Justice died on January 2, 1934 (The Post Dispatch, January 4, 1934).

\section{DATA RECOVERY INVESTIGATIONS}

\section{Historical Research}

Limited historical research during the data recovery phase was aimed at further defining the 
details of the construction, use, and abandonment of the dugout. This research included an informant interview and examination of Garza County tax records to identify the construction date of the ranch house and its associated complex. Newly acquired historical data were then cross-checked with the previously compiled site history and information on the Justice family published in Garza County histories.

Mr. Odie Hood, the current landowner and great-grandson of Jeff D. Justice, provided additional information on the site history (Hood 1992, 1993). Like the other informants interviewed, Mr. Hood had no recollection of the dugout but remembered the housesite well. His grandmother, Alice Justice Williams, had lived in the dugout, however, and recorded the birthdates of all of the family members in her Bible (Table 35). Jefferson Davis Justice was born in 1860 or 1861 , and according to a 1932 newspaper article (Dallas Morning News, December 11, 1932) provided by Hood (1993), moved from Alabama to Marshall, Texas, with his family when he was 4 years old. Jeff Justice married Mattie Humphreys on December 8, 1878, and they had eight children between 1880 and 1892 (Hood 1993). Hood (1992) thought that all eight of the children must have lived in the dugout when Jeff and Mattie moved to Garza County in 1899 or 1900 (see Table 35). Hood (1992) also suggested that the ranch house was occupied by various tenants in the 1930s and 1940s and was finally abandoned in the late 1940s and then torn down in the 1950s. The ranch house appears on an aerial photo taken in 1941 (Texas Highway Department 1941:Photo CVC-3B191), but Hood (1992) stated that the house was gone by the time he acquired the land in 1960 .

Much of this information is corroborated by information on the Justice family published in two different Garza County histories. One version indicates that the family (Jeff, Mattie, and eight children) lived in Snyder until 1899, but "when homesteading began in Garza County, Jeff Justice filed on land here and moved his family to a oneroom shack on the south bank of the Brazos River and started his beautiful ranch home. The family moved into this home in 1900" (Didway 1973:24).
TABLE 35

THE JEFF JUSTICE FAMILY, CA. 1900*

\begin{tabular}{|l|l|c|} 
& Date of Birth & $\begin{array}{c}\text { Approximate } \\
\text { Age }\end{array}$ \\
\hline Jefferson Davis Justice & December 7, 1860** & 40 \\
Mattie Humphreys Justice & August 22, 1856 & 44 \\
Lillie Justice (Jones) & April 28, 1880 & 20 \\
Pearl Justice (Williams) & April 28, 1882 & 18 \\
Nannie Justice (Boren) & January 26, 1883 & 17 \\
Appleton Justice & July 23, 1885 & 15 \\
efferson Davis Justice, Jr. & January 26, 1887 & 13 \\
Alice Justice (Williams) & October 8, 1888 & 12 \\
Georgia Justice (Porter) & February 21, 1891 & 9 \\
Cameron Justice & May 1892*** & 8 \\
\hline
\end{tabular}

*Information in this table is derived from handwritten notes in Alice Justice Williams's Bible. Minor contradictions with these data, noted below, are found in published family histories.

*Didway (1973:23) states that Jeff Justice was born on December 7, 1861.

**Women's Division of Post Chamber of Commerce (n.d.:89) states that Cameron Justice was bom on May 8, 1890.

The other version states that "Jeff D. Justice, his wife, and their eight children . . . moved to the area of what is now known as Justiceburg in 1900 and established the Justice Ranch" (Women's Division of Post Chamber of Commerce n.d.:89). Both of these histories suggest that the Justice family moved to 41GR474 in 1899 or 1900 and that the large ranch house was built within a year.

The tax records for Garza County (Garza County Ad Valorem Tax Roll 1899, 1900, 1901, $1902,1903,1904)$, however, tell a slightly different story about when the ranch house was built. Jeff Justice first appears as a landowner in Garza County in the 1901 tax roll, but no improvements to the land show up until 1907, when the value of Section 20 increased by $\$ 1,280$. The 1906 tax rolls were compiled in August, and the 1907 records were compiled in November; thus, it appears that the ranch house was constructed between August 1906 and November 1907. Jeff Justice was a successful rancher and businessman since he steadily increased his Garza County holdings from 1,600 acres in 1901 to over 12,000 acres in 1907, and his total land, property, and livestock value increased from ca. $\$ 5,000$ to $\$ 28,000$ during this same time. His success must have continued because by 1910 Jeff Justice had become a prominent businessman and was 
actively promoting the community of Justiceburg.

Taking all of the archival and informant records into account, the following chronology for 41GR474 is suggested. It appears that Jeff Justice was camping in the area by December 1899 in order to establish himself on the land. He filed a claim on the land in February 1900 and presumably built the dugout around this time while the rest of the family was living in Snyder. The dugout probably was constructed early in 1900, and the Justice family moved in during that year. It appears that Justice only intended for the dugout to serve as a temporary dwelling until a larger ranch house could be built, but the dugout appears to have been occupied for longer than most of the family members recollect. The ranch house may have been under construction as early as 1906, but it probably was not completed and occupied until 1907.

The dugout appears to have been occupied for only 5-7 years, but its abandonment history is less clear. Since all of the informants agreed that the the dugout was unknown to the later inhabitants of the site, it is likely that the dugout quickly fell into ruins and was filled with trash soon after its final abandonment. From the archival and informant records, however, it is unclear whether the dugout was reused for any other purposes immediately after the family moved out.

\section{Archeological Investigations}

The archeological investigation of 41GR474 conducted in November 1992 targeted the early historic occupation and was confined to the dugout. The primary archeological objectives were to detail the architectural construction of the dugout and recover the material culture associated with this early occupation period. A secondary objective was to obtain a sample of the material culture associated with the later house occupation for comparative purposes. It was clear from the testing data that the trash fill inside the dugout depression would provide a suitable sample of late occupation artifacts, and thus no further work was done on the house complex itself.

Since the 1988 test excavations had been backfilled, the dugout looked much the same in 1992 as it did when it was first discovered in 1987 (see Figure 62). The dugout appeared as a low swale covered with dense vegetation, with a surface scatter of artifacts and a few large sandstone rocks vaguely outlining the depression. A rock alignment along the east edge of the depression marked the presumed location of the back wall of the dugout. The 1988 datum, which had been assigned an arbitrary elevation of $100.00 \mathrm{~m}$, was re-located, and a more detailed topographic map of the dugout area was made (Figure 64). The 1988 test units and test trench were reexcavated, and the site grid, which was oriented with the structure (rather than to true or magnetic north) was reestablished with grid center located in the east-central part of the depression. To facilitate excavation, all of the vegetation was cleared and the dugout area was divided into large collection/excavation units. In the field, each unit was designated as an excavation unit and given directional names (e.g., northeast, north-central). In theory, each of the units was to be approximately $2 \times 2 \mathrm{~m}$ and to extend to the edge of the structure, but in practice the units were of various sizes because the dugout orientation was slightly different than expected. The excavation unit designations are not relevant for analysis purposes, however, and they are not presented here.

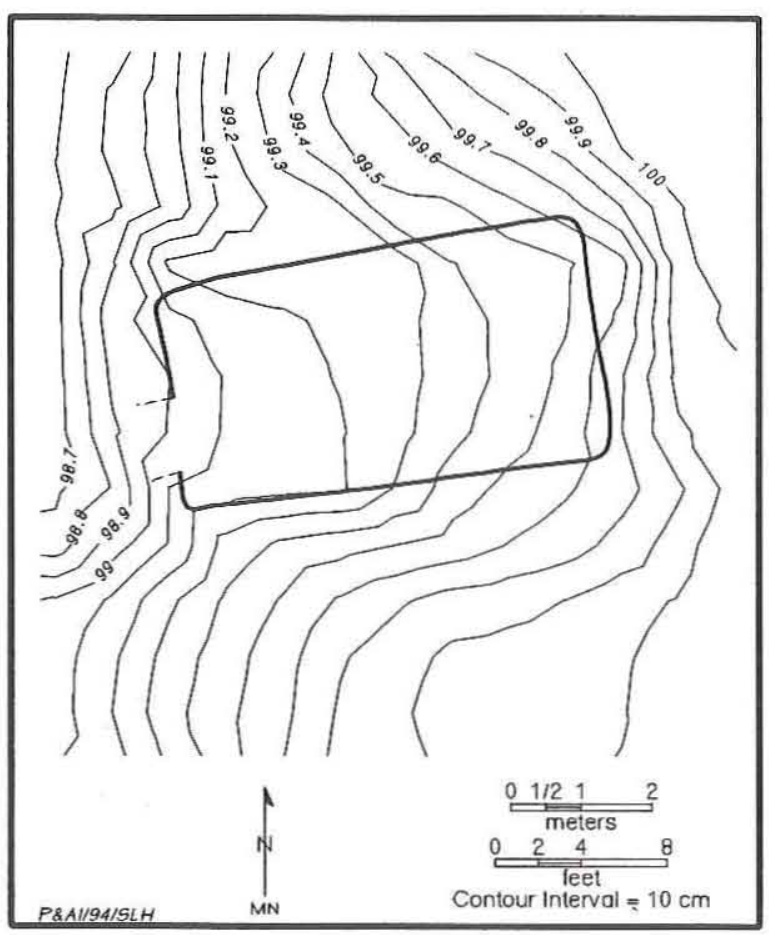

Figure 64. Topographic map of dugout area, 41GR474.

The dugout was excavated in levels corresponding to the fill stratigraphy and proceeded in four phases. The first phase was the excavation of the central portion of the structure to approximately 10 
$15 \mathrm{~cm}$ above the floor. It began by taking all of the units down from the surface to elevation $98.90 \mathrm{~m}$ while leaving all of the large rocks in place. This first level was designated as the Upper Fill. A second level, designated as the Lower Fill, was excavated to $98.80-98.70 \mathrm{~m}$, and the large rocks again were left in place. During the excavation of the Upper and Lower Fill levels, it was obvious that the large rocks represented partial rock walls that had collapsed inward (Figure 65a). All of the cultural materials found on the surface and in these first two levels clearly represented fill associated with the housesite rather than the dugout occupation. The artifacts were not distributed evenly throughout the fill levels but were concentrated in a basin-shaped lens that conformed to the contours of the dugout depression. Detailed maps of the rock fall around the perimeter of the dugout were made to reconstruct their relationships to each other and to the intact portion of the structure walls.

Because the later trash fill was not a major focus of investigation and the large quantities of materials made it impractical to recover everything, only a sample was collected. All of the artifacts in the Lower Fill were collected, but the Upper Fill sample was limited to four of the six units. In the Upper Fill of those units, all nonmetal artifacts and the more unusual or diagnostic metal items were collected, while large bulky items (e.g., windmill blades and gear housing), the ubiquitous tin cans and and other containers, and many pounds of unidentifiable corroded iron fragments were described and discarded in the field.

The first excavation phase continued with the excavation of one additional level within the rock fall perimeter. The central area from $98.70-98.45 \mathrm{~m}$ was designated as Dugout Zone 1. The sparse cultural materials encountered in this level are attributed to the dugout occupation and were clearly below the trash dump layer (i.e., Upper and Lower Fill). Zone 1 also included large segments of logs lying below the level of the rock fall, which are undoubtedly remnants of the roof. The first excavation phase ended with the central portion of the dugout exposed to $98.45 \mathrm{~m}$, while the rock fall perimeter was left in situ.

At the end of the first phase, the need to determine the depth of the floor and the location of the north and south structure walls became apparent. To accomplish this, the second phase consisted of excavating a $1-\mathrm{m}$-wide, north-south trench across the dugout to expose the floor and cut through the north and south walls. When this trench was completed (Figure $65 \mathrm{~b}$ ), the floor of the structure was clearly visible at $98.35-98.30 \mathrm{~m}$, and the locations of the north and south walls were defined. The lowest excavation level, from $98.45 \mathrm{~m}$ to the floor, was designated as the Dugout Floor Zone and includes all materials on or near the floor. Artifacts found in situ were mapped in place and collected separately, while others were collected within 1-m segments along the trench.

The third phase consisted of excavating the Dugout Floor Zone across the central portion of the dugout, with the rock fall perimeter still being left in place (except for the 1-m-wide trench that had removed rocks along the north and south walls). All artifacts found in situ on or near the floor were mapped in place and collected separately, while others were collected within excavation units.

The fourth phase consisted of removing the rock fall around the perimeter of the dugout and excavating all remaining fill inside the structure. The layer that includes all of the rocks was designated as the Rock Fall Zone, and the artifacts found in this zone correspond to the Upper and Lower Fill. Logs and artifacts found below the rock fall were clearly associated with the structure, and these sediments were excavated in layers corresponding to the Dugout Zone 1 (98.70-98.45 m) and the Dugout Floor Zone (98.45 m-floor). When the excavation was completed, ca. 37 person-days of effort had been expended to remove approximately $25 \mathrm{~m}^{3}$ of sediment and expose the dugout (Figure 66).

\section{STRATIGRAPHY AND DEFINITION OF COMPONENTS}

Stratigraphic cross sections of the dugout (Figure 67) reveal two main strata within the fill, while the sterile substrate is significantly different. The latter is a firm blocky reddish brown (2.5YR 4/3 to 4/4) loam or sandy loam containing many $\mathrm{CaCO}_{3}$ nodules and filaments which increase with depth. Besides being slightly redder, it is considerably harder than the loose sandy fill inside the structure. Hence, defining the floor and lower walls (i.e., below $98.80 \mathrm{~m}$ ) of the structure was quite easy. Above $98.80 \mathrm{~m}$, it was more difficult to distinguish the upper dugout fill from the undisturbed sediment because the latter is sandier and structureless above that elevation. 


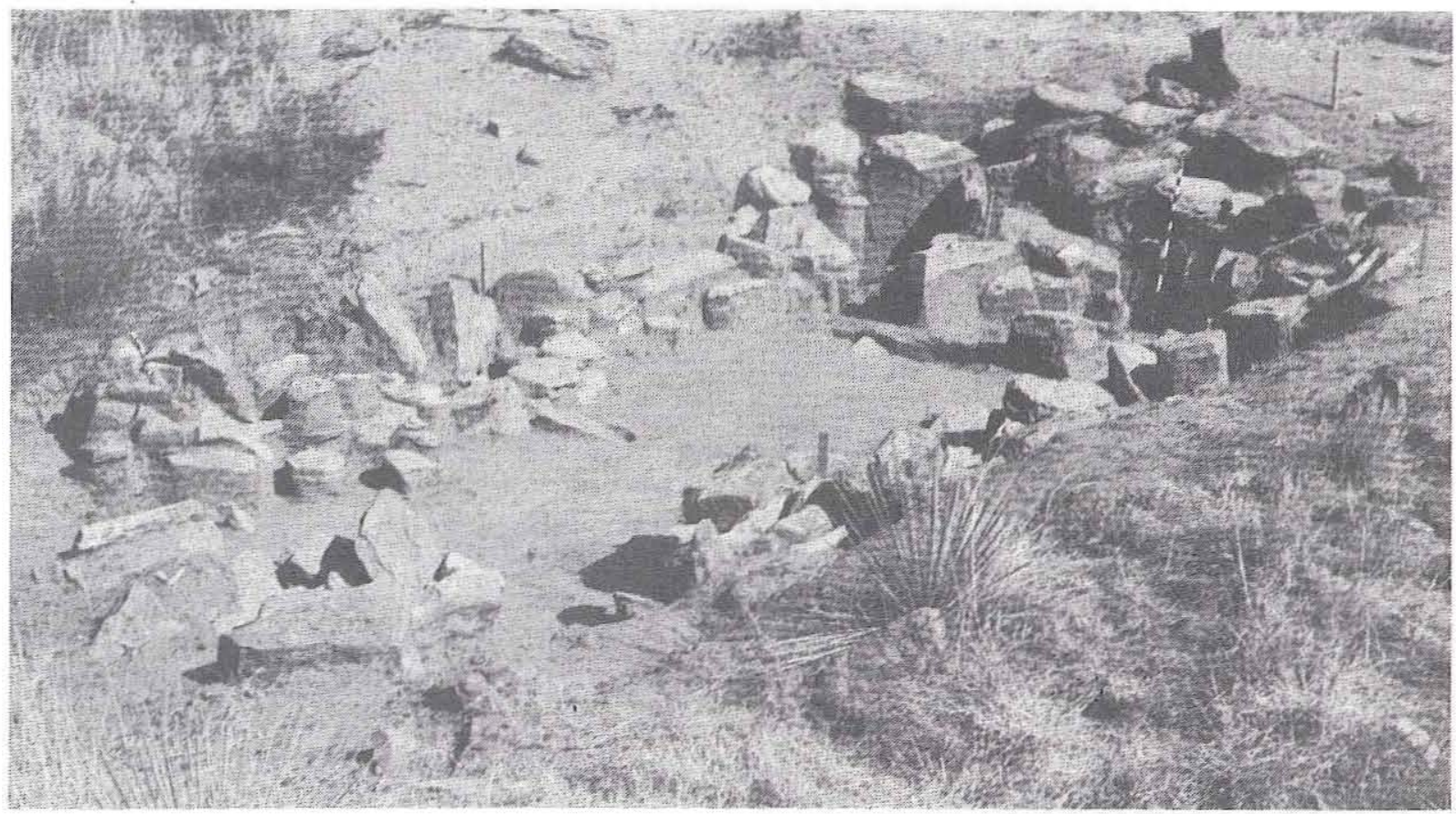

a

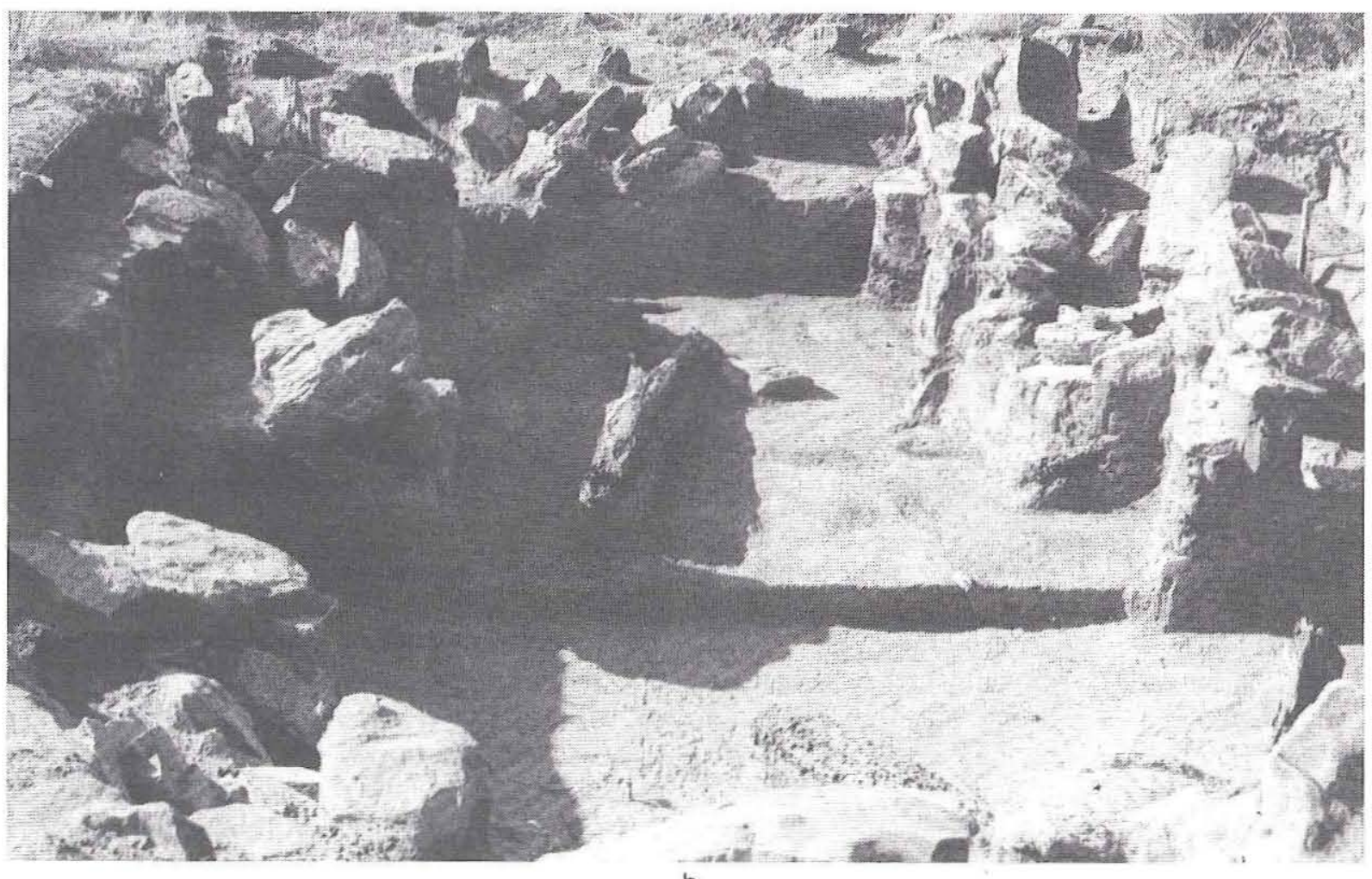

b

Figure 65. Photographs of excavation of the dugout at 41GR474 in progress with rocks in situ. (a) View northeast during the first excavation phase; the central area is excavated to $98.70 \mathrm{~m}$; $(b)$ view west during second excavation phase; the 1-m-wide trench extends from left center to right center, and the foreground is exposed to the structure floor at $98.35-98.30 \mathrm{~m}$; the west half of the structure is exposed to $99.45 \mathrm{~m}$ except for the front wall; note the large log lying $20-25 \mathrm{~cm}$ above floor level. 

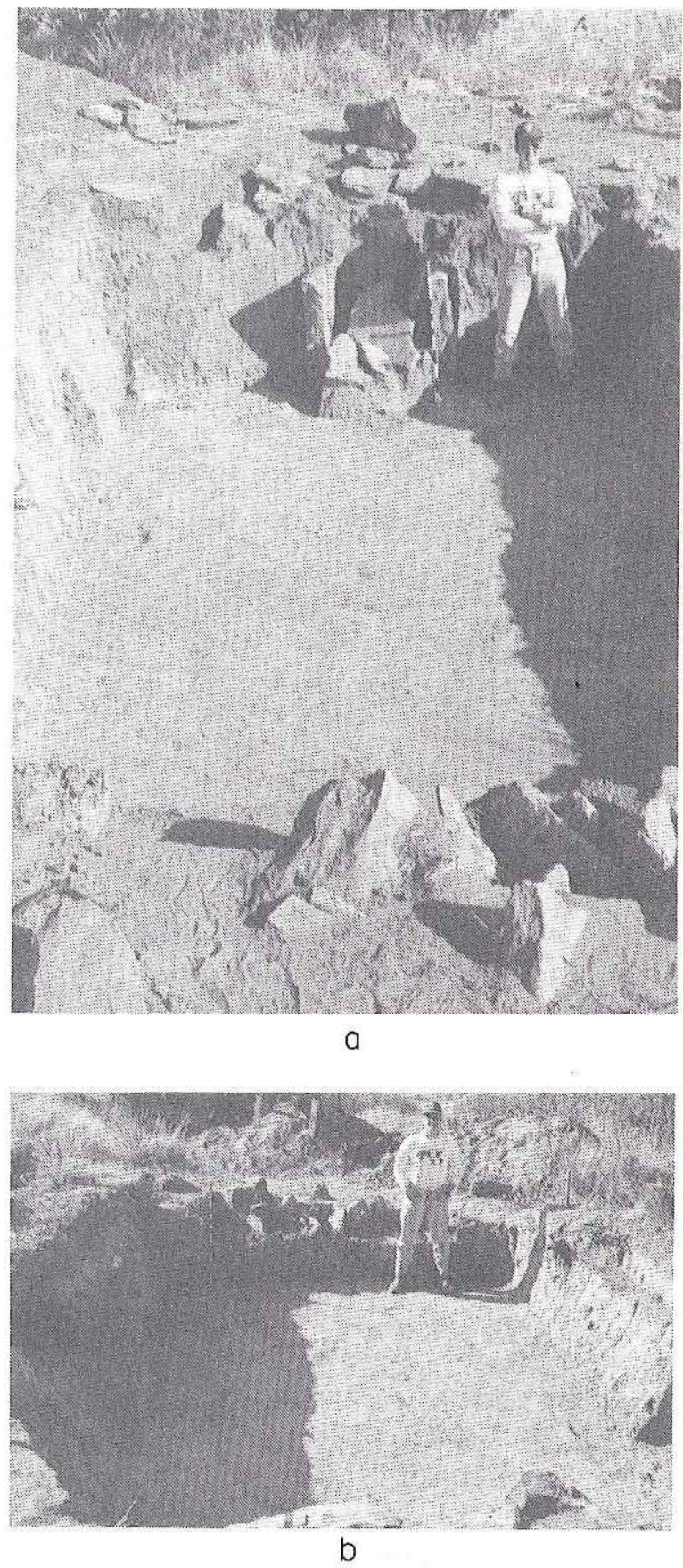

Figure 66. Photographs of the completed excavation of the dugout at 41GR474. (a) View east toward back wall with firebox in center and Ash Stain 2 in northeast corner; $(b)$ view west toward front wall; the rock jumble along the west wall is inside the entryway.

The lower stratum inside the dugout, which includes Dugout Zone 1 and the Dugout Floor Zone, is a loosely consolidated reddish brown to yellowish red (5YR $4 / 6$ to 4/6) structureless fine sand or sandy loam. Numerous artifacts were recovered from the lowest part of this zone, i.e., within $20-25 \mathrm{~cm}$ of the floor, but very few were encountered above that. This stratum represents an eolian sand that accumulated after the structure was abandoned. It continued to accumulate, probably at a rapid rate, during and after the collapse of the roof and rock walls.

The upper stratum in the dugout, which includes the Upper and Lower Fill, is the same sandy sediment as the stratum below it, but it contains a considerable concentration of historic artifacts. In some places, the artifacts were packed quite densely; otherwise, they were scattered throughout this zone. This stratum is characterized by cultural debris mixed with eolian sands and follows the contours of the dugout depression. It represents a trash disposal area which also was filling in with wind-blown sand.

Although the lower boundary of the upper stratum undulated considerably, the trashy Lower Fill disappeared by ca. $98.75 \mathrm{~m}$. In the approximate center of the dugout, a 5-cm-thick lens of laminated sandy loam (see Figure 67) was observed at 98.90$98.70 \mathrm{~m}$ within a $1.5-\mathrm{m}$-diameter area roughly in the center of the dugout. Although the sediment in this layer is the same color as the surrounding sediment, its consistency is slightly different. Fine laminations observed in profile suggest that this layer represents an accumulation of ponded sediments in the bottom of a depression. It is interpreted as a rainwater trap in the dugout depression prior to its use as a dump.

From the preceding discussions, it is clear that the artifacts recovered from the dugout are from two different contexts that are separable vertically. All materials recovered above $98.70 \mathrm{~m}$, including the excavation levels designated as Upper Fill, Lower Fill, and Rock Fall Zone, clearly postdate occupation of the dugout. All of the other cultural materials, from excavation levels designated as Dugout Zone 1 and Dugout Floor, constitute an artifact collection associated with the habitation or last use of the dugout. The presence of a substantial accumulation of eolian sediments and temporary ponding of water in the depression indicate that some amount of time separates the dugout artifacts from the trash fill. Consequently, it is appropriate for analytical purposes to consider the lower artifacts as representing materials associated with the habitation and/or use of the dugout and the upper artifacts as representing a postoccupational trash dump. 

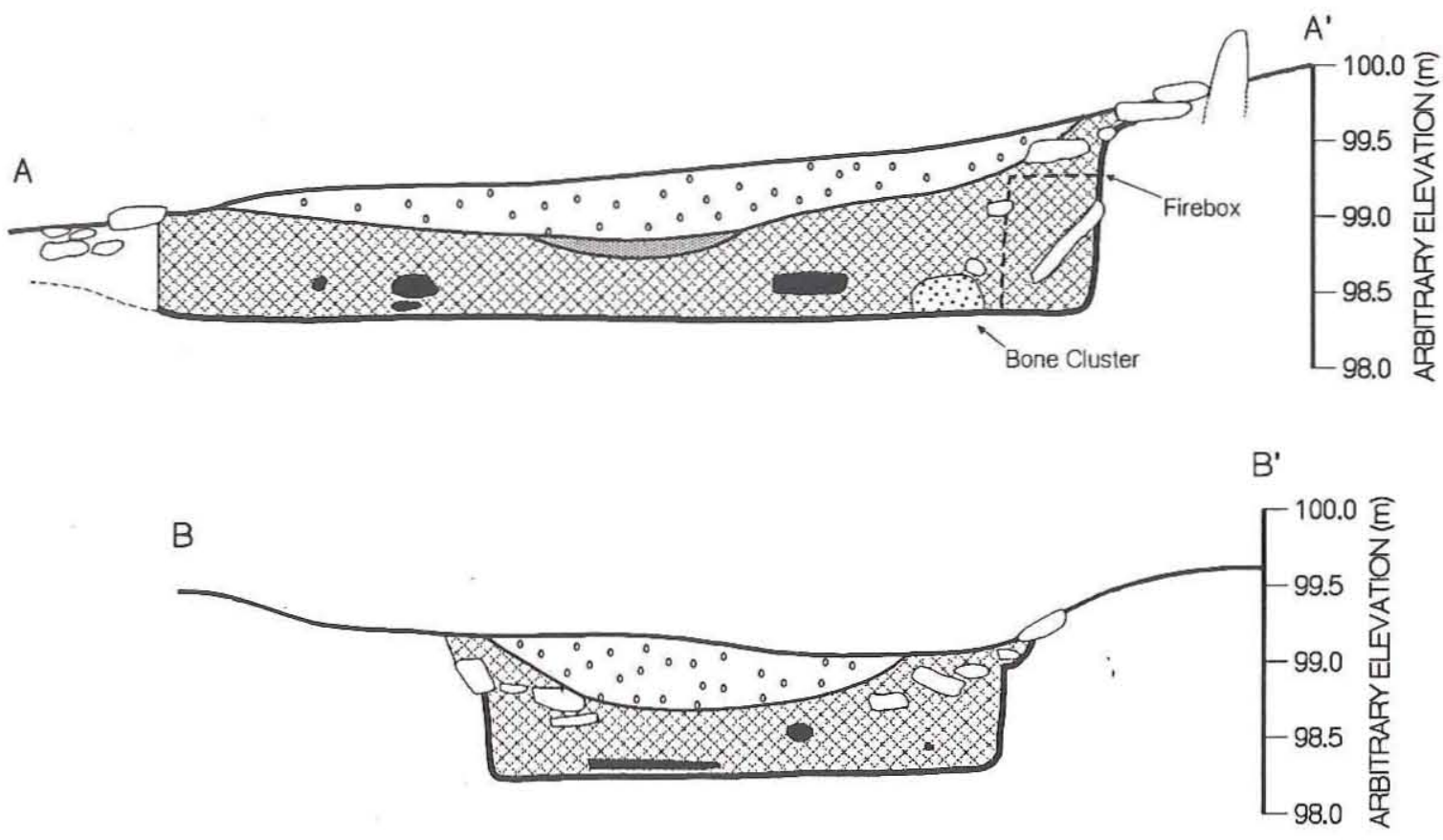

LEGEND

खx又 Sandy Fill

$0 \% 0$ Trash Fill

[i.].] Laminated Sandy Loam

Rock

- Log

Dugout FloorMall
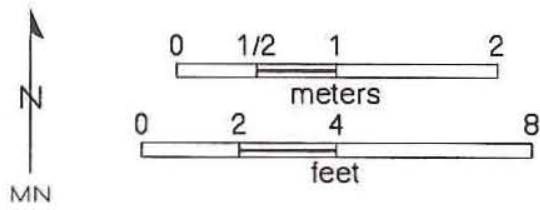

MN

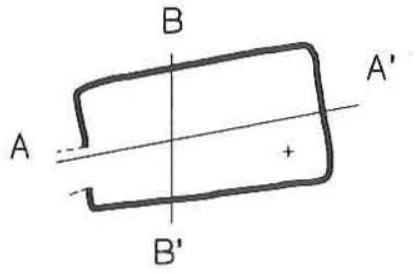

P\&AI/94/SLH

Figure 67. Stratigraphic cross sections of the dugout at 41GR474.

\section{THE DUGOUT AND RELATED FEATURES}

The excavations revealed that the dugout was roughly rectangular with an entryway that faced generally west and downslope toward the Double Mountain Fork of the Brazos River. At floor level, the dugout measured 3.3 to $3.5 \mathrm{~m}$ north-south by 6.0 to $6.2 \mathrm{~m}$ east-west, or approximately $11 \times 20 \mathrm{ft}$. Before excavation, the surface sloped westward with a very shallow depression marking the approximate center of the structure (see Figure 64). When excavated, the floor was found to be as deep as $130 \mathrm{~cm}$ below the surface on the east end and $70 \mathrm{~cm}$ below the surface on the west end (see Figure 67), with the average depth of the fill being ca. $100 \mathrm{~cm}$. Consequently, approximately $20.7 \mathrm{~m}^{3}$ of fill was removed from the dugout.
The dugout consists of a number of different structural elements (Figure 68). The floor, lower walls, and entryway of the dugout were hard sediment, while the rock fall around the edges of the structure indicates that the upper walls were constructed of stacked rocks. Logs found below the rocks are interpreted as remnants of the roof. Inside the dugout, a fireplace is the only feature that is integrated into the structure, while other features are limited to concentrations of materials on or near the floor (i.e., bone and ash concentrations near the fireplace). Each of these structural components is described in more detail below, followed by a discussion of the nonstructural interior features.

\section{Structural Components}

The floor and lower walls were formed when 


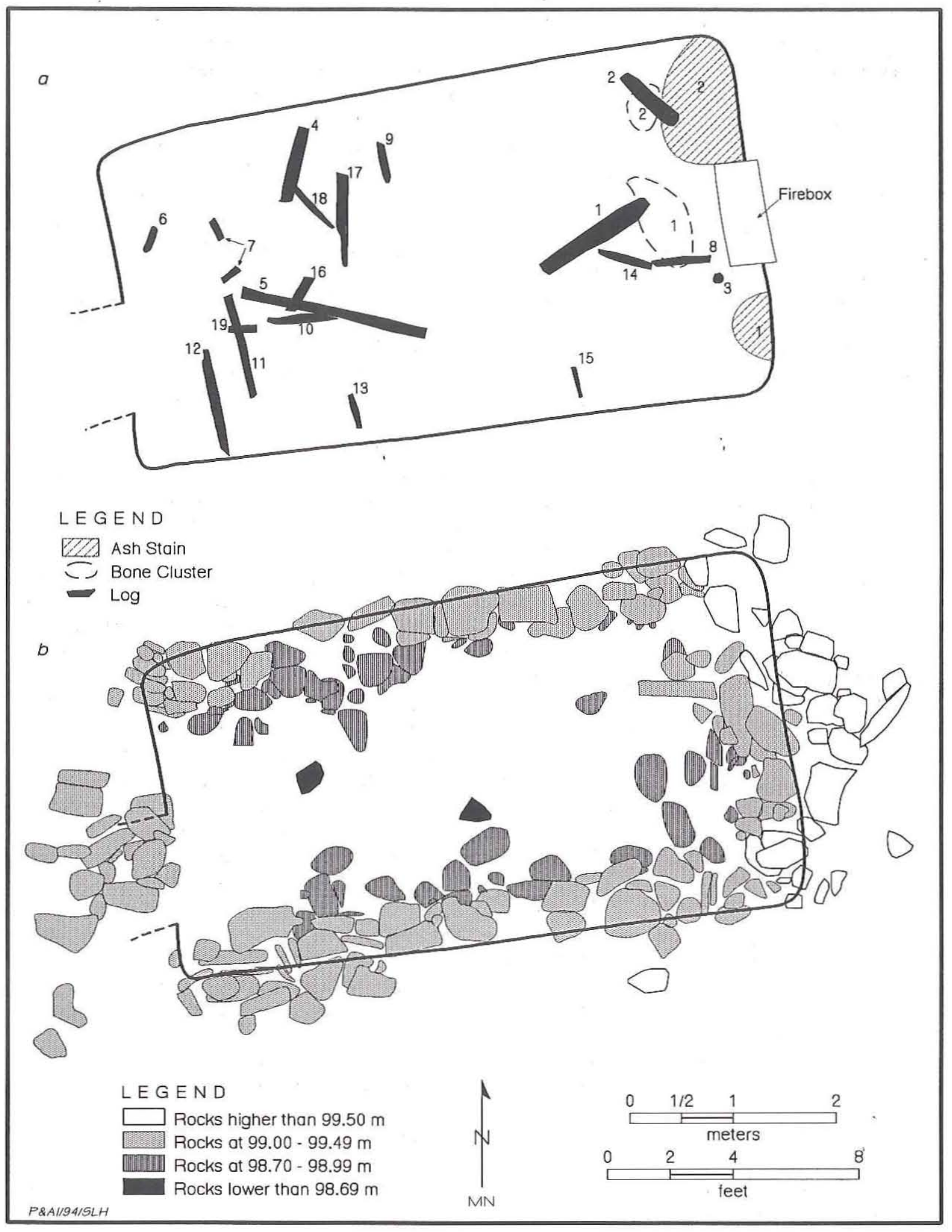

Figure 68. Plan views of the dugout at 41GR474 showing various structural elements. (a) Outline of lower walls showing the fireplace, floor features, and logs; $(b)$ horizontal and vertical distribution of upper wall rockfall. 
the dugout was excavated into the hard sediment. The floor undulates slightly but is generally level, with less than a 5-cm difference across the structure. It is slightly lower toward the center, as would be expected due to removal and/or compaction of the central floor by foot traffic. There were no permanent interior room dividers, and even if temporary walls were used, the central path from the entryway to the fireplace probably was left open.

Although the lower walls are slightly irregular, it is clear that the structure was a rectangle of approximately $11 \times 20 \mathrm{ft}$ (see Figure 68a). It was a half dugout placed to take advantage of the natural slope, with the entryway facing west toward the river. Because the upper rocks stabilized the slope, it is doubtful that erosion has removed much, if any, of the sediment walls. Some of the uppermost rocks along the back appeared to be in place on top of undisturbed sediment (see east end of Figure 67), indicating that the sediment portion of the wall was approximately $4.2 \mathrm{ft}$ high. The north and south sediment walls slope westward to a height of approximately 2 to $3 \mathrm{ft}$ at the front wall. The lower sediment walls appear to be relatively intact, and there is no evidence that any portion has slumped under the weight of the upper rock walls. The floor along the front wall lips upward to form a sediment wall only a few centimeters high, indicating that the front wall was constructed of other materials. Notably, no rocks were found in place along the front wall. A slight break in the lipping of the floor indicates where the entryway was located.

There is no hint that the floor or lower walls were plastered or otherwise covered. While unimproved dirt floors and walls apparently were common for dugouts (Freeman 1990:78), many families used some type of covering. Had the floor and/or lower walls been covered with starched gunny sacks, canvas, paper, or wooden planks (Archer 1978:11-14; Freeman 1990:78, Table 6; Lewis 1976:54), there probably would be no archeological evidence left. Fabric or paper coverings would have deteriorated completely, and wooden planks could have been removed and reused upon abandonment.

The upper walls of the dugout consisted of stacked rocks which collapsed inward toward the center of the structure after abandonment (see Figures 67 and $68 b$ ). Some short segments of the rock wall, such as in the northwest corner, collapsed as a unit with the stacked layers remaining partially intact; otherwise, the rock fall around the perimeter is jumbled. At the time the walls collapsed, a significant amount of sediment already had accumulated inside the structure, suggesting that the dugout had been abandoned for some time. The rock fall approximates the contours of the depression at the time of collapse, with the lowest rocks being nearest the center and others being progressively higher toward the walls. The few rocks found in the center were $5-15 \mathrm{~cm}$ above the floor, while those closest to the north and south walls generally were over $50 \mathrm{~cm}$ above the floor. This same pattern was observed in a tested dugout in Payne County, Oklahoma, which also had upper rock walls that had collapsed inward (Young 1978:19-24, Figure 4).

The distribution of the rocks shows that three walls - the back and two sides - were made of stacked rocks. The rock pile at the front of the structure, however, is not large enough to account for a full rock or even half-rock front wall, and it is likely that most or all of the front wall was constructed of wood or some other material. Several alternative explanations could account for the rock pile in the entryway. The rocks could have lined the sides of an entryway trench, which may have sloped or stepped down into the dugout. Similarly, there may have been a partial rock wall along the front or a doorway outlined by rocks. In either case, the rocks could have collapsed into the entryway. Conversely, there may have been no rocks along the front wall, and the rock pile in the entryway may have been placed there after abandonment. Perhaps rocks were robbed from the side walls and intentionally piled in the entryway after the dugout collapsed to prevent access. A dilapidated or collapsed dugout would have attracted rodents and snakes and would been a safety hazard for children. Along the north and south walls, there appear to be gaps in the rock fall (see Figure 68b) which may support the theory that some wall rocks were removed. Nevertheless, the total amount of rocks and the height of the lower walls suggest that, assuming a total wall height of approximately $6 \mathrm{ft}$, the upper rock walls were about $2 \mathrm{ft}$ tall at the back and perhaps 3 to $4 \mathrm{ft}$ tall at the front.

All of the rocks used in the construction of the dugout walls are tabular sandstone slabs obtained from Triassic outcrops in the immediate vicinity. None show evidence of having been shaped, although there clearly was some intentional selection in their placement. Larger, flatter slabs were selected for the lower rocks, and as would be expected, the rocks used on the upper portions of the walls tend to be 
smaller and more blocky. No evidence of plastering on the rock walls was found, but it is likely that they were at least chinked with mud on the interior. There is no archeological evidence that there were any windows in the structure.

The nature of the materials and construction of the dugout roof is unclear, but wood sections found in the Lower Fill, at the bottom or below the level of the rock fall, are almost certainly remnants of the roof (see Figure 68a). The wood was identified as Juniperus sp. by Phil Dering (Palynology Laboratory at Texas A\&M University) and represents rough-hewn logs (Table 36) which, like the rocks, were obtained locally. The wood could be from red-berry juniper (Juniperus pinchotii), oneseed juniper ( $J$. monosperma), ashe juniper (J. ashe), or Rocky Mountain juniper ( $J$. virginiana, $J$. scopulorum, or $J$. virginiana scopulorum). All of these species are found in the region today and are often generically called "cedar trees" (Hutchins 1968:29-30; Rowell 1967:38-39; Vines 1982:1118). Given the large size of some fragments (e.g., Logs 1 and 5), one-seed juniper or Rocky Mountain juniper are most likely since they grow much larger than the other species (Vines 1982:11-18). Flores (1990:108) suggests that Rocky Mountain juniper, found only in relict populations in the canyonlands today, is extremely rare because it was harvested selectively by early settlers and pioneer loggers.

Not enough wood was found to account for a complete wooden roof, but the two largest fragments (Logs 1 and 5) are oriented longitudinally and may represent parts of a ridge beam, while the smaller logs are generally perpendicular and may have served as rafters. The presence of a longitudinal ridge beam and rafters suggests that the roof was a moderate- to low-pitched, front-gabled style, which is quite common for dugouts (McAlester and McAlester 1990:76, 86). The absence of nails or wooden pegs or pegholes in the logs does not necessarily mean that none were used, but it is possible that the roof components (i.e., the ridge beam and rafters) were laid without the aid of fasteners. The log frame may
TABLE 36

SUMMARY OF LOGS IN THE DUGOUT AT 41GR474

\begin{tabular}{|c|c|c|c|c|}
$\begin{array}{l}\text { Maximum } \\
\text { Diameter } \\
(\mathrm{cm})\end{array}$ & $\begin{array}{l}\text { Length } \\
(\mathrm{cm})\end{array}$ & $\begin{array}{l}\text { Top } \\
\text { Elevation } \\
(\mathrm{m})\end{array}$ & $\begin{array}{l}\text { Bottom } \\
\text { Elevation } \\
(\mathrm{m})\end{array}$ & $\begin{array}{l}\text { Cm above } \\
\text { Floor* }\end{array}$ \\
\hline 15 & 122 & 98.70 & 98.49 & 17 \\
8 & 63 & 98.90 & 98.76 & $41-46$ \\
7 & 30 & 98.94 & 98.64 & $29-34$ \\
10 & 75 & 98.78 & 98.63 & 26 \\
14 & 180 & 98.69 & 98.42 & $18-20$ \\
9 & 28 & 98.50 & 98.43 & $8-13$ \\
6 & 32 & 98.48 & 98.42 & $7-12$ \\
7 & 47 & 98.51 & 98.42 & $7-12$ \\
8 & 35 & 98.65 & 98.52 & $17-22$ \\
5 & 62 & 98.56 & 98.50 & $15-20$ \\
8 & 100 & 98.60 & 98.56 & 24 \\
10 & 103 & $98.47 ;$ & 98.35 & $0-2$ \\
6 & 30 & 98.54 & 98.39 & $4-10$ \\
5 & 43 & 98.38 & 98.34 & $0-2$ \\
5 & 30 & 98.46 & 98.38 & $3-8$ \\
6 & 34 & 98.43 & 98.37 & 0 \\
9 & 90 & 98.41 & 98.33 & $0-3$ \\
4 & 5 & 98.42 & 98.37 & $2-5$ \\
4 & 27 & 98.40 & 98.32 & 0 \\
\hline
\end{tabular}

have been covered over with more logs and branches, milled lumber, hides, sod, grass, or some combination of these (Freeman 1990:78). It also is possible that some of the sterile sediment in the lower dugout fill came from a sod-covered roof. The scarcity of nails ( $n=13$, see Dugout Assemblage) suggests that they were not used in the construction of the roof (or the front wall, if it was constructed of wood).

\section{Interior Features}

\section{Fireplace}

The dominant interior feature is a fireplace sunk into the back wall opposite the doorway (Figure 69). While some structures had stoves, fireplaces commonly were used for heating and cooking in dugouts and typically were recessed into the back wall (Freeman 1990:78). In the Justice dugout, two large flat slabs were placed vertically to form the sides of the firebox. Each slab is recessed about 6 to 8 inches into the back wall, and a large horizontal 


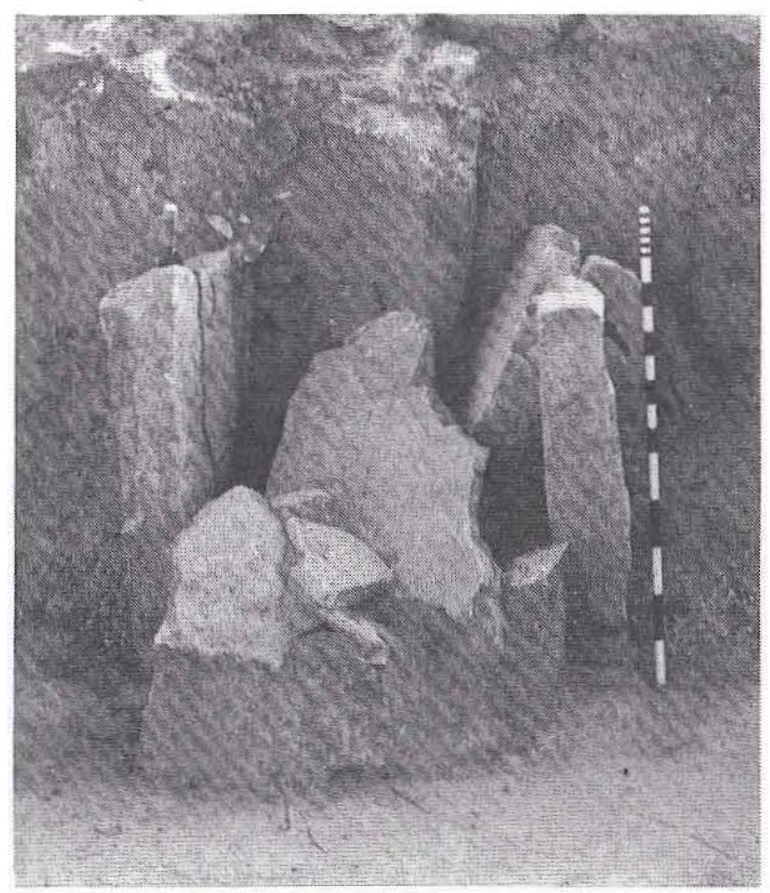

Figure 69. View east of firebox along the back wall of the dugout at 41GR474. Vertical slabs are in place, while central rocks are collapsed from above.

rock located immediately in front of the firebox originally may have lain on the vertical rocks to form the top of the box. Its orientation, lying horizontal at a slight angle to the west, suggests that it may have fallen forward from this position. Other slabs found in the center of the firebox appear to have collapsed from above. It is not clear what the rest of the fireplace may have looked like. Smoke may have been vented through the ceiling with a flue pipe connected to the top of the firebox, or perhaps a sod chimney was used. There do not appear to be enough rocks along the back wall, however, to account for a full rock chimney.

\section{Ash Stains and Bone Concentrations}

Four interior features are nonstructural. Two ash stain concentrations and two bone clusters were found in the back of the dugout close to the fireplace (see Figure 68a). The two ash stains are located on the floor on either side of the fireplace. Both consist of fine gray ashy sediment with occasional charcoal flecks, and they appear to represent burned debris that was dumped onto the floor from the fireplace. Ash Stain 1 is the smaller of the two. It covers a $70-\mathrm{x}-40-\mathrm{cm}$ area and is $5-8 \mathrm{~cm}$ thick in the southeast comer of the structure. Ash Stain 2 is larger and covers a $125-\mathrm{x}-85-\mathrm{cm}$ area; it is ca. $10-15 \mathrm{~cm}$ thick and is located in the northeast comer of the structure.

The two bone clusters also were lying on the floor near the fireplace, and like the ash stains, they appear to have been dumped on the floor. Bone Cluster 1 is a $95-\mathrm{x}-50-\mathrm{cm}$ concentration located immediately in front of the fireplace. It is a $16-\mathrm{cm}-$ thick pile consisting of 77 bones in a random arrangement. Only 12 specimens are identifiable to genus or species, and only five taxa- duck, pronghorn, coyote, cow, and pig - are represented (Table 37). Seven of the bones exhibit saw marks, but none are burned. Bone Cluster 2 consists of four bones within a $40-\mathrm{x}-25-\mathrm{cm}$ area immediately adjacent to Ash Stain 2. All of the bones were within $8 \mathrm{~cm}$ of the floor, and they appear to have been dumped near the ash stain. None of the bones are modified or burned, and only one specimen, a complete pronghorn humerus, was identifiable to species (see Table 37).

Given the variety of species represented and the presence of saw marks on a few specimens, it is assumed that these bones represent the butchered remains of animals that were eaten. Since it is unlikely that butchered animal remains or fireplace debris would have been discarded inside the dugout when it was being occupied, it is likely that these remains were dumped there after the dugout had been abandoned. These nonstructural features are interpreted as representing activities postdating use of the dugout as a domicile.

\section{MATERIALS RECOVERED}

The data recovery investigations produced 2,321 artifacts (Table 38). As discussed in Chapter 2 , these historic artifacts are classified into material groups and functional classes following South (1977). The artifacts are separated and described in two distinct groups, called the dugout and trash fill assemblages. The dugout assemblage accounts for $20 \%$ of the materials and includes all artifacts recovered from the lower portion of the dugout (i.e., at and below the roof fall in excavation levels designated as Dugout Zone 1 and Dugout Floor). The trash fill assemblage accounts for $80 \%$ of the materials and includes all artifacts recovered from the upper portion of the dugout (i.e., above the roof fall in excavation levels designated as Upper Fill, Lower Fill, and Rock Fall Zone). These assemblages are 


\begin{tabular}{|c|c|c|c|}
\hline \multicolumn{4}{|c|}{$\begin{array}{l}\text { TABLE } 37 \\
\text { AUNAL REMAINS IN BONE CLUSTERS } 1 \text { AND 2, 41GR474 }\end{array}$} \\
\hline Taxon & Common Name & Elements Represented & $\begin{array}{l}\begin{array}{l}\text { No. of } \\
\text { Specimens }\end{array} \\
\end{array}$ \\
\hline $\begin{array}{l}\text { Bone Cluster } \mathbb{1} \\
\text { Anatidae } \\
\text { Antilocapra americana } \\
\text { Canis latrans } \\
\text { cf. Bos } \text { sp. } \\
\text { cf. Sus scrofa } \\
\text { Medium-large Artiodactyla } \\
\text { Unidentified mammal* }\end{array}$ & $\begin{array}{l}\text { duck } \\
\text { pronghom } \\
\text { coyote } \\
\text { cow } \\
\text { pig } \\
\text { deer-sized } \\
\text { - }\end{array}$ & $\begin{array}{l}\text { tarsometatarsus } \\
\text { scapula, humerus, calcaneus, metacarpal, phalange } \\
\text { radius, ulna, femur } \\
\text { cranium fragments } \\
\text { carpal } \\
\text { humerus, carpals, metacarpals } \\
\text { - }\end{array}$ & $\begin{array}{r}1 \\
5 \\
3 \\
2 \\
1 \\
5 \\
60 \\
77 \\
\end{array}$ \\
\hline $\begin{array}{l}\text { Bone Custer } 2 \\
\text { Antilocapra americana } \\
\text { Large Artiodactyla } \\
\text { Unidentified mammal }\end{array}$ & $\begin{array}{l}\text { pronghom } \\
\text { deer-sized } \\
-\end{array}$ & $\begin{array}{l}\text { complete humerus } \\
\text { complete lumbar vertebra } \\
\text { - }\end{array}$ & $\begin{array}{l}1 \\
1 \\
2 \\
4\end{array}$ \\
\hline
\end{tabular}

\begin{tabular}{|l|r|r|r|}
\hline \multicolumn{4}{|c|}{ TABLE 38} \\
\multicolumn{4}{|c|}{ HISTORIC ARTIFACTS RECOVERED DURING } \\
DATA RECOVERY, 41GR474 \\
\hline Material Group & Dugout & Trash Fill & Totals \\
\hline Metal & 155 & 253 & 408 \\
Ceramic & 1 & 468 & 469 \\
Glass & 27 & 948 & 975 \\
Rubber & 2 & 1 & 3 \\
Leather & - & 30 & 30 \\
Bone & 280 & 142 & 422 \\
Shell & 2 & 3 & 5 \\
Stone & - & 4 & 4 \\
Brick & - & 1 & 1 \\
Other & 1 & 3 & 4 \\
\hline Totals: & 468 & 1,853 & 2,321 \\
\hline
\end{tabular}

separated by the roof fall, an event that marked the end of the dugout's use life. Hence, the dugout assemblage represents an accumulation of artifacts that is associated with the dugout's habitation and/or subsequent use episodes, while the trash fill assemblage represents a secondary deposit of artifacts that is associated with the habitation of the later ranch house. Each of these artifact assemblages is described separately below.

\section{Dugout Assemblage}

The dugout assemblage contains 468 items (Table 39). Bone is the most frequent material, representing $60 \%$ of the assemblage, followed by metal $(33 \%)$, and glass $(6 \%)$. All other materials make up only $1 \%$, and it is noteworthy that the ceramic category, which is usually a well-represented material group, consists of only a single specimen.

\section{Metal}

Metal artifacts consist of 155 specimens representing a range of activities (Table 40). Firearms-related artifacts are well represented, with several brass cartridge cases being identifiable. These include .45-caliber Colt and .40-.70-caliber Sharps centerfire cases and a .44-caliber Henry rimfire case. None have base stamps, and their identifications are based on case measurements which are not absolutely precise due to corrosive build-up on the cases.

The measurements for eight of the cases (length $1.27-1.30$ inches, base diameter $0.47-0.48$ inch, and rim diameter $0.50-0.52$ inch) closely fit the dimension given for .45-caliber Colt cartridges introduced in 1873 (Barnes 1980:187, 193). The largest cartridge (length 2.5 inches, base diameter 0.46 inch, 
TABLE 39

SUMMARY OF THE DUGOUT ARTIFACT ASSEMBLAGE, 41GR474

\begin{tabular}{|l|r|c|c|c|c|c|r|r|}
\hline Functional Category & Metal & Ceramic & Glass & Rubber & Bones & Shell & Eggshell & Totals \\
\hline Kitchen & 4 & - & - & - & - & - & 1 & 5 \\
Bone & - & - & - & - & 279 & - & - & 279 \\
Architecture & 13 & - & - & - & - & - & - & 13 \\
Furniture & - & - & - & - & - & - & - & 0 \\
Arms & 28 & - & - & - & - & - & - & 28 \\
Clothing & 1 & 1 & - & - & 1 & 2 & - & 5 \\
Personal & 1 & - & - & 1 & - & - & - & 2 \\
Tobacco & 2 & - & - & 1 & - & - & - & 3 \\
Activities & 7 & - & - & - & - & - & - & 7 \\
Unidentified & 99 & - & 27 & - & - & - & - & 126 \\
\hline Totals: & 155 & 1 & 27 & 2 & 280 & 2 & & 1 \\
\hline
\end{tabular}

\begin{tabular}{|c|c|c|}
\hline \multicolumn{3}{|c|}{$\begin{array}{c}\text { TABLE } 40 \\
\text { METAL ARTIFACTS IN THE DUGOUT ASSEMBLAGE, 41GR474 }\end{array}$} \\
\hline Functional Category & Artifact Description* & No. of Specimens \\
\hline Kitchen $(n=4)$ & $\begin{array}{l}\text { 1-gallon can, round } \\
\text { wire loop and cap for bottle closure (for cork or rubber stopper) } \\
\text { drinking cup }\end{array}$ & $\begin{array}{l}1 \\
1 \\
2\end{array}$ \\
\hline Architecture $(n=13)$ & square-cut nails, various sizes $(3 d-20 d)$ & 13 \\
\hline Arms $(n=28)$ & $\begin{array}{l}.45 \text {-caliber Colt brass cartridge cases } \\
.40 \text { - } 70 \text {-caliber Sharps Straight brass cartridge case } \\
.44-\text { caliber Henry Flat brass cartridge case } \\
\text { Boxer primer, small }(0.212-0.217 \text {-inch diameter }) \\
\text { Boxer primer, large }(0.259-0.265 \text {-inch diameter }) \\
\text { Berdan primer, small }(0.215 \text {-inch diameter }) \\
\text { round lead ball }(0.34-0.35 \text {-inch diameter })\end{array}$ & $\begin{array}{r}8 \\
1 \\
1 \\
12 \\
2 \\
1 \\
3\end{array}$ \\
\hline Clothing $(n=1)$ & compound disk button, 4-hole/checkered pattern & 1 \\
\hline Personal $(n=1)$ & brass/wood harmonica & 1 \\
\hline Tobacco $(n=2)$ & round tobacco plug trademarks & 2 \\
\hline Activities $(\mathrm{n}=7)$ & $\begin{array}{l}\text { wagon brace (?) } \\
\text { wagon tongue plate (?) } \\
\text { horseshoe nails (sizes \#5 and \#6) } \\
\text { bolt fragment ( } 3-4 \text { inch) } \\
\text { single-bit axe head }(4.5 \mathrm{lbs})\end{array}$ & $\begin{array}{l}1 \\
1 \\
3 \\
1 \\
1\end{array}$ \\
\hline Unidentified $(\mathrm{n}=99)$ & $\begin{array}{l}\text { iron fragments } \\
\text { brass fragment } \\
\text { lead fragment } \\
\text { iron object } \\
\text { brass object, possible harmonica plate } \\
\text { miscellaneous large can fragment } \\
\end{array}$ & $\begin{array}{r}92 \\
1 \\
1 \\
2 \\
1 \\
2 \\
\end{array}$ \\
\hline Total: & & 155 \\
\hline
\end{tabular}


and rim diameter 0.55 inch) measures closest to the $.40-.70$-caliber Sharps Straight which was introduced in 1879 (Bames 1980:109, 134). One cartridge (length 0.95 inch, base diameter 0.46 inch, and rim diameter 0.52 inch) measures closest to the .44caliber Henry Flat cartridge introduced about 1860 and manufactured until ca. 1934 (Barnes 1980:296, 299). This specimen also has the distinctive double firing pin marks left by the Henry rifle (Herskovitz 1978:49).

The primers in the dugout assemblage probably relate to reloading of cartridges and are of three types (Bames 1980:318-323). Two are large Boxertype primers which were used in centerfire rifle cartridges, and 13 specimens are smaller primers used in centerfire pistol or small rifle cartridges. Of the latter, 1 is a Berdan primer, and the other 12 are Boxer primers. Boxer and Berdan primers were invented at about the same time (1867 and 1866, respectively), but in the United States, Boxer primers became the preferred type and are still in use today, while American companies phased out the manufacture of Berdan primers by the 1920s (Barnes 1980: 318-319).

Of the remaining metal artifacts, only the tobacco plug marks are identifiable. Both specimens are round plugs identical in size (0.52-inch diameters) with ears on opposite edges. One is completely rusted, while the other has one surface painted red with black lettering and reads "CLIMAX" and "GRADE" in block letters along the top and bottom, with "P LORILLARD Co" in script letters across the center (Figure 70). Campbell (1964:102-104) states that $\mathrm{P}$. Lorillard is the oldest tobacco company in the world (founded in 1760) and that they were the first to use pronged tin tags stamped with their company name to identify their tobacco plugs. A similar trademark, "LORILLARD'S CLIMAX PLUG," is illustrated in the 1897 Sears Roebuck Co. catalog (Israel 1967:n.p.), and a plug mark identical to the 41GR474 specimen is reported at a late nineteenth/ early twentieth-century dugout site in Lamb County (Guffee 1980:31, Figure 14a). Heinmann (1960) notes that the peak popularity of chewing tobacco consumption was ca. 1890 , but that smoking tobacco (cigars and cigarettes) become much more popular during the 1910s and 1920s.

Other metal artifacts of interest are the architectural and activities artifacts and include square cut nails, horseshoe nails, wagon parts, a harmonica reed plate (and a possible harmonica name

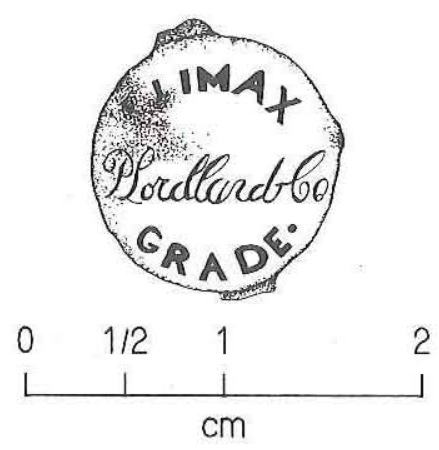

Figure 70. Tobacco plug trademark in the dugout assemblage at $41 \mathrm{GR} 474$.

plate), and a $77 / 8$-inch-long axe head of the same style as the single-bit "Wisconsin Pattern" axe heads in the 1908 Sears, Roebuck and Co. catalog (Schroeder 1971:500). The possible wagon parts are a $13-x-31 / 4-$ inch flat metal plate similar to wagon tongue or bolster plates illustrated in Spivey $(1979: 21,27)$ and the 1895 Montgomery Ward and Co. catalog (Dover Publications, Inc. 1969:595) and an 183/4-x-3-inch bar similar to cutter shoes illustrated by Spivey (1979:154). Metal artifacts also include four kitchen items and one clothing item (i.e., a button).

The paucity of nails in the dugout assemblage is particularly interesting and has some bearing on the construction and life history of the structure. For example, Earls (1993a:394) suggests that the low density of nails $\left(\mathrm{N}=360\right.$, or 4.2 nails $\left./ \mathrm{m}^{2}\right)$ in one excavated structure complex in the Texas Panhandle is related to its short use life and/or the dismantling and salvaging of materials after abandonment. Since the Justice dugout apparently was occupied for 6-7 years (a rather long occupation for a dugout), the extremely low density of nails $(\mathrm{N}=13$, or ca. 0.6 nails $/ \mathrm{m}^{2}$ ) probably is not related to its short use life. The near absence of nails more likely indicates that nails were not used or were used sparingly in the original construction. It also is possible that any components that were constructed of nails (e.g., part or all of the front wall or door could have been constructed of nailed lumber) subsequently were dismantled and salvaged.

\section{Ceramics and Glass}

Ceramics and glass are poorly represented in the dugout assemblage. A Prosser-type china button (Pool 1987:281) is the only ceramic specimen, and 
the glass is limited to colorless, light green, and brown fragments (Table 41). A square container base appears to be from a snuff bottle, but it is too fragmentary to be certain. It has the raised number " 2 ..." in its center, but this mark is incomplete.

\section{Bone}

Bone is the most abundant functional category in the dugout assemblage but includes only one artifact-a four-hole button (see Table 41). The remaining 279 specimens are faunal remains that apparently represent discarded bone refuse. Of these, $22 \%(n=60)$ exhibit evidence of burning and $4 \%$
( $n=10$ ) have butchering marks (i.e., cut or saw marks). A wide range of animals is represented (see Appendix C), although not all of them definitely represent food resources. The only bones with cut marks are those of rabbit and cow, but it is likely that many of the other groups, particularly antelope, duck, and domestic pig, also represent food resources. It is not certain whether the coyote, dog, prairie dog, and snake remains represent discarded food refuse. In addition to the date recovery bones, one cow and two pronghom were identified among the 11 bones recovered during the 1988 testing (Freeman and Boyd 1990:146).

\begin{tabular}{|c|c|c|c|}
\hline & $\begin{array}{l}\text { CERAMIC, GLAS } \\
\text { ARTIFACTS }\end{array}$ & $\begin{array}{l}\text { TABLE } 41 \\
\text { BONE, AND MISCELLANEOUS MA } \\
\text { THE DUGOUT ASSEMBLAGE, } 41 \mathrm{G}\end{array}$ & \\
\hline Functional Category & Material Group & Artifact Description & No. of Specimens \\
\hline Personal $(n=1)$ & Ceramic & porcelain button, Prosser type, 4-hole & 1 \\
\hline Unidentified $(n=27)$ & Glass & $\begin{array}{l}\text { container fragments, colorless } \\
\text { container fragments, light green } \\
\text { container fragments, brown } \\
\text { square container base fragment, brown } \\
\text { unidentified fragment, colorless }\end{array}$ & $\begin{array}{r}11 \\
5 \\
9 \\
1 \\
1\end{array}$ \\
\hline Clothing $(\mathrm{n}=1)$ & Bone & disk button, 4-hole & 1 \\
\hline Bone $(n=279)$ & Bone & $\begin{array}{l}\text { antelope } \\
\text { cottontail rabbit } \\
\text { cow } \\
\text { coyote } \\
\text { dog or dog family } \\
\text { duck } \\
\text { medium-size bird } \\
\text { pig } \\
\text { pit viper } \\
\text { prairie dog } \\
\text { unidentified mammal } \\
\text { unidentified medium/large mammal } \\
\text { unidentified ungulate } \\
\text { unidentified medium/large ungulate }\end{array}$ & $\begin{array}{r}14 \\
5 \\
3 \\
4 \\
5 \\
5 \\
1 \\
4 \\
1 \\
2 \\
4 \\
4 \\
211 \\
3 \\
18\end{array}$ \\
\hline Personal $(n=1)$ & Rubber & comb handle & 1 \\
\hline Tobacco $(n=1)$ & Rubber & smoking pipe mouthpiece fragment & 1. \\
\hline Clothing ( $\mathrm{n}=2$ ) & Shell & $\begin{array}{l}\text { disk button, 4-hole } \\
\text { disk button, 2-hole }\end{array}$ & $\begin{array}{l}1 \\
1\end{array}$ \\
\hline Unidentified $(n=1)$ & Other & eggshell fragment & 1 \\
\hline \multicolumn{3}{|l|}{ Total: } & 313 \\
\hline
\end{tabular}




\section{Other Materials}

Rubber, shell, and eggshell are the only other materials represented in the dugout assemblage (see Table 41). The two rubber artifacts are a smoking pipe fragment and a comb handle. The latter has engraved letters "... COMB" on one side and "THE BUTLER H . . ." on the other, but these markings have not been identified. Both of the shell artifacts are buttons, and the eggshell fragment probably represents a food resource.

\section{Trash Fill Assemblage}

The trash fill assemblage is represented by 1,853 items (Table 42). In contrast to the dugout assemblage, glass, ceramic, and metal artifacts are dominant and comprise $90 \%$ of the materials. Bones comprise only $8 \%$ of the assemblage, and all other materials account for the remaining $2 \%$.

\section{Metal}

The trash fill assemblage is dominated heavily by metal, although this is not readily apparent from the artifacts analyzed (see Table 38). Metal is the only material class that was not collected consistently, and 270 pounds of unidentifiable scrap metal encountered in the Upper Fill were weighed and discarded in the field and are not included in the analysis. The metal assemblage consists of 253 specimens (Table 43) that were included in the analysis, but some of these $(n=154,61 \%)$ were recorded in the field and discarded. Hence, metal as a material category is underrepresented in Table 42. In functional terms, however, metal is not underrepresented since the only materials excluded were fragments that would not have been assigned to a specific functional class.

Metal artifacts assigned to the kitchen functional class include a variety of cans, cooking pot and kettle fragments, a cup, a flour sifter, a serving utensil, parts for wood-burning and kerosene stoves, and a food grinder. The latter is a cylindrical snap case (minus the crank handle and grinding blade) that is very similar to the 6-inch-cylinder "Home Meat Chopper and Stuffer" illustrated in the 1895 Montgomery Ward and Co. catalog (Dover Publications, Inc. 1969:390). The most ubiquitous cans are of the hole-in-cap variety $(n=54)$ followed by holein-top cans $(n=21)$, both of which were common in the late nineteenth century (Busch 1981; Rock 1984). The absence of modern sanitary cans (in common use after 1904) may be misleading, however, since some of the unidentifiable scraps in the Upper Fill looked like modern can debris (i.e., with double end seams) but could not be positively identified. Conversely, the presence of diagnostic solder insured that holein-cap and hole-in-top specimens were identifiable.

Some of the kitchen items have identifiable markings. Two large cans are stamped "CALUMET" "BAKING POWDER" "5 LBS," and three snap-on lids from the same size cans also are stamped with product labels. One lid is marked "CAL. .." "5 lbs" "BAKING POWDER" "FULL WEIGHT" "ABSOLUTELY PURE"; a second lid is

TABLE 42

SUMMARY OF THE TRASH FILL ARTIFACT ASSEMBLAGE, 41 GR474

\begin{tabular}{|l|r|r|r|r|r|r|r|r|r|r|r|}
\hline Functional Category & Metal & Ceramic & Glass & Rubber & Leather & Bone & Shell & Other & Stone & Brick & Totals \\
\hline Kitchen & 127 & 467 & 209 & - & - & - & - & 1 & - & - & 804 \\
Bone & - & - & - & - & - & 142 & - & - & - & - & 142 \\
Architecture & 4 & - & 19 & - & - & - & - & - & - & 1 & 24 \\
Furniture & 13 & - & - & - & - & - & - & - & - & - & 13 \\
Arms & 22 & - & - & - & - & - & - & - & - & - & 22 \\
Clothing & 19 & 1 & - & - & 29 & - & 3 & - & - & - & 52 \\
Personal & 2 & - & - & - & - & - & - & - & - & - & 2 \\
Tobacco & 6 & - & 18 & - & - & - & - & - & - & - & 24 \\
Activities & 45 & - & 1 & - & - & - & - & 2 & - & - & 48 \\
Unidentified & 15 & - & 701 & 1 & 1 & - & - & - & 4 & - & 722 \\
\hline Totals: & 253 & 468 & 948 & 1 & 30 & 142 & 3 & 3 & 4 & 1 & 1,853 \\
\hline
\end{tabular}


TABLE 43

METAL ARTIFACTS IN THE TRASH FILL ASSEMBLAGE, 41GR474

\begin{tabular}{|c|c|c|}
\hline Functional Category & Artifact Description* & No. of Specimens \\
\hline Kitchen $(n=127)$ & $\begin{array}{l}\text { round hole-in-top cans, ca. 16-ounce size } \\
\text { round hole-in-cap cans, 12-32-ounce sizes } \\
\text { rectangular key-wind can (sardine type) } \\
\text { oval hole-in-cap key-wind can } \\
\text { key-wind openers } \\
\text { baking powder can, 32-ounce size } \\
\text { baking powder can, 5-pound size } \\
\text { baking powder can lids, 5-pound size } \\
\text { unidentified round flat can } \\
\text { cast iron kettle fragment } \\
\text { cooking pot, } 1 / 2 \text {-quart size } \\
\text { drinking cup with handle } \\
\text { food grinder, snap-case } \\
\text { flour sifter fragments } \\
\text { serving utensil handle (spoon or fork?) } \\
\text { kerosene stove burner mechanism } \\
\text { cast iron burner grill } \\
\text { cast iron wood stove fragments }\end{array}$ & $\begin{array}{r}21 \\
53 \\
4 \\
1 \\
2 \\
1 \\
2 \\
3 \\
1 \\
6 \\
1 \\
1 \\
1 \\
2 \\
1 \\
2 \\
1 \\
24\end{array}$ \\
\hline Architecture $(n=4)$ & $\begin{array}{l}\text { square-cut nails, } 8 \mathrm{~d}-10 \mathrm{~d} \\
\text { wire nail, } 8 \mathrm{~d} \\
\text { electrical fuse, socket type }\end{array}$ & $\begin{array}{l}2 \\
1 \\
1\end{array}$ \\
\hline Fumiture $(n=13)$ & $\begin{array}{l}\text { kerosene lantern part } \\
\text { bedspring coils }\end{array}$ & $\begin{array}{r}1 \\
12\end{array}$ \\
\hline Arms $(n=22)$ & $\begin{array}{l}.45 \text {-caliber Colt brass cartridge cases } \\
.44-\text { caliber Henry Flat brass cartridge cases } \\
\text { conical lead bullet, large caliber } \\
\text { flattened conical lead bullet, large caliber } \\
\text { conical bullet, } .44 \text { or } .45 \text { caliber }\end{array}$ & $\begin{array}{r}16 \\
3 \\
1 \\
1 \\
1\end{array}$ \\
\hline Clothing $(n=19)$ & $\begin{array}{l}\text { overall buttons } \\
\text { brass suspender buckle } \\
\text { brass shoe eyelets } \\
\text { disk button, } 4 \text {-hole } \\
\end{array}$ & $\begin{array}{r}12 \\
1 \\
5 \\
1\end{array}$ \\
\hline Personal $(\mathrm{n}=2)$ & $\begin{array}{l}\text { toy baby carriage fragment } \\
\text { medicine container (salve) }\end{array}$ & $\begin{array}{l}1 \\
1\end{array}$ \\
\hline Tobacco $(n=6)$ & oval tobacco tins & 6 \\
\hline Activities $(n=45)$ & $\begin{array}{l}\text { wagon part, wheel hub band } \\
\text { wagon part, sunshade fragment } \\
\text { doubletree clip } \\
\text { horseshoe nails, sizes } \# 5-7 \\
\text { harness buckles } \\
\text { stimup frame } \\
\text { brass rivet/burrs } \\
\text { brass burr } \\
\text { chain link (repair link) }\end{array}$ & $\begin{array}{l}2 \\
1 \\
1 \\
4 \\
2 \\
1 \\
5 \\
1 \\
1\end{array}$ \\
\hline
\end{tabular}




\begin{tabular}{|c|c|c|}
\hline \multicolumn{3}{|l|}{ Table 43, continued } \\
\hline Functional Category & Artifact Description & No. of Specimens \\
\hline Activities, cont'd. & $\begin{array}{l}\text { barbed wire fragments } \\
4-1 / 4 \text {-inch bolt with } 1 / 2 \text {-inch nut } \\
1 \text {-3/16-inch nut } \\
1 \text {-inch nut } \\
\text { wash pan, } 10 \text {-inch size } \\
\text { enamelware wash pan, } 10 \text {-inch size } \\
\text { washtub fragments } \\
\text { barrel band } \\
\text { paint can, } 1 / 2 \text {-gallon size } \\
\text { bucket with wire handle, } 1 \text {-gallon size } \\
\text { kerosene can, } 1 / 2 \text {-gallon size } \\
\text { kerosene can, } 1 \text {-gallon size } \\
\text { screw cap (for kerosene can) } \\
\text { square can, } 5 \text {-gallon size } \\
\text { windmill vein with blades }\end{array}$ & $\begin{array}{l}2 \\
1 \\
1 \\
1 \\
1 \\
2 \\
2 \\
1 \\
1 \\
8 \\
2 \\
2 \\
1 \\
1 \\
1 \\
1\end{array}$ \\
\hline Unidentified $(n=15)$ & $\begin{array}{l}\text { lead strip } \\
\text { iron fragments } \\
\text { round iron disk } \\
\text { molded brass object } \\
\text { hooklike iron object } \\
\text { iron appliance gear }\end{array}$ & $\begin{array}{r}1 \\
10 \\
1 \\
1 \\
1 \\
1\end{array}$ \\
\hline \multicolumn{2}{|l|}{ Total: } & 253 \\
\hline
\end{tabular}

marked "HIGHEST [quality ?]" "JACK . . ." "BAKING POWDER" "[ ? ]" "FULL WEIGHT"; and the third is marked "FULL WEIGHT" "80" "Ozs" "KC" "BAKING POWDER" "[ ? ]" "80" "ABSOLUTELY PURE." Calumet Baking Powder is still made today, and the archivist for Kraft General Foods, Inc. in Morton Grove, Illinois (Becky Haglund Tousey, personal communication 1993), suggested that this specimen dates between 1916 and the late 1920 s but could not be more specific. According to Ward et al. (1977:240), KC Baking Powder was registered with the U.S. Patent Office on November 20, 1911 (Serial No. 59816) but apparently had been on the market since ca. 1890. The exact date of this particular label has not been determined, but the style of the name is most similar to labels used from 1925-1950 (i.e., the "TRUE HEIGHT CAN" style).

Other kitchen artifacts with diagnostic markings are cast iron stove parts with name banners incorporated into their ornate curvilinear designs. Among the stove fragments are pieces labeled "WARMING CLOSET" and ". . . K's STOVE \& RANGE CO."

Architectural artifacts are mainly nails, but an electric socket fuse may be one of the latest artifacts in the assemblage. While it is not known exactly when electricity was brought into the project area, it probably was not before the Rural Electrification Administration began in the early 1930s. The nails include both machine-cut and wire types, neither of which are particularly sensitive chronological indicators since both were manufactured before and after the turn of the century, but wire nails became the dominant type during the 1890s (Nelson 1968). The furniture class is represented only by a kerosene lantern part and bedspring coils.

Firearms-related artifacts include sixteen .45caliber Colt cartridges and three .44-caliber Henry Flat cartridges. None of these have base stamps, and their identifications are based on case measurements. The .45-caliber Colt cases measure 1.27-1.30 inches in length, $0.47-0.48$ inch in base diameter, and 0.50 0.52 inch in rim diameter; the .44-caliber Henry cases measure $0.90-0.95$ inch in length, 0.46 inch in base diameter, and 0.52 inch in rim diameter (Barnes 1980:193, 299). The .44-caliber cartridges also exhibit the distinctive double firing pin marks of the Henry rifle (Herskovitz 1978:49). The conical lead 
bullets could come from either of these calibers but could not be positively identified.

Clothing artifacts consist of buttons, shoe eyelets, and a buckle, but only two personal items - a toy baby carriage and a medicinal container-were recovered. Most of the overall buttons are plain or have a crosshatched (i.e., checkered) pattern, but two are stamped "BLUE BUCKLE" and one is stamped "LONE STAR." These trade names have not been identified. The suspender buckle also is stamped with a name that has not been identified: "W.K. \& CO." The medicinal container is a $2 \frac{1}{2}$-inch, snapon can lid stamped with "BUCKLEN'S" "ARNICA" "COMPOUND" "SALVE." Arnica salve was used to treat sprains and bruises of both people and animals and appears in the index to the 1908 Sears, Roebuck and Co. catalog (Schroeder 1971:529), but no specific information on the Bucklen's brand could be found.

The tobacco class consists of six oval tobacco tins with hinged snap lids. Activities artifacts related to ranching include wagon parts and horse gear (i.e., horseshoe nails, stirrup frame, hamess buckles, doubletree clip, and burrs and rivet/burrs used on leather equipment), barbed wire fragments, and miscellaneous hardware (e.g., nuts and a bolt). Artifacts related to more general household activities include a windmill vein (the other half of which was found near the concrete windmill platform adjacent to the ranch house) and a variety of items for cleaning (e.g., wash pans and tubs) and storage (e.g., a barrel band and kerosene cans).

\section{Ceramics}

The trash fill ceramic assemblage consists of one Prosser-type china button (Pool 1987:281), assigned to the clothing category, and 467 fragments of ceramic vessels, assigned to the kitchen functional category (Table 44). Of the kitchen ceramics, $60 \%$ $(\mathrm{n}=278)$ are stoneware, $36 \%(\mathrm{n}=169)$ are whiteware, and $4 \%(n=20)$ are semiporcelain. Eighty-eight percent $(n=410)$ of these sherds could be assigned to a specific vessel (Table 45), and 32 distinct vessels were identified (Table 46). In some cases, vessels were defined by a single sherd (e.g., Vessel 4), but many of the vessels were partially reconstructed (e.g., Vessel 3). Vessel forms represented are butter churn, jug, wide-mouth crock/jar, unidentified crock/jar, a variety of bowls (e.g., mixing, serving, sugar, and soup), plate, and

\begin{tabular}{|c|c|c|}
\hline \multicolumn{3}{|c|}{$\begin{array}{c}\text { CERAMIC ARTIFACTS IN THE TRASH FILL } \\
\text { ASSEMBLAGE, } 41 \mathrm{GR} 474\end{array}$} \\
\hline $\begin{array}{l}\text { Functional } \\
\text { Category }\end{array}$ & Artifact Description* & No. \\
\hline $\begin{array}{l}\text { Kitchen } \\
(\mathrm{n}=467)\end{array}$ & $\begin{array}{l}\text { stoneware, vessel sherds } \\
\text { stoneware, nonvessel sherds } \\
\text { whiteware, vessel sherds } \\
\text { whiteware, nonvessel sherds } \\
\text { semiporcelain, vessel sherds } \\
\text { semiporcelain, nonvessel sherds }\end{array}$ & $\begin{array}{r}251 \\
27 \\
144 \\
25 \\
15 \\
5\end{array}$ \\
\hline $\begin{array}{l}\text { Clothing } \\
(\mathrm{n}=1)\end{array}$ & $\begin{array}{l}\text { porcelain button, Prosser type, } \\
\text { 4-hole }\end{array}$ & 1 \\
\hline \multicolumn{2}{|l|}{ Total: } & 468 \\
\hline \multicolumn{3}{|c|}{$\begin{array}{l}\text { *Vessel sherds are those that can be attributed } \\
\text { confidently to a vessel of known form. Nonvessel } \\
\text { sherds are those that cannot be attributed to a } \\
\text { single vessel. }\end{array}$} \\
\hline
\end{tabular}

cup (Figure 71). Stoneware vessels are dominant, accounting for $50 \%$ of the vessels, and generally represent plain utilitarian forms with a variety of Albany slips and salt and/or Bristol glazes. In addition, two mixing bowls (Vessels 2 and 18) have raised decorations along their rims, and two vessels (nos. 20 and 22) have raised decorations and are partially covered by a runny blue paint (similar to the blue cobalt paint and Bristol glaze on a pitcher from the San Antonio Pottery illustrated in Greer 1981: 174).

Only one recognizable marking was found on the stoneware vessels (Figure 72a). It is a partial mark on the exterior side of Vessel 9; it is missing the upper half, but its lower portion has "San Antonio, Texas" stenciled in blue. This mark has not been identified positively, but it could be either a maker's mark from a San Antonio pottery such as the San Antonio Pottery mentioned in Greer (1981:174) or a San Antonio merchant's mark such as the one on a stoneware jug in Greer (1981:175).

The stoneware vessels represent common late nineteenth/early twentieth-century forms (except for unusual wire-handled Vessel 22). In particular, jugs and wide-mouth jars are extremely common stoneware vessel forms (Greer 1981:75-86). Albany slips and Bristol glazes are the most common types of slip glazes and were used widely on stonewares in the late nineteenth and early twentieth centuries. The 


\begin{tabular}{|c|c|c|c|c|}
\hline \multicolumn{5}{|c|}{$\begin{array}{r}\text { TABLE } 45 \\
\text { SUMMARY OF CERAMIC VESSELS IN THE TR }\end{array}$} \\
\hline Vessel No. & Vessel Form & Vessel Description & \multicolumn{2}{|c|}{ Diagnostic Markings } \\
\hline \multicolumn{5}{|c|}{ Stoneware } \\
\hline 1 & butter chum & $\begin{array}{l}\text { ca. } 40 \% \text { reconstructed ( } 67 \text { sherds), Bristol glaze } \\
\text { interior and exterior, straight sided, flat base, lug } \\
\text { handles and lid ledge, } 370 \mathrm{~mm} \text { tall, } 240-\mathrm{mm}- \\
\text { diameter; similar forms are illustrated in Ketchum } \\
(1983: 60) \text { and Greer (1981:149) }\end{array}$ & none & \\
\hline 2 & mixing bowl & $\begin{array}{l}\text { rim only ( } 4 \text { sherds), Bristol glaze interior and } \\
\text { exterior with raised exterior geometric design, } \\
295-\text { mm rim diameter; similar form is illustrated in } \\
\text { Ketchum (1983:219) }\end{array}$ & none & \\
\hline 4 & crock/jar? & $\begin{array}{l}\text { large base fragment only, Albany slip interior and } \\
\text { Bristol glaze exterior, } 220-\mathrm{mm} \text { base diameter }\end{array}$ & none & \\
\hline 9 & wide-mouth jar & $\begin{array}{l}\text { ca. } 20 \% \text { reconstructed ( } 31 \text { sherds), Albany slip } \\
\text { interior and tan salt glaze exterior, straight sided, } \\
\text { flat base, } 212 \mathrm{~mm} \text { tall, } 220-\mathrm{mm} \text { diameter }\end{array}$ & "San & xas" \\
\hline 11 & butter chum & $\begin{array}{l}\text { rim sherds only }(n=4) \text {, Bristol glaze interior and } \\
\text { exterior with lug handle and lid ledge }\end{array}$ & none & \\
\hline 14 & wide-mouth jar & $\begin{array}{l}\text { base and rim sherds }(\mathrm{n}=8) \text {, reddish Albany slip } \\
\text { interior and tan salt glaze exterior, } 260-\text { mm base } \\
\text { diameter }\end{array}$ & none & \\
\hline 16 & jug & $\begin{array}{l}\text { mouth with handle and base sherds }(n=7) \text {, Albany } \\
\text { slip interior and Bristol glaze exterior, 300-mm } \\
\text { diameter, } 30-\text { mm interior mouth diameter; similar } \\
\text { form is illustrated in Ketchum (1983:97) }\end{array}$ & none & \\
\hline 17 & crock/jar? & $\begin{array}{l}\text { base sherds }(n=12) \text {, Bristol glaze interior and } \\
\text { exterior, flat base, 195-mm base diameter }\end{array}$ & none & \\
\hline 18 & mixing bowl & $\begin{array}{l}\text { ca. } 20 \% \text { reconstructed ( } 29 \text { sherds), Bristol glaze } \\
\text { interior and exterior with raised exterior geometric } \\
\text { design, } 152 \mathrm{~mm} \text { tall, } 290-\mathrm{mm} \text { rim diameter }\end{array}$ & none & \\
\hline 20 & small bowl? & $\begin{array}{l}\text { base and body sherds }(n=3) \text {, Bristol glaze interior } \\
\text { and exterior with raised floral decoration and blue } \\
\text { paint on exterior }\end{array}$ & none & \\
\hline 22 & wide-mouth jar & $\begin{array}{l}\text { ca. } 70 \% \text { reconstructed ( } 53 \text { sherds), large jar with } \\
\text { lugs for wire bail handle, Bristol glaze interior and } \\
\text { exterior with raised geometric and floral design and } \\
\text { blue paint on exterior, } 205 \mathrm{~mm} \text { tall, } 215 \text {-mm } \\
\text { maximum body diameter, } 195 \text {-mm rim diameter; } \\
\text { roughly similar jar form with wire handle is } \\
\text { illustrated in Greer (1981:92) }\end{array}$ & none & $\cdot$ \\
\hline
\end{tabular}




\begin{tabular}{|c|c|c|c|}
\hline \multicolumn{4}{|c|}{ Table 45, continued } \\
\hline Vessel No. & Vessel Form & Vessel Description & Diagnostic Markings \\
\hline 23 & crock/jar? & $\begin{array}{l}\text { base sherds only }(\mathrm{n}=8) \text {, Bristol glaze interior and } \\
\text { exterior, } 195-\mathrm{mm} \text { base diameter }\end{array}$ & none \\
\hline 24 & crock/jar? & $\begin{array}{l}\text { base sherds only }(n=7) \text {, Bristol glaze interior and } \\
\text { exterior, } 200-\mathrm{mm} \text { base diameter }\end{array}$ & none \\
\hline 26 & crock/jar? & $\begin{array}{l}\text { base and body sherds }(\mathrm{n}=7) \text {, Albany slip interior } \\
\text { and exterior, } 150-\mathrm{mm} \text { base diameter }\end{array}$ & none \\
\hline 31 & bowl & $\begin{array}{l}\text { base and body sherds }(n=5) \text {, gray Bristol glaze } \\
\text { interior and exterior, } 115-\mathrm{mm} \text { footring diameter }\end{array}$ & none \\
\hline 32 & crock/jar? & $\begin{array}{l}\text { base sherds }(n=5) \text {, tan Bristol glaze interior and } \\
\text { exterior, } 240-\text { mm base diameter }\end{array}$ & none \\
\hline \multicolumn{4}{|c|}{ Whiteware } \\
\hline 3 & cup with handle & $\begin{array}{l}\text { ca. } 80 \% \text { reconstructed ( } 26 \text { sherds), green floral } \\
\text { transfer print with hand-painted gold-gilded accents } \\
\text { covers entire exterior and one portion of the interior, } \\
\text { raised decoration along base and rim, scalloped- } \\
\text { edge footring, } 62 \mathrm{~mm} \text { tall, } 92-\mathrm{mm} \text { rim diameter; } \\
\text { same transfer design as Vessels } 10 \text { and } 27\end{array}$ & "22" on base \\
\hline 5 & $\begin{array}{l}\text { unknown } \\
\text { (pitcher?) }\end{array}$ & $\begin{array}{l}\text { handle fragments only }(n=2) \text {, raised design, } \\
\text { unpainted, from large vessel }\end{array}$ & none \\
\hline 6 & $\begin{array}{l}\text { unknown large } \\
\text { vessel (pitcher?) }\end{array}$ & $\begin{array}{l}\text { rim and body sherds only }(n=6) \text {, undecorated, } \\
\text { scalloped rim }\end{array}$ & none \\
\hline 7 & plate & $\begin{array}{l}\text { rim sherd only, raised floral/scroll/beaded design, } \\
\text { unpainted }\end{array}$ & none \\
\hline 8 & bowl & rim sherd only, raised floral design, unpainted & none \\
\hline 10 & plate & $\begin{array}{l}\text { rim sherds }(n=3) \text {, scalloped edge with same green } \\
\text { floral transfer print as Vessels } 3 \text { and } 27\end{array}$ & none \\
\hline 12 & $\begin{array}{l}\text { unknown (cup or } \\
\text { vase?) }\end{array}$ & $\begin{array}{l}\text { base fragment only, undecorated, scalloped-edge } \\
\text { footring, 64-mm base diameter }\end{array}$ & $\begin{array}{l}\text { Royal Firenze China and } \\
\text { crown }\end{array}$ \\
\hline 13 & soup bowl & $\begin{array}{l}\text { ca. } 30 \% \text { reconstructed ( } 26 \text { sherds), raised scroll/ } \\
\text { floral decoration along scalloped rim, unpainted, } \\
43 \mathrm{~mm} \text { tall, } 241-\mathrm{mm} \text { diameter; similar form is } \\
\text { illustrated in Ketchum (1983:201) }\end{array}$ & Taylor, Smith \& Taylor \\
\hline 15 & large bowl & base sherds only $(n=2)$, undecorated & $\begin{array}{l}\text { Royal Coat of Arms, } \\
\text { possibly Johnson Bros. } \\
\text { Ltd., England }\end{array}$ \\
\hline 19 & sugar bowl & $\begin{array}{l}\text { ca. } 30 \% \text { reconstructed ( } 27 \text { sherds), double handles, } \\
\text { raised nonpainted fluted decoration, scalloped-edge } \\
\text { rim and footring, } 160-\mathrm{mm} \text { maximum body diameter, }\end{array}$ & $\begin{array}{l}\text { Johnson Bros. Ltd., } \\
\text { England }\end{array}$ \\
\hline
\end{tabular}




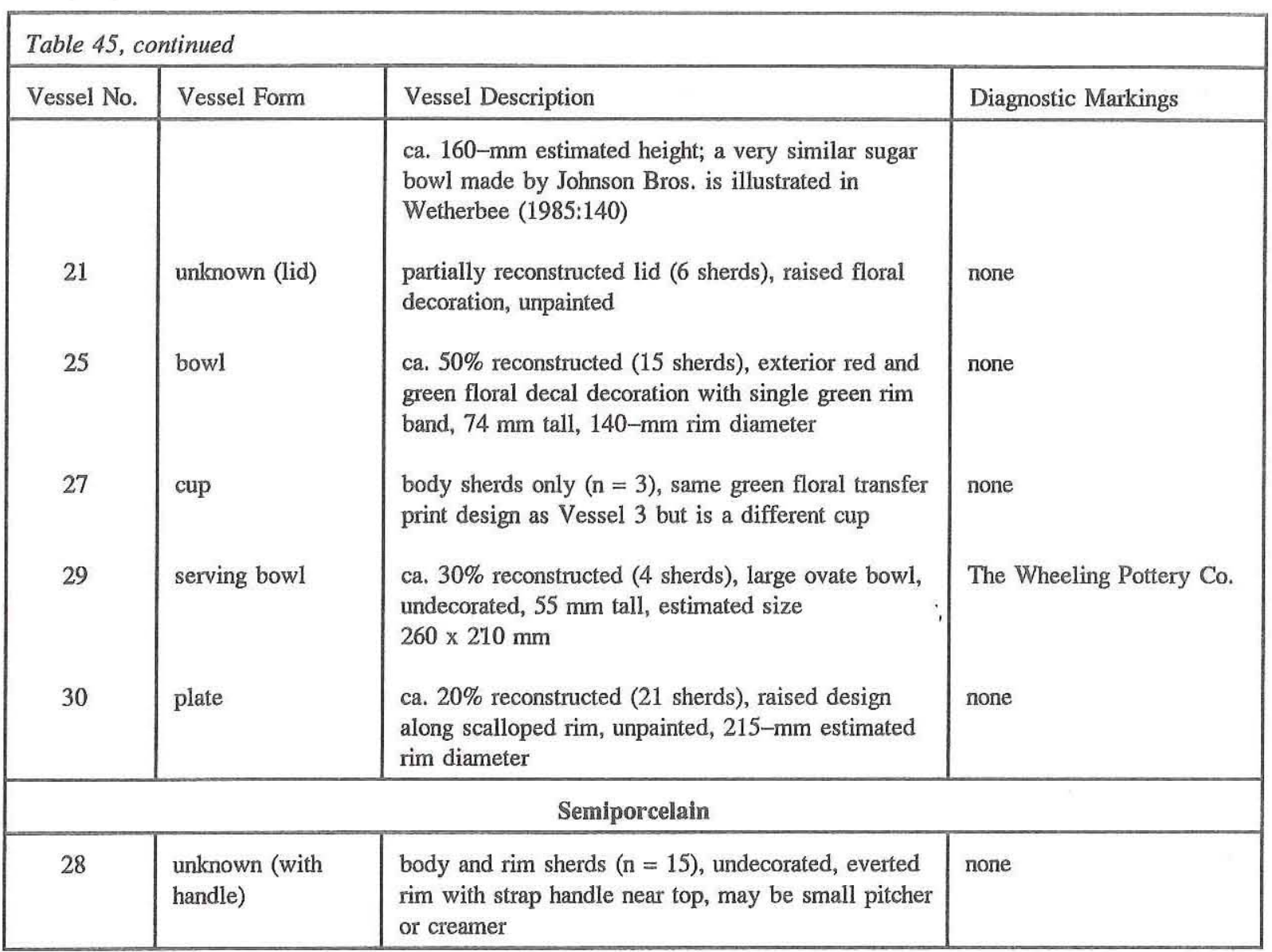

use of Albany slip (Vessels 9, 14, and 26) is generally earlier than the use of Bristol glazes, which became popular after 1890 . The combination of Albany and Bristol decorations (Vessels 4 and 16) was common until 1915 when Albany slips were dropped in favor of Bristol glaze inside and out (Vessels 1, 2, 11, 17, 23, 24, and 25) (Greer 1981:263-264). Thus, it appears likely that most of the stonewares were manufactured no earlier than the 1890s and dominantly after ca. 1915.

A single vessel of semiporcelain (3\% of all vessels) is undecorated and represents a rather plain utilitarian ware, but whitewares account for $47 \%$ of the identifiable vessels and exhibit a variety of decorative techniques. Of the 15 semiporcelain vessels, only 4 (Vessels $6,12,15$ and 29) exhibit no decorations, and only 1 of these (Vessel 29) is complete enough to be certain that it is undecorated. Seven vessels have unpainted raised decorations (Vessels 5, 7, 8, 13, 19, 21, and 30), three have transfer-printed decorations (Vessels 3, 10, and 27), and one has a hand-painted decal decoration (Vessel 25).

Two cups and a plate (Vessels 3, 10, and 27) have the same green floral transfer-printed pattem, beaded and scroll molding, and hand-painted gold accents and represent the same set of dishes. Only the reconstructed cup (Vessel 3) has a complete base; it does not have a maker's mark, but a stamped number "22" in green underglaze could be a mold size or pattern identification number. The maker of these transfer-printed wares is unknown, but the gold accents suggest that they represent a more expensive ware than any of the others in the assemblage.

Five whiteware vessels have makers' marks. A small cup or vase base (Vessel 12) has a green stamped mark with "ROYAL" above a crown and "FIRENZE" "CHINA" below it (Figure 72b). The name Firenze probably refers to Florence, Italy (Kovel and Kovel 1986:234), but this mark has not been identified. It could indicate that the ware was made in Florence prior to 1891, when the McKinley 


\begin{tabular}{|l|c|c|c|c|}
\hline \multicolumn{5}{|c|}{ TABLE 46 } \\
\multicolumn{5}{|c|}{ TRASH FILL ASSEMBLAGE, 41GR474 } \\
\hline Vessel Forms* & Stoneware & Whiteware & Semiporcelain & Totals \\
\hline Butter chum & 2 & - & - & 2 \\
Jug & 1 & - & - & 1 \\
Unidentified form & 6 & - & - & 6 \\
$\quad$ crock/jar) & 3 & - & - & 3 \\
Wide-mouth crock/jar & 2 & - & - & 2 \\
Mixing bowl & - & 1 & - & 1 \\
Serving bowl & - & 1 & - & 1 \\
Soup bowl & - & 1 & - & 1 \\
Sugar bowl & 2 & 3 & - & 5 \\
Unspecified bowl & - & 3 & - & 3 \\
Plate & - & 2 & - & 2 \\
Cup & - & 4 & 1 & 5 \\
\hline Unidentified & 16 & 15 & 1 & 32 \\
\hline Totals: & & & \\
\hline *Vessel form terminology follows Greer (1981) and Ketchum (1983) & \\
\hline
\end{tabular}

mark postdates the McKinley Tariff Act of 1891 because some companies used England in their marks as early as 1880 (Kovel and Kovel 1986:229). These vessels may come from the same set of dishes, and Johnson Bros. wares are quite prominent in the 1895 Montgomery Ward and Co. catalog (Dover 1969:526-530).

A partial maker's mark on a large oval serving bowl (Vessel 29) is that of the Wheeling Pottery Company (Figure 72f) and may have been used on whiteware and semiporcelain ca. 1896 (Kovel and Kovel 1986:70-C). This particular mark, illustrated and described by Lehner (1988:517, no. 4), must date between 1879

Tariff Act began requiring imported items to be marked with their country of origin. Alternatively, and perhaps more likely, Firenze may only be a pattern name used by a particular manufacturer. The other names are not particularly diagnostic, but Kovel and Kovel (1986:233) suggest that "ROYAL" was used most commonly in English marks. Notably, a mark used between 1912 and 1941 on wares made by E. Hughes and Company of Fenton, England (Godden 1964:338-339) is similar in that it depicts a crown and includes the words "ROYAL" and "CHINA" along with other elements (i.e., a globe and "STAFFORDSHIRE").

A soup bowl (Vessel 13) has a partial mark identified as one of the marks used by the Taylor, Smith and Taylor Company (Figure 72c). This mark is illustrated in Gates and Ormerod (1982:Figure 245c) and was used on semivitreous wares from 1901 to 1930 . This vessel was manufactured after 1901 since the company name was not changed to Smith, Taylor \& Smith until that year (Gates and Ormerod 1982:267-268).

A large bowl and a sugar bowl (Vessels 15 and 19) have similar makers' marks (Figure $72 d, e$ ). Although both marks are incomplete, the mark on the sugar bowl is identified as one used on ironstone wares by Johnson Bros. Ltd. of England from 18831913 (Kovel and Kovel 1986:12-I). The use of the country name does not necessarily indicate that the (the year that the Wheeling Pottery Company was founded) and 1903 (the year that the company changed its name to the Wheeling Potteries Company). DeBolt (1988:77) dates this mark from 1896 to ca. 1903 . It identifies this ware as "WHITE GRANITE" (ironstone) and also notes that it was "MADE IN AMERICA."

\section{Glass}

The glass (Table 47) consists of 948 specimens, of which 209 (22\%) are glassware and food containers or medicine and liquor containers assigned to the kitchen group, 19 (2\%) are window glass (with thicknesses varying from $0.09-0.137$ inches) assigned to the architectural category, and 18 (2\%) are snuff bottles and fragments assigned to the tobacco group. The largest percentage of the glass, however, is assigned to the unidentified function category $(74 \%)$. Fifty-nine different vessels are recognized in these groups and account for $24 \%(\mathrm{n}=$ 228) of the 948 specimens (Table 48). Only four of the vessels are complete (one of these was completely reconstructed from two fragments), some are partially reconstructed from fragments, and others are represented by single diagnostic fragments (i.e., a mouth or base fragment).

Not all of the glass vessels exhibit diagnostic attributes, but $29 \%(\mathrm{n}=17)$ are hand finished and 


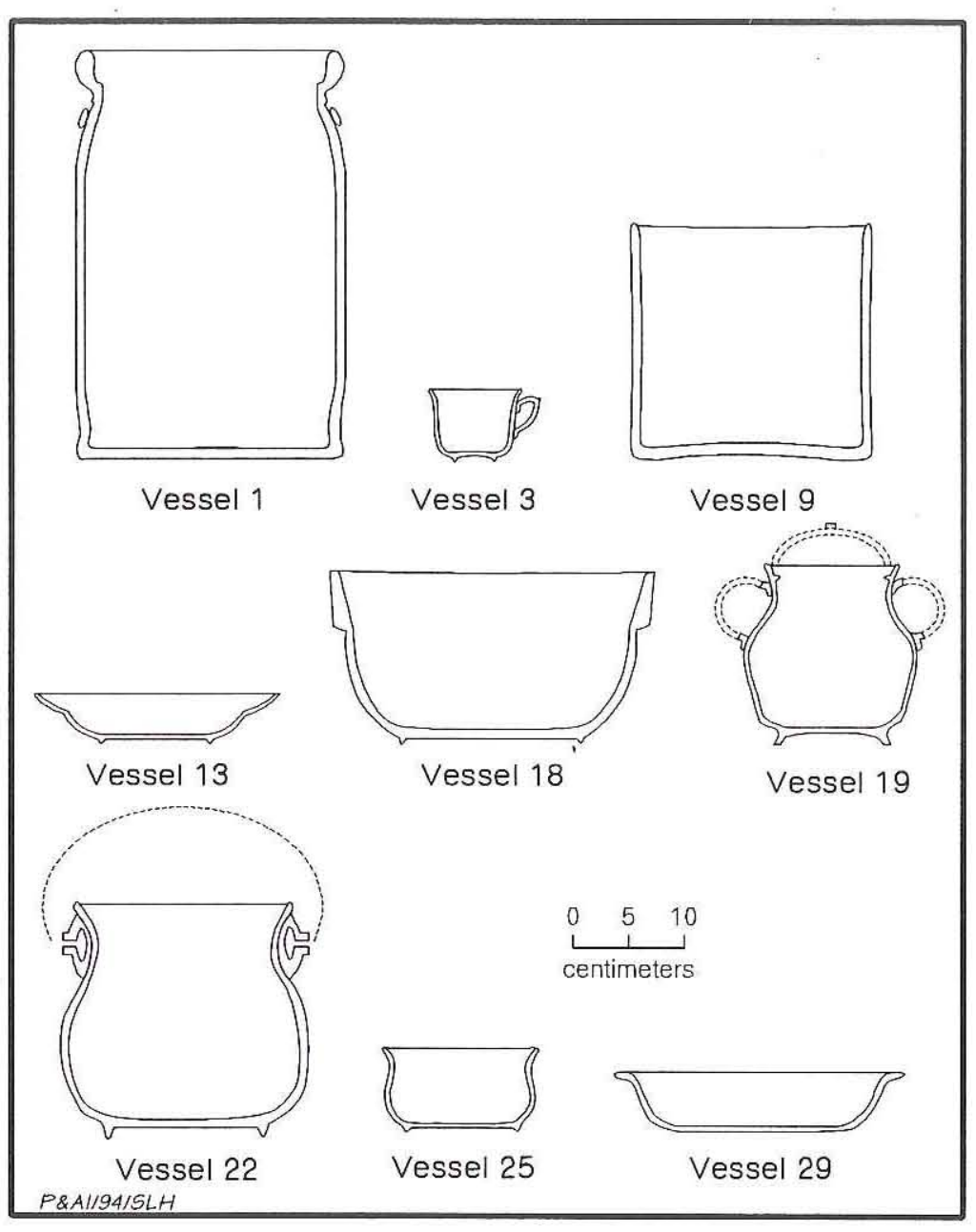

Figure 71. Ceramic vessel forms represented in the trash fill assemblage, $41 \mathrm{GR} 474$.

$22 \%(n=13)$ are completely machine made (Table 49). A fully automatic bottle machine was patented in 1903 , and by 1920 all commercial glass production was automated (Lorrain 1969:43). Given the nearly equal percentages of hand-finishing and machinemade attributes, the glass assemblage appears to span this transition period.

Only one diagnostic marking was found on a glass food container in the kitchen category. Incomplete markings on a rectangular clear glass panel bottle (Vessel 23) consist of an eagle emblem and "TRADE MARK" on the front panel, ". . . ARDT EAGLE" on one side panel, "CHILI POWDER" on the other side panel, and "DESIGN" "PATENTED" "8" on its base. This is Gebhardt Eagle brand chili powder. Similarly embossed panel bottle fragments have been found in an early twentieth-century dump in San Antonio (Clark
1984:44, Table 3), and two specimens found at the McKinney Homestead in Travis County have Illinois Glass Company maker's marks that date them to 1916-1929 (McEachern and Ralph 1980a:52, 1980b:154). Gebhardt Eagle brand chili powder is still made today, and the labels of the modern rectangular plastic containers (similar to the old glass panel bottles) indicate that Gebhardt has been in operation since 1896 . The Gebhardt Products Business Manager for Hunt Wesson, Inc. in Fullerton, California, notes that while the trademark was registered in 1896, Gebhardt Chili Powder was first produced in San Antonio around 1890 but that these bottle fragments probably date to the 1920s (Jeff Fried, personal communication 1993).

Several medicine vessels have diagnostic markings. Two (Vessels 1 and 18) are opaque white glass, screw-top jars with "MENTHOLATUM" "REG" "TRADE" "MARK" on their bases. A large brown glass panel bottle (Vessel 22) has recessed panels with "FOLEY'S KIDNEY \& BLADDER REMEDY" on the front panel, "CHICAGO, U.S.A." on a side panel, and "FOLEY \& CO" on the opposite side panel. The back panel is flat and may have had a paper label. According to Fike (1987:59), Foley and Company was established in 1890 and operated at least through the 1950s, but it is not clear when the "Kidney and Bladder Remedy" name was used. The final specimen is a completely reconstructed medicine bottle (Vessel 32) with the number "12" on its base.

In the tobacco group, a complete brown glass snuff jar (Vessel 55) has a single dot and the number "1" on its base. While the dots on the bottom of snuff jars are often thought to indicate the strength of the tobacco (e.g., Ferraro and Ferraro 1964:66), other researchers (e.g., Boice 1980:168; McKearin and Wilson 1978:261) suggest that the dots were not strength indicators but were some type of maker's mark. 

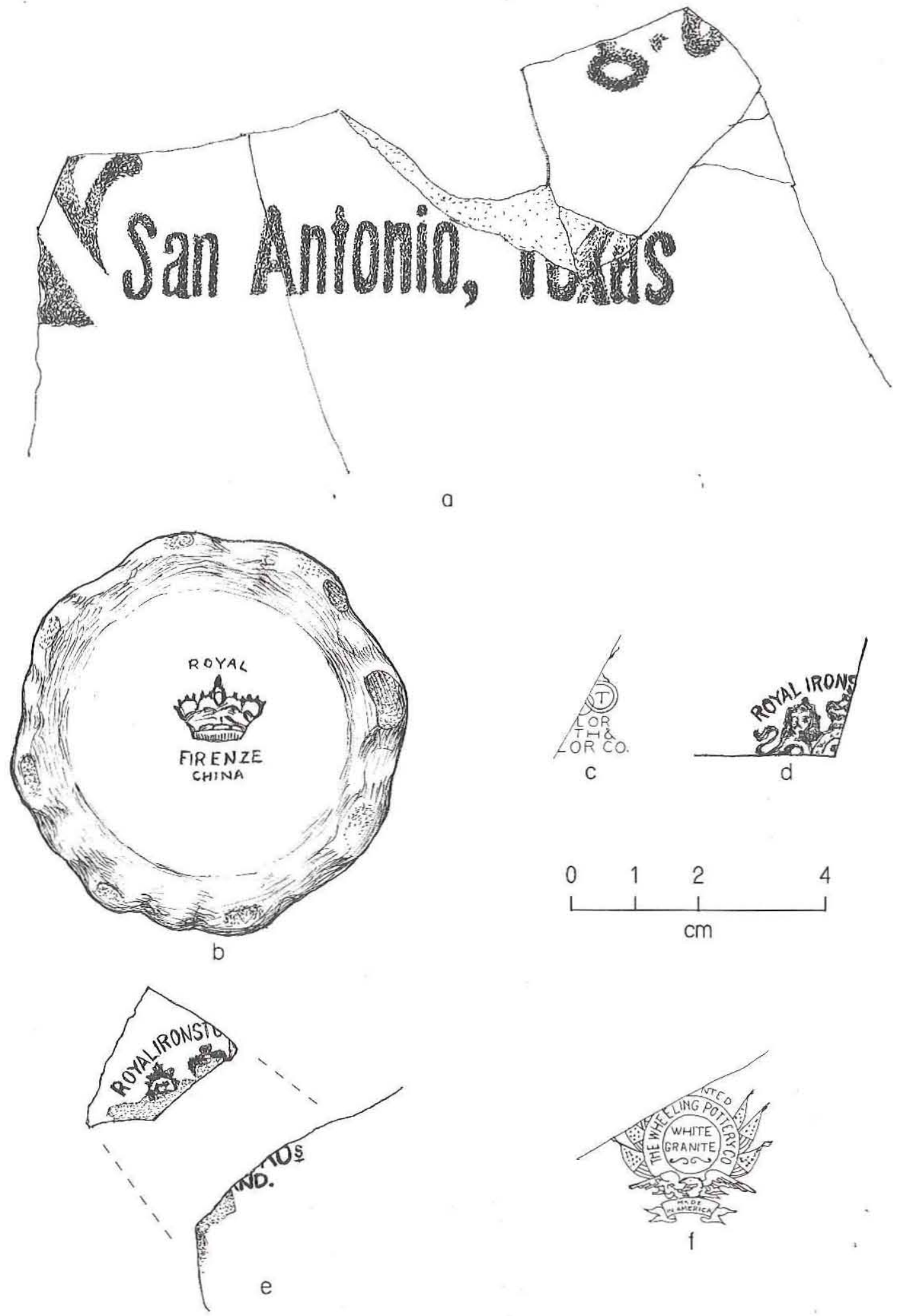

Figure 72. Diagnostic ceramic marks in the trash fill assemblage, 41GR474. (a) Unidentified mark on Vessel 9; (b) unidentified mark on Vessel 12; (c) Taylor, Smith, and Taylor Co. mark on Vessel 13; (d) Royal Coat of Arms mark (possibly Johnson Bros. Ltd.) on Vessel 15; (e) Johnson Bros. Ltd. mark on Vessel 19; (f) Wheeling Pottery Co. mark on Vessel 29. 


\begin{tabular}{|c|c|c|}
\hline \multicolumn{2}{|r|}{ TABLE 47} & \\
\hline $\begin{array}{l}\text { Functional } \\
\text { Category }\end{array}$ & Artifact Description* & No. \\
\hline $\begin{array}{l}\text { Kitchen } \\
(n=209)\end{array}$ & $\begin{array}{l}\text { vessel fragments (glassware and food container) } \\
\text { nonvessel fragments (glassware and food container) } \\
\text { complete medicine jar } \\
\text { vessel fragments (medicine and liquor) } \\
\text { nonvessel fragments (medicine and liquor) }\end{array}$ & $\begin{array}{r}98 \\
45 \\
1 \\
52 \\
13\end{array}$ \\
\hline $\begin{array}{l}\text { Architecture } \\
(\mathrm{n}=19)\end{array}$ & window glass fragments & 19 \\
\hline $\begin{array}{l}\text { Tobacco } \\
(n=18)\end{array}$ & $\begin{array}{l}\text { complete snuff bottle } \\
\text { vessel fragments } \\
\text { nonvessel fragments }\end{array}$ & $\begin{array}{l}1 \\
8 \\
9\end{array}$ \\
\hline $\begin{array}{l}\text { Activities } \\
(n=1)\end{array}$ & complete poison bottle & 1 \\
\hline $\begin{array}{l}\text { Unidentified } \\
(\mathrm{n}=701)\end{array}$ & $\begin{array}{l}\text { vessel fragments } \\
\text { nonvessel fragments }\end{array}$ & $\begin{array}{r}67 \\
634 \\
\end{array}$ \\
\hline Total: & & 948 \\
\hline
\end{tabular}

with 1,2 , or 3 dots; several unidentified bottle bases with various numbers and one with a letter (i.e., "3," "5," "8," "10," "13," and "V"); an unidentified bottle base with the number "2" and a diamond enclosing the number "273"; a panel bottle fragment with ". . BOLE . . ." "(REGISTERED)" ". . . E . . ."; and the incomplete patent markings "PAT . .." "DEC.2 . . ." "JULY.17 . .." "H 2" on a round bottle base. Three marks on nonvessel glass fragments were identifiable. A clear glass panel bottle fragment with ". . . GEBHARD . . ." is probably a chili powder container (kitchen group) of the Gebhardt Eagle brand mentioned above. It could be from Vessel 23 or from a different bottle. A clear glass rectangular panel bottle base (unidentified group) has a diamond encircling the letter "I" above the word "LYRIC." The diamond "I" mark was used by

A single complete bottle (Vessel 21) in the activities category has diagnostic markings. It is a small brown glass bottle with "POISON" on its side and "67" and " $F$ " on its base. The specific type of poison that this bottle contained is not known. Deviating slightly from South's (1977:Table 4) classification which groups all glass in the kitchen category, it is more appropriate to classify this specimen in the activities group than the kitchen group.

One panel bottle (Vessel 16) in the unidentified function category has a base mark consisting of the number "34" inside a diamond and "TRADE MARK" with a horizontal line through it on one panel. An incomplete word, "W . . ." on another panel, may have identified the contents. The diamond mark was used by several glass manufacturing companies and could be a variation of marks used by the Illinois Glass Company or the Diamond Glass Company (Toulouse 1971:52, 264-268, 550-552).

Other marks noted on nonvessel glass (i.e., glass fragments that could not be assigned to specific vessels) include snuff bottle bases (tobacco group) the Illinois Glass Company of Alton, Illinois, from 1916-1929 (Toulouse 1971:264).

Several fruit jar body fragments (kitchen group) exhibit the label "K . . $\mathrm{r}$ " "Self Sealing" in script lettering and "TRADEM . . ." in block letters. This mark was used on canning jars manufactured by the Kerr Glass Manufacturing Company (Toulouse 1971:306-307). The script "Kerr" name was used after 1912, and this particular "Self Sealing" trademark was used on jars with a distinctive twopiece lid (a screw top band and a heat-softening lid ring) that was patented in 1915.

\section{Bone}

The bone assemblage (Table 50) consists of 142 specimens that apparently represent discarded food refuse. Of these, $8 \%(n=11)$ exhibit evidence of burning and $1 \%(n=2)$ have butchering marks (see Appendix C). All of the identifiable species probably were utilized as food resources and include both wild (antelope, deer, and squirrel) and domestic (chicken and pig) animals. In addition to the data recovery 


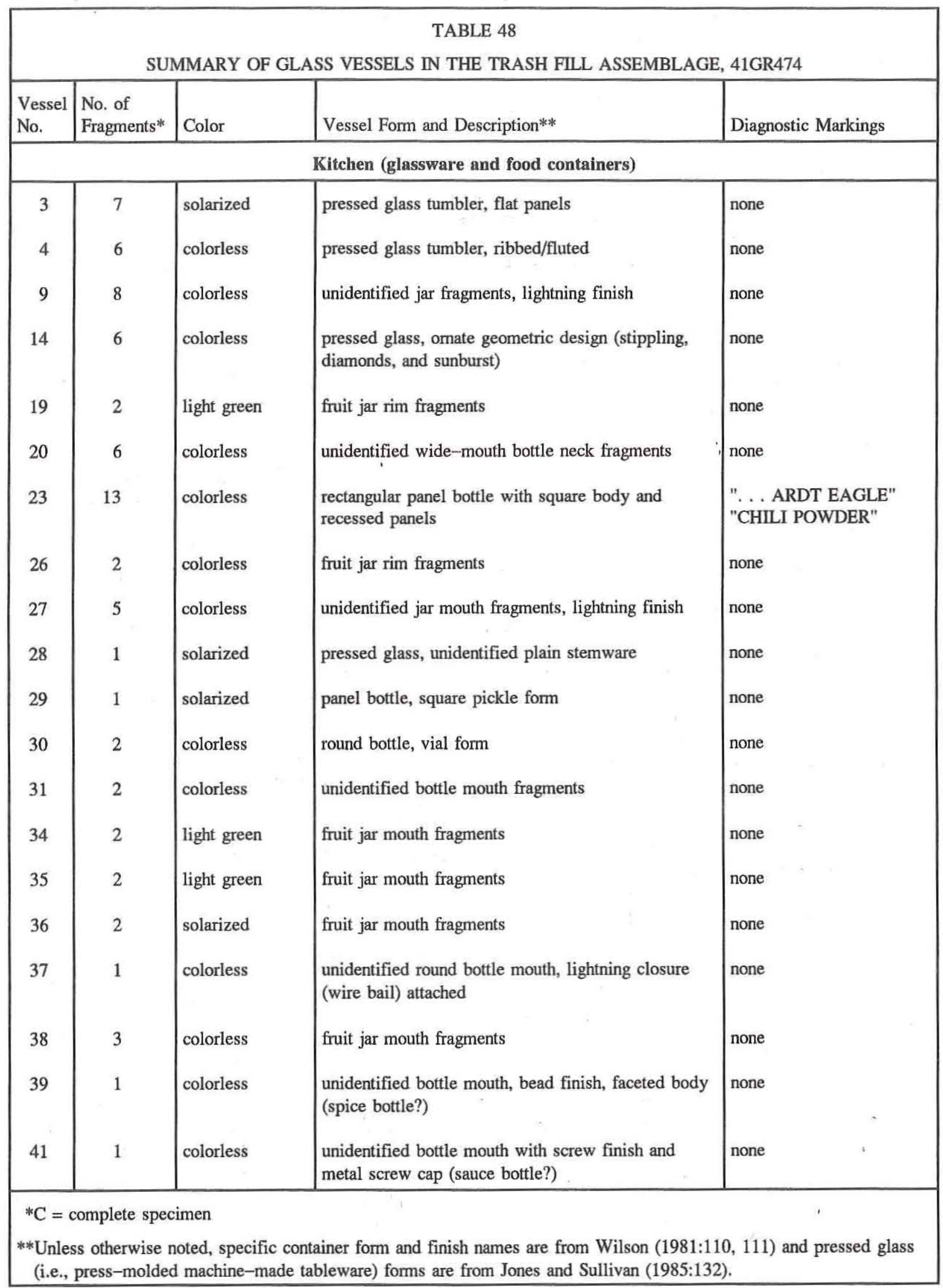




\begin{tabular}{|c|c|c|c|c|}
\hline \multicolumn{5}{|c|}{ Table 48 , continued } \\
\hline $\begin{array}{l}\text { Vessel } \\
\text { No. }\end{array}$ & $\begin{array}{l}\text { No. of } \\
\text { Fragments }\end{array}$ & Color & Vessel Form and Description & Diagnostic Markings \\
\hline 43 & 1 & colorless & pressed glass pitcher, faceted body & none \\
\hline 44 & 1 & colorless & $\begin{array}{l}\text { pressed glass, unidentified form with handle, } \\
\text { starburst pattem on base (drinking mug?) }\end{array}$ & none \\
\hline 45 & 1 & solarized & $\begin{array}{l}\text { pressed glass, unidentified form, faceted lid } \\
\text { fragment with knob handle }\end{array}$ & none \\
\hline 46 & 4 & colorless & pressed glass tumbler, faceted & none \\
\hline 47 & 2 & solarized & pressed glass tumbler, fluted & none \\
\hline 48 & 4 & colorless & pressed glass tumbler, plain round body & none \\
\hline 49 & 1 & solarized & pressed glass tumbler, ribbed/fluted & none \\
\hline 50 & 1 & colorless & pressed glass tumbler, faceted & none \\
\hline 51 & 1 & solarized & pressed glass tumbler, ribbed/fluted & none \\
\hline 52 & 1 & purple & $\begin{array}{l}\text { pressed glass tumbler, plain round body with } \\
\text { starburst pattern on base }\end{array}$ & none \\
\hline 53 & 6 & solarized & pressed glass, unidentified stemware base, plain & none \\
\hline 57 & 2 & solarized & pressed glass, unidentified stemware base, plain & none \\
\hline \multicolumn{5}{|c|}{ Kitchen (liquor and medicinal containers) } \\
\hline 1 & 1 & opaque white & small screw-top medicine jar & $\begin{array}{l}\text { "MENTHOLATUM } \\
\text { REG TRADE MARK" }\end{array}$ \\
\hline 2 & $\mathrm{C}$ & opaque white & $\begin{array}{l}\text { small screw-top medicine jar; same size and style } \\
\text { as Vessel } 1 \text { but no markings }\end{array}$ & none \\
\hline 8 & 8 & solarized & $\begin{array}{l}\text { 3-ounce panel bottle, single-bead patent medicine } \\
\text { finish, rectangular shape }\end{array}$ & ounce marks on sides \\
\hline 11 & 3 & solarized & liquor bottle neck with brandy finish & none \\
\hline 12 & 18 & brown & liquor bottle base and body fragments & none \\
\hline 13 & 6 & black/dark green & wine bottle base fragments with kickup & none \\
\hline 18 & 1 & opaque white & small screw-top medicine jar base & $\begin{array}{l}\text { "MENTHOLATUM } \\
\text { REG TRADE MARK" }\end{array}$ \\
\hline 22 & 9 & brown & $\begin{array}{l}\text { large panel bottle fragments, rectangular with } \\
\text { three recessed panels }\end{array}$ & $\begin{array}{l}\text { "FOLEY'S KIDNEY \& } \\
\text { BLADDER REMEDY" } \\
\text { etc. }\end{array}$ \\
\hline
\end{tabular}




\begin{tabular}{|c|c|c|c|c|}
\hline \multicolumn{5}{|c|}{ Table 48, continued } \\
\hline $\begin{array}{l}\text { Vessel } \\
\text { No. }\end{array}$ & $\begin{array}{l}\text { No. of } \\
\text { Fragments }\end{array}$ & Color & Vessel Form and Description & Diagnostic Markings \\
\hline 24 & 1 & brown & liquor bottle neck with crown finish & none \\
\hline 32 & 2 & colorless & $\begin{array}{l}\text { small medicine bottle, pomade/morphine shape, } \\
\text { single-bead finish (completely reconstructed) }\end{array}$ & "12" on base \\
\hline 42 & 1 & brown & liquor bottle neck with brandy finish & none \\
\hline 56 & 2 & brown & liquor bottle neck with brandy finish & none \\
\hline \multicolumn{5}{|c|}{ Tobacco } \\
\hline 5 & 2 & brown & snuff jar mouth fragments & none \\
\hline 6 & 2 & brown & snuff jar mouth fragments & none \\
\hline 7 & 2 & brown & snuff jar, mouth fragments & none \\
\hline 17 & 2 & brown & snuff jar mouth fragments & none \\
\hline 55 & $\mathrm{C}$ & brown & snuff jar & "1" and single dot \\
\hline \multicolumn{5}{|c|}{ Activities } \\
\hline 21 & $\mathrm{C}$ & brown & $\begin{array}{l}\text { small poison bottle, spherical triangle shape (Fike } \\
\text { 1987:Figure 3.27), prescription finish }\end{array}$ & $\begin{array}{l}\text { "POISON" } \\
\text { "67" "F" }\end{array}$ \\
\hline \multicolumn{5}{|c|}{ Unidentified } \\
\hline 10 & 28 & light green & $\begin{array}{l}\text { small panel bottle with recessed panels, stacked- } \\
\text { ring neck finish (Fike 1987:Figure 2.23) }\end{array}$ & none \\
\hline 15 & 2 & light green & round container base and body fragments & none \\
\hline 16 & 10 & colorless & $\begin{array}{l}\text { panel bottle, rectangular with one recessed panel } \\
\text { with partial label }\end{array}$ & $\begin{array}{l}\text { "34" inside diamond and } \\
\text { "TRADE MARK" }\end{array}$ \\
\hline 25 & 1 & solarized & small bottle neck, prescription finish & none \\
\hline 33 & 1 & colorless & unidentified bottle neck & none \\
\hline 40 & 1 & colorless & unidentified jar neck fragment & none \\
\hline 54 & 1 & solarized & $\begin{array}{l}\text { panel bottle neck, prescription finish, ball-neck } \\
\text { panel form }\end{array}$ & none \\
\hline 58 & 22 & colorless & unidentified oval bottle, prescription finish & none \\
\hline 59 & 1 & colorless & $\begin{array}{l}\text { unidentified panel bottle, Philadelphia oval, } \\
\text { double-bead finish }\end{array}$ & none \\
\hline
\end{tabular}




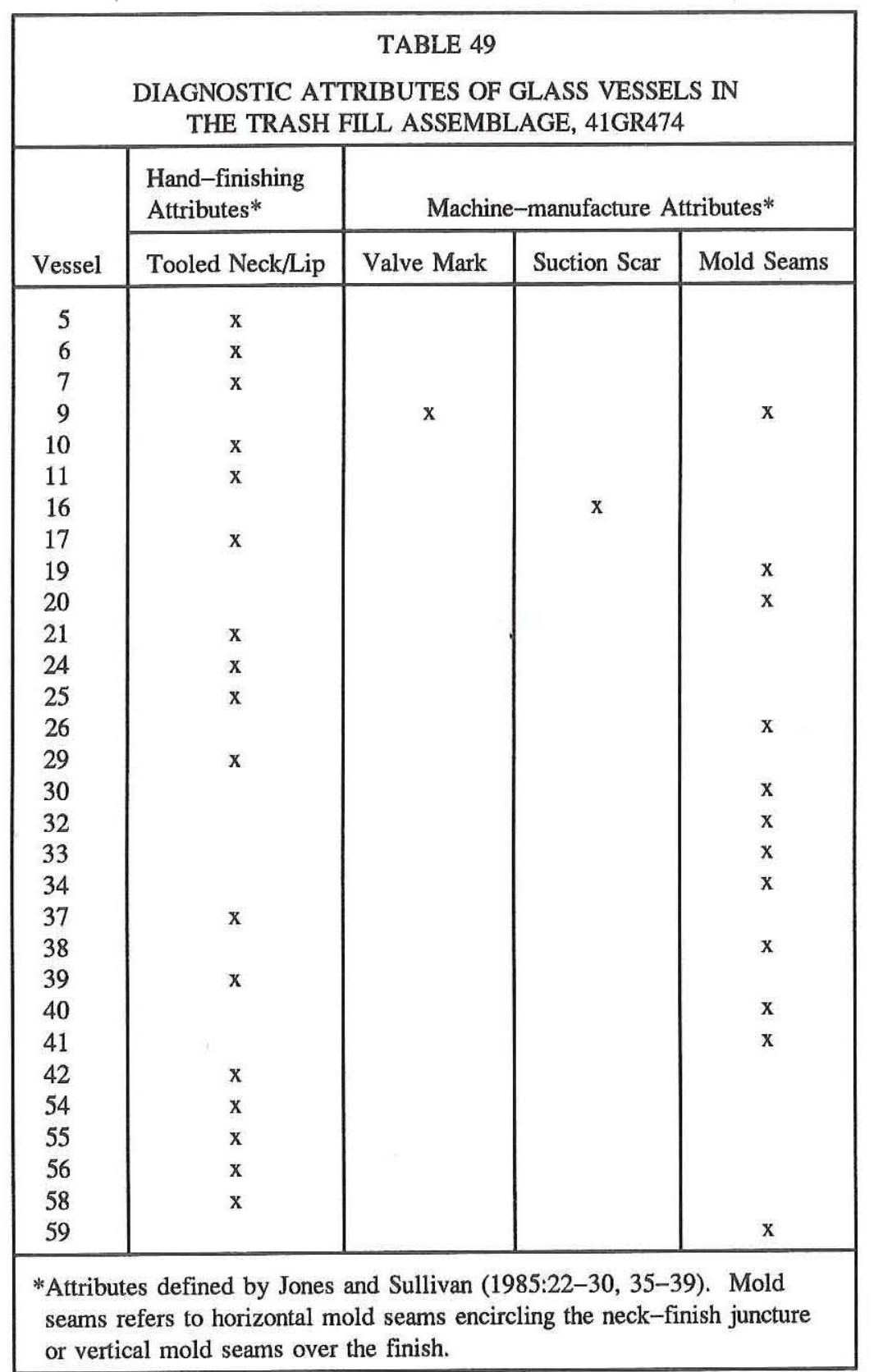

undiagnostic, although three specimens exhibit attributes of mass-produced footwear (Anderson 1968). The boot sole is completely machine stitched, and the heel is machine nailed; one shoe sole is cemented, while the other is machine stitched with a nailed heel. Complete machine stitching became common after 1862 , but cementing of shoe soles did not become common until after 1926 (Anderson 1968:64).

The shell artifacts are limited to buttons, while the other materials include a coal lump (perhaps indicating the use of coal fuel), a $2 \frac{1}{4}$-inch-thick brick fragment, and two battery carbon rods. The latter are from large dry-cell batteries such as those illustrated in the 1908 Sears, Roebuck and Company catalog (Schroeder 1971:205). One is a 3/4-inch-diameter smooth rod at least 4 inches long, while the other is a 1-inch-diameter fluted rod (with six deep longitudinal channels) at least $33 / 4$ inches long. Both specimens are similar to the round and fluted dry-cell cores found in a trash-filled cellar (deposited between 1912 and 1930) at a turn-of-the-century farmstead in Arkansas (StewartAbernathy 1986:96, Figure 64d and e). The final other material class - stone - includes one quartzite and three chert flakes. These specimens are probably specimens, 70 bones recovered during the 1988 testing include specimens identified as pheasant, cow, and rabbit (Freeman and Boyd 1990:152).

\section{Other Materials}

Other materials in the assemblage are rubber, leather, shell, and other (i.e., coal, brick, carbon, and stone) (see Table 50). A single rubber artifact is a bottle stopper for a lightning-type closure. Leather boot and shoe fragments are generally small and from the prehistoric component at the site, and their occurrence in the dugout fill is likely to be incidental.

\section{SUMMARY AND CONCLUSIONS}

\section{Site Chronology}

The archival and archeological evidence suggests the following sequence of events pertaining to the Justice dugout: (1) construction, occupation, and initial abandonment; (2) reuse and final 


\begin{tabular}{|c|c|c|c|}
\hline BON & AND OTHER M & $\begin{array}{l}\text { TABLE } 50 \\
\text { ALS IN THE TRASH FILL ASSEMBLAGE, }\end{array}$ & \\
\hline Functional Category & Material Group & Artifact Description & No. of Specimens \\
\hline Bone $(n=142)$ & Bone & $\begin{array}{l}\text { antelope } \\
\text { chicken } \\
\text { deer } \\
\text { large bird } \\
\text { pig } \\
\text { squirrel } \\
\text { unidentified } \\
\text { unidentified mammal } \\
\text { unidentified medium/large mammal } \\
\text { unidentified medium/large ungulate }\end{array}$ & $\begin{array}{r}5 \\
18 \\
3 \\
5 \\
7 \\
1 \\
14 \\
1 \\
78 \\
10\end{array}$ \\
\hline Unidentified $(n=1)$ & Rubber & bottle stopper fragment & 1 \\
\hline Clothing $(\mathrm{n}=29)$ & Leather & $\begin{array}{l}\text { boot heel/sole, machine stitched } \\
\text { shoe sole, cemented } \\
\text { shoe heel/sole fragments, machine stitched } \\
\text { shoe upper fragment with eyelets } \\
\text { miscellaneous shoe sole/heel fragments }\end{array}$ & $\begin{array}{r}1 \\
1 \\
4 \\
1 \\
22\end{array}$ \\
\hline Unidentified $(n=1)$ & Leather & unidentified fragment & 1 \\
\hline Clothing $(\mathrm{n}=3)$ & Shell & $\begin{array}{l}\text { disk button, 2-hole } \\
\text { compound button with brass loop shank }\end{array}$ & $\begin{array}{l}2 \\
1\end{array}$ \\
\hline Kitchen $(n=1)$ & Other & coal lump (fuel) & 1 \\
\hline Architecture $(n=1)$ & Other & brick fragment & 1 \\
\hline Activities $(n=2)$ & Other & dry-cell battery carbon rod fragments & 2 \\
\hline Unidentified $(n=4)$ & Other & $\begin{array}{l}\text { chert flake } \\
\text { quartzite flake }\end{array}$ & $\begin{array}{l}3 \\
1\end{array}$ \\
\hline \multicolumn{3}{|l|}{ Total: } & 184 \\
\hline
\end{tabular}

abandonment; and (3) collapse and filling of the structure. The first phase began in late 1899 or early 1900 when the dugout was constructed. It was built by Jeff Justice, probably with help from his brother, W. B. Justice, and neighbors T. E. and T. J. Payne. Jeff probably moved his family into the dugout in 1900 , soon after it was constructed, and they began building a larger ranch house several years later. Jeff, his wife Mattie, and their eight children lived in the dugout until the ranch house was completed. The first period ended when the family moved out of the dugout and into the ranch house when its construction was completed in 1906 or 1907 (according to archival records).

At the time of initial abandonment, the dugout ceased to function as a dwelling, but material culture evidence (discussed in more detail below) suggests that the structure continued to be used by the Justices for other purposes for some time. The chronology of its reuse and final abandonment is not well documented, but these events probably occurred within a relatively short time. Quite likely, little or no effort was expended to maintain the dugout during its reuse, and it probably deteriorated rapidly. At some point, it would have become more of a hazard than a resource. The second phase ended when the dugout ceased to be used for any purpose.

The third phase began with the collapse of the roof and rock walls. The collapse may have occurred naturally due to deterioration or it may have been 
intentional. In either case, it may have occurred at or soon after the time of the dugout's final abandonment. How the dugout collapsed is not known, but an interpretation of intentional destruction has some merit. The dugout would have been about 7 years old at the time the family moved out, and allowing a few years for reuse, the structure probably would have needed major renovations by ca. 1910, if not earlier. At some point, it probably became impractical to maintain the structure because it was too great an investment of labor and because it was so far away from the house, barn, and corral area. At the time the structure was finally abandoned, it was probably unstable and it could have been left to fall on its own, or perhaps the logical choice may have been to salvage any useful materials (e.g., the door, roof beams, and windows, if any) and collapse the roof and walls to eliminate the safety hazard. It appears that some wall rocks may have been robbed and used to plug up the entryway, but this could have been done regardless of whether the structure was destroyed intentionally or deteriorated naturally.

Soon after the collapse of the superstructure, the dugout began to fill with wind-blown sands, forming a basin-shaped depression that held enough rainwater, at least temporarily, to form laminated deposits in its center. The depression then was used as a trash dump and was filled in with a considerable quantity of refuse generated by the Justice family while they were living in the ranch house.

There is little structural or stratigraphic evidence to suggest how long the dugout may have been reused or how quickly the natural and cultural filling might have occurred, but the material culture does provide some answers. The artifacts from the dugout are separated into two assemblages - one representing the occupation and/or reuse of the dugout and the other representing trash dumping in the depression. These assemblages provide temporal evidence in the form of manufacturing dates and dates of common usage that corroborate the site chronology outlined above.

The dugout assemblage does not contain many temporally sensitive artifacts, but those that can be dated are typical of the turn of the century. Many of the datable artifacts are firearms related and consist of 10 cartridge cases and 15 primers. The cartridge cases represented are .45-caliber Colt (developed in 1873), .40-.70-caliber Sharps Straight (developed in 1879), and .44-caliber Henry Flat (developed in 1860 and in use until ca. 1934), while the primers include
Boxer (developed in 1867 and still in use) and Berdan (developed in 1866 and used in the United States until the 1920s) types. In addition, a tobacco plug marked with the P. Lorillard Company name and a china button of the Prosser type are typical of the late nineteenth and early twentieth centuries. Unfortunately, none of these artifacts are extremely sensitive time indicators, and the remainder of the dugout materials have little or no chronological value.

The dugout assemblage is perhaps more temporally diagnostic by what is not represented than by what is represented. It contains no artifacts that definitely were manufactured after the turn of the century, and none of the artifacts are out of place for 1900 to ca. 1910. This is in sharp contrast to the trash fill assemblage, which is much more variable and contains numerous artifacts that probably were manufactured from the 1910 s to 1930 s.

The trash fill assemblage contains some of the same artifacts (i.e., .45-caliber Colt and .44-caliber Henry Flat cartridges and a Prosser-type button) as the dugout assemblage, but it also contains many other temporally sensitive items. While many holein-cap and hole-in-top cans (both common in the late nineteenth and early twentieth centuries) were identified because of their distinctive lead solder, the apparent absence of modern sanitary cans (which were used almost exclusively by ca. 1920) is probably due to deterioration and the inability to recognize them. Some of the large 5-pound baking powder cans are more datable. The datable specimens are stamped with a style of Calumet Baking Powder label that was used from 1916 to the late 1920s and a style of KC Baking Powder label that was used from 1925-1950. The "Jack . . ." baking powder label has not been specifically identified. An electrical socket fuse cannot be dated absolutely but does indicate that at least some of the trash was deposited after electricity had come to Garza County, probably no earlier than the 1930s.

Ceramics that provide chronological evidence include four partial vessels with identifiable makers' marks. One is identified as the mark used on ironstone by Johnson Bros. Ltd. from 1883-1913, a distinctive mark of the Taylor, Smith, and Taylor Company dates from 1901-1930, and a mark of the Wheeling Pottery Company dates from 1896-1903. In addition, several stoneware vessels have glaze combinations that are somewhat time sensitive. While the use of Albany and Bristol glaze together on vessels was common from the late 1800 s to 1915 , the 
exclusive use of Bristol glaze, evident on seven vessels in the trash fill assemblage, was much more common after 1915.

Temporally diagnostic glass markings include a distinctive mark used from 1916-1929 by the Illinois Glass Company and a Foley and Company mark that is no older than 1890. A fruit jar fragment with the diagnostic "Self-Sealing" label of the Kerr Glass Company indicates that it was manufactured after 1915. Other materials of relatively recent vintage (i.e., dating to the early twentieth century) include dry-cell battery carbon rods and a cemented shoe sole.

The trash fill assemblage clearly contains a mix of artifacts manufactured before and after the turn of the century. Many of the artifacts could have been manufactured at or before the turn of the century, and the oldest datable artifact (i.e., a ceramic bowl with the Wheeling Pottery mark) in the assemblage was manufactured between 1896 and 1903. Allowing for some lag time, even the oldest materials could have been deposited after the turn of the century or after the 1907 abandonment date for the dugout (based on the archival data). Several of the artifacts, however, indicate that a portion of the assemblage was deposited after 1915 and perhaps as late as the 1920s or 1930s. Allowing for some lag time, these materials probably were deposited during the 1920 s or 1930 s or perhaps as late as the 1940s. This assessment is consistent with the archival data which suggest that the trash fill assemblage must have been deposited prior to the site's final abandonment in the 1940s.

In summary, the dugout assemblage apparently dates from ca. 1899 to ca. 1910, while the trash fill assemblage dates from ca. 1910 to ca. 1940 . Within this chronological context, the material culture data are examined to infer site function, subsistence and technology, and socioeconomic conditions to provide a greater understanding of cultural changes during the closed-range ranching period in the western Rolling Plains.

\section{Site Function}

The archival data indicate that 41GR474 served as a homestead and ranching headquarters for Jeff Justice and his family from 1900 through the 1920 s and subsequently was occupied by tenants who used the land in a similar manner until the late 1940s. The site was associated generally with agriculture (i.e., mainly cattle ranching) throughout its history, and the historical documents suggest that there were no significant changes in site function that would be reflected in the material culture. Notably, only a few artifacts in the dugout and trash fill assemblages are related strictly to ranching activities. As a cautionary note, an overly simplistic interpretation might suggest that ranching was relatively unimportant, which is certainly not the case.

In his archeological model of Texas cattle ranching, Martin (1983) notes the importance of artifacts that he considers to be linked exclusively and directly to specific ranching tasks (i.e., what he terms acquisition, maintenance, and processing activities) and suggests that their presence can be used to verify site function. While this is true to an extent, this model does not take archeological or preservation biases into account, and a paucity or absence of ranching artifacts does not necessarily equate to an absence of ranching. Martin (1983:8390) presents the George Jowell Ranch Complex in Palo Pinto County as an example of a late nineteenth-century Anglo ranch. Test excavations there (Jackson 1975) yielded only two artifacts (strands of barbed wire) that were considered to be evidence of ranch maintenance, yet the archival data clearly indicated that it was a ranching site (Martin 1983:93).

At 41GR474, the dugout assemblage contains only two cow bones (one from testing and one from data recovery) that might be considered direct evidence of cattle ranching. The trash fill assemblage contains a range of general hardware (e.g., bolts), wagon parts, and horse tack that could be indirectly related to ranching, but only a few cow bones (from testing), the windmill, and barbed wire are specifically and exclusively associated with ranching (according to Martin 1983:55). Obviously, the recovery of minimal ranching evidence at 41GR474 does not mean that ranching was unimportant. Although only a few cow bones were recovered in each of the assemblages (minimum number of individuals $=1$ for each assemblage), the Garza County Ad Valorem Tax Rolls for 1900-1908 indicate that Jeff Justice had a minimum of 300 head of cattle each year and had as many as 1,000 head of cattle in 1907. He also had from 20-32 horses and up to 35 hogs or goats each year from 1900-1908. Clearly, the material culture from 41GR474 must be interpreted within the context of the historical data indicating that the site functioned primarily as a cattle 
ranching headquarters. Another example of a ranching site that yielded few ranch-related artifacts is Johnny Ward's Ranch (Fontana and Greenleaf 1962), where an unspecified number of cow bones constitute the only direct evidence of ranching.

A functional comparison of the artifacts (following South 1977) indicates that there are significant differences between the dugout and trash fill assemblages (Table 51). The most obvious differences when comparing the assemblages are the extremely variable frequencies of kitchen artifacts and bones and the more moderate variations in furniture, arms, clothing, and personal artifacts. At face value, these variations suggest functional differences between the two assemblages. This may be true to some extent, but the nature of the individual assemblages must be taken into account before such interpretations are made.

The trash fill assemblage is dominated by kitchen artifacts (mainly glass, ceramic, and metal food containers and utensils) and bone, which account for $83 \%$ of the artifacts. Clothing and activities artifacts make up another $10 \%$, while the other five groups (architecture, furniture, arms, personal, and tobacco) account for only $7 \%$. Its archeological context indicates that this assemblage represents a secondary refuse deposit, and this interpretation is consistent with the material culture.

In contrast, while $84 \%$ of the dugout assemblage is kitchen artifacts and bone, bone is predominant and kitchen artifacts are very poorly represented. This relationship is opposite that observed in the trash fill assemblage, and the paucity of kitchen artifacts makes the dugout assemblage unusual. The arms category is well represented (8\%), while all of the architecture artifacts (4\%) are cut nails which may be directly related to the dugout structure. The other five groups (furniture, clothing, personal, tobacco, and activities) account for only 5\% of the assemblage. With its low frequency of kitchen artifacts and high frequency of firearms-related artifacts, the dugout assemblage is unusual for a turn-of-the-century domestic site. It is suggested that the dugout assemblage represents a more specialized set of activities than those represented by the trash fill assemblage.

The archeological context indicates that the dugout assemblage is not a trash dump. A dump area associated with the dugout occupation would be directly comparable and probably much more similar to the trash fill assemblage, but no such dump was found. Debris generated by the dugout occupation probably was discarded downslope and long since has washed away. While some of the dugout assemblage

\begin{tabular}{|c|c|c|c|c|c|c|}
\hline \multicolumn{7}{|c|}{$\begin{array}{l}\text { FUNCTIONAL COMPARISON OF HISTORIC ARTIFACTS IN THE DUGOUT } \\
\text { AND TRASH FILL ASSEMBLAGES, 41GR474* }\end{array}$} \\
\hline \multirow[b]{2}{*}{ Functional Category** } & \multicolumn{2}{|c|}{ Dugout Assemblage } & \multirow{2}{*}{$\begin{array}{l}\text { Adjusted Standardized } \\
\text { Residuals for Dugout } \\
\text { Assemblage*** }\end{array}$} & \multicolumn{2}{|c|}{ Trash Fill Assemblage } & \multirow[b]{2}{*}{ Totals } \\
\hline & No. & $\%$ & & No. & $\%$ & \\
\hline Kitchen & 5 & 1.4 & -23.28 & 1,068 & 69.5 & 1,073 \\
\hline Bone & 290 & 82.2 & +26.22 & 212 & 13.8 & 502 \\
\hline Architecture & 13 & 3.7 & +1.78 & 32 & 2.1 & 45 \\
\hline Furniture & - & - & -2.15 & 20 & 1.3 & 20 \\
\hline Arms & 28 & 7.9 & +6.86 & 22 & 1.4 & 50 \\
\hline Clothing & 5 & 1.4 & -3.86 & 103 & 6.7 & 108 \\
\hline Personal & 2 & 0.6 & +1.61 & 2 & 0.1 & 4 \\
\hline Tobacco & 3 & 0.8 & -1.23 & 27 & 1.8 & 30 \\
\hline Activities & 7 & 2.0 & -1.31 & 51 & 3.3 & 58 \\
\hline Totals: & 353 & 100.0 & & 1,537 & 100.0 & 1,890 \\
\hline \multicolumn{7}{|c|}{$\begin{array}{l}\text { *Excludes all artifacts in the unknown function category but includes } 417 \text { artifacts from the } 1988 \text { testing } \\
\text { (see Boyd and Freeman 1990:Table 15). }\end{array}$} \\
\hline \multicolumn{7}{|c|}{ **Follows South (1977:92-102). } \\
\hline
\end{tabular}


may have been and probably was deposited as secondary refuse, other materials are interpreted as representing primary activities that occurred during the dugout occupation or during a brief period of reuse. An assemblage composed entirely of secondary materials might be expected to be different from an assemblage that is a mix of secondary and primary materials, even if overall site function and material access remained unchanged. Consequently, the differences between the dugout and trash fill assemblages probably are related more to the nature of the deposits than to temporal changes in site function or availability of material goods.

The intrastructural distributions of artifacts and features have important implications for understand- ing the nature of the dugout assemblage and support the interpretation that much of it was deposited during a reuse episode (Figure 73). As mentioned above, it is not likely that the ash stains and bone clusters were deposited inside the dugout during the time that it was used as a domicile. This is particularly true since they are clustered around the fireplace along the dugout's back wall, an area that probably would have been kept clean. Since a large family probably would not have dumped trash inside the small dugout, these features most likely represent dumping during a reuse episode. The same is true for the larger artifacts found on and near the floor (i.e., the possible wagon parts, the tin cans, and the axe head) since they are not likely to have been lost or discarded in the dugout

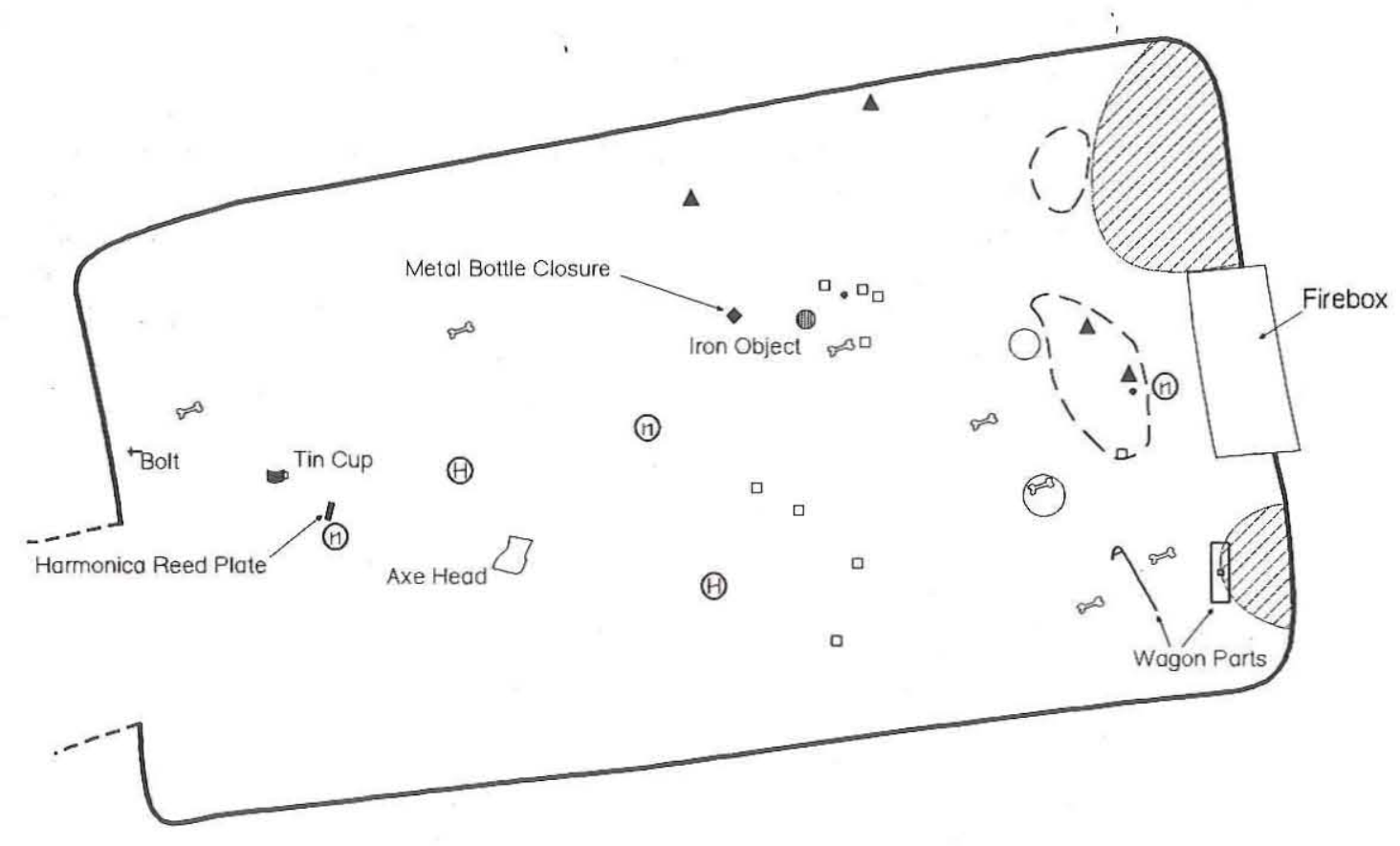

LEGEND

Ash Stain
B Tin Can
$\Delta$ Cartridge Case
Drimer
- Lead Ball
$\infty$ Bone
(1) Nail
(1) Horseshoe Nail
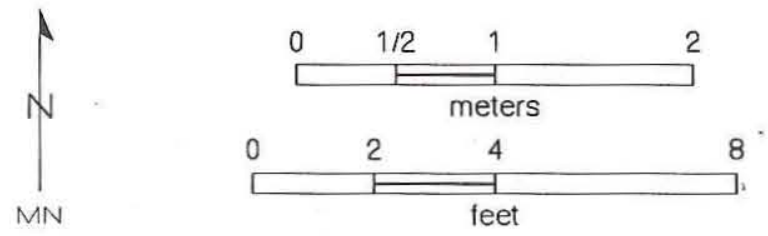

P\&AI/94/SLH

Figure 73. Distribution of cultural features and artifacts on and near the floor of the dugout at 41 GR474. 
during its occupation. And finally, artifacts found on the floor include four cartridges, eight primers, and two lead balls. These firearms-related items, particularly the primers since they are indicative of reloading activities, also are unlikely to have been lost or discarded inside a structure during its occupation.

The dugout assemblage is unusual and is interpreted as representing a set of specialized activities postdating the initial abandonment of the structure. The most plausible explanation is that after the Justice family moved out of the dugout in 19061907 , the structure was used for a few more years, probably for a variety of different activities. It may have served as a storage area, tack room, or tool shed (as evidenced by horseshoe nails and an axe head); as a butchering area and smokehouse (as evidenced by the butchered bones and aș stains); and/or as a workshop where machinery was repaired and cartridge cases were reloaded (as evidenced by possible wagon parts, a bolt, and firearms artifacts). It probably was used until more-permanent structures (e.g., barns and worksheds) were constructed near the ranch house. However, this interpretation does not necessarily imply that the reuse episode was characterized by a continuous period of multifunctional use. Alternatively, its reuse may have been in the form of multiple intermittent and temporary use episodes, each including a single or limited set of activities.

Further comparisons of the 41GR474 artifact data with established artifact patterns support the interpretations that the dugout artifacts are a specialized assemblage and the trash fill artifacts are a typical dump assemblage (Table 52). The 41GR474 trash fill pattern is much more similar to a contemporaneous (1894-1920s) dump assemblage from north San Antonio (Clark 1984:6, 114) than it is to the dugout assemblage. Both the trash fill and San Antonio dump assemblages are characterized by very high frequencies of kitchen artifacts. These assemblages should not be expected to be identical, and the minor differences between them (e.g., a higher frequency of clothing in the trash fill assemblage) may be attributed to behavioral differences in household dumping vs. community dumping.

Conversely, the dugout assemblage appears more similar in some ways to South's (1977:145) Frontier Pattern (both are characterized by relatively high percentages of architecture and arms artifacts and relatively low percentages of kitchen artifacts) than it does to either the trash dump or San Antonio dump assemblages. If, as has been suggested, the dugout assemblage is a mixed deposit of primary and secondary artifacts representing multiple specialized activities, it might be expected that its artifact pattern would not conform to others. While this interpretation is reasonable given the nature of the dugout

\begin{tabular}{|c|c|c|c|c|c|}
\hline \multicolumn{6}{|c|}{$\begin{array}{l}\text { TABLE 52 } \\
\text { NCTIONAL COMPARISON BETWEEN 41GR474 ARTIFACTS AND } \\
\text { VARIOUS ARTIFACT PATTERNS AND ASSEMBLAGES }\end{array}$} \\
\hline \multirow[b]{2}{*}{ Functional Category } & \multicolumn{2}{|c|}{ 41GR474* } & \multirow{2}{*}{$\begin{array}{l}\text { 41BX171, North } \\
\text { San Antonio Dump } \\
\text { (Clark 1984:114) }\end{array}$} & \multirow[b]{2}{*}{$\begin{array}{l}\text { Carolina Pattern } \\
\text { (South 1977:107) }\end{array}$} & \multirow[b]{2}{*}{$\begin{array}{l}\text { Frontier Pattem } \\
\text { (South 1977:145 }\end{array}$} \\
\hline & $\begin{array}{l}\text { Dugout } \\
\text { Assemblage }\end{array}$ & $\begin{array}{l}\text { Trash Fill } \\
\text { Assemblage }\end{array}$ & & & \\
\hline Kitchen & $7.9 \%$ & $80.6 \%$ & $84.4 \%$ & $63.1 \%$ & $27.6 \%$ \\
\hline Architecture & $20.6 \%$ & $2.4 \%$ & $4.8 \%$ & $25.5 \%$ & $52.0 \%$ \\
\hline Furniture & - & $1.5 \%$ & $2.3 \%$ & $0.2 \%$ & $0.2 \%$ \\
\hline Arms & $44.4 \%$ & $1.7 \%$ & $0.2 \%$ & $0.5 \%$ & $5.4 \%$ \\
\hline Clothing & $7.9 \%$ & $7.7 \%$ & $0.8 \%$ & $3.0 \%$ & $1.7 \%$ \\
\hline Personal & $3.2 \%$ & $0.2 \%$ & $1.6 \%$ & $0.2 \%$ & $0.2 \%$ \\
\hline Tobacco & $4.8 \%$ & $2.0 \%$ & $0.3 \%$ & $5.8 \%$ & $9.2 \%$ \\
\hline Activities & $11.1 \%$ & $3.9 \%$ & $5.6 \%$ & $1.7 \%$ & $3.7 \%$ \\
\hline No. of Artifacts & 63 & 1,325 & 4,429 & 77,610 & 48,519 \\
\hline
\end{tabular}


assemblage, changing economic conditions on the western Rolling Plains during the early twentieth century also may have played a role. Since South's (1977) Frontier Pattern reflects physical isolation and limited access to sources of mass-manufactured goods, the possibility that some of the patterning evident in the dugout assemblage could be related to these factors must be considered.

\section{Subsistence and Technology}

Although they are somewhat tenuous, two alternative interpretations of subsistence and technology can be offered based on the 41GR474 artifact assemblages (assuming, as discussed above, that there were no significant changes in site function). First, the observed differences between the dugout and trash fill assemblages could be related exclusively to differences in their depositional contexts. If this is the case, the material culture differences are attributed mainly to the fact that one assemblage is a general household dump while the other represents a mix of primary and secondary deposits related to specialized activities. Alternatively, some or much of the variability between the assemblages could be due to changes in subsistence and technology that are related to changing economic patterns during the early twentieth century (i.e., 1900-1930). This scenario implies that there were significant changes during the early twentieth century in the availability of massmanufactured goods, transportation, and access to markets. These two explanations are, of course, oversimplified, and the 41GR474 material culture probably reflects both to some degree. The extent to which changing economic patterns in the early twentieth century may have affected the 41GR474 material culture is not known, but the latter explanation merits some consideration.

The western Rolling Plains was being settled rapidly by the turn of the century, and it cannot be considered to have been frontier at that time, although there were still remote areas where access to material goods was limited (Anderson 1990:104; Freeman 1990:96). In the early 1880 s, towns such as Abilene, Sweetwater, and Colorado City sprang up all along the Texas and Pacific Railroad from Fort Worth to El Paso and became the commercial trade centers providing the region with supplies and access to markets. By 1898, the community of Snyder was linked to this system by the Roscoe, Snyder and Pacific Railroad. When Jeff Justice and his family settled at 41GR474 in 1900, they came from Snyder and were connected to this transportation network rather than the more distant system of railroads and regional trade centers (e.g., Amarillo and Lubbock) in the Texas Panhandle-Plains. The Justice homestead could only be reached by wagon during the first decade of the twentieth century. From 1900-1906, Snyder ( 40 miles to the south) was the closest town, but small communities not connected to the railroads, such as Post and Polar (Webb 1952), sprang up in Garza County in 1906-1907 and became rural trade centers. Access to commercial goods, however, did not change dramatically until 1910-1911, when the Santa Fe Railroad built a line connecting Lubbock and Coleman. This line went through Post and also spurred the development of the community of Justiceburg. While the Justice homestead certainly was not a frontier outpost prior to the railroad, after 1911 the presence of a local community and a railway link to the market centers in the Panhandle and Southern Plains probably affected the availability of mass-produced goods to some extent.

There may be evidence of this in the 41GR474 assemblages. The overall paucity of artifacts in the dugout assemblage could be related to limited access to mass-manufactured goods. Other researchers have noted that low artifact densities are characteristic of isolated dugouts (Earls 1993b:474, 483) and prerail road townsites (Cheek 1977:147; Lees 1977:i). Also in the dugout assemblage, the unusually high frequency of firearms-related artifacts may not be strictly a function of the specialized reuse of the dugout but could be related in part to an emphasis on hunting. One possible explanation is that, prior to 1911 , the Justice family may have hunted more and consumed more wild game because the availability of other foods was limited by the frequency of their wagon trips to market (e.g., an 80-mile round trip to Snyder prior to ca. 1907 or a 30-mile round trip to Post prior to 1911). With the coming of the railroad and after the community of Justiceburg was established in 1910-1911, a wider variety of goods was immediately available to the Justice family. While the 41GR474 faunal assemblages (see Appendix C) do not support this interpretation since wild animals appear in approximately equal percentages in both assemblages, the trash fill assemblage clearly indicates that nonlocal foods (i.e., commercially purchased or mass-produced foods) were important.

Unfortunately, without a more detailed regional data base from other prerailroad and postrailroad 
sites for comparative purposes, it is impossible to assess the impacts that the railroad may have had on the material culture. It is likely, however, that there may be an increase in the volume and variability of material culture at postrailroad sites in the western Rolling Plains. The greater variability in artifact types seen in the 41GR474 trash fill assemblage probably is related to the increasing availability of mass-produced goods in the early twentieth century, which in turn is related to the expanding commercial transportation and marketing networks (i.e., railroads). Earls (1993a:395, 424, 1993b:472-474) suggests a similar scenario to explain artifact patterns from two historic ranching sites in the northern Texas Panhandle. Both sites, 41HF8 and 41HF113 (a dugout), predate the railroad and yielded sparse assemblages with low variability in artifact types.

\section{Socioeconomic Considerations}

One final consideration related to interpreting site $41 \mathrm{GR} 474$ is the socioeconomic aspects of the material culture. The archival data clearly demonstrate a changing economic status for Jeff Justice during the early twentieth century. While he lived in the dugout from 1900-1907, Justice's landholdings and property increased considerably, and by 1907 he was able to build an impressive ranch house. The family's wealth evidently continued to increase during the rest of the time that they occupied 41GR474, from 1907 to the 1920s. Consequently, the material culture might be expected to reflect this increasing socioeconomic status through time, with the dugout assemblage generally containing more-utilitarian and less-expensive materials than the subsequent trash fill assemblage. At face value, this seems to be the case because the earlier assemblage is meager compared to the later assemblage.

Unfortunately, there are many problems with this simplistic view, and because of the small sample sizes and lack of a substantial comparative regional data base, detailed socioeconomic interpretations of the material culture cannot be supported. There also are problems with the comparability and temporal integrity of the assemblages. The dugout assemblage, reflecting various specialized activities, is not directly comparable to the trash fill assemblage. Consequently, the apparent lack of high-status items in the dugout assemblage does not necessarily mean that they were not in use at the time. Notably, the archival data suggest that the apparent shift in economic scaling (i.e., the Justice's transition from low or moderate income to high income status) may have occurred mainly during the time the dugout was occupied and used rather than after it was abandoned. Thus, a representative artifact sample from the dugout occupation period might include a mix of items indicating different socioeconomic status levels. Furthermore, the economic status of subsequent tenants presumably was less than that of the Justices, and artifacts that may date to the 1930 s or 1940 s suggest that tenants may have made contributions (although perhaps only minimal) to the trash fill assemblage. If this is the case, the mixing of materials from different economic levels would render the trash fill assemblage useless for detailed socioeconomic comparisons.

A final note is that, without sufficient knowledge of regional commercial exchange networks, interpretations of material culture are limited (Majewski and O'Brien 1987:181). For example, ceramics commonly are used as socioeconomic or status indicators because variability in decorative styles often was linked to cost (e.g., Miller 1980), but the relative popularity and pricing of various ceramic decorative styles changed considerably through time. For example, decal-decorated ceramics were more costly than transfer-printed wares prior to 1900 but became the lower priced wares after that time (Majewski and O'Brien 1987:147). The situation was very complex after ca. 1850; consequently, Majewski and O'Brien (1987:134-135) suggest that in order to understand the economic scaling of late nineteenthand early twentieth-century ceramics, ware types (i.e., based on paste characteristics and composition) are as important as decorative styles. Unfortunately, the definition and recognition of valid ware types (e.g., "semivitreous" vs. "semiporcelain") is difficult, and the nature of ceramic pricing relative to decorative and ware types is poorly understood (Majewski and O'Brien 1987:134). Consequently, given the small sample sizes, lack of comparative regional data, and the limitations of historic artifact analyses, the assemblages from 41GR474 are not particularly useful as socioeconomic indicators at this time. 



\section{BOREN SHELTER \#1, 41GR546}

Jay Peck and Karl W. Kibler

\section{SITE SETTING}

Site 41GR546 is a rockshelter on the uppermost sandstone bluff forming the east canyon rim of an unnamed drainage, ca. $40 \mathrm{~m}$ north of its confluence with the Double Mountain Fork of the Brazos River and ca. $500 \mathrm{~m}$ upstream from the mouth of Sand Creek. Facing west and overlooking the drainage, it is ca. $60 \mathrm{~m}$ south of rockshelter 41GR559. Formed by erosion of friable sandstone directly beneath the harder sandstone canyon rim, the shelter extends $4 \mathrm{~m}$ back into the bedrock. The ceiling is a hard conglomerate sandstone layer; from the present ground surface, its height varies from $0.6 \mathrm{~m}$ at the back to $1.8 \mathrm{~m}$ along the dripline. The shelter has a small protected floor area of ca. $12 \mathrm{~m}^{2}$. The open floor is immediately south of a section of collapsed roof which covers a space equally as large as the open area. The floor slopes down gently to the west, and the talus slope drops off sharply at the front. Historic graffiti sites 41GR536 and 41GR537 occur nearby, but there is no evidence of vandalism to the shelter. Site elevation is $2250 \mathrm{ft} \mathrm{msl}$.

The sheltered area is devoid of vegetation except along the front edge where small viney plants are encroaching. A dense row of agarita along the dripline helps protect the talus slope from erosion and shield the shelter from view. Junipers, mesquites, grasses, and various cacti are found on the upland above and in the valley below the shelter. Hackberries are present along the drainage.

\section{PREVIOUS INVESTIGATIONS}

When this site was recorded in 1987, possible smoke-blackening of the ceiling, sparse lithic debris on the floor, and cultural materials in a shovel test indicated that the shelter had been occupied. Mapping and testing were recommended (Boyd et al. 1989). As a result of these recommendations, mapping of the general site area, detailed mapping and profiling of the shelter, and excavation of a shovel test in the area of the collapsed ledge and a single $1-x-1-m$ test unit in the shelter floor were conducted (Boyd et al. 1990). Shovel Test 2, excavated to $20 \mathrm{~cm}$, yielded no cultural materials, indicating that the ledge collapse probably predates the occupation of the shelter. The test unit, located over the 1987 shovel test, was excavated to bedrock at $70-96 \mathrm{~cm}$ below the surface. Cultural materials were recovered in the upper $80 \mathrm{~cm}$, and portions of two burned rock features were found.

Cultural materials recovered from the single test unit consisted of 6 pieces of lithic debitage, 7 pieces of microdebitage, 43 pieces of burned and unburned bones, eggshell fragments, 7 burned rocks, and a snail shell. The lithic materials have since been reanalyzed, and most have been determined to be noncultural. Much of the material was recovered by flotation or fine screening. The only identifiable burned bone is a snake vertebra; unburned bones represent snake, rabbit, bird, and rodent. It was thought at the time that multiple occupations probably 
were represented, but a calibrated radiocarbon date range of A.D. 1430-1950 with a single intercept at A.D. 1643 obtained on charcoal from Feature 2 provided the only chronological evidence since no diagnostic artifacts were found. The testing suggested that macrobotanical and faunal remains were relatively abundant and well preserved. Burned wood from features represented several species (oak, juniper, hackberry, rose, and unidentified hardwood). Evidence of probable food resources included a burned snake vertebra, unidentifiable burned bone fragments, and relatively abundant Chenopodium fruits from Features 1 and 2. While it was recognized that the macrobotanical and faunal remains could represent both cultural activities and natural scavenger or predator utilization of the shelter, it was felt that cautious interpretations based on careful data recovery and comparative flotation sample analysis could identify culturally introduced materials and also provide considerable environmental data. The combination of enhanced preservation of organic remains, horizontally discrete activities, and vertically separable components represented in this rockshelter is not found at open campsites in the project area, and hence further work was recommended.

\section{DATA RECOVERY INVESTIGATIONS}

Excavation and mapping of 41GR546 was undertaken over a period of 2 work-weeks and required 10 person-days of effort. A total of $3.25 \mathrm{~m}^{3}$ of sediment was excavated and screened through $1 / 4$-inch-mesh hardware cloth. Initially an arbitrary grid was reestablished based on a grid point designated N100/E100, which was set during the 1988 testing. This grid point was marked with a length of rebar driven into the ground and assigned an arbitrary elevation of $100 \mathrm{~m}$ above an imaginary datum plane. All subsequent three-dimensional measurements were taken relative to that point. The grid increases north and east from an imaginary zero point $100 \mathrm{~m}$ south and $100 \mathrm{~m}$ west of the rebar. The grid was oriented to magnetic north and projected through the interior of the rockshelter, and this orientation was maintained during excavation.

Six units were excavated at 41GR546 in 1992; when Test Unit 1 excavated in 1988 (renumbered as Excavation Unit [EU] 3) is added, seven units have been excavated (Figure 74). Initially, Test Unit 1 was shoveled out, and the south and west walls were profiled to complement profiles of the north and east walls drawn during the testing phase. Subsequently, EUs 1, 2, and 4 were excavated to bedrock to the north, west, and south of EU 3. It was then determined that the remaining units along the east wall would be smaller than $1 \mathrm{~m}$ in width (east-west), would not require a great deal of effort to excavate, and might contain cultural materials cached or lost along the back wall of the rockshelter. These partial units were then excavated.

All of the units were excavated in 10-cmthick arbitrary levels, although levels less than or slightly greater than $10 \mathrm{~cm}$ in thickness sometimes were used for the upper proveniences to level off the excavations at even increments relative to the site datum. The units were excavated to depths ranging from 53 to $93 \mathrm{~cm}$, with the mean depth being $73 \mathrm{~cm}$.

\section{SEDIMENTS AND;STRATIGRAPHY}

The rockshelter is formed in soft, friable, finegrained, cross-stratified gray sandstone of the Upper Triassic Dockum Group. It underlies more-resistant Dockum gravel and mud clast conglomerates and coarse-grained,cross-stratifiedsandstones representing a sequence of channel fills deposited by braided streams (McGowen et al. 1979). Several boulders of these overlying rock units, representing brow retreat and earlier shelter collapse episodes, litter the surface in front of the shelter and constitute a substantial amount of the fill in the lower levels of the rockshelter.

The unconsolidated fill of the rockshelter is over $100 \mathrm{~cm}$ thick at the front and becomes progressively thinner, as well as younger, toward the back wall. The rockshelter fill consists of a fine sandy loam matrix with many gravel-, pebble-, and cobble-sized clasts which tend to be flat to lenticular in shape with sharp angular edges. The fill is considered to be endogenous, representing the grain by grain disintegration of the sandstone walls and ceiling and the spalling off of larger clasts. The larger clasts or rockfall are derived through the widening of joints and bedding planes in the rock of the ceiling or walls by water, freeze-thaw cycles, biological activity, and other agents (Donahue and Adovasio 1990:236). The release of individual sand grains is poorly understood, but to a large extent it probably is related to the type of cement holding the sand grains together and most likely involves both physical and chemical weathering. Although exogenous sediments may be present in the fill, their 


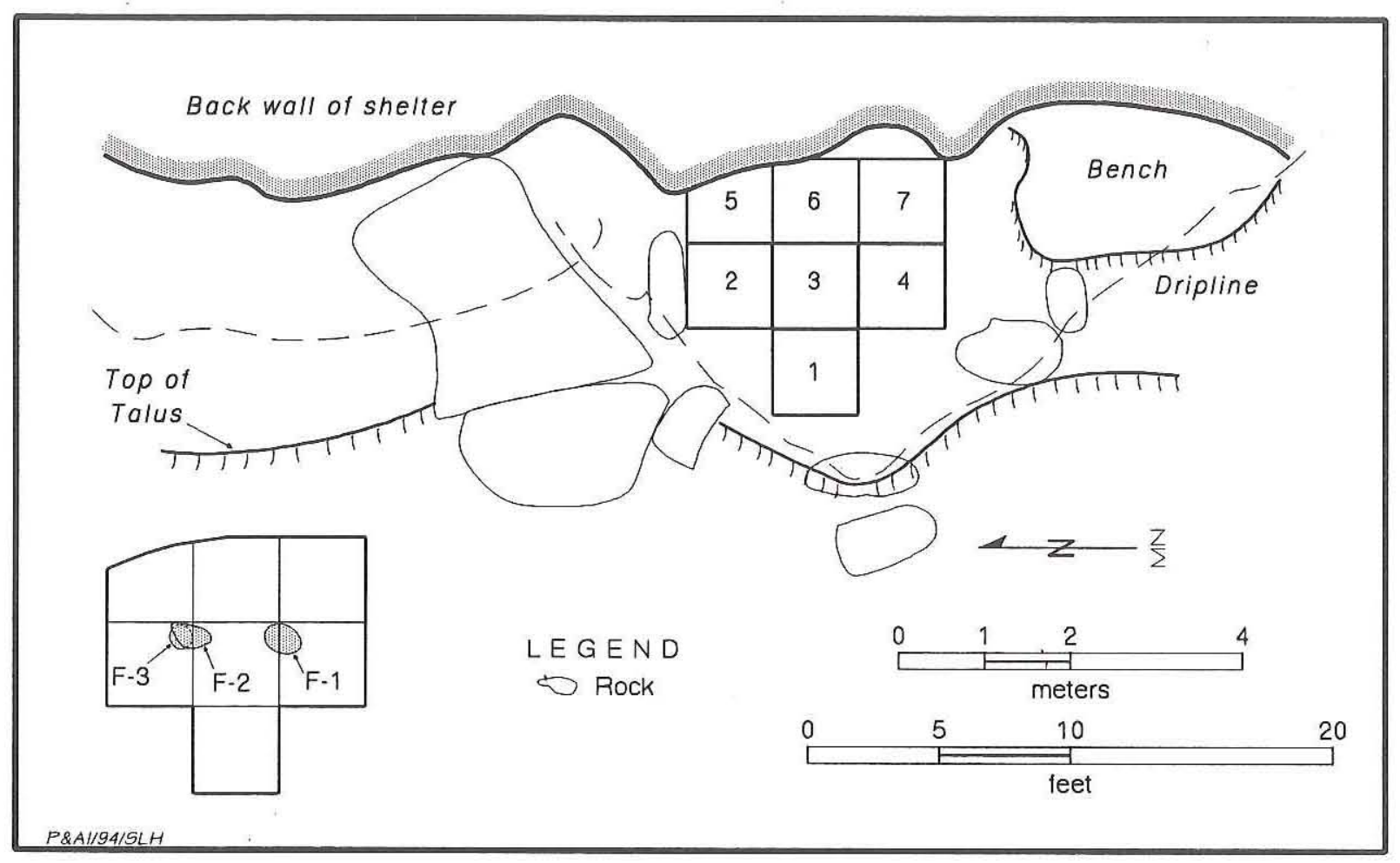

Figure 74. Plan of rockshelter and excavation units, 41GR546.

presence is very slight and not discemible on a macroscopic level. Based on a single radiocarbon assay from Feature 2 (see Boyd et al. 1990:144), the shelter has been filling for at least the last 300 years. However, it is more likely that the shelter fill is at least 1,000 years old based on the provenience of Feature 2 in the upper levels of the fill and some similarities with nearby Boren Shelter \#2 (see Chapter 7).

The sediments and stratigraphy of the shelter fill were documented in detail from the north wall profile of EU 3 (Figure 75; see Appendix A). Three zones were identified in the $62-\mathrm{cm}$ profile. Zone 1 $(0-38 \mathrm{~cm})$ is a $\mathrm{Cu}$ horizon consisting of a massive light yellowish brown (10YR 6/4) fine sandy loam with many gravel- and pebble-sized clasts. Rodent burrows are common throughout the zone. Remnants of Feature 2, which was excavated by Boyd et al. (1990), are present in the profile at the bottom of the zone. Zones 1 and 2 are separated by a clear, smooth boundary. Zone $2(38-51 \mathrm{~cm})$ is a massive light yellowish brown (2.5Y 6/4) fine sandy loam with many gravel-, pebble-, and cobble-sized clasts. It also is a $\mathrm{Cu}$ horizon. A conglomerate boulder representing roof fall rests on the contact between Zones 2 and 3. Gravel-sized clasts of conglomerate are also common at this clear, wavy boundary. Zone $3(51-62+\mathrm{cm})$ represents the bedrock floor of the shelter and is an R horizon. It consists of a gray sandstone.

Interestingly, the amount of gravel- and pebble-sized clasts increases with depth throughout the profile. Donahue and Adovasio (1990:237) note that increased frequencies of larger clasts or rockfall are associated with major roof fall or slab failure events, which is certainly represented in Zone 2 by the large conglomerate boulder. They also note that rockfall is generally represented in greater amounts during the initial stages of shelter formation, which is also represented in Zone 2 since it rests on the bedrock floor of the shelter.

\section{CULTURAL FEATURES}

Three stone-lined hearths were recorded as features. Portions of two of these hearths (Features 1 and 2) were recorded during the 1988 testing (Boyd et al. 1990).

\section{Feature 1}

Feature 1 consists of a concentration of ca. 19 


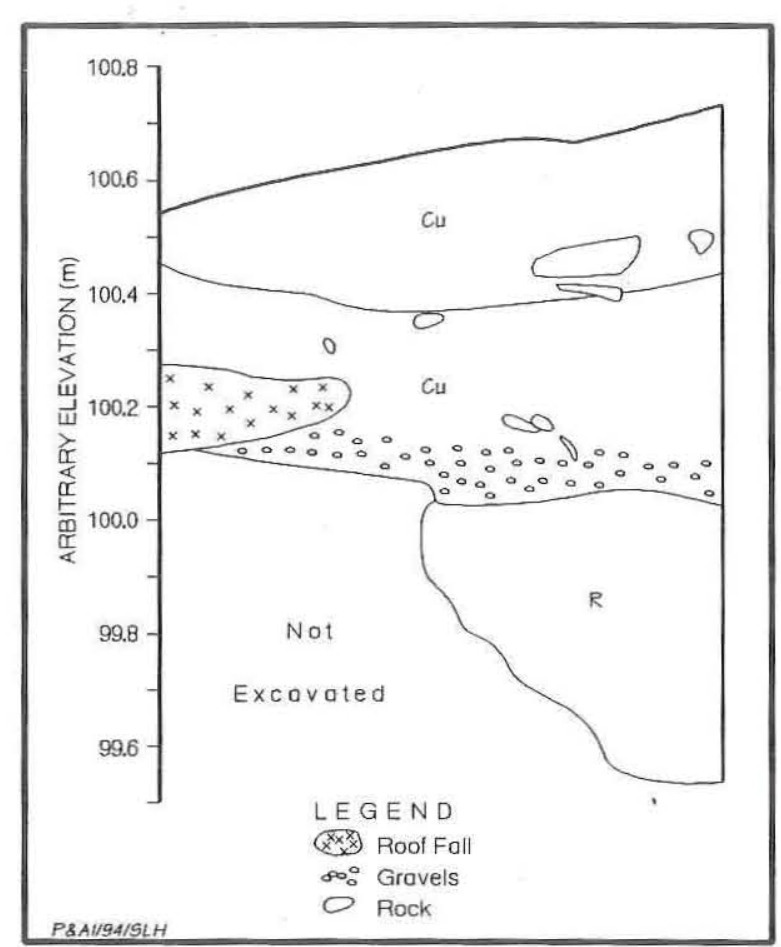

Figure 75. Profile of EU 3, 41GR546.

burned sandstone and sandstone conglomerate rocks in EUs 3 and 4. The total weight of the rocks is $18.2 \mathrm{~kg}$. The feature occurs at a depth of $25-35 \mathrm{~cm}$ below the modern ground surface. Feature 1 was initially recorded during the 1988 testing when the north half of the concentration was discovered in the southern portion of Test Unit 1 (EU 3). Subsequent Phase III excavations revealed the south half of the feature in EU 4 at $20-28 \mathrm{~cm}$ below the modern ground surface. The total concentration extends $50 \mathrm{~cm}$ north-south and $40 \mathrm{~cm}$ east-west, extending $20 \mathrm{~cm}$ north into EU 3 and $30 \mathrm{~cm}$ south into EU 4. The feature sits on top of a large fallen conglomerate roof block, and the eastern margin is $25 \mathrm{~cm}$ from the edge of another fallen conglomerate roof block that constitutes the lower back (east) wall of the rockshelter. A large, flat slab of white sandstone $50 \times 45 \times 3 \mathrm{~cm}$ in dimension was found lying flat just south of the hearth. The slab is lightly burned and probably was used as a wind deflector. Other than this slab, all associated rocks are slanted inward toward the center of the feature. No soil staining was recognized within or around the feature, and only a sparse scatter of small charcoal flecks was observed. Feature 1 is interpreted as a stone-lined hearth.

\section{Feature 2}

Feature 2 consists of a concentration of ca. 27 burned sandstone and sandstone conglomerate rocks in EUs 2 and 3. The total weight of the rocks is $24.5 \mathrm{~kg}$. Feature 2 was initially recorded during the 1988 testing when the south half of the concentration was discovered in the northern portion of Test Unit 1 (EU 3) at $20-40 \mathrm{~cm}$ below the modern ground surface. Subsequent Phase III excavations revealed the north half of the feature in EU 2 at $12-29 \mathrm{~cm}$ below the modern ground surface. The total concentration extends $85 \mathrm{~cm}$ north-south and $50 \mathrm{~cm}$ east-west, extending $50 \mathrm{~cm}$ north into EU 2 and $35 \mathrm{~cm}$ south into EU 3. The eastern margin of the feature is directly adjacent to the fallen conglomerate roof block that constitutes the lower back (east) wall of the rockshelter. All associated rocks are slanted inward toward the center of the feature.

No soil staining was recognized within or around the feature, and only a sparse scatter of small charcoal flecks was observed. Feature 2 is dated by a corrected radiocarbon assay on charcoal recovered during the 1988 testing to $275 \pm 210$ B.P. (see Boyd et al. 1990:371); this radiocarbon age was calibrated according to Stuiver and Reimer (1986) to A.D. 1450 (1643) 1950. A thermoluminescence assay yielded no date, probably due to insufficient heating of the sample. Feature 2 is interpreted as a stone-lined hearth.

\section{Feature 3}

Feature 3 was discovered during the Phase III excavations and consists of a concentration of five burned sandstone and sandstone conglomerate rocks in EU 2. The total weight of the rocks is $5.5 \mathrm{~kg}$. The feature occurs at a depth of $29-41 \mathrm{~cm}$ below the modern ground surface and is directly beneath Feature 2. The concentration extends $35 \mathrm{~cm}$ northsouth and $40 \mathrm{~cm}$ east-west and is in the southeast quadrant of EU 2. The eastern margin of the feature is within $5 \mathrm{~cm}$ of the fallen conglomerate roof block that constitutes the lower back (east) wall of the rockshelter. The vertical surface of this wall is burned at this depth. The two easternmost rocks of the feature are slanted inward toward the center of the hearth. No soil staining was recognized within or 
around the feature, and only a sparse scatter of small charcoal flecks was observed. Feature 3 is interpreted as a stone-lined hearth.

\section{MATERIALS RECOVERED}

Two artifacts were found during the Phase III excavations. Because of this low recovery, the materials obtained in the 1987 survey and the 1988 testing were reexamined; 2 pieces from the survey and all 13 pieces from the testing that originally were thought to be debitage were found to be noncultural.

\section{Stone Artifacts}

One unifacial tool was recovered from 41GR546. It is a side scraper made on a small rectangular cobble of Potter chert which was recovered from Level 7 of EU 1. The cortex has been removed along the working edge of the ventral side of the tool, and five flakes have been removed from the working edge on the dorsal side. Some rounding exists on the working edge as well as five small flake removals that appear to be use related. The specimen is $80 \mathrm{~mm}$ long, $48 \mathrm{~mm}$ wide, and $23 \mathrm{~mm}$ thick.

One broken hammerstone was recovered from the backfill of Test Unit 1 (EU 3) during the 1992 excavation. The fragment is of purple quartzite with postdepositional calcium carbonate encrustations on its surfaces. Three areas on three different surfaces appear to be ground, one surface exhibits breakage, and four surfaces exhibit battering severe enough to be the result of use as a hammerstone. The specimen is $54 \mathrm{~mm}$ long, $51 \mathrm{~mm}$ wide, and $33 \mathrm{~mm}$ thick; it weighs $122 \mathrm{~g}$.

\section{Manuports}

Manuports are any specimens that do not fit into artifactual categories but do not occur naturally within the site limits. One unburned manuport was recovered from Level 5 of EU 1 during the 1992 excavations. This specimen is a rectangular piece of $\tan$ quartzite measuring $95 \mathrm{~mm}$ in length, $62 \mathrm{~mm}$ in width, and $27 \mathrm{~mm}$ in thickness; it weighs $207 \mathrm{~g}$. There is no recognizable modification on this specimen and no indication of its possible use. Also in this category are one small piece of hematite from Level 3 of EU 4 and one small piece of limonite from Level 4 of EU 7. Also recovered were two pieces of burned Potter chert less than $10 \mathrm{~cm}$ in maximum dimension from the backfill of Test Unit 1 (EU 3).

\section{Faunal Remains}

The 1992 excavations recovered three pieces of small unburned rodent bones; one is from the Test Unit 1 (EU 3) backfill, and the other two are from Levels 5 and 8 of EU 2. As these bones are not burned and appear fresh, they are considered to be intrusive and recent. Also recovered were three eggshell fragments. It is not possible to identify these specimens.

Three gastropod shells were recovered during the 1992 excavations. All three are of Family Succineidae-terrestrial snails preferring moist, wooded areas. These shells are considered noncultural and intrusive.

\section{CONCLUSIONS}

The very limited cultural remains at 41GR546 indicate that the rockshelter was occupied possibly on a few occasions for very short periods of time. The single radiocarbon assay from Feature 2 indicates that at least one of these use episodes took place within the period of time ranging from A.D. 1430 1950. Feature 3, which underlies Feature 2, was not dated due to the scarcity of charcoal; it apparently predates Feature 2, but it is uncertain how much time elapsed between these occupations. The single intercept of A.D. 1643 on the Feature 2 radiocarbon date coincides with the single intercept of A.D. 1642 on the bison bone collagen date from Features 9 and 12 at nearby site 41GR559. It is possible that both sites were utilized concurrently and that people occupying 41GR546 were responsible for the animal bones in the talus area at 41GR559.

The very low density of cultural materials argues for short occupations, possibly only overnight at most. The small size of the shelter would preclude occupation by more than one or two individuals at any one time. Macrobotanical samples of Chenopodium and burned pieces of oak, juniper, hackberry, and rose wood recovered during the 1988 testing, along with the presence of three burned rock features, indicate fire building and possibly cooking activities. On the whole, however, it is clear that a very limited range of activities was performed at this site. 

BOREN SHELTER \#2, 41GR559

Jay Peck, Steve A. Tomka, and Karl W. Kibler

\section{SITE SETTING}

Site 41GR559 is a rockshelter on the uppermost sandstone bluff forming the east canyon rim of an unnamed drainage on the north side of the Double Mountain Fork of the Brazos River, ca. $100 \mathrm{~m}$ north of the confluence of the drainage and the Double Mountain Fork (Figure 76). Facing south and overlooking the mouth of the drainage, 41GR559 is ca. $60 \mathrm{~m}$ north of rockshelter 41GR546. It was formed by erosion of the friable sandstone immediately below the hard bluff-forming conglomerate sandstone. The ceiling height from the present ground surface ranges from $0.4 \mathrm{~m}$ at the back of the shelter to about $1.5 \mathrm{~m}$ at the lower dripline to over $4 \mathrm{~m}$ at the upper dripline in some places, with an average height of over $1 \mathrm{~m}$. The lower dripline marks the extent of the protection afforded the inside of the shelter by the immediately overlying sandstone. The upper dripline marks the extension of this protection by about $1 \mathrm{~m}$ in the east end of the shelter as a result of the further extension of the upper sandstone strata. The shelter floor covers ca. $30 \mathrm{~m}^{2}$, and an additional ca. $30-\mathrm{m}^{2}$ area in front of the shelter is protected from erosion by a line of boulders ca. $2-5 \mathrm{~m}$ out from the dripline. Beyond this line of boulders, the talus slope drops sharply into the valley. The floor is quite level, but a rill has developed recently where rainwater drips into the east and west ends of the shelter and runs along the back wall. Site elevation is $2240-2250 \mathrm{ft}$ msl.

Adjacent to the shelter on the east is a collapsed section of ledge that once was a sizable shelter. There is little ground exposure in this area, most being covered by the large jumble of boulders. Historic graffiti (i.e., four sets of initials, the years 1918 and 1926, and unreadable dates) in this area indicate that the site has been visited, but there is no evidence of vandalism.

Vegetation is limited to a few spindly vines and buried tubers inside the shelter and a row of agarita and catclaw acacia along the dripline. The latter help control erosion and hide the shelter from view. Upland vegetation above the shelter is mainly junipers, mesquites, grasses, and cacti, while the valley below contains mainly tall grasses, mesquites, junipers, and hackberries.

\section{PREVIOUS INVESTIGATIONS}

When 41GR559 was recorded in 1987, two shovel tests yielded cultural materials at depths of 30 to $75 \mathrm{~cm}$, but no cultural materials were present on the surface. Mapping and testing were recommended (Boyd et al. 1989). The 1988 testing investigations included mapping of the general site area, detailed mapping and profiling of the shelter, and excavation of two shovel tests and three $1-\mathrm{x}-1-\mathrm{m}$ test units (Boyd et al. 1990). Shovel Test 3, in the east end of the shelter adjacent to a pile of collapsed ledge boulders, was excavated to $90 \mathrm{~cm}$ and yielded no cultural materials, although bedrock was not reached. Additional probing into the cracks and crevices of the collapsed ledge produced no cultural materials, but once again bedrock was not reached. Shovel Test 4 was excavated to $55 \mathrm{~cm}$ in front of the shelter and 


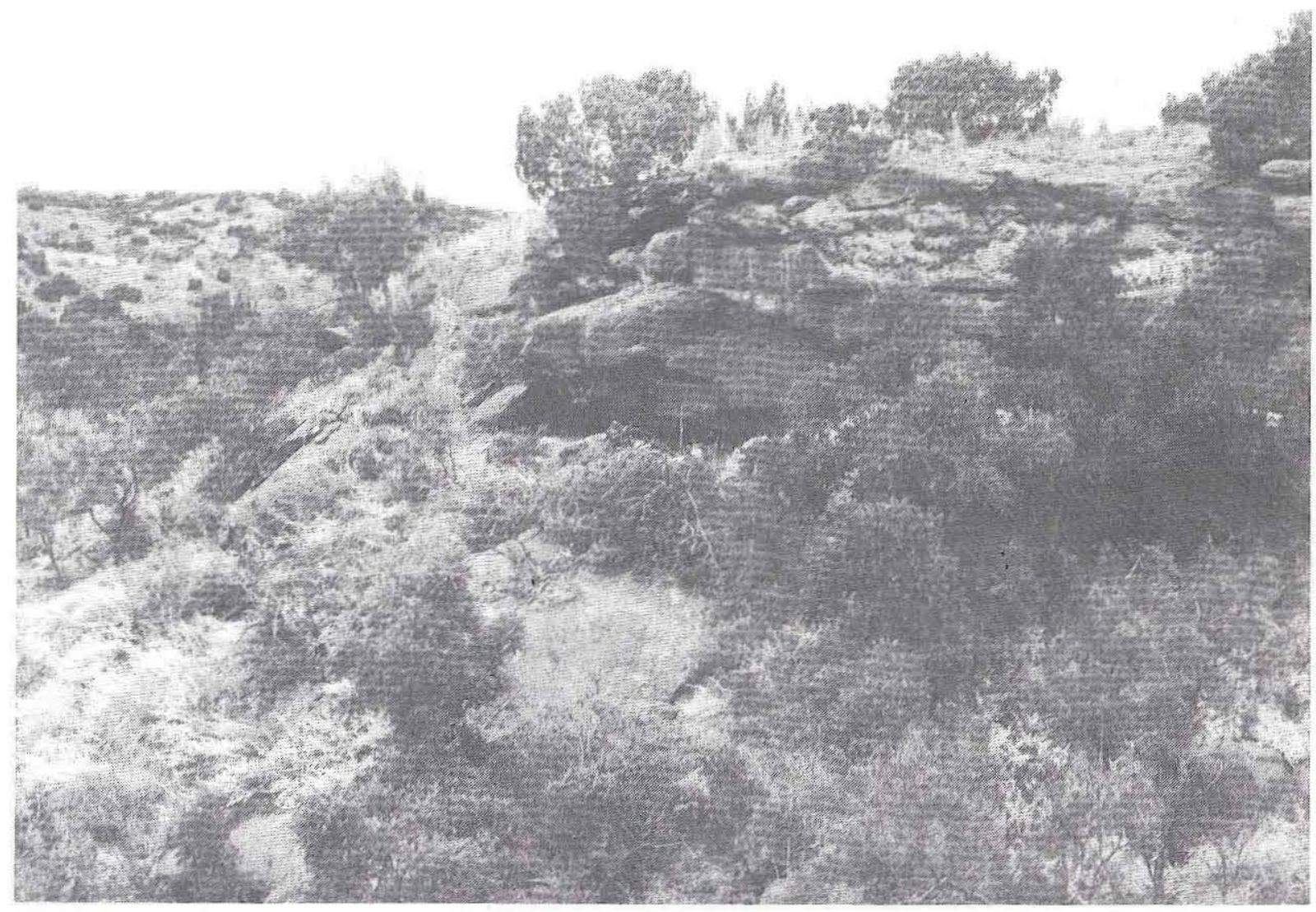

Figure 76. Rockshelter 41GR559; view is to the northwest from above and north of 41GR546.

yielded a considerable amount of rodent and rabbit bones, perhaps indicating the presence of a midden area. Test Units 1 and 2 were excavated inside the shelter, and Test Unit 3 was excavated at the dripline. They were dug to various depths, and all yielded cultural materials. Features 1-5 (burned rock concentrations) were exposed in Test Units 2 and 3, and a cluster of bones (Feature 6) was encountered in Test Units 1 and 2.

The following materials were recovered from the 1/4-inch screens: 1 snail shell from Test Unit 1; 2 hammerstones, 5 pieces of unmodified debitage, 53 bone fragments, and 2 snail shells from Test Unit 2; 1 core, 17 pieces of unmodified debitage, 10 pieces of microdebitage, 8 burned rocks, 4 bones or bone fragments, and 4 snail shells from Test Unit 3; 12 bones or bone fragments from Feature 6 in Test Units 1 and 2; 8 bones or bone fragments and 1 snail shell from Shovel Test 3; and 31 bones or bone fragments from Shovel Test 4. Flotation and fine screening of matrix samples produced 28 bone fragments from
Test Unit 2 and 113 bone fragments, 1 Uniomerus declivus shell fragment, and 116 snail shells from Test Unit 3 (see Boyd et al. 1990:563-569).

Macrobotanical remains were well preserved, but only the burned wood fragments were definitely of cultural origin. Taxa represented include juniper, oak, hackberry, and unidentified hardwood. It was noted that the noncharred plant remains and unburned bones could be of cultural or natural origin, and two medium-sized mammal rib fragments with canine teeth marks showed that animal predation had contributed to the assemblage.

\section{DATA RECOVERY INVESTIGATIONS}

Excavation of 41GR559 was undertaken over a period of ca. 6 work-weeks and required ca. 129 person-days of effort. A total of $20.87 \mathrm{~m}^{3}$ of sediment was extracted and screened through $1 / 4$-inchmesh hardware cloth. Initially, an arbitrary grid was reestablished based on a grid point designated 
N100/E100, which was set during the 1988 testing. This grid point was marked in 1988 with a piece of rebar driven into the ground and assigned an arbitrary elevation of $100 \mathrm{~m}$ above an imaginary datum plane. All subsequent three-dimensional measurements were taken relative to that point. The grid increases north and east from an imaginary zero point $100 \mathrm{~m}$ south and $100 \mathrm{~m}$ west of the rebar. The grid, oriented to magnetic north, was projected through the interior of the rockshelter (Figure 77) and across the entire area in front of the rockshelter. A permanent horizontal and vertical marker consists of an aluminum cap set in concrete at N100/E105.

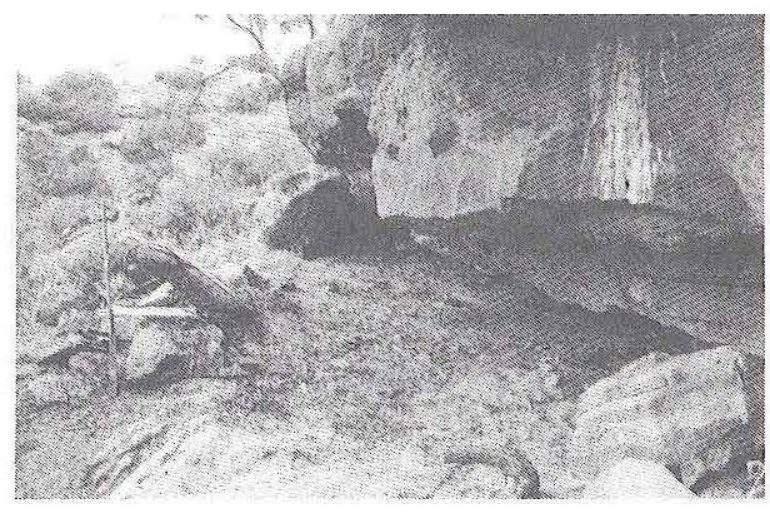

Figure 77. View west-northwest of the interior of the shelter at 41GR559.

Twenty-seven units were excavated in two areas of the site in 1992 (Figure 78). Initially, four units (EUs 17, 18, 20, and 21) were excavated and Test Units 2 and 3 (renumbered as EUs 19 and 22) from the 1988 testing were shoveled out to expose a 6-m-long profile extending east-west across the front of the rockshelter. To relate the stratigraphy inside the rockshelter to that of the suspected midden area in front, a line of units (EUs 2, 5, 8, and 11) was excavated along the north-south E100 gridline. This line was terminated on the north by a large roof block extending below the ground surface across the northern portion of EU 11. Excavation Units 12, 13, and 15 were excavated in an attempt to breach this blockage, but an impenetrable barrier of roof blocks extending the entire width in front of the rockshelter was encountered. Subsequently, seven more units were excavated to complete the midden block, and nine more units were excavated inside the rockshelter to investigate the area most productive of cultural materials as evidenced by the east-west trench.

The units were excavated in $10-\mathrm{cm}$-thick arbitrary levels, although levels less than or slightly greater than $10 \mathrm{~cm}$ in thickness were used for the upper proveniences to level off the excavations at even increments relative to the site datum. Units were excavated to depths ranging from 33 to $120 \mathrm{~cm}$, with the mean depth being $84 \mathrm{~cm}$. When the 1988 test units are added (renumbered as EUs 16, 19, and 22), a total of 30 units was excavated at 41GR559.

\section{SEDIMIENTS AND STRATIGRAPHY}

Recent rockshelter investigations have attempted to analyze the sediments and stratigraphy by various methods to interpret site formation, depositional processes, and paleoenvironments (e.g., Coffman et al. 1986; Colcutt 1979; Collins 1991; Courty et al. 1989; Donahue and Adovasio 1990; Farrand 1985; Laville et al. 1980). Simple variations of some of these methods were used to analyze the sediments and stratigraphy at 41GR559 to identify the endogenous and exogenous depositional processes responsible for sedimentation. This in turn allows interpretations of site formation and paleoenvironments to be made.

The sediments and stratigraphy at 41GR559 were observed and analyzed in the profiles of the archeological excavation units. Detailed descriptions of two of these excavation unit profiles are provided in Appendix A. Seven sediment samples from one of the profiles (EU 20) were subjected to analyses of organic matter and carbonate content and sediment grain size by hydrometer (see Appendix B). Twenty sediment samples from four other excavation units across the rockshelter were separated by sieve analysis into coarse (larger than -1.0 phi) and fine (smaller than -1.0 phi) fractions (also see Appendix B). The coarse fraction was further separated into two fractions consisting of clasts larger than -1.0 phi to smaller than -1.25 phi (coarse fraction II) and clasts larger than -1.25 phi (coarse fraction I).

The 41GR559 rockshelter is formed in soft, friable, fine-grained, cross-stratified, gray sandstone of the Upper Triassic Dockum Group. It underlies more-resistant Dockum gravel and mud clast conglomerates and coarse-grained, cross-stratified sandstones representing a sequence of channel fills deposited by braided streams (McGowen et al. 1979). Several boulders of these overlying rock units, representing brow retreat and earlier shelter collapse episodes, litter the surface in front of the shelter and downslope. 


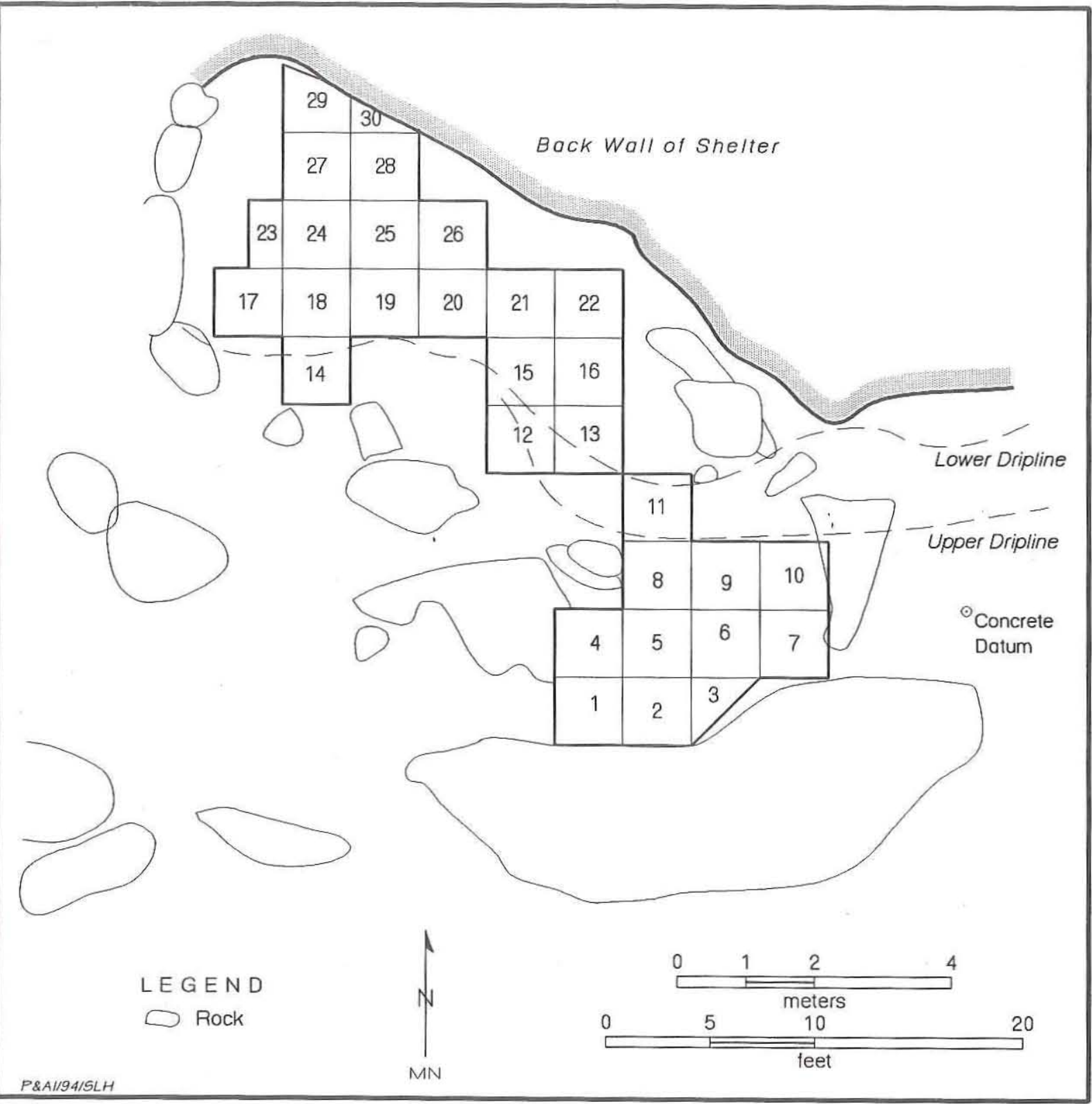

Figure 78. Plan of rockshelter and excavation units, 41GR559.

The deposits within the shelter vary in thickness due to the nature of the undulating bedrock floor. The fill varies from $100 \mathrm{~cm}$ in thickness in the middle and the back of the shelter to less than $40 \mathrm{~cm}$ along the western margin. Based on radiocarbon assays from the cultural features, sedimentation within the shelter has been ongoing for approximately the last 2,000 years. Visually, the fill can be divided into two general zones. The upper one is a $20-50-\mathrm{cm}-$ thick zone of reddish brown sediments which thickens and dips to the east, and the lower one is a zone of light brown sediments (Figure 79). Temporally, the stratigraphic break between the two zones is dated to ca. 900 B.P. based on radiocarbon assays from two features (Features 3 and 27) that occur just above the break or straddle it.

The rockshelter fill consists of a fine sandy loam matrix (fine fraction) with sandstone cobbles, pebbles, and gravels (coarse fraction). The fine fraction constitutes $70-99 \%$ of the sediment. Based on the hydrometer analysis of the fine fraction, sandsized particles are predominant. The percentages of 


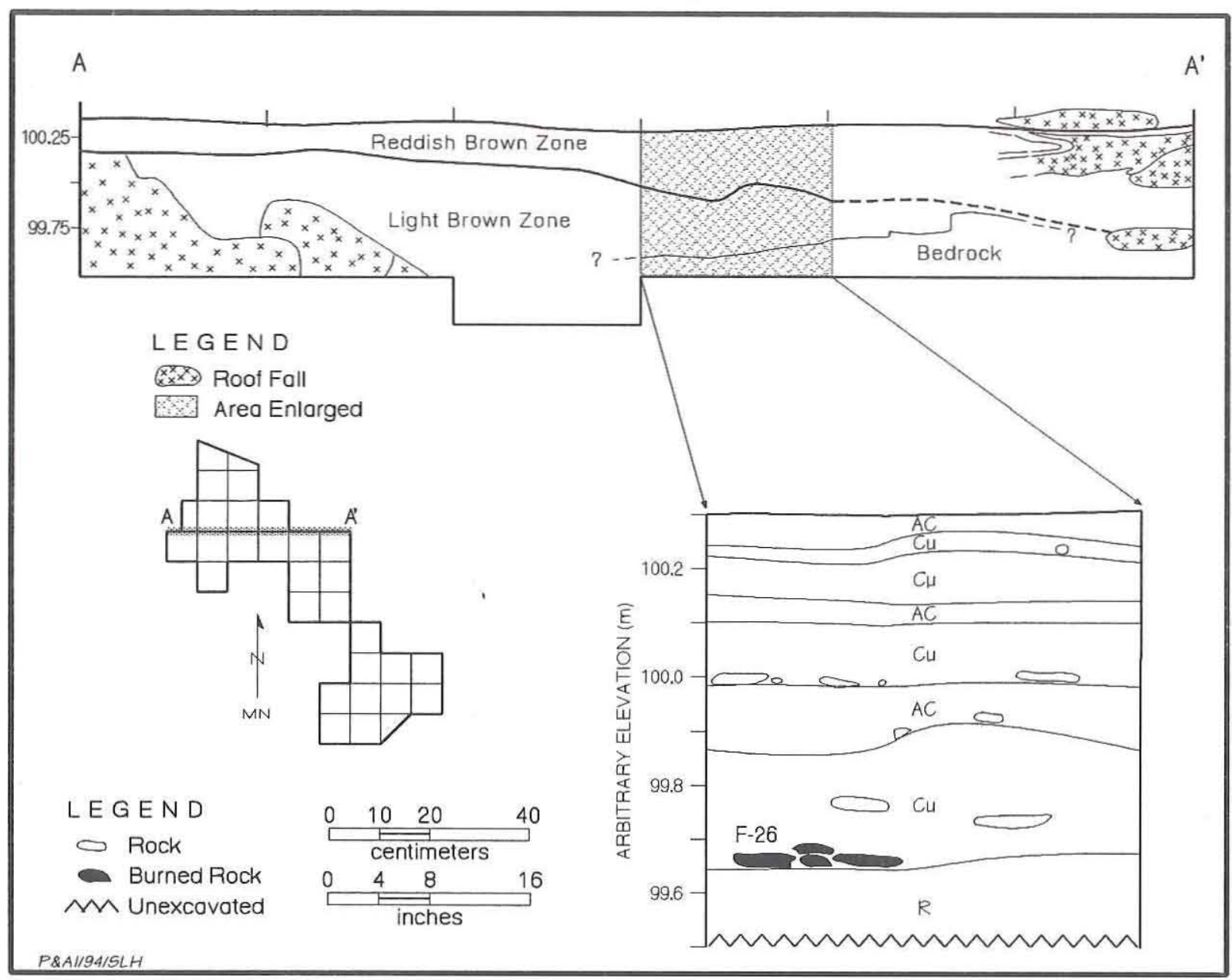

Figure 79. East-west profile of rockshelter fill depicting the two zones and detailed profile of EU 20, 41 GR559.

sand vary little through the vertical sequence of samples analyzed $(\overline{\mathrm{x}}=63.6 \%, \sigma=3.7)$, which include both the upper reddish brown zone and the underlying light brown zone. The percentages of siltand clay-sized particles vary even less. These factors make it difficult to further separate distinct zones and to interpret depositional agents, rates of accumulation, and change through time based on texture or granulometric data from the fine fraction. Since this is not unusual for rockshelter sediments (Farrand 1985:21), other methods were utilized to help determine the kinds of depositional agents, the rate of deposition and how it changed over time, and the presence of secondary or postdepositional weathering of the sediments.

Granulometric separation of the fine and coarse fractions and microscopic examination (100x) of the sand fractions of these samples reveal that most of the fill is endogenous, representing the grain by grain disintegration of the sandstone walls and ceiling and the spalling off of larger clasts. This is characteristic of samples from both the light brown zone and the overlying reddish brown zone. Donahue and Adovasio (1990) report that the Meadowcroft Rockshelter consists predominantly of similar sediments, rockfall, and sand grains delivered to the floor by the granular disintegration of the shelter's walls and ceiling. Rockfall is defined as the release of gravel-sized or larger clasts from the ceiling or wall through the widening of joints and bedding planes by water, freeze-thaw cycles, , biological activity, or any other agent (Donahue and Adovasio 1990:236). The release of individual sand grains, however, is poorly understood, although it probably is determined to a large extent by the type of cement holding the sand grains together and most likely 
involves both physical and chemical weathering. Most of the coarse fraction of the fill consists of gravel- and pebble-sized sandstone clasts. Many are flat in shape with sharp angular edges and probably were deposited through spalling brought on by a number of weathering and erosional processes. A major episode of roof fall is evident from a large slab located at the western margin of the shelter and most likely predates the ca. 2,000-year-old basal sediments. This large slab partially protects or closes the western reentrant of the shelter, creating an opening toward the back of the shelter. The coarse fraction also contains rare quartzitic gravels and rock fragments. These are present throughout the light brown zone in the front and back of the shelter. Quartzite gravels and pebbles litter the surface above the rockshelter and are probably lag deposits of the Lingos Formation (see Caran and Baumgardner 1990). The quartzitic gravels in the shelter deposits most likely represent colluvium washed downslope into the western reentrant and into the back of the shelter. Dessication cracks on the surface in the back of the shelter indicate the presence of water-laid deposits.

Run-off funneled into the back of the shelter may also explain why the fill in that area is as thick as or thicker than the fill in the front of the shelter. As a shelter forms, its back wall retreats and moves up due to the accumulation of sediment along the back wall which protects its lower portions from weathering. The continuous influx of water into the back of Boren Shelter \#2 probably left the sediments there saturated for long periods, leaving the back wall more susceptible to chemical and physical weathering. Water entering the shelter also removed unconsolidated sediments as it drained out the front of the shelter along its eastern margin. This appears to have been a more common occurrence prior to ca. 900 B.P., as the channel along the eastern margin of the shelter is filled with the reddish brown sediments of the upper zone.

Microscopic examination of the sand fractions of a vertical sequence of samples from EU 18 also indicates a predominance of endogenous sediments throughout the shelter fill. The samples are dominated by fine angular quartz sand grains, coarse and very coarse sand-sized aggregrates of quartz and mica, and mica flecks. Rounded fine quartz sand grains are common in the sample from Level 2 but decrease substantially in frequency in the samples from lower levels. The rounded fine sand grains are indicative of eolian deposition and are represented in the upper reddish brown zone, which postdates ca. 900 B.P. The reddish brown hue probably comes from eolian dust consisting of clays and silts derived from the Permian red beds or possibly the Blackwater Draw Formation to the west. Unlike 41GR546, Boren Shelter \#2 is open to the prevailing wind and therefore conducive to capturing eolian sediment.

Sedimentation within the shelter is dominated by endogenous deposition, represented by the in situ weathering of the ceiling and walls. Colluvial and eolian processes are exogenous agents responsible for minor contributions of sediment into the shelter; however, these processes were not synchronic. Colluvial processes were considerably more active during the early period of shelter sedimentation, while eolian processes became more prevalent after ca. 900 B.P. It probably is not a coincidence that eolian sedimentation increased markedly after ca. 900 B.P., for a shift to increasingly xeric conditions is well documented across the Southern Plains and adjacent regions (e.g., Abbott 1990; Blum and Valastro 1989; Ferring 1986; Hall 1982, 1988; Holliday 1985; Meltzer 1991).

Based on their work in the Perigord region of France, Laville et al. (1980) suggest that changes in the amount and average size of the gravel-sized or larger clasts in the fill of rockshelters represent fluctuating climatic conditions. However, a direct correlation between their work and the Boren Shelter \#2 fill is tenuous because of differences in shelter lithology (i.e., limestone vs. sandstone). Donahue and Adovasio (1990:237, 240) note that increases in the amount of rockfall within a sandstone shelter's fill are not related to climatic changes but rather to major roof fall events and the initial development of the shelter. Preliminary observations of the coarse- and fine-fraction percentages from a vertical sequence (EU 18) of samples in the 41GR559 shelter do reveal a decrease in the amount of coarse fraction from the lower light brown zone to the upper reddish brown one. Cobble-sized clasts were not collected as part of the fill to be analyzed from 41GR559, but field observations noted that these larger clasts generally are limited to the deeper deposits immediately above the bedrock floor. However, the up-profile decrease in rockfall observed in the front of the shelter (EU 18) is opposite that observed in the back of the shelter (EU 29). Given the great fluctuations in the coarse-fraction percentages $(\bar{x}=9.5 \%, \sigma=9.3)$ from level to level in EU 29, it is probable that the record 
is distorted due to mixing of the endogenous sediments with colluvium or the removal of sediments. Clearly, the preliminary study of the coarse fraction tends to support Donahue and Adovasio's concepts of sandstone shelter development.

Because the fine fraction is predominant throughout the fill, it appears that grain by grain disintegration of the walls and ceiling was the dominant process of endogenous deposition in the shelter rather than the spalling off of larger clasts. However, this interpretation does not consider whether secondary weathering has had any effect on the deposits.

Separation of the coarse fraction into larger size (coarse fraction I) and smaller size (coarse fraction II) components was accomplished to see if secondary weathering of larger sandstone clasts is responsible for the predominance of sand-sized particles. If this is the case, then coarse fraction II sediments should occur in higher percentages in the older deposits. A vertical sequence of coarse-fraction samples taken from EU 18 reveals that the coarse fraction II percentages do increase with depth (see Appendix B). Further, the EU 18 percentages tend to be higher than those in samples taken from EU 29 in the back of the shelter. This suggests that a considerable amount of the sand within the fine fraction may be the result of secondary weathering of the coarse fraction through the disintegration of carbonate cement and that the spalling off of larger clasts may be more prevalent than indicated in the geologic record. It also implies that the disintegration of larger clasts is chronologically controlled, regardless of whether the environment was aggrading or stable.

Postdepositional weathering related to hiatuses in sedimentation also is evident in the shelter. Pauses in sedimentation within the rockshelter can be delineated from the carbonate percentages of the samples from EU 20 (see Appendix B), which provide evidence of leaching. Correlated with zonal boundary characteristics within the profile, at least three episodes of decreased sedimentation and weathering are suspected, all of which occur within the upper reddish brown zone.

Detailed profile documentation and description of the north wall of EU 20 identified eight zones within the $66-\mathrm{cm}$ profile (see Figure 79 and Appendix A). The upper seven zones consist of brown, dark brown, and dark yellowish brown friable fine sandy loams. Zones 1-6 (0-44 cm) are within the upper reddish brown zone, while Zone 7
(44-66 cm) is in the underlying light brown zone; Zone $8(66+\mathrm{cm})$ is weathered bedrock. Zones 1,4 , and 6 represent weakly developed soils or structureless AC horizons. Buried soils, Zones 4 and 6 , are separated by abrupt boundaries from the overlying sediments, which represent the return to intervals of deposition. Carbonate percentages tend to increase with depth below each $\mathrm{AC}$ horizon, implying that leaching is prevalent. The occurrence of these soils in the upper reddish brown zone indicates the following: (1) sedimentation and probably climatic conditions prior to ca. 900 B.P. were continuous and consistent; and (2) sedimentation, particularily eolian sedimentation, occurred in pulses or episodes after ca. 900 B.P., with the climatic regime probably being highly variable.

In sum, Boren Shelter \#2 has been filling for ca. 2,000 years. The sedimentary fill can be divided into two general zones based on sediment color changes. The stratigraphic break between these two zones is tentatively dated at ca. 900 B.P. Both zones, the upper reddish brown and the underlying light brown, consist predominantly of endogenous sediments. These sediments consist of cobble-, pebble-, gravel-, and sand-sized clasts representing the in situ weathering of the shelter's ceiling and walls, although larger clasts are more frequent in the lower light brown zone and most likely are associated with a major episode of roof fall prior to 2000 B.P. Sandsized particles dominate the fill but may not accurately reflect the depositional processes at work in the shelter as they may be a result of postdepositional disintegration of larger clasts.

Two exogenous agents also have contributed sediments. A minor colluvial component is represented in the light brown zone by rare quartzitic gravels and rock fragments. Prior to ca. 900 B.P., slopewash entering the back of the shelter and draining out the front along its eastem margin was common. This not only removed small amounts of unconsolidated sediments from the back of the shelter but also probably accelerated the weathering of the back wall.

A climatic shift to more-xeric conditions is evidenced by episodic eolian deposition after ca. 900 B.P. This correlates with other regional data suggesting that the late Holocene environment became more xeric around 1000 B.P. (e.g., Hall 1982, 1988; Holliday 1985; Meltzer 1991). Although eolian sedimentation occurred more frequently after ca. 900 B.P., in situ weathering of the ceiling and walls 
continued to be the predominant agent of sedimentation, while the influx of slopewash or colluvium became less common and more variable. Intervals of stability and leaching are evident from three weakly developed soils within the fill. All three soils formed in the upper reddish brown zone, implying a change from continuous sedimentation to more variable sedimentation at ca. 900 B.P.

\section{CULTURAL FEATURES}

Twenty-seven anomalies were recorded as features in the field (Table 53 and Figure 80). Six of these (Features 1-6) were discovered during the 1988 testing (Boyd et al. 1990). Feature 21 was recorded in the field as a deflector slab, but it was determined during the postfield analysis to be noncultural. Two features (13 and 26) in adjoining excavation units were found to be portions of the same feature. Thus, 25 features are considered to be cultural in origin.
The cultural features consist of two unlined hearths, seven stone-lined hearths, five burned rock scatters or disaggregated hearths, one burned rock concentration, two hearth dumps, one wind deflector slab, and seven bone clusters.

Features $7,9,10$, and 12 were recorded in the field as separate features because they were encountered as separate small bone clusters. However, they were found in close proximity, and they may not represent discrete units. Nine radiocarbon dates (including two from the 1988 testing) were obtained from the features (Table 54).

\section{Feature 1}

Feature 1 consists of a dispersed concentration of burned sandstone and sandstone conglomerate rocks in EUs 19, 20, and 24. The feature was originally recorded in Test Unit 3 (EU 19) during the 1988 testing (Boyd et al. 1990), and subsequent

\begin{tabular}{|c|c|c|c|c|}
\hline \multicolumn{5}{|c|}{ TABLE 53} \\
\hline Feature No. & Type & Provenience & Top Elevation (m) & Bottom Elevation (m) \\
\hline 1 & Disaggregated hearth & EUs 19,20 , and 24 & 100.12 & 100.04 \\
\hline 2 & Disaggregated hearth & EUs 19,20 , and 26 & 100.04 & 99.90 \\
\hline 3 & Stone-lined hearth & EU 22 & 99.61 & 99.49 \\
\hline 4 & Disaggregated hearth & EU 19 & 99.71 & 99.60 \\
\hline 5 & Disaggregated hearth & EUs 19,24 , and 25 & 99.60 & 99.52 \\
\hline 6 & Bone cluster & EUs 15,16 , and 22 & 100.21 & 100.08 \\
\hline 7 & Bone cluster & EU 9 & 99.65 & 99.60 \\
\hline 8 & Stone-lined hearth & EUs 17,18 , and 23 & 99.87 & 99.67 \\
\hline 9 & Bone cluster & EUs 7 and 10 & 99.65 & 99.60 \\
\hline 10 & Bone cluster & EUs 6 and 7 & 99.72 & 99.62 \\
\hline 11 & Disaggregated hearth & EUs 20 and 21 & 99.96 & 99.86 \\
\hline 12 & Bone cluster & EU 7 & 99.65 & 99.60 \\
\hline $13 / 26$ & Stone-lined hearth & EUs 20 and 26 & 99.80 & 99.65 \\
\hline 14 & Bone cluster & EU 2 & 99.68 & 99.62 \\
\hline 15 & Burned rock concentration & EU 17 & 100.00 & 99.50 \\
\hline 16 & Unlined hearth & EUs 19 and 25 & 99.33 & 99.31 \\
\hline 17 & Bone cluster & EUs 1 and 2 & 99.28 & 99.21 \\
\hline 18 & Stone-lined hearth & EU 28 & 100.04 & 99.89 \\
\hline 19 & Hearth dump & EUs 1 and 4 & 99.12 & 98.88 \\
\hline 20 & Wind deflector & EU 14 & 100.16 & 99.26 \\
\hline 22 & Stone-lined hearth & EU 24 & 100.08 & 99.95 \\
\hline 23 & Hearth dump & EU 23 & 100.14 & 100.09 \\
\hline 24 & Unlined hearth & EU 27 & 99.62 & 99.60 \\
\hline 25 & Stone-lined hearth & EU 27 & 99.54 & 99.50 \\
\hline 27 & Stone-lined hearth & EU 24 & 100.25 & 100.14 \\
\hline
\end{tabular}




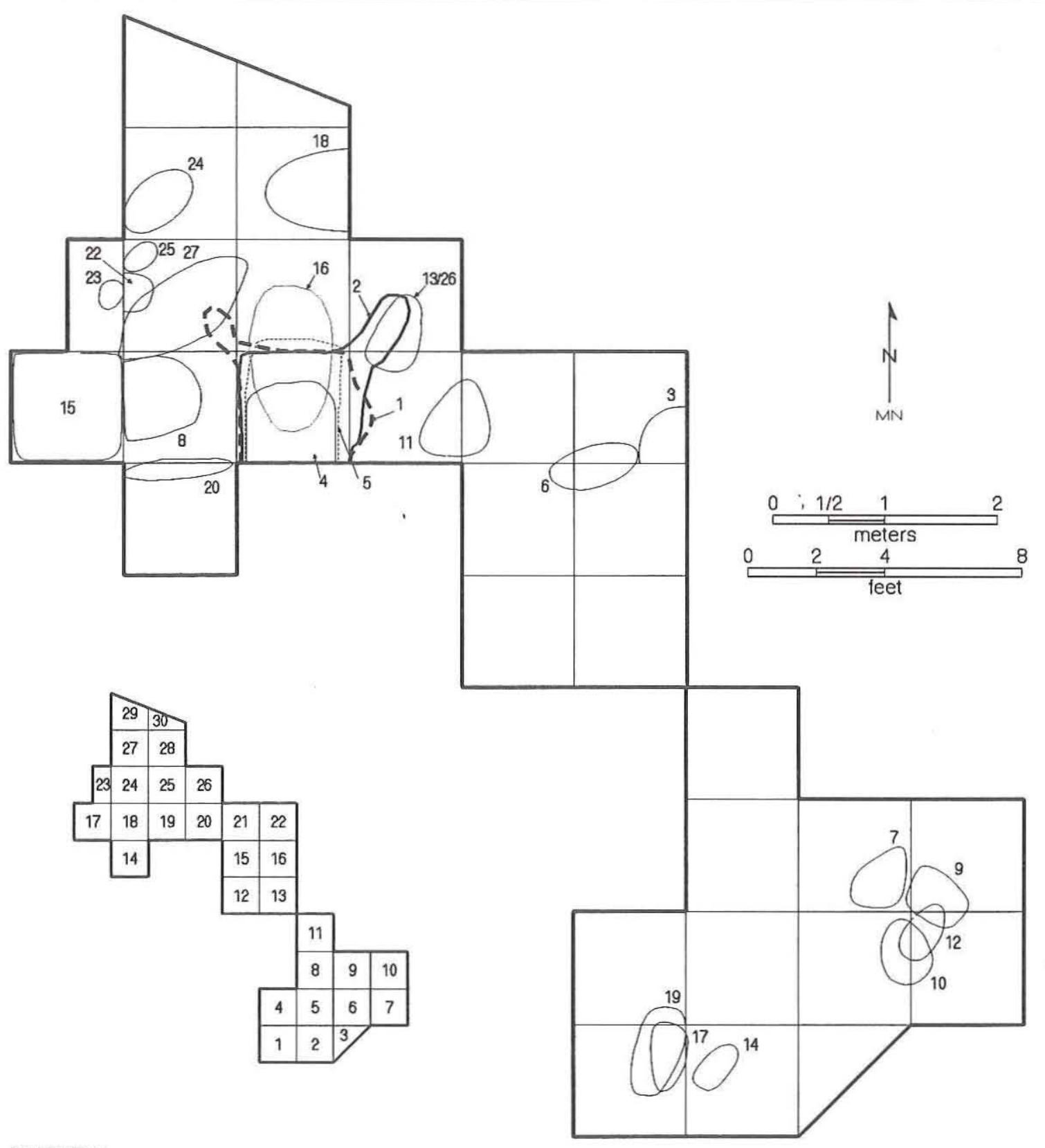

P\&AI/94/SLH

Figure 80. Plan of cultural features, 41GR559.

excavation in 1992 revealed additional parts of the feature in two adjacent units. The weight of the rocks is $6.3 \mathrm{~kg}$ (ca. 20 rocks) in EU $19,5.0 \mathrm{~kg}$ (ca. 23 rocks) in EU 20 , and $2.5 \mathrm{~kg}$ (ca. 10 rocks) in EU 24, for a total weight of $13.8 \mathrm{~kg}$. The feature occurs at a depth of $20-28 \mathrm{~cm}$, and the overall rock scatter extends $150 \mathrm{~cm}$ southeast-northwest and $100 \mathrm{~cm}$ southwest-northeast. Feature 1 is topmost in a stack of hearths and disaggregated hearths in EU 19, overlying Features 2, 4, 5, and 16.

No soil discoloration was observed, but some scattered charcoal was found in association with the feature. Several laboratory tests were run on the feature contents after the 1988 testing. Pollen analysis of the fill resulted in no interpretable results due to poor preservation. Phytolith analysis yielded 


\begin{tabular}{|c|c|c|c|c|}
\hline \multicolumn{5}{|c|}{ TABLE 54} \\
\hline Lab No. & Provenience & $\begin{array}{l}\text { Uncorrected } \\
\text { Age B.P. }\end{array}$ & $\begin{array}{l}\text { Corrected Age } \\
\text { B.P.* }\end{array}$ & $\begin{array}{l}\text { Calibrated Date (Intercepts and } \\
\text { 1-Sigma Range)** }\end{array}$ \\
\hline GX 14448 & Feature 3 & $815 \pm 190$ & $855 \pm 190(-22.7)$ & A.D. 990 (1191) 1290 \\
\hline GX 14449 & Feature 5 & $1155 \pm 210$ & $1180 \pm 210(-23.3)$ & A.D. $650(880) 1030$ \\
\hline Beta-60261 & Feature 16 & $1920 \pm 70$ & $1930 \pm 70(-24.9)$ & 2 B.C. (A.D. 72) A.D. 129 \\
\hline Beta-60262 & Feature $13 / 26$ & $1730 \pm 80$ & $1720 \pm 80(-25.4)$ & A.D. $227(265,281,333) 412$ \\
\hline Beta-59829 & Feature 18 & $790 \pm 80$ & $790 \pm 80(-24.9)$ & A.D. 1169 (1252) 1277 \\
\hline Beta-59831 & Feature 22 & $1320 \pm 110$ & $1300 \pm 110(-26.5)$ & A.D. 640 (681) 860 \\
\hline Beta-59832 & Feature 24 & $1520 \pm 90$ & $1530 \pm 90(-24.5)$ & A.D. 420 (544) 622 \\
\hline Beta-59830 & Feature 27 & $950 \pm 60$ & $930 \pm 60(-26.4)$ & A.D. $1021(1043,1105,1112,1150) 1169$ \\
\hline Beta-61502*** & $\begin{array}{l}\text { Features } 9 \\
\text { and } 12\end{array}$ & $30 \pm 70^{\circ}$ & $280 \pm 70(-9.7)$ & A.D. 1506 (1642) 1663 \\
\hline $\begin{array}{l}\text { *Ages uncali } \\
{ }^{* *} \text { Calibrations } \\
* * * \text { Date is on } b\end{array}$ & $\begin{array}{l}\mathrm{d} ; \delta^{13} \mathrm{C} \text { val } \\
20 \text {-year re } \\
\text { collagen; al }\end{array}$ & $\begin{array}{l}\text { parenthese } \\
\text { f Stuiver ar } \\
\text { rs are on ch }\end{array}$ & imer (1986). & \\
\hline
\end{tabular}

mostly festucoid and elongate varieties. Feature 1 is interpreted as a disaggregated hearth because of the dispersed nature of the concentration.

\section{Feature 2}

Feature 2 consists of a dispersed concentration of burned sandstone and conglomerate rocks in EUs 19,20 , and 26, with small clusters in each unit and scattered rocks in between. The weight of the rocks is $10.5 \mathrm{~kg}$ (ca. 22 rocks) in EU 19 (Test Unit 3), $2.0 \mathrm{~kg}$ (ca. 9 rocks) in EU 20, and $6.5 \mathrm{~kg}$ (ca. 5 rocks) in EU 26, for a total weight of $19.0 \mathrm{~kg}$. Feature 2 occurs at a depth of $28-42 \mathrm{~cm}$. The associated rock scatter extends $150 \mathrm{~cm}$ southwestnortheast and $100 \mathrm{~cm}$ southeast-northwest.

The portion of Feature 2 in EU 19 was discovered during the 1988 testing (Boyd et al.1990), and the parts in EUs 20 and 26 were found during the Season 3 excavations. Extensive charcoal stains and nodules of charcoal were found in EUs 19 and 20. Twelve pieces of debitage were found in the feature. Flotation of the fill after the 1988 testing recovered burned juniper and hackberry wood, a burned rabbit tooth fragment, and numerous remains that are probably noncultural (i.e., noncharred plant remains, rodent bones, a rabbit tooth, and snail shells). Seven unidentifiable fragments of bones of small mammals are burned and appear to be cultural remains (Boyd et al. 1990:564). Due to the dispersed nature of the concentration, Feature 2 is interpreted as a disaggregated hearth.

\section{Feature 3}

Feature 3 is a cluster of burned and unburned sandstone and unburned conglomerate rocks in EU 22 (Test Unit 2) investigated during the 1988 testing (Boyd et al. 1990). Of the eight rocks constituting the feature, five weighing ca. $2 \mathrm{~kg}$ are burned. The feature occurs at a depth of $71-83 \mathrm{~cm}$. The cluster extends $50 \mathrm{~cm}$ north-south and $50 \mathrm{~cm}$ east-west and into the east and south walls of the unit. The rocks lie in a semicircle near the margin of an area of burned gravels and charcoal found in the southeastern quadrant of the unit. A radiocarbon assay on charcoal yielded a calibrated date of A.D. 990 (1191) 1290 (see Table 54). A thermoluminescence assay on burned sandstone yielded a date of A.D. $1470 \pm 105$, but the thermoluminescence study done during the 
Phase II testing indicates that such dates on burned rocks are not reliable.

Postfield analysis yielded evidence of chloridoid and elongate phytoliths, plant matter in an organic extraction from a burned rock, and flotationrecovered burned oak and hackberry wood, noncharred hackberry seeds, and snail shells, all considered to be noncultural (Boyd et al. 1990:564). Phytolith analysis also revealed the presence of Opuntia sp. calcium oxylate crystals in samples from this feature, indicating that prickly pear pads may have been processed there (Cummings 1990:541). Feature 3 also produced 54 rodent bones and a possibly cultural burned snake vertebra during the 1988 testing (Boyd et al. 1990:564).

The area of the site containing Feature 3 is highly disturbed, with the feature lying directly beneath a large block of roof fall. The feature lies within and near the eastern terminus of an erosional channel cut along the back of the rockshelter by runoff. Feature 3 lies just beneath the contact between the upper reddish brown and lower light brown sediments, and its radiocarbon date helps bracket this depositional change. The late date for Feature 3 and the overlying roof fall lead to the possibility that the feature was constructed after the change in the depositional regime and was then pushed downward into the light brown sediments by the weight and impact of the roof-fall event. Feature 3 is interpreted as a stone-lined hearth due to its circular shape.

\section{Feature 4}

Feature 4 consists of an amorphous cluster of eight burned sandstone rocks and a scatter of eight burned sandstone rocks in Test Unit 3 (EU 19) discovered during the 1988 testing (Boyd et al. 1990). A possible extension of the feature in the southeast corner of EU 24 was discovered during the 1992 excavations. It consists of a 20-cm-diameter area of red burned soil and a small amount of associated charcoal nodules. The main portion of the feature occurs at a depth of $61-72 \mathrm{~cm}$, while the extension occurs at $56-66 \mathrm{~cm}$. The rock scatter extends $80 \mathrm{~cm}$ north-south and $85 \mathrm{~cm}$ east-west and into the south wall of the unit. The total weight of the ca. 16 rocks is $5.5 \mathrm{~kg}$.

No soil discoloration was observed in EU 19, but a large scatter of charcoal flecks was noted in the northwestern quadrant of the unit connecting the rock scatter to the red stain in EU 24. Twenty-six bones1 canid tooth, 3 long bones of medium-sized mammal, and 22 rodent bones - were recovered from the charcoal scatter in EU 19 during the 1988 testing (Boyd et al 1990:150). Nine fragments of unidentifiable rodent bones and one rodent calcaneus are burned. Four bones identifiable only as vertebrate were recovered from a comparable depth in EU 24 during the 1992 excavations (see Appendix C). A macrobotanical sample from the Feature 4 extension in EU 24 produced five seeds of dropseed and burned ring-porous hardwood (Table 55; see Appendix E). Feature 4 is interpreted as a disaggregated hearth due to the dispersed nature of the rock cluster.

\section{Feature 5}

Feature 5 consists of an amorphous cluster of burned sandstone rocks and charcoal-stained soil spread across all of Test Unit 3 (EU 19). The feature was discovered during the 1988 testing (Boyd et al. 1990) and was found during the 1992 excavations to extend into EU 25. The charcoal stain also extends into EU 24, along with small fragments of burned sandstone. The weight of the rocks is $3.2 \mathrm{~kg}$ (10 rocks) in EU 19 and $1.5 \mathrm{~kg}$ (3 rocks) in EU 25. The feature occurs at a depth of $72-80 \mathrm{~cm}$. The rock scatter and charcoal stain extend $120 \mathrm{~cm}$ north-south and $85 \mathrm{~cm}$ east-west.

A radiocarbon assay on charcoal yielded a calibrated date of A.D. 650 (880) 1030 (see Table 54); a thermoluminescence assay on burned sandstone yielded no date, probably due to insufficient heating of the specimen. Analyses of materials recovered during the testing confirmed poor pollen preservation, the presence of festucoid and elongate phytoliths, and probable plant residue in an organic extraction from a burned rock. Four recovered unidentifiable bone fragments are unburned and do not appear to be cultural (Boyd et al 1990:565). Feature 5 is interpreted as a disaggregated hearth because of the scattered nature of the rocks.

\section{Feature 6}

Feature 6 is a bone cluster in the upper levels of EUs 16, 21, and 22. During the 1988 testing, a left mandible of an adult deer and three maxillary fragments were recovered from Test Units 1 and 2 (EUs 16 and 22), along with seven fragments of mammal bones and one unidentifiable fragment (Boyd 


\begin{tabular}{|c|c|c|c|c|c|}
\hline \multicolumn{6}{|c|}{$\begin{array}{l}\text { TABLE } 55 \\
\text { SUMMARY OF FEATURE FLOTATION SAMPLES AND } \\
\text { MACROBOTANICAL RECOVERY, 41GR559 }\end{array}$} \\
\hline $\begin{array}{l}\text { Feature } \\
\text { No. }\end{array}$ & $\begin{array}{l}\text { Flotation } \\
\text { Sample No. }\end{array}$ & $\begin{array}{l}\text { Description of } \\
\text { Archeological Context }\end{array}$ & $\begin{array}{l}\text { Sediment Volume } \\
\text { Processed (liters) }\end{array}$ & $\begin{array}{l}\text { No. of Charred } \\
\text { Seeds Recovered }\end{array}$ & $\begin{array}{l}\text { Carbonized } \\
\text { Wood } \\
\text { Identified }\end{array}$ \\
\hline 4 & 43 & EU $24,99.70-99.80 \mathrm{~m}$ & 0.5 & 5 & single taxa \\
\hline 6 & 22 & EUs 16 and $22,100.06-100.21 \mathrm{~m}$ & 3.5 & - & multiple taxa \\
\hline 8 & $\begin{array}{l}18 \\
21 \\
31 \\
35 \\
45\end{array}$ & $\begin{array}{l}\text { EU } 18,99.70-99.80 \mathrm{~m} \\
\text { EU } 18,99.60-99.70 \mathrm{~m} \\
\text { EU } 18,99.50-99.60 \mathrm{~m} \\
\text { EU } 18,99.60-99.70 \mathrm{~m} \\
\text { EU } 24,99.50-99.60 \mathrm{~m}\end{array}$ & $\begin{array}{l}4.0 \\
2.5 \\
4.0 \\
3.0 \\
5.0\end{array}$ & $\begin{array}{r}5 \\
5 \\
10 \\
- \\
2\end{array}$ & $\begin{array}{l}\text { multiple taxa } \\
\text { multiple taxa } \\
\text { multiple taxa } \\
\text { multiple taxa } \\
\text { single taxa }\end{array}$ \\
\hline 12 & 28 & EU 7, 99.60-99.65 m & 3.5 & 4 & - \\
\hline 13 & 27 & EU $20,99.60-99.70 \mathrm{~m}$ & 5.0 & $\dot{-}$ & single taxa \\
\hline 16 & $\begin{array}{l}36 \\
37\end{array}$ & $\begin{array}{l}\text { EU } 19,99.30-99.40 \mathrm{~m} \\
\text { EU } 25,99.30-99.40 \mathrm{~m}\end{array}$ & $\begin{array}{l}5.0 \\
5.0\end{array}$ & $\begin{array}{r}8 \\
15\end{array}$ & $\begin{array}{l}\text { multiple taxa } \\
\text { single taxa }\end{array}$ \\
\hline 18 & 39 & EU $28,99.90-100.00 \mathrm{~m}$ & 5.5 & 5 & multiple taxa \\
\hline 19 & 41 & EU $1,99.00-99.10 \mathrm{~m}$ & 4.0 & 3 & single taxa \\
\hline 24 & 48 & EU $27,99.50-99.60 \mathrm{~m}$ & 0.5 & - & single taxa \\
\hline 25 & 50 & EU $27,99.50-99.60 \mathrm{~m}$ & 2.5 & 1 & multiple taxa \\
\hline 26 & $\begin{array}{l}69 \\
70 \\
71\end{array}$ & $\begin{array}{l}\text { EU } 26,99.70-99.80 \mathrm{~m} \\
\text { EU } 26,99.65-99.70 \mathrm{~m} \\
\text { EU } 26,99.60-99.65 \mathrm{~m}\end{array}$ & $\begin{array}{l}2.0 \\
5.0 \\
6.0\end{array}$ & $\begin{array}{r}- \\
7 \\
17\end{array}$ & $\begin{array}{l}\text { single taxa } \\
\text { single taxa } \\
\text { multiple taxa }\end{array}$ \\
\hline- & 53 & EU $29,100.20-100.30 \mathrm{~m}$ & 3.5 & 1 & - \\
\hline- & 58 & EU $29,99.90-100.00 \mathrm{~m}$ & 3.5 & 9 & - \\
\hline- & 67 & EU $26,99.90-100.00 \mathrm{~m}$ & 3.5 & - & multiple taxa \\
\hline - & 68 & EU $26,99.80-99.90 \mathrm{~m}$ & 3.0 & - & multiple taxa \\
\hline
\end{tabular}

et al. 1990). During the 1992 excavations, the feature was found to extend into EU 15 with the discovery of a right mandible from an adult deer and six teeth from a deer at least 7 years old at the same level (see Appendix C). A macrobotanical sample from the feature area of EU 15 produced a sample of ring-porous hardwood (see Table 55 and Appendix E). The feature occurs at a depth of $12-25 \mathrm{~cm}$. The total bone cluster extends $70 \mathrm{~cm}$ east-west and $37 \mathrm{~cm}$ north-south.

Rodent bones also were scattered throughout the test unit at this level. Canine tooth marks were discovered on unidentified mammal rib heads found associated with the feature during the testing (Boyd et al. 1990:564). This feature may be cultural, or it may be the result of carnivore predation. Evidence of gnawing and no evidence of butchering or burning point to the latter. No soil discoloration or charcoal were noted in association with Feature 6.

\section{Feature 7}

Feature 7 (Figure 81) is a cluster of 313 bones of wolf, dog, bobcat, rodent, and unidentified 
carnivore, mammal, and vertebrate at a depth of 54$59 \mathrm{~cm}$ in EU 9 (see Appendix C). The bone cluster extends $50 \mathrm{~cm}$ north-south and $25 \mathrm{~cm}$ east-west and is in the upper reddish brown sediment zone. In an area of overlap between Features 7, 9, 10, and 12, 82 bones of rodent, mammal, and vertebrate were recovered (see Appendix C). Only one bone of the complex of Features 7, 9, 10, and 12 bears any trace of cultural modification (i.e., a spirally fractured rodent bone), and it came from Feature 9.

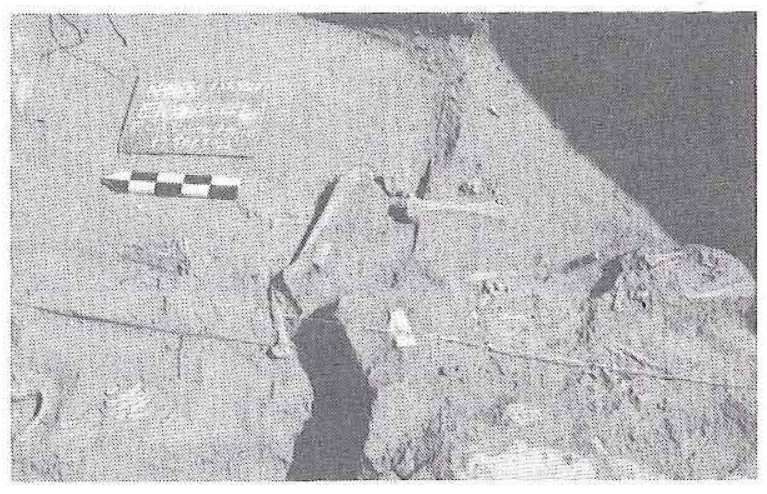

Figure 81. View to the east of Features 7, 9, 10, and 12, 41 GR559.

No soil discoloration or charcoal was observed directly associated with the feature; however, a change in soil composition was noted from west to east in EU 9 that carries into EUs 7 and 10. West of that line, the soil is hard packed and gravelly, whereas to the east the soil is a darker sandy loam with few gravels which suggests a pit, although no pit outline could be discemed. The feature is east of the change and in an area that may represent a natural deposit of clean fill in a cavity. Two hammerstones and a chert core were recovered from the edge of the feature at this point of change. The bones of Feature 7 were found jumbled and not in natural osteological association, although some partial articulation was present (see Appendix C).

\section{Feature 8}

Feature 8 consists of a circle of burned sandstone and sandstone conglomerate rocks in EU 18. The total weight of the rocks in EU 18 is $8.8 \mathrm{~kg}$ (ca. 12 rocks). Burned rocks were found in adjacent units at the same level as Feature 8. Eighteen small burned rocks weighing $3.8 \mathrm{~kg}$ were recovered from EU 23 to the north of Feature 8, and many small burned rocks weighing a total of $12.3 \mathrm{~kg}$ were recovered from EU 17 to the west of Feature 8. Also possibly related is a large upright slab of sandstone which may be a wind deflector (Feature 20) that is southeast of the feature in EU 14.

Feature 8 occurs at a depth of $49-69 \mathrm{~cm}$ and extends $85 \mathrm{~cm}$ north-south and $75 \mathrm{~cm}$ east-west. It does not extend into any adjacent units. No soil discoloration was present, but a large charcoal sample was collected from the feature. One cobble tool and a hammerstone were found in direct association with this feature. Also from within this feature, eight bones of Canis sp. and unidentified mammal and vertebrate were recovered (see Appendix C). Of these, one mammal bone is charred and one other mammal bone exhibits spiral fracturing. The three Canis sp. bones are probably from a coyote, based on their size. A macrobotanical sample from the feature produced seven hackberry seeds, two mesquite seeds, two Chenopodium sp. seeds, five skullcap seeds, six dropseed seeds, and burned juniper, mesquite, and ring-porous hardwood (see Table 55 and Appendix E). Feature 8 is interpreted as a stone-lined hearth due to the presence of burned rocks in a circular arrangement.

\section{Feature 9}

Feature 9 is a bone cluster in EU 10 (see Figure 81) that consists of 139 bones of bison, bobcat, wolf, rodent, and unidentified carnivore, mammal, and vertebrate occurring at a depth of 55$60 \mathrm{~cm}$ in the upper reddish brown sediment zone (see Appendix C). Feature 9 measures $40 \mathrm{~cm}$ north-south and $50 \mathrm{~cm}$ east-west and extends slightly into EU 7 , adjoining the northeast boundary of Feature 10. No soil discoloration was noted, and no charcoal was found in association with the feature. A radiocarbon assay on bison bone collagen from Features 9 and 12 produced a calibrated date of A.D. 1506 (1642) 1663 (see Table 54).

Feature 9 is in loamy sediments similar to those containing Feature 7; however, a few more gravels are present in this area south of Feature 7. The bones constituting Feature 9 were jumbled and not arrayed in natural osteological association. Only one rodent bone exhibits spiral fracturing, and none are burned. Bones overlapping this feature and Features 7 and 10 are listed separately under Feature 7. 


\section{Feature 10}

Feature 10 is a bone cluster in EUs 6 and 7 at a depth of 51-61 cm (see Figure 81). The feature consists of 469 bones of bison, bobcat, wolf, rabbit, and unidentified carnivore, mammal, and vertebrate (see Appendix C). Feature 10 extends $60 \mathrm{~cm}$ northsouth and $20 \mathrm{~cm}$ east-west. No soil discoloration or charcoal were observed, and the feature is contained in a gravelly matrix that is part of the upper reddish brown sediment zone. The Feature 10 bones were jumbled and not arrayed in natural osteological association, and no bones from this feature exhibit cultural modification. Bones found overlapping this feature and Features 7 and 9 are discussed under Feature 7. A cow/bison calcaneus which probably relates to the bison lower leg elements in Features 9 and 12 was recovered from this feature.

\section{Feature 11}

Feature 11 is a concentration of burned and unburned sandstone rocks in EUs 20 and 21. The total weight of the ca. 10 rocks is $10 \mathrm{~kg}$. Feature 11 occurs at a depth of 34-44 cm and extends $62 \mathrm{~cm}$ north-south and $40 \mathrm{~cm}$ east-west; a small scatter of burned rocks in EU 26 to the north may be related to Feature 11. Feature 2 occurs at a similar depth in EU 19 and, along with the scatter in EU 26, may be an extension of Feature 11.

Dark red-stained soil was discovered beneath the rocks of Feature 11, and charcoal-stained soil was found between the rocks. A chert core was found directly above the feature. Recovered from Feature 11 were 50 bones of rabbit, rodent, and unidentified mammal and vertebrate (see Appendix C). Seven specimens-one rabbit and six vertebrate- exhibit spiral fractures, and three rabbit, seven vertebrate, one rodent, and two mammal bones are burned. Only one vertebrate fragment is both burned and spirally fractured. Feature 11 is interpreted as a disaggregated hearth because the rocks have been scattered and there is no longer any recognizable shape.

\section{Feature 12}

Feature 12 is a bone cluster at a depth of 58$63 \mathrm{~cm}$ in EU 7 (see Figure 81). It consists of 176 bones of bison, bobcat, wolf, dog, rodent, and unidentified carnivore, mammal, and vertebrate (see
Appendix C). Feature 12 extends $50 \mathrm{~cm}$ north-south and $40 \mathrm{~cm}$ east-west and is in the upper reddish brown sediment zone. A radiocarbon assay on bison bone collagen from Features 12 and 9 produced a calibrated date of A.D. 1506 (1642) 1663 (see Table 54). No soil discoloration or charcoal was observed, and the matrix was very gravelly. The Feature 12 bones were jumbled and not arrayed in natural osteological association. No bones from this feature bear any trace of cultural modification. A macrobotanical sample yielded two hackberry seeds and two prickly pear seeds (see Table 55 and Appendix E).

\section{Feature 13/26}

Originally recorded as two individual featuresFeature 13 at 52-66 cm in;EU 20 and Feature 26 at $57-67 \mathrm{~cm}$ in EU 26 - these burned rock clusters were later determined to be parts of the same feature. It is an ovate ( $75 \mathrm{~cm}$ north-south by $60 \mathrm{~cm}$ east-west) cluster consisting of 16-20 burned sandstone rocks weighing $16 \mathrm{~kg}$. The majority of the rocks are closely spaced and generally dip toward the center of the feature, in the south-central portion of EU 26. Two larger slabs, however, were found above the main cluster and could cap the feature or they may be unrelated. Feature 13/26 is interpreted as a stonelined hearth.

One unifacial tool, one chert core, and five flakes were found in the feature, along with 43 bones of rodent, toad or frog, and unidentified mammal and vertebrate (see Appendix C). Of these, two vertebrate bones are burned, and one mammal bone is both burned and spirally fractured. Macrobotanical samples produced 12 hackberry seeds, 2 Chenopodium sp. seeds, 7 skullcap seeds, 2 Panicum sp. seeds, 1 Portulaca sp. seed, and burned juniper and ring-porous hardwood (see Table 55 and Appendix E). A radiocarbon assay on charcoal produced a calibrated date of A.D. 227 (265, 281, 333) 412 (see Table 54).

\section{Feature 14}

Feature 14 is a bone cluster discovered in EU 2 (Figure 82). It occurs at a depth of $25-31 \mathrm{~cm}$ and extends $30 \mathrm{~cm}$ north-south and $30 \mathrm{~cm}$ east-west. Within this feature were 390 bones of wolf, bobcat, bird, rodent, and unidentified carnivore, mammal, and vertebrate (see Appendix C). No soil discoloration 


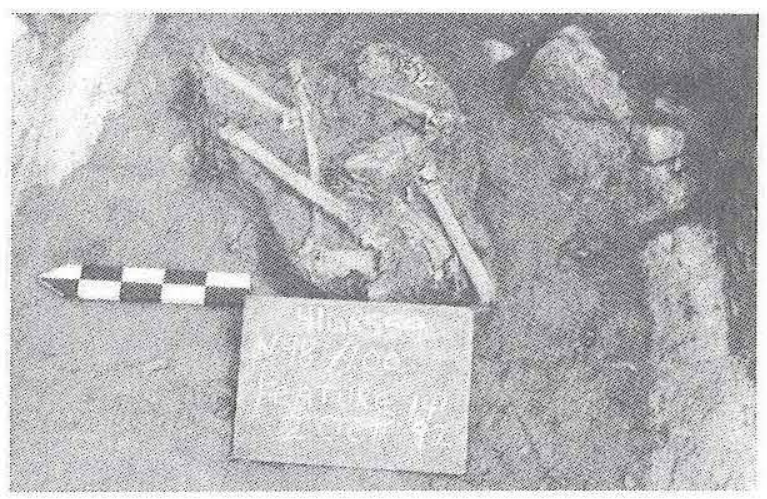

Figure 82. View to the east of Feature 14, 41GR559.

or charcoal was observed among these bones, which were seriously disturbed by roots growing through and amongst them. The Feature 14 bones were jumbled and not arrayed in natural osteological association. One fragment of a vertebrate bone exhibits the effects of burning.

\section{Feature 15}

Feature 15 consists of a concentration of burned rocks in EU 17 scattered throughout a jumble of large roof spalls. The total weight of the burned rocks is $31.8 \mathrm{~kg}$, including many rocks of all sizes. The feature occurs at $37-87 \mathrm{~cm}$ and extends horizontally across the entire $1-x-1-m$ unit but is concentrated in the eastern half of the unit. It is difficult to interpret Feature 15, but it may be a dump for exhausted hearthstones or boiling stones associated with Feature 8. Small flecks of charcoal were observed distributed throughout the feature. A unifacial tool, three chert cores, and three flakes were recovered from among the burned rocks.

\section{Feature 16}

Feature 16 appears to be an unlined hearth at a depth of $97-100 \mathrm{~cm}$ in EUs 19 and 25 . It is an amorphous area extending $115 \mathrm{~cm}$ north-south and $75 \mathrm{~cm}$ east-west that is tan sediment heavily mottled with red and contains a moderate amount of charcoal. The area is level, and the matrix has been thermally altered to a thickness of $2-3 \mathrm{~cm}$. A radiocarbon assay on charcoal from Feature 16 yielded a calibrated date of 2 B.C. (A.D. 72) A.D. 129 (see Table 54). No thermally altered rocks were associated, and no basin shape could be ascertained in the matrix. One chert core was found in association with the feature, along with 11 bones of snake, rabbit, rodent, and unidentified mammal and vertebrate (see Appendix C). Two of the vertebrate bones exhibit spiral fracturing. A macrobotanical sample produced 19 Chenopodium sp. seeds, 2 skullcap seeds, 2 Panicum sp. seeds, and burned juniper, hardwood, and ring-porous hardwood (see Table 55 and Appendix E).

\section{Feature 17}

Feature 17 is a bone cluster at a depth of 65$72 \mathrm{~cm}$ in EUs 1 and 2. It consists of 834 bones of Canis sp., fox, skunk, rabbit, and unidentified carnivore and mammal (see Appendix C). Most of the bones are of two juveniles that are probably wolf. The feature extends $50 \mathrm{~cm}$ north-south and $40 \mathrm{~cm}$ east-west. This feature occurs in an area that is quite disturbed. No soil discoloration or charcoal was directly associated with the feature, but it was located immediately above a large circular charcoal stain that was later investigated as Feature 19.

Thirty-one bones of bird, rabbit, and unidentified mammal and vertebrate were recovered from the overlap area between Features 17 and 19 (see Appendix C). Six vertebrate bones from this overlap area exhibit spiral fracturing, and one bird bone and six vertebrate bones show the effects of burning. These modified bones probably bear more relationship to Feature 19, which is a hearth dump, than to Feature 17. The Feature 17 bones were jumbled and not arrayed in natural osteological association.

\section{Feature 18}

Feature 18 is a circular concentration of burned sandstone and sandstone conglomerate rocks in EU 28. Large pieces of burned sandstone that are probably associated with this concentration were found at the same level in EU 27 to the west. The total weight of the burned rocks in EU 28 is $8.3 \mathrm{~kg}$ (ca. 12 rocks), and those in EU 27 weigh $2.0 \mathrm{~kg}$ (ca. 9 rocks), for a total weight from both units of 10.3 $\mathrm{kg}$. The feature occurs at $30-45 \mathrm{~cm}$ and is directly overlain by $4 \mathrm{~cm}$ of coarse red sediment containing many small pea-sized gravels. The more discrete portion of the feature in EU 28 extends $72 \mathrm{~cm}$ northsouth and $65 \mathrm{~cm}$ east-west. This area expands to $100 \mathrm{~cm}$ north-south and over $200 \mathrm{~cm}$ east-west if the extension in EU 27 is considered. 
No soil discoloration was observed, but a small amount of charcoal was obtained directly from the feature and the immediately surrounding area. A radiocarbon assay on charcoal from this feature produced a calibrated date of A.D. 1169 (1252) 1277 (see Table 54). One chert core and one flake were associated with Feature 18. Thirty-three bones of rodent and unidentified mammal and vertebrate also were recovered from the feature (see Appendix C). Three of the mammal bones exhibit spiral fracturing. A macrobotanical sample produced one mesquite seed, three Chenopodium sp. seeds, one Bromus sp. seed, and burned juniper, hardwood, and ring-porous hardwood (see Table 55 and Appendix E). Because of the U-shaped pattern of the burned rocks, Feature 18 is interpreted as a stone-lined hearth.

\section{Feature 19}

Feature 19 is a depression in EUs 1 and 4 filled with a sandy matrix containing charcoal, burned bones, and burned hackberry seeds found prior to flotation. The flotation procedure produced three dropseed seeds and burned hardwood (see Table 55 and Appendix E). The bones consist of 94 specimens of wolf, rabbit, bird, snake, rodent, and unidentified mammal and vertebrate (see Appendix C). Of these, one rabbit, one bird, one mammal, and six vertebrate bones are burned. The culturally modified bones discussed under Feature 17 as coming from the overlap area with Feature 19 probably belong to Feature 19. The feature occurs at a depth of 81$105 \mathrm{~cm}$ and extends $80 \mathrm{~cm}$ north-south and $60 \mathrm{~cm}$ east-west. The sides and bottom of the depression were not thermally altered, and it appears to be a hearth dump predating deposition of the Feature 17 bones.

\section{Feature 20}

Feature 20 is a vertical sandstone slab in EU 14 at a depth of $23-113+\mathrm{cm}$. The slab measures $76 \mathrm{~cm}$ east-west, $13 \mathrm{~cm}$ north-south, and at least $90 \mathrm{~cm}$ in thickness. The slab is much too heavy to have been carried into the shelter, and so it probably represents a detached piece of the shelter roof or wall which may have been repositioned to deflect winds from the south away from Feature 8.

\section{Feature 22}

Feature 22 is a circular concentration of burned sandstone in EU 24 (Figure 83). The total weight of the ca. 12 rocks in EU 24 is $3.5 \mathrm{~kg}$. More burned sandstone rocks which may be extensions of this feature were recorded in adjacent EUs $23(0.5 \mathrm{~kg})$ and 27 (ca. $1.0 \mathrm{~kg}$ ). A charcoal stain in the southwestern comer of EU 28 also may be related. The feature occurs at a depth of $28-41 \mathrm{~cm}$ and extends $35 \mathrm{~cm}$ north-south and $37 \mathrm{~cm}$ east-west in EU 24 . It sits on a sandstone bench which is a large fallen roof block.

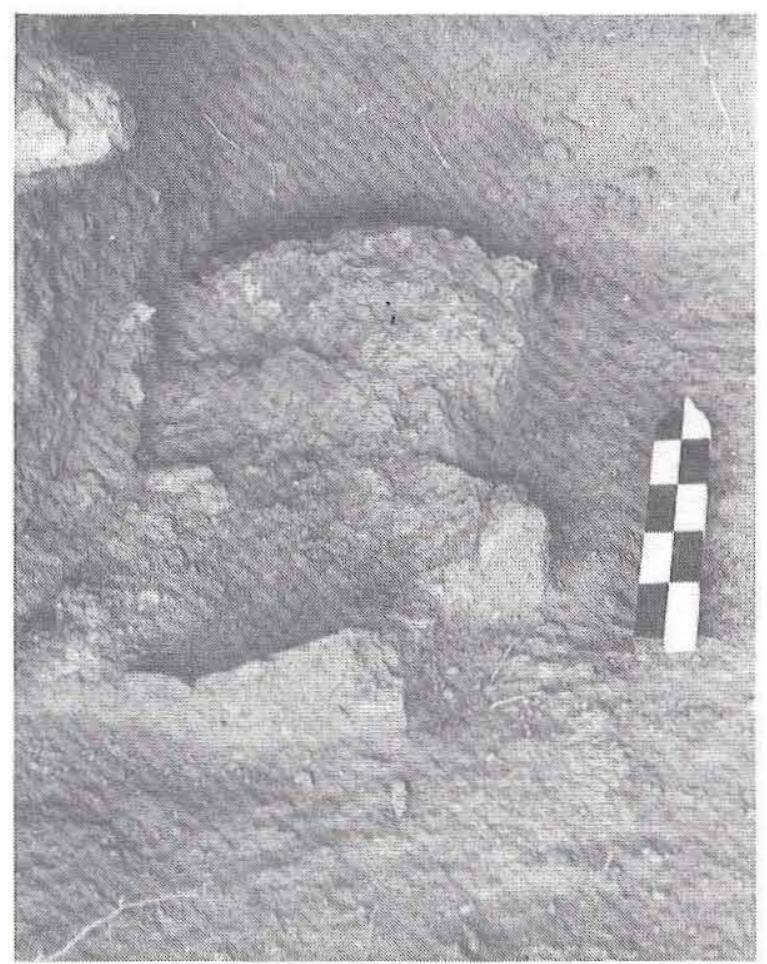

Figure 83. View to the north of Feature 22, 41GR559.

The feature was indicated from above by an ash stain which continued downward the full depth of the feature. Charcoal, ash, and small pieces of firecracked rocks were abundant throughout. One chert flake was found in association. Bones recovered from the feature consist of 24 specimens of rabbit, rodent, and unidentified mammal and vertebrate (see Appendix C). No modification was found on any of these specimens. A radiocarbon assay on charcoal from Feature 22 produced a calibrated date of A.D. 640 (681) 860 (see Table 54). Feature 22 is interpreted as a stone-lined hearth due to its circular shape.

\section{Feature 23}

Feature 23 is a dish-shaped depression filled 
with sandy matrix containing small charcoal nodules and charcoal powder in the eastern part of EU 23. It occurs at a depth of $23-28 \mathrm{~cm}$ and extends $18 \mathrm{~cm}$ north-south and $18 \mathrm{~cm}$ east-west. Five unmodified fragments of unidentified vertebrate bones were recovered from the feature. Feature 23 is interpreted as a hearth dump possibly related to nearby Feature 22.

\section{Feature 24}

Feature 24 is an unlined hearth represented by a lens of burned, ashy soil with considerable charcoal in EU 27. Three pieces of burned but randomly aligned sandstone which weigh ca. $2 \mathrm{~kg}$ are associated with the feature. At the south end of the feature, a large slab of sandstone intrudes from EU 24 to the south, and the north end of this slab appears to be thermally altered. The feature occurs at a depth of $74-76 \mathrm{~cm}$. It extends $40 \mathrm{~cm}$ north-south and $50 \mathrm{~cm}$ east-west and is $2-3 \mathrm{~cm}$ thick. Thirteen bones of rabbit and unidentified vertebrate were recovered from the feature (see Appendix C). None are culturally modified. A macrobotanical sample produced burned ring-porous hardwood (see Table 55 and Appendix E). A radiocarbon assay on charcoal yielded a calibrated date of A.D. 420 (544) 622 (see Table 54).

\section{Feature 25}

Feature 25 is a circular arrangement of five burned, flat sandstone slabs in EU 27. The three pieces of sandstone on the perimeter of the feature tilt inward toward the center. Three more pieces of burned sandstone are close by and probably associated. The total weight of the rocks is $0.8 \mathrm{~kg}$. The feature occurs at a depth of $82-86 \mathrm{~cm}$ and extends $20 \mathrm{~cm}$ north-south and $20 \mathrm{~cm}$ east-west. It appears to have been dug down into the soft gray sandstone roof slab on which it sits. The soil in the southwestern part of the feature was discolored red, and charcoal was present in the form of nodules, powder, and staining. No artifacts or faunal remains were recovered; however, a macrobotanical sample produced one bottle gourd seed and burned juniper, hardwood, and ring-porous hardwood (see Table 55 and Appendix E). The lack of cultural materials and the position of the feature toward the rear of the shelter indicate that it may have been built for heating rather than for food processing. Because of its circular shape, Feature 25 is interpreted as a stonelined hearth.

\section{Feature 27}

Feature 27 is a U-shaped concentration of large burned sandstone slabs in EU 24. The open end of the $U$ faces southwest. The total weight of the ca. 12 rocks is $30 \mathrm{~kg}$. The feature occurs at a depth of $11-22 \mathrm{~cm}$ and extends $80 \mathrm{~cm}$ north-south and $105 \mathrm{~cm}$ east-west. An extension of the feature occurs in EU 18 to the south. Two large sandstone slabs stacked in the northeast corner of the unit and one in the northwest corner complete the arms of the $U$ and extend the feature $25 \mathrm{~cm}$ south. These slabs weigh ca. $5 \mathrm{~kg}$ and occur at a depth of $2-15 \mathrm{~cm}$.

Small fire-cracked rocks and flecks of charcoal were scattered across the unit. Also within the unit, in Level 3 near the feature, a piece of a sandstone metate was found. It refits with two other fragments found in Level 3 of EUs 14 and 24 to form a portion of a metate. A radiocarbon assay on charcoal produced a calibrated date of A.D. 1021 (1043, 1105, 1112, 1150) 1169 (see Table 54). Feature 27 is interpreted as a stone-lined hearth because of its morphology.

\section{Discussion}

Twenty-five cultural features were found at 41GR559. Inside the shelter were two unlined hearths, seven stone-lined hearths, five disaggregated hearths, one burned rock concentration, one hearth dump, one wind deflector, and one bone cluster; these point chiefly to food processing or the production of heat for warmth.

In contrast, the talus area in front of the shelter contained six bone clusters and one hearth dump, which are suggestive of refuse disposal. The hearth dump was found beneath the bone clusters but is thought to have been deposited at approximately the same time. Although its contemporaneity cannot be confirmed since it is undated, the hearth dump is definitely cultural in origin while the bone clusters are more difficult to interpret (see Appendix C). They consist predominantly of taxa (i.e., canids, bobcats, and rodents) that might be expected to occur naturally in rockshelters, and only 22 of the specimens recovered in $1992(<1 \%)$ exhibit any evidence of cultural modification. On these grounds, the bone clusters might be interpreted as noncultural remains 
redeposited from the adjacent shelter, perhaps as a result of erosion or shelter cleaning by humans.

On the other hand, there is no evidence of carnivore gnawing on the bones from this part of the site, and most of the bones occur in discrete clusters suggesting cultural deposition. Most importantly, however, the lack of axial skeletal elements and most femurs suggests that the meat-bearing portions of these animals were removed, which points to processing by humans. In any case, the presence of individual animals in multiple clusters (i.e., bison bones representing one individual in Features 9, 10, and 12 and bobcat bones representing three individuals spread across Features 7, 9, 10, 12, and 14) indicates that all of the bone clusters were deposited over a short span of time (i.e., as a single event or a few closely spaced events) and represent a single bone midden.

\section{MATERIALS RECOVERED}

This section discusses the materials recovered from 41 GR559 during the Season 3 excavations. Of the 147 artifacts, 135 are chipped stones, 8 are hammerstones, and 3 are refittable ground stone fragments which make up a partial metate. One mussel shell fragment, possibly utilized as a scraper, also was recovered. Table 56 provides distributional information for these artifacts; more-detailed provenience data for selected classes of artifacts are presented in Appendix H. The total faunal assemblage recovered in 1992 consists of 3,580 vertebrate specimens, 14 fragments of freshwater mussel shells, and 27 gastropod shells.

\section{Chipped Stone Artifacts}

Three arrow points and fragments, 1 dart point fragment, 1 cobble tool, 6 unifaces and fragments, 16 cores, 7 edge-modified flakes, and 101 pieces of unmodified lithic debitage were recovered.

\section{Arrow Points}

One of the arrow points is complete, one is a proximal fragment, and one is a distal fragment. The complete point is of Tecovas jasper, and the fragments are of fine-grained chert. The complete specimen is a Washita arrow point, and the two fragments are untypeable.

\section{WASHITA}

This specimen is a triangular side-notched arrow point with a concave base (Figure 84a). Although it is missing a barb and ear, it is characteristic of triangular side-notched Washita points recovered from Late Prehistoric sites from northem Texas, Oklahoma, and eastern New Mexico (Bell 1958). Its blade edges are straight but have undergone one reworking episode to rejuvenate a distal blade break that appears to have occurred during use; the cause of the ear and barb breaks cannot be determined. Although it is almost certain that the point was made on a flake blank, no remnant features of the parent flake are present. The maximum length is $24 \mathrm{~mm}$, its blade is $15 \mathrm{~mm}$ long, and its haft is $9 \mathrm{~mm}$ long. It has a 7-mm-wide neck; blade and stem widths could not be measured due to their incompleteness. The base concavity is $2 \mathrm{~mm}$ deep.
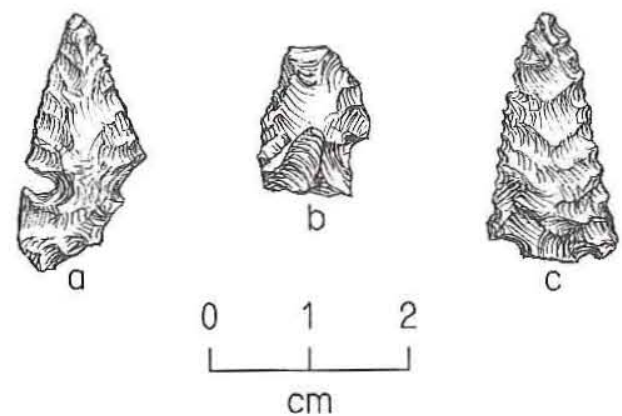

Figure 84. Arrow points, 41GR559. (a) Washita; (b) untypeable expanding stem, straight base; $(c)$ untypeable serrated distal fragment.

\section{UNTYPEABLE FRAGMENTS}

A proximal fragment and a distal fragment are included in this category. The proximal fragment, of local fine-grained chert (Color 32), is missing a small portion of its distal blade and both ears (Figure $84 b$ ). The blade appears to have been reworked at least once prior to the break; however, no rejuvenation of the latest breaks was attempted. The fragment has a straight base, and judging from the angle of the notch, it may have been corner notched. The specimen retains portions of the ventral and dorsal surfaces of the parent flake. Coupled with the fact that the blade break is use related, the flake blank remnants suggest that the specimen was manufactured relatively expediently. Although it 


\begin{tabular}{|c|c|c|c|c|c|c|c|c|c|c|}
\hline \multicolumn{11}{|c|}{$\begin{array}{c}\text { TABLE } 56 \\
\text { DISTRIBUTION OF DATA RECOVERY ARTIFACTS, } 41 \text { GR559 }\end{array}$} \\
\hline $\begin{array}{l}\text { Unit/Maximum } \\
\text { Depth (cm } \\
\text { below surface) }\end{array}$ & \begin{tabular}{|l|} 
Projectile \\
Points
\end{tabular} & $\begin{array}{l}\text { Cobble } \\
\text { Tools } \\
\end{array}$ & Unifaces & Cores & $\begin{array}{l}\text { Edge-modified } \\
\text { Flakes }\end{array}$ & $\begin{array}{l}\text { Unmodified } \\
\text { Debitage }\end{array}$ & $\begin{array}{l}\text { Ground } \\
\text { Stones }\end{array}$ & Other Lithics & Other Materials & Totals \\
\hline EU $2,83 \mathrm{~cm}$ & - & - & - & - & - & 1 & - & - & - & 1 \\
\hline EU $8,84 \mathrm{~cm}$ & 1 & - & - & 1 & - & 2 & - & 3 hammerstones & - & 7 \\
\hline EU $14,113 \mathrm{~cm}$ & - & - & - & - & - & 1 & 1 & - & |- & 2 \\
\hline EU $17,87 \mathrm{~cm}$ & - & - & 1 & 3 & - & 3 & - & |- & - & 7 \\
\hline EU $18,106 \mathrm{~cm}$ & 1 & 1 & - & 1 & 1 & 4 & - & 1 hammerstone & 1 mussel shell scraper? & 10 \\
\hline EU $20,90 \mathrm{~cm}$ & - & - & 1 & 3 & 1 & 8 & - & - & - & 13 \\
\hline EU 21, $68 \mathrm{~cm}$ & - & - & - & 1 & - & 7 & - & - & |- & 8 \\
\hline EU $23,82 \mathrm{~cm}$ & - & - & - & - & - & 5 & - & |- & - & 5 \\
\hline EU $24,96 \mathrm{~cm}$ & 1 & - & - & - & 2 & 9 & 1 & - & - & 14 \\
\hline EU 30,103 & - & - & - & - & - & 2 & - & - & |- & 2 \\
\hline Miscellaneous* & - & - & - & 2 & 1 & 6 & - & - & - & 9 \\
\hline Totals: & 4 & 1 & 6 & 16 & 7 & 101 & $3 * *$ & 8 hammerstones & 1 mussel shell scraper? & 147 \\
\hline
\end{tabular}


cannot be determined with certainty, it is possible that the ears also were broken in use. Few metric attributes can be recorded on this fragment. The haft is $6 \mathrm{~mm}$ long, the neck is $9 \mathrm{~m}$ wide, and the maximum thickness is $2 \mathrm{~mm}$.

The distal fragment is broken at the neck but retains the entire blade portion (Figure $84 c$ ). One of the blade edges is slightly convex, the other is recurved, and both are lightly serrated. The blade edges have been reworked to rejuvenate a use break, which also removed one of the barbs. The reworking resulted in slightly beveled blade edges on one face. Although the specimen probably was made on a flake blank, the fine initial workmanship and subsequent reworking have removed all diagnostics of the nature of the blank used in its manufacture. The specimen has a maximum blade length of $22 \mathrm{~mm}$, a neck width of $6 \mathrm{~mm}$, and a maximum thickness of $4 \mathrm{~mm}$. The point is of locally available fine-grained chert (Color 6).

\section{Dart Point}

A proximal dart point fragment, typed as Ellis (Miller and Jelks 1952; Suhm et al. 1954), was recovered. It is of nonlocal fine-grained chert (Color 47). The specimen has a moderately expanding stem and a convex base (Figure 85). One of the blade edges is straight, and the other is recurved. Both were resharpened at least once prior to the recent break that resulted in the removal of the distal end and one of the barbs. The remaining barb flares down and out. The breaks are use related and, judging from the asymmetrical base outline, represent at least the second time the point was broken. The stem edges and base are not ground. Although the specimen is well made, one of its faces retains a small portion of the ventral surface of the parent flake. Few metric attributes could be recorded. The point has a $21-\mathrm{mm}$-wide base, a 16-mm-wide neck, and a 9-mm-long haft. It is $5 \mathrm{~mm}$ in maximum thickness.

\section{Cobble Tool}

The single complete cobble tool is of coarsegrained quartzite (Figure 86). Based on its weight $(467 \mathrm{~g})$ and size $(93 \times 78 \times 58 \mathrm{~mm})$, the specimen falls in the heavy cobble tool category identified in the collections from both 41GR291 and 41GR303B. The tool has two slightly convex working edges, both

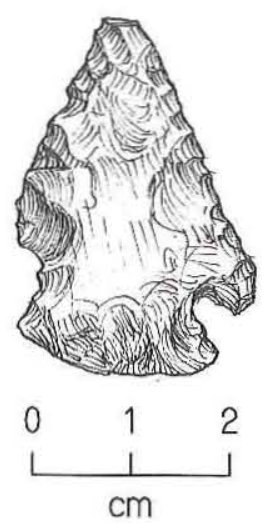

Figure 85. Ellis dart point, 41GR559.

of which are cortex backed. As is characteristic of the majority of heavy cobble tools from other sites, both working edges are heavily step fractured and rounded as a result of heavy crushing.

\section{Unifaces}

Six unifaces and fragments were recovered. They consists of three end scrapers, one minimally retouched side scraper, and two indeterminate unifaces.

\section{END SCRAPERS}

Two of the three complete end scrapers are of fine-grained chert; the third is of opalized caliche. All three were made on short and relatively broad, trapezoidal secondary flake blanks. One specimen was made by retouching only the distal end or working edge of the flake blank (Figure 87a). The thinness of the working edge suggests that the scraper was not resharpened many times. No haft wear is evident on the specimen. The lack of haft wear and marginal retouch suggest that the specimen was a hand-held scraper which might have been made expediently rather than transported to the site and discarded there following an extensive period of curation. The fact that the tool is of local finegrained chert (Color 5) supports this interpretation. The specimen has a maximum length of $38 \mathrm{~mm}$, a maximum width of $50 \mathrm{~mm}$ near the working edge, and a maximum thickness of $18 \mathrm{~mm}$.

The second complete end scraper was made by retouching both lateral margins and the distal end of the flake blank (Figure 87b). Smoothing and polish on flake scar ridges on both the ventral and dorsal 

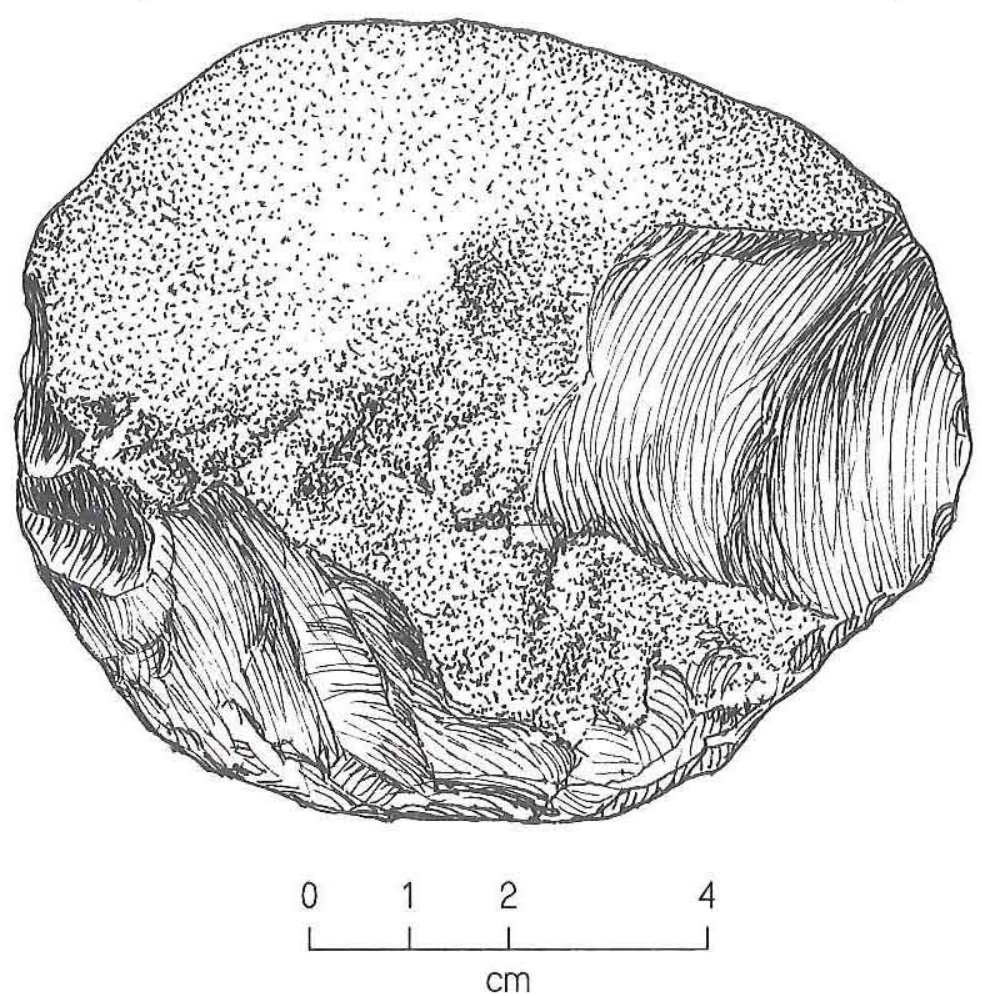

\section{MINIMALLY RETOUCHED SIDE SCRAPER}

A single complete minimally retouched side scraper was recovered. It is a large, secondary, coarse-grained quartzite flake with marginal retouch along one edge of its ventral face (Figure 87d). Due to the coarseness of the raw material, it is not possible to determine whether haft wear is present. In addition, although it is classified as a scraper, the lack of use wear makes it impossible to determine with certainty whether the specimen was used as a scraper or as a knife. The tool is $58 \mathrm{~mm}$ long, $39 \mathrm{~mm}$ wide, and $16 \mathrm{~mm}$ thick.

\section{INDETERMINATE UNIFACES}

The two specimens consist of one distal fragment and one indeterminate uniface edge. The distal fragment (Figure $87 e$ ) is made on a local finegrained chert (Color 5) pebble blank.

Figure 86. Cobble tool, 41GR559.

surfaces suggest that the scraper was hafted. Haft wear appears to extend for $21 \mathrm{~mm}$ along the proximal portion of the tool. The presence of haft wear, coupled with the more extensive marginal retouch, suggests that this specimen was a curated tool that was brought to the site rather than manufactured there. The fact that the tool is of nonlocal finegrained chert (Color 41) supports this interpretation. The specimen has a maximum length of $35 \mathrm{~mm}$, a maximum width of $41 \mathrm{~mm}$, and a maximum thickness of $13 \mathrm{~mm}$.

The third end scraper is of opalized caliche. It is retouched on both lateral margins and the distal working edge (Figure 87c). As in the case of the second end scraper, light rounding and polish on flake scar ridges on the dorsal face of the tool suggest that the specimen was hafted. Haft wear extends along $29 \mathrm{~mm}$ of the proximal end. It is $38 \mathrm{~mm}$ long, $36 \mathrm{~mm}$ wide, and $10 \mathrm{~mm}$ thick. Although it is of opalized caliche, a locally available raw material (see Boyd et al. 1992:24), the degree of marginal and distal end retouch and the hafted nature of the tool suggest that this end scraper represents a curated tool.
It has been heavily reworked on its ventral surface and a portion of the break face. However, small patches of cortex are present on both the ventral and dorsal surfaces. Judging from the polish and light smoothing present on flake scar ridges on both the ventral and dorsal faces, the specimen appears to have been hafted. Due to the reworking of a portion of the break face, the cause of the break cannot be determined with certainty. In addition, due to the heavy reworking of the original specimen, based on its present form it cannot be established with certainty whether the tool was an end scraper or a gouge. Although the original length of the specimen cannot be obtained, its maximum width is $38 \mathrm{~mm}$ and its maximum thickness is $23 \mathrm{~mm}$.

The remaining specimen is an indeterminate edge fragment made on a corticate local fine-grained chert (Color 5) flake blank. Neither the presence of haft wear nor the cause of the break could be determined. No metric attributes could be recorded.

\section{Cores}

Sixteen cores and core fragments were 

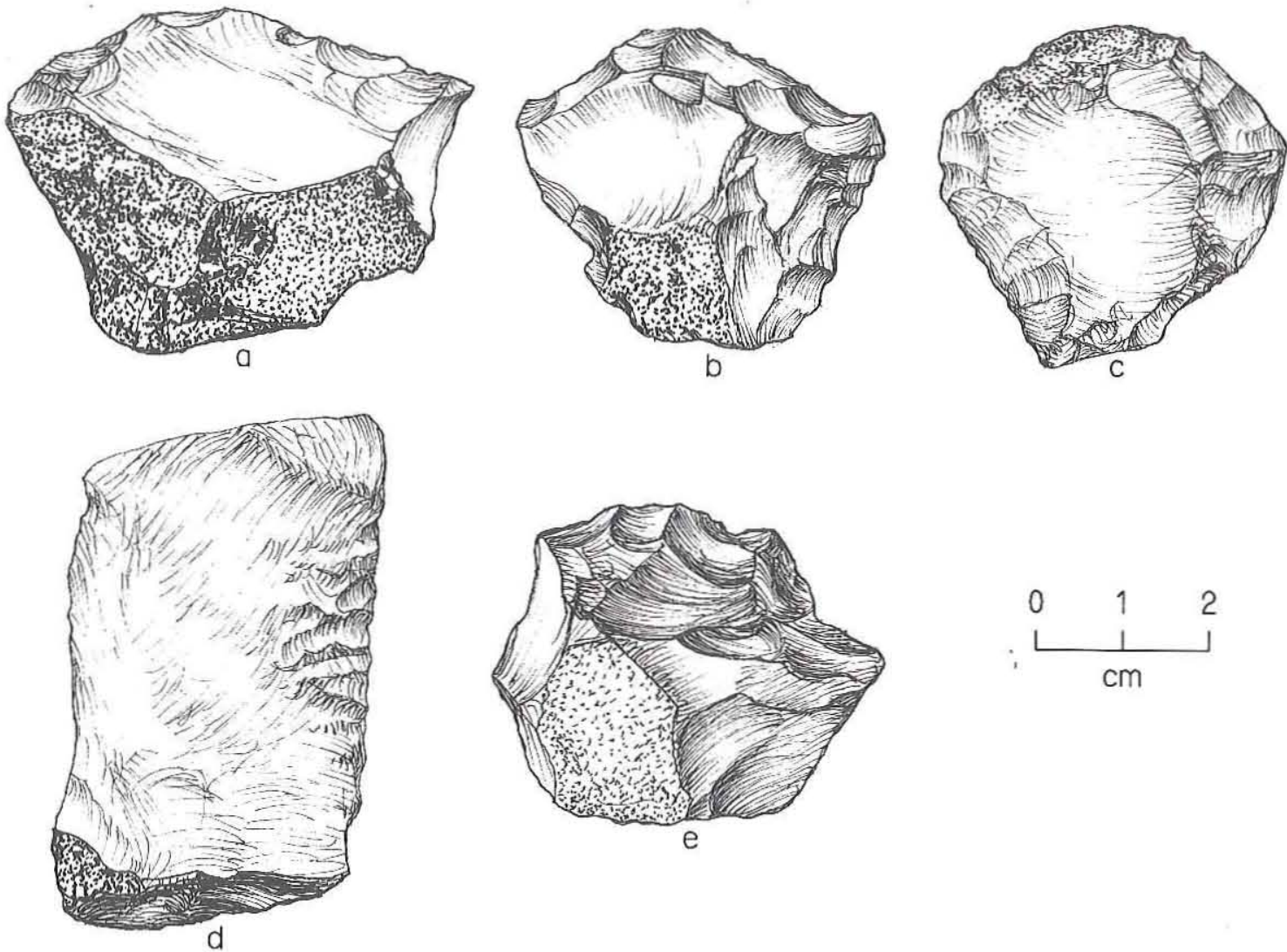

Figure 87. Unifaces, 41GR559. (a-c) End scrapers; $(d)$ minimally retouched side scraper; $(c)$ indeterminate uniface.

recovered. More than half $(n=10,63 \%)$ of these are of fine-grained chert. Fine-grained Potter chert $(n=$ $2,13 \%)$, coarse-grained Potter chert $(n=2,13 \%)$, coarse-grained quartzite $(n=1,6 \%)$, and coarsegrained chert $(\mathrm{n}=1,6 \%)$ constitute the remaining specimens.

Eight of the 10 fine-grained chert specimens are complete; the other 2 are fragmentary. Nine are pebble cores, and a single specimen is a flake core. Multidirectional cores are the most common $(n=6)$, followed by unidirectional specimens $(n=3)$. The remaining specimen is a bidirectional core. Cortex is present on all but one of the cores.

The number of flake removals ranges from 1 to 20 , with the mean being $7.9(\sigma=6.3)$. Three cores have a single flake removal and probably represent tested pebbles. Six have eight or more flake removals. None of the cores appear to represent artifacts recycled into cores from other tool categories. The 10 fine-grained chert cores have a mean length of $52.6 \mathrm{~mm}(\sigma=17.6)$, a mean width of $43.7 \mathrm{~mm}(\sigma=15.6)$, and a mean thickness of
$27.6 \mathrm{~mm}(\sigma=14.0)$. All of the fine-grained chert cores are of local origin (Colors 2, 6 [n=2], 8, 21, $27,31,32$, and $55[n=2])$.

Because only a few nonchert cores were recovered, the discussion of the remaining cores combines the six nonchert specimens into a single group. Of these, five (83\%) are complete and one $(17 \%)$ is fragmentary. All but one are pebble cores. Bidirectional $(\mathrm{n}=3,50 \%)$ and unidirectional $(\mathrm{n}=2$, $33 \%)$ specimens are the most common. Unlike the fine-grained chert cores, a single nonchert core is multidirectional. All of the nonchert cores are corticate, and none have been recycled into cores from other artifact categories.

The number of flake removals on the nonchert cores ranges from 1 to 13 , with a mean of $6.5(\sigma=$ 4.9). None have only one flake removal sçar, while two $(33 \%)$ have more than eight removals. Nonchert cores are larger than the fine-grained chert specimens. They have a mean length of $72.6 \mathrm{~mm}(\sigma=$ $29.6)$, a mean width of $58.8 \mathrm{~mm}(\sigma=28.7)$, and a mean thickness of $43.3 \mathrm{~mm}(\sigma=27.9)$. A compari- 
son of the fine-grained chert and nonchert cores shows that the fine-grained chert cores are more intensively reduced than the coarse-grained specimens.

\section{Edge-modified Flakes}

Only seven edge-modified flakes were recovered. Six $(86 \%)$ of these are complete tools, and one (14\%) is fragmentary. All of the complete tools represent complete flakes. Two $(29 \%)$ of the edge-modified flakes are tertiary specimens, four $(57 \%)$ have between $1-50 \%$ cortex, and one (14\%) has between $51-99 \%$ cortex. Three $(43 \%)$ of the edge-modified flakes are between $31-40 \mathrm{~mm}$ in maximum size, one (14\%) falls between $11-20 \mathrm{~mm}$, two $(29 \%)$ are between $21-30 \mathrm{~mm}$, and one is between $41-50 \mathrm{~mm}$ in maximum size.

Nine working edges were noted on the seven edge-modified flakes. The majority $(n=5,71 \%)$ of the tools have single working edges; only two (29\%) have two working edges. Working edges used for scraping tasks alone occur on six $(86 \%)$ of the edgemodified flakes. A single specimen with one working edge was used in sawing/cutting tasks.

Six $(86 \%)$ of the edge-modified flakes are of fine-grained chert. A single specimen (14\%) is of fine-grained Potter chert. All but one of the finegrained chert specimens are of locally available cherts (Colors 3, 6, 20, 21, and 55). The remaining specimen is of nonlocal fine-grained chert (Color 42).

\section{Unmodified Debitage}

A total of 101 pieces of unmodified lithic debitage were recovered. The majority $(n=67,66 \%)$ are of fine-grained chert. Fine-grained Potter chert, coarse-grained quartzite, chalcedony, and silicified caliche are the next most common raw material categories, with each containing six specimens (6\%). Specimens of fine-grained quartzite $(n=4,4 \%)$, coarse-grained Potter chert $(n=3,3 \%)$, silicified wood $(\mathrm{n}=1,1 \%)$, Tecovas jasper $(\mathrm{n}=1,1 \%)$, and unidentified material types $(n=1,1 \%)$ occur in even lower numbers. Table 57 shows the breakdown of the debitage attributes by raw material type. Because the sample size for each nonchert material type is so small, for analytical purposes the nonchert raw materials are combined into one group.

Complete flakes represent the largest percentages among both the fine-grained chert and nonchert materials. Chips constitute approximately one-fourth of both raw material groups. In general, this pattern is different from the patterns noted in both the 41GR291 and 41GR303B debitage collections in which chips tend to outnumber the other flake completeness categories. Chunks are infrequent or absent in all raw material types. Complete flakes and flake fragments with $1-50 \%$ cortex are the most common category in the fine-grained chert debitage. In addition, the percentage of flakes with $51-99 \%$ cortex (21\%) is almost identical to the percentage of entirely decorticate flakes (25\%). Although entirely decorticate debitage makes up slightly more than one-third $(36 \%)$ of the nonchert debitage collection, corticate specimens combined constitute the bulk of the pieces. These percentages suggest that chipped stone artifacts were not reduced substantially at the site. Although such a pattern might be characteristic of raw material procurement sites, the paucity of locally available raw materials suggests that 41 GR559 did not serve as a raw material procurement area. Rather, it is likely that the low percentage of entirely decorticate debitage reflects the rather expedient manufacture strategies employed in the making of chipped lithic artifacts at the shelter. It also may indicate that few entirely decorticate artifacts were introduced to and further reduced or rejuvenated at the site. If these suggestions are correct, they imply an opportunistic occupation strategy associated with the shelter.

A large majority $(81 \%)$ of the platform-bearing fine-grained chert flakes (i.e., complete flakes) and flake fragments (i.e., proximal fragments) have entirely decorticate striking platforms. On the other hand, only $35 \%$ of the nonchert platform-bearing debitage collection has decorticate striking platforms. This pattern may be indicative of differences in reduction strategies (i.e., bifacial for fine-grained chert and multidirectional for nonchert) between the two groups of raw materials. Ground striking platforms are infrequent $(2 \%)$ in the fine-grained chert debitage. None of the nonchert debitage have ground striking platforms.

Large percentages of both the fine-grained chert $(85 \%)$ and nonchert $(96 \%)$ platform-bearing debitage are single faceted. However, debitage with two or more striking platforms represents a larger percentage of the fine-grained chert specimens $(16 \%)$ than of the nonchert debitage $(4 \%)$. These differences parallel the patterns noted in platform cortex breakdowns and suggest that a large portion of the nonchert debitage 


\begin{tabular}{|c|c|c|c|c|c|}
\hline \multicolumn{6}{|c|}{$\begin{array}{l}\text { BREAKDOWN OF DEBITAGE ATTRIBUTES WITHIN THE FINE-GRAINED CHERT } \\
\text { AND NONCHERT RAW MATERIALS, 41GR559 }\end{array}$} \\
\hline \multirow[b]{2}{*}{ Attribute } & \multicolumn{2}{|c|}{ Fine-grained Chert } & \multicolumn{2}{|c|}{ Nonchert } & \multirow[b]{2}{*}{ Totals } \\
\hline & $\#$ & $\%$ & $\#$ & $\%$ & \\
\hline $\begin{array}{l}\text { Flake Completeness } \\
\text { Complete } \\
\text { Proximal } \\
\text { Chips } \\
\text { Chunks } \\
\text { Totals: } \\
\end{array}$ & $\begin{array}{r}50 \\
2 \\
15 \\
- \\
67 \\
\end{array}$ & $\begin{array}{r}25 \\
36 \\
21 \\
18 \\
100 \\
\end{array}$ & $\begin{array}{r}17 \\
6 \\
10 \\
1 \\
34 \\
\end{array}$ & $\begin{array}{r}50 \\
18 \\
29 \\
\quad 3 \\
100 \\
\end{array}$ & $\begin{array}{r}67 \\
8 \\
25 \\
1 \\
101 \\
\end{array}$ \\
\hline $\begin{array}{c}\text { Cortex Percent } \\
0 \\
1-50 \\
51-99 \\
100 \\
\text { Totals: }\end{array}$ & $\begin{array}{l}17 \\
24 \\
14 \\
12 \\
67\end{array}$ & $\begin{array}{r}25 \\
36 \\
21 \\
18 \\
100\end{array}$ & $\begin{array}{r}12 \\
10 \\
6 \\
5 \\
33\end{array}$ & $\begin{array}{r}36 \\
30 \\
18 \\
-15 \\
99\end{array}$ & $\begin{array}{r}29 \\
34 \\
20 \\
17 \\
100\end{array}$ \\
\hline $\begin{array}{l}\text { Chunk Cortex } \\
\text { Absent } \\
\text { Present } \\
\text { Totals: }\end{array}$ & $\begin{array}{l}\overline{\overline{0}} \\
\overline{0}\end{array}$ & $\begin{array}{l}\overline{\overline{0}} \\
\overline{0}\end{array}$ & $\frac{-}{1}$ & $\frac{-}{100}$ & $\begin{array}{l}0 \\
1 \\
1\end{array}$ \\
\hline $\begin{array}{l}\text { Platform Cortex } \\
\text { Absent } \\
\text { Present } \\
\text { Totals: }\end{array}$ & $\begin{array}{l}42 \\
10 \\
52\end{array}$ & $\begin{array}{r}81 \\
19 \\
100\end{array}$ & $\begin{array}{r}8 \\
15 \\
23\end{array}$ & $\begin{array}{r}35 \\
65 \\
100\end{array}$ & $\begin{array}{l}50 \\
25 \\
75\end{array}$ \\
\hline $\begin{array}{l}\text { Platform Grinding } \\
\text { Absent } \\
\text { Present } \\
\text { Totals: }\end{array}$ & $\begin{array}{r}51 \\
1 \\
52\end{array}$ & $\begin{array}{r}98 \\
\frac{2}{100}\end{array}$ & $\begin{array}{l}23 \\
\frac{-}{23}\end{array}$ & $\frac{100}{\overline{100}}$ & $\begin{array}{r}74 \\
\frac{1}{75}\end{array}$ \\
\hline $\begin{array}{l}\text { Platform Facet Count } \\
1 \\
2 \\
>2 \\
\text { Totals: }\end{array}$ & $\begin{array}{r}44 \\
6 \\
2 \\
52\end{array}$ & $\begin{array}{r}85 \\
12 \\
4 \\
101\end{array}$ & $\begin{array}{r}22 \\
1 \\
-\overline{2}\end{array}$ & $\begin{array}{r}96 \\
4 \\
- \\
100\end{array}$ & $\begin{array}{r}66 \\
7 \\
2 \\
75\end{array}$ \\
\hline $\begin{array}{l}\text { Maximum Dimension } \\
1-10 \mathrm{~mm} \\
11-20 \mathrm{~mm} \\
21-30 \mathrm{~mm} \\
31-40 \mathrm{~mm} \\
>40 \mathrm{~mm} \\
\text { Totals: }\end{array}$ & $\begin{array}{r}1 \\
29 \\
28 \\
5 \\
4 \\
67\end{array}$ & $\begin{array}{r}1 \\
43 \\
42 \\
7 \\
6 \\
99\end{array}$ & $\begin{array}{r}- \\
13 \\
11 \\
7 \\
3 \\
34\end{array}$ & $\begin{array}{r}- \\
38 \\
32 \\
21 \\
\frac{9}{100}\end{array}$ & $\begin{array}{r}1 \\
42 \\
39 \\
12 \\
7 \\
101\end{array}$ \\
\hline $\begin{array}{l}\text { Flake Type } \\
\text { Biface } \\
\text { Uniface } \\
\text { Indeterminate } \\
\text { Totals: }\end{array}$ & $\begin{array}{r}1 \\
18 \\
48 \\
67\end{array}$ & $\begin{array}{r}1 \\
27 \\
72 \\
100\end{array}$ & $\begin{array}{r}- \\
\frac{33}{33}\end{array}$ & $\begin{array}{r}- \\
- \\
100 \\
100\end{array}$ & $\begin{array}{r}1 \\
18 \\
81 \\
100\end{array}$ \\
\hline
\end{tabular}


may have derived from multidirectional core reduction. On the other hand, at least some of the fine-grained chert debitage appears to be the result of bifacial reduction.

The analysis of debitage maximum dimensions indicates that the nonchert debitage contains a larger percentage of specimens in the $31-40-\mathrm{mm}$ and the $>40-\mathrm{mm}$ categories compared to the fine-grained chert collection. This pattern suggests that the nonchert cores, in general, might have been larger than the fine-grained chert cores.

The platform cortex and faceting attributes suggest that a relatively large percentage of the finegrained chert debitage may have derived from bifacial reduction strategies. The flake type breakdown shows that, among the fine-grained chert debitage, a large percentage of the flakes that could be categorized derive from uniface reduction. None of the nonchert debitage could be grouped into flake type categories. While the fine-grained chert flake type breakdowns appear to contradict the suggestions above, indeterminate specimens constitute a large percentage of the collection, and it is possible that the indeterminate flakes and flake fragments contain a large percentage of specimens produced during bifacial reduction.

The majority ( $n=63,94 \%)$ of the fine-grained chert debitage is of local origin. This percentage is slightly higher than that noted in both the 41GR291 and 41GR303B fine-grained chert debitage collections. Nonlocal-origin fine-grained chert specimens comprise only $4 \%(n=3)$ of the $41 \mathrm{GR} 559$ collection, while indeterminate-origin $(\mathrm{n}=1,1 \%)$ fine-grained chert debitage is rare.

\section{Ground and Battered Stone Artifacts}

Eleven ground and battered stone artifacts were recovered. Contrary to the patterns noted in the other two prehistoric sites, hammerstones $(n=8)$ constitute the largest group. Metates $(n=3)$ are few in number.

\section{Metates}

All three of the sandstone metates are fragmentary. Although they refit into a single incomplete ground stone tool, for analytical purposes they are treated as three specimens because they were recovered from three distinct proveniences. Each fragment exhibits unifacial use wear and pecking and lacks battering. The metate fragments have planoconcave transverse cross sections. The original shape of the metate could not be determined. The mean thickness of the three specimens is $30.0 \mathrm{~mm} \mathrm{(} \sigma=$ 5.0). Other metric attributes could not be recorded. None of the fragments bear evidence of burning or recycling into hearthstones.

\section{Hammerstones}

Eight hammerstones were recovered. All are complete and are of coarse-grained quartzite. The eight specimens can be divided into light $(n=3)$, medium $(n=2)$, and heavy $(n=3)$ hammerstones in terms of weight. The three light hammerstones weigh between 100-140 $\mathrm{g}$ and have a mean weight of $107.5 \mathrm{~g}(\sigma=3.5)$. The two medium specimens weigh between $170-185 \mathrm{~g}$ and have a mean weight of $177.0 \mathrm{~g}(\sigma=7.1)$. The three heavy hammerstones weigh between $230-430 \mathrm{~g}$ and have a mean weight of $333.0 \mathrm{~g}(\sigma=95.0)$.

Two of the three light hammerstones exhibit light battering around their peripheries (Figure $88 a$ ); the remaining specimen has moderate battering concentrated primarily on one end (Figure $88 b$ ). Both of the medium-weight specimens have moderate battering concentrated on the comers or ends (Figure $88 c$ ). Two of the heavy hammerstones exhibit only light battering; the remaining specimen has moderate battering concentrated on one end (Figure $88 d$ ). The eight hammerstones have a mean length of $71.1 \mathrm{~mm}$ ( $\sigma=10.5)$, a mean width of $56.4 \mathrm{~mm}(\sigma=12.4)$, and a mean thickness of $39.6 \mathrm{~mm}(\sigma=9.3)$. Their mean weight is $213.2 \mathrm{~g}(\sigma=114.5)$.

\section{Burned and Fire-cracked Rocks}

The burned and fire-cracked rocks from 41 GR559 are considered to be manuports, and all were found in association with cultural features. They are discussed in the Cultural Features section of this chapter. All of the burned rocks are either sandstone or sandstone conglomerate, and all could have been obtained in the immediate vicinity. The total weight of the burned rocks is $180.8 \mathrm{~kg}$. With the exception of the Feature 15 rocks, which were not counted, and some of the innumerable small fragments, 242 burned rocks were contained in the cultural features.

\section{Vertebrate Faunal Remains}

The vertebrate faunal assemblage from 41GR559 is large and represents the overwhelming 

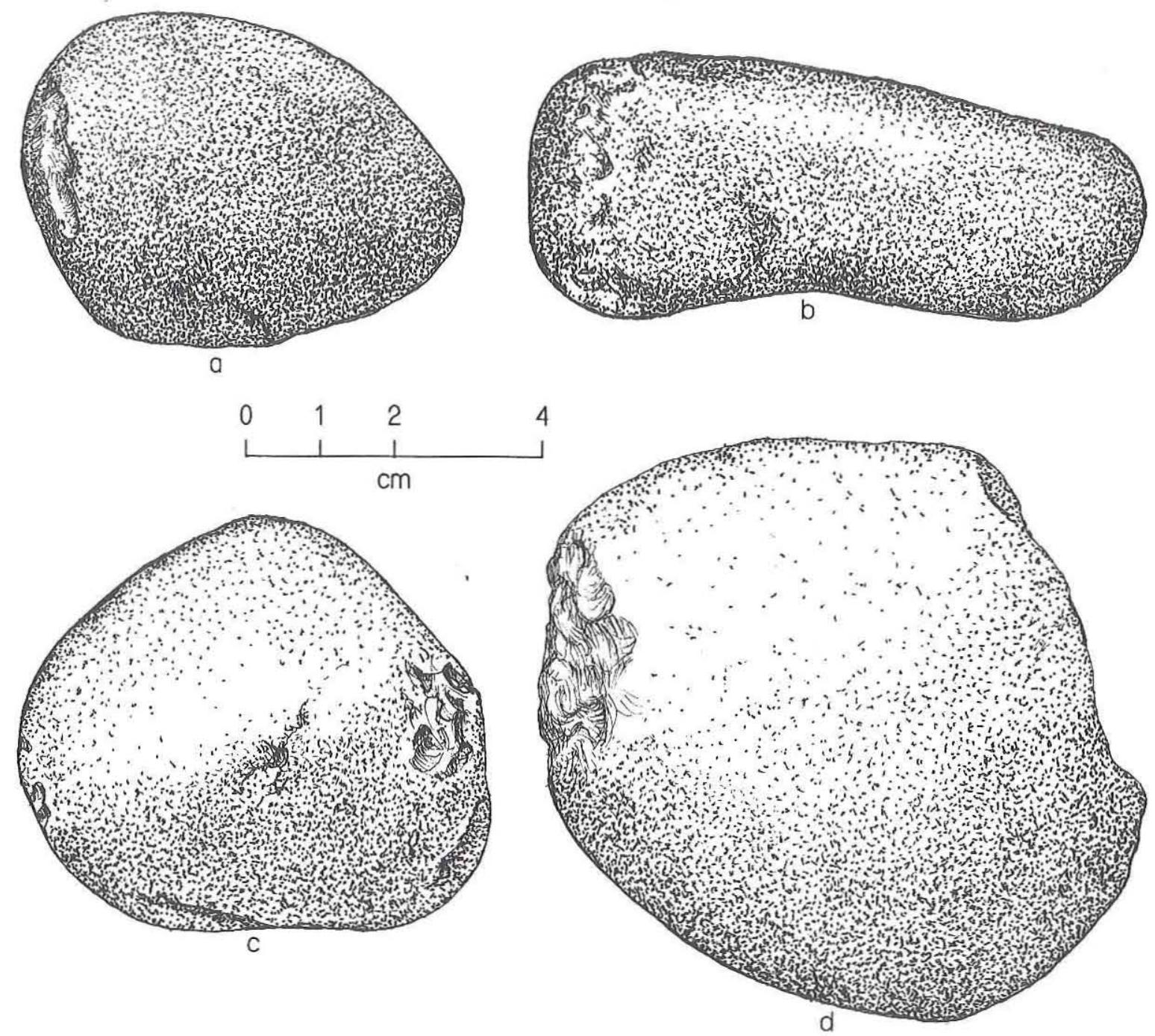

Figure 88. Hammerstones, 41GR559.

majority of the materials recovered from the site. All of the specimens discussed here were recovered in the 1992 excavations. Data on the vertebrate faunal specimens recovered in the 1988 testing can be found in Boyd et al. (1990:563). Of this collection, 3,033 $(85 \%)$ are from the area in front of the rockshelter and $547(15 \%)$ are from excavation units inside the rockshelter. Of the total, 2,722 were recovered from feature contexts and 858 were recovered from nonfeature contexts. Bones recovered from features inside the rockshelter total 194. Bones from bone midden features total 2,528. The Season 3 assemblage is listed and fully discussed in Appendix C.

Inside the rockshelter, the most common taxon is Vertebrata, represented by 311 specimens. Mammalia is represented by 91 bones. Bones identified to genera are 19 Odocoileus sp., 4 Canis sp., 2 Lepus sp., and 42 Sylvilagus sp. Various rodent bones total 61. Also inside the rockshelter were three bones of Colubridae and one each of Carnivora, Anura, Serpentes, Aves (small and medium), Emberizidae, and Lacertilia. Fifty-two bones $(10 \%)$ from inside the rockshelter exhibit evidence of cultural modification, and 29 of these are from hearth features. The most common are rabbit and unidentified mammals and vertebrates.

The bone midden consists of the area in front of and downhill from the mouth of the rockshelter that is contained in EUs 1-11. Six bone clusters were recovered in this area. The most common taxon is medium/large Mammalia, represented by 1,588 specimens. All Mammalia specimens total 1,765. 
Unidentified Vertebrata number 402. Bones of specified genera include 568 Canis sp., 7 Vulpes sp., 6 Lepus sp., 9 Sylvilagus sp., and 72 bones of various rodent species. Bones of identified species are 78 Felis rufus, 10 Bison bison, 12 Mephitis mephitis, and 1 Mustela vison. Bones of unidentified Carnivora total 49, and 31 Canidae, 12 Leporidae, 4 Colubridae, and 6 Aves complete the assemblage.

It appears that these bone midden features are a result of cultural behavior and that this behavior was related to subsistence activities. Twenty-two modified bones $(<1 \%)$ occur in the bone midden, and the assemblage is not sufficiently mixed to indicate that it was deposited as a result of erosion from inside the shelter or intentional cleaning of the shelter.

\section{Invertebrate Faunal Remains}

Fourteen fragments of freshwater mussel shells that appear similar to those identified as Uniomerus declivus by Raymond Neck (Boyd et el. 1990:566) were collected at 41GR559 (see Appendix D). One rectangular fragment from Level 4 of EU 17 appears to be use modified. It exhibits rounding and smoothing on the outer edge of the shell and may have been utilized lightly as a scraper. The specimen is $74 \mathrm{~mm}$ in length, $25 \mathrm{~mm}$ in width, and $3 \mathrm{~mm}$ in maximum thickness; it weighs $7.7 \mathrm{~g}$. Of the remaining 13 fragments, all were recovered from Level 5 of EU 23. None exhibit any signs of modification, and it appears that their presence results from utilization as a food source.

The 1992 excavations yielded 27 snail shells of the Succineidae family (see Appendix D). All but one were recovered from units inside the shelter. Succineidae are terrestrial and prefer moist, wooded areas. There is no evidence to suggest that their presence is cultural.

\section{Modern Artifact}

One piece of rusty, six-strand wire $80 \mathrm{~cm}$ long was recovered from EU 9, Level 2. As the rust is surficial, the stratigraphic location shallow, and the wire still springy, this specimen is considered recent and intrusive.

\section{CHRONOLOGY}

The primary technique employed for defining the chronology of $41 \mathrm{GR} 559$ is radiocarbon dating of organic remains associated with features. Thermoluminescence dating of burned rocks was utilized in a previous phase of the project, but the results were inconsistent with the radiocarbon dates and are not considered to be reliable. Also discussed here are the three temporally sensitive artifacts recovered. These artifacts are characteristic of broad time periods and geographic areas, but they are not sufficiently diagnostic to be very useful in defining the chronology of the site.

\section{Absolute Chronology}

The cultural remains at 41GR559 are dated by nine radiocarbon assays associated with cultural features (see Table 54; Figure 89). Eight of these dates are on charcoal samples, and one is on bone collagen. Two dates are from charcoal samples recovered in the 1988 testing (GX-14448/14449; Boyd et al. 1990:149), and seven are from samples recovered during the 1992 excavations. As Figure 89 shows, the 1-sigma ranges of these nine assays cover a long span of time from 2 B.C. to A.D. 1663. They are discussed here in three groups.

First, five dates were obtained from hearths (Features 5, 13/26, 16, 22, and 24) in the lower light brown fill of the rockshelter. The date range represented is 2 B.C. to A.D. 1030. The earliest date, 2 B.C.-A.D. 129 , is from Feature 16 in the front-central portion of the shelter. It is the deepest feature at 97-100 cm (Figure 90). A few of the other dates appear to be out of stratigraphic order, with some later features occurring lower than earlier ones, but this is the result of the construction of hearths on natural benches and large pieces of roof fall. For example, Feature 5, the latest dated feature in the lower zone (A.D. 650-1030), is lower than Feature 13/26 (A.D. 227-412) and Feature 22 (A.D. $640-860$ ), but Feature 5 is in the center portion of the shelter while Features $13 / 26$ and 22 are on sandstone benches. It is assumed that the undated features in the lower zone (Features 2, 4, 8, 11, 15, 20, 23, and 25) all date between 2 B.C. and A.D. 1030 .

Four burned rock features appear to have originated in the upper reddish brown fill of the shelter (Features 1, 3, 18, and 27), and three of these features produced date ranges of A.D. 990-1290, A.D. 1021-1169, and A.D. 1169-1277. Features 1, 18 , and 27 crosscut the boundary between the lower light brown and upper reddish brown sediment zones; 


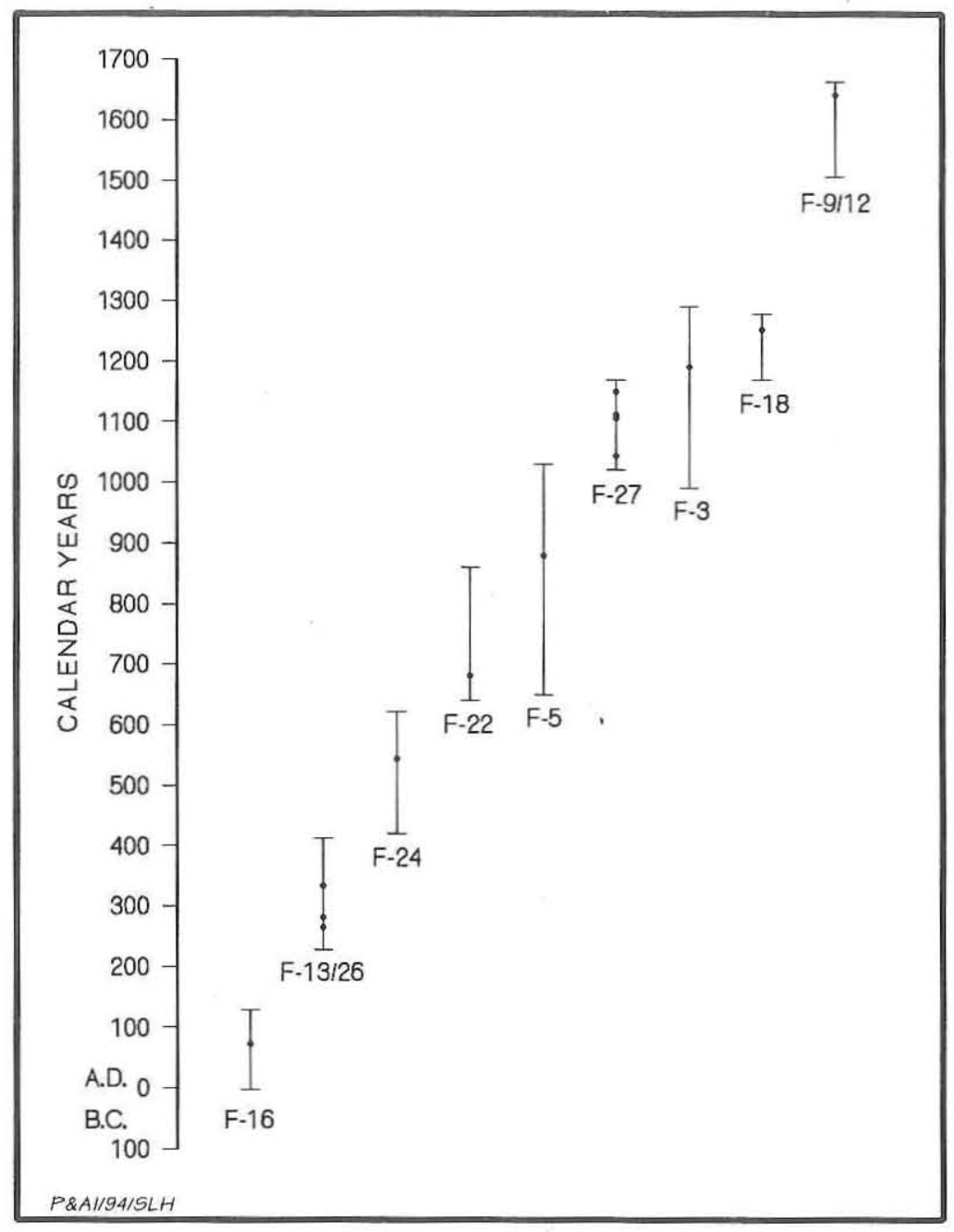

Figure 89. Graph of calibrated radiocarbon dates (intercepts and 1-sigma ranges) from features, 41GR559.

Feature 3 appears below this boundary, but it probably was forced down into the lower zone by the weight of a large piece of roof fall lying on top of it. As a group, these dates suggest that the cultural materials in the upper reddish brown fill of the shelter date to ca. A.D. 1000-1300.

The third dated context relates to the talus area in front of the shelter. A single date on bison bones from Features 9 and 12 has a 1-sigma range of A.D. 1506-1663. These two features, as well as Features 7 and 10, are clearly contained in the upper reddish brown sediment zone (Figure 91). Their stratigraphic relationships, proximity to one another, and sharing of elements from the same animals suggest that these four bone clusters were deposited over a short time span and represent a single bone midden. Thus, the date from Features 9 and 12 probably applies to all of these remains. This also may be the case for the other three features found to the west in front of the shelter (Features 14, 17, and 19), since the taxa contained in the two bone clusters (Features 14 and 17) are quite similar to those in Features 7 , 9,10 , and 12 . These three western features are in an area disturbed by runoff, however, and they cannot be related to Features 7, 9, 10, and 12 stratigraphically.

\section{Relative Chronology}

There are few temporally diagnostic artifacts from 41GR559. The single dart point is typed as Ellis, which supposedly is diagnostic of the late, or terminal, Archaic period on the Texas High Plains, as Ellis points have been found at Deadman's Shelter in Stratum D with dates of A.D. 120 and 210 and at the Canyon City Club Cave in Level 4 with dates of A.D. 300 and 680 (Hughes 1991:21). Ellis side-notched points are reported to be diagnostic of the nonceramic Hueco phase of the eastern Jornada Mogollon dating up to A.D. 950 (Leslie 1978:129) and of the Brantley phase of the Carlsbad Basin dating to A.D. 1-750 (Katz and Katz 1985:67). The type was originally defined to include a time span of 2,000 years (Davis 1991:74; Suhm and Jelks 1962:187; Turner and Hester 1985:93), and the single specimen from 41GR559 is of little help in dating the site. The Ellis point was recovered from Level 3 of EU 24, $1.5 \mathrm{~cm}$ below Feature 27, dated to A.D. $1021-1169$, and $4.5 \mathrm{~cm}$ above Feature 22, dated to A.D. $640-860$. These date ranges bracket the endings of the time ranges attributed to the type, so it is not out of place as a late holdover or a recycled artifact manufactured earlier. The resharpening on this specimen supports this contention.

The distal arrow point fragment from Level 7 of EU 28 has lost its base. It appears Scallorn-like, but without the base its identity is uncertain. Scallorn-like corner-notched arrow points are 


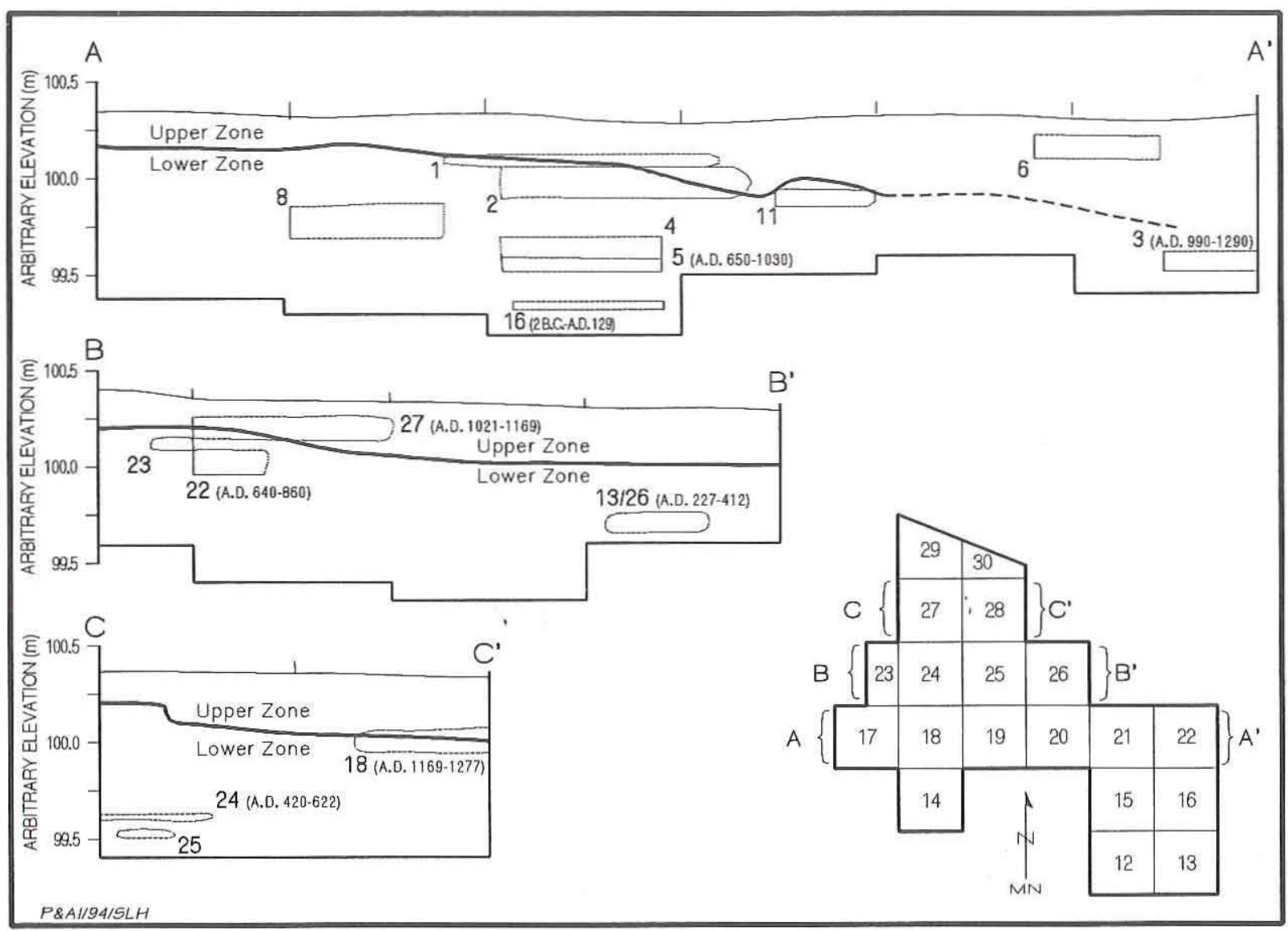

Figure 90. Collapsed east-west profiles indicating the stratigraphic positions of the features inside the rockshelter, 41 GR559.

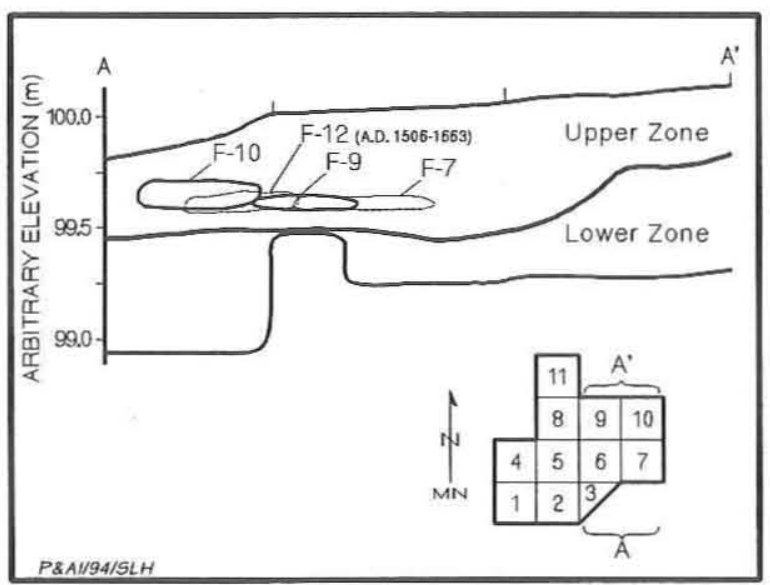

Figure 91. Collapsed north-south profile of the talus area and bone midden features in front of the shelter, 41GR559.

characteristic of the period from about A.D. 200 to 1100 (the Early Neoindian substage of Hughes 1991:26; the Late Prehistoric I of Boyd et al. 1993:7) on the Southern Plains and have been recovered in association with Ellis dart points in Lake Creek complex sites (Hughes 1991:26). The dated feature in the rockshelter closest to the location of this arrow point is Feature 24 in Level 8 of EU 27, dated to A.D. 420-622.

The side-notched arrow point recovered from Level 2 of EU 18 is classified as a Washita. Sidenotched arrow points are considered a Plains type and are thought to have been adapted specifically for bison hunting. Kehoe (1966:832) suggests that sidenotched points date no earlier than A.D. 1300 on the Northern Plains. Gunnerson (1960:Plates 12 and 13) dates similar points of the Dismal River aspect to post-A.D. 1000. The specimen from 41GR559 compares well with Leslie's (1978:101) Type 2-D, which he states first appeared in the extreme southeastern portion of New Mexico after A.D. 1350. The 41GR559 specimen was recovered from high in the upper sediment zone close to Feature 27, dated to A.D. 1021-1169. 


\section{INTERPRETIVE SUMMARY}

Chronological data from Boren Shelter \#2 confirm that the site was utilized intermittently over a long span of time. The lower sediment zone within the shelter contains evidence of occupation through the entire first millenium A.D. The upper sediment zone retains traces of occupations for another 300 years, ca. A.D. 1000-1300. The talus area in front of the shelter produced one radiocarbon assay indicating deposition of a bone midden during the sixteenth or seventeenth century, and it is likely that all of the remains in this part of the site date to this interval. In total, the site represents a series of occupations over a span of approximately 1,600 years. At no time, however, was 41GR559 occupied intensely or for an extended period of time. This has resulted in a lack of readily definable and separable components. Nonetheless, three gross use episodes or occupational periods can be identified, as listed above. This summary section examines the cultural features and the material cultural at 41GR559 from the perspective of these three periods of use/ occupation.

\section{Cultural Features}

The 25 cultural features can be separated into use episodes or periods of occupation based on radiocarbon dates, stratigraphic relationships, or similarities in content to other features (Table 58). All but four of the hearth and burned rock features are assigned to the lower sediment zone in the rockshelter, which dates to the Late Prehistoric I period. Four burned rock features and a possibly noncultural bone cluster are assignable to the upper sediment zone, which has been dated to A.D. 10001300 , or the early part of the Late Prehistoric II period. Similarities in the taxa contained in the bone clusters in front of the shelter and the presence of elements apparently representing the same animal in more than one cluster suggest that all of the talus area bone clusters, as well as the hearth dump underneath, are contemporaneous, dating to ca. A.D. $1500-1700$, or the Protohistoric period.

\section{Lower Shelter}

The early occupations in the rockshelter, dated to 2 B.C.-A.D. 1030, are represented by two unlined hearths, four stone-lined hearths, four disaggregated hearths, a burned rock concentration, a hearth dump, and a wind deflector. These features are contained in an approximately 1-m-thick deposit of primarily endogenous sediments derived from the degradation of the soft sandstone walls and ceiling inside the rockshelter. Activities that may have been associated with these features include cooking of both animal and plant materials and building fires for warmth in colder seasons. Because the rockshelter mouth faces south, it would have provided shelter from cold winds out of the north and allowed collection of solar radiation during most of the daytime hours. The dark surface of the hard sandstone and sandstone conglomerate bedrock absorbs solar heat at a higher rate than lighter colored rock, increasing its effectiveness as a solar collector (Hunt 1972:90). For these reasons, 41GR559 would have served well as a winter

\begin{tabular}{|l|c|c|c|c|}
\hline \multicolumn{5}{|c|}{ TABLE 58 } \\
\hline \multirow{4}{*}{ SUMMARY OF FEATURES BY TYPE AND TEMPORAL AFFILIATION, 41GR559 } \\
\cline { 2 - 5 } Feature Type & \multicolumn{3}{|c|}{ Feature Nos. } \\
\hline Unlined hearth & 16,24 & & Total No. of Features \\
Stone-lined hearth & $8,13 / 26,22,25$ & $3,27,18$ & & 2 \\
Disaggregated hearth & $2,4,5,11$ & 1 & & 7 \\
Burned rock concentration & 15 & & 19 & 5 \\
Hearth dump & 23 & & & 2 \\
Wind deflector & 20 & 6 & $7,9,10,12,14,17$ & 1 \\
Bone cluster & 13 & 5 & 7 & 7 \\
\hline Total No. of Features & & & & 25 \\
\hline
\end{tabular}


campsite but poorly as a summer location for almost any activities.

Macrobotanical samples and modified bones from features in this sediment zone produced limited information relating to the diet of the inhabitants during this period. Seeds of possibly economic plants were found in Features 4, 8, 13/26, 16, and 25 (see Appendix E). The strongest vegetal evidence is the presence of 19 Chenopodium sp. seeds in Feature 16. Other identified seeds from features in this zone are dropseed, hackberry, skullcap, Panicum sp., and one bottle gourd seed; none were found in great amounts. Culturally modified animal remains from this occupation zone include burned or spirally fractured bones from Features 2, 4, 8, 11, 13/26, and 16 . Animals represented are rabbits, rodents, snakes, and unidentified mammals and vertebrates. There is no clear evidence for the procurement of medium or large mammals such as deer, antelope, or bison. Overall, the evidence from the features of the Late Prehistoric I period occupations indicates small-scale hunting and gathering as possible activities.

\section{Upper Shelter}

The late occupations in the rockshelter, dated to A.D. 1000-1300, are represented by three stone-lined hearths, one disaggregated hearth, and one bone cluster (see Table 58). The bone cluster consists of 15 , possibly 19 , deer bones not associated with burned rock features or tools. Gnawing marks were found on four associated ribs from a medium mammal, and although these bones may have been deposited originally by humans, they also could be the result of carnivore activities. These features are contained mostly within the reddish brown, partly eolian sediments which vary in thickness from approximately $20 \mathrm{~cm}$ in the west end of the shelter to over $50 \mathrm{~cm}$ in the east end. The fact that all four burned rock features crosscut the boundary between the upper and lower sediment zones indicates that they were constructed shortly after the depositional regimes changed within the shelter. This change may have been associated with increasingly xeric climatic conditions after ca. A.D. 1000.

Activities associated with these features appear to have been the same as for the lower zone, i.e., cooking of both plant and animal materials and building fires to keep warm. The kinds of features and their contents bear witness to continuity in how the shelter was used from the early period of occupation. Macrobotanical samples from these features suggest the processing of prickly pear in Feature 3 (Cummings 1990:541) and possible processing of seeds of mesquite, Chenopodium sp., and Bromus sp. in Feature 18. Burned wood samples from cultural features indicate continued use of small pieces of juniper, hardwoods, and ring-porous hardwoods as fuel.

Generally, the evidence from the upper zone features indicates a continuation of the small-scale hunting and gathering implied in the lower zone. Animals taken during this period of occupation appear to have been rabbits, rodents, snakes, and perhaps deer, along with unidentified mammals and vertebrates. Plants that were processed may have included cactus, mesquite, Chenopodium sp., and grass seeds. While the sedimentological evidence points to changes in climate, the feature contents indicate continuity in subsistence strategies.

\section{Talus Area}

The talus area in front of the shelter is dominated by a bone midden dated to A.D. 15061663. Of the 3,033 bones from this area, 2,528 were recovered directly from features. The other 505 bones from this area are probably feature-associated but were not collected as part of the clusters that are designated as features. Of the seven features in this area, six are bone clusters and one is a hearth dump located beneath one of the bone clusters. The following observations are derived from the distributions of the bones (see Appendix C).

Feature 7 is dominated by bones of the heads and feet of wolves and dogs. The juvenile bones in Feature 7 are doglike and not from the same individuals found in Feature 17. Features 10 and 12 are dominated by head and feet elements of wolf and limb elements of bobcat. Bobcat is represented in all of the bone clusters except Feature 17. Feature 14 is dominated by head and feet elements of wolf. Feature 17 contains juvenile wolflike elements. All of the fox elements from the bone midden occur in this feature; these are a mandible and the accompanying teeth. The skunk elements in the bone midden, consisting of right and left mandibles and accompanying teeth, were in or near Feature 17. Bison elements are all of lower rear legs, i.e., left leg elements in Feature 9 and right leg elements in Feature 12. A matching calcaneous was recovered from Feature 10. The rodent elements in Level 1 of 
EU 4, the cotton rat elements in Level 1 of EU 10, and the Neotoma elements in Levels 1 and 2 of EUs 8 and 9 and Level 1 of EU 12 are considered intrusive.

Although the hearth dump is cultural in origin, interpreting the bone midden remains problematical. The assemblage is unusual in the high percentage of mixed carnivores, i.e., animals that might be found naturally in rockshelter deposits. However, it is not unusual to find carnivores in small quantities in cultural contexts. Wolves have been documented as a starvation food for frontier Anglos (Young 1944:171-4), ethnographically for historic Arikara, Arapahoe, Cheyenne, Crow, and Sioux groups (Young 1944:176-185), and in archeological deposits in Texas (Creel 1990:177; Duffield 1970:246; Hughes 1969:126) and elsewhere (e.g., Driver 1985; Frison 1973:72; Walker 1980:347; Walker and Frisonn 1979; Walter 1987:119; Wood 1968:174). A butchered wolf skeleton recovered from a Garza occupation at the Lubbock Lake site (Green 1962; Johnson et al. 1977:95) is approximately contemporaneous with the 41GR559 bone midden deposits.

While there are few butchering marks, neither are there many indications of gnawing as would be expected if the bone midden was the result of carnivore predation. Canine tooth marks on four mammal rib heads associated with Feature 6 inside the shelter constitute the only evidence of animal predation at 41GR559, and these bones and the deer elements from Feature 6 and Level 1 of EU 20 could provide evidence for denning by carnivores in the shelter. While denning is unlikely in the excavated area of the rockshelter because it is open and lacks a constricted, defensible entranceway (Young 1944:98), it is more conceivable in the jumbled rocks which limit entry to the collapsed part of the shelter east of the site.

Further, most of the bones were found in discrete piles rather than scattered as would be expected if they were the result of carnivore predation (Binford 1981:Chapter 5), and several of the clusters contain elements apparently representing the same animal. This suggests that the bone clusters are cultural in origin, and the lack of axial skeletal elements and most femurs, representing meaty portions, suggests that these animals were killed nearby and processed at 41GR559 before transport of the meat elsewhere.

\section{Material Culture}

The total artifact collection from 41GR559 consists of 146 stone artifacts and 1 possibly utilized mussel shell from the 1992 mitigative excavations and 22 stone artifacts from the 1988 testing. While the sample sizes are too small for statistical comparisons, the following paragraphs discuss the distributions of these remains using the three contexts discussed above, i.e., lower shelter, upper shelter, and talus area. Also discussed here are the 3,580 vertebrate remains recovered in 1992 and 241 specimens found inside the shelter in the 1988 testing.

\section{Lower Shelter}

Of the total artifact collection from testing and excavation, 116 (69\%) are from the lower sediment zone in the shelter (Table 59). The low overall artifact density $\left(7.5 / \mathrm{m}^{2}\right)$ indicates that the shelter was used in a limited fashion and probably on a shortterm basis. This is supported by the lack of ceramics and ground stones. The collection is dominated by unmodified debitage $(76 \%)$, with cores $(9 \%)$, unifaces (4\%), edge-modified flakes (4\%), and hammerstones (3\%) occurring in smaller numbers; cobble tools, bifaces, and projectile points are even less frequent ( $1 \%$ each). Also recovered from this zone was the single possibly modified mussel shell. This collection is suggestive of a limited range of procurement, processing, and maintenance activities. While both

\begin{tabular}{|l|c|c|c|}
\hline \multicolumn{4}{|c|}{ TABLE 59 } \\
ARTIFACTS RECOVERED FROM ALL \\
PHASES OF WORK AT 41GR559 \\
\hline Artifact Type & Upper & Lower & Talus \\
\hline Shelter & Shelter & Area \\
Unifaces & 1 & 5 & - \\
Hammerstones & 2 & 3 & 5 \\
Coround stones & 3 & - & - \\
Edge-modified flakes & 2 & 11 & 3 \\
Cobble tool & - & 5 & - \\
Biface & - & 1 & - \\
Projectile points & 2 & 1 & - \\
Unmodified debitage & 24 & 88 & 7 \\
Modified (?) shell & - & 1 & - \\
\hline Totals: & $37(22 \%)$ & $116(69 \%)$ & $16(9 \%)$ \\
\hline
\end{tabular}


unifacial and bifacial lithic reduction strategies are represented, expedient multidirectional core reduction appears to have been predominant. The vast majority of the lithic artifacts (109 of $115,95 \%)$ are of local materials, with only 4 pieces of unmodified debitage, 1 edge-modified flake, and 1 uniface being of nonlocal materials.

The total osteological assemblage from the lower zone consists of 482 specimens (Table 60). The best represented taxa are rabbits and rodents. This suggests a hunting strategy focused on small mammals, although some of these remains could be noncultural in origin. Fifty-seven bones exhibit cultural modification. Forty-two elements (5 rabbit, 11 rodent, 14 unidentified mammal, and 12 unidentified vertebrate) are burned; 17 elements (1 rabbit, 1 rodent, 4 unidentified mammal, and 11 unidentified vertebrate) have spiral fractures; and, 1 canid metapodial has cut marks.

\section{Upper Shelter}

Only 37 artifacts (22\%) were recovered from the upper fill of the shelter (see Table 59). The very low overall artifact density $\left(2.0 / \mathrm{m}^{2}\right)$ indicates extremely infrequent and short-term use. The stone tool assemblage is dominated by unmodified debitage (65\%), but it contains a number of tool types as well. Ground stones and cores are equally frequent $(8 \%$ each), followed by hammerstones, edge-modified flakes, and projectile points (5\% each); only a single uniface $(3 \%)$ was found, and the collection lacks cobble tools and bifaces. In spite of its small size, this collection is suggestive of a suite of procurement, processing, and maintenance tasks. Most of the lithics (34 of 37, 92\%) are of local materials; the only items of nonlocal materials are an arrow point, a dart point, and a piece of unmodified debitage.

The total faunal assemblage consists of 275 specimens (see Table 60). The most common taxa are rabbits, rodents, and deer. This suggests greater reliance on medium-sized mammals than during the occupations represented by the lower shelter deposits, although gnawing on some of the possible deer bones raises the possibility that these are the result of carnivore predation rather than human subsistence remains. Only 12 bones (1 rabbit, 4 unidentified vertebrate, and 7 unidentified mammal) show evidence of cultural modification in the form of spiral fracturing.

\begin{tabular}{|c|c|c|c|}
\hline \multicolumn{4}{|c|}{$\begin{array}{l}\text { TABLE } 60 \\
\text { FAUNAL REMAINS FROM } \\
\text { S OF WORK AT } 41 \text { GR559 }\end{array}$} \\
\hline Taxon & $\begin{array}{l}\text { Upper } \\
\text { Shelter }\end{array}$ & $\begin{array}{l}\text { Lower } \\
\text { Shelter }\end{array}$ & $\begin{array}{l}\text { Talus } \\
\text { Area }\end{array}$ \\
\hline Vertebrata & 89 & 243 & 402 \\
\hline Anura & - & 1 & - \\
\hline Lacertilia & 1 & - & - \\
\hline Serpentes & 1 & 1 & - \\
\hline Colubridae & 2 & 1 & 4 \\
\hline Aves & - & 2 & 6 \\
\hline Emberizidae & 1 & - & - \\
\hline Mammalia & 45 & 71 & 1,782 \\
\hline Leporidae & 2 & 8 & 12 \\
\hline Lepus sp. & 1 & 1 & 6 \\
\hline Sylvilagus sp. & 14 & 25 & 9 \\
\hline cf. Sylvilagus sp. & $; 1$ & 2 & - \\
\hline Rodentia & 65 & 103 & 10 \\
\hline Sciuridae & - & 1 & 1 \\
\hline Geomyidae & 3 & 1 & - \\
\hline Cricetidae & 2 & 1 & - \\
\hline cf. Liomys irroratus & - & 2 & - \\
\hline Perognathus sp. & - & - & 3 \\
\hline Dipodomys sp. & - & - & 1 \\
\hline Peromyscus sp. & - & - & 2 \\
\hline Sigmodon sp. & 4 & - & 16 \\
\hline Neotoma sp. & 19 & 14 & 38 \\
\hline cf. Neotoma sp. & - & - & 1 \\
\hline Camivora & 1 & - & 49 \\
\hline cf. Mustela vison & - & - & 1 \\
\hline cf. Mephitis mephitis & - & - & 12 \\
\hline Canidae & - & 1 & 22 \\
\hline cf. Canidae & - & - & 15 \\
\hline Vulpes sp. & - & - & 7 \\
\hline Canis sp. & - & 4 & 275 \\
\hline cf. Canis sp. & - & - & 60 \\
\hline cf. Canis lupus & - & - & 241 \\
\hline Felis rufus & - & - & 68 \\
\hline cf. Felis rufus & - & - & 10 \\
\hline Odocoileus sp. & 15 & - & - \\
\hline cf. Odocoileus sp. & 9 & - & - \\
\hline Bison bison & - & - & 10 \\
\hline Bos/Bison & - & - & 1 \\
\hline Totals: & 275 & 482 & 3,064 \\
\hline
\end{tabular}

\section{Talus Area}

The talus area in front of the shelter produced only 16 artifacts, or $9 \%$ of the total collection (see Table 59). This extremely low density of artifacts $\left(1.4 / \mathrm{m}^{2}\right)$ points to nonintensive, probably ephemeral, 
use. The collection consists predominantly of unmodified debitage (44\%) and hammerstones (31\%), followed by cores (19\%) and a projectile point (6\%). Artifacts in direct association with the bone features are few. Three hammerstones, one arrow point, one chert core, and one flake were recovered from between the boulders in EU 8 west of Feature 7 at elevations comparable to the bone cluster, and two hammerstones and a core were recovered from EU 9 on the western boundary of Feature 7 just below the bone clusters. The assemblage is suggestive of a limited range of activities, and the high frequency of hammerstones is interesting given their association with the bone clusters. These tools may have been used to crush bones for marrow extraction, although no direct evidence of this was noted in the bone assemblage. All of the lithic artifacts are of local materials.

The total osteological assemblage from the testing and mitigative excavations consists of 3,064 specimens (see Table 60). The collection is unusual in that the most common taxa are canids and bobcats; other notable taxa include rabbit, bison, skunk, and rodents. Assuming that these are human subsistence remains, and this is supported by their occurrence mostly in discrete clusters and the lack of axial skeletal elements and most femurs, this collection indicates use of the site during the Protohistoric period for the processing of carnivores and a few other animals before the meat was transported elsewhere, i.e., as a hunting camp/processing location. Twenty-three bones exhibit spiral fracturing and/or burning indicating cultural modification. One canid metapodial shaft has cut marks; a Sigmodon sp. femur from Feature 9 has spiral fracturing; one fragment of a vertebrate bone from Feature 14 is burned; one small/medium mammal fragment, six vertebrate fragments, one bird tarsometatarsus, and a cottontail calcaneus from Feature 19 are burned; six vertebrate bones from the area of overlap between Features 17 and 19 are burned and spirally fractured and a bird ulna from this area is burned; and one vertebrate fragment and one tibia and two metatarsals of cottontail rabbit from Level 8 of EU 4 are burned. 


\section{SUMIMARY AND CONCLUSIONS}

Douglas K. Boyd, Steve A. Tomka, and Jay Peck

This report documents the third and final season of Phase III data recovery at Lake Alan Henry. The Season 3 data recovery consisted of archeological investigations at one historic and four prehistoric sites. The first two sections of this chapter summarize the historic archeological and archival findings for $41 \mathrm{GR} 474$ and relate the site to the regional historic context "Agriculture in Texas: Ranching in the Western Rolling Plains, 1877-1945" (see Chapter 2). To evaluate the propositions put forth in the prehistoric research design, the last two sections of this chapter summarize the archeological findings at 41GR291, 41GR303B, 41GR546, and 41GR559 and relate these sites to the "Model of Late Holocene Human Adaptation" (see Chapter 2).

\section{HISTORIC SITE INVESTIGATION}

Homesteaded by prominent rancher and businessman Jeff D. Justice in 1899, site 41GR474 contains a dugout depression dating to the turn of the century and an early twentieth-century housesite surrounded by a complex of related buildings and features (e.g., barn and corrals). Although the site also has a prehistoric component, the data recovery investigations concentrated on the historic remains. During the 1988 testing, the dugout was found to be largely intact, while the integrity of the later ranch house complex was limited because the house and many related features were razed during the 1950 s. Consequently, data recovery was restricted to archeological excavation of ca. $25 \mathrm{~m}^{3}$ of sediment in and around the dugout depression; 2,321 artifacts have been recovered from all phases of work.

The excavations revealed that the structure was an $11-\mathrm{x}-20-\mathrm{ft}$ rectangular half dugout with partial rock walls that was dug into the slope adjacent to the Double Mountain Fork of the Brazos River. The dugout fill consisted of three zones that produced two distinct and separable artifact assemblages. These zones, from bottom to top, can be characterized as follows: (1) the dugout floor zone, which contained native cedar (i.e., Juniper) logs from the roof and yielded artifacts associated with the dugout's occupation/use; (2) the rock fall zone, which contained numerous large native sandstone rocks that had formed the upper walls of the structure and subsequently collapsed inward; and (3) the trash fill zone, which contained a large quantity of historic debris that had been discarded into the dugout depression by the occupants of the later ranch house.

Although historic data indicate that the site functioned continuously as a ranch/farm headquarters throughout its history (i.e., site function remained unchanged), the two artifact assemblages are quite different in the activities that they represent. Rather than reflecting overall changes in site function, the differences between these assemblages are interpreted as representing the differential nature of the deposits. The dugout artifact assemblage is interpreted as a mix of primary and secondary materials associated with the dugout's occupation and a brief but specialized reuse period when the structure served as a multifunctional workshop. The later trash assemblage is interpreted as a generalized household dump and compares favorably with a contemporaneous dump 
assemblage from San Antonio (Clark 1984). Chronologically, the dugout assemblage dates from ca. 1900-1910, while the trash assemblage dates from ca. 1910-1940. Both assemblages are associated with the closed-range ranching period in the western Rolling Plains, but the higher frequency and greater diversity of artifacts in the later assemblage may be related in part to improved access to railroads and material goods in the early twentieth century.

\section{THE JEFF JUSTICE HOMESTEAD: CLOSED-RANGE RANCHING IN THE WESTERN ROLLING PLAINS}

Historic occupation at site 41GR474 came as a result of the 1895 Four-Section Act, which was intended to promote settlement by offering homesteads to anyone who filed a claim. Like many others, Jeff Justice took full advantage of the legislation which allowed an individual to file a claim for one homestead section and three additional sections of land for grazing or agriculture. Justice homesteaded the land that now includes 41GR474 between 1899 and 1903. He built the dugout, probably in 1899 or 1900, and after meeting the 3year occupancy requirement, took possession of the land. He apparently moved his wife, Mattie, and their eight children onto the land soon after 1900. In 1906-1907, the Justice family built and moved into a large ranch house, which they occupied until the 1920s. After moving away from 41GR474, the Justices rented the property to various tenants; the ranch house was occupied until the late 1940s but was subsequently torn down, apparently in the 1950s.

In many ways, site $41 \mathrm{GR} 474$ is a typical example of turn-of-the-century homesteads that sprang up all over the Rolling Plains as a result of the Four-Section Act. While many individuals homesteaded lands with the intent of leasing or selling them back to the large-scale ranchers who previously had leased them (Freeman 1989:142), others homesteaded with the intent of settling on their own farm or ranch. Jeff Justice built a dugout to serve as his first home, and he probably intended it only as a temporary residence. The temporary use of dugouts appears to have been a common practice at isolated homesteads, and quite frequently they were replaced by more-permanent structures of milled lumber or stone (Freeman 1990:79). Dugouts are the most common structure type associated with the initial settlement period (Freeman 1990:71). In the Lake Alan Henry project area, four historic sites with dugouts (41GR263, 41GR392, 41GR586, and 41KT150) were first settled as homesteads around 1900, while a fifth turn-of-the-century homestead (41GR443) includes a dugout and corral that may have been built and occupied initially by sheepherders in the late nineteenth century (Boyd et al. 1989:142143, 146-147; Boyd et al. 1991:25-29; Freeman and Boyd 1990:125-128). Only one dugout in the project area (at 41KT84) appears to have been built and occupied prior to 1899 , but it served as a ranching line camp rather than a homestead (Boyd et al. 1989:139-140, 146).

Architecturally, the Justice dugout is typical of Plains-style dugouts in some ways, but it also is unique in other respects. It has been said that no two dugouts were exactly alike (Archer 1978:27), and archeological data confirm the wide range of variability in "size, shape, function, and construction features" (Earls 1993b:482). Dugouts do conform to general patterns, however, and they can be classified by type, building materials, construction techniques, and environmental setting (Freeman 1990:71). Information on dugouts in the western Rolling Plains was compiled by Freeman (1990:Table 6), and O'Neill (1993:Table J.2) expanded on these data to include the Panhandle area. Consequently, O'Neill's (1993) data include information on 82 dugouts and are useful for comparing architectural details on a regional basis. Of 47 structures that could be defined as either full or half dugouts, $45(96 \%)$ were identified as half dugouts, indicating that this was the preferred style. Of 27 half dugouts for which data are available, $15(56 \%)$ were, like the Justice dugout, excavated into the slope, while the other 12 were excavated straight down from a level surface.

A variety of different materials was used in the construction of the upper walls and roofs of dugouts (O'Neill 1993:Table J.2). Of the 29 half dugouts for which data are available, the most common upper wall materials were milled lumber $(n=9)$, native wood $(n=6)$, milled lumber and sod $(n=5)$, dirt $/$ sod $(n=4)$, or a combination of milled lumber and native wood $(n=2)$. Only three examples of dugouts with upper walls of stone $(n=1)$ or stone and mud/adobe $(\mathrm{n}=2)$ are listed, and one of these was later plastered with stucco (see 41HF75 below). Of the 29 half dugouts for which data are available, most had some form of wood and/or sod roof. Milled lumber $(n=8), \operatorname{sod}(n=8)$, native wood $(n=3)$, milled 
lumber and sod $(\mathrm{n}=1)$, and native wood and sod ( $n=1)$ account for $72 \%$ of the examples. Milled lumber was also used in conjunction with tarpaper $(n=1)$ and shingles $(n=4)$. A fifth example of shingles is listed without reference to other materials, but since its upper walls were of milled wood, the roof frame presumably also was wood. One half dugout had a roof constructed of native wood and buffalo hides, while the final example had a roof constructed of layers of milled lumber, stucco, and corrugated metal, having been remodeled at a later date (see 41HF75 below).

The selection of an appropriate site for a dugout was extremely important, and several factors such as distance to water, neighbors, and towns were taken into account (Archer 1978:52; Freeman 1990:78-79). The choice of the specific location for a dugout may have been dictated by the local topography and drainage conditions, but factors such as ease of construction and availability of wall and roofing materials also must have been important considerations. O'Neill's (1993) data clearly indicate a preference for half dugouts, possibly because they were easier to build than full dugouts. Similarly, it may have been even easier to construct half dugouts along a slope, provided that sufficient materials for walls and roofing were available. It is possible that full dugouts were constructed mainly when construction materials were in limited supply. One probable disadvantage of full dugouts on the open plains - the tendency for their roofs to blend in with the surrounding landscape and for livestock to unexpectedly drop in (Archer 1978:19) - may help explain the apparent preference for half dugouts built into natural slopes.

Although other factors (e.g., intended length of occupation and intended function) probably were considered, the choice of materials for building dugout walls and roofs may have been dictated mainly by the availability of commercial materials. Although O'Neill's (1993) list includes sufficient data on only 7 half dugouts out of his sample of 82 , there appears to be a correlation between construction date and building materials. The superstructure of the oldest dugout, built in 1879, was constructed entirely of native wood, while the walls and roofs of the other six were constructed of milled lumber between 1902 and 1918. This suggests that native materials were used initially but that commercial building materials became more readily available through time. During the late nineteenth and early twentieth centuries, milled lumber became increasingly available because of improved transportation and marketing networks (i.e., more railroads and towns), resulting in dugouts slowly giving way to aboveground frame houses (Freeman 1990:79-80).

Dugouts were the dominant form of vernacular architecture in the Texas Panhandle-Plains during the late nineteenth and early twentieth centuries, and their regional importance is evidenced by two nearby dugout sites that are listed on the National Register of Historic Places (Steely 1984:28, 69). The Post West Dugout (41GR189), nominated to the National Register in 1976 (Williams and Clark 1976), is located in Garza County on the west side of Post, just below the Caprock Escarpment. The site consists of a large dugout depression, obviously having been excavated into a slope, and a surface scatter of late nineteenth-century artifacts. No investigations have been undertaken at the site, but its archeological integrity is good. Although it is presumed to be one of the earliest settlements in Garza County, no archival research was done to confirm its site history.

The Mayfield Dugout (41BI52), nominated to the National Register in 1973 (Hume et al. 1973), is located in Briscoe County, along the margin of the High Plains, 7 miles northwest of Silverton. It is one of the best preserved dugouts anywhere in the Southern Plains and was still close to its original condition in 1973. Built in 1889 , it has been protected and maintained by the Mayfield family since it was abandoned early in the twentieth century. The interior dimensions of the intact structure are $15 \times 20 \mathrm{ft}$, and the ceiling is $8-9 \mathrm{ft}$ above the floor. It is a half dugout that was built into a sloping embankment; its back wall extends about $3 \mathrm{ft}$ above ground, while its front wall extends 5-6 ft above ground. The doorway is centrally located along the front wall and is sunk about $2 \mathrm{ft}$ below ground level. In the interior, the floor is dirt and the lower walls are lined with rocks and plastered with mud. The upper walls are composed of horizontally laid logs chinked with mud. The superstructure is a lowpitched, front-gabled roof composed of native wood and sod. Photographs of the structure also show corrugated metal between the wood frame and the sod, but this was probably added later. The wooden frame consists of three central longitudinal beams, one being the central ridge beam, with smaller logs laid transversely to serve as rafters extending from the ridgepole to each side wall. Although they are not specifically identified, the large beams may be cottonwood and the smaller logs may be cedar. 
Inside the structure, a vertical central pole provides additional support for the superstructure. The Mayfield dugout had no fireplace, and a metal stovepipe coming through the roof suggests that a wood-burning stove was used for heating and cooking.

The Mayfield and Justice dugouts exhibit some similarities and differences in style and construction. They are roughly the same size, although the Mayfield dugout is larger than the Justice dugout $\left(300 \mathrm{ft}^{2}\right.$ vs. $\left.220 \mathrm{ft}^{2}\right)$. The upper walls are composed of different materials, with native wood logs used in the Mayfield dugout and native sandstone slabs used in the Justice dugout. There is no evidence that milled lumber or nails were used in the construction of the Justice dugout, and its superstructure, like Mayfield's, may have been made entirely of native wood and sod. Another major difference is that the Mayfield dugout presumably had a wood stoṿe, while the Justice dugout had a fireplace along its back wall. Freeman (1990:78) suggests that such fireplaces were common in dugouts.

The A. E. Powers Ranch headquarters (41HF75), located ca. $350 \mathrm{~km}$ north of Justiceburg in Hansford County, is a good example of a turn-ofthe-century homestead (Freeman et al. 1990:77-78, 148-167). Thorough archival and architectural documentation of the site (but no archeological investigation) demonstrate that site $41 \mathrm{HF} 75$ is similar to 41GR474 in many ways. In response to the FourSection Act, pioneer cattleman "Ace" Powers established his homestead between 1899 and 1903 and apparently built his dugout in 1899 . Like the Justice dugout, the Powers dugout was relatively large (18 x $20 \mathrm{ft})$ and apparently accommodated Ace, his wife, and their two daughters and four sons. Powers apparently intended the dugout as a temporary residence, and by 1904 he had already built a one-story ranch house with three bedrooms, a living room, kitchen, and dining room. Although its floor plan is different, the Powers house was comparable to the house that Justice built (compare Freeman et al. 1990:Figure 27 and Freeman 1990:Figure 6). The Powers dugout, however, was remodeled extensively in the 1920s (Freeman et al. 1990:78, 154), at which time the walls and roof were covered with stucco, corrugated metal was added to the roof, and a layer of concrete was put on the floor. Because of these alterations, it is impossible to determine the original architectural details of the dugout. Site 41HF75 was occupied and functioned as the Powers Ranch headquarters until ca. 1945 (Freeman et al. 1990:
153).

Sites 41GR474 and 41HF75 are two of the best documented closed-range ranching homesteads in the Texas Panhandle-Plains. Both sites were settled around the turn of the century as a direct result of the Four-Section Act. Dugouts served as the original dwellings at both locations, but larger and more permanent wood-frame houses soon replaced them. In both cases, the dugouts apparently were reused for nonhabitation purposes after they were initially abandoned. The main difference between the two sites is that the Justice dugout was reused for only a short time, eventually deteriorating as a result of permanent abandonment, while the Powers dugout was maintained and was still in use as late as ca. 1945. This difference probably is related to the locations of the dugouts relative to the later housesites. The Justice dugout was located ca. $500 \mathrm{ft}$ away from the main house and may have been permanently abandoned because it was not practical to maintain it. Conversely, the Powers dugout was only $30 \mathrm{ft}$ behind the main house, and because of its proximity, it may have been practical to maintain it as a functioning structure.

Turn-of-the-century homesteads such as sites 41GR474 and 41HF75 were once ubiquitous throughout the plains of Texas (Freeman and Boyd 1990:96-97, 120; Freeman et al. 1990:69), but beyond the fact that they existed, not much else is known about them. Many of these sites have long since disappeared, while others remain to be documented. Typical of the closed-range ranches of the period, 41GR474 and 41HF75 exemplify the homesteads that sprang up when settlers poured into the sparsely occupied plains in response to the FourSection Act of 1895 (Freeman 1990:96; Freeman et al. 1990:33). The breaking up of large ranches and the tremendous influx of people, accompanied by the expansion of railroads and construction of new towns, brought the free-range era of ranching to an end. In spite of the limited amount of archival and archeological research that has been done on these kinds of sites, the Justice Homestead and the Powers Ranch are illustrative of an early twentieth-century settlement pattern that appears to have been repeated many times over a vast area.

\section{PREHISTORIC SITE INVESTIGATIONS}

\section{Data Recovery at 41GR291}

Data recovery at the Sam Wahl site, 41GR291, 
included extensive mechanical blading and trenching and hand excavation of ca. $45 \mathrm{~m}^{3}$ of sediment. Combined with the efforts of the previous phases of work, 3 blade cuts, 37 backhoe trenches, 1 shovel and 24 auger tests, and 94 complete and 18 partial units covering $106 \mathrm{~m}^{2}$ have been excavated. A total of 3,970 artifacts have been recovered from all phases of work, and 37 cultural features have been exposed. The archeological evidence indicates that three temporally distinct cultural events or periods of occupation are represented.

The earliest cultural event is a human burial that occurred ca. A.D. 240-400. It is a caim-covered burial of a middle-aged adult male with no obvious pathological conditions. The fragmentation and orientation of the bones indicate a secondary interment of an incomplete, disarticulated skeleton, and the remains may have been wrapped or placed inside an organic container (e.g., hide or basket). A 6-cm-long Scallorn arrow point and a hematite paint stone are the only definite grave inclusions. There is no evidence of occupation of the site associated with this early burial.

The next human activities evident at the site are two sequential periods of residential occupation which postdate and are apparently unrelated to the burial. Only the radiocarbon-dated cultural features can be assigned to these occupation periods with complete confidence. Other features and artifacts are assigned based on arbitrary excavation levels and/or associations with dated features. Consequently, there is some ambiguity in the division of the artifacts into the two assemblages. Notably, the temporal division between the occupation periods, ca. A.D. 1050-1150, is based on a slight gap in the radiocarbon dates associated with cultural features. This temporal gap may be more apparent than real (i.e., there may have been continuous or intermittent cultural occupations during this time), but the cultural significance of the break is a hypothesized shift in site function and subsistence strategies.

The early occupation period at 41GR291 occurred ca. A.D. $600-1050$ and is characterized by a pithouse, storage pits, hearths/baking pits, and bedrock mortars. Large metates, a relative abundance of ground stones and frequent reuse of fragments, and large chopping tools that may have been used to refurbish ground stones indicate an intensive grinding technology probably oriented toward native plant resources such as mesquite. Although there is no definitive archeological evidence for the use of cultigens, limited horticulture may have been practiced during this early occupation period. Also notable is the relatively low frequency of arrow points, perhaps indicating that hunting was relatively unimportant while the inhabitants were occupying 41 GR291.

The second period of occupation occurred from ca. A.D. 1150-1400, and it appears to have been significantly different from the preceding period. This late period lacks the variety of features seen in the early period and is characterized by a single feature type-charcoal/burned rock scatters. The functions of these amorphous features are unknown, although one may represent the floor of a surface house. There are no pithouses, storage pits, or baking pits associated with the late occupation period. Other notable differences include the following: (1) the late occupation period may be characterized by smaller and less frequent ground stones that indicate a less intensive grinding technology; and (2) a relatively high frequency of arrow points that may indicate that hunting was more important than in the early occupation period.

Ceramic sherds, apparently associated with both occupation periods, represent at least three vessels of two different types (cf. Jornada Brown and South Pecos Brown) and indicate that pottery tempered with crushed volcanic rocks was imported from southern New Mexico and/or western Texas. A pendant fragment, made of an unusual type of kaolinite that may have originated in the Big Bend region of Texas, cannot be associated confidently with either occupation period. Extraregional lithic materials are represented by two obsidian flakes, identified by trace element analysis as being from the Malad source area in southern Idaho, that could belong to both occupation periods. Baugh and Nelson (1987: 322-325) indicate that Malad obsidian is well represented in Late Prehistoric sites in the Southern Plains but is rare at Protohistoric period sites, the latter being dominated by obsidian from sources in the Jemez Mountains of north-central New Mexico. Hall et al. (1986:516-520, Table 53) note that Malad obsidian is fairly common in late Archaic and Late Prehistoric sites in central and south Texas. Both of these studies interpret their data as evidence of a north-south Plains trade network that eventually was superceded, beginning around A.D. 1450, by the Plains/Pueblo trade (Baugh and Nelson 1987:324; Hall et al. 1986:519-520).

Chipped stone tools that characterize both 
occupation periods include a variety of rather expedient scrapers and edge-modified flakes, nonprojectile point bifaces, and gouges (including the Clear Fork variety). The chipped stone tools and debitage indicate that local materials, derived from the Lingos Formation gravels that are ubiquitous throughout the project area, were utilized extensively in both periods. Besides obsidian, nonlocal lithics include two materials, Tecovas jasper $(n=35)$ and fine-grained cherts of nonlocal Cretaceous origin $(n=$ 172), that appear to be associated with both occupation periods and a single flake of Alibates agate that may be from the early period. Altogether, nonlocal lithic materials (i.e., obsidian, Alibates agate, Tecovas jasper, and nonlocal varieties of Cretaceous chert) account for nearly equal percentages of the early $(7 \%)$ and late $(6 \%)$ occupation chipped stone assemblages. Consequently, there do not seem to be any significant differences in lithic procurement strategies between the two periods.

The early occupation period appears to have entailed the use of Scallorn (eddy and coryell varieties) and Bonham arrow points and perhaps Deadman's points as well. A few surface-collected dart points could be associated with the early occupation period, or they might represent procurement and reuse of lithic materials from nearby Archaic sites. The late occupation period assemblage contains Scallorn points but also includes a late-style, multiple-side-notched arrow point (cf. Huffaker). The presence of a side-notched arrow point is significant because the shift from corner- to sidenotched arrow points in the Southem Plains is thought to have occurred at ca. A.D. 1100 (Hughes 1991:29).

Other archeological evidence at 41GR291 also points to cultural changes around A.D. 1100. While there is a certain degree of continuity between the assemblages (e.g., the continuous use of predominantly local lithic materials), there appear to be significant changes in feature types and in the frequencies of some artifact types (ground stones and arrow points, in particular) that are interpreted as evidence of changes in subsistence strategies and site function.

The inhabitants of 41GR291 during the early occupation period appear to have focused on the intensive utilization of plant resources, involving the procurement, processing, and storage of some key native plant or plants and perhaps incorporating limited farming. Conversely, the late occupation inhabitants may have been more generalized hunter- gatherers who placed a greater emphasis on hunting. There is no good archeological evidence for seasonality of the occupations during either period, but circumstantial evidence suggests that during the early period the site served as a temporary residential base that was seasonally occupied on an intermittent basis. The use of a pithouse, storage pits, and baking pits is consistent with a seasonal subsistence strategy oriented toward specific plant resources that probably were harvested in the summer and/or fall, and the surplus produce may have been intended for use during the winter.

\section{Data Recovery at 41GR303B}

Two test units and 35 excavation units covering $37 \mathrm{~m}^{2}$ were completed during the testing and data recovery excavations at; the Cat Hollow site, 41GR303B. Four cultural features were exposed, and 2,796 stone artifacts were recovered. Two generalized periods of occupation are proposed for 41GR303B, each having a different stone tool assemblage and a different purpose in terms of site function.

The early period is not well dated. Radiocarbon assays from below (A.D. 71-197) and within (A.D 681-852) Feature 8 bracket the complex of burned rock features and indicate that these features and the associated artifacts date to the Late Prehistoric I period. This occupation is represented by two baking pits and a sitewide scatter of burned and unburned sandstone slabs. All of the metates recovered are attributable to this period of occupation. Calcium oxylate crystals recovered from Feature 8 suggest that prickly pear was processed on-site.

The later occupation of 41GR303B is represented by stone artifacts in the upper levels above the burned rock zone. A radiocarbon assay of A.D. 1020-1382 from Feature 5, which was $29 \mathrm{~m}$ east of the main excavations and cannot be attributed directly to either the lower or upper occupation zone, suggests that the later occupation period may date to the latter part of the Late Prehistoric I period or the early part of the Late Prehistoric II period.

With the exception of the projectile points, the tool kits from both zones appear to be informal and functionally broad based. There are no stone tools from either zone for which a definite function, such as that of snub-nosed scrapers or four-beveled knives, may be ascribed. Most of the stone tools, $94 \%$ of the 
lower zone debitage, and $92 \%$ of the upper zone debitage are of local origin. The generalized nature of the tool collections and the predominance of local materials indicate that many of the tools were produced on-site and then discarded.

The few temporally sensitive artifacts are from the upper zone or were found on the surface. Two specimens are dart points; one is untyped, and the other is classified as a Darl. Arrow points include two classified as Scalloms (coryell variety) and one that is Scallorn-like (possible sattler variety). A triangular arrow point fragment may be a preform, or it may be a complete specimen similar to the Fresno type.

Unifaces are the most numerous stone tools at 41GR303B. Sixty-eight unifaces constitute $27 \%$ of all of the stone tools. If the gouges that are primarily unifacial are included, this increases to $31 \%$. Of the unifaces, 30 are scrapers, 1 is a graver, and 37 are tools of indeterminate usage. Forty-eight unifaces came from the block, and 30 of these are from the upper zone. Together with the arrow points and the lack of ground stones, this may constitute evidence of hide processing by the later occupants of the site. Twice as many edge-modified flakes came from the upper zone than the lower zone; they may represent expedient cutting tools that also were used for hide processing, but the lack of osteological remains leaves this contention unsupported.

Of the eight light cobble tools, seven were recovered from the lower zone. It has been suggested that these tools were wedges which may have been used to split wood for various purposes, e.g., for production of wooden tools (i.e., digging sticks) or to create a wooden vise to hold a tool being worked. Heavy cobble tools bear wear patterns that indicate their possible utilization in ground stone rejuvenation by pecking. The three broken distal ends of heavy cobble tools appear to be a result of this high-impact utilization.

The debitage from the lower $(n=539)$ and upper $(n=1,114)$ zones indicates more late-stage reduction by the later occupants, while the raw material sources used for both occupations were predominantly local. The greater percentage of Tecovas jasper in the upper zone may indicate greater mobility or trade connections to the north.

The portion of the site excavated indicates that, for both zones, the site functioned as a specialpurpose location. Although there is a possibility that part of the bench was utilized for habitation, this is not apparent in the excavation block or on the surface of the deflated area to the east of the block. In the block and in the scattered test units in the western portion of the bench, all evidence suggests that the earlier occupations focused on plant processing and the later occupations perhaps focused on hunting, stone tool manufacturing and modification, and possibly hide processing.

In the absence of further supporting evidence, the presence of the Darl dart point does not constitute a case for a late Archaic presence at 41GR303B. It is interpreted as either a holdover heirloom or an intrusion. The earliest use of the site is interpreted as a Late Prehistoric I period plant-processing station. The later occupations appear to represent a shift to hunting.

\section{Data Recoveriy at 41 GR546}

One shovel test, one test unit, and six excavation units covering $7 \mathrm{~m}^{2}$ were completed in all phases of work at Boren Shelter \#1, 41GR546. Three cultural features were exposed, and just four artifacts were recovered. One radiocarbon assay on charcoal from Feature 2 produced a calibrated date range of A.D. 1450-1950 with a single intercept at A.D. 1643. This assay suggests a possible relationship with the nearby bone midden at 41GR559, dated to A.D. $1506-1663$.

Three stone-lined hearths were found inside the rockshelter. Two hearths, Features 1 and 2, were relatively shallowly buried. Feature 1 is considered to be contemporaneous with the dated feature. Feature 3 was discovered beneath Feature 2 and must date earlier, but it did not produce enough charcoal for radiocarbon assay.

None of the four artifacts are temporally sensitive. An expedient uniface made of Potter chert, an edge-modified flake fragment of Edwards chert, a small piece of unmodified debitage also of Edwards chert, and a hammerstone were recovered; a sandstone manuport completes the assemblage. A burned snake vertebra and Chenopodium sp. seeds in the macrobotanical samples are the only indications of possible subsistence items in the rockshelter.

The rockshelter is so small that it could have functioned only as a temporary haven for a few individuals. The only activities that could have been performed inside it in comfort are fire building for warmth and possibly cooking and sleeping. The radiocarbon date associating 41GR546 with 41GR559 
suggests the possibility that it was used for shelter during the deposition of the 41GR559 bone midden $60 \mathrm{~m}$ to the north.

\section{Data Recovery at 41GR559}

Four shovel tests, three test units, and 27 excavation units covering $30 \mathrm{~m}^{2}$ were completed in all phases of work at Boren Shelter \#2, 41GR559. Twenty-five cultural features were exposed; a large collection of vertebrate faunal remains but only 168 stone artifacts were recovered. Evidence from radiocarbon dating of nine features indicates that the site was occupied sporadically during the Late Prehistoric I, Late Prehistoric II, and Protohistoric periods. Horizontal and vertical stratigraphic evidence indicate that the span of occupation or use of the shelter may be subdivided into three periods.

The earliest period of occupation is represented by 13 cultural features contained in the lower stratum inside the rockshelter. Five radiocarbon assays on charcoal from these features date the early occupations from 2 B.C.-A.D. 1030. Eight more features contained below the stratigraphic break between the upper reddish brown sediments and the lower light brown sediments are also dated to this period by association. Features characterizing the period are a wind deflector and unlined, stone-lined, and disaggregated hearths. No temporally sensitive artifacts were recovered from these levels. Faunal and macrobotanical samples indicate subsistence based on generalized hunting and gathering.

The second period of occupation is marked by a shift in the stratigraphy inside the rockshelter. Four stone-lined or disaggregated hearths are associated with this stratum, and the period is dated by three radiocarbon assays on charcoal to ca. A.D. 10001300. A Washita arrow point and a reworked Ellis dart point are the only temporally sensitive artifacts recovered. The stratigraphic break may represent a change to a drier climatic regime, but culturally modified bones of small animals and vegetal evidence from features suggest the continuation of the generalized hunting and gathering subsistence strategy proposed for the earlier occupations.

The third use episode is represented by a bone midden in the talus area in front of the shelter. A single radiocarbon assay on bison bone collagen dates the midden to A.D. 1506-1663. Because of relationships among individual bone clusters, this single assay appears to date accurately most or all of the bone deposition, as well as a hearth dump below one of the bone clusters. The dated bison lower rear limb bones represent the only ungulate found in the midden. More characteristic of the midden is the presence of a broad array of carnivores, mainly wolves. The scarcity of axial skeletal portions and femurs indicates that these animals probably were utilized for food, with the meaty portions of the butchered animals transported off-site for consumption elsewhere.

The Washita arrow point found in the upper shelter fill is made of Tecovas jasper and may represent northward mobility/contact or visiting hunters from the north. The Ellis dart point, which is not very temporally diagnostic, is made of chert sourced possibly to Nolan County, southeast of the study area. Other artifacts of nonlocal chert include a unifacial scraper, an edge - modified flake, and three unmodified flakes, all most likely from the Callahan Divide, $100-160 \mathrm{~km}$ south of the study area.

The projectile points, scrapers, edge-modified flakes, and debitage indicative of expedient manufacture and rejuvenation are characteristic of short-term camps probably related to hunting. The cobble tool associated with hearth Feature 8 in the lower zone bears wear patterns similar to the heavy cobble tools at 41GR291 and 41GR303B. This type of wear has been associated with metate rejuvenation but also may result from heavy utilization as a bone crusher. The metate fragments are associated with Feature 27 in the upper zone. The primary association of ground stones is vegetal, but metates also have served as bases for grinding and pounding meat into pemmican. The high frequency of hammerstones, especially in the bone midden, suggests that these tools could have been used to process meat.

Seventy-four cases of cultural modification exist among the 3,580 bone specimens recovered from 41 GR559 in 1992 . These modified bones, along with their provenience and the absence of certain elements, indicate that much of the bone assemblage, including the bone clusters in the talus area, is the result of cultural behavior. A large mussel shell fragment bearing possible indications of utilization as a scraper was recovered from inside the rockshelter. The few other scraps of mussel shells recovered do not indicate that aquatic resources were utilized for subsistence.

Two other rockshelters in the Texas Southern Plains have yielded similar evidence of periodic occupations. Willey and Hughes (1978:189-190) 
note that radiocarbon dates at Deadman's Shelter "indicate an intermittent 600 year occupation of the shelter during which relatively few changes in material culture occurred" (ca. A.D. 120-710). At the Canyon City Club Cave in Randall County, periodic occupations occurred from late Archaic times, at various times during the Late Prehistoric period (including Level 4 at ca. A.D. 300-680), and perhaps into Protohistoric times (Hughes 1969:39-40, 143-149).

Although some of the macrobotanical remains may provide evidence of summer-fall occupations at 41GR559, it is interpreted as primarily a winteroccupied site. Like 41GR559, Deadman's Shelter (Willey and Hughes 1978:190) and the Canyon City Club Cave (Hughes 1969:145-146) also are interpreted as winter occupations. Greater quantities of artifacts indicate that Deadman's Shelter was occupied much more intensively than either 41GR559 or the Canyon City Club Cave. The more ephemeral nature of the occupations at 41GR559 indicates that it was utilized on a short-term basis.

\section{THE PREHISTORIC SITES IN REGIONAL PERSPECTIVE}

The Phase III, Season 3 investigations at two open sites, 41GR291 and 41GR303B, and two rockshelters, 41GR546 and 41GR559, yielded evidence of human occupations dating mainly to the Late Prehistoric period, although there is limited evidence of occupations dating to late Archaic and Protohistoric times. Calibrated radiocarbon dates on charcoal, bone, and soil humates indicate that the occupations occurred from as early as 2 B.C. to as late as ca. A.D. 1700 but were most intensive between ca. A.D. 500-1300 (Figure 92).

Evidence of possible Archaic occupations is limited to a few dart points that appear to be late or transitional Archaic styles. Dart points have been found at 41GR291 $(n=3), 41 G R 303 B(n=2)$, and 41 GR559 $(n=1)$, and only two types, Darl and Ellis, are represented. The occurrence of dart points in each of these sites, however, is problematic. None of the dart points are from good archeological contexts that definitely date to late Archaic times. It is uncertain whether they represent in situ evidence of late Archaic occupations, the use of dart points during a transitional late Archaic/Late Prehistoric period, or recycling of points picked up elsewhere and brought to the sites by Late Prehistoric peoples. Hence, no archeological remains from any of the sites can be associated unequivocally with late Archaic occupations.

There is evidence of Protohistoric period activity at rockshelters 41GR546 and 41GR559. A single hearth at 41GR546, radiocarbon dated to ca. A.D. 1643, provides evidence that this small shelter was occupied contemporaneously with the deposition of a series of bone clusters in front of rockshelter 41GR559, dated to ca. A.D. 1642. Although evidence of cultural modification of the 41GR559 bones is minimal, the unusual nature and composition of the bone assemblage suggests cultural involvement, and it is interpreted as a probable single-event (or at least short-term) bone midden. None of the cultural remains inside the 41GR559 shelter are definitely attributed to the Protohistoric period, although some occupation during this period could have occurred there. Alternatively, the bone midden may be related to Protohistoric occupation of nearby shelter 41GR546. A bone concentration found in the upper levels of 41GR559 may be the result of animal predation, and none of the upper-level hearths can be associated with Protohistoric period occupation. A Washita arrow point from the upper levels could be related to either Late Prehistoric or Protohistoric occupations.

Consequently, the evidence from 41GR546 and 41GR559 indicates that these rockshelters were utilized sporadically in the Protohistoric period, but the material culture evidence is sparse and difficult to interpret. The faunal evidence from the 41GR559 bone midden provides the best evidence of subsistence activities and suggests that a wide range of animals was hunted. Animals that may have been killed and eaten by Protohistoric peoples at 41GR559 include gray wolf, dog, bobcat, skunk, fox, mink, bison, rodents, birds, and snakes. The bison elements suggest that a single bison was killed but that only its hide and lower legs were brought back to the shelter.

The Protohistoric period activities at 41GR546 and 41GR559 were contemporaneous with, and perhaps related to, occupations at two other sites in the Lake Alan Henry project area. Sites 41KT51 and 41KT53 are interpreted as encampments of Plains bison-hunting peoples (Boyd et al. 1993). The presence of bison at 41GR559 is provocative and suggests that the rockshelters and campsites may represent different aspects of the bison-oriented Protohistoric settlement pattern discussed in Boyd et al. (1993). None of these late occupations in the 


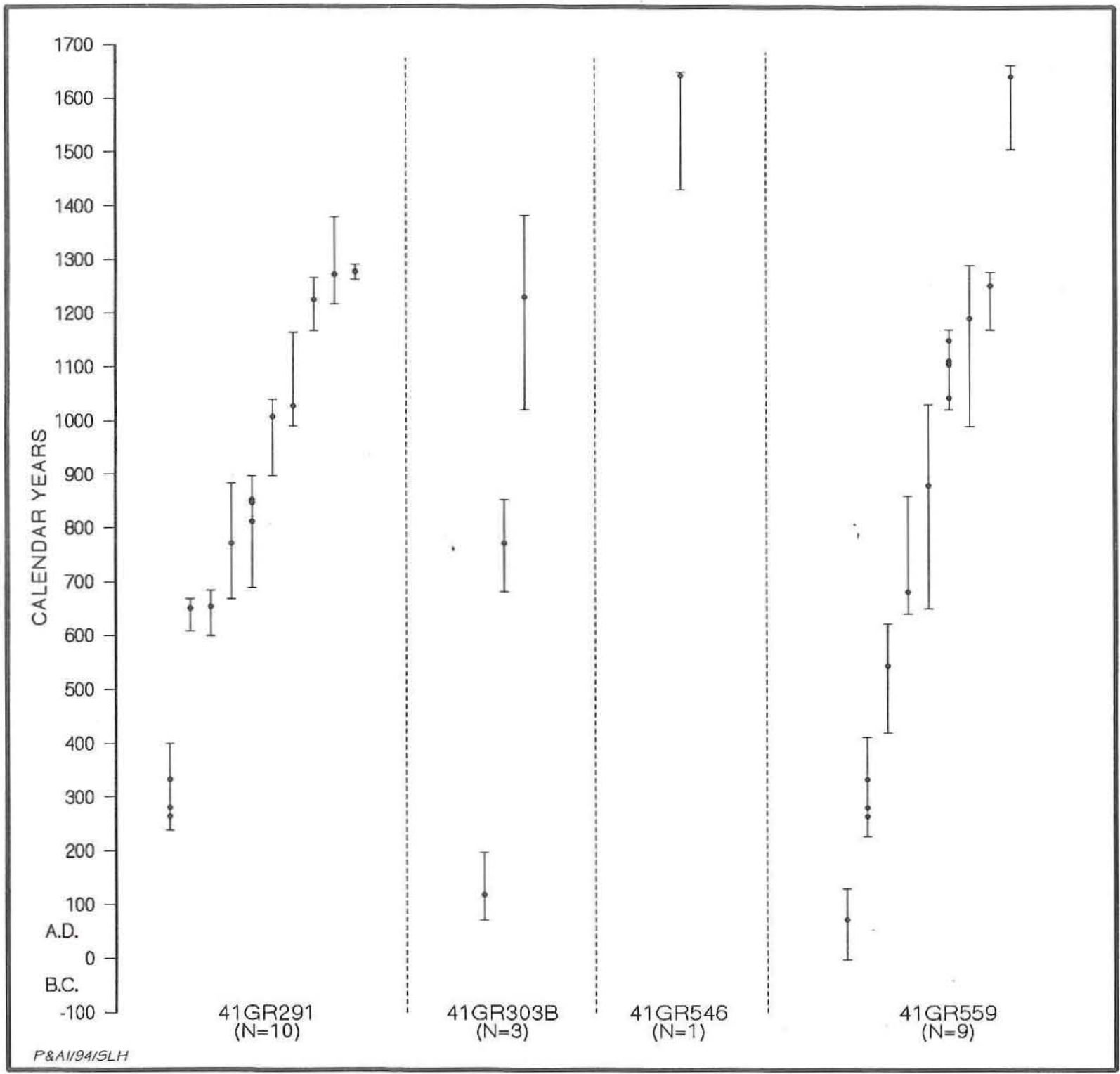

Figure 92. Comparison of calibrated radiocarbon dates from prehistoric sites 41GR291, 41GR303B, 41GR546, and 41GR559.

Lake Alan Henry project area can be assigned to any regional archeological complex. While the Protohistoric occupations at sites 41GR546 and 41GR559, as well as those at $41 \mathrm{KT} 51$ and $41 \mathrm{KT} 53$, are contemporaneous with the bison-oriented Garza Complex, there is no good evidence for a cultural link. The Protohistoric period is discussed at length in Boyd et al. (1993) and is not considered further here.

Except for the minimal evidence for Protohistoric and possible late Archaic period occupations mentioned above, the sites investigated during Season
3 mainly yielded evidence of Late Prehistoric human occupations. Consequently, the remainder of this chapter is concerned only with the Late Prehistoric period occupations and their contexts within the region.

\section{Late Prehistoric Archeological Taxonomy}

The prehistory of the Texas Lower Plains during the first millenium A.D. is not well understood, but considerable progress has been made in the last decade. In the data recovery research design for 
Lake Alan Henry, it was suggested that the Late Prehistoric period should be divided into a Late Prehistoric I period, ca. 2000-1000 B.P., and a Late Prehistoric II period, ca. 1000 B.P. to European contact. More recent archeological data from the project area, however, seem to support the assessment by Hughes (1991:24) that A.D. 1100 is a more appropriate time for dividing the Late Prehistoric period, or NeoIndian substage as he calls it. Hughes (1991:24, 29) suggests that the Early NeoIndian substage dates from ca. A.D. 200-1100 and that the Late NeoIndian substage dates from A.D. 11001541. The beginning of the Early NeoIndian substage is not well dated, but A.D. 1100 does seem to be a better dividing date than A.D. 1000, given the significant cultural changes that occurred over most of the region at that time. Hughes (1991:24) suggests that A.D. 1100 splits the difference between the Plains Woodland to Plains Village transition at ca. A.D. 1000 in the northern Panhandle and the pithouse to surface house transition at ca. A.D. 1200 in western Texas and southern New Mexico. Thus, for this discussion, the Late Prehistoric period is divided into Late Prehistoric I, A.D. 1-1100, and Late Prehistoric II, A.D. 1100-1541.

Late Prehistoric archeological manifestations pertinent to the discussion of the Season 3 investigations include the Lake Creek complex (Hughes 1962, 1991:25-26), the Palo Duro complex or phase (Cruse 1992; Hughes 1991:26-27; Willey and Hughes 1978), the Blowout Mountain phase (Creel 1990:15-18), and the Querecho, Maljamar, and Ochoa phases of the eastern extension of the Jornada Branch of the Mogollon (Corley 1965a, 1965b; Hughes 1991:27-29; Leslie 1979). A critical review of these constructs is not attempted (for a brief summary, see Hughes 1991), but it is important to note that all of these complexes and phases are minimally defined on the basis of scant archeological data. Only a handful of sites can be attributed to each, and even fewer sites have been adequately investigated and reported. These constructs should be viewed in their proper context, i.e., as preliminary attempts to define the culture history of two vast regions, the southern High Plains and Lower Plains, in which little archeological research has been done.

Plains Woodland sites in the Texas Panhandle are assigned to the Lake Creek complex (Hughes 1991:25-26) and are generally confined to the northern Panhandle and the Canadian River valley (Couzzourt 1985:68-69). The Lake Creek complex is characterized by the presence of cordmarked pottery of the Plains Woodland tradition but apparently of local manufacture and Scallorn-like arrow points; however, corner-notched dart points are sometimes associated, and some sites have high frequencies of ground stone tools (Hughes 1991:2526). Jornada Mogollon brownware sherds, resembling Alma Plain, also are found occasionally. With the exception of arrow points and pottery, the other material culture is similar to that of late Archaic sites and appears to represent a generalized hunter-gather lifestyle. No evidence of structures has been found, and rock-lined hearths and refuse dumps are the only feature types recognized. Bison remains appear to be scarce. The Lake Creek complex is not well dated but appears to span several hundred years, from A.D. $200-800$ or 900 .

Palo Duro complexisites may be found as far south as the upper Brazos River drainage (Boyd et al. 1989:111-112) but are most common in the upper Red River drainage and are occasionally found as far north as the Canadian River (Couzzourt 1985:67; Etchieson 1979). The Palo Duro complex (Hughes 1991:26-27) or phase (Cruse 1992:145-149) spans most of the first millenium A.D. and is characterized by Mogollon brownwares resembling Jornada Brown and its variants, Scallorn-like arrow points, and the distinctive basal-notched Deadman's arrow points. Three types of sites recognized for the Palo Duro complex are rockshelters, open camps, and villages. Two excavated pithouses at the Kent Creek site constitute the only evidence for structures, and Cruse (1992:125-129) suggests that they are stylistically similar to pithouses in the Jornada Mogollon region. Unlined and rock-lined hearths or baking pits are present at Palo Duro complex sites, but bison remains appear to be scarce. No definite evidence of agriculture has been found, but Cruse (1992:146) and Hughes (1991:27) suggest that horticulture may have been practiced. Notably, remains of Microtus ochrogaster, a prairie vole that no longer lives in the region, found at three sites may indicate that moister conditions prevailed during the first millenium A.D. (Hughes 1991:27).

The relationship between the Lake Creek and Palo Duro complexes is a subject of controversy. As Couzzourt (1985:66-77) notes, the archeological remains of these contemporaneous complexes are extremely similar and are separable only by differences in points, pottery, and geographic locations. An overly simplistic view is that Lake 
Creek has Woodland pottery and Palo Duro does not, Palo Duro has Deadman's arrow points and Lake Creek does not, and the two complexes overlap geographically in the Canadian River valley. Of considerable importance, Mogollon brownwares are present in both Lake Creek and Palo Duro sites but are much more common in, and diagnostic of, the latter. The relationship of Mogollon brownwares to Woodland occupations in the northern Panhandle is not clearly understood, and it is possible that these ceramics were obtained indirectly via trade between Woodland and Palo Duro complex peoples (Couzzourt 1985:75-76). Couzzourt (1985:76-77) and Hughes (1991:29) suggest that the Canadian River valley was utilized by both Lake Creek and Palo Duro complex peoples and hence represents a cultural boundary between two groups coming from very different traditions (i.e., Plains Woodland vs. Jornada Mogollon).

The influence of the Jornada Mogollon culture on the peoples of the Southern Plains is of considerable importance to understanding the region's archeology and has a direct bearing on the Mogollon brownware problem mentioned above. As originally defined by Lehmer (1948:Figure 22), the culture area for the Jornada Branch of the Mogollon was confined to northern Chihuahua, far western Texas, and southcentral New Mexico, and it did not extend to the Pecos River. Corley (1965a, 1965b) and Leslie (1979) later proposed that a variant, called the eastem extension of the Jornada Mogollon, extends east of the Pecos River across all of southeastern New Mexico and onto the southwestern Llano Estacado as far east as Midland and Lamesa. They suggest that the eastern Jornada Mogollon manifestations are divisible into three phases (Querecho, A.D. 950-1100; Maljamar, A.D. 1100-1300; and Ochoa, A.D. 1300-1450) characterized by cultural changes that are roughly analogous to those represented by Lehmer's (1948) northern (San Andres, Three Rivers, and Capitan) and southern (Mesilla, Dona Ana, and El Paso) Jornada phases.

Jelinek's (1967) work on the middle Pecos River suggests that the people there were similar, if not related, to the Jornada Mogollon, and recent work in the Roswell area by Wiseman $(1981,1988$, personal communication 1993) has demonstrated that Jornada Mogollon sites are present in the Pecos River valley to the south. The nature of the eastern Jornada Mogollon, however, is not well understood. Leslie $(1979: 179)$ proposes that these manifestations represent "groups of/or related to the Jornada Branch of the Mogollon" but that they retained a more nomadic hunter-gatherer lifestyle, did not practice agriculture, and relied heavily on shin oak acoms and mesquite beans. Collins (1968:153-157, 1971:88) and Hughes (1991:28) indicate that this scenario is likely and suggest that these sites represent either local adaptations under considerable influence from the Jornada Mogollon or Jornada Mogollon groups that moved onto the plains. In either case, they may have successfully merged a hunting-foraging existence and a semisedentary lifestyle without having practiced agriculture (Collins 1971:89). While semipermanent seasonal settlements apparently began early in the pithouse period (at least by A.D. 800) in the Jornada Mogollon area (Carmichael 1990; Whalen 1980a), Collins (1971:89) and Hughes (1991:28) suggest that semipermanent settlements on the southern Llano Estacado did not occur until after A.D. 1200 and that bison hunting was a key element. Notably, however, pithouses associated with the Kent Creek site (Cruse 1992), radiocarbon dated to A.D. 710, and 41GR291, radiocarbon dated to ca. A.D. 1000 , indicate that semipermanent settlements along the eastern edge of the High Plains occurred earlier and apparently were not related to bison hunting.

Based on a reassessment of Cyrus Ray's (1929:12-15) Sand Dune culture and E. B. Sayles's (1935:47-52) Brazos River culture (Ray and Sayles [1941] subsequently agreed on the name Brazos culture) and work at 41TG91, Creel (1990:15-18) defined the Blow Out Mountain phase, ca. A.D. 8001300. The geographic area for the Blow Out Mountain phase is west-central Texas, and it includes most of the drainage basins of the Clear Fork of the Brazos and the Concho rivers and the portion of the Colorado River drainage between them. Diagnostic features include stone-lined hearths, flexed burials in stone cists and with rock caims (and occasional secondary bundle burials and cremations), and mussel shell dumps. Distinctive arrow points include Albaand/or Bonham-like specimens (often with serrated blades), diminutive forms of Zephyr or Darl, and stemmed points resembling Cliffton but with rounded bases and well-defined shoulders. Washita points may occur late in the sequence, but no pottery has been found at any Blow Out Mountain phase site.

The preceding discussion of the Late Prehistoric archeological complexes and phases provides the background necessary for considering the cultural 
affiliations of the prehistoric sites investigated during the 1992 data recovery at Lake Alan Henry. Much of the archeological evidence for these sites, however, is ambiguous when it comes to assigning remains to regional complexes. For example, while Scallorn points were found at 41GR291 and 41GR303B, Hughes (1991:29) notes that "Scallorn-like and other barbed arrowpoints blanketed the entire Southern High Plains and bordering area" during the Late Prehistoric I period. Because of their broad geographic and temporal span, Scallom points, which can be associated with either Woodland or Palo Duro complex occupations, are not particularly diagnostic for defining cultural affiliations. Unfortunately, the absence of temporally sensitive or culturally diagnostic arrow points, pottery, and features at 41GR303B, 41GR546, and 41GR559 makes it difficult to assign these sites to specific archeological complexes.

The greater range of diagnostic artifacts and features encountered at 41GR291, however, warrants more careful consideration. The burial (Feature 23) at 41GR291 is somewhat similar to secondary cairn burials that are tentatively associated with the Blow Out Mountain phase (Creel 1990:17). Several bundle burials are attributed to the Blow Out Mountain phase, but only two have diagnostic grave inclusions. Ray (1933) and Forrester (1951) report bundle burials (near Abilene and in Shackelford County, respectively) with associated Alba-like arrow points. Since none of the Blow Out Mountain burials (primary or secondary) have been independently dated, their reported association with this phase implies that they date to A.D. 800-1300. This makes them 400-560 years younger than the burial at 41GR291 (dated to A.D. 240-400), but Creel (1990:17) acknowledges that some of the burials may actually predate the Blow Out Mountain phase. If the unusually large Alba-like points represent early-style arrow points, then these bundle burials might be approximately contemporaneous with the 41GR291 burial with its associated large Scallorn point (see Figure 21d). Although these similarities are intriguing, it is not suggested that the 41 GR2 21 burial is affiliated with the Blow Out Mountain phase. Contemporaneity cannot be demonstrated since none of the Blow Out Mountain phase burials have been dated. It is also likely that secondary burial was not a culture-specific mortuary trait but was practiced by various groups under certain conditions (see Chapter 3, Interpretive Summary).
Of the constructs discussed above, 41GR291 is in closest geographic proximity to the areas defined for the Palo Duro complex and the Blow Out Mountain phase. The early occupation period at 41GR291 includes arrow points that are stylistically similar to the types described for both of these archeological manifestations. The Deadman's point fragment from 41GR291 suggests an affiliation with the Palo Duro complex since this type is not found in any other complex. While Scallorn points also are attributed to the Woodland Lake Creek complex, the 41GR291 specimens (eddy and coryell varieties) are most similar to specimens associated with the Palo Duro complex from the Kent Creek site (compare Figure $21 b$ and $c$ with Cruse 1992:Figure 18h-k) and Deadman's Shelter (compare Figure 21e with Willey and Hughes 1978:Figure 54h). The Bonham specimen is similar to the Alba- or Bonham-like specimens of the Blow Out Mountain phase (compare Figure 21a with Creel 1990:Figure 42, Groups E and F).

The Mogollon brownware pottery from 41GR291 suggests an affiliation with the Palo Duro complex. While Mogollon brownwares are occasionally found in Woodland sites in the Texas Panhandle, they generally are rare and are greatly outnumbered by cordmarked pottery of local manufacture. The absence of Woodland-tradition cordmarked pottery at 41GR291 indicates that the site probably is not associated with the Lake Creek complex. Conversely, Mogollon brownwares imported from the Jornada region are an important aspect of the Palo Duro complex.

The features at 41GR291 include some that are not culturally diagnostic and others that are similar to features described for the Palo Duro complex and the eastern Jornada Mogollon. Although baking pits are ubiquitous in the archeological record, their morphologies are too poorly defined in the region to be temporally or culturally diagnostic; however, the 41GR291 pithouse and storage pits are more distinctive. These features previously were compared with similar features from other sites (see Chapter 3), but some important points are worth emphasizing. No definitive conclusion can be reached regarding the cultural or archeological distinctiveness of the 41GR291 storage pits and pithouse. Cylindrical storage pits are an extremely common form and were used by many different peoples in the surrounding regions, but the 41GR291 pits are unusual in that they were dug into bedrock. Clustering of pits, probably indicating a definable extramural activity area used 
specifically for storage, is documented only at 41GR291 and at the Coyote site, a Late Prehistoric site on the Red River in Montague County (Woodall 1967:17-20, Figures 18 and 19). Although intriguing, the fact that storage pits occur in clusters at these sites does not indicate a cultural connection.

There are no good structural analogies suggesting that the 41GR291 pithouse is associated with Plains Woodland or Plains Village occupations in the Texas Panhandle or western Oklahoma (see Chapter 3, Interpretive Summary). The 41GR291 pithouse is unlike the rectangular pithouses at the nearby Kent Creek site (Cruse 1991), although there are other similarities between these sites. A circular pithouse at the Merchant site in Lea County, New Mexico (Leslie 1965), is similar to but somewhat larger than the 41GR291 pithouse. Notably, however, in addition to the ovate pithouse, a rectangular pithouse similar to and approximately the same size as Structure 2 at the Kent Creek site (Cruse 1992:51) also was found at the Merchant site (Leslie 1965:26). Thus, it is not unreasonable to suggest that the morphological differences may be functional or temporal rather than cultural, and other researchers (e.g., Lehmer 1948) have noted a great deal of variability in Jornada Mogollon pithouses. The 41GR291 pithouse is most similar to structures in the Jornada Mogollon region, particularly the ovate or circular pithouses in the Keystone Dam area near El Paso (O'Laughlin 1980:136-145; Carmichael 1985:173-182), and at the King Ranch site and the Fox Place on the Pecos River in the vicinity of Roswell (Wiseman 1981, 1988, personal communication 1993).

In conclusion, the early occupation at 41GR291 (representing the Late Prehistoric I period) exhibits strongest similarities with the Palo Duro complex, which is probably related in some way to the Querecho (and perhaps Maljamar) phase of the eastern Jornada Mogollon. Because of the paucity of Deadman's arrow points at 41GR291 and the architectural differences, however, the association between 41GR291 and the Palo Duro complex is tentative. Some of the discrepancies (e.g., architectural differences between the pithouses at 41GR291 and the Kent Creek site) and uncertainties certainly are a product of the limited archeological work in the region. It is clear that the Palo Duro complex or phase, as it is currently defined (Cruse 1992:145-149; Hughes 1991:26-27; Willey and Hughes 1978:185190 ), is not well understood and probably does not account for the full range of variability within the culture. In light of the recent findings, one particularly important future research problem for the Late Prehistoric I period is the relationship between the Palo Duro pithouse villages along the eastern edge of the High Plains and the eastern Jornada Mogollon pithouse villages in southeastern New Mexico and on the southern High Plains of Texas.

The late occupation period at 41GR291 (representing the Late Prehistoric II period) cannot be assigned to a regional archeological complex, in part because the archeological evidence for this time period at 41GR291 is more limited and in part because this time period is poorly understood throughout the region. The only archeological complexes defined for the period from A.D. 11001541 in the southern part of the High Plains are the Maljamar (A.D. 1100-1300) and Ochoa (A.D. 13001450) phases of the eastem, Jornada Mogollon, which Hughes (1991:27-29, 33-34) admits are poorly known and dated. Although the Palo Duro complex appears to terminate around A.D. 1100, human occupation in the Lower Plains did not come to an end. Since significant cultural changes appear to have occurred at 41GR291 around A.D. 1100, the late occupation period could represent an influx of new people or, perhaps more likely, could represent indigenous Palo Duro complex peoples who were in the process of adapting to regional changes in the climate, flora, and fauna. One of the more intriguing research problems for the Late Prehistoric II period is the relationships between the post-Palo Duro complex occupations in the Lower Plains and the Maljamar and Ochoa phases of the eastern Jornada Mogollon in the southwestem High Plains. Notably, some of the cultural changes that may have occurred in the Lower Plains at ca. A.D. 1100 are contemporaneous with, and probably related to, cultural changes that occurred elsewhere in the Southern Plains and the Southwest; these are discussed in more detail in the final sections of this chapter.

\section{Late Prehistoric I Settlement Patterns and Subsistence}

The radiocarbon dates show considerable overlap among the occupations at 41GR291, 41GR303B, and 41GR559 during much of the Late Prehistoric period. Most of the calibrated dates $(\mathrm{n}=$ 21) have intercepts that fall between A.D. 50-1300, indicating that the sites were occupied within the same general time frame (see Figure 92). While it is 
not suggested that the all of the occupations dating to this time period are attributable to a single group of people, they are considered to be representative of regional settlement patterns and subsistence strategies. Notably, 17 of the radiocarbon dates have intercepts that fall in the A.D. 500-1300 range and indicate more intensive use of these sites during that time. Clearly, much of the Season 3 data recovery evidence relates to the Late Prehistoric I period, ca. A.D. 1-1100.

Cultural activities during the early part of the Late Prehistoric I period are represented by only four dates from A.D. 50-500. At 41GR559, early dates associated with deeply buried hearths (Features 16 and 13/26) indicate that sporadic occupation of the rockshelter had begun by ca. A.D. 50 and that periodic use of the shelter still occurred at ca. A.D. 260-330. An early date at 41GR303B indicates that cultural activities had begun there by ca. A.D. 100, but since the date is not associated with a feature, it is unclear what those activities may have been. Intensive plant processing at 41GR303B had begun by A.D. 770 (a date associated with a baking pit), but the temporal gap between these dates does not necessarily represent an absence of cultural activities since so few dates were obtained from the site. It is possible, although it cannot be proven with only two dates, that the site was used intermittently (but certainly not continuously) as a specialized plant-processing area prior to A.D. 500.

At 41GR291, the only cultural activity prior to A.D. 500 is a human burial (Feature 23) radiocarbon dated at A.D. 240-400. This burial appears not to be associated with the later occupations at the site, but this possibility cannot be ruled out. A Scallorn point associated with the burial denotes the use of the bow and arrow at a time when other sites in the region show evidence that late Archaic bison hunters were still present (Hughes 1977, 1989; Hughes 1991; Lintz et al. 1991). Hughes (1991:21) acknowledges that "pitifully few" late Archaic components have been radiocarbon dated, and Lintz, Speth et al. (1991:262) note that many of the dates are questionable and suggest that a long late Archaic/Late Prehistoric transition period and contemporaneous use of the atlatl and the bow and arrow may have occurred.

Not much is known about human subsistence and settlement patterns in the Texas Lower Plains from A.D. 500-1100, but new archeological discoveries of pithouses and storage pits in the region (see Chapter 3; Cruse 1992) provide evidence useful for inferring residential mobility and settlement patterns. Much of the recent archeological research in the southwestern United States has emphasized the importance of understanding residential mobility (e.g., Whalen and Gilman 1990). Shifting away from the view that pithouses are evidence of sedentism, most researchers now embrace the view that the pithouse period may have been characterized by considerable and varying degrees of residential mobility (e.g., O'Laughlin 1980, 1993; Whalen 1977, 1978, 1980a). Bearing in mind that "hunter-gatherer adaptations come in many kinds, with many levels of mobility" (Whalen and Gilman 1990:73), some inferences can be made about the nature of residential mobility in the Texas Southern Plains during the first millenium A.D. Gilman (1987:548) states that "pithouse structures are the most adaptive and useful choice of habitation under conditions of biseasonal settlement systems, dependence on stored foods, and cold season sedentism." If this idea holds true beyond the Southwest, then the early occupation period at 41GR291 and the pithouse occupation(s) at the Kent Creek site (Cruse 1992) can be interpreted as seasonal occupations.

Repeated use of 41GR291 over a long period of time is adequately demonstrated by several lines of evidence, including the wide range of radiocarbon dates, the diversity of activities represented by features and stone tools, and the extensive reworking/reuse of stone tools. Continuous occupation of the site over several hundred years cannot be supported, however, given the low density of cultural materials. Thus, the occupations at 41GR291 appear to have been seasonal and intermittent.

While there is no direct evidence for when during the year 41GR291 was occupied, the seasonal nature of the occupations is supported by circumstantial evidence. First, the early occupation period from A.D. 600-1050 appears to have been characterized by a subsistence strategy heavily weighted toward plant resources, and both native and domesticated plant foods are seasonal in occurrence. The availability of food resources in temperate environments is very seasonal, resulting in hunter-gatherer adaptations involving a high degree of seasonal mobility. Human populations respond to seasonal environments by adopting a forager strategy to exploit a diverse range of unpredictable low-yield resources, by adopting a logistical collector strategy and storage to exploit a single dominant high-yield resource, or by adopting 
a mix of the two strategies, but the overall settlement pattern is characterized by high mobility because the resource base changes on a seasonal basis (Binford 1980; Hayden 1986, 1987; Kelly 1980, 1983).

The presence of storage pits at 41GR291 also provides evidence for seasonally oriented residential mobility. In general, storage implies seasonal changes in resource availability (Binford 1990:140 146; Goland 1983:93-98). The old idea that storage must always equate with sedentism, however, has been shown to be erroneous (Goland 1983:1-5, 101), as there are numerous examples of storage of various kinds and intensities among nomadic, seminomadic, and semisedentary peoples (e.g., DeBoer 1988; Gilman 1987; Ingold 1983; Raymer 1988). Goland (1983:93-98) suggests that food storage among hunter-gatherers occurs in response to predictable periods or seasons of resource abundance and scarcity. It has been observed that bulk food storage generally occurs at the locality where the resource is procured or harvested and that often the locality where the food is stored is also where it is later consumed (Binford 1990:145; Goland 1983:97). Since it was impractical for most prehistoric huntergatherers to transport bulk foods great distances beyond the point of procurement or storage, this behavioral generalization has important implications for interpreting the nature of archeological storage facilities. When storage facilities occur at a residential base, it can be inferred that the stored resources were procured nearby and probably were intended for subsequent consumption at or near the site (Binford 1990:144-146). While food storage away from residences (often called caching) is documented in some circumstances, it apparently was rare unless an efficient means of transporting the resources (e.g., horses) was available (Goland 1983:97, 105).

Researchers have suggested functional differences between aboveground storage and subsurface pit storage. While aboveground storage may have been practiced at 41GR291, it probably was limited to the intervals during which the site was occupied. Conversely, subsurface storage could have been used during intervals of temporary site abandonment. Based on a survey of worldwide ethnographic data in Murdock's (1967) Ethnographic Atlas, Gilman (1987:558-559) has proposed a strong correlation between seasonal residential mobility and pit storage for the purpose of concealment. Using a different set of Plains and Woodland ethnographic data, DeBoer
(1988:3) concludes that "subterranean storage serves primarily to conceal foodstuffs." Archeological studies in various parts of the United States also support this interpretation (e.g., O'Laughlin 1993; Ward 1985:98-99). If a primary function of storage pits was for concealment, then the presence of storage pits at 41GR291 may be evidence that the site was abandoned periodically and that occupations occurred on a seasonal basis.

Ethnographic data compiled by Binford (1990:140-146) suggest that storage strategies are directly correlated to environmental variables (also see Ingold 1983). Using effective temperature (ET) as a measure of environmental variability, he suggests that "groups in settings warmer than ET $14.6\left[{ }^{\circ} \mathrm{C}\right]$ exhibit some specialization in the storage of plant foods, which are used as reserves primarily during the winter" (Binford 1990:142). The dominance of plant food storage in temperate zones is predictable due to the difficulty of keeping meat from spoiling during warm periods. While meat is the primary product stored in colder climates with an ET lower than 14.2, storage of both plant foods and meat may be expected in areas with an ET of 14.2-14.6. Of the ethnographic accounts in his data set, however, "almost all cases falling between ET 15.3 and 14.6 store only plant foods" (Binford 1990:141-142). Thus, assuming that the modern effective temperature of $14.85^{\circ} \mathrm{C}$ at Lake Alan Henry is representative of the effective temperature during the Late Prehistoric I period, storage of plant foods should be expected. The ethnographic data also indicate that subsurface pits are common storage features in a broad range of environments, from ET 12.2-15.9 (Binford 1990:144145). Hence, it is reasonable to suggest that the storage pits at 41GR291 were used primarily, if not exclusively, for storing plant foods. Binford's (1990) data also support the interpretation that 41GR291 may have been occupied during winter but cannot be used to predict whether native plants or cultigens were used and stored.

It has been suggested that 41GR291 was a residential site that was occupied seasonally and periodically during the Late Prehistoric I period, but it clearly represents only a portion of the broader settlement pattern and yearly round of the huntergatherers who lived there. Consequently, other kinds of sites, presumably related to ranges of activities at different times of the year, might be expected in the area. Sites 41GR303B and 41GR559 appear to be examples of these kinds of sites. 
At 41GR559, a meager artifact assemblage and evidence of ephemeral occupations suggest that the rockshelter served as a temporary camp, perhaps occupied mainly in the winter, and radiocarbon dates indicate intermittent occupations throughout much of the Late Prehistoric I period. Hunting and plant foraging activities seem to be represented, but there is no indication that one activity was particularly dominant.

The Late Prehistoric I cultural activities at 41GR303B, as evidenced by a massive burned rock accumulation and baking pits in the lower zone, are not well dated, but they occurred contemporaneously with the use of hearths/baking pits and storage pits at 41GR291 by, and probably before, A.D. 770 . Rather than representing a residential base, however, 41GR303B is interpreted as a specialized activity site where intensive plant processing (i.e., pit baking of plant foods) occurred. The only direct evidence of the plants that may have been processed is prickly pear calcium oxylate phytoliths recovered from baking pit Feature 8 (Cummings 1990:541).

A comparison of the early occupation artifacts from 41GR291 and 41GR303B, using adjusted standardized residuals to take varying sample sizes into account, indicates that the assemblages are quite similar (Table 61). The only significant difference is the higher frequency of ground stones at 41GR291. However, the arrow point to ground stone ratios for the early occupation period at 41GR291 (11:78) and the lower zone at 41GR303B (0:11) are both low. This suggests intensive plant processing at both sites, especially 41GR291, during Late Prehistoric I times (relative to the Late Prehistoric II period discussed below). A major difference between 41GR291 and 41GR303B, however, is the much greater quantity of lower-zone burned rocks $(689 \mathrm{~kg})$ relative to ground stones $(n=11)$ at 41GR303B compared to the early occupation period at 41GR291, which yielded $221 \mathrm{~kg}$ of burned rocks and 78 ground stones. Assuming that many of the burned rocks are related to baking pits, the activities at 41GR303B may have been oriented more toward cooking, while plant food processing through grinding was relatively important at 41GR291. Perhaps the greater emphasis on grinding activities at 41GR291 is related to site function (i.e., a multifunctional residential site) and processing of plant resources for storage. It also has been suggested that a high frequency of complete and fragmentary ground stones should be expected at residential sites occupied for longer durations (Schlanger 1990), and this seems to be the case at 41GR291. Thus, the subsistence patterns proposed for the Late Prehistoric I occupations at these sites are consistent in representing intensive plant processing and utilization, but the site functions appear to have been different. It is proposed that 41GR291 was a residential base where a variety of activities took place, including grinding and storage of plant foods, while 41GR303B was a specialized

\begin{tabular}{|l|c|c|c|c|}
\hline \multicolumn{5}{|c|}{ TABLE 61 } \\
\hline & COMPARISONS OF EARLY OCCUPATION STONE ARTIFACTS FROM 41GR291 AND 41GR303B \\
\hline Artifact Type & 41GR291 & Adjusted Standardized & 41GR303B & \\
\hline Arrow point & Early Occupation Period & Residuals for 41GR291* & Lower Zone & Totals \\
Biface & 4 & +1.32 & - & 4 \\
Uniface & 5 & -0.93 & 4 & 9 \\
Core & 31 & -1.00 & 18 & 49 \\
Edge-modified flake & 33 & -1.59 & 22 & 55 \\
Cobble tool & 16 & -0.92 & 10 & 26 \\
Perforator & 17 & -1.57 & 13 & 30 \\
Ground stone & 1 & +0.66 & - & 1 \\
Hammerstone & 78 & +3.76 & - & .89 \\
Gouge & 1 & +0.66 & 4 & 7 \\
Unmodified debitage & 3 & -1.55 & 539 & 1,781 \\
\hline Totals: & 1,242 & 0.00 & 621 & 2,052 \\
\hline *Everitt (1977:46-48). Numbers above +1.95 or below -1.95 are statistically significant at .05 level of confidence. \\
\hline
\end{tabular}


activity locality where plant foods were processed in baking pits.

While the major difference in the Late Prehistoric I occupation periods at the two sites is the higher frequency of ground stones at 41GR291, several artifact categories show tendencies to be over- or underrepresented. At 41GR291, for instance, cores, cobble tools, and gouges are underrepresented, and some artifact classes at 41GR291 are absent at 41GR303B (i.e., arrow points, perforators, hammerstones, modified shells, and ceramics). Some of this variability could be due to differences in site function. The greater overall diversity of the 41GR291 assemblage is more characteristic of residential base camps, while the less diverse assemblage recovered from 41GR303B is most characteristic of specialized activity sites and/or campsites occupied for shorter periods.

Unfortunately, little direct data concerning the plant foods utilized during the Late Prehistoric I period have been acquired. Macrobotanical remains recovered from flotation of sediments from 41GR559 are not particularly diagnostic because the charred seeds representing edible plants (i.e., hackberry, mesquite, pigweed, and knotweed) were recovered in very small amounts. Calcium oxylate crystals recovered from sediments associated with a baking pit (Feature 8) at 41GR303B and a stone-lined hearth (Feature 3) at 41GR559 are interpreted as representing prickly pear (see Cummings 1990:541). This is intriguing given that the ethnographic evidence indicates that baking pits were used for cooking desert succulents (see Chapter 4, Cultural Features). The most common plants associated with baking pits are agave and sotol (Basehart 1960:41; Castetter and Opler 1936:38; Pennington 1969:110, 141; Sonnichsen 1958:16-20; Tunnell and Madrid 1990:145-162), but other plants known to have been processed in a similar manner include prickly pear (Basehart 1960:38-39), yucca (Basehart 1960:40; Castetter and Opler 1936:39), and agave (Medsger 1966:163; Pennington 1963:130). All of these plants, except agave, are documented for the Lower Plains region and are likely to have been present prehistorically. Unfortunately, the distribution and relative abundance of these plants during the Late Prehistoric period are not known.

Prickly pear is ubiquitous today throughout the Texas Panhandle-Plains and was present in historic times prior to Anglo settlement of the area in the late nineteenth century (e.g., Galvin 1970:36; Parker
1984:183). Conversely, the modern distribution and density of sotol in the Lower Plains appear to be very limited. It is abundant in the Edwards Plateau and Trans-Pecos regions of Texas, but McMahan et al. (1984:10) note the presence of sotol as far north as Garza County along the Caprock Escarpment, although they do not provide any information on its abundance. No sotol has been observed in the Lake Alan Henry area during any of the archeological investigations, nor is it mentioned as occurring in the Lower Plains in four important botanical studies for the state (Hatch et al. 1990:39), the region (Kirkpatrick 1992; Rowell 1967), and Garza County (Hutchins 1968). This implies that it is quite rare in the Lower Plains. The low density of sotol may be a modern phenomenon, however, and it could have been more abundant in the Lower Plains prior to Anglo ranching. While livestock grazing has spread some plants extensively, such as the spread of yucca and mesquite documented in southern New Mexico by York and Dick-Peddie (1969), sotol is one of the plants that has been adversely affected because it was used extensively as food for livestock during drought periods, and thus densities of sotol may have been seriously modified in some areas (David Riskind, personal communication 1993). Wilson (1930:61) suggests that sotol was virtually eliminated in some areas by "early cattlemen feeding it to their cattle in dry winters." Yucca, on the other hand, is abundant in the project area and is listed for the area in all four of the botanical studies mentioned above. Although it may be more abundant in some areas because of cattle grazing, its modern distribution is probably representative of its historic and Late Prehistoric distributions.

There is one possible ethnographic account for the use of sotol by native peoples in the Lower Plains. At the headwaters of the Double Mountain Fork of the Brazos River along the edge of the Llano Estacado, a band of Comanches may have been processing sotol in 1875 (Wallace 1978:238-239). An observer reported that they were searching for "a certain kind of vegetable of which they are very fond and which they put in these rock lined pitts [sic] and bake" (Wallace 1978:239). Although the vegetable is not specifically identified, Wallace (1978:239) suggests that it was sotol, but other plants such as yucca and prickly pear are also possibilities. In the Southem Plains, the heart of the yucca plant was used for food by the Kiowas (Vestal and Schultes 1939:18-19), and prickly pears are reported to have 
been used as food by Comanches and Kiowas (Carlson and Jones 1940:527). Hence, it is possible that baking pits in the Lower Plains might be related to sotol, yucca, or prickly pear and that any of these plants could have been important food resources. Also, the possibility that some cultigens were processed in the baking pits must be considered, especially since corn is known to have been cooked in pits (Castetter and Opler 1936:29).

At 41GR291, the macrobotanical remains from features include a large concentration of charred Chenopodium seeds recovered from one storage pit (Feature 19) that could represent a stored food resource. At least four species of Chenopodium, including C. album, apparently are native to the region (Rowell 1967:81), and it is likely that the seeds from 41GR291 are from local native species rather than domesticated species such as those found in the eastern United States (Ford 1985:348). Unfortunately, there is no way to confirm the depositional origin of the pit fill, and the seeds could have been introduced by other cultural, natural, or postdepositional processes (see Storage Pits in Chapter 3). Other charred seeds from storage pits were recovered in relatively low frequencies and could have been introduced by various processes. Consequently, there is no unequivocal evidence for the types of plants that were stored in the 41GR291 storage pits.

The recovery of charred mesquite beans from 41GR291 and 41GR559 provides evidence that mesquite probably was utilized as a food resource during Late Prehistoric I times. The recovery of numerous charred mesquite seeds on the floor of the 41GR291 pithouse, in particular, provides good evidence for their use as a food resource at ca. A.D. 1000 .

Two main species of Prosopis were considered staple resources for many native peoples in the southwestern United States and northern Mexico (Bell and Castetter 1937:21-33), along the Texas coast and in central Texas (Covey 1961:66, 86-87, 100), in the Rio Grande delta (Salinas 1990:117-119), and in the Texas South Plains (Carlson and Jones 1940:530; Vestal and Schultes 1939:33-34). Honey mesquite ( $P$. juliflora var. glandulosa or $P$. glandulosa) is the common variety in northern Texas, while screwbean mesquite ( $P$. pubescens) or tornillo is not common in Texas but is found in many parts of the Southwest (Vines 1960:515-517, 1982:261-263). Mesquite also has been suggested as an important prehistoric resource in the Jomada Mogollon region, although archeological evidence for its use is limited (Brethauer 1979; Leslie 1979:186). Even the historic Comanches and Kiowas, whose homeland was in the Texas Panhandle-Plains, utilized mesquite beans for food (Carlson and Jones 1940:530; Galvin 1970:3031; Vestal and Schultes 1939:33-34). Nutritional data compiled by Hiles (1993) suggest that mesquite seeds are very high in protein and that pods are high in carbohydrates and fiber.

The often-told story that mesquite was not present or was rare in the Southern Plains until recent times has little basis in fact. While mesquite certainly has been spread because of historic and modern agricultural activities such as cattle grazing (and severe overgrazing), root plowing and chaining, and control of natural range fires (Flores 1990:60-61; Kirkpatrick 1992:141), there is ample evidence that it existed in some abundance in parts of the region prior to these activities. Kirkpatrick (1992:141) suggests that prior to Anglo ranching and farming, mesquite was confined to the canyonlands and riparian zones and was not present in the grasslands. The presence of mesquite in the Lower Plains prior to historic ranching is documented by the Texan Santa Fe expedition of 1841 and by Captain Marcy's explorations of the headwaters of the Red and Brazos rivers in the 1850s. During the 1841 trip from the new Republic of Texas capitol at Austin to Santa Fe, New Mexico, expedition members described areas in Knox and Motley counties as being "a fine rolling prairie covered with mesquite" and "a beautiful mesquite prairie country" (Bailey 1951:105, 121). In 1852, Marcy noted that mesquite trees were abundant along the Red River (probably in the vicinity of Hall County) and that they were relatively abundant as far north as the "thirty-sixth degree of north latitude" (Foreman 1937:97). In 1854, an observer traveling with Marcy was impressed by the "immense quantity" and large size of mesquite trees in the Brazos River valley (probably in the vicinity of Garza County) and along the Caprock Escarpment (Parker 1984:170171). During this same trip, "mesquite flats" were noted near the Double Mountain Fork of the Brazos, probably north of Abilene in the general vicinity of Jones or Haskell counties (Parker 1984:183). Clearly, mesquite was present in appreciable quantities, if not abundant, in parts of the Lower Plains during the mid nineteenth century prior to any extensive land alterations or cattle grazing. If its mid-nineteenth-century distribution and density are 
any indication, mesquite may have been sufficiently abundant during Late Prehistoric times to constitute an important food resource.

The occurrence of charred mesquite beans in the prehistoric pithouse at 41GR291 suggests that this may have been the case, and the co-occurrence of a broken pestle suggests that bedrock mortars were in use at the same time. This is intriguing because mortars were used extensively in the processing of mesquite beans (Bell and Castetter 1937:14, 21-33; Carlson and Jones 1940:530). The processing of mesquite beans usually involved crushing the whole pods in wooden or bedrock mortars and grinding them into flour on metates, although some processes involved parching them prior to grinding. Charring of beans may have been incidental to the process, or intentionally charred beans might have been accidentally lost, but in either case, charred mesquite beans should not be expected to be recovered frequently in archeological contexts. Notably, the use of rocklined baking pits for cooking mesquite beans is not mentioned in Bell and Castetter (1937).

The mesquite beans in the 41GR291 pithouse may represent their earliest occurrence in the region, but other archeological evidence indicates that mesquite beans and wood were used at various times by prehistoric peoples in the Texas Panhandle-Plains. Charred mesquite beans have been found at Landergin Mesa, a Panhandle aspect site along the Canadian River in the northern Texas Panhandle (Glenna Dean, personal communication 1993). In two cases, mesquite beans from Structure 1 are clearly associated with uncorrected charcoal radiocarbon dates of A.D. 1250-1390 (Feature 23) and A.D. 1260-1440 (Lintz 1990:Table 5, personal communication 1993). Fossil mesquite seeds, although not from cultural contexts, were recovered at the Lubbock Lake site in Stratum 5A, dated to approximately A.D. 1200-1500 (E. Johnson 1987:20; Thompson 1987:33-34, Table 4.2). Charred fragments of wood identified as Prosopis/Acacia from Mitchell Reservoir site 41HM49 could represent mesquite and are associated with deposits radiocarbon dated to A.D. 705-1030 (Quigg et al. 1993:Table 10.15, 158, personal communication 1993). Late Archaic-age deposits (i.e., Zones 7A and 8A) at 41TG91 yielded charred wood (identified as Acacia/Prosopis by Holloway 1990:Table 69) that may represent the use of mesquite around 2,500 years ago (Creel 1990:35-43, 217). These few examples suggest that mesquite was present in the Texas Panhandle-Plains for at least the last 2,500 years and that it was utilized for firewood and food by prehistoric peoples.

Since it is likely that mesquite beans were used by the prehistoric occupants of 41GR291, the possibility that they were kept in storage pits should be considered. Basehart (1960:37) noted that the Mescalero Apache placed a high value on mesquite as a food resource, in part because of "the storage properties of the mesquite bean." Ethnographic accounts indicate that some groups procured sufficient quantities to enable a surplus to be traded or stored on platforms or on house-tops (Bell and Castetter 1937:21-33). Mesquite beans sometimes were stored "for winter use" (Bell and Castetter 1937:21), and a paste made from mesquite bean flour mixed with water could be "kept for an indefinite period without spoiling or becoming wormy" (Bell and Castetter 1937:22). Mesquite beans also were stored in ceramic vessels buried in the ground, and at least one variety of mesquite was sometimes buried in grasslined pits for up to a month to slowly "cook" or cure the pods to counter bitterness (Bell and Castetter 1937:26, 32-33). Processing by burial apparently was done with only the screwbean variety of mesquite, which tends to be bitter unless cured in the ground, and this process is not documented in connection with honey mesquite.

A few cucurbit (i.e., bottle gourd) seeds recovered from 41GR291 and 41GR559 constitute the only possible evidence of horticulture at Lake Alan Henry, and this evidence is tenuous. These seeds could not be identified to species and could be from native or domesticated plants, and bottle gourds could have been used as containers rather than as a food resource. Although there is no good archeological evidence of cultigen use at any sites in the Lower Plains region, both Hughes (1991:27) and Cruse (1992:146) hint that horticulture may have been practiced during the first millenium A.D.

Notably absent from the discussion above of Late Prehistoric I subsistence are faunal remains. The Season 3 data recovery yielded few significant faunal remains from Late Prehistoric I contexts due in part to poor preservation. It is also possible that the paucity of animal remains may be due partly to limited reliance on hunting since the artifactual and feature evidence suggest a considerable degree of plant dependence. Faunal remains recovered in the 41 GR559 rockshelter, where poor preservation is not a factor, indicate that small animals (e.g., rabbits, rodents, and snakes) were eaten, but there is no 
evidence for the hunting of big game.

In summarizing subsistence strategies and settlement patterns during Late Prehistoric I times, three kinds of sites representing different suites of cultural activities are recognized. The primary type of site is a residential base camp occupied periodically on a seasonal basis for the purpose of exploiting selected native and/or domesticated plants. There may have been a single plant, or perhaps two or three plants, that were particularly abundant and served as key dietary staples. Regardless of the particular plants utilized, it is likely that a logistical collector strategy was employed during the procurement and processing of the resources. Residential base camps with pithouses (such as 41GR291 and the Kent Creek site [Cruse 1992]) may have been occupied at two or more times during different seasons of the year. One occupation was most likely during summer and/or fall for plant harvest, and surplus foods were put into storage pits. Following a period of abandonment, a second occupation probably occurred in the winter, the lean season when the stored foods would have been most valuable. Of a wide range of possible plants that could have been utilized, mesquite and prickly pear are the best candidates for having been prehistoric dietary staples based on ethnographic and archeological evidence. If limited farming was incorporated into the activities that occurred at residential bases, this would have required at least a brief occupation during the spring or early summer planting season and probably a second occupation at harvest time in the fall and perhaps into winter.

The second type of site recognized for the Late Prehistoric I period is rockshelters. Several rockshelters in the region have evidence of intermittent occupations, perhaps serving as temporary winter residences, but the intensity of the occupation episodes varies considerably. Archeological evidence (i.e., the relative densities of cultural debris) indicates minimal use of the 41GR559 rockshelter, moderate use of the Canyon City Club Cave (Hughes 1969), and intensive use of Deadman's Shelter (Willey and Hughes 1978). The activities that occurred at these rockshelters are also variable. Generalized huntinggathering activities are represented at 41GR559, in contrast to the specialized hunting activities at the Canyon City Club Cave and a broad range of residential and specialized activities (e.g., a burial) at Deadman's Shelter.

Open campsites constitute the third type of site recognized for the Late Prehistoric I period, and they exhibit evidence of intensive plant processing (e.g., high ratios of ground stones relative to projectile points and high frequencies of burned rocks and baking pits). Some sites, such as 41GR303B, appear to represent specialized plant-processing localities, while others such as the Gobbler Creek Bridge site (Boyd et al. 1992:51-80) appear to be multifunctional campsites with an emphasis on plant processing.

The reconstruction of the Late Prehistoric I period above contradicts what was originally proposed in the research design for data recovery at Lake Alan Henry (see Chapter 2). Late Prehistoric I land use was originally viewed as a residentially mobile forager system geared toward exploiting a variety of resources at different times of the year. Underlying this model was the assumption that, in the absence of significant bison populations in the region, no other resources would have been available in sufficient quantity or predictability to allow the adoption of logistical resource exploitation strategies.

Archeological data from the Season 3 investigations, however, strongly suggest that one or more plant resources were procured in large quantities and stored at residential base camps, like the Sam Wahl site, which were occupied on a seasonal basis. This implies that the Late Prehistoric I inhabitants were logistically organized collectors during part of the year. Other contemporaneous sites are assumed to be part of the Late Prehistoric I settlement pattern (i.e., 41GR303B and 41GR559) and appear to have been generated by residentially mobile foragers during other parts of the year. Thus, it is proposed here that both of these resource acquisition patterns were employed by Late Prehistoric I peoples in response to seasonal shifts in the resource base. Based on this new evidence, it is suggested that, in order to adapt to a continually changing resource base, human populations oscillated between residentially mobile foraging and logistically oriented collecting.

Many of the details of the land-use systems proposed for the Late Prehistoric I period remain unknown. There is little evidence of the specific plant resources that were exploited or of the organization of the annual round and seasonal settlement patterns. In addition, incipient agriculture may have been incorporated into the subsistence pattern and might have contributed to increased logistical organization. The recognition of a logistical collector component and increased seasonal sedentism within the Late Prehistoric I period on the Lower Plains is a radical departure from previous percep- 
tions of hunter-gatherer subsistence during the first millenium A.D.

\section{Late Prehistoric II Settlement Patterns and Subsistence}

Three of the sites investigated during Season 3 (41GR291, 41GR303B, and 41GR559) were occupied during the Late Prehistoric II period (ca. A.D. 11001541). Seven radiocarbon dates from these sites fall into this time period (see Figure 92), and the features and artifacts associated with these dates are significantly different from those of the preceding period. These differences are interpreted as representing a change in subsistence strategies that occurred around A.D. 1100 at 41GR291 and 41GR303B. At 41GR559, geomorphic evidence suggests that a shift in depositional regimes inside the rockshelter may have occurred at ca. A.D. 1000, and this shift may be related to regional climatic changes.

It has been proposed that the Late Prehistoric II occupations at $41 \mathrm{GR} 291$ (i.e., late occupation period) and 41GR303B (i.e., upper zone) reflect a shift away from the intensive and specialized plant processing of the previous period toward a more generalized forager adaptation with a greater emphasis on hunting. Interestingly, at both sites significantly fewer burned rocks are associated with the late occupations than with the earlier occupations. Only
$93 \mathrm{~kg}$ of burned rocks are associated with the late occupation period at 41GR291, and few burned rocks are associated with the upper zone in the 41GR303B excavation block. For the earlier occupations, it has been suggested that the burned rocks are related to plant processing activities (i.e., baking pits), and hence the relative paucity of burned rocks in the later occupations may reflect less intensive plant processing.

A comparison of the late occupation artifact assemblages from 41GR291 and 41GR303B, using adjusted standardized residuals to take varying sample sizes into account, suggests that there are significant differences in only two artifact categories-projectile points and edge-modified flakes (Table 62). These data suggest that similar activities were performed at both sites, and the greater number of arrow points and smaller, number of edge-modified flakes at 41GR291 could be related to minor differences in site function. It has been suggested that the upper zone at 41GR303B could represent use of the site as a short-term camp, while the late occupation period at 41GR291 was a more substantial residential base. If this is the case, a greater number of projectile points might be expected at a residential camp where hunting-related activities occurred (i.e., resulting in more points having been manufactured, refurbished, used, and discarded more frequently) than at a short-term campsite where

\begin{tabular}{|c|c|c|c|c|}
\hline \multicolumn{5}{|c|}{$\begin{array}{c}\text { TABLE } 62 \\
\text { COMPARISONS OF LATE OCCUPATION STONE ARTIFACTS FROM 41GR291 AND 41GR303B }\end{array}$} \\
\hline Artifact Type & $\begin{array}{c}\text { 41GR291 } \\
\text { Late Occupation Period }\end{array}$ & $\begin{array}{l}\text { Adjusted Standardized } \\
\text { Residuals for 41GR291* }\end{array}$ & $\begin{array}{l}\text { 41GR303B } \\
\text { Upper Zone }\end{array}$ & Totals \\
\hline Arrow point*** & 18 & +2.31 & 3 & 21 \\
\hline Biface & 11 & +0.97 & 4 & 15 \\
\hline Uniface & 48 & +0.21 & 29 & 77 \\
\hline Core & 31 & +0.12 & 19 & 50 \\
\hline Edge-modified flake & 19 & -1.96 & 22 & 41 \\
\hline Cobble tool & 15 & +1.59 & 4 & 19 \\
\hline Perforator & 2 & +1.13 & - & 2 \\
\hline Ground stone & 18 & +1.69 & 5 & 23 \\
\hline Hammerstone & 2 & +1.13 & - & 2 \\
\hline Gouge & 9 & -0.09 & 6 & 15 \\
\hline Unmodified debitage & 1,728 & -1.43 & 1,114 & 2,842 \\
\hline Totals: & 1,901 & & 1,206 & 3,107 \\
\hline
\end{tabular}


expedient flake tools might have been important.

Besides the difference in arrow point frequencies, ground stones, cobble tools, and bifaces tend to be overrepresented at 41GR291, while unmodified debitage tends to be underrepresented. In addition, artifact classes present at 41GR291 and absent at 41GR303B (i.e., perforators, hammerstones, and ceramics) suggest some functional differences between the sites. As for the Late Prehistoric I period, a comparison of the Late Prehistoric II assemblages from 41GR291 and 41GR303B indicates that the former is more diverse and more characteristic of residential base camps, while the latter is more characteristic of specialized activity sites and/or campsites occupied for shorter periods.

Late Prehistoric II land use was originally viewed as a combination of logistical collector and residentially mobile forager strategies alternating on a seasonal basis. It was proposed that bison was the key resource that allowed the operation of the resource acquisition system in a collector mode. During seasons of bison abundance, human populations employed logistically organized acquisition practices, shifting to residentially mobile foraging and exploitation of a variety of other resources during seasons of bison scarcity. Underlying this model was the assumption that the Late Prehistoric II period was characterized by climatic changes that favored the proliferation of bison in the Southern Plains (e.g., Dillehay 1974).

The Season 3 archeological data suggest that significant cultural changes did occur in the Late Prehistoric II period and that land-use practices were altered in response to environmental changes. While evidence of bison procurement in the Southern Plains is minimal until the terminal Late Prehistoric II period, an increased emphasis on hunting is noted in the Lake Alan Henry archeological record beginning at around A.D. 1100. The hunting component during the early part of the Late Prehistoric II period appears to have functioned within a broad forager system, and bison populations in the Southern Plains appear not to have increased dramatically until around A.D. 1200-1300 (Bison Presence Period III; Dillehay 1974:184-185). The Season 3 archeological data suggest that bison was not a significant resource in the project area between A.D. 1100 and 1300, and the limited evidence for the early part of the Late Prehistoric II period suggests that people operated in a foraging mode utilizing a broad mix of plant and animal resources.

\section{Late Prehistoric Cultural Dymamics in the Lower Plains}

Archeological features associated with the Late Prehistoric I period are significantly different from those of the Late Prehistoric II period. The pithouse/ storage pit/baking pit complex which characterizes the Late Prehistoric I at 41GR291 denotes increased sedentism and intensive use of plant resources. In contrast, the Late Prehistoric II period lacks these features, and the material evidence suggests that subsistence strategies were quite different from those of the preceding period (see Tables 25 and 33). In general, ground stones are less frequent in the Late Prehistoric II period $(0.9 \%$ at 41 GR2 21 and $0.4 \%$ are 41GR303B) than in the Late Prehistoric I period (5.5\% at 41GR291 and $1.8 \%$ at 41GR303B). Conversely, arrow points are more frequent in the Late Prehistoric II period $(0.9 \%$ at $41 \mathrm{GR} 291$ and $0.2 \%$ at $41 \mathrm{GR} 303 \mathrm{~B}$ ) than in the Late Prehistoric I period $(0.3 \%$ at $41 \mathrm{GR} 291$ and $0 \%$ at $41 \mathrm{GR} 303 \mathrm{~B})$. The arrow point to ground stone ratios for the late occupation period at 41GR291 (18:18) and the upper zone at 41GR303B (3:5) are considerably different from those of the earlier occupations (4:78 at 41GR291 and 0:11 at 41GR303B) and indicate an increased emphasis on hunting and/or a decreased emphasis on the grinding of plant foods. Notably, no large basin metates such as those associated with the early occupation pithouse and storage pits at 41GR291 are represented in the late occupations from either site.

Overall, the archeological evidence from these sites appears to indicate changing land-use patterns beginning around A.D. 1100. The Late Prehistoric I period is characterized by seasonal sedentism, plant resource specialization, and storage. On the other hand, land-use patterns during the Late Prehistoric II period are characterized by increased residential mobility and decreased specialization in resource procurement. This shift in land-use patterns may be related to environmental changes evident in the archeological and paleoenvironmental data for the Southern Plains. Geoarcheological evidence suggests that at A.D. 1000-1100 the depositional processes inside the 41GR559 rockshelter changed dramatically and may indicate a period of increased aridity. This does not appear to have been an isolated event, and there is considerable evidence for widespread cultural and environmental changes throughout the Southern Plains and in the Southwest at A.D. 1100-1200. 
Although theoretical models conflict (e.g., Bryson et al. 1970; Gunn 1987; Hall 1982), there is ample evidence of drought during the twelfth and thirteenth centuries that affected the Texas South Plains and surrounding regions. Ferring's (1986:63) paleoecological studies, Hall's (1977:31, 1980:45) pollen and geomorphic studies, Duffield's (1970:241) work with Panhandle faunal assemblages, tree-ring analysis by Dean et al. (1985:543), and cross-disciplinary work by Albert (1981) all produced results indicating a period of dessication beginning at ca. A.D. 1100 but having slightly different intensities and beginning and ending dates in each region.

Cultural changes also occurred throughout the Southern Plains at about this time, as evidenced by a widespread shift from corner-notched to side-notched arrow points and the appearance of beveled knives at A.D. 1100-1200 (Hughes 1991:29-30). It has been suggested that side-notched arrow points, 'beveled knives, and Plains-style end scrapers constitute an effective bison-hunting tool kit which spread rapidly throughout the region because of an influx of large numbers of bison, perhaps returning to the Southern Plains after a period of absence (Dillehay 1974; Hughes 1991:30). It probably is not coincidental that many groups of people on the Southern Plains became bison-hunting farmers during this time. Along the Canadian River in the northern Texas Panhandle, the local Woodland peoples (i.e., Lake Creek complex) began to settle into becoming the bison-hunting farmers known as the Antelope Creek phase (Brooks 1989:80-82; Lintz 1986:29-37). Similarly, local Woodland peoples of the Washita River phase (Brooks 1989:77-80) in western Oklahoma intensified their agriculture and incorporated large-scale seasonal bison hunts. In the eastern Jornada Mogollon area, Ochoa phase people on the southern High Plains apparently developed a semisedentary village lifestyle based on bison hunting and foraging (Collins 1971:89; Hughes 1991:28). An influx of bison is noted in the middle Pecos region during the Late McKenzie phase (A.D. 1250-1350) (Jelinek 1967:138, 158) and at approximately the same time in the Lincoln phase in the Pecos River valley near Roswell (Kelley 1984:51-56, 489). It has been suggested that the ultimate abandonment of the southeastern puebloan area (i.e., the Sierra Blanca, the middle Pecos, and the Jornada Mogollon regions) during the fourteenth century might be related to factors such as changing environmental conditions and corresponding changes in human subsistence strategies, the influx of new peoples into the Southern Plains, and increasing hostilities between the puebloan peoples and Plains bison hunters (Jelinek 1967:162163; Kelley 1984:156-158; Whalen 1980b:446-448).

The Season 3 archeological investigations at Lake Alan Henry produced a considerable amount of data that are compatible with this broad regional perspective. Within the project area, the archeological evidence parallels the cultural and environmental changes seen over the entire Southern Plains. As postulated for the Southern Plains and surrounding regions, the Lake Alan Henry data indicate that major cultural changes occurred around A.D. 1100. Increased aridity may have resulted in changes in floral and faunal communities necessitating the adoption of new survival strategies by local human populations. Prior to A.D. 1100, some groups in the Lower Plains, perhaps locally recognizable as the Palo Duro complex, lived semisedentary lifestyles by relying predominantly on a narrow range of plant resources. After A.D. 1100, local groups may have abandoned their heavy dependence upon plant resources in favor of a broad-based hunter-gatherer adaptation in response to changing environmental conditions. By around A.D. 1200-1300, bison may have been present in sufficient numbers in the region to allow a seasonal dependence upon bison hunting, although there is no evidence of this in the Lake Alan Henry project area itself until the terminal Late Prehistoric II period. While some Southem Plains groups emerged as semisedentary Plains Villagers combining horticulture and bison hunting, others incorporated seasonal bison hunting into a mobile hunter-gatherer lifestyle. 


\section{REFERENCES CITED}

Abbott, James T.

1990 Geomorphic and Geoarcheological Investigations. Chapter 4 in Phase II Investigations at Prehistoric and Rock Art Sites, Justiceburg Reservoir, Garza and Kent Counties, Texas, by Douglas K. Boyd, James T. Abbott, William A. Bryan, Colin M. Garvey, Steve A. Tomka, and Ross C. Fields, pp. 23-62. Reports of Investigations No. 71, vol. I. Prewitt and Associates, Inc., Austin.

Adair, Mary J.

1983 Prehistoric Agriculture in the Central Plains. University of Kansas Publications in Anthropology No. 16. Lawrence.

Adams, Jenny L.

1993 Toward Understanding the Technological Development of Manos and Metates. Kiva 58(3):331-344.

Agogino, George, and Van Tries Button

1985 A Secondary Burial of Human Cranial Fragments from 34GR6, a Prehistoric Cemetery on the Shoreline of Lake Altus, Greer County, Oklahoma. U.S. Department of the Interior, Bureau of Reclamation, Southwest Region, Amarillo.

Albert, Lois E.

1981 Ferndale Bog and Natural Lake: Five Thousand Years of Environmental Change in Southeastern Oklahoma. Studies in Oklahoma's Past No. 7. Oklahoma Archeological Survey, Norman.
Alex, Thomas C.

1990 The Search for the Elusive Kaolinite: Prehistoric Utilization of a Lithic Resource for Omamental Artifacts. In Papers from the Third Symposium on Resources of the Chihuahuan Desert Region, pp. 163-168. Chihuahuan Desert Research Institute, Alpine, Texas.

Alexander, Robert K.

1982 Archaeological Site Reconnaissance in the Proposed Justiceburg Reservoir Area, Garza County, Texas. Grand River Consultants, Inc., Grand Junction, Colorado.

Amsden, Charles Avery

1949 Prehistoric Southwesterners from Basketmaker to Pueblo. Southwest Museum, Los Angeles.

Anderson, Adrienne

1968 The Archaeology of Mass-Produced Footwear. Historical Archaeology 2:56-65.

Anderson, Charles G.

1990 Reflections: An Album of West Texas History, 1840-1990. Snyder Publishing Company, Inc., Snyder, Texas.

Anyon, Roger

1980 The Late Pithouse Period. Chapter 5 in An Archeological Synthesis of South-Central and Southwestern New Mexico, edited by Steven A. LeBlanc and Michael E. Whalen, pp. 142-204. Office of Contract Archeology, University of New Mexico, Albuquerque. 
Archer, Grace Emily

1978 In Search of the Dugouts. Ms. in the Collections of the Panhandle-Plains Historical Museum, Canyon, Texas.

Bailey, Carroll H.

1951 The Texan Santa Fe Trail. PanhandlePlains Historical Review 24 (entire volume).

Bames, Frank C.

1980 Cartridges of the World. 4th ed. D.B.I. Books, Inc., Northfield, Illinois.

Bames, Virgil E. (project director)

1967 Geologic Atlas of Texas, Lubbock Sheet. Bureau of Economic Geology, The University of Texas at Austin.

Basehart, Harry W.

1960 Mescalero Apache Subsistence Patterns and Socio-Political Organization, Section I: Mescalero-Chiricahua Land Claims Project. The University of New Mexico, Albuquerque.

Baugh, Timothy G., and Fred W. Nelson, Jr.

1987 New Mexico Obsidian Sources and Exchange on the Southem Plains. Journal of Field Archaeology 14:313-329.

Bell, Robert E.

1958 Guide to the Identification of Certain American Indian Projectile Points. Special Bulletin No. 1. Oklahoma Anthropological Society, Norman.

1960 Guide to the Identification of Certain American Indian Projectile Points. Special Bulletin No. 2. Oklahoma Anthropological Society, Norman.

Bell, Willis H., and Edward F. Castetter

1937 The Utilization of Mesquite and Screwbean by the Aborigines in the American Southwest. University of New Mexico Bulletin No. 314. Ethnobiological Studies in the American Southwest, Biology Series 5(2). University of New Mexico Press, Albuquerque.

Bender, Margaret M., David A. Baerreis, and Raymond L. Steventon

1981 Further Light on Carbon Isotopes and Hopewell Agriculture. American Antiquity 46(2):346-353.

Bettis, E. Arthur, III

1984 New Conventions for the Designation of Soil Horizons and Layers. Plains Anthropologist
29(103):57-59.

Binford, Lewis R.

1980 Willow Smoke and Dogs' Tails: HunterGatherer Settlement Systems and Archaeological Site Formation. American Antiquity 45(1):4-20.

1981 Bones: Ancient Men and Modern Myths. Academic Press, New York.

1990 Mobility, Housing and Environment: A Comparative Study. Journal of Anthropological Research 46(2):119-152.

Birkeland, Peter W.

1984 Soils and Geomorphology. Oxford University Press, Oxford, England.

Blair, W. Frank

1950 The Biotic Provinces of Texas. Texas Journal of Science 2(1):93-117.

Blum, Michael D.

1989 Geoarcheological Investigations. Chapter 7 in Phase I Cultural Resources Investigations at Justiceburg Reservoir on the Double Mountain Fork of the Brazos River, Garza and Kent Counties, Texas, by Douglas K. Boyd, Martha Doty Freeman, Michael D. Blum, Elton R. Prewitt, and J. Michael Quigg, pp. 81-106. Reports of Investigations No. 66, vol. I. Prewitt and Associates, Inc., Austin.

Blum, Michael D., James T. Abbott, and

Salvatore Valastro, Jr.

1992 Evolution of Landscapes on the Double Mountain Fork of the Brazos River, West Texas: Implications for Preservation and Visibility of the Archaeological Record. Geoarchaeology: An International Journal 7(4):339-370.

Blum, Michael D., and Salvatore Valastro, Jr.

1989 Response of the Pedemales River of Central Texas to Late Holocene Climatic Change. Annals of the Association of American Geographers 79(3):435-456.

Boice, Nancy

1980 Cistern One Glass. Appendix G in Archeological Investigations at the Thomas $\mathrm{F}$. McKinney Homestead, Travis County, Texas: An Experiment in Historic Archeology, by Michael McEachern and Ronald W. Ralph. Bulletin of the Texas Archeological Society 51:168-208. 
Bousman, C. Britt

1991 Geoarcheological Investigations. Chapter 4 in Archeological Survey of Wildlife Mitigation Lands, Justiceburg Reservoir, Garza County, Texas, by Douglas K. Boyd, C. Britt Bousman, and Martha Doty Freeman, pp. 4549. Reports of Investigations No. 79. Prewitt and Associates, Inc., Austin.

1992 Off-Site Geological Investigations. Chapter 8 in Data Recovery at Justiceburg Reservoir (Lake Alan Henry), Garza and Kent Counties, Texas: Phase III, Season 1, by Douglas K. Boyd, Steve A. Tomka, C. Britt Bousman, Karen M. Gardner, and Martha Doty Freeman, pp. 135-142. Reports of Investigations No. 84. Prewitt and Associates, Inc., Austin.

Boyd, Douglas K.

1982 Human Skeletal Remains from 34GR6, Greer County, Oklahoma. Department of the Interior, Bureau of Reclamation, Southwest Region, Amarillo.

Boyd, Douglas K., James T. Abbott, William A. Bryan, Colin M. Garvey, Steve A. Tomka, and Ross C. Fields

1990 Phase II Investigations at Prehistoric and Rock Art Sites, Justiceburg Reservoir, Garza and Kent Counties, Texas. Reports of Investigations No. 71.2 vols. Prewitt and Associates, Inc., Austin.

Boyd, Douglas K., C. Britt Bousman, and Martha Doty Freeman

1991 Archeological Survey of Wildlife Mitigation Lands, Justiceburg Reservoir, Garza County, Texas. Reports of Investigations No. 79. Prewitt and Associates, Inc., Austin.

Boyd, Douglas K., Martha Doty Freeman, Michael D. Blum, Elton R. Prewitt, and J. Michael Quigg

1989 Phase I Cultural Resources Investigations at Justiceburg Reservoir on the Double Mountain Fork of the Brazos River, Garza and Kent Counties, Texas. Reports of Investigations No, 66. 2 vols. Prewitt and Associates, Inc., Austin.

Boyd, Douglas K., and Karl W. Kibler

1993 Rock Art Site Investigations, Monitoring and Survey, and Off-Site Geomorphological Investigations. Chapter 8 in Data Recovery at Justiceburg Reservoir (Lake Alan Henry), Garza and Kent Counties, Texas: Phase III, Season 2, by Douglas K. Boyd, Jay Peck,
Steve A. Tomka, and Karl W. Kibler, pp. 237-240. Reports of Investigations No. 88. Prewitt and Associates, Inc., Austin.

Boyd, Douglas K., Jay Peck, Steve A. Tomka, and Karl W. Kibler

1993 Data Recovery at Justiceburg Reservoir (Lake Alan Henry), Garza and Kent Counties, Texas: Phase III, Season 2. Reports of Investigations No. 88. Prewitt and Associates, Inc., Austin.

Boyd, Douglas K., Steve A. Tomka, C. Britt Bousman, Karen M. Gardner, and Martha Doty Freeman

1992 Data Recovery at Justiceburg Reservoir (Lake Alan Henry), Garza and Kent Counties, Texas: Phase III, Season 1. Reports of Investigations No. 84. Prewitt and Associates, Inc., Austin.

Brethauer, Douglas Paul

1979 The Possible Role of Mesquite as a Food Resource in the Jornada Mogollon Region. In Jornada Mogollon Archaeology: Proceedings of the First Jornada Conference, edited by Patrick $\mathrm{H}$. Beckett and Regge N. Wiseman, pp. 67-80. Historic Preservation Bureau, State Planning Office, Santa Fe, and Cultural Resources Management Division, New Mexico State University, Las Cruces.

Bronson, Bennet

1977 The Earliest Farmers: Demography as Cause and Consequence. In Origins of Agriculture, edited by Charles A. Reed, pp. 23-48. Mouton, The Hague.

Brooks, Robert L.

1989 Village Farming Societies. Chapter 6 in From Clovis to Comanchero: Archeological Overview of the Southern Great Plains, by Jack L. Hofman, Robert L. Brooks, Joe S. Hays, Douglas W. Owsley, Richard L. Jantz, Murray K. Marks, and Mary H. Manhein, pp. 71-90. Research Series 35. Arkansas Archeological Survey, Fayetteville.

Brown, Theodore M., Kay L. Killen, Helen Simons, and Virginia A. Wulfkuhle

1982 Resource Protection Planning Process for Texas. Texas Historical Commission, Austin.

Bryson, Reid A., David A. Baenreis, and Wayne A. Wendland

1970 The Character of Late-Glacial and PostGlacial Climatic Changes. In Pleistocene and Recent Environments of the Central Great 
Plains, edited by Wakefield Dort, Jr., and J. Knox Jones, Jr., pp. 53-77. Special Publications 3. Department of Geology, University of Kansas, Lawrence.

Buol, S. W., F. D. Hole, and R. J. McCracken

1980 Soil Genesis and Classification. 2nd ed. The Iowa State University Press, Ames.

Bureau of Economic Geology

1992 Geologic Map of Texas. Virgil E. Barnes, Project Supervisor. Bureau of Economic Geology, The University of Texas at Austin.

Busch, Jane

1981 An Introduction to the Tin Can. Historical Archaeology 15(1):95-104.

Button, Van Tries, and George Agogino

1987 Human Skeletal Material Exposed by Reservoir-Induced Erosion at Lake Altus, Southwestem Oklahoma. Bulletin of the OklahomaAnthropological Society 36:15-37.

Butzer, Karl W.

1982 Archaeology as Human Ecology. Cambridge University Press, Cambridge.

Calamia, Mark A.

1991 Ground Stone Variability among Jornada Mogollon Sites and Its Implications for Interpreting Residential Mobility. In Mogollon V, edited by Pat Beckett, pp. 119132. COAS Publishing and Research, Las Cruces.

Callahan, Everett

1979 The Basics of Biface Knapping in the Eastern Fluted Point Tradition: A Manual for Flintknappers and Lithic Analysts. Archaeology of Eastern North America 7:1-180.

Campbell, Hannah

1964 Why Did They Name It . . . ? Fleet Publishing Corporation, New York.

Campbell, Robert G.

1975 Archaeological Reconnaissance of Portions of the South Plains Region. Texas Tech University, Lubbock.

1977 Archaeological Reconnaissance of Portions of the South Plains Planning Region. Report No. 2: Corrections and Additions. Texas Tech University, Lubbock.
Campbell, Robert G., and Sharon J. Judd

1977a Archaeological Reconnaissance of Portions of the South Plains Planning Region. Report No. 3: Tabulation of Archaeological Sites and Materials. Department of Anthropology, Texas Tech University, Lubbock.

1977b Archaeological Reconnaissance of Portions of the South Plains Planning Region. Report No. 4: Analysis of Archaeological Materials. Department of Anthropology, Texas Tech University, Lubbock.

Campbell, Thomas Jefferson

1983 The Greenbelt Site: An Example of Variation among Prehistoric Plains Village Sites in the Texas Panhandle. Unpublished Master's thesis, Department of Anthropology, Texas Tech University, Lubbock.

Caran, S. Christopher, and Robert W. Baumgardner, Jr.

1988 The Lingos formation, westem Rolling Plains of Texas. In Decade of North American Geology: Geological Society of America, South-Central Section, Centennial Field Guidebook 4:287-291. Edited by O. T. Hayward.

1990 Quatemary Stratigraphy and Paleoenvironments of the Texas Rolling Plains. Geological Society of America Bulletin 102:768-785.

Carlson, Gustav G., and V. H. Jones

1940 Some Notes on Uses of Plants by the Comanche Indians. Papers of the Michigan Academy of Science, Arts, and Letters 25:517-542.

Carmichael, David

1985 Archeological Excavations at Two Prehistoric Campsites Near Keystone Dam, El Paso, Texas. Occasional Papers No. 14. New Mexico State University, Las Cruces.

Castetter, Edward F., and Morris E. Opler

1936 The Ethnobiology of the Chiricahua and Mescalero Apache. A. The Use of Plants for Foods, Beverages, and Narcotics. The University of New Mexico Bulletin, Biological Series 4(5). University of New Mexico Press, Albuquerque.

Cerling, T. E., J. Quade, Y. Wong, and J. R. Bowman 1989 Carbon Isotopes in Soils and Palaeosols as Ecology and Palaeoecology Indicators. Nature 341:138-139. 
Cerling, Thure E., and Richard L. Hay

1986 An Isotopic Study of Paleosol Carbonates from Olduvai Gorge. Quaternary Research 25:63-78.

Cheek, Annetta

1977 The Panhandle Plains Assemblage. In Investigations at Tx-33, Old Hardesty, Texas County, Oklahoma, by William Lees, pp. 146-148. Report No. 11. Archeological Research Associates, Tulsa.

Clark, John W., Jr.

1984 Archaeological Test Excavations at an Early Twentieth Century Dump in North San Antonio, Bexar County, Texas. Publications in Archaeology Report No. 26. State Department of Highways and Public Transportation, Highway Design Division, Austin.

Coffman, Robert J., Margaret C. Trachte, and Michael B. Collins

1986 Cultural Ecology of the Kenyon Rockshelter and the Cunningham Site, Canyon Creek Development, Travis County, Texas. Reports of Investigations No. 53. Prewitt and Associates, Inc., Austin.

Colcutt, S. N.

1979 The Analysis of Quaternary Cave Sediments. World Archaeology 10:290-301.

Collins, Michael B.

1968 The Andrews Lake Locality: New Archeological Data from the Southern Llano Estacado, Texas. Unpublished Master's thesis, The University of Texas at Austin.

1971 A Review of Llano Estacado Archaeology and Ethnohistory. Plains Anthropologist 16(52): 85-104.

1991 Archaeological Record in the Americas. In The First Americans: Search and Research, edited by Tom D. Dillehay and David J. Meltzer, pp. 157-182. CRC Press, Boca Raton.

Corley, John A.

1965a Proposed Eastern Extension of the Jornada Branch of the Mogollon. Transactions of the First Regional Symposium for Southeastern New Mexico and Western Texas:31-36.

1965b Proposed Eastern Extension of the Jornada Branch of the Mogollon. Bulletin of the Lea County Archeological Society 1:30-36.
Courty, Marie Agnes, Paul Goldberg, and Richard MacPhail 1989 Soils and Micromorphology in Archaeology. Cambridge University Press, Cambridge.

Couzzourt, Jim

1985 Preliminary Report: Testing at the Tascosa Creek Site, Oldham County, Texas. Transactions of the Twentieth Regional Archeological Symposium for Southeastern New Mexico and Western Texas:65-142.

Covey, Cyclone (translator)

1961 Cabeza De Vaca's Adventures in the Unknown Interior of America. University of New Mexico Press, Albuquerque.

Crabtree, Don E.

1972 An Introduction to Flintworking. Occasional Papers of the Idaho State Museum No. 28. Pocatello.

Creel, Darrell

1990 Excavations at 41TG91, Tom Green County, Texas. Publications in Archaeology Report No. 38. Texas State Department of Highways and Public Transportation, Highway Design Division, Austin.

Cruse, Jimmy Brett

1992 Archeological Investigations at the Kent Creek Site (41HL66): Evidence of Mogollon Influence on the Southern Plains. Panhandle Archeological Society Publication No. 6.

Cummings, Linda Scott

1990 Phytolith and Pollen Analysis: A Feasibility Study. Appendix I in Phase II Investigations at Prehistoric and Rock Art Sites, Justiceburg Reservoir, Garza and Kent Counties, Texas, by Douglas K. Boyd, James T. Abbott, William A. Bryan, Colin M. Garvey, Steve A. Tomka, and Ross C. Fields, pp. 533-550. Reports of Investigations No. 71, vol. II. Prewitt and Associates, Inc., Austin.

Dallas Morning News

Davis, Dan R., Jr.

1991 Prehistoric Artifacts of the Texas Indians. Pecos Publishing Company, San Antonio.

Dean, Jeffrey S., Robert C. Euler, George J. Gumerman, Fred Plog, Richard H. Hevly, and Thor N. V. Karlstrom

1985 Human Behavior, Demography, and Paleoenvironment on the Colorado Plateaus. American Antiquity 50(3):537-554. 
DeBoer, Warren R.

1988 Subterranean Storage and the Organization of Surplus: The View from Eastern North America. Southeastern Archaeology 7(1):120.

DeBolt, C. Gerald

1988 The Dictionary of American Pottery Marks: Whiteware and Porcelain. Charles E. Tuttle Company, Rutland, Vermont.

Dickens, Roy S., Jr.

1985 The Form, Function, and Formation of Garbage-filled Pits on Southeastem Aboriginal Sites: An Archaeological Analysis. Chapter 2 in Structure and Process in Southeastern Archaeology, edited by Roy S. Dickens and H. Trawick Ward, pp. 34-59. University of Alabama Press, University.

Didway, Charles (editor)

1973 Wagon Wheels: A History of Garza County. Pioneer Book Publishers, Inc., Seagraves, Texas.

Dillehay, Tom D.

1974 Late Quaternary Bison Population Changes on the Southem Plains. Plains Anthropologist 19(65):180-196.

Donahue, Jack, and James Adovasio

1990 Evolution of Sandstone Rockshelters in Eastern North America: A Geoarchaeological Perspective. In Archaeological Geology of North America, edited by Norman P. Lasca and Jack Donahue, pp. 231-251. Centennial Special Volume 4. Geological Society of America, Boulder, Colorado.

Dover Publications, Inc.

1969 Montgomery Ward and Co. Catalogue and Buyer's Guide, No. 57, Spring and Summer 1895. Dover Publications, Inc., New York.

Driver, Jonathan C.

1985 Zooarchaeology of Six Prehistoric Sites in the Sierra Blanca Region, New Mexico. Technical Reports No. 17, Research Reports in Archaeology Contribution 12. Museum of Anthropology, University of Michigan, Ann Arbor.

Duffield, Lathal Flay

1970 Some Panhandle Aspect Sites: Their Vertebrates and Paleoecology. Unpublished $\mathrm{Ph} . \mathrm{D}$. dissertation, University of Wisconsin. University Microfilms, Ann Arbor.
Dulaney, Alan R., and John D. Pigott

1977 Preliminary Technological Analysis of El Paso Brownware and Design for Further Research. In Part II: Special Archeological Studies in $A$ Cultural Resource Inventory and Assessment of McGregor Guided Missile Range, Otero County, New Mexico, by Michael R. Beckes, Alan R. Dulaney, John D. Pigott, Robert F. Scott, IV, and Nancy Kays Smiley, pp. 263289. Research Report No. 65/II. Texas Archeological Survey, The University of Texas at Austin.

Earls, Amy C.

1993a Results of Historic Site Investigations. Chapter 7 in Historic and Prehistoric Data Recovery at Palo Duro Reservoir, Hansford County, Texas, by J. Michael Quigg, Christopher Lintz, Fred M. Oglesby, Amy C. Earls, Charles D. Frederick, W. Nicholas Trierweiler, Douglas Owsley, and Karl W. Kibler, pp. 357-424. Technical Report No. 485. Mariah Associates, Inc., Austin.

1993b Historic Issues. Part 2 of Research Design Issues. Chapter 8 in Historic and Prehistoric Data Recovery at Palo Duro Reservoir, Hansford County, Texas, by J. Michael Quigg, Christopher Lintz, Fred M. Oglesby, Amy C. Earls, Charles D. Frederick, W. Nicholas Trierweiler, Douglas Owsley, and Karl W. Kibler, pp. 472-483. Technical Report No. 485. Mariah Associates, Inc., Austin.

Etchieson, Gerald Meeks

1979 Archeological Testing at the South Ridge Site, Lake Meredith Recreation Area, Hutchinson County, Texas. Archeological Research Laboratory, Killgore Research Center, West Texas State University, Canyon.

Etchieson, Gerald Meeks, Roberta D. Speer, and Jack T. Hughes

1979 Archeological Investigations in the Crowell Reservoir Area, Cottle, Foard, King and Knox Counties, Texas. Archeological Research Laboratory, Killgore Research Center, West Texas State University, Canyon.

Everitt, B. S.

1977 The Analysis of Contingency Tables. Halstead Press, Chapman and Hall Ltd., London.

Farrand, William R.

1985 Rockshelters and Cave Sediments. In 
Archaeological Sediments in Context, edited by Julie K. Stein and William R. Farrand, pp. 21-39. Peopling of the Americas, vol. 1. Center for the Study of Early Man, Orono, Maine.

Ferraro, Pat, and Bob Ferraro

1964 The Past in Glass. Western Printing and Publishing Co., Sparks, Nevada.

Ferring, C. Reid

1986 Late Holocene Cultural Ecology in the Southem Plains: Perspectives from Delaware Canyon, Oklahoma. In Current Trends in Southem Plains Archaeology, edited by Timothy G. Baugh, pp. 55-82. Plains Anthropologist 31(114, Pt. 2) Memoir 21.

Fields, Ross C., Eloise F. Gadus, L. Wayne Klement, C. Britt Bousman, and Jerrilyn B. McLerran

1993 Excavations at the Tick, Spike, Johns Creek, and Peerless Bottoms Sites, Cooper Lake Project, Delta and Hopkins Counties, Texas. Reports of Investigations No. 91. Prewitt and Associates, Inc., Austin.

Fields, Ross C., and Jeffrey S. Girard

1983 Investigations at Site 32 (41EP325), Keystone Dam Project: A Multicomponent Archeological Site in Western El Paso County, Texas. Reports of Investigations No. 21. Prewitt and Associates, Inc., Austin.

Fields, Ross C., L. Wayne Klement, C. Britt Bousman, Steve A. Tomka, Eloise F. Gadus, and Margaret A. Howard

1991 Excavations at the Bottoms, Rena Branch, and Moccasin Springs Sites, Jewett Mine Project, Freestone and Leon Counties, Texas. Reports of Investigations No. 82. Prewitt and Associates, Inc., Austin.

Fike, Richard E.

1987 The Bottle Book: A Comprehensive Guide to Historic, Embossed Medicine Bottles. Peregrine Smith Books, Salt Lake City.

Flores, Dan

1990 Caprock Canyonlands. University of Texas Press, Austin.

Fontana, Bernard L., and J. Cameron Greenleaf 1962 Johnny Ward's Ranch: A Study in Historic Archaeology. Kiva 28(1-2):1-115.

Ford, Richard I.

1985 Patterns of Prehistoric Food Production in
North America. In Prehistoric Food Production in North America, edited by Richard I. Ford, pp. 341-364. Anthropological Papers No. 75. Museum of Anthropology, University of Michigan, Ann Arbor.

Foreman, Grant (editor)

1937 Adventures on the Red River: Report on the Exploration of the Headwaters of the Red River by Captain Randolph B. Marcy and Captain G. B. McClellan. University of Oklahoma Press, Norman.

Forrester, Robert E.

1951 A Series of Eighteen Indian Skeletons Excavated in Shackelford County, Texas. Bulletin of the Texas Archeological and Paleontological Society 22:132-143.

1987 The Moran Point from North-Central Texas. Bulletin of the Oklahoma Anthropological Society 36:131-136.

Fredlund, Glen

1993 A Holocene Phytolith Record from Morgan Playa. Appendix L in Data Recovery at Justiceburg Reservoir (Lake Alan Henry), Garza and Kent Counties, Texas: Phase III, Season 2, by Douglas K. Boyd, Jay Peck, Steve A. Tomka, and Karl W. Kibler, pp. 481-489. Reports of Investigations No. 88. Prewitt and Associates, Inc., Austin.

Freeman, Martha Doty

1989 Historic Resources in Justiceburg Reservoir. Chapter 9 in Phase I Cultural Resources Investigations at Justiceburg Reservoir on the Double Mountain Fork of the Brazos River, Garza and Kent Counties, Texas, by Douglas K. Boyd, Martha Doty Freeman, Michael D. Blum, Elton R. Prewitt, and J. Michael Quigg, pp. 133-156. Reports of Investigations No. 66, vol. 1. Prewitt and Associates, Inc., Austin.

1990 Agriculture in Texas: Ranching on the Western Rolling Plains, 1877-1945. Chapter 3 in Phase II Historical Investigations at Justiceburg Reservoir, Garza and Kent Counties, Texas, by Martha Doty Freeman and Douglas K. Boyd, pp. 51-97. Reports of Investigations No. 72. Prewitt and Associates, Inc., Austin.

Freeman, Martha Doty, and Douglas K. Boyd

1990 Phase II Historical Investigations at Justiceburg Reservoir, Garza and Kent 
Counties, Texas. Reports of Investigations No. 72. Prewitt and Associates, Inc., Austin.

Freeman, Martha Doty, Robert A. Steinbomer, and Joe C. Freeman

1990 Historic Architectural Resources of the Palo Duro Reservoir, Hansford County, Texas. RioGroup Architects and Planners, Austin, Texas.

Frison, George C.

1973 The Wardell Buffalo Trap-48SU301: Communal Procurement in the Upper Green River Basin, Wyoming. Anthropological Papers No. 48. Museum of Anthropology, University of Michigan, Ann Arbor.

Galvin, John (editor)

1970 Through the Country of the Comanche Indians in the Fall of the Year 1845: The Journal of a U.S. Army Expedition Led by Lieutenant James W. Abert of the Topographical Engineers. John Howell Books, San Francisco.

Garza County

Ad Valorem Tax Rolls

Deed Records

Map and Plat Records

Gates, William C., and Dane E. Ormerod

1982 The East Liverpool Pottery District: Identification of Manufacturers and Marks. Historical Archaeology 16(1-2):1-358.

Gettys, Francie

1991 Salvage Excavation in Roger Mills County. Oklahoma Archeological Survey Newsletter 11(3).

Gillette, Dale A.

1981 Production of Dust that May Be Carried Great Distances. In Desert Dust: Origin, Characteristics, and Effect on Man, edited by Troy L. Pewe, pp. 11-26. Geological Society of America Special Paper 186.

Gillette, Dale A., and Theodore R. Walker

1977 Characteristics of Airborne Particles Produced by Wind Erosion of Sandy Soil, High Plains of West Texas. Soil Science 123:97-110.

Gilman, Patricia A.

1983 Changing Architectural Forms in the Prehistoric Southwest. Unpublished Ph.D. dissertation, Department of Anthropology, University of New Mexico, Albuquerque.
1987 Architecture as Artifact: Pit Structures and Pueblos in the American Southwest. American Antiquity 52(3):538-564.

Godden, Geoffrey A.

1964 Encyclopedia of British Pottery and Porcelain Marks. Crown Publishers, Inc., New York.

Goland, Carol Ann

1983 The Ecological Context of Hunter-Gatherer Storage Strategies. Unpublished Master's thesis, Department of Anthropology, University of North Carolina, Chapel Hill.

Green, F. Earl

1955 Excavations near Gran Quivira, New Mexico. Bulletin of the Texas Archeological Society 26:182-185.

1962 The Lubbock Lake Reservoir Site: 12,000 Years of Human Prehistory. The Museum Journal 6:83-123. West Texas Museum Association, Lubbock.

Greer, Georgeanna H.

1981 American Stonewares: The Art and Craft of Utilitarian Potters. Schiffer Publishing Limited, Exton, Pennsylvania.

Greer, John W.

1965 A Typology of Midden Circles and Mescal Pits. Southwestern Lore 31:41-55.

1968 Notes on Excavated Ring Midden Sites, 19631968. Bulletin of the Texas Archeological Society 38:39-44.

Guffee, Eddie J.

1980 The Soda Lake Ruins: An Archeological Investigation of Anglo Occupation in the Sand Hills of Lamb County, Texas. Archeological Resarch Laboratory, Llano Estacado Museum, Plainview, Texas.

Gunn, Joel

1987 Middle Pecos Culture Sequence and Holocene Climate. In Advances in Middle Pecos Archaeology: Proceedings of a Symposium Held at the Fourth Jornada Mogollon Conference, Tularosa, New Mexico, on October 12, 1985, edited by Susanna R. Katz and Paul Katz, pp. 65-86. U.S. Department of the Interior, Carlsbad.

Gunnerson, James H.

1960 An Introduction to Plains Apache Archaeol- 
ogy - The Dismal River Aspect. Bureau of American Ethnology Bulletin 173:131-260.

Gustavson, Thomas C., and William W. Simpkins

1989 Geomorphic Processes and Rates of Retreat Affecting the Caprock Escarpment, Texas Panhandle. Reports of Investigations No. 180. Bureau of Economic Geology, The University of Texas at Austin.

Hall, Grant D., Thomas R. Hester, and Stephen L. Black

1986 The Prehistoric Sites at Choke Canyon Reservoir, Southern Texas: Results of the Phase II Archeological Investigations. Choke Canyon Series, vol. 10. Center for Archaeological Research, The University of Texas at San Antonio.

Hall, Stephen A.

1977 Geology and Palynology of Archaeological Sites and Associated Sediments.' In The Prehistory of the Little Caney River: 1976 Field Season, edited by Donald O. Henry, pp. 13-41. Contributions in Archaeology 1. Laboratory of Archaeology, University of Tulsa.

1980 Paleoenvironmental Synthesis of Hominy Creek Valley: Pollen and Land Snail Evidence. In The Prehistory and Paleoenvironment of Hominy Creek Valley: 1978 Field Season, edited by Donald O. Henry, pp. 44-55. Contributions in Archaeology 6. Laboratory of Archaeology, University of Tulsa.

1982 Late Holocene Paleoecology of the Southem Plains. Quaternary Research 17:391-407.

1988 Environment and Archaeology of the Central Osage Plains. Plains Anthropologist 33(120):203-218.

Hammack, Laurens C.

1962 LA 5599: A Pithouse Village Near Rincon, New Mexico. Laboratory of Anthropology Notes No. 8. Museum of New Mexico, Santa Fe.

Hard, Robert J.

1990 Agricultural Dependence in the Mountain Mogollon. In Perspectives on Southwestern Prehistory, edited by Paul E. Minnis and Charles L. Redman, pp. 135-149. Westview Press, Boulder, Colorado.
Harrison, Billy R., and Bob T. Griffin

1973 An Infant Burial in the Texas Panhandle. Bulletin of the Texas Archeological Society 44:61-68.

Hatch, Stephan L., Kancheepuram N. Gandhi, and Larry E. Brown

1990 Checklist of the Vascular Plants of Texas. Publication No. MP-1655. Texas Agricultural Experiment Station, College Station.

Hayden, Brian

1986 Resource Models of Inter-Assemblage Variability. Lithic Technology 15(3):82-89.

1987 Reply to Conaty. Lithic Technology 16(23):62.

Heinmann, Robert K.

1960 Tobacco and. Americans. McGraw-Hill Book Company', Inc., New York.

Herskovitz, Robert M.

1978 Fort Bowie Material Culture. University of Arizona Press, Tucson.

Hester, Thomas R., Delbert Gilbow, and Alan D. Albee

1973 A Functional Analysis of "Clear Fork" Artifacts from the Rio Grande Plain, Texas. American Antiquity 38(1):90-96.

Hiles, Harold

1993 Guide to Protein and Nutritional Values of Plants Used by Native Americans of the Southwest. Southwest Research Native, Fairaces, New Mexico.

Hofman, Jack L.

1978 The Development and Northem Relationships of Two Archeological Phases in the Southem Plains Subarea. In The Central Plains Tradition: Internal Development and External Relationships, edited by Donald J. Blakeslee, pp. 6-35. Report No. 11. Office of the State Archeologist, Iowa City.

1984a The Plains Villagers: The Custer Phase. In Prehistory of Oklahoma, edited by Robert E. Bell, pp. 287-305. Academic Press, New York.

1984b The Western Protohistoric: A Summary of the Edwards and Wheeler Complexes. In Prehistory of Oklahoma, edited by R. E. Bell, pp. 347-362. Academic Press, New York. 
Hofman, Jack L., and Robert L. Brooks

1989 Prehistoric Culture History: Woodland Complexes in the Southem Great Plains. Chapter 5 in From Clovis to Comanchero: Archeological Overview of the Southern Great Plains, by Jack L. Hofman, Robert L. Brooks, Joe S. Hays, Douglas H. Owsley, Richard L. Jantz, Murray K. Marks, and Mary H. Manhein, pp. 61-70. Research Series No. 35. Arkansas Archeological Survey, Fayetteville.

Holliday, Vance T.

1983 Stratigraphy and Soils of the Lubbock Lake Landmark Area. In Guidebook to the Central Llano Estacado, edited by Vance $\mathrm{T}$. Holliday, pp. 25-80. Intemational Center for Arid and Semi-Arid Land Studies and The Museum, Texas Tech University, Lubbock.

1985 Holocene Soil-geomorphological Relations in a Semi-arid Environment: The Southem High Plains of Texas. In Soils and Quaternary Landscape Evolution, edited by J. Boardman, pp. 325-357. John Wiley and Sons, New York.

1989 Middle Holocene Drought on the Southem High Plains. Quaternary Research 31:74-82.

Holliday, Vance T., and Curtis M. Welty

1981 Lithic Tool Resources of the Eastern Llano Estacado. Bulletin of the Texas Archeological Society 52:201-214.

Holloway, Richard G.

1990 Identification of Wood Charcoal. Appendix II in Excavations at 41TG91, Tom Green County, Texas, by Darrell Creel, pp. 249253. Publications in Archaeology, Report No. 38. Texas State Department of Highways and Public Transportation, Highway Design Division, Austin.

Honea, Kenneth

1965 The Caballo Highway Salvage Project. Laboratory of Anthropology Notes No. 35. Museum of New Mexico, Santa Fe.

Hood, Odie A.

1992 Interview with Mr. Odie A. Hood by Douglas K. Boyd, October 31, 1992, at site 41GR474, Garza County, Texas.

1993 Letter to Douglas K. Boyd, January 18, 1993. On file, Prewitt and Associates, Inc., Austin.
Hooton, Emest A.

1933 Notes on Five Texas Crania. Bulletin of the Texas Archeological and Paleontological Society 3:25-38.

Hughes, David T.

1977 Analysis of Certain Prehistoric Bison Kills in the Texas Panhandle and Adjacent Areas. Unpublished Master's thesis, University of Arkansas, Fayetteville.

1989 Terminal Archaic Bison Kills in the Texas Panhandle. In In the Light of Past Experience: Papers in Honor of Jack T. Hughes, edited by Beryl Cain Roper, pp. 183-204. Panhandle Archeological Society Publication No. 5. Amarillo.

Hughes, Jack T.

1942 An Archeological Report on the Harrell Site of North-Central Texas. Unpublished Master's thesis, The University of Texas at Austin.

1962 Lake Creek: A Woodland Site in the Texas Panhandle. Bulletin of the Texas Archeological Society 32:65-84.

1969 The Canyon City Club Cave in the Panhandle of Texas. Ms. on file, Office of the State Archeologist, Texas Historical Commission, Austin.

1991 Prehistoric Cultural Developments on the Texas High Plains. Bulletin of the Texas Archeological Society 60(for 1989):1-55.

Hughes, Jack T., and Patrick S. Willey

1978 Archeology at Mackenzie Reservoir. Office of the State Archeologist Survey Report 24. Texas Historical Commission, Austin.

Hume, Gary L., Marsha F. Jackson, and Roberta Speer

1973 National Register Nomination Form for Mayfield Dugout (41BI52). On file, Texas Historical Commission, Austin.

Hunt, Charles B.

1972 Geology of Soils: Their Evolution, Classification, and Uses. W. H. Freeman and Company, San Francisco.

Hutchins, Charles Robert

1968 Vascular Flora of Garza County, Texas. Unpublished Master's thesis, Biology 
Department, Texas Technology College, Lubbock.

Ingold, Tim

1983 The Significance of Storage in Hunting Societies. Man 18:553-571.

Israel, Fred L.

19761897 Sears Roebuck Catalogue. Chelsea House Publishers, New York.

Jackson, Marsha F.

1975 The George Jowell Ranch Complex: A Report on the Preliminary Testing and Evaluation of the Archeological and Architectural Features. Office of the State Archeologist Report No. 27. Texas Historical Commission, Austin.

Jelinek, Arthur J.

1967 A Prehistoric Sequence in the Middle Pecos Valley, New Mexico. Anthropological Papers No. 31. Museum of Anthropology, University of Michigan, Ann Arbor.

Jelks, Edward B.

1962 The Kyle Site: A Stratified Central Texas Aspect Site in Hill County, Texas. Archaeology Series No. 5. Department of Anthropology, The University of Texas at Austin.

Jennings, J. D.

1940 A Variation of Southwestern Pueblo Culture. Technical Series Bulletin No. 10. Laboratory of Anthropology, University of New Mexico, Santa Fe.

Johnson, Donald L.

1989 Subsurface Stone Lines, Stone Zones, Artifact-Manuport Layers, and Biomantles Produced by Bioturbation Via Pocket Gophers (Thomomys Bottae). American Antiquity 54(2):370-389.

Johnson, Eileen

1987 Lubbock Lake: Late Quaternary Studies on the Southern High Plains. Texas A\&M University Press, College Station.

Johnson, Eileen, Vance To Holliday, Michael J. Kaczor, and Robert Stuckenrath

1977 The Garza Occupation at the Lubbock Lake Site. Bulletin of the Texas Archeological Society 48:83-109.

Johnson, Jay K.

1979 Archaic Biface Manufacture: Production
Failures, a Chronicle of the Misbegotten. Lithic Technology 8(2):25-35.

1981

Further Additional Biface Production Failures. Lithic Technology 10(2-3):26-28.

Johnson, LeRoy, Jr.

1987 A Plague of Phases. Bulletin of the Texas Archeological Society 57(for 1986):1-26.

Jones, Olive, and Catherine Sullivan

1985 The Parks Canada Glass Glossary for the Description of Containers, Tableware, Flat Glass, and Closures. National Historic Parks and Sites Branch, Parks Canada, Environment Canada. Canadian Govemment Publishing Centre, Hull, Quebec.

Judd, Sharon J.

1977 Prehistoric Cültural Resources in the Central Llano Estacado and Western Rolling Plains of Texas. Unpublished Master's thesis, Department of Anthropology, Texas Tech University, Lubbock.

Katz, Susana R., and Paul Katz

1985 The Prehistory of the Carlsbad Basin, Southeastern New Mexico. Department of the Interior, Bureau of Reclamation, Southwest Regional Office, Amarillo.

Katzenberg, M. Anne, and Jane H. Kelley

1991 Stable Isotope Analysis of Prehistoric Bone from the Sierra Blanca Region of New Mexico. In Mogollon $\mathrm{V}$, edited by Jane $\mathrm{H}$. Kelley and Patrick H. Beckett, pp. 207-219. COAS Publishing and Research, Las Cruces, New Mexico.

Keeley, Lawrence $\mathrm{H}$.

1980 Experimental Determination of Stone Tool Use: A Microwear Analysis. The University of Chicago Press, Chicago.

Kehoe, Thomas R.

1966 The Small Side-Notched Point System of the Northern Plains. American Antiquity 31(6): 827-841.

Kelley, Jane Holden

1984 The Archaeology of the Sierra Blanca Region of Southeastern New Mexico. Anthropological Paper 74. University of Michigan, Museum of Anthropology, Ann Arbor.

Kelly, Robert L. 1980 Hunter-Gatherer Settlement Systems. Mas- 
ter's thesis, Department of Anthropology, University of New Mexico, Albuquerque.

1983 Hunter-Gatherer Mobility Strategies. Journal of Anthropological Research 39(3):277-306.

Kenmotsu, Ray D.

1981 An Assessment and Overview of the Flora of the Brazos Natural Salt Pollution Control Project Area. Appendix VII in A Preliminary Assessment of the Cultural Resources in the Brazos Natural Salt Pollution Control Project, Kent, King, and Stonewall Counties, Texas, by J. Peter Thurmond, Martha Doty Freeman, and Susan L. Andrews, pp. 339351. Reports of Investigations No. 18. Prewitt and Associates, Inc., Austin.

Ketchum, William C., Jr.

1983 Pottery and Porcelain. Knopf Collector's Guides to American Antiques. Alfred A. Knopf, New York.

Kirkpatrick, Zoe Merriman

1992 Wildflowers of the Western Plains: A Field Guide. University of Texas Press, Austin.

Kovel, Ralph M., and Terry H. Kovel

1986 New Dictionary of Marks. Crown Publishers, Inc., New York.

Krueger, Harold W., and Charles H. Sullivan

1984 Models for Carbon Isotope Fractionation Between Diet and Bone. In Stable Isotopes in Nutrition, edited by Judith R. Tumlund and Phyllis E. Johnson, pp. 205-220. ACS Symposium Series No. 258.

Laville, Henri, Jean-Philippe Rigaud, and James Sacket 1980 Rockshelters of the Perigord: Geological Stratigraphy and Archaeological Succession. Academic Press, New York.

LeBlanc, Steve A., and Michael E. Whalen (editors) 1980 An Archaeological Synthesis of SouthCentral and Southwestern New Mexico. Office of Contract Archaeology, University of New Mexico, Albuquerque.

Lees, William

1977 Investigation at Tx-33, Old Hardesty, Texas County, Oklahoma. Report No. 11. Archaeological Research Associates, Tulsa.

Lehmer, Donald J.

1948 The Jornada Branch of the Mogollon. University of Arizona Bulletin 19(2); Social
Science Bulletin 17. Tucson.

Lehner, Lois

1988 Lehner's Encyclopedia of U.S. Marks on Pottery, Porcelain, and Clay. Collector Books, Schroeder Publishing Company, Inc., Paducah, New York.

Leonhardy, Frank C.

1966 Test Excavations in the Mangum Reservoir Area of Southwestern Oklahoma. Contributions of the Museum of the Great Plains, No. 2. Lawton, Oklahoma.

Leslie, Robert H.

1965 The Merchant Site: L.C.A.S. E-4. Transactions of the First Regional Archeological Symposium for Southeastern New Mexico and Western Texas:23-29.

1978 Projectile Point Types and Sequence of the Eastern Jornada-Mogollon, Extreme Southeastem New Mexico. Transactions of the 13th Regional Archeological Symposium for Southeastern New Mexico and Western Texas:81-157.

1979 The Eastern Jornada Mogollon, Extreme Southeastern New Mexico (A Summary). In Jornada Mogollon Archaeology: Proceedings of the First Jornada Conference, edited by Patrick H. Beckett and Regge N. Wiseman, pp. 179-199. New Mexico State University, Cultural Resources Management Division, Las Cruces.

LeVick, Gene, and Lawrence LeVick

1966 The Case of the Aching Ulna or Would you Believe a Projectile in an Elbow? Oklahoma Anthropological Society Newsletter 14(9):910.

Lewis, Willie Newbury

1976 Between Sun and Sod: An Informal History of the Texas Panhandle. Revised edition, originally published 1938. Texas A\&M University Press, College Station.

Lintz, Christopher

1978 Flake Blank Production Strategy of the Heerwald Site Cache. Oklahoma Anthropological Society XXVII:179-206.

1982 An Overview of the Antelope Creek Focus. Transactions of the 17th Regional Archeological Symposium for Southeastern New Mexico and Western Texas:37-56. 
1986

Architecture and Community Variability within the Antelope Creek Phase of the Texas Panhandle. Studies in Oklahoma's Past No. 14. Oklahoma Archeological Survey, Norman.

1990 The Landergin Mesa: The 1984 Phase II Field Results. Draft report prepared for the Texas Historical Commission, Austin.

Lintz, Christopher, Karen Kramer, Amy Earls, W. N. Trierweiler, Terry Del Dene, John Acklen, Fred Nials, and Jack Bertram

1988 Class II Survey and Testing of Cultural Resources at the Melrose Air Force Range, Curry and Roosevelt Counties, New Mexico. Mariah Associates, Inc., Albuquerque.

Lintz, Christopher, John D. Speth, David T. Hughes, and Jeffrey A. Huebner

1991 Additional Radiocarbon Dates from the Twilla Bison Kill Site, Hall County, Texas. Bulletin of the Texas Archeological Society 60:257266.

Lintz, Christopher, Abby Treece, Fred M. Oglesby, Karl Kibler, Patrick O'Neill, W. Nicholas Trierweiler, Charles Frederick, J. Michael Quigg, and A. J. Taylor

1994 Cultural Resource Investigations in the O. H. Ivie Reservoir, Concho, Coleman, and Runnels Counties, Texas. Technical Report 346-II. Mariah Associates, Inc., Austin.

Lobeck, A. K.

1948 Physiographic Diagram of the United States. The Geographical Press, Maplewood, New Jersey.

Lorrain, Dessamae

1967 The Glass Site. In A Pilot Study of Wichita Indian Archeology and Ethnohistory, assembled by Robert E. Bell, Edward B. Jelks, and W. W. Newcomb, pp. 24-44. Final report to the National Science Foundation, Grant GS-964.

1969 An Archeologist's Guide to Nineteenth Century American Glass. Historical Archaeology 2:35-44.

Majewski, Teresita, and Michael J. O'Brien

1987 The Use and Misuse of Nineteenth-Century English and American Ceramics in Archaeological Analysis. Chapter 3 in Advances in Archaeological Method and Theory, vol. 11, edited by Michael B. Schiffer, pp. 97-209. Academic Press, Inc., San Diego.
Martin, Joseph P.

1983 An Historical Overview and Archaeological Model of Texas Cattle Ranching. Master's thesis, Department of Anthropology, University of Idaho, Moscow.

Martin, Paul S.

1979 Prehistory: Mogollon. In Southwest, edited by Alfonso Ortiz, pp. 61-74. Handbook of North American Indians, vol. 9, William C. Sturtevant, general editor. Smithsonian Institution, Washington, D.C.

Matson, R. G., and Brian Chisholm

1991 Basketmaker II Subsistence: Carbon Isotopes and Other Dietary Indicators from Cedar Mesa, Utah. American Antiquity 56(3):444459.

Matthews, William H., III :

1969 The Geologic Story of Palo Duro Canyon. Guidebook 8. Bureau of Economic Geology, The University of Texas at Austin.

Mauldin, Raymond

1991 Agricultural Intensification in the Mogollon Highlands. In Mogollon $\mathrm{V}$, edited by Jane $\mathrm{H}$. Kelley and Patrick H. Beckett, pp. 62-75. COAS Publishing and Research, Las Cruces, New Mexico.

1993 The Relationship between Groundstone and Agricultural Intensification in Western New Mexico. Kiva 58(3):317-330.

Mauldin, Raymond, and Steve A. Tomka

1988 Informe Preliminar sobre Experimentos en el Uso de Moledoras de Piedra (Khona) en la Communidad de Alota, Nor Lipez, Potosí, Bolivia. Ms. submitted to the Museo Nacional de Etnografia y Folklore, La Paz, Bolivia.

McAlester, Virginia, and Lee McAlester

1990 A Field Guide to American Houses. Alfred A. Knopf, New York.

McEachem, Michael, and Ronald W. Ralph

1980a Archeological Investigations at the Thomas F. McKinney Homestead, Travis County, Texas: An Experiment in Historical Archeology, Part I. Bulletin of the Texas Archeological Society 51:5-127.

1980b Texas Parks and Wildlife Excavations at the McKinney Homestead (41TV289): Description of House Artifacts and Recommendations 
for Architectural Reconstruction. Appendix D to Archeological Investigations at the Thomas F. McKinney Homestead, Travis County, Texas: An Experiment in Historical Archeology, Part 1 , by Michael McEachern and Ronald W. Ralph. Bulletin of the Texas Archeological Society 51:146-161.

McGowen, J. H., G. E. Granata, and S. J. Seni

1979 Depositional Framework of the Lower Dockum Group (Triassic), Texas Panhandle. Report of Investigations No. 97. Bureau of Economic Geology, The University of Texas at Austin.

McKearin, Helen, and Kenneth M. Wilson

1978 American Bottles and Flasks and Their Ancestry. Crown Publishers, Inc., New York.

McMahan, Craig A., R. G. Frye, and K. L. Brown

1984 The Vegetation Types of Texas, including Cropland. Texas Parks and Wildlife Department, Austin.

Medsger, Oliver Perry

1966 Edible Wild Plants. Collier Books, New York.

Meltzer, David J.

1991 Altithermal Archaeology and Paleoecology at Mustang Springs on the Southern High Plains of Texas. American Antiquity 56(2):236-267.

Mera, H. P.

1943 An Outline of Ceramic Developments in Southern and Southeastern New Mexico. Technical Series Bulletin 11. New Mexico Archaeological Survey, Laboratory of Anthropology, Santa Fe.

Miller, E. O., and E. B. Jelks

1952 Archeological Excavations at the Belton Reservoir, Coryell County, Texas. Bulletin of the Texas Archeological Society 23:168-217.

Miller, George L.

1980 Classification and Economic Scaling of 19th Century Ceramics. Historical Archaeology $14: 1-40$

Miller, Miles R., III, and Trace Stuart

1991 The NASA-STGT Excavations: Short-Term Mesilla Phase Settlements Along the Southern San Andres Mountain Bajada. Cultural Resources Report No. 125. Batcho and Kauffman Associates, Las Cruces.
Minnis, Paul E.

1985 Domesticating People and Plants in the Greater Southwest. In Prehistoric Food Production in North America, edited by Richard I. Ford, pp. 309-339. Anthropological Papers No. 75. Museum of Anthropology, University of Michigan, Ann Arbor.

Morris, Donald H.

1990 Changes in Groundstone Following the Introduction of Maize into the American Southwest. Journal of Anthropological Research 46:177-194.

Murdock, G. P.

1967 Ethnographic Atlas. University of Pittsburg Press, Pittsburg, Pennsylvania.

Muto, Guy R.

1971 A Technological Analysis of the Early Stages in the Manufacture of Lithic Artifacts. Unpublished Master's thesis, Idaho State University, Pocatello.

Nelson, Lee H.

1968 Nail Chronology: An Aid to Dating Old Buildings. Technical Leaflet 48 of the American Association for the Advancement of State and Local History, Nashville (or) History News 24(11):detachable leaflet.

New Mexico Geological Society

1982 New Mexico Highway Geologic Map. New Mexico Geologic Society, New Mexico Bureau of Mines and Mineral Resources, Socorro.

Newton, Richard E., and Steadman Upham

1988 Projectile Points as Chronological Markers?: A Reassessment of Morphological Attributes as Time-sensitive Indicators. In Fourth Jornada Mogollon Conference (Oct. 1985) Collected Papers, edited by Meliha S. Duran and Karl W. Laumbach, pp. 93-105. Human Systems Research, Inc., Tularosa, New Mexico.

Odell, George H., and Frank Cowan

1986 Experiments with Spears and Arrows on Animal Targets. Journal of Field Archaeology 13(2):195-212.

Oklahoma Archeological Survey

1993 Radiocarbon Dates for RM-668. Oklahoma Archeological Survey Newsletter 12(3). 
O'Laughlin, Thomas C.

1979 Excavations at the Transmountain Campus, El Paso Community College, El Paso, Texas. El Paso Centennial Museum Publications in Anthropology No. 7. The University of Texas at El Paso.

1980 The Keystone Dam Site and Other Archaic and Formative Sites in Northwest El Paso, Texas. El Paso Centennial Museum Publications in Anthropology No. 8. The University of Texas at El Paso.

1993 The Long and Short of It: Jomada Pueblo Occupational History. Paper presented at the Jornada Mogollon Conference VIII, March 18-21, 1993, Tularosa, New Mexico.

O'Neill, Patrick L.

1993 Archival Documentation. Appendix $J$ in Historic and Prehistoric Data Recovery at Palo Duro Reservoir, Hansford County, Texas, by J. Michael Quigg, Christopher Lintz, Amy C. Earls, Charles D. Frederick, W. Nicholas Trierweiler, Douglas Owsley, and Karl W. Kibler, pp. J1-J11. Technical Report No. 485. Mariah and Associates, Inc., Austin.

Owsley, Douglas W.

1989 The History of Bioarcheological Research in the Southern Great Plains. Chapter 10 in From Clovis to Comanchero: Archeological Overview of the Southern Great Plains, by Jack L. Hofman, Robert L. Brooks, Joe S. Hays, Douglas W. Owsley, Richard L. Jantz, Murray K. Marks, and Mary H. Manhein, pp. 123-136. Research Series No. 35. Arkansas Archeological Survey, Fayetteville.

Parker, W. B.

1984 Notes Taken during the Expedition Commanded by Capt. R. B. Marcy, U.S.A., through Unexplored Texas, in the Summer of 1854. Texas State Historical Association, Austin.

Pass, Fred

1981 Texas Almanac and State Industrial Guide, 1982-1983. A. H. Belo, Dallas.

Pennington, Campbell W.

1963 The Tarahumar of Mexico. University of Utah Press, Salt Lake City.

1969 The Tepehuan of Chihuahua: Their Material Culture. University of Utah Press, Salt Lake City.
Perttula, Timothy K.

1992 The Caddo Nation: Archaeological and Ethnohistoric Perspectives. University of Texas Press, Austin.

Perttula, Timothy K., and James E. Bruseth

1983 Early Caddoan Subsistence Strategies, Sabine River Basin, East Texas. Plains Anthropologist 28(99):9-21.

Pool, Juliann C.

1987 Fanthorp Inn: A Study of Nineteenth and Twentieth Century Buttons. Appendix V in Archeological Excavations at Fanthorp Inn State Historic Site (41GM79), Grimes County, Texas: Spring and Fall 1982, edited by J. David Ing and John Hart, pp. 277-293. Texas Parks and Wildlife Department, Historic Sites and Restoration Branch, Austin.

Post Dispatch, The

Pough, Frederick $\mathrm{H}$.

1960 A Field Guide to Rocks and Minerals. The Peterson Field Guide Series. Houghton Mifflin Company, Boston.

Prikryl, Daniel J.

1990 Lower Elm Fork Prehistory: A Redefinition of Cultural Concepts and Chronologies along the Trinity River, North-Central Texas. Office of the State Archeologist Report 37. Texas Historical Commission, Austin.

Prinz, Martin, George Harlow, and Joseph Peters (editors) 1978 Simon and Schuster's Guide to Rocks and Minerals. Simon and Schuster, New York.

Quigg, J. Michael, Christopher Lintz, Fred M. Oglesby, and Abby C. Treese

1993 The Testing Phase at Mitchell Reservoir, Mitchell County, Texas. Technical Report 820. Mariah Associates, Inc., Austin.

Raisz, Erwin

1957 Landforms of the United States. Map, 6th revised edition, to accompany Atwood's "Physiographic Provinces of North America."

Ray, Cyrus N.

1929 A Differentiation of the Prehistoric Cultures of the Abilene Section. Bulletin of the Texas Archeological and Paleontological Society 7:70-88.

1933 Multiple Burials in Stone Cist Mounds of the Abilene Region. Bulletin of the Texas 
Archeological and Paleontological Society 3:14-24, and 32 (Plate 8).

Ray, Cyrus N., and E. B. Sayles

1941 An Agreement on Abilene Region Terminology. Bulletin of the Texas Archeological and Paleontological Society 13:175-176.

Raymer, Leslie E.

1988 The Form and Function of Subterranean Food Storage Structures: A Cross-Cultural Study of the Conditions Affecting Pit Storage in the Ethnographic Records. Paper presented at the symposium "The Economics of Storage: Developing an Interpretive Framework for Archaeology" at the 53rd Annual Meeting of the Society for American Archaeology, April 29, 1988, Phoenix, Arizona.

Richardson, Wayne E., Darrell G. Grice, and Lee A. Putnam 1965 Soil Survey of Garza County, Texas! United States Department of Agriculture, Soil Conservation Service, in cooperation with the Texas Agricultural Experiment Station.

Rock, James T,

1984 Cans in the Countryside. Historical Archaeology 18(2):97-111.

Rowell, Chester Momison

1967 Vascular Plants of the Texas Panhandle and South Plains. Unpublished Ph.D. dissertation, Oklahoma State University, Stillwater.

Runkles, Frank A.

1964 The Garza Site: A Neo-American Campsite near Post, Texas. Bulletin of the Texas Archeological Society 35:101-125.

Runyan, John W., and John A. Hedrick

1973 Pottery Types of the SWFAS Area. Transactions of the 8th Regional Archeological Symposium for Southwestern New Mexico and Western Texas:19-45.

1987 Pottery Types of the Southwest Federation of Archaeological Societies (SWFAS) Area. The Artifact 25(4):23-59.

Salinas, Martín

1990 Indians of the Rio Grande Delta: Their Role in the History of Southern Texas and Northeastern Mexico. University of Texas Press, Austin.

Sayles, E. B.

1935 An Archaeological Survey of Texas.
Medallion Papers 17. Gila Pueblo, Globe, Arizona.

Schlanger, Sarah H.

1990 Artifact Assemblage Composition and Site Occupation Duration. In Perspectives on Southwestern Prehistory, edited by Paul E. Minnis and C. L. Redman, pp. 103-121. Westview Press, Boulder, Colorado.

1991 On Manos, Metates, and the History of Site Occupations. American Antiquity 56(3):460 474.

Schroeder, Joseph J., Jr. (editor)

1971 Sears, Roebuck and Co. 1908 Catalogue No. 117: The Great Price Maker. Reprinted by Digest Books, Inc., Northfield, Illinois.

Schroedl, Gerald F.

1980 Structures and Village Pattern at the Historic Overhill Cherokee Towns of Chota and Tanasee. Paper presented at the 37th annual meeting of the Southeastem Archaeological Conference, New Orleans.

Schurr, Mark R.

1992 Isotopic and Mortuary Variability in a Middle Mississippian Population. American Antiquity 57(2):300-320.

Schwarcz, Henry P., F. Jerome Melbye, M. Anne Katzenberg, and Martin Knyf

1985 Stable Isotopes in Human Skeletons of Southem Ontario: Reconstructing Paleodiet. Journal of Archaeological Sciences 12:187206.

Shiner, Joel L.

1975 The Clear Fork Gouge Revisited. Bulletin of the Texas Archeological Society 46:179-188.

Sonnichsen, C. L.

1958 The Mescalero Apaches. University of Oklahoma Press, Norman.

South, Stanley

1977 Method and Theory in Historical Archeology. Academic Press, New York.

Spivey, Towana

1979 A Historical Guide to Wagon Hardware and Blacksmith Supplies. Contributions of the Museum of the Great Plains No. 9. Lawton, Oklahoma. 
Steely, James Wright

1984 A Catalog of Texas Properties in the National Register of Historic Places. Texas Historical Commission, Austin.

Stein, Julie K.

1992 Organic Matter in Archaeological Contexts. In Soils in Archaeology: Landscape Evolution and Human Occupation, edited by Vance T. Holliday, pp. 193-216. Smithsonian Institution Press, Washington, D.C.

Stewart-Abernathy, Leslie C.

1986 The Moser Farmstead - Independent but Not Isolated: The Archeology of a Late Nineteenth Century Ozark Farmstead. Research Series No. 26. Arkansas Archeological Survey, Fayetteville.

Stickney, Francis, and Teddy Stickney

1984 Crane County: Sites 41CR'1-41CR9. Transactions of the 19th Regional Archeological Symposium for Southeastern New Mexico and Western Texas:37-73.

Stuiver, Minze, and Gordon W. Pearson

1986 High-Precision Calibration of the Radiocarbon Time Scale, A.D. 1900-500 B.C. Radiocarbon 28(2B):805-838.

Stuiver, Minze, and Paula J. Reimer

1986 A Computer Program for Radiocarbon Age Calibration. Radiocarbon 28(2B):1022-1030.

Suhm, Dee Ann, and Edward B. Jelks

1962 Handbook of Texas Archeology: Type Descriptions. Texas Memorial Museum Bulletin No. 4. Austin.

Suhm, Dee Ann, Alex D. Krieger, and Edward B. Jelks 1954 An Introductory Handbook of Texas Archeology. Bulletin of the Texas Archeological Society 25.

Taylor, James W.

1987 Oklahoma's Oldest Arrowpoint? Salvage of an Ancient Hearth at the Canyon Road Site (34CN46), Canadian County. Oklahoma Anthropological Society Newsletter 35(7):79.

Texas. General Land Office

1936 Garza County. File 58825. Abstract 928. H\&GN Railway Company Certificate No. 12/2415. Patented to T. E. Payne, Assignee, November 14, 1936.
1939 Garza County. File 59132. Abstract 903. H\&GN Railway Company Certificate No. 12/2412. Patented to Jeff Justice, Assignee, August 16, 1939.

Texas Highway Department

1941 Areal Photo Series Taken October 17, 1941. Texas Highway Department, Highway Planning Survey, Garza County 86. Austin.

Thompson, Jerome L.

1987 Modern, Historic, and Fossil Flora. Chapter 4 in Lubbock Lake: Late Quaternary Studies on the Southern High Plains, edited by Eileen Johnson, pp. 26-35. Texas A\&M University Press, College Station.

Tomka, Steve A.

1986 Biface Manufacture Failures. In Intensive Excavations of the State Highway 71 Rightof-Way, 41BP19, Bastrop County, Texas, by Leland C. Bement, pp. 110-129. Ms. on file, Texas Archeological Research Laboratory, The University of Texas at Austin.

Tomka, Steve A., and Ross C. Fields

1990 Chipped Stone Artifacts, 41LN29A and 41LN106. Chapter 9 in Excavations at the Charles Cox, Lambs Creek Knoll, and Buffalo Branch Sites, Jewett Mine Project, Leon and Freestone Counties, Texas, edited by Ross C. Fields, pp. 141-287. Reports of Investigations No. 70, vol. I. Prewitt and Associates, Inc., Austin.

Toulouse, Julian Harrison

1971 Bottle Makers and Their Marks. Thomas Nelson, Inc., New York.

Tringham, R., G. Cooper, G. Odell, B. Voytek, and A. Whitman

1974 Experimentation in the Formation of Edge Damage: A New Approach to Lithic Analysis. Journal of Field Archaeology 1:171-196.

Tunnell, Curtis, and Enrique Madrid

1990 Making and Taking Sotol in Chihuahua and Texas. In Papers from the Third Symposium on Resources of the Chihuahuan Desert Region, United States and Mexico, 10-12 November 1988, edited by A. Michael Powell, Robert R. Hollander, Jon C. Barlow, W. Bruce McGillivray, and David A. Schmidly, pp. 145-162. Chihuahuan Desert Research Institute, Alpine, Texas. 
Tumer, Ellen Sue, and Thomas R. Hester

1985 A Field Guide to Stone Artifacts of Texas Indians. Texas Monthly Press, Austin.

Vehik, Susan C.

1984 The Woodland Occupations. Chapter 8 in Prehistory of Oklahoma, edited by Robert E. Bell, pp. 175-197. Academic Press, Orlando.

Vestal, Paul A., and Richard Evans Schultes

1939 The Economic Botany of the Kiowa Indians as it Relates to the History of the Tribe. Botanical Museum, Harvard University, Cambridge, Massachusetts.

Vines, Robert A.

1960 Trees, Shrubs, and Woody Vines of the Southwest. University of Texas Press, Austin.

1982 Trees of North Texas. University of Texas Press, Austin.

Walker, Danny N.

1980 The Garnsey Site Canid. In Late Prehistoric Bison Procurement in Southeastern New Mexico: The 1978 Season at the Garnsey Site (LA 18399), edited by John D. Speth and William J. Parry, pp. 344-369. Technical Reports No. 7, Research Reports in Archaeology Contribution 7. Museum of Anthropology, University of Michigan, Ann Arbor.

Walker, D. N., and G. C. Frison

1979 The Continual Domestication of Wolf/Dog Hybrids on the Northwestern Plains. Paper presented at the 44th Annual Meeting of the Society for American Archaeology.

Wallace, Ernest (editor)

1978 The Journal of Ranald S. Mackenzie's Messenger to the Kwahadi Comanches. Red River Valley Historical Review 3(2):227-246.

Wallace, Emest, and E. Adamson Hoebel

1986 The Comanches: Lords of the South Plains. University of Oklahoma Press, Norman.

Walter, Emest G.

1987 The Gowan Site: Cultural Adaptation on the Northern Plains During the Altithermal Period. In Man and the Mid-Holocene Climatic Optimum: Proceedings of the Seventeenth Annual Conference of the Archaeological Association of the University of Calgary, edited by Neil A. McKinnon and Glen S. L. Stewart, pp. 111-122. University of Calgary Archaeological Association, Calgary, Alberta.
Ward, Albert E., Emily K. Abbink, and John R. Stein

1977 Ethnohistorical and Chronological Basis of the Navaho Material Culture. Chapter 9 in Settlement and Subsistence along the Lower Chaco River: The CGP Survey, edited by Charles A. Reher, pp. 217-278. Office of Contract Archeology, University of New Mexico, Albuquerque.

Ward, H. Trawick

1985 Social Implications of Storage and Disposal Pattems. Chapter 4 in Structure and Process in Southeastern Archaeology, edited by Roy S. Dickens and H. Trawick Ward, pp. 82101. University of Alabama Press, University.

Waters, Michael R.

1992 Principles of Geoarchaeology: A North American Perspective. The University of Arizona Press, Tucson.

Watts, W. C.

1972 Token and Multi-notched Points of the South Plains of Texas. Transactions of the Seventh Regional Archeological Symposium for Southeastern New Mexico and Western Texas:21-37.

Webb, Walter Prescott (editor)

1952 The Handbook of Texas, vol. 2. Texas State Historical Association, Austin.

Wedel, Waldo R.

1975 Chalk Hollow: Culture Sequence and Chronology in the Texas Panhandle. Actas del $X L I$ Congreso Internacional de Americanistas 1:271-278. Mexico, D.F.

1986 Central Plains Prehistory: Holocene Environments and Culture Change in the Republican River Basin. University of Nebraska Press, Lincoln.

Weltfish, Gene

1965 The Lost Universe. Basic Books, New York.

Wetherbee, Joan

1985 A Second Look at White Ironstone. Wallace-Homestead Book Co., Lombard, Illinois.

Whalen, Michael E.

1977 Settlement Patterns of the Eastern Hueco Bolson. El Paso Centennial Museum Publications in Anthropology No. 4. The University of Texas at El Paso. 
1978 Settlement Patterns of the Western Hueco Bolson. El Paso Centennial Museum Publications in Anthropology No. 6. The University of Texas at El Paso.

1980a The Pithouse Periods of South-Central New Mexico. Chapter 8 in An Archeological Synthesis of South-Central and Southwestern New Mexico, edited by Steven A. LeBlanc and Michael E. Whalen, pp. 318-386. Office of Contract Archaeology, University of New Mexico, Albuquerque.

1980b The Pueblo Periods of South-Central New Mexico. Chapter 9 in An Archaeological Synthesis of South-Central and Southwestern New Mexico, edited by Steven A. LeBlanc and Michael E. Whalen, pp. 388-448. Office of Contract Archaeology, University of New Mexico, Albuquerque.

1981 Origin and Evolution of Ceramics in Western Texas. Bulletin of the Texas Archeological Society 52:215-229.

1985 Chronological Studies in the Jomada Area. In Views of the Jornada Mogollon: Proceedings of the Second Jornada Mogollon Archaeology Conference, edited by Colleen Beck, pp. 136-142. Eastem New Mexico University Press, Portales.

Whalen, Michael E., and Patricia A. Gilman

1990 Introduction: Transitions to Sedentism. In Perspectives on Southwestern Prehistory, edited by Paul E. Minnis and Charles L. Redman, pp. 71-75. Westview Press, Boulder, Colorado.

Willey, Patrick S., and Jack T. Hughes

1978 The Deadman's Shelter Site. In Archeology at Mackenzie Reservoir, edited by Jack T. Hughes and Patrick S. Willey, pp. 149-190. Archeological Survey Report No. 24. Texas Historical Commission, Austin.

Williams, Joe, and John W. Clark

1976 National Register Nomination Form for Post West Dugout. On file, Texas Historical Commission, Austin.

Wilson, Emest W.

1930 Bumed Rock Mounds of Southwest Texas. Bulletin of the Texas Archeological and Paleontological Society 2:59-63.
Wilson, Gilbert L.

1987 Buffalo Bird Woman's Garden: Agriculture of the Hidatsa Indians. Minnesota Historical Society Press, St. Paul. Originally published in 1917 as Agriculture of the Hidatsa Indians: An Indian Interpretation by the University of Minnesota Press, Minneapolis.

Wilson, Jack H., Jr.

1985 Feature Zones and Feature Fill: More than Trash. Chapter 3 in Structure and Process in Southeastern Archaeology, edited by Roy S. Dickens and H. Trawick Ward, pp. 60-81. University of Alabama Press, University.

Wilson, Rex L.

1981 Bottles on the Western Frontier. University of Arizona Press, Tucson.

Wiseman, Regge N.

1981 Further Investigations at the King Ranch Site, Chaves County, New Mexico. The Artifact 19(3 and 4):169-198.

1988 The Continuing Saga of the King Ranch Site (LA 26764): Update and Summaries of Findings. In Fourth Jornada Mogollon Conference (Oct. 1985) Collected Papers, edited by Meliha S. Duran and Karl W. Laumbach, pp. 223-254. Human Systems Research, Inc., Tularosa, New Mexico.

1991 The Bent Project: An Archaeological Excavation at the Bent Site (LA 10835), Otero County, Southern New Mexico. COAS Publishing and Research, Las Cruces.

1993 Comments on pottery from the Sam Wahl site, 41GR291, Justiceburg Reservoir, Garza and Kent Counties, Texas. Letter to Doug Boyd dated May 15, 1993. On file, Prewitt and Associates, Inc., Austin.

Witte, Adolph Henry

1955 A Double Indian Burial from Donley County, Texas. Panhandle-Plains Historical Review 28:82-86.

Women's Division of Post Chamber of Commerce (compiler and editor)

n.d. Foot Prints: A History of Garza County and Its People. Taylor Publishing Company, n.p.

Wood, Raymond

1968 Mississippian Hunting and Butchering Patterns: Bones from the Vista Shelter, 
23SR20, Missouri. American Antiquity 33(2):170-179.

Woodall, J. Ned

1967 The Coyote Site. In A Pilot Study of Wichita Indian Archeology and Ethnohistory, assembled by Robert E. Bell, Edward B. Jelks, and W. W. Newcomb, pp. 15-23. Final report to the National Science Foundation, Grant GS-964.

Woodbury, Richard A., and Ezra B. W. Zubrow

1979 Agricultural Beginnings, 2000 B.C.A.D. 500. In Southwest, edited by Alfonso Ortiz, pp. 43-60. Handbook of North American Indians, vol. 9, William C. Sturtevant, general editor. Smithsonian Institution, Washington, D.C.

Wulfkuhle, Virginia A.

1986 Investigations into the Prehistory of the Upper Clear Fork of the Brazos River, Fisher and Jones Counties, Texas. Master's thesis, The University of Texas at Austin.
Yerkes, Richard W.

1987 Prehistoric Life on the Mississippi Floodplain: Stone Tool Use, Settlement Organization, and Subsistence Practices at the Labras Lake Site, Illinois. University of Chicago Press, Chicago.

York, John C., and William A. Dick-Peddie

1969 Vegetation Changes in Southern New Mexico during the Past Hundred Years. In Arid Lands in Perspective, edited by W. B. McGinnies and B. J. Goldman, pp. 157-166. AAAG, University of Arizona Press, Tucson.

Young, Stanley P.

1944 Their History, Habits, Economic Status, and Control. Part I in The Wolves of North America, pp. 1-389. American Wildlife Institute, Washington.

Young, Wayne C.

1978 Archaeological Testing in the Camp Creek Locale, Payne and Pawnee Counties, Oklahoma. Project Report Series 1. Archaeological Research and Management Center, University of Oklahoma, Norman. 


\section{APPENDIX A: Geomorphic Profile Descriptions for 41GR291, 41GR303B, 41GR546, and 41GR559}

Karl W. Kibler 

The geomorphic profiles are described and classified according to the procedures and criteria presented by Buol et al. (1980:21-43), Birkeland (1984), and Bettis (1984). The color (Munsell Soil Color Chart) and consistency (loose, very friable, friable, firm, very firm, and extremely firm) of a zone or sediment are recorded from a moist condition. Field definitions of texture consist of (1) sand (loose, single-grained, moist cast will crumble); (2) sandy loam (mostly sand with enough silt and clay to be somewhat coherent, individual sand grains are visible, moist cast bears careful handling); (3) loam (even mixture of sand, silt, and clay, gritty yet fairly smooth and slightly plastic, moist cast handles freely); (4) silt loam (predominantly silt with small amounts of fine sand and clay, moist cast bears heavy handling but will not ribbon); (5) clay loam (predominantly clay with small amounts of fine sand and silt, moist ribbon breaks easily, moist cast bears heavy handling, slightly plastic, kneaded heavy compact mass will not crumble); and (6) clay (very plastic and sticky when wet, flexible ribbon). The terms sandy clay, sandy clay loam, loamy sand, silty clay, silty clay loam, and silt loam are used when the texture of a zone could not be confidently placed into one of the above categories. The structure or soil aggregation of a zone or horizon is described by grade, size, and type. The grade is shown as weak, moderate, or strong. The size of the peds is shown as fine, medium, or coarse, depending on ped morphology or type. The type, referring to the shape of the peds, is identified as blocky (subangular and angular), platy, prismatic, columnar, or granular. Soil horizons not containing these characteristics are considered structureless. Final soil horizon classifications were made based on the terminology and criteria presented by Birkeland (1984) and Bettis (1984).

In the absence of soil formation, the sedimentary structures of a zone are presented. Types of sedimentary structures include, but are not limited to, planar laminations, graded beds, cross-stratifications, trough cross-stratifications, ripples, climbing ripples, and massive structures.

Mottles are described by color, abundance, contrast, and size. Abundance is shown as few $(<2 \%)$, common $(2-20 \%)$, and many $(>20 \%)$, while contrast is described as' faint, distinct, or prominent. Size ranges are given as fine $(<0.5 \mathrm{~cm})$, medium $(0.5-1.5 \mathrm{~cm})$, or coarse $(>1.5 \mathrm{~cm})$. Terms pertaining to abundance also are used to describe the occurrence of inclusions or intrusive objects, such as gravels and charcoal. The lower boundary of each zone or horizon is described in terms of distinctiveness - very abrupt $(<0.1 \mathrm{~cm})$, abrupt $(0.1-2.5 \mathrm{~cm})$, clear $(2.5-$ $6.4 \mathrm{~cm})$, gradual $(6.4-12.7 \mathrm{~cm})$, and diffused $(>12.7 \mathrm{~cm})$ - and topography — smooth, wavy, irregular, and broken. 
Zone

Depth (cm)

Description

41GR291

EU 83, South Wall

$1 \quad 0-20$

Brown to dark brown (10YR 4/3) friable fine sandy loam, common rootlets, few sandstone gravels, few charcoal flecks, moderate medium subangular blocky structure, clear smooth lower boundary, A horizon.

2

$20-31$

3

$31-36+$

2

19-33

3

$33-53$

4

$53-60+$

\section{EU 95, South Wall}

$1 \quad 0-7$

2

$7-22$
Brown to dark brown (10YR 4/3) friable fine sandy loam, common rootlets, common sandstone gravels, few insect burrows, moderate medium subangular blocky structure, clear to gradual smooth lower boundary, A horizon.

Brown to dark brown (10YR 4/3) friable fine sandy loam, common rootlets, common charcoal flecks, few insect burrows, few rodent burrows, common sandstone gravels and pebbles, moderate medium subangular blocky structure, clear smooth lower boundary, AB horizon.

Yellowish brown (10YR 5/4) friable sandy clay loam, few rootlets, common rodent burrows, common sandstone gravels, few manganese nodules, many voids and cracks are filled with illuvial clays, moderate to strong medium subangular blocky structure, abrupt wavy lower boundary, Bt horizon.

Light yellowish brown (10YR 6/4) friable loam, few rootlets, few rodent burrows, many sandstone gravels, massive, lower boundary not observed, $\mathrm{Cr}$ horizon.

Brown to dark brown (10YR 4/3) friable fine sandy loam, common rootlets, common insect burrows, structureless, few sandstone gravels, clear smooth lower boundary, A horizon.

Brown to dark brown (10YR 4/3) friable fine sandy loam, few rootlets, common sandstone gravels and pebbles, few insect burrows, moderate medium subangular blocky structure, clear smooth lower boundary, $\mathrm{AB}$ horizon. 


\begin{abstract}
Zone Depth (cm)
Description

$3 \quad 22-33+$

Yellowish brown (10YR 5/4) to dark yellowish brown (10YR 4/4) friable sandy clay loam, common sandstone gravels, few rodent burrows, common to many illuvial clays, moderate medium subangular blocky structure, lower boundary not observed, Bt horizon.
\end{abstract}

\title{
Backhoe Trench 1992-1, East End of South Wall
}

$1 \quad 0-7$

Yellowish brown (10YR 5/4) friable fine sandy loam, common rootlets, common insect burrows, weak planar-laminated beds, few sandstone gravels, abrupt smooth lower boundary, $\mathrm{Cu}$ horizon.

$2 \quad 7-22$

Yellowish brown (10YR 5/4) friable fine sandy loam, common rootlets, common sandstone gravels, common insect burrows, few small vertical cracks, one rodent burrow at $21 \mathrm{~cm}$ filled with many sandstone gravels, moderate medium to coarse subangular blocky structure, abrupt wavy lower boundary, $2 \mathrm{Ab}$ horizon.

$3 \quad 22-47$

Strong brown (7.5YR 4/6) friable sandy clay loam, few rootlets, few hackberry seeds, common insect burrows, few manganese nodules, common rodent burrows, common sandstone gravels, few carbonized plant remains, strong coarse subangular blocky structure, abrupt wavy lower boundary, $2 \mathrm{Bt}$ horizon.

$4 \quad 47-65$

Light yellowish brown (10YR 6/4) friable sandy clay loam to fine sandy loam, many sandstone gravels and pebbles, massive, clear wavy lower boundary, $2 \mathrm{Cr}$ horizon.

$5 \quad 65-140+\quad$ Triassic Dockum Group sandstones and mudstones, R horizon.

\section{Backhoe Trench 1992-3, North End of East Wall}

$1 \quad 0-49$

$2 \quad 49-84$

$3 \quad 84-109$

$4 \quad 109-156+$
Dark yellowish brown (10YR 4/4) friable fine sandy loam, common rootlets, few sandstone gravels, common insect burrows, few manganese nodules, moderate medium subangular blocky structure, gradual smooth lower boundary, A horizon.

Strong brown (7.5YR 4/6) friable fine sandy loam, common roots and rootlets, common sandstone gravels, common insect burrows, few hackberry seeds, few siliceous gravels, moderate medium subangular blocky structure, gradual smooth lower boundary, Bt horizon.

Strong brown (7.5YR 4/6) friable fine sandy loam, few charcoal chunks at $91 \mathrm{~cm}$, few charcoal flecks, common roots and rootlets, common sandstone gravels and pebbles, few siliceous gravels and pebbles, weak fine subangular blocky structure, clear wavy lower boundary, Bw horizon.

Brownish yellow (10YR 6/6) very friable to friable fine sandy loam to loamy sand, few rootlets, common sandstone gravels, few siliceous gravels, massive, common prominent coarse mottles $(2.5 \mathrm{Y} 8 / 4)$, lower boundary not observed, $\mathrm{Cr}$ horizon. 
Zone Depth (cm) Description

Backhoe Trench 1992-3, South End of West Wall

$1 \quad 0-23$

Yellowish brown (10YR 5/4) friable fine sandy loam, common roots and rootlets, few charcoal flecks, common sandstone gravels, common insect burrows, weak fine subangular blocky structure, clear smooth lower boundary, AC horizon.

2 23-35 Light yellowish brown (10YR 6/4) friable sandy clay loam, common rootlets, common to many sandstone gravels, few insect burrows, massive, abrupt wavy lower boundary, $\mathrm{Cr}$ horizon.

$3 \quad 35-80+\quad$ Triassic Dockum Group sandstone, weathered and decomposing, R horizon.

\section{Backhoe Trench 1992-4, Middle of North Wall}

$1 \quad 0-40$

$2 \quad 40-72$

$3 \quad 72-81$

$4 \quad 81-97+$
Dark yellowish brown (10YR 4/4) very friable fine sandy loam, common rootlets, fẹ insect burrows, few sandstone gravels; common charoal flecks, moderate medium to coarse subangular blocky structure, clear smooth lower boundary, A horizon.

Brown to dark brown (7.5YR 4/4) friable sandy clay loam, common rootlets, few insect burrows, few sandstone gravels, few charcoal flecks, few manganese nodules, common distinct fine mottles (10YR 4/4), moderate medium subangular blocky structure, abrupt smooth lower boundary, $\mathrm{Bt}$ horizon.

Yellowish brown (10YR 5/4) friable to firm loam, many sandstone gravels, common rootlets, few insect burrows, massive, clear smooth lower boundary, $\mathrm{Cu}$ horizon.

Very pale brown (10YR 7/4) very friable gravelly sandy clay loam, massive, lower boundary not observed, $\mathrm{Cr}$ horizon.

\section{GR303B}

\section{EU 1, East Wall}

$1 \quad 0-16$

$2 \quad 16+$

\section{EU 6, West Wall}

$1 \quad 0-68$
Brown to dark brown (10YR 4/3) friable fine sandy loam, common rootlets, few sandstone gravels, structureless, few weak fine planar laminations, abrupt wavy lower boundary, AC horizon.

Triassic Dockum Group sandstone, $\mathrm{R}$ horizon.

Reddish brown (5YR 4/3) friable fine sandy loam, common rootlets, one rodent burrow, many sandstone gravels and pebbles, common insect burrows, weak fine blocky structure, clear smooth lower boundary, A horizon. 
Zone

$$
\text { Depth (cm) }
$$

2

$68-114$

3

$114+$

EU 27. West Wall

1

$0-62$

2

$62-125$

3

$125+$

EU 32, West Wall

$1 \quad 0-48$

$2 \quad 48-81$

3

$81+$

EU 35, North Wall

$1 \quad 0-35$

$2 \quad 35-68$

3

$68+$

\section{Description}

Yellowish red (5YR 4/6) firm fine sandy loam, few rootlets, few rodent burrows, many sandstone gravels and pebbles, moderate medium blocky structure, many fine $\mathrm{CaCO}_{3}$ filaments, abrupt wavy lower boundary, Bwk horizon.

Triassic Dockum Group sandstone, $\mathrm{R}$ horizon.

Brown to dark brown (10YR 4/3) friable fine sandy loam, common rootlets, common humic materials, many sandstone gravels and pebbles, few rodent burrows, structureless, gradual smooth lower boundary, A horizon.

Brown to dark brown (7.5YR 4/4) friable fine sandy loam, common rootlets, many sandstone gravels and pebbles, common $\mathrm{CaCO}_{3}$ filaments, few rodent burrows, moderate medium blocky structure, abrupt wavy lower boundary, Bwk horizon.

Triassic Dockum Group sandstone, $\mathbf{R}$ horizon.

Brown to dark brown (10YR 4/3) friable fine sandy loam, common charcoal flecks, many sandstone gravels and pebbles, common rootlets, common insect burrows, sandstone cobble pavement at $25 \mathrm{~cm}$, weak fine planar laminations ( $5 \mathrm{~cm}$ thick) above and below pavement, weak fine blocky structure, gradual smooth lower boundary, A horizon.

Strong brown (7.5YR 4/6) friable fine sandy loam, common rootlets, many sandstone gravels and pebbles, common $\mathrm{CaCO}_{3}$ filaments, moderate medium blocky structure, abrupt smooth lower boundary, Bwk horizon.

Triassic Dockum Group sandstone, $\mathrm{R}$ horizon.

Brown to dark brown (10YR 4/3) friable fine sandy loam, common rootlets, common humic materials, many sandstone gravels and pebbles, few rodent burrows, structureless, gradual smooth lower boundary, A horizon.

Strong brown (7.5 YR 4/6) friable fine sandy loam, common roots and rootlets, many sandstone gravels and pebbles, few rodent burrows, few $\mathrm{CaCO}_{3}$ filaments, moderate medium blocky structure, abrupt wavy lower boundary, Bw horizon.

Triassic Dockum Group sandstone, R horizon. 
Phase III Data Recovery, Season 3, Lake Alan Henry

Zone Depth (cm) Description

41GR546

EU 3, North Wall

$1 \quad 0-38$

$2 \quad 38-51$

3

51-62+ Triassic Dockum Group sandstone, R horizon.

Light yellowish brown (10YR 6/4) friable fine sandy loam, massive, common rootlets, many gravels and pebbles, common rodent burrows, clear smooth lower boundary, $\mathrm{Cu}$ horizon.

Light yellowish brown (2.5Y 6/4) friable loamy sand, massive, few roots and rootlets, common rodent burrows, many gravels, pebbles, and cobbles, few boulders, clear wavy lower boundary, Cu horizon.

\section{GR559}

EU 11, North Wall

$1 \quad 0-44$

$2 \quad 44-67$

Brown to dark brown (10YR 4/3) friable fine sandy loam, massive, few bone fragments (bird?), common insect burrows, common rootlets, common to many gravels and pebbles that increase in number down-profile, gradual smooth lower boundary, $\mathrm{Cu}$ horizon.

Brown to dark brown (10YR 4/3) friable fine sandy loam, massive, many gravels, common rootlets, few rodent burrows, abrupt broken lower boundary, $\mathrm{Cu}$ horizon.

$3 \quad 67-80+$

Triassic Dockum Group sandstone, R horizon.

\section{EU 20, North Wall}

$1 \quad 0-6$

Brown to dark brown (10YR 4/3) friable fine sandy loam, fine weak planar laminated beds, common humic materials, few rootlets, few gravels, abrupt wavy lower boundary, AC horizon.

$2 \quad 6-9$

Brown to dark brown (10YR 4/3) friable fine sandy loam, massive, few rootlets, few gravels and pebbles, few insect burrows, common charcoal flecks, abrupt wavy lower boundary, $\mathrm{Cu}$ horizon.

$3 \quad 9-16$

Dark yellowish brown (10YR 4/4) firm fine sandy loam, structureless, common insect burrows, many gravels, few rootlets, abrupt smooth lower boundary, AC horizon.

$4 \quad 16-20$

Dark yellowish brown (10YR 4/4) friable fine sandy loam, structureless, few rootlets, common small gravels, few insect burrows, clear smooth lower boundary, AC horizon. 


$\begin{array}{cr}\text { Zone } & \text { Depth (c } \\ 5 & 20-32 \\ & \\ 6 & 32-44 \\ 7 & 44-66 \\ & \\ 8 & 66+\end{array}$

\section{Description}

Dark yellowish brown (10YR 4/4) friable fine sandy loam, massive, few rootlets, few insect burrows, one rodent burrow, few siliceous gravels, common gravels, pebbles, and cobbles in the bottom one-third of zone representing roof spall, abrupt smooth lower boundary, $\mathrm{Cu}$ horizon.

Dark yellowish brown (10YR 4/4) friable fine sandy loam, structureless, common charcoal flecks and chunks, common gravels, pebbles, and cobbles, few rootlets, one rodent burrow, clear wavy lower boundary, AC horizon.

Brown (10YR 5/3) friable fine sandy loam, common gravels and pebbles, common charcoal flecks and chunks, one rodent burrow, few rootlets, common burned cobbles at $66 \mathrm{~cm}$ representing an old occupation surface, clear smooth lower boundary, Cu horizon.

Brown (10YR 5/3) friable fine sandy loam, massive, representing weathered sandstone of the Triassic Dockum Group, $\mathrm{R}$ horizon.

\section{REFERENCES CITED}

Bettis, E. Arthur, III

1984 New Conventions for the Designation of Soil Horizons and Layers. Plains Anthropologist 29(103):57-59.

Birkeland, Peter W.

1984 Soils and Geomorphology. Oxford University Press, Oxford, England.
Buol, S. W., F. D. Hole, and R. J. McCracken

1980 Soil Genesis and Classification. 2nd ed. The Iowa State University Press, Ames. 
APPENDIX B: Textural and Chemical Analyses of Sediment Samples from 41GR291, 41GR303B, and 41GR559

Karl W. Kibler 

Sixty sediment samples were analyzed by the Soils and Physical Geography Laboratory, Department of Geography, University of WisconsinMilwaukee for grain size and organic matter and carbonate content (Table 63). Grain sizes were measured by hydrometer analysis. Organic matter and carbonate percentages were measured by loss on ignition (Broadbent 1965; Nelson 1982).

The determination of clastic sediment sizes is based on the Wentworth scale (Wentworth 1922). This scale uses millimeters to determine class size and divisions; for example, the lower size limit of sand is $0.0625 \mathrm{~mm}$ (fine sand), while the upper size limit is $2 \mathrm{~mm}$ (very coarse sand). This scale can be converted to a logarithmic scale (e.g., Krumbein 1934), which is known as the phi scale. Thus, a phi value of zero equals $1 \mathrm{~mm},-1$ phi equals $2 \mathrm{~mm}$, 1 phi equals $0.5 \mathrm{~mm}$, and so on. The use of the logarithimic ratio scales for grain sizes' results in more-normally distributed sediment populations.

The textural boundaries utilized in this analysis in terms of the phi scale are as follows: sand, finer than -1.0 to coarser than 4.0 ; silt, finer than 4.0 to coarser than 9.0; and clay, finer than 9.0. The textural phi values are percentages based on weight. The percentages of sand, silt, and clay were calculated in relation to each other.

In addition to the 60 samples analyzed by the Soils and Physical Geography Laboratory, 20 samples from Boren Shelter \#2 (41GR559) were separated by dry sieve analysis into coarse (coarser than -1.0 phi) and fine (finer than -1.0 phi) fractions in the laboratory of Prewitt and Associates, Inc. Each sample, approximately $2-3 \mathrm{~kg}$, was weighed, separated, and the coarse fraction weighed again. The coarse fraction (primarily gravels) was separated further into two components: Coarse Fraction I (coarser than -1.25 phi) and Coarse Fraction II (finer than -1.25 but coarser than -1.0 phi). The percentages of the three size classes-Coarse Fraction I, Coarse Fraction II, and Fine Fraction- are based on weight and were calculated in relation to each other (Table 64). 


\begin{tabular}{|c|c|c|c|c|c|}
\hline $\begin{array}{l}\text { GRAIN SIZE, } \\
\text { OF SEDIM }\end{array}$ & \multicolumn{3}{|c|}{ TABLE 63} & $\begin{array}{l}\text { ANALYSIS } \\
\text { 1GR559 }\end{array}$ & \\
\hline Provenience & $\%$ Sand & $\%$ Silt & $\%$ Clay & $\%$ Organic Matter & $\%$ Carbonate \\
\hline $\begin{array}{l}41 \mathrm{GR} 291, \text { EU } 83 \\
0-5 \mathrm{~cm} \\
10-15 \mathrm{~cm} \\
18-22 \mathrm{~cm} \\
26-30 \mathrm{~cm} \\
32-36 \mathrm{~cm}\end{array}$ & $\begin{array}{l}62 \\
63 \\
64 \\
65 \\
55\end{array}$ & $\begin{array}{l}28 \\
24 \\
21 \\
18 \\
24\end{array}$ & $\begin{array}{l}10 \\
13 \\
15 \\
17 \\
21\end{array}$ & $\begin{array}{l}2.5 \\
2.3 \\
2.3 \\
2.6 \\
2.9\end{array}$ & $\begin{array}{l}1.5 \\
2.5 \\
3.7 \\
3.5 \\
3.6\end{array}$ \\
\hline $\begin{array}{l}41 \text { GR291, EU } 88 \\
0-6 \mathrm{~cm} \\
12-17 \mathrm{~cm} \\
20-24 \mathrm{~cm} \\
26-30 \mathrm{~cm} \\
34-37 \mathrm{~cm} \\
42-46 \mathrm{~cm} \\
50-60 \mathrm{~cm}\end{array}$ & $\begin{array}{r}62 \\
64 \\
59 \\
60 \\
55 \\
52 \\
52\end{array}$ & $\begin{array}{l}28 \\
22 \\
25 \\
22 \\
23 \\
24 \\
30\end{array}$ & $\begin{array}{l}10 \\
14 \\
16 \\
18 \\
22 \\
24 \\
18\end{array}$ & $\begin{array}{l}2.2 \\
1.9 \\
2.1 \\
2.6 \\
2.7 \\
3.1 \\
2.0\end{array}$ & $\begin{array}{r}2.1 \\
3.2 \\
4.4 \\
4.7 \\
5.8 \\
5.3 \\
16.4\end{array}$ \\
\hline $\begin{array}{l}41 \mathrm{GR} 291, \text { Backhoe Trench } 1992-1 \\
0-5 \mathrm{~cm} \\
10-14 \mathrm{~cm} \\
17-21 \mathrm{~cm} \\
24-28 \mathrm{~cm} \\
30-34 \mathrm{~cm} \\
37-41 \mathrm{~cm} \\
44-48 \mathrm{~cm} \\
52-56 \mathrm{~cm} \\
60-65 \mathrm{~cm}\end{array}$ & $\begin{array}{l}65 \\
61 \\
58 \\
54 \\
55 \\
57 \\
53 \\
52 \\
60\end{array}$ & $\begin{array}{l}26 \\
22 \\
22 \\
22 \\
20 \\
20 \\
23 \\
24 \\
22\end{array}$ & $\begin{array}{r}9 \\
17 \\
20 \\
24 \\
25 \\
23 \\
24 \\
24 \\
18\end{array}$ & $\begin{array}{l}1.8 \\
4.1 \\
3.5 \\
3.9 \\
3.8 \\
3.4 \\
3.8 \\
3.1 \\
2.7\end{array}$ & $\begin{array}{l}1.6 \\
2.7 \\
3.2 \\
3.1 \\
3.2 \\
2.7 \\
2.5 \\
5.0 \\
5.5\end{array}$ \\
\hline $\begin{array}{l}41 \mathrm{GR} 291, \text { Backhoe Trench 1992-3a } \\
0-5 \mathrm{~cm} \\
8-11 \mathrm{~cm} \\
16-20 \mathrm{~cm} \\
24-29 \mathrm{~cm} \\
31-35 \mathrm{~cm} \\
38-43 \mathrm{~cm} \\
47-51 \mathrm{~cm} \\
54-59 \mathrm{~cm} \\
61-65 \mathrm{~cm} \\
67-71 \mathrm{~cm} \\
74-78 \mathrm{~cm} \\
84-89 \mathrm{~cm} \\
92-96 \mathrm{~cm} \\
102-106 \mathrm{~cm} \\
110-114 \mathrm{~cm} \\
118-122 \mathrm{~cm} \\
126-130 \mathrm{~cm} \\
135-143 \mathrm{~cm} \\
148-156 \mathrm{~cm}\end{array}$ & $\begin{array}{l}63 \\
70 \\
69 \\
70 \\
71 \\
67 \\
68 \\
67 \\
66 \\
65 \\
64 \\
66 \\
64 \\
69 \\
71 \\
75 \\
74 \\
77 \\
81\end{array}$ & $\begin{array}{l}29 \\
22 \\
21 \\
20 \\
18 \\
21 \\
19 \\
21 \\
20 \\
22 \\
22 \\
22 \\
22 \\
19 \\
18 \\
16 \\
17 \\
15 \\
14\end{array}$ & $\begin{array}{r}8 \\
8 \\
10 \\
10 \\
11 \\
12 \\
13 \\
12 \\
14 \\
13 \\
14 \\
12 \\
16 \\
12 \\
11 \\
9 \\
9 \\
8 \\
5\end{array}$ & $\begin{array}{l}2.7 \\
2.1 \\
2.2 \\
2.2 \\
2.2 \\
2.1 \\
2.4 \\
2.4 \\
2.3 \\
2.1 \\
2.0 \\
2.1 \\
2.1 \\
1.8 \\
1.5 \\
1.5 \\
1.6 \\
1.4 \\
1.3\end{array}$ & $\begin{array}{l}1.2 \\
1.3 \\
1.9 \\
3.0 \\
2.6 \\
2.9 \\
2.9 \\
3.2 \\
3.2 \\
3.8 \\
4.0 \\
4.6 \\
7.2 \\
5.9 \\
5.4 \\
4.8 \\
3.7 \\
3.2 \\
1.7\end{array}$ \\
\hline
\end{tabular}


Appendix B: Textural and Chemical Analyses of Sediment Samples

\begin{tabular}{|l|c|c|c|c|c|}
\hline \multicolumn{7}{|l|}{ Table 63, continued } & \% Sand & \% Silt & \% Clay & \% Organic Matter & \% Carbonate \\
\hline Provenience & & & & & \\
\hline 41 GR291, Feature 17 & 58 & 24 & 18 & 3.1 & 3.2 \\
$99.60-99.55 \mathrm{~m}$ & 57 & 21 & 22 & 3.1 & 4.4 \\
$99.40-99.35 \mathrm{~m}$ & 59 & 22 & 19 & 2.8 & 4.1 \\
$99.20-99.15 \mathrm{~m}$ & & & & & \\
\hline $41 \mathrm{GR} 303 \mathrm{~B}$, EU 6 & 63 & 27 & 10 & 2.0 & 1.0 \\
$0-5 \mathrm{~cm}$ & 67 & 20 & 13 & 0.0 & 2.3 \\
$11-15 \mathrm{~cm}$ & 65 & 21 & 14 & 1.8 & 3.3 \\
$20-24 \mathrm{~cm}$ & 65 & 21 & 14 & 1.9 & 2.7 \\
$30-34 \mathrm{~cm}$ & 61 & 26 & 13 & 1.5 & 4.3 \\
$40-44 \mathrm{~cm}$ & 63 & 24 & 13 & 1.8 & 3.4 \\
$50-54 \mathrm{~cm}$ & 61 & 23 & 16 & 2.2 & 4.3 \\
$61-66 \mathrm{~cm}$ & 59 & 27 & 14 & 1.9 & 5.2 \\
$71-76 \mathrm{~cm}$ & 57 & 28 & 15 & 1.9 & 5.0 \\
$81-85 \mathrm{~cm}$ & 59 & 27 & 14 & 1.7 & 4.9 \\
$94-100 \mathrm{~cm}$ & & & & & \\
\hline $41 \mathrm{GR} 559$, EU 20 & 62 & 25 & 13 & 3.5 & 5.0 \\
$0-5 \mathrm{~cm}$ & 61 & 29 & 10 & 2.2 & 5.4 \\
$7-9 \mathrm{~cm}$ & 68 & 23 & 9 & 1.6 & 8.6 \\
$12-15 \mathrm{~cm}$ & 59 & 32 & 9 & 1.4 & 6.3 \\
$17-20 \mathrm{~cm}$ & 66 & 24 & 10 & 1.1 & 7.9 \\
$25-29 \mathrm{~cm}$ & 68 & 21 & 11 & 1.3 & 6.6 \\
$35-41 \mathrm{~cm}$ & 61 & 28 & 11 & 1.7 & 5.2 \\
$47-51 \mathrm{~cm}$ & & & & \\
\hline
\end{tabular}


TABLE 64

DRY SIEVE ANALYSIS OF SEDIMENTS FROM BOREN SHELTER \#2, 41GR559

\begin{tabular}{|l|c|c|c|}
\hline Provenience & Coarse I \% & Coarse II \% & Fine \% \\
\hline EU 18 & & & \\
$100.3-100.2 \mathrm{~m}$ & 13.6 & 1.2 & 85.2 \\
$100.2-100.1 \mathrm{~m}$ & 12.8 & 2.8 & 84.4 \\
$100.1-100.0 \mathrm{~m}$ & $10.8^{*}$ & 2.5 & 86.7 \\
$100.0-99.9 \mathrm{~m}$ & $11.7^{*}$ & 3.8 & 84.5 \\
$99.9-99.8 \mathrm{~m}$ & $15.2^{*}$ & 4.1 & 80.7 \\
\hline EU 24 & & & \\
$99.5-99.4 \mathrm{~m}$ & 12.6 & 3.9 & 83.5 \\
\hline EU 27 & & & \\
$99.5-99.4 \mathrm{~m}$ & 4.8 & 1.7 & 93.5 \\
\hline EU 29 & & & \\
$100.37-100.30 \mathrm{~m}$ & 3.2 & 0.8 & 96.0 \\
$100.30-100.20 \mathrm{~m}$ east & 8.3 & 1.3 & 90.4 \\
$100.30-100.20 \mathrm{~m}$ west & 25.9 & 4.2 & 95.9 \\
$100.20-100.15 \mathrm{~m}$ & 3.8 & 0.5 & 73.6 \\
$100.15-100.10 \mathrm{~m}$ & $23.0^{*}$ & 3.4 & 93.6 \\
$100.10-100.00 \mathrm{~m}$ & 5.5 & 0.9 & 93.8 \\
$100.00-99.90 \mathrm{~m}$ & $5.3^{*}$ & 0.9 & 92.2 \\
$99.90-99.80 \mathrm{~m}$ & 6.3 & 1.5 & 93.8 \\
$99.80-99.70 \mathrm{~m}$ & 5.7 & 0.5 & 99.2 \\
$99.70-99.65 \mathrm{~m}$ & 0.5 & 0.3 & 97.7 \\
$99.65-99.60 \mathrm{~m}$ & 1.8 & 0.5 & 92.8 \\
$99.60-99.56 \mathrm{~m}$ & $16.3^{*}$ & 0.9 & \\
$99.56-99.50 \mathrm{~m}$ & 2.1 & 0.6 & \\
\hline$*$ Rare quartzitic gravels present & & & \\
\hline & \multicolumn{3}{|c|}{} \\
\hline
\end{tabular}

\section{REFERENCES CITED}

Broadbent, F. E.

1965 Organic Matter. In Methods of Soil Analysis, edited by C. A. Blank, pp. 1397-1400. American Society of Agronomy, Inc., Soil Science Society of America, Inc., Madison, Wisconsin.

Krumbein, W. C.

1934 Size Frequency Distributions of Sediments. Journal of Sedimentary Petrology 4:65-77.
Nelson, R. E.

1982 Carbonate and Gypsum. In Methods of Soil Analysis, Part 2: Chemical and Microbiological Properties, 2nd ed., edited by A. L. Page, R. H. Miller, and D. R. Keeney, pp. 181-197. American Society of Agronomy, Inc., Soil Science Society of America, Inc., Madison, Wisconsin.

Wentworth, C. K.

1922 A Scale of Grade and Class Terms for Clastic Sediments. Journal of Geology 30:377-392. 


\title{
APPENDIX C: Analysis of the Vertebrate Remains from 41GR291,
} 41GR303B, 41GR474, and 41GR559

\author{
Brian S. Shaffer \\ Department of Anthropology \\ Texas A\&M University \\ College Station, Texas
}





\section{INTRODUCTION}

The goals of the analyses of the faunal remains from 41GR291, 41GR303B, 41GR474, and 41GR559 were to provide identifications of the faunal materials and to identify cultural attributes. Each sample is unique in composition and attributes. Site 41GR474 represents the only historic site of the group. Sites 41GR291, 41GR303B, and 41GR474 are all represented by relatively small samples, whereas 41GR559 consists of a large sample. Each site is discussed below.

\section{METHODS}

Specimens from each of the sites were analyzed after being washed and sorted. Identifications were made by using the Texas A\&M University Department of Anthropology's Zooarchaeological' Research Collection. Osteological guides used to aid in the identification include Auffenberg (1969) for snakes and Olsen (1968) for other herpetofauna, Lawrence (1951) for deer and pronghom, and McCuaig and Cumbaa (1992) and Olsen (1960) for cattle and bison. Additional mammal remains were identified with the aid of Glass (1951), Hillson (1986), and Olsen (1964). No identifications were made solely upon criteria provided in the guides. Attribute recording was accomplished using a vertebrate coding system (Shaffer and Baker 1992). Attributes addressed include provenience, taxon, element, portion of element, side, aging criteria, age of the animal, and basic taphonomic information. The taphonomic information varies slightly among the sites but includes weathering, breakage, burning, cut marks, and any additional information. Unique observations, such as pathological disorders or types of cut marks, were recorded in a comments field. Unique or culturally modified specimens were observed under magnification by either a $10 \mathrm{x}$ hand lens or a light microscope up to $30 \mathrm{x}$.

Specimens that could not be identified to the level of order or below were identified on the basis of size and recognizable attributes. For example, specimens that are too small to identify to the level of class were identified simply as "Vertebrata." Bird sizes identified include small (sparrow sized) and medium (jay or dove sized). Mammal sizes identified include micro (mouse sized), small/medium (rabbit/canid sized), medium/large (canid/deer sized), and large/very large (deer/bison sized). While these levels of identification do not provide detailed taxonomic information, they do provide an estimate of the sizes of taxa represented by the unidentifiable elements.

\section{SITE 41GR291}

A small sample of 183 specimens was recovered from 41GR291 (Table 65). Of this sample, only two could be identified to order or below-one medium-sized artiodactyl naviculo-cuboid and one jack rabbit innominate. Most of the sample is heavily weathered ( 124 specimens, $68 \%$ ), as indicated by fine-line fractures, flaking in planes, and general degradation of the bone surfaces. No gnaw marks or cut marks were noted on any specimens. No spiral fracturing was identified, indicating that fragmentation of the assemblage occurred after the bones had lost collagen (Johnson 1985) or that fresh-break surfaces had not survived. Burning was noted on 27 (15\%) specimens, 8 of which are charred (burned black) and 19 of which are calcined (burned white). Aside from the burning and the presence of these materials in an archeological site, no other cultural or possibly cultural attributes were identified on the assemblage.

\section{SITE 41GR303B}

Sixteen specimens were recovered from 41GR303B (Table 66). Of this sample, nine (56\%) could be identified to order or below. Identified taxa include a jack rabbit (Lepus sp.) innominate, a gopher (Geomys sp.) mandible and four associated teeth, a medium-sized artiodactyl (deer sized) tooth fragment, a large artiodactyl (bison sized) proximal radius, and a bison astragalus. No gnaw marks or cut marks were noted on the assemblage. Of the 16 specimens, $4(25 \%)$ have marked weathering. The only specimen with a spiral fracture is the large artiodactyl radius. Burning was noted on five (31\%) specimens - three are charred and two are calcined. Burning, spiral breakage, and presence in the site are the only possible cultural indicators identified.

\section{SITE 41GR474}

The historic dugout at 41GR474 is represented by 421 faunal specimens (Table 67). Specimens identified to the level of order or below total 104 (25\%). Identified taxa include viperid snake (pit viper), duck (Anatidae), chicken (Gallus gallus), 


\begin{tabular}{|l|l|l|r|}
\hline \multicolumn{3}{|c|}{ TABLE 65 } \\
VERTEBRATE TAXA AND TAPHONOMIC \\
ATTRIBUTES, 41GR291 \\
\hline Taxon & Breakage & Burning & Totals \\
\hline Vertebrata & Angular & Unburned & 16 \\
Vertebrata & Angular & Charred & 7 \\
Vertebrata & Angular & Calcined & 16 \\
Mammalia (small/medium) & Angular & Charred & 1 \\
Mammalia (medium/large) & Angular & Unburned & 121 \\
Mammalia (medium/large) & Angular & Calcined & 3 \\
Mammalia & Angular & Unburned & 17 \\
Lepus sp. & Angular & Unburned & 1 \\
Artiodactyla (medium) & Angular & Unburned & 1 \\
& & & 183 \\
\hline
\end{tabular}

\begin{tabular}{|l|l|l|c|}
\hline \multicolumn{3}{|c|}{ TABLE 66 } \\
VERTEBRATE TAXA AND TAPHONOMIC \\
ATTRIBUTES, 41GR303B \\
\hline Taxon & Breakage & Buming & Totals \\
\hline Vertebrata & Angular & Charred & 1 \\
Mammalia (medium/large) & Angular & Unburned & 3 \\
Mammalia (medium/large) & Angular & Charred & 2 \\
Mammalia & Angular & Calcined & 1 \\
Lepus sp. & Angular & Unburned & 1 \\
Geomys sp. & Unbroken & Unburned & 4 \\
Geomys sp. & Angular & Unburned & 1 \\
Artiodactyla (medium) & Angular & Calcined & 1 \\
Artiodactyla (large) & Spiral & Unburned & 1 \\
Bison bison & Angular & Unburned & 1 \\
\multicolumn{2}{|c|}{} & \\
\hline
\end{tabular}

fractures, and 32 (8\%) are unbroken. Charring was noted on 1 specimen, and another $70(17 \%)$ specimens are calcined. Gnawing was not identified on the specimens from this sample.

Historic cut marks were identified on 19 specimens, and another 3 specimens were identified with cuts that apparently are trowel damage. These recent cut marks expose unstained bone and are usually clean, retaining little or no matrix. Historic cut marks that apparently were made by a bladed instrument, such as a knife, are present on four specimens; the staining inside the cuts is similar to that on the outside of the bones, indicating that the antiquity of the cuts corresponds to when the bones were discarded.

Sawing was identified on 10 specimens, with 1 specimen having been sawed by hand. Hand-sawed specimens can be recognized by their crosshatched but straight saw marks, as opposed to a band saw, which produces parallel straight saw marks, or a circular saw, which produces curved and parallel saw marks. The type of sawing on the other nine specimens could not be identified unequivocally due to root etching and bone fragmentation. The only actual cuts recognized are the short rib cuts identified on four large/very large mammal ribs (probably from cow) (cf. Dansie 1979). These specimens are rib mid-shafts, truncated on each end by

cottontail rabbit (Sylvilagus sp.), prairie dog (Cynomys sp.), various canids, pig (Sus scrofa), deer (Odocoileus sp.), pronghom (Antilocapra americana), and cow (Bos sp.). Each of these taxa are represented by one individual only, except for the chicken and cottontail rabbit which are represented by two individuals each. The most pronounced taphonomic factor aside from fragmentation is root etching, which was observed on $337(80 \%)$ of the total sample. Many bones still retain rootlets adhering to their surfaces. In several instances, it is clear that the rootlets have completely obliterated the original surfaces of the bones, although the damage is actually quite shallow.

Fragmentation of the specimens is common. Three hundred and forty-four $(82 \%)$ specimens exhibit angular fractures, $45(11 \%)$ have spiral the saw marks. Other specimens with cut marks include medium/large mammals (3 specimens), large/very large mammals (6 specimens, including short ribs), a cottontail pelvis fragment with $5+$ cuts, a large artiodactyl (1 cow-sized femur shaft fragment), and one cow ulna that exhibits cut marks on the proximal articular surface and trowel marks on the shaft.

The only unique specimen identified in this assemblage is a pig (Sus scrofa) mandible and associated teeth. The second lower permanent molar exhibits two depressions in the exposed dentine that match the description given by Colyer (1936:616). Colyer describes pig caries as being deep, cuplike depressions in the teeth. The specimen was sent to veterinary doctor Elbert D. Derrick in Houston for confirmation. Dr. Derrick concurred that the unusual 


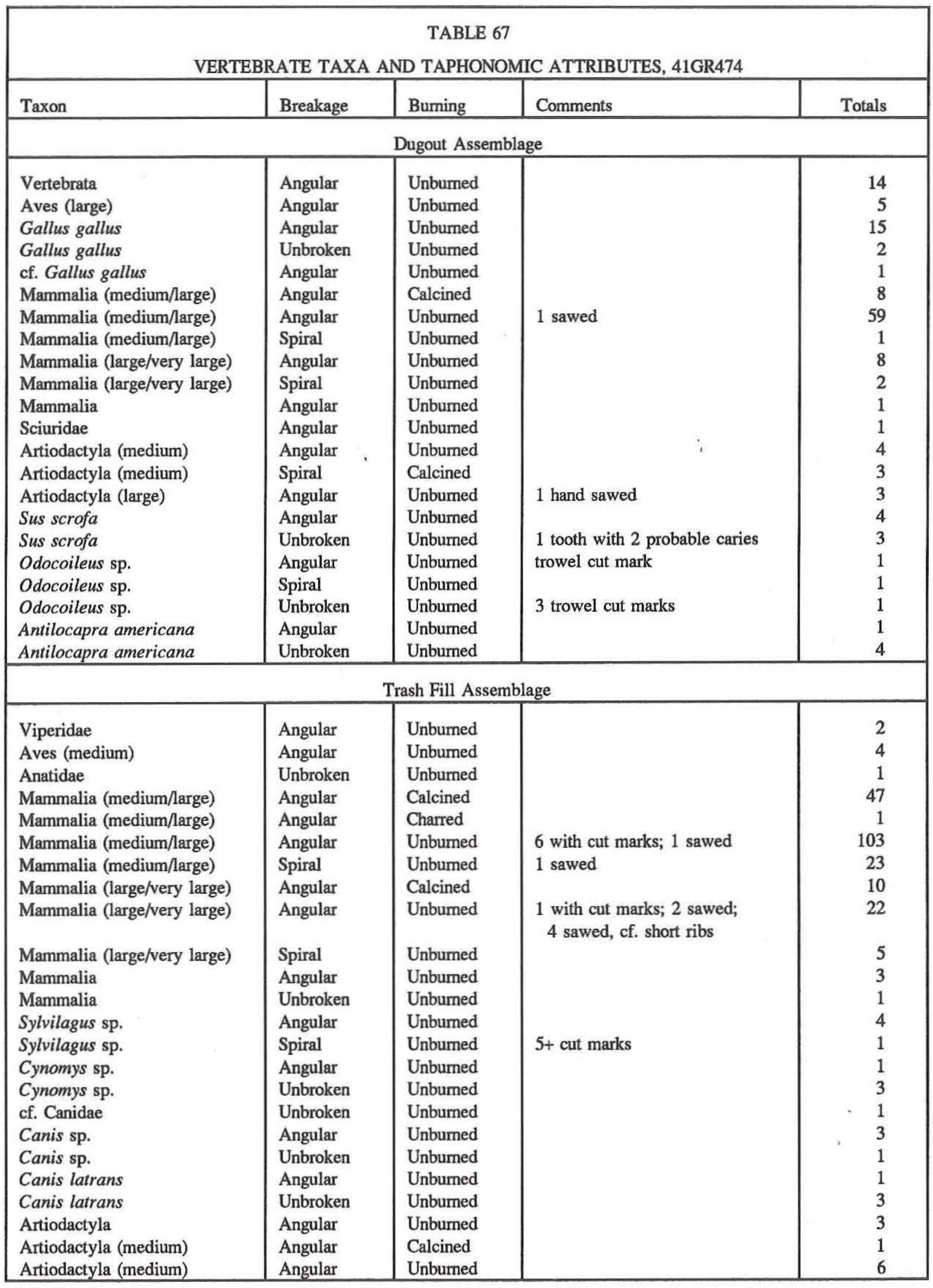




\begin{tabular}{|l|l|l|l|c|}
\hline \multicolumn{2}{|l|}{ Table 67, continued } \\
\hline Taxon & Breakage & Burning & Comments & Totals \\
\hline Artiodactyla (medium) & Spiral & Unburned & & 5 \\
Artiodactyla (medium) & Unbroken & Calcined & & 1 \\
Artiodactyla (large) & Angular & Unburned & & 1 \\
Artiodactyla (large) & Unbroken & Unburned & & 4 \\
cf. Sus scrofa & Angular & Unburned & & 1 \\
Antilocapra americana & Angular & Unburned & & 6 \\
Antilocapra americana & Spiral & Unburned & 1 with trowel mark & 4 \\
Antilocapra americana & Unbroken & Unburned & Cut marks on articular surface/ & 4 \\
Bos sp. & Angular & Unburned & trowel mark on shaft & 1 \\
cf. Bos sp. & Angular & Unburned & & 2 \\
\hline Total: & & & 421 \\
\hline
\end{tabular}

depressions in the tooth are not abnormal wear patterns but are indeed cavities.

\section{SITE 41GR559}

The rockshelter and talus area excavated at 41 GR559 produced 3,580 bone and tooth specimens, of which 1,003 specimens (28\%) could be identified to order or below (Table 68). This assemblage is unusual in that most of the identifiable assemblage is derived from canid and felid remains. Additionally, the scarcity of obvious cultural modification of the assemblage is perplexing given the number of hearth features identified within the shelter (see Chapter 7).

\section{Taphonomy and Assemblage Composition}

In looking at the general condition of the assemblage, 2,954 (83\%) specimens are broken. Spiral fractures were identified on only $39(1 \%)$ specimens, and therefore most fractures apparently occurred after the bone had lost collagen. In contrast to the high frequency of breakage, 3,368 (94\%) specimens are lightly weathered, and bone and tooth surfaces are generally intact. No rodent gnawing, carnivore gnawing, or cut marks were identified on any of the bones.

The identified assemblage is dominated by carnivores, primarily canids (see Table 68), both in terms of the number of identified specimens (NISP) and the minimum number of individuals (MNI). Minimum number of individuals was computed treating the site as three aggregates and based upon the most frequent element recovered and age assessments of the individuals represented by the elements from the Lower Shelter, Upper Shelter, and Talus Area Bone Midden. Although the site apparently was occupied sporadically over a long period (see Chapter 7), the complex nature of the deposits did not allow for ready separation of individual episodes.

Nine canids, representing one fox, one juvenile canid that is too young for further identification, two Canis sp. juveniles, three Canis sp. adults (apparently not wolf), and two old adult cf. Canis lupus (wolf) individuals are represented. The Canis sp. juveniles are represented by long bones with unfused epiphyses, podials that are incompletely formed, and mandibles and maxillae with deciduous teeth, some underlain by permanent teeth that were still forming at the time of death. The old wolves are represented by mandibles and maxillae with extremely worn teeth. Some incisors and cheek teeth are worn to the root. Canines are often worn to a smooth, rounded surface. Adult wolf bones, probably from the old adults, were recovered and have fused epiphyses with obliterated metaphyses, indicating that fusion was complete.

Identification of cf. Canis lupus was made based on the size of the elements, comparison with a skeletonized wolf provided by Bonnie Yates at the National Fish and Wildlife Forensics Laboratory in Ashland, Oregon, and comparison with metric data from Goldman (1944). Due to the fragmented nature of the wolf assemblage, cranial metrics could not be assessed. The only measurement that could be taken consistently was on the lower carnassial or first molar. These measurements of $26.65,26.45,27.5$, and $27.9 \mathrm{~mm}$ are larger than those listed for Texas 


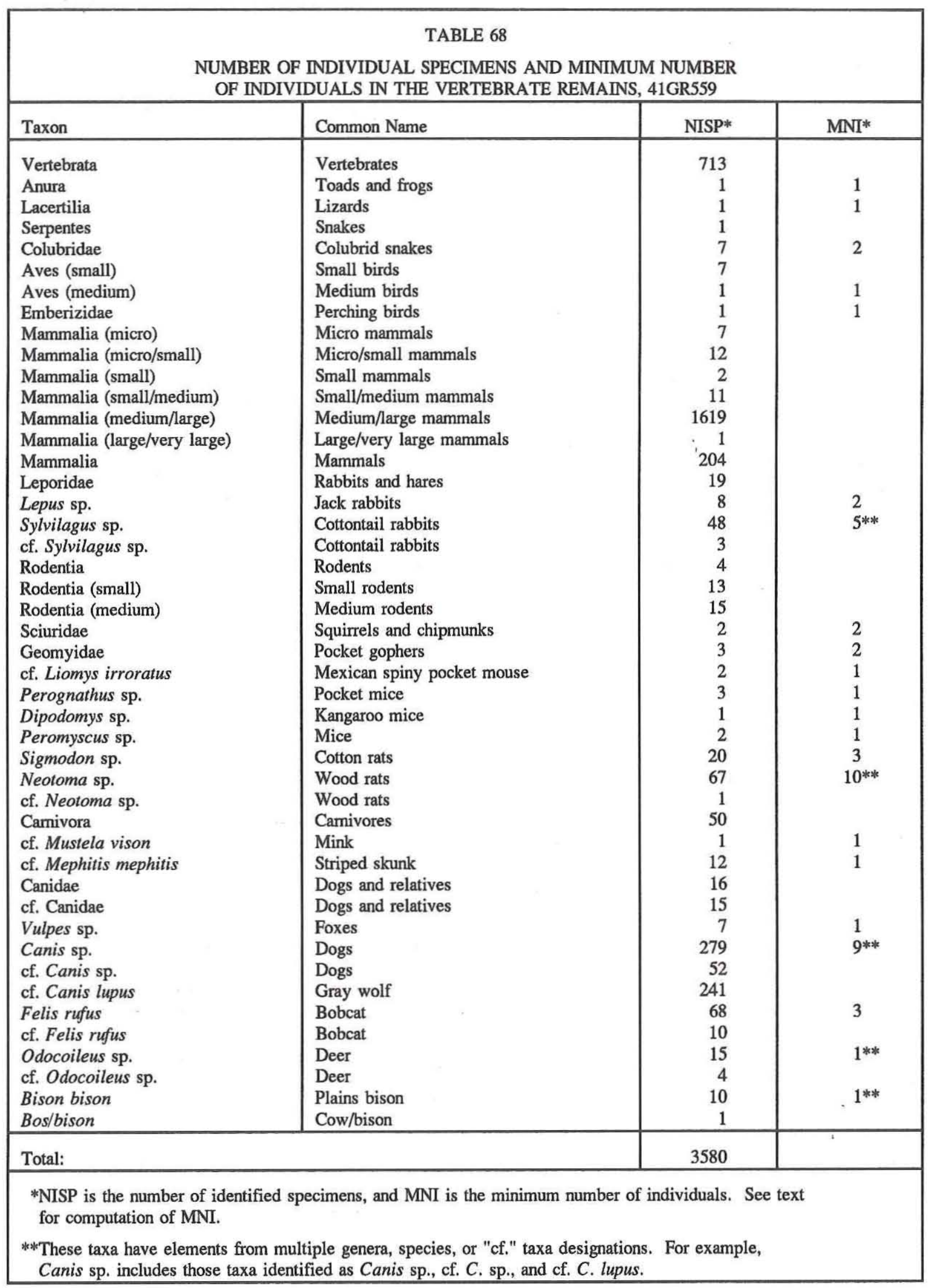


male and female $C$. niger rufus (red wolf) specimens (Goldman 1944:Tables 5 and 7). These measurements are too small for males of $C$. lupus (Goldman 1944:Table 4) and fall within the range of females (Goldman 1944:Table 6).

Additionally, three bobcats, one skunk, and one cf. mink are represented. This high frequency of carnivores was not expected in a rockshelter occupied by humans. Additionally, the low frequency of modification to the bones that would normally be associated with cultural assemblages (e.g., burning, spiral breakage, cut marks) necessitated closer examination of the composition to identify those specimens associated with the cultural deposits. This process was neither apparent nor without ambiguity.

The assemblages from inside the rockshelter and from the talus bone midden outside the shelter (Table 69) differ substantially. The bone midden sample is much larger, with 3,033 specimens. The shelter assemblage consists of 547 specimens. Major taxonomic differences include the following: (1) most of the carnivore and rodent remains and all of the bison remains are from the bone midden; and (2) all of the deer remains and most of the leporids (rabbit and hare specimens) are from the rockshelter.

In looking at the taphonomic factors affecting each assemblage, marked weathering was noted on 209 specimens in the bone midden but on only 3 in the shelter. More-heavily weathered specimens would be expected in the area outside the shelter. Burning was observed on 21 specimens from the bone midden and 24 from inside the shelter. Spiral fracturing was identified on only 9 specimens from the bone midden but on 30 from the shelter. Lastly,
TABLE 69

VERTEBRATE TAXA FROM 41GR559

\begin{tabular}{|c|c|c|c|}
\hline \multicolumn{2}{|c|}{ Talus Area Bone Midden } & \multicolumn{2}{|l|}{ Shelter } \\
\hline Taxon & Totals & Taxon & Totals \\
\hline Vertebrata & 402 & Vertebrata & 311 \\
\hline Colubridae & 4 & Anura & 1 \\
\hline Aves (small) & 6 & Lacertilia & 1 \\
\hline Mammalia (micro) & 2 & Serpentes & 1 \\
\hline Mammalia (small/medium) & 6 & Colubridae & 3 \\
\hline Mammalia (medium/large) & 1588 & Aves (small) & 1 \\
\hline Mammalia (large/very large) & 1 & Aves (medium) & 1 \\
\hline Mammalia & 168 & Emberizidae & 1 \\
\hline Leporidae & 12 & Mammalia (micro) & 5 \\
\hline Lepus sp. & 6 & Mammalia (micro/small) & 12 \\
\hline Sylvilagus sp. & 9 & Mammalia (small) & 2 \\
\hline Rodentia (small) & 6 & Mammalia & 5 \\
\hline Rodentia (medium) & 4 & (small/medium) & 31 \\
\hline Sciuridae & 1 & Mammalia (medium/large) & 36 \\
\hline Perognathus sp. & 3 & Mammalia & 7 \\
\hline Dipodomys sp. & 1 & Leporidae & 2 \\
\hline Peromyscus sp. & 2 & Lepus sp. & 39 \\
\hline Sigmodon sp. & 16 & Sylvilagus sp. & 3 \\
\hline Neotoma sp. & 38 & cf. Sylvilagua sp. & 4 \\
\hline cf. Neotoma sp. & 1 & Rodentia & 7 \\
\hline Camivora & 49 & Rodentia (small) & 11 \\
\hline cf. Mustela vison & 1 & Rodentia (medium) & 1 \\
\hline cf. Mephitis mephitis & 12 & Sciuridae & 3 \\
\hline Canidae & 16 & Geomyidae & 2 \\
\hline cf. Canidae & 15 & cf. Liomys irroratus & 4 \\
\hline Vulpes sp. & 7 & Sigmodon sp. & 29 \\
\hline Canis sp. & 275 & Neotoma sp. & 1 \\
\hline cf. Canis sp. & 52 & Carnivora & 4 \\
\hline cf. Canis lupus & 241 & Canis sp. & 15 \\
\hline Felis rufus & 68 & Odocoileus sp. & 4 \\
\hline cf. Felis rufus & 10 & cf. Odocoileus sp. & \\
\hline Bison bison & 10 & & \\
\hline Bos/Bison & 1 & & \\
\hline Totals: & 3033 & & 547 \\
\hline
\end{tabular}

gastric acid etching (as described by Andrews 1990:45-90) was identified on nine specimens from within the shelter. These specimens are obviously not cultural and are probably the remains of either carnivore scats or raptor pellets.

Another aspect to consider is the provenience of the remains (Tables 70 and 71). In the bone midden area, Features $7,9,10,12,14$, and 17 are bone clusters with few associated artifacts and no charcoal. As noted above, a very small percentage of the faunal specimens in the midden have possible cultural traits. Additionally, the talus area contains only sparse 


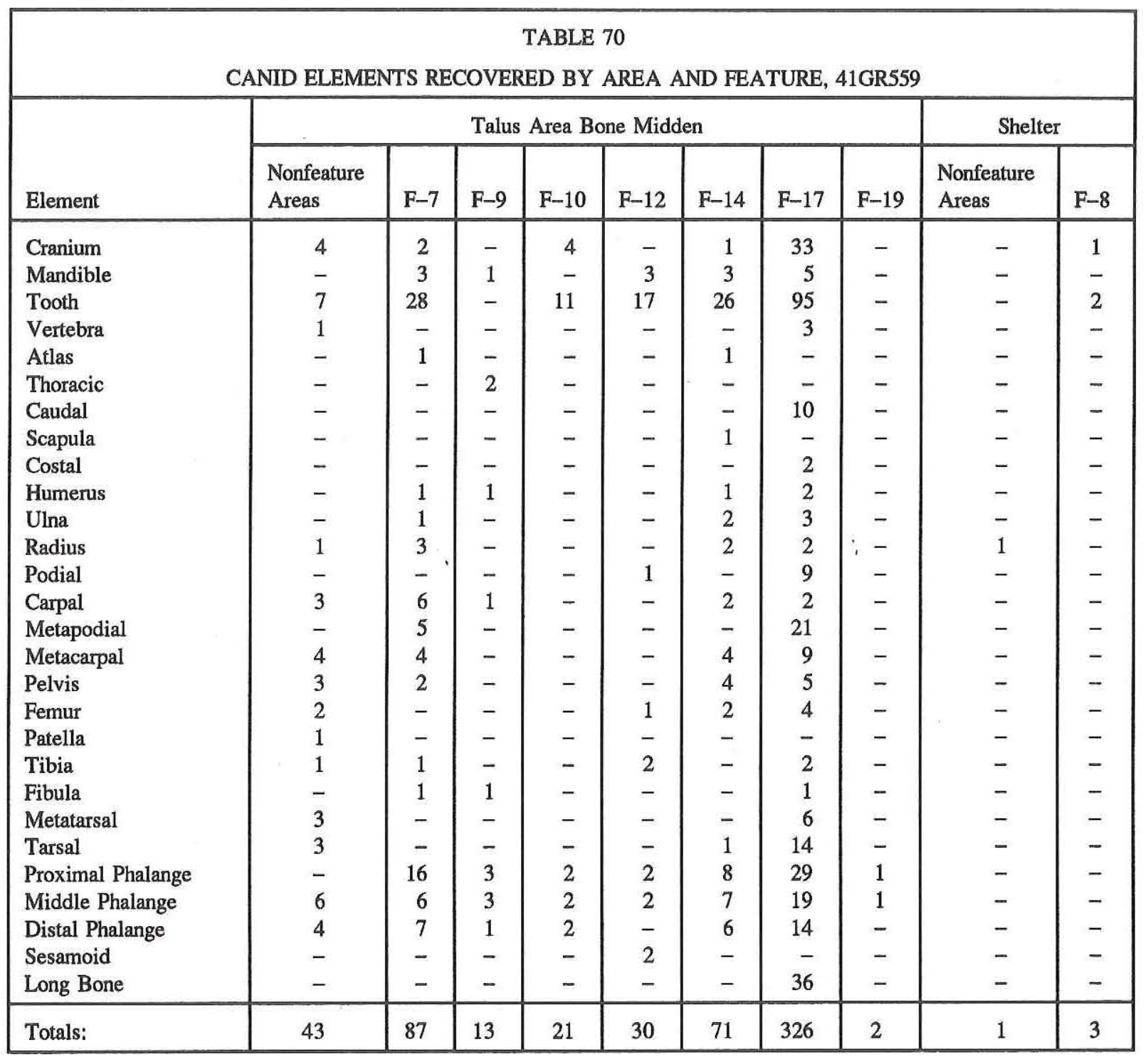

artifacts, i.e., two hammerstones and one core indirectly associated with Feature 7 in EU 9 and three hammerstones, one arrow point, one core, and one flake indirectly associated with Feature 7 in EU 8 (see Chapter 7). Conversely, the shelter area has a higher proportion of probable cultural bones (burning and spiral breakage) and is associated with numerous hearth features.

\section{The Talus Area Assemblage}

In looking at the talus area taxa, several of the canids are represented by articulated or partially articulated elements. This is reflected in the elements identified and in the feature descriptions (see Chapter
7). In fact, Feature 7 contained the remains of a cf. Canis lupus foot, including the carpals, metacarpals, and phalanges. This feature also contained the juvenile identified as Canidae. Both Canis sp. juveniles came from Feature 17 and are represented by numerous elements. Features 9 and 12 contained the remains of two subadult bison hind legs, each with an associated metapodial, podials, and some phalanges. The bison remains show no signs of butchering, carnivore gnawing, or rodent gnawing.

What is interesting about the canid remains is the obvious exclusion of most of the elements of the thorax. Notably missing are most of the cervical and thoracic vertebrae, all of the lumbar vertebrae, sacrae, ribs, and scapulae. Even rib shaft fragments that 
TABLE 71

VERTEBRATE TAXA BY FEATURE AND NONFEATURE AREAS, 41 GR559

\begin{tabular}{|c|c|c|}
\hline Provenience & Taxon & No. of Identified Specimens \\
\hline Feature 6 & Odocoileus sp. & 7 \\
\hline Feature 7 & $\begin{array}{l}\text { Vertebrata } \\
\text { Mammalia (medium/large) } \\
\text { Mammalia } \\
\text { Neotoma } \text { sp. } \\
\text { Camivora } \\
\text { cf. Mustela vison } \\
\text { Canidae } \\
\text { cf. Canidae } \\
\text { cf. Canis lupus } \\
\text { Felis rufus }\end{array}$ & $\begin{array}{r}25 \\
179 \\
11 \\
2 \\
4 \\
1 \\
15 \\
15 \\
57 \\
4\end{array}$ \\
\hline Features 7,9 , and 10 & $\begin{array}{l}\text { Vertebrata } \\
\text { Mammalia (medium/large) } \\
\text { Mammalia } \\
\text { Rodentia (medium) } \\
\text { Sciuridae }\end{array}$ & $\begin{array}{r}50 \\
2 \\
28 \\
1 \\
1\end{array}$ \\
\hline Feature 8 & $\begin{array}{l}\text { Vertebrata } \\
\text { Mammalia (micro/small) } \\
\text { Mammalia (medium/large) } \\
\text { Canis sp. }\end{array}$ & $\begin{array}{l}3 \\
1 \\
1 \\
3\end{array}$ \\
\hline Feature 9 & $\begin{array}{l}\text { Vertebrata } \\
\text { Mammalia (small/medium) } \\
\text { Mammalia (medium/large) } \\
\text { Mammalia (large/very large) } \\
\text { Rodentia (small) } \\
\text { Rodentia (medium) } \\
\text { Sigmodon sp. } \\
\text { Camivora } \\
\text { cf. Canis lupus } \\
\text { Felis rufus } \\
\text { Bison bison } \\
\end{array}$ & $\begin{array}{r}37 \\
2 \\
71 \\
1 \\
1 \\
3 \\
1 \\
2 \\
13 \\
2 \\
6 \\
\end{array}$ \\
\hline Feature 10 & $\begin{array}{l}\text { Vertebrata } \\
\text { Mammalia (medium/large) } \\
\text { Mammalia } \\
\text { Lepus sp. } \\
\text { Carnivora } \\
\text { cf. Canis lupus } \\
\text { Felis rufus } \\
\text { cf. Felis rufus } \\
\text { Bos/Bison }\end{array}$ & $\begin{array}{r}64 \\
300 \\
11 \\
5 \\
8 \\
21 \\
50 \\
9 \\
1 \\
\end{array}$ \\
\hline Feature 11 & $\begin{array}{l}\text { Vertebrata } \\
\text { Mammalia (micro) } \\
\text { Mammalia (micro/small) } \\
\text { Sylvilagus sp. } \\
\text { cf. Sylvilagus sp. } \\
\text { Rodentia (small) }\end{array}$ & $\begin{array}{r}38 \\
1 \\
3 \\
5 \\
2 \\
1\end{array}$ \\
\hline
\end{tabular}




\begin{tabular}{|c|c|c|}
\hline \multicolumn{3}{|l|}{ Table 71 , continued } \\
\hline Provenience & Taxon & No. of Identified Specimens \\
\hline Feature 12 & $\begin{array}{l}\text { Vertebrata } \\
\text { Mammalia (small/medium) } \\
\text { Mammalia (medium/large) } \\
\text { Mammalia } \\
\text { Rodentia (small) } \\
\text { Peromyscus sp. } \\
\text { Carnivora } \\
\text { Canis sp. } \\
\text { cf. Canis lupus } \\
\text { Felis rufus } \\
\text { cf. Felis rufus } \\
\text { Bison bison }\end{array}$ & $\begin{array}{r}18 \\
3 \\
75 \\
33 \\
1 \\
2 \\
3 \\
3 \\
27 \\
6 \\
1 \\
4\end{array}$ \\
\hline Feature $13 / 26$ & $\begin{array}{l}\text { Vertebrata } \\
\text { Anura } \\
\text { Mammalia (micro) } \\
\text { Mammalia (micro/small) } \\
\text { Mammalia (small/medium) } \\
\text { Mammalia (medium/large) } \\
\text { Mammalia } \\
\text { Geomyidae } \\
\text { Neotoma } \mathrm{sp} .\end{array}$ & $\begin{array}{r}29 \\
1 \\
2 \\
1 \\
2 \\
4 \\
2 \\
1 \\
1\end{array}$ \\
\hline Feature 14 & $\begin{array}{l}\text { Vertebrata } \\
\text { Aves (small) } \\
\text { Mammalia (medium/large) } \\
\text { Mammalia } \\
\text { Neotoma sp. } \\
\text { Carnivora } \\
\text { cf. Canis lupus } \\
\text { Felis rufus }\end{array}$ & $\begin{array}{r}33 \\
1 \\
218 \\
50 \\
2 \\
14 \\
71 \\
1\end{array}$ \\
\hline Feature 16 & $\begin{array}{l}\text { Vertebrata } \\
\text { Serpentes } \\
\text { Mammalia (micro/small) } \\
\text { Mammalia (small) } \\
\text { Leporidae } \\
\text { Neotoma } \mathrm{sp.}\end{array}$ & $\begin{array}{l}4 \\
1 \\
1 \\
1 \\
2 \\
2\end{array}$ \\
\hline Feature 17 & $\begin{array}{l}\text { Mammalia (medium/large) } \\
\text { Sylvilagus sp. } \\
\text { Carnivora } \\
\text { cf. Mephitis mephitis } \\
\text { Vulpes sp. } \\
\text { Canis } \text { sp. } \\
\text { cf. Canis sp. }\end{array}$ & $\begin{array}{r}500 \\
1 \\
1 \\
5 \\
7 \\
268 \\
52\end{array}$ \\
\hline Features 17 and 19 & $\begin{array}{l}\text { Vertebrata } \\
\text { Aves (small) } \\
\text { Mammalia (medium/large) } \\
\text { Leporidae }\end{array}$ & $\begin{array}{r}16 \\
1 \\
3 \\
11\end{array}$ \\
\hline
\end{tabular}


Phase III Data Recovery, Season 3, Lake Alan Henry

\begin{tabular}{|c|c|c|}
\hline Provenience & Taxon & No. of Identified Specimens \\
\hline Feature 18 & $\begin{array}{l}\text { Vertebrata } \\
\text { Mammalia } \\
\text { Rodentia (small) } \\
\text { Rodentia (medium) } \\
\text { Sigmodon sp. } \\
\text { Neotoma sp. }\end{array}$ & $\begin{array}{r}23 \\
3 \\
1 \\
2 \\
3 \\
1\end{array}$ \\
\hline Feature 19 & $\begin{array}{l}\text { Vertebrata } \\
\text { Colubridae } \\
\text { Aves (small) } \\
\text { Mammalia (micro) } \\
\text { Mammalia (small/medium) } \\
\text { Mammalia (medium/large) } \\
\text { Leporidae } \\
\text { Sylvilagus sp. } \\
\text { Rodentia (small) } \\
\text { Neotoma sp. } \\
\text { cf. Canis lupus }\end{array}$ & $\begin{array}{r}54 \\
1 \\
1 \\
2 \\
1 \\
25 \\
1 \\
3 \\
1 \\
3 \\
2\end{array}$ \\
\hline Feature 22 & $\begin{array}{l}\text { Vertebrata } \\
\text { Mammalia (micro/small) } \\
\text { Mammalia (medium/large) } \\
\text { Sylvilagus sp. } \\
\text { Rodentia } \\
\text { Rodentia (small) }\end{array}$ & $\begin{array}{l}8 \\
1 \\
5 \\
5 \\
4 \\
1\end{array}$ \\
\hline Feature 23 & Vertebrata & 5 \\
\hline Feature 24 & $\begin{array}{l}\text { Vertebrata } \\
\text { Leporidae }\end{array}$ & $\begin{array}{l}9 \\
4 \\
\end{array}$ \\
\hline Nonfeature Areas & $\begin{array}{l}\text { Vertebrata } \\
\text { Lacertilia } \\
\text { Colubridae } \\
\text { Aves (small) } \\
\text { Aves (medium) } \\
\text { Emberizidae } \\
\text { Mammalia (micro) } \\
\text { Mammalia (micro/small) } \\
\text { Mammalia (small) } \\
\text { Mammalia (small/medium) } \\
\text { Mammalia (medium/large) } \\
\text { Mammalia } \\
\text { Leporidae } \\
\text { Lepus sp. } \\
\text { Sylvilagus sp. } \\
\text { cf. Sylvilagus sp. } \\
\text { Rodentia (small) } \\
\text { Rodentia (medium) } \\
\text { Sciuridae } \\
\text { Geomyidae } \\
\text { cf. Liomys irroratus } \\
\text { Perognathus } \text { sp. } \\
\text { Dipodomys sp. }\end{array}$ & $\begin{array}{r}297 \\
1 \\
6 \\
4 \\
1 \\
1 \\
2 \\
5 \\
1 \\
3 \\
236 \\
66 \\
1 \\
3 \\
34 \\
1 \\
7 \\
9 \\
1 \\
2 \\
2 \\
3 \\
1\end{array}$ \\
\hline
\end{tabular}




\begin{tabular}{|l|l|c|}
\hline Table 71, continued & Taxon & No. of Identified Specimens \\
\hline Provenience & Sigmodon sp. & 16 \\
& Nonfeature Areas, continued & 56 \\
& cf. Neotoma sp. & 1 \\
& Carnivora & 18 \\
& cf. Mephitis mephitis & 7 \\
& Canidae & 1 \\
& Canis sp. & 5 \\
& cf. Canis lupus & 50 \\
& Felis rufus & 8 \\
& Odocoileus sp. & 4 \\
\hline Total: & cf. Odocoileus sp. & 3580 \\
\hline
\end{tabular}

could only be identified as medium/large mammal number just 28; however, cranial, mandibular, and limb elements are common. The reason for this representation is not apparent. Without any signs of rodent gnawing, degradation such as by acids, cut marks, or cultural modifications to the assemblage, it is difficult to attribute the missing elements to a particular cause. It is possible that, since the vertebrae are not as durable as other elements such as long bones, they might have been destroyed with the ribs by some unrecognized process. However, portions of the ribs should have survived, such as the articular ends. Additionally, had such processes acted upon the canid assemblage, then the skull elements and juvenile canid remains should not have survived either. Many of these are quite delicate, but they were recovered and show no signs of chemical, camivore, or rodent degradation. The skull remains were highly fragmented after removal but were virtually complete before removal from the matrix.

The assemblage of Felis rufus remains is similar to that of the canids. All of the felid remains were recovered from the bone midden. In fact, 59 of the 78 specimens came from Feature 10 (see Table 71); the remaining 19 elements are from nonfeature areas or from Features 7, 9, 12, and 14. The one major difference with the felid assemblage is that it contains five rib specimens. Other carnivore remains are represented by few elements, mostly dental related (teeth or mandibles).

One possibility for the accumulation of carnivores in the talus area and not inside the shelter is that the human occupants of the site cleaned the shelter of various debris and dumped it in the midden area. This debris may have included the remains of various carnivores that may have inhabited the shelter and subsequently died there. Some specimens undoubtedly would have been missed in such cleaning efforts, and this would explain the few canid remains inside the shelter. This scenario might be especially likely for the old cf. Canis lupus individuals and for the Canis sp. juveniles; however, this does not explain why so many thoracic elements are missing.

Predation by humans on the carnivores is another possibility. It is plausible that humans did prey upon the old and young canids (those being the easiest to dispatch) and then selectively butchered the animals for bones, organs, or tissues in the thoracic body cavity. However, the lack of cut marks makes this explanation difficult to assess.

Regardless of the cause of demise of the carnivores, their inclusion in the talus area predominantly as bone clusters, as opposed to being found in the shelter where their remains would be expected, indicates that some agent other than natural causes was responsible for their deposition. Although water may have entered the shelter in the past, there is no indication that it washed the remains into the talus area (Jay Peck, personal communication 1993). Had these animals been preyed upon by other carnivores and deposited in the talus area, then highly fragmented (with spiral fractures) and scattered bones would be expected. Based on these lines of reasoning, it appears that the carnivore remains were intentionally deposited in front of the shelter by humans, possibly as a result of removing the remains 
from inside the shelter.

\section{Taxa and Elements With Possible Human Modification}

Assuming that the spiral fracturing and burning identified on specimens in the assemblage are the result of human activity, then the taxa hunted were mostly small. As can be seen in Table 72, no taxa larger than cottontail (Sylvilagus sp.), which is the most frequently identified taxon, were identified to the species level. Rodents and birds, all small taxa, also were identified. Only five specimens from medium/large-sized mammals are modified.

\section{Unique Observations}

Several specimens - all cf. Canis lupus - were identified with pathological disorders. These are an unidentified vertebra centrum, two atlases, and one thoracic vertebra with arthritic lipping. Arthritic lipping also was noted on one proximal phalange. Other disorders noted on foot elements include a fourth metacarpal with reactive bone along its shaft and a middle phalange that appears to have a callous from a healed fracture. The one scapula recovered exhibits several enthesophytes, indicating pronounced muscle use. With the exception of the fracture and reactive bone, the arthritic lipping and enthesophytes are probably from one or both of the two old wolves and are a function of their advanced age.

\section{Summary}

The 3,580 faunal specimens recovered from the shelter and talus area of 41GR559 show few signs of being a cultural assemblage. Only 74 specimens show possible cultural modification of burning or

TABLE 72

VERTEBRATE TAXA WITH POSSIBLE CULTURAL MODIFICATIONS, 41GR559

\begin{tabular}{|c|c|c|c|c|}
\hline Taxon & Element & Breakage & Burning & Totals \\
\hline Vertebrata & Indeterminate & Angular & Charred & 18 \\
\hline Vertebrata & Indeterminate & Spiral & Unburned & 16 \\
\hline Vertebrata & Indeterminate & Spiral & Charred & 7 \\
\hline Vertebrata & Indeterminate & Spiral & Calcined & 1 \\
\hline Aves (small) & Ulna & Angular & Charred & 1 \\
\hline Aves (small) & Tarsometatarsus & Angular & Charred & 1 \\
\hline Mammalia (micro/small) & Indeterminate & Angular & Charred & 1 \\
\hline Mammalia (micro/small) & Vertebra & Angular & Charred & 2 \\
\hline Mammalia (micro/small) & Metapodial & Angular & Charred & 1 \\
\hline Mammalia (micro/small) & Proximal phalange & Unbroken & Charred & 1 \\
\hline Mammalia (small/medium) & Indeterminate & Spiral & Calcined & 1 \\
\hline Mammalia (small/medium) & Vertebrata & Angular & Charred & 1 \\
\hline Mammalia (medium/large) & Indeterminate & Spiral & Unburned & 5 \\
\hline Mammalia & Indeterminate & Spiral & Unburned & 4 \\
\hline Mammalia & Indeterminate & Spiral & Charred & 1 \\
\hline Sylvilagus sp. & Mandible & Angular & Charred & 1 \\
\hline Sylvilagus sp. & Permanent tooth & Unbroken & Charred & 1 \\
\hline Sylvilagus sp. & Humerus & Spiral & Unburned & 1 \\
\hline Sylvilagus sp. & Tibia & Angular & Charred & 1 \\
\hline Sylvilagus sp. & Tibia & Spiral & Unburned & 1 \\
\hline Sylvilagus sp. & Metatarsal 2 & Angular & Charred & 1 \\
\hline Sylvilagus sp. & Metatarsal 3 & Angular & Charred & -2 \\
\hline Sylvilagus sp. & Metatarsal 4 & Angular & Charred & 1 \\
\hline Sylvilagus sp. & Calcaneus & Angular & Charred & 1 \\
\hline Rodentia (mouse sized) & Cranium & Angular & Charred & 1 \\
\hline Rodentia (rat sized) & Femur & Spiral & Unburned & 1 \\
\hline Sigmodon sp. & Femur & Spiral & Unburned & $\frac{1}{74}$ \\
\hline
\end{tabular}


spiral fractures, and most of these are on animals the size of cottontails or smaller. This is not to say that the assemblage is not the result of human activity. The presence of numerous hearths indicates that the shelter was occupied several times over a long period of time. However, there is little direct evidence for human interaction with the faunal remains. Further interpretation of human involvement with the 41GR559 faunal remains must be based upon their archeological contexts (see Chapter 7).

The presence of the carnivores in the area outside the shelter is problematic. It would not be expected that all of these remains would be located outside the shelter, but rather they more likely would occur inside the shelter. Therefore, the carnivore remains, whether they represent shelter occupants that died of natural causes or as a result of human predation, may have been removed from the shelter by the human inhabitants as part of a cleaning process.

The bison remains are nearly as problematic as the canid and felid remains. Neither bison leg shows any sign of modification by humans or other animals. Therefore, the reason for their inclusion in the assemblage cannot be determined.

Probably due to the advanced age of two of the wolves, several specimens show degenerative disorders. The most frequent is arthritic lipping on the vertebrae and a phalange. Additionally, reactive bone was identified on a metacarpal and a healed fracture on another phalange. 


\section{REFERENCES CITED}

Andrews, $\mathrm{P}$.

1990 Owls, Caves, and Fossils. University of Chicago Press, Chicago.

Auffenberg, W.

1969 The Fossil Snakes of Florida. Tulane Studies in Zoology 10:131-216.

Colyer, Frank

1936 Variations and Diseases of the Teeth of Animals. John Bale, Sons and Danielsson, Ltd., London.

Dansie, A.

1979 Beef, Bobcat and Other Beast Bones: Faunal Remains from Lovelock's Chinatown. Part III, Chapter V in Archaeological and Historical Studies at Ninth and Amherst, Lovelock, Nevada, edited by Eugene M. Hattori, Mary K. Rusco, and Donald R. Tuohy, pp. 348-510. Nevada State Museum, Carson City.

Glass, B. P.

1951 A Key to the Skulls of North American Mammals. Burgess Publishing, Minneapolis, Minnesota.

Goldman, E. A.

1944 Part II: Classification of Wolves. In The Wolves of North America, by Stanley P. Young and Edward A. Goldman pp. 389-507. American Wildlife Institute, Washington, D.C.

Hillson, S.

1986 Teeth. Cambridge University Press, New York.

Johnson, E.

1985 Current Developments in Bone Technology. In Advances in Archaeological Method and Theory, vol. 8, edited by M. B. Schiffer, pp.
157-235. Academic Press, New York.

Lawrence, B.

1951 Post-cranial Skeletal Characters of Deer, Pronghom, and Sheep-goat with Notes on Bos and Bison. Papers of the Peabody Museum of Archaeology and Ethnology 35(3):9-43. Harvard University, Cambridge.

McCuaig, D. and S. L. Cumbaa

1992 A Guide to the Identification of Postcranial Bone of Bos taurus and Bison bison. Syllogeus No. 71. Canadian Museum of Nature, Ottowa.

Olsen, S. J.

1960 Post-Cranial Skeletal Characters of Bison and

Bos. Papers 'of the Peabody Museum of Archaeology and Ethnology Vol. 35, No. 4. Harvard University, Cambridge.

1964 Mammal Remains from Archaeological Sites. Part 1: Southeastem and Southwestern United States. Papers of the Peabody Museum of Archaeology and Ethnology Vol. 56, No. 1. Harvard University, Cambridge.

1968 Fish, Amphibian and Reptile Remains from Archaeological Sites. Part 1: Southeastern and Southwestem United States. Papers of the Peabody Museum of Archaeology and Ethnology Vol. 56, No. 2. Harvard University, Cambridge.

Shaffer, B. S., and B. W. Baker 1992

A Vertebrate Faunal Analysis Coding System: With North American Taxonomy and dBase Support Programs and Procedures (Version 3.3). Museum of Anthropology Technical Report 23. University of Michigan, Ann Arbor. 
APPENDIX D: Analysis of Fish Otoliths and Invertebrate Fauna from 41GR291, 41GR303B, and 41GR559

\author{
Karen M. Gardner \\ and
}

Jay Peck 



\section{FISH OTOLITHS}

Two fish otoliths were recovered from the Sam Wahl site (41GR291). Both specimens (from EU 58, Level 2 and EU 62, Level 3) are identified as Aplodinotus grunniens Rafinesque, commonly known as freshwater drum, belonging to the family Sciaenidae. Freshwater drum is a deep-bodied silver fish with a high back and long dorsal fin, with a preference for large silty lakes and rivers (Lee 1980:756). It is primarily a bottom feeder, consuming insect larva, crayfish, clams, and other fish, and it commonly ranges in size from 5-15 pounds, growing to a length of 3-4 feet (Eddy 1969; Lee 1980:756). The sciaenids are a large family of mostly coastal fishes; freshwater drum is the only freshwater species of this family, and it has a widespread distribution from central Manitoba southward to the Yucatan Peninsula and into Guatemala (Knapp 1953:134; Tomelleri and Eberle 1990:161). With the exception of parts of the Panhandle, freshwater drum is commonly found today throughout Texas (Hubbs 1982; Lee 1980:756).

\section{INVERTEBRATE REMAINS}

The invertebrate fauna recovered from the three prehistoric sites consist of freshwater mussels and terrestrial gastropods. Species identification was checked by comparison with specimens recovered during previous seasons of work in the project area (Neck and Boyd 1992:203-206; Quigg 1990:566).

The majority of the mussel shell fragments are fragmentary, although some are identifiable as Uniomerus declivus (Table 73), a common freshwater species on the High Plains that lives in slow-moving streams, sloughs, and ponds (Neck and Boyd 1992: 206). The mussel shells are generally considered to be cultural, with four of the shell fragments from 41GR291 and one fragment from 41GR559 showing evidence of intentional modification. These are discussed in detail in Chapters 3 and 7.

Few gastropods came from the three sites, with 27 from 41GR559, 5 from 41GR291, and 4 from 41GR303B. The gastropods recovered may belong to the Family Succineidae, possibly Catinella sp. Definite identification of these taxa is based primarily on the soft parts of the snail, making identification based on shell characteristics tentative (Allen and Cheatum 1961:299; Neck 1987:316). Several species of Succineidae are found in North Texas, with habitat preferences including moist wooded areas, seepage slopes, and dry terrace areas (Allen and Cheatum 1961:299; Neck 1987:316). The gastropods are interpreted as being noncultural in origin. 
Phase III Data Recovery, Season 3, Lake Alan Henry

TABLE 73

INVERTEBRATE FAUNAL REMAINS FROM 41GR291, 41GR303B, AND 41 GR559

\begin{tabular}{|c|c|c|c|c|}
\hline Provenience & No. of Fragments & Weight $(\mathrm{g})$ & No. of Uniomerus declivus & No. of Modified Specimens \\
\hline 41GR291 & & & & \\
\hline EU 1 & 9 & 1.7 & - & - \\
\hline EU 2 & 5 & 1.8 & - & - \\
\hline EU 4 & 9 & 13.1 & 1 & - \\
\hline EU 5 & 10 & 1.7 & 1 & - \\
\hline EU 6 & 1 & 0.1 & - & - \\
\hline EU 7 & 3 & 1.5 & - & 1 \\
\hline EU 8 & 4 & 4.9 & 1 & - \\
\hline EU 9 & 2 & 2.0 & 1 & - \\
\hline EU 10 & 10 & 10.1 & - & - \\
\hline EU 11 & 7 & 1.4 & - & - \\
\hline EU 12 & 3 & 1.2 & - & - \\
\hline EU 13 & 2 & 0.9 & - & - \\
\hline EU 16 & 1 & 0.1 & - & - \\
\hline EU 18 & 2 & 0.4 & - & - \\
\hline EU 19 & 1 & $0.1^{\prime}$ & - & - \\
\hline EU 20 & 2 & 0.2 & - & - \\
\hline EU 21 & 1 & $<0.1$ & - & - \\
\hline EU 23 & 5 & 0.7 & - & - \\
\hline EU 24 & 3 & 2.0 & - & - \\
\hline EU 26 & 10 & 4.3 & 3 & - \\
\hline EU 27 & 20 & 2.1 & 3 & - \\
\hline EU 29 & 9 & 1.0 & - & - \\
\hline EU 30 & 9 & 2.1 & 2 & - \\
\hline EU 31 & 9 & 3.1 & 1 & - \\
\hline EU 32 & 11 & 12.9 & - & 1 \\
\hline EU 33 & 6 & 1.2 & 1 & - \\
\hline EU 34 & 10 & 1.7 & - & - \\
\hline EU 35 & 16 & 3.5 & 1 & - \\
\hline EU 37 & 9 & 2.6 & - & 1 \\
\hline EU 38 & 11 & 1.7 & - & - \\
\hline EU 39 & 5 & 0.3 & - & - \\
\hline EU 40 & 6 & 2.1 & - & - \\
\hline EU 41 & 28 & 8.9 & 1 & 1 \\
\hline EU 42 & 5 & 0.5 & - & - \\
\hline EU 43 & 2 & 0.8 & - & - \\
\hline EU 44 & 2 & 0.3 & - & - \\
\hline EU 45 & 4 & 0.3 & - & - \\
\hline EU 46 & 5 & 2.4 & 1 & - \\
\hline EU 47 & 4 & 1.1 & - & - \\
\hline EU 48 & 11 & 3.2 & - & - \\
\hline EU 49 & 3 & 0.9 & 1 & - \\
\hline EU 50 & 6 & 1.6 & - & - \\
\hline EU 51 & 11 & 1.4 & - & - \\
\hline EU 52 & 3 & 0.9 & - & - \\
\hline EU 53 & 1 & 0.3 & - & - \\
\hline EU 54 & 1 & 0.1 & - & - \\
\hline EU 55 & 1 & 0.1 & - & - \\
\hline EU 57 & 9 & 4.1 & 1 & - \\
\hline EU 58 & 16 & 3.7 & 2 & - \\
\hline EU 59 & 10 & 2.2 & - & - \\
\hline
\end{tabular}




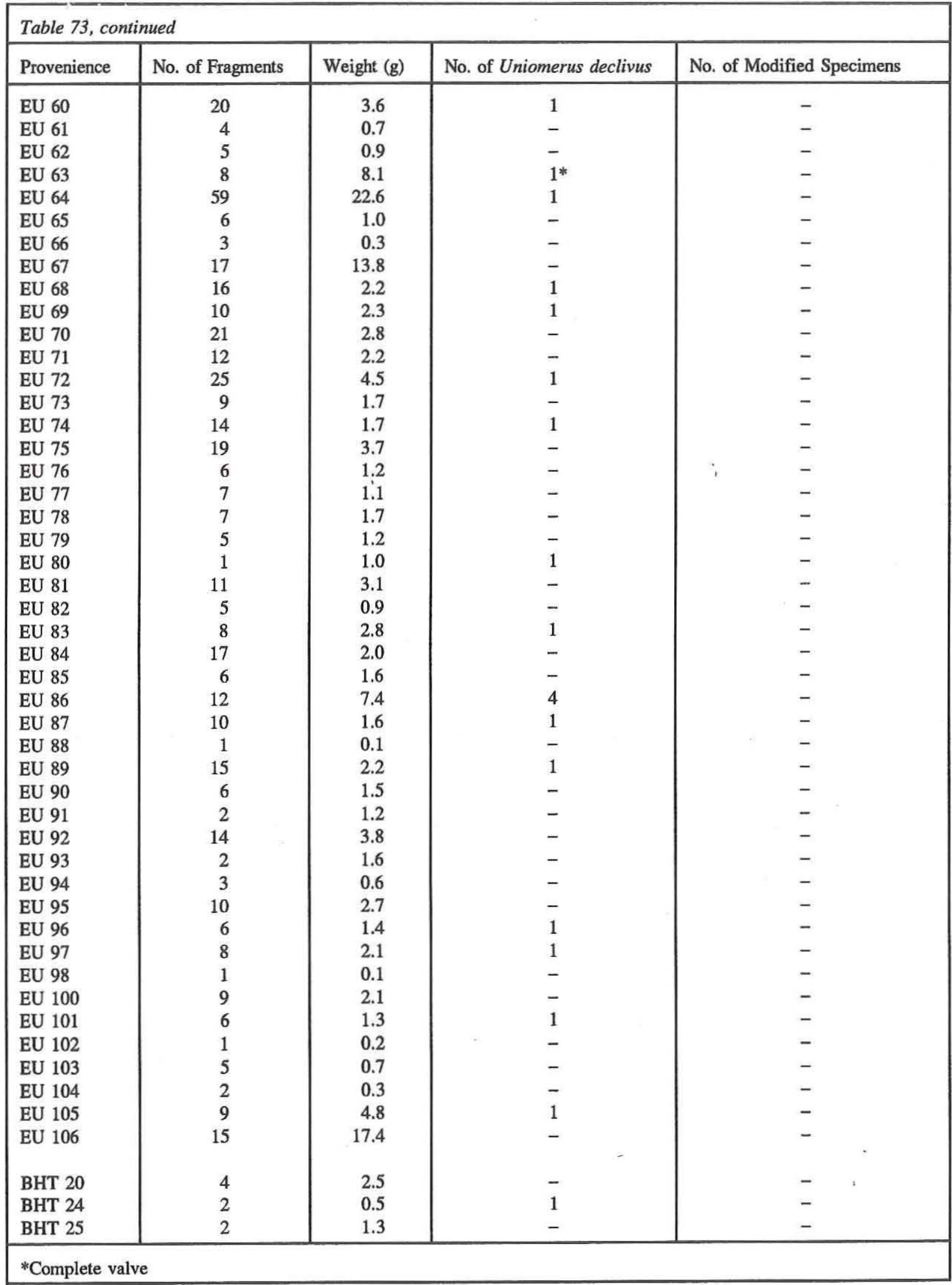


Phase III Data Recovery, Season 3, Lake Alan Henry

\begin{tabular}{|c|c|c|c|c|}
\hline \multicolumn{5}{|c|}{ Table 73, continued } \\
\hline Provenience & No. of Fragments & Weight $(\mathrm{g})$ & No. of Uniomerus declivus & No. of Modified Specimens \\
\hline Feature 17 & 7 & 0.6 & - & - \\
\hline Feature 18 & 2 & 1.4 & - & - \\
\hline Feature 19 & 2 & 0.7 & 1 & - \\
\hline Feature 20 & 3 & 0.6 & - & - \\
\hline Feature 23 & 1 & 2.5 & - & - \\
\hline Feature 25 & 3 & 0.3 & - & - \\
\hline \multicolumn{5}{|l|}{ 41GR303B } \\
\hline EU 2 & 1 & 0.5 & - & \multirow{20}{*}{$\begin{array}{l}- \\
- \\
- \\
- \\
- \\
- \\
- \\
- \\
- \\
- \\
- \\
- \\
- \\
- \\
- \\
- \\
- \\
- \\
- \\
- \\
-\end{array}$} \\
\hline EU 3 & 1 & 0.6 & - & \\
\hline EU 5 & 1 & 1.4 & - & \\
\hline EU 6 & 1 & 0.2 & - & \\
\hline EU 8 & 1 & 0.8 & - & \\
\hline EU 11 & 1 & 0.2 & - & \\
\hline EU 12 & 2 & 5.0 & - & \\
\hline EU 13 & 3 & 2.4 & - & \\
\hline EU 22 & 1 & 0.4 & - & \\
\hline EU 23 & 2 & $0.3^{\circ}$ & - & \\
\hline EU 24 & 2 & 0.5 & - & \\
\hline EU 25 & 1 & 0.2 & - & \\
\hline EU 28 & 7 & 5.8 & - & \\
\hline EU 29. & 3 & 1.0 & - & \\
\hline EU 30 & 5 & 1.8 & - & \\
\hline EU 31 & 8 & 6.1 & - & \\
\hline EU 32 & 4 & 4.0 & - & \\
\hline EU 34 & 2 & 0.4 & - & \\
\hline EU 35 & 3 & 0.4 & - & \\
\hline EU 36 & 2 & 1.1 & - & \\
\hline \multicolumn{5}{|l|}{ 41GR559 } \\
\hline EU 17 & 1 & 7.7 & - & \multirow{2}{*}{$\begin{array}{r}1 \\
-\end{array}$} \\
\hline EU 23 & 13 & 2.3 & - & \\
\hline
\end{tabular}




\section{REFERENCES CITED}

Allen, D. C., and E. P. Cheatum

1961 Ecological Implications of Fresh-water and Land Gastropods in Texas Archeological Studies. Bulletin of the Texas Archeological Society 31:291-316.

Eddy, Samuel

1969 The Freshwater Fishes. 2nd ed. Wm. C. Brown Company, Douglas, Iowa.

Hubbs, Clark

1982 A Checklist of Freshwater Fishes. Technical Series No. 11. Texas Parks and Wildlife Department, Austin.

Knapp, Frank T.

1953 Fishes Found in the Freshwater of Texas. Ragland Studio and Litho Printing Company, Georgia.

Lee, David S.

1980 Atlas of North American Freshwater Fishes. Publication \#1980-12 of North Carolina Biological Survey. North Carolina StateMuseum of Natural History.
Neck, Raymond W.

1987 Changing Holocene Snail Faunas and Environments Along the Eastern Caprock Escarpment of Texas. Quaternary Research 27:312-322.

Neck, Raymond W., and Douglas K. Boyd

1992 Faunal Remains from Prehistoric Sites. Appendix C in Data Recovery at Justiceburg Reservoir (Lake Alan Henry), Garza and Kent Counties, Texas: Phase III, Season 1, by Douglas K. Boyd, Steve A. Tomka, C. Britt Bousman, Karen M. Gardner, and Martha Doty Freeman, pp. 201-206. Reports of Investigations No. 84. Prewitt and Associates, Inc., Austin.

Quigg, J. Michael

1990 Faunal Analysis. Appendix J in Phase II Investigations at Prehistoric and Rock Art Sites, Justiceburg Reservoir, Garza and Kent Counties, Texas, by Douglas K. Boyd, James T. Abbott, William A. Bryan, Colin M. Garvey, Steve A. Tomka, and Ross C. Fields, pp. 551570. Reports of Investigations No. 71, Vol. II. Prewitt and Associates, Inc., Austin.

Tomelleri, Joseph R., and Mark E. Eberle

1990 Fishes of the Central United States. University Press of Kansas. 



\section{APPENDIX E: Analysis of Plant Remains from 41GR291 and 41GR559}

\section{J. Philip Dering}

Palynology Laboratory Department of Anthropology Texas A\&M University

College Station, Texas 



\section{INTRODUCTION}

This macrobotanical study is based on the analysis of 29 flotation samples from 41GR291 and 22 flotation samples from 41GR559. In addition to the macrobotanical study, five sediment samples from 41GR291 were examined for pollen/phytolith content.

The study area is located near the upper reaches of the Double Mountain Fork of the Brazos River, in the western section of the Rolling Plains vegetation area of Texas (Hatch et al. 1990). The dominant vegetation types in the area are mesquitelotebush brush and juniper-mixed brush (McMahan et al. 1984). Important woody species of the region include mesquite, juniper, lotebush, catclaw acacia, sumac, and agarito. In prehistoric times, the woody species probably were confined to erosional breaks, and the uplands may have been dominated by grasslands composed of bluestems, grama grasses, needlegrass, and wildrye.

\section{RESEARCH QUESTIONS}

The main goal of this analysis is to describe and interpret the macrobotanical assemblages recovered from the two sites. In connection with this objective, the following specific research questions are addressed:

(1) Are cultivated plants present in the samples?

(2) What plants were gathered and what is their significance to subsistence regimes?

(a) What gathered seeds and fruits are present and what was their importance to the prehistoric diet?

(b) What wood types are present and what was their importance?

(3) What are the inter- and intrasite distributions of plant remains?

(4) What effects have formation processes had on the macrobotanical remains, and how do these affect interpretation of the assemblages? Which plant remains are intrusive, and which are a part of the archeological record?

\section{MACROBOTANICAL METHODS}

\section{Sample Processing, Laboratory Sorting, and Identification}

Flotation sediment samples collected during the 1992 data recovery excavations at 41GR291 and
41GR559 were processed by personnel at Prewitt and Associates, Inc. Provenience data and sediment volumes for the processed samples are presented for each site in Chapter 3 (see Table 7) and Chapter 7 (see Table 55).

The flotation samples were submitted to the Paleoethnobotanical Laboratory at Texas A\&M University for analysis. Prewitt and Associates, Inc. divided each sample into three categories - light fraction, heavy fraction coarse, and heavy fraction fine. The heavy fractions were scanned at a magnification of $8 \mathrm{x}$ using a binocular dissecting microscope. No identifiable carbonized plant parts, however, were recovered from the heavy fraction subsamples. The light fraction first was sorted through a series of nested geological screens with mesh sizes of $0.5,1.0,3.0$, and $4.0 \mathrm{~mm}$. Seeds, fruits, and floral parts were sorted from all four mesh sizes. Carbonized wood was removed from the 3and 4-mm sieves. The first 25 pieces of carbonized wood were identified and counted, similar to Miksicek's (1985) procedure. Identification was accomplished at low magnifications $(<75 \mathrm{x})$ using the reference collections at Texas A\&M University.

\section{Quantification}

Two methods are utilized to describe the quantity of plant remains recovered from the flotation samples: (1) "seed" density; and (2) wood charcoal frequencies. The "seed" density value is simply the number of carbonized seeds, fruits, and edible plant parts other than wood divided by the volume of the flotation sample from which they were sorted. The frequency of occurrence of carbonized wood taxa was determined by dividing the number of fragments counted for each taxon by the total number of wood fragments examined for each sample.

\section{POLLEN/PHYTOLITH EXTRACTION METHODS}

Five sediment samples (Table 74) were processed in the same manner so that their data would be comparable. First, $20 \mathrm{ml}$ of each sample was mixed with a dilute dispersing agent (DARVAN). The rinse from this process was placed in a large beaker, and $30 \% \mathrm{HCl}$ was added to remove carbonates. At this time, one Lycopodium tracer tablet containing $11,300 \pm 400$ spores was added to each sample to monitor the progress of the processing. 


\begin{tabular}{|c|c|l|}
\hline \multicolumn{3}{|c|}{ TABLE 74 } \\
\multicolumn{3}{|c|}{ SEDIMENT SAMPLES SUBMITTED FOR } \\
POLLEN/PHYTOLITH ASSESSMENT, \\
41GR291
\end{tabular}

After the carbonate reaction ceased, each beaker was filled with distilled water and then stirred in all directions to enable pollen to remain suspended. The liquid fraction was then quickly poured into another beaker and saved. This process was repeated several times for each sample before the remaining rocks and large-grained silicates were checked and discarded. This was followed by heavy-density separation with zinc bromide at a specific gravity of 2.35. This process removed much of the remaining detritus from the pollen and phytoliths. At this point, the samples were examined for the presence of pollen and phytoliths.

\section{RESULTS OF THE MACROBOTANICAL ANALYSIS}

\section{Botanical Assemblage}

Twenty-four different plant taxa are represented in the macrobotanical assemblage (Table 75). Seeds and fruits are represented by 15 different taxa, and 11 different wood taxa were identified. Both seeds and wood of hackberry, lotebush, and mesquite were identified in the samples. The average seed concentration for the flotation samples from 41GR291 is 2.0 seeds/liter, somewhat higher than the samples from 41KT51 and 41KT53 in adjacent Kent County (Dering 1993). The seed density at 41GR291 is more typical of arid lands (Miksicek 1987:215) and may be a function of slightly better conditions for charcoal deposition and preservation in the pithouse structure and the storage pits. On the other hand, the higher seed densities at 41GR291 could reflect a greater intensity of occupation.

\section{Carbonized Seeds}

The assemblage of carbonized seeds is represented by 15 genera from 12 families. No positively identified cultigens were recovered. Eight peduncle (fruit stem) fragments of a cucurbit (cf. Lagenaria) were found, but the identification of these fragments is problematic.

A total of 455 seeds was recovered from the 41GR291 samples and 97 seeds from the 41GR559 samples. Fourteen genera, including those of the edible gathered plants goosefoot, pigweed, knotweed, mesquite, and hackberry are present in the samples from 41GR291. The seed assemblage from 41GR559 is much smaller and less diverse, and the edible plants include only hackberry, mesquite, pigweed, and knotweed. Seed counts, sample volumes, and seed concentrations for each sample are presented in Tables 76 and 77.

\section{Carbonized Wood}

The carbonized wood assemblage is fairly diverse compared to the samples from 41KT51 and 41KT53 in Kent County (Dering 1993). Eleven genera of carbonized wood are present in the samples from 41GR291 and four genera in the samples from 41GR559. In addition, significant quantities of very small carbonized hardwood twigs, most likely from xeric-adapted hardwood shrubs, are present. These appear under the categories "ring-porous hardwood" and "hardwood."

The most frequently occurring wood type in the samples from 41GR291 is juniper, followed by the categories hardwood and ring-porous hardwood. At 41GR559, the hardwood and ring-porous hardwood types are the most abundant, followed by juniper. Mesquite, lotebush, and acacia are present in the samples from both sites. The counts of carbonized wood for each sample are presented in Tables 78 and 79.

\section{RESULTS OF THE POLLEN/ PHYTOLITH ASSESSMENT}

Examination of the five samples from 41GR291 


\begin{tabular}{|c|c|c|c|}
\hline \multicolumn{4}{|c|}{$\begin{array}{l}\text { TABLE } 75 \\
\text { ANT REMAINS RECOVERED } \\
\text { GR291 AND 41GR559 }\end{array}$} \\
\hline Scientific Name & Common Name & 41GR291 & 41GR559 \\
\hline $\begin{array}{l}\text { Seeds and Fruits } \\
\text { Chenopodium } \text { sp. } \\
\text { Amaranthus } \mathrm{sp} . \\
\text { Polygonum sp. } \\
\text { Argemone } \mathrm{sp} . \\
\text { Portulaca } \text { sp. } \\
\text { Cucurbitaceae (cf. Lagenaria } \text { sp.) } \\
\text { Mollugo verticillata } \\
\text { Scutellaria } \text { sp. } \\
\text { Prosopis glandulosa } \\
\text { Ziziphus obtusifolia } \\
\text { Celtis reticulata } \\
\text { Bromus } \text { sp. } \\
\text { Paspalum } \text { sp. } \\
\text { Sporobolus } \mathrm{sp} . \\
\text { Panicum } \mathrm{sp} . \\
\text { Unknown "B" }\end{array}$ & $\begin{array}{l}\text { Goosefoot } \\
\text { Pigweed } \\
\text { Knotweed } \\
\text { Prickly poppy } \\
\text { Purslane } \\
\text { Bottle gourd } \\
\text { Carpet weed } \\
\text { Skullcap } \\
\text { Mesquite } \\
\text { Lotebush } \\
\text { Netleaf hackberry } \\
\text { Brome grass } \\
\text { Paspalum } \\
\text { Dropseed } \\
\text { Panic grass }\end{array}$ & $\begin{array}{l}\mathrm{x} \\
\mathrm{x} \\
\mathrm{x} \\
\mathrm{x} \\
\mathrm{x} \\
\mathrm{x} \\
\mathrm{x} \\
\mathrm{x}\end{array}$ & $\begin{array}{l}x \\
x \\
x \\
x \\
x \\
x \\
x\end{array}$ \\
\hline $\begin{array}{l}\text { Wood } \\
\text { Juniperus sp. } \\
\text { Prosopis glandulosa } \\
\text { Salix } \mathrm{sp} . \\
\text { Quercus } \text { sp. } \\
\text { Celtis } \text { sp. } \\
\text { Acacia } \text { sp. } \\
\text { Ziziphus obtusifolia } \\
\text { Rhus } \text { sp. } \\
\text { Berberis trifoliata } \\
\text { Forestieria } \text { sp. } \\
\text { Rosaceae (cf. Prunus } \text { sp.) } \\
\text { Hardwood } \\
\text { Diffuse-porous hardwood } \\
\text { Ring-porous hardwood }\end{array}$ & $\begin{array}{l}\text { Juniper, cedar } \\
\text { Mesquite } \\
\text { Willow } \\
\text { Oak } \\
\text { Hackberry } \\
\text { Acacia } \\
\text { Lotebush } \\
\text { Sumac } \\
\text { Agarito } \\
\text { Ironwood } \\
\text { Wild plum }\end{array}$ & $\begin{array}{l}\mathrm{x} \\
\mathrm{x} \\
\mathrm{x} \\
\mathrm{x} \\
\mathrm{x} \\
\mathrm{x} \\
\mathrm{x} \\
\mathrm{x} \\
\mathrm{x} \\
\mathrm{x} \\
\mathrm{x} \\
\mathrm{x} \\
\mathrm{x} \\
\mathrm{x}\end{array}$ & $\begin{array}{l}\mathrm{x} \\
\mathrm{x} \\
\mathrm{x}\end{array}$ \\
\hline
\end{tabular}

some other plants), and thus the potential for their preservation cannot be evaluated. Since calcium oxylate crystals are preserved in archeological sediment samples from other prehistoric contexts at Lake Alan Henry (Cummings 1990: 535), their potential should be considered.

In conclusion, it appears that further pollen studies at 41GR291 are not warranted. This assessment is consistent with the results of several other pollen studies that found poorly preserved pollen in archeological samples from Lake Alan Henry (Bryant 1993; Dering and Bryant 1992; Jones 1990). The utility of phytolith analysis, however, cannot be evaluated fully at present, and the technique could have some potential for archeological interpretation at sites in the area. Detailed interpretation of phytolith data, however, requires an extensive regional reference collection of modern plant samples, which currently does not exist for the Southern Plains.

\section{DISCUSSION}

pollen. A few spicate, cross, and club-shaped phytoliths were observed in the samples, but the concentrations appear to be very low, and the samples may be biased by differential preservation. Although opal phytoliths do not appear to be present in great quantities, useful data could be obtained if the few phytoliths present could be identified to genus or species. Merely knowing whether or not certain plants, various cultigens for example, are present at a site could be valuable information. It also should be noted that the extraction technique used to process these samples may have destroyed calcium oxylate crystals (a type of phytolith found in prickly pear and

\section{Seeds, Storage Pits, and Evidence of Prehistoric Mesquite Utilization in the Upper Brazos River Drainage}

Charred seeds were not recovered from 41GR559 in sufficient quantities to support detailed interpretations of human utilization of plant resources, but those from edible plant species, i.e., hackberry, mesquite, pigweed, and knotweed, could represent food resources. All of these species are also represented in the samples from 41GR291. The distribution of carbonized seeds among the feature 


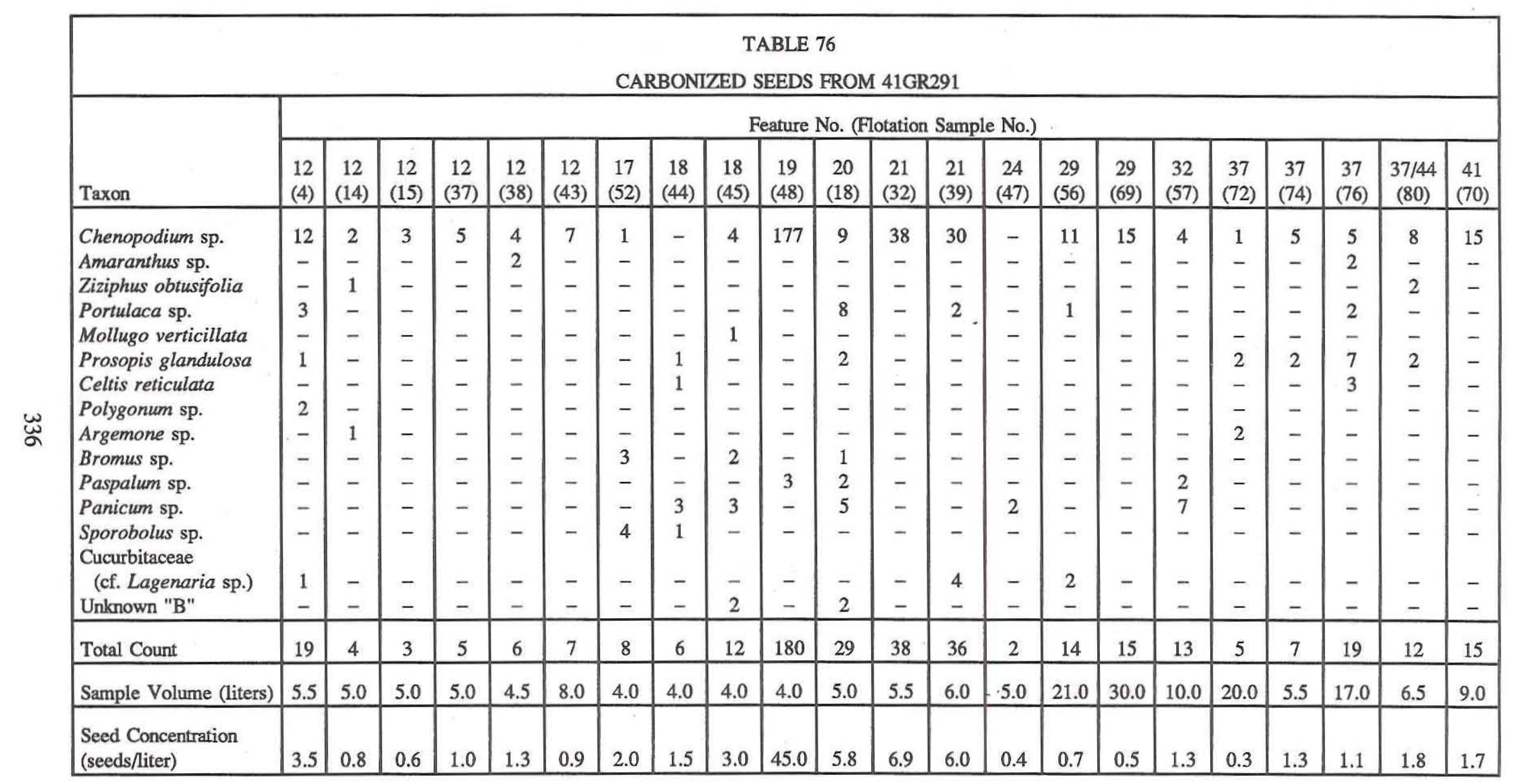




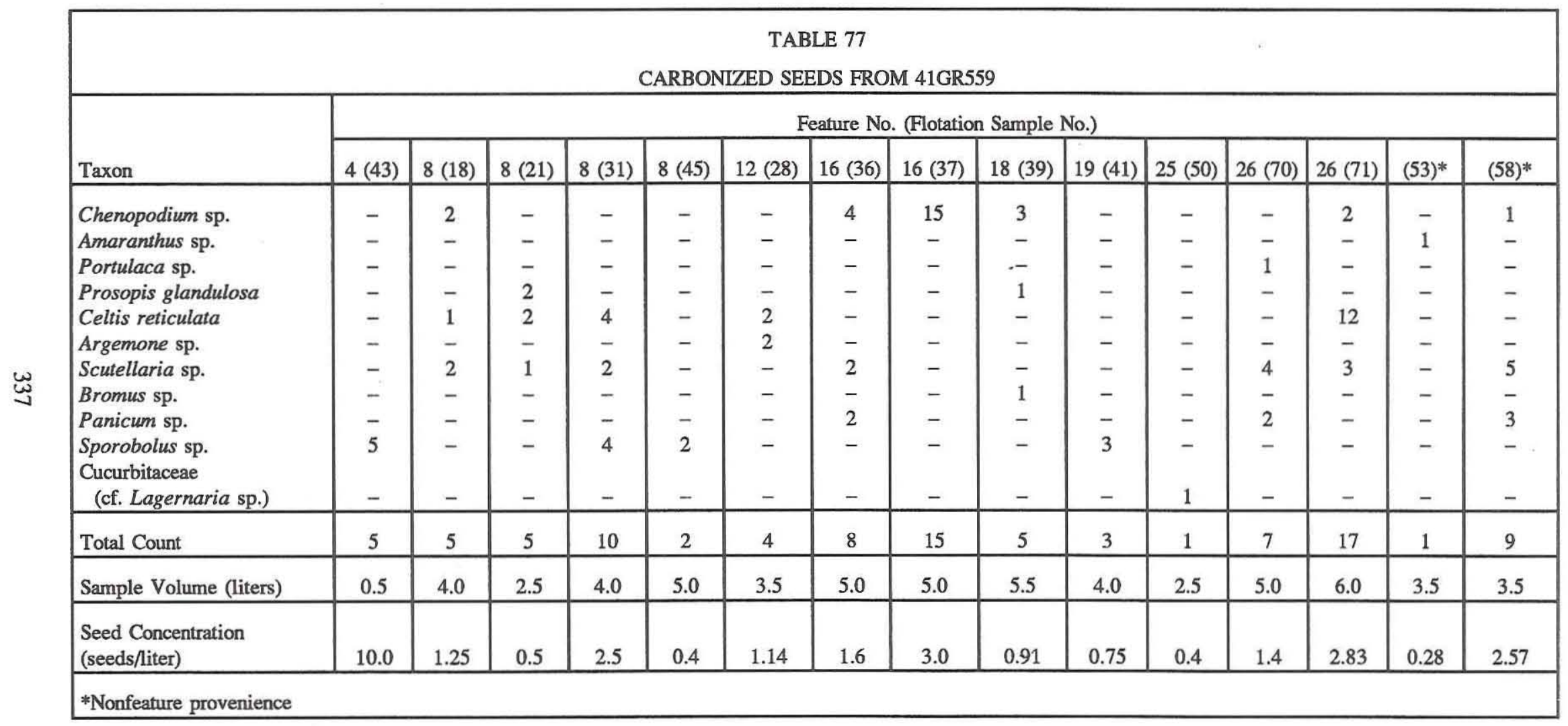




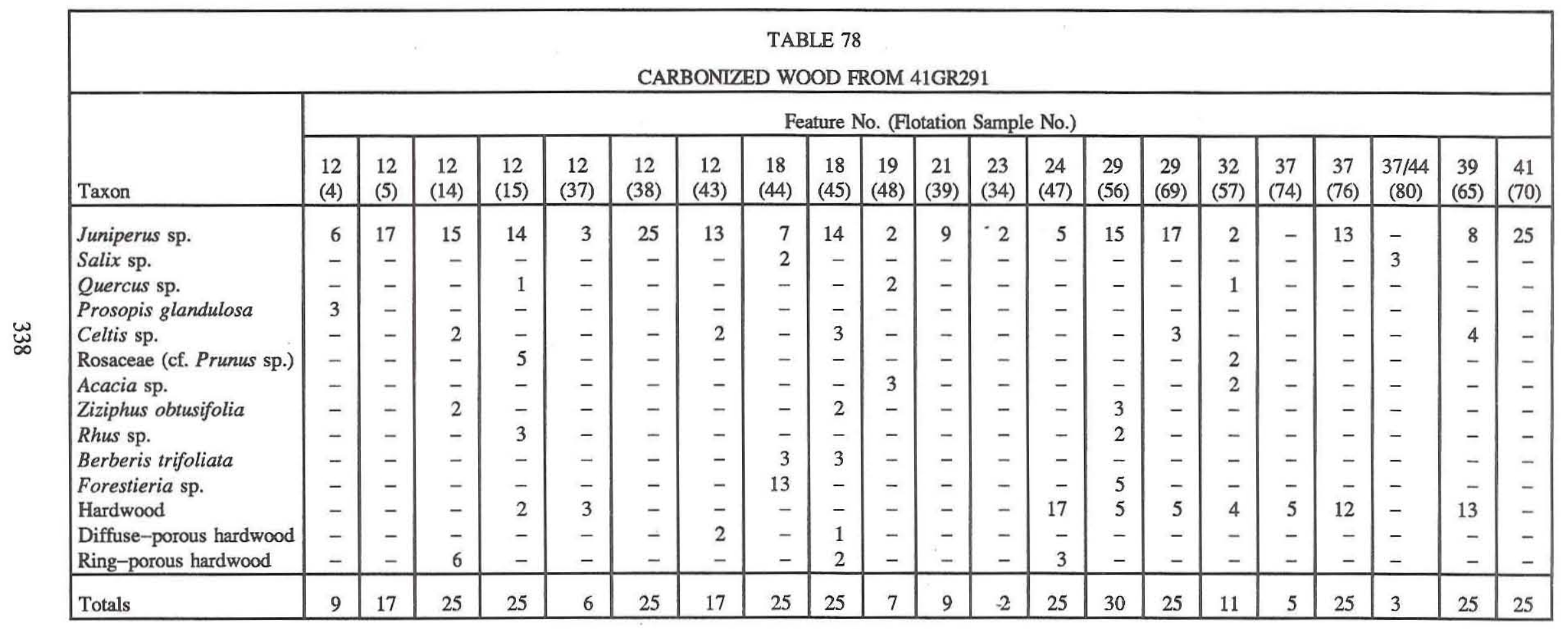




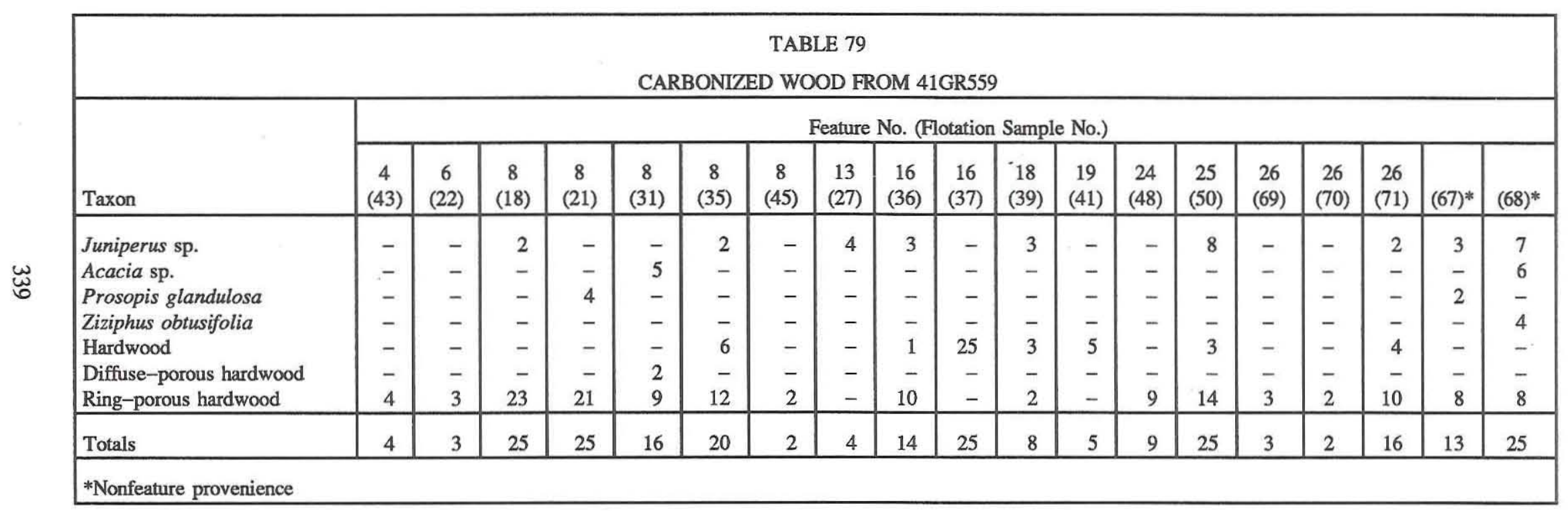


types at $41 \mathrm{GR} 291$ is presented in Table 80. Small seeds, mostly from grasses and goosefoot, were concentrated in the pit features. Mesquite seeds, the largest seeds in the sample, were concentrated within the pithouse. The presence of mesquite in the pithouse and large numbers of grass seeds in the pits warrants discussion.

The prehistoric distribution and utilization of mesquite in the Southern Plains and northern Chihuahuan Desert have received little attention (e.g., Johnston 1963). With the exception of its positive identification in the rockshelters near the confluence of the Rio Grande and the Pecos River (Dering 1979), mesquite had not been positively identified from archeological sites until recently (Dering 1993). Previous analysts have not separated the mesquite wood type from other leguminous shrubs, including the acacias. The positive identification of mesquite wood is possible, however, and has been practiced for several years at archeological sites in Arizona (Miksicek 1985). Complicating the problem is the widely accepted argument among plant ecologists that the distribution of mesquite was limited to the Rio Grande Valley or the South Texas plains until after European contact.

The recovery of charred mequite seeds from the pithouse at 41GR291 establishes beyond question that not only was mesquite growing far north of its expected prehistoric distribution in Texas, but it also was a resource that was utilized by the prehistoric population at ca. A.D. 1000 (see Chapter 3).

The importance of mesquite to the Native Americans in arid southwestern North America is well-documented (Castetter and Bell 1937). The pods were collected at different stages of ripening. Both immature and mature pods were considered an important crop. The Akmiel people of the Sonoran Desert called it the "tree of life." Mature pods were pounded into flour in stone mortars, and seeds were parched and consumed outright (Crosswhite 1981:6465). The charred mesquite seeds in the pithouse
TABLE 80

SUMMARY OF CHARRED SEEDS BY FEATURE TYPE, 41GR291

\begin{tabular}{|c|c|c|c|c|}
\hline \multirow[b]{2}{*}{ Plant Taxa } & \multicolumn{4}{|c|}{ Feature Type (No. of Flotation Samples) } \\
\hline & $\begin{array}{l}\text { Storage } \\
\text { Pits (11) } \\
\end{array}$ & $\begin{array}{l}\text { Pithouse } \\
\text { Fill and } \\
\text { Floor (4) } \\
\end{array}$ & $\begin{array}{l}\text { Hearth/ } \\
\text { Baking } \\
\text { Pits (3) } \\
\end{array}$ & $\begin{array}{l}\text { Charcoal/ } \\
\text { Burned Rock } \\
\text { Scatters (10) } \\
\end{array}$ \\
\hline Chenopodium sp. & 195 & 19 & 41 & 101 \\
\hline Amaranthus sp. & - & 2 & - & 2 \\
\hline Polygonum sp. & - & - & - & 2 \\
\hline Argemone sp. & - & 2 & - & 1 \\
\hline Portulaca sp. & 8 & 2 & 1 & 5 \\
\hline Cucurbitaceae & & & & \\
\hline (cf. Lagenaria sp.) & - & - & 2 & 5 \\
\hline Mollugo verticillata & 1 & - & - & - \\
\hline Prosopis glandulosa & 3 & 13 & - & 1 \\
\hline Ziziphus obtusifolia & - & 2 i & - & 1 \\
\hline Celtis reticulata & 1 & 3 & - & - \\
\hline Bromus sp. & 6 & - & - & - \\
\hline Paspalum sp. & 7 & - & - & - \\
\hline Sporobolus sp. & 5 & - & - & - \\
\hline Panicum sp. & 20 & - & - & - \\
\hline Unknown "B" & 4 & - & - & - \\
\hline Total Count & $\begin{array}{c}250 \\
(70)^{*}\end{array}$ & 43 & 44 & 118 \\
\hline $\begin{array}{l}\text { Total Volume of } \\
\text { Samples (liters) }\end{array}$ & $\begin{array}{c}54.0 \\
(50.0)^{*} \\
\end{array}$ & 49.0 & 60.0 & 55.5 \\
\hline $\begin{array}{l}\text { Concentration } \\
\text { (seeds/liter) }\end{array}$ & $\begin{array}{c}4.6 \\
(1.5)^{*}\end{array}$ & 0.9 & 0.7 & 2.1 \\
\hline \multicolumn{5}{|c|}{$\begin{array}{l}\text { *Figures in parentheses reflect seed counts and concentrations for } \\
\text { pits that exclude Sample } 48 \text { (which had } 177 \text { Chenopodium seeds). } \\
\text { The lower figure of } 1.5 \text { seeds/liter is a more accurate reflection of } \\
\text { the seed recovery from pits. }\end{array}$} \\
\hline
\end{tabular}

probably resulted from accidental charring during parching.

The plant content of the features interpreted as storage pits is somewhat more difficult to interpret. For example, during the course of site occupation, the function of such pits often changes from storage to refuse disposal. Sometimes pits are cleaned out when the site is abandoned, and the fill is related to postabandonment formation processes :(Dickens 1985:44-45). To complicate matters, the archeological evidence for the function of the pit features at 41GR291 and their postuse histories (see Chapter 3) is not definitive. None of the features under study had been burned catastrophically resulting in the 
preservation of charred plant remains in their primary ex facto context (Hally 1981; Miksicek 1987), and no charred cultigens or other commonly stored plants such as dried mesquite pods were recovered from the pit features. Therefore, the content of these pits may be background scatter that is related to general site activities instead of any specific original use of the pits.

The pits did contain grass and chenopod seeds. Functional storage pits, however, often are lined with grass. This grass usually serves to absorb moisture and to keep the stored material, which was often contained in skin bags, above the bottom of the storage pit (Young 1992). This could explain the presence of grass seeds. Many groups also stored grasses and chenopod seeds in pits (Young 1992). Feature 19 at $41 \mathrm{GR} 291$ contained 177 chenopod seeds. Whether this is a result of a natural rain of seeds carbonized by grass fires falling into a pit or a result of food preparation activities is difficult to determine. More sites need to be analyzed to more completely document the utilization of goosefoot and pigweed by prehistoric groups in the Southern Plains region. It is interesting to note that no carbonized goosefoot was recovered from 41KT51 and 41KT53 (Dering 1993), increasing the possibility that the goosefoot from 41GR291 is the result of food preparation activities.

\section{Carbonized Wood as an Environmental Indicator}

Both sites produced an excellent record of carbonized wood fragments, especially from the hearths at 41GR559 and from the midden, pithouse, and hearth areas at 41GR291. The carbonized wood frequencies from hearths at 41GR559 are presented in Table 81, and the frequencies from all features at 41GR291 are presented in Table 82. The sites reflect very distinct patterns of wood utilization. At 41GR291, juniper wood was overwhelmingly utilized, especially in the hearths. At 41GR559, twigs of hardwood, most likely from the xeric shrubs surrounding the site, are much more abundant than juniper. Because both sites are located in generally similar vegetation zones, it is difficult to interpret these differences in the wood assemblage.

The hearth features at 41GR559 did contain especially high figures for the "hardwood" wood type, which is composed almost exclusively of pith-bearing hardwood stem fragments. In addition, most of the juniper wood type at 41GR291 was recovered in twig form that measures less than $5 \mathrm{~cm}$ in diameter. The size of the source material as reflected by the carbonized wood may indicate a local environment that produced a marginal supply of firewood at both sites.

Paleoenvironmental assessments of archeological sites often have been based on the analysis of archeological wood recovered from hearth features (Miksicek 1985; Weir and Dering 1986). The analysis is based on the assumption that the macrobotanical assemblage in the hearths reflects the immediate availability of the wood in the area. In the study of the Lomas of Paloma (Weir and Dering 1986), decreasing stem size of the carbonized wood in the hearths over a period of 2,000 years was interpreted to reflect environmental degradation, most likely due to human impact. Miksicek's (1985) analysis spanned a period of a few hundred years. These studies indicate the feasibility of interpreting the hearth assemblages at 41GR291 and 41GR559 as an indication of environmental stress. Lacking diachronic data, however, it is difficult to make a definitive statement regarding the nature and extent of environmental degradation.

\section{CONCLUSIONS}

The following conclusions can be offered regarding the specific archeological questions posed at the beginning of this appendix.

(1) Are cultivated plants present in the samples? Other than the possible remains of bottle gourd, no cultivated plants were recovered from the flotation samples. This by no means rules out the utilization of cultigens by the inhabitants of the sites. It does greatly reduce the possibility that macrobotanical remains of cultigens will be recovered at the sites unless a burned structure or a much larger site area is excavated. Larger flotation samples may or may not produce an increase in species recovery. In order to determine the efficacy of increasing flotation sample size, a taxa recovery curve would need to be constructed. This could be accomplished by processing flotation samples in a series of increasing volumes from similar sites and features in the same region. By counting the average number of taxa from each sample volume size, a curve could be constructed. For example, at Hohokam sites Miksicek (1987:232) has shown that processing more than 4 liters of sample volume does not produce an 


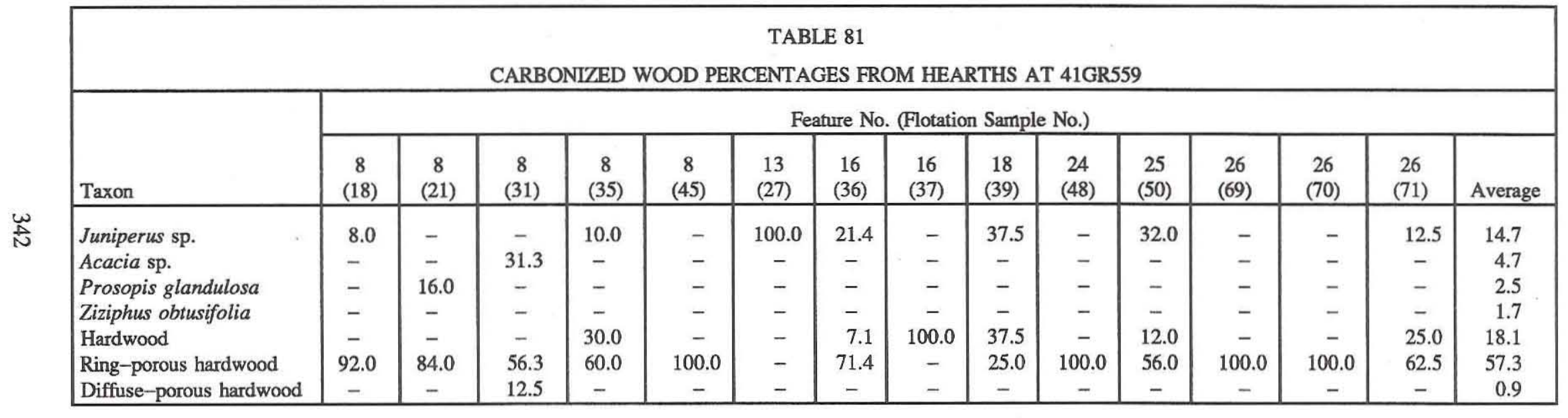




\begin{tabular}{|c|c|c|c|c|c|c|c|c|}
\hline \multicolumn{9}{|c|}{$\begin{array}{c}\text { TABLE } 82 \\
\text { SUMMARY OF CHARRED WOOD RECOVERY BY FEATURE TYPE AT 41GR291 }\end{array}$} \\
\hline \multirow[b]{2}{*}{ Taxon } & \multicolumn{2}{|c|}{ Storage Pits $(11)^{*}$} & \multicolumn{2}{|c|}{$\begin{array}{l}\text { Pithouse Fill and } \\
\text { Floor (4) }\end{array}$} & \multicolumn{2}{|c|}{$\begin{array}{c}\text { Hearths/Baking } \\
\text { Pits (3) } \\
\end{array}$} & \multicolumn{2}{|c|}{$\begin{array}{c}\text { Charcoal/Burned } \\
\text { Rock Scatters (10) }\end{array}$} \\
\hline & No. & $\%$ & No. & $\%$ & No. & $\%$ & No. & $\%$ \\
\hline Juniperus sp. & 38 & 32.2 & 13 & 39.4 & 57 & 71.3 & 93 & 75.0 \\
\hline Salix sp. & 2 & 1.7 & 3 & 9.1 & - & - & - & - \\
\hline Quercus sp. & 3 & 2.5 & - & - & - & - & 1 & 0.8 \\
\hline Prosopis glandulosa & - & - & - & - & - & - & 3 & 2.4 \\
\hline Celtis sp. & 7 & 5.9 & - & - & 3 & 3.8 & 4 & 3.2 \\
\hline Rosaceae (cf. Prunus sp.) & 2 & 1.7 & - & - & - & - & 5 & 4.0 \\
\hline Acacia sp. & 5 & 4.2 & - & - & - & - & - & - \\
\hline Ziziphus obtusifolia & 2 & 1.7 & - & - & 3 & 3.8 & 2 & 1.6 \\
\hline Rhus sp. & - & - & - & - & 2 & 2.5 & 3 & 2.4 \\
\hline Berberis trifoliata & 6 & 5.1 & - & - & - & - & - & - \\
\hline Forestieria sp. & 13 & 11.0 & - & - & 5 & 6.3 & - & - \\
\hline Hardwood & 34 & 28.8 & 17 & 51.5 & 10 & 12.5 & 5 & 4.0 \\
\hline Diffuse-porous hardwood & 1 & 0.8 & - & - & - & - & 2 & 1.6 \\
\hline Ring-porous hardwood & 5 & 4.2 & - & - & - & - & 6 & 4.8 \\
\hline Totals & 118 & 99.8 & 33 & 100.0 & 80 & 100.2 & 124 & 99.8 \\
\hline
\end{tabular}

effective increase in taxa represented in the samples. This figure is specific for Hohokam sites and does not necessarily apply to sites in other regions.

(2) What plants were gathered and what is their significance to the subsistence regimes? Charred mesquite seeds were recovered from the lower fill and floor of the pithouse at 41GR291. The context and charred condition of the seeds indicate that mesquite was utilized in the upper Brazos River drainage ca. 1,000 years ago (see Chapter 3). Goosefoot seeds were also present in significant numbers in the charcoal/burned rock scatters and pit features at 41GR291, indicating the possibility that this may have been an important wild resource. Other indications of gathered plants included the charred seeds of hackberry at 41GR559 and small numbers of seeds of knotweed, purslane, and several grasses. Wood types recovered indicate that juniper was most important at 41GR291 and that twigs of xeric hardwood shrubs were very important at 41GR559. The carbonized wood remains indicate that the fuel wood resource base probably was very limited in the region.

(3) What were the inter- and intrasite distributions of plant remains? Site 41GR291 contains much more variety and quantity in both the seed assemblage and the wood assemblage than does 41GR559. This is probably due to the greater intensity and duration of occupations at 41GR291. The intrasite patterning of the seed remains among features at 41GR291 shows concentrations of grass and goosefoot seeds in the pits and mesquite seeds in the pithouse. The charcoal/burned rock scatters reflect components from all over the site in lower numbers, although the high number of goosefoot seeds tends to push the seed concentrations higher in that feature type.

(4) What effects have formation processes had on the samples, and how does this affect interpretation of the macrobotanical assemblage? Which plant remains are intrusive, and which are part of the archeological record? Formation processes operating at both sites dictate that only carbonized seeds should be considered as part of the archeological record. The presence of carbonized grass seeds in the pits could be a function of human utilization of the seeds as food or as part of the storage process. On the other hand, the materials may have been deposited as part of a background scatter of seeds while the pit was being filled. 


\section{REFERENCES CITED}

Bryant, Vaughn M.

1993 Pollen Analysis of Archeological Sediments from 41KT53. Appendix I in Data Recovery at Justiceburg Reservoir (Lake Alan Henry), Garza and Kent Counties, Texas: Phase III, Season 2, by Douglas K. Boyd, Jay Peck, Steve A. Tomka, and Karl W. Kibler, pp. 435-443. Reports of Investigations No. 88. Prewitt and Associates, Inc., Austin.

Castetter, E. F., and W. H. Bell

1937 The Utilization of Mesquite and Screwbean in the American Southwest. University of New Mexico Biological Series No. 314. Albuquerque.

Crosswhite, Frank S.

1981 Desert Plants, Habitat and Agriculture in Relation to the Major Pattern of Cultural Differentiaton in the O'odham People of the Sonoran Desert. Desert Plants 3(2):47-76.

Cummings, Linda Scott

1990 Phytoliths and Pollen Analyses: A Feasibility Study. Appendix I in Phase II Investigations at Prehistoric and Rock Art Sites, Justiceburg Reservoir, Garza and Kent Counties, Texas, by Douglas K. Boyd, James T. Abbott, William A. Bryan, Colin M. Garvey, Steve A. Tomka, and Ross C. Fields, pp. 533-550. Reports of Investigations No. 71. Prewitt and Associates, Inc., Austin.

Dering, J. Philip

1979 Pollen and Plant Macrofossil Vegetation Record from Hinds Cave, Val Verde County, Texas. Master's thesis, Texas A\&M University, College Station.

1993 Plant Remains. Appendix $\mathrm{H}$ in Data Recovery at Justiceburg Reservoir (Lake Alan Henry), Garza and Kent Counties, Texas: Phase III, Season 2, by Douglas K. Boyd, Jay Peck, Steve A. Tomka, and Karl W. Kibler, pp. 419-434. Reports of Investigations No. 88. Prewitt and Associates, Inc., Austin.

Dering, Phil, and Vaughn M. Bryant, Jr.

1992 Pollen Analysis of Archeological Sediments from Justiceburg Reservoir. Appendix E in Data Recovery at Justiceburg Reservoir (Lake Alan Henry), Garza and Kent
Counties, Texas: Phase III, Season 1, by Douglas K. Boyd, C. Britt Bousman, Steve A. Tomka, Karen M. Gardner, and Martha Doty Freeman, pp. 211-220. Reports of Investigations No. 84. Prewitt and Associates, Inc., Austin.

Dickens, Roy S.

1985 Form, Function and Formation of GarbageFilled Pits on Southeastern Aboriginal Sites: An Archaeological Analysis. Chapter 2 in Structure and Process in Southeastern Archaeology, edited by Roy S. Dickens and H. Trawick Ward, pp. 34-59. University of Alabama Press, University.

Hally, David J.

1981 Plant Preservation and the Content of Paleobotanical Samples: A Case Study. American Antiquity 46(4):723-742.

Hatch, Stephan L., K. N. Gandhi, and Larry E. Brown

1990 Checklist of the Vascular Plants of Texas. Publication No. MP-1655. Texas Agricultural Experiment Station, College Station.

Johnston, Marshall C.

1963 Past and Present Grasslands of Southem Texas and Northeastem Mexico. Ecology 44 (3): 456-466.

Jones, John G.

1990 Assessment of Pollen Preservation and Macrobotanical Analysis. Appendix $\mathrm{H}$ in Phase II Investigations at Prehistoric and Rock Art Sites, Justiceburg Reservoir, Garza and Kent Counties, Texas, by Douglas $\mathrm{K}$. Boyd, James T. Abbott, William A. Bryan, Colin M. Garvey, Steve A. Tomka, and Ross C. Fields, pp. 521-532. Reports of Investigations No. 71. Prewitt and Associates, Inc., Austin.

McMahan, Craig A., Roy G. Frye, and Kirby L. Brown 1984 Vegetation Types of Texas, Including Cropland. Bulletin 7000-120. Texas Parks and Wildlife Department, Austin.

Miksicek, Charles H.

1985 Paleoecology: The Charcoal Evidence. In Excavations at the Valencia Site, edited by William H. Doelle, pp. 235-238. Anthropological Papers 3. Institute for American 
Research, Tucson.

1987 Formation Processes of the Archaeobotanical Record. In Advances in Archaeological Method and Theory, vol. 10, edited by M. B. Schiffer, pp. 215-247. Academic Press, New York.

Weir, Glendon H., and J. Philip Dering 1986 The Lomas of Paloma: Human-Environment Relations in a Central Peruvian Fog Oasis:
Archaeobotany and Palynology. In Andean Archaeology: Papers in Memory of Clifford Evans, edited by Ramiro Matos M., Solveig Turpin, and Herbert H. Eling, Jr., pp. 18-44. Monograph XXVII. Institute of Archaeology, University of California, Los Angeles.

Young, Lisa C.

1992 Pits, Rooms, Baskets, Pots: Storage Among Southwestern Farmers. Paper presented at the Southwest Symposium, Tucson, Arizona, 



\title{
APPENDIX F: Analysis of Human Skeletal Remains from 41GR291
}

\author{
D. Gentry Steele
}

and

Barry W. Baker

Department of Anthropology

Texas A\&M University

College Station, Texas 77843-4352 



\section{INTRODUCTION}

The skeletal remains of one individual, a middle-aged adult male approximately $166 \mathrm{~cm}$ tall, were recovered during the 1992 field season at Lake Alan Henry (recorded as archeological Feature 23 at 41GR291). No discemible postcranial medical disorders other than dental wear and alveolar resorption could be detected in the skeletal remains.

\section{TAPHONOMY}

The remains shipped to the Physical Anthropology Laboratory at Texas A\&M University indicate that a nearly complete but highly comminuted and eroded skeleton was recovered. Portions of most of the skeletal elements were preserved and recovered, including elements from the cranium, the axial skeleton, the arms, hands, legs, and feet (Table 83). While most of the elements are present, virtually all elements have been highly fragmented postmortem. The angle of the fractures and their number indicate dry bone breakage, fragmentation which occurred after the protein matrix of the bone had deteriorated. Further, the surfaces of all cortical bones are extensively degraded. The degradation appears to be the result of chemical erosion and root etching while the skeleton was shallowly buried. Although the orientation of the bones in the grave strongly indicates that the burial represents a secondary interment (see Chapter 3), the degraded condition of the cortical bones does not provide supportive evidence of this conclusion.

\section{ESTIMATION OF SEX}

The individual appears to have been a male. The preserved remains of the lateral aspect of the left brow ridge, the sciatic region of both ilia, and the shape of the chin all indicate the individual was a male. It should be noted, however, that these features are not markedly masculine in expression. Body size is a further indication that the individual was a male.

Stature, based upon the tibia, indicates the individual was approximately $166 \mathrm{~cm}$ tall, well within the typical height range of prehistoric American Indian males from the Trans-Pecos region and western Texas but slightly shorter than the average height of males from central Texas and northeastern Texas (Table 84).

Also supporting this assessment is the size of the talus. The discriminant function score for the talus (after Steele 1976), based upon talus length, width, height, and trochlear surface length and width, is 49.6. Such a discriminant function score indicates that the individual's talus was slightly smaller than the mean for a sample of prehistoric males from a population of relatively large American Indians from the Upper Missouri region of the central plains, but larger than the average male talus of a population of relatively small people from the Pecos Site in New Mexico (Steele 1976). The talus of the Lake Alan Henry individual is larger than the means for females of both populations.

\section{ESTIMATION OF AGE}

The degree of comminution of the skeleton precludes evaluation of age based upon the more diagnostic elements, such as the pubic symphysis. However, several lines of evidence support the assessment that the individual was an adult in his late 20 s to mid 40 s. All articular surfaces preserved show obliterated epiphyseal fusion lines. The third molars had erupted, and occlusal wear had proceeded to the point where dentin is exposed on more than $20 \%$ of these teeth. All of these observations indicate the individual was past adolescence and at least into early adulthood. On the other hand, there is no evidence of marked osteoporosis or degenerative joint diseases typically associated with old age. Therefore, the best assessment is that the individual was an adult, between his late 20 s to mid 40 s.

\section{BODY SIZE AND SHAPE}

Although the skeleton is highly comminuted, there is sufficient material to get an impression of the individual's body size and shape (Tables 85 and 86 provide skeletal and dental measurements). Most of the left tibia (segments 2-5) is preserved intact, permitting an estimation of its complete length following Steele and Bramblett (1988). The estimated complete length is $37.1 \mathrm{~cm}$. A tibia of this length suggests the individual was $166 \pm 2.8 \mathrm{~cm}$ tall, following Genovés's (1967) formulae for estimating stature based upon recent Mesoamericans. The tibia length and the estimate of stature of the Lake Alan Henry male corresponds well with average tibia lengths and stature estimates of males of the TransPecos, Lower Pecos, and Tamaulipas regions of Texas and Mexico, but he was slightly shorter than 
TABLE 83

HUMAN SKELETAL INVENTORY, 41GR291

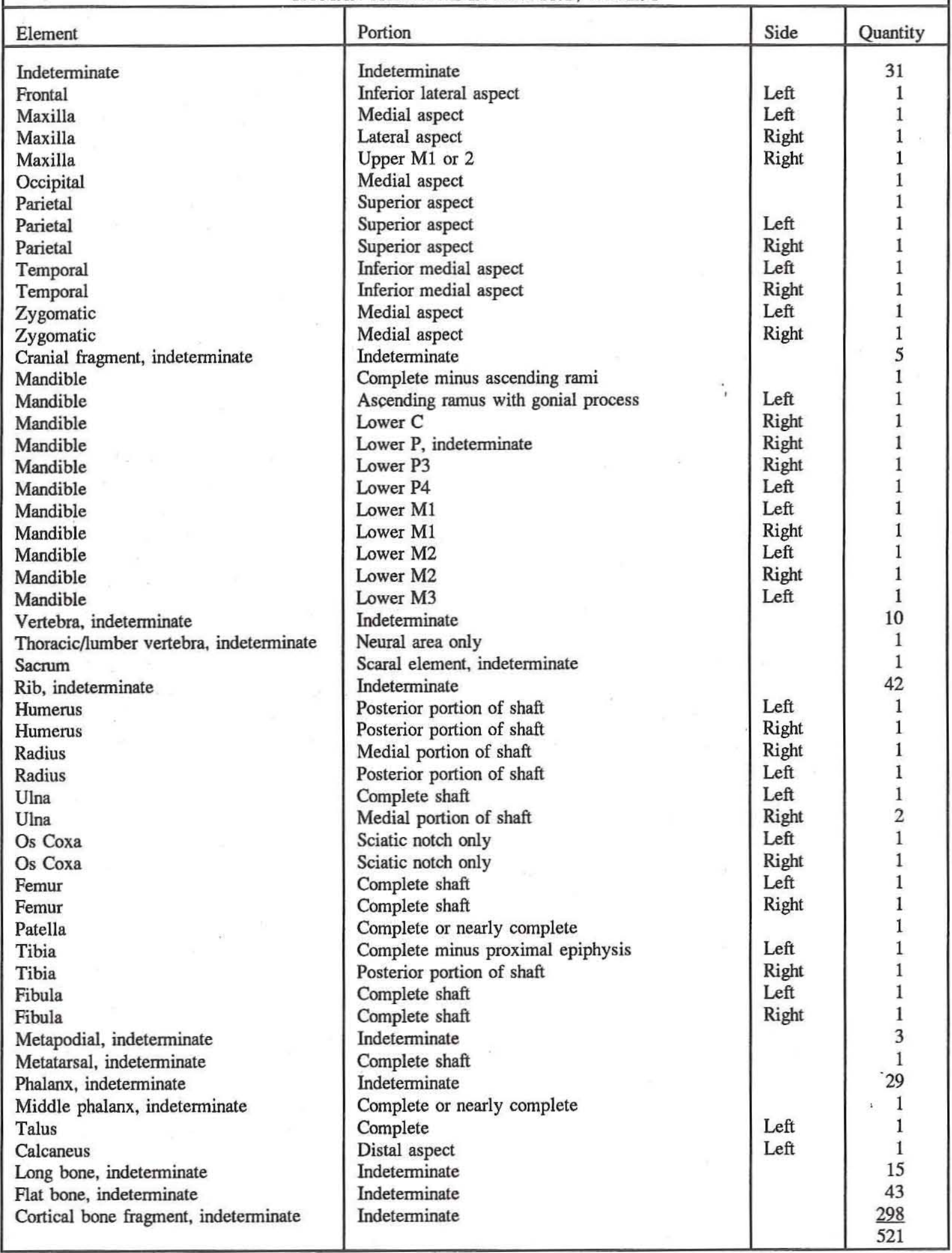


TABLE 84

TIBIA LENGTH AND STATURE OF PREHISTORIC TEXAS POPULATIONS

\begin{tabular}{|l|c|c|c|c|c|c|}
\hline \multirow{2}{*}{ Locality } & \multicolumn{4}{|c|}{ Tibia Length } & \multicolumn{3}{c|}{ Stature } \\
\cline { 2 - 8 } & No. & $\overline{\mathrm{x}}(\mathrm{cm})$ & Standard Deviation & No. & $\overline{\mathrm{x}}(\mathrm{cm})$ & Standard Deviation \\
\hline Lake Alan Henry & 1 & 37.1 & - & 1 & 166.0 & 2.8 \\
\hline Trans-Pecos & 2 & 37.2 & 0 & 6 & 167.4 & 2.6 \\
\hline Lower-Pecos & 12 & 37.9 & 1.2 & 21 & 166.0 & 4.2 \\
\hline Tamaulipas & - & - & - & 8 & 166.7 & 4.8 \\
\hline Loeve-Fox & - & - & - & 12 & 168.7 & - \\
\hline Caddo & - & - & - & 14 & 169.0 & - \\
\hline
\end{tabular}

\begin{tabular}{|c|c|c|}
\hline $\begin{array}{c}\text { TABLE } 85 \\
\text { POSTCRANIAL METRICS, 41GR291 }\end{array}$ & & \\
\hline Element and Measurement (mm) & Left & Right \\
\hline $\begin{array}{l}\text { Humerus } \\
\text { Minimum circumference }\end{array}$ & 60 & 61 \\
\hline $\begin{array}{l}\text { Femur } \\
\text { Segment } 2 \\
\text { Midshaft circumference } \\
\text { Anterior-posterior diameter } \\
\text { Transverse diameter } \\
\text { Anterior subtrochanteric diameter } \\
\text { Transverse subtrochanteric diameter }\end{array}$ & $\begin{array}{l}- \\
86 \\
28 \\
27 \\
25 \\
30\end{array}$ & $\begin{array}{r}265 \\
88 \\
28 \\
28 \\
25 \\
33\end{array}$ \\
\hline $\begin{array}{l}\text { Tibia } \\
\text { Maximum length (excludes spines) } \\
\text { Segment } 2 \\
\text { Segment } 3 \\
\text { Segment } 4 \\
\text { Segment } 5 \\
\text { Midshaft circumference } \\
\text { Circumference at nutrient foramen } \\
\text { Minimum circumference } \\
\text { Anterior-posterior diameter (nutrient foramen) } \\
\text { Transverse diameter (nutrient foramen) }\end{array}$ & $\begin{array}{r}(371) \\
70 \\
192 \\
92 \\
10 \\
100 \\
99 \\
83 \\
38 \\
23 \\
\end{array}$ & $\begin{array}{l}- \\
- \\
- \\
- \\
- \\
- \\
- \\
-\end{array}$ \\
\hline $\begin{array}{l}\text { Talus } \\
\text { Maximum length } \\
\text { Maximum width } \\
\text { Body height } \\
\text { Trochlear maximum length } \\
\text { Trochlear maximum width }\end{array}$ & $\begin{array}{l}51 \\
41 \\
29 \\
32 \\
35\end{array}$ & $\begin{array}{l}- \\
- \\
- \\
- \\
-\end{array}$ \\
\hline
\end{tabular}




\begin{tabular}{|l|c|c|}
\hline \multicolumn{3}{|c|}{ TABLE 86 } \\
\multicolumn{3}{|c|}{ DENTAL METRICS, 41GR291 } \\
\cline { 2 - 3 } Tooth & \multicolumn{2}{|c|}{ Cervical } \\
\cline { 2 - 3 } $\mathrm{RM}_{2}$ & 10.0 & 9.5 \\
\hline $\mathrm{RM}_{1}$ & 10.0 & 9.5 \\
\hline $\mathrm{RP}_{3}$ & 5.5 & 7.5 \\
\hline $\mathrm{RC}_{1}$ & 5.5 & 7.5 \\
\hline $\mathrm{LP}_{4}$ & 5.0 & 7.0 \\
\hline $\mathrm{LM}_{1}$ & 9.5 & 9.5 \\
\hline $\mathrm{LM}_{2}$ & 10.0 & 10.0 \\
\hline $\mathrm{LM}_{3}$ & 10.0 & 10.0 \\
\hline
\end{tabular}

the average stature reported for Indian males of central and east Texas (see Table 84).

Two other features of the postcranial skeleton could be documented - the shapes of the femur and tibia shafts. A common osteometric index compares the anterior/posterior diameter of a long bone shaft to its transverse diameter. The anterior/posterior diameter of the femur shaft of the Lake Alan Henry male is relatively flat (platymeric), while the transverse diameter of the tibia is relatively narrow (platycnemic). Both of these shapes are common in American Indian populations.

Little could be discerned about the health of the individual during life or the cause of death. There are no observable traumatic injuries to the skeleton and no postcranial evidence of past medical disorders.

The teeth are wom (Table 87) but not to the degree that they represent a pathological condition, and no caries were observed. The alveolar borders of all teeth are resorbed, exposing the cervical margin of the crown and the uppermost part of the roots of the teeth. There is no subperiosteal bone deposition indicative of infection of the gums.

\begin{tabular}{|l|c|c|c|}
\hline \multicolumn{4}{|c|}{ TABLE 87 } \\
TOOTH WEAR AND ALVEOLAR \\
RESORPTION, 41GR291
\end{tabular}




\section{REFERENCES CITED}

Genovés, S.

1967 Proportionality of Long Bones and Their Relation to Stature among Mesoamericans. American Journal of Physical Anthropology 26:67-78.

Scott, E. C.

1979 Dental Wear Scoring Technique. American Journal of Physical Anthropology 51:213-218.

Smith, B. H.

1984 Patterns of Molar Wear in Hunter-Gatherers and Agriculturists. American Journal of Physical Anthropology 63:39-56.

Steele, D. G.

1976 The Estimation of Sex on the Basis of the Talus and Calcaneus. American Journal of Physical Anthropology 45:581-588.

Steele, D. G., and C. A. Bramblett

1988 The Anatomy and Biology of the Human Skeleton. Texas A\&M University Press, College Station.

Steele, D. G., and J. F. Powell

n.d. An Osteological Examination of Prehistoric Hunters and Gatherers of the Southern Desert and Semi-Arid Regions of North America. In The Archaic of the Southern North American Desert, edited by H. J. Shafer, D. L. Carlson, and K. D. Sobolik. In Review, Texas A\&M Press, College Station. 



\title{
APPENDIX G: Petrographic Analysis of Plainwares from 41GR291
}

\author{
David G. Robinson
}

Texas Archeological Research Laboratory The University of Texas at Austin 



\section{INTRODUCTION}

A petrographic analysis was performed on five ceramic potsherds recovered from the Sam Wahl site, 41GR291, at Lake Alan Henry in the Texas South Plains region. It was thought that the collected sherds, all plainwares, might be nonlocal types, and the research was designed to examine this hypothesis and augment the typological analysis of the pottery. Specifically, the sherds were examined petrographically and compared with other forms of geological data to attempt to identify the regional sources of the constituents of the pottery, and hence the regions of origin of the ceramics. Further, the sherds were examined for similarities and differences with the ceramic assemblages excavated and studied from other sites at Lake Alan Henry and in the surrounding region (Boyd and Reese-Taylor 1993; Robinson 1992). It was hoped that this examination might reveal pattems of site and ceramic function among the regional sites and suggest the nature of regional and interregional exchange systems.

\section{METHODOLOGY}

The petrographic analysis was accomplished by thin section analysis, pioneered in archeology by Shepard $(1942,1954)$. Comparisons of the determined mineralogy with ceramics from New Mexico were made by reference to petrographic studies of ceramics there (Garrett 1988a, 1988b, 1988c; Hill 1988, 1990; Rugge 1985; Southward 1979). The thin sections were prepared at the Bureau of Economic Geology, The University of Texas at Austin, Balcones Research Center. Rock and mineral identifications and point-counting of 200 bodies or attributes were accomplished on a stereo petrographic microscope following the method of Chayes (1949). All physical attributes of the thin sections, including matrix and pore space, entered the point-counting as they all contribute importantly to ceramic patterning. The result of this procedure and methodology is a consistent and systematically collected body of data comparable to similarly studied data sets in the region and New Mexico. Point-count data also may be manipulated by a variety of statistical measures.

\section{RESULTS}

All five plainware sherds from the Sam Wahl site have mineral and rock constituents entirely of crushed angular and subangular igneous rocks. Furthermore, the size distributions of the particles in the thin sections are all seriate, ranging from very fine to granule size. These basic features of the collection give it clear affinities with heavily volcanic areas to the west, in New Mexico or in Trans-Pecos Texas, rather than the Texas South Plains. The angularity and seriate size distributions of the sections also are consonant with the aboriginal Southwestern ceramic tradition of crushed mineral tempering, as opposed to other materials and modes of tempering practiced in West and Central Texas. The present study collection has strong westward affinities, in keeping with other plainware (Robinson 1992) and painted tradeware (Boyd and ReeseTaylor 1993) collections unearthed from archeological sites at Lake Alan Henry.

Table 88 presents the mineral and ceramic attribute data of the collection, all expressed as percentages of the 200-point count. The rock and mineral data are organized as nearly as possible according to similar rocks and the minerals that constitute or are associated with them. This was done as an aid to petrological grouping.

Based on petrology alone, the thin sections fall into two groups. Group 1 consists of Sherds 367-3, 521-1, and 472-1. Group 2 contains Sherds 306-1 and $8-0$. Group 1 is defined by the significant presence of a granitic rock and its attendant minerals; Group 2 is keyed by basaltic rock and related minerals as a major tempering agent. It is noteworthy that all of the sherds have at least $44 \%$ coverage of the section as ceramic matrix and from $7 \%$ to $17 \%$ coverage as pore space. It is apparent that in this case, perhaps due to the small size of the assemblage, matrix and pore space alone are insufficient to discern differences in the ceramics. It is also notable that the maximum size of particles in both groups is the same (granule), and this attribute similarly has little discriminating value. The potential significance of the groupings in terms of the research questions is discussed below.

Sherd 367-3: This sherd is the most typical of the Group 1 sherds in having granitic rock fragments (Granitic Rock A), attendant free particles of quartz and orthoclase, and a trace of nonferrous opaques. The quartz and orthoclase are constituent minerals of the granitic rock.

Sherd 521-1: This sherd shows the same constituents as Sherd $367-3$, but the proportions of common quartz to orthoclase are reversed. This may 


\begin{tabular}{|l|c|c|c|c|c|}
\hline \multicolumn{5}{|c|}{ TABLE 88 } \\
\hline \multicolumn{5}{|c|}{ CERAMIC ATTRIBUTES AND POINT COUNT DATA, 41GR291* } \\
\hline Attributes & Sherd & Sherd & Sherd & Sherd & Sherd \\
\hline Matrix & $367-3$ & $521-1$ & $472-1$ & $306-1$ & $8-0$ \\
Pore Space & 44.0 & 57.5 & 46.0 & 44.5 & 47.5 \\
Common Quartz & 17.0 & 7.0 & 14.0 & 15.0 & 12.5 \\
Granitic Rock A & 23.5 & 8.0 & - & 11.0 & 12.5 \\
Orthoclase & 9.0 & 6.5 & 5.5 & - & - \\
Biotite & 6.0 & 20.5 & 33.5 & - & 12.0 \\
Celadonite & - & - & 1.0 & 1.5 & - \\
Nonferrous Opaques & - & - & - & 15.5 & - \\
Basaltic Rock A & T & T & - & - & - \\
Basaltic Rock B & - & - & - & 2.0 & - \\
Plagioclase & - & - & - & - & 3.0 \\
Pyroxene & - & - & - & 8.0 & - \\
Amphibole & - & - & - & - & 1.0 \\
Tourmaline & - & - & - & - & 1.0 \\
Chlorite & - & - & - & 2.5 & - \\
Unknown Rock A & - & - & - & - & 1.0 \\
Ferrous Opaques and & - & - & - & - & 8.5 \\
Iron Stain & - & - & T & - & 1.0 \\
\hline Maximum Grain Size (mm) & 1.5 & 2.5 & 1.9 & 1.5 & 2.5 \\
\hline *Data are given as percentages of the 200-point count; T = trace. \\
\hline
\end{tabular}

clase, sanidine, and a trace of an amphibole. Quartz, orthoclase, and amphibole are distributed throughout the matrix as well. Pyroxene as an accessory mineral of basaltic rocks also was observed in the matrix. The basaltic rock in this sherd varies from the more common composition of the basaltic rock in Sherd 306-1 in having some orthoclase and amphibole. Sherd $8-0$ is the only thin section with Unknown Rock A, a rock with specimens in the section containing either orthoclase and a firstorder yellow accessory mineral or quartz with some chlorite. If fragments of these rocks were observed together, they would meet the criteria of definition of a granitic rock. If this were the case, the original pottery vessel would have had a mixture of

or may not be due to the vagaries of temper crushing and the mixing of ceramic pastes, but it does not materially affect group membership as established here.

Sherd 472-1: This sherd has granitic rock fragments (Granitic Rock A), orthoclase, biotite in association, and ferrous opaques. A peculiarity is the lack of common quartz as a species broken free from the rock.

Sherd 306-1: This is the typical Group 2 specimen, having basaltic rock fragments (Basalt A) composed of plagioclase, quartz, some pyroxene, and an amphibole, possibly basaltic hornblende. Quartz and plagioclase were counted as free species as well. There is a small amount of biotite with a large proportion of a related mineral, celadonite, well distributed through the slide. Celadonite generally occurs in cavities of basaltic rocks (American Geological Institute 1962:76). Tourmaline also was found in the thin section, the only instance of its occurrence in the study collection.

Sherd 8-0: This sherd was assigned to Group 2 , but it has problematic aspects. It contains a basaltic rock (Basalt B) composed of quartz, ortho- constituents from variant igneous petrogenetic sources. The section also has ferrous opaques and iron stain distributed tightly along one edge of the ceramic fabric; this appears superficially to be the remains of an iron-based slip or red paint. The material is worked well into the ceramic fabric, however, and is not sitting on the surface in a discrete layer. The material may have been concentrated there by polishing or smoothing the surface. The layer is near the interior surface of the aboriginal vessel. If the reddish iron stain was added deliberately to the vessel during manufacture as a colorant and part of its decoration (and this must remain hypothetical), then the original vessel was a bowl in which the interior surface was visible.

\section{DISCUSSION}

The typology and volcanic character of the collection strongly suggest two different nonlocal sources for the sherds. In the Texas South Plains region, the surface geology apart from Quaternary deposits is comprised of sedimentary rocks and deposits of the Permian Quartermaster Formation and 
Triassic Dockum Group. Minor fractions of stream-rolled volcanic sands are found, however, in modem stream deposits throughout the PanhandlePlains area. These small, rounded fractions cannot account for the subangular and angular coarse-sandsized volcanics in the study collection. The nearest region where fresh igneous rocks are abundant is the Middle Pecos River valley of eastern New Mexico. The river receives sediments from numerous mountain ranges that expose volcanic and metamorphic rocks. Pleistocene terraces in the Middle Pecos valley contain granite and metamorphic gravels in abundance (Jelinek 1967). As an outside possibility, the Wichita Mountains of southwestern Oklahoma also expose igneous rocks.

It remains now to make comparisons and attempt to make more-precise estimates of regions of ceramic origin. A summary of pertinent Southwestern petrological studies (Garrett 1988a, 1988b, 1988c; Hill 1988, 1990; Rugge 1985; Southward 1979) shows no clear identifications with the Lake Alan Henry collection but does yield several useful observations. The Group 1 granitic sherds compare moderately well with studies by Garrett (1988b) of sherds from eastern New Mexico, which have high amounts of monzonite, a volcanic rock related to granite. The three brownware sherds she studied vary from the Group 1 sherds in also having syenite and homblende but very few free crystals of quartz or feldspars. Garrett compared her sherds with similarly studied thin sections from Conchas Lake in northeastem New Mexico. Her comparisons show great dissimilarities, principally that the Conchas Lake sherds are tempered with metamorphic rock. Garrett (1988c:G-5) also cites a Quaternary basalt flow located $30 \mathrm{~km}$ north of Conchas Lake as a potential source of basaltic rocks.

Garrett (1988a) found that a majority of sherds examined by thin section analysis from a site in $\mathrm{El}$ Paso were manufactured from nonlocal rocks, and the nearest natural outcrops of these materials were in southern New Mexico. Interestingly, both the El Paso Brown and El Paso Polychrome sherds in her sample have virtually identical rocks and minerals in their ceramic fabrics, and the crushed monzonite used as a tempering agent was available no closer than the eastern flanks of the Organ Mountains east of Las Cruces, New Mexico. This is a clear example of both a painted type and a plain type being used as tradewares, or at least the two types being transported comparable distances.
Hill (1988) identified granite temper in eight sherds from Pickup Pueblo in northeast El Paso. By comparison with thin sections and published data on granites outcropping in the Franklin Mountains, Hill makes a strong case for the ceramic temper originating in the Red Bluff Granite in the eastern flanks of the Franklin Mountains and their outwash drainages. The perthic feldspars, microcline, and biotite in the Pickup Pueblo thin sections set this granite apart from those in the present study collection as well as those of Garrett's (1988a) study. Hill $(1988,1990)$ also suggests that, since granite cracks readily when heated, it would crush easily for tempering material after use as hearthstones.

Rugge (1985) conducted a petrographic analysis on unspecific brownwares (16 sherds), El Paso Brown (1 sherd), and El Paso Polychrome (1 sherd) from southem New Mexico. He compared them with petrologic data on Jornada Brown sherds from the vicinity of Three Rivers, New Mexico. Two granitic rocks were identified as the tempering agents in the collections, the El Paso Brown and El Paso Polychrome sherds being virtually identical in their paste and mineral compositions. The granites were assigned tentatively to source outcrops in the southem San Andres Mountains; direct thin section analysis of granite samples from there lay beyond the scope of Rugge's (1985:128) study. Moderate to heavily altered microperthite in both the granites gives them clear distinctions from the granitic rocks in the Lake Alan Henry collection.

Southward (1979) reports a petrographic study by Dale Rugge of ceramics from a site in the Tularosa Basin, just south of Three Rivers Petroglyph Park. Six sherds each of six ceramic types were analyzed, including Jornada Brown. The Jornada Brown sherds are tempered with granite-gneiss and granite. Microperthite, quartz, and plagioclase are the major constituents of the minerals. Hornblende, biotite, and muscovite are minor and trace contributors to the thin sections. This composition shows strong contrasts with the Lake Alan Henry sample. Southward suggests that these granites and granitegneisses were available naturally all along the eastern escarpment of the San Andres Mountains. Further, among the El Paso Polychrome sherds are two granitic tempering agents. Granite A is closely similar to the granite in the other types in the sample, but Granite B contains the rare mineral xenotime, for which no source area could be identified. From this Southward suggests that El Paso Polychrome had 
different sources, locales of manufacture, and modes of introduction to the site than the other five types studied (Southward 1979:100).

At Lake Alan Henry and its vicinity, this small collection is notable for the virtual lack of biotite, a signature attribute of many of the plainware sherds from 41KT33, 41SW23, and all but the Jornada Brown sherds from 41HL66 (Robinson 1992). There, biotite helped confirm the typological analysis of origin in the Middle Pecos region, where abundant micas enter ceramics frequently. The reasons for these differences in origin may well involve differing forms or fluctuations of exchange systems, but the issue is difficult to pursue with such a small collection. In conclusion, the five thin sections examined in this analysis are from sherds tempered with crushed volcanic rocks and represent wares of a
Southwestem ceramic tradition. Two distinct compositional groups - one characterized by granitic rocks and the other dominated by basaltic rocks appear to represent two different manufacturing source areas. Specific source areas for these groups cannot be defined, however, based on the evidence currently available.

\section{ACKNOWLEDGMENTS}

The help, facilitation, forebearance, and good humor of Dr. Shirley Dutton of the Bureau of Economic Geology, Balcones Research Center, are gratefully acknowledged. Mr. Arten Avakian, also of the Bureau, provided enlightening discussions on igneous petrology and helped identify some of the minerals in the thin sections. 


\section{REFERENCES CITED}

American Geological Institute

1962 Dictionary of Geological Terms. Dolphin Books, Doubleday and Company, Inc., Garden City, New York.

Boyd, Douglas K., and Kathryn Reese-Taylor

1993 Petrographic Analysis of Ceramic Sherds and Clay Samples. Appendix D in Data Recovery at Justiceburg Reservoir (Lake Alan Henry), Garza and Kent Counties, Texas: Phase III, Season 2, by Douglas K. Boyd, Jay Peck, Steve A. Tomka, and Karl W. Kibler, pp. 339-378. Reports of Investigations No. 88. Prewitt and Associates, Inc., Austin.

Chayes, F.

1949 A Simple Point-Counter for Thin Section Analysis. American Mineralogist 34:1-11.

Garrett, Elizabeth M.

1988a Petrographic Analysis of Twelve Sherds from Site 41EP2611, El Paso, Texas. Appendix C in Class III Survey and Testing of Cultural Resources in Proposed Flood Control System Rights-of-way, Southeastern El Paso, El Paso County, Texas, by Amy C. Earls and Darryl C. Newton. Prepared for United States Army Corps of Engineers, Albuquerque District, by Mariah Associates, Inc., Albuquerque.

1988b Petrographic Analysis of Prehistoric Ceramics from the Melrose Range Project, ACOE, 1987. Appendix $\mathrm{H}$ in Class II Survey and Testing of Cultural Resources at the Melrose Air Force Range, Curry and Roosevelt Counties, New Mexico, by Christopher Lintz, Karen Kramer, Amy Earls, W. Nicholas Trierweiler, Terry Del Bene, John Acklen, Fred Nials, and Jack Bertram. Mariah Associates, Inc., Albuquerque.

1988c Petrographic Analysis of Selected Conchas River Ceramics. Appendix G in Report of the 1986 and 1987 Class II Surveys and Testing of Cultural Resources at Conchas Lake, New Mexico, by Karen Kramer, Christopher R. Lintz, W. Nicholas Trierweiler, Stephen Lent, John Frizell, Mary Stiner, John C. Acklen, and Steven Kuhn. Mariah Associates, Inc., Albuquerque.

Hill, David V.

1988 Petrographic Analysis of El Paso Polychrome Pottery from Pickup Pueblo. Appendix D in
Pickup Pueblo: A Late Prehistoric House Ruin in Northeast El Paso. The Artifact 26(2):75-84.

1990 Ceramic Production and Settlement Pattems in the Paso del Norte. In Historia Regional Comparada 1990. Actas del Segundo Congreso, pp. 29-40. Universidad Autonoma de Ciudad Juarez, Mexico.

Jelinek, Arthur J.

1967 A Prehistoric Sequence in the Middle Pecos Valley, New Mexico. Anthropological Papers 31. Museum of Anthropology, University of Michigan, Ann Arbor.

Robinson, David G.

1992 Petrographic Analysis of Nonlocal Plainwares. Appendix F in Data Recovery at Justiceburg Reservoir (Lake Alan Henry), Garza and Kent Counties, Texas: Phase III, Season 1, by Douglas K. Boyd, Steve A. Tomka, C. Britt Bousman, Karen M. Gardner, and Martha Doty Freeman, pp. 221-227. Reports of Investigations No. 84. Prewitt and Associates, Inc., Austin.

Rugge, Dale R.

1985 Petrographic Analysis of Ceramic Technology. In Archeological Testing at the Fairchild Site (LA45732), Otero County, New Mexico, edited by Roger Anyon, pp. 121-135. Office of Contract Archeology, University of New Mexico, Albuquerque.

Shepard, Anna O.

1942 Rio Grande Glaze Paint Ware: A Study Illustrating the Place of Ceramic Technological Analysis in Archaeological Research. Contributions to American Anthropology and History Vol. 7, No. 30. Carnegie Institution of Washington, Washington, D.C.

1954 Ceramics for the Archaeologist. Publication 609. Carnegie Institution of Washington, Washington, D.C.

Southward, Judith A.

1979 A Summary of Ceramic Technology, Plant Remains, and Shell Identification Analyses from LA 4921, Three Rivers, New Mexico. In Jornada Mogollon Archaeology: Proceedings of the First Jornada Conference, edited by Patrick H. Beckett and Regge N. Wiseman, pp. 91-102. New Mexico State University, Las Cruces. 

APPENDIX H: Provenience of Selected Artifact Classes from 41GR291, 41GR303B, 41GR546, and 41GR559 



\begin{tabular}{|c|c|c|c|c|c|}
\hline \multicolumn{6}{|c|}{$F 80$} \\
\hline \multicolumn{6}{|c|}{ PROVENIENCE OF PROJECTILE POINTS FROM 41GR291, 41GR303B, AND 41GR559 } \\
\hline Major Category & Type/Group & Raw Material & EU & Level & Feature \\
\hline \multicolumn{6}{|c|}{ Site 41GR291 } \\
\hline Arrow point & $\begin{array}{l}\text { Bonham } \\
\text { Scallom } \\
\text { Scallom } \\
\text { Scallom } \\
\text { Scallom } \\
\text { untyped triangular } \\
\text { untyped triangular } \\
\text { untypeable fragment } \\
\text { untypeable fragment } \\
\text { untypeable fragment } \\
\text { untypeable fragment } \\
\text { untypeable fragment } \\
\text { untypeable fragment } \\
\text { untypeable fragment } \\
\text { untypeable fragment } \\
\text { untypeable fragment } \\
\text { untypeable fragment } \\
\text { untypeable fragment } \\
\text { untypeable fragment }(n=2) \\
\text { preform } \\
\text { preform } \\
\text { preform } \\
\text { preform } \\
\text { preform } \\
\text { preform }\end{array}$ & $\begin{array}{l}\text { fine-grained chert } \\
\text { fine-grained chert } \\
\text { fine-grained chert } \\
\text { fine-grained chert } \\
\text { fine-grained chert } \\
\text { fine-grained chert } \\
\text { fine-grained chert } \\
\text { fine-grained chert } \\
\text { fine-grained chert } \\
\text { fine-grained chert } \\
\text { fine-grained chert } \\
\text { fine-grained chert } \\
\text { fine-grained chert } \\
\text { fine-grained Potter chert } \\
\text { fine-grained chert } \\
\text { fine-grained chert } \\
\text { fine-grained chert } \\
\text { fine-grained chert } \\
\text { fine-grained chert } \\
\text { fine-grained chert } \\
\text { fine-grained chert } \\
\text { fine-grained chert } \\
\text { fine-grained chert } \\
\text { fine-grained chert } \\
\text { fine-grained chert }\end{array}$ & $\begin{array}{r}81 \\
38 \\
47 \\
88 \\
- \\
61 \\
62 \\
20 \\
22 \\
23 \\
24 \\
47 \\
52 \\
64 \\
69 \\
77 \\
79 \\
87 \\
* \\
48 \\
71 \\
77 \\
83 \\
88 \\
*\end{array}$ & $\begin{array}{l}3 \\
2 \\
1 \\
3 \\
- \\
2 \\
2 \\
2 \\
2 \\
1 \\
2 \\
1 \\
2 \\
2 \\
2 \\
2 \\
2 \\
2 \\
* \\
1 \\
4 \\
1 \\
3 \\
2 \\
*\end{array}$ & $\begin{array}{l}21 \\
21\end{array}$ \\
\hline Dart point & $\begin{array}{l}\text { expanding stem, convex base } \\
\text { expanding stem, convex base }\end{array}$ & $\begin{array}{l}\text { fine-grained chert } \\
\text { fine-grained chert }\end{array}$ & $\begin{array}{r}88 \\
* \\
\end{array}$ & $\begin{array}{l}3 \\
* \\
\end{array}$ & \\
\hline \multicolumn{6}{|c|}{ 41GR303B } \\
\hline Arrow point & $\begin{array}{l}\text { Scallom } \\
\text { Scallom } \\
\text { expanding stem, straight base } \\
\text { untypeable fragment } \\
\text { preform } \\
\text { preform }\end{array}$ & $\begin{array}{l}\text { Tecovas jasper } \\
\text { fine-grained chert } \\
\text { fine-grained chert } \\
\text { fine-grained chert } \\
\text { fine-grained chert } \\
\text { fine-grained chert }\end{array}$ & $\begin{array}{r}26 \\
* \\
22 \\
* \\
13 \\
*\end{array}$ & $\begin{array}{l}1 \\
* \\
2 \\
* \\
2 \\
*\end{array}$ & \\
\hline Dart point & $\begin{array}{l}\text { Darl } \\
\text { expanding stem, convex base }\end{array}$ & $\begin{array}{l}\text { fine-grained chert } \\
\text { fine-grained chert }\end{array}$ & $\begin{array}{l}15 \\
23 \\
\end{array}$ & $\begin{array}{l}4 \\
3 \\
\end{array}$ & \\
\hline \multicolumn{6}{|c|}{ 41GR559 } \\
\hline Arrow point & $\begin{array}{l}\text { Washita } \\
\text { untypeable fragment } \\
\text { untypeable fragment }\end{array}$ & $\begin{array}{l}\text { Tecovas jasper } \\
\text { fine-grained chert } \\
\text { fine-grained chert }\end{array}$ & $\begin{array}{r}18 \\
8 \\
28 \\
\end{array}$ & $\begin{array}{l}2 \\
5 \\
7\end{array}$ & - \\
\hline Dart point & Ellis & fine-grained chert & 24 & 3 & \\
\hline
\end{tabular}


TABLE 90

PROVENIENCE OF PERFORATORS AND GOUGES FROM 41GR291 AND 41GR303B

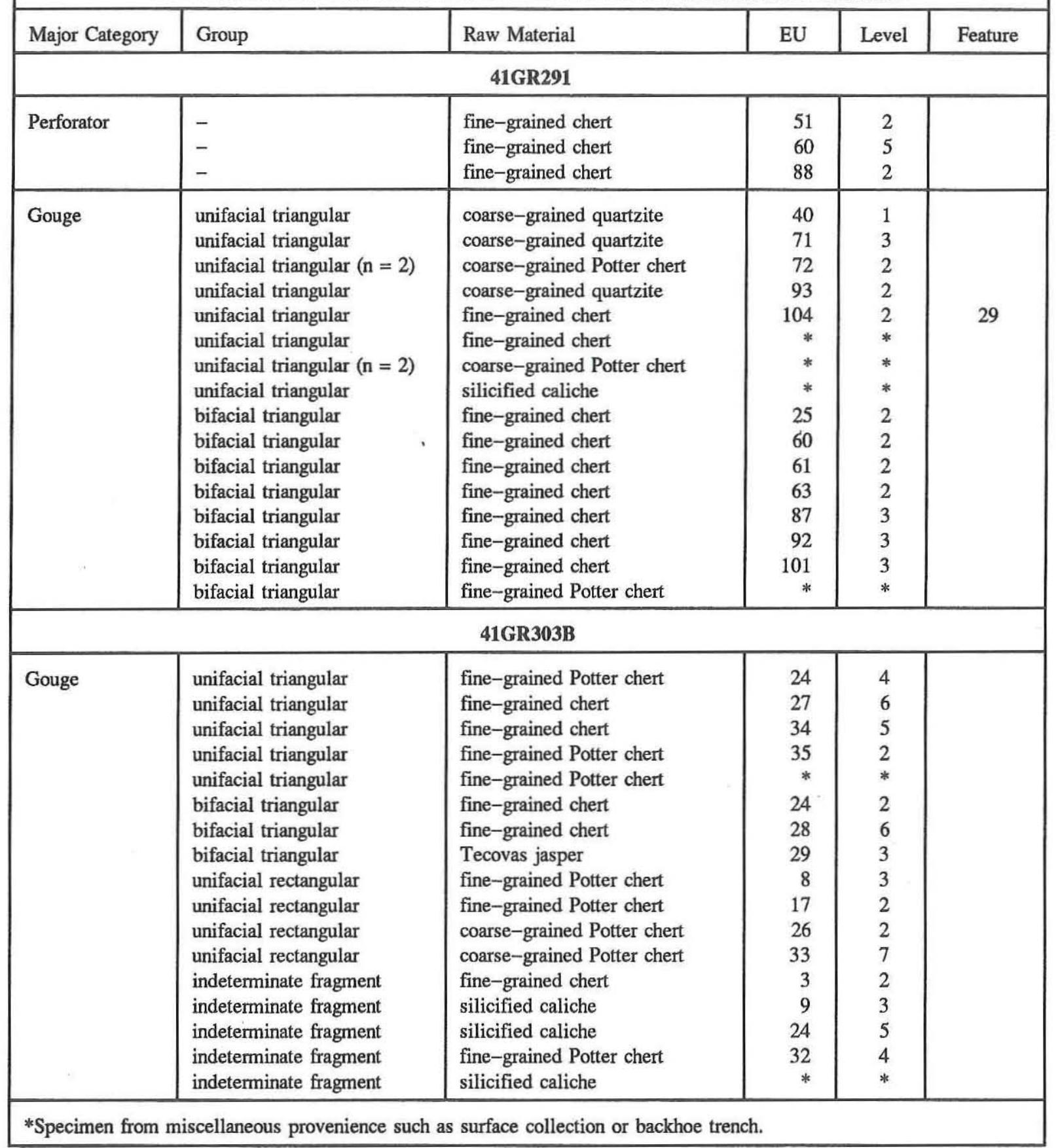


TABLE 91

PROVENIENCE OF NONPROJECTILE POINT BIFACES FROM 41GR291 AND 41GR303B

\begin{tabular}{|c|c|c|c|c|}
\hline Group & Raw Material & $\mathrm{EU}$ & Level & Feature \\
\hline \multicolumn{5}{|c|}{ 41GR291 } \\
\hline Unutilized & $\begin{array}{l}\text { fine-grained chert } \\
\text { fine-grained chert } \\
\text { fine-grained Potter chert }\end{array}$ & $\begin{array}{r}47 \\
98 \\
*\end{array}$ & $\begin{array}{l}2 \\
3 \\
*\end{array}$ & \\
\hline Bifacial knife & $\begin{array}{l}\text { fine-grained chert } \\
\text { fine-grained chert } \\
\text { fine-grained chert }\end{array}$ & $\begin{array}{l}27 \\
47 \\
74\end{array}$ & $\begin{array}{l}1 \\
1 \\
2\end{array}$ & 12 \\
\hline Indeterminate & $\begin{array}{l}\text { fine-grained chert } \\
\text { fine-grained chert } \\
\text { fine-grained chert } \\
\text { fine-grained chert } \\
\text { fine-grained chert } \\
\text { fine-grained chert } \\
\text { fine-grained chert } \\
\text { fine-grained chert } \\
\text { fine-grained chert } \\
\text { fine-grained chert }\end{array}$ & $\begin{array}{l}46 \\
48 \\
51 \\
72 \\
73 \\
74 \\
78 \\
86 \\
88 \\
97\end{array}$ & $\begin{array}{l}3 \\
2 \\
2 \\
1 \\
1 \\
1 \\
1 \\
2 \\
3 \\
2 \\
4\end{array}$ & \\
\hline \multicolumn{5}{|c|}{ 41GR303B } \\
\hline Unutilized & fine-grained chert & 19 & 5 & \\
\hline Indeterminate & $\begin{array}{l}\text { fine-grained chert } \\
\text { fine-grained chert } \\
\text { fine-grained chert }(n=2) \\
\text { silicified caliche } \\
\text { fine-grained chert } \\
\text { fine-grained chert }\end{array}$ & $\begin{array}{r}4 \\
12 \\
13 \\
27 \\
31 \\
36\end{array}$ & $\begin{array}{l}2 \\
5 \\
6 \\
1 \\
2 \\
5\end{array}$ & \\
\hline
\end{tabular}

*Specimen from miscellaneous provenience such as surface collection or backhoe trench. 
TABLE 92

PROVENIENCE OF COBBLE TOOLS FROM 41GR291, 41GR303B, AND 41GR559

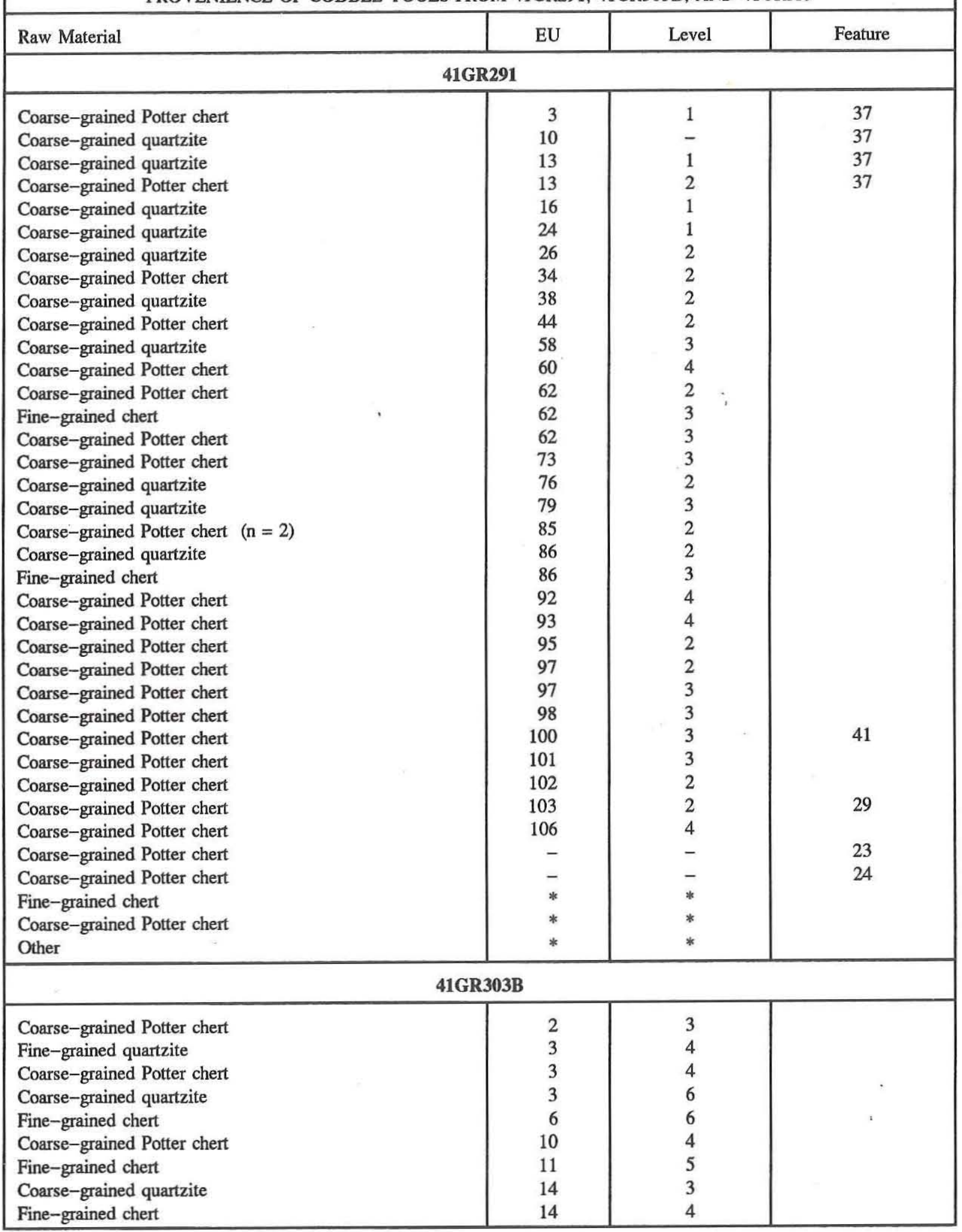


Table 92, continued

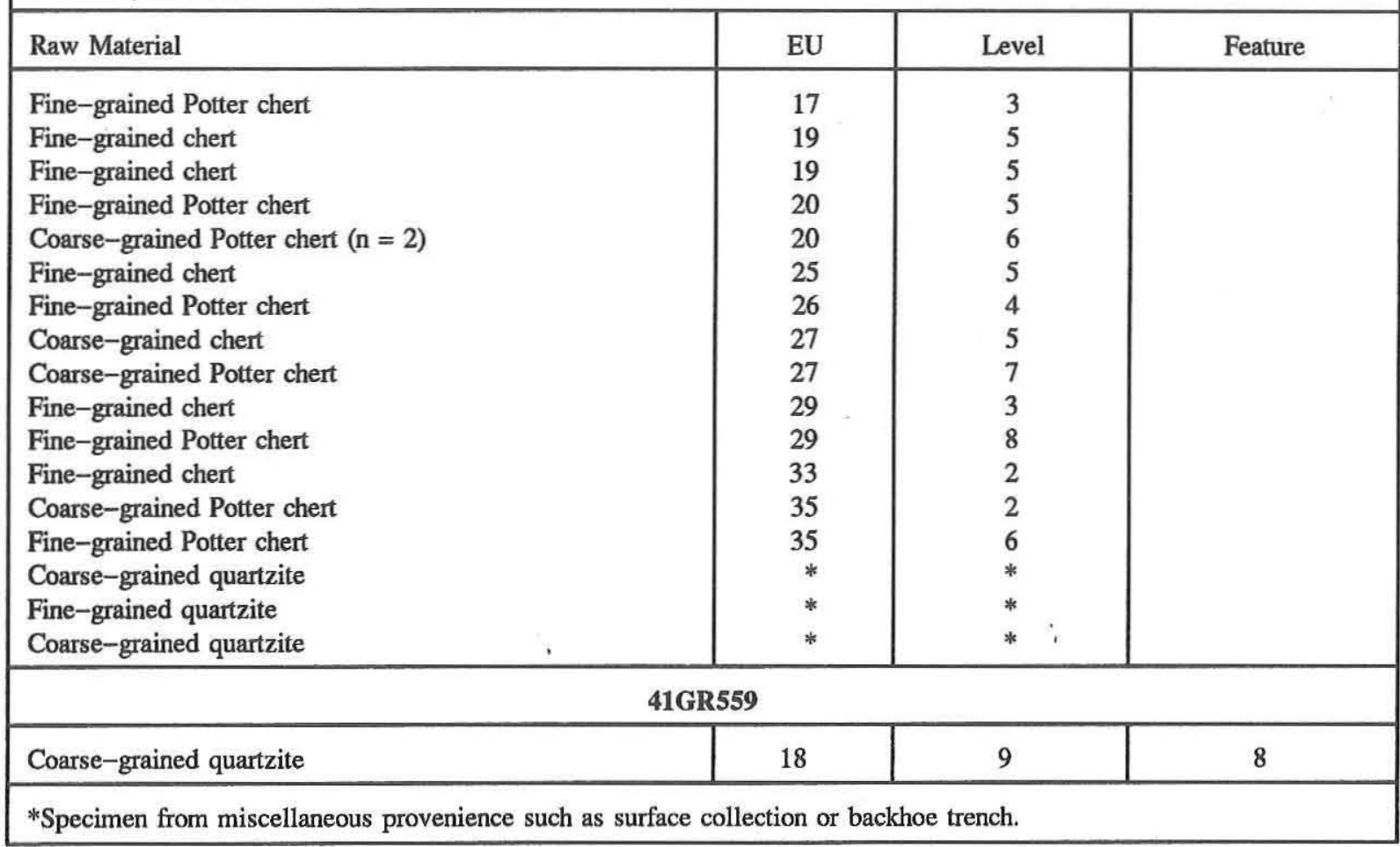


TABLE 93

PROVENIENCE OF UNIFACES FROM 41GR291, 41GR303B, 41GR546, AND 41GR559

\begin{tabular}{|c|c|c|c|c|}
\hline Group & Raw Material & EU & Level & Feature \\
\hline \multicolumn{5}{|c|}{ 41GR291 } \\
\hline End scraper & $\begin{array}{l}\text { fine-grained chert } \\
\text { fine-grained chert } \\
\text { fine-grained Potter chert } \\
\text { coarse-grained quartzite } \\
\text { fine-grained chert } \\
\text { fine-grained chert } \\
\text { fine-grained chert } \\
\text { Tecovas jasper }\end{array}$ & $\begin{array}{r}7 \\
24 \\
25 \\
27 \\
33 \\
60 \\
67 \\
69\end{array}$ & $\begin{array}{l}4 \\
1 \\
2 \\
2 \\
1 \\
2 \\
4 \\
2\end{array}$ & 37 \\
\hline Side scraper & $\begin{array}{l}\text { coarse-grained quartzite } \\
\text { coarse-grained quartzite } \\
\text { fine-grained quartzite } \\
\text { fine-grained chert } \\
\text { coarse-grained Potter chert } \\
\text { silicified wood }\end{array}$ & $\begin{array}{r}54 \\
62 \\
67 \\
83 \\
96 \\
- \\
\end{array}$ & $\begin{array}{l}3 \\
2 \\
3 \\
3 \\
2 \\
-\end{array}$ & 29 \\
\hline Combination end and side scraper & $\begin{array}{l}\text { fine-grained chert } \\
\text { Tecovas jasper } \\
\text { fine-grained chert }\end{array}$ & $\begin{array}{l}24 \\
37 \\
70 \\
\end{array}$ & $\begin{array}{l}2 \\
5 \\
2 \\
\end{array}$ & \\
\hline Minimally retouched end scraper & $\begin{array}{l}\text { fine-grained chert } \\
\text { fine-grained chert } \\
\text { fine-grained chert } \\
\text { fine-grained Potter chert } \\
\text { fine-grained chert } \\
\text { coarse-grained quartzite } \\
\text { coarse-grained quartzite } \\
\text { coarse-grained Potter chert } \\
\text { fine-grained chert } \\
\text { coarse-grained quartzite } \\
\text { fine-grained chert }\end{array}$ & $\begin{array}{r}2 \\
3 \\
7 \\
21 \\
28 \\
44 \\
46 \\
78 \\
95 \\
95 \\
96 \\
\end{array}$ & $\begin{array}{l}2 \\
2 \\
1 \\
1 \\
2 \\
1 \\
2 \\
2 \\
3 \\
3 \\
2 \\
\end{array}$ & $\begin{array}{l}37 \\
37 \\
37\end{array}$ \\
\hline Minimally retouched side scraper & $\begin{array}{l}\text { coarse-grained quartzite } \\
\text { fine-grained chert } \\
\text { fine-grained chert } \\
\text { fine-grained chert } \\
\text { fine-grained chert } \\
\text { fine-grained chert } \\
\text { opalized caliche } \\
\text { fine-grained chert } \\
\text { fine-grained chert } \\
\text { fine-grained Potter chert }\end{array}$ & $\begin{array}{r}2 \\
5 \\
18 \\
24 \\
24 \\
31 \\
48 \\
51 \\
61 \\
63 \\
\end{array}$ & $\begin{array}{l}2 \\
3 \\
1 \\
1 \\
2 \\
1 \\
2 \\
1 \\
3 \\
2 \\
\end{array}$ & $\begin{array}{l}37 \\
37\end{array}$ \\
\hline Graver & $\begin{array}{l}\text { fine-grained chert } \\
\text { silicified wood }\end{array}$ & $\begin{array}{l}4 \\
* \\
\end{array}$ & $\begin{array}{l}1 \\
* \\
\end{array}$ & 37 \\
\hline Indeterminate & $\begin{array}{l}\text { fine-grained chert } \\
\text { other } \\
\text { fine-grained chert } \\
\text { coarse-grained quartzite }\end{array}$ & $\begin{array}{r}5 \\
6 \\
12 \\
18\end{array}$ & $\begin{array}{l}4 \\
3 \\
3 \\
1\end{array}$ & $\begin{array}{l}37 \\
37 \\
37\end{array}$ \\
\hline
\end{tabular}




\begin{tabular}{|c|c|c|c|c|}
\hline \multicolumn{5}{|l|}{ Table 93, continued } \\
\hline Group & Raw Material & EU & Level & Feature \\
\hline Indeterminate, continued & $\begin{array}{l}\text { fine-grained chert } \\
\text { fine-grained chert } \\
\text { fine-grained Potter chert } \\
\text { fine-grained Potter chert } \\
\text { coarse-grained Potter chert } \\
\text { fine-grained Potter chert } \\
\text { coarse-grained quartzite } \\
\text { fine-grained chert } \\
\text { fine-grained chert } \\
\text { fine-grained chert } \\
\text { fine-grained chert } \\
\text { fine-grained chert } \\
\text { fine-grained chert } \\
\text { fine-grained chert } \\
\text { Tecovas jasper } \\
\text { fine-grained chert } \\
\text { fine-grained chert } \\
\text { fine-grained chert } \\
\text { fine-grained chert } \\
\text { fine-grained chert ( } n=2) \\
\text { fine-grained chert } \\
\text { coarse-grained Potter chert } \\
\text { fine-grained chert ( } n=2 \text { ) } \\
\text { fine-grained chert } \\
\text { coarse-grained quartzite } \\
\text { fine-grained chert } \\
\text { coarse-grained quartzite } \\
\text { fine-grained chert } \\
\text { fine-grained Potter chert } \\
\text { fine-grained chert } \\
\text { fine-grained chert } \\
\text { fine-grained chert } \\
\text { fine-grained Potter chert } \\
\text { fine-grained chert }\end{array}$ & $\begin{array}{r}19 \\
23 \\
23 \\
25 \\
26 \\
30 \\
34 \\
39 \\
47 \\
47 \\
51 \\
58 \\
61 \\
62 \\
64 \\
65 \\
70 \\
71 \\
74 \\
76 \\
76 \\
77 \\
78 \\
81 \\
82 \\
87 \\
88 \\
90 \\
93 \\
93 \\
101 \\
105 \\
- \\
* \\
\end{array}$ & $\begin{array}{l}2 \\
1 \\
1 \\
2 \\
1 \\
2 \\
2 \\
3 \\
1 \\
2 \\
1 \\
2 \\
2 \\
3 \\
2 \\
1 \\
2 \\
3 \\
1 \\
2 \\
3 \\
2 \\
2 \\
2 \\
1 \\
3 \\
3 \\
3 \\
2 \\
3 \\
3 \\
5 \\
- \\
\end{array}$ & 21 \\
\hline \multicolumn{5}{|c|}{ 41GR303B } \\
\hline End scraper & $\begin{array}{l}\text { fine-grained chert } \\
\text { fine-grained chert } \\
\text { fine-grained chert } \\
\text { fine-grained chert } \\
\text { fine-grained Potter chert } \\
\text { fine-grained Potter chert }\end{array}$ & $\begin{array}{l}18 \\
20 \\
25 \\
25 \\
33 \\
33 \\
\end{array}$ & $\begin{array}{l}3 \\
2 \\
3 \\
4 \\
5 \\
6\end{array}$ & \\
\hline Side scraper & fine-grained chert & 6 & 5 & \\
\hline Combination end and side scraper & $\begin{array}{l}\text { fine-grained chert } \\
\text { fine-grained chert }\end{array}$ & $\begin{array}{l}12 \\
26 \\
\end{array}$ & $\begin{array}{l}5 \\
4 \\
\end{array}$ & . \\
\hline Minimally retouched end scraper & $\begin{array}{l}\text { fine-grained chert } \\
\text { fine-grained Potter chert } \\
\text { fine-grained chert } \\
\text { fine-grained chert } \\
\text { coarse-grained quartzite } \\
\text { fine-grained chert }\end{array}$ & $\begin{array}{r}6 \\
8 \\
8 \\
16 \\
25 \\
26 \\
\end{array}$ & $\begin{array}{l}4 \\
2 \\
3 \\
2 \\
6 \\
5\end{array}$ & : \\
\hline
\end{tabular}


Phase III Data Recovery, Season 3, Lake Alan Henry

\begin{tabular}{|c|c|c|c|c|}
\hline Group & Raw Material & EU & Level & Feature \\
\hline Minimally retouched end scraper, continued & $\begin{array}{l}\text { fine-grained Potter chert } \\
\text { fine-grained chert } \\
\text { fine-grained chert }(n=2) \\
\text { silicified caliche }\end{array}$ & $\begin{array}{r}32 \\
34 \\
36 \\
*\end{array}$ & $\begin{array}{l}6 \\
1 \\
3 \\
*\end{array}$ & \\
\hline Minimally retouched side scraper & $\begin{array}{l}\text { fine-grained quartzite } \\
\text { coarse-grained quartzite } \\
\text { fine-grained chert } \\
\text { coarse-grained quartzite } \\
\text { fine-grained Potter chert }\end{array}$ & $\begin{array}{r}2 \\
13 \\
29 \\
* \\
*\end{array}$ & $\begin{array}{l}3 \\
9 \\
4 \\
* \\
*\end{array}$ & \\
\hline $\begin{array}{l}\text { Minimally retouched combination end } \\
\text { and side scraper }\end{array}$ & $\begin{array}{l}\text { fine-grained chert } \\
\text { fine-grained chert } \\
\text { fine-grained Potter chert } \\
\text { Tecovas jasper } \\
\text { fine-grained chert }\end{array}$ & $\begin{array}{r}9 \\
23 \\
26 \\
28 \\
32 \\
\end{array}$ & $\begin{array}{l}3 \\
3 \\
3 \\
3 \\
4\end{array}$ & \\
\hline Graver & fine-grained chert & 23 & 4 & \\
\hline Indeterminate & $\begin{array}{l}\text { Tecovas jasper } \\
\text { fine-grained chert } \\
\text { fine-grained chert } \\
\text { coarse-grained Potter chert } \\
\text { fine-grained chert } \\
\text { silicified caliche } \\
\text { fine-grained chert } \\
\text { coarse-grained quartzite } \\
\text { fine-grained chert } \\
\text { fine-grained chert } \\
\text { fine-grained chert } \\
\text { fine-grained chert } \\
\text { fine-grained chert } \\
\text { fine-grained chert } \\
\text { fine-grained chert } \\
\text { fine-grained chert } \\
\text { fine-grained chert } \\
\text { coarse-grained chert } \\
\text { fine-grained chert } \\
\text { fine-grained chert } \\
\text { fine-grained chert } \\
\text { fine-grained chert } \\
\text { silicified caliche } \\
\text { fine-grained chert } \\
\text { fine-grained chert } \\
\text { fine-grained chert } \\
\text { fine-grained Potter chert } \\
\text { opalized caliche } \\
\text { fine-grained chert } \\
\text { fine-grained chert } \\
\text { fine-grained chert } \\
\text { fine-grained chert } \\
\text { fine-grained chert } \\
\text { fine-grained chert } \\
\text { fine-grained chert } \\
\text { coarse-grained Potter chert }(\mathrm{n}=2 \text { ) }\end{array}$ & $\begin{array}{r}1 \\
5 \\
7 \\
11 \\
11 \\
13 \\
13 \\
13 \\
13 \\
13 \\
16 \\
16 \\
17 \\
18 \\
18 \\
20 \\
23 \\
23 \\
24 \\
25 \\
27 \\
27 \\
27 \\
30 \\
30 \\
30 \\
33 \\
33 \\
33 \\
34 \\
34 \\
36 \\
36 \\
36 \\
* \\
*\end{array}$ & $\begin{array}{c}2 \\
6 \\
2 \\
2 \\
4 \\
1 \\
2 \\
4 \\
7 \\
10 \\
3 \\
4 \\
4 \\
3 \\
4 \\
6 \\
3 \\
3 \\
2 \\
3 \\
1 \\
6 \\
7 \\
3 \\
4 \\
5 \\
1 \\
4 \\
7 \\
2 \\
3 \\
6 \\
7 \\
9 \\
* \\
*\end{array}$ & . \\
\hline
\end{tabular}




\begin{tabular}{|c|c|c|c|c|}
\hline \multicolumn{5}{|l|}{ Table 93 , continued } \\
\hline Group & Raw Material & EU & Level & Feature \\
\hline \multicolumn{5}{|c|}{ 41HR546 } \\
\hline Side scraper & fine-grained Potter chert & 1 & 7 & \\
\hline \multicolumn{5}{|c|}{ 41GR559 } \\
\hline End scraper & $\begin{array}{l}\text { opalized caliche } \\
\text { fine-grained chert } \\
\text { fine-grained chert }\end{array}$ & $\begin{array}{l}12 \\
24 \\
27\end{array}$ & $\begin{array}{l}3 \\
7 \\
7\end{array}$ & \\
\hline Minimally retouched side scraper & coarse-grained quartzite & 17 & 7 & 15 \\
\hline Indeterminate & $\begin{array}{l}\text { fine-grained chert } \\
\text { fine-grained chert }\end{array}$ & $\begin{array}{l}26 \\
20\end{array}$ & $\begin{array}{l}6 \\
6\end{array}$ & 26 \\
\hline
\end{tabular}




\begin{tabular}{|c|c|c|c|c|}
\hline & \multicolumn{4}{|c|}{$\begin{array}{l}\text { PROVENIENCE OF GROUND AND BATTERED STONES FROM } \\
\text { 41GR291, 41GR303B, 41GR546, AND 41GR559 }\end{array}$} \\
\hline Group & Raw Material & EU & Level & Feature \\
\hline \multicolumn{5}{|c|}{ 41GR291 } \\
\hline Mano & $\begin{array}{l}\text { sandstone } \\
\text { coarse-grained quartzite } \\
\text { coarse-grained quartzite } \\
\text { coarse-grained quartzite }(n=2) \\
\text { coarse-grained quartzite } \\
\text { sandstone } \\
\text { coarse-grained quartzite } \\
\text { coarse-grained quartzite } \\
\text { sandstone } \\
\text { coarse-grained quartzite } \\
\text { sandstone } \\
\text { coarse-grained quartzite } \\
\text { coarse-grained quartzite } \\
\text { sandstone } \\
\text { coarse-grained quartzite } \\
\text { coarse-grained quartzite } \\
\text { coarse-grained quartzite } \\
\text { coarse-grained quartzite } \\
\text { coarse-grained quartzite } \\
\text { coarse-grained quartzite } \\
\text { sandstone } \\
\text { coarse-grained quartzite }(n=2) \\
\text { coarse-grained quartzite }(n=5) \\
\text { coarse-grained quartzite } \\
\text { sandstone }(n=6)\end{array}$ & $\begin{array}{r}5 \\
19 \\
30 \\
32 \\
56 \\
58 \\
69 \\
70 \\
71 \\
73 \\
75 \\
82 \\
82 \\
86 \\
95 \\
95 \\
96 \\
98 \\
100 \\
- \\
- \\
- \\
* \\
* \\
* \\
\end{array}$ & $\begin{array}{l}- \\
3 \\
3 \\
1 \\
1 \\
3 \\
2 \\
2 \\
3 \\
3 \\
3 \\
2 \\
3 \\
2 \\
3 \\
4 \\
4 \\
4 \\
3 \\
- \\
- \\
- \\
* \\
* \\
* \\
\end{array}$ & $\begin{array}{l}16 \\
16 \\
\\
41 \\
29 \\
29 \\
37\end{array}$ \\
\hline Metate & $\begin{array}{l}\text { sandstone } \\
\text { sandstone } \\
\text { sandstone } \\
\text { sandstone }(\mathrm{n}=2) \\
\text { sandstone } \\
\text { sandstone } \\
\text { sandstone } \\
\text { sandstone }(\mathrm{n}=2) \\
\text { sandstone } \\
\text { sandstone } \\
\text { sandstone } \\
\text { sandstone } \\
\text { sandstone } \\
\text { sandstone } \\
\text { sandstone } \\
\text { sandstone } \\
\text { sandstone } \\
\text { sandstone }(\mathrm{n}=4) \\
\text { sandstone }(\mathrm{n}=4) \\
\text { sandstone }(\mathrm{n}=2) \\
\text { sandstone }\end{array}$ & $\begin{array}{r}1 \\
2 \\
4 \\
5 \\
10 \\
11 \\
24 \\
47 \\
47 \\
59 \\
62 \\
70 \\
71 \\
75 \\
80 \\
95 \\
95 \\
100 \\
100 \\
100 \\
103 \\
\end{array}$ & $\begin{array}{l}- \\
3 \\
- \\
- \\
- \\
- \\
2 \\
2 \\
3 \\
4 \\
3 \\
2 \\
4 \\
2 \\
4 \\
3 \\
4 \\
3 \\
4 \\
5 \\
4 \\
\end{array}$ & $\begin{array}{r}16 \\
\cdot 16 \\
41 \\
41 \\
41\end{array}$ \\
\hline
\end{tabular}




\begin{tabular}{|c|c|c|c|c|}
\hline \multicolumn{5}{|l|}{ Table 94 , continued } \\
\hline Group & Raw Material & EU & Level & Feature \\
\hline Metate, continued & $\begin{array}{l}\text { sandstone } \\
\text { sandstone } \\
\text { sandstone } \\
\text { sandstone } \\
\text { sandstone } \\
\text { sandstone } \\
\text { sandstone }(n=2) \\
\text { sandstone }(n=29) \\
\text { sandstone }(n=2) \\
\text { sandstone }(n=4)\end{array}$ & $\begin{array}{r}105 \\
105 \\
106 \\
- \\
- \\
- \\
- \\
- \\
- \\
*\end{array}$ & $\begin{array}{l}3 \\
5 \\
4 \\
- \\
- \\
- \\
- \\
- \\
- \\
*\end{array}$ & $\begin{array}{l}17 \\
18 \\
19 \\
24 \\
29 \\
37\end{array}$ \\
\hline Pestle & $\begin{array}{l}\text { sandstone } \\
\text { sandstone } \\
\text { sandstone } \\
\text { sandstone } \\
\text { sandstone } \\
\text { sandstone }\end{array}$ & $\begin{array}{r}10 \\
40 \\
68 \\
94 \\
100 \\
*\end{array}$ & $\begin{array}{l}2 \\
1 \\
2 \\
3 \\
4 \\
*\end{array}$ & $\begin{array}{l}37 \\
21 \\
16 \\
41\end{array}$ \\
\hline Anvil & sandstone & - & - & 17 \\
\hline Hammerstone & $\begin{array}{l}\text { coarse-grained quartzite } \\
\text { coarse-grained quartzite } \\
\text { coarse-grained quartzite } \\
\text { coarse-grained quartzite }\end{array}$ & $\begin{array}{r}25 \\
38 \\
74 \\
* \\
\end{array}$ & $\begin{array}{l}1 \\
2 \\
3 \\
*\end{array}$ & 12 \\
\hline Indeterminate & $\begin{array}{l}\text { sandstone } \\
\text { sandstone } \\
\text { sandstone } \\
\text { sandstone } \\
\text { sandstone } \\
\text { sandstone } \\
\text { sandstone } \\
\text { sandstone } \\
\text { sandstone } \\
\text { sandstone }(n=2)\end{array}$ & $\begin{array}{r}8 \\
11 \\
27 \\
31 \\
38 \\
41 \\
58 \\
75 \\
106 \\
-\end{array}$ & $\begin{array}{l}- \\
6 \\
2 \\
1 \\
3 \\
2 \\
2 \\
4 \\
3 \\
-\end{array}$ & $\begin{array}{l}37 \\
37 \\
12 \\
\\
\\
29 \\
\end{array}$ \\
\hline \multicolumn{5}{|c|}{ 41GR303B } \\
\hline Mano & $\begin{array}{l}\text { coarse-grained quartzite } \\
\text { sandstone } \\
\text { sandstone } \\
\text { sandstone } \\
\text { coarse-grained quartzite } \\
\text { coarse-grained quartzite } \\
\text { coarse-grained quartzite } \\
\text { coarse-grained quartzite } \\
\text { coarse-grained quartzite } \\
\text { coarse-grained quartzite } \\
\text { coarse-grained quartzite } \\
\text { coarse-grained quartzite } \\
\text { coarse-grained quartzite } \\
\text { coarse-grained quartzite }(n=3)\end{array}$ & $\begin{array}{r}8 \\
10 \\
11 \\
13 \\
13 \\
24 \\
25 \\
27 \\
30 \\
31 \\
33 \\
34 \\
35 \\
*\end{array}$ & $\begin{array}{c}3 \\
4 \\
4 \\
5 \\
11 \\
4 \\
5 \\
5 \\
5 \\
2 \\
3 \\
5 \\
3 \\
*\end{array}$ & , \\
\hline
\end{tabular}


Phase III Data Recovery, Season 3, Lake Alan Henry

\begin{tabular}{|c|c|c|c|c|}
\hline \multicolumn{5}{|c|}{ Table 94, continued } \\
\hline Group & Raw Material & EU & Level & Feaiure \\
\hline Metate & $\begin{array}{l}\text { sandstone } \\
\text { sandstone } \\
\text { sandstone } \\
\text { sandstone }(\mathrm{n}=2) \\
\text { sandstone } \\
\text { sandstone } \\
\text { sandstone }\end{array}$ & $\begin{array}{l}10 \\
12 \\
24 \\
25 \\
29 \\
31 \\
33 \\
\end{array}$ & $\begin{array}{l}4 \\
7 \\
5 \\
6 \\
3 \\
5 \\
2 \\
\end{array}$ & \\
\hline Pestle & sandstone & 24 & 4 & \\
\hline Hammerstone & $\begin{array}{l}\text { coarse-grained quartzite } \\
\text { coarse-grained quartzite }\end{array}$ & $\begin{array}{r}2 \\
32 \\
\end{array}$ & $\begin{array}{l}4 \\
2 \\
\end{array}$ & \\
\hline \multicolumn{5}{|c|}{ 41GR546 } \\
\hline Hammerstone & coarse-grained quartzite & 3 & - & \\
\hline \multicolumn{5}{|c|}{ 41GR559 } \\
\hline Metate & $\begin{array}{l}\text { sandstone } \\
\text { sandstone } \\
\text { sandstone }\end{array}$ & $\begin{array}{l}14 \\
24 \\
25\end{array}$ & $\begin{array}{l}3 \\
3 \\
3\end{array}$ & 27 \\
\hline Hammerstone & $\begin{array}{l}\text { coarse-grained quartzite } \\
\text { coarse-grained quartzite }(n=2) \\
\text { coarse-grained quartzite }(n=2) \\
\text { coarse-grained quartzite } \\
\text { coarse-grained quartzite } \\
\text { coarse-grained quartzite }\end{array}$ & $\begin{array}{r}8 \\
8 \\
9 \\
12 \\
18 \\
28\end{array}$ & $\begin{array}{l}5 \\
7 \\
9 \\
- \\
8 \\
6\end{array}$ & $\begin{array}{l}7 \\
8\end{array}$ \\
\hline
\end{tabular}




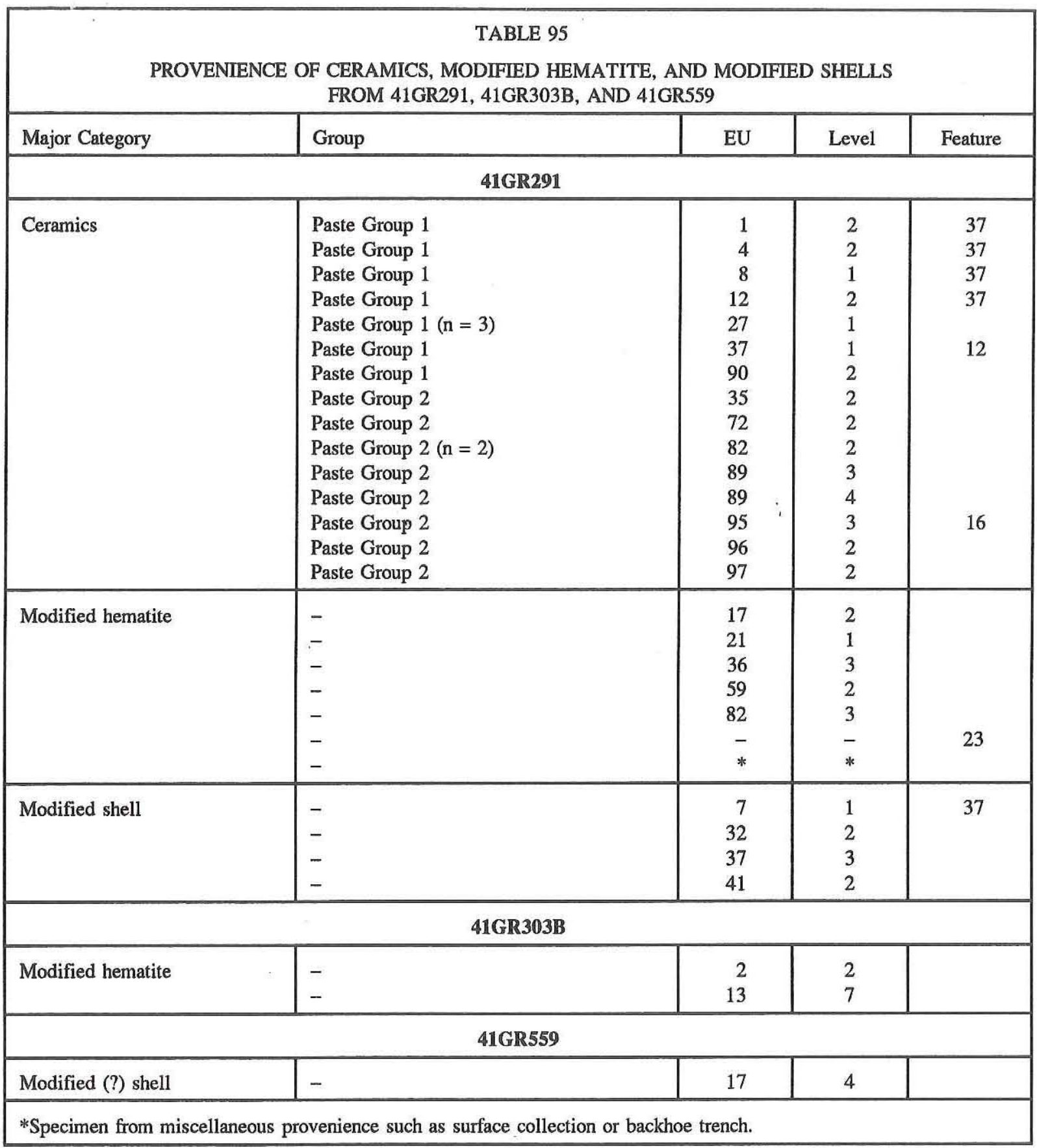





\section{APPENDIX I: Discovery and Documentation of the Callahan Petroglyph Site, 41KT164}

Douglas K. Boyd 



\section{INTRODUCTION}

The Callahan site is a rock art site located near the emergency spillway at Lake Alan Henry. It was found by Granite Construction Company employee Bryan Callahan, his wife Jeanette, and his father Lee, during the summer of 1993 . Bryan Callahan, operator of the pug mill near the emergency spillway, reported the site to Granite Construction Company's project manager, David Smith, who made sure that construction activities in the area avoided the site. It was reported to Freese and Nichols, Inc., and Prewitt and Associates, Inc. was subsequently notified of the new discovery. The site was inspected and documented on August 17 and 18, 1993. Had it not been for its fortuitous discovery by Bryan Callahan, the site might have been destroyed by construction activities and never documented.

The site consists of a bluff-edge overhang that protects a series of Euro-American inscriptions (one historic and numerous modern) and six panels of Native American petroglyphs. The initial documentation of the site consisted of measured sketches of the shelter in plan and profile views, recording of the historic and modern Euro-American inscriptions and vandalism, and measured sketches of the Native American petroglyph panels. Numerous color slide and black-and-white photographs of the shelter and the rock art panels also were taken. Subsequent to the field recording, the site was designated as $41 \mathrm{KT} 164$, and it was recommended that it be assessed as eligible for listing on the National Register of Historic Places. Some additional documentation of the Native American petroglyphs also was recommended.

Representatives of the U.S. Army Corps of Engineers and the Texas Historical Commission concurred with the above assessment and recommendation. Additional documentation was done during a second visit to the site on October 21, 1993. This investigation consisted of producing full-size acetate tracings of the petroglyphs, taking additional color slide and black-and-white photographs, and making additional measured drawings and observations. The rock art line drawings in this appendix are reproduced from the acetate tracings.

The author sincerely appreciates the comments of rock art specialists Mike Bilbo, Polly Schaafsma, and Dr. Kay Sutherland, who took the time to comment on drawings of the Callahan site petroglyphs. Their observations, based on many years of experience in rock art research, were extremely enlightening and provide a better frame of reference for understanding Southwestern influence in Texas Panhandle-Plains rock art. The author also appreciates the help of two individuals, Mike Gilliland (City of Lubbock) and Jeanine McDonald (Prewitt and Associates, Inc.), who assisted in the recording of the site.

\section{SITE SETTING}

Site $41 \mathrm{KT} 164$ is a prominent overhang shelter that is located along the bluff edge on the east canyon rim of Grape Creek, 100-200 m north of the Lake Alan Henry emergency spillway. At this point, the bluff edge trends northwest to southeast and the overhang faces southwest, overlooking a wide segment of the Grape Creek valley. The overhang (at $2260 \mathrm{ft} \mathrm{msl})$ is $90-110 \mathrm{ft}(27.4-33.5 \mathrm{~m})$ above the floodplain (at $2150-2170 \mathrm{ft} \mathrm{msl}$ ) and is approximately $27 \mathrm{~m}$ in length and varies from $0-5 \mathrm{~m}$ deep and from 0-4 $\mathrm{m}$ in height (Figure 93). A small, 1-m-high, wind-worn alcove just south of the overhang also contains a petroglyph and extends the site length to $35 \mathrm{~m}$ (northwest to southeast).

The topography of the project area is the result of the variable erosion of alternating layers of Triassic-age Dockum Group sandstones and mudstones (McGowen et al. 1979). The overhanging ledge at the Callahan site was formed when the friable sandstone stratum eroded out from underneath the harder ledge-forming sandstone stratum, probably because of spring sapping and seepage (Gustavson and Simpkins 1989). Shelter formation also may have been accelerated by eolian processes and various biological agents (e.g., vegetation growth along the bluff edges). As the underlying stratum eroded, the overlying hard sandstone fractured under its own weight, and some portions of the overhanging ledge have collapsed recently, probably within the last few hundred years. The floor of the overhang is alternately exposed bedrock or a thin veneer of sands derived from in situ weathering of the friable sandstone. Water channels, originating at driplines below cracks in the capping sandstone layer, converge in the central portion of the overhang. Consequently, most of the overhang has been scoured out to bedrock, and there is little potential for subsurface cultural deposits. It is doubtful that the overhang was ever occupied as a shelter.

The overhang is immediately adjacent to the 


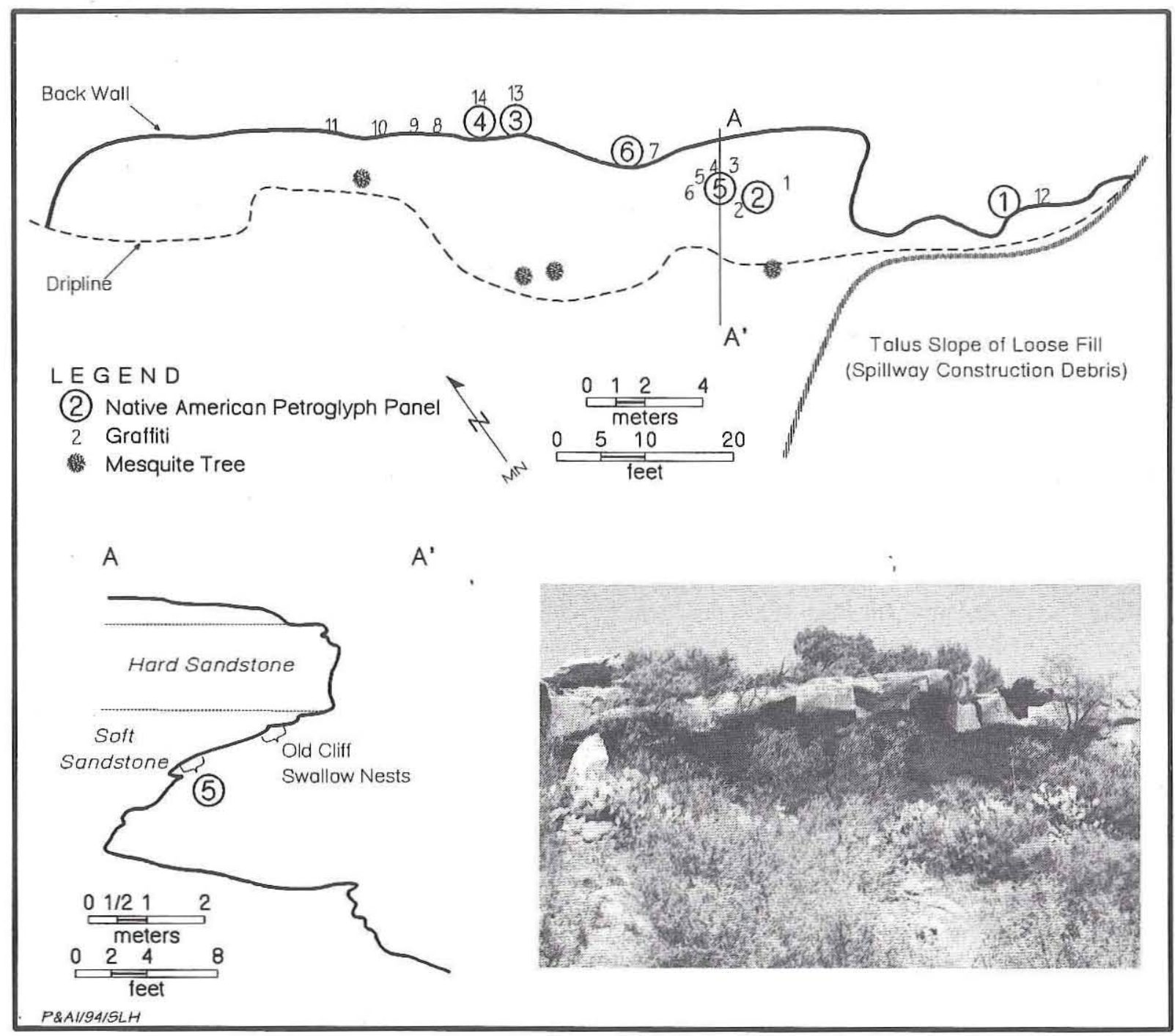

Figure 93. Plan and profile views and photograph of the overhang shelter, 41KT164. Photograph is looking uphill to the northeast at the front of the overhang.

area where Granite Construction Company disposed of loose fill, excavated from the emergency spillway, by pushing it over the edge of the bluff. The result is a substantial talus accumulation of sand and sandstone (i.e., upland sandy sediments, unconsolidated sand derived from sandstone, and sandstone fragments). The northern edge of the debris talus butts up against the southern end of the overhang, and loose talus sediment has partially filled the southern alcove. The dumping of construction debris, however, has not seriously impacted the site.

\section{DESCRIPTION OF PETROGLYPHS}

Manmade images at the site consist of several occurrences of modern graffiti and vandalism, one historic inscription, and six groups of Native American petroglyphs (see Figure 93 for locations within shelter). The latter are designated as Panels 1-6, and individual glyphs within each panel are designated by consecutive decimal numbers from left to right (e.g., Glyphs 3.1, 3.2, and 3.3). Of the 14 occurrences of Euro-American glyphs, only one inscription, "BOWEN DANIEL 1927" (Table 96), is definitely of historic age, but this person has not been identified. All of the other inscriptions appear to be of recent age, and five occurrences are dated 1990. One of the 1990 inscriptions has damaged the Panel 2 petroglyphs, and the petroglyphs on Panels 3 and 4 also have been damaged or altered recently. Each 


\begin{tabular}{|c|l|l|}
\hline \multicolumn{2}{|c|}{} \\
\hline \multicolumn{2}{|c|}{ TISTORIC AND MODERN EURO-AMERICAN INSCRIPTIONS AND VANDALISM AT 41KT164 } \\
\hline Map Key & Location in Shelter & Inscription \\
\hline 1 & ceiling & "RAUl" \\
2 & ceiling & "ROSY 1990" superimposed on Panel 2 \\
3 & ceiling & "S2 JWH" \\
4 & ceiling & "BOWEN DANIEL 1927" immediately above Panel 5 \\
5 & ceiling & faint and illegible, could be "JAMES" \\
6 & ceiling & "MIChaEL 1990" with a geometric symbol and a longhom head \\
7 & back wall & "CH RW 1966" \\
8 & back wall & "MIChaEL WAS HERE 1990" \\
9 & back wall & indistinct initials and "1990" \\
10 & back wall & "AMA 1990" \\
11 & back wall & "WM" and indistinct date, possible 194[?]" \\
12 & back wall of small alcove & "TM 79" \\
13 & back wall & alteration of Panel 3; Glyph 3.5 \\
14 & back wall & indistinct "N" superimposed on Panel 4 \\
\hline
\end{tabular}

panel containing Native American petroglyphs is described below.

Panel 1 is in the southern alcove and contains a single small geometric design (Figure 94). The soft sandstone face is strongly concave but very smooth and covers over $1 \mathrm{~m}^{2}$. The isolated glyph is centrally located within the alcove and consists of a $12-\mathrm{cm}-$ diameter circle enclosing three horizontal and three vertical lines with two short segments extending beyond the circle (one vertical on top and one horizontal on the right). The glyph is composed of 4-6-mm-wide, 2-2.5-mm-deep, shallow, U-shaped grooves that are patinated equally with the surrounding rock face.

Panel 2 is the most intriguing and contains two large stylized figures carved into the soft sandstone on the sloping ceiling, $1.8 \mathrm{~m}$ above the floor in the southern part of the overhang (Figure 95). Panel 2 consists of a smooth, slightly convex face bracketed on all sides by spalling or cliff swallow nests. Spalling along the lower edge of the panel is particularly severe due to burrowing insects. Various species of burrowing wasps and bees actually dig small holes into the rocks and lay their eggs inside them, thereby weakening the rock face and greatly accelerating spalling (Raymond Neck, personal communication 1993). Burrowing insects have been noted as the culprits accounting for damage in many other rock art sites in Texas. In Garza County, for example, insect burrowing was noted as having caused spalling damage to rock art at the Ward

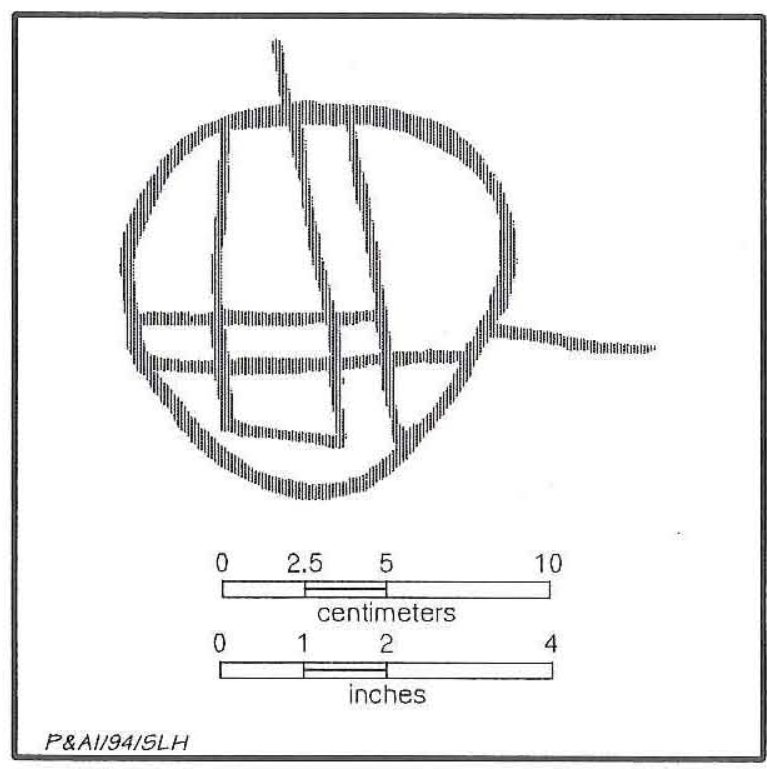

Figure 94. Line drawing of Panel 1 petroglyph, 41KT164.

petroglyph site (Boyd and Garvey 1990:215), and Bilbo (1986:9) mentions the severe spalling caused by "bee bores" all along the bottom of the large sandstone face at Cowhead Mesa. Turpin (1982:194) and Silver (1985:21) also note "burrowing wasp" damage at Seminole Canyon rock art sites in Val Verde County. Panel 2 also has been vandalized (i.e., "ROSY 1990" inscribed on the lower left side of the panel), but no critical parts of the glyphs have been destroyed. The original images are patinated to the same reddish color (i.e., 5YR 5/4 reddish brown 


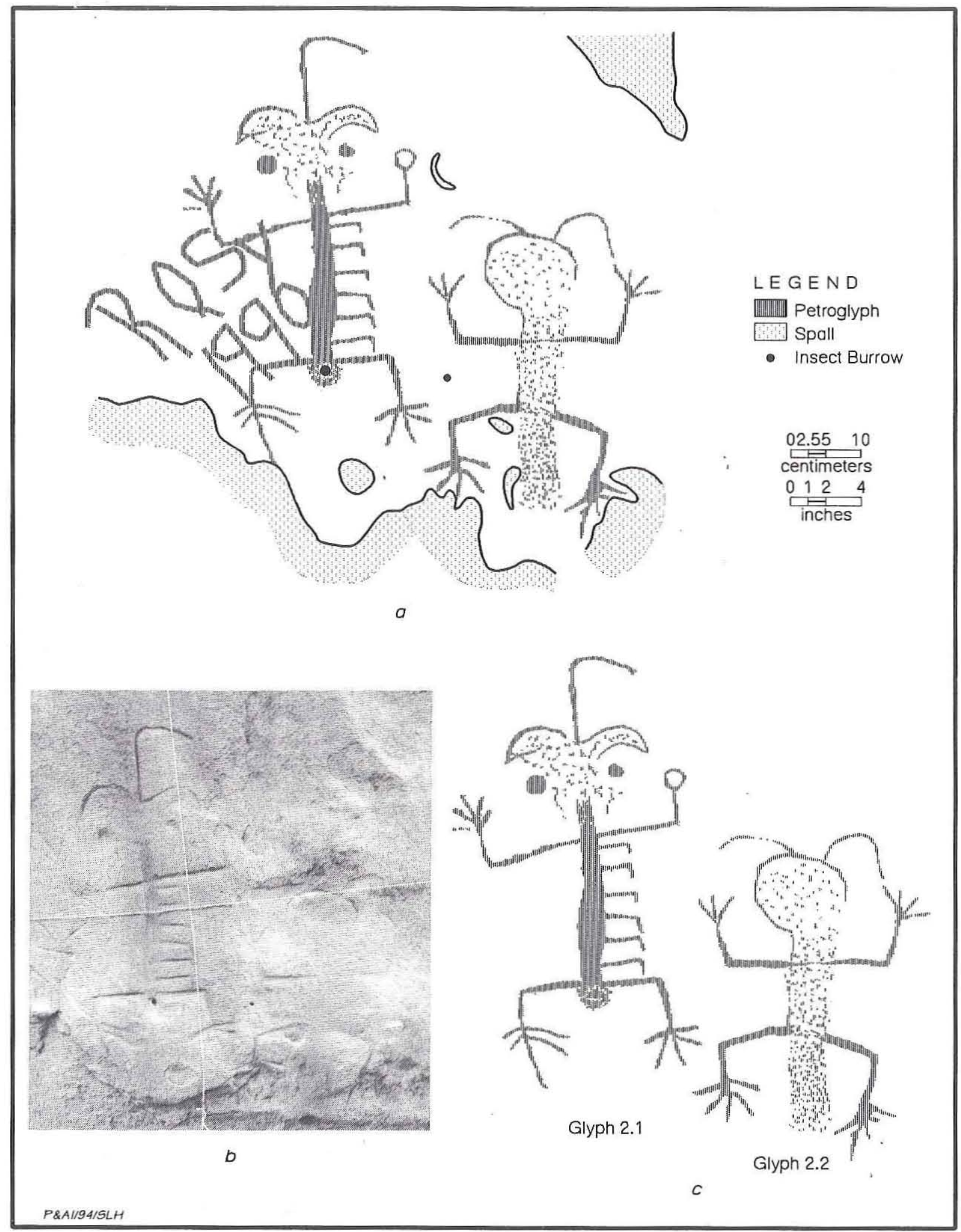

Figure 95. Petroglyph Panel 2,41KT164. (a) Line drawing of petroglyphs showing recent vandalism and natural deterioration of the rock face; $(b)$ photograph of panel; and $(c)$ line drawing of petroglyphs with natural deterioration and recent alterations removed. 
to $5 Y R$ $6 / 6$ reddish yellow) as the rest of the rock face, but the incised lines of the recent inscription are a light gray $(2.5 \mathrm{Y} 7 / 2)$, the unweathered natural color of the sandstone.

The figures on Panel 2 are presumably stylized human representations although they could represent zoomorphs. The two figures are similar in that both have extended arms, bent and up-turned at right angles, and extended legs, bent and down-turned at right angles. With one exception, the arms each have three or four fingers, and all of the feet have five toes. There is no evidence that either of these glyphs were painted, and they both appear to have been produced using a combination of wide shallow grooves, fine line grooves, and smoothing of broad areas.

Glyph 2.1 has a slender stick-figure body composed of a 23-26-mm-wide, 5-mm-deep groove with riblike appendages coming off one side. Its arms and legs have fingers and toes except for a small circle at the end of one arm, which may represent an object held in its hand. Its head consists of two prominent eyes $(25 \mathrm{~mm}$ in diameter and 3-4-mm-deep depressions) on either side of a smoothed area and underneath two hornlike projections or eyebrows. A single antenna projects upward from the head. The lines comprising the arms, rib appendages, homlike projections, and antenna are 7-11-mm-wide, 2-2.5-mm-deep, U-shaped grooves that are considerably smaller than that of the main body.

It is possible that Glyph 2.1 represents a female since there is a prominent, $45-\mathrm{mm}$-diameter circular depression in the crotch area. An insect burrow inside this depression makes the feature stand out, and its occurrence there probably is not a coincidence. Most of the other insect burrows are found along the margins of the surrounding rock face where the exposed rock face is softer. Since the patinated rock face tends to be harder and more compact, this circular depression probably provided a softer area into which the insect could burrow. The depression could have been a natural pebble cast that was incorporated into the glyph, but more likely it was an intentional modification that was part of the petroglyph. In either case, it may have been intended to represent female genitalia.

Glyph 2.2 is similar in that it has stick arms and legs (composed of 5-11-cm-wide, 1.5-2.5-mmdeep, U-shaped grooves) that are oriented the same as those of Glyph 2.1, but the head and body of
Glyph 2.2 are different. Glyph 2.2 has a round head with no obvious facial features and two hanging antennaelike projections, one of which is rather faded and indistinct. Its body is depicted by a shallow (less than 1.0-mm deep), elongated rectangular (5060-mm wide), smoothed area. The edges of the smoothed body area are not clearly definable, but it is patinated like the rest of the rock face. The lower end of the body clearly extends below the crotch, and although its termination is not distinct, it could be a phallic representation.

Panel 3 is on a hard sandstone layer, ca. $2 \mathrm{~m}$ above the floor in the central portion of the overhang. It is immediately below a large hollow tree cast (a common occurrence in the Triassic sandstones) that extends back into the bedrock and currently houses an owl's nest. Panel 3 consists of six human figures and two simple geometric designs (Figure 96). While the owl's nest has not impacted the rock art panel, a pebble stringer (i.e., a horizontal line of small pebble casts along a thin conglomerate lense within the finegrained sandstone) extending across the entire length of the panel obscures some of the glyphs. Three simple rectangular-bodied human figures (Glyphs 3.1-3.3) have upper bodies that have been truncated by spalling related to the pebble inclusions. A fourth human figure (Glyph 3.8) is similarly truncated but has parallel lines across its body and fringe hanging off it. A fifth rectangular-bodied human figure (Glyph 3.4) is complete and has a round head with up-turned arms that appear to be holding something. The sixth one (Glyph 3.5) is a larger rectangularbodied human figure that has been altered recently. The two geometric glyphs are a simple "+" (Glyph 3.6) that may be related to the adjacent human figure (Glyph 3.5) and an upward-pointing arrow (Glyph 3.7). All of the human figures in Panel 3 are similar in style, being rectangular-bodied with triangular legs and, when present, having square shoulders with rounded heads.

While all of the glyphs are neatly executed in bold (5-7-mm-wide and 2-mm-deep) V-shaped grooves, Glyph 3.5 has been altered by the addition of elements executed in thin, fine line $(1.0-1.5-\mathrm{mm}-$ wide and ca. 1-mm-deep) grooves that are of recent origin. It appears that the original glyph was subsequently altered by the addition of the wings (Figure 97). The only uncertainty is whether the original glyph had two arms or only one. The original arm on the left is obvious, but the arm on the right is problematic. It is possible that the figure did 


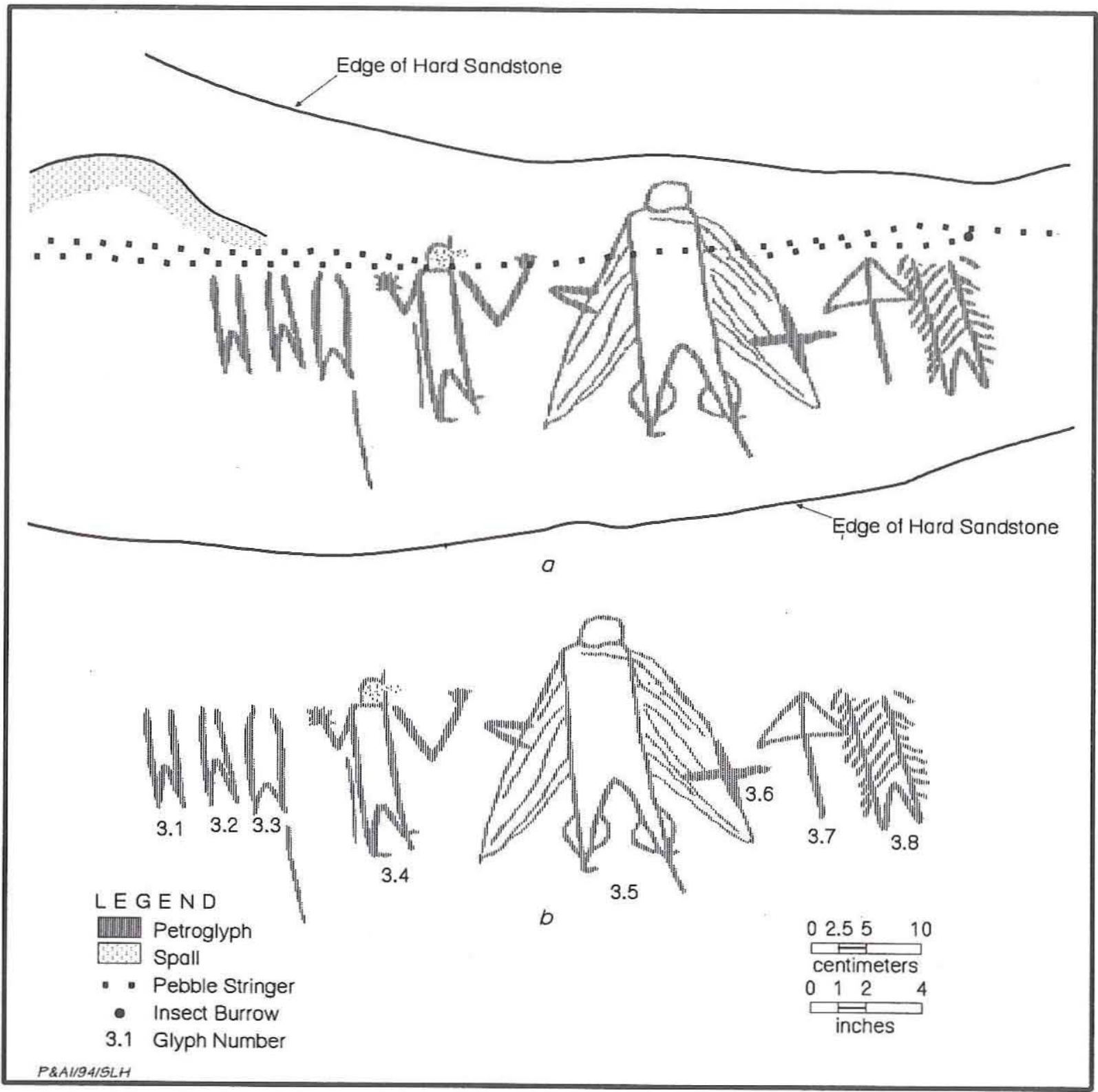

Figure 96. Line drawings of Panel 3 petroglyphs, 41KT164. (a) With natural features shown; (b) with natural features removed and individual glyphs numbered.

not have an arm on this side, but it is more likely that this arm extended from the shoulder downward to Glyph 3.6. While the arm on the left clearly has a recent incised line within the broader original groove, there are only subtle hints that a broader groove existed on the right side prior to the recent addition of the incised wing. In addition to the alterations of Glyph 3.5, two other vertical lines (one below Glyph 3.3 and one to the left of Glyph 3.4) appear to be recent additions.
Panel 4 is a single glyph of a horse (Figure 98) located on the same hard sandstone layer as the Panel 3 petroglyphs, $2 \mathrm{~m}$ above the floor in the central portion of the overhang. The horse is a fairly simple image with short triangular legs, a short tail, and ears, but no facial features or mane are depicted. The horse glyph is composed of 4-6-mm-wide, 1.01.5-mm-deep, V-shaped grooves centered within a $25-\mathrm{x}-40-\mathrm{cm}$ smoothed area on the hard sandstone face. The natural surface of this rock is rough and 

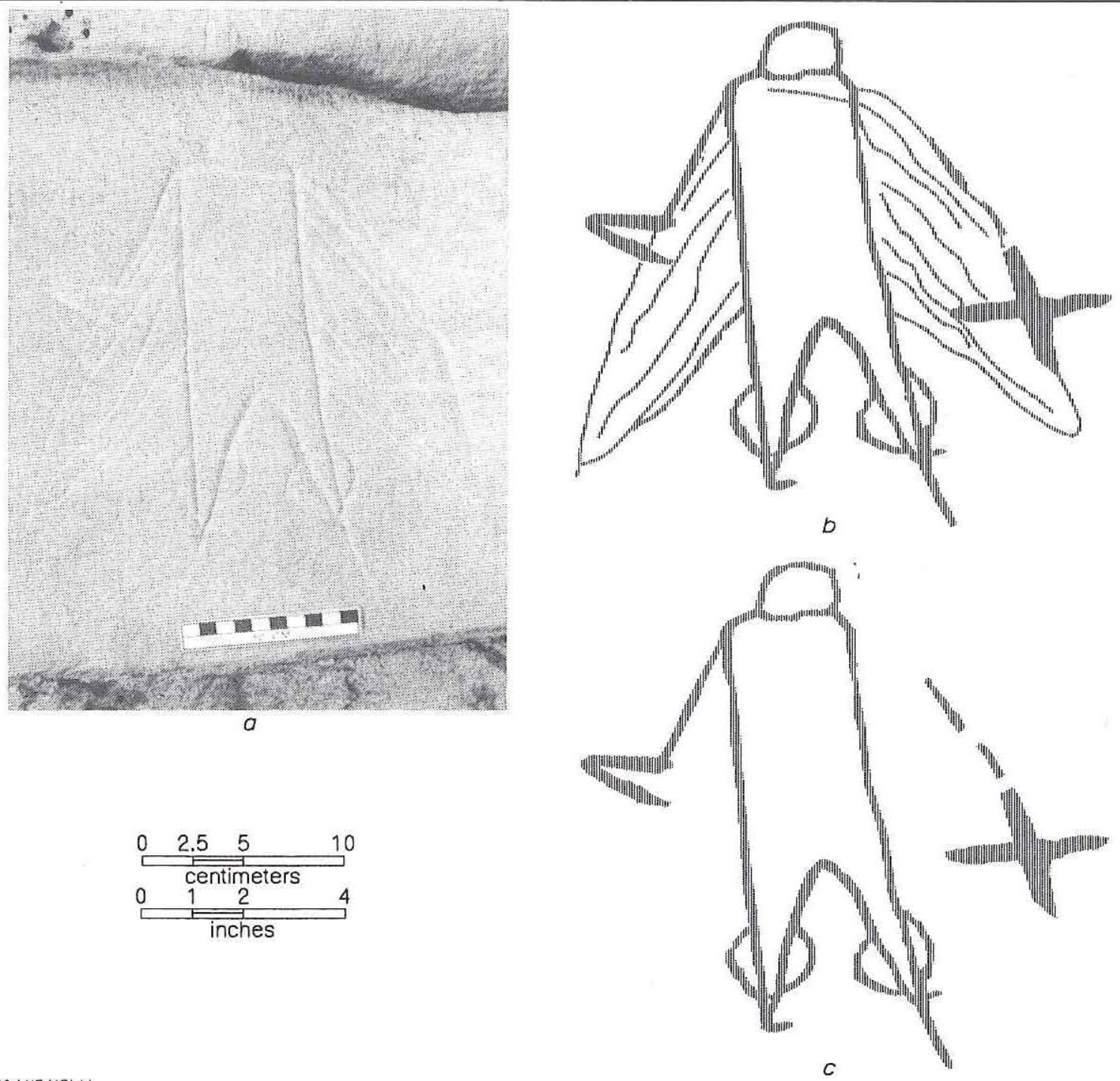

P\&A1/94/SLH

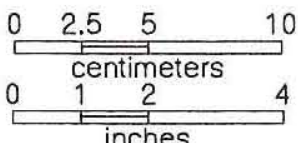

Figure 97. Close-up views of Glyphs 3.5 and 3.6, 41KT164. (a) Photograph of glyph; $(b)$ line drawing with recent modifications shown; $(c)$ line drawing with recent modifications removed.

undulating, and the smoothed area is an intentionally prepared flat surface. The horse is an obvious historic motif and suggests that most, if not all, of the petroglyphs in nearby Panel 3 (located $1 \mathrm{~m}$ to the northwest of Panel 4) are of protohistoric or historic age. The horse glyph has been partially obscured by some recent scratchings that obviously crosscut the original glyph. The crude " $\mathrm{N}$ " that overlies the rear one-quarter of the horse glyph is composed of much wider $(7-10-\mathrm{mm})$, U-shaped grooves of variable depths $(0.5-2.0 \mathrm{~mm})$ that are less patinated than the incised lines comprising the horse glyph.

Panel 5 is a group of six indistinct glyphs on the ceiling (Figure 99), $1.7 \mathrm{~m}$ above the floor in the central portion of the overhang shelter. The Panel 5 glyphs are immediately below the historic inscription "BOWEN DANIEL 1927" and include both angular and curvilinear images. They appear to be simple geometric designs, but none of the images can be identified with any degree of confidence. Because of spalling of the friable sandstone, no glyph line measurements were taken.

Panel 6 is a single glyph that apparently depicts the upper portion (torso and head only) of a human (Figure 100). It is located on a sloping surface inside a small cavity along on the back wall, 


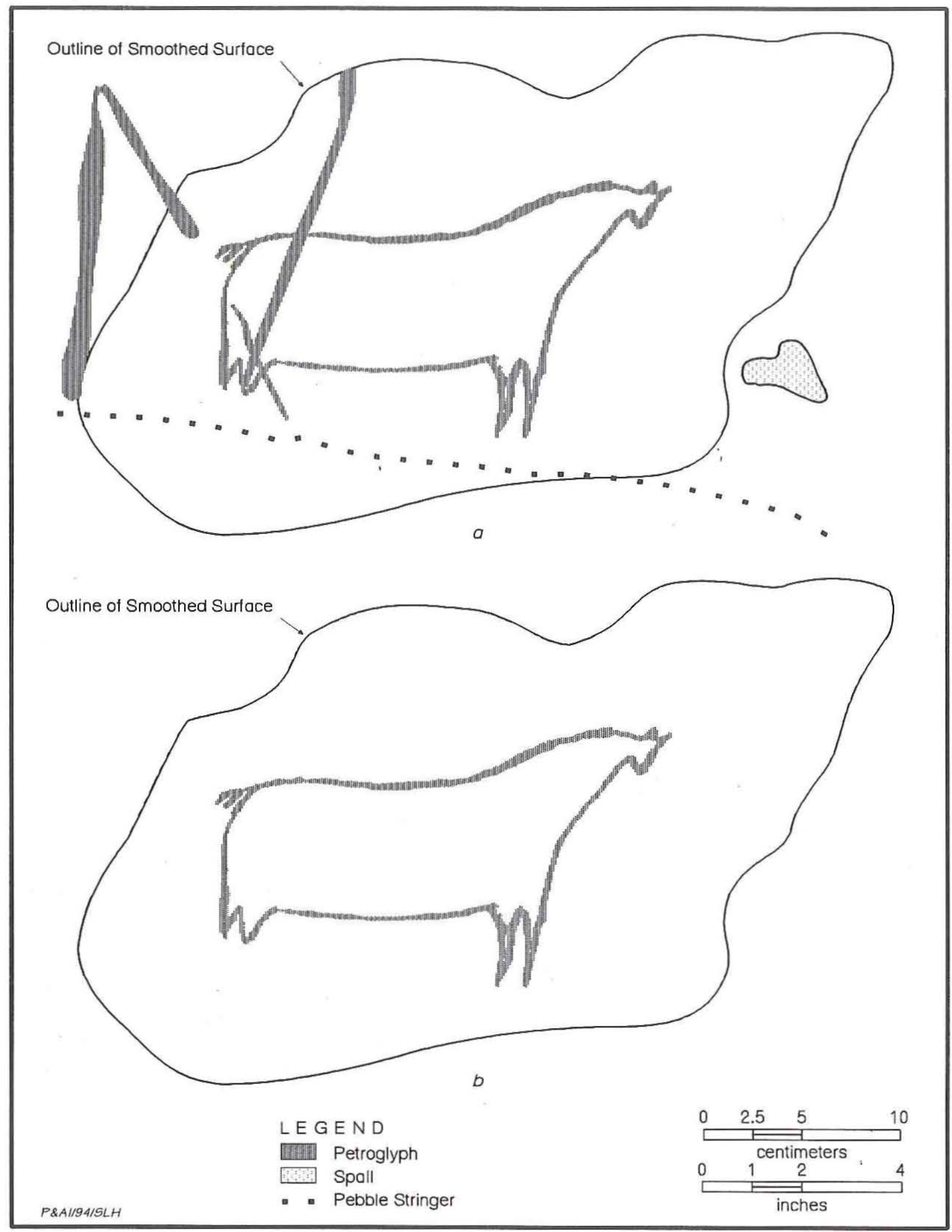

Figure 98. Line drawings of Panel 4 petroglyph, 41KT164. (a) With recent modification and natural features shown; $(b)$ with recent modification and natural features removed. 


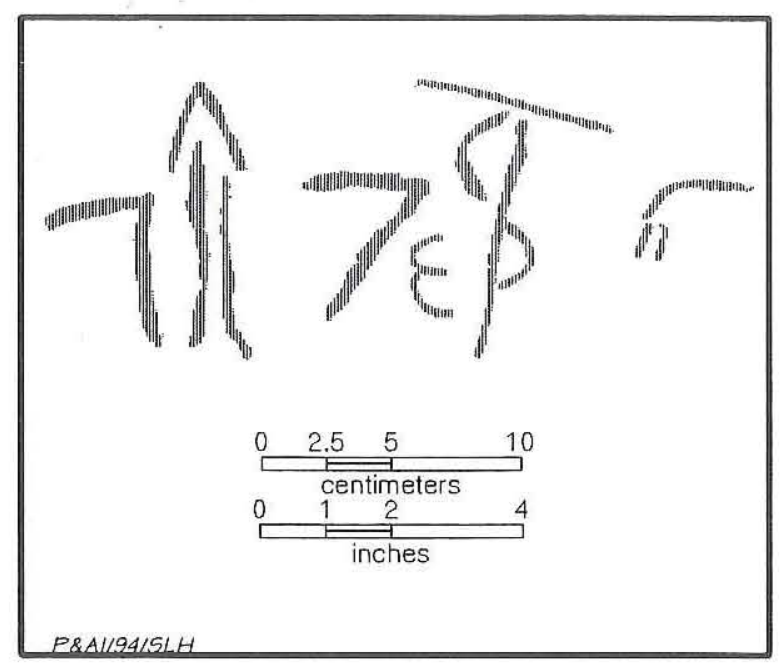

Figure 99. Line drawing of Panel 5 petroglyphs, 41KT164.

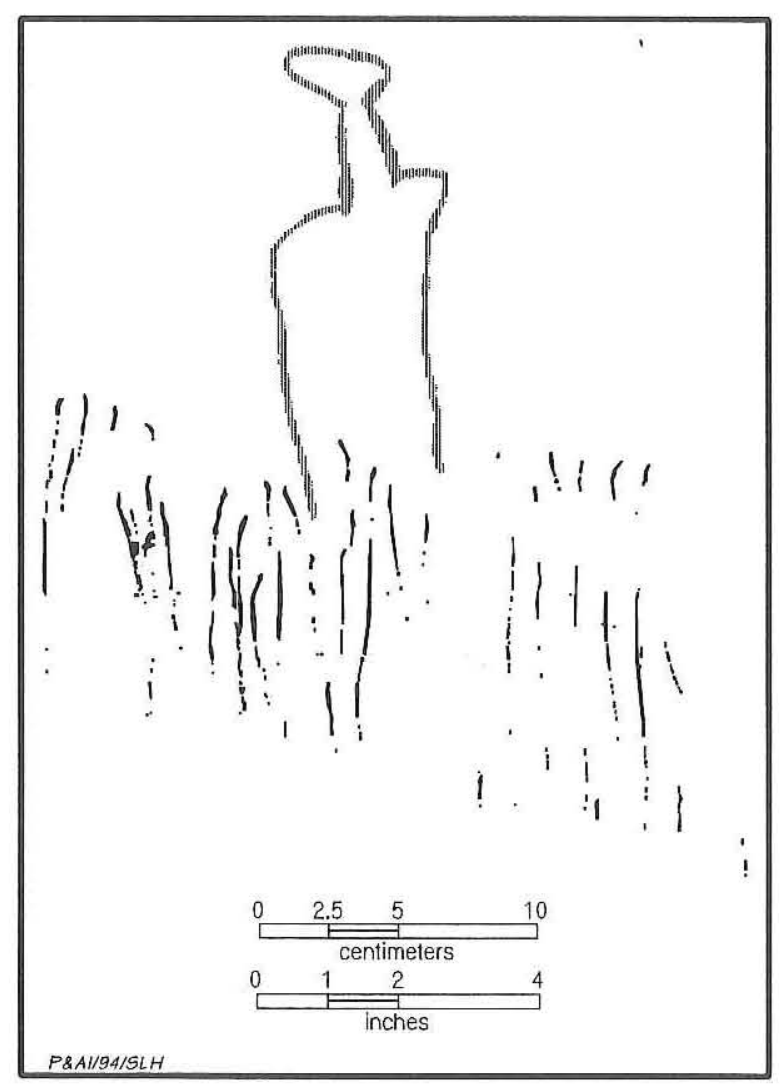

Figure 100. Line drawing of Panel 6 petroglyph, 41KT164.

$1.9 \mathrm{~m}$ above the floor in the central portion of the overhang. This simple anthropomorphic figure has a rectangular body, square shoulders with no arms, an elongated neck, and an ovate head. It is composed of relatively thin $(2-3 \mathrm{~mm})$ and shallow $(1.0-1.5 \mathrm{~mm})$ grooves that are equally patinated as the surrounding rock face. Immediately below the glyph is a series of very faint, irregular scratches, most of which are vertical. These scratches apparently were made by birds sitting inside the small cavity that contains the glyph.

\section{DISCUSSION}

There appear to be two very different styles represented in the Native American petroglyphs at this site. The petroglyphs on Panels 1 and 3-6 are rather typical of the rock art in the project area, and some or all of these glyphs may represent Plains Biographic Style imagery (Connor and Connor 1971; Keyser 1987). The Plains Biographic Style includes a wide variety of motifs produced by many different groups of protohistoric and historic Plains Indians as a means of relating personal or group biographies (e.g., personal coup recounting or depictions of significant historical events). Some variation of this rock art style is found all across the Great Plains, and it appears to be well represented in the Texas Panhandle-Plains (Boyd 1990). Unfortunately, the Plains Biographic Style in the Southern Plains is not as well documented and has not received as much attention as it has in the Northern Plains.

The Panel 1 glyph is similar to simple circular petroglyphs at 41GR315, 41GR445, and 41KT68 (Boyd 1989:Figures 31c, 32f, and 39d; Boyd and Garvey 1990:Figure 59). Some similar images found in conjunction with human figures, such as the pictograph at 41GR390 (Boyd 1989:Figure 36a; Boyd and Garvey 1990:Figure 51a), are thought to represent shield-bearing warriors. Shield-bearing warriors, including pedestrian and mounted versions, are a common motif in Plains ceremonial and protohistoric art (Gebhard 1966:721; Keyser 1987:45-47), and shields are sometimes depicted alone (Gebhard 1966:723). Unfortunately, it is impossible to determine whether the isolated Panel 1 glyph was intended to represent a shield. Likewise, since it is an isolated occurrence, the Panel 1 glyph cannot be definitely associated, or considered contemporaneous, with any of the other petroglyphs at the site, although this could well be the case.

Panels 3 and 4 are in close proximity to each other and also are similar to other petroglyphs in the project area and elsewhere in the region. The horse glyph on Panel 4 is similar to horses at a number of sites throughout the region, although the most common horse form is open-faced and riders are 
quite common (Boyd 1990:Figure 11.5). Mike Bilbo (personal communication 1993) suggests that the Panel 4 horse might be a Comanche petroglyph and that it is stylistically different from Apache horse depictions. He also suggests that it may even depict a large draft horse and hence might be datable to the late 1800 s when they were introduced to Texas. While intriguing, these interpretations are difficult to evaluate because there has been little systematic study of Southern Plains rock art and only recently have there been any serious attempts to define Comanche rock art (e.g., Pelon 1993).

The horse, one of the most common elements in the Plains Biographic Style (Keyser 1987:52-54), is the only glyph at the Callahan site that definitely denotes historic influence, but stylistic attributes of the Panel 3 glyphs suggest that they also are historic. Panel 3 includes several rectangular-bodied human figures that show many similarities to the historic petroglyphs at the Ward petroglyph site (Boyd 1989:Figure 43, 1992:Figure 5; Boyd and Garvey 1990:Figure 49) and at the Verbena site (Boyd 1992: Figure 3; Parsons 1987:Figure 11), both in Garza County. They also are similar to human figures at historic rock art sites in the Canadian River valley such as Mujares Creek, Brown's Camp, and Alibates Creek (Kirkland and Newcomb 1967:Plates 153, 155, and 158). Although no definite historic motifs are associated, human figures at another site in the project area, 41KT65 (Boyd and Garvey 1990:Figure $58 \mathrm{~d}$ and e), are very similar to those at the Callahan site, especially in the nature of the up-turned arms, squared shoulders, and triangular legs.

One unusual stylistic feature in Panel 3, the parallel body lines and hanging fringe in Glyph 3.8, may be indicative of historic period petroglyphs. Rectangular-bodies with fringe on one side of the body and chevron lines in the chest area are depicted on standing and mounted figures at Mujares Creek in Oldham County and at Harrell Ranch site A in Armstrong County (Kirkland and Newcomb 1967: Plates 153 and 157; Upshaw 1972:Plate III). These features are interpreted as representing historic Plains Indian feathered headdresses (i.e., war bonnets) and bone bead breastplates, respectively (Kirkland and Newcomb 1967:212; Upshaw 1972:59). Two standing figures in a historic petroglyph panel at Brown's Camp in Oldham County are even more similar in that they have fringe coming off both sides of their bodies (Kirkland and Newcomb 1967:Plate 155). In this case, the parallel fringe lines hanging off the figure probably represent some type of clothing. Based on these comparisons, it is suggested that the parallel body lines and hanging fringe seen in Glyph 3.8 at the Callahan site represent clothing and that the image is of protohistoric or historic age. Mike Bilbo (personal communication 1993) also suggests that Glyph 3.8 might be a "Comanche filiform element."

None of the Panel 5 images can be identified positively, but Glyph 5.2 appears similar to other simple human figures in the project area (e.g., Boyd 1989:Figure 35a). The human form in Panel 6 is consistent with the simple rectangular-bodied human petroglyphs that are common in the region. While the geometric glyphs in Panel 5 are not particularly distinctive, they are consistent with the wide range of geometric designs found throughout the project area (e.g., Boyd 1989:Figures 31-34).

The Panel 2 petroglyphs, however, are quite different from all of the others at this site and elsewhere in the area. While the other petroglyphs were executed using only narrow V- or U-shaped grooves, the Panel 2 glyphs also include deep wide grooves and broad areas of shallow smoothing. While the limbs, fingers, and toes are relatively narrow grooves, they are still generally wider than those in most of the petroglyphs throughout the region, and the main bodies and faces of these anthropomorphs are depicted by deep wide grooves or as smoothed areas. This combination of petroglyph techniques (i.e., variable ine widths and smoothing) has not been observed at any sites in Garza County nor is it described for any other sites in the region. Although rubbing or smoothing of the interior of historic petroglyphs has been observed in some Texas Panhandle-Plains sites (Boyd 1992:84), these images are outlined by incised lines, and the smoothing technique observed at the Callahan site (with no incised lines outlining the smoothed area) appears to be unique.

The Panel 2 glyphs not only are technically different but also are stylistically unusual and have a Southwestern look to them. Several rock art researchers agreed that these images probably represent some degree of Southwestern influence or might even be the work of some displaced Southwestern peoples. Polly Schaafsma (personal communication 1993) notes that this type of rectilinear stick figure is a common, perhaps diagnostic, attribute of Anasazi rock art between ca. A.D. 900 and 1300. The Panel 2 figures exhibit stylistic 
attributes which, when taken individually, may not appear particularly diagnostic but when viewed in combination are suggestive of Anasazi influence. While no systematic review of all of the pertinent Southwestern rock art literature was attempted, a few important similarities warrant mention.

One of the more distinctive traits of the Panel 2 figures is the orientation of their arms and legs (extended and bent at right angles), which is similar to that observed in animal and human representations found in rock art throughout much of New Mexico. This posture most commonly occurs with stick figure representations but also occurs with broad rectangular-bodied figures. Anasazi rock art in northwestern New Mexico (generally pre-A.D. 1300) has many similar stick figures depicting animals (lizards are most common) and humans with arms and legs bent at right angles (e.g., Schaafsma 1980: Figures 97, 98, and 99, 1992:Figures 9, 11, 14, 20, and 24-26). These types of stick figures are particularly abundant at Chaco Canyon (e.g., Steed $1980: 8,31,35,40,51,78,121)$. Although less frequent, a similar arm and leg orientation is seen in Anasazi rock art of north-central New Mexico (generally post-A.D. 1300) attributed to the Rio Grande Style (e.g., Boyd and Ferguson 1988:Figures 21b and 26d; Crotty 1990:Figure 12.11; PattersonRudolph 1993:Figures 31, 40, and 58; Schaafsma 1980:Figure 201, 1992:Figures 163 and 167). Many of these Southwestern stick figures clearly depict male and female personages, as may be the case in the Panel 2 Callahan site petroglyphs. Schaafsma (1992:13) notes that Pueblo II and III period glyphs sometimes depict female stick figure anthropomorphs along with humpbacked and/or phallic fluteplayers in fertility scenes.

Another possible Anasazi attribute is the antennaelike projections depicted on the heads of the Panel 2 figures, particularly Glyph 2.2. Antennaelike projections are present in Anasazi petroglyphs (e.g., Boyd and Ferguson 1988:Figures 21 and 22; Schaafsma 1992:Figure 20) and particularly in depictions of fluteplayers (e.g., Patterson-Rudolph 1993:Chart 2; Steed 1980:87-88).

Mike Bilbo (personal communication 1993) suggests that, while the Panel 2 glyphs look Anasazi and the up-raised arms suggest shamanic depictions, the face on Glyph 2.1 has a Jornada look to it. While there are no strong parallels, its face is highly stylized and is vaguely reminiscent of Jornada Style masks and anthropomorphs, especially the "google- eyed" figures which are highly variable but characteristically have large eyes as the dominating and sometimes exclusive facial feature (Crotty 1990:148150; Schaafsma 1980:203, 1992:67). In the Jomada Style, however, these large-eyed faces generally occur with wide rectangular-bodied figures. Stick figures that are quite similar to these also are found in the Jomada Style, although less frequently than in Anasazi rock art. Such similarities should be expected, however, since Anasazi rock art (e.g., the Rio Grande Style) may have evolved directly out of the Jornada Style (Schaafsma 1992:116). Throughout the Jornada Mogollon area, stick figures are found that exhibit the same posture (i.e., extended rightangle arms and legs) as the Panel 2 glyphs at the Callahan site (e.g., Jackson 1938:Plate CCLLII-8; Schaafsma 1980:Figure 155; Steed 1976:Drawings 44 and 115, 1978:Drawing 98).

An alternative theory, suggested by Dr. Kay Sutherland (personal communication 1993), is that these images are neither distinctively Anasazi nor Jornada Mogollon but that they could represent the work of protohistoric or historic Apaches. Sutherland feels that, along with the distinctive posture, the three- and four-fingered hands might also be a diagnostic Apachean attribute because of similarities with rock art at Hueco Tanks known to be of Mescalero Apache origin (e.g., Kirkland and Newcomb 1967:Plate 125 [site 2-C], Plate 128 [sites 6-C and 6-D], Plate 147 [site 26-A]). Since the Apaches came under a great deal of Anasazi influence, their rock art should be expected to show a strong Southwestem influence rather than a Plains influence, which seems to be the case (Kirkland and Newcomb 1967:189-190).

The possibility that some of the Callahan site petroglyphs are Apachean is intriguing because two nearby archeological sites, the Longhom and Headstream sites (Boyd et al. 1993), contain evidence of protohistoric occupations. These sites, which yielded large amounts of late Puebloan pottery (e.g., Rio Grande Glaze V and Tewa Polychrome), are located in the valley about $0.5 \mathrm{~km}$ south of, and clearly visible from, the Callahan site. The occupations were primarily during the seventeenth century, a time when various Apachean groups are known to have been in the Southern Plains and actively participating in Plains-Pueblo interactions (Boyd et al. 1993:241-284). While it is tempting to propose a connection between the Panel 2 rock art, the nearby protohistoric camps, and seventeenth-century 
Apaches, there is no concrete evidence to support such a theory. There are always inherent problems connecting rock art, archeological remains, and ethnic groups, and the ethnicity of the occupants of the nearby sites cannot be proven since all of the evidence for an Apache affiliation is circumstantial (i.e., based solely on ethnographic accounts). Further, much more research is needed to demonstrate convincingly that the Panel 2 glyphs at the Callahan site were made by Apachean peoples as opposed to some displaced Anasazi or Mogollon group or some Plains group that interacted with Anasazi or Mogollon peoples.

To further confuse matters, at least one of the stylistic conventions evident in Panel 2 is unknown, or at least very rare, in the Southern Plains and in the Southwest. The riblike appendages on one side of its body are an intriguing feature of Glyph 2.1, and they appear to represent some form of " $\mathrm{x}$-ray" image or "skeletonization" found in many parts of the world, such as in Australian Aborigine rock art (Kirkland and Newcomb 1967:31) and in the Pecos River Style pictographs in Texas (Boyd 1993; Carolyn Boyd, personal communication 1993). Mike Bilbo (personal communication 1993), noting this attribute, suggests that Glyph 2.1 is simlar to the "rib cage" appendages in Mexican Huichol art (e.g., Furst 1986:220-221). In most cultures, skeletonization is considered to be a representation of an individual, frequently a shaman, in an altered state (i.e., trance) or someone who had made the journey to the spirit world (Boyd 1993; Furst 1986:220). While intriguing, the possible cultural affiliation of symbolic significance of Glyph 2.1 is far from clear. At this point, it would be premature to invoke a shamanic trance-vision interpretation, such as that proposed for Huichol and Lower Pecos art (e.g., Boyd 1993; Furst 1986:217225; Kirkland and Newcomb 1967:79-80), as an explanation for these isolated images in the Texas Plains.

\section{CONCLUSIONS}

A strong Southwestern influence is well represented in the Late Prehistoric archeology of the Texas Panhandle-Plains. Beginning with the appearance of nonlocal brownware ceramics (and possibly other traits such as pithouse styles) before A.D. 500, some form of Jomada Mogollon influence is present in the region until at least A.D. 1200-1400 (see Chapter 3 for detailed discussion of Jornada
Mogollon influence). Following the demise of the Jornada Mogollon culture, the Southwestern influence in the Southern Plains seems to have shifted to the Anasazi as they entered into a long and intensive trade and raid relationship with various Plains groups that lasted from ca. A.D. 1200 until the nineteenth century (see Boyd et al. 1993). Imported objects found throughout the Southern Plains, such as Puebloan ceramics, shell beads from the Pacific coast, and New Mexican obsidian and turquoise, hint at the intensity of the exchange between the Plains peoples and the Southwestern cultures.

Southwestern influence in the Texas Panhandle-Plains also is present in the form of isolated rock art occurrences. Although their ages are unknown, Southwestern-style fluteplayer images (often generically called Kokopelli) include a red monochrome pictograph at the Giant Boulder site in Randall County (Upshaw 1972:76, 85-89) and a petroglyph at the O.S. Ranch or Yellowhouse Crossing Mesa site in Garza County (Riggs 1969). In one case, Puebloan Indians are known to have produced rock art at a Texas Panhandle site. While U.S. Army Lieutenant Whipple was encamped at a place called Rocky Dell (in Oldham County) during an 1853 surveying expedition, a group of Pueblo Indians visited the site and told him that their ancestors had, in Whipple's words, "recorded their thoughts and deeds upon the rocks" (Whipple et al. 1856:38). Among the pictographs that the Pueblo Indians attributed to their people is a 13-ft-long plumed serpent, "the great watersnake" of Pueblo mythology (Kirkland and Newcomb 1967:203-208; Whipple et al. 1856:38). The plumed serpent and the fluteplayer commonly are depicted in the rock art of the Southwest and often are associated with such themes as water, rainmaking, plant germination, and fertility of humans and animals (Schaafsma 1980:136, 140-141, 238, 1992:19, 64, 67, 100, 111).

Clearly, the presence of Southwestern style images in Southern Plains rock art is of considerable interest to rock art scholars, archeologists, and ethnographers studying the interaction between the Plains and Pueblo peoples. Even the general public finds the subject fascinating, as evidenced by a popular article entitled "In Search of Kokopelli" in Texas Parks and Wildlife magazine (Flores 1992). Given the paucity of recorded rock art sites in the Texas Panhandle-Plains (see Boyd 1990:133-137), the small percentage of sites that contain motifs of possible Southwestern origin or influence is of great 
importance for understanding the nature of the PlainsPueblo relationship.

The Callahan site is an unusual PanhandlePlains rock art site in that it contains both Plains and Southwestern style petroglyphs. The majority of the images appear to represent typical Plains iconography, and some correspond to the historic Plains Biographic Style, but the Panel 2 glyphs have a decidedly Southwestem look to them. They do not fit clearly into any single Southwestern rock art style, however, and specific attributes of these glyphs might be considered representative of several different styles, while other attributes do not seem to fit any particular style. The inability to relate these images to a specific style may be due in part to the complexity and interrelatedness of much of the Southwestern rock art. It also is likely that
Southwestern motifs found in peripheral Plains areas may not represent pure and unadulterated Southwestern iconography. Perhaps it is more realistic to expect that at least some of these images might be slightly, or even greatly, modified versions of Southwestern imagery. At this point, it would be purely speculative to suggest whether such images were produced by wandering or displaced Southwestern peoples or by Southwestern-influenced Plains peoples. Thus, it does not seem necessary or desirable to attempt to force these petroglyphs into a Southwestern rock art classification or attribute them to a specific ethnic group. The Callahan site is certainly a significant addition to the regional data base, and it is hoped that continued research will enable a better understanding of the Southwestern images found there. 


\section{REFERENCES CITED}

Bilbo, Michael

1986 Retum to Cowhead Mesa: The Proposed Cowhead Mesa National Register Archeological District. Transactions of the 21st Regional Archeological Symposium for Southeastern New Mexico and Western Texas 21, pp. 1-23. El Paso Archaeological Society.

Boyd, Carolyn

1993 Pictographic Evidence of the Peyote Cult in the Lower Pecos Archaic. Paper presented at "Shamanism and Rock Art: Interpretations from Around the World," a public lecture series sponsored by Trinity University and the Witte Museum, San Antonio, Texas, February 26-27, 1993.

Boyd, Douglas K.

1989 Rock Art Resources. Chapter 10 in Phase I Cultural Resources Investigations at Justiceburg Reservoir on the Double Mountain Fork of the Brazos River, Garza and Kent Counties, Texas, by Douglas K. Boyd, Martha Doty Freeman, Michael D. Blum, Elton R. Prewitt, and J. Michael Quigg, pp. 157-194. Reports of Investigations No. 66. Prewitt and Associates, Inc., Austin.

1990 Aboriginal Rock Art of Justiceburg Reservoir: A Regional Perspective for the Texas PanhandlePlains. Chapter 11 in American Indian Rock Art 16:123-145. Proceedings of the Intemational Rock Art Conference and 16th Annual Meeting of the American Rock Art Association.

1992 Historic Native American Rock Art of Garza County, Texas. In Guidebook to the Ethnohistory and Selected Protohistoric Sites of the Southern Plains, edited by Eileen Johnson, pp. 69-89. Lubbock Lake Landmark Quatemary Research Center Series, No. 3. Museum of Texas Tech University, Lubbock.

Boyd, Douglas K., and Bobbie Ferguson

1988 Tewa Rock Art in the Black Mesa Region: Cultural Resources Investigations, Velarde Community Ditch Project, Rio Arriba County, New Mexico. U.S. Department of the Interior, Bureau of Reclamation, Southwest Region, Amarillo.

Boyd, Douglas K., and Colin M. Garvey

1990 Investigations at Rock Art Sites. Chapter 7 in Phase II Investigations at Prehistoric and Rock
Art Sites, Justiceburg Reservoir, Garza and Kent Counties, Texas, by Douglas K. Boyd, James T. Abbott, William A. Bryan, Colin M. Garvey, Steve A. Tomka, and Ross C. Fields, pp. 209234. Reports of Investigations No. 71. Prewitt and Associates, Inc., Austin.

Boyd, Douglas K., Jay Peck, Steve A. Tomka, and Karl W. Kibler

1993 Data Recovery at Justiceburg Reservoir (Lake Alan Henry), Garza and Kent Counties, Texas: Phase III, Season 2. Reports of Investigations No. 88. Prewitt and Associates, Inc., Austin.

Connor, Stuart W., and Betty Lu Connor

1971 Rock Art of the Montana High Plains. The Art Galleries, University of California, Santa Barbara.

Crotty, Helen K.

1990 Formal Qualities of the Jornada Style and Pueblo IV Anasazi Rock Art: A Comparison with Implications for the Origins of Pueblo Ceremonialism. Chapter 12 in American Indian Rock Art 16:147-166. Proceedings of the International Rock Art Conference and 16th Annual Meeting of the American Rock Art Association.

Flores, Dan

1992 In Search of Kokopelli: Vanishing Rock Art of the Texas High Plains. Texas Parks and Wildlife 50(1):28-35.

Furst, Peter T.

1986 Shamanism, the Ecstatic Experience, and Lower Pecos Art: Reflections on Some Transcultural Phenomena. Chapter 7 in Ancient Texans: Rock Art and Lifeways Along the Lower Pecos edited by Harry J. Shafer, pp. 210-225. Texas Monthly Press, Austin.

Gebhard, David

1966 The Shield Motif in Plains Rock Art. American Antiquity 31(5):721-732.

Gustavson, Thomas C., and William W. Simpkins

1989 Geomorphic Processes and Rates of Retreat Affecting the Caprock Escarpment, Texas Panhandle. Reports of Investigations No. 180. Bureau of Economic Geology, The University of Texas at Austin.

Jackson, A. T

1938 Picture-writing of Texas Indians. Bureau of Research in the Social Studies, Study No. 37. 
University of Texas Publication No. 3809.

Keyser, James D.

1987 Lexicon for Historic Plains Indian Rock Art: Increasing the Interpretive Potential. Plains Anthropologist 32(115):43-71.

Kirkland, Forrest, and W. W. Newcomb, Jr.

1967 The Rock Art of Texas Indians. University of Texas Press, Austin.

McGowen, J. H., G. E. Granata, and S. J. Seni

1979 Depositional Framework of the Lower Dockum Group (Triassic), Texas Panhandle. Reports of Investigations No. 97. Bureau of Economic Geology, The University of Texas at Austin.

Parsons, Mark L.

1987 Plains Indian Portable Art as a Key to Two Texas Rock Art Sites. Plains Anthropologist 32(117): 257-274.

Patterson-Rudolph, Carol

1993 Petroglyphs and Pueblo Myths of the Rio Grande. Avanyu Publishing, Inc., Albuquerque.

Pelon, Linda Nash

1993 An Attempt to Use Rock Art as an Independent Source in Ethnohistorical Research. Chapter 10 in Issues in Penatuhkah Comanche Ethnohistory, pp. 80-90. Unpublished Master's thesis in Anthropology, The University of Texas at Arlington.

Riggs, Aaron D., Jr.

1969 Yellowhouse Crossing Mesa Petroglyphs. Transactions of the Fifth Regional Archeological Symposium for Southeastern New Mexico and Western Texas 5, pp. 25-33. Llano Archeological Society, Portales, New Mexico.

Schaafsma, Polly

1980 Indian Rock Art of the Southwest. School of American Research, Southwest Indian Art Series. University of New Mexico Press, Albuquerque.
1992 Rock Art in New Mexico. University of New Mexico Press, Albuquerque.

Silver, Constance S.

1985 The Rock Art of Seminole Canyon State Historical Park: Deterioration and Prospects for Conservation. Publication No. PWD-BK4503-082C-2/85. Texas Parks and Wildlife Department, Austin, Texas.

Steed, Paul P., Jr.

1976 Rock Art on Alamo Mountain, Otero County, N.M. The Artifact, Vol. 14, No. 4. El Paso Archaeological Society.

1978 Rock Art on Alamo Mountain, Otero County, N.M.: Part II. The Artifact, Vol. 17, No. 3. El Paso Archaeological Society.

1980 Rock Art In Chaco Canyon. The Artifact, Vol 18, No. 3. E1 Paso Archaeological Society.

Sutherland, Kay

1978 Petroglyphs at Three Rivers, New Mexico: A Partial Survey. The Artifact, Vol. 16, No. 2. El Paso Archaeological Society.

Turpin, Solveig A.

1982 Seminole Canyon: The Art and the Archeology. Research Report No. 83. Texas Archeological Survey, The University of Texas at Austin.

Upshaw, Emily

1972 Palo Duro Rock Art: Indian Petroglyphs and Pictographs. Unpublished Master's thesis, West Texas State University, Canyon.

Whipple, A. W., Thomas Eubank, and William W. Tumer

1856 Report Upon the Indian Tribes. In Reports of Explorations and Surveys to Ascertain the Most Practicable and Economical Route for a Railroad from the Mississippi River to the Pacific Ocean. 33rd Congress, 2nd Session, Senate Executive Document No. 78. Government Printing Office, Washington, D.C. 

